UNIVERSITAT
POLITÉCNICA
DE VALËNCIA P.

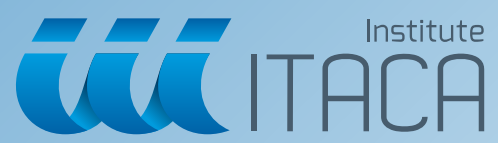

Information and Communication Technologies

\title{
AMPERE 2019
}

17th International

Conference on Microwave and High Frequency Heating

Valencia, Spain

September 9-12, 2019

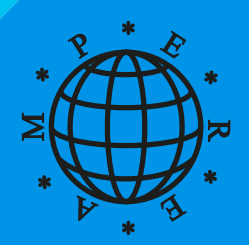




\section{Congress UPV}

\section{7th International Conference on Microwave and High Frequency Heating}

The contents of this publication have been evaluated by the Scientific Committee which it relates and the procedure set out

http://ocs.editorial.upv.es/index.php/AMPERE2019/AMPERE2019/about/editorialPolicies

\section{(C) Scientific Editor \\ Beatriz García Baños}

\section{(c) Publisher}

Editorial Universitat Politècnica de València, 2019

www.lalibreria.upv.es / Ref.:6475_01_01_01

ISBN: 978-84-9048-719-8 (print version)

Print on-demand

DOl: http://dx.doi.org/10.4995/AMPERE2019.2019.10535

\section{(C) $(1) \Theta(\Theta$}




\section{AMPERE 2019 \\ 17th International Conference on Microwave and High Frequency Heating}
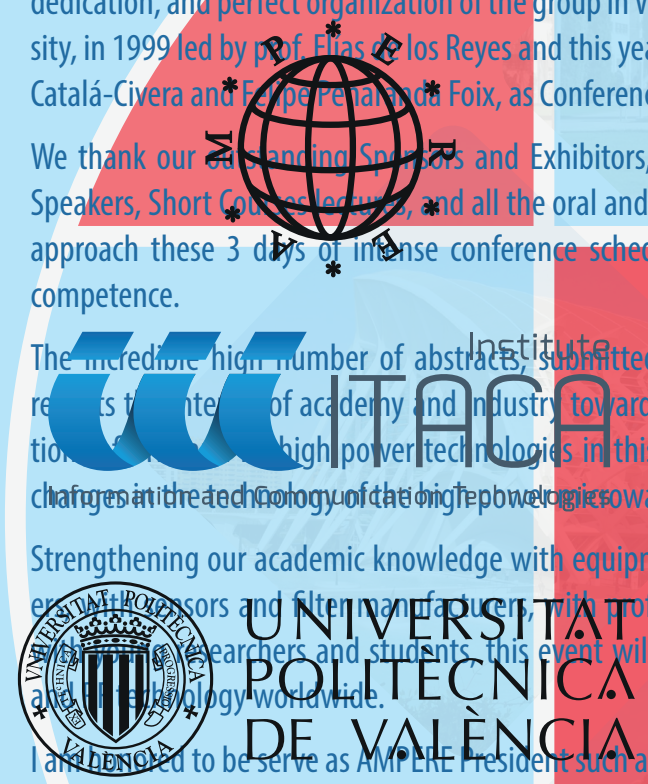

Editor:

Beatriz García Baños

ITACA Research Institute

Universitat Politècnica de València

Valencia, September 9-12, 2019 


\section{7th International Conference on Microwave}

and High Frequency Heating

\section{Welcome}

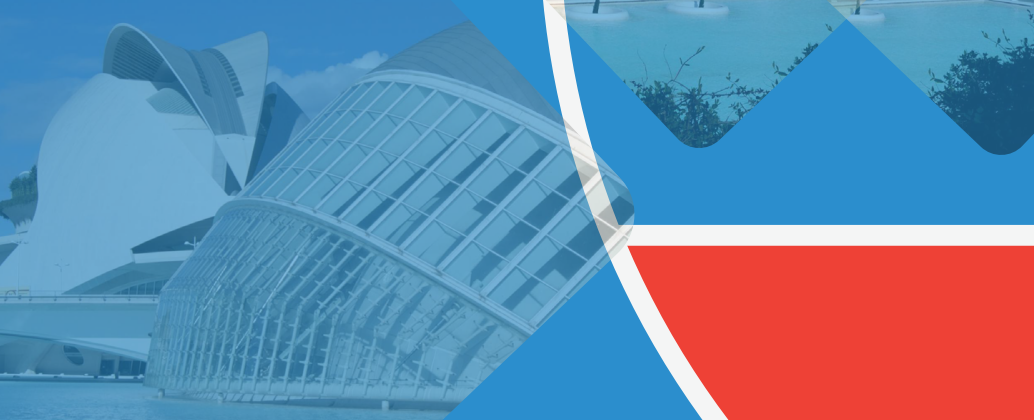




\section{WELCOME LETTER FROM THE AMPERE PRESIDENT}

Dear AMPERE Association Members;

Dear Conference Attendees;

Dear Conference Organizers;

I am delighted to share this important event with all of You!

Those of You who are longtime members might have already participate to the previous editions of the conference series: "International Conference on Microwave and High Frequency Heating" organized by AMPERE EUROPE members every two years.

This is the second time we benefit of extraordinary hospitality, unwavering dedication, and perfect organization of the group in Valencia Polytechnic University, in 1999 led by prof. Elías de los Reyes and this year led by proff. José Manuel Catalá-Civera and Felipe Peñaranda Foix, as Conference Chairs.

We thank our outstanding Sponsors and Exhibitors, our Plenary and Keynote Speakers, Short Courses lectures, and all the oral and poster presenters that will approach these 3 days of intense conference schedule with enthusiasm and competence.

The incredible high number of abstracts, submitted from all over the globe, reflects the interest of academy and industry towards the most recent applications of MW and RF high power technologies in this peculiar time of ongoing changes in the technology of the high power microwave generators.

Strengthening our academic knowledge with equipment and software producers, with sensors and filter manufacturers, with professionals and consultants, with young researchers and students, this event will contribute to diffuse MW and RF technology worldwide.

I am honored to be serve as AMPERE President such a vivid community.

Warm welcome to all of you!

Cristina Leonelli, AMPERE President 


\section{WELCOME LETTER FROM THE AMPERE CHAIRS}
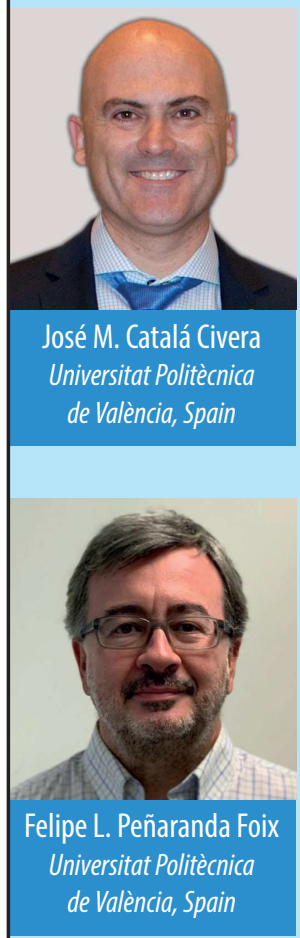

Dear Colleagues,

On behalf of the AMPERE2019 Steering Committee, we are honored and delighted to welcome you to the 17th International Conference on Microwave and High Frequency Heating at Valencia, Spain.

The conference will be held on September, 9-12, 2019 at the Polytechnic City of Innovation (CPI) of the Universitat Politècnica de València. This Science Park is conceived as a space to connect university, business and society in order to streamline the generation of knowledge-intensive activities. Moreover, the city of Valencia has many attractions making it one great location for congresses, tourists and trade in Europe.

Over the years, the AMPERE conference has evolved to become surely one of the most important international events dedicated to microwave and radiofrequency power systems, technology and applications. As in previous years, AMPERE2019 provides an excellent forum for researchers and engineers from academia and industry to exchange innovative ideas, networking, discuss collaborations and to meet with international experts in a wide variety of specialties at both scientific and industrial scale.

The conference this year has brought together a tremendous and rich diversity of authors and speakers from universities, government and industry. Our technical program will feature more than 190 technical presentations including oral, posters, plenary and keynote talks, industrial exhibits and student competitions. Besides, the conference offers 2 short courses designed to increase the knowledge in the microwave science while offering the experience of highly regarded experts.

As conference chairs of AMPERE2019, we know that the success of the AMPERE conference depends ultimately on the many people who have worked with us in planning and organizing both the technical and social programs. In particular, we would like to thank the Scientific Committee of the conference for their thorough and timely reviewing of the papers, their wise advice and brilliant suggestion on organizing the technical program and to our exhibitors and sponsors who have helped us to keep down the costs of AMPERE2019 for all participants. Special recognition should go to the Local Organizing Committee members who have all worked extremely hard for the details of important aspects of the conference programs and social activities to create an event that we hope will be memorable for you.

We are looking forward to meeting you in Valencia during AMPERE2019 and to sharing a most pleasant, interesting and fruitful conference.

José M. Catalá Civera and Felipe L. Peñaranda Foix. 


\section{(IIUECGE DGIrOC}
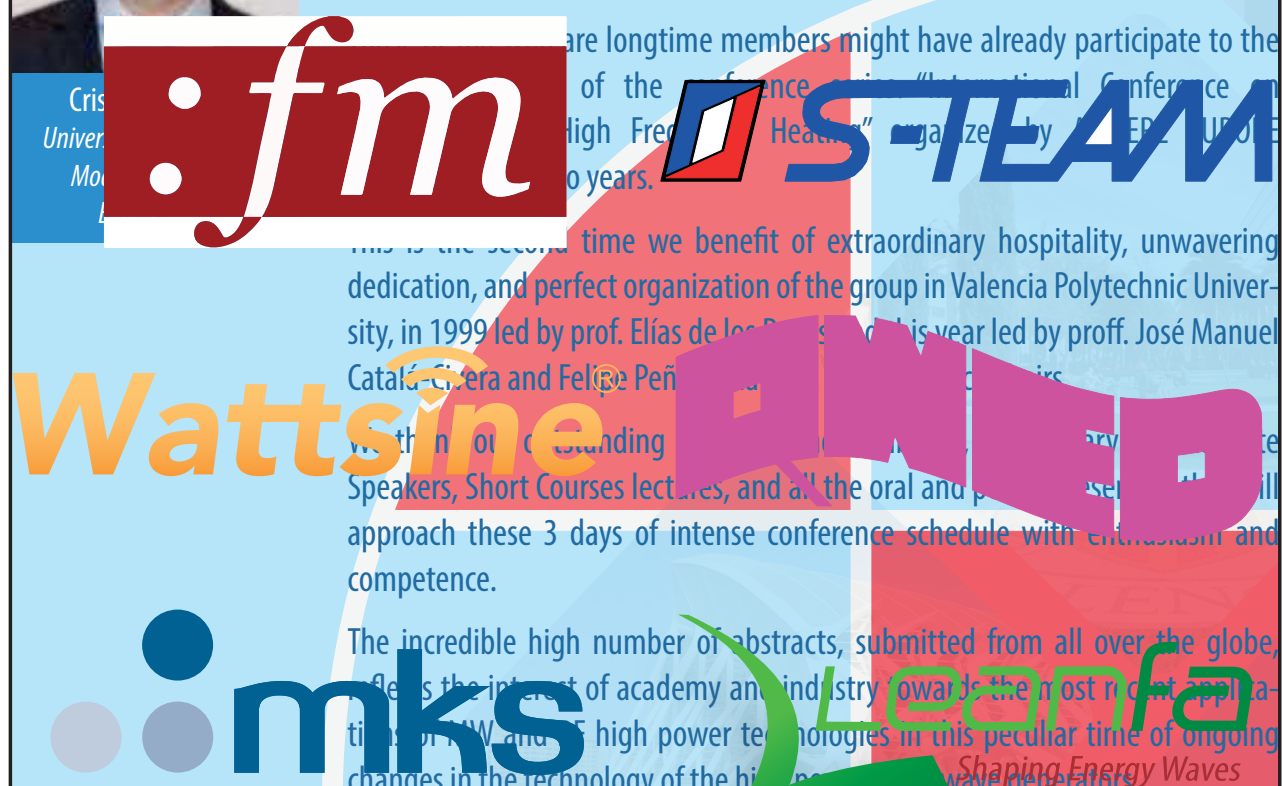
Alter ${ }^{\oplus}$ Products

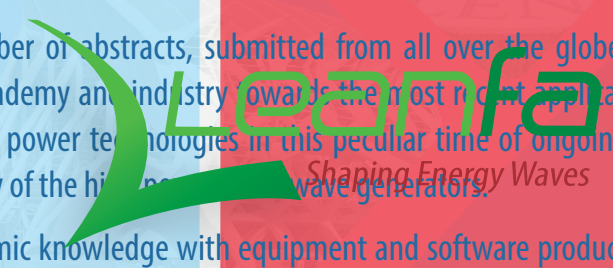

$\mathrm{HBH}$ 


\section{7th International Conference on Microwave}

and High Frequency Heating

\section{Committees}
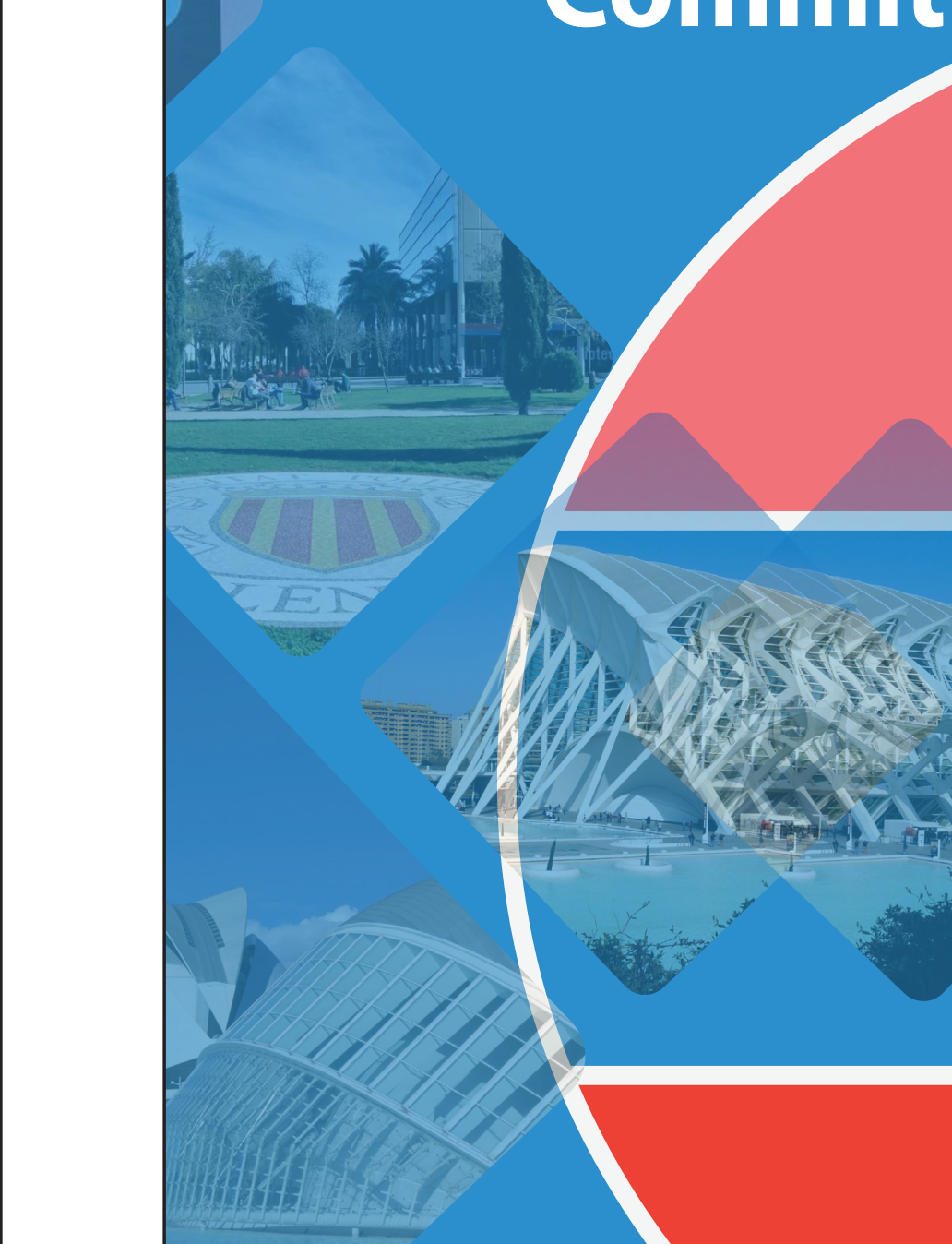


\section{CONFERENCE CHAIRS}

José M. Catalá-Civera, ITACA Research Institute - Universitat Politècnica de València

Felipe L. Peñaranda-Foix, ITACA Research Institute - Universitat Politècnica de València

\section{STEERING COMMITTEE}

A.C. Metaxas, AC Metaxas and Associates, UK

Andrzej Stankiewicz, TU Delft, The Netherlands

Antoni J. Canós-Marín, UPV, Spain

Beatriz García Baños, UPV, Spain

Birgitta Raaholt, RISE, Sweden

Cristina Leonelli, UNIMORE, Italy

Darek Bogdal, Cracow University of Technology, Poland

Eli Jerby, Tel Aviv University, Israel

Elías de los Reyes Davó, UPV, Spain

Georgios Dimitrakis, The University of Nottingham, UK

Georgios Stefanidis, KU Leuven, Belgium

Guido Link, KIT, Germany

Isidora Navarro Milla, UPV, Spain
Jean Paul Bernard, SAIREM, France

José Daniel Gutiérrez Cano, UPV, Spain

Juan R. Sánchez Marín, UPV, Spain

Junwu Tao, University of Toulouse, France

Kama Huang, Sichuan University, China

Koen Van Reusel, KU Leuven, Belgium

Marco Garuti, MKS Instruments, Italy

Marilena Radoiu, Microwave Technologies

Consulting, France

Paolo Veronesi, UNIMORE, Italy

Pedro Plaza González, UPV, Spain

Vaidhy Vaidhyanathan, Loughborough University, UK

\section{SCIENTIFIC COMMITTEE}

\section{A.C. Metaxas, AC Metaxas and Associates, UK}

Antoni J. Canós-Marín, UPV, Spain

Beatriz García Baños, UPV, Spain

Cristina Leonelli, UNIMORE, Italy

Daniel R. Slocombe, Cardiff University, UK

Darek Bogdal, Cracow University of Technology, Poland

Eli Jerby, Tel Aviv University, Israel

Erik Esveld, Wageningen University \& Research, The

Netherlands

Felipe L. Peñaranda-Foix, UPV, Spain

Gary Bond, University of Central Lancashire, UK

Georgios Dimitrakis, The University of Nottingham, UK

Georgios Stefanidis, KU Leuven, Belgium

Guido Link, KIT, Germany

Guido Sturm, TU Delft, Germany

Ioan Calinescu, Polytechnic University of Bucharest, Romania
Isabelle Polaert, INSA de Rouen, France

Jean Paul Bernard, SAIREM, France

José Daniel Gutiérrez Cano, UPV, Spain

José M. Catalá-Civera, UPV, Spain

Juan Monzó-Cabrera, UPCT, Spain

Junwu Tao, University of Toulouse, France

Kama Huang, Sichuan University, China

Koen Van Reusel, KU Leuven, Belgium

Luigi Vaccaro, University of Perugia and Green

Chemistry Group of Società Chimica Italiana, Italy

Marco Garuti, MKS Instruments, Italy

Marilena Radoiu, Microwave Technologies

Consulting, France

Paolo Veronesi, UNIMORE, Italy

Reyes Mallada, UNIZAR, Spain

Yoshio Nikawa, Kokushikan University, Japan

Vaidhy Vaidhyanathan, Loughborough University, UK 


\section{MICROWAVE MATTER INTERACTION}

Experimental investigations of microwave effects on rock breakage system via SEM analysis

K. Teimoori, F. Hassani, A.P. Sasmito, S.A. Ghoreishi-Madiseh

Quantum chemistry molecular modeling for radio-frequency and microwave- frequency thermo-upconversion heating of metal oxides of $\mathrm{NiO}$ and $\mathrm{Fe}_{2} \mathrm{O}_{3}$

S. Yanagida, T. Matsumura

Elucidation of electromagnetic wave effect and outgoing of future trend in microwave chemistry and biology

S. Horikoshi

\section{PLASMA PROCESSING INDUCED BY MICROWAVES}

Generation and modelling of gaseous plasmas using microwave power

A high-power source of optical radiation with microwave excitation

G. Churyumov, A. Denisov, T. Frolova, N. Wang, J. Qiu

Synergetic effect of microwave plasma and catalysts in $\mathrm{CO} 2$ hydrogenation into high added-value molecules

B. Alrafei, J. Delgado, A. Ledoux, I. Polaert

In-liquid plasma using microwave power for applications

S. Sawada and S. Horikoshi

Numerical simulation of the activation process of supersonic gas flows by a microwave discharge

M.S. Bobrov, M.U. Hrebtov, A.K. Rebrov

Microwave influenced laminar premixed hydrocarbon flames: spectroscopic investigations

S. Eckart, R. Behrend, H. Krause 


\section{MICROWAVE HYBRID HEATING PROCESSES}

Microwave treatment of materials in low pressure

R. Parosa, A. Brożyński, P. Grześkowiak, K. Kowalczyk, M. Natoński, P. Ziętek, J.

Żytkiewicz.

Microwave and induction heating on fibre-reinforced cementitious materials for the demolition of structures

R. Borinaga-Treviño, A. Orbe, J. Cuadrado, I.Crespo, J. Norambuena-Contreras

Joining of $\mathrm{Cu}$ to SS304 by microwave hybrid heating with $\mathrm{Ni}$ an interlayer

S. Tamang, S. Aravindan

Microwave and ultrasounds together - A challenge

M. Vinatoru, I. Calinescu

Analysis of high-frequency c-core magnetic flux leakages for bone tumor with induction heating by using multi-coil

M. Jokpudsa, S. Kotchapradit, C. Thongsopa, T. Thosdeekoraphat

\section{MICROWAVE INDUSTRIAL EQUIPMENT AND SCALE-UP}

Subcritical hydrothermal conversion process supported by microwaves R. Parosa

Experimental study of microwave slow wave comb and ceramic applicators for soil treatment at frequency $2.45 \mathrm{GHz}$

G. Brodie and G. Torgovnikov

Microwave wood chip treatment use in chemical pulp manufacturing (technical-economic assesment)

A. Leshchinskaya

Microwave generated plasma railway track treatment

J. Swan, M. Candy, M. Radoiu, G. Richardson

Solid-state microwave processor for food treatment

M.Fiore, N. Di Modugno, F. Pellegrini, M. Roselli

Hybrid microwave with heat recovery for an efficient drying process 
Homogeneous dielectric heating in large microwave ovens by excitation of multiple eigenmodes at their resonance frequencies

D. Neumaier, S. Sanseverino, G. Link, J. Jelonnek

Developing microwave chemistry under process engineering principles

E.Tioni, J. Breysse, P.Rousseaux

\section{MICROWAVE PROCESSING IN ORGANIC CHEMISTRY}

Microwave pretreatment of lignocellulosic biomass to release maximum phenolic acids

A. Bichot, M. Radoiu, N. Bernet, V. Mechin, J-P. Delgenès, D. García-Bernet

Continuous industrial-scale microwave-assisted extraction of high-value ingredients from natural biomass

M. Radoiu, S. Splinter, T. Popek

Microwave pyrolysis of biomass in a rotatory kiln reactor: deep characterization and comparative analysis of pyrolytic liquids products

L. Ubiera, I. Polaert, L. Abdelouahed, B. Taouk

Growth of nannochloris algae in the presence of microwaves (continuous reactor)

I. Calinescu, A. Vintila, A. Diacon, M. Vinatoru, P. Chipurici, Ana-Maria Galan, Sanda Velea

Scalable microwave waste-to-fuel conversion

Optimization of microwave assisted delignification of wood residues by surface response methodology

A. Trifan, I. Calinescu, M. Vinatoru, A. I. Gavrila

\section{MICROWAVE APPLICATIONS IN FOOD PROCESSING}

Effectiveness of a microwave fluidised bed dryer in eradication of seedborne botrytis grey mold of lentils

S. Taheri, G. Brodie, D. Gupta 
Microwave drying of corn seeds: effect of temperature on drying time, energy consumption and germination rate

Á. H. Moreno, R. Hernández-Maqueda, I. Ballesteros, C. Torres-Miño

Generalisation and evaluation of macroscopic models for microwave susceptors in contact with heated foods

M. Celuch, K. Wilczynski, M. Olszewska-Placha

Enhanced microwave assisted processing of 2D cylindrical porous food dielectric

S. Kumari, S. K. Samanta, K. Patel

Microwave processing of food samples: influence of cavity design and dielectric properties

C. D. Albuquerque, S. Curet, L. Boillereaux

\section{EM MODELLING AND NUMERICAL TECHNIQUES}

Numerical investigations on the effects of single-mode microwave treatment on rock breakage system

K. Teimoori, F. Hassani, A.P. Sasmito, S.A. Ghoreishi-Madiseh

Theoretical analysis on efficient microwave warming of human blood

Mathematical modeling of microwave heating of products with central symmetry

V. A. Karelin, VI. V. Salomatov

Plane wave irradiation of a layered system: resonance-based control over thermal runaway

A.A. Mohekar, B.S. Tilley, V.V. Yakovlev

A new concept to improve microwave heating uniformity through datadriven process modelling

Jing Zhou, Yingguang Li, Di Li

\section{EXPERIMENTAL METHODS}

Millimeter wave absorption in hydroxyapatite and 3 YSZ ceramics in wide temperature range 
Microwave rapid heating system using carbon heating tube

T. Sameshima, T. Kikuchi, T. Uehara, T. Arima, M. Hasumi, T. Miyazaki, G. Kobayashi, and I. Serizawa

Low-cost measurement system and filter for reduction of EMC interferences in radio-frequency applications

R. Sallier, L. Nietner, U. Roland, U. Trommler, M. Kraus, F. Holzer, C. Hoyer, and D.

Schlayer

Do a science experiment for future scientists

Y. Kanematsu, T. Matsumura

Microwave annealing of powder metals without sintering

R. Bureš, M. Fáberová, M. Dilyova

Optimizing asphalt mixtures to be heated by microwaves

J. Gallego, F. Gulisano, L. Picado, J. Crucho

\section{TECHNIQUES FOR MONITORING MICROWAVE PROCESSES}

H2-reduction behavior of FeS-CaO mixture during microwave heating

A. Amini, K. Ohno, T. Maeda, K. Kunitomo, K. Kashimura

Dielectric monitoring of the pan fiber stabilization process

J. Hofele, M. Jung, G. Link and J. Jelonnek

In-situ spectroscopy and two-color thermography during microwave irradiation in materials processing

J. Fukushima, H. Takizawa.

\section{MICROWAVE CHEMISTRY-SYNTHESIS}

Faster plasticizers production by microwave irradiation

Rapid synthesis of highly luminescent carbon quantum dots using lowpressurized microwave solvothermal heating

K. Hagiwara, S. Horikoshi

Microwave-assisted synthesis and spectroscopic properties of novel pyridine-based fluorescent molecular probes

P. Fiedor, J. Ortyl, M. Galek 
Tungsten carbide nanopowder synthesis under the exposure of $24 \mathbf{~ G H z}$ gyrotron radiation on the nanocomposite of the $\mathrm{W}-\mathrm{C}$ system obtained in a thermal plasma

A. Vodopyanov, A. Samokhin, N. Alekseev, M. Sinayskiy, A. Sorokin, S. Sintsov

High efficiency microwave flow chemistry towards synthesis of functional materials and pharmaceutical cores

J. P. Barham, E. Koyama, J. Sugiyama, Y. Norikane, H. Egami, Y. Hamashima

Microwave-assisted preparation of 1D NCs for photodegradation process of organic dyes

A. Żaba, K. Matras-Postolek, S. Sovinska, D. Bogdal

\section{MICROWAVE CHEMISTRY-SINTERING}

Low temperature degradation behaviour of $10 \mathrm{Ce}-\mathrm{TZP} / \mathrm{Al}_{2} \mathrm{O}_{3}$ bioceramics obtained by microwave sintering technology

L. Gil, M. D. Salvador, F. L. Penaranda-Foix, R. Rosa, P. Veronesi, C. Leonelli, A. Borrell

Comparison in mechanical properties of zirconium titanate (ZrTiO4) synthetized by alternative routes and sintered by microwave (MW)

R. Guillén, A. Borrell, M. Salvador, F. L. Penaranda-Foix, R. Moreno

Features of microwave fired utilitarian stoneware

T. Santos, N. F. Santos, C. S. F. Gomes, L. Hennetier, V. A. F. Costa, L. C. Costa

Fe/MgO powder composite sintered by microwave heating

R. Bureš, M. Fáberová, Z. Birčáková, V. Koval, P. Kollár, J. Füzer, M. Jakubčin, P.Slovenský

Sintering of MLCC's barium titanate with microwaves J.A. Aguilar-Garib, O. Tijerina-García, J. Garza-Guajardo

\section{MICROWAVE CHEMISTRY-CATALYTIC REACTIONS}

Selective formation of linear alpha-olefins via microwave catalytic cracking of straight-chain alkanes 
A comparative study of microwave and barrier discharge plasma for the regeneration of spent zeolite catalysts

A. Halman, G. Bond, H. Eccles, R. Mao, S. Pollington, P. Hinde, V. Demidyuk, A. Gkelios

\section{DESIGN OF MICROWAVE APPLICATORS AND CIRCUITS}

Automatic measurement of magnetron Rieke diagrams

V. Bilik

Development of coaxial type flow microwave reactor

T. Matsumura, M. Kishihara, H.Urushihara

\section{DIELECTRIC MATERIAL PROPERTIES MEASUREMENTS}

On the possibilities of permittivity calculation in a certain bandwidth from single frequency results.

A. J. Lozano Guerrero, J. Monzó-Cabrera, A. Díaz-Morcillo

Application of dielectric resonators to surface impedance measurements of microwave susceptors

M. Celuch, J. Rudnicki, J. Krupka, W. Gwarek

Measurement of the dielectric properties of liquid crystal material for microwave applications

J.R. Sánchez, V. Nova, C. Bachiller, B. Villacampa, A. de la Rua, R. Kronberger, F. Peñaranda, and V. E. Boria 


\title{
EXPERIMENTAL INVESTIGATIONS OF MICROWAVE EFFECTS ON ROCK BREACKAGE USING SEM ANALYSIS
}

\author{
K. Teimoori ${ }^{1}$, F. Hassani ${ }^{1}$, A.P. Sasmito ${ }^{1}$, and S.A. Ghoreishi-Madiseh ${ }^{2}$, \\ ${ }^{I}$ Department of Mining and Materials Engineering, McGill University, 3450 University, \\ Frank Dawson Adams Bldg., Montreal, QC H3A OE8, Canada \\ ${ }^{2}$ Norman B Keevil Institute of Mining Engineering, University of British Colombia, \\ Vancouver, BC, V6T 1Z4, Canada \\ khashayar.teimoori@mail.mcgill.ca \\ ferri.hassani@mcgill.ca \\ agus.sasmito@mcgill.ca \\ ali.madiseh@ubc.ca
}

\begin{abstract}
Preconditioning of hard rocks by microwave energy has recently been considered a potentially effective technology in mechanical rock breakage for civil and mining engineering. To obtain the amount of mechanical damage that a single-mode microwave treatment produces in rocks, it is necessary to analyze and evaluate the thermal cracking process by microwave heating at different power levels, exposure times, and distances from the antenna. The current study employs the scanning electron microscopy imaging technique to capture images from surfaces of irradiated rock specimens and to compare them with a nontreated specimen. To evaluate and quantify the amount of cracking (i.e. crack density, crack size, etc.) in a rock specimen after microwave irradiation with different microwave input operating parameters, the following steps were evaluated. First, several experiments of single-mode microwave treatments with different operating parameters were performed on rectangular specimens of basalt. Then, cylindrical core samples with a dimension of $\mathrm{r}=0.5 \mathrm{~cm}, \mathrm{~h}=2 \mathrm{~cm}$, were drilled from the center of the irradiated specimens and prepared for image processing. ${ }^{1}$ The results of the present study show that there are significant differences between the number of microcracks present in samples irradiated at different power levels and distances from the antenna. Also, longer exposure times result in more severe cracks.
\end{abstract}

Keywords: microwave-assisted rock breakage, rock preconditioning, scanning electron microscopy (SEM), image analysis, single-mode microwave, basalt

\section{Introduction}

Microwave applications have primarily been investigated for mineral processing to potentially reduce the required energy consumption during comminution and to increase mechanical stresses through the particles of rocks for easier separation of rock pieces [1]. The technology of applying microwave irradiation on hard rocks has recently been assessed as a potential approach for rock fracturing prior to breakage by mechanical means such as the Tunnel Boring Machine (TBM) [2]. The purpose of microwave treatment of hard rocks is to reduce their mechanical strength by weakening key mechanical and physical properties. This process is referred to as "rock preconditioning". A conceptual illustration of microwave-assisted rock breakage as an alternative to conventional methods is shown in Fig. 1.

\footnotetext{
${ }^{1}$ In this study, the term 'specimen' refers to rectangular basalt extractions of size $40 \mathrm{~cm} \times 40 \mathrm{~cm}$, while the term 'sample' refers to cylindrical basalt extractions of size $\mathrm{r}=0.5 \mathrm{~cm}$ from the center of the irradiated specimens.
} 
Understanding the complex process of rock breakage by microwave energy requires a careful consideration of various aspects. To identify the effects of applying microwave treatments at different power levels, exposure times, and distances from the antenna, a powerful microanalysis technique should be employed. Among different available methods for analyzing the number of microcracks produced in a rock specimen by microwave treatment, the scanning electron microscopy (SEM) technique is comparatively more useful for analyzing microstructural characteristics because the SEM instrument has a greater depth of field and high spatial resolution [3].

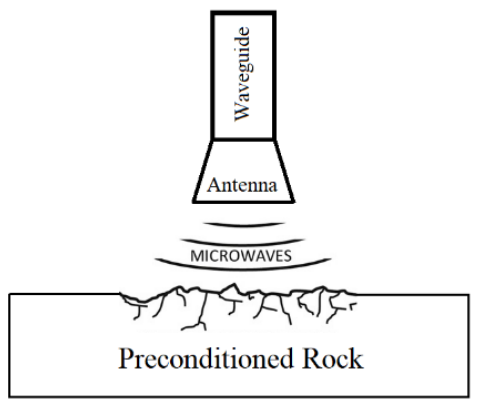

Fig. 1. The concept of microwave-assisted rock breakage

Many studies on the mechanical behavior of rocks verify that the effect of microwave treatment on hard rocks is significant [4,5]. For example, since 2005, several studies on microwave-assisted rock breakage have been conducted by the McGill microwave research team. They introduced the application of microwave energy which is considered a novel technique for rock breakage, in rock drilling, cutting, and breaking. It was shown that high-power microwave treatment resulted in an increased temperature and, therefore, the formation of mechanical damages to the rock surface, better facilitation of breakage operations for the mechanical disc cutter of an excavation machine, and reduction of disc cutter wear [4-7]. These results opened a new horizon for future breakage operations by applying microwave energy on one of the available rock excavation machines, i.e. full-face TBM. In another recent study, the authors of this paper investigated the influences of different microwave operating parameters, such as power level, exposure time, and the distance from the antenna (for single-mode microwave), on the surface temperatures of hard rocks by comparing results obtained through experiments and numerical simulations. Their results demonstrated that microwave energy has the potential to damage hard rocks by inducing multiple microcracks [6].

Although thermal stress and thermal cracking mechanisms by microwaves have been studied for many years, microstructural analysis of the processes of crack initiation, intensity, and their relevance to the applied microwave operating parameters has not been extensively carried out. Therefore, this challenge needs to be addressed through a fundamental understanding of how microwaves interact with hard rocks. It is essential to explore comprehensively the microstructural characteristics of rocks before and after microwave treatments using a more accurate analysis. The current study investigates and analyzes the amount of damage produced by microwave treatments on basalt specimens with different power levels, distances from the antenna, and exposures. By taking account of the features of the scanning electron microscopy imaging technique, the present study investigates the microstructural behavior of basaltic rock specimens after microwave treatments by using the captured images from the SEM device. 


\section{Methodology}

The method utilized in this study involves the following four steps:

1- Microwave irradiation experiments with different operating parameters on rectangular specimens of basalt,

2- Preparing cylindrical core samples from the center of the irradiated basalt specimens using a drill bit with an inner radius of $0.5 \mathrm{~cm}$,

3- Image processing on the cylindrical cored samples using an SEM instrument,

4- Crack quantification analysis on the SE images using ImageJ software.

The experimental tests were performed in the following steps. First, multiple rectangular basalt specimens were placed together in a series on an insulator slab inside a single-mode microwave cavity to arrive at the desired distance from the antenna (see Fig. 2b). Then, microwave irradiation was operated at different experimental conditions for a total of twelve case studies. The input operating parameters of the microwaves for the experiments are given in Table 1. For better evaluation of the impact of the different microwave operating parameters on microcracking behavior, case no. 1 was designated a nontreated basalt sample.

\begin{tabular}{cccc}
\hline Case no. & Power $(\mathrm{KW})$ & Distance from the antenna $(\mathrm{cm})$ & Exposure time $(\mathrm{s})$ \\
\hline 1 & nontreated & nontreated & nontreated \\
2 & 3 & 5 & 60 \\
3 & 3 & 15 & 60 \\
4 & 3 & 20 & 60 \\
5 & 3 & 25 & 60 \\
6 & 9 & 15 & 60 \\
7 & 9 & 20 & 60 \\
8 & 9 & 25 & 60 \\
9 & 15 & 20 & 60 \\
10 & 15 & 25 & 60 \\
11 & 3 & 5 & 120 \\
12 & 9 & 15 & 120 \\
\hline
\end{tabular}

Table 1. Input operating parameters of microwave for the experiments

By referring to a recent paper by the authors [6], the current study by performing crack quantification analysis provides a valuable way to understand the changes in the microstructure of the basalt rock specimen after microwave irradiation with different operating parameters. When specimens of basalt were subjected to different microwave treatments, it was expected that several microcracks would be induced because of the increased thermal expansion inside the irradiated specimens. However, the intensity of microcracks was highly dependent on the input parameters of the applied microwaves. The results from crack quantification analysis on irradiated cylindrical cored samples were compared with the nontreated sample.

\subsection{Microwave treatment experiments}

Microwaves are designed in two different types as follows: single-mode and multimode. Single-mode microwaves consist of a metallic enclosure and a waveguide horn. These metallic parts were designed so that the electromagnetic field polarization from a microwave signal would undergo several reflections. The superposition of the propagated waves in different directions gives rise to a standing wave pattern [8]. This pattern of waves is very well defined inside the cavity's space. Consequently, the precise knowledge of different 
configurations of the electromagnetic waves enables the rock specimen to be placed in the position at which maximum heating rate and subsequent breakage can be achieved. With that information in mind, a single-mode microwave cavity was chosen for the experiments of the present study. An illustration of this single-mode cavity system is given in Fig. 2.

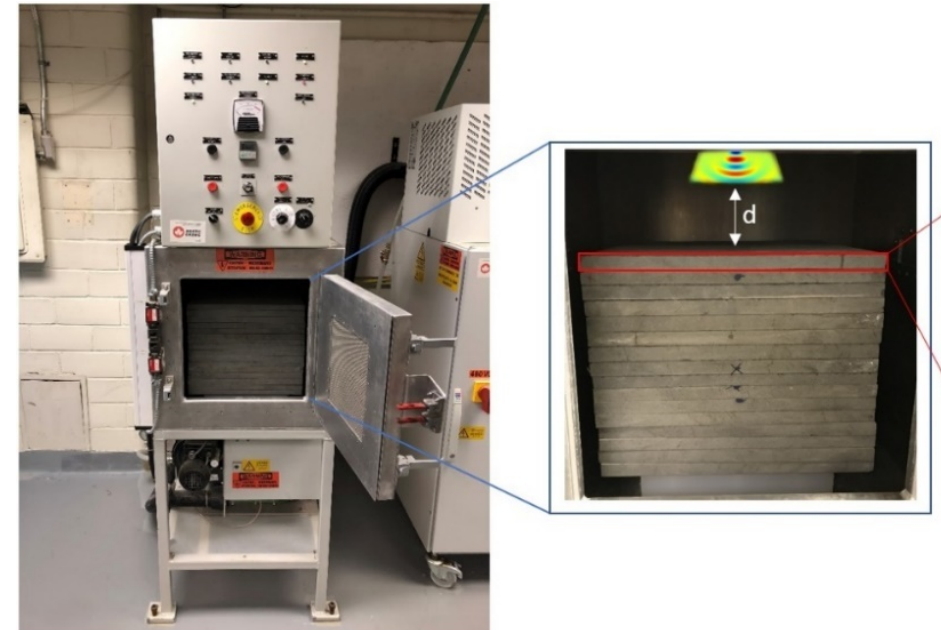

(a) (b)

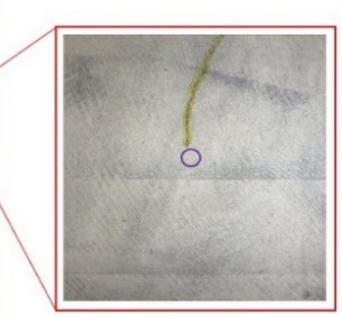

(c)

Fig. 2. (a) Single-mode microwave cavity system, (b) Microwave equipment set-up with the rock specimens placed inside the microwave cavity and below the horn; the distance from the waveguide horn (antenna), $\mathrm{d}(\mathrm{cm})$, is shown by a white arrow, and (c) the first irradiated specimen from top after the experiment. Note here that the first specimen from the top is used for drilling cored samples from its center (shown by a purple circle).

The blocks of basalt were cut at the mine into rectangular specimens with dimensions of $40 \mathrm{~cm} \times 40 \mathrm{~cm}$. The specimens were intact and without visible cracks. They were used to conduct microwave tests according to the input conditions of Table 1. After the experiments, the first specimen from the top was used for the drilling of cylindrical core samples (see Fig. 2(c)). Subsequently, the SEM instrument was employed to capture SE images from the top center of the cored samples and for further image processing purposes. Finally, crack quantification analysis was performed on the rendered images using ImageJ software. The results were used to compare the effects of different microwave operating conditions and to analyze the microcracking behavior of basalt.

\subsection{Core samples' preparation process}

After microwave treatment tests, the irradiated specimens were drilled using a drill bit with an inner radius of $r=0.5 \mathrm{~cm}$; cylindrical core samples, with a dimension of $r=0.5 \mathrm{~cm}$ and $h=2 \mathrm{~cm}$, were drilled into the surface of the irradiated specimens for SEM image analysis. The drill bit and cored samples' size are shown in Fig. 3(a) and 3(b), respectively. For a better visual understanding of the SEM imaging process, the SEM device (type SU3500) is illustrated in Fig. 3(c). 


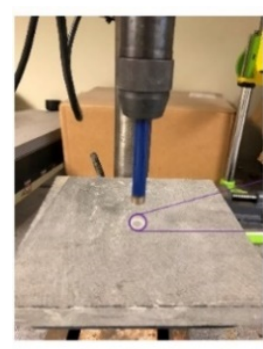

(a)

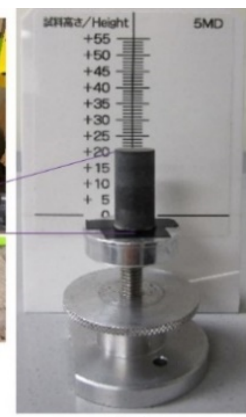

(b)

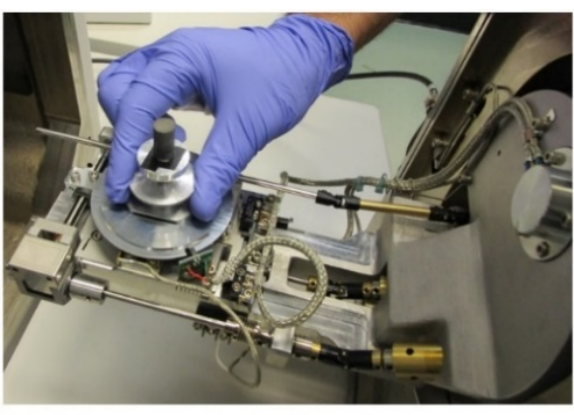

(c)

Fig. 3. (a) The drill bit for making core samples, (b) cylindrical cored samples with a dimension of $\mathrm{r}=0.5 \mathrm{~cm}$ and $\mathrm{h}=2 \mathrm{~cm}$, and (c) installation process of the core sample in SEM-SU3500 instrument for image processing

\subsection{SEM analysis}

Scanning electron microscopy (SEM) is one of the most advanced technologies for the quantification and analysis of microstructural responses of solid materials. SEM analysis is comparatively more useful in studies that investigate quantification of microstructural properties such as microcracks and voids. Fig. 4 shows the Hitachi SEM SU3500 instrument used for SEM imaging.

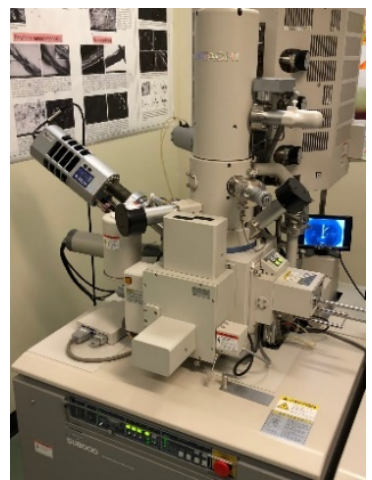

(a)

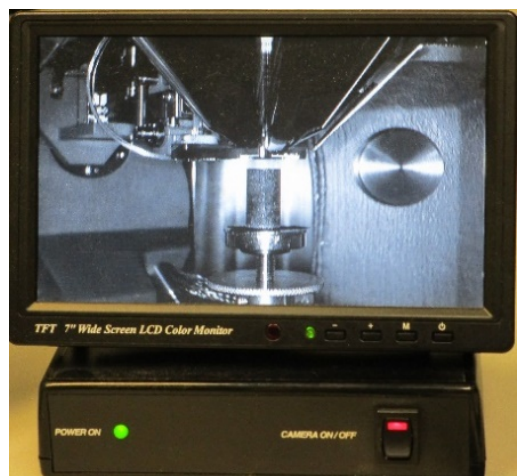

(b)

Fig. 4. (a) The Hitachi SEM-SU3500 Variable Pressure Scanning Electron Microscope (VP-SEM) instrument located at McGill University, Department of Mining and Materials Engineering, (b) position monitoring of sample

The Hitachi SU3500 Variable Pressure Scanning Electron Microscope (VP-SEM) used in the present study can provide up to $7 \mathrm{~nm}$ SE images with a resolution at $3 \mathrm{kV}, 10 \mathrm{~nm}$, and backscattered electrons (BSE) image resolution at $5 \mathrm{kV}$. It is equipped with an $80 \mathrm{~mm}$ 2X-Max SDD EDS detector with the EBSD Aztec HKL Advanced system. The high probe of this microscope with $1 \mu \mathrm{A}$ current allows the automatic EDS-EBSD mapping to create a montage of all the regions of a given specimen automatically at very high speed [9]. The BSE detector can produce quantitative three-dimensional maps of the surface of a specimen to quantify complicated and rough surfaces. The low vacuum mode characterizes nonconductive materials without coatings.

In this study, the SE images were captured with a resolution at $2 \mathrm{kV}$ and $200 \mu \mathrm{m}$. After acquiring the images from the irradiated specimens with the conditions given in Table 1, image processing was performed to view microcracks in basalt. 
ImageJ software developed by the National Institute of Health (NIH) was used to conduct image processing [10]. This software has the capability to perform various operations such as binary thresholding and area measurement based on Java language.

\section{Results and discussion}

The results from the SE images are shown in Fig. 5. The plots follow the case studies listed in Table 1. The specified case numbers are shown under each plot. It can be seen that the intensity of microcracks reduces when the power level decreases from $15 \mathrm{KW}$ to $3 \mathrm{KW}$, the exposure time decreases from 120 -second to 60 -second, and the distance from the antenna increases from $5 \mathrm{~cm}$ to $25 \mathrm{~cm}$.

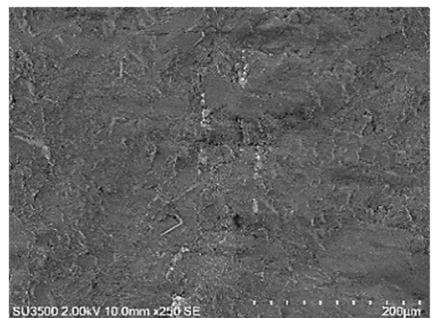

Case no.1

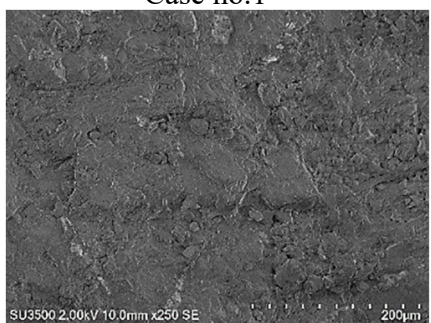

Case no. 4

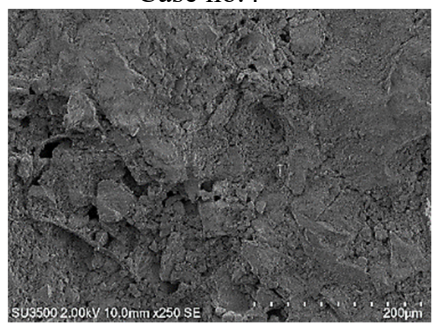

Case no.7

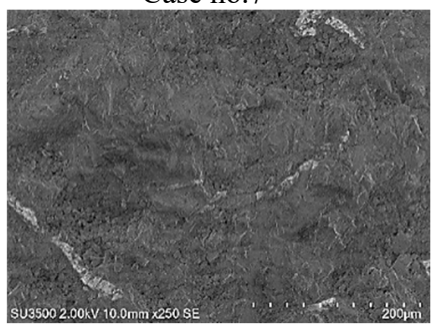

Case no.10

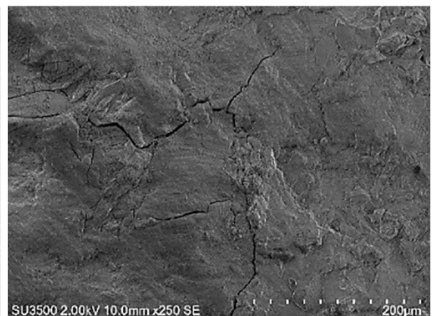

Case no. 2

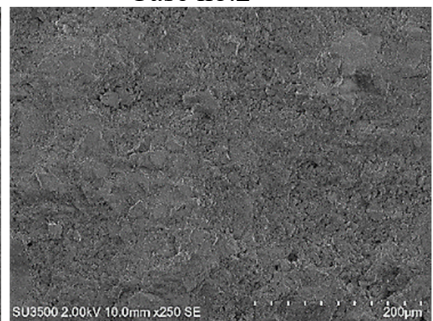

Case no.5

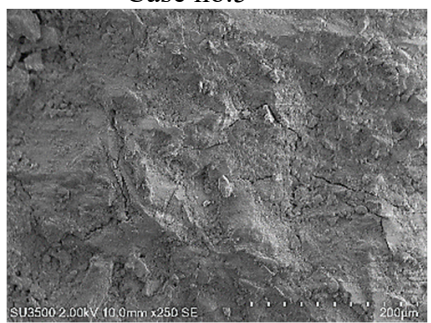

Case no. 8

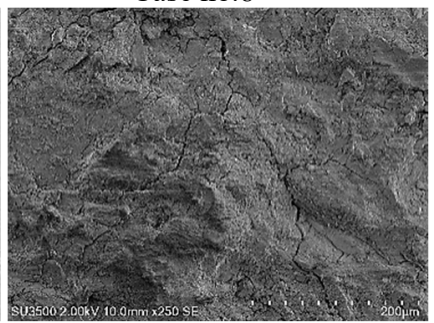

Case no.11

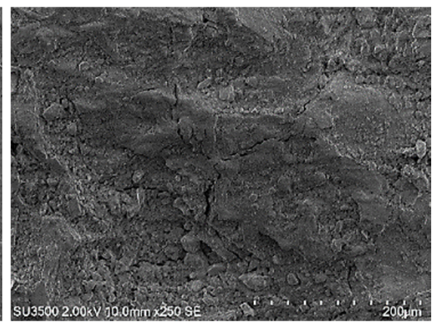

Case no. 3

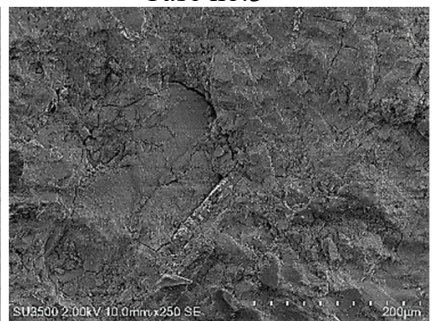

Case no.6

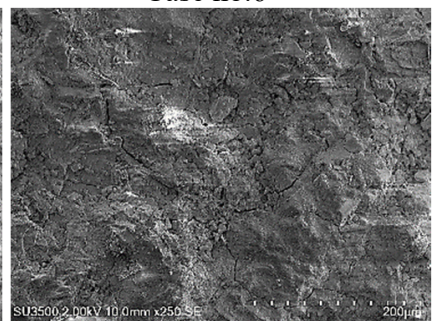

Case no. 9

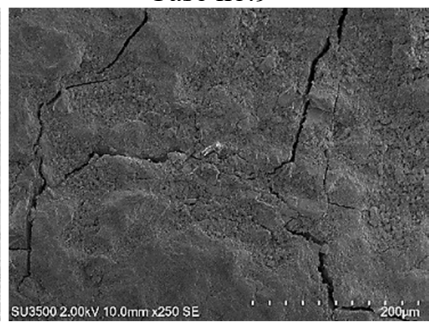

Case no. 12

Fig. 5. The SEM images of cylindrical cored samples after microwave treatments with different operating parameters 
The first step of image processing was to conduct segmentation of the images into binary images through the brightness thresholding approach. This makes the SE images black and white. By this process, ImageJ changes the intensities of image pixels that will highlight the regions of interests - in this study, are microcracks. For each plot of Fig. 5, the low threshold level was set at zero and the best threshold level was determined by comparing the original gray image with the image after application of manual thresholding on the binary image. After being converted to binary mode, all images were manually mapped, and the total number of crack lengths and crack density for all the case studies was obtained. However, it is worth mentioning here that microcracks are very elongated structures; therefore, length to width (referred to as shape factor) analysis is applied for different geometries. This means that the structures with a shape factor of 2.7 or greater were considered microcracks. In this case, the remaining structures should be assumed to be voids. The corresponding data obtained for the length and density of cracks for the different case numbers after image processing on the SE images are summarized in Table 2.

\begin{tabular}{cccc}
\hline Case no. & Cracks length $(\mu \mathrm{m})$ & Image field & Crack density $\left(\mu \mathrm{m} / \mu \mathrm{m}^{2}\right)$ \\
\hline 1 & 432.15 & $183 \mu \mathrm{m} \times 250 \mu \mathrm{m}$ & 0.009445 \\
2 & 1310.64 & $183 \mu \mathrm{m} \times 250 \mu \mathrm{m}$ & 0.028647 \\
3 & 1182.07 & $183 \mu \mathrm{m} \times 250 \mu \mathrm{m}$ & 0.025837 \\
4 & 943.74 & $183 \mu \mathrm{m} \times 250 \mu \mathrm{m}$ & 0.020628 \\
5 & 780.42 & $183 \mu \mathrm{m} \times 250 \mu \mathrm{m}$ & 0.017058 \\
6 & 1670.72 & $183 \mu \mathrm{m} \times 250 \mu \mathrm{m}$ & 0.036518 \\
7 & 1322.91 & $183 \mu \mathrm{m} \times 250 \mu \mathrm{m}$ & 0.028916 \\
8 & 1107.085 & $183 \mu \mathrm{m} \times 250 \mu \mathrm{m}$ & 0.024198 \\
9 & 1432.11 & $183 \mu \mathrm{m} \times 250 \mu \mathrm{m}$ & 0.031302 \\
10 & 1216.32 & $183 \mu \mathrm{m} \times 250 \mu \mathrm{m}$ & 0.026586 \\
11 & 1540.02 & $183 \mu \mathrm{m} \times 250 \mu \mathrm{m}$ & 0.033661 \\
12 & 1861.81 & $183 \mu \mathrm{m} \times 250 \mu \mathrm{m}$ & 0.038909 \\
\hline
\end{tabular}

Table 2. Crack quantification analysis for basalt samples

The above results indicate the presence of microcracks in the given sample. Maximum lengths of cracks were $1670.72 \mu \mathrm{m}$ and $1861.81 \mu \mathrm{m}$, for 60 -second and 120 -second exposure times, respectively. These two values were achieved when a $3 \mathrm{KW}$ power level was applied at a $5 \mathrm{~cm}$ distance from the antenna. Therefore, it can be concluded that the effect of exposure time was significant; longer exposure times were responsible for more extensive cracks. In addition, for a better understanding of the cracking mechanism, a crack quantification analysis was done on the nontreated sample compared to the treated samples. The result of the nontreated sample is shown in case no. 1 in Table 2. When comparing results from the irradiated samples with the nontreated sample, the effect of microwave input operating parameters is more visible. For example, by comparing cases 4 and 5, in which the amount of crack length was under $1000 \mu \mathrm{m}$, the effect of the distance from the antenna can be observed. As the distance from the antenna increases, the number of microcracks declines. The results from crack quantification analysis yield a better understanding of the mechanism of crack propagation in regard to differently applied microwave operating parameters. The density of cracks at the captured area differs, depending upon different microwave input operating parameters. By comparing the data in Table 2, it can be observed that a higher level of microwave power results in greater crack lengths and densities. On closer inspection, however, at the same power level, a shorter distance from the antenna results in denser cracking. 


\section{Conclusion}

A previous study conducted by the authors [6] showed that microwave energy can thermally break hard rocks by inducing several microcracks; however, the actual mechanisms in such a complex system with different multiphysics interactions are still hard to investigate. The objective of this study was to analyze the microcracking behavior of rocks under microwave treatments with different operating parameters using the SEM technique. For a better visual understanding of the mechanism of crack development, including spallation/disintegration, the SEM images were captured from the top center of the cylindrical cored samples.

The previous study also showed that rock failure mechanism under microwave irradiation tests is closely related to the input operating parameters of the applied microwave energy. More significantly, a higher input power level of microwaves contributes to more complex physical and mechanical reactions, which include the elevation of internal pressure and decomposition of particles in the basalt specimen. The results both studies are highly consistent with each other.

\section{Acknowledgements}

Financial support for this work from the Natural Sciences and Engineering Research Council of Canada (NSERC), De Beers Group, Argex, Metso and the McGill Engineering Doctoral Award (MEDA) Award is gratefully acknowledged.

\section{References}

[1] Lovas, M., Znamenackova, I., Zubrik, M., Kovacova, M., and Dolinska, S., "The Application of Microwave Energy in Mineral Processing - a Review”, Acta Montanistica Slovaca, 2011, 16(2), pp. 137-148.

[2] Lu, G. ming, Li, Y. hui, Hassani, F., and Zhang, X., “The Influence of Microwave Irradiation on Thermal Properties of Main Rock-Forming Minerals", Appl. Therm. Eng., 2017, 112, pp. 1523-1532.

[3] Nemati, K. M., "Fracture Analysis of Concrete Using Scanning Electron Microscopy”, Scanning, 2006, 19(6), pp. 426-430.

[4] Motlagh, P. N., "Physical and Mechanical Properties of Rocks Exposed: To Microwave Irradiation: Potential Application to Tunnel Boring”, McGill Univ., 2015.

[5] Hassani, F., Nekoovaght, P. M., and Gharib, N., "The Influence of Microwave Irradiation on Rocks for Microwave-Assisted Underground Excavation", J. Rock Mech. Geotech. Eng., 2016, 8(1), pp. 1-15.

[6] Teimoori, K., Hassani, F., and Sasmito, A. P., "Multiphysics Study of Microwave Irradiation Effects on Rock Breakage System”, IJRMMS, 2019, 1(514), pp. 1-32.

[7] Lu, G., Feng, X., Li, Y., Hassani, F., and Zhang, X., "Experimental Investigation on the Effects of Microwave Treatment on Basalt Heating, Mechanical Strength and Fragmentation," Rock Mech. Rock Eng., 2019, 1(Feb), pp. 1-19.

[8] Whittles, D. ., Kingman, S. ., and Reddish, D. ., "Application of Numerical Modelling for Prediction of the Influence of Power Density on Microwave-Assisted Breakage”, Int. J. Miner. Process., 2003, 68(1-4), pp. 71-91.

[9] Brodusch, N., Demers, H., and Gauvin, R., "Imaging with a Commercial Electron Backscatter Diffraction (EBSD) Camera in a Scanning Electron Microscope: A Review”, J. Imaging, 2018, 4(7), p. 88.

[10] Abramoff, M. D., Magalhães, P. J., and Ram, S. J., “Image Processing with ImagJ”, Biophotonics Int., 2004, 11(7), pp. 36-42. 


\title{
QUANTUM CHEMISTRY MOLECULAR MODELING FOR RADIO- FREQUENCY AND MICROWAVE- FREQUENCY THERMO-UPCONVERSION HEATING OF TRANSITION METAL OXIDES OF NiO AND Fe2O3
}

\author{
Shozo Yanagida ${ }^{1}$, and Takeko Matsumura ${ }^{2}$

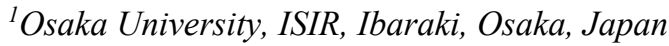 \\ ${ }^{2}$ Minerva Light Laboratory, Kyoto, Japan \\ yanangida@mls.eng.osaka-u.ac.jp
}

Keywords: DFT, molecular modeling, Spartan, IR/FIR, thermo-upconversion, dielectric heating

\section{Introduction}

Most solid-state inorganic materials heat up by microwave (MW) induced conductivity and dielectric loss heating, and the MW heating power to transition metal oxides, which have unpaired electron (spin), was considered to be proportional to dielectric heating relating to magnetic loss factor relating to spin in transition metal oxides. We know, however, that, nickel oxide ( $\mathrm{Ni}(\mathrm{II}) \mathrm{O}, 25 \mathrm{gr})$ ) having 2 unpaired electron, heat up to $1,300^{\circ} \mathrm{C}$ in 6 min under $1 \mathrm{~kW}-\mathrm{MW}$ irradiance, and that. ferric oxide ( $\left.\mathrm{Fe}(\mathrm{III})_{2} \mathrm{O}_{3}, 25 \mathrm{gr}\right)$ having 10 unpaired electron, up to only $88^{\circ} \mathrm{C}$ in $30 \mathrm{~min}$ under the same $1 \mathrm{~kW}-\mathrm{MW}$ irradiance. ${ }^{1)}$ The big difference in MW heating is now attempted to rationalize on the basis of density functional theory-based molecular modeling (DFT/MM). Recently, we reported that DFT/MM verifies that water aggregates undergo absorption and emission in radio-frequency wave, MW and FIR region, resulting in thermal absorption and dissipation, i.e., heat of IR radication. We proposed that MW and radio frequency heating of water should instantly proceed thermo-upconversion mechanism under MW energy irradicance. ${ }^{2)}$

For density functional theory-based molecular modeling (DFT/MM, B3LYP, 6$\left.31 \mathrm{G}^{*}\right) \mathrm{DFT} / \mathrm{MM}$, we take into account that both nickel and ferrite oxides should be composed of covalent-bonding metal oxides of respective $\mathrm{Ni}(\mathrm{II}) \mathrm{O}$ or $\mathrm{Fe}(\mathrm{III})_{2} \mathrm{O}_{3}$, and their van der Waals $(\mathrm{vdW})$ aggregates of $[\mathrm{Ni}(\mathrm{II}) \mathrm{O}]_{\mathrm{n}}(\mathrm{n}=2 \sim 4)$ and $\left[\mathrm{Fe}(\mathrm{III})_{2} \mathrm{O}_{3}\right]_{\mathrm{n}}(\mathrm{n}=2 \sim 4)$. We obtain their equilibrium three-dimensional molecular-aggregate structures, showing them with electron density in Table 1 and 2. Electron energy structures result in giving infrared (IR), far infrared (FIR), MW, and, radio-frequency wave- energy absorption spectra. It should be noted that stretching and bending and concerted back and forth movement between composed elements can be visualized for molecular aggregates of [Ni(II)O $]_{n}(n=2 \sim 4)$ and $\left[\mathrm{Fe}(\mathrm{III})_{2} \mathrm{O}_{3}\right]_{\mathrm{n}}(\mathrm{n}=2 \sim 4)$ by clicking strong absorption peaks especially in $500 \sim 0 \mathrm{~cm}^{-1}$ region. It is very interesting to recognize that radio frequency wave, MW and FIR absorption induce back and forth movement of atoms in vdW aggregates and thermo-upconversion starts very quickly to FIR and IR absorption and emission. IR emission is heating.

DFT/MM of molecular vdW aggregates gives heat of formation ( $\square \mathrm{E}$ ), the energy level of the lowest unoccupied molecular orbital (ELUMO) and energy level of the highest occupied molecular orbital (EHOMO), dipole, and electron transfer gap ( $\square \mathrm{Et}$ ) that can be calculated from ELUMO (0) and ELUMO (+1). The bandgap will be a measure of conductivity. They are all summarized with IR and FIR absorption peaks with high intensity in Table 1 and 2. Clicking intense IR and FIR absorption peaks makes us understand that thermoupconversion starts at concerted back and forth movement of atoms in molecular aggregates. 


\section{DFT/MM for quick MW heating of nickel oxides}

Table 10 DFT/MM for IR and FIR absorption spectrum analysis of Ni(II) oxides

\begin{tabular}{|c|c|c|c|c|c|c|}
\hline $\begin{array}{l}\text { Nickel(II) } \\
\text { oxide }\end{array}$ & $\begin{array}{l}\text { Equilibrium } \\
\text { geometry with } \\
\text { density }\end{array}$ & $\begin{array}{l}\text { Heat of } \\
\text { formation } \\
\text { (E/au) }\end{array}$ & $\begin{array}{l}\text { Dipole } \\
\text { (debye) }\end{array}$ & $\begin{array}{c}\text { Conductivity } \\
\text { measure: } \\
\begin{array}{c}\Delta E=\alpha L U M O\{+1\} \\
-\alpha L U M O^{* *}\end{array}\end{array}$ & $\begin{array}{l}\text { FIR region } \\
\left(0 \sim 500 \mathrm{~cm}^{-1}\right) \\
\mathrm{cm}^{-1} \text { (intensity) }\end{array}$ & $\begin{array}{c}\text { IR region } \\
\left(3500 \sim 500 \mathrm{~cm}^{-1}\right) \\
\mathrm{cm}^{-1} \text { (intensity) }\end{array}$ \\
\hline $\begin{array}{c}\mathrm{NiO} \\
\text { Unpaired } \\
\text { electron } 2\end{array}$ & & -1583.20835 & 2.01 & 0.23 & $379(33.57$ & - \\
\hline $\begin{array}{c}\mathrm{Ni}_{2} \mathrm{O}_{2} \\
\text { Unpaired } \\
\text { electron } 4\end{array}$ & & -0.24919 & 0 & 1.30 & $\begin{array}{l}198(58.1) \\
472(194)\end{array}$ & 639(99.3) \\
\hline $\begin{array}{c}\mathrm{Ni}_{4} \mathrm{O}_{4} \\
\text { Unpaired } \\
\text { electron } 8\end{array}$ & & -0.71603 & 0.01 & 1.51 & $\begin{array}{c}172(0.2), 178(2.97) \\
183(3.02), 184(2.49) \\
430(1.89), 455(164) \\
456(170), 461(167)\end{array}$ & $\begin{array}{l}535(58.4) \\
539(62.1)\end{array}$ \\
\hline $\begin{array}{c}\text { Single point } \\
\text { vdW dimer } \\
*\left(\mathrm{Ni}_{4} \mathrm{O}_{4}\right)_{2} \\
\text { Unpaired } \\
\text { electron } 16\end{array}$ & If & $-1.3948 *$ & 0 & 0.42 & $\begin{array}{c}133(4.35), 169(3.11) \\
169(3.12), 170(4.6) \\
264(0.06), 428(0.62)\end{array}$ & $\begin{array}{c}518(477), 531(192) \\
536(398), 536(398) \\
580(4.87)\end{array}$ \\
\hline
\end{tabular}
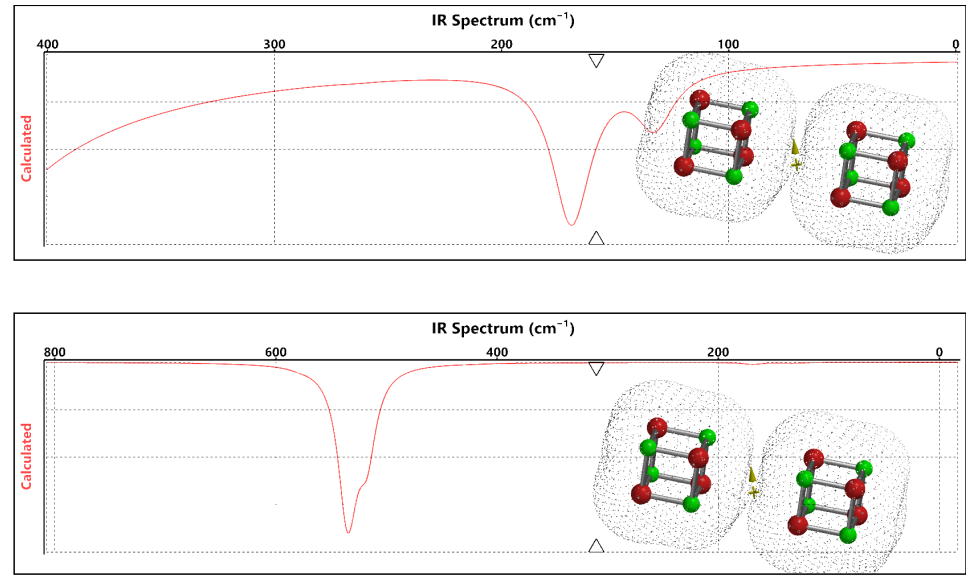

Fig. 1. DFT/MM-determined FIR and IR absorption spectra of singlepoint vdW dimer of $*\left[\left(\mathrm{Ni}_{4} \mathrm{O}_{4}\right)_{2}\right]_{2}$

DFT/MM of nickel oxides gives a dimer of $\mathrm{Ni}_{2} \mathrm{O}_{2}$, a tetramer $\left(\mathrm{Ni}_{4} \mathrm{O}_{4}\right)$ and vdW aggregate of the tetramer (Table 1) when unpaired electron 2 for each nickel (II) atom is taken into consideration. Two of them are shown covalent bonding structures with surface electron density (dots style) and are thermally stable as proved by negative heat of formation. All of them have a small dipole. Previously we reported that conductivity of molecular aggregates should be evaluated by bandgap between electron running states (biased state) of molecules and aggregates. ${ }^{3)}$ At electron running states of nickel oxides, $\alpha$ LUMO (0) will become singly occupied molecular orbital (SOMO) and $\alpha \mathrm{LUMO}(+1)$ will become $\alpha \mathrm{LUMO}(0)$ of the electron-running-state nickel oxides (" $\alpha$ " stands for alpha spin). Conductivity measure of $\Delta \mathrm{E}=\alpha \mathrm{LUMO}\{+1\}-\alpha \mathrm{LUMO}$ verifies that they have semi-conductivity, predicting that conductivity may contribute to MW heating at elevated temperature. 
The covalent-bonding $\mathrm{Ni}(\mathrm{II}) \mathrm{O}$ dimer, $\mathrm{Ni}_{2} \mathrm{O}_{2}$, the covalent-bonding $\mathrm{Ni}(\mathrm{II}) \mathrm{O}$ tetramer, $\mathrm{Ni}_{4} \mathrm{O}_{4}$, and the vdW dimer of $\left(\mathrm{Ni}_{4} \mathrm{O}_{4}\right)_{2}$ are molecular modeled to know if thermo-upconversion mechanism is operating in the MW heating of nickel oxides. The single point energy structure of $\left(\mathrm{Ni}_{4} \mathrm{O}_{4}\right)_{2}$ is obtained and analyzed although heat of formation $\mathrm{f}$ is positive. Because the equilibrium geometry could not be molecular modeled contrast to the case of $\mathrm{Fe}(\mathrm{III})_{2} \mathrm{O}_{3}$ (see Table 2).

Table 1 shows that all of $\mathrm{Ni}$ (II)O aggregates have absorption with high intensity in 500 0 $\mathrm{cm}^{-1}$ region including MW and radio-frequency wave. In addition, the stronger absorption expands to the IR absorption and dissipation region. Typical FIR and IR absorption spectra shown in Fig. 1 verifies and predicts the thermo-upconversion occurs effectively during MW heating of nickel oxides.

\section{DFT/MM for MW heating of ferric oxides}

DFT/MM is extended to ferric oxides by taking into account of unpaired electron 5 for each $\mathrm{Fe}(\mathrm{III})$ atom . The equilibrium geometry of covalent-bonding of $\mathrm{Fe}_{2} \mathrm{O}_{3}$ and single pint energy structure of vdW aggregates of $\left[\mathrm{Fe}(\mathrm{III})_{2} \mathrm{O}_{3}\right]_{2}$ are successfully modeled and their heat of formation, dipole and conductivity measure $\Delta \mathrm{E}=\alpha \mathrm{LUMO}\{+1\}-\alpha \mathrm{LUMO}$ are summarized in Table 2. They have no dipole, but have semi-conductivity comparable to the nickel oxides. Interestingly, the equilibrium geometry structure $\mathrm{Fe}_{2} \mathrm{O}_{3}$ and the single pint energy structure of vdW aggregates have no absorption in IR region of more than $500 \mathrm{~cm}^{-1}$. These calculation results well verify slow MW heating of ferrite as reported. ${ }^{1)}$

Table 20 DFT/MM for IR and FIR absorption spectrum analysis of $\mathrm{Fe}(\mathrm{III})_{2} \mathrm{O}_{3}$

\begin{tabular}{|c|c|c|c|c|c|c|}
\hline $\mathrm{Fe}(\mathrm{III})_{2} \mathrm{O}_{3}$ & $\begin{array}{l}\text { Equilibrium } \\
\text { geometry with } \\
\text { density }\end{array}$ & $\begin{array}{l}\text { Heat of } \\
\text { formation } \\
\text { (E/au) }\end{array}$ & $\begin{array}{l}\text { Dipole } \\
\text { (debye) }\end{array}$ & $\begin{array}{c}\text { Conductivity } \\
\text { measure: } \\
\Delta E=\alpha L U M O\{+1\}- \\
\alpha L U M O * *\end{array}$ & $\begin{array}{l}\text { FIR region } \\
\left(0 \sim 500 \mathrm{~cm}^{-1}\right) \\
\mathrm{cm}^{-1} \text { (intensity) }\end{array}$ & $\begin{array}{c}\text { IR region } \\
\left(3500 \sim 500 \mathrm{~cm}^{-1}\right) \\
\mathrm{cm}^{-1} \text { (intensity) }\end{array}$ \\
\hline $\begin{array}{c}\mathrm{Fe}(\mathrm{III})_{2} \mathrm{O}_{3} \\
\text { Unpaired electron } \\
10\end{array}$ & & -2752.8048 & 0 & 1.25 & $\begin{array}{l}102(2.65) \\
117(2.78) \\
387(81.8)\end{array}$ & $549(0.09)$ \\
\hline $\begin{array}{c}\text { *vdW dimer } \\
{\left[\mathrm{Fe}(\mathrm{III})_{2} \mathrm{O}_{3}\right]_{2}} \\
\text { Unpaired electron } \\
20\end{array}$ & & 0.24497 & 0 & 0.26 & $\begin{array}{c}118(122) .155(17.1) \\
253(31.5), 279(24.7) \\
302(12.7), 356(10.84)\end{array}$ & No absorption \\
\hline $\begin{array}{c}\text { vdW dimer } \\
{\left[\mathrm{Fe}(\mathrm{III})_{2} \mathrm{O}_{3}\right]_{2}} \\
\text { Unpaired electron } \\
20\end{array}$ & & -0.2593 & 0 & 0.79 & $\begin{array}{c}62(9.72), 175(39.9) \\
208(29.1), 231(98.2) \\
301(0.17), 393(0.21)\end{array}$ & $\begin{array}{l}535(30.8), 585(166) \\
644(426), 722(606)\end{array}$ \\
\hline $\begin{array}{c}\mathrm{Fe}(\mathrm{III})_{4} \mathrm{O}_{6} \\
\text { Unpaired electron } \\
20\end{array}$ & & -0.25926 & 0.05 & 0.79 & $\begin{array}{c}58(9.5), 174(40.0) \\
208(29.7), 231(98.2) \\
301(0.17), 393(0.21)\end{array}$ & $\begin{array}{c}534(31.1), 584(166) \\
644(426), 666(7.5) \\
722(607)\end{array}$ \\
\hline $\begin{array}{c}\text { vdW dimer } \\
{\left[\mathrm{Fe}(\mathrm{III})_{4} \mathrm{O}_{6}\right]_{2}} \\
\text { Unpaired electron } \\
20\end{array}$ & & -0.7066 & 0 & 0.35 & $\begin{array}{c}54(0.02), 61(3.3), \\
72(7.4), 86(7.1) \\
139(0.53), 142(49.2) \\
205(15.4), \\
217(24.3)\end{array}$ & $\begin{array}{l}534(31.1), 584(166) \\
644(426), 666(426) \\
653(177), 658(374)\end{array}$ \\
\hline
\end{tabular}

* Single point calculation, ** “ $\alpha$ ” stands for alpha spin. 
Interestingly, DFT/MM for an equilibrium geometry of a vdW dimer of [ $\left.\mathrm{Fe}(\mathrm{III})_{2} \mathrm{O}_{3}\right]_{2}$ leads to covalent bonding $\mathrm{Fe}(\mathrm{III})_{4} \mathrm{O}_{6}$. The vdW dimer $\left[\mathrm{Fe}(\mathrm{III})_{2} \mathrm{O}_{3}\right]_{2}$ is structurally identical with the covalent bonding $\mathrm{Fe}(\mathrm{III})_{4} \mathrm{O}_{6}$ as shown in Table 2. Further DFT/MM is successful for obtaining an equilibrium geometry for the vdW dimer of $\left[\mathrm{Fe}(\mathrm{III})_{4} \mathrm{O}_{6}\right]_{2}$. They all have negative heat of formation and then the structure ae thermally stabilized.
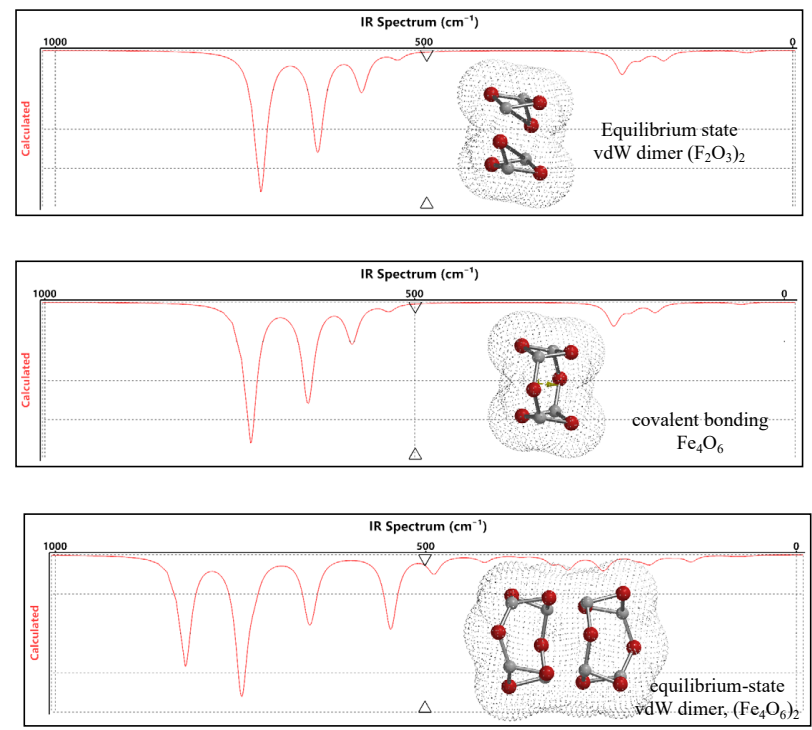

Fig. 2. DFT/MM-determined FIR and IR absorption spectra of equilibriumstate vdW dimer $\left(\mathrm{F}_{2} \mathrm{O}_{3}\right)_{2}, \mathrm{Fe}_{4} \mathrm{O}_{6}$ and equilibrium-state vdW dimer, $\left(\mathrm{Fe}_{4} \mathrm{O}_{6}\right)_{2}$

Fig. 2 shows IR and FIR absorption spectra of the equilibrium-state vdW dimer of $\left[\mathrm{Fe}(\mathrm{III})_{2} \mathrm{O}_{3}\right]_{2}$, covalent bonding $\mathrm{Fe}(\mathrm{III})_{4} \mathrm{O}_{6}$ and its equilibrium-state vdW dimer, $\left[\mathrm{Fe}(\mathrm{III})_{4} \mathrm{O}_{6}\right]_{2}$. Contrary to the case of $\mathrm{Fe}(\mathrm{III})_{2} \mathrm{O}_{3}$, Covalently bonding $\mathrm{Fe}(\mathrm{III})_{4} \mathrm{O}_{6}$ structures are verified to undergoes thermo-upconversion. On careful examination, Koleini and Barani reported that $\mathrm{Fe}_{2} \mathrm{O}_{3}$ heats up to $1000{ }^{\circ} \mathrm{C}$ at 6 -min interval. ${ }^{4}$ Then we conclude that ferrite heat up accompanying structural change under MW irradiance.

\section{References}

1. S.L. McGill and J.W. Walkiewicz, J. Microwave Power Electromag. Energy, Symp. Summ., 1987, 175.

2. S. Yanagida and T. Matsumura, AMPERE Newsletter, 95, 27, 2018

3. S. Yanagida, et al, J. Electrochem. Soc., 162 (10) E263-E270 (2015)

4. S.M.J. Koleini and K. Barani, "Microwave Heating Applications in Mineral Processing" (http://dx.doi.org/10.5772/45750) 


\title{
ELUCIDATION OF ELECTROMAGNETIC WAVE EFFECT AND OUTGOING OF FUTURE TREND IN MICROWAVE CHEMISTRY AND BIOLOGY
}

\section{S. Horikoshi}

Sophia University, 7-1 Kioi-cho, Chiyoda-ku, Tokyo

horikosi@sophia.ac.jp

Keywords: Electromagnetic field, semiconductor microwave generator, organic synthesis, photocatalyst, plant breeding, intelligent cooker, aged beef

\begin{abstract}
Can we use microwaves as electromagnetic energy in the chemical and biological fields? We have been researching this question in many aspects and in a bird's-eye view. In response to this question, in some chemical reactions, it turned out that the advantage of microwaves is heat. Note that we also found that this efficient thermal energy transfer method is useful in the chemical field. On the other hand, it was found that electromagnetic waves other than thermal energy can be used in limited chemical reactions (mainly radical reactions). On the other hand, in biological use, there are not many things that require more heat energy than the atmosphere in the first place. However, we have found that depending on the microwave irradiation conditions, it can be activated as a stimulus other than heat. In other words, we discovered a phenomenon that would not have appeared without microwaves. In this paper, it will explain the electromagnetic wave effects of microwaves using photocatalysts, enzymes, and plants as examples. Furthermore, the importance of microwave control will be explained from the development of intelligent cooker.
\end{abstract}

\section{Introduction}

The German chemist Theodor Grotthuss was the first to formulate the first law of photochemistry in 1817; he postulated that a reaction could be driven by light when the energy of light is absorbed by molecules [1]. Grotthuss' idea remained unknown for many years until John W. Draper, an expert in chemistry and photographic processes, proposed in 1842 that only absorbed light rays can produce chemical changes. For this reason, Grotthuss' postulate is better known today as the Grotthuss-Draper law or the first law of photochemistry [2]. The photographic technique developed by Louis Jacques Mandé Daguerre in 1839 as a practical photographic method was to become the starting point of photochemical developments. In this regard, the first researcher to develop true photochemical concepts that distinguished between primary and secondary processes taking place in a chemical system under light absorption was Johannes Stark (1908), who defined the primary process as the immediate absorption of a photon by a molecule or an atom followed by secondary processes. In effect, the law states that in a photochemical process (such as a photochemical reaction) one photon that is absorbed by a molecule causes the main photochemical process. In some circumstances, a molecule having absorbed a photon initiates a process that may involve several other molecules. The Stark-Einstein law is the second law of photochemistry named after Johannes Stark and Albert Einstein. This law states that for each photon of light absorbed by a chemical system, no more than one molecule is activated for a photochemical reaction, as defined by the quantum yield [2]. These two laws have elevated photochemistry as an academic (science) discipline over the last one hundred years. In addition, because of advances in light sources 
and various devices (engineering), such materials and processes as photocatalysts, organic solar cells, photopolymerization, quantum dots, and photochromism (among others) are currently being applied in various other fields.

The next significant surge in chemistry is microwave chemistry wherein microwaves, which represent electromagnetic waves other than light, were introduced as a driving force in the chemical reaction domain in the late 1980s. Since then, thousands of articles have appeared that pertain to microwave-assisted organic syntheses. There are three characteristics in this chemistry when using microwaves. The first is the high heating efficiency caused by the energy of the microwaves that directly reach and are absorbed by the substance. The second is the selectivity with which a specific substrate is heated, while the third characteristic is the enhancement of chemical syntheses by the microwaves' electromagnetic wave energy, often referred to as the microwave effect (or non-thermal effect). Together with the first and second efficient heat effects, these open a new path in microwave chemistry (see e.g., ref. 3 ). The phenomenon of the microwave effect (third characteristic) impacting chemical reactions has been summarized in much of the relevant literature (see e.g., ref. 4). Many researchers who have tackled the mechanism suggest either the presence or the absence of the microwave effect [5]. The reason why the microwave effect has not been clarified to anyone's satisfaction is that the term microwave effect in microwave chemistry includes numerous factors. Accordingly, the microwave phenomenon, which is not yet well understood, is bundled by the word microwave effect and thus has failed to being classified properly.

Thermal energy can be exchanged instantaneously via several mechanisms such as dipolar polarization, ionic conduction, and the Maxwell-Wagner effect (among others) when a substance absorbs microwaves. Consequently, is the microwave involvement in chemical reactions an electromagnetic wave effect (non-thermal effect) or is it a temperature effect (thermal effect)? Experience has shown that it is difficult to separate between the two. Such problems do not occur in photochemistry. Moreover, microwaves (e.g., at a frequency of $2.45 \mathrm{GHz}$; wavelength $=12.24 \mathrm{~cm}$ ) cause electromagnetic waves to be distributed unevenly in the sample, thereby resulting in a non-uniform heating of the sample [6]. Hence, it is necessary to have a proper appreciation of microwave engineering (e.g., fabrication of a microwave apparatus) and to pay close attention to sample size and microwave irradiation methods. Failure to do so can result in erroneous reporting of microwave effects originating from incorrect temperature measurements. Consequently, it is necessary to implement the following points in order to clarify the microwave effect: (i) elucidate the meaning of the microwave effect, (ii) establish an experimental system from which we can observe the microwave effect on the sample using electromagnetic waves and heat, and (iii) build an experimental system (microwave engineering) such that measurement errors of temperatures are avoided.

To achieve some of the above points, we have examined photocatalyzed reactions enhanced by microwaves for nearly two decades during which we demonstrated the microwave effect by simultaneously irradiating the metal-oxide photocatalyst $\mathrm{TiO}_{2}$ with UV light and microwave radiation in reactions taking place during wastewater treatments, something that could not and cannot be achieved by conventional heating [7]. As a non-thermal effect, the microwave effect in photocatalyzed reactions was demonstrated by establishing that the lifetime and utilization efficiency of electrons excited by UV light in the photocatalyst are enhanced by the microwave radiation [8]. Other researchers have also investigated such phenomena. For instance, Kishimoto and coworkers reported that an applied microwave 
field can enhance the photocatalytic reduction of bipyridinium ion using $\mathrm{CdS}$ quantum dots (QDs) via an acceleration of the electron transfer process [9].

In this paper, the use of "microwave energy is introduced as electromagnetic energy" in the chemical, environmental, biological and food fields, taking photocatalytic reactions as an example. On the other hand, microwaves can deliver energy to matter in pulses [10]. The pulsed microwave irradiation will also deal with various phenomena caused by irradiation in chemical reaction and enzyme reaction. The use of microwave energy as these electromagnetic waves is made possible by using a semiconductor generator.

\section{Results and discussions}

\subsection{Coupled Microwave/Photoassisted Methods for Environmental Remediation}

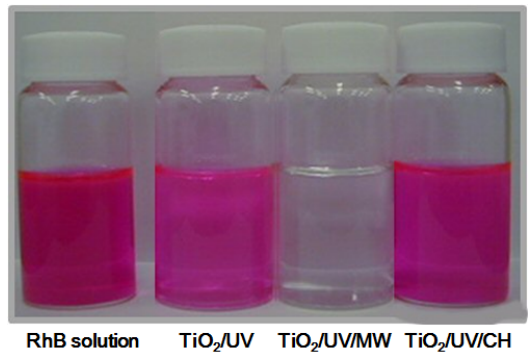

Figure 1. Visual comparison of color fading in the degradation of RhB solutions $(0.05 \mathrm{mM})$ subsequent to being subjected to various degradation methods for $150 \mathrm{~min}$.

From left to right: initial RhB solution; RhB subjected to photoassisted degradation (UV); RhB subjected to integrated microwave-/photo-assisted degradation (UV/MW); $\mathrm{RhB}$ subjected to thermal- and photo-assisted degradation $(\mathrm{UV} / \mathrm{CH})$. Reproduced from [18]. Copyright 2009 by Elsevier B.V.

The photoassisted oxidative (and reductive) decomposition of pollutants by means of $\mathrm{TiO}_{2}$ semiconductor nanoparticulates is an effective and attractive oxidation (reduction) method in the general area of Advanced Oxidation Technologies. Several review articles have appeared that summarize environmental protection using $\mathrm{TiO}_{2}$ materials as the photomediators, if not as photocatalysts [11-14]. Applications of photoassisted treatments to air pollution have been developed by $\mathrm{TiO}_{2}$ fixation on such suitable substrate supports as filters in air conditioners, for instance [15]. However, this photoassisted degradation methodology is not suitable for large-scale wastewater treatment because the degradation rates of organic compounds dissolved in wastewaters tend to be rather slow. In this regard, relatively little has been done in this area in the last several decades as large-scale treatments of organic pollutants in aquatic environments have not been without some problems, not least of which is the low photodegradation efficiency, a result of several factors.

The rate of photodegradation of the cationic dye rhodamine- $\mathrm{B}(\mathrm{RhB})$ is slow in acidic aqueous media because the surface of $\mathrm{TiO}_{2}$ particles is positively charged $\left(\mathrm{Ti}-\mathrm{OH}_{2}{ }^{+}\right.$; $\mathrm{pI}=6.3$ ). However, $\mathrm{RhB}$ has proven as an interesting model compound to examine the microwave effect. In earlier studies, our major focus was on the degradation of organic pollutants, as exemplified by the degradation of the rhodamine- $\mathrm{B}(\mathrm{RhB})$ dye catalyzed by $\mathrm{TiO}_{2}$ semiconductor particles under both UV and microwave irradiation $[16,17]$. Changes in color intensity of the $\mathrm{RhB}$ dye solutions occurring under various conditions are illustrated in Figure 1. The photodegradation of $\mathrm{RhB}$ is clearly evident on using the $\mathrm{TiO}_{2}$-assisted $\mathrm{UV}$ 
and microwave (UV/MW) method. These observations demonstrate that a method that can treat large quantities of pollutants in wastewaters by a hybrid combination of microwaves and $\mathrm{TiO}_{2}$ photoassisted technologies is conceivable. The photodegradation by this metal oxide is unaffected by conventional heating $(\mathrm{CH})$; compare, for example, the results from the UV and the $\mathrm{UV} / \mathrm{CH}$ methods in the presence of $\mathrm{TiO}_{2}$ (Figure 1) [18].

An integrated microwave-/photoassisted methodology presents certain advantages in wastewater treatment. In this technique, a feature of the reaction on the $\mathrm{TiO}_{2}$ surface involves thermal and specific effects (e.g., non-thermal effects) originating from the absorption of microwave radiation by the metal-oxide nanoparticulates. Differences between various $\mathrm{TiO}_{2}$ batches with regard to microwave-specific effect(s) were examined using microwaves of different frequencies [19], and by examining the effects of the microwaves' magnetic and electric fields [20] in photoassisted processes involving $\mathrm{TiO}_{2}$ and $\mathrm{ZnO}$ nanomaterials. The microwave specific effect, not encountered when the photocatalyzed reaction is subjected to conventional heating, inferred that the composition and the electronic characteristics of the $\mathrm{TiO}_{2}$ nanomaterials were important factors. We researched from various angles other than this. Among them, what is particularly remarkable is that they are not responsive to microwaves except for Evonik $\mathrm{P} 25 \mathrm{TiO}_{2}$ particle (commerciale $\mathrm{TiO}_{2}$ particles).

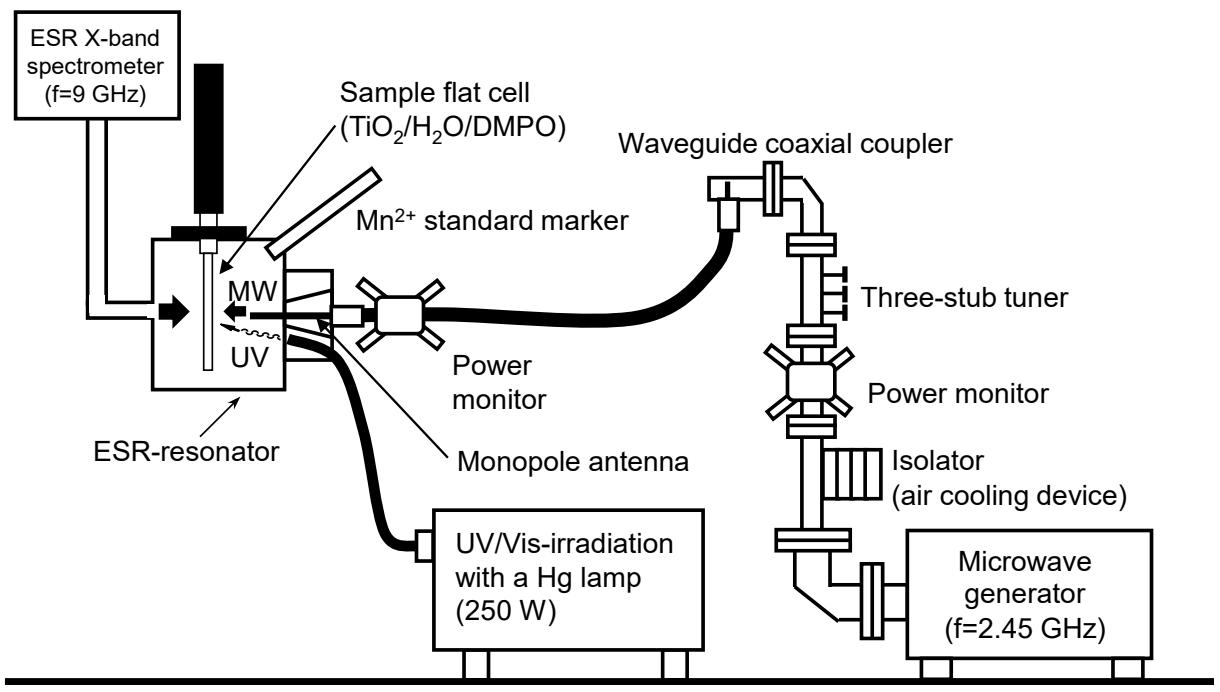

Figure 2. Setup used to generate $\cdot \mathrm{OH}$ radicals in water alone under $\mathrm{MW}$ irradiation, in an aqueous $\mathrm{TiO}_{2}$ dispersion by $\mathrm{MW}$ irradiation alone, and by the UV and UV/MW methods. Reproduced from [21]. Copyright 2003 by Elsevier B.V.

For the photocatalytic reaction mechanism, Titanium dioxide has a bandgap of $3.20 \mathrm{eV}$ (anatase crystal), which corresponds to a wavelength of $387 \mathrm{~nm}$. This means that electronhole pairs are created when $\mathrm{TiO}_{2}$ is radiated with UV-light with wavelengths shorter than $387 \mathrm{~nm}$ (Reaction (1)). To the extent that not all the photogenerated electrons and holes recombine, some of the holes can migrate to the surface and react with surface-bound -OH groups and/or water molecules surrounding the particles that lead ultimately to the formation of hydroxyl radicals (Reaction (2)). Dissolved oxygen molecules react with conduction band electrons $\left(\mathrm{e}^{-}\right)$to yield superoxide radical anions $\left(\mathrm{O}_{2}^{-\bullet}\right.$; 
Reaction (3)), which on protonation generate the hydroperoxy radicals ${ }^{\bullet} \mathrm{OOH}$ (Reaction (4)). Accordingly, the photooxidation of organic substrates with the $\mathrm{UV} / \mathrm{TiO}_{2}$-driven photoassisted process (Reaction (5)) depends on the concentration of ${ }^{\bullet} \mathrm{OH}\left(\right.$ and/or $\left.{ }^{\bullet} \mathrm{OOH}\right)$ radicals produced by the photooxidation of surface hydroxyl groups and/or chemisorbed $\mathrm{H}_{2} \mathrm{O}$. Here, the following questions arise. Does the amount of ${ }^{\bullet} \mathrm{OH}$ radicals generated from $\mathrm{TiO}_{2}$ change under microwave irradiation?

$$
\begin{aligned}
& \mathrm{TiO}_{2}+\mathrm{h} v \rightarrow \mathrm{TiO}_{2}\left(\mathrm{e}^{-}+\mathrm{h}^{+}\right) \rightarrow \mathrm{e}^{-}+\mathrm{h}^{+} \\
& \mathrm{h}^{+}+{ }^{-} \mathrm{OH}_{\text {surf. }}\left(\text { and } / \text { or } \mathrm{H}_{2} \mathrm{O}\right) \rightarrow{ }^{\bullet} \mathrm{OH}\left(+\mathrm{H}^{+}\right) \\
& \mathrm{e}^{-} \text {cb }+\mathrm{O}_{2} \rightarrow \mathrm{O}_{2}^{-} \cdot \\
& \mathrm{O}_{2}{ }^{-}+\mathrm{H}^{+} \rightarrow \cdot{ }^{\bullet} \mathrm{OOH}
\end{aligned}
$$

$\bullet \mathrm{OH}\left(\right.$ or $\left.^{\bullet} \mathrm{OOH}\right)+$ organic pollutant $\rightarrow$ Oxidative products

The possible enhancement of the photoactivity of metal-oxide specimens subsequent to being exposed to microwave radiation from the viewpoint of the amount of - $\mathrm{OH}$ radicals generated was also investigated [21]. Formation of ${ }^{\bullet} \mathrm{OH}$ radicals during $\mathrm{TiO}_{2}$ assisted photooxidations that were driven simultaneously by UV light and microwave radiation was probed by electron spin resonance spectroscopy employing a novel setup in which the ESR sample (contained the DMPO spin-trap agent and $\mathrm{TiO}_{2}$ particles in aqueous media) was irradiated by both UV light and microwave radiation [21]. Figure 2 shows a special ESR system that can observe ${ }^{\bullet} \mathrm{OH}$ radicals in situ while simultaneously irradiating microwaves and ultraviolet light.

The number of ${ }^{\bullet} \mathrm{OH}$ radicals generated under various experimental conditions is summarized in Table 1. For P25 titania, the number of ${ }^{\circ} \mathrm{OH}$ radicals produced by the UV/MW method was nearly $30 \%$ greater than the quantity generated by the UV method alone [21]. A fivefold increase in incident microwave power from 3 to $16 \mathrm{~W}$ led to a significant increase ( $\mathrm{ca} .40 \%)$ in the number of ${ }^{\circ} \mathrm{OH}$ radicals. Such an increase was sufficient to increase the efficiency of the photooxidation of the organic pollutant in water.

Table 1. Number of DMPO- ${ }^{\circ} \mathrm{OH}$ spin adducts produced in the various heterogeneous systems under microwave irradiation, UV irradiation, and $\mathrm{MW} / \mathrm{UV}$ irradiation relative to those formed in the rutile $\mathrm{TiO}_{2}$ specimen for the $\mathrm{TiO}_{2} / \mathrm{H}_{2} \mathrm{O} / \mathrm{MW}$ heterogeneous system. Reproduced from [21]. Copyright 2003 by Elsevier B.V.

\begin{tabular}{|c|c|c|c|c|}
\hline & P25 & UV100 & Anatase & Rutile \\
\hline UV & 182 & 45 & 110 & 110 \\
\hline $\begin{array}{c}\text { UV/MW } \\
(3 \mathrm{~W})\end{array}$ & 259 & 51 & 92 & 76 \\
\hline $\begin{array}{c}\text { UV/MW } \\
(16 \mathrm{~W})\end{array}$ & 369 & - & - & - \\
\hline
\end{tabular}


For the UV100 $\mathrm{TiO}_{2}$ sample (anther commercial $\mathrm{TiO}_{2}$ particles), the increase in the number of ${ }^{\bullet} \mathrm{OH}$ radicals produced was only $10 \%$ greater on increasing the MW power five times. On the other hand, the number of ${ }^{\bullet} \mathrm{OH}$ radicals generated for the pristine anatase and rutile $\mathrm{TiO}_{2}$ samples decreased under microwave irradiation. The P25 specimen was clearly influenced by the microwaves and generated ${ }^{\bullet} \mathrm{OH}$ radicals efficiently under the influence of microwave effects. Therefore, the rate of decomposition was enhanced when P25 was used to decompose the wastewater sample by the UV/MW method. On the other hand, to the extent that the quantity of $\bullet \mathrm{OH}$ radicals produced by the other $\mathrm{TiO}_{2}$ does not increase even when irradiated with microwaves, the rate of decomposition is not enhanced.

Why can only Evonik P25 $\mathrm{TiO}_{2}$ particles benefit from microwaves? P25 might be a hint that it is a mixed crystal of anatase and rutile. The effects of $2.45-\mathrm{GHz}$ microwave radiation on the Raman-active lattice phonons of two selected (Wako) anatase and rutile specimens and on Degussa P-25 $\mathrm{TiO}_{2}$ nanoparticles were probed by in-situ microscopic Raman spectroscopy in the solid phase with samples subjected to microwave irradiation (MW) or in combination with UV illumination (UV/MW) [22]. Significant changes were seen in the Raman band intensities for the pure anatase $E_{g}$ mode at $143 \mathrm{~cm}^{-1}$ and for the rutile vibration at $446 \mathrm{~cm}^{-1}$, whereas only negligible changes in intensity were observed for the $144 \mathrm{~cm}^{-1}$ band of P-25 nanoparticles exposed to MW and UV/MW radiation. It is deduced that microwaves have a negligible impact on the lattice vibrational modes (phonons) of P-25 titania, even though this specimen was thermally heated by the microwaves. The photodegradation of phenol was re-visited to examine the photoactivity of the $\mathrm{TiO}_{2}$ specimens selected for the $i n$-situ Raman study, as attested by the number of $\bullet \mathrm{OH}$ radicals produced (DMPO spin trap ESR) under the UV versus UV/MW irradiations, and by the related enhanced dynamics under UV/MW irradiation relative to $\mathrm{UV}$ alone or $\mathrm{UV} / \mathrm{CH}$. Figure 3 shows a special Raman spectroscopy system that can observe Raman spectral radicals in situ while simultaneously irradiating microwaves and ultraviolet light.

Scheme 1 illustrates a simplistic view of the proposed effects of microwave and microwave/ultraviolet irradiations on the anatase and rutile polymorphs. Under MW/dark conditions, we deduce that the Raman intensities decrease owing to formation of oxygen vacancies $\left(V_{O}\right)$ induced by the microwave radiation fields as attested for the $\mathrm{P}-25 \mathrm{TiO}_{2}$ sample yielding $F$-type color centers [23]. By contrast, under MW/UV irradiation the Raman intensities promptly increase at first, owing to the UV photogenerated mobile charge carriers in the conduction band (electrons) that lead to an overall increase in polarizability, subsequent to which the intensity ratios decrease back to ca. 1 for anatase and close to 1 for rutile owing to opposing effects between generation of photoelectrons by UV and formation of oxygen vacancies (or other defects) by the microwave radiation fields that trap the electrons to form color centers such as $F$-centers and $\mathrm{Ti}^{3+}$ centers [23]. 


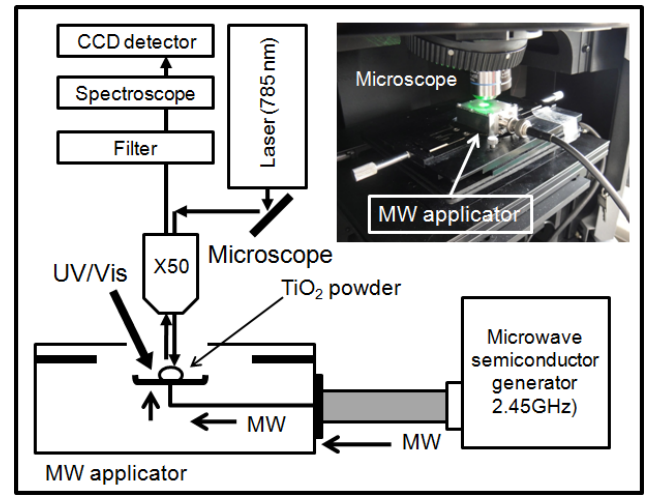

Figure 3. Experimental setup for the in-situ Raman spectroscopy of $\mathrm{TiO}_{2}$ powdered specimens using an integrated Microwave and UV-Vis radiation system. Reproduced from [22]. Copyright 2003 by Elsevier B.V.

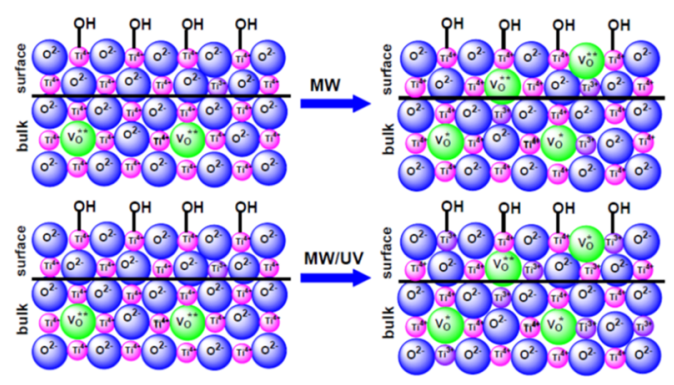

Scheme 1. Simplified view of the effects of microwave and microwave/ultraviolet irradiation on the anatase and rutile polymorphs that lead to formation of $\mathrm{F}$ and $\mathrm{Ti3}+$ color centers. Reproduced from [22]. Copyright 2003 by Elsevier B.V.

Unlike the anatase and rutile polymorphs, only negligible changes occurred in the Raman spectral intensities of $\mathrm{P}-25 \mathrm{TiO}_{2}$ under both $\mathrm{MW}$ and $\mathrm{MW} / \mathrm{UV}$ irradiations. That is, the Raman spectral intensities are severely curtailed by the microwave effect with or without concomitant UV radiation. This is likely the result of the nature of the $\mathrm{P}-25 \mathrm{TiO}_{2}$ heteroarchitecture of a rutile domain (or particle) strongly coupled (fused) to anatase [24] that resulted from the manner by which $\mathrm{P}-25$ is produced in the high temperature $\mathrm{H}_{2}$ flame in the presence of oxygen (precursor, $\mathrm{TiCl}_{4}$ ). The interfacial construct between the rutile and the anatase is perhaps the major defect in $\mathrm{P}-25 \mathrm{TiO}_{2}$ which, under applied microwave radiation fields, likely causes the accumulation of photogenerated charge carriers at this interface (interfacial or space-charge polarization [25]).

In summary, we deduce from the presently available data and the above discussion that the microwave energy absorbed by the $\mathrm{P}-25 \mathrm{TiO}_{2}$ system was consumed to bring about changes at the interfacial boundaries between the coupled rutile and anatase polymorphic structures. Further, such changes may lead to generation of oxygen vacancies, well known to trap conduction band electrons to yield $F$-type color centers, thereby allowing the valence band holes to produce additional ${ }^{\circ} \mathrm{OH}$ radicals as evidenced by the ESR results of Table 1, unlike the anatase and rutile samples where the number of ${ }^{\circ} \mathrm{OH}$ radicals decreased on UV/MW combined irradiation relative to UV irradiation alone. 


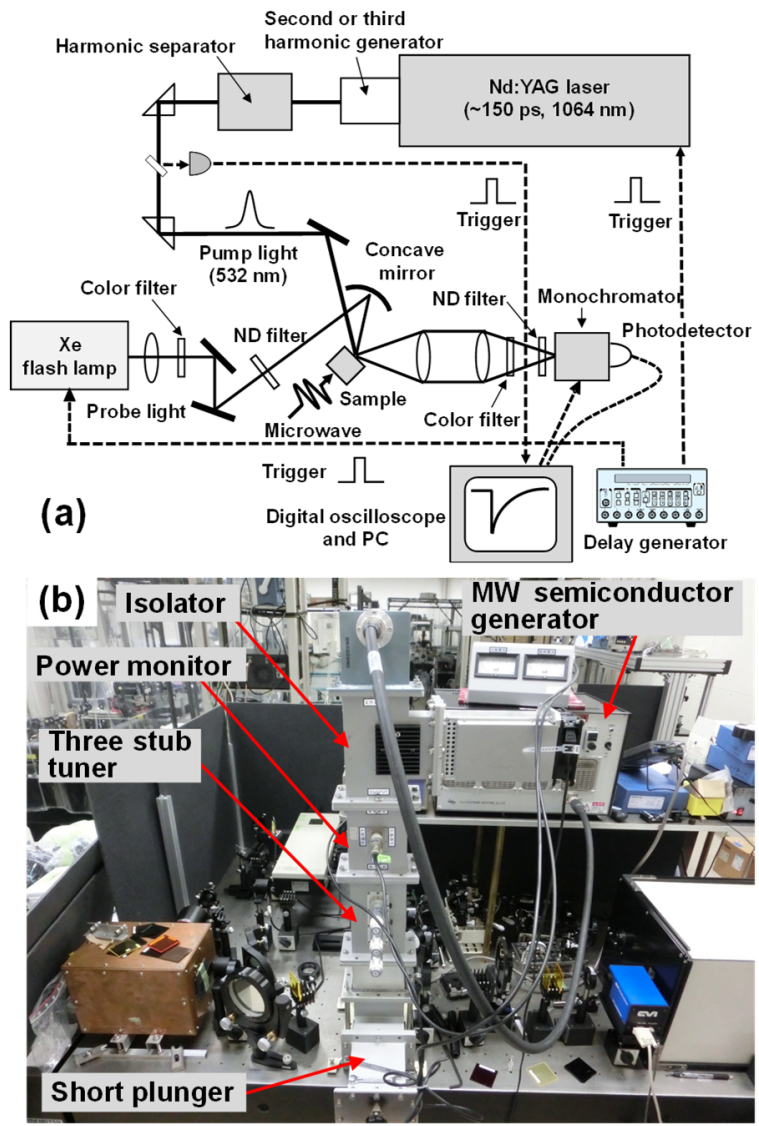

Figure 4. Schematic diagram illustrating the overall diffuse reflectance setup; (b) photograph of the microwave irradiation setup. Reproduced from [26]. Copyright 2015 by Royal Society of Chemistry.

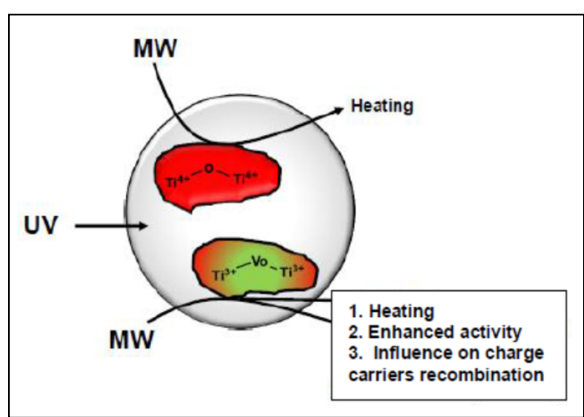

Figure 5. Cartoon depicting the influence of microwave radiation on a regular ST01 particle and on Vo-ST01 particle. Reproduced from [25]. Copyright 2015 by Royal Society of Chemistry. 
The next question is, where does the microwave affect Evonik $\mathrm{P} 25 \mathrm{TiO}_{2}$ particles? Transient decay kinetics at $550 \mathrm{~nm}$ for all three samples were determined in situ using a 150 ps Nd-YAG pulsed laser system $(10 \mathrm{~Hz})$ and a Xe flash lamp (2 ms pulses) probe while samples were being microwave-irradiated (2.45 GHz) [26] (Figure 4). The transient(s) absorbing at the probe wavelength displayed double exponential decay kinetics: a fast decay that occurred within ca. 5-12 ns ascribed to recombination of photogenerated shallowtrapped or free conduction band electrons with valence band holes, and a slower decay that occurred from hundreds of nanoseconds to several microseconds attributable to recombination of electrons trapped in deep traps (e.g., either as $\mathrm{Ti}^{3+}$ or as $\mathrm{F}$ color centers) with free holes.

Contrary to conventional heating, which heats up the titania particle from the surface to the bulk, microwave dielectric heating occurs inside-out from the bulk to the surface while the samples are being simultaneously UV-irradiated. Accordingly, it is not unreasonable to infer that the surface of the titania particles was modified, particularly by the former treatment $(\mathrm{UV} / \mathrm{CH})$. Hence, to the extent that diffuse reflectance spectra probe the surface of the particles, the greater absorption intensity of oxygen vacancies $\mathrm{TiO}_{2}$ subjected to UV/CH is sensible. The presence of oxygen vacancies within the crystal lattice of ceramics has been reported to enhance microwave heating through Joule heating that brings about changes of the microscopic electrical resistance in ceramics. Although conversion of microwave energy to thermal energy may be affected by the presence of oxygen vacancies, it may be possible (under some conditions) to control the recombination of charge carriers and increase the catalyst photoactivity as inferred in Figure 5.

\subsection{Electromagnetic wave effects on living organisms and biomaterials}

The interaction of electromagnetic fields with various life processes has intrigued scientists since the $1800 \mathrm{~s}$. Of current interest are the electromagnetic fields present in microwave radiation, which spans a frequency from $300 \mathrm{GHz}$ to $300 \mathrm{MHz}$ (i.e., from a wavelength of $1 \mathrm{~mm}$ to $1 \mathrm{~m}$ ). Microwaves are used widely in communications and in heating, particularly in the heating of foodstuffs. This non-ionizing electromagnetic radiation is absorbed at the molecular level causing changes in the vibrational energy of the molecules; it also manifests itself as heat [28]. Identifying and evaluating biological effects of microwaves have been rather complex and controversial. In this regard, enzymatic reactions involving microwaves have been the object of active investigations in the latter half of the 20 th century, with the first such study reported in the early 1970s. For instance, microwave radiation to inactivate the enzymes in the brain of animals has been used widely since Stavinoha and coworkers first introduced it in 1970 [29]. Inactivating the enzymes makes it possible to sample and measure many enzymatically destroyed brain neurochemicals thereby reducing the influence of postmortem changes [30]. Usage of microwaves has succeeded in stopping enzymatic activity selectively with minimal damage to other cells and/or proteins under in vivo conditions, for which equipment is commercially available.

Aging and quality improvement of meat [31]: Our research strategy is an attempt to discover phenomena that other energies cannot imitate and apply them to the food sector. In particular, because of the need for control of these precise microwaves, semiconductorbased generators are very effective microwave sources. One experimental example of our research using direct heating of a substance by microwaves and an electromagnetic wave effect is shown in Figure 6. The papain enzyme, used this research as a model enzyme, is a cysteine protease enzyme largely used as a meat tenderizer via the breakdown of tough meat fibers, thereby rendering the meat easier to cook. This enzyme is used extensively in the 
textile, pharmaceutical, and cosmetic industries. If the enzymatic activity of papain could be enhanced by the microwaves' electromagnetic fields, it would lead to significant industrial benefits.

Possible changes in the degree of enzymatic activity in the papain-assisted hydrolysis of proteins present in beef samples were examined by comparing microwave heating relative to conventional heating [4]. The hydrolysis in beef samples whose surface was covered with the papain enzyme was used as the model process $(20 \times 20 \times 50 \mathrm{~mm}$ rectangularly-shaped specimens). The papain-treated beef samples were subjected to pulsed microwave irradiation (PMI; 20 -millisecond pulses microwave irradiation; Figure 6b) and to continuous-wave microwave irradiation (CMI; continuous microwave irradiation Figure $6 \mathrm{c}$ ). Under microwave heating, the papain-treated samples of Figure $6 \mathrm{~b}$ and Figure $6 \mathrm{c}$ softened in a manner suggestive of liquefied jelly when compared to the non-irradiated papain-free initial sample (Figure 6a). That is, it shows that exposing the papain-coated beef specimens to microwave heating enhanced the decomposition of the protein(s) on the beef surface. We also observed that the degree of softness of the beef surface under PMI conditions was, to some extent, greater than under CMI. By contrast, upon being subjected to heating in an electric furnace at $45^{\circ} \mathrm{C}$ for $10 \mathrm{~min}$, the papain-treated beef sample of Figure $6 \mathrm{~d}$ softened much less than under microwave irradiation. Moreover, after the conventional heat treatment, the specimen's surface was unlike the jelly-like surface displayed by the samples of Figure $6 \mathrm{~b}$ and Figure $6 \mathrm{c}$. Experiments were also conducted at $50^{\circ} \mathrm{C}\left(+5^{\circ} \mathrm{C}\right)$ and $40^{\circ} \mathrm{C}\left(-5^{\circ} \mathrm{C}\right)$ in the electric furnace to examine whether errors in temperature measurements could explain this difference. There were no differences at both these temperatures. Accordingly, despite the same temperature conditions $\left(45^{\circ} \mathrm{C}\right)$, the microwaves promoted the activity of papain on the beef surface that we attribute to the microwaves' electromagnetic fields.

Plant growth promotion technology: Microwave-induced growth acceleration of plants is reported in this research. The response of plants (Arabidopsis thaliana) to microwave irradiation was investigated to specify the conditions that enhanced plant growth. Microwave irradiation accelerated the phase transition from vegetative to reproductive growth, the growth of the inflorescence stem, as well as seed germination. However, microwave irradiation did not affect plant diameter. In addition, it was found that microwave irradiation enhanced the expression of a gene that regulates growth phase transition, suggesting that microwave irradiation could indeed enhance the growth of plants by modulating the expression of gene. On the other hand, the expression of genes involved in the response of plants to heat stress were not clearly altered by microwave irradiation. Our results suggest that the effects of microwave irradiation on plant growth is not from the thermal effect. By understanding the key factor behind the microwave-dependent growth stimulation, it makes it possible to apply this finding to the growth of other plants (potato, etc). 


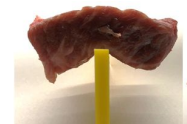

(a)

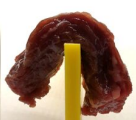

(b)

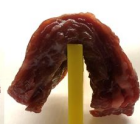

(c)

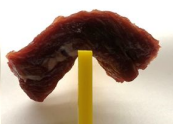

(d)

Figure 6. Observations of the surface changes and the degree of beef samples after applying papain to the beef: (a) control experiment with no papain used on the beef, (b) papain-treated beef sample after heating with pulsed microwave irradiation (PMI) and maintaining it at $45^{\circ} \mathrm{C}$ for $10 \mathrm{~min}$ (c) papain-treated beef sample subjected to continuous microwave irradiation (CMI) under otherwise identical conditions as in (b), and (d) papain-treated beef sample subjected to heating in an electric furnace at $45^{\circ} \mathrm{C}$ for 10 min. Reproduced from [33]. Copyright 2019 by Nature Springer.

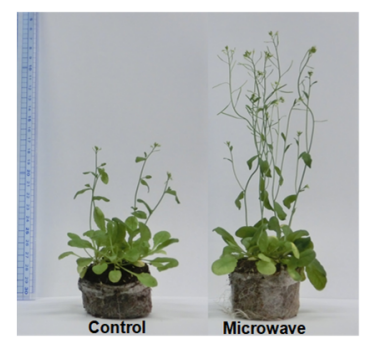

Figure 7. Grown comparison of Arabidopsis thaliana with no radiation (control) and microwave radiation (Microwave)

When either microwave heating or conventional heat treatment was applied to A thaliana, a significant change in the growth of the leaves could not be observed. However, the shift to the reproductive stage and the growth of the inflorescence stem were dramatically promoted in the microwave treatment condition (Figure 7). Because such phenomena were not observed under ordinary high temperature $\left(40^{\circ} \mathrm{C}\right)$ conditions, a specific effect that was only observed in the case of microwave irradiation was expected. When the expression of the gene that regulates growth or stress response was investigated, the expression of the gene which controls the growing stage was affected by microwave irradiation.

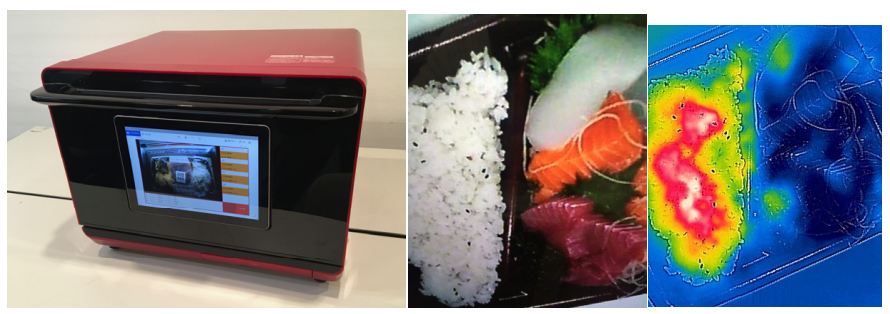

Figure 8. (Top) Photo of intelligent cooker, (bottom left) photo of Sushi (Raw fish) lunch box, (bottom right) The rice warms up to the set temperature and the sushi (raw fish) doesn't warm up at all (thermographic image). 
Intelligent cooker: Currently, eating habits taking advantage of individual personality advances, food of various kinds and form is being sold. On the other hand, the advances of food heating method is not added to most meals. Especially the microwave oven is convenient to heat cooked food in supermarkets and convenience stores. However, it is impossible for individuals to set the temperature, and heating of the microwave oven is adjusted by the arrangement on the food, water concentration and salt concentration. In order to practice this innovation, we switched the microwave source of the microwave oven to the RF high power semiconductor generator (semiconductor type generator) (Figure 8). There have been attempts to utilize semiconductor type generator for microwave from long ago, but there was no one available with the size and price of the device, and it could not be put into practical use. However, in recent years we succeeded in ultra-miniaturization of the generator, and it is estimated that selling price will be sold at the same price as the current price if mass-produced. We will introduce "intelligent cooker" such as new microwave cooking oven and new warm catering cart that operate by partial heating of foods in lunch box and hospital plate meal using semiconductor type generator. Furthermore, it also shows that combinations with internet of things (IoT) are effective when implementing and sale at a market.

\section{References}

[1] C. F. Buchholz, Catechismus d. Apothekerkunst. Erf. 1810, Vermehrt herausgeg. v. Brandes. Erfurt 1820, 2 Bde.

[2] J. G. Calvert, J. N. Pitts, Photochemistry. Wiley \& Sons; New York, USA, 1966.

[3] S. Horikoshi, R. F. Schiffmann, J. Fukushima, N. Serpone, Microwave chemical and materials processing, A tutorial. Springer, Japan, 2017; ISBN 978-981-10-6465-4.

[4] M. A. Rodriguez, P. Prieto, A. de la Hoz, Ý. Diaz-Ortiz, D. R. Martin, J. I. Garcia, ChemistryOpen, 2015, 4, 308-317.

[5] J. Ma, J. Phys. Chem. A, 2016, 120, 7989-7997.

[6] T. Razzaq, J. M. Kremsner, C. O. Kappe, J. Org. Chem., 2008, 73, 6321-6329.

[7] S. Horikoshi, N. Serpone, Catal. Today, 224, 225-235 (2014).

[8] S. Horikoshi, H. Tsutsumi, H. Matsuzaki, A. Furube, A. V. Emelinec, N. Serpone, J. Mater. Chem. C, 2015, 3, 5958-5969.

[9] F. Kishimoto, T. Imai, S. Fujii, D. Mochizuki, M. M. Maitani, E. Suzuki, Y. Wada, Sci. Rep., 2015 5, 11308.

[10] S. Horikoshi, T. Watanabe, A. Narita, Y. Suzuki, N. Serpone, Sci. Rep., 2018, 8:5151, DOI:10.1038/s41598-018-23465-5.

[11] K. Hashimoto, H. Irie, A. Fujishima, Jpn. J. Appl. Phys., 2005, 44, 8269-8285.

[12] K. Kabra, R. Chaudhary, R. L. Sawhney, Ind. Eng. Chem. Res., 2004, 43, 7683-7696.

[13] K. Konstantinou, T. A. Albanis, Appl. Catal. B: Environ., 2003, 42, 319-335.

[14] D. S. Bhatkhande, V. G. Pangarkar, A. A. Beenackers, J. Chem. Technol. Biotechnol., 2002, 77, 102-116. 
[15] A. Fujishima, K. Hashimoto, T. Watanabe, $\mathrm{TiO}_{2}$ Photocatalysis: Fundamentals and Applications; BKC Incorporated: Tokyo, Japan, 1999.

[16] S. Horikoshi, H. Hidaka, N. Serpone, Environ. Sci. Technol., 2002, 36, 1357-1366.

[17] S. Horikoshi, H. Hidaka, N. Serpone, Environ. Sci. Technol., 2002, 37, 5813-5822.

[18] S. Horikoshi N. Serpone, J. Photochem. Photobiol. C: Photochem. Rev., 2009, 10, 96110.

[19] S. Horikoshi, F. Sakai, M. Kajitani, M. Abe, N. Serpone, Chem. Phys. Lett., 2009, 470, 304-307.

[20] S. Horikoshi, A. Matsubara, S. Takayama, M. Sato, F. Sakai, M. Kajitani, M. Abe, N. Serpone, Appl. Catal. B: Environ., 2009, 91, 362-367.

[21] S. Horikoshi, H. Hidaka, N. Serpone, Chem. Phys. Lett., 2003, 376, 475-480.

[22] S. Horikoshi, M. Abe, S. Sato, N. Serpone, J. Photochem. Photobiol. C: Photochem. Rev., 2011, 220, 94-101.

[23] V. N. Kuznetsov, N. Serpone, J. Phys. Chem. C, 2009, 113, 15110-15123.

[24] S.A. Chambers, C. M. Wang, S. Thevuthasan, T. Droubay, D. E. McCready, A. S. Lea, V. Shutthanandan, C. F. Windisch, Thin Solid Films, 2002, 418, 197-210.

[25] S. K. Arora, V. Patel, B. Amin, A. Kothari, Bull. Mater. Sci., 2004, 27, 141-147.

[26] S. Horikoshi, H. Tsutsumi, H. Matsuzaki, A. Furube, A. V. Emeline, N. Serpone, J. Mater. Chem. C, 2015, 3, 5958-5969.

[27] A. V. Emeline, L. G. Smirnova, G. N. Kuzmin, L. L. Basov, N. Serpone, J. Photochem. Photobiol., A, 2002, 148, 97-102.

[28] S. Banik, S. Bandyopadhyay, S. Ganguly, Bioresource Technol., 2003, 87, 155-159.

[29] W. B. Stavinoha, B. Pepelko, P. W. Smith, Pharmacologist ,1970, 12, 257.

[30] W. B. Stavinoha, S. T. Weintraub, A. T. Modak, J. Neurochem., 1973, 20, 361-371.

[31] S. Horikoshi, K. Nakamura, M. Yashiro, K. Kadomatsu, N. Serpone, Sci. Rep., 2019, 9:8945 | https://doi.org/10.1038/s41598-019-45152-9. 
17th International Conference on Microwave and High Frequency Heating

\section{Plasma processing induced by microwaves}
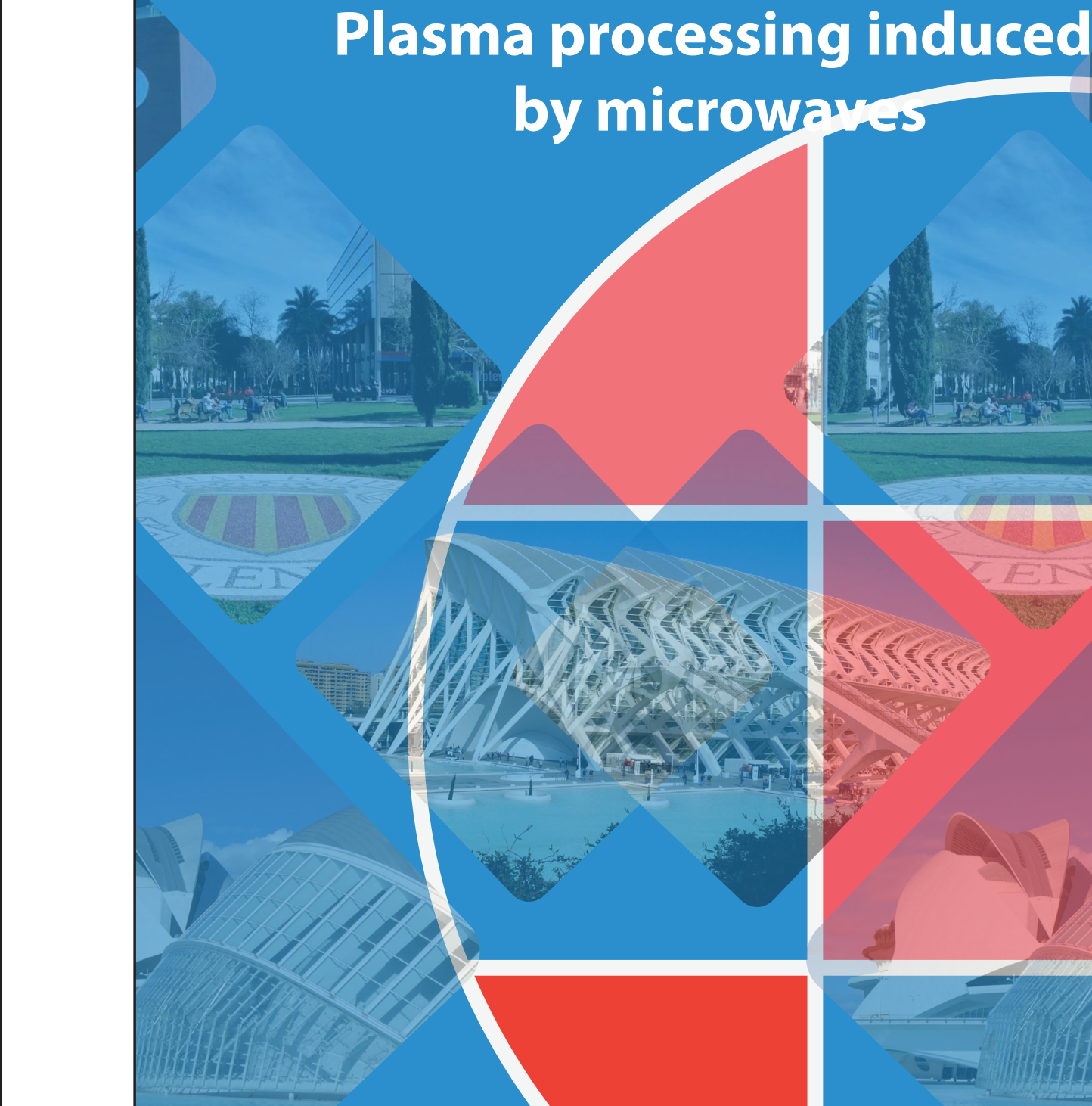


\title{
GENERATION AND MODELING OF GASEOUS PLASMAS USING MICROWAVE POWER
}

\author{
M. Moisan ${ }^{1}$, H. Nowakowska ${ }^{2}$ \\ ${ }^{1}$ Groupe de physique des plasmas, Université de Montréal, Montréal H3C 3J7, Québec \\ ${ }^{2}$ Institute of Fluid Flow Machinery, Polish Academy of Sciences, 80-952, Gdansk, Poland \\ Michel.moisan@umontreal.ca
}

Keywords: microwave sustained plasmas, modeling, power balance, similarity laws

\section{Introduction}

In contrast to RF produced plasmas, in the case of microwave (MW) sustained plasmas the energy from the electromagnetic (EM) field is communicated only to electrons since ions, being a few thousand times much heavier than electrons, cannot respond to the periodic changes in the direction of the $\boldsymbol{E}$-field of microwaves (frequency range $300 \mathrm{MHz}-300 \mathrm{GHz}$ ) and therefore cannot gain energy in the EM field. The energy thus acquired by electrons is essentially transferred to heavy particles either through collisional absorption (high enough gas pressures) or, in the presence of a static applied magnetic field, by collisionless (lowpressure: $10^{-4}-10^{-3}$ mbar) absorption through electron-cyclotron resonance (ECR), providing in both cases excitation and ionization of the discharge gas. In order to analyze the power transfer from the EM $\boldsymbol{E}$-field, instead of the usual global power balance, it is much more direct and informative, as will be discussed, to turn to the concept of power per electron, more specifically of power absorbed per electron $\theta_{\mathrm{A}}$ and of power loss in the plasma on a per electron basis $\theta_{\mathrm{L}}$.

This approach was initially based on the experimental results from Glaude et al. [1] who, when characterizing the properties of surface-wave sustained plasmas (at $300 \mathrm{MHz}$ ), noticed that electron density (under ambipolar diffusion regime) was proportional to absorbed microwave power. Another important step was later on contributed by Zakrzewski [2] and Ferreira [3] who, considering the global power balance, underlined that, under steady-state conditions, the level of MW power absorbed simply adjusts to compensate for plasma losses. Losses are finally mainly due to the recombination of charged particles (electrons, ions) on tube walls, which depend on operating conditions ${ }^{1}$.

The properties of surface-wave discharges will be utilized in what follows to illustrate the discharge modelling developed, in the end suitable for MW discharges in general, and, also, to provide corresponding examples. Figure 1 displays a schematic representation of the surface-wave launcher with its EM field interstice (a 2-3 mm wide gap in the case of a surfatron), which is the essential part of the field applicators used to achieve tubular surfacewave discharges (SWDs). The EM field emerging from this interstice allows generating a plasma column sustained ultimately by an EM surface wave in both directions from the gap ${ }^{2}$. However, the SWDs do not start at the wave launcher immediate exit, but at some distance from it [4].

\footnotetext{
${ }^{1}$ Operating conditions are: nature and pressure of discharge gas, EM field frequency, and discharge tube inner and outer diameters as well as permittivity. Absorbed MW power is excluded.

${ }^{2}$ The back column emerging from the surfatron interstice is much shorter in length than the forward column as a result of the presence of the surfatron body, a tight conducting enclosure surrounding the discharge tube.
} 


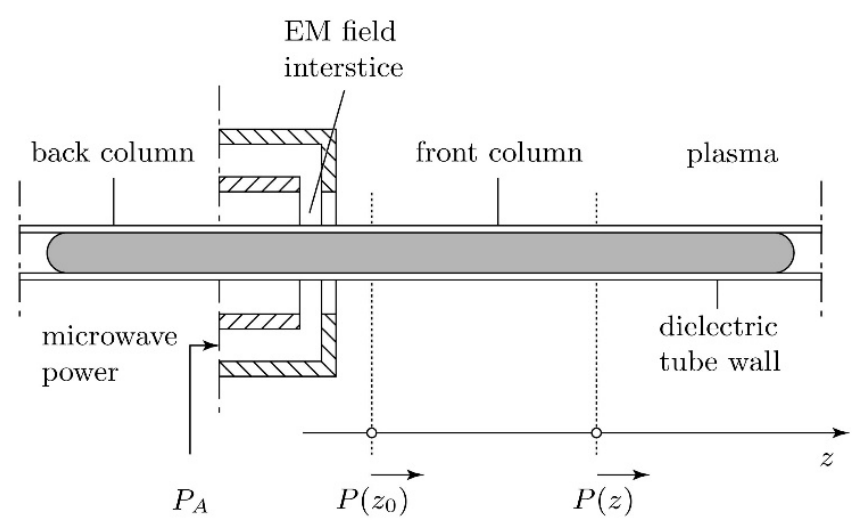

Figure 1. Schematic representation of an EM field applicator with a circular aperture (of the type developed in Montréal) used for achieving a tubular SW discharge, highlighting its essential part, namely the EM field radiating interstice, typically here a 2-3 mm wide gap. As a result of the EM field emerging from the interstice, a SW is launched in both directions (past the $P\left(z_{0}\right)$ point for the front wave), sustaining a plasma column enclosed in a dielectric tube. The impedance matching system of the feed line with the power generator is not represented.

\section{An example of a SW sustained plasma column}

Figure 2 shows photographs of the plasma column generated at atmospheric pressure in argon with a $915 \mathrm{MHz}$ surfatron in a $6 / 8 \mathrm{~mm}$ id/od fused silica tube. The plasma column extends away from the $\boldsymbol{E}$-field applicator as a result of an EM SW using the plasma column and its dielectric medium as the propagating medium. In a), there is no surrounding Faraday cage (FC) at all while in b) and c), the discharge is enclosed in a $22.5 \mathrm{~mm}$ radius FC ensuring wave cut-off in a circular waveguide ${ }^{3}$. The cage length in b) is $30 \mathrm{~mm}$, which was found to be the minimum FC length averting space-wave radiation in the room (see further) from affecting much our measurements; the axial slot in the FC allows making field intensity and spectroscopic measurements along the plasma column. In c), the FC length is longer than the plasma column. Absorbed power being $300 \mathrm{~W}$ in each photo, clearly, the plasma column length is the longest with the full length FC: this is because the power loss due to spacewave radiation generated by the $\boldsymbol{E}$-field applicator has been confined within the FC, giving rise to additional electrons [6].

\footnotetext{
${ }^{3}$ A circular waveguide enclosing (coaxially) at cut-off (on its fundamental mode) the plasma tube prevents waves from propagating within this conducting cage (but not the SW, which uses the discharge tube and the plasma as its propagating medium). The fundamental mode of a circular waveguide (i.e., the lowest frequency at which a wave can propagate within it) is the $\mathrm{TE}_{11}$ mode. The minimum FC radius to achieve cut-off is given by $R_{\mathrm{Fc}(\mathrm{co})}=1.841 \lambda_{0} / 2 \pi$, where $\lambda_{0}$ is the free-space wavelength. At $915 \mathrm{MHz}$, a circular waveguide (acting as a Faraday cage) with a radius smaller than $R_{\mathrm{Fc}(\mathrm{co})}=96.1 \mathrm{~mm}$ is at cut-off, i.e., no wave can propagate within it. At $2450 \mathrm{MHz}, R_{\mathrm{Fc}(\mathrm{co})}$ is $35.9 \mathrm{~mm}$ [5].
} 


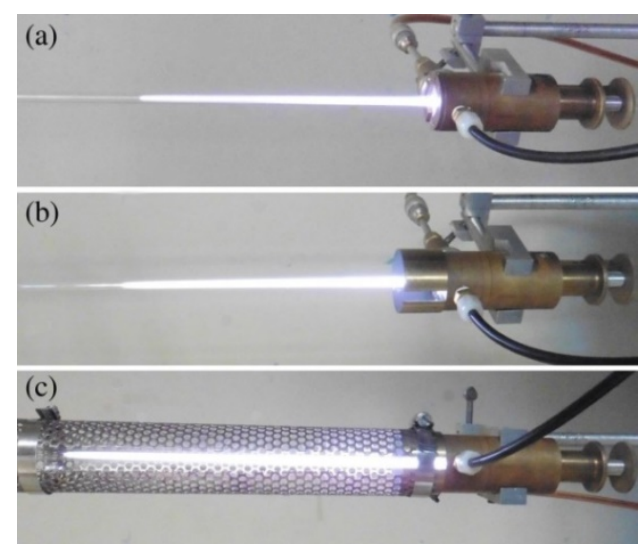

Figure 2. Photographs showing the plasma column obtained with a $915 \mathrm{MHz}$ surfatron: a) with no surrounding Faraday cage at all; b) and c) when enclosed in a $22.5 \mathrm{~mm}$ radius FC corresponding to wave cut-off in a circular waveguide. In b), the cage length is $30 \mathrm{~mm}$ while in c) it is $305 \mathrm{~mm}$ long, extending beyond the present plasma column length. The MW power coupled to the surfatron is $300 \mathrm{~W}$ in each photo [6].

\section{Defining the power absorbed per electron by considering the decrease of power flow along a SW sustained plasma column}

Figure 3 shows the axial distribution of electron density along a SWD at reduced-gas pressure and at different applied-field frequencies. The arrows in the figure point at the value of the power flowing out from the surfatron interstice: at $100 \mathrm{MHz}$, it indicates that raising the MW power from 36 to $58 \mathrm{~W}$ simply increases the plasma column length without affecting the properties of the plasma column pre-existing at $36 \mathrm{~W}$, and thus that the added power all went into the extension of the previously created plasma column. From this, we infer that any increase in wave power from $P_{\mathrm{a}}(z)$ to $P_{\mathrm{a}}(z+\mathrm{d} z)$ is absorbed in the differential segment of plasma column $z, z+\mathrm{d} z$. It thus means that the amount of power taken away from the wave power flow sustaining the discharge is equal to the power absorbed in the additional plasma slab $\mathrm{d} z$ generated at $z$, hence experimentally the following relationship:

$$
\mathrm{d} P_{\mathrm{a}}(z) / \mathrm{d} z=\theta_{\mathrm{A}} \bar{n}_{\mathrm{e}}(z) S
$$

where we define $\theta_{\mathrm{A}}$ as the power absorbed per electron averaged over the plasma column cross-section $S$ at $z$ with a corresponding average electron density $\bar{n}_{\mathrm{e}}(z)$.

\section{The power absorbed per electron: an insightful and far-reaching parameter Power consumption along the SW plasma column}

Figure 4 shows measured values of $\theta_{\mathrm{A}} / p$ as functions of the axial distance from the end of the SW plasma column sustained at $200 \mathrm{MHz}$, for three different gas pressures $p$. For a given gas pressure, $\theta_{\mathrm{A}}$ is observed not to vary with axial position except close to the column very end [4]. Introducing $\theta_{\mathrm{A}}$ in relation (1) is thus really insightful as it shows that the power cost for maintaining an electron in the discharge is almost the same all along the plasma column, hence that it is independent of electron density. This behavior is characteristic of plasmas operated under ambipolar diffusion regime ${ }^{4}$ (as opposed to that of volume recombination of

4 This is the usual regime describing the movement of charged particles in low-pressure rare-gas discharges $(0.5-10$ Torrs, $65-1300 \mathrm{~Pa})$. 
charged particles occurring at higher discharge gas pressures), the simplest regime to deal with when investigating the features of the power balance per electron.

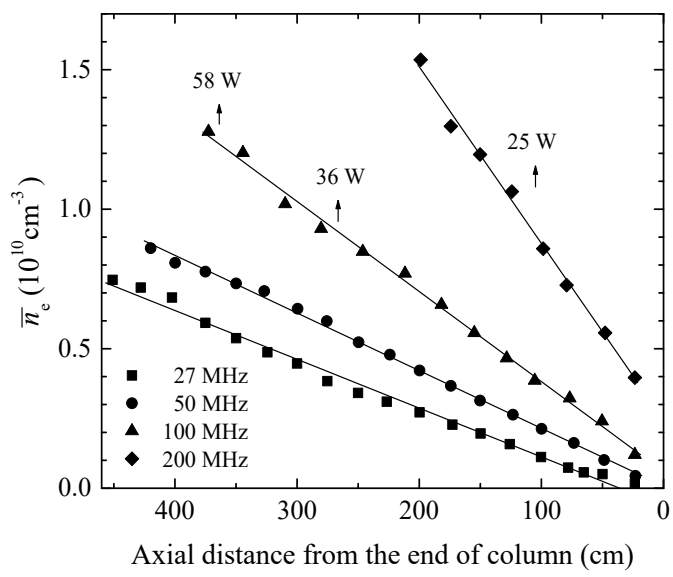

Figure 3. Measured axial variation of the electron density averaged over the radial crosssection of the plasma column sustained by the propagation of the electromagnetic surface wave at four different field frequencies, in argon gas at a pressure of $30 \mathrm{mTorr}(\approx 4 \mathrm{~Pa})$ in a tube of $64 \mathrm{~mm}$ inner diameter [7].

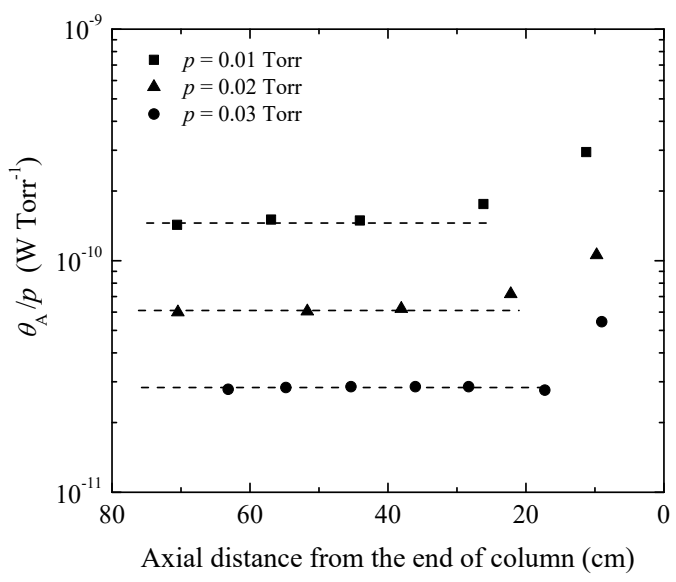

Figure 4. Measured values of $\theta_{\mathrm{A}} / p$ as functions of the axial distance from the end of the SW plasma column sustained at $200 \mathrm{MHz}$, for three different gas pressures $p$. For a given gas pressure, $\theta_{\mathrm{A}}$ is observed not to vary with axial position except very close to the column end $[8]$.

\section{Expression for the absorbed power $\theta_{A}$ (case of collisional absorption)}

The value of $\theta_{A}$, the average power (over a MW field period) absorbed per electron in the discharge, is given by [9]:

$$
\theta_{A}(E)=e^{2} \overline{E^{2}} v /\left[m_{e}\left(v^{2}+\omega^{2}\right)\right],
$$


where $v$ is the electron-heavy particle collision frequency for momentum transfer, $\omega$, the wave angular frequency, $e / m_{e}$, the electron charge to mass ratio, and $\overline{E^{2}}$, the mean squared value of the EM $\boldsymbol{E}$-field. Collisional absorption implies that $v / \omega$ is not much smaller than unity.

\section{The power $\theta_{\mathrm{A}}$ and the casting of similarity law diagrams}

Similarity laws, by definition, involve combined variables. It suffices to know the values of a limited number of these combined variables, obtained experimentally or theoretically from a given set of operating conditions, to span the full extent of these combined variables without the need for further measurements or calculations. It has long been known, for instance, that $E / p$ vs. $p R$ (where $E$ is the intensity of the discharge maintenance field) constitutes a similarity law for the DC positive column. Ferreira [3] showed, by modeling under diffusion regime, that this similarity law could be extended to include SWDs, provided it then reads $\theta / p$ vs. $p R$. It is verified experimentally in figure 5, at a fixed wave frequency, for different values of the tube radius $R$.

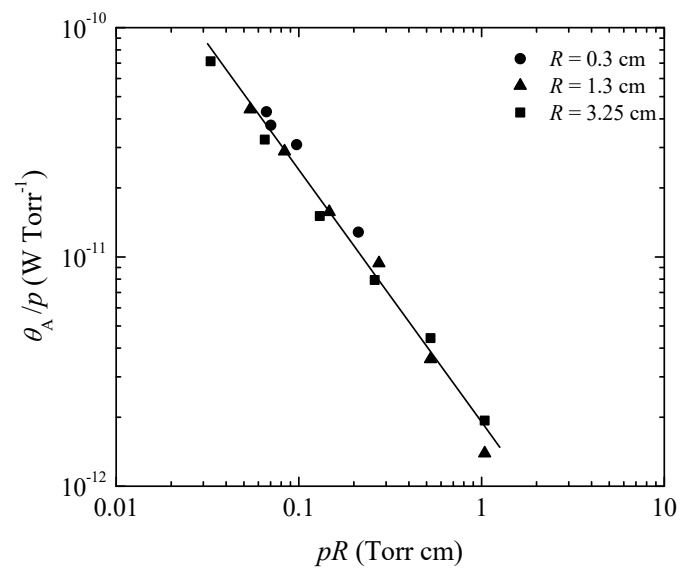

Figure 5. Measured $\theta_{\mathrm{A}} / p$ values as functions of the $p R$ product at $200 \mathrm{MHz}$, for three values of the discharge tube inner radius [3], establishing that $\theta_{\mathrm{A}} / p$ vs. $p R$ constitutes a similarity law.

\section{Expression for the absorbed power $\theta_{A}$ (case of collisionless absorption at ECR)}

MW power transfer at electron cyclotron resonance (ECR) requires a static magnetic field, of given intensity $B$, assumed in the present case to be parallel to the SW direction of propagation ( $z$ coordinate). Usual ECR conditions are two-fold: a) $\omega_{c e} / \omega=1$ where $\omega_{c e}=$ $/ e / B / m_{e}$ is the electron cyclotron angular frequency; b) very low discharge gas pressure such that $v / \omega \square 1$. Physically speaking it means that the EM electric field vector, which is oriented perpendicularly to $\boldsymbol{B}$ ( $y$ coordinate), is rotating around $\boldsymbol{B}$ field lines ( $z$ coordinate) at the same frequency as that of the electrons (characterized by $\omega_{c e}$ ), hence the electron sees a constant $\boldsymbol{E}$-field! Therefore, the lower the collision frequency disrupting this process, the higher the power gained by electrons in the MW field during the time elapsed between successive collisions.

The expression for $\theta_{\mathrm{A}}$ can be shown [9] to be given by: 


$$
\theta_{\mathrm{A}} \equiv \frac{P_{\mathrm{a}}}{n_{\mathrm{e}}}=-\frac{e}{2} \mathfrak{R}\left[v_{y} E_{y}^{*}\right]=\frac{e^{2} E_{0}^{2}}{2 v m_{\mathrm{e}}}\left[\frac{1}{2} \frac{v^{2}}{\left(\omega-\omega_{\text {ce }}\right)^{2}+v^{2}}+\frac{1}{2} \frac{v^{2}}{\left(\omega+\omega_{\text {ce }}\right)^{2}+v^{2}}\right],
$$

where it is assumed that a plane EM wave is propagating along the $z$ axis (parallel to the static $\boldsymbol{B}$-field) with the electric field component $\boldsymbol{E}=\boldsymbol{E} \exp (i \omega t)$ taken along $y$, of intensity $E_{0}^{2} ; v_{y}$ is the electron velocity along the $\boldsymbol{E}$-field while $n_{e}$ is the electron density, assumed uniform; (3) clearly reduces to (2) for $\omega_{c e}=0$. Expression (3) in a condensed form:

$$
\theta_{\mathrm{A}} \equiv \frac{P_{\mathrm{a}}}{n_{\mathrm{e}}}=\frac{1}{2 n_{\mathrm{e}}} \mathfrak{R}\left(\sigma_{y y} E_{0}^{2}\right)
$$

or equivalently:

$$
\theta_{\mathrm{A}}=\frac{E_{0}^{2}}{2 n_{\mathrm{e}}} \mathfrak{R}\left(\sigma_{y y}\right),
$$

where $\mathfrak{R}\left(\sigma_{y y}\right)$ means the real part of the electron-conductivity tensor of component $y y$ (recall that the $y$ coordinate is directed along $\boldsymbol{E})$. The expression for $\mathfrak{R}\left(\sigma_{y y}\right)$ is readily obtained from (5) by keeping only the ECR frequency-condition term $\left(\omega_{\mathrm{ce}}=\omega\right)$, yielding [9]:

$$
\mathfrak{R}\left(\sigma_{y y}\right)=\frac{n_{\mathrm{e}} e^{2}}{m_{\mathrm{e}}}\left[\frac{1}{2} \frac{v}{\left(\omega-\omega_{\mathrm{ce}}\right)^{2}+v^{2}}\right] .
$$

Figure 6 shows experimentally that: i) the higher the value of $\omega_{\mathrm{ce}}$, i.e. the higher the static magnetic field intensity, the lower the charged particles losses as a result of their increased confinement; ii) there is no maximum of the $\theta_{\mathrm{A}}$ value at $\omega_{\mathrm{ce}} / \omega=1$ as the value of pressure $p$ is decreased such as to meet gradually condition b) above ( $v / \omega \square 1)$.

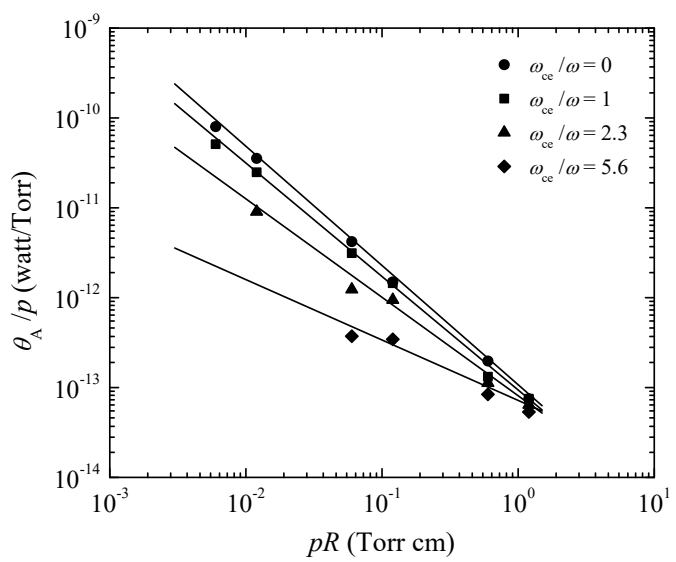

Figure 6. Measured absorbed power per electron as a function of the $p R$ product in a SW sustained argon discharge immersed in a static magnetic field $\boldsymbol{B}$ directed along the plasma column (i.e. along the SW direction of propagation), considering different values of the ratio $\omega_{\text {ce }} / \omega$ (including ECR frequency condition $\omega_{\text {ce }} / \omega=1$ ). The EM surface wave propagates on the $\mathrm{HE}_{01}$ fundamental mode (magnetized plasma case [10]) at the frequency $\omega / 2 \pi=600 \mathrm{MHz}$. The tube inner radius is $R=13 \mathrm{~mm}$ while the pressure domain is varied between $5 \times 10^{-3}$ and 1 Torr $(\approx 0.67-133 \mathrm{~Pa})$ in order to achieve the $p R$ values plotted in the figure [10]. 
The value of $\mathfrak{R}\left(\sigma_{y y}\right)$ in (6) increases as $\omega$ tends toward $\omega_{\mathrm{ce}}=\omega$ (passing through a maximum at $\omega_{\mathrm{ce}} / \omega=1$ ), but the value of $\theta_{\mathrm{A}}$ goes on decreasing linearly as pressure is reduced (no maximum or minimum in $\theta_{\mathrm{A}}$ : figure 6): it requires that the intensity of the EM electric field in (5) must decrease correspondingly to compensate for the increase in $\mathfrak{R}\left(\sigma_{y y}\right)$ to ensure the observed behaviour of $\theta_{\mathrm{A}}$. It therefore can be concluded that there is no increase but rather a decrease in the intensity of the maintenance field at ECR in contrast to what has long been accepted [11]. The fact that there are no changes in the value of $\theta_{\mathrm{A}}$ as the MW power absorption mechanism is varied proves once again that the plasma losses are controlling the discharge behavior and that the absorbed power necessarily adjusts to compensate these losses.

\section{Using the properties of SWDs to develop and extend the power per electron concept to various microwave discharges}

\section{a) Case of homogeneous plasmas}

By homogeneous plasmas, it is meant that the differential volume in which the wave power is absorbed is the same as the one in which this power is spent.

\section{Expression for the power loss on a per electron basis $\theta \mathrm{L}$}

The absorbed power $\theta_{\mathrm{A}}$ gained by electrons is mainly lost through various collisions (elastic and inelastic) with heavy particles, designated $\theta_{\mathrm{Lc}}$, a possible form of which is:

$$
\theta_{\mathrm{Lc}}\left(\left\langle U_{\mathrm{eV}}\right\rangle\right)=\frac{2 m_{\mathrm{e}}}{M}\left\langle v\left(U_{\mathrm{eV}}\right) U_{\mathrm{eV}}\right\rangle+\sum_{j}\left\langle v_{j}\left(U_{\mathrm{eV}}\right)\right\rangle V_{j}+\left\langle v_{i}\left(U_{\mathrm{eV}}\right)\right\rangle V_{i}
$$

where $m_{\mathrm{e}} / M$ is the mass ratio of the electron to that of the atom (molecule), $v\left(U_{\mathrm{eV}}\right)$ representing the microscopic collision frequency for an electron of energy $U_{\mathrm{ev}}$, which results in a transfer of momentum to heavy particles, while $v_{j}$ and $v_{i}$ are the microscopic collision frequencies generating atomic (molecular) excitation to level $j$ (threshold energy $V_{j}$ ) or ionisation (threshold energy $V_{i}$ ), respectively; the symbol \langle\rangle represents the average of the quantity within the brackets taken over the electron energy distribution function (EEDF). Expression (7) illustrates the case of excitation and ionisation by a single collision from the ground state ("direct collision"). In the case where the EEDF is Maxwellian, the average values in (7) are completely determined by the sole electron temperature $T_{\mathrm{e}}$ and gas pressure. In general, $\theta_{\mathrm{Lc}}\left(\left\langle U_{\mathrm{eV}}\right\rangle\right)$ is an increasing function of $\left\langle U_{\mathrm{eV}}\right\rangle$. When discharge- gas pressure is increased, direct collisions must be completed by multi-step excitation and ionisation processes. Although the power lost in collisions $\theta_{\mathrm{Lc}}$ is the main loss mechanism, some of the power gained by electrons in the wave electric field is ultimately lost in the sheath ${ }^{5}$ and in sustaining the ambipolar DC field, hence the total power loss needs to be expressed on a per electron basis designated as $\theta_{\mathrm{L}}$.

Ultimately, the power thus transferred from electrons to heavy particles is lost in ion-electron recombination (at the tube wall under diffusion regime or, at higher pressures, in volume recombination within the plasma), in light emission (photons) through de-excitation or recombining electron-ion emission, and in heating the discharge tube wall,...

\footnotetext{
${ }^{5}$ The plasma sheath is that non-neutral region connecting the plasma with the discharge tube. In contrast to RF sheaths, it does not vary with the EM field and it is much smaller in width (because the electron density is higher).
} 
Setting the per-electron power balance

In the case of homogenous plasmas, the power balance then simply reads:

$$
\theta_{A}=\theta_{L}
$$

and more explicitly as:

$$
\theta_{A}(E)=\theta_{L}\left(U_{e V}\right)
$$

Since the value of $\theta_{A}$ adjusts so as to compensate exactly for $\theta_{L}$, as repeatedly mentioned, the intensity of the maintenance $\boldsymbol{E}$-field sustaining the discharge comes out as an internal parameter. It means that it is operator-independent, in contrast to what is commonly believed, ${ }^{6}$ whatever the kind of $\boldsymbol{E}$-field sustained discharges.

\section{b) Case of inhomogeneous plasmas}

It means considering the possibility that MW power be absorbed in a smaller volume $V_{l}$ than the plasma volume $V_{2}$ : in such a case, the smaller the volume in which power is absorbed with respect to the volume in which it is spent, the higher the intensity of the maintenance $\boldsymbol{E}$-field. This can be seen from the global power balance:

$$
\theta_{A} n_{e 1} V_{1}=\theta_{L} n_{e 2} V_{2},
$$

where $n_{\mathrm{e} 1}$ and $\mathrm{n}_{\mathrm{e} 2}$ are the electron density in the absorption and plasma volumes, respectively. Since the total number of electrons in volume $\mathrm{V}_{l}$ must be equal to that in volume $V_{2}$, then:

$$
\theta_{A} / \theta_{L}>n_{e 2} / n_{e 1}>1 \text {. }
$$

A smaller absorption volume thus provides higher excitation and ionization rates (including molecular dissociation). In particular, it explains the very high ionization rate in microdischarges [4].

\section{Reference}

1. Glaude V.M.M., M. Moisan, R. Pantel, P. Leprince, J. Marec, J. Appl. Phys. 1980, 57, 5693.

2. Zakrzewski Z, J. Phys. D: Appl. Phys. 1983, 16, 171-80.

3. Ferreira CM, J. Phys. D: Appl. Phys. 1983, 16, 1673-85.

4. Moisan, M., H. Nowakowska, Plasma sources Sci. Technol., 2018, 27, 07301 (43pp).

5. Collin R. E., Foundations for Microwave Engineering, 2nd ed., 2001, (John Wiley \& Sons, Inc.: New York).

6. Moisan, M, H. Nowakowska, AMPERE Newsletter, 2019, issue 98. Also Moisan, M. P. Levif, H. Nowakowska, Microwave Discharges: fundamentals and applications, $X^{\text {th }}$ International Workshop (Zvenigorod, Russia), 2018, 97-109 (Yanus-K: Moscow).

7. Chaker, M,. M. Moisan, Z. Zakrzewski, Plasma Chem. Plasma Process., 1986, 6, 79-96.

8. Moisan, M., C. Barbeau, R. Claude, C.M. Ferreira, J. Margot, J. Parasczak, A. B. Sa, G. Sauvé, M. R. Wertheimer, J. Vac. Sci. Technol., 1991, B 9, 8-25.

9. Moisan, M., J. Pelletier, Physics of collisional plasmas: introduction to high-frequency discharges, 2012 (Berlin: Springer).

10. Margot, J. M. Moisan, J. Phys. D Appl. Phys., 1991, 24, 1765-1788.

11. Allis W.P. Handbuch der Physik, 1956, ed. S. Flügge (Berlin: Springer).

\footnotetext{
${ }^{6}$ For example, it is of no use to call on a resonant cavity with a high $Q$-factor (hence a very high local $\boldsymbol{E}$-field) to achieve a discharge with a higher maintenance $\boldsymbol{E}$-field. However, in such a case, starting the discharge is easier!
} 


\title{
MODELLING AND STUDY OF A MICROWAVE PLASMA SOURCE FOR HIGH- RATE ETCHING
}

\author{
S. Pauly ${ }^{1}$, A. Schulz 1 , M. Walker ${ }^{1}$, M. Gorath², K.-M. Baumgärtner ${ }^{2}$, G.E.M. Tovar ${ }^{1}$ \\ ${ }^{1}$ Institute of Interfacial Process Engineering and Plasma Technology (IGVP), \\ University of Stuttgart, Stuttgart, Germany \\ ${ }^{2}$ Muegge GmbH, Reichelsheim (Odenwald), Germany \\ Steffen.Pauly@igvp.uni-stuttgart.de
}

\begin{abstract}
The aim of this study is to investigate and to optimize an existing microwave-powered remote plasma source (RPS) with respect to the etching rate and gas temperature and to simplify the setup to save production costs. To achieve these goals, a FEM-based model of the RPS has been developed in order to investigate the microwave coupling into the plasma chamber and the microwave field distribution as well as the plasma itself. Different examples of FEM-based microwave simulations at different conditions and their experimental validations will be presented.
\end{abstract}

Keywords: Microwave Plasma, Remote Plasma Source, Modelling, Plasma Etching.

\section{Introduction}

Photoresists are photo-susceptible polymer layers used in the semi-conductor industry to manufacture surface structures in the sub-micrometer range. This polymer layer acts as shaping die for the envisaged microstructures and has to be removed in the final step of the manufacturing process. Here, conventional wet etching of the cured resist pattern is not a process of choice, as the microstructures must not be damaged, which is not guaranteed with wet etching processes. In contrast, dry plasma chemical etching by means of radicals generated in the plasma chamber of a remote plasma source (RPS) is a suitable method for avoiding damages to the microstructures made of metals like nickel, copper or gold. Another advantage is, that the products does not have to be dried and cleaned after the manufacturing process and can be directly processed.

Plasma is often called the fourth state of matter, because usually every matter goes through the known states of matter from solid to liquid to gaseous as the energy input is raised. By further increasing the energy input, atoms and molecules become partly ionized and molecules dissociate until the plasma state arises. Hence, plasma is an ionized gas in which the same amount of negative charge in the form of electrons exists as positive charge in the form of ions. When a high-frequency electrical field is applied, only the electrons can follow the fast electromagnetic waves. Thus, only the electrons can gain energy. It's then called a nonequilibrium, or non-isothermal plasma, which means that the electron temperature is much higher than the gas and the ion temperature $\left(T_{e} \gg T_{i} \approx T_{g}\right)$. The advantage of such a non-isothermal plasma is that high temperatures are avoided, and thus even sensitive surfaces can be etched. However, the accelerated electrons collide with neutral atoms and molecules, which leads to excited atoms, excited molecules, ions or radicals. During plasma etching, the highly reactive radicals then react with the substrate surface atoms forming gaseous molecules. The plasma etching is a purely chemical etching process. A schematic view of the etching process is shown in figure 1.

The aim of the study is to investigate and to optimize the RPS with respect to the etching rate and gas temperature. Therefore, a finite element method (FEM) based plasma model of 
the RPS has been developed to investigate the microwave coupling into the plasma chamber and the microwave field distribution as well as the plasma itself.

\section{Experimental Setup}

Figure 1 shows a schematic view of the Remote Plasma Source (RPS) flanged on top of a reactor. The RPS consists of a magnetron with a frequency of $2.45 \mathrm{GHz}$ and a power up to $3 \mathrm{~kW}$, and waveguides to feed the microwaves from the magnetron into the plasma chamber. The operating pressure in the reactor and the RPS is between $1 \mathrm{~Pa}$ and $150 \mathrm{~Pa}$. There is atmospheric pressure in the waveguides. A vacuum window made of ceramic is between the rectangular waveguide and the plasma chamber. Together with the tuning stubs and the mode coupler, the configuration is such that the plasma ignites and operates without readjusting the microwave coupling. A quartz glass window is arranged on the top of the RPS for optical investigations of the plasma. The gas used in this work is oxygen, hence the resulting products of the etching process are not dangerous for the environment such that no waste gas treatment is needed. The gas is fed in above the plasma chamber via a mass flow controller. One important advantage of the RPS is its compact design, so that it can be easily flanged on existing production lines.

To validate the numerically calculated microwave field configuration in the plasma chamber when there is no plasma in the RPS (ignition condition), polymethylmethacrylate (PMMA) plates were placed into the plasma chamber and heated up with the microwave. After removing the plates from the RPS, the heat distribution was visualized with a thermal camera system and liquid crystal sheets and compared with the numerical simulation.

To validate the electric field distribution when there is plasma in the RPS (operational condition), photographs through the observation window were taken and compared with the electric field distribution calculated with the later explained Drude model.

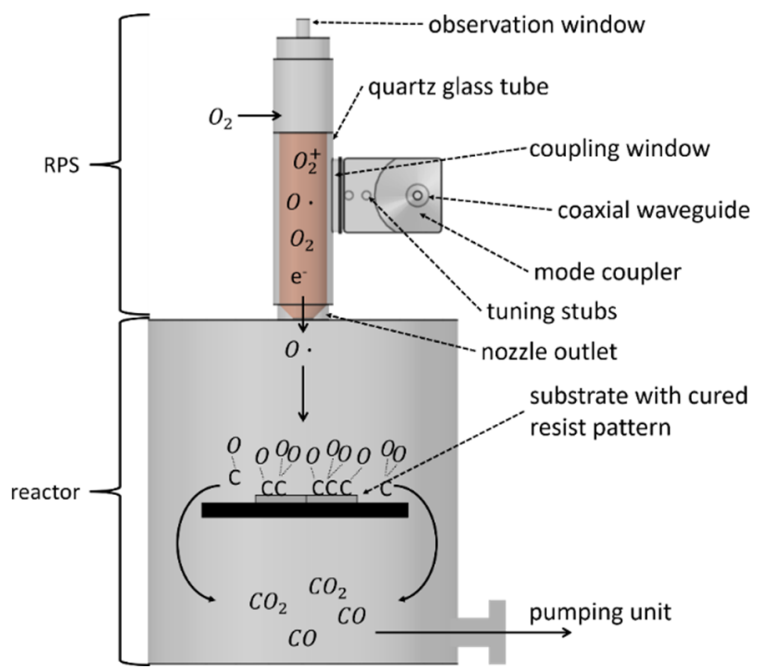

Figure 1: Schematic view of the plasma etching process of the cured resin.

The resin on top of the substrate is etched by oxygen radicals generated by microwaves in the orange-colored plasma chamber of the RPS. 


\section{Simulation Setup}

To investigate and to optimize the RPS, a 3D FEM model based on the engineering drawings of the RPS was developed which is shown in figure 2. For the investigation of this study, there are two essential process conditions of the plasma source. The first one is the ignition condition of the RPS when the microwave power is turned on, but there is no plasma in the chamber yet, so the microwaves propagate like in free space. The second one is the operational condition when the plasma has been ignited. In this case the plasma affects the microwave field distribution in the RPS. In the following section, the equations for both conditions as well as the boundary conditions for the calculations will be presented.

To calculate the electromagnetic wave propagation at the ignition condition, the wave equation for the electric field $\vec{E}$ has to be solved:

$$
\nabla \times\left(\frac{1}{\mu_{r}} \nabla \times \vec{E}\right)-k_{0}^{2} \epsilon_{r} \vec{E}=0,
$$

with the relative permeability $\mu_{r}$, the vacuum wave number $k_{0}$ and the relative permittivity $\epsilon_{r}$ which is $\epsilon_{r}=1$ for vacuum.

For the operational condition of the RPS, when a plasma is assumed, eqn. (1) is complemented with the Drude model. As mentioned in the introduction, only the electrons in the plasma can follow the high-frequent electromagnetic waves. This behavior can be treated like the free electron gas in a metal which the Drude model [1] describes. However, eqn. (1) has to be adapted with a frequency-dependent permittivity $\epsilon(\omega)$. By converting the equation of motion of an electron $e$ with the mass $m_{e}$, an expression for the conductivity $\sigma(\omega)$ containing the electron density $n_{e}$ can be derived as follows [2]:

with the collision frequency [3]

$$
\sigma(\omega)=\frac{e^{2} n_{e}}{m_{e}(v+i \omega)^{\prime}}
$$

$$
v=v_{e N}=n_{g a s} \overline{v_{e}} \sigma_{e N} .
$$

The collision frequency depends on the mean velocity of an electron $\bar{v}_{e}$, the neutral gas density $n_{g a s}$ and the collision cross section $\sigma_{e N}$. Inserting eqn. (2) into the mathematical term for the permittivity with the plasma frequency known as [4]

$$
\omega_{p}=\sqrt{\frac{n_{e} e^{2}}{m_{e} \epsilon_{0}}}
$$

the permittivity becomes [5]

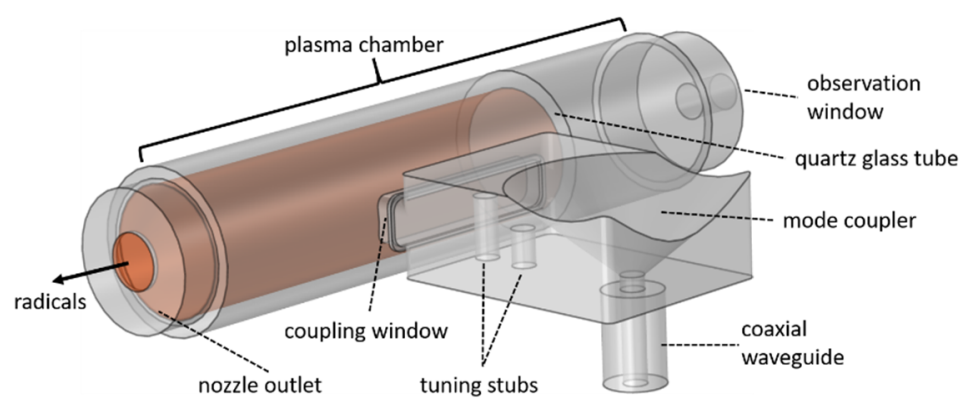

Figure 2: Schematic image of the RPS. The orange-colored volume shows where the plasma exists and, where the Drude model is implemented in the simulation. 


$$
\epsilon(\omega)=1-\frac{i \sigma(\omega)}{\omega \epsilon_{0}}=1-\frac{\omega_{p}^{2}}{\omega^{2}\left(1-\frac{i v}{\omega}\right)} .
$$

$i$ is the imaginary number and $\epsilon_{0}$ the vacuum permittivity. This leads to the frequencydependent wave equation for the electric field:

$$
\nabla \times\left(\frac{1}{\mu_{r}} \nabla \times \vec{E}\right)-k_{0}^{2}\left(\epsilon_{r}-\frac{i \sigma(\omega)}{\omega \epsilon_{0}}\right) \vec{E}=0 .
$$

If the frequency $\omega$ and the plasma frequency $\omega_{p}$ are equal, the cut-off density can be derived from eqn. (4) as follows:

$$
n_{\text {cutoff }}=\frac{m_{e} \epsilon_{0}}{e^{2}} \cdot \omega_{p}^{2}
$$

If collisions in the plasma are neglected, the cut-off density represents a threshold that indicates whether the injected microwaves are affected by the plasma or not. Is the electron density below the cut-off density, the electromagnetic waves can enter the plasma. If the electron density exceeds the cut-off density, the wave gets repelled. This effect will be presented in the results and can be seen in figure 5.

In the finite element method, the boundary conditions are the setting of known values on specific nodes, lines or areas. In the case of the RPS (figure 2), all walls enclosing the simulation domain are set as perfect electric conductors, so that there are no losses considered. The nozzle outlet is defined as scattering boundary condition, which is transparent for incoming scattered waves. The coaxial waveguide, where the microwaves are fed into the system, is set as a coaxial port with an input power of $3 \mathrm{~kW}$ and a microwave frequency of $2.45 \mathrm{GHz}$.

\section{Results and Discussion}

In the following section, the simulations at ignition conditions with the experimental validations will be presented at first and the results of the simulations under operational conditions with its validations discussed afterwards.

Ignition condition means that there is no plasma in the RPS but only the electric field. In order to do so, the whole geometry of the RPS is represented as free space according to eqn. (1). Figure 3 shows the distribution of the calculated normalized electric field

$$
E_{\text {norm }}=|\bar{E}|=\sqrt{E_{x}^{2}+E_{y}^{2}+E_{z}^{2}}
$$




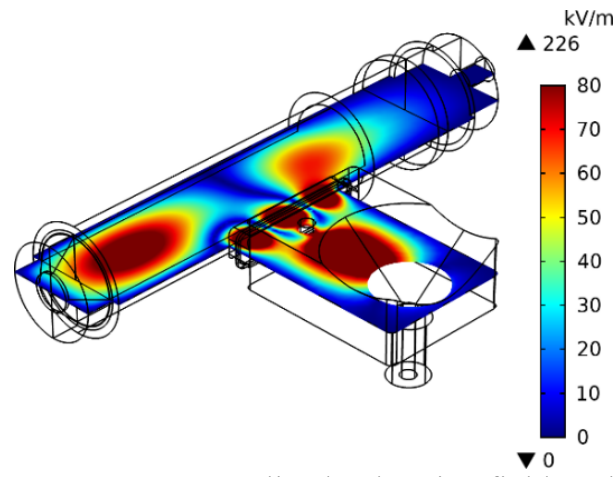

Figure 3: Normalized electric field. The electric field distribution is calculated for ignition conditions, so no plasma is inside the plasma chamber of the RPS.

In the RPS. The microwave is fed into the system via the coaxial waveguide and then tuned with the mode coupler and the tuning stubs for coupling into the plasma chamber through the vacuum window. Figure 3 shows a high electric field above the tuning stubs due to the permanent ignition and operational condition configuration. Because of the high relative permittivity of the material of the vacuum window, the propagation velocity of the microwave is reduced, leading to three field maxima. In the plasma chamber are two field maxima, a higher one near the nozzle and a smaller one at the top. To validate the calculated electric field distribution in the plasma chamber at ignition conditions, a PMMA plate was placed into the plasma chamber of the RPS in an experiment and implemented in the model which is shown in figure 4 a).

In the experiment, the PMMA plate was heated up for 30 seconds with the microwave power of $\mathrm{P}=3 \mathrm{~kW}$. After removing the plate from the RPS, the heat distribution was visualized by two different methods, a thermal camera system b) and liquid crystal sheets c). Liquid crystal sheets consist of crystals 3 to $5 \mu \mathrm{m}$ in size which are dispersed within a polymer matrix. The crystals exhibit a color spectrum response with the change of temperature [6]. Because of the different visualization systems in the experiment and the associated color spectra of them, the different colors in b) and c) can be neglected. Only the position of the maxima and their geometry are considered. As can be seen, the images of the experiment and the image of the simulation show a high maximum at the top and a smaller one at the bottom. On the right side of the images b), c), d), where the microwaves are fed into the system, all three images show a hotspot, too. Considering the time dependence of the experiment, the geometry of the electric field distribution in the experiment fits very well with the calculated field distribution at ignition conditions.

As mentioned before, there are two conditions which are essential for that study. The following simulations were performed under operational conditions, which means that there is plasma in the RPS. For simulations with plasma, the frequency-dependent conductivity $\sigma(\omega)$ has to be included into eqn. (1), which leads to eqn. (6). The frequency-dependent wave equation is determined by the electron density which can be used as a parameter to define a plasma and to implement it into the simulation model [4]. Eqn. (6) is valid in the volume of the orange-colored inner part of the plasma chamber in figure 2, whereas eqn. (1) is valid for the remaining simulation model of the RPS. To investigate the frequency dependence of the assumed plasma, the electron density is then varied around the 


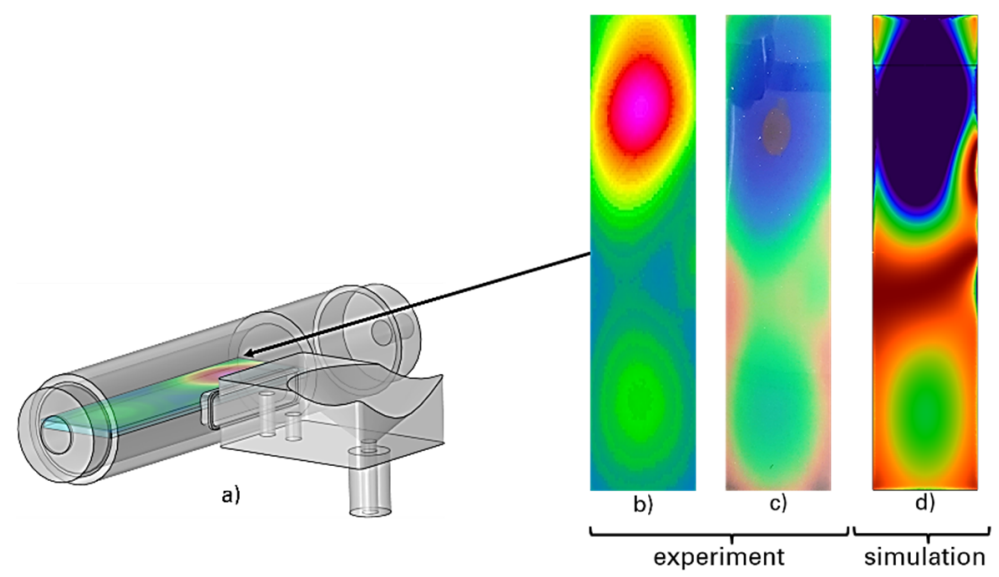

Figure 4: Integrated PMMA substrate in the RPS simulation model a). To compare the electric field distribution: PMMA substrate heated-up with $3 \mathrm{~kW}$ microwave power for 30 seconds and visualized with b) infrared camera system, c) liquid crystal sheet and in comparison, d) the calculated field.
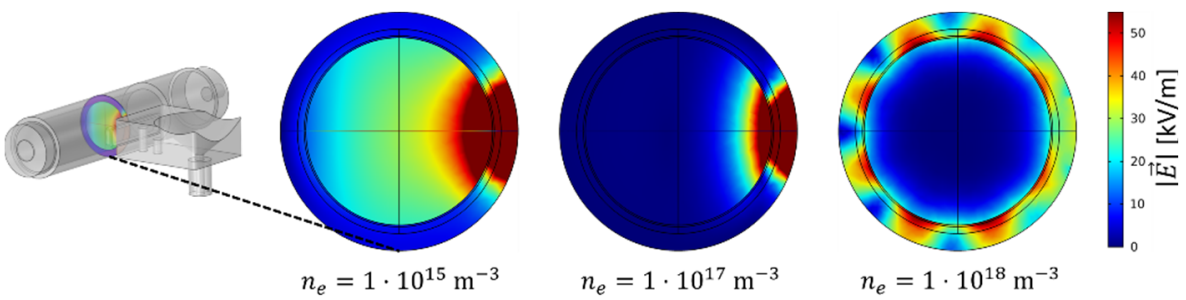

Figure 5: Normalized electric field in a cross section of the plasma chamber at operational conditions for different electron densities.

cut-off density of the plasma. In addition, collisions are considered by defining a collision frequency, see eqn. (3). The collision frequency is determined by the neutral gas density $n_{\text {gas }}$, a typical electron velocity $\overline{v_{e}}$ and the collision cross section $\sigma_{e N}$. The considered collision frequency for oxygen is $v=9.31 \cdot 10^{7} \mathrm{~s}^{-1}$, with the assumed values for operational condition: $n_{\text {gas }}=1.94 \cdot 10^{22} \mathrm{~m}^{-3}, \quad \bar{v}_{e}=1.06 \cdot 10^{6} \frac{\mathrm{m}}{\mathrm{s}}$ and $\sigma_{e N}=4.5$. $10^{-20} \mathrm{~m}^{2}$. Langmuir probe and double probe measurements will follow to validate the assumed data and to implement an electron density distribution profile in the simulation model. Figure 5 shows the normalized electrical field $E_{\text {norm }}$ of a cross section of the plasma chamber at different electron densities, where the microwaves are fed in the system from the right side. The cut-off density at $2.45 \mathrm{GHz}$ is $7.45 \cdot 10^{16} \mathrm{~m}^{-3}$. For the electron density below the cut-off density at $n_{e}=1 \cdot 10^{15} \mathrm{~m}^{-3}$, the microwaves can penetrate the plasma area comparable to figure 3 , where no plasma is assumed. If the cut-off density is exceeded, the microwaves are repelled, and the electric field gets displaced by the plasma which can be seen at $n_{e}=1 \cdot 10^{17} \mathrm{~m}^{-3}$. By further increasing the electron density to the value $n_{e}=1$. $10^{18} \mathrm{~m}^{-3}$, which can be assumed for the RPS, the microwaves get displaced out of the plasma chamber and propagate through the quartz glass tube like in a waveguide around the plasma chamber. At that density the electric field in the quartz glass tube has eight maxima. The plasma is field-free, except for a small penetration depth which is the plasma skin depth. 
To validate the simulations with the implemented Drude model, photos of the plasma were taken through the observation window on the top of the RPS. The gas which is used for the experiments is oxygen at a pressure of $100 \mathrm{~Pa}$ and a gas flow of $3 \mathrm{slm}$. The microwave has a frequency of $2.45 \mathrm{GHz}$ and $3 \mathrm{~kW}$ power. The photo of the plasma can be seen in figure 6 a). It shows eight bright shining regions arranged in a circle.

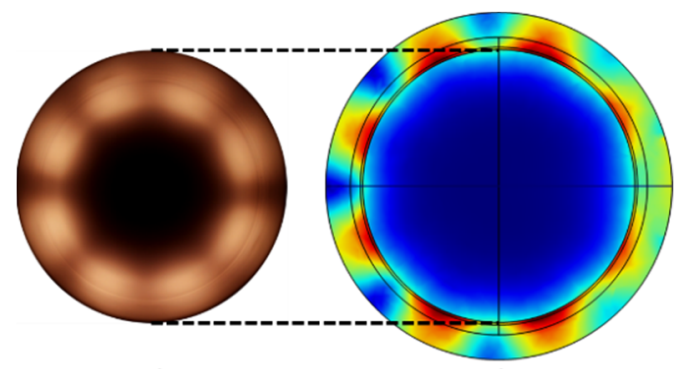

a)

b)

Figure 6: Photo of an oxygen plasma a) compared with the normalized electric field $b$ ) of the calculated RPS at operational conditions for $n_{e}=1 \cdot 10^{18} \mathrm{~m}^{-3}$.

To compare the photo of the experiment with the simulations, b) shows again the normalized electric field $E_{\text {norm }}$ simulated under operational conditions at an electron density of $n_{e}=$ $1 \cdot 10^{18} \mathrm{~m}^{-3}$. As already mentioned, at that density the microwaves propagate through the quartz glass tube around the plasma, and the field distribution has eight maxima. Figure 6 a) only shows the plasma chamber of the RPS but not the quartz glass tube. So, the section of the photo a) corresponds to the inner area of the simulation b) which is indicated by the dashed lines. It suggests that at the eight maxima of the electric field around the plasma area, the plasma shines brighter, because there is also more energy available for the ionization and excitation. To confirm that assumption, more detailed simulations will follow in that study based on experimental investigations with Langmuir probe and double probe measurements of the plasma.

\section{Conclusion}

To investigate the remote plasma source regarding its two essential operating states, the ignition condition and the operational condition, a FEM based simulation model has been developed. The ignition condition represents the simulation case, when there is no plasma in the RPS yet. For that condition, the electric field distribution was calculated with the wave equation in free space. To simulate the RPS under operational conditions, which means there is plasma existing, the wave equation was adapted with a frequency dependent conductivity based on the Drude model.

In order to validate the simulations under ignition conditions, PMMA plates were heated up in the RPS, and the heat distribution was visualized with different methods. The heat distribution was then compared with the calculated electric field distribution on a PMMA plate, which was integrated in the model. The idea behind this comparison was that at positions of higher electric field the PMMA plate should be heated up more than at positions of lower electric field. The results show that the experiment and the simulation are in good agreement. 
The simulation with the implemented Drude model shows at the assumed electron density an electric field distribution with eight maxima arranged circular around the plasma chamber. In order to validate this simulation, photos of the plasma were taken and compared with the simulations of the normalized electric field. The plasma photo shows a structure with eight bright shining regions arranged circular in the plasma chamber, which suggests that they correspond to the eight electric field maxima around the plasma chamber in the simulation. To confirm that assumption, further investigations of the plasma with Langmuir probe and double probe measurements as well as optical investigations with the infrared camera system will follow.

\section{References}

1. Drude, P.: Annalen der Physik. Zur Elektronentheorie der Metalle, 1900, 306: 566-613.

2. Janzen, G., Plasmatechnik: Grundlagen, Anwendungen, Diagnostik, 1992, 3.

3. Bergmann, L., Schaefer, C., Lehrbuch der Experimentalphysik: VielteilchenSysteme, 1992, $\mathbf{5}$.

4. Gaiser, S., Diagnostik und Modellierung eines Mikrowellen-Plasmabrenners bei Atmosphärendruck, Dissertation, University of Stuttgart, 2017.

5. Moisan, M., Pelletier, J., Plasma Technology, 4, Microwave Excited Plasmas, Elsevier, Amsterdam-London-New York-Tokyo, 1992.

6. Edmund Optics, Temperature Sensitive Liquid Crystal Sheets: https://www.edmundoptics.com/f/temperature-sensitive-liquid-crystalsheets/11878/ [Date accessed 05/06/19]. 


\title{
A HIGH-POWER SOURCE OF OPTICAL RADIATION WITH MICROWAVE EXCITATION
}

\author{
G. Churyumov ${ }^{1,2}$, A. Denisov ${ }^{1}$, T. Frolova ${ }^{2}$, N. Wang ${ }^{1}$, J. Qiu $^{1}$ \\ ${ }^{1}$ 1Harbin Institute of Technology, 92, West Dazhi Street, Nan Gang District, Harbin, China \\ 2 Kharkiv National University of Radio Electronics, 14, Nauky Ave., 61166, Kharkiv, \\ Ukraine \\ g.churyumov@ukr.net
}

Keywords: microwave heating, magnetron, electrodeless sulfur lamp, plasma, microwave excitation

\section{Introduction}

For more than 50 years, interest to the microwave heating technology has not weakened. In addition to the traditional areas of its application, which described in detail in [1], recently there has been an expansion of technological possibilities for the use of microwave energy associated with the impact of electromagnetic waves of the microwave range on various materials (sintering of metal and ceramic powders) and media, including plasma [2]. One such new direction is the creation of high-power and environmentally friendly sources of optical radiation on the basis of the electrodeless sulfur lamp with microwave excitation [2, $3]$.

As it is known, Michael Ury and his associates at Fusion Systems invented this radically new lamp in 1990, but the lamp was not ideal because of the complexity of its design [4]. Therefore, it was not put into production. However, every year the scientific interest was growing. An analysis of scientific publications shows that every 5 years a new country is joined to this issue. Now more than in 10 countries of all would including USA, Great Britain, South Korea, Netherlands, Germany, Russia, and so on where there are research teams that are carried out an investigation concerning the electrodeless lamps with microwave excitation.

The plasma lighting plant with a sulfur lamp is a high-power light source providing light fluxes of $\sim 140 \mathrm{klm}$, and color temperature of about $6400 \mathrm{~K}$. The main advantage of such lamps is its quasi-solar emission spectrum as well as the ability controlling by radiation power in process of its operation. As a result, it allows imitating the modes of sunrise and sunset and may be of interest in future for creating the solar simulator [5]. The main spectral and color characteristics of the electrodeless sulfur lamps with microwave excitation are presented in Figs. 1 and 2. For comparison in the same place the standard solar spectrum AM $1.5 \mathrm{G}$ is shown.

The purpose of this paper is to the further development of the theory and practice of microwave excitation by the electrodeless sulfur lamps, improving the energy efficiency during energy conversion into the optical radiation and widening the application of new light sources in real practice.

\section{Physical processes in the two-component microwave plasma}

The principle of ionization of gases under the influence of microwave energy is the base for the plasma lamp. Microwaves generated by the magnetron excites sulfur vapor in argon within the bulb. Highly ionized gas becomes a plasma state, which begins to emit light constantly when a certain operating temperature is reached. 


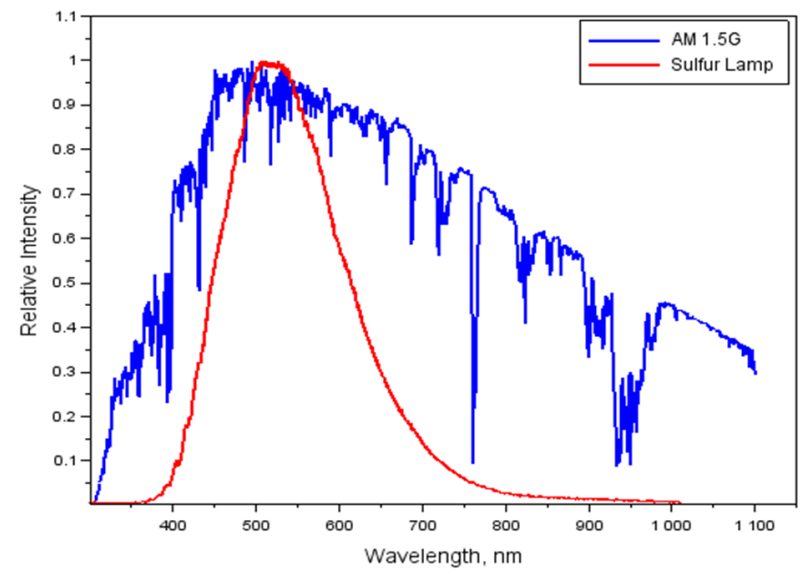

Fig. 1. Spectral characteristics of the electrodeless sulfur lamp with microwave excitation and spectral profile of the standard solar radiation [6]

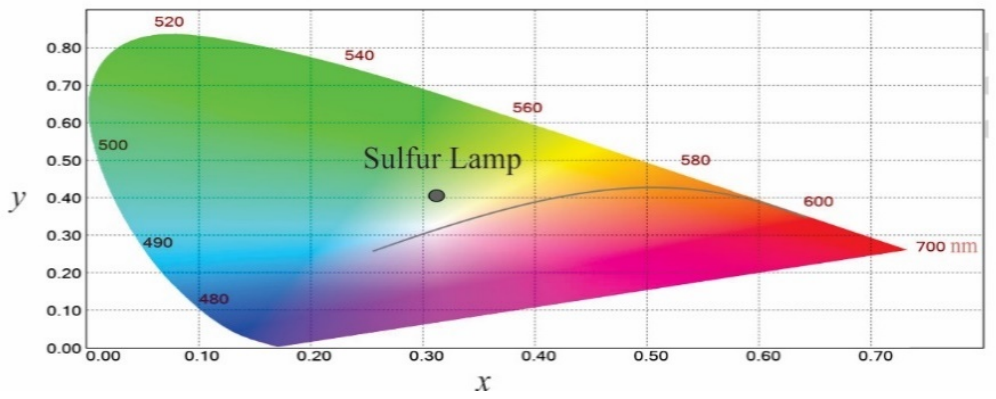

Fig. 2. Color characteristics of the electrodeless sulfur lamp with microwave excitation

For understanding the mechanism of converting microwave energy into optical radiation and for further improvement of the characteristics optical radiation sources it is necessary to study the physical processes occurring in the spherical quartz bulb filled with an inert gas (e.g., Ne or Ag) and having a small number of main component (sulfur in solid state). Impacting the microwave radiation causes a glowing discharge in the buffer gas having a low breakdown threshold on low initial pressure. At the same time with an increase in the quartz bulb filling temperature, the state of sulfur changes from solid to gaseous and at temperatures greater than $600 \mathrm{C}$, most of the sulfur has dissociated to $\mathrm{S}_{2}$ [7]. In the resulting high-pressure gas mixture, the microwave field converts the atoms (as well as dimers and other particles) of sulfur to excited states. When this occurs, the processes of direct atomic and molecular absorption, and the collisional mechanism. Finally, reradiation from excited states forms the observed optical emission spectrum.

Characteristic lines in the spectrum of the sulfur atom are in the open portion of the optical spectrum of a molecule 867, 921 and $1045 \mathrm{~nm}$ [8]. The ionization potential of each radiating state is less than $3 \mathrm{eV}$. To get some idea of the energetic interactions between sulfur dimer and atom, consider a simplified diagram of the energy levels of the two systems which shown in Fig. 3. As illustrated by Fig. 3, we use the dissociation energy of the ground state of the dimer, and $4.4 \mathrm{eV}$ - energy offset between the systems. 


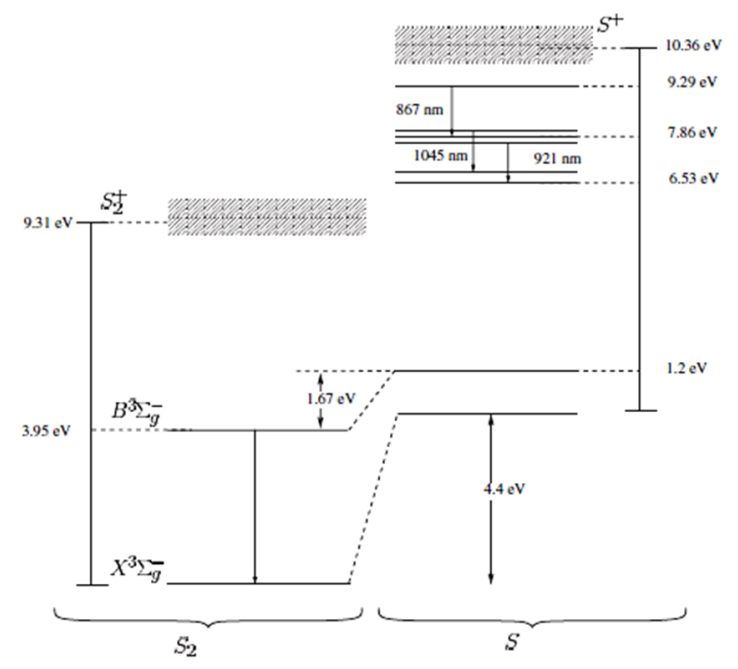

Fig. 3. The diagram of the energy levels of sulfur [8]

The ionization energy of the molecule is more attractive since the ionization potential of the molecular state is less than half of the ground state of the atom. Thus, if the degree of dissociation is relatively low, $\mathrm{S}_{2}{ }^{+}$ions will be the dominant species. As the level of the emitting atoms is about $11 \mathrm{eV}$ and higher molecular state, and their density is exponentially sensitive to temperature, they are ideal for study.

Studies show that the emission spectrum of the sulfur lamp in the visible region is formed mainly by radiative transitions between the excited level $B^{3} \sum \bar{u}$ and the ground state $X^{3} \sum \bar{g}$ of diatomic sulfur molecules $\mathrm{S}_{2}$.

\section{Mathematical description of the states of plasma}

Due to the diversity of processes occurring in spatially inhomogeneous plasma, an analytical description of the actual plasma is generally very difficult. Therefore, it is usually considered a simplified model of plasma, and then specifying how real plasma is close to the accepted model.

Condition of real plasma in an arbitrary pressure is determined by:

a) the concentrations of particles of all kinds (the number of particles in unit volume);

b) their velocities distribution function;

c) the population of excited levels (the number of particles per unit volume in the excited state);

d) the spatial distributions of these quantities.

Getting information on all the above characteristics is extremely difficult, as the theoretical studies of the plasma state require setting up and solving the system of equations connecting these quantities with the external conditions.

For description inside an electrodeless sulfur lamp of gas-discharge plasma, formed as a result impacting the electromagnetic field, it is advisable to use the local thermodynamic equilibrium (LTE) model. This model allows us to qualitatively and quantitatively describe the continuous spectrum of the lamp, as well as the distribution of basic physical quantities of sulfur plasma. 
According to the model of the LTE temperature in the different elements of the volume of the medium is different, but the plasma can be described (locally) by dint of the Boltzmann and Maxwell distributions and Saha equation in each volume element where the local temperature is the same for all types of particles.

The LTE model for most stationary cases of generating plasmas received in the laboratory conditions is typical. Under the conditions of the LTE model of plasma, the detailed equilibrium for optical transitions it is broken, so it is advisable to consider radiation and absorption separately.

\section{Description of the software product}

The optical radiation source may present itself a spherical or cylindrical flask of transparent anhydrous quartz glass filled with metered amounts of sulfur (and possibly to include contaminants, such as $\mathrm{CaBr} 2$ ) or another substance (e.g. indium iodide $\mathrm{InI}$ ) as well as buffer gas (argon, neon, krypton). Theoretical studies of output spectral characteristics of the optical radiation of such source and their dependence on the microwave power to the pump wavelength from $\lambda$ to $\lambda+d \lambda$, the temperature distribution inside the bulb, the electrical conductivity of the plasma, and so on can be carried out by changing the composition of the bulb filling.

The buffer gas (argon) is used to obtain the initial ionization and glow discharge (gas pressure is set at the initial stage). We get the dependence of the dynamics of change of pressure in the bulb temperature.

The interface developed software named HID Lamps v.2.0 is shown in Fig. 4. All output parameters and characteristics of the charts have the opportunity to be saved in separate files for further analysis and comparison of the results with experimental data.

The simulation was performed for the electrodeless discharge lamps filled with sulfur $\mathrm{S}$ and indium iodide InI as active components with the buffer gas argon or neon. We studied the effect of the amount of filling in the luminous efficacy and color rendering index behavior. These results were tested and experimental studies. However, it was noticed that there are threshold values for the buffer gas below and above which can not be taken since it is impossible to receive the gas discharge. For easier ignition, it is necessary to choose a pressure of the buffer gas close to the lower boundary.

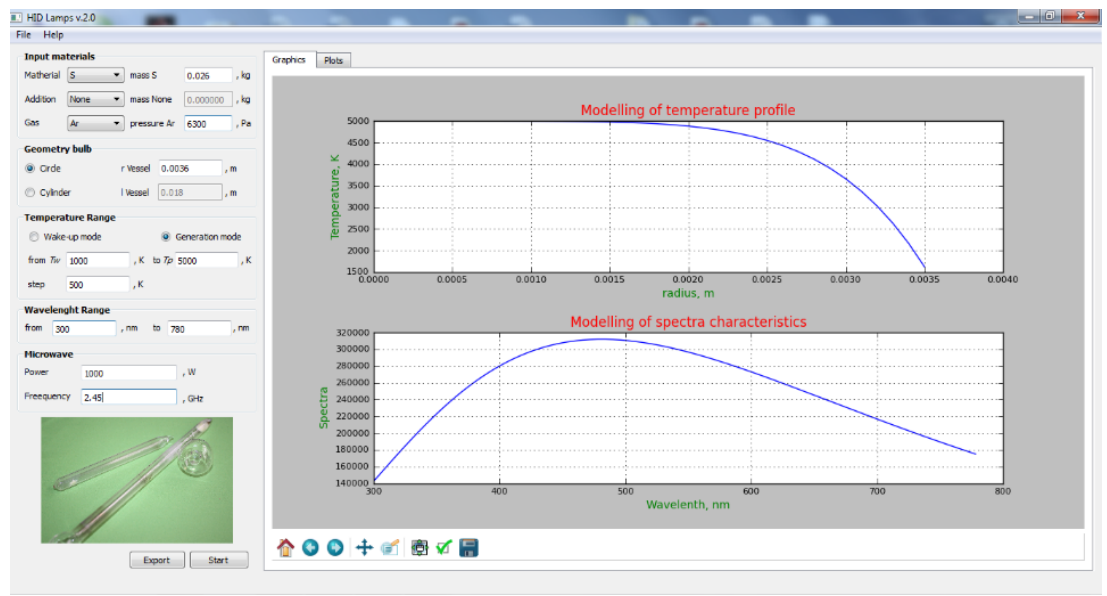

Fig. 4. The interface of the software HID Lamps v.2.0 
There were also carried out the theoretical studies of the emission spectrum depending on the type of microwave pumping source (microwave devices), the pressure in the flask and the concentration of the substance. As a result, the following assumption can be made that using the LTE model to describe the gas-discharge plasma, the pressure change will primarily affect the spectral characteristics of the system. Therefore, the work was to study the behavior of the spectrum lamp for different values of the buffer gas pressure of argon.

\section{Vistas of application}

The electrodeless sulfur lamps with microwave excitation can be used together with other electronic devices for creating the power energy-efficient lighting systems. It is proposed to use a lighting facility based on an electrodeless sulfur lamp with microwave excitation combined with solar panels that are located indoors (for example, greenhouses) [9]. This gives several advantages: first, it facilitates the maintenance of solar panels (protection from various natural phenomena); secondly, with the reduction in the number of sunny days, increases the need to use artificial light (for example, for supplementary lighting plants in greenhouses), however, if the lighting devices have a spectrum close to the Sun, then solar panels can more efficiently produce electricity.

It is shown that additional use of the solar panels for the reverse conversion of the optical radiation into DC energy with follow-up its using in the circuits of secondary power supply allows improving the energy efficiency of the light source.

Table 1 compares the photovoltaic characteristics of the solar panels under solar irradiation $(1000 \mathrm{~W} / \mathrm{m} 2)$ and the sulfur lamp (170 W/ m2).

Table 1. Comparison photovoltaic parameters.

\begin{tabular}{ccccccc}
\hline \hline \multirow{2}{*}{ Light source } & \multicolumn{7}{c}{ Parameters } \\
\cline { 2 - 7 } & $\boldsymbol{I}_{\boldsymbol{s c}, \boldsymbol{A}}$ & $\boldsymbol{V}_{\boldsymbol{o c}}, \boldsymbol{W}$ & $\begin{array}{c}\boldsymbol{J}_{\boldsymbol{s c}}, \\
\boldsymbol{A} / \boldsymbol{m}^{2}\end{array}$ & $\eta, \boldsymbol{\%}$ & $\boldsymbol{F F}$ & $\boldsymbol{P}_{\text {out }}, \boldsymbol{W}$ \\
\hline Outdoor (Sun) & 8,94 & 38,5 & 5,5 & 16,6 & 0,78 & 165,2 \\
Sulfur Lamp & 0,97 & 36,4 & 0,6 & 8,15 & 0,79 & 17,3 \\
\hline \hline
\end{tabular}

The fill factor finds as

$$
F F=\frac{V_{m} \cdot I_{m}}{V_{O C} \cdot I_{s c}}=\frac{P_{\max }}{V_{O C} \cdot I_{s c}},
$$

where $V_{m}$ - the voltage and current at peak power, respectively; $P_{\max }$ - the maximum output power of solar cell; $V_{O C}-$ the open circuit voltage; $I_{S C}-$ the short circuit current. The solar efficiency of the solar cell can be written as

$$
\eta=\frac{V_{m} \cdot I_{m}}{S \cdot E} \times 100 \%
$$

where $S-$ the area of solar cell on which radiation falls $\left[\mathrm{m}^{2}\right] ; E-$ the irradiance $\left[\mathrm{W} / \mathrm{m}^{2}\right]$. 
The electrical power output of the solar cell is given by

$$
P_{\text {out }}=F F \cdot J_{S C} \cdot V_{o C}
$$

Fig. 5 shows the thermal images taken from the back solar panel under the real sun and the electrodeless sulfur lamp with microwave excitation.
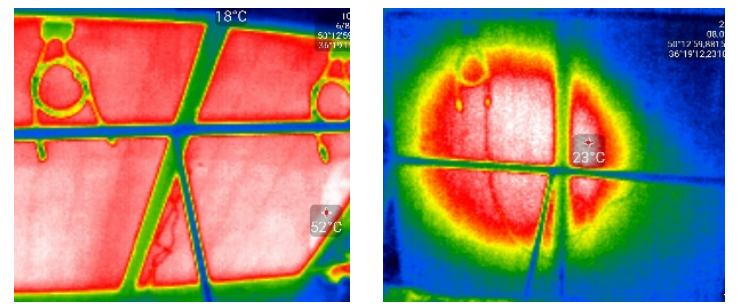

Fig. 5. Thermal imaging of the solar panel with the back side

When using a tungsten halogen lamp together with a sulfur lamp, the spectral response of solar cells in the near-infrared region can be improved. In Fig. 6 shows the spectra of sulfur and halogen lamps separately and their combined radiation, as well as the external quantum efficiency for silicon.

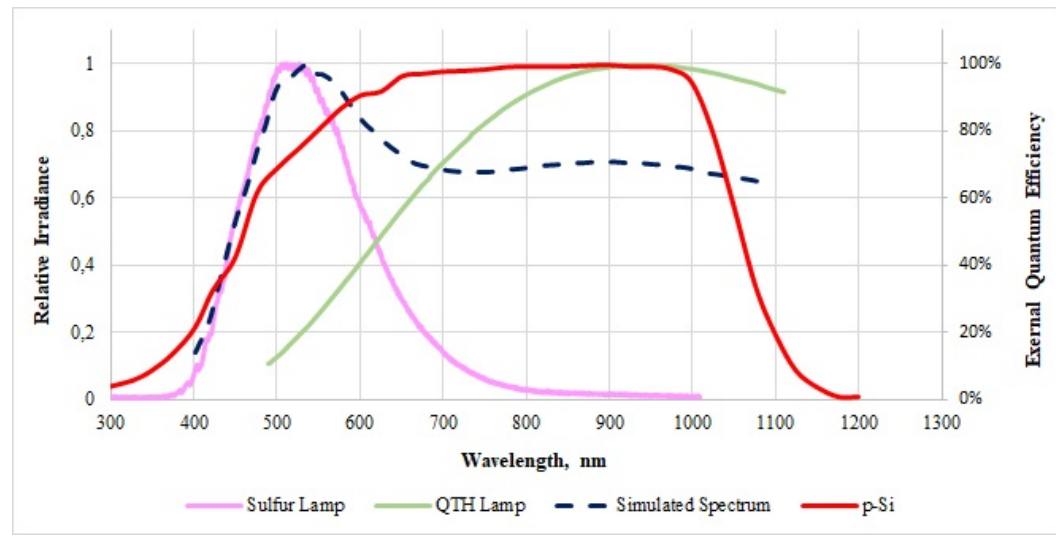

Fig. 6. Spectral profiles of lamps and spectral response curve for silicon

The Fig. 7 and 8 show a general block diagram of a lighting device based on the connection of an electrodeless sulfur lamp with microwave excitation to solar batteries and a diagram of connecting solar panels with additional devices: the solar panels, a charge controller, the batteries, an inverter, an automatic transfer switch, an electric network, a light systems and other electronic devices. 


\section{Conclusions}

Thus, an application of the electrodeless sulfur lamps with microwave excitation allows creating new electronic plants and systems for the effective decision of different technology problems. It is shown, that the output optical radiation of an electrodeless sulfur lamp has a

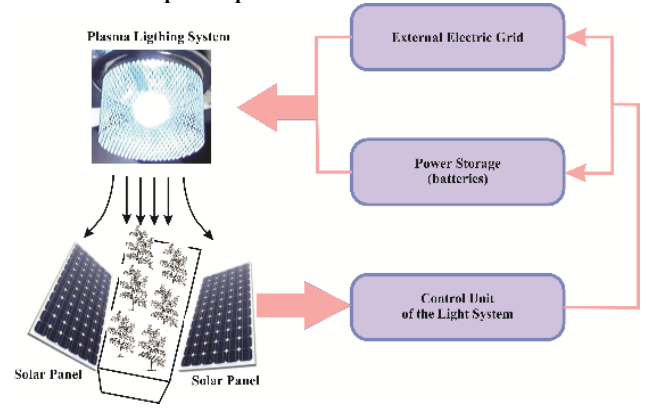

Fig. 7. General block diagram of a lighting device on the basis of linking an electrodeless sulfur lamp with microwave excitation and solar panels

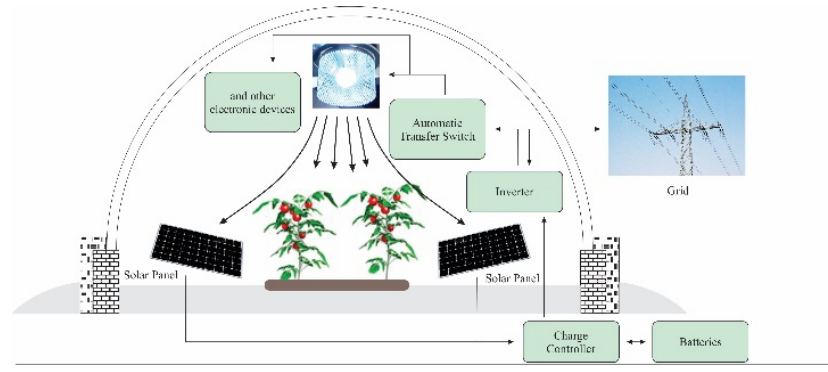

Fig. 8. Scheme of connection of the solar panels

continuous quasi-solar spectrum close to the spectrum of the Sun in the wavelength interval, where the spectral sensitivity of the solar cells is the most effective. The possibility of using plasma lighting systems based on an electrodeless solar lamp with microwave excitation in combination with solar batteries has an additional regeneration of electricity by converting its optical radiation into electric current using solar panels. Regenerated energy can be used in the future for the operation of both the lighting device itself and other electrical equipment or for its return to the external electrical grid. This will save energy and reduce the cost of production in general (for example, when growing agricultural products).

\section{References}

1. Microwave Power Engineering. Edited by E.C. Okress. V. 1, 2. Academic Press, New York \& London. 1968.

2. A. N. Didenko, SVCh-energetika. Teoriya i praktika. - Moscow: Nauka. 2003. $445 \mathrm{~s}$.

3. G. Churyumov, T. Frolova, "Microwave Energy and Light Energy Transformation: Methods, Schemes and Designs. Microwave Energy and Light Energy Transformation: Methods, Schemes and Designs" // In the book "Emerging Microwave Technologies in Industrial, Agricultural, Medical and Food Processing.” Edited by Kok Yeow You, IntechOpen, 2018. pp. 75-91. 
4. Dolan I.T., Ury M.G., Wood C.H. A Novel High Efficacy Microwave Powered Light Source. Presented as a Land-mark.- In: Sixth International Symposium on the Science and Technology of Light Sources, 1992, Technical University of Budapest.

5. Frolova T. Analysis of a Solar Simulator Based on the Electrodeless Sulfur Lamp for Photovoltaic Devices // T. Frolova, A. Frolov / Telecommunications and Radio Engineering, № 77 (6), - 2018. - P. 525-539. DOI: 10.1615/TelecomRadEng.v77.i6.50

6. ASTM G173-03(2012), Standard Tables for Reference Solar Spectral Irradiances: Direct Normal and Hemispherical on $37^{\circ}$ Tilted Surface, ASTM International, West Conshohocken, PA, 2012, DOI: 10.1520/G0173-03R12

7. D.A. Peterson, L.A. Schlie. Stable pure sulfur discharges and associated spectra // The Journal of Chemical Physics, Vol. 73, \# 4. 1980. P. 1551-1566.

8. C. W. Johnston, J. Jonkers, and J. J. A. M. van der Mullen, "Operational trends in the temperature of a high-pressure microwave powered sulfur lamp," J. Phys. D: Appl. Phys., 2002. pp. 2578-2585.

9. Frolova T.I., Churyumov G.I. Lighting Devices based on the Electrodeless Sulfur Lamp with Microwave Excitation / Patent of Ukraine \# 123127; Publication 12 February 2018 (2018) (in Ukrainian) 


\section{SYNERGETIC EFFECT OF MICROWAVE PLASMA AND CATALYSTS IN CO HYDROGENATION INTO HIGH ADDED-VALUE MOLECULES}

B. Alrafei, J. Delgado, A. Ledoux, I. Polaert.

${ }^{I}$ Normandie University, INSA Rouen, LSPC, EA 4704, Avenue de l'Université - Saint-

Étienne-du-Rouvray cedex 76801 France

e-mail: isabelle.polaert@insa-rouen.fr

Keywords: Microwave, Plasma, $\mathrm{CO}_{2}$ methanation, Catalyst.

\section{Introduction}

The concept known as Power-to-gas (PtG) has shown an outstanding importance in terms of renewable energy storage and $\mathrm{CO}_{2}$ conversion into high added-value products such as methane and methanol. The production of electricity from renewable energy is very dependent on weather and environmental conditions, thus the large excess of electricity can be used in water electrolysis in order to produce hydrogen and oxygen, which can be used directly or as intermediates in chemical reactions. The complexity of hydrogen storage due to its explosive properties make the Power-to-gas concept a very suitable process to store the hydrogen and reduce $\mathrm{CO}_{2}$ emissions at the same time by reacting hydrogen with $\mathrm{CO}_{2}$ to form methane or methanol.

$\mathrm{CO}_{2}$ is a very stable molecule due to its inert electronic structure, and its conversion requires various forms of energy supply (heat, light, etc.) to overcome kinetic limitations. Conventional processes operate under high temperatures and pressures, which leads to fast deactivation of the catalyst and temperature control issues in the reactor due to the high exothermicity of the reaction.

The coupling of non-thermal plasma with $\mathrm{CO}_{2}$ hydrogenation reaction is considered very promising in terms of chemical reaction improvement; especially that renewable energy can be the driving force of this technology [1]. The first works were carried out by Russians [2,3] and Italians $[4,5]$. Different types of plasma were used in order to dissociate $\mathrm{CO}_{2}$ molecule such as Luminescent, Microwave (MWP), Dielectric Barrier Discharge (DBD) and Corona plasma. Among the different types of plasma, microwave plasma is the most interesting. Fig. 1 shows the energy efficiency as a function of $\mathrm{CO}_{2}$ conversion for the different types of plasma. The energy efficiency is defined as: $\eta=\frac{\mathrm{X}_{T} \cdot \Delta H_{r}}{S E I}$

With $\mathrm{X}_{T}$ is the total conversion: $\mathrm{X}_{T}=y_{\mathrm{CO}_{2} \text { in }} \cdot X_{\mathrm{CO}_{2}}+y_{\mathrm{H}_{2} \text { in }} \cdot X_{\mathrm{H}_{2}}$

and SEI is the specific energy input : $\operatorname{SEI}(\mathrm{eV} /$ molecule $)=\frac{\operatorname{SEI}\left(\mathrm{J} \cdot \mathrm{cm}^{-3}\right)}{3.92}=\frac{P / \mathrm{F}}{3.92}$

where $\mathrm{P}$ is the power in $\mathrm{W}$, and $\mathrm{F}$ is the input flow rate in $\mathrm{cm}^{3} \cdot \mathrm{s}^{-1}$.

It is clear that higher conversion and efficiency could be achieved using microwave plasma compared to other discharges [6].

A microwave discharge does not require any electrodes to be generated and therefore, the cost of these electrodes and their maintenance is reduced compared to that of glow discharge plasmas or DBD. Microwave plasma can also be generated over a wide pressure range (between 3 mbar and 1 bar). In addition, in the case of microwave plasma, more electrons 
and active species are produced compared to other types of plasma $[7,8]$. This ensures an appropriate environment for a chemical reaction and allows effective dissociation of $\mathrm{CO}_{2}$.

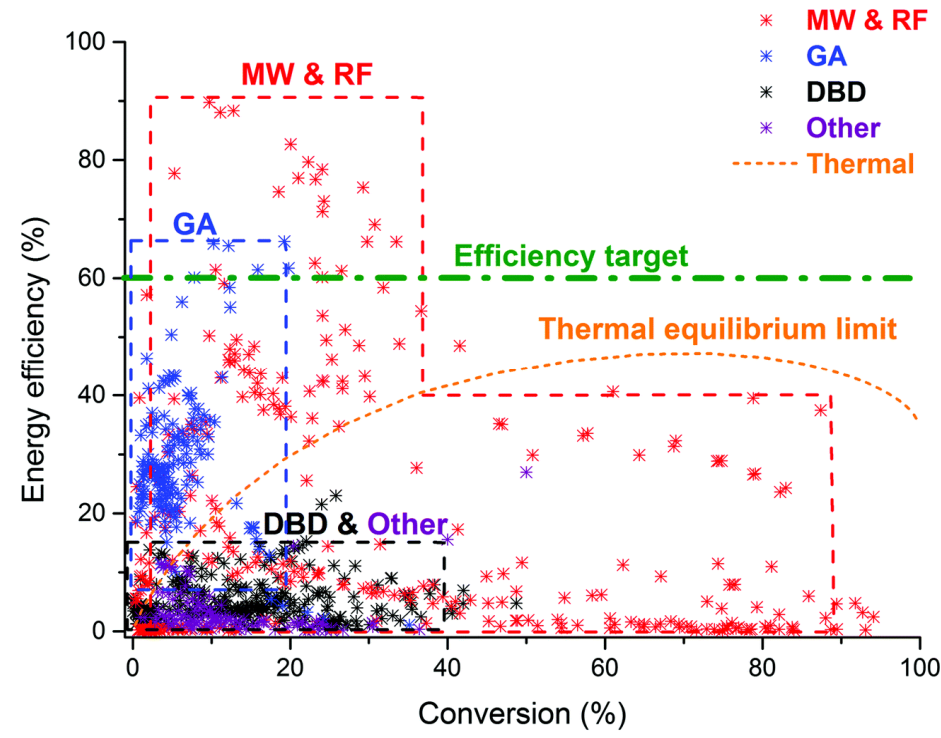

Fig. 1. Comparison of the energy efficiency of $\mathrm{CO}_{2}$ conversion using different plasma types [6]

This work focuses on the hydrogenation of $\mathrm{CO}_{2}$ into light hydrocarbons $\left(\mathrm{CH}_{4}, \mathrm{CH}_{3} \mathrm{OH}\right)$ under microwave plasma. The synergy between plasma and $\mathrm{Ni}$ catalysts supported on alumina was studied. First, the reaction was carried out without any catalyst and the effect of the $\mathrm{CO}_{2} / \mathrm{H}_{2}$ ratio, total flow rate and incident power were evaluated. Then, MWP was coupled with $\mathrm{Ni}$ catalysts that we prepared, characterized and tested on conventional $\mathrm{CO}_{2}$ methanation reaction [9]. Several configurations were studied by changing the position of the catalyst bed. The results obtained were compared to conventional catalytic tests performed with the same catalysts.

\section{Experimental set-up}

The MWP set-up used in the present work is illustrated in Figure 1. This laboratory scale set-up can be divided into two parts: the plasma generation and the gas feed and circuit.

The plasma generation set up includes a microwave generator (magnetron) with a maximum power of $3000 \mathrm{~W}$ working at $2.45 \mathrm{GHz}$ frequency. It includes an isolator with a reflected power crystal detector to protect the magnetron from reflected power. Manual impedance tuners and a manual sliding short-circuit were used to ensure a high energy supply into the plasma zone. The tuners and the sliding short-circuit are used to optimize the transfer of energy from the source to the load. A microwave cavity plasma generator (downstream plasma source) was used to ignite the plasma in a quartz tube with $30 \mathrm{~mm}$ inner diameter. Two tubes with the same diameter were used, one with a glass frit (pore size 2) and onother without the frit. The cavity was cooled simultaneously with compressed air and water. The tube wall was cooled using compressed air. A vaccum pump with a minimum pressure of 0.15 mbar was connected to the tube. The operating pressure was varied between 70 and 800 $\mathrm{Pa}$. 
The gaseous reactants were introduced into the tube using three mass flowmeters. A capacitif pressure sensor was used to measure the pressure into the tube. The temperature of the outgoing air from the cavity was measured using a $\mathrm{K}$ type thermocouple, and the one at the surface wall was measured using an optic fiber.

The outlet gas was analysed using gas chromatograph equipped with three columns and two detectors (FID, TCD).

\section{Experimental procedure}

First, the plasma was ignited in the reactor using a low flow of nitrogen while applying a power between $800 \mathrm{~W}$ and $1000 \mathrm{~W}$. The pressure in the reactor at this stage was between 100 and $200 \mathrm{~Pa}$. After ignition, the plasma was centered using the manual sliding shortcircuit, and the reflected power was minimized.

Later, the reactants $\left(\mathrm{CO}_{2}\right.$ and $\left.\mathrm{H}_{2}\right)$ were introduced into the reactor, and nitrogen flow was adjusted to keep a $\mathrm{CO}_{2} / \mathrm{H}_{2} / \mathrm{N}_{2}$ ratio equal to $1 / 4 / 5$. Then, the reflected power was minimized again by means of the sliding short-circuit and the tuners if necessary.

As the analysis was done using an off-line GC, the gas at the outlet of the pump was collected using sampling bags and then analyzed in the GC.

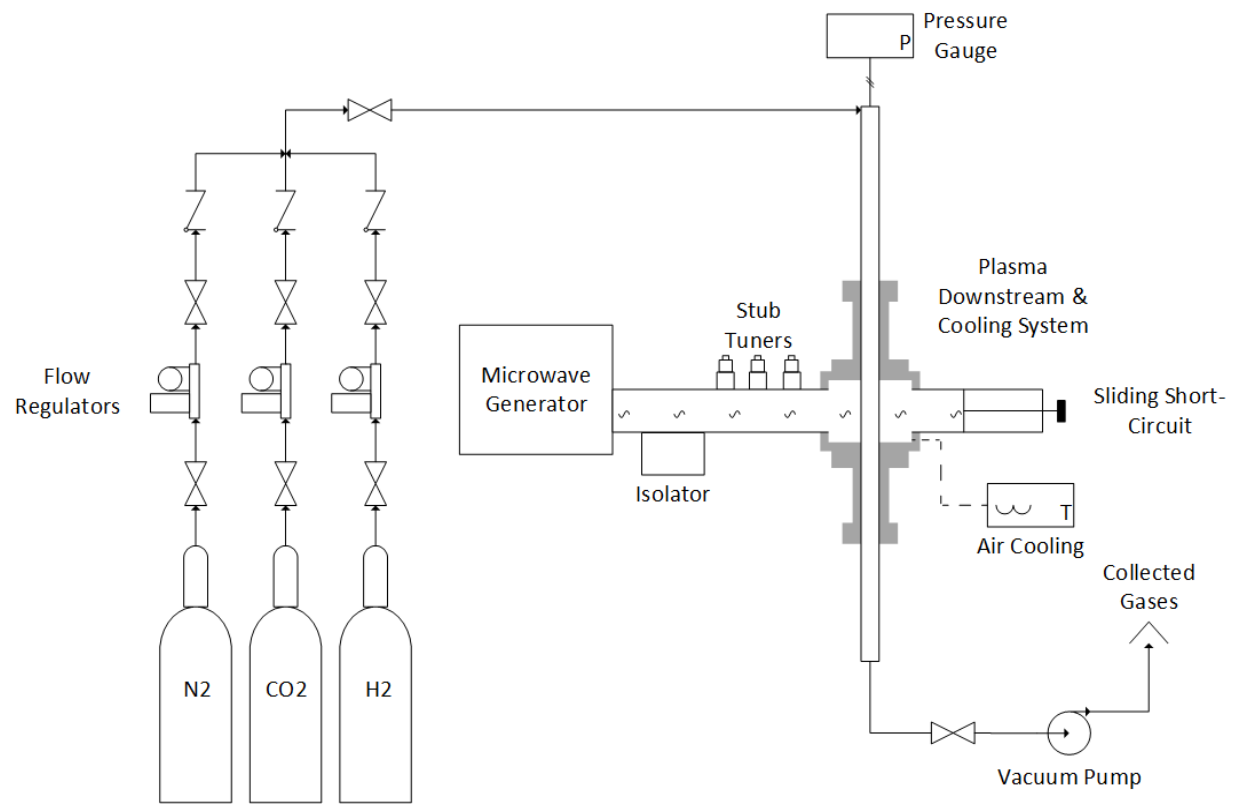

Fig 1. Schematic of the laboratory scale MWP set-up 


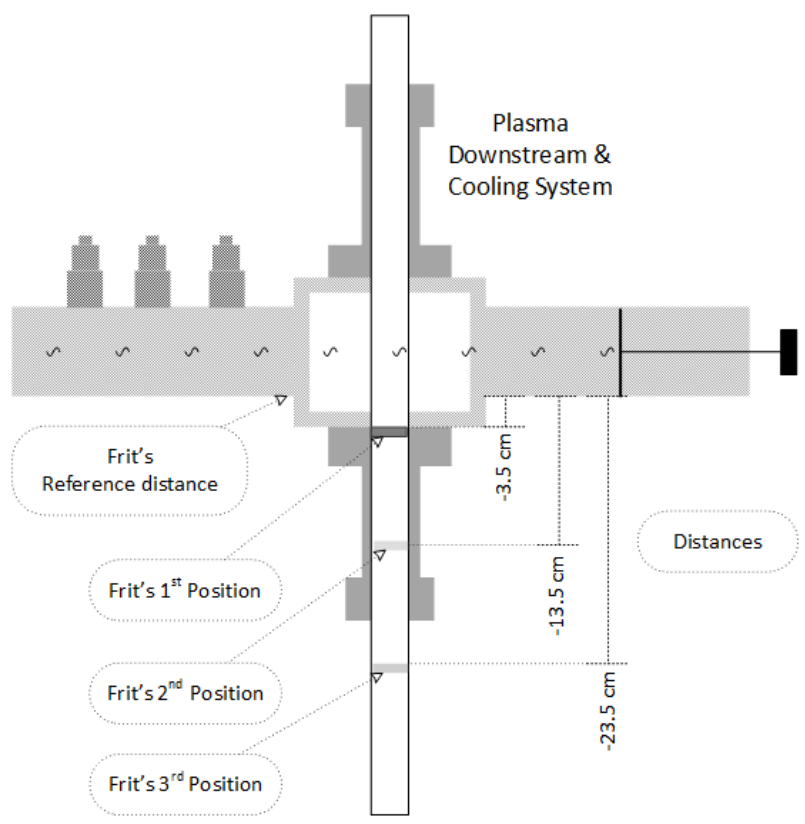

Fig 2. Different frit positions in the quartz tube

\section{Results and discussion}

\section{Catalyst-free $\mathrm{CO}_{2}$ hydrogenation with MWP}

First, $\mathrm{CO}_{2}$ hydrogenation was evaluated in the hollow tube without any catalyst. Figure 1 and 2 present the effect of total flow rate and incident power on $\mathrm{CO}_{2}$ conversion and $\mathrm{CO}$ selectivity. A fixed $\mathrm{H}_{2} / \mathrm{CO}_{2} / \mathrm{N}_{2}$ ratio equal to $4 / 1 / 5$ was used, and the total flow rate was varied between 100 and $400 \mathrm{~mL} / \mathrm{min}$. The results showed that the increase in total flowrate induces a strong decrease in $\mathrm{CO}_{2}$ conversion. This can be directly related to the decrease in reactants residence time in the plasma column when the total flow rate increases. The increase of total flow rate induced an increase of the pressure in the reactor, which results in more contracted plasma, shorter plasma column was then observed.

$\mathrm{CO}_{2}$ conversion is also greatly affected by the microwave input power. It is worth to mention that the absorbed power is very close to the incident power thanks to the optimization via the short circuit and the tuners. $\mathrm{CO}_{2}$ conversion is favored at higher input power between $300 \mathrm{~W}$ and $500 \mathrm{~W}$. At $600 \mathrm{~W}$, nearly the same conversion was obtained, which means that an equilibrium of $\mathrm{CO}_{2}$ dissociation in the plasma was achieved. The increase of $\mathrm{CO}_{2}$ conversion with input power can be related to a higher specific energy input, which implies highly energetic electrons that collide with gas molecules and dissociate $\mathrm{CO}_{2} . \mathrm{CO}_{2}$ conversion reached a maximum value of $84 \%$ using a total flowrate of $100 \mathrm{~mL} / \mathrm{min}$ and an incident power equal to $600 \mathrm{~W}$. 


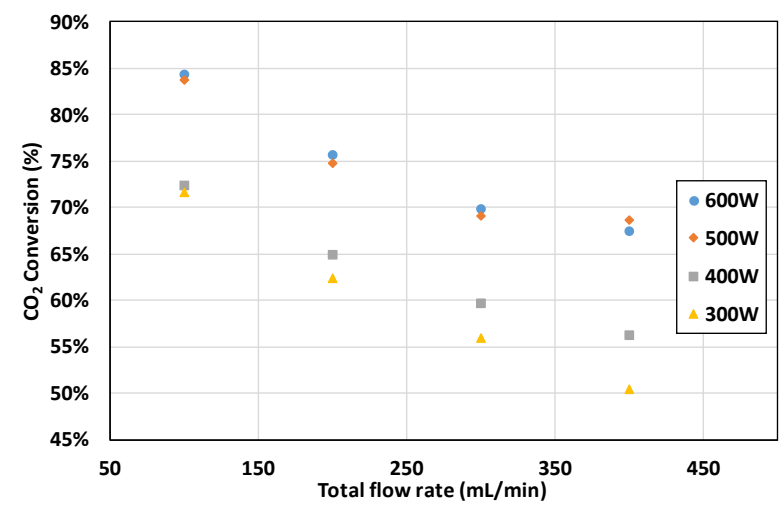

Fig 3. Effect of total flow on $\mathrm{CO}_{2}$ conversion, Hollow tube, $\mathrm{H}_{2} / \mathrm{CO}_{2} / \mathrm{N}_{2}=4 / 1 / 5$

Looking at $\mathrm{GC}$ analysis results, it was observed that $\mathrm{CO}$ is the main product of the reaction. Carbon dioxide was mainly reduced into $\mathrm{CO}$ in the plasma. Fig.4 shows that $\mathrm{CO}$ selectivity was not much affected neither by the total flow rate, nor by the incident power, and reaches about $90 \%$ in each case. However, it also means that about $10 \%$ of the converted carbon dioxide leads to other carbonated species that can be of great interest.

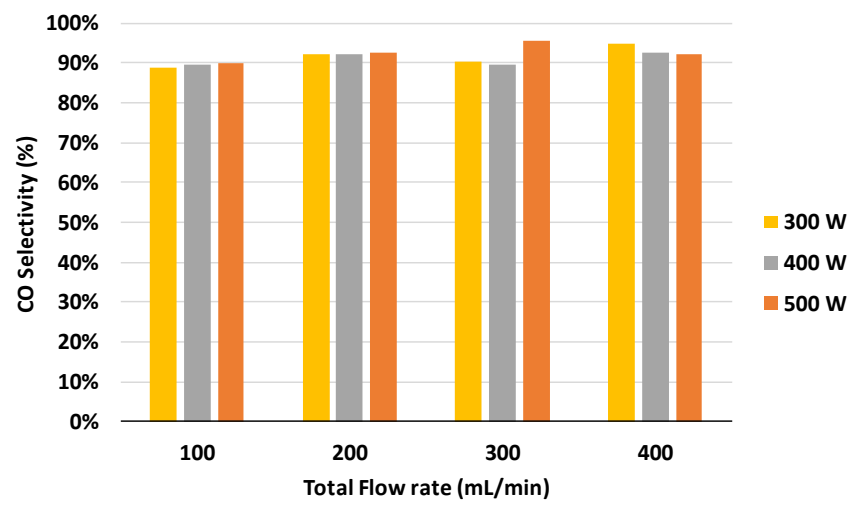

Fig 4. Effect of total flow rate on $\mathrm{CO}$ selectivity, Hollow tube, $500 \mathrm{~W}, \mathrm{H}_{2} / \mathrm{CO}_{2} / \mathrm{N}_{2}=4 / 1 / 5$

Figure 5 shows methane and methanol production in ppm. Methanol production was higher than methane $(\sim 1500 \mathrm{ppm})$ during $\mathrm{CO}_{2}$ hydrogenation in the plasma. The production of methane was not much affected by microwave power, but more affected by the total flow: it increased by decreasing total flow rate from 400 to $100 \mathrm{~mL} / \mathrm{min}$. Methanol production was more dependent on the power. It showed a maximum of $1632 \mathrm{ppm}$ at $400 \mathrm{~W}$.

Small amounts of propane (100-150 ppm) were also observed during GC analysis. 

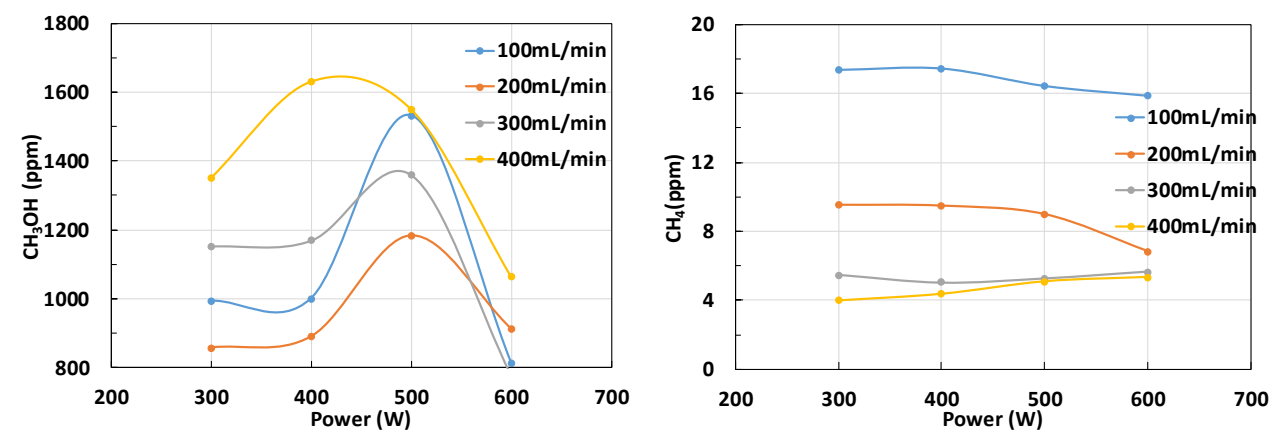

Fig 5.Methanol and methane production in ppm

The fraction of nitrogen in the feed gas was varied between $20 \%$ and $50 \%$ in order to study the effect of inlet gas dilution on $\mathrm{CO}_{2}$ conversion. The flow rate of $\mathrm{H}_{2}$ and $\mathrm{CO}_{2}$ remained fixed at $160 \mathrm{~mL} / \mathrm{min}$ and $40 \mathrm{~mL} / \mathrm{min}$ respectively, and a power of $300 \mathrm{~W}$ was used (Figure 6). It was found that the increase in $\mathrm{N}_{2}$ fraction in the inlet gas induced a moderate decrease in $\mathrm{CO}_{2}$ conversion. This can be explained by a competitive absorbance of energy between $\mathrm{N}_{2}$ and the other molecules. $\mathrm{N}_{2}$ is known as a high energetic molecule in plasma, it can store energy by vibration and rotation and thus, a part of the incident power is not used to dissociate $\mathrm{CO}_{2}$, but is consumed by $\mathrm{N}_{2}$.

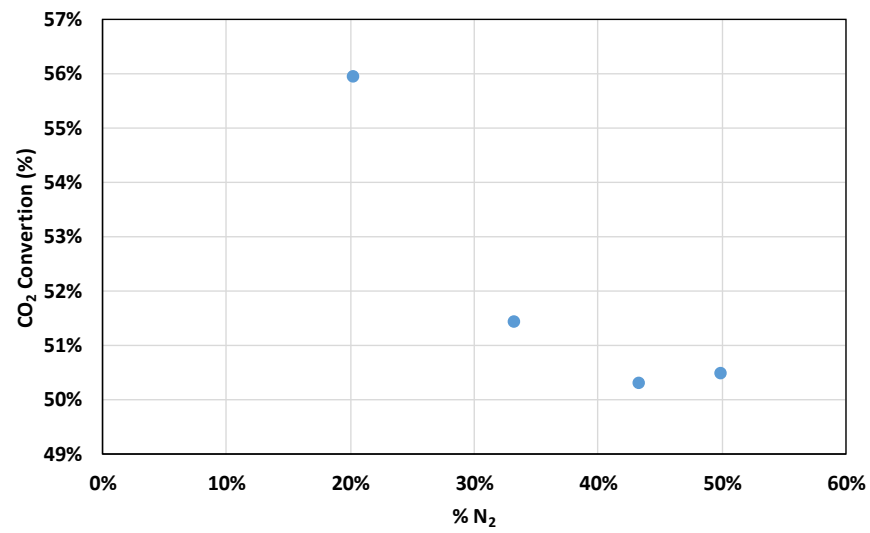

Fig 6. Nitrogen dilution effect on $\mathrm{CO}_{2}$ conversion, Hollow tube, $300 \mathrm{~W}, \mathrm{~F}_{\mathrm{H} 2}=160 \mathrm{~mL} / \mathrm{min}$, $\mathrm{F}_{\mathrm{CO} 2}=40 \mathrm{~mL} / \mathrm{min}$

\section{Plasma-assisted catalytic $\mathrm{CO}_{2}$ hydrogenation}

The catalyst $20 \% \mathrm{Ni} / \mathrm{Al}_{2} \mathrm{O}_{3}$ calcined at $450{ }^{\circ} \mathrm{C}$ prepared and characterized in [9] was used in the present study. A comparison between experiments made with the hollow tube, $1 \mathrm{~g}$ and $5 \mathrm{~g}$ of the catalyst is presented in Figure 7 in terms of $\mathrm{CO}_{2}$ conversion, $\mathrm{CH}_{4}$ and $\mathrm{CH}_{3} \mathrm{OH}$ production, and $\mathrm{CH}_{3} \mathrm{OH} / \mathrm{CH}_{4}$ ratio. $\mathrm{CO}_{2}$ conversion increased from $75 \%$ to more than $90 \%$ when using nickel catalyst with plasma. This increase in $\mathrm{CO}_{2}$ conversion results from the effect of coupling plasma with nickel catalyst. As well, methane and methanol production increased after catalyst addition. Methanol production increased from $900 \mathrm{ppm}$ to $1900 \mathrm{ppm}$, and methane production from $6 \mathrm{ppm}$ to $25 \mathrm{ppm}$. 
The $\mathrm{CH}_{3} \mathrm{OH} / \mathrm{CH}_{4}$ ratio decreased when coupling the plasma with catalyst. This means that the use of catalyst increased the rate of methane production more than methanol one. The amount of methane produced using plasma-catalyst is 5 times greater than the amount produced using the plasma alone, while that of methane is 2 times greater than the amount produced using plasma.
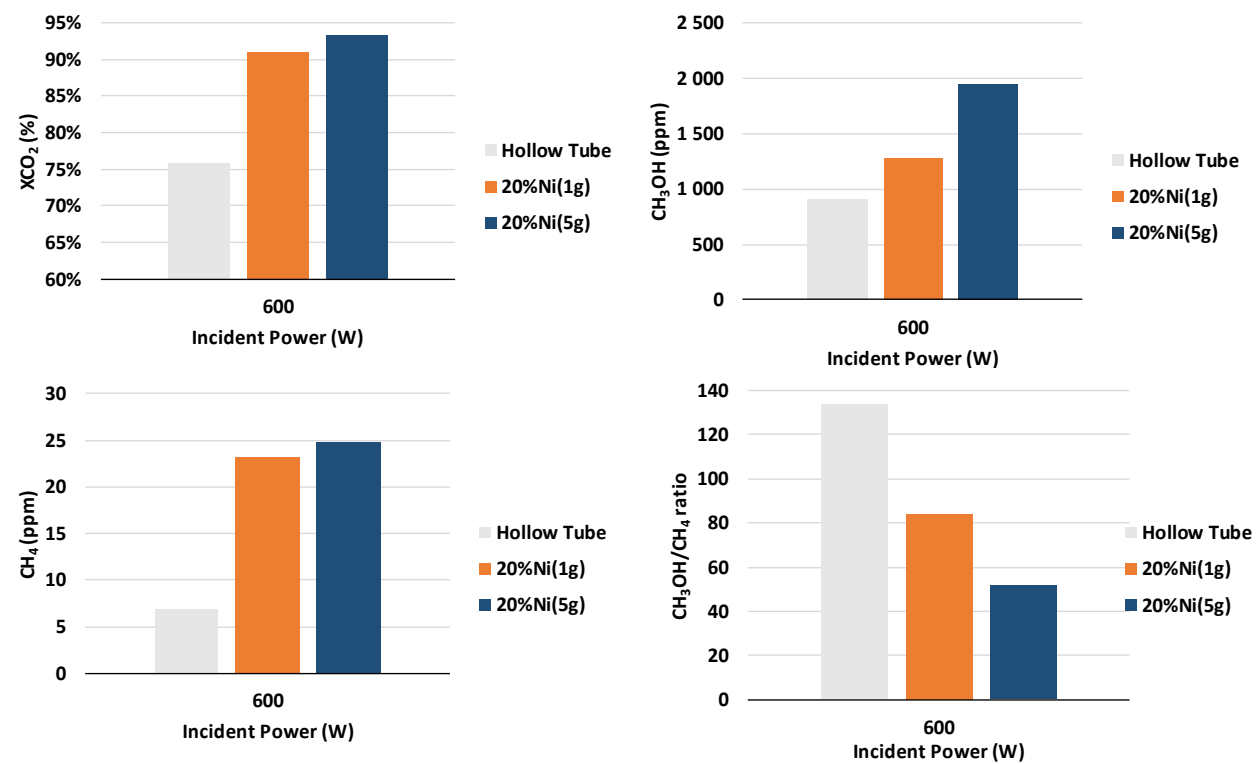

Fig 7.Catalytic $\mathrm{CO}_{2}$ hydrogenation using MWP, $600 \mathrm{~W}, 400 \mathrm{~mL} / \mathrm{min}, \mathrm{H}_{2} / \mathrm{CO}_{2} / \mathrm{N}_{2}=4 / 1 / 5$

The catalyst bed position was varied in order to evaluate the effect of plasma on catalyst activity and reported in Table 1 . A higher $\mathrm{CH}_{3} \mathrm{OH} / \mathrm{CH}_{4}$ ratio was found when putting the catalyst in the position $-13.5 \mathrm{~cm}$, which is further from the center of plasma. This can be explained by more favorable thermal conditions in this position for $\mathrm{CO}_{2}$ hydrogenation into methanol.

Table 1.Effect of catalyst bed position on $\mathrm{CH}_{4} / \mathrm{CH}_{3} \mathrm{OH}$ ratio

\begin{tabular}{lcc}
\cline { 2 - 3 } & \multicolumn{2}{c}{ Catalyst position $(\mathrm{cm})$} \\
\cline { 2 - 3 } $\mathrm{CH}_{3} \mathrm{OH} / \mathrm{CH}_{4}$ & -10.3 & $-13,5$ \\
\hline
\end{tabular}

\section{Conclusion}

Microwave plasma technology can be efficiently used to convert carbon dioxide into carbon monoxide. By adding Ni catalyst into the plasma, a synergetic effect is observed and light hydrocarbons such as methanol and methane, propane are produced. The results of this study clearly show that the reduction of $\mathrm{CO}_{2}$ is governed by a combined effect between the plasma induced electronic excitation and catalysis at the reduced nickel particles surface. Optimization is possible by varying the energy input, the flowrates and the relative position between the catalytic bed and the plasma. 


\section{References}

[1] Y. Qin, G. Niu, X. Wang, D. Luo, Y. Duan, Status of CO2 conversion using microwave plasma, Journal of CO2 Utilization. 28 (2018) 283-291. doi:10.1016/j.jcou.2018.10.003.

[2] A. Fridman, Plasma Chemistry by Alexander Fridman, Cambridge Core. (2008). doi:10.1017/CBO9780511546075.

[3] V.A. Legasov, V.K. Zhivotov, E.G. Krasheninnikov, M.F. Krotov, B.I. Patrushev, V.D. Rusanov, G.V. Rykunov, A.M. Spektor, A.A. Fridman, G.V. Sholin, Nonequilibrium plasma-chemical process of the decomposition of $\mathrm{CO} 2 \mathrm{in} \mathrm{HF}$ and UHF discharges, Akademiia Nauk SSSR Doklady. 238 (1978) 66-69.

[4] P. Capezzuto, F. Cramarossa, R. D’Agostino, E. Molinari, Contribution of vibrational excitation to the rate of carbon dioxide dissociation in electrical discharges, J. Phys. Chem. 80 (1976) 882-888. doi:10.1021/j100549a024.

[5] M. Capitelli, E. Molinari, Kinetics of dissociation processes in plasmas in the low and intermediate presssure range, in: Plasma Chemistry II, Springer Berlin Heidelberg, 1980: pp. 59-109.

[6] R. Snoeckx, A. Bogaerts, Plasma technology - a novel solution for CO2 conversion?, Chem. Soc. Rev. 46 (2017) 5805-5863. doi:10.1039/C6CS00066E.

[7] J.F. de la Fuente, S.H. Moreno, A.I. Stankiewicz, G.D. Stefanidis, Reduction of CO2 with hydrogen in a non-equilibrium microwave plasma reactor, International Journal of Hydrogen Energy. 41 (2016) 21067-21077. doi:10.1016/j.ijhydene.2016.08.032.

[8] A. Vesel, M. Mozetic, A. Drenik, M. Balat-Pichelin, Dissociation of CO2 molecules in microwave plasma, Chemical Physics. 382 (2011) 127-131. doi:10.1016/j.chemphys.2011.03.015.

[9] B. Alrafei, I. Polaert, A. Ledoux, F. Azzolina-Jury, Remarkably stable and efficient $\mathrm{Ni}$ and Ni-Co catalysts for CO2 methanation, Catalysis Today. (2019). doi:10.1016/j.cattod.2019.03.026. 


\title{
IN-LIQUID PLASMA USING MICROWAVE POWER FOR APPLICATIONS
}

\author{
S. Sawada and S. Horikoshi
}

Sophia University, 7-1 Kioi-cho, Chiyoda-ku, Tokyo

sawada-1112@eagle.sophia.ac.jp,horikosi@sophia.ac.jp

Keywords: in-liquid plasma, semiconductor microwave generator, pulse irradiation, wastewater treatment, crosslinking agent/initiator-free polymer-gel synthesis

\begin{abstract}
The research of in-liquid plasma that uses microwave as an energy source can be generated without depending on the electric conductivity of the solution, so it has wide application. However, it is not practical because the microwave antenna (plasma electrode) is deteriorated immediately. We were noticed that the microwave is pulsed to solve this problem. In this study, its optimum conditions was investigated. The best parameters of pulse microwave power was investigated using the generator (peak power; $380 \mathrm{~W}$, pulse width ; $13.4 \mathrm{msec}$ and pulse duty cycle ; $16.7 \mathrm{msec}$ ). Stable in-liquid plasma could be generated in this conditions. This optimised system of in-liquid plasma tried to use applications for wastewater treatment and gel synthesis. In first application (wastewater treatment), tetrabromobisphenol A (TBBPA) was ionized and minerated by using in-liquid plasma method for only $20 \mathrm{~min}$, respectivily. In second one (gel synthesis), in-liquid plasma can synthesize gel-synthesis without crosslinking agent and initiator. This new method could synthesize gel of dimethyl polysiloxane (silicone) and polyvinilypirroridone (PVP) without both crosslinking agent and initiator, it suggested that in-liquid plasma method is effective as a green chemistry process.
\end{abstract}

\section{Introduction}

Historically, in-liquid plasma was generated in electrolytic aqueous media in the late 1980s by Clements et al. [1] who used needle and plate electrodes together with an alternating current (AC) power supply under gas bubbling conditions and was then proposed as a means of water treatments. Studies followed on generating in-liquid plasma by a discharge device using direct current (DC) or an AC power supply and two electrodes [2]. However, many of these devices required the conductive solution medium to generate inliquid plasma. Over a decade ago, Nomura et al. [3, 4] observed the ignition and maintenance of stable plasma in a liquid hydrocarbon solvent exposed to a combination of ultrasonic waves and microwave radiation. Though one of the problems in generating plasma continuously in a liquid medium with microwave radiation at high-applied microwave power levels is the deterioration of the tungsten antenna tip because both plasma heating, which can reach a temperature above the melting point of tungsten $\left(3422{ }^{\circ} \mathrm{C}\right)$, together with ultrasound waves, melted the electrode. In the last few years, our interest in this area has focused on finding ways (our challenges) to improve on the generation of stable in-liquid plasma in aqueous media and its applications. In this regard, the first challenge resolved successfully [5] was reduction of the electrical power from 700 to $220 \mathrm{~W}$ to produce the microwave radiation load on the electrode through the utilization of an integrated hybrid antenna system consisting of a supersonic horn $(19.5 \mathrm{kHz})$ and a microwave radiation $(2.45$ $\mathrm{GHz}$ ) source [5]. Unfortunately, while it was possible to continuous generate the in-liquid plasma; it was not possible to bring about improvements on the complex device. The second challenge resolved was that, in the absence of the supersonic horn and by reducing the 
pressure inside the reaction vessel with an aspirator, the irradiation power of the microwaves could be low significantly from 700 to $150 \mathrm{~W}$ [6]. Most importantly, the latter method led to the generation of stable, permanent in-liquid plasma. In order to advance the miniaturization of the device, we replaced the magnetron microwave generator used earlier [6] by a semiconductor microwave generator and discovered that, unlike the former, irradiation with 150-W microwaves no longer generated in-liquid plasma. Even if the microwave power increased to $300 \mathrm{~W}$, no in-liquid plasma generated-a curious result that led us to query the causes that might explain these observations.

To the extent that a semiconductor microwave generator, by its very nature, emits continuous microwaves that pose a problem in generating in-liquid plasma, we herein reexamine this issue by using a prototype high power semiconductor generator and compare its characteristics to the pulsed microwaves emitted by a magnetron to generate in-liquid plasma [7]. Accordingly, the aim of the present study was to investigate the optimal pulsed microwave conditions to generate long-lived and stable in-liquid plasma, and its applications toward the degradation of a TBBPA wastewater [8], and gel-synthesis of PVP and silicone [9].

\section{Experimental methods}

\subsection{Development of in-liquid plasma device}

Photograph and schematic illustration of in-liquid plasma device are shown in Fig. 1a and 1b, while Fig. 1c displays the photograph of the microwave-generated in-liquid plasma [7]. The microwave device was constructed using an Ampreon M2A-R semiconductor generator that emits either continuous or pulsed microwaves (maximal power, $1200 \mathrm{~W}$; microwave frequency, $2.45 \mathrm{GHz}$ ), together with an isolator (air cooling device), a power monitor, a three-stub tuner, and a short-circuit plunger. The liquid medium was continuously irradiated with microwaves through the tungsten electrode antenna (diameter, $1.0 \mathrm{~cm}$; length, $20.0 \mathrm{~cm}$ ). Metal cones were used in the waveguide to focus the microwaves onto the tip of the antenna electrode, through which the microwaves irradiated the aqueous solution and generated the in-liquid plasma (Fig. 1b). The tungsten antenna was isolated from both the reactor and the waveguide using a ceramic spacer, so that the microwave irradiated only the liquid. The cylindrically shaped reactor consisted of a quartz vessel (diameter, $32.0 \mathrm{~mm}$; length, $160.0 \mathrm{~mm}$ ) and fabricated such that the pressure reduced with a water aspirator through the top of the reactor. A three-stub tuner adjusted the irradiating microwaves such that there were no reflected waves.

\subsection{Application in wastewater treatment}

High-purity grade tetrabromobisphenol-A (TBBPA) was purchased from Wako Pure Chemical Industries, Ltd. The aqueous TBBPA solution $(50 \mathrm{~mL} ; 0.10 \mathrm{mM} \approx 54.4 \mathrm{mg}$ $\mathrm{L}^{-1}$ ) was placed in the quartz reactor and subsequently subjected to irradiation with the nerated in-liquid plasma [8]. The solution was adjusted to $\mathrm{pH}=9,11$ and 13 with sodium hydroxide. Samples of the decomposed TBBPA solution were collected at various time intervals and then analyzed to assess the extent of debromination of the TBBPA (formation of Br- ions) using a Thermo Scientific ${ }^{\mathrm{TM}}$ Dionex $^{\mathrm{TM}}$ Integrion $^{\mathrm{TM}}$ HPIC ${ }^{\mathrm{TM}}$; loss of total organic carbon (TOC) was determined using a Shimadzu TOC-L analyser. Computer simulations were carried out using a CAChe Worksystem version 3.2 (Fujitsu Co. Ltd) implemented on an Intel P-III and Windows 7 system. Frontier electron densities for radical attack in the heteroaromatic substrate was also calculated by a MOPAC/PM3 wavefunction. A geometrical configuration was determined by pre-optimization calculation in the Mechanics using augmented MM3, followed by geometrically optimized calculation in MOPAC using PM3 parameters; solvation effects in water were also simulated using COSMO. 
Intermediates species formed during the debromination and mineralization of TBBPA were identified by direct injection into the mass spectral detector of a JEOL Ltd., JMS-T100LC LC-TOFMS (electrospray ionization: ESI) system operated in the negative ion mode; orifice voltage was $30 \mathrm{~V}$. The eluent was a solution of methanol. Note that the LC column was not used in this study. The spectrometer was scanned from $\mathrm{m} / \mathrm{z}=100$ to 10,000 for recording mass spectra.

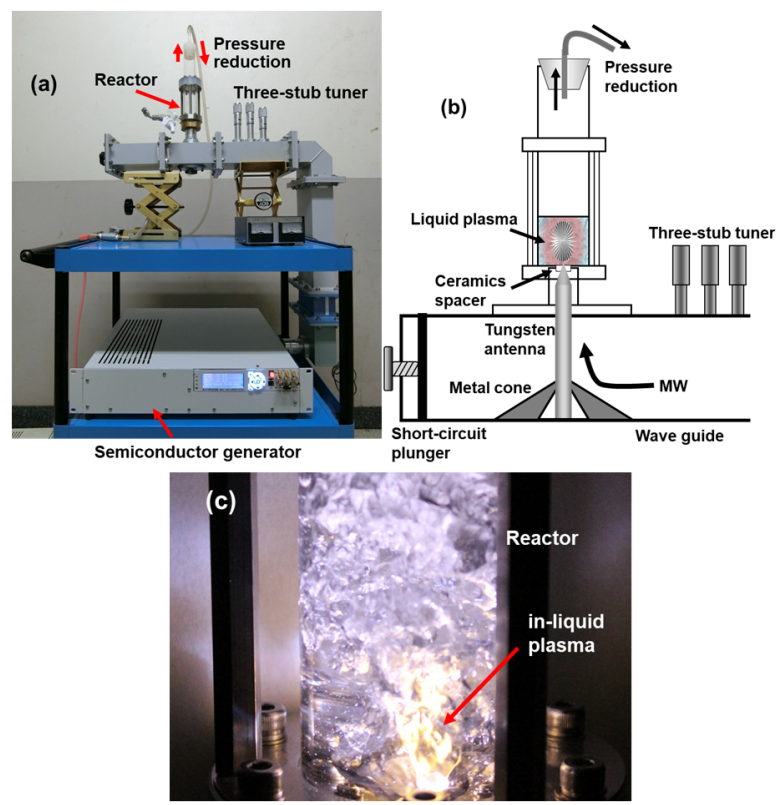

Fig. 1. (a) Photograph and (b) schematic image of in-liquid plasma device, and (c) photograph of the generated in-liquid plasma in the reactor

\subsection{Application in crosslinking agent/initiator-free polymer-gel synthesis}

High-purity grades of dichlorodimethylsilane and polyvinylpyrrolidone K30 (PVP) as starting materials for silicone gel (HySi-gel) and polyvinylpyrrolidone gel (PVP-gel) were purchased from Tokyo chemical industry Co., Ltd. In the synthesis of HySi-gel, dichlorodimethylsilane was used for monomer of dimethylpolysiloxane (silicone). $10 \mathrm{~g}$ dichlorodimethysilane was added in $50 \mathrm{~mL}$ ionized water and was stirred at room temperature $\left(25^{\circ} \mathrm{C}\right)$ for $1 \mathrm{~h}$. After that, the solution was stirred in $60^{\circ} \mathrm{C}$ for $5 \mathrm{~h}$ and silicone was synthesized in ionized water. Silicone-gel was synthesized from the silicone solution by using in-liquid plasma method at $30 \mathrm{sec}$-irradiation and $150 \mathrm{~W}$ of microwave power in the same pulse-microwave condition of the wastewater treatment. In the synthesis of PVP-gel, $3 \mathrm{~g}$ PVP was dissolved in $30 \mathrm{~mL}$ ionized water and PVP-gel was synthesized from the PVP solution by using in-liquid plasma method at 5 min-irradiation and $150 \mathrm{~W}$ of microwave power in the same pulse-microwave condition of wastewater treatment. the functional groups of PVP (before in-liquid plasma treatment) and PVP-gel (after in-liquid plasma treatment) were analyzed by using FT-IR. 


\section{Experimental results and discussions}

\subsection{Development of in-liquid plasma device}

The pulsed condition of microwave power was investigated to generate more stable plasma in aqueous solution and to extend the life of the electrode. The investigation parameters were amplitude of microwave power pulses, pulse width and pulse period. Maintaining the minimum threshold of the microwave applied power at $380 \mathrm{~W}$, the pulse width and the pulse period were then altered to achieve optimal conditions with which to generate in-liquid plasma. A matrix of the results to generate in-liquid plasma under the various conditions are presented in Table 1.

Table 1. Status of in-liquid plasma generation under different pulse conditions for a pulse period (ms : milisecond) and percent pulse duration (period) at afixed applied microwave power of $380 \mathrm{~W}$ (pulse amplitude) NG: no generation of in-liquid plasma, G: generation of in-liquid plasma, S: slight generation of sound waves, B: generation of fine bubbles

\begin{tabular}{|c|c|c|c|c|c|c|c|c|c|}
\hline \multirow{2}{*}{ Pulse period / ms } & \multicolumn{9}{|c|}{ Period /\% } \\
\cline { 2 - 10 } & 90 & 80 & 70 & 60 & 50 & 40 & 30 & 20 & 10 \\
\hline 10.0 & NG & NG & NG & NG & NG & NG & NG & NG & NG \\
\hline 11.0 & NG & NG & NG & NG & NG & NG & NG & NG & NG \\
\hline 12.5 & NG & NG & NG & NG & NG & NG & NG & NG & NG \\
\hline 14.3 & NG & NG & NG & NG & NG & NG & NG & NG & NG \\
\hline 16.7 & G & G & G & G & G & S & S & S & S \\
\hline 20.0 & S & S & S & S & S & S & B & B & B \\
\hline 25.0 & B & B & B & B & B & B & B & B & B \\
\hline
\end{tabular}

Only experiments conducted at a pulse width of $16.7 \mathrm{~ms}$ and a pulse period at $50 \%(8.35$ $\mathrm{ms})$ through $90 \%(15.03 \mathrm{~ms})$ led to the generation of stable plasma in the liquid. Consideration of power consumption, the optimal pulse irradiation conditions were a microwave irradiation power of $380 \mathrm{~W}$ (pulse amplitude), a pulse period of $16.7 \mathrm{~ms}$ and a pulse width of $13.4 \mathrm{~ms}$ (see Fig. 2). Stable generated in-liquid plasma could be sustained for $1 \mathrm{~h}$ with no visible deterioration of the tungsten antenna tip observed under these optimized conditions. Summary, we clarified the device conditions and the microwave irradiation conditions that can use the in-liquid plasma continuously for practical use, and this enabled stable experiments.

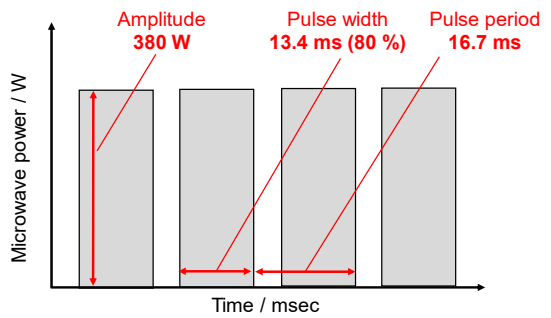

Fig. 2. Optimized conditions for pulsed microwave irradiation with a semiconductor microwave generator

\subsection{Application in wastewater treatment}

The time course of the debromination of aqueous TBBPA solutions under various alkaline conditions of $\mathrm{pH}(\mathrm{pH} \sim 9,11$ and 13) was examined with the optimised in-liquid plasma system (Fig. 1a) [8]. When decomposition was carried out at $\mathrm{pH} 13$ for an irradiation time of $20 \mathrm{~min}$, complete debromination occurred (ca. 100\%), whereas at pHs 11 and 9, the 
extent of debromination was $87 \%$ and $83 \%$, respectively (see Fig. 3a). Debromination followed first-order kinetics: $k=0.107 \pm 0.008 \mathrm{~min}^{-1}(\mathrm{pH} \mathrm{13}), 0.079 \pm 0.003 \mathrm{~min}^{-1}(\mathrm{pH} 11)$, and $0.079 \pm 0.006 \mathrm{~min}^{-1}(\mathrm{pH}$ 9). By comparison, the extent of mineralization of TBBPA was also complete $(100 \%)$ within 20 min of irradiation at $\mathrm{pH} \mathrm{13,} \mathrm{while} \mathrm{the} \mathrm{extent} \mathrm{of} \mathrm{TOC} \mathrm{loss}$ was $81 \%$ at $\mathrm{pH} 11$ and $76 \%$ at $\mathrm{pH} 9$; the corresponding first-order kinetics were: $k=0.28 \pm$ $0.05 \mathrm{~min}^{-1}$ (pH 13), $0.37 \pm 0.08 \mathrm{~min}^{-1}$ (pH 11), and $0.39 \pm 0.11 \mathrm{~min}^{-1}$ (pH 9) - see Fig. 3b. Clearly, the optimal condition to degrade and mineralize the TBBPA flame retardant is in aqueous alkaline media at $\mathrm{pH} 13$.
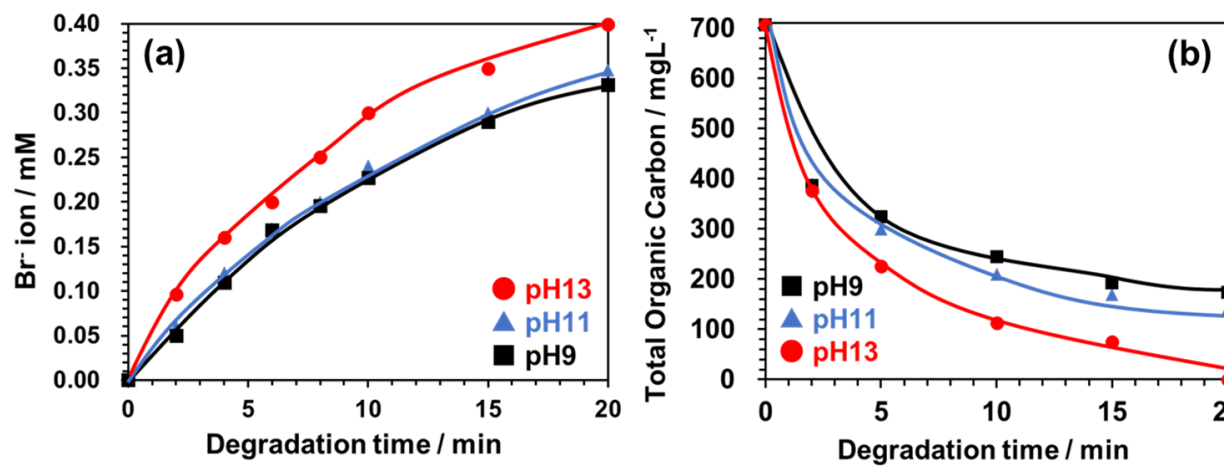

Fig. 3. (a) Dependence of the debromination of tetra-bromobisphenol-A (TBBPA) at various alkaline $\mathrm{pHs}(\mathrm{pH}=9,11$ and 13) and on irradiation time; (b) plot showing the time course of the loss of total organic carbon (TOC) under in-liquid plasma irradiation at various $\mathrm{pHs}$ of 9,11 , and 13 . Other conditions: volume of aqueous medium, $50 \mathrm{~mL}$; concentration of TBBPA, $0.10 \mathrm{mM}$ or ca. $54.4 \mathrm{mg} \mathrm{L}^{-1}$

In the graph of Fig. 3a, bromo-ion concentration of the samples depended on the $\mathrm{pH}$ value. The strongest basic solution ( $\mathrm{pH} \mathrm{13)}$ promoted to desert bromo-ion from TBBPA and the solution which in-liquid plasma had treated for $20 \mathrm{~min}$ in the $\mathrm{pH}$ value of 13-desert bromo-ion completely. On the other hands, the graph of Fig. 3b was about evaluation of TOC concentration of samples. The decrease of TOC concentration promoted to be decomposed in order of $\mathrm{pH} 13, \mathrm{pH} 11$ and $\mathrm{pH} 9$ because reactions between $\mathrm{OH}$ radical and bromo-ion occurred when in-liquid plasma generates. These reactions are shown below [10]. $\mathrm{OH}$ radical was synthesized in reaction of (eq. 1) by using in-liquid plasma method. $\mathrm{Br}^{-}$ion was ionized and $\mathrm{Br}$ radical was synthesized from TBBPA in solution by using in-liquid plasma. After that, $\mathrm{BrOH}^{-}$radical could synthesized from $\mathrm{Br}$ radical and $\mathrm{Br}^{-}$ion in reaction of (eq. 2). $\mathrm{Br} 2$ - radical, $\mathrm{Br}$ radical and $\mathrm{OH}^{-}$ion were combined into $\mathrm{BrOH}^{-}$radical in reactions of (eq.3) and (eq. 4). $\mathrm{BrOH}^{-}$radical was ionized to $\mathrm{OH}$ radical and $\mathrm{Br}^{-}$ion (eq. 5) so $\mathrm{OH}$ radical promoted to decompose TBBPA in $\mathrm{pH}$ value of 13 during generating in-liquid plasma.

$$
\begin{aligned}
& \mathrm{H}_{2} \mathrm{O}+\mathrm{e}^{*} \rightarrow \mathrm{H}^{\bullet}+{ }^{\bullet} \mathrm{OH}+\mathrm{e} \\
& \mathrm{Br}^{\bullet}+\mathrm{Br}^{-} \rightleftarrows \mathrm{Br}_{2}^{--} \\
& \mathrm{BrOH}^{\bullet-} \rightleftarrows \mathrm{Br}^{\bullet}+\mathrm{OH}^{-} \\
& \mathrm{BrOH}^{--}+\mathrm{Br}^{-} \rightleftarrows \mathrm{Br}^{\circ-}+\mathrm{OH}^{-} \\
& { }^{\circ} \mathrm{OH}+\mathrm{Br}^{-} \rightleftarrows \mathrm{BrOH}^{--}
\end{aligned}
$$

The degradation mechanism of TBBPA (Scheme 1) is investigated by computer simulation and analysis of intermediates. As an initial decomposition, ROSs such as $\mathrm{OH}$ radicals generated from in-liquid plasma, attack the position of $\mathrm{C}-\mathrm{Br}$ and debromination proceeds. Then intermediates, assigned as products (II)-(IV), were identified by LC-MS. 
The product intermediates can be mainly classified into (II) triBBPA (2,6-dibromo-4-[1-(3bromo-4-hydroxyphenyl)-1-hydroxyethyl]phenol), (III) diBBPA, (IV) monoBBPA. Finally, all bromine is ionized to form (V) BPA (bis(3-bromo-4-hydroxyphenyl)methanone). Thereafter, the ring opening of the benzene ring for BPA proceeds by further ROSs attack. Along with that, intermediates (VI) (2E,6Z)-8-hydroxy-3-methyl-5-methylidene-4-oxoocta2,6-dienal, (VII) (3Z)-5-hydroxy-2-methylidenepent-3-enoic acid, (VIII) 5hydroxypentanoic acid are formed and finally mineralized into $\mathrm{CO}_{2}$ gas and water. Oxidative degradation took place through scission, debromination, hydroxylation, or cleavage of the aromatic rings to achieve the final mineralization.
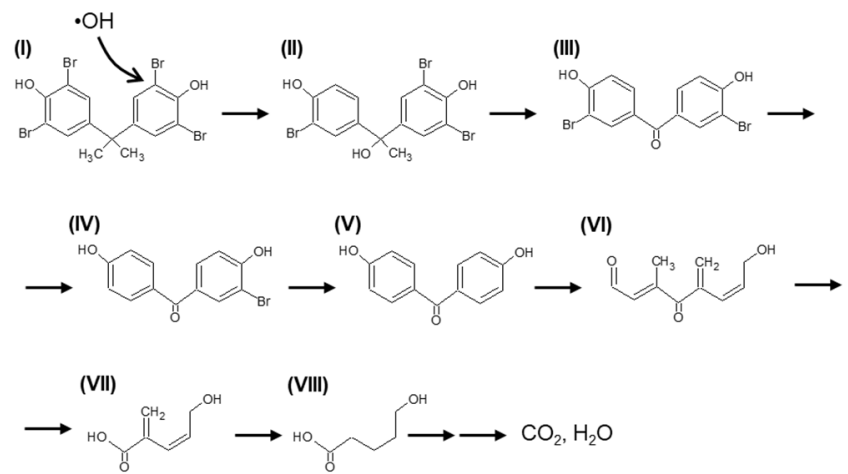

Scheme 1. Degradation pathway of tetrabromobisphenol-A with using in-liquid plasma.

Degradation of TBBPA in alkaline aqueous media by a newer advanced oxidation method - namely, the in-liquid plasma method - has been demonstrated in this study. Although there are already several oxidative decomposition methods (photocatalyst, Fenton, ROS, etc.), it has been clarified that the in-liquid plasma method can decompose them sufficiently quickly. This methodology, which is a new member of AOPs, provides a simple and green approach to detoxify TBBPA in aqueous media requiring only electric power and no such chemicals as catalysts and oxidizing agents to achieve it.

\subsection{Application in crosslinking agent/initiator-free polymer-gel synthesis}

In the case of no cross-linking agent and initiator, HySi-gel and PVP-gel is synthesized by using in-liquid plasma with $150 \mathrm{~W}$-power of microwave for $30 \mathrm{sec}$ or $5 \mathrm{~min}$ irradiation respectively.

Photographs of silicone and silicone-gel is shown in Fig. 4. Methyl groups of this gel was attacked by $\mathrm{OH}$ radical which was dissociated from $\mathrm{H}_{2} \mathrm{O}$ in reaction of (eq. 1) by inliquid plasma method and the macroradical attached with each other. Then, silicone-gel is synthesized through this process and could have some hydroxy groups because $\mathrm{OH}$ radicals attacked the silicone and combined with the gel. 


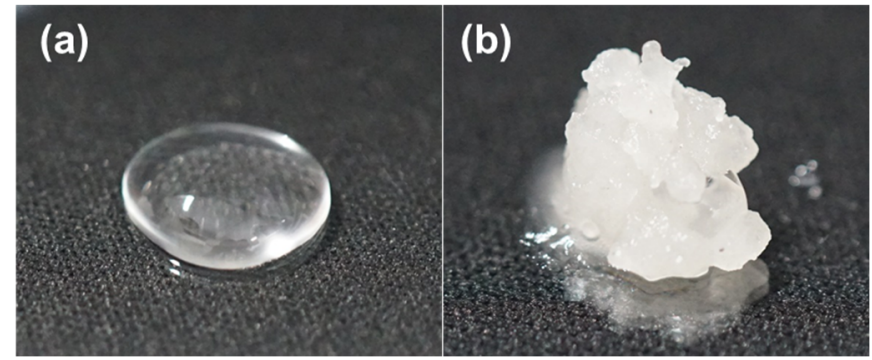

Fig. 4. Photographs of (a) silicone sample before in-liquid plasma method and (b) silicone-gel $30 \mathrm{sec}$ after in-liquid plamsa method treated silicone solution without crosslinking agent and initiator

In the condition of no cross-linking agent and initiator, PVP-gel was synthesized by using in-liquid plasma at 5 min-irradiation and $150 \mathrm{~W}$. FT-IR spectra of PVP-gel which was a sample after PVP was treated by using in-liquid plasma method and the chemical mechanism of PVP-gel synthesis are shown in Fig. 5.

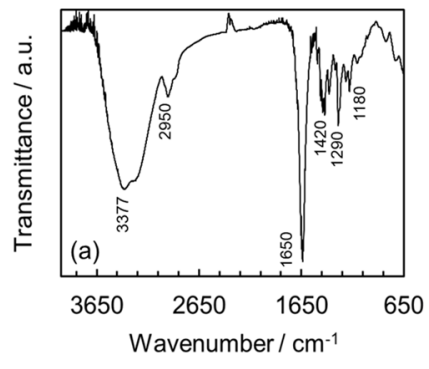

(b)
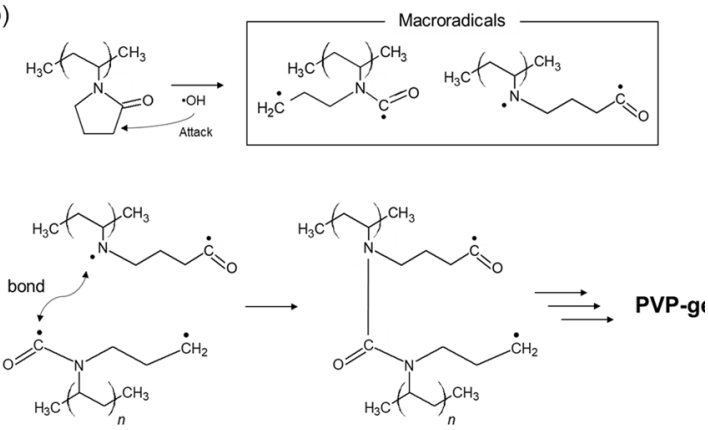

Fig. 5. Photographs of (a) FT-IR spectra of PVP-gel which was a sample 5 min after PVP was treated by using inliquid plasma method, and (b) a chemical mechanism of the PVP-gel

In the spectra (Fig. 5a), PVP-gel has hydroxy groups $\left(3377 \mathrm{~cm}^{-1}\right)$, carboxyl group $\left(2950 \mathrm{~cm}^{-}\right.$ $1,1420 \mathrm{~cm}^{-1}$ and $\left.1180 \mathrm{~cm}^{-1}\right)$ and tertiary amide group $\left(1650 \mathrm{~cm}^{-1}, 1290 \mathrm{~cm}^{-1}\right)$. According to the spectra, $\mathrm{OH}$ radical, which dissociated from $\mathrm{H} 2 \mathrm{O}$ by using in-liquid plasma, opened fivemember ring of PVP and the PVP became the macroradicals. In the chemical structure, these macroradicals attacked each other and these chemical reactions occurred for many times (Fig. 5b). After that, PVP-gel was synthesized [11].

They are the first attempt to synthesize gel only by the power of electricity. Since the initiator and crosslinking agent are very toxic materials, LP method is effective as a green chemistry process.

\section{Conclusions}

The best conditions of pulsed microwave (pulse width: $16.7 \mathrm{~ms}$, pulse period: $80 \%$ ) was investigated to generate stable plasma in liquid and was tried to use in-liquid plasma method for applications of wastewater treatment and gel-synthesis. In the application of wastewater treatment, it took only $20 \mathrm{~min}$ for in-liquid plasma method to be able to decompose and mineralize $0.1 \mathrm{M}$ TBBPA solution and in-liquid plasma method has high potential for 
wastewater treatment. In the applications of wastewater treatment, we could synthesize HySi-gel and PVP-gel without crosslinking agent and initiator. In-liquid plasma method will be a new gel-synthesis of green chemistry process.

\section{References}

1. J. S. Clements, M. Sato, R. H. Davis, IEEE Trans. Ind. Appl., 1987, 23, 224-235.

2. S. Horikoshi, N. Serpone, RSC Adv., 2017, 7, 47196-47218.

3. S. Nomura, H. Toyota, Appl. Phys. Lett., 2003, 83, 4503-4505.

4. S. Nomura, H. Toyota, S. Mukasa, Y. Takahashi, T, Maehara, A. Kawashima, H. Yamashita, Appl. Phys. Express, 2008, 1, 046002.

5. S. Horikoshi, S. Sato, M. Abe, N. Serpone, Ultrason. Sonochem., 2011, 18, 938-942.

6. A. Tsuchida, T. Shimamura, S. Sawada, S. Sato, N. Serpone, S. Horikoshi, Rad. Phys. Chem., 2018, 147, 53-58.

7. S. Horikoshi, S. Sawada, S. Sato, N. Serpone, Plasma Chem. Plasma Process., 2019, 39, 51-62

8. S. Horikoshi, S. Sawada, A. Tsuchida, N. Serpone, Environ. Sci. Water Res. Technol., 2019, submitted

9. S. Horikoshi, S. Sawada, 2019, will be submitted

10. Y. Guo, Jun Zhou, Xiaoyi Lou, Renlan Liu, Dongxue Xiao, Changling Fang, Zhaohui Wang, Jianshi Liu, Chem. Eng. J., 2014, 254, 538-544.

11. G.J.M. Fechine, J.A.G. Barrros, M.R. Alacantra, L. H. Catalani, Polymer, 2006, 47, 2629-2633. 


\title{
NUMERICAL SIMULATION OF THE ACTIVATION PROCESS OF SUPERSONIC GAS FLOWS BY A MICROWAVE DISCHARGE
}

\author{
M.S. Bobrov ${ }^{1,2}$, M.Y. Hrebtov ${ }^{1}$, A.K. Rebrov ${ }^{1}$, \\ ${ }^{1}$ Kutateladze Institute of Thermophysics SB RAS, Novosibirsk, Russia \\ ${ }^{2}$ Novosibirsk State University, Novosibirsk, Russia \\ maximbobrov20@gmail.com
}

Keywords: microwave plasma, resonant chamber, supersonic gas flow, simulations

Diamond films are widely used due to their unique physical and chemical properties, such as a large Young's modulus, the highest among all the materials known in nature, thermal conductivity, high breakdown voltage, transparency in the wavelength range from ultraviolet to infrared and chemical resistance. In the past few decades, there has been an intensive development of the technology of chemical vapor deposition (CVD) of diamond films. Activation of the gas mixture is usually carried out by the methods of hot filament, microwave plasma, arc plasma torches and glow discharge. The presence of electrodes or filament near the substrate affects the quality of the diamond. When using such activation methods, an additional impurity gets into the deposited diamond layer. In this regard, the method of activation of the gas mixture using microwave plasma is one of the most suitable from the point of view of the purity of the diamonds obtained. At the same time, this method allows to achieve high growth rates of diamond films, which makes it even more attractive.

The use of microwave radiation to activate gas mixtures containing hydrogen and carbon in the diamond synthesis is widespread. In the process of diamond film deposition, generation of a plasma cloud above the substrate is most frequently used. From that cloud active molecular fragments diffuse to the surface of the substrate [1]. However, a new deposition method has recently emerged, in which active fragments formed in a plasma cloud flow from a resonant chamber into a vacuum deposition chamber in form of a supersonic jet [2]. This method allows the use of plasma at very high pressures.

The study and optimization of microwave plasma generation process is a difficult task for modern experimental physics, due to the presence of extreme temperatures, high rate reactions and complex geometry of the setup. In this regard, numerical simulation may be very helpful as it allows testing the effect of many different parameters on the efficiency of the system in a short time. A popular tool for simulating the microwave plasma dynamics is COMSOL Multiphysics. Previously, this program was used for modeling of diamond deposition with microwave plasma activation [3]. Also, this software package was used to simulate the flow in a space thrusters [4].

It should be noted that the presence of atomic hydrogen in the active zone ensures efficient growth of diamond films. Atomic hydrogen quickly converts graphite, formed in the process of the reactions, again into gaseous hydrocarbon, practically without affecting the diamond. Atomic hydrogen is created mainly by thermal dissociation and electron impact reactions with $\mathrm{H}_{2}$ molecules. Each of the processes prevails in its temperature range. When using microwave radiation of high power (about $3000 \mathrm{~W}$ ), the resulting plasma has a temperature of about 3000-6000 degrees. Under such conditions, the process of thermal dissociation of hydrogen is dominant.

It is well known that the microwave radiation field has inhomogeneous distribution in the volume of resonant chamber. Because of this, with the fixed net radiation power, the magnitude of the electric field may vary significantly in different parts of the chamber. Thus, 
in the optimization of microwave heating device, the right choice of the resonant chamber geometry plays an important role.

In this paper, we present the results of optimization of the geometrical parameters of the resonant chamber achieved by means of numerical simulation. A baseline chamber design was chosen to mimic an experimental setup from [2].

The baseline case was a cylindrical chamber with an outlet nozzle in the bottom wall in a shape of a $1 \mathrm{~mm}$ hole. To optimize the microwave heating process, additional calculations were performed with an outlet nozzle in the shape of a truncated cone and a hemisphere playing the role of a microwave field (and heating) concentrator in a resonant chamber.
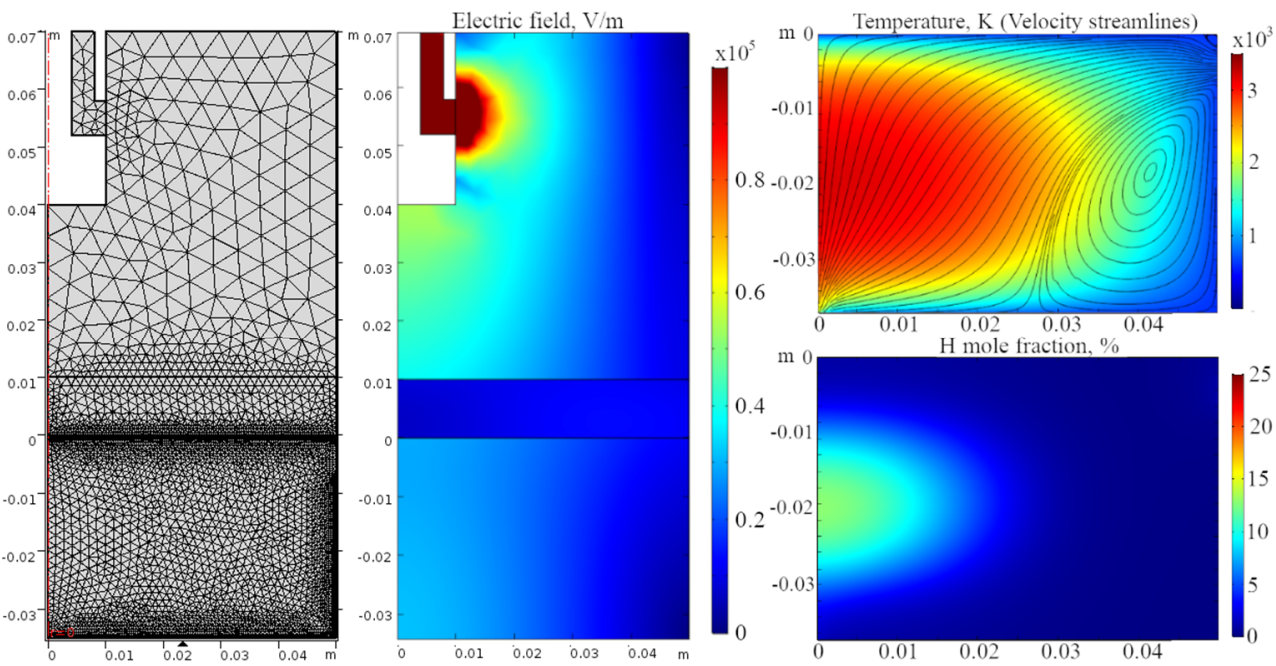

Fig. 1. Simulation grid (left), electric field (middle), temperature and dissociation degree (right) distributions for the case without the nozzle modifications.

The simulations were conducted with the finite element software COMSOL Multiphysics. The simulations were carried out in axisymmetric formulation. A cylindrical resonant chamber was chosen as computation domain (Fig. 1). The diameter and height of the chamber were $100 \mathrm{~mm}$ and $145 \mathrm{~mm}$, respectively. Microwave radiation was supplied through a coaxial port located in the center of the top base of the resonant chamber. The radiation frequency was $2.45 \mathrm{GHz}$, and the deposited power was $\sim 1.5 \mathrm{~kW}$. Walls of the chamber were set perfectly conducting. Microwave radiation was used to heat the working gas. Molecular hydrogen was chosen as the working gas. The gas was flowing through the bottom part of the resonant chamber, separated by a quartz wall. The gas enters the chamber through a hole in the side wall, with a constant flow rate of $10 \mathrm{l} / \mathrm{m}$. The diameter of the exit hole was $1 \mathrm{~mm}$. A fixed pressure of 2 Torr was set at the exit hole, while inside the chamber the pressure was sustained at the level of about 300 torr. At the metal boundaries a fixed temperature of $500 \mathrm{~K}$ was set (according to preliminary experimental measurements). The computational grid consisted of about 16,000 triangular nodes (Fig. 1 left). The grid was refined toward the domain boundaries. The turbulent fluid dynamics was simulated using the standard $k$ - $\varepsilon$ model. Due to the fact that the flow behind the nozzle was not resolved, and the gas velocity in the chamber didn't exceed $300 \mathrm{~m} / \mathrm{s}$, it was possible to use the sub-sonic compressible fluid approximation $(\mathrm{Ma}<0.3)$. The rates of hydrogen dissociation reactions were taken from [5] presented in Table 1. Dissociation energy, denoted in the table as $D$, 
was $435 \mathrm{~kJ} / \mathrm{mol}$. Forward sticking coefficient for surface reaction $H+H=H_{2}$ was 0.03 . This value of a forward sticking coefficient is typical for some metals. At the initial moment of time, the whole chamber was filled with molecular hydrogen, all atomic hydrogen was obtained as a result of reactions.

Table 1. Rate coefficients for hydrogen dissociation and recombination reactions.

\begin{tabular}{ll}
\hline Reaction & Rate coefficient, $\mathrm{cm}^{3}$ mole $^{-1} \mathrm{sec}^{-1}$ \\
\hline$H_{2}+H_{2}=H_{2}+2 H$ & $k=1.8 * 10^{17} T^{-1.5} \exp (-D / R T)$ \\
$H_{2}+H=3 H$ & $k=1.2 * 10^{20} T^{-0.5} \exp (-D / R T)$ \\
$H+H=H_{2}$ & $k=1.66 * 10^{-3} T^{2}$ \\
\hline
\end{tabular}

The simulations were carried out in a segregated manner. At the first stage, the stationary velocity distribution in the chamber was found for the given temperature field distribution inside the chamber. At the second step, the equations describing the microwave heating of the gas, the equations of thermal dissociation and recombination of hydrogen, and the equations of the heat transfer for the mixture were jointly solved, taking into account the velocity distribution obtained at the first stage. Then these two steps were repeated until the equilibrium solution was found.

To verify the model, we simulated the equilibrium thermal dissociation of hydrogen for various temperatures in the interval from 2000 to $5000 \mathrm{~K}$. The simulation results showed a good convergence with reference data [6]. Comparison of the equilibrium values of the degree of thermal dissociation for different temperatures with the data from the literature are presented in Table 2.

Table 2. Equilibrium thermal dissociation.

\begin{tabular}{ccc}
\hline Temperature, $\mathrm{K}$ & $\begin{array}{c}\mathrm{H}_{2} \text { dissociation degree } \\
\text { (reference) }\end{array}$ & $\begin{array}{c}\mathrm{H}_{2} \text { dissociation } \\
\text { degree(simulated) }\end{array}$ \\
\hline 2000 & 0.088 & 0.13 \\
2500 & 1.31 & 1.33 \\
3000 & 8.34 & 8.38 \\
3500 & 29.6 & 29.5 \\
4000 & 63.9 & 63.8 \\
4500 & 82.3 & 81.6 \\
5000 & 95.8 & 93.1 \\
\hline
\end{tabular}

At the first stage, simulations were carried out for the surrounding space of the nozzle in the form of a hole in the center of the lower base of the computational domain. Under such conditions, the electric field is not focused near the exit nozzle, but is almost uniformly distributed over the entire chamber with a magnitude of $15 \mathrm{kV} / \mathrm{m}$. In this case, the temperature distribution is determined by the distance from the walls (due to the wall heat losses), and therefore the maximum temperature and degree of dissociation, equal to 3190 $\mathrm{K}$ and $12 \%$ respectively, are located in the center of the chamber, which significantly reduces the amount of atomic hydrogen at the nozzle exit. Near the exit nozzle in this case, the temperature was only $2350 \mathrm{~K}$ and the degree of dissociation was $2.1 \%$. 
To achieve greater focusing of the electric field near the exit nozzle, it was decided to change the shape of the exit nozzle by adding a hemisphere or a truncated cone, that both also had a $1 \mathrm{~mm}$ exit hole (same as the initial nozzle). Figure 2 presents the simulation results for cases with nozzles in the form of a hemisphere and a truncated cone with different values of the radius of the upper base. It was found that the field focuses on the edge of a truncated cone, and as its upper base radius increases, the field maximum moves away from the exit hole, which indicates a decrease in the efficiency of microwave heating. It was found that optimal focusing of the electric field near the exit hole for a cone type nozzle takes place when the upper base radius is $2 \mathrm{~mm}$. The smaller upper base radius would be harder to achieve from an engineering point of view because the hole should be made in a thin tungsten foil wrapped around the cone and it would not give much of additional electric field strength. The field strength near the nozzle for this case was about $65 \mathrm{kV} / \mathrm{m}$, the maximum temperature was $3420 \mathrm{~K}$ and the corresponding value of the dissociation degree near the nozzle exit was $23.2 \%$.
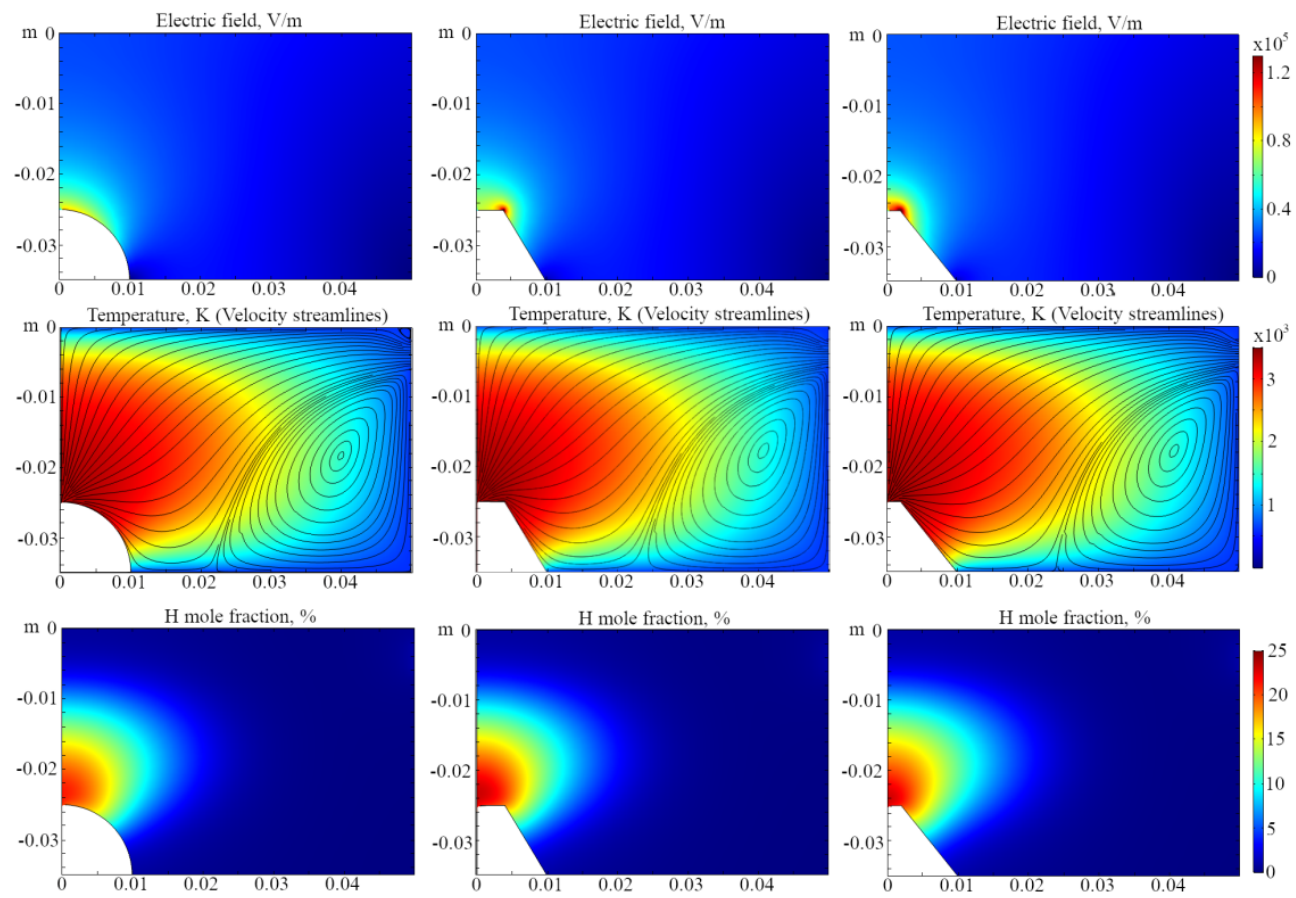

Fig. 2. Electric field, velocity, temperature and dissociation degree distributions for cases with hemisphere and a truncated cone (with $2 \mathrm{~mm}, 4 \mathrm{~mm}$ top base radius) nozzles.

A hemisphere nozzle was considered attractive as it reduces the recombination losses on the inner walls of the nozzle compared to a cone and at the same time maintains high field strength. It is observed that the maximum field strength in this case reaches 60 $\mathrm{kV} / \mathrm{m}$. The maximum temperature in this case was $3400 \mathrm{~K}$ and the corresponding value of the degree of dissociation was $22.8 \%$ at the nozzle exit which is only slightly less than for a cone. Figure 2 shows that the change in flow of gas does not significantly affect the temperature distribution and concentration of atomic hydrogen for the used flow rates. This is associated with a significant difference in the characteristic time scales for fluid dynamics and for the processes of dissociation and recombination. 

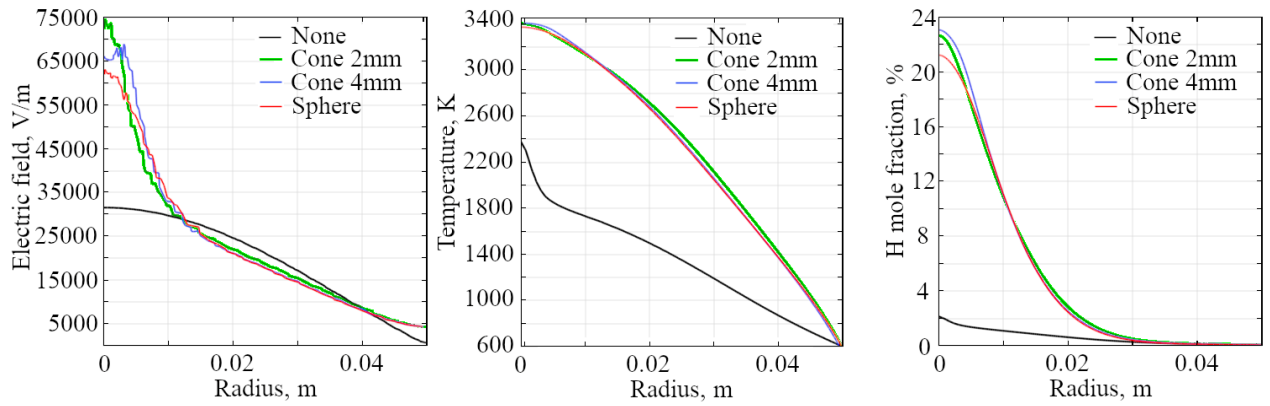

Fig. 3. Electric field, temperature, $\mathrm{H}$ mole fraction profiles through a section located $2 \mathrm{~mm}$ from the exit hole.

The profiles in Figure 3 show the efficiency of using additional nozzles. Using a truncated cone or hemisphere as a nozzle gives a gain in the electric field and temperature by a factor of two relative to the base case. It is important to note that the dissociation degree depends on temperature non-linearly, and when using an additional nozzle, the degree of dissociation increases by almost 10 times.

Also, it is necessary to make a nozzle (or at least a part of it near the exit hole) from a thin and very heat-resistant material (e.g. tungsten). In this case, higher temperatures could be achieved and the recombination rate on such hot wall will be much lower.

The preliminary experimental tests with setup from [2] showed that such modification (either cone or hemisphere) increased a working temperature inside the chamber significantly and made the discharge more stable even for higher pressures (up to 300 torr. and more).

First experimental results on the diamond deposition with using analyzed external nozzle configurations have been positive and encouraging. These tests with setup from [2] showed that such modification (either cone or hemisphere) increased a working temperature inside the chamber significantly and made the discharge more stable even for higher pressures (up to 300 torr. and more).

\section{References:}

1. Bolshakov, A. P., Ralchenko, V. G., Yurov, V. Y., Popovich, A. F., Antonova, I. A., Khomich, A. A., Diamond and Related Materials, 2016, 62, 49-57.

2. Rebrov, A. K., Isupov, M. V., Litvintsev, A. Y., Journal of Applied Mechanics and Technical Physics, 2018, 59(5),771-777.

3. Alcantar-Peña, J. J., Montes, J., Arellano-Jimenez, M. J., Aguilar, J. O., BermanMendoza, Diamond and Related Materials, 2016, 69, 207-213.

4. Gao, Enyu, and Sven G. Bilén, Department of Electrical Engineering, The Pennsylvania State University, University Park, PA,(Excerpt from the Proceedings of the COMSOL Conference 2008 Boston), 2008.

5. Gardiner Jr, W. C., \& Kistiakowsky, G. B., The Journal of Chemical Physics, 1961, 35(5), 1765-1770.

6. Nekrasov, B.V. Basics of general chemistry. Ripol Classic, 1965. 


\title{
MICROWAVE INFLUENCES LAMINAR PREMIXED HYDROCARBON FLAMES: SPECTROSCOPIC INVESTIGATIONS
}

\author{
S. Eckart ${ }^{1}$, R. Behrend ${ }^{1}$, H. Krause ${ }^{1}$ \\ ${ }^{I}$ Institute of Thermal Engineering, TU Bergakademie Freiberg, Freiberg, Germany \\ Sven.eckart@iwtt.tu-freiberg.de
}

Keywords: microwave, low calorific gas, hydrocarbon flames, combustion, spectroscopy

\begin{abstract}
Low laminar burning velocities' and slow reactions propagation are among the key problem in combustion processes with low calorific gas mixtures. These mixtures have a laminar burning velocity of $10 \mathrm{~cm} / \mathrm{s}$ to $15 \mathrm{~cm} / \mathrm{s}$ or even below which is $37 \%$ of natural gas. Thermal use of these gases could save considerable amounts of fossil fuel and reduce $\mathrm{CO} 2$ emissions. Due to low burning velocities and low enthalpy of combustion, ignition and stable combustion is complex, often preventing utilization of these gases. Microwaveassisted combustion can help to solve these problems. With microwave assistance, these gas mixtures could be burned with a higher burning velocity without preheating or cofiring. Therefore, this effect could be used for flame stabilization processes in industry applications. Microwaves could also change the combustion properties, for example radical formation and flame thickness.

In this paper, we explore a possibility of using microwaves to increase the burning velocity of propane as one component in low calorific gas mixtures and also show higher productions of $\mathrm{OH}^{*}$ and $\mathrm{CH}^{*}$ radicals with an increase of the input microwave power. Different compositions of low calorific fuels were tested within a range of equivalence ratios from $\varphi=0.8$ to $\varphi=1.3$ for initial temperatures of $298 \mathrm{~K}$ and atmospheric conditions and microwave powers from $120 \mathrm{~W}$ to $600 \mathrm{~W}$.
\end{abstract}

For the experiments, a standard WR340 waveguide was modified with a port for burner installation and filter elements allowing for flue gas exhaust and optical access from the side. A $2.45 \mathrm{GHz} \mathrm{CW}$ magnetron was used as microwave source, microwave measurements were carried out with a 6-port- reflectometer with integrated three-stub tuner. An axisymmetric premixed burner was designed to generate a steady conical laminar premixed flame stabilized on the outlet of a contoured nozzle under atmospheric pressure. The burner was operated with a propane mass flow of $0.2-0.4 \mathrm{nl} / \mathrm{min}$ at an equivalence ratio of $\varphi=0.8$ to $\varphi=1.3$. The optical techniques used in the current study are based on the flame contours detection by using the $\mathrm{OH}^{*}$ chemiluminescence image technique. For every experimental case, 150 pictures were taken and averaged. Additionally, spectroscopic analysis of the flames was undertaken.

The results suggest that production of $\mathrm{OH}^{*}$ radicals in the flame front increases with microwave power. For evaluation, a picture based $\mathrm{OH}^{*}$ chemiluminescence and a spectrographic method were used. In addition, a $9.9 \%$ increase of the burning velocity was observed in the premixed propane-air mixture for a 66 Watt absorbed microwave power. This effect is attributed to the increased $\mathrm{OH}^{*}(\sim 310 \mathrm{~nm})$ and $\mathrm{CH}^{*}(\sim 420 \mathrm{~nm})$ radical formation, which also reduces the flame thickness. It was found that absorption of microwaves in flames is generally low, but could be improved by a customized applicator design.

\section{Introduction}

The field of plasma-assisted combustion aroused increased interest over the last years and new approaches for the development of combustion systems were investigated to optimize 
fuel and energy consumption. The current focus of science is on the reduction of ignition, mixing and flame stability problems and the understanding of their fundamental influences. In particular, hypersonic combustion processes with devices such as plasma burners, microwave resonators and other discharge technologies are faced with complex problems.

Previous work has shown that resonant microwaves are capable of coupling into a hydrocarbon flame front, leading to a noticeable improvement in laminar burning velocity. Furthermore, the influence of a standing wave, and therefore the field maximum of the electric field at the position of the flame have been investigated. [1, 2, 11-13] In addition to experiments with laminar flames, further studies were carried out with turbulent flames with different equivalence ratios. [11] For these conditions, microwave irradiation was studied for both continuous and pulsed. $\mathrm{Li}$ et al. investigated direct microwave coupling into the reaction zone, as well as the influence of plasma on the flame form. Furthermore, they found out that there was an increase in flame propagation speed due to the increase in temperature. [7] In the experiments of Shinohara, an increase of the $\mathrm{OH}$ intensity and burning velocity was measured, but no rotational temperature increase in the flame. Therefore, it was assumed that the change in the combustion velocity is directly related to the electron heating. [10] Further experiments focused on the strength of the electric field and the power dependence. In addition, the electron concentration and the resulting increase in reactivity of the system has been investigated experimentally and computationally with PLIF and LES. The investigations were carried out in microwave stimulation of a turbulent low-swirl flame. The experiments also focused on turbulent flames, temperature fields at ignition, burning velocity and changes in the flame position under pulsed microwave input. [6] Ju et al. made a sensitivity analysis of the free electron number for different equivalence ratios. Their results indicated that the exhaust gas could possibly absorb the majority of the microwave electron heating. [8]

\section{Experimental Method}

\section{a. Laminar premixed burner setup}

The burner assembly and optical measurement technology used to produce and analyze the combustion are illustrated in Fig 1. An axisymmetric premixed burner is designed to generate a steady conical laminar premixed flame stabilized on the outlet of a contoured nozzle under atmospheric pressure. The shape of the axisymmetric central contracting nozzle is designed to reduce the boundary layer thicknesses by accelerating the flow and providing a flat velocity profile at the nozzle outlet. The contoured nozzle has an outlet diameter of $\mathrm{d} 1=10 \mathrm{~mm}$ and an inlet length of the premixing region of $120 \mathrm{~mm}$. One type $\mathrm{K}(0.5 \mathrm{~mm})$ thermocouple is placed at the inlet position of the burner to monitor the temperature of combustion reactants. The current setup uses calibrated thermal mass flow controllers (MFC, EL-Flow, Bronkhorst) for controlled fuel gas supply with an uncertainty of $0.8^{\circ} \%$ of the reading value and $0.2 \%$ of the full scale value. MFCs are controlled via software from a personal computer via Ethernet connection. Combustion air is supplied using compressed filtered air controlled by a MFC with $\mathrm{O}_{2} / \mathrm{N}_{2}$ volume ratio of $21 / 79$. In order to create a homogenous mixture of the fuel and air a mixing chamber is used. Therefore, the temperature is controlled with a second thermocouple type $\mathrm{K}(\mathrm{d}=0.5 \mathrm{~mm})$. The outlet of the burner has been integrated into the chamber structure so that there are no irregularities between the wall and the nozzle. 


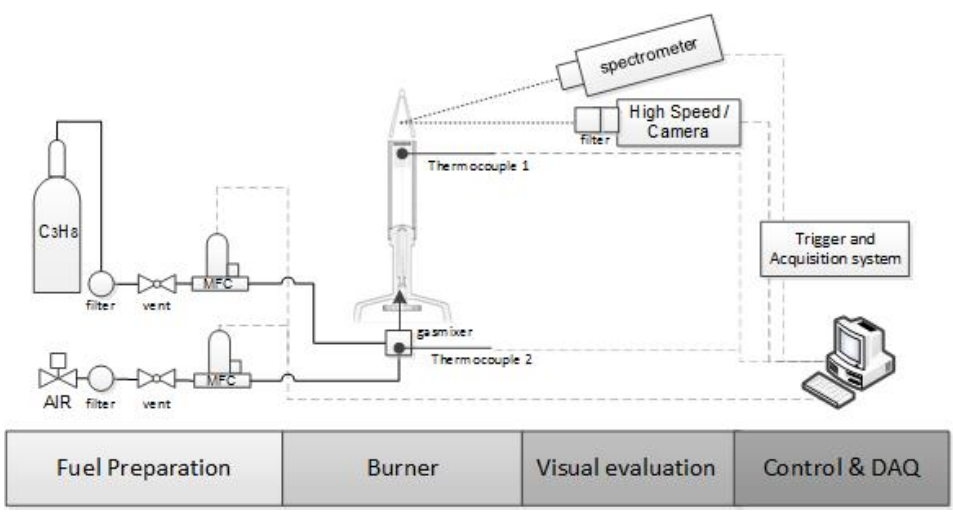

Fig. 1 Axisymmetric premixed Burner assembly at the TUBAF

\section{b. Microwave set up}

A $2.45 \mathrm{GHz}$ magnetron with $1200 \mathrm{~W}$ nominal power was connected to a WR340 waveguide via standard launcher. A switching power supply from ALTER (CM440) was used for continuous controllable power adjustment from $120 \mathrm{~W}$ to $1200 \mathrm{~W}$. Microwave measurements and impedance matching was carried out using a MW2104H-260EC automatic tuner from MUEGGE. The system combines an automatic impedance and power measurement system via a 6-Port-Reflectometer with a motorized three-stub tuner for load impedance matching. During the experiments, the three-stub-tuner was set to automatic impedance matching with a minimum reflection coefficient as target parameter.

For equipment safety, a circulator was installed between magnetron and tuner as well as a microwave window made from high purity fused silica between tuner and load to ensure equipment safety against flue gas condensates (water vapor).

The burner was installed in a modified WR340 waveguide build from high temperature resistant steel at quarter wavelength before the shorting wall. For exhaust gas, exit and optical access two filters with a diameter of $40 \mathrm{~mm}$ and length of $80 \mathrm{~mm}$ were integrated over the burner and lateral to the burner (see Fig. 2). The burner inlet was additionally sealed with aluminum tape for reduced microwave leakage. The waveguide and the adjacent waveguide were cooled with water-cooled aluminum cooling plates for safety. The setup was checked regularly for microwave leakage during operation via MUEGGE microwave survey meter MM3001B-110AB in order to comply with relevant health and safety norms.

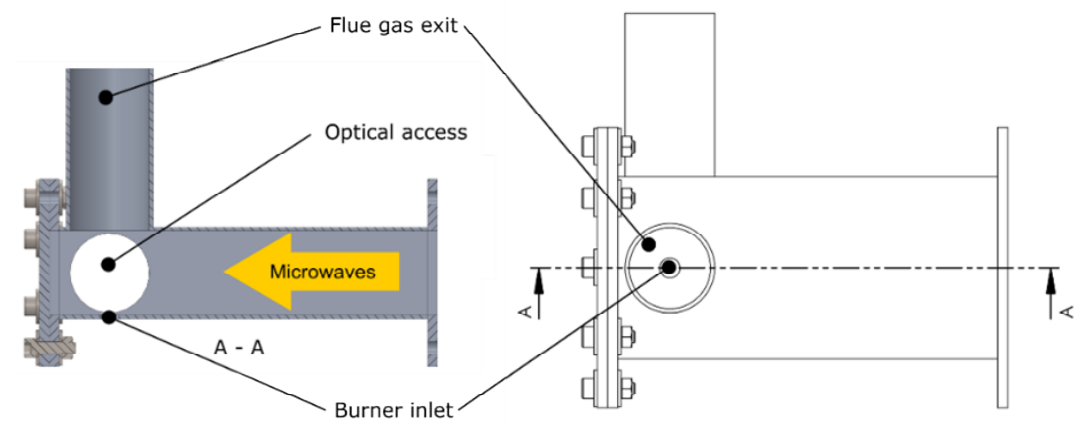

Fig. 2 Schematic representation of the microwave setup with burner installation 


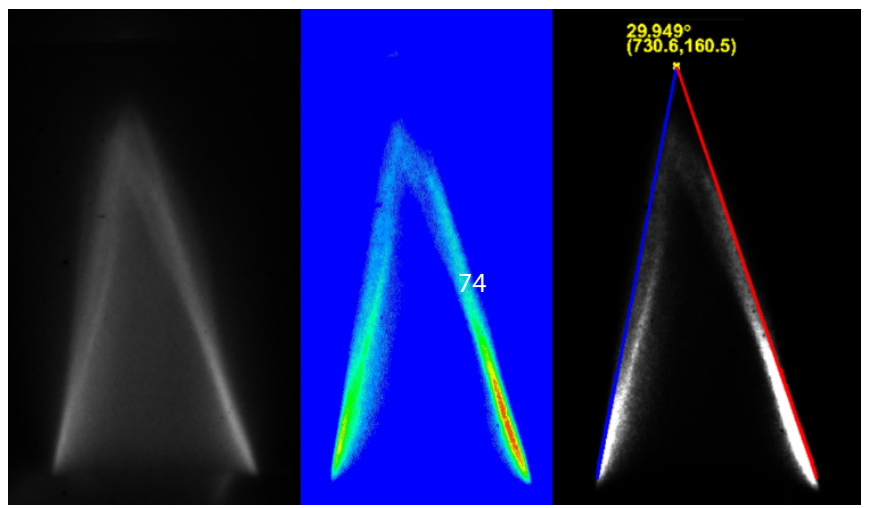

Fig. 3 The flame preparation for a picture of a propane flame with $0.3 \mathrm{ln} / \mathrm{min}$ fuel stream and at a microwave intensity of $300 \mathrm{~W}$, left $\mathrm{OH}^{*}$ spherical picture, middle Abel transformation and peak detection, right interpolated lines at the criteria value of 0.99 and angle calculation

\section{c. Spectrometer and $\mathrm{OH}^{*}$ chemiluminescence setup}

One of the optical techniques used in the current study is based on the flame contours detection by using the $\mathrm{OH}^{*}$ chemiluminescence image technique [4]. The camera used to record the $\mathrm{OH}^{*}$ radical emission is an 8-bit intensified CCD camera (mvBlueFox-224G) with a $1600 \times 1200$ array. The camera is equipped with an $\mathrm{f} / 3.8, \mathrm{f}=100 \mathrm{~mm}$, achromatic UV lens (CERCO 2073, Sodern) combined with a short pass optical filter centered at $307 \mathrm{~nm}$ and having a bandwidth of $\pm 8 \mathrm{~nm}$. An Image Light Amplifier (ILA, C9546-03L3, Hamamatsu) is integrated in the optical system. The acquisition repetition rate of the camera is kept at $8.5 \mathrm{~Hz}$. For every experimental case, 150 pictures were taken and averaged, to eliminate fluctuations. For detection of the flame angle and reaction zone, the $\mathrm{OH}^{*}$ chemiluminescence image technique was used. Unlike laser-induced-fluorescence (LIF), chemiluminescence imaging is a technique, which uses the chemical excitation instead of a laser beam. The camera records the light emitted from the chemically excited $\mathrm{OH}$, denoted $\mathrm{OH}^{*}$, where * represents the electronically excited state of a given radical or molecule. As in LIF imaging of $\mathrm{OH}^{*}$, a narrow optical band pass filter was placed in front of the camera lens so that the detection system only recorded light around $309 \pm 5 \mathrm{~nm}$. This interference filter passed the optical emission corresponding to the $\mathrm{A}^{2} \Sigma^{+}-\mathrm{X}^{2} \Pi$ transition of $\mathrm{OH}$. The picture preparation for the spherical $\mathrm{OH}^{*}$ picture (Fig. 3), followed by a Abel transformation to a $2 \mathrm{D}$ distribution of the radical and a peak detection to interpolate one line for each side of the flame at the criteria of 0.99 of the maximal radical distribution in this area. The interpolated lines are used to calculate the angle between both flame fronts.

A spectrometer was used as the second examination method. The spectrometer was installed at a fixed position with a line of sight to the flame by means of a glass fiber cable. The spectrometer covers a wavelength range from $200 \mathrm{~nm}$ to $700 \mathrm{~nm}$ with a resolution of $0.2 \mathrm{~nm}$. A background superposition was done to reduce background noise. This allows the detection of Signal/Noise with $300: 1$ and a sensitivity of 470,000 counts/ $\mu \mathrm{W}$ per ms integration time.

\section{Results and discussion}

The experiments carried out in this thesis shall show that the laminar burning speed of propane air flames can be increased by microwave energy and that the influence on the 


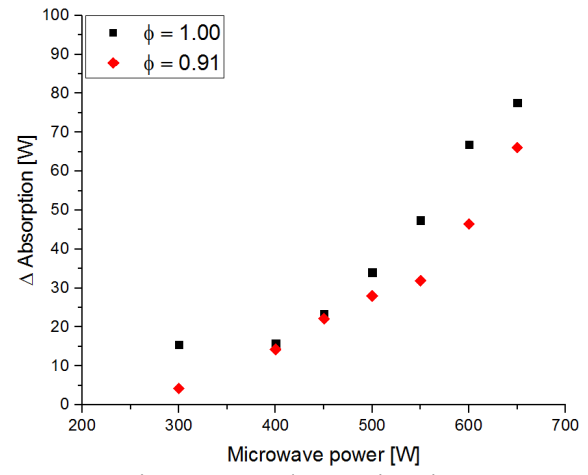

Fig. 4 Microwave absorption in a propane flame with volume flow of $0,25 \mathrm{ln} / \mathrm{min}$

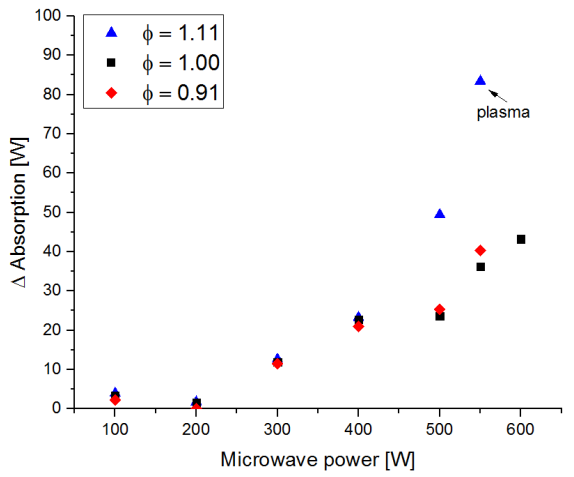

Fig. 5 Microwave absorption in a propane flame with volume flow of $0,3 \ln / \mathrm{min}$

radical formation in the flame front can be investigated. Results were obtained for propane-air flames at various flow conditions and equivalence ratios. The operating conditions of the volume flow rate were varied between $0.25-0.3 \mathrm{nl} / \mathrm{min}$ at fixed equivalence ratio of $0.87,0.91,1.0$ and 1.11 . For a quantification of the microwave influence, the absorption of the flame was measured for the empty WR340 waveguide and with a stabilized flame. For this procedure, the microwave power was varied. The results can be seen in Fig. 4 and Fig. 5. As in Stockman $[11,12]$ the absorption is a non-linear trend, this could be shown for all equivalence ratios. For the flames with a fuel rich mixture and a high fuel stream after $500 \mathrm{~W}$ microwave power the flame became unstable and plasma was ignited at several occasions.

Fig. 6. presents a propane flame spectrum in the wavelength between $200 \mathrm{~nm}$ and $700 \mathrm{~nm}$ for equivalence ratio $\Phi=0.91$ and fuel stream of $0.3 \mathrm{nl} / \mathrm{min}$. From the combustion process and the initial reactions some radicals emit light in significant wavelengths, therefore this wavelength are markers for combustion radicals such as $\mathrm{OH}^{*} \mathrm{CH}^{*}$ and $\mathrm{C} 2 *$ which are nonstable intermediates in the combustion process.

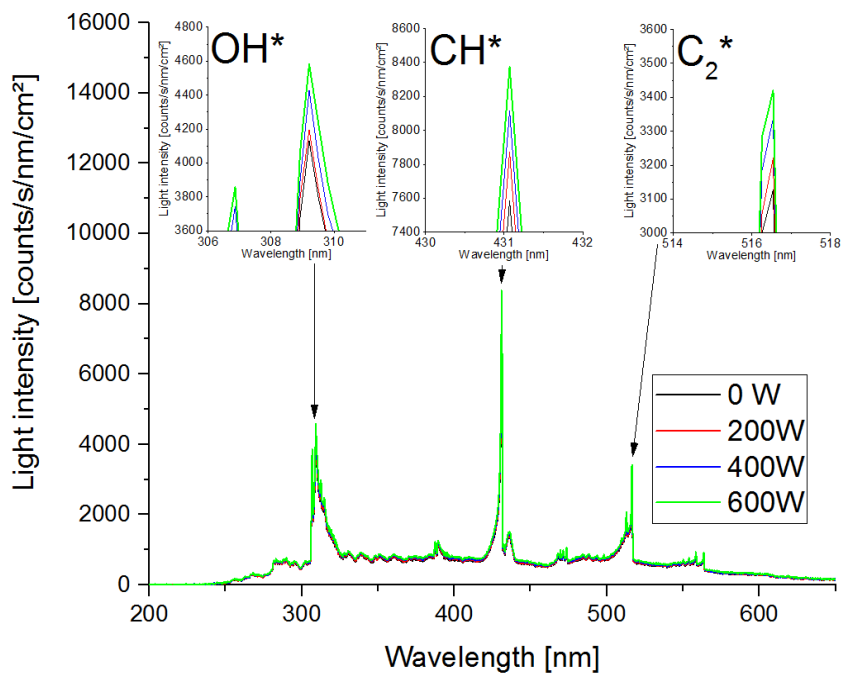

Fig. 6 Evaluation of the spectral analysis in the range from $200 \mathrm{~nm}$ to $700 \mathrm{~nm}$ at equivalence ratio of 0.91 and a fuel volume flow of $0.3 \mathrm{nl} / \mathrm{min}$ as well as the microwave influence of 0 to 600 Watt. 


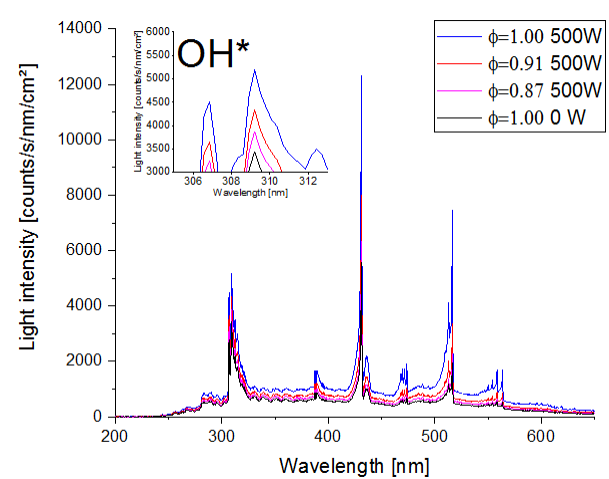

Fig. 7 Evaluation of the spectral analysis in the range from $200 \mathrm{~nm}$ to $700 \mathrm{~nm}$ at a fuel flow of $0.25 \mathrm{nl} / \mathrm{min}$ as well as the microwave influence of 0 and 500 Watt

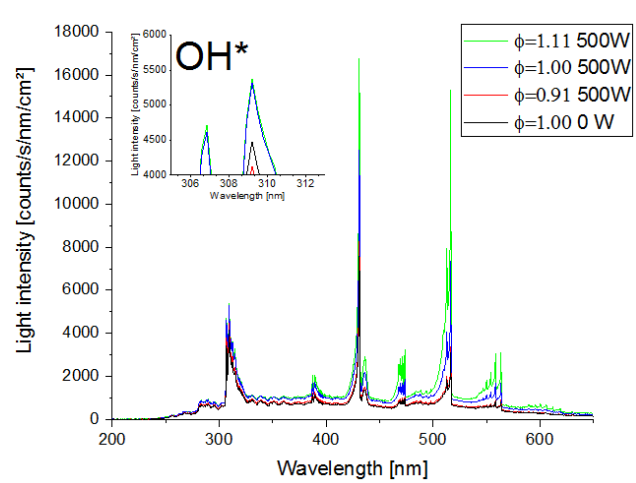

Fig. 8 Spectral analysis at a fuel flow of $0.3 \mathrm{nl} / \mathrm{min}$ as well as the microwave influence of 0 and 500 Watt for different equivalence ratios

The significant wavelengths for $\mathrm{OH}^{*}$ radicals are $307 \mathrm{~nm}$ and $309 \mathrm{~nm}$, forming a double maximum peak. They were analyzed to show the increase in the optical emission intensity when applying microwave power. $\mathrm{OH}^{*}$ is typical used as a marker for the flame front, the maximal $\mathrm{CH}^{*}(\mathrm{~A} 2 \Delta-\mathrm{X} 2 \Pi)$ emission was found to be at the wavelength $431 \mathrm{~nm}$. At $516.6 \mathrm{~nm}$ the maximum peak for $\mathrm{C} 2 *\left(\mathrm{a}^{3} \Pi \mathrm{u}-\mathrm{d}^{3} \Pi \mathrm{g}\right)$ radicals was found. $\mathrm{C} 2 *$ radicals can provide information about areas susceptible to soot formation. For all three investigated wavelengths it could be shown, that with an increase of microwave power, the discovered light intensity also increases at the exact same wavelength. This implies an increase in emissions in the flame front, coupled with an increase in the number of reactions in the flame. Furthermore, the influence gas mixture and volume stream was investigated. In Fig 7 the emission spectra for $200 \mathrm{~nm}$ to $700 \mathrm{~nm}$ are shown. The microwave power was fixed to $500 \mathrm{~W}$ and the equivalence ratio was varied. It was observed that with an increase of the equivalence ratio in the tested area the $\mathrm{OH}^{*}, \mathrm{CH}^{*}$ and $\mathrm{C} 2 *$ radical detection increases also. Furthermore, the $\mathrm{OH}^{*}$ radicals for an equivalence ratio of $\Phi=0.87$ at $500 \mathrm{~W}$ are still higher than the $\mathrm{OH}^{*}$ concentration at $\Phi=1.0$ without microwave input. For the case with higher fuel stream, the situation shows the similar trend.

In Fig. 8 the result of the spectral analysis of a propane flame with a fuel volume flow of $0.3 \mathrm{nl} / \mathrm{min}$ are shown. Again, the microwave power was fixed to $500 \mathrm{~W}$ and the equivalence ratio was varied. It can be seen that the maximum light intensity for all wavelength increases as expected: Due to the higher amount of fuel, the reaction intensity also increases. Furthermore, it can be shown that the maximum peak value of $\mathrm{OH}^{*}$ radicals rises with in the tested range. $\Phi=1.11$ shows the highest $\mathrm{OH}^{*}$ emission, while $\Phi=0.91$ shows the lowest, the value for $\Phi=1.00$ rises from 4440 counts $/ \mathrm{s} / \mathrm{nm} / \mathrm{cm}^{2}$ without microwave influence to 5380 counts $/ \mathrm{s} / \mathrm{nm} / \mathrm{cm}^{2}$ with influence of $500 \mathrm{~W}$ microwave power. The same behavior can be shown for the entire considered wavelength range. The $\mathrm{OH}^{*}$ concentration of $\Phi=1.11$ is slightly higher than at $\Phi=1.0$, but the difference is significantly lower than the difference in $\mathrm{OH}^{*}$ concentrations between $\Phi=0.91$ and $\Phi=1.0$. This could show a similar trend to the laminar burning velocity, which also has a platoon in the area of $\Phi=1.0$ to $\Phi=1.1$ and decreases after the value of $\Phi=1.1$. For this case the maximum $\mathrm{OH}^{*}$ concentration at $\Phi=0.91$ was lower than the concentration of $\Phi=1.0$ without microwave influence. As expected, the values with higher fuel streams are for all three investigated radical cases increased.

Furthermore, with the determined flame tip angles from the $\mathrm{OH}^{*}$ images the laminar burning velocity was determined. 


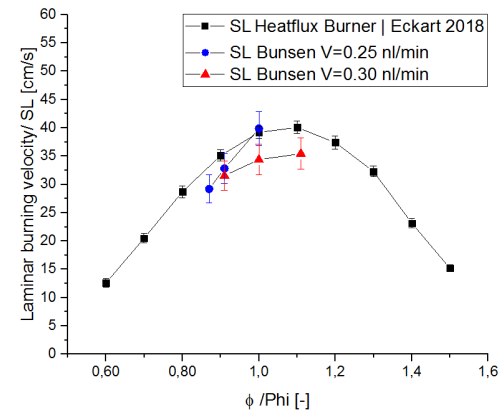

Fig. 9 Comparison of the laminar burning velocity of the cone method with the results of previous work at a Heat flux burner setup [5, 9]

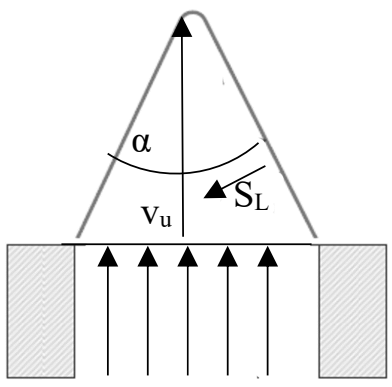

Fig. 10 Scheme for determining the laminar burning velocity $\left(\mathrm{S}_{\mathrm{L}}\right)$ from the flame angle $(\alpha)$ of the cone flame and the flow velocity of the unburned fuel-air mixture $\left(\mathrm{v}_{\mathrm{u}}\right)$

For analysis the formula after Rallis was used [3]: $S_{L}-\vartheta_{w} \cdot \sin \left(\frac{\pi}{2}\right)$ with flame angle $(\alpha)$ of the cone flame and the flow velocity of the unburned fuel-air mixture $\left(v_{u}\right)$ as shown in Fig. 10. The results were compared with previous work at TUBAF with the Heatflux burner in Fig. 9. The present results for both fuel stream values are lower but show a similar trend. The accuracy of the determined burning velocity is $\pm 11 \%$ and depends mainly on the uncertainty of the mass flow controller and the fluctuations of the flame tip during the experiment, resulting to an uncertainty of the flame angle. The shortened flame length, the increase of the flame tip angle, indicate the increases in the burning velocities in the flames. The shortened flame length can be interpreted as increased consumption of propane gas in the flame area closer to the burner nozzle. The burning velocity $S_{L}$ was obtained experimentally by Stockman $[11,12]$ and Zaidi [13] with a flat flame method and pulsed microwave. In the present experiments, the cone method was used. In Fig. 11 the results for a propane flame with 3 equivalence ratio $\Phi=0.87,0.91$, 1.0 and a fuel stream of $0.25 \mathrm{nl} / \mathrm{min}$ are shown. It was observed that with a higher microwave, input power the flame was shortened and the illustrated flame angle increased nonlinear. Since the flame tip fluctuated during the averaging of 150 images, this has an effect on the error bars. Thus, in this case, the error detected does not provide an accurate interpretation of the trend. In Fig. 12 the volume flow was increased to $0.3 \mathrm{nl} / \mathrm{min}$. Because of the higher unburned gas mixture speed at the nozzle the angle of the flame decreases without microwave input. 


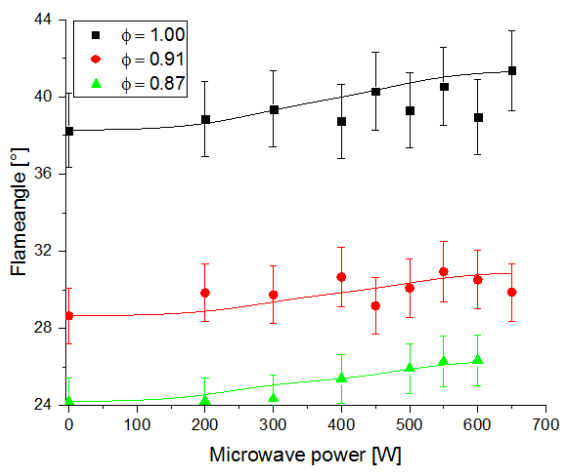

Fig. 11 Evaluation of the determined flame angles at equivalence ratios of $\Phi=0.87$ to 1.0 and a fuel flow of $0.25 \mathrm{nl} / \mathrm{min}$

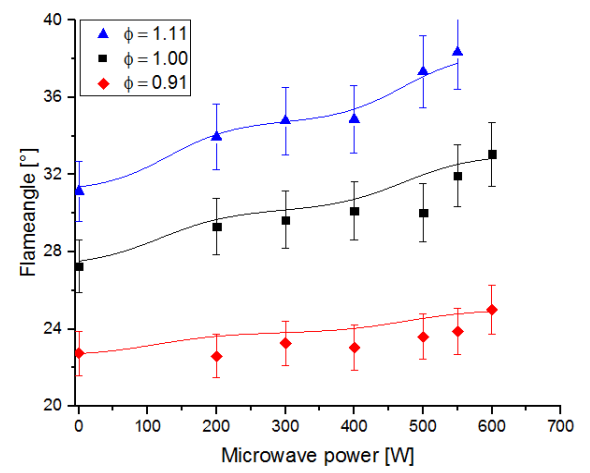

Fig. 12 Evaluation of the determined flame angles at equivalence ratios of $\Phi=0.91$ to 1.11 and a fuel flow of $0.3 \mathrm{nl} / \mathrm{min}$

After this, the input microwave power parameter was set as before. In this second case the influence of the microwave and the trend seems to be more clear, especially for the fuel rich conditions at $\Phi=1.11$. With the increase of the microwave power, the flame angle rises for all three equivalence ratios. Higher equivalence ratios in the fuel rich case could be of further interest in the future.

\section{Conclusions \& Outlook}

We observed the enhancements of the burning velocity in a premixed propane-air flame by irradiating microwave power in a wide range of equivalence ratios at atmospheric conditions. In addition, the intensity of the microwave power has a significant influence on the optical emission intensity of $\mathrm{OH}^{*}$ radicals. This has been thoroughly investigated by spectrometer and $\mathrm{OH}^{*}$ image investigations. Therefore, it was shown that microwave energy couples in the reaction front of a premixed propane flame and significantly influences the inter reaction chains. From this can be concluded that the emission behavior of the flame will also be influenced. For that reason, further investigations on the reaction paths will follow. The measurement of the temperature distribution inside the flame zone, the estimation of the electron energy distribution function and the measurements of radical densities will be helpful for understanding the mechanism of burning velocity enhancement. In addition, the effects of microwave input on the exhaust gas composition are of further interest.

\section{Acknowledgement}

The authors gratefully acknowledge the financial support by the European Union and the state of Saxony in the ESF project (project number 040606100).

\section{References}

[1] 43rd AIAA Aerospace Sciences Meeting and Exhibit. American Institute of Aeronautics and Astronautics, Reston, Virigina.

[2] 45th AIAA Aerospace Sciences Meeting and Exhibit. American Institute of Aeronautics and Astronautics, Reston, Virigina.

[3] C. J. Rallis and A.M. Garforth. The determination of laminar burnig velocity. Proceedings of the Combustion Institute 1980, 303-329.

[4] Dieke, G. H. and Crosswhite, H. M. 1962. The ultraviolet bands of $\mathrm{OH}$ Fundamental data. Journal of Quantitative Spectroscopy and Radiative Transfer 2, 2, 97-199. 
[5] Eckart, S., Penke, C., Voss, S., and Krause, H. 2017. Laminar burning velocities of low calorific and hydrogen containing fuel blends. Energy Procedia 120, 149-156.

[6] Ehn, A., Petersson, P., Zhu, J. J., Li, Z. S., Aldén, M., Nilsson, E.J.K., Larfeldt, J., Larsson, A., Hurtig, T., Zettervall, N., and Fureby, C. 2017. Investigations of microwave stimulation of a turbulent low-swirl flame. Proceedings of the Combustion Institute 36, 3, 4121-4128.

[7] Hong-Yuan Li, Yei-Chin Chao. 2017. Study of the Thermal Effects of Microwave induced Plasma on Premixed Methane-Air Flames. Proceedings of International Colloquium on the Dynamics of Explosions and Reactive Systems.

[8] Ju, Y., Macheret, S., Miles, R., and Sullivan, D. 07112004. Numerical Study of the Effect of Microwave Discharge on the Premixed Methane-air Flame. In 40th AIAA/ASME/SAE/ASEE Joint Propulsion Conference and Exhibit. American Institute of Aeronautics and Astronautics, Reston, Virigina, 179. DOI $=10.2514 / 6.2004-3707$.

[9] S. Eckart, H. K. 2018. Renewable hydrogen in gas grids, Effects on laminar burning velocities. International Journal of Energy Engineering 2018, 2, 1-9.

[10] Shinohara, K., Takada, N., and Sasaki, K. 2009. Enhancement of burning velocity in premixed burner flame by irradiating microwave power. J. Phys. D: Appl. Phys. 42, $18,182008$.

[11] Stockman, E., Zaidi, S., and Miles, R. 06252007. Hydrocarbon Flame Speed Enhancement with High-Power Pulsed Microwaves. In 38th Plasmadynamics and Lasers Conference. American Institute of Aeronautics and Astronautics, Reston, Virigina, 265. DOI=10.2514/6.2007-4601.

[12] Stockman, E. S., Zaidi, S. H., Miles, R. B., Carter, C. D., and Ryan, M. D. 2009. Measurements of combustion properties in a microwave enhanced flame. Combustion and Flame 156, 7, 1453-1461.

[13] Zaidi, S., Qin, X., Macheret, S., Ju, Y., Miles, R., Sullivan, D., and Evans, M. 01102005. Microwave-assisted Hydrocarbon Flame Speed Enhancement. In 43rd AIAA Aerospace Sciences Meeting and Exhibit. American Institute of Aeronautics and Astronautics, Reston, Virigina, 57. DOI=10.2514/6.2005-992. 


\section{MICROWAVE TREATMENT OF MATERIALS IN LOW PRESSURE}

Ryszard Parosa, Andrzej Brożyński, Piotr Grześkowiak, Krzysztof Kowalczyk, Marek Natoński, Piotr Ziętek and Janusz Żytkiewicz ${ }^{1}$

\section{${ }^{1}$ PROMIS-TECH, Poland}

Keywords: microwave treatment, low pressure heating, microwave drying

Uniquely favourable characteristics of biological product can be obtained through the use of the microwave method in vacuum heating process. Microwave-vacuum drying is superior to other methods in terms of dried products' structure, flavour, colour and biological active compounds contents. But applications of such a methods seems to be much wider: drying of fruits and vegetables for consumption, drying of herbs for extraction of valuable biological compounds, for modification of seed structure (sunflowers seeds, pumpkin seeds), for pasteurisation etc.

A universal system was designed for testing such processes in laboratory scale and several industrial scale system have been developed.

The most important advantages of this method are:

- possibility of heating the dried material in its entire volume (however, it requires special solutions of process chambers),

- very short drying time,

- relatively low temperature of the material due to the lowering of the boiling point of water at low pressure,

- favorable structure of the dried material caused by the effect of "loosening" the material as a result of water heating in capillaries and inside cell structures (puffing effect).

Process of thermal treatment can be carried out with plastic drum installed inside of multi-mode microwave cavity and cavity which is connected by microwave line with reflectometer and circulator - to microwave generator. In laboratory unit generator 2.45 $\mathrm{GHz}$ with controlled power (from $50 \mathrm{~W}$ to $800 \mathrm{~W}$ ) was applied. System was equipped with vacuum pump with pressure control and is controlled by computer. Most important technical parameters, like: microwave power, time of treatment, pressure inside of drum, temperature of steam - are controlled and recorded. Laboratory scale unit is shown below. 


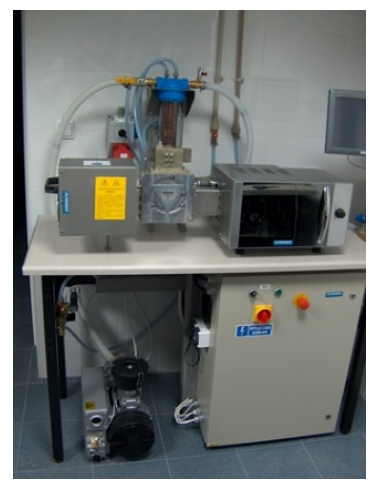

Fig. 1. Photo of a laboratory microwave vacuum station for testing drying processes. The device has been ordered by Institute of Fruit Farming in Skierniewice, Poland. Similar units have been delivered to several universities and research institutions and to industrial laboratories as well. Are mainly used for drying, but also for pasteurization and recently for seed structure modification. Very promising research has been carried out for drying of hemp before extraction.

Basing on laboratory scale test several technologies in industrial scale was developed. Industrial scale unit equipped with 8 generators of $3 \mathrm{~kW}(2.45 \mathrm{GHz})$ was constructed and for last 8 years has been successfully used for "production" of crispy chips which are now popular in Polish market. Exemplary industrial scale installation is shown in photo below.

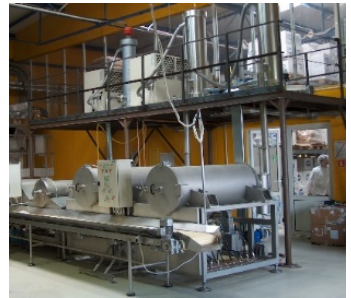

a)

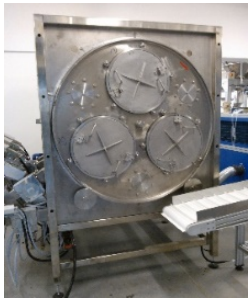

b)

Fig. 2. a) Two cavity microwave industrial drier, b) Multi - drum microwave drier

Another system for modification of seed is now constructed - ordered by big industrial producer of batons and sweet snacks. Process will be carried out inside of dielectric drum in low pressure and reactor will be equipped with 8 generators of $3 \mathrm{~kW}(2.45 \mathrm{GHz})$. Treatment time will be reduced to 3-4 minutes and next material (seeds) will be cooled down.

Last project which now is realized connected with drying of scobs(wood chips) applied in composite material production. System will work continuously with two airlocks and with dielectric drum and will be connected with 4 microwave generators $(3 \mathrm{~kW}, 2.45$ $\mathrm{GHz}$ ). In next step planed installation will be equipped with microwave high power generator ca. $60 \mathrm{~kW}$ with frequency $915 \mathrm{MHz}$. 


\section{Microwave vacuum installation in technical scale - two-chamber version}

The above, briefly described process of drying vegetables, fruit and herbs has for many years been applied in technical scale at PAULA company in Kalisz, Poland. To meet the company's needs, four separate lines were constructed, consisting of pulse-and-fluidization driers for initial drying of fruit and vegetables, as well as two-chamber microwave vacuum driers. The functional system of microwave driers is illustrated by the block diagram in figure 3 and 4 .

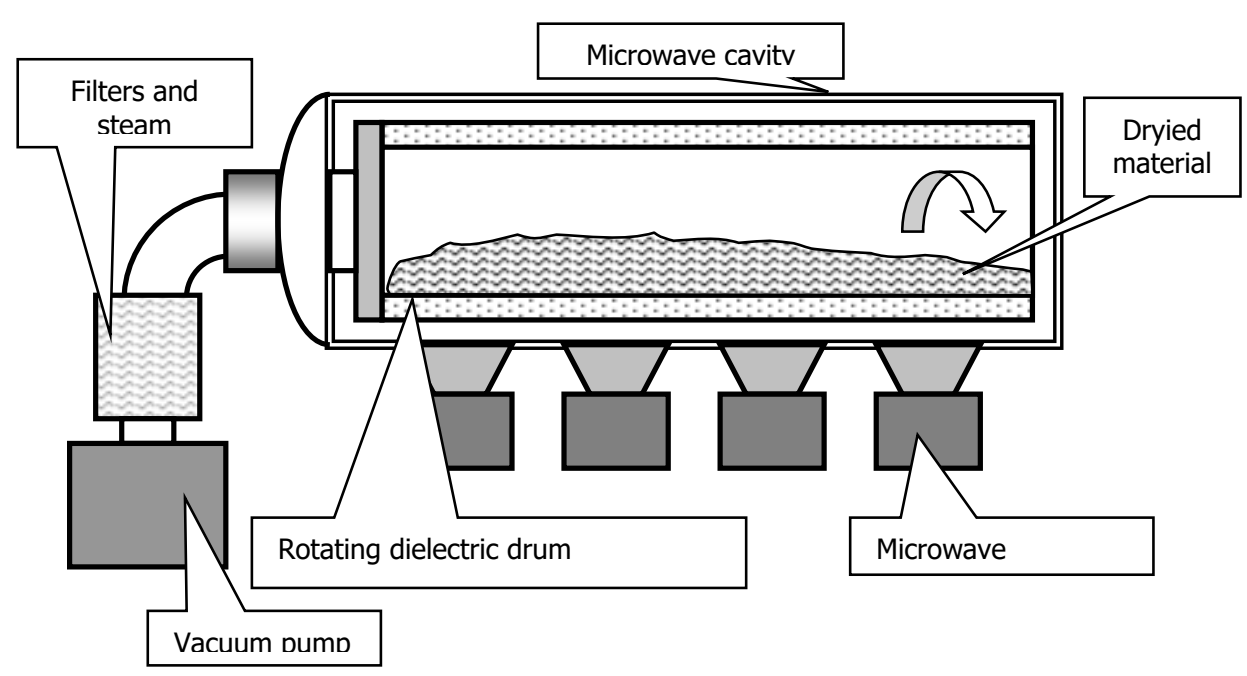

Fig. 3. Simplified diagram of the industrial microwave vacuum drier.

In the described process, the humidity of the inserted material is usually approx. $15-25 \%$ (per weight), and as a result of the microwave drying, the humidity is reduced to about 2$4 \%$. The efficiency of an installation operating in such a way is approx. $30-45 \mathrm{~kg}$ of dried material an hour.

Photographs in fig. 4 present two lines made by PROMIS-TECH: one with manual loading and unloading of material, and a line with a set of automatic loading and unloading of the material from drums in both chambers.

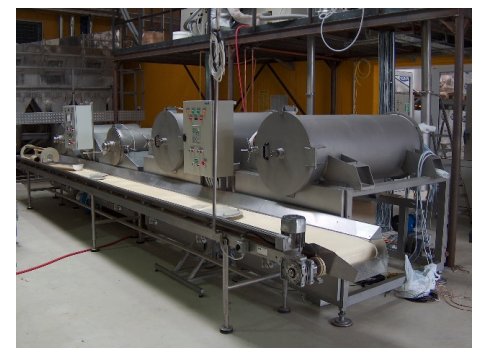

a)

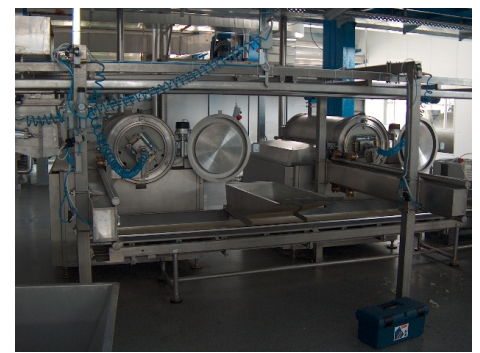

b)

Fig. 4. Photographs of microwave vacuum driers (installation made for PAULA company in Kalisz, Poland): a) version for manual loading, b) version with automatic loading and unloading of the material. 


\section{Multi-drum vacuum drier}

The previously described structure of a drier is relatively expensive and requires complex control system with the so-called switch throw enabling to switch power supply of a set of microwave generators installed on both chambers. Moreover, for quite a long time the devices remain inactive when the process of stabilizing the product in the chamber takes place, after previous heating up with microwaves.

These inconveniences have been removed in a new, largely modified structure defined as the multi-drum vacuum drier. The structure utilizes only a single set of microwave generators instead of two sets installed in the previously described system; in addition, specially developed chargers for microwave generators were applied, which provide low power rippling with a simple and inexpensive structure.

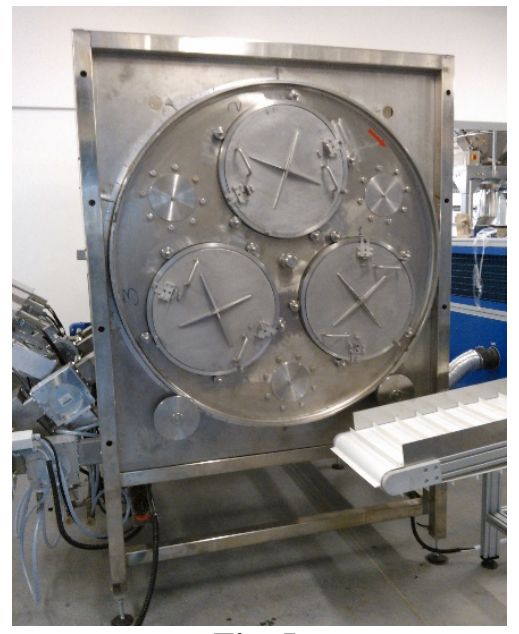

Fig. 5.

Other example for the use of microwave vacuum system is industrial installations analogous to two-chamber designs used to change the structure of pumpkin seeds, sunflower seeds and nuts to reduce their hardness and to obtain a more fragile structure. This process was used to modify these seeds and nuts used in the production of bars.

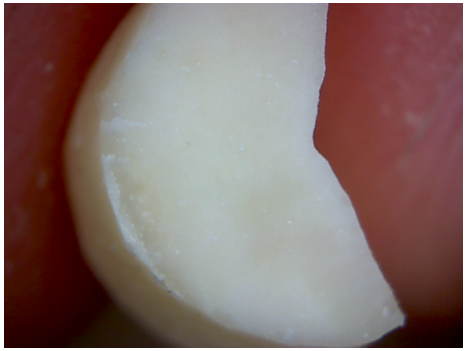

a)

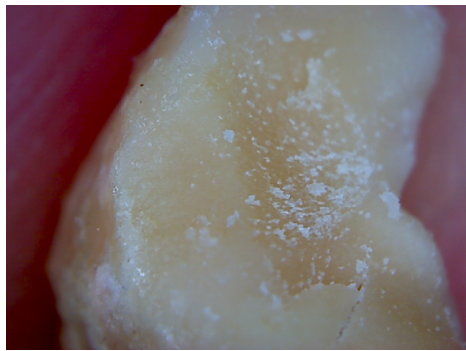

b)

Fig. 6. Photographs of cracked peanut a) before and b) after the process under microscope. 
Another project based on the use of microwaves in drying processes under reduced pressure is the technology of drying scobs. This product, after drying to a relative humidity below $1 \%$, is used for the production of special composites with resins.

As part of laboratory tests, the rate of scobs drying was compared by conventional method with microwave drying. In both cases, the process was carried out at reduced pressure (about $60 \mathrm{hPa}$ ).

Conventional drying of scobs to achieve a very low final relative humidity (about $1-2 \%$ ) is a difficult and potentially dangerous process. With conventional drying in a stream of hot air, the limitations are:

- dust removal effects (fine fractions from finely divided shavings),

- possibility of dust explosion (eg due to a spark between electrostatically charged particles).

In addition, as a result of the conventional drying of scobs, the drying product can be characterized by significant differences in relative humidity (some, for example, larger fragments may remain more moist than the smallest fractions).

It is intended that these drawbacks can be removed by using a drying process under reduced pressure, heating the dried material with microwave energy and through the hot walls of the process chamber.

The application of a reduced pressure in the dryer chamber results in a lower water evaporation temperature, and the lack of oxygen in the chamber prevents the explosion of dry scobs. However, it is necessary to supply energy to the dried material and in this process it is preferable to use microwave energy properly emitted to the dryer chamber. In addition, partial energy transmission to the dried material can be provided by heating scobs by contact with the hot wall of the feeder.

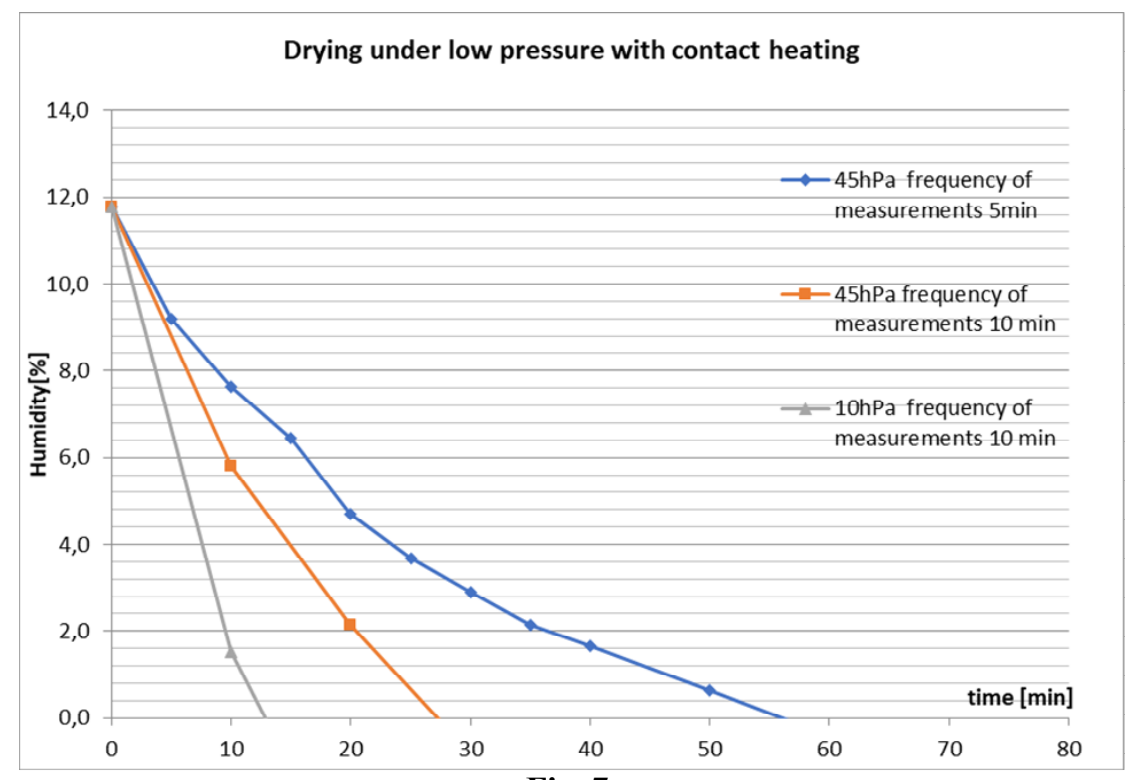

Fig. 7. 


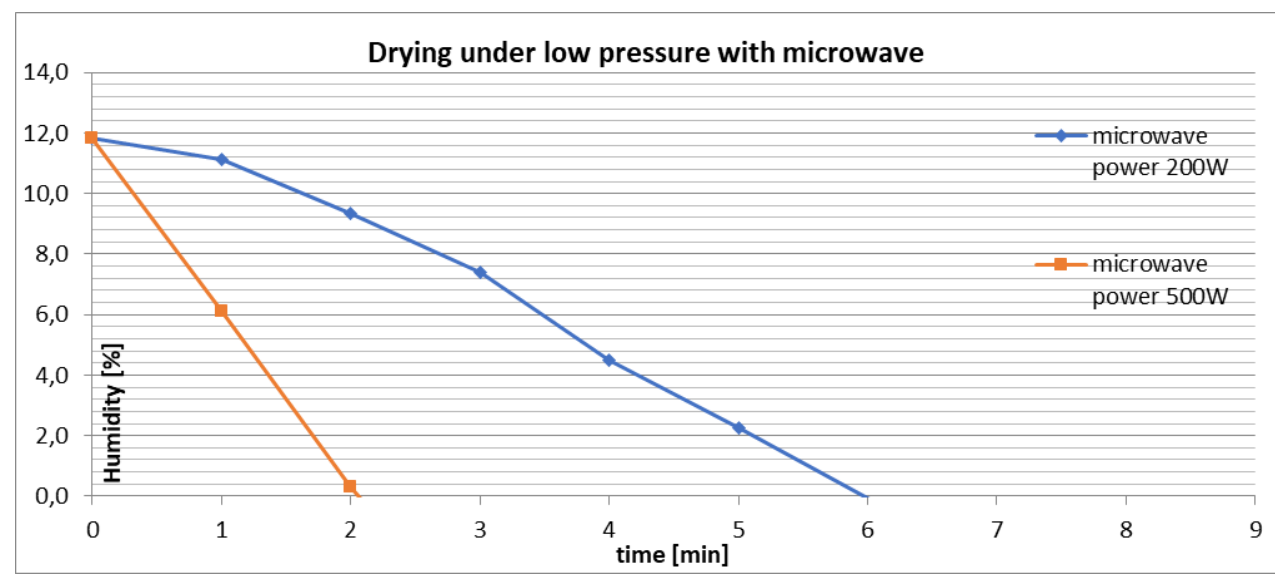

Fig. 8.

The use of microwaves has allowed the drying process to be accelerated many times from about 20-40 minutes with contact heating to 2-6 minutes in the case of microwave heating.

\section{Conclusions}

The process of microwave vacuum drying has found its valuable use in technical scale, enabling to obtain dried material with unique qualities, impossible to be obtained with the use of conventional methods. All quality research carried out so far has shown very favourable characteristics in dried material obtained this way: preserving valuable biologically active components and very good organoleptic assessment.

The quality of obtained dried material is comparable to the material dried through sublimation methods, which methods are costly, require long time for drying and large spaces.

In both mentioned cases of industrial application of microwave vacuum driers, the investment process was preceded by tests with the use of laboratory driers. Such driers are also very useful during operation of industrial installations, as they allow to seek optimal technological parameters with the use of small amounts of raw material, and they eliminate the risk of significant losses caused by improper selection of process parameters.

However, it should be stressed that the described designs both in laboratory scale and industrial installations allow optimal process of drying by applying adequate technical solutions resulting from the specificity of the microwave technology: special radiators emitting microwaves to the material being dried, their proper location in the chamber, and microwave generators with low power rippling. Proper selection of working pressure values and the use of special microwave generators also enables elimination of the threat of spontaneous discharges.

To sum up, vacuum microwave driers are a technological solution with very large application potential; however, their implementation requires design works and starting such lines taking into account the specificity resulting from wave-related phenomena, characteristic for the microwave band. 


\title{
MICROWAVE AND INDUCTION HEATING ON FIBRE-REINFORCED CEMENTITIOUS MATERIALS FOR THE DEMOLITION OF STRUCTURES
}

\author{
R. Borinaga-Treviño ${ }^{1}$, A. Orbe ${ }^{1}$, J. Cuadrado ${ }^{1}$, Crespo, I., ${ }^{2}$, J. Norambuena-Contreras ${ }^{3}$ \\ ${ }^{I}$ Department of Mechanical Engineering, University of the Basque Country UPV/EHU, \\ Plaza Ingeniero Torres Quevedo 1, 48013 Bilbao, Spain \\ ${ }^{2}$ TECNALIA Research and innovation, Paseo Mikeletegi 7, 2009, San Sebastian, Spain \\ ${ }^{3}$ LabMAT, Department of Civil and Environmental Engineering, University of Bio-Bio, \\ Concepción, Chile
}

*Corresponding author: R. Borinaga-Treviño. Email: roque.borinaga@ehu.eus

Keywords: microwave, induction heating, demolition, concrete, fibre

\begin{abstract}
Nowadays, strengthening and refurbishing of existing structures on urban areas has gained interest in order to reduce costs by avoiding both the use of construction raw materials and the disposal of construction and demolition waste on landfill. Usually, existing structures need to be locally demolished in order to replace either the existing corroded reinforcement or strengthen the existing structure. Among all the existing demolition methods, microwave and induction heating have been proven as a good alternative to generate a local damage with little noise and dust production, which is a desirable feature when structures are in urban areas. Nevertheless, there is a lack of information about the behaviour of both damaging methods when steel fibre-reinforced concrete is involved.
\end{abstract}

In this context, this paper studies the influence of the steel fibre addition on the damaging capacity of both microwave and induction heating demolition methods. For that purpose, mortars containing two different steel fibres were added in three different proportions and exposed to microwave and induction heating technologies. With the aim of evaluating the damage caused by both heating methods, test specimens were visually checked, and mechanical properties were determined via flexural and compressive strength tests.

According to the results, cement mortar specimens exposed to either microwave or induction heating suffered a sudden, violent disintegration without noticing any previous damage on the mortar specimens. Results proved that a pressure increment on water-saturated pores caused the failure regardless of the fibre type used as a reinforcement. However, for microwave heating, the reflection of microwaves on the fibres tended to concentrate the heating effect on the specimen surface, resulting in higher surface temperatures, but lower damaging potential of the demolition method. Furthermore, similar failure mechanism was observed on oven-dried mortars exposed to microwave heating. Nevertheless, oven dried mortars exposed to induction heating suffered a highly variable, silent and controlled damage, presenting a highly varying quantity of fracture planes that decreased its flexural and compressive strengths by up to $38 \%$ and $31 \%$, respectively. 


\section{Introduction}

With the aim of reducing construction and material costs on the construction industry, reuse of old structures has gained interest. At the same time, facades, pavements, roofs, and other non-structural parts of the buildings must be frequently replaced during the refurbishing process of old buildings. In any case, mortar and concrete materials often need to be replaced in order to guarantee a minimum quality on the refurbished building. For the removal of those materials, there are numerous alternatives [1-3], which could be classified according to the demolition mechanism used: impact, cutting, shear, bursting, abrading, high water pressure and heating. On the last, flame lance, laser beam, electron beam, arc heating, highvoltage pulses, microwave heating, induction heating, and microwave-drilling are the main existing alternatives. Among the existing thermal methods, this paper studies the use of microwave and induction heating to cause a local damage on mortar specimens.

Regarding the microwave heating technology, Ong and Akbarnezhad [3] and Makul et al. [4] summarised the different uses of microwave radiation on cement-based materials. As well as other non-destructive uses, microwaves have also been used to remove contaminated superficial concrete, to provide a contactless drilling method [5] or even to improve the recycled aggregate quality by eliminating the superficial cement paste of the recovered aggregate [6-8].

Moreover, regarding induction heating technology, it is widely being studied as a healing mechanism on several bituminous materials by Norambuena-Contreras and co-workers [9], or as a non-destructive method to detect corroded rebars [10-12]. Although Kasai [2] already indicated that demolition by induction heating of the rebar is a feasible demolition technique, the topic was not further studied for a long time due to its high cost. Nevertheless, recent studies indicate that there is an increasing interest on this demolition method $[13,14]$. Anh and co-workers [13] introduced a wire mesh as a reinforcement on cementitious finish joints to ease its dismantling when required, with successful results.

Lim and Lee [14] compared induction heating demolition method to other methods based on the mechanical dismantling of the reinforced-concrete structure. They concluded that induction heating is $12 \%$ more expensive and significantly slower than the conventional crushing methods, but they also indicate that the generation of dust and pollutants is significantly reduced which could counterbalance, at least, the increase in cost. Nonetheless, there is few literature regarding the study of the behaviour of steel fibre reinforced cementitious materials subjected to induction heating.

This paper analyses the effect of using microwave or induction heating technologies as a demolition process on steel fibre reinforced mortars. On the particular, the paper analyses their influence on the mechanical properties of steel fibre reinforced cement mortars.

\section{Materials and methods}

This section describes the materials and experimental programme followed in this study.

Fig. 1 shows the different test methods used on the cement mortar specimens with, and without, steel fibres. 
a)

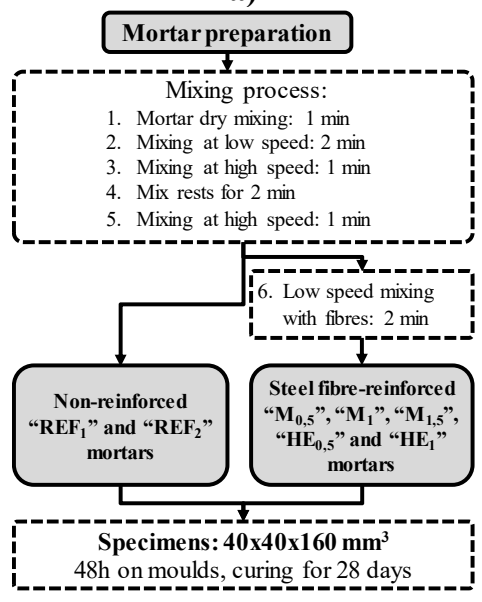

b)

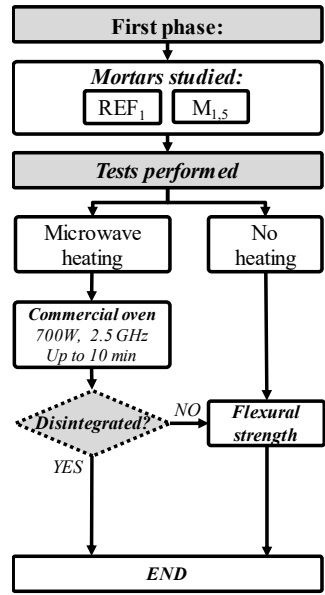

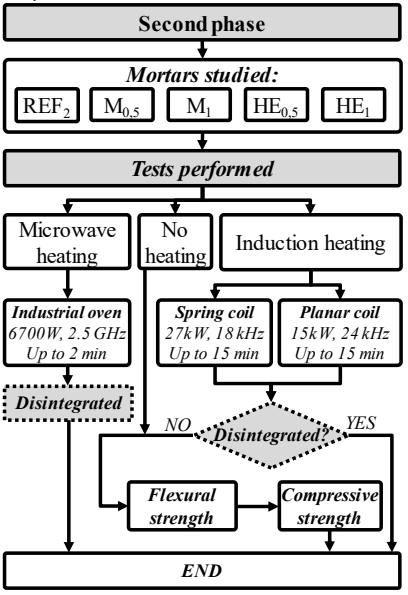

Fig. 1. Experimental programme followed in this study: a) Mixing process of the mortars and b) Tests performed on each research phase.

\section{Materials, mix proportions and specimen preparation}

On the first phase, Mapei Planitop HPC floor ${ }^{\circledR}$ mortar was used with and without the DRAMIX $^{\circledR}$ OL $13 / .20$ brass covered steel fibres $(\boldsymbol{M})$ proposed by the manufacturer as a reinforcement with the aim of evaluating the influence of the fibre presence on the damage caused by microwave heating. $\boldsymbol{M}$ fibres were $13 \mathrm{~mm}$ long and $0.21 \mathrm{~mm}$ in diameter, with a tensile strength and an elastic modulus of $2750 \mathrm{MPa}$ and $200000 \mathrm{MPa}$, respectively. The $d r y$ mortar:water:fibre proportions by weight set were the following:

(1) 1:0.12:0 for the reference $\left(\boldsymbol{R} \boldsymbol{E} \boldsymbol{F}_{1}\right)$ non-reinforced mortar.

(2) 1:0.12:0.065 for the $\boldsymbol{M}_{1.5}$ mortar, reinforced with $1.5 \%$ of $\boldsymbol{M}$ fibres by mortar volume as proposed by the manufacturer.

On the second phase, an ordinary mortar was used, as the high strength of the previous mortar could partially hinder the damaging effect expected due to both heating methods. CEM II-B (L)/32.5R cement (c) and 0/2 mm grain size limestone sand (s) were used as mortar main constituents. CRISOL 550 superplasticizer (sp) was added to all the mortars to reduce the water $(\boldsymbol{w})$ required to achieve self-compacting consistency. Finally, two types of fibres were also used to reinforce the mortar. Firstly, $\boldsymbol{M}$ fibres were again used in this second phase, since they are more appropriate to reinforce mortars due to the more reduced length and diameter. Alternatively, $\boldsymbol{H E}$ hooked structural steel fibres $(\boldsymbol{H E})$ were used, as they are used to reinforce structural concretes. $\boldsymbol{H E}$ fibres were $50 \mathrm{~mm}$ long and $1 \mathrm{~mm}$ in diameter. Five different mortars were created by varying both the fibre type and the fibre proportion used. Thus, the c:s:sp:w:fibre weight proportions set were the following:

1:3:0.01:0.5:0 for the reference $\left(\boldsymbol{R} \boldsymbol{E} \boldsymbol{F}_{2}\right)$ mortar.

$>$ 1:3:0.01:0.5:0.1 and 1:3:0.01:0.5:0.2 for the $\boldsymbol{M}_{0.5}$ and $\boldsymbol{M}_{1}$ mortars, reinforced with 0.5\% and $1 \%$ of $\boldsymbol{M}$ fibres by mortar volume, respectively.

$>$ 1:3:0.01:0.5:0.1 and 1:3:0.01:0.5:0.2 for the $\boldsymbol{H} \boldsymbol{E}_{0.5}$ and $\boldsymbol{H} \boldsymbol{E}_{\boldsymbol{1}}$ mortars, reinforced with $0.5 \%$ and $1 \%$ of $\boldsymbol{H E}$ fibre by mortar volume, respectively. 
All the tests were carried out in $40 \times 40 \times 160 \mathrm{~mm}^{3}$ prismatic test specimens prepared in compliance with EN 1015-10 [16]. The mortar mixing was similar for all the mortars studied. During the first 24 hours, test specimens were cured on moulds under ambient laboratory conditions. After removing the moulds, test specimens were submerged in water at $20^{\circ} \mathrm{C}$ for 27 days in order to reach 28 days curing age.

\section{Heating of specimens via microwave radiation}

It is known that microwave heating penetration depth on cementitious materials mainly depends on the material dielectric properties and on the heating frequency used [3]. The higher the frequency, the more superficial and local is the heating effect. At the same time, the higher the water content, the higher both the dielectric constant and the loss factor. In this way, for lower frequencies, microwaves cause the disaggregation of the cementitious material by increasing the pore water pressure until its internal collapse, while for higher frequencies, the fracture is more superficial and caused by the high thermal gradients generated. With that in mind, specimens exposed to microwave heating were oven-dried for $72 \mathrm{~h}$ at $105^{\circ} \mathrm{C}$. Afterwards, microwave ovens, with a power of $700 \mathrm{~W}$ and $6700 \mathrm{~W}$, and a frequency of $2.5 \mathrm{GHz}$, were used to ensure a uniform heating of the specimens [6,17]. Six test specimens were heated via microwave for $10 \mathrm{~min}$ and compared to another six nonheated samples. During the heating time, test specimens were surrounded by rockwool insulation and silicone moulds to protect the microwave heater from possible explosions.

\section{Heating of specimens via electromagnetic induction}

As opposed to microwave heating, non-reinforced cementitious materials are usually immune to induction heating as their ferromagnetic material content is negligible. With that in mind, ferromagnetic additives such as steel bars and steel fibres are frequently added into the cement matrix. These materials could be heated via electromagnetic induction. Thus, mortar could be indirectly heated mainly in the areas where the ferromagnetic material is, providing a way of triggering a local, selective mortar cracking. As for the microwave heating, there are two main thermal mechanisms that could fracture the mortar. Firstly, mortar could be damaged by increasing the pore water pressure until its internal collapse. Also, mortar could also be broken due to internal stress caused by excessive thermal gradients. Oven-dried and water saturated $\boldsymbol{M}_{0.5}, \boldsymbol{M}_{1}, \boldsymbol{H} \boldsymbol{E}_{0.5}$ and $\boldsymbol{H E}_{1}$ mortars were exposed to two different induction heating processes as it is summed up in Fig. 2: 
a)

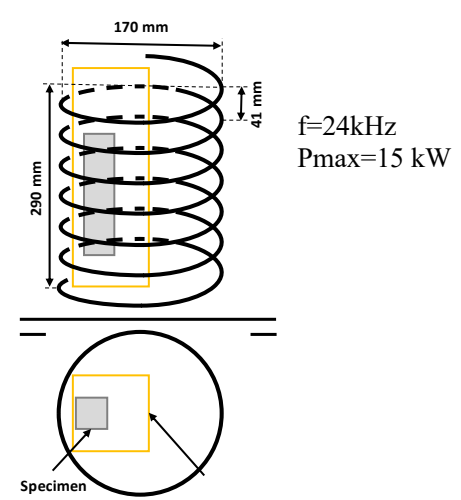

b)

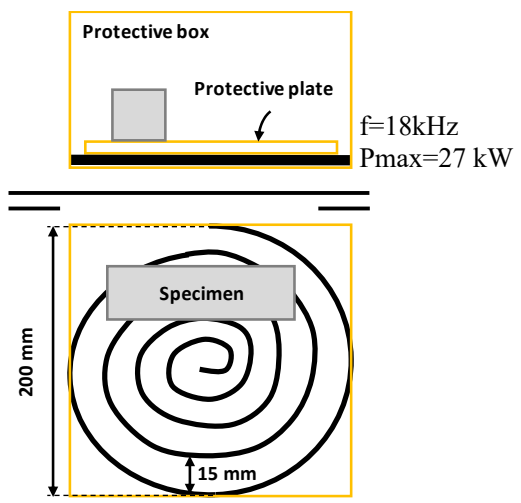

Fig. 2. Disposition of the specimens on each induction heating equipment: a) spring inductor, and b) spiral planar inductor.

\section{Flexural and compressive strength tests}

Flexural and compressive strength tests were carried out according to the standard EN 101511 to determine the damage caused by the thermal process applied on each test specimen. Flexural test was displacement-controlled, with a displacement speed ratio set to 0.5 $\mathrm{mm} / \mathrm{min}$. Compressive test was load controlled, with a load increment force ratio of $0.5 \mathrm{kN} / \mathrm{s}$. Finally, for each mortar type and thermal process combination studied, the resulting flexural strength was calculated as the mean value of all the specimens available on each case.

\section{Results and discussion}

\section{Microwave heating effect on the mechanical properties of the hardened mortar}

Fig.3a shows the flexural strength of the $\boldsymbol{R E} \boldsymbol{F}_{1}$ and $\boldsymbol{M}_{1.5}$ mortars with, and without the microwave heating process carried out at $700 \mathrm{~W}$ and $2.5 \mathrm{GHz}$. For the $\boldsymbol{R E F}_{1}$ mortar, flexural strength of the non-heated mortar samples was $14.9 \mathrm{MPa}$, while the value after $10 \mathrm{~min}$ of heating could not be measured as the specimens violently collapsed after 300-400s (Fig. 3b). To determine if the flexural strength suffers a gradual decrease until its failure, 6 more $\boldsymbol{R} \boldsymbol{E} \boldsymbol{F}_{\boldsymbol{1}}$ specimens were also microwave heated during $4 \mathrm{~min}$. In this case, flexural strength was reduced to nearly $50 \%$ of the value observed for the non-heated samples. 
a)

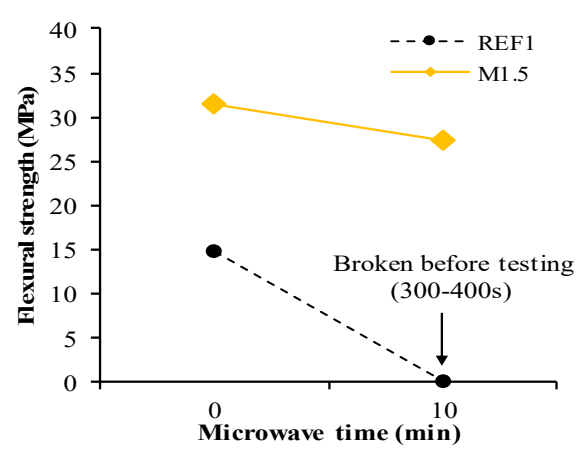

b)

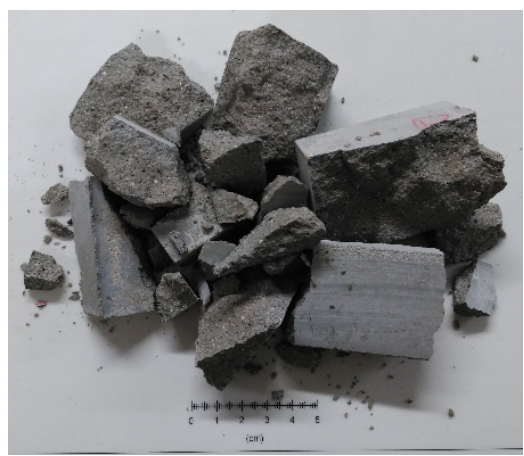

Fig. 3. Influence of microwave heating on the hardened mortar: a) evolution of the flexural strength with the microwave heating time; b) $\mathrm{REF}_{1}$ mortar situation after its disaggregation

$$
\text { (300-400s). }
$$

Additionally, surface temperature of the specimens was also measured by using a "Fluke 561 " infrared thermometer. As the $\boldsymbol{R E F}_{1}$ mortars collapsed before reaching the $10 \mathrm{~min}$ initially set, collapsed non-reinforced mortar sample temperatures were determined immediately after cancelling the heating process. According to the results, it seems that in $\boldsymbol{R E} \boldsymbol{F}_{1}$ the temperature reached on the newly exposed surfaces was higher than the $250.9^{\circ} \mathrm{C}$ measured, as there was a time lapse of 15-30s between the occurrence of the collapse and the moment when the photographs were taken. Besides, the microwave walls were wet when the collapse of the $\boldsymbol{R E} \boldsymbol{F}_{1}$ mortars occurred, but dry when the $\boldsymbol{M}_{1.5}$ mortars were heated, which indicated that the main cause of the collapse for the $\boldsymbol{R} \boldsymbol{E} \boldsymbol{F}_{1}$ mortars was the rise of the pore water (vapor) pressure as there was a sudden release of water in the disaggregation process. Regarding $\boldsymbol{M}_{1.5}$ mortar, the temperature observed on the surface of the specimens was $348.7^{\circ} \mathrm{C}$, thus significantly higher than that observed on the $\boldsymbol{R E} \boldsymbol{F}_{\boldsymbol{1}}$ mortar. Despite the higher surface temperature, specimens were not significantly damaged, as the flexural strength was only reduced by $13 \%$ due to all the heating process. As the steel reflects the microwave radiation [3], most of the heating effect was focused on the specimen surface for the $\mathrm{M}_{1.5}$ mortar. Therefore, a high temperature gradient was generated on the surface, but not enough to cause the collapse with the power of $700 \mathrm{~W}$ applied. However, some specimens were also tested in a more powerful microwave $(6700 \mathrm{~W}$, nearly 10 times the power previously used). In this case, both mortar types broke after a heating time of 90-120s. For the $\boldsymbol{R E F}_{\boldsymbol{1}}$ mortar, the appearance of the specimen after the collapse was similar to that observed previously. However, $\boldsymbol{M}_{1.5}$ mortars tended to suffer little collapses sequentially. With the first explosion, there was a superficial removal of the material surrounding the $40 \mathrm{~mm}$ edges of the specimens. After a few seconds, a second explosion occurred, removing more material on the newly created surface. In short, collapses were more controlled and gradual than that observed for the non-reinforced mortars.

\section{Induction heating effect on the mechanical properties of the hardened mortar}

Fig. 4 shows the flexural and compressive strength of the oven-dried mortars as a function of the heating treatment used. According to the results obtained, both flexural and compressive strengths were clearly reduced due to the induction heating when spring 
inductor was used. For the mortars reinforced with $\boldsymbol{H E}$ fibres, flexural strength and compressive strength were reduced by up to $38 \%$ and $31 \%$, respectively. In addition, the strength reduction could be considered similar for both fibre proportions studied. In the same way, mortars reinforced with $\boldsymbol{M}$ fibres suffered a decrease on the flexural and compressive strengths of up to $35 \%$ and $15 \%$, respectively. However, after a visual inspection of both mortar types, it is clear that the damage is only visually appreciable on the $\boldsymbol{H E}$ mortars (see Fig. 5a), as the $\boldsymbol{M}$ mortars did not present any surface crack that could indicate this weakening effect caused by induction heating. Due to their greater fibre content for the same volume due to their smaller length, fibres were more effective for crack arrest. Accordingly, $\boldsymbol{H E}$ mortars subjected to flexural strength test with visual cracks tended to break following the previously generated crack paths (see Fig. 5b).

a)

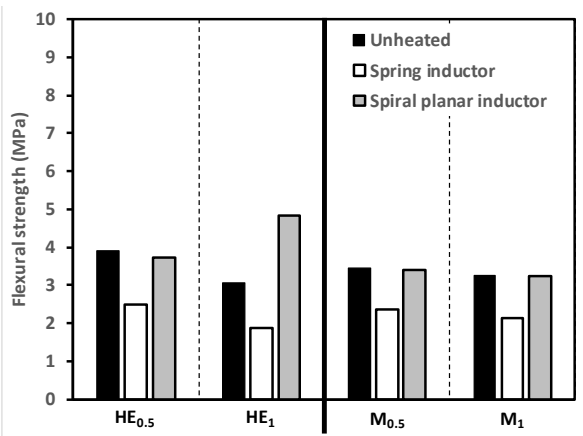

b)

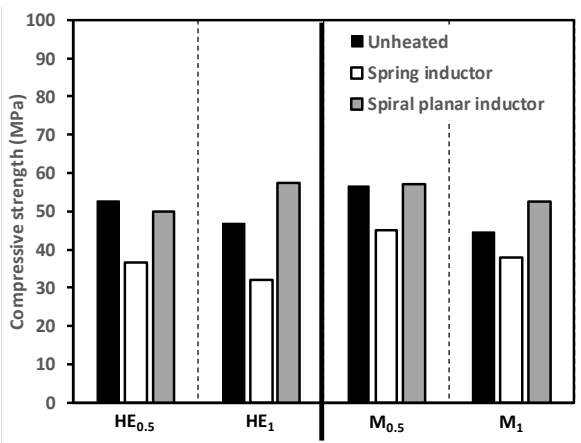

Fig. 4. Influence of the fibre type, proportion and induction heating process on mechanical properties of the hardened mortar: a) flexural strength; b) compressive strength.

Moreover, regarding the results obtained on oven-dried specimens heated by a spiral planar inductor, results are not conclusive. Spiral planar inductor is not as effective as the spring inductor, since it only affects one face of the specimens, instead of enclosing it. For both $\boldsymbol{H E}$ and $\boldsymbol{M}$ mortars, reductions of flexural strength and compressive strength are negligible. Even in some cases, an increase on the mechanical properties of the tested specimens was observed, which is attributed to the known statistical variations of the cementitious matrix and to the randomness of the fibre distribution and orientation.

a)

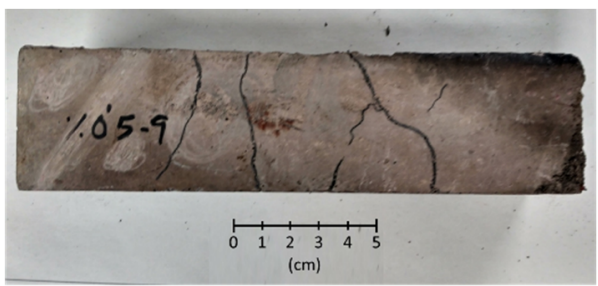

b)

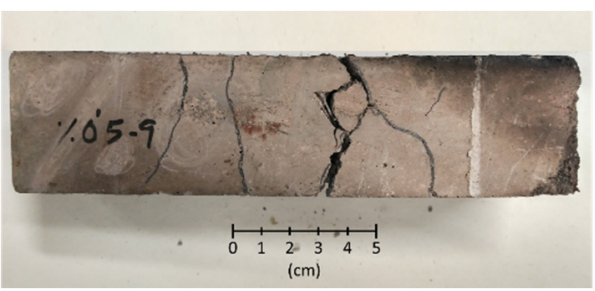

ortar: a) after spring induction heating; b) after flexural strength test.

Finally, one water saturated mortar specimen per mix was heated with the spiral planar inductor. In this case, $\boldsymbol{H E}_{\boldsymbol{1}}$ and $\boldsymbol{M}_{\boldsymbol{1}}$ mortars broke after heating them for $2 \mathrm{~min}$ and $9 \mathrm{~min}$, respectively. Consonant with the microwave heated specimens, the excessive pore water 
pressure generated caused the sudden release of the vapour generated during the heating process. Thus, both specimens suffered a sudden, very violent collapse due to the high porewater pressure. Fig. 6 shows the appearance of the $\boldsymbol{H E}_{\boldsymbol{1}}$ specimen. $\boldsymbol{M}_{\boldsymbol{1}}$ specimen is not shown as it was completely disintegrated during the process. Nevertheless, $\boldsymbol{M}_{\boldsymbol{I}}$ test specimen also took a significantly higher heating time that $\boldsymbol{H} \boldsymbol{E}_{\boldsymbol{l}}$ mortar, which is attributed to the higher specific surface of the fibres related to their smaller diameter, which makes the coupling of the fibres with the inductor less efficient. For the $\mathrm{M}$ fibres, the heating efficiency would increase by increasing the frequency of the induction equipment. Anyway, water saturated $\boldsymbol{M}_{0.5}$ and $\boldsymbol{H} \boldsymbol{E}_{0.5}$ test specimenmortars did not explode, which indicated the importance of the fibre content on the whole process.

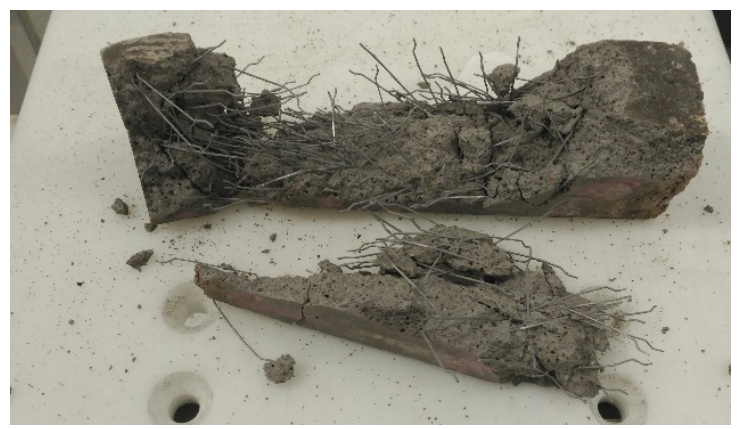

Fig. 6. Appearance of the water saturated $\mathrm{HE}_{1}$ mortar after 2 min under induction heating.

\section{Conclusions}

This paper analysed the effect of using microwave or induction heating as a potential demolition thermal process on steel fibre-reinforced mortars. The influence of both heating technologies on the mechanical properties of steel fibre reinforced mortars was studied. For that, non-reinforced mortars were compared to mortars with the same constituents and proportions, but with the addition of two different types of fibres (Hooked structural steel fibres and brass covered steel needles) in different proportions $(0.5 \%$ and $1 \%$ and $1.5 \%$ by mortar/vol.). Based on the results obtained, the authors reached the following conclusions:

With respect to the microwave heating demolition method:

$>$ For a heating power and a frequency of $700 \mathrm{~W}$ and $2.5 \mathrm{GHz}$, non-reinforced mortars collapsed after $300-400$ s heating time, because of the high pore-water pressure generated during the heating process, causing a sudden, explosive collapse, even when the specimens were previously oven-dried.

$>$ For the same heating power and frequency combination, mortars reinforced with brass covered steel fibres did not collapse and suffered a negligible reduction (13\%) of their flexural strength. Fibres tended to concentrate the heating effect on the specimen surface due to their microwave shielding effect, which resulted in surface temperatures $50 \%$ higher than those observed on the non-reinforced mortars.

$>$ Both mortars collapsed after increasing the heating power to $6700 \mathrm{~W}$. The failure mechanism on non-reinforced mortar was similar to that previously observed. For the mortar reinforced with brass-covered steel fibres, failure mechanism was the same but occurred via consecutive superficial removals nearby the shorter edges of the specimens. 
With respect to the induction heating demolition method:

$>$ On oven-dried specimens, both flexural and compressive strengths were only clearly reduced due to the induction heating when spring inductor was used. The damage was only visually appreciable on the mortars reinforced with hooked structural steel fibres.

$>$ On water-saturated specimens, the fibre proportion used had a significant influence on the damage observed. No matter the fibre type used, mortars with the lowest fibre proportion did not suffer significant damage. However, damage on the mortars with the highest fibre proportion suffered a similar failure mechanism to that observed on nonreinforced mortar samples exposed to microwave heating. In this case, the collapse was even more violent and effective to that observed with microwaves.

\section{Acknowledgements}

This work has been partly financed within the European Horizon 2020 Joint Technology Initiative Shift2Rail through contract no.730841 (IN2TRACK) and no. 826255 (IN2TRACK2). This work was also co-financed with the projects Elkartek 2018 ref. KK2018/00073 (GOLIAT) and Elkartek 2019 ref. KK-2019/00023 (GOLIAT2). The authors also wish to thank the Basque Government for financial assistance through IT919-16.

\section{References}

[1] Lindsell P. Demolition Techniques for Concrete Structures. In: Kreijger PC, editor. Adhesion Problems in the Recycling of Concrete, Boston, MA: Springer US; 1981, p. 201-215.

[2] Kasai Y. Demolition of concrete structure by heating. Concr Int 1989;11:33-8.

[3] Ong K, Akbarnezhad A. Microwave-Assisted Concrete Technology: Production, Demolition and Recycling. 1st ed. 6000 BROKEN SOUND PARKWAY NW, STE 300, BOCA RATON, FL 33487-2742 USA: CRC Press TAYLOR \& FRANCIS GROUP, 2015.

[4] Makul N, Rattanadecho P, Agrawal DK. Applications of microwave energy in cement and concrete - A review. Renewable Sustainable Energy Rev 2014;37:715-33.

[5] Jerby E, Aktushev O, Dikhtyar V. Theoretical analysis of the microwave-drill near-field localized heating effect. J Appl Phys 2005;97.

[6] Akbarnezhad A, Ong KCG, Zhang MH, Tam CT, Foo TWJ. Microwave-assisted beneficiation of recycled concrete aggregates. Constr Build Mater 2011;25:3469-79.

[7] Tsujino M, Noguchi T, Kitagaki R, Nagai H. Ompletely recyclable concrete of aggregate-recovery type by using microwave heating. J Struct Constr Eng 2011;76:2239.

[8] Menard Y, Bru K, Touze S, Lemoign A, Poirier JE, Ruffie G et al. Innovative process routes for a high-quality concrete recycling. Waste Manage 2013;33:1561-5.

[9] Norambuena-Contreras J, Garcia A. Self-healing of asphalt mixture by microwave and induction heating. Mater Des 2016;106:404-14.

[10] Kobayashi K, Banthia N. Corrosion detection in reinforced concrete using induction heating and infrared thermography. J Civ Struct Health Monit 2011;1:25-35.

[11] Baek S, Xue W, Feng MQ, Kwon S. Nondestructive Corrosion Detection in RC Through Integrated Heat Induction and IR Thermography. J Nondestr Eval 2012;31:18190.

[12] Liu L, Zheng D, Zhou J, He J, Xin J, Cao Y. Corrosion detection of bridge reinforced concrete with induction heating and infrared thermography. Int $\mathrm{J}$ Rob Autom 2018;33:379-85. 
[13] Ahn J, Noguchi T, Kitagaki R. Disassembly properties of cementitious finish joints using an induction heating method. Mater 2015;8:2433-53.

[14] Lim M, Lee JS. Economic feasibility of the induction heating method for dismantling structures: Analysis of reinforcement recovery costs based on required demolition equipment. Int J Appl Eng Res 2017;12:13077-83.

[15] UNE-EN 1015-10. Methods of Test for Mortar Masonry. Part 10: Determination of Dry Bulk Density of Hardened Mortar, . 2007.

[16] Norambuena-Contreras J, Gonzalez-Torre I. Influence of the microwave heating time on the self-healing properties of asphalt mixtures. Appl Sci 2017;7. 


\title{
JOINING OF Cu TO SS304 BY MICROWAVE HYBRID HEATING WITH Ni AS INTERLAYER
}

\author{
S. Tamang ${ }^{1}$, S. Aravindan ${ }^{1}$ \\ ${ }^{1}$ InstituteIndian Institute of Technology Delhi, Department of Mechanical Engineering, \\ Hauz Khas, New Delhi \\ aravindan@mech.iitd.ac.in
}

Keywords: microwave heating, $\mathrm{Cu}, \mathrm{SS} 304$, joining, $\mathrm{Ni}$

\begin{abstract}
Joining of dissimilar pure Copper $(\mathrm{Cu})$ to Stainless Steel (SS304) is necessitated in many industrial applications such as heat exchangers and electrical contacts. Advantages of both the materials such as high electrical conductivity of copper and better corrosion resistance of SS304 can be harnessed by way of joining both the metals. However, joining to $\mathrm{Cu}$ to itself or other materials is a challenge since the input heat is dissipated rapidly. Most of the conventional welding methods such as arc and gas are incompetent and unconventional methods such as Explosion Welding, EBW, and Diffusion Bonding are very expensive.

In this study a new economical process of joining of dissimilar metals i.e. $\mathrm{Cu}$ to SS304 by microwave hybrid heating is investigated. Microwave joining is made possible by applying a powder (in this work, Nickel metal powder) as an interlayer and exposing to microwave surrounding the interlayer with a susceptor.

The interlayer of $\mathrm{Ni}$ powder having average size $200 \mathrm{~nm}$ and $45 \mu \mathrm{m}$ was used. The microstructure of the joint was studied by optical microscope and scanning electron microscope. The joints formed with $200 \mathrm{~nm} \mathrm{Ni}$ powder were observed to have a defect free microstructure. The EDS and XRD analysis determine the formation of solid solution between $\mathrm{Cu}-\mathrm{Ni}$ interface and an intermetallic compound at $\mathrm{Fe}-\mathrm{Ni}$ interface. The diffusion of elements across the joint was further analyzed by EDS line scan. The hardness variation was studied by Vickers' micro-hardness. It can be concluded that smaller size Ni heats up faster in microwave and produce stronger joint of $\mathrm{Cu}$ to $\mathrm{SS} 304$ by microwave hybrid heating.
\end{abstract}

\section{Introduction}

Dissimilar material joining is gaining interest nowadays due to the application potentials in terms of flexibility, functionality and energy savings. Different industries such as chemical, nuclear, automobile, rail, aviation, electronics and power have requirements for dissimilar material joining. Many difficulties that occur during dissimilar joining are due to the difference in mechanical, thermal and electrical properties of the materials to be joined. Failures to join dissimilar combinations are due to the difference in thermal expansion coefficients, fusion zone (FZ) solidification or thermal fatigue. Bimetallic corrosion may also occur. Hence, it is difficult to fabricate dissimilar material combinations using conventional welding methods like Shielded Metal Arc Welding (SMAW), Gas Tungsten Arc Welding (GTAW), Gas Metal Arc Welding (GMAW), and Submerged Arc Welding (SAW) etc. There are other methods for making dissimilar joints such as explosive welding, laser welding, electron beam welding, diffusion bonding, brazing, soldering and friction welding. These methods follow stringent processing conditions, and are expensive and time consuming. Faster processing method is always the necessity of the industry owing to energy saving and improved productivity. 
Microwaves are the part of the electro-magnetic spectrum having wavelength between $1 \mathrm{~m}$ to $1 \mathrm{~mm}$ for frequencies ranging from $300 \mathrm{MHz}$ to $300 \mathrm{GHz}$. Faster and selective heating and energy saving are the well-known advantages of microwave processing [1]. The molecules interact with the microwaves and coverts the microwave energy to heat energy. Due to this, the materials heat up faster compared to conventional heating. On the other hand, in conventional heating the convection, conduction and radiation help in heating a sample from outside to inside. Microwave heating is used in the past for a number of applications such as heating fruits, beans, meat and cooking food. It is also used in other industries to dry paper, tobacco, leather, textiles, ceramics, cure adhesives, vulcanise rubber, waste management and etch semiconductors ${ }^{[2]}$ and in processing of ceramics, polymers and metals $^{[3]}$.

It is a well-known fact that metals reflect back most of the incident microwaves and penetration depth is limited to some microns. But when metals are used in powdered form it will heat up when subjected to microwave due to its small particle size being nearly equal to the penetration depth ${ }^{[4],[5]}$. The heating metal powders by microwaves was first reported by Walkiewicz et al ${ }^{[6]}$ of heating $\mathrm{Mg}, \mathrm{Fe}$ upto $120^{\circ} \mathrm{C}$ and $768^{\circ} \mathrm{C}$ respectively. Later Roy et al ${ }^{[7]}$ in 1999 conducted sintering to obtain full density components from metal powders with the formation of finer microstructures. This had resulted in better properties at lower cost.

Joining by microwave hybrid heating of similar bulk metals have been done by some researchers ${ }^{[8]-[15]}$. Limited work on dissimilar metals has been reported i.e. Srinath et al ${ }^{[16]}$ joined Stainless steel to mild steel and Bansal et al ${ }^{[17]}$ joined Inconel718 to SS316L.

The joining of copper and stainless steel can be used in many applications like in heat exchangers where corrosive fluids are used. The high conductivity of copper is a boon and a bane when it is to be joined with other material. Hence many problems are to be surpassed when welding copper to stainless steel. Tungsten inert gas (TIG) welding of copper is a challenge due to its high conductivity since all the heat generated is dissipated towards the base metal very fast. Copper has low miscibility with iron and no intermetallic compounds are expected. With preheating, TIG welding of the $\mathrm{Cu}$ and SS sheet is possible but with non-homogeneous joints, owing to resolidification time ${ }^{[18]}$. The high cooling rates in TIG and Electron beam welding cause bulk cooling of the molten pool. The molten metal solidifies to form $\mathrm{Fe}$ rich spherulites embedded in $\varepsilon-\mathrm{Cu}$ matrix and $\mathrm{Cu}$ rich spherulites embedded in $\alpha-\mathrm{Fe}$ matrix ${ }^{[18]}$. These phases will tend to transform to equilibrium microstructure on application of thermal stress during service life, thereby affecting the mechanical and corrosion properties of the joint ${ }^{[19]}$. Magnabosco et al ${ }^{[20]}$ confirmed the formation of non-equilibrium phases which are rich in $\mathrm{Cu}$ and $\mathrm{Fe}$ with $\mathrm{Fe}-\mathrm{Cr}-\mathrm{Ni}$ phase. The porosities in the copper-rich phase is due to the shrinkage of copper from inside the steel globule and intergranular microfissures at the interface towards the stainless steel are reported.

Diffusion welding was used to join $\mathrm{Cu}$ to SS304 by Yilmaz et al ${ }^{[21]}$. Micro-voids are seen along the grain boundaries on the $\mathrm{Cu}$ side of the bond region and it also has microcracks. Fracture can be observed at the microvoids on the copper side rather than the interface of $\mathrm{Cu}$ to SS. It is also reported that there is a thick band of intermetallic compounds formation. This is the limitation of the diffusion bonding process that leads to reduced joint strength. Using the same diffusion bonding process with nickel as an interlayer Sabetghadam et al [22] reported voids at both interfaces, that were of corrugated pattern. Intermetallic compounds were formed and its extent increased with temperature.

Explosion welding of $\mathrm{Cu}$ and stainless steel enhanced the tensile shear strength and flexural strength of joints ${ }^{[23]}$. Intermetallic compounds are not formed at the interface but the hardness is increased due collision of two plates. This process faces the limitation of being expensive and the lack in joint configuration flexibility. Moreover, it can be applied 
only to lap joint. Friction welding is another process used for joining copper to Stainless steel but this too is limited only to joining of cylindrical work piece. Sahin ${ }^{[24]}$ reported that the tensile strength of joint to be proportionally dependent on friction time and friction pressure until a certain point $(75 \mathrm{MPa})$ after which intermetallic compounds were observed to be formed at prolonged friction time.

By assembling a slope butt (scarf assembly) joint and focussing the laser on the steel side Yao et al ${ }^{[25]}$ achieved joint of $\mathrm{Cu}$ to $\mathrm{SS}$ with good tensile strength. Defect free joints can be obtained when limited copper is dissolved in steel matrix.

Hence it can be observed that there is a need of a joining process that can give good metallurgical joint with flexibility in joint configurations. Joining of Copper to Stainless Steel using microwave hybrid heating followed by analysis of the joints are presented in the subsequent sections.

\section{Materials and Methods}

Stainless steel SS304 and pure copper plates were cut into small section $6 \mathrm{~mm} \times 7 \mathrm{~mm} \times$ $25 \mathrm{~mm}$. Pure Nickel powder of average size $45 \mu \mathrm{m}$ and $200 \mathrm{~nm}$ was mixed with $20 \mathrm{wt} \%$ epoxy resin (bisphenol A diglycidyl ether) to form a slurry. The joint prepared was a simple lap joint with the prepared slurry in between as interlayer and clamped by a force $\sim 15 \mathrm{MPa}$ as observed in previous work ${ }^{[26]}$. A graphite crucible is placed inside a multimode microwave oven with $2.45 \mathrm{GHz}$ frequency and $3200 \mathrm{~W}$ power rating with the power set to $40 \%$. The sample is placed in top of graphite crucible and covered with graphite susceptor and microwave is set with a heating rate of $50^{\circ} \mathrm{C}$ per min.

\section{Results and Discussion}

The microstructure of the joints formed by $45 \mu \mathrm{m}$ and $200 \mathrm{~nm}$ Ni powder were observed under optical microscope. Fig. 1(a,b) clearly shows that the thickness of the interlayer using $45 \mu \mathrm{m} \mathrm{Ni}(140-170 \mu \mathrm{m})$ is larger than the interlayer formed by $200 \mathrm{~nm}$ Ni powder $(30-50 \mu \mathrm{m})$. It also contains voids which are not observed for the smaller Ni powder. This difference in interlayer thickness is due to the large difference in size of the powder which also affects the heating rate.

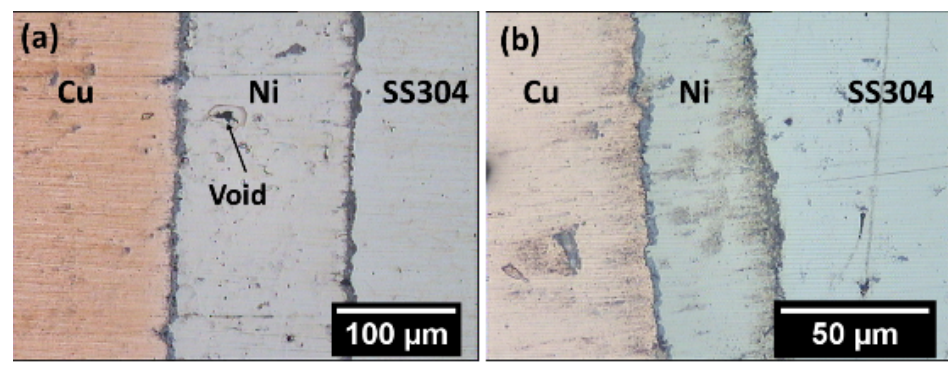

Fig. 1. Optical micrograph of the microwave joined $\mathrm{Cu}$ to SS034 samples (a) $45 \mu \mathrm{m} \mathrm{Ni}$ (b) $200 \mathrm{~nm}$ Ni powder

The experimental details are shown in Table 1 . The $200 \mathrm{~nm}$ Ni powder coupled with microwave at a soaking temperature of $900^{\circ} \mathrm{C}$ and $5 \mathrm{~min}$ soaking time. Microwave uniformly heat the metal powder if its size is smaller than the skin depth ${ }^{[27]}$. So the heating of metal powder depends on the skin depth,

$$
d=\sqrt{1 / \pi f \mu_{0} \mu^{r} \sigma}
$$


Where, $\sigma=$ electrical conductivity of a material $(\mathrm{S} / \mathrm{m})$

$f=$ frequency of the microwave $(\mathrm{Hz})$,

$\mu_{\circ}=$ permeability of free space and

$\mu^{\prime}=$ permeability $(\mathrm{H} / \mathrm{m})$.

Table 1. Experiment with varying temperature

\begin{tabular}{|c|c|c|c|c|c|c|c|}
\hline Size of & \multicolumn{2}{|c|}{ Soak time at $700{ }^{\circ} \mathrm{C}$} & \multicolumn{2}{|c|}{ Soak time at $800^{\circ} \mathrm{C}$} & \multicolumn{3}{|c|}{ Soak time at $900^{\circ} \mathrm{C}$} \\
\hline & $5 \mathrm{~min}$ & $10 \mathrm{~min}$ & $5 \mathrm{~min}$ & $10 \mathrm{~min}$ & $5 \mathrm{~min}$ & $10 \mathrm{~min}$ & $15 \mathrm{~min}$ \\
\hline $45 \mu \mathrm{m}$ & No joint & No joint & No joint & No joint & $\begin{array}{c}\text { No } \\
\text { joint }\end{array}$ & $\begin{array}{c}\text { No } \\
\text { joint }\end{array}$ & $\begin{array}{c}\text { Good } \\
\text { Joint }\end{array}$ \\
\hline $200 \mathrm{~nm}$ & No joint & No joint & No joint & No joint & $\begin{array}{l}\text { Good } \\
\text { Joint }\end{array}$ & $\begin{array}{c}\text { Good } \\
\text { Joint }\end{array}$ & $\begin{array}{c}\text { Good } \\
\text { Joint }\end{array}$ \\
\hline
\end{tabular}

Thus 200nm Ni powder heat up faster by microwave hybrid heating than $45 \mu \mathrm{m}$ Ni powder. The SEM image of the joints representing the different points across them are shown in Fig.2. The elements present on all these points are represented in Table 2. From the EDS data, the diffusion of $\mathrm{Cu}$ and $\mathrm{Fe}$ across the interlayer is very prominent in addition to some diffusion of $\mathrm{Ni}$ and $\mathrm{Cr}$. Since $\mathrm{Ni}$ and $\mathrm{Cu}$ are fully soluble ${ }^{[28]}$ in each other and diffusion of $\mathrm{Cu}$ is high. The interlayer forms into a solid solution of $\mathrm{Ni}$ and $\mathrm{Cu}$ by substitution method. A reaction layer of $\mathrm{FeNi}_{3}$ is formed between $\mathrm{SS} 304$ and the $\mathrm{Ni}$ interlayer. The higher diffusion of $\mathrm{Cu}$ and $\mathrm{Fe}$ for $200 \mathrm{~nm}$ Nickel is due to the faster heating obtained by the smaller size of the powder particle. The XRD analysis of the joint cross-section verify the formation of $\mathrm{FeNi}_{3}$ also represented in Fig 3.
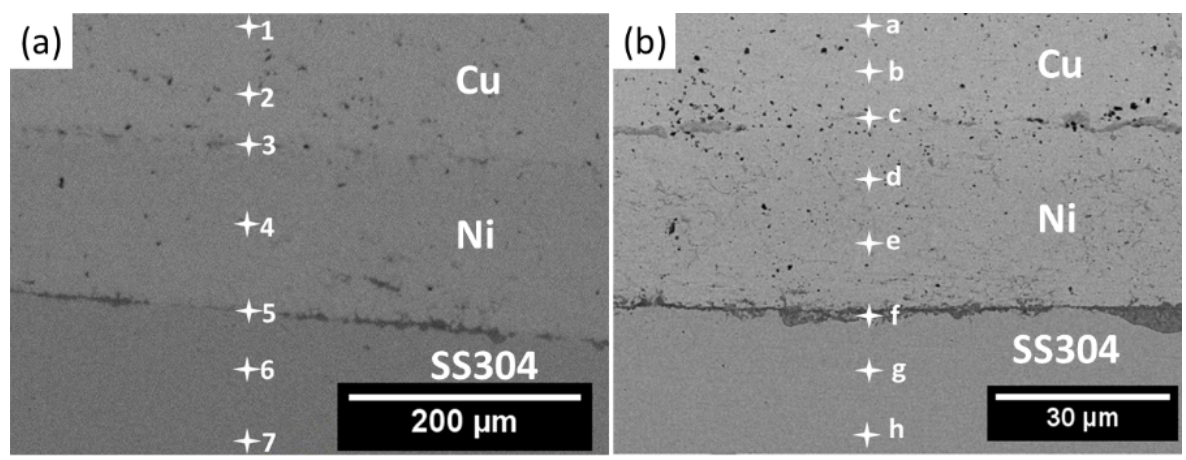

Fig 2. The SEM image of (a) $45 \mu \mathrm{m} \mathrm{Ni}$ and (b) $200 \mathrm{~nm}$ Ni powder 


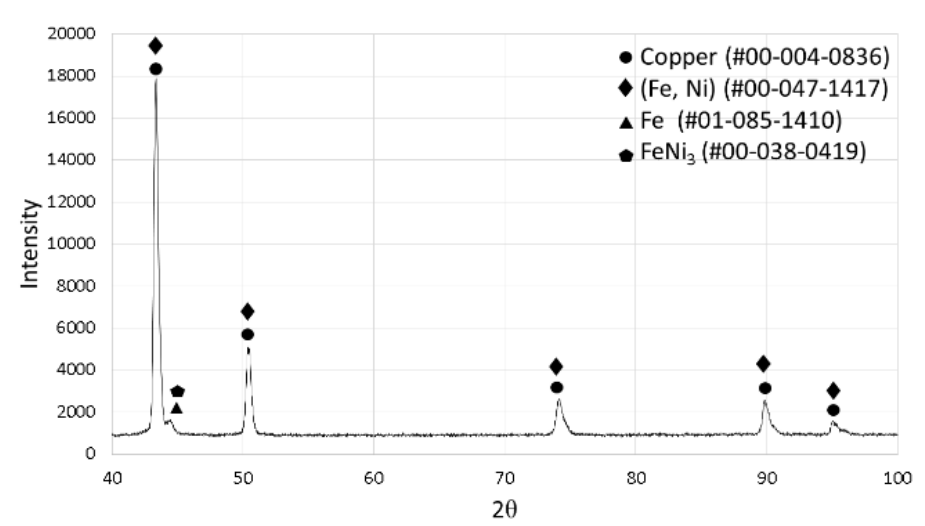

Fig. 3.XRD analysis across the joint zone

Table 2. wt $\%$ of elements obtained by EDS of points shown in Fig 2

\begin{tabular}{|c|c|c|c|c|c|c|c|}
\hline & Point & $\mathrm{O}$ & $\mathrm{Cr}$ & $\mathrm{Fe}$ & $\mathrm{Ni}$ & $\mathrm{Cu}$ & Probable compound \\
\hline \multirow{7}{*}{$\begin{array}{c}45 \\
\mu \mathrm{m}\end{array}$} & 1 & 1.333 & 0.794 & 2.144 & 2.430 & 93.299 & $\mathrm{Cu}$ \\
\hline & 2 & 1.278 & 0.806 & 2.388 & 2.961 & 92.567 & $\mathrm{Cu}$ \\
\hline & 3 & 1.367 & 1.088 & 2.730 & 26.939 & 67.875 & $\mathrm{Ni}-\mathrm{Cu}$ solid solution \\
\hline & 4 & 0.378 & 1.273 & 4.050 & 60.579 & 33.719 & $\mathrm{Ni}-\mathrm{Cu}$ solid solution \\
\hline & 5 & 5.027 & 5.114 & 18.994 & 39.760 & 31.106 & $\mathrm{FeNi}_{3}+\mathrm{Cu}$ \\
\hline & 6 & 1.473 & 14.436 & 51.843 & 9.252 & 22.996 & SS304 \\
\hline & 7 & 0.653 & 15.197 & 53.531 & 8.850 & 21.769 & SS304 \\
\hline \multirow{8}{*}{$\begin{array}{l}200 \\
\mathrm{~nm}\end{array}$} & $\mathrm{~A}$ & 0.605 & 1.218 & 4.323 & 2.692 & 91.162 & $\mathrm{Cu}$ \\
\hline & $\mathrm{B}$ & 0676 & 1.197 & 4.538 & 4.051 & 89.537 & $\mathrm{Cu}$ \\
\hline & $\mathrm{C}$ & 2.689 & 1.414 & 4.989 & 19.949 & 70.958 & $\mathrm{Ni}-\mathrm{Cu}$ solid solution \\
\hline & $\mathrm{D}$ & 1.051 & 1.431 & 5.642 & 56.196 & 35.680 & $\mathrm{Ni}-\mathrm{Cu}$ solid solution \\
\hline & $\mathrm{E}$ & 2.951 & 1.763 & 10.683 & 53.302 & 31.301 & $\mathrm{Ni}-\mathrm{Cu}$ solid solution \\
\hline & $\mathrm{F}$ & 4.677 & 4.372 & 20.102 & 40.006 & 30.843 & $\mathrm{FeNi}_{3}+\mathrm{Cu}$ \\
\hline & $\mathrm{G}$ & 1.572 & 14.786 & 50.177 & 6.901 & 26.564 & SS304 \\
\hline & $\mathrm{H}$ & 0.914 & 14.219 & 54.203 & 6.665 & 23.999 & SS304 \\
\hline
\end{tabular}

The Microhardness across the two types of joint obtained after soaking at $900^{\circ} \mathrm{C}$ for $15 \mathrm{~min}$ was measured as represented in Fig 4. The hardness of the Ni-Cu solid solution is higher for $200 \mathrm{~nm}$ powder than $45 \mu \mathrm{m}$. The hardness of the heat affected zone is also higher for $200 \mathrm{~nm}$ powder. With decrease in metal powder size the heating rate of the Ni interlayer increases and also reaches a higher temperature. All this is attributed due to total necking and melting of nano Ni powder. The lower hardness of $45 \mu \mathrm{m}$ is due to the partial fusion of the interlayer and presence of pores. 

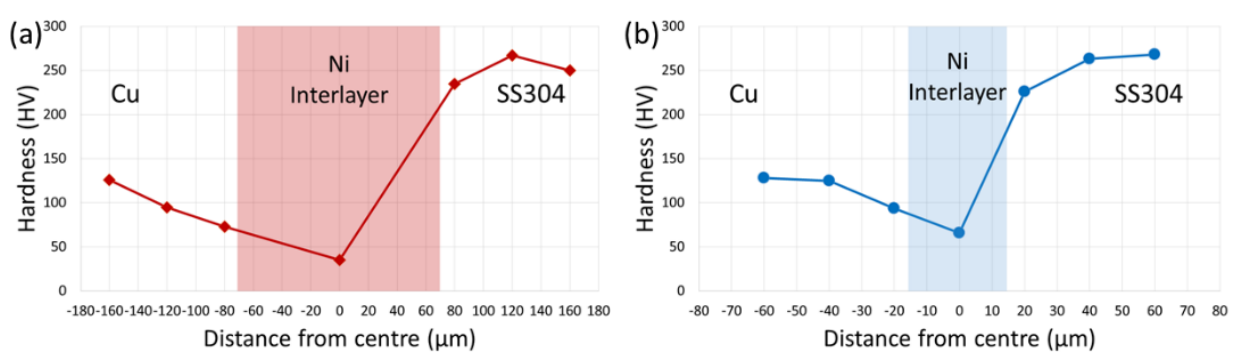

Fig. 4. Microhardness of the joint cross-section with joints formed using (a) $45 \mu \mathrm{m}$ size Ni powder and (b) $200 \mathrm{~nm}$ size Ni powder

\section{Conclusion}

The following conclusion are made for the study of joining of $\mathrm{Cu}$ to SS304 by microwave hybrid heat using Ni metal powder:

1. The joining was successful by using both $45 \mu \mathrm{m}$ and $200 \mathrm{~nm}$ Ni metal powder as interlayer

2. The $200 \mathrm{~nm}$ Ni formed the joint faster than $45 \mu \mathrm{m} \mathrm{Ni}$.

3. The thickness of the interlayer formed by $200 \mathrm{~nm} \mathrm{Ni}$ is almost 3 times smaller than the formed by $45 \mu \mathrm{m}$.

4. The microhardness of the interlayer is higher for the $200 \mathrm{~nm} \mathrm{Ni}$, with porosity in turn exhibiting higher strength.

\section{References}

1. Siores, E. \& Do Rego, D. Journal of Materials Processing Technology, 1995, 48, 619-625.

2. Clark, D. E. \& Sutton, W. H. Annual reviews Matterial Science, 1996, 26, 299331.

3. Thostenson, E. T. \& Chou, T. W. Composites Part A: Applied Science and Manufacturing , 1999, 30, 1055-1071.

4. Sheinberg, H., Meek, T. T. \& Blake, R. D. Patent Number: 4,942,278, 1990, 1-3.

5. Huey, H. E. \& Morrow, M. S. in Fourth World Congress on Microwave and Radio Frequency Applications (eds. Schulz, R. L. \& Folz, D. C.) , 2004, 286-293.

6. Walkiewicz, J. W., Kazonich, G. \& McGill, S. L. in Mineral Metallurgy Proceedings 5, 1988, 39-42.

7. Roy, R., Agrawal, D., Cheng, J. \& Gedevanlshvili, S. Nature , 1999, 399, 668670.

8. Srinath, M. S., Sharma, A. K. \& Kumar, P. Materials \& Design, 2011, 32, 26852694.

9. Srinath, M. S., Sharma, a. K. \& Kumar, P. Proceedings of the Institution of Mechanical Engineers, Part B: Journal of Engineering Manufacture, 2011, 225, 1083-1091. 
10. Srinath, M. S., Murthy, P. S., Sharma, A. K., Kumar, P. \& Kartikeyan, M. V. International Journal of Engineering, Science and Technology, 2012, 4, 152-158.

11. Badiger, R. I., Narendranath, S. \& Srinath, M. S. Journal of Manufacturing Processes , 2015, 18, 117-123.

12. Bansal, A., Sharma, A. K., Kumar, P. \& Das, S. Materials Characterization, 2014, 91, 34-41.

13. Bansal, A., Sharma, A. K., Kumar, P. \& Das, S. JOM , 2015, 67, 2087-2098.

14. Singh, S., Suri, N. M. \& Belokar, R. M. Materials Today: Proceedings , 2015, 2, 1340-1346.

15. Gupta, P., Kumar, S. \& Kumar, A. Materials and Manufacturing Processes , 2013, 28, 601-604.

16. Srinath, M. S., Sharma, A. K. \& Kumar, P. Journal of Manufacturing Processes , 2011, 13, 141-146.

17. Bansal, A., Sharma, A. K., Das, S. \& Kumar, P. Proceedings of the Institution of Mechanical Engineers, Part L: Journal of Materials Design and Applications, 2015, 1-10.

18. Munitz, A. Journal of Materials Science, 1995, 30, 2901-2910.

19. Tosto, S., Nenci, F., Jiandong, H., Corniani, G. \& Pierdominici, F. Material Science and Technology, 2003, 19, 519-522.

20. Magnabosco, I., Ferro, P., Bonollo, F. \& Arnberg, L. Materials Science and Engineering A , 2006, 424, 163-173.

21. Yilmaz, O. Journal of Materials Processing Technology, 2003, 141, 67-76.

22. Sabetghadam, H., Hanzaki, A. Z. \& Araee, A. Materials Characterization, 2010, 61, 626-634.

23. Durgutlu, A., Gülenç, B. \& Findik, F. Materials and Design, 2005, 26, 497-507.

24. Sahin, M. Industrial Lubrication and Tribology, 2012, 61, 319-324.

25. Yao, C. et al. Optics and Lasers in Engineering , 2009, 47, 807-814.

26. Tamang, S. \& Aravindan, S. Journal of Manufacturing Processes , 2017, 28, 94 100.

27. Mishra, R. R. \& Sharma, A. K. Critical Reviews in Solid State and Materials Sciences , 2016, 41, 217-255.

28. Baker, H. Alloy Phase Diagrams. ASM Handbook, 1998. 


\title{
MICROWAVE AND ULTRASOUNDS TOGETHER - A CHALLENGE
}

\author{
M. Vinatoru, I. Calinescu
}

University Politehnica of Bucharest, Faculty of Applied Chemistry and Materials Science, Bioresources and Polymer Science Department, 1-7 Gh. Polizu Str., 011061, Bucharest, Romania

Emails: mircea.vinatoru@upb.ro;ioan.calinescu@upb.ro;

Keywords: microwave, ultrasound, equipment, combined MW-US

\section{Introduction.}

If we are looking on each energy effects (MW, US) on a system, we could easily see that:

- Microwave effect on a homogenous system produce volumetric heating as well as quick heating;

- Microwave effect on a heterogenous system produce selective heating of phases.

- Ultrasonic effect on a homogenous system produce radical active species (influencing many chemical reactions, especially those implying electron transfer);

- Ultrasonic effect on a heterogenous system produce mass transfer intensification and surface effects (erosion, cleaning, catalyst activation, etc.).

Based on these facts it is obvious that a combination of these two forms of energy and their effects are quite exciting among chemists, and from here to making such a combination was just a step. The idea of combining MW with US was probably older than 1999 when the first paper on this topic was published [1], but the accessibility to shelf acquirable apparatus was not possible. Like in many other laboratory trials the first apparatus of this kind was inhouse build.

It was the paper of Lionelli and Mason [2], which after separately introducing theoretical and scientific facts for microwaves and sonochemistry, along with their applications as an individual sources of energy (totally different: microwave is an electromagnetic energy while acoustic energy is mostly a mechanical energy, however generated via electromagnetic waves), several pictures of combination between a microwave and an ultrasonic device described already in the literature were presented. In their paper is stated that two styles of combining microwave with ultrasound were used at that time:

- $\quad$ Separate reactors (MW and US) with a pump to allow the liquid to be transferred from one reactor to another and

- $\quad$ Single reactor with both US and MW inside.

Both type of reactors is nowadays available for laboratory lab scale.

Searching the literature using Scopus with as key words: microwave, ultrasound, combined and chemistry returns 192 documents result. Adding to the keywords "simultaneous" the number of documents drop to 86. Of course, the more general searching engines returns much more documents, but many of them irrelevant to the search topic. The main challenge in making a machine to be able to use combined and/or simultaneously both source of energy is related to the concept of inserting ultrasound into a microwave unit or vice versa.

In the first report on combining ultrasound with microwaves published in 1999 by Lagha et. al. [1], it was shown (see figure 1 [1]) how a magnetron insert microwaves in a rector having an ultrasonic cup-horn device mounted on the bottom of the wave guide, decalin being used as US coupling liquid. 


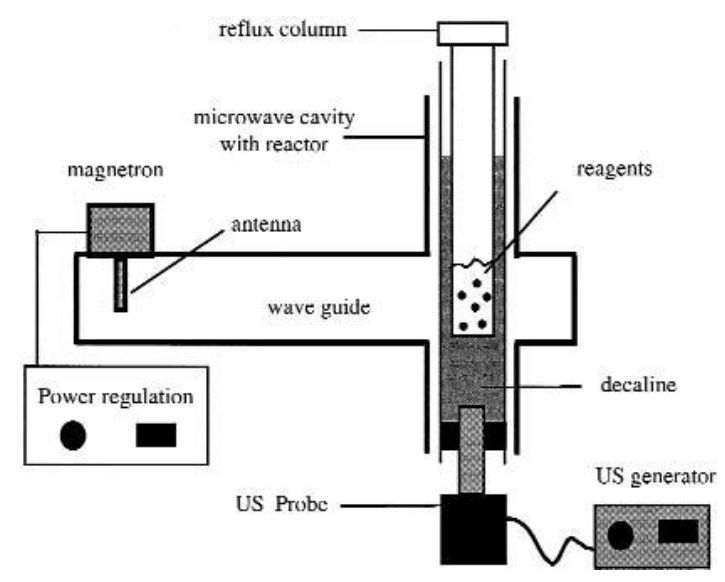

Figure 1. Schematic drawing of the single mode microwave system combined with ultrasound (after Lagha et. al. [1])

The combined microwave and ultrasound apparatus decreased the digestion and dissolution time of samples for analytical purposes. Similar device was employed for analytical sample preparation purpose (determination of copper in edible oil and total Kjeldahl nitrogen) via digestion assisted by microwave and ultrasound apparatus [3].

In a paper published in 2001 another arrangement of the two sources of energy in one machine is proposed by Peng et. al. figure 2 [4]:

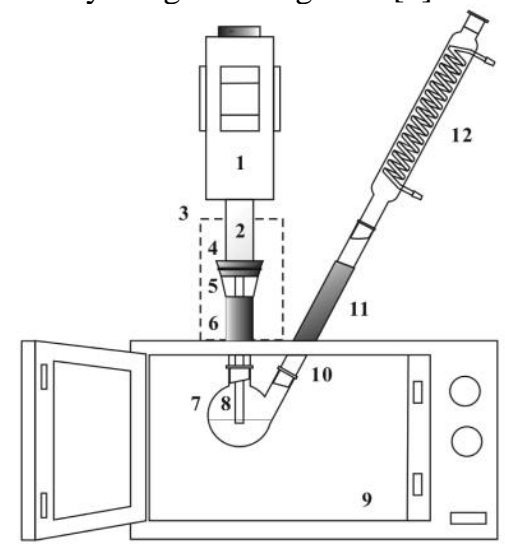

1. Transducer housing;

2. Upper horn;

3. Copper mesh screen;

4. Rubber stopper;

5. Adaptor;

6. Port;

7. Two-necked Pyrex flask;

8. Detachable horn;

9. Multimode cavity;

10. Adaptor;

11. Chock;

12. Condenser

Figure 2. Apparatus for microwave-ultrasound combined reactions (after Peng et. al. [4]).

In this approach a microwave oven (multimode cavity) was transformed to adapt to it a classic ultrasonic horn device. To prevent radiation leakage a copper mesh screen was settled around the ultrasonic horn. This device was successfully used to prepare hydrazides by hydrazinolysis of the corresponding esters with hydrazine monohydrate. The same device was used to synthesis of 3-aryl acrylic acids in good yields via Knoevenagel-Doebner reaction [5] in a solvent-free protocol.

Cravotto et. al. used US and MW in a different way [6, 7]. The ultrasonic probe reactor is placed outside of microwave oven and a tube connect the reaction mixture to both sources of energy (figure 3): 


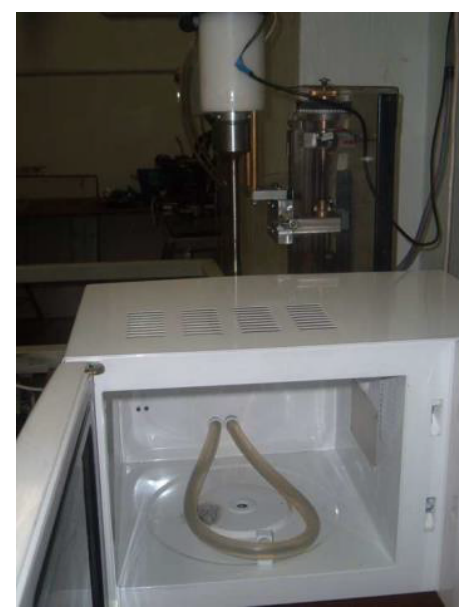

Figure 3. Reactor prototype featuring combined US/MW irradiation [6].

The such of combined devices was used to carry out ligand-free Pd/C-catalyzed Suzuki cross- and homocouplings reaction in aqueous media.

Cintas and Cravotto wrote in 2007 a paper about Ultrasound and Microwaves in Chemistry: A Relay Race or Tandem? The Combined Use of Microwaves and Ultrasound: Improved Tools in Process Chemistry and Organic Synthesis [8]. Among devices that combine microwave with ultrasound, perhaps the simplest is the one in which ultrasonic irradiation is inside of a single vessel provided by a quartz (or glass) made horn which can be inserted into microwave oven (figure 4 [8]):

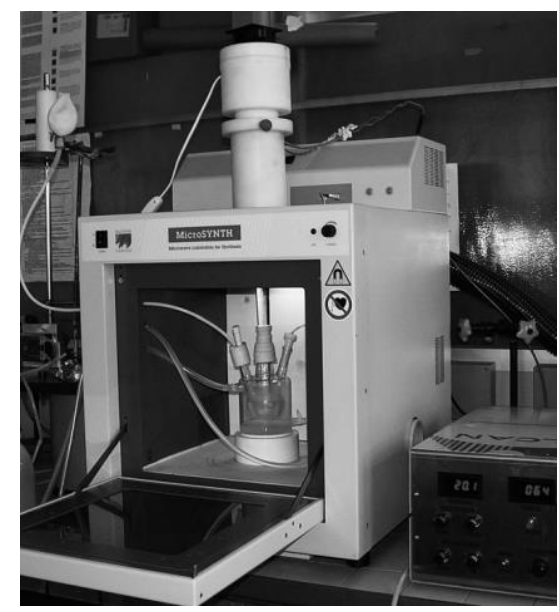

Figure 4. Simultaneous irradiation in a multimode MW oven equipped with a Pyrex horn [8]. 
Although quartz or glass is fragile and the ultrasonic power entering into a reaction mixture cannot be too high, it is probably the best combination for simultaneous uses of both energies. Though there are not too many papers published so far in this field, MW coupled with US in one machine (or a sequential one) the attention for having two energies interacting differently with materials, is raising.

Cravotto, prosed in the same time period (2007) another type of combined MW-US reactor [9], a "flow" reactor for the treatment of polluted water. The word "flow" was not adequately used for this reactor, actually it is a batch type reactor, with a limited tank volume holding the initial solution to treat. It is having not inlet/outlet points for solution to make it a real "flow" reactor, but clearly makes possible treatment using both energies features.

Using a cup-horn or probe ultrasonic device, with different frequencies (19, 25, 40 and 300 $\mathrm{kHz}$ ), Cravotto et. al. successfully extracted soybean germs or algae oil in a simultaneous US/MW (multimode oven) developed apparatus. The authors concluded that techniques using US or combined US/MW irradiation should be well suited for other processes, such as two step one: extraction and transesterification for biofuel purpose [10].

In an apparatus similar with those described in figure 1, Shen [11] proposed a rapid synthesis of $\mathrm{Pb}(\mathrm{OH}) \mathrm{Br}$ nanowires. Heating alone provide $0.02-0.03 \mathrm{~mm}$ diameter and $2-3 \mathrm{~mm}$ length nanowires, while MW $(50 \mathrm{~W})$ the diameter is ten times smaller and rather short nanowires. US alone further reduce the diameter as well as the length of the nanowires. Combining MW and US $(50 \mathrm{~W}-50 \mathrm{~W})$ the diameter drops to $80-800 \mathrm{~nm}$, and the length to $50-100 \mu \mathrm{m}$. If the MW power is higher $-250 \mathrm{~W}$, (higher heating contribution) and US power maintained the same, the diameter narrowed $(100-500 \mathrm{~nm})$ while the length became rather short Table 1 in reference [11]).

After 2010 a number of papers were published having as topic either two steps processes, like metal organic framework IRMOF-1 synthesis [12], (in a separate reactors as stated by Lionelli and Mason), synthesis of polysubstituted pyridines under combined microwave and ultrasound irradiation [13], porous manganese dioxide $\left(\mathrm{MnO}_{2}\right)$ synthesized via an ultrasound-microwave-intensified precipitation [14] (perhaps in a single reactor - the papers do not offers details of equipment), or even for biodiesel preparation emphasizing the synergetic effects of combining both source of energy in one device [15]. Several papers shown how to build or review the simultaneous ultrasound and microwave new reactor [1619], others how to use this combination for adding value to some byproducts (like glycerol from biodiesel synthesis) [20]. Clearly there are efforts in developing such a combined device in which MW and US could be used together in a controllable mode.

Our group is having at least one of the equipment capable to work in single reactor with both US and MW [2], but different from the apparatus presented in the paper. This device is produced by SAIREM and is described in a paper published few years ago by Sacco et. al., [17] as a hybrid technology applicable for a specific process (ring closing metathesis) and is in use in our laboratory from a couple of years. This device is having as ultrasonic source a modified ultrasonic cleaning bath to be attachable to the MW unit (see figure 5). 


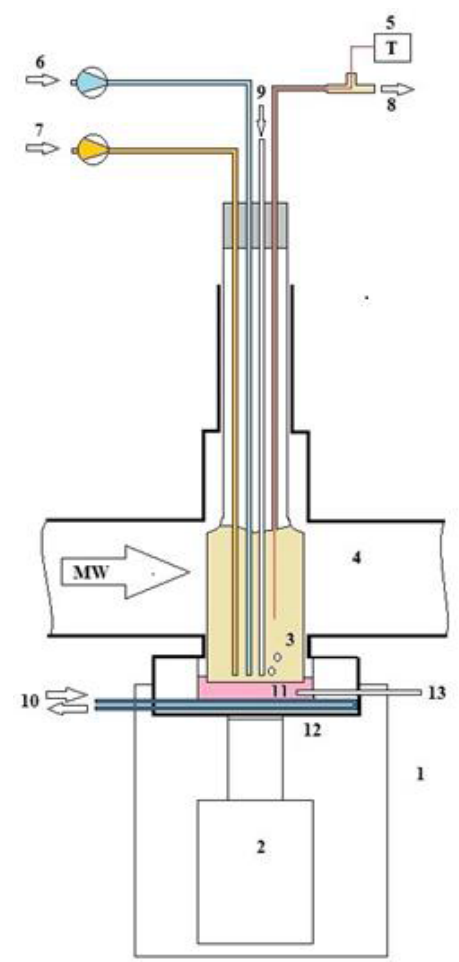

1. Ultrasonic bath

2. Transducer

3. Reactor

4. Waveguide

5. Temperature control

6. Methanol inlet

7. Oil inlet

8. Reaction products outlet

9. Gas flow

10. Cooling agent

11. Paraffin oil

12. Cooling coil

13. Thermometer

Figure 5. Miniflow US + MW simultaneous microwaves-ultrasound irradiation.

This setup was successfully employed for biodiesel synthesis using simultaneous MW-US irradiation. The conversion of sunflower oil to methyl fatty acids esters (FAME) being over 97\% (EU norm for an accepted for a transesterification process [21] imposes at least $96.5 \%$ concentration of FAME at the end of process).

The only problem that we encountered using this device in some of laboratory experiments was that the US power control is lacking and therefore the device is working on full US power and adjustable MW power. Fortunatley the development in the ultrasonic devices construction allow a new type of ultrasonic device (clamp-on [22]) to be attachable to a flow through pipe, like in the figure 6 below (device existent in our laboratory), and therefore building a hybrid machine able to work with both powers on full control. 


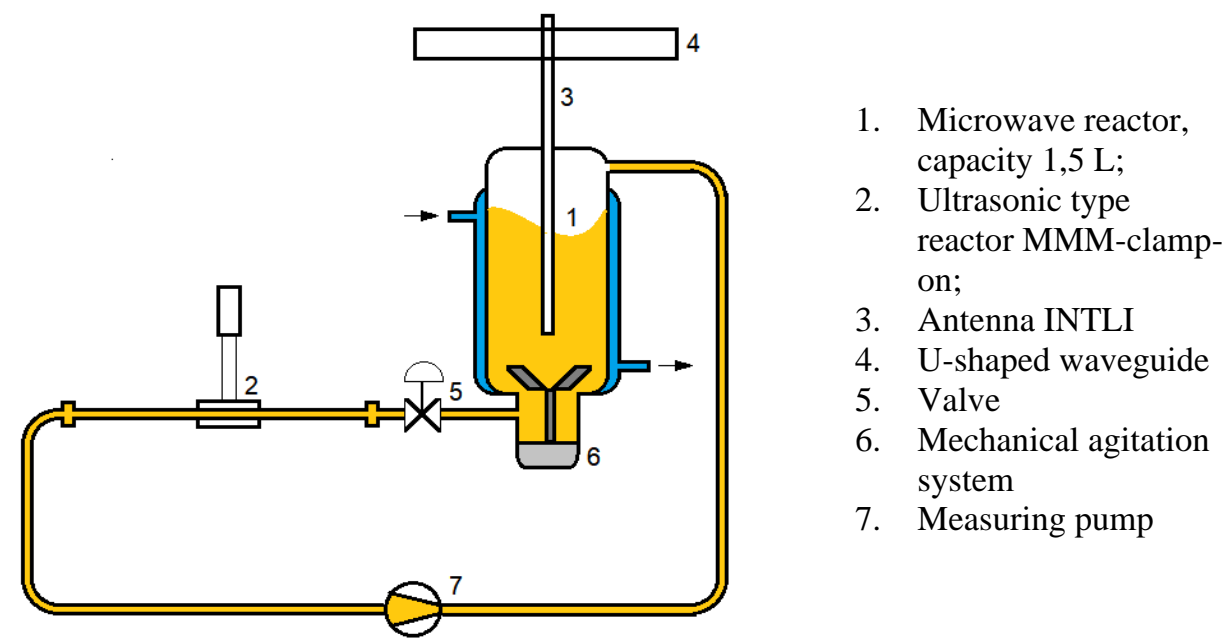

Figure 6. MW and US in a close loop configuration

\section{Conclusions}

The simultaneous or combined uses of microwaves and ultrasound is a solution (perhaps a very good one) for physical and/or chemical processes intensification [23, 24]. The microwaves ensure a speeding of the reaction rate via selective or volumetric heating (depending of the reaction mixture types: homogenous or heterogenous), while ultrasound enhance the mass transfer between phases, ensures better mixing and allow a better distribution of temperature in homogenous media. Combining these two sources of energy it may be possible to assist to the naissance of a synergetic hybrid technology [2], fully controllable and usable in the laboratories, pilot and why not industrial scales.

The difficulties we face now are challenging and hopefully at least at laboratory scale will be overcome. The first challenge is that microwaves heat up the reaction mixture, while ultrasound is known by its paradoxical effects - lower the temperature better effects. What kind of reactions/processes will benefit from this hybrid technology? The time and reactions/processes selections and experiments will show which one is privileged by this. We may advance here the idea that heterogenous reactions/processes could be the ideal candidate for such of technology.

There is obvious that we need to overcome also the lack of coherent mathematical models to be used for simulation MW and US features in one application.

Scaling up of both techniques is very difficult and this difficulty increases when we will put together MW and US. However, using the new concept of numbering up instead of scaling up [25] we are confident that soon we will step towards a new exciting hybrid technology, applicable to any scale.

\section{Aknowledgements}

The authors acknowledge the financial support received from the Competitiveness Operational Programme 2014-2020, Action 1.1.4: Attracting high-level personnel from abroad in order to enhance the RD capacity, project: P_37_471, „Ultrasonic/Microwave Nonconventional Techniques as new tools for nonchemical and chemical processes", financed by contract: 47/05.09.2016 


\section{References}

[1]. A. Lagha, S. Chemat, P. V. Bartels and F. Chemat, Analusis, 27(5) (1999) 452457 https://doi.org/10.1051/analusis:1999124.

[2]. C. Leonelli and T. J. Mason, Chemical Engineering and Processing: Process Intensification, 49(9) (2010) 885-900 https://doi.org/10.1016/j.cep.2010.05.006

[3]. S. Chemat, A. Lagha, H. Ait Amar and F. Chemat, Ultrasonics Sonochemistry, 11(1) (2004) 5-8 https://doi.org/10.1016/S1350-4177(03)00128-7.

[4]. Y. Peng and G. Song, Green Chemistry, 3(6) (2001) 302-304 https://doi.org/10.1039/b108878p.

[5]. Y. Peng and G. Song, Green Chemistry, 5(6) (2003) 704-706 https://doi.org/10.1039/b310388a.

[6]. G. Cravotto, M. Beggiato, A. Penoni, G. Palmisano, S. Tollari, J. M. Lévêque and W. Bonrath, Tetrahedron Letters, 46(13) (2005) 2267-2271 https://doi.org/10.1016/i.tetlet.2005.02.015.

[7]. G. Cravotto and P. Cintas, Chemical Society Reviews, 35(2) (2006) 180-196.

[8]. G. Cravotto and P. Cintas, Chemistry - A European Journal, 13(7) (2007) 19021909 https://doi.org/10.1002/chem.200601845.

[9]. G. Cravotto, S. Di Carlo, M. Curini, V. Tumiatti and C. Roggero, Journal of Chemical Technology and Biotechnology, 82(2) (2007) 205-208 https://doi.org/10.1002/jctb.1658.

[10]. G. Cravotto, L. Boffa, S. Mantegna, P. Perego, M. Avogadro and P. Cintas, Ultrasonics Sonochemistry, 15(5) (2008) 898-902 https://doi.org/10.1016/j.ultsonch.2007.10.009.

[11]. X. F. Shen, Journal of Chemical Technology and Biotechnology, 84(12) (2009) 1811-1817 https://doi.org/10.1002/jctb.2250.

[12]. R. Sabouni, H. Kazemian and S. Rohani, Chemical Engineering Journal, 165(3) (2010) 966-973 https://doi.org/10.1016/j.cej.2010.09.036.

[13]. H. Feng, Y. Li, E. V. Van Der Eycken, Y. Peng and G. Song, Tetrahedron Letters, 53(9) (2012) 1160-1162 https://doi.org/10.1016/j.tetlet.2011.12.103.

[14]. P. Wang, Y.-J. Zhao, L.-X. Wen, J.-F. Chen and Z.-G. Lei, Industrial \& Engineering Chemistry Research, 53(52) (2014) 20116-20123 dx.doi.org/10.1021/ie5025485.

[15]. E. Martinez-Guerra and V. G. Gude, Fuel, 137 (2014) 100-108 https://doi.org/10.1016/j.fuel.2014.07.087.

[16]. V. Ragaini, C. Pirola, S. Borrelli, C. Ferrari and I. Longo, Ultrasonics Sonochemistry, 19(4) (2012) 872-876 https://doi.org/10.1016/j.ultsonch.2011.12.008.

[17]. M. Sacco, C. Charnay, F. De Angelis, M. Radoiu, F. Lamaty, J. Martinez and E. Colacino, RSC Advances, 5(22) (2015) 16878-16885 https://doi.org/10.1039/c4ra14938f.

[18]. P. Cintas, S. Tagliapietra, M. Caporaso, S. Tabasso and G. Cravotto, Ultrasonics Sonochemistry, 25(1) (2015) 8-16 https://doi.org/10.1016/j.ultsonch.2014.12.004.

[19]. K. Martina, S. Tagliapietra, A. Barge and G. Cravotto, Combined Microwaves/Ultrasound, a Hybrid Technology, in Topics in Current Chemistry. 2016.

[20]. P. Cintas, S. Tagliapietra, E. Calcio Gaudino, G. Palmisano and G. Cravotto, Green Chemistry, 16(3) (2014) 1056-1065 https://doi.org/10.1039/c3gc41955j. 
[21]. Liquid petroleum products - Fatty acid methyl esters (FAME) for use in diesel engines and heating applications - Requirements and test methods in DIN EN 14214, E. Standards, Editor. 2014.

[22]. Ultrasonics.com Available from: https://www.mpiultrasonics.com/content/clamp-ultrasonic-systems.

[23]. A. Stankiewicz and J. A. Moulijn, Industrial \& Engineering Chemistry Research, 41(8) (2002) 1920-1924 https://doi.org/10.1021/ie011025p.

[24]. A. I. Stankiewicz and J. A. Moulijn, Chemical engineering progress, 96(1) (2000) 22-34.

[25]. D. Fernandez Rivas and S. Kuhn, Synergy of Microfluidics and Ultrasound: Process Intensification Challenges and Opportunities, in Topics in Current Chemistry. 2016. 


\section{ANALYSIS OF HIGH-FREQUENCY C-CORE MAGNETIC FLUX LEAKAGES FOR BONE TUMOR WITH INDUCTION HEATING BY USING MULTI-COIL}

Metharak Jokpudsa, Supawat Kotchapradit, Chanchai Thongsopa, Thanaset Thosdeekoraphat

School of Electronic Engineering, Suranaree University of Technology, Nakhonratchasima 30000, Thailand

m6040062@g.sut.ac.th

Keywords: high-frequency magnetic core, multi-coil induction heating, bone tumor

High-frequency magnetic field has been developed pervasively. The induction of heat from the magnetic field can help to treat tumor tissue to a certain extent. Normally, treatment by the low-frequency magnetic field needed to be combined with magnetic substances. To assist in the induction of magnetic fields and reduce flux leakage [1]. However, there are studies that have found that high frequencies can cause heat to tumor tissue [2]. In this paper present, a new magnetic application will focus on the analysis of the high-frequency magnetic nickel core with multi-coil. In order to focus the heat energy using a high-frequency magnetic field into the tumor tissue. The magnetic coil was excited by $915 \mathrm{MHz}$ signal and the combination of tissues used are muscle, bone, and tumor as shown in table 1 . The magnetic power on the heating predicted by the analytical model in Fig. 1 , the power loss density $\left(2.98 \mathrm{e}^{-6} \mathrm{w} / \mathrm{m}^{3}\right)$ was analyzed using the CST microwave studio.

Table 1. Material properties of tissue in simulation.

\begin{tabular}{cccc}
\hline Tissue & Density $\left(\mathrm{kg} / \mathrm{m}^{3}\right)$ & $\begin{array}{c}\text { Conductivity }(\sigma, \\
\mathrm{S} / \mathrm{m})\end{array}$ & $\begin{array}{c}\text { Heat capacity } \\
(\mathrm{kJ} / \mathrm{K} / \mathrm{kg})\end{array}$ \\
\hline Muscle & 1050 & 0.37 & 3.54 \\
Bone & 1040 & 0.13 & 1.3 \\
Tumor & 1050 & 2.7 & 3.82 \\
\hline
\end{tabular}
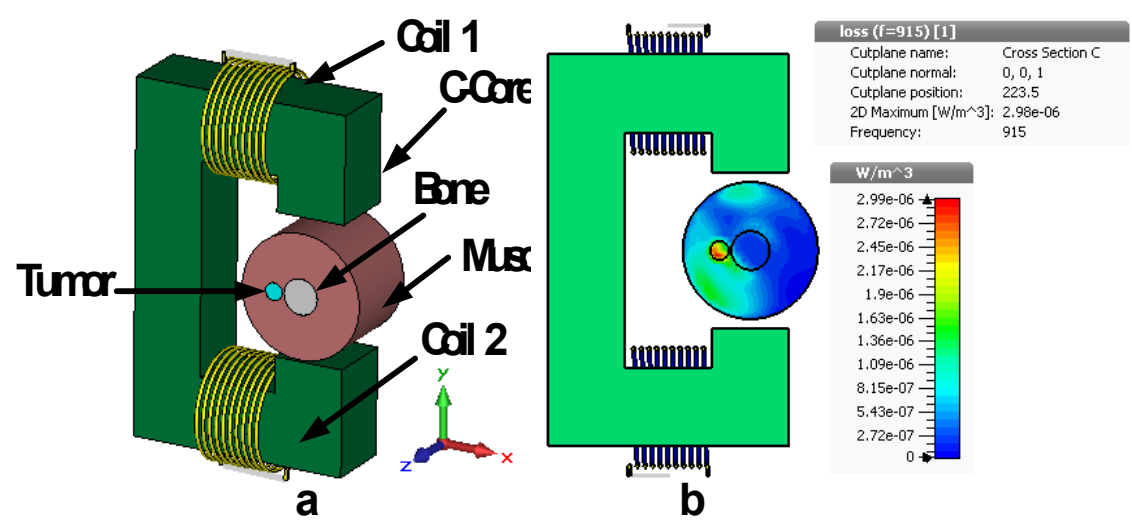

Fig. 1. a) Magnetic induction with multi-coil design and b) power loss density in tissue. 


\section{Introduction}

Cancer is the leading cause of death in the world. Cancer is a genetic disease that can be inherited from parents to children. Cancer tissue is caused by some changes in genes. Cancer cells are divided rapidly and cannot be controlled. Causing tumors in the body. The tumor is divided into 2 types, Is benign tumors and malignant tumors. Benign tumors will have a mild disease that does not spread to other organs. Malignant tumors will destroy the cells of the organ. And spread to neighboring organs and can also spread through the bloodstream or the lymphatic system to other organs throughout the body such as lungs, liver or bones $[3,4]$. Bone cancer can occur with bones in all parts of the body. Most of it occurs in the bones that are long, such as the bones, arms, and legs. Bone cancer makes the bones that are close to the cancer cells very strong. Causing the bones to break easily Bone cancer can be divided into 2 types, Primary bone cancer and Secondary bone cancer the primary bone cancer begins to cause abnormalities in the cells that are attached to the bone. And secondary bone cancer will cause abnormalities of cells from other places Is already another type of cancer and the spread of cancerous tissue to the bone [5].

Due to the above treatment has many side effects and use the time to rest the floor for a long time the authors, therefore, propose methods of treatment with hyperthermia. Is a method of heating within cancer cells used for primary bone cancer in the early stages without damage to normal tissues. Hyperthermia therapy uses the principle of highfrequency transmission. Induce the target tissue to increase the temperature up to $42-43{ }^{\circ} \mathrm{C}$ [6]. For a period of not less than 40 minutes, the cancerous tumor will be destroyed. Causing cancer cells to atrophy cannot grow and spread to other parts of the body within the primary cancer cells there is a large concentration of blood vessels. Causing poor blood circulation like normal body cells which will make the cancer cells sensitive to temperature changes and retain heat the form of cancer treatment by heat therapy has many characteristics, such as radio frequency, microwave, or ultrasonic waves. The main components of the system of heat treatment include Generator, Computer, Applicator, and feedback temperature to process on the computer in order to be able to control the temperature within the cancerous tumor [7].

Treatment of malignant tumor cells by heating with magnetic fields. In most cases, magnetic nanoparticles are added into the target tissue as well to increase the induction of heat. Can use hyperthermia treatment in combination with other forms of treatment for better performance $[8,9]$. In the past year, people have designed the magnets for magnetic hyperthermia applications in large models. In the form of a c-core magnetic core using a frequency of $200 \mathrm{kHz}$ [10]. The selection of frequencies will affect the heating of the tissues for the hyperthermia system at a very high frequency. It is necessary to have magnetic nanoparticles. Helps to cause heat Because the normal tissue of the body has the ability to absorb magnetic fields is not good. But if using high frequency, the heat generated from eddy current can be achieved and can generate enough heat to destroy cancerous tumor cells without having to give magnetic nanoparticles enter the body before hyperthermia heat.

\section{Materials and Methods}

For winding the coil on the ferrite core in a circular motion when a current is supplied to the coil, where the core made from the ferromagnetic material causes the intensity of the magnetic field Increased from zero. Which the density of this magnetic force line will increase until the density of the magnetic force line is constant which are saturated. 
Calculations for magnetic circuits have a calculation principle similar to the calculation of electrical circuit from Ampere's Law. If there is no effect of the leakage line outside the ferrite core. And the magnetic force line is constant in the ferrite core along the length of the ferrite core, resulting in the density of the magnetic force line constant. And when the ferrite core has air gap in Fig. 2.
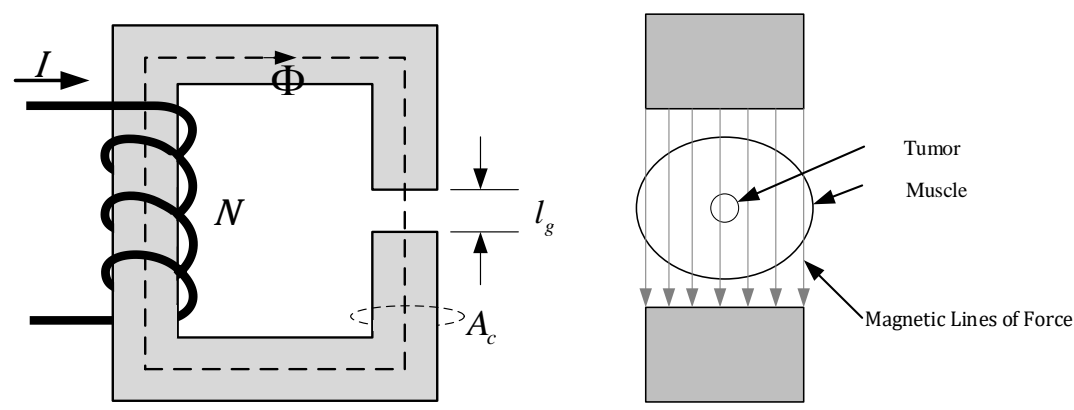

Fig. 3. a) The Schematic of c-core magnetic induction heating system.

b) Modeling the muscles between the air gap.

will receive the magnetic force equation within the magnetic core and in the air gap as

$$
\Phi=\frac{N I\left(\mu_{0} A_{c}\right)}{l_{g}}
$$

where $\Phi$ is magnetic lines of force (Wb), $N$ is number of turns (Turns), $I$ is current (A), $\mu_{0}$ is permeability of free space $(\mathrm{H} / \mathrm{m}), A_{c}$ is cross-sectional area of ferrite core $\left(\mathrm{m}^{2}\right)$, and $l_{g}$ is the length of air gap. Therefore, the density of the magnetic force line moving through this air gap can be obtained from equation 2, but the area where the magnetic force line moves through this air area is uncertain. Which will vary according to the length of the air and will change according to the pattern of the cross-sectional area of the magnetic core as well

$$
H=\frac{\Phi}{\mu_{0} A_{g}}
$$

where $H$ is magnetic flux density $\left(\mathrm{Wb} / \mathrm{m}^{2}\right)$. Induction heating is heated by the magnetic force that moves through the ferrite core. The basic properties of human tissue are the infiltration of the magnet near the vacuum. Causes the magnetic field to have a slight effect on the heat generated by human tissue. A common method for creating magnetic stimulation models from Ampere's Circuital Law with the Coulomb gauge [11]. Will receive the magnetic field produced by the coil current and modified using the equation of Laplace vector

$$
-\nabla^{2} A=\mu_{0} \times J
$$

where $J$ is the current density vector that flows within the source coil and $A$ is the vector magnetic potential $\left(H=\frac{1}{\mu_{0}} \nabla \times A\right)$. High frequency alternating magnetic field produces heat 
inside the body. When the magnetic is present, eddy currents conductive power loses. Which is created from the current density vector shown as [12]

$$
P=\frac{J^{2}}{\sigma}
$$

where $P$ is power density $\left(\mathrm{W} / \mathrm{m}^{3}\right)$, and $\sigma$ is conductivity of tissue $(\mathrm{S} / \mathrm{m})$.

In human body has a circulatory system, there are a lot of blood vessels in many parts of the body. The circulatory system will maintain the body's temperature and $\mathrm{pH}$. The circulatory system will have blood as a heat carrier. In which the blood is liquid consisting of blood, red blood cells, white blood cells, and platelets. Which the heart functions through the tube system when there is a part of the body that has abnormal temperature from other parts the circulatory system will adjust the body temperature. Where the heart will pump blood throughout the body consisting of various parts to transfer heat through the blood to various parts of the body and make the body temperature balance. The Penne's heat equation is the basis of the heat diffusion equation. Which is used very well for heat transfer of various biological tissues [13-14]. The Penne's equation combines the main factors that affect temperature change only. Which there are some factors that are not used in the calculation will get the equation of heat dissipation as equation [15]

$$
\rho C \frac{\partial T}{\partial t}=\nabla(k \nabla T)+\rho_{B} C_{B} \omega_{B}\left(T-T_{B}\right)+A+P
$$

where $\rho$ is tissue density $\left(\mathrm{kg} / \mathrm{m}^{3}\right), C$ is specific heat of tissue $\left(\mathrm{J} / \mathrm{kg}^{\circ} \mathrm{C}\right), T$ is temperature of tissue $\left({ }^{\circ} \mathrm{C}\right), t$ is time $(\mathrm{s}), k$ is heat conduction of tissue $\left(\mathrm{W} / \mathrm{m}^{\circ} \mathrm{C}\right), \rho_{B}$ is blood density $\left(\mathrm{kg} / \mathrm{m}^{3}\right), C_{B}$ is specific heat blood $\left(\mathrm{J} / \mathrm{kg}^{\circ} \mathrm{C}\right), \omega_{B}$ is blood distribution rate $(\mathrm{Hz}), T_{B}$ is temperature of blood $\left({ }^{\circ} \mathrm{C}\right)$, and $A$ is heat from the energy metabolism of the body $\left(\mathrm{W} / \mathrm{m}^{3}\right)$. For this experiment, it is not thought of as the heat from the energy metabolism of the body fields because of the small amount compared to the results from other parts.

The design of the ferrite core in the form of a c-core with air space the c-core ferrite core is a loop. There is a section that allows the magnetic force line to move through the airless. Causing the loss of the magnetic force line as well and the air gap is the direct release of the magnetic field to the simulation tissue causing the c-core ferrite core to have a very small loss. The purpose of the use is a set of magnetic fields created for the human body in the organ that found bone cancer by focusing on the bones, arms and leg bones, so the size of the ferrite core that acts as a magnetic resonator must be the size that is appropriate for that particular organ. Therefore, designed the size of the ferrite core to be the form of c-core, the size that can control the part of the human arm and has the shape of the model and size shown as Fig. 4. 


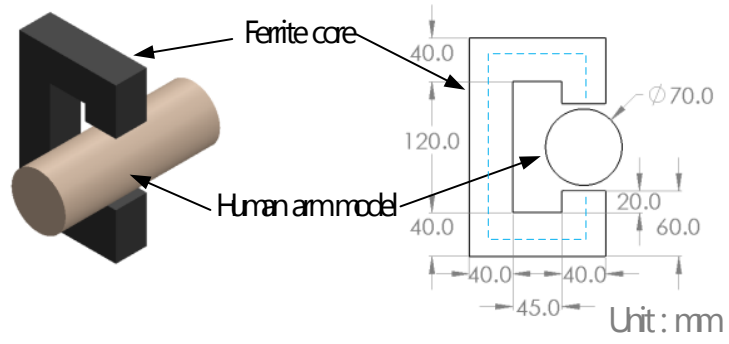

Fig. 4. The analytical model of c-core magnetic induction heating system.

\section{Results}

In this section, we investigate the magnetic flux density, which can be controlled by ccore magnetic induction heating system. In order to resolve the problem of the heating region and local heating can be controlled by varying the air gap size of the c-core magnetic induction system. Moreover, the investigation showed that the position of the heating region can be relocated by changing the position of the tumor tissue with c-core magnetic induction system in the $\mathrm{x}$-axis direction. The construction of $\mathrm{c}$-core magnetic induction heating system to verify the field distribution on the heating model, full wave 3-D numerical simulation was performed using the finite difference time domain method.

From these theoretical investigations, one effective method to control a heating region in the bone was found. Hence, the temperature in the heating body can be controlled by the size of the air gap size of the c-core magnetic. Electric loss density for the heating model was evaluated. The ferrite core is excited by $915 \mathrm{MHz}$ signal. The air gap sizes in the simulation are $5 \mathrm{~cm}$. Electric loss density images for heating region shown in Figure 5.

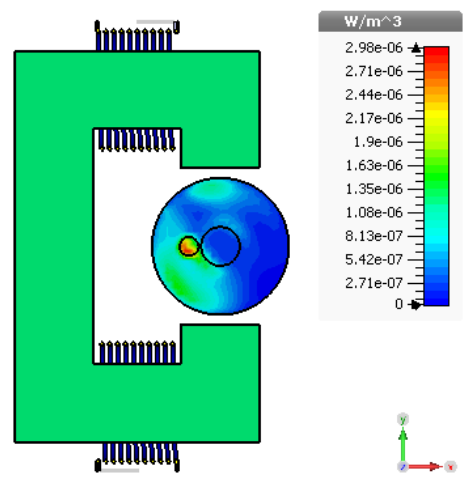

Fig. 5. power loss density in tissue in left-position. 
Fig. 5 the simulation results show that the heating efficiency is related to the c-core magnetic. It can be seen that the maximum heat occurs between c-core magnetic in the position of tumor tissue. Furthermore, the result of c-core magnetic also has a role in reducing heat distribution in neighboring tissues of breast phantom but midpoint heating are increasing. As mentioned, if the diameter of the tumor tissue is changed as left, right and center. The distributions of the power absorption per volume are changed as shown in Fig. 6
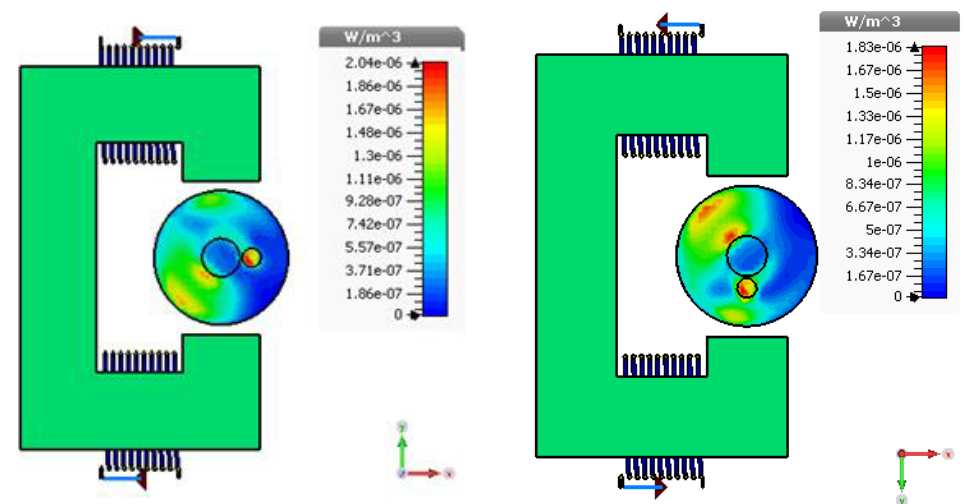

Fig. 6. a) power loss density in tissue in right-position, b) power loss density in tissue in center-position.

According, Fig. 5, and 6 the heating results show that the efficiency is related to the tumor tissue position, which the maximum heating area was inside the bone.

\section{Conclusion}

In this paper, the induction heating for hyperthermia with high-frequency c-core magnetic system was conducted to determine the heat distribution in the dielectric loads or tumor tissue. For analyzing the process, the c-core magnetic was excited by frequency 915 $\mathrm{MHz}$, Then the c-core magnetic is considered by an air gap to investigate the difference of power loss density in the dielectric material. The simulation found that power loss density can be increased while the heating area is narrowed. Nevertheless, the power loss density changes as a function of tumor tissue diameter also. This research is believed to be effectively applied to control the size of the heating area for hyperthermia cancer treatment.

\section{Acknowledgment}

This work was supported by Suranaree University of Technology (SUT) and by the Office of the Higher Education under NRU project of Thailand 


\section{References}

[1] S. L. Ho, S. Niu and W. N. Fu, Design and Analysis of Novel Focused Hyperthermia Devices.IEEE Transactions on Magnetics, 2012, 48, no. 11, 3254-3257.

[2] A. Ishikawa and Y. Nikawa, New microwave heating methodology with noninvasive temperature measurement using Magnetic Resonance equipment. The 40th European Microwave Conference, 2010, 40, 1441-1444.

[3] Ottewell, Penelope D., The role of osteoblasts in bone metastasis. Journal of Bone Oncology, 2016, 5, no. 3, 124-127.

[4] Weilbaecher, K.N., Guise, T.A., McCauley, L.K., Cancer to bone: A fatal attraction. Nature Reviews Cancer, 2011, 11, no. 6, 411-425.

[5] Weinberg, Robert A., How cancer arises. Scientific American, 1996, 275, no. 3, 62-70.

[6] Luk, K.H., Hulse, R.M., Phillips, T.L., Hyperthermia in cancer therapy. Western Journal of Medicine, 1980, 132, no. 3, 179-185.

[7] R. B. Riadh W. Y. Habash, Thermal Therapy Part 2: Biomedical Engineering, 2016, 34, no. 6, 491-542.

[8] Shah, B.P., Pasquale, N., De, G., Tan, T., Ma, J., Lee, K.-B., Core-shell nanoparticlebased peptide therapeutics and combined hyperthermia for enhanced cancer cell apoptosis. ACS Nano, 2014, 8, no. 9, 9379-9387.

[9] Tonthat, L., Yamamoto, Y., Aki, F., Saito, H., Mitobe, K., Thermosensitive Ferromagnetic Implant for Hyperthermia Using a Mixture of Magnetic MicroNanoparticles. IEEE Transactions on Magnetics, 2018, 54, no. 7.

[10] Nomura, S., Isobe, T., Design Study on High-Frequency Magnets for Magnetic Hyperthermia Applications. IEEE Transactions on Applied Superconductivity, 2018, 28, no. 3.

[11] W.Wang and S. R. Eisenberg, Athree-dimensional finite elementmethod for computing magnetically induced currents in tissues. IEEE Trans. Magn., 1994, 30, no. 6, 50155023.

[12] A. Miaskowski and B. Sawicki, Magnetic Fluid Hyperthermia Modeling Based on Phantom Measurements and Realistic Breast Model. IEEE transactions on bio-medical engineering, 2013, 60, no. 7, 1806-1813.

[13] J. Lienhard, A Heat Transfer Textbook, 2005.

[14] E. H. Wissler, Pennes' 1948 paper revisited. Journal of Applied Physiology, 1998, 85, no. $1,35-41$.

[15] D. Yang, M. C. Converse, D. M. Mahvi and J. G. Webster, Expanding the Bioheat Equation to Include Tissue Internal Water Evaporation During Heating. IEEE Transactions on Biomedical Engineering, 2007, 54, no. 8, 1382-1388. 


\section{SUBCRITICAL HYDROTHERMAL CONVERSION (SHC) PROCESS SUPPORTED BY MICROWAVES}

Ryszard Parosa

PROMIS-TECH, Poland

\section{Introduction}

The process described in this article uses the change of the property of water in the socalled critical state in very high pressure - about 22,131,360 Pa (over $200 \mathrm{~atm}$ ). In the critical state, water becomes a very effective solvent, causing the biomass to break down into simple hydrocarbons, including mainly bio-diesels (liquid hydrocarbons), gases (methane, ethane, etc.), and the so-called bio-char. The process is illustrated in fig. 1.

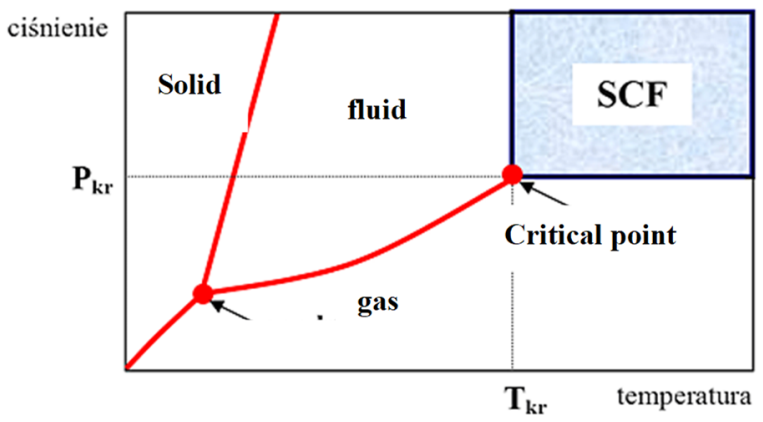

Fig. 1. Graph

Until now, the process in question has been used in a system with conventional heating of the mass being utilised through heating the walls of the high-pressure chamber. It requires the heating of thick metal walls and the material inside needed a long heating time as a result of thermal conductivity.

The essence of the process in question is to use microwave energy for controlled maintenance (stabilisation) of temperature in the process chamber in which the biomass is fed under very high pressure. In critical conditions, water ceases to be a polar liquid and dielectric parameters of water change rapidly (dielectric permeability and the so-called loss angle tangent). Thanks to that property, the introduction of microwaves (in the frequency band from $900 \mathrm{MHz}$ to $2.5 \mathrm{GHz}$ ) into the process chamber makes it possible to precisely maintain critical conditions through the control of the reflected wave in the microwave line. The essence of the process is presented in the flow chart (below). 




Fig. 2.

The biomass (sludge) is pressed into the heat exchanger, where it is initially heated and then fed to the high-pressure process chamber, where the biomass is heated up to required high temperature of about $260-300^{\circ} \mathrm{C}$. After hydrothermal conversion of the biomass in the process chamber, the products of conversion are pressed through the heat exchanger, in which they are cooled down, and the recovered thermal energy is used for the heating of another portion of biomass to be utilised. Cooled down products of conversion of the biomass are then separated into gaseous fractions (biogas), liquid fractions (bio-diesel), and solid fractions (biochar).

The use of microwave energy is aimed at enhancing (accelerating) the process of heating of biomass, ensuring uniform heating throughout the volume (and not only along the walls, as is the case in the conventional method).

The implementation process is divided into three stages:

- tests in a laboratory scale for the determination of optimal process conditions;

- studies and tests in a semi-technical installation;

- construction of a prototype industrial installation.

Tests in a laboratory scale with the use of a metal chamber fitted with a connection allowing microwaves to be introduced inside the chamber.

The laboratory installation was supplied from the microwave generator with frequency of $2.45 \mathrm{GHz}$ and continuous regulated power in the range from $300 \mathrm{~W}$ to $3 \mathrm{~kW}$. In the microwave supply line, a reflectance mater was installed for the measurement of reflected power, together with a ferrite circulator (cf. figure 3). 


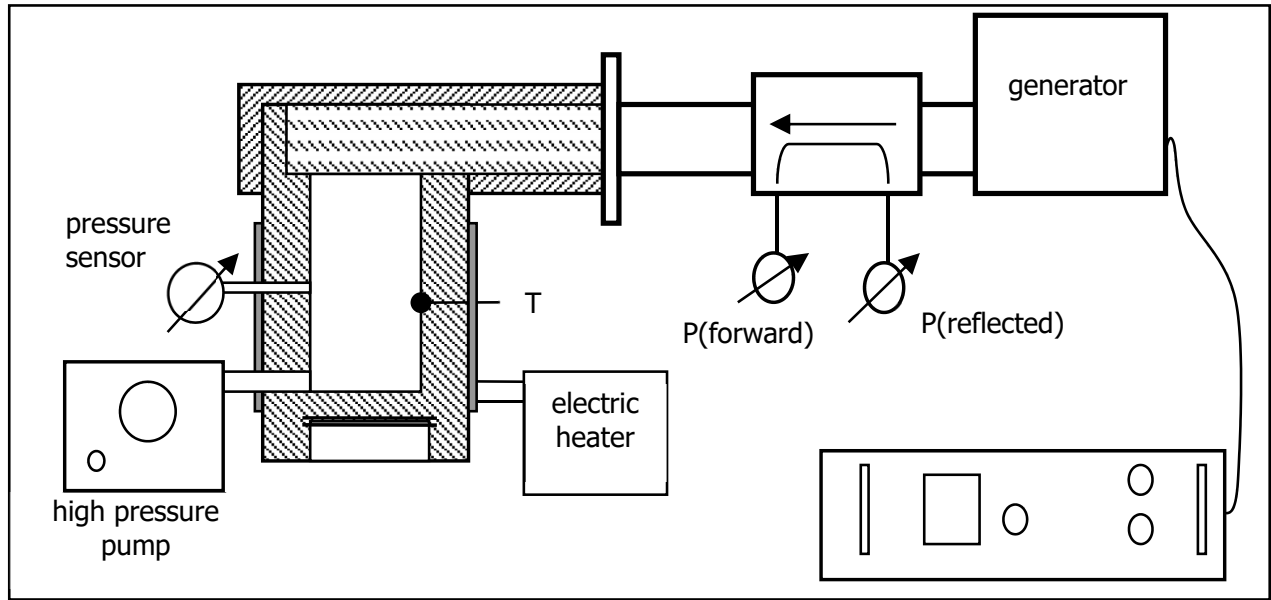

Fig. 3.

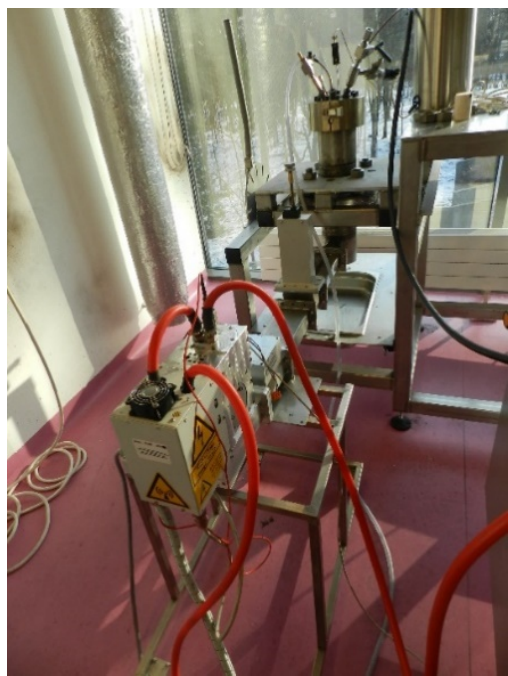

Fig. 4.

In the photograph, a laboratory installation is shown. The process was conducted with stabilised sludge with initial moisture of 50\%-70\%.

A series of tests allowed the determination of optimal conditions of the process, i.e. pressure, temperature in the process chamber, and microwave heating time — in order to obtain as large volume of liquid as possible with composition including flammable hydrocarbon substances. Examples of results are presented in table 1. 


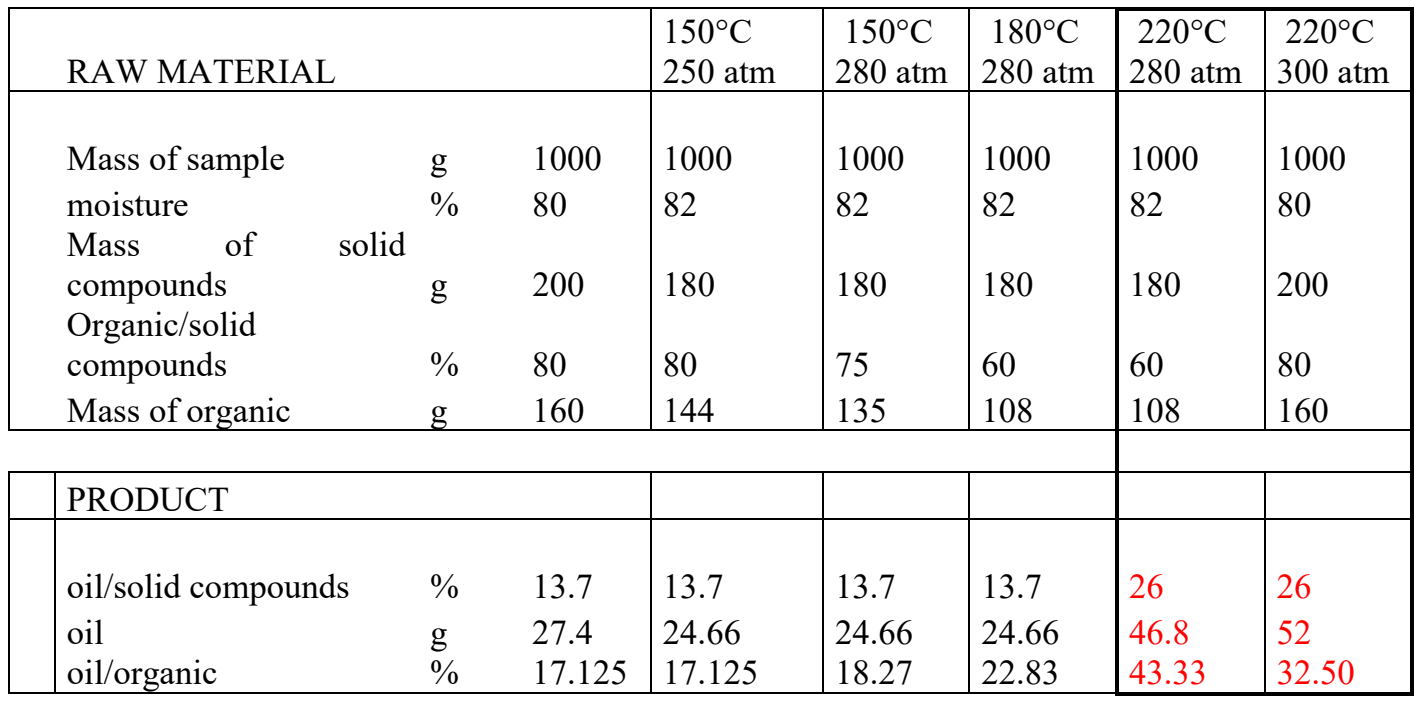

Based on the results of tests conducted on the laboratory installation, a semi-technical installation was developed. The installation was installed inside the standard container cf. photographs.

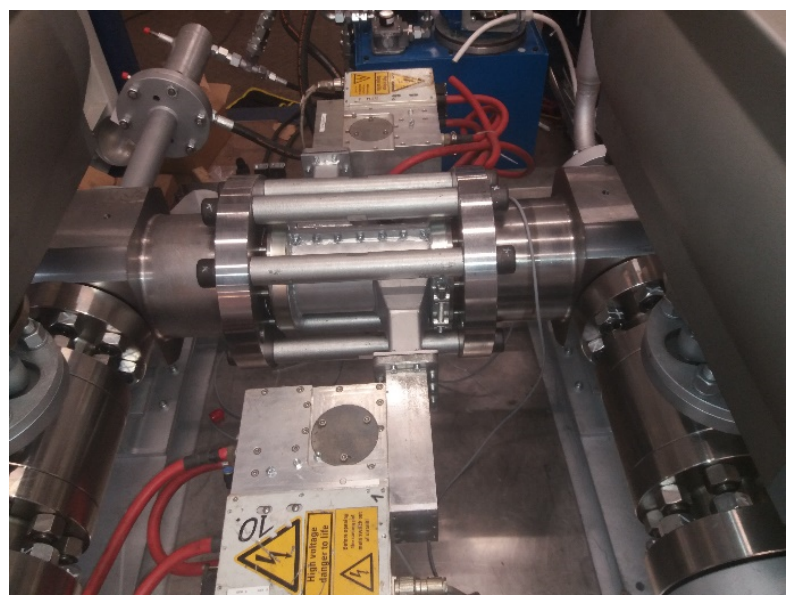

Fig. 5. 


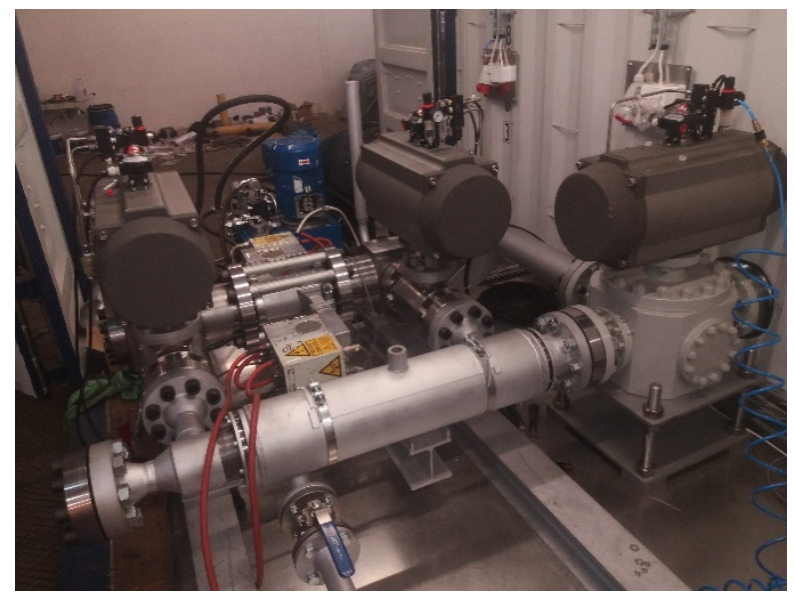

Fig. 6.

The high-pressure chamber was made of zirconium ceramics (pipe $\Phi 140 \mathrm{~mm} / \mathrm{ext}$.) with a metal cover, on which two waveguide radiators were installed, connected with generators of $3 \mathrm{~kW}(\mathrm{CW})$ power each $(\mathrm{f}=2.45 \mathrm{GHz})$. The structure with a high-pressure pump and a system of valves allowed the obtainment of required pressure in the process chamber. Before being fed to the process chamber, the processed sludge was pre-heated to a temperature of over $160^{\circ} \mathrm{C}$. Further heating was performed with the use of microwaves. In the course of the heating process, thermal insulation aimed at reducing energy losses was placed on the metal cover, and in further experiments an additional electric heater was installed in order to accelerate the process of heating of sludge. As a result, sludge was heated in the area next to the walls of the process chamber due to the absorption of microwaves and additionally due to thermal conductivity.

In the course of the process, on one microwave line between the generator and the radiator, a microwave reflectance meter was installed, and the size of the wave reflected during the heating process was recorded. A small increase of the reflected wave after the heating of sludge to the temperature of $200^{\circ} \mathrm{C}$ was found. With transmission of microwaves of power of about $2980 \mathrm{~W}$ into the process chamber, the power of the reflected wave changed from about $130 \mathrm{~W}$ to slightly over $160-180 \mathrm{~W}$ after heating the sample to a temperature of $200^{\circ} \mathrm{C}$. It was connected with changes in dielectric parameters of the material in the process chamber after achieving conditions close to the critical state.

Tests in that installation allowed the obtainment of up to 6 litres of bio-oil in one cycle of about 30-40 minutes. The technological problem is the selection of material for the highpressure pipe that would be transparent to microwaves. The use of zirconium pipes proved suboptimal - such pipes absorbed a part of the microwave energy, heating up unevenly, mainly directly next to microwave radiators. Those experiences have led to a search for another method of transmission of microwaves to the biomass being heated up in relation to previously planned method presented in the figure below. 


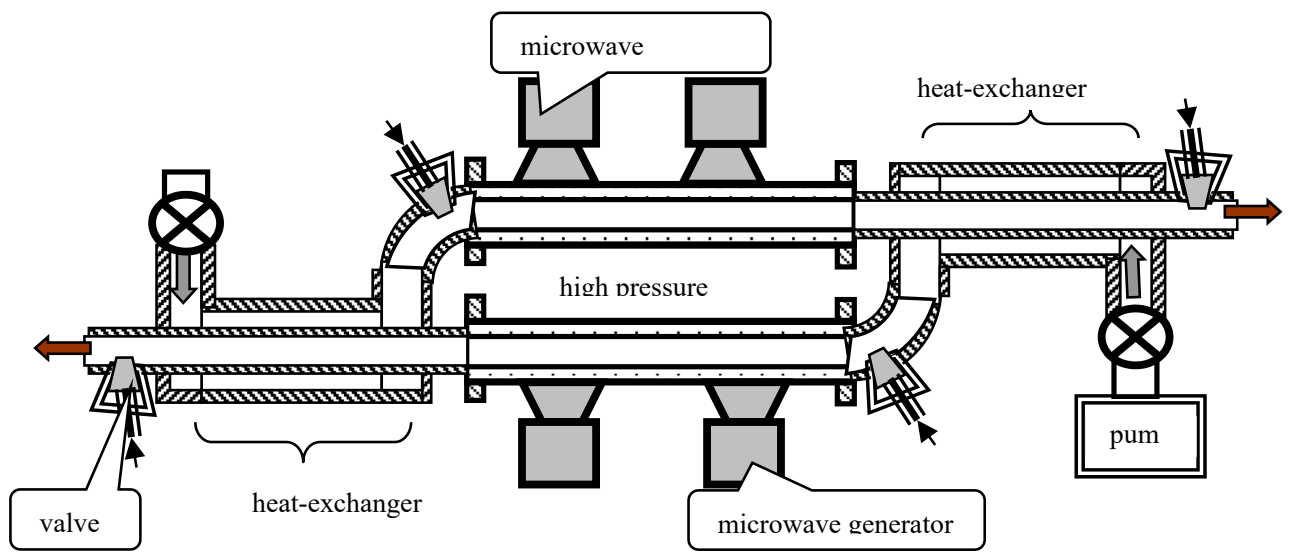

Fig. 7.

The structure of the industrial installation being currently prepared assumes the use of rod microwave radiators placed inside the metal high-pressure chamber. This will make it possible to use alund ceramics for the cover of radiators and to eliminate inconveniences connected with the use of zirconium pipes.

\section{Summary:}

It has been confirmed that the described process of application of microwave energy for utilisation of biomass with the method of hydrothermal conversion is practicable in laboratory conditions and in an industrial scale. It is confirmed by results of tests and an initial analysis of viability of the process. However, it is necessary to remove technical inconveniences connected with the problem of lossless transmission of microwaves through special ceramic materials and to ensure the most uniform distribution of the electromagnetic field inside the process chamber. Such works are being continued and a new reactor in a technical scale is being designed. The reactor is intended to make it possible to carry out a process competitive to known conventional methods of utilisation of sludge. 


\title{
EXPERIMENTAL STUDY OF MICROWAVE SLOW WAVE COMB AND CERAMIC APPLICATORS FOR SOIL TREATMENTAT AT FREQUENCY 2.45 GHZ
}

G. Brodie and G. Torgovnikov

\author{
University of Melbourne, 4 Water St, Creswick, Victoria 3363, Australia; \\ e-mail: grigori@unimelb.edu.au
}

Keywords: ceramic applicator, comb applicator, microwave, slow wave, soil microwave treatment

\begin{abstract}
In many cases in industry it is required to heat or treat surface layers of different material (soil, timber, concrete, plastics and so on) with microwaves (MW). Traditional MW irradiators (antennas) cannot provide heating only to the surface areas and energy penetrates deep into the material, where it decays exponentially due to normal attenuation. To reduce energy losses it was required to develop special MW applicators for surface treatment to increase process efficiency. To address this problem, a slow wave ("surface wave") comb and ceramic applicators were designed, built and studied. The main property of slow waves is that the energy concentration is very near impedance electrode - comb or ceramic plate surface. Comb and ceramic slab applicators for frequency $2.45 \mathrm{GHz}$ operation were designed for the soil treatment and studied using soil with moisture content range $32-174 \%$ and density range $590-1070 \mathrm{~kg} / \mathrm{m}^{3} .30 \mathrm{~kW}$ MW plant was used for experiments.
\end{abstract}

Results of the experiments showed that a ceramic applicator provides better uniformity of energy distribution across the width of the applicator. It reduces overheating of the soil surface and energy losses. The depth of energy penetration provided by ceramic applicator is lower compared with the comb applicator. It means that the ceramic applicator provides better energy localization and more energy absorption in the soil surface layers compared with the comb applicator. The ceramic applicator is more effective for MW treatment of the soil surface areas and is recommended for practical use in machines for thermal treatment and sterilization of surface layers of the soil and other materials.

\section{Introduction}

Traditional MW irradiators (antennas) cannot provide heating only to the surface areas and energy penetrates deep into the material, where it decays exponentially due to normal attenuation. Therefore, energy losses are very significant when heating depth of $20-40 \mathrm{~mm}$ (for example to heat soil for killing weed seeds) is all that is required. Therefore, it is required to develop special MW applicators for surface treatment to increase process efficiency.

To address this problem, a slow wave (which is sometimes called a "surface wave" applicator) comb and ceramic structures, were studied. The main property of slow waves is that the energy concentration is very near impedance electrode - comb or ceramic plate surface. Previously, slow wave structures (SWS) were used mostly as delay lines [4] and as interaction circuits in MW vacuum devices, and their properties were explored only for these specific applications [3]. Extending MW technologies to industry, medicine, and army initiated a study of slow wave structures, properties and peculiarities which can be used for developing novel technologies for industrial, medical, domestic and military applications [1], [5]. It was shown by the full-wave analysis, as well as by experiments, and practical 
realization that the SWSs have many previously unknown peculiarities, which can be used for creating novel technologies for domestic and industrial heating, plasma generating, etc. The work objectives of this study were:

1. design slow wave, ceramic and comb structure applicators for soil treatment at frequency $2.45 \mathrm{GHz}$;

2. experimentally study the energy distribution from slow wave applicators in the soil;

3. examine opportunities to use slow wave structures for surface soil layer heating; and

4. recommendations for practical use of new slow wave applicators.

\section{Applicators design}

On the base of the theoretical study [2] and computer modelling slow wave comb and ceramic slab applicators for frequency $2.45 \mathrm{GHz}$ were designed. Comb applicator made from aluminium and ceramic applicator made from alumina are shown in Fig 1. Main dimensions of comb and ceramic applicators are displayed in Table 1.

Table 1. Applicator parameters.

\begin{tabular}{|l|c|c|}
\hline Parameters & Comb, mm & Ceramic, $\mathrm{mm}$ \\
\hline Working length & 356 & 356 \\
\hline Applicator body thickness & 23 & 23 \\
\hline Applicator body width & 150 & 150 \\
\hline Comb electrode width & 100 & 100 \\
\hline Ceramic slab width & 16 & 13 \\
\hline Comb electrode thickness & 185 & \\
\hline Ceramic slab thickness & $13 / 3$ & \\
\hline $\begin{array}{l}\text { Comb electrode conic part } \\
\text { length }\end{array}$ & 3 & $\begin{array}{c}\text { Alumina slab (DC=9.8, } \\
\text { loss tangent 0.0002) }\end{array}$ \\
\hline Grove depth/ width & Aluminium & \multicolumn{2}{|c|}{$0.0002)$} \\
\hline Comb tooth thickness & $\begin{array}{l}\text { Alumina (99\%) ceramic plate size 3x84x146 } \\
\text { mm (4 pieces), (DC=9.8, loss tangent }\end{array}$ \\
\hline $\begin{array}{l}\text { Material } \\
\text { Ceramic plates covering } \\
\text { comb and ceramic slab }\end{array}$ & \multicolumn{2}{|c|}{} \\
\hline
\end{tabular}

In experiments the comb and ceramic slab (Fig. 1) surfaces were covered by $3 \mathrm{~mm}$ thickness alumina plates. 


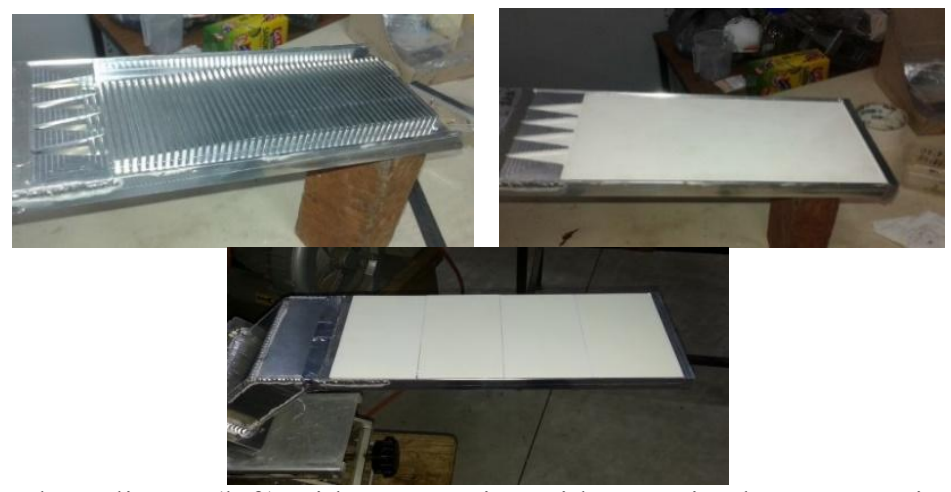

Fig. 1. Comb applicator (left) without covering with ceramic plates. Ceramic applicator (middle) without covering by ceramic plates. Comb and ceramic applicators view (right) covered by ceramic plates $(3 \times 84 \times 146 \mathrm{~mm}-4 \mathrm{pc})$.

In experiments a ceramic block made from alumina was inserted into the applicator body instead of aluminium comb electrode. The ceramic block (13 mm thickness) with $3 \mathrm{~mm}$ thickness ceramic plates formed ceramic part of the applicator with thickness $16 \mathrm{~mm}$.

\section{Experimental study. Material and methods.}

\section{Material}

The soil "Potting Mix Hortico" was used for tests. The soil had 3 different moisture contents (MC) and densities:

$\mathrm{MC}=32 \%$, density $=586 \mathrm{~kg} / \mathrm{m}^{3}$,

$\mathrm{MC}=89 \%$, density $=710 \mathrm{~kg} / \mathrm{m}^{3}$,

$\mathrm{MC}=174 \%$, density $=1070 \mathrm{~kg} / \mathrm{m}^{3}$.

The soil (mixture of organic and mineral substances) used in experiments had significant percentage of organic particles of different sizes (wood, bark, grass) therefore dielectric parameters of the soil at frequencies $2.45 \mathrm{GHz}$, temperatures from 15 to $80^{\circ} \mathrm{C}$ were in the range: dielectric constant - from 4 to 19 and loss tangent - from 0.2 to 0.3 . Only the most significant properties for $\mathrm{MW}$ heating of soil: moisture content and density were measured. The soil was placed into polypropylene containers (Fig.2) with sizes 160x250x300 mm.

\section{Experimental installation and procedure}

MW plant $30 \mathrm{~kW}(2.45 \mathrm{GHz})$ was used for experiments (Fig. 2). The applicator was connected to the MW generator by waveguides and placed into the metal box 400x500x1120 $\mathrm{mm}$ for leakage protection. Position of the container with soil on the applicator is shown in Fig. 2 (middle). Auto tuners used in MW systems provided good matching for the generator and applicators (with soil) practically without power reflection. 


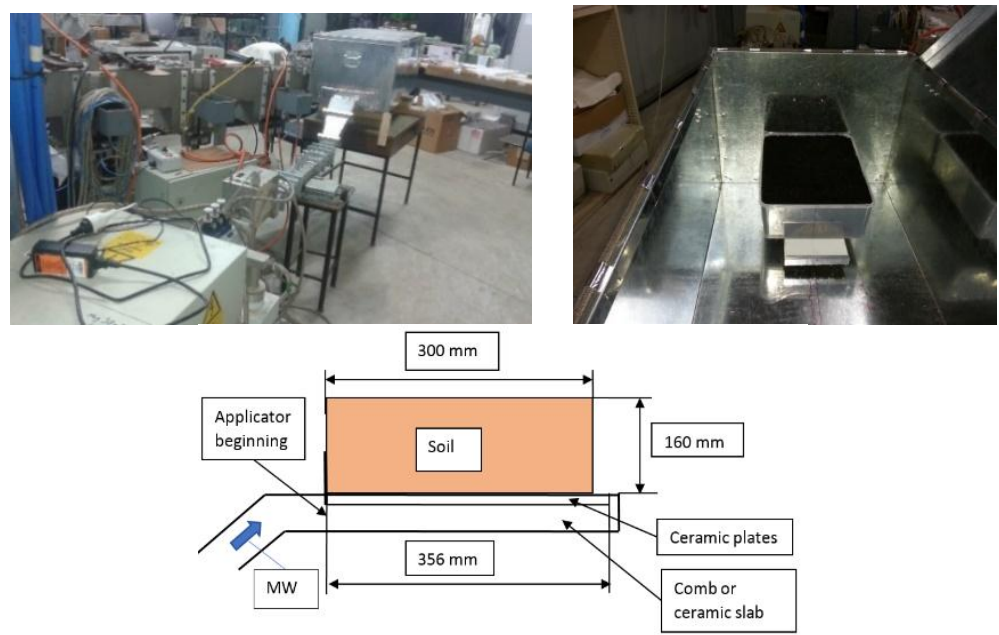

Fig 2. Applicator (in metal box) connection with $30 \mathrm{~kW} \mathrm{MW} \mathrm{generator,} 2.45 \mathrm{GHz}$ (left).

Container with soil on the applicator inside of the metal box (middle). Scheme of applicator with soil in the container (right).

Temperatures in the soil were measured by thermocouples after MW heating on the depth $10,30,50,80,100$ and $140 \mathrm{~mm}$ along applicator at distances from applicator beginning 30, $60,90,120,150,180,210,240,270$ and $350 \mathrm{~mm}$, and across the applicator in the central vertical plane and at distances from central vertical plane $37.5,75$, and $112.5 \mathrm{~mm}$ in both cross directions. Distribution of measuring points covered all volume of the soil along and across the soil samples. Scheme of container with soil position on applicator is shown in Fig.2. Lay-on jig was used for thermocouples positioning during measurements. To get reliable results of MW heating at every soil moisture content four repeats were performed. MW power of $3.5 \mathrm{~kW}$ was applied to the soil for $15 \mathrm{sec}$ and after that the temperatures were measured by thermocouples. Energy applied to the soil during experiments was 53 $\mathrm{kJ}$.

\section{Results and discussion}

\section{Temperature distribution in the soil by comb applicator}

We assume that the temperature distribution in the soil reflects energy release in different spots of the soil volume and allows assessment of the energy distribution by MW applicators. Fig. 3 shows typical temperature distribution in the soil volume at the depth of $10 \mathrm{~mm}$ by comb applicator after applying MW power $3.5 \mathrm{~kW}$ for $15 \mathrm{sec}$. 


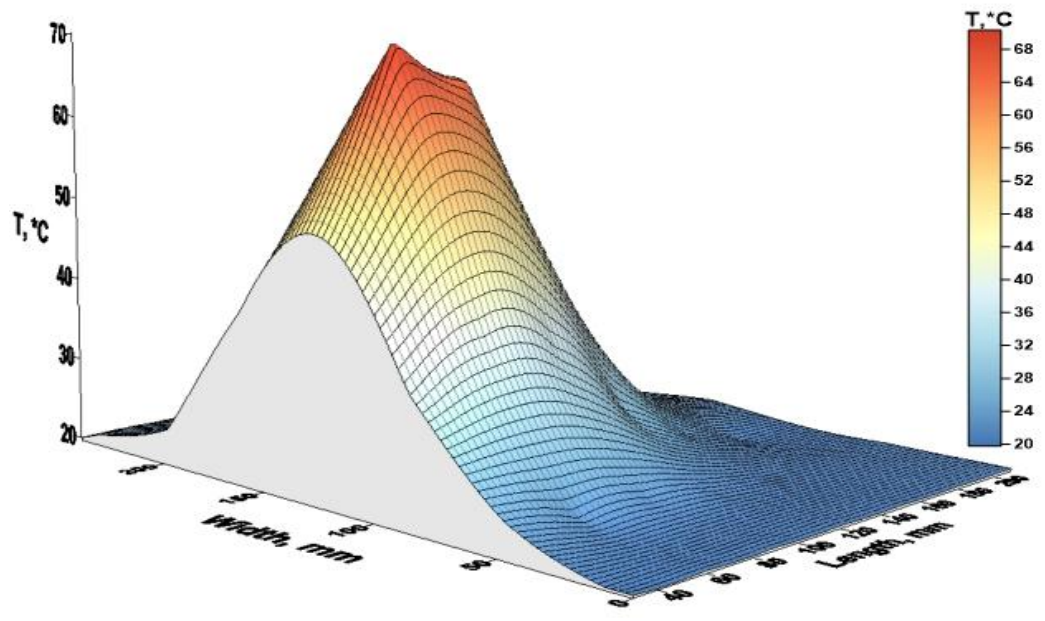

Fig. 3. Temperature distribution in the soil at the depth of $10 \mathrm{~mm}$ by comb applicator at $\mathrm{F}=2.45 \mathrm{GHz}, \mathrm{P}=3.5 \mathrm{~kW}$, time of $\mathrm{MW}$ heating $15 \mathrm{sec}$, To $=20^{\circ} \mathrm{C}$, applied energy $53 \mathrm{~kJ}$. Soil moisture content $\mathrm{MC}=89 \%$, density $710 \mathrm{~kg} / \mathrm{m}^{3}$.

Temperature distribution in the soil along the comb applicator central vertical plane for soil with $\mathrm{MC}=174 \%$, initial temperature $\mathrm{To}=20^{\circ} \mathrm{C}$, power $\mathrm{P}=3.5 \mathrm{~kW}$, duration of $\mathrm{MW}$ heating $15 \mathrm{sec}$ is shown in Fig. 4.

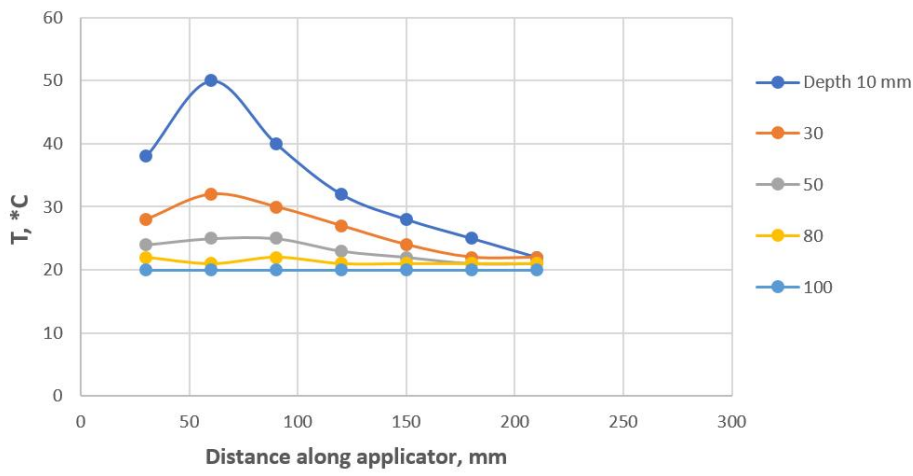

Fig. 4. Temperature distribution in the soil along the comb applicator central plane at $\mathrm{F}=2.45 \mathrm{GHz}, \mathrm{P}=3.5 \mathrm{~kW}$, duration of $\mathrm{MW}$ heating $15 \mathrm{sec}, \mathrm{To}=20^{\circ} \mathrm{C}$, applied energy $53 \mathrm{~kJ}$. Moisture content $\mathrm{MC}=174 \%$, density $1070 \mathrm{~kg} / \mathrm{m}^{3}$.

Maximum energy absorption takes place at a distance of about $60 \mathrm{~mm}$ from the beginning of the applicator. Almost all of the energy was absorbed at the distance of $200 \mathrm{~mm}$ along the applicator for all of the tested soil moisture contents. Maximum energy release takes place in the zone between 60 to $90 \mathrm{~mm}$ from the applicator beginning.

Practically all energy irradiated by applicator was absorbed at the width of about $150 \mathrm{~mm}$ across the applicator. This was consistent across different moisture content of the soil samples. 
Temperature distribution in the central plane of the comb applicator at soil depth between 10 and $100 \mathrm{~mm}$ is illustrated by Fig. 5 .

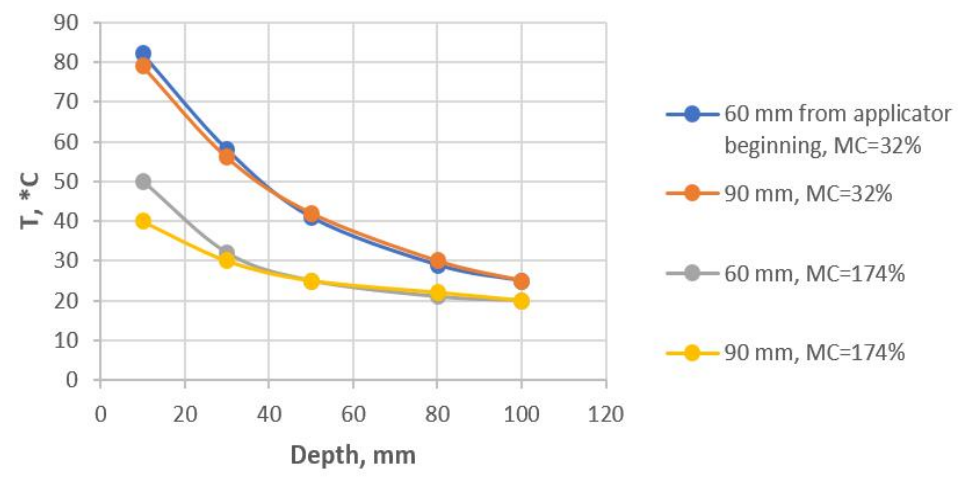

Fig. 5. Temperature distribution in the central vertical plane of the comb applicator at soil depth between 10 and $100 \mathrm{~mm}$ after heating. $\mathrm{MC}=32$ and $174 \%, \mathrm{~F}=2.45 \mathrm{GHz}, \mathrm{P}=3.5 \mathrm{~kW}$, time of MW heating $15 \mathrm{sec}, \mathrm{To}=21^{\circ} \mathrm{C}$.

The most significant part of the energy is absorbed by the soil, with moisture content in the range 32 to $174 \%$, up to the depth of $50 \mathrm{~mm}$ measured in the central vertical plane of the comb applicator. In other volume zones of the soil the share of energy absorbed by surface layers is higher. Almost all of the energy is absorbed at applicator length $200 \mathrm{~mm}$ and width $150 \mathrm{~mm}$ covering soil surface $300 \mathrm{~cm} 2$.

\section{Temperature distribution in the soil by ceramic applicator}

Ceramic block with $3 \mathrm{~mm}$ ceramic plates on the top forms ceramic applicator with thickness $16 \mathrm{~mm}$ from alumina (99\%) material with dielectric parameters: $\mathrm{DC}=9.8$, loss tangent 0.0002. When microwaves travel through alumina they reduce in wave length.

The wave length in the material (ceramics):

$$
\lambda=\lambda_{0} / \sqrt{\dot{\varepsilon}},
$$

where $\lambda_{0}$-wavelength in vacuum (or in air) and

$\dot{\varepsilon}$ - dielectric constant of material.

In our case at $2.45 \mathrm{GHz}$ wave length is $122 \mathrm{~mm}$ and at $\dot{\varepsilon}=9.8$ the wave length in alumina is $\lambda=122: 3.13=40 \mathrm{~mm}$.

This means a wave length in ceramic block is about $40 \mathrm{~mm}$ and microwaves in the ceramics will provide two energy maximums (peak volumes) on the applicator width (comb applicators provide one maximum because wave length in it is $122 \mathrm{~mm}$ ).

Fig. 6 shows typical temperature distribution in the soil at the depth of $10 \mathrm{~mm}$ by ceramic applicator after applying MW power $3.5 \mathrm{~kW}$ for $15 \mathrm{sec}$. 


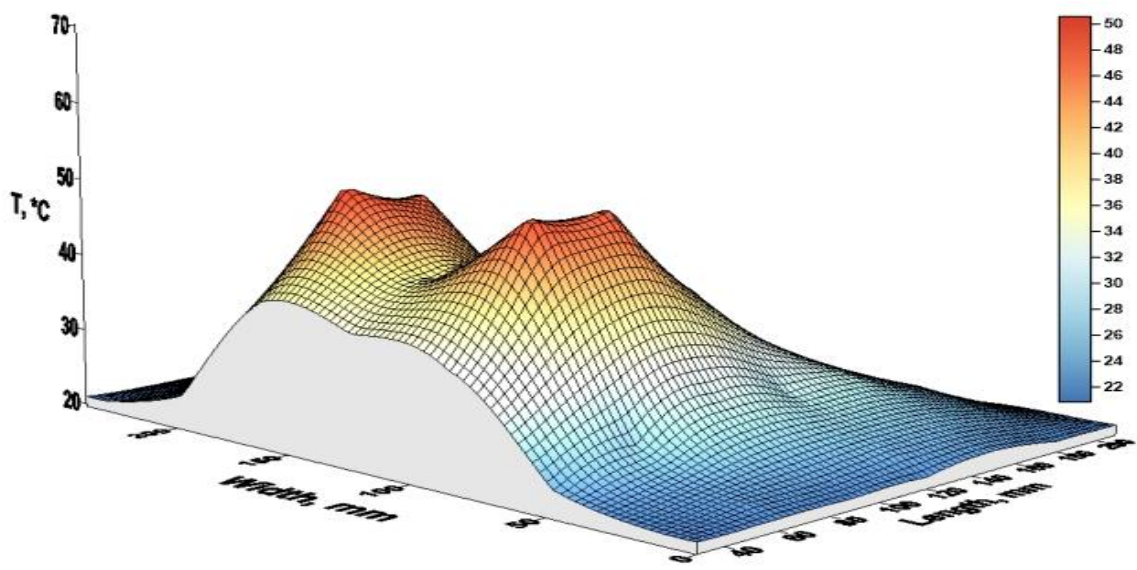

Fig. 6. Temperature distribution in the soil at the depth of $10 \mathrm{~mm}$ by ceramic applicator after MW heating at $\mathrm{F}=2.45 \mathrm{GHz}, \mathrm{P}=3.5 \mathrm{~kW}$, time of $\mathrm{MW}$ heating $15 \mathrm{sec}$, To $=20^{\circ} \mathrm{C}$. Soil moisture content $89 \%$, density $710 \mathrm{~kg} / \mathrm{m}^{3}$.

Temperature distribution along ceramic applicator in the peak vertical plane $(38 \mathrm{~mm}$ from central vertical plane) is shown in Fig. 7 (left).
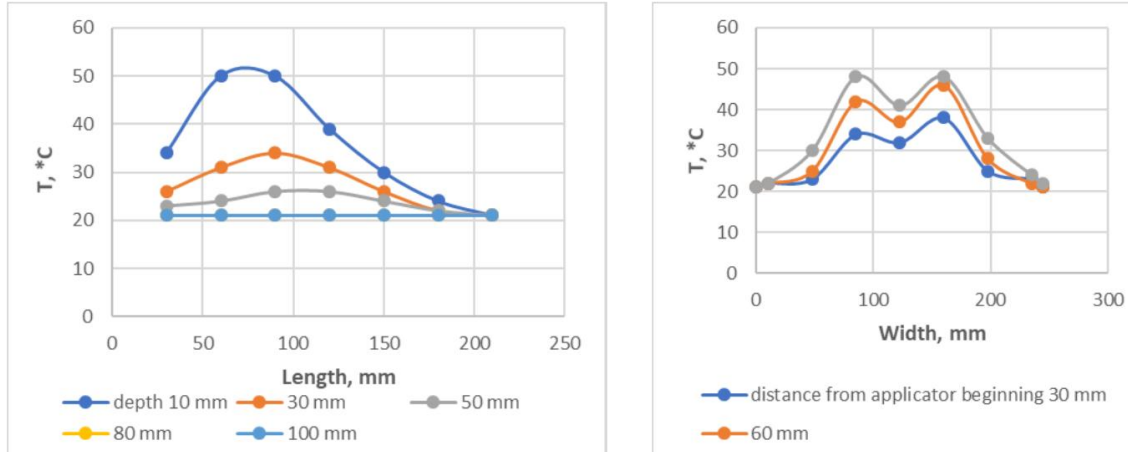

$\rightarrow$ distance from applicator beginning $30 \mathrm{~mm}$

$-60 \mathrm{~mm}$

$-90 \mathrm{~mm}$

Fig. 7. Left. Temperature distribution along ceramic applicator in the peak vertical plane (38 mm from central plane) at different depths after MW heating of the soil with moisture contents $89 \%$. Right. Temperature distribution across ceramic applicator at the depth of 10 mm after MW heating soil with moisture contents $32 \%$. $\mathrm{F}=2.45 \mathrm{GHz}, \mathrm{P}=3.5 \mathrm{~kW}$, time of $\mathrm{MW}$ heating $-15 \mathrm{sec}, \mathrm{To}=21^{\circ} \mathrm{C}$.

For soil $\mathrm{MC}=89$ and $174 \%$ practically all energy is absorbed at the applicator length of 200 $\mathrm{mm}$. For soil $\mathrm{MC}=32 \%$ energy spreads along all of the applicator length. The temperature distribution in a soil sample with $\mathrm{MC}=32 \%$ across the applicator after $\mathrm{MW}$ heating is displayed in Fig.7 (right). Temperature curves show two peaks at a distance of $40 \mathrm{~mm}$ on each side of the central vertical applicator plane and lowest at the applicator central vertical plane.

Temperature distribution in the peak vertical plane of the ceramic applicator at soil depths and $\mathrm{MC}=32,89$ and $174 \%$ is shown in Fig. 8 . 


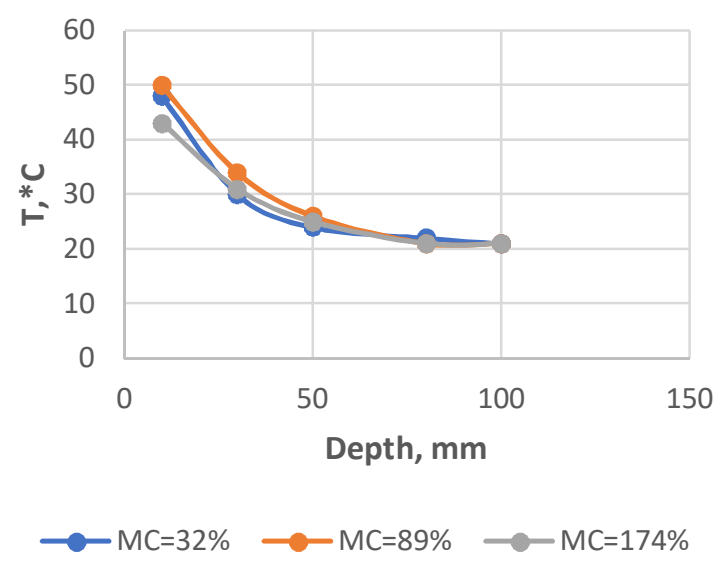

Fig. 8. Temperature distribution by the soil depths at the peak vertical pane of the ceramic applicator at the distance of $90 \mathrm{~mm}$ from applicator beginning after heating and $\mathrm{MC}=32$, 89 and $174 \% . \mathrm{F}=2.45 \mathrm{GHz}, \mathrm{P}=3.5 \mathrm{~kW}$, time of heating $15 \mathrm{sec}, \mathrm{To}=21^{\circ} \mathrm{C}$.

The most significant part of the energy is absorbed by the soil at the peak vertical planes on the depth up to $50 \mathrm{~mm}$, and practically all of the energy is absorbed at the depth up to 80 $\mathrm{mm}$. In other volumes of the soil the share of energy absorbed by surface layers is higher.

\section{Comparison of the temperature distribution in the soil by comb and ceramic applicators}

Fig. 3 with one temperature peak and Fig. 6 with two temperature peaks illustrate principal difference in energy distribution in the soil by comb and ceramic applicators. Comb applicator provides maximum soil heating in the central vertical applicator plane because the transporting electromagnetic wavelength is $122 \mathrm{~mm}$. Alumina ceramic block transforms electromagnetic wave from $122 \mathrm{~mm}$ to the wavelength of $40 \mathrm{~mm}$ and provides a different pattern of energy distribution in the soil with two peaks in the vertical cross planes. Fig. 9 illustrates the difference of temperature distribution in a soil sample at the vertical cross section of the two applicators. 


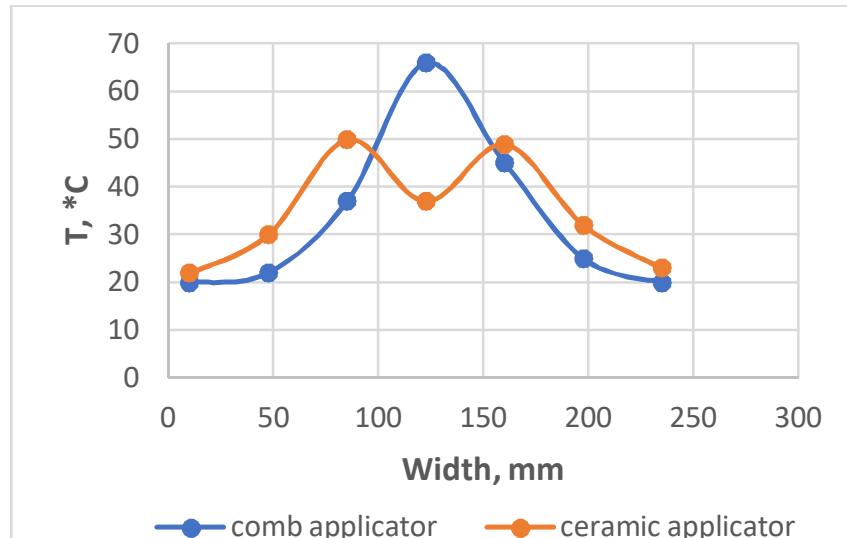

Fig. 9. Temperature distribution across comb and ceramic applicators at the soil depth of $10 \mathrm{~mm}$ and distance of $90 \mathrm{~mm}$ from applicator beginning after MW heating of the soil with moisture contents $89 \%$. $\mathrm{F}=2.45 \mathrm{GHz}, \mathrm{P}=3.5 \mathrm{~kW}$, time of MW heating $15 \mathrm{sec}, \mathrm{To}=21^{\circ} \mathrm{C}$.

Peak temperature in the soil provided by the ceramic applicator is significantly lower compared to peak temperature provided by the comb applicator. This reduces overheating of the soil surface and energy losses. Test results of comb and ceramic applicators in similar conditions are shown in Table 2.

Table 2. Test results of comb and ceramic applicators for frequency $2.45 \mathrm{GHz}$.

\begin{tabular}{|l|l|c|c|}
\hline Items & Units & Comb applicator & Ceramic applicator \\
\hline $\begin{array}{l}\text { Soil moisture content and } \\
\text { density }\end{array}$ & & \multicolumn{2}{|c|}{$\mathrm{MC}=32-174 \%$, density $586-1070 \mathrm{~kg} / \mathrm{m}^{3}$} \\
\hline $\begin{array}{l}\text { Majority of the applied MW } \\
\text { energy was absorbed: }\end{array}$ & $\mathrm{mm}$ & $200-210$ & $\begin{array}{c}200(\mathrm{MC}=89-174 \%) \\
350(\mathrm{MC}=32 \%)\end{array}$ \\
\hline on the applicator length & $\mathrm{mm}$ & 150 & $200-210$ \\
\hline on the applicator width up to & $\mathrm{mm}$ & $100-130$ & 80 \\
\hline on the material depth up to & $\mathrm{mm}$ & 30 & 70 \\
\hline $\begin{array}{l}\text { Length of zone of high intensity } \\
\text { heating along applicator } \\
\text { (distance from applicator } \\
\text { beginning) }\end{array}$ & & & 60 to 90$)$ \\
\hline $\begin{array}{l}\text { Average MW specific energy } \\
\text { applied to the soil }\end{array}$ & $\mathrm{kJ} / \mathrm{cm} 2$ & 0.18 & 0.13 \\
\hline
\end{tabular}

Measuring the maximum degree of non-uniformity of the temperature in the soil by ratio of peak temperatures to initial temperatures, the comb applicator provides non-uniformity 3.1 , ceramic applicator -2.3 . 
The ceramic applicator provides better uniformity of energy distribution on the width of the applicator due to two temperature peaks. In addition, depth of energy penetration is lower. This means the ceramic applicator provides more energy absorption within the soil surface layers compared to comb applicator and therefore is more effective. For practical use, instead of ceramic block (thickness $13 \mathrm{~mm}$ ) + ceramic plates (thickness $3 \mathrm{~mm}$ ), it is better to use one alumina ceramic block with thickness of $16 \mathrm{~mm}$.

To improve the uniformity of the energy distribution by ceramic applicators it can be recommended to use ceramics with higher dielectric constant 15-25 (PD- 15, PD-20, PD25). This creates 3 or $4 \mathrm{MW}$ power peaks along the applicator width keeping the energy closer to the applicator surface. This will increase efficiency of MW energy use.

In terms of effective energy use for thermal treatment and sterilization of soil surface layers, using a ceramic applicator has the following advantage compared to a comb applicator:

- better uniformity of energy distribution on the width of the applicator,

- better energy localization in the soil surface layers,

- the soil moisture content in the range between $32-174 \%$ does not have a significant effect on energy localization in the surface layers.

Ceramic applicators can be recommended for practical use in machines for thermal treatment and sterilization of the soil and other materials surface layers.

\section{Conclusion}

Comb applicator provides maximum energy release into the soil at the central vertical plane. Ceramic applicator from alumina forms two temperature peaks in the vertical planes at a distance of about $40 \mathrm{~mm}$ each side of the central applicator plane and minimum in the applicator central plane. Ceramic applicator provides better uniformity of the energy distribution along the width of the applicator due to two temperature peaks. The depth of energy penetration is lower compared to comb applicator. Thus, the ceramic applicator provides better energy localization and more energy absorption in soil surface layers compared to a comb applicator. To provide better uniformity of energy distribution across ceramic applicator it is recommended to use ceramics with higher dielectric constant. Using ceramics with dielectric constant of 15-25 would provide 3 or 4 temperature peaks on the applicator width, keeping more energy closer to the applicator surface. This will increase efficiency of MW energy use.

The ceramic applicator is more effective for MW treatment of the soil surface areas and is recommended for practical use in machines for thermal treatment and sterilization of surface layers of the soil and other materials.

\section{References}

1. Pchelnikov Yu. N. Features of slow waves and potentials for their nontraditional application. J. of Communications Technology and Electronics, 2003, Vol 48, (4), 450-462.

2. Pchelnikov Yu. N. SWS - based applicators for agriculture application.

Unpublished Report of Company "Pchelnikov Consulting" for Melbourne University. 2014, pp 39.

3. Silin R. A. Periodic Waveguides. Fazis, Moscow, 2002, [in Russian].

4. Watkins D. A. Topics in Electromagnetic Theory. Willy \& Sons Inc., N.Y.,1958.

5. Yelizarov A. A., Pchelnikov Yu. N. Radio-Wave Elements of Technological Devices and Equipment on Slow-Wave Structures. Radio and Communication, Moscow, 2002, [in Russian]. 


\title{
MICROWAVE WOOD CHIP TREATMENT USE IN CHEMICAL PULP MANUFACTURING (TECHNICAL-ECONOMIC ASSESMENT)
}

\author{
A. Leshchinskaya \\ Plekhanov Russian University of Economics. 36 Stremyannyy Pereulok, 115093 Moscow, \\ Russia, \\ e-mail: alixfl@mail.ru]
}

\begin{abstract}
.
Low permeability of many wood species causes problems in the chemical pulp industry. These include: very long cooking times, high chemical consumption, large material losses, high energy consumption, and environment pollution. New microwave (MW) wood modification technology can provide an increase in wood permeability for liquids and gases that solves many of these problems. MW wood pre-treatment can increase pulp mill throughput, reduce chemical and power consumption, increase pulp quality and yield, and improve the environmental performance. Economic modelling of this new technology use in different chemical pulp mill conditions allowed to asses the effect of capital costs, electricity costs, labour costs and other cost components to specific total costs of MW chip processing. MW chip treatment costs for pulp mills with output 50,000 to 500,000 ait dry ton (ADT) per year at electricity cost range US\$0.04 to US\$0.24/kWh vary in the range from US\$17.7 to US\$60.8 per air dry ton of pulp. Electricity costs form the most significant part - 51- $69 \%$ of the total specific costs of MW chip processing at electricity costs US $\$ 0.08$ to US $\$ 0.12 / \mathrm{kWh}$. New technology application in different Russian conditions can provide benefits up to $7-22$ Mil US\$ per year for pulp mills with output more than 200,000 ADT/year. Ecological effect and high economic advantages of this MW technology provide good opportunity for commercialisation.
\end{abstract}

Keywords: chemical pulping, microwave wood modification, pulp, softwood, wood chips.

\section{Introduction}

The global production of pulp in the world is around 180 mil tons per year [3]. USA is the most significant pulp manufacturer producing 50 mil tons per year. The volumes of pulp products in Russia over the last few years has increased by $5.2-6.9 \%$ per year and reached 8.1 mil tons in 2017. The consumption of pulp and paper products is growing steadily in the world. A quarter of the pulp produced in Russia is for export. Therefore, it is necessary to use advanced knowledge-based technologies in pulp industry, which Russian Government provides funding for development and commercialization of such technologies.

Large volumes of cellulose are produced from wood chips using chemical methods which have many shortcomings: high energy and chemical consumption, significant raw material losses, and high level of environment pollution [4]. One of the new technologies is microwave wood structure modification technology [7] which changes wood properties and radically increases wood permeability by liquids and gases. Industrial application of this technology can significantly improve technological processes of the pulp production leading to great economic and ecological benefits. 


\section{Technology}

The MW wood modification technology works in the following way. Intensive MW power applied to green wood generates steam pressure within the wood cells. High internal pressure destroys the weak elements of wood structure, opens pores and forms micro and macro cracks. A several thousand-fold increase in wood permeability can be achieved in species previously found to be impermeable by liquids and gases. Fig. 1 demonstrates how MW modification changes wood structure and converts wood to a highly porous material: the left side of the sample - wood before modification, the right side - after modification with a lot of visible and invisible voids.

Fig 1. Wood sample after intensive MW modification. Left side - natural wood, right side MW modified wood.

MW wood modification significantly increases the speed of the pulp cooking and improves the production processes. The MW technology $[1,2,7]$ showed potential improvements in the pulp industry through:

- increase in mill throughput

- $\quad$ significant reduction of chemical consumption

- significant reduction of power consumption

- increase in pulp quality and yield

- improvement of environmental performance.

One of the proposed pulp process schemes with chip MW treatment is shown in Fig 2.

1

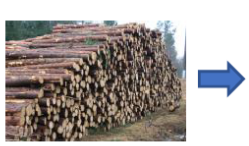

4

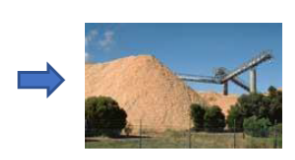

7

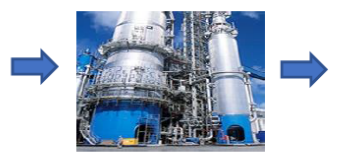

2

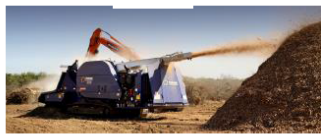

5

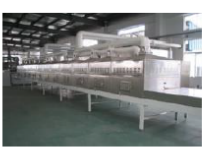

8

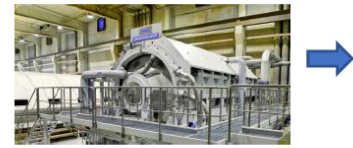

3

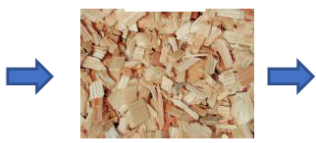

6

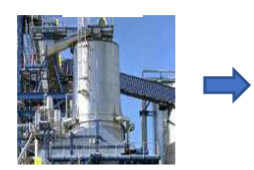

9

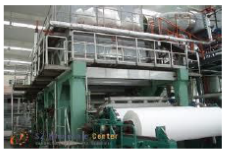

Fig 2. Pulp manufacturing process scheme. 1 - logs, 2 - chipping, 3 - chips for pulping, 4 chip storage, 5- chip MW treatment, 6 - steaming, 7 - cooking, 8 - washing, 9 - pulp making. 
In addition, the raw material can be in the form of large chips, wood pieces, parts of boards or logs. MW wood modification technology can be used by pulp mills with batch and continuous digesting and not limited by mill throughput.

\section{New process advantages}

The potential impacts and benefits of chemical pulp manufacturing using this new MW technology was assessed in Oak Ridge National Laboratory $[1,2,8]$ and University of Melbourne [7] and outlined in the Table 1.

Table 1. Microwave technology benefits.

\begin{tabular}{|l|l|}
\hline Potential impacts & Benefits \\
\hline Increase in throughput & $\begin{array}{l}20-34 \% \text { increase in throughput for digester- } \\
\text { limited mills }\end{array}$ \\
\hline $\begin{array}{l}\text { Chemical savings } \\
\text { (increased speed of } \\
\text { chemical chip cooking } \\
\text { reactions) }\end{array}$ & $\begin{array}{l}\text { on chemical recovery processes, especially lime kiln } \\
\text { and recover boiler }\end{array}$ \\
\hline Material savings & $\begin{array}{l}\text { Reject reduction from } 3.8 \text { to } 0.3 \% \\
\text { Screened pulp yield increase } 2.7 \%\end{array}$ \\
\hline $\begin{array}{l}\text { Larger chip size can be } \\
\text { utilised as pulping liquor is } \\
\text { more readily absorbed }\end{array}$ & $\begin{array}{l}\text { Reduction of wood loses associated with } \\
\text { oversize chips } \\
2 \% \text { increase in pulp strength due to fewer cut } \\
\text { fibres from chipping }\end{array}$ \\
\hline $\begin{array}{l}\text { Environmental aspects } \\
\text { Improved environmental performance due to } \\
\text { reduction of fuel usage }\end{array}$ \\
\hline
\end{tabular}

MW modified wood has a more even structure meanings that pulping chemicals are absorbed more evenly, thereby cooking more consistently. This means less variation in the degree of cooking across the chip, and therefore less quality variations due to overcooking or undercooking. Handsheet properties of the pulp from MW modified wood are comparable to the properties of the pulp from untreated wood. An increase in the length of chip will reduce the number of cut fibres, improving the yield of longer fibres by reducing the proportion of fines. Both microwave modified hardwood and softwood can be used for pulping.

MW technology reduces chemical, energy and raw material consumption in pulp production. This allows the chemical pulp mills to significantly reduce pollution loads to the environment.

\section{Microwave plant parameters and costs}

Softwood microwave modification requires less energy and provides lower costs compared to hardwoods, therefore the first cost estimation was done for softwoods. The MW plant power required for pulp mills with different throughput, electric energy consumption, and MW plant costs are outlined in Table 2. All specific data of pulp units is in ADT (air dried ton) and calculations are based on world prices of MW equipment (MW generators with frequency $0.922(0.915) \mathrm{GHz})$. All costs are in US\$. 
Table 2. MW power plant parameters and costs.

\begin{tabular}{|c|l|l|l|l|}
\hline $\begin{array}{l}\text { Plant output, } \\
\text { ADT(air dry } \\
\text { ton) /year }\end{array}$ & $\begin{array}{l}\text { Output, } \\
\text { ADT/hour } \\
\text { (7500 working } \\
\text { hours per } \\
\text { year) }\end{array}$ & $\begin{array}{l}\text { Microwave } \\
\text { plant power, } \\
\mathrm{kW}\end{array}$ & $\begin{array}{l}\text { Electric energy } \\
\text { consumption, } \\
\mathrm{kWh} / \mathrm{ADT}\end{array}$ & $\begin{array}{l}* \text { MW plant } \\
\text { cost (only } \\
\text { technological } \\
\text { equipment } \\
\text { cost), US\$ }\end{array}$ \\
\hline 50,000 & 6.7 & 1000 & 192 & $1,665,000$ \\
\hline 100,000 & 13.3 & 2000 & 192 & $3,330,000$ \\
\hline 200,000 & 26.6 & 4000 & 192 & $6,290,000$ \\
\hline 500,000 & 66.7 & 10200 & 192 & $14,688,000$ \\
\hline
\end{tabular}

* MW plant costs in the Table 3 include only technological equipment (MW generators, applicators, mechanical, electrical facilities) and do not include buildings.

Economic modelling of this technology application has been done for pulp mill throughput range 50,000 to 500,000 ADT/year, 3 working shifts per day (930 shifts per year) and electricity cost range $\$ 0.04$ to $\$ 0.24 / \mathrm{kWh}$. Microwave plants were operated by workers (not automatic).

\section{Costs of microwave chip treatment}

The cost assessment was based on analysis of specific MW treatment costs which include capital costs (depreciation rate 17\%), energy, maintenance, magnetron replacement costs, and labour costs. The specific costs do not include building, electrical connections, mechanical installation, on costs (overheads) and taxes.

MW chip processing costs depends significantly from capital costs, electricity and labour costs. Fig. 3 shows a dependence of MW treatment specific costs on electricity costs at different sawmill throughput.

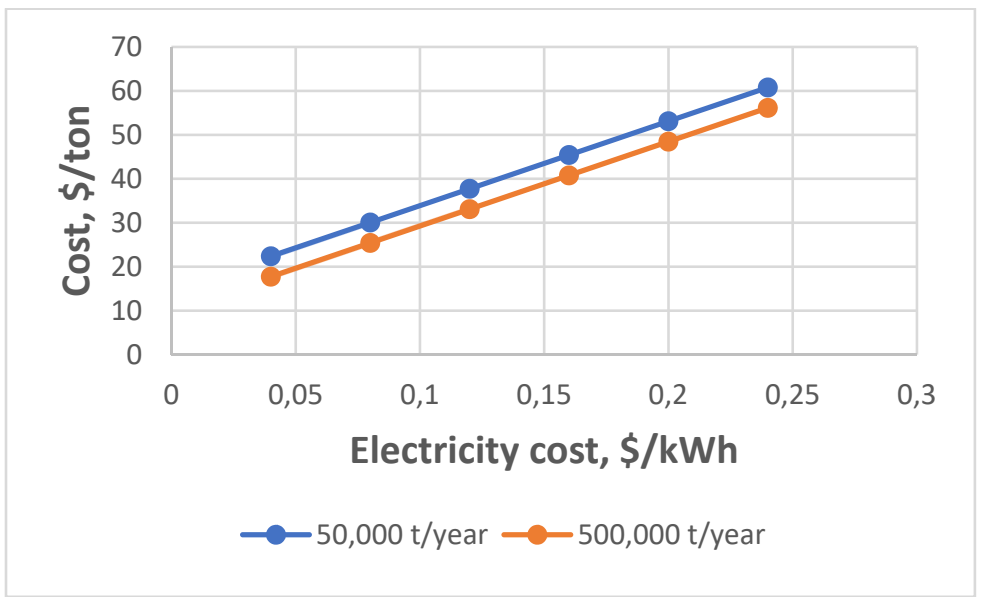

Fig. 3. Specific MW chip treatment costs depending on electricity costs at pulp mill output 50,000 and 500,000 ADT/year.

The electricity cost increase from $\$ 0.04$ to $\$ 0.24 / \mathrm{kWh}$ provides specific MW processing cost rise by 2.7 to 3.1 times at pulp mill output range of 50,000 to 500,000 ADT/year. 
Effect of the pulp mill throughput on MW treatment specific costs is shown in Fig. 4. Throughput increase lowers costs because the portion of the capital and labour costs are reduced in the total specific costs.

An increase in the pulp mill throughput from 50,000 to 500,000 ADT/y at electricity cost range $\$ 0.04$ to $\$ 0.24 / \mathrm{kWh}$ reduces specific MW treatment costs by 21 to $8 \%$. Specific cost components: capital cost (CC), magnetron replacement cost (RC), maintenance cost (MC), electricity cost (EC), labour cost (LC) at pulp mill output from 50,000 to 500,000 ADT/year at electricity cost $\$ 0.12 / \mathrm{kWh}$ are shown in Fig. 5.

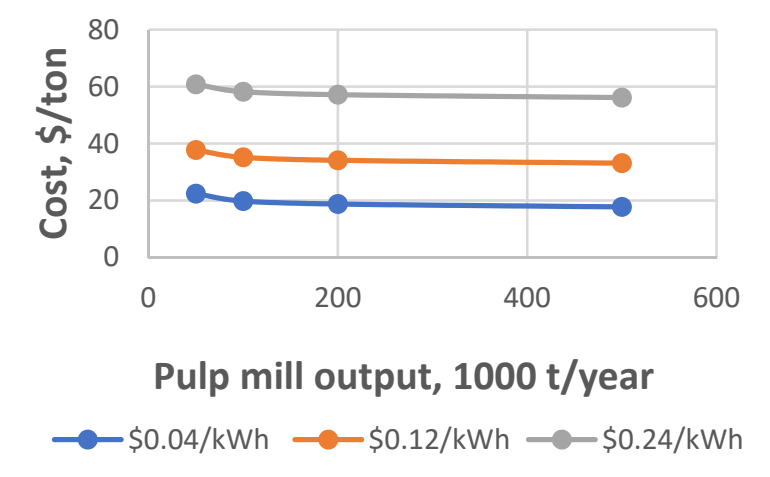

Fig. 4. Specific MW chip treatment costs depending on pulp mill output at electricity cost $\$ 0.04,0.12$ and $\$ 0.24 / \mathrm{kWh}$.

Electricity costs form the most significant part of the total specific costs of MW processing. Electricity costs (at electricity price $\$ 0.12 / \mathrm{kWh}$ ) form $62-69 \%$ of the total specific costs. Capital costs form $15-16 \%$ of the total specific costs. Labour costs form $5-14 \%$ of the total specific costs. At electricity price of $\$ 0.08 / \mathrm{kWh}$ the electricity costs form $51-60 \%$, capital costs $-19-20 \%$, labour costs - 6-18\% of the total specific costs of MW treatment.

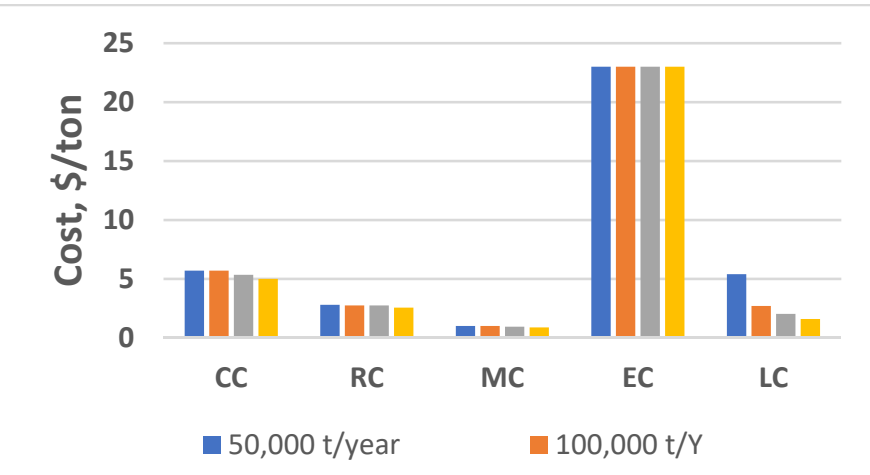

Fig. 5. Specific cost components: CC- capital cost, RC- magnetron replacement cost, MC - maintenance cost, EC- electricity cost, LC- labour cost, at pulp mill output from 50,000 to $500,000 \mathrm{ADT} /$ year at electricity cost $\$ 0.12 / \mathrm{kWh}$. 


\section{Results}

MW chip treatment costs for pulp mills with output 50,000 to 500,000 t/year at electricity cost range $\$ 0.04$ to $\$ 0.24 / \mathrm{kWh}$ vary in the range from $\$ 17.7$ to $\$ 60.8$ per air dry ton of pulp. Electricity costs form the most significant part of the total specific costs of MW chip processing. They form $62-69 \%$ of the total specific costs of MW processing at electricity price $\$ 0.12 / \mathrm{kWh}$. Capital costs forms $15-16 \%$, labour costs forms $5-14 \%$ of the total specific costs. At electricity price $\$ 0.08 / \mathrm{kWh}$ the electricity costs form $51-60 \%$, capital costs - 19$20 \%$, labour costs $-6-18 \%$ of the total specific costs of MW treatment.

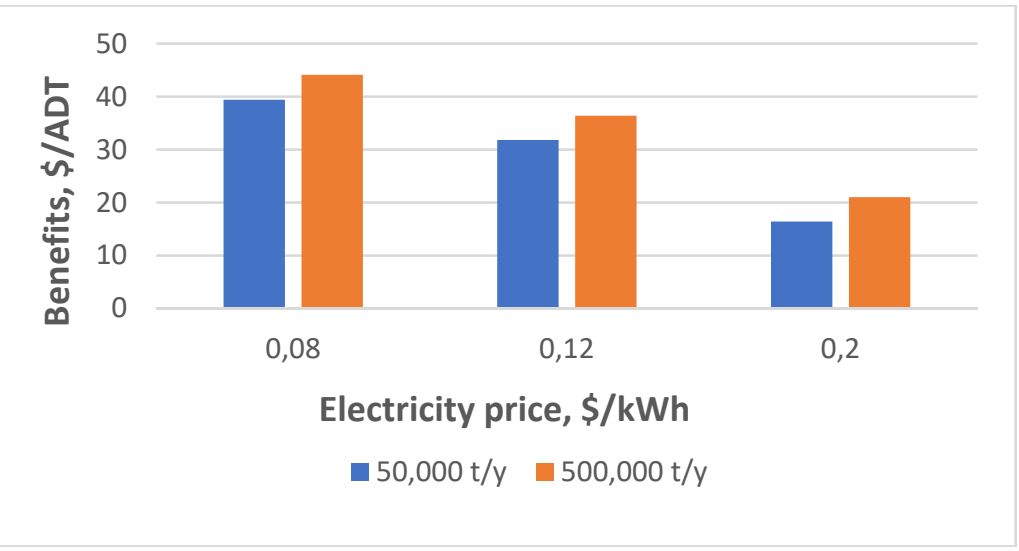

Fig.6. New MW technology benefits depending on electricity costs and pulp mill output.

Cost savings connected with advantages of MW technology use in chemical pulp production (outlined in Table1)

make up $\$ 65.0$ per air dry tone (ADT) of pulp for softwoods (this value does not include larger chip size usage, water use reduction and other environment aspects). Depending on electricity costs in the range of $\$ 0.08$ to $\$ 0.20 / \mathrm{kWh}$ (after withdrawal costs associated with MW treatment) the benefits of applying the new technology application range from $\$ 44$ to \$16/ADT (Fig.6).

Economic modelling of MW technology application in different Russian conditions, depending on mill throughput, raw material, chemical costs, energy costs, and capital costs, gave the following benefits for pulp mill with output 50,000 to 500,000 ADT/year at world pulp price US\$860/ADT:

\begin{tabular}{|c|c|c|c|}
\hline \multirow{2}{*}{$\begin{array}{c}\text { Pulp mill } \\
\text { output, } \\
\text { ADT/year }\end{array}$} & \multicolumn{3}{|c|}{ Electricity price, $\$ / \mathrm{kWh}$} \\
\cline { 2 - 4 } & 0.08 & 0.12 & 0.20 \\
\hline 50,000 & 2.0 & 1.6 & 0.8 \\
\hline 100,000 & 4.2 & 3.4 & 1.9 \\
\hline 200,000 & 8.6 & 7.1 & 4.0 \\
\hline 500,000 & 22.1 & 18.2 & 10.5 \\
\hline
\end{tabular}

It is difficult to assess ecological advantages in dollars because they are determined on the individual conditions of the chemical pulp mill. Developed by the author, "Methodology of Knowledge-based High Technology Financing" [5] outlines the mechanism for making optimal decisions in concrete conditions for technology commercialization. 


\section{Conclusions}

MW wood modification technology use in chemical pulping provides significant advantages: increase in mill throughput, reduction of chemical and power consumption, increase in pulp quality and yield, improvement of environmental performance. Specific costs of softwood chip processing in MW plant at electricity costs $\$ 0.08-\$ 0.12 / \mathrm{kWh}$ are $\$ 25.4-\$ 33.7$ /ADT of pulp. Electricity costs form the most significant part of the total specific costs of MW processing and form 51-69\% share in the total specific costs at electricity prices $\$ 0.08$ to $\$ 0.12 / \mathrm{kWh}$. At the same conditions capital costs form $15-20 \%$ share, labour costs forms $5-18 \%$ share of the total specific costs. The electricity cost increase from $\$ 0.04$ to $\$ 0.24 / \mathrm{kWh}$ leads specific MW processing cost rise by 2.7 to 3.1 times at pulp mill output range 50,000 to 500,000 ADT/year.

New technology application in different Russian conditions can provide a significant reduction in the softwood chemical pulp production costs and result in profit up to $7-22$ Mil US\$ per year for pulp mills with output more 200,000ADT/year at electricity costs $\$ 0.08-0.12 \$ / \mathrm{kWh}$. High economic advantages of this microwave technology application in pulp and paper industry recommend it for commercialisation. The technology can be used by pulp mills with batch and continuous digesting and not limited by mill throughput.

\section{References}

1. Compere A. L. et al. Increasing yield and quality of low-temperature, low-alkali kraft cooks with microwave pretreatment. US Department of Energy. Industrial Technologies Program. FY06 ITP Forest Products Portfolio Peer Review. April 56, 2006. Atlanta, Georgia, USA. Presentation. 19 pp. 2006.

2. Compere A. L. et al. Microwave Pretreatment to Decrease Pulping Energy and Chemicals. TAPPI Pulping Conference, 7 pp. 2007, www.tappi.org/Downloads/unsorted/UNTITLED--PS0442pdf.aspx

3. FAO Statistics. Yearbook of Forest Products. 2016. http://www.fao.org/3/ai7304m.pdf. Pages 144-145.

4. Lagutina T F., Bogolitsin K G., Gusakova M A., Ecological assessment of pulp mill activities in cellulose paper industry. Prospect ways of waste utilization. Journal of Russian Chemical Society (Mendeleev's name) LV (1), UDK 574-676, 2001. 5. Leshchinskaya A. F. Methodology of Knowledge-based High Technology

Financing. RGTEU, Moscow. ISBN 978-5-87827-506-4 (UДК 336.645.1 L54), 2012

6. Oak Ridge National Laboratory. Industrial Technologies Program. Completed R\&D. Increasing Yield and Quality of Low-Temperature, Low-Alkali Kraft Cooks with Microwave Pretreatment, 2007, http://www.ornl.gov/sci/ees/itp/CompletedRD.shtml.

7. Torgovnikov G. and Vinden P., Microwave wood modification technology and its applications. Forest Products Journal. 2010, Vol 60, (4), 173-182.

8. US Department of Energy. Industrial Technologies Program. Forest Products.. Increasing yield and quality of low-temperature, low-alkali kraft cooks with microwave pretreatment. Project Fact Sheet. pp. 1-2. 2007. 


\title{
MICROWAVE GENERATED PLASMA RAILWAY TRACK TREATMENT
}

Julian Swan ${ }^{1}$, Matt Candy ${ }^{1}$, Marilena Radoiu ${ }^{2}$, Gareth Richardson ${ }^{3}$

${ }^{I}$ The Imagination Factory, Power Road Studios, 114 Power Road, City, London, UK

${ }^{2}$ Microwave Technologies, 30 rue Marguerite 69100, Lyon, France

${ }^{3}$ Industrial Microwave Systems, 20 Alston Drive, Bradwell Abbey, Milton Keynes, Buckinghamshire, MK13 9HA, UK

julian@imaginationfactory.co.uk

Keywords: microwave, plasma, high power, thermal effects, mobile, rail, real-world testing

\begin{abstract}
Braking conditions are a fundamental issue for the railway and have been a limiting factor in network capacity \& timetabling. This work was focused on taking high power microwave generated plasma out of the laboratory into a railway environment.

The Imagination Factory with no experience in microwave generated plasma has partnered with experts in this field to develop a mobile system which delivered $15 \mathrm{~kW} 2.45 \mathrm{GHz}$ microwave generated plasma - Fig. 1. The plasma was created within a dielectric tube placed in a monomode microwave cavity; the atmospheric plasma sustained in different inert gases (nitrogen, argon) as well as mixtures of inert gases with reactive molecules was jetted directly onto the railhead as to change the conditions for the wheel-rail interface. This technology is hoped to be a game changer in enabling predictable \& optimized braking on the railway network. Challenges encountered during the demonstration phase will be discussed.
\end{abstract}

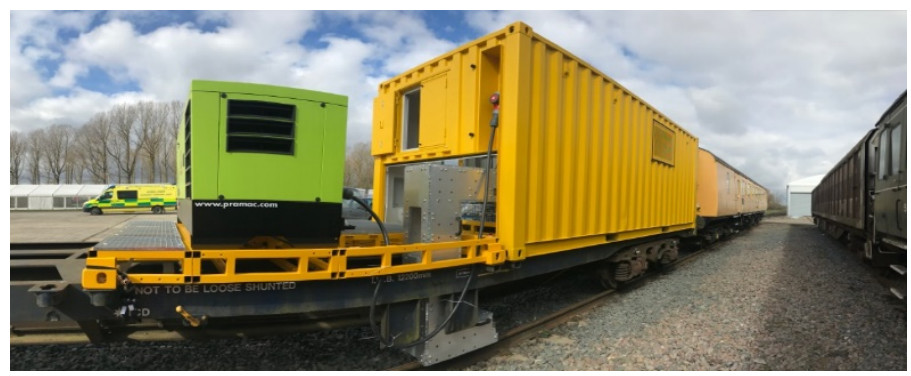

Fig. 1. Fullscale Demonstrator

\section{Introduction}

Effective railway acceleration and braking is reliant on the small contact patch between wheel and rail. This is roughly $1 \mathrm{~cm}^{2}$ and must support high loads under numerous different conditions. Traction in the contact is generated because of torque being applied through the driving wheelsets and low levels of traction, often known as low adhesion, between wheel and rail can occur under certain conditions and cause difficulties when accelerating or braking.

The wheel-rail contact is an open system and therefore exposed to several conditions and contaminants, $3^{\text {rd }}$ layer, that may influence adhesion. This can include natural contaminants such as leaves and organic debris, iron oxides and wear particles, as well as artificial contaminants such as sand, oil and salt. Some of the causes of low adhesion in the wheel- 
rail contact are well understood and can be predicted and mitigated, whilst others remain hard to prevent. Different environmental conditions such as temperature, precipitation and humidity change the properties of this third body layer and therefore change adhesion conditions on the railway. Low adhesion can lead to wheel slides and slips during acceleration and deceleration, which can cause large amounts of damage to the wheel and rail as well as causing safety issues and delays if a train cannot accelerate or decelerate when necessary.

The starting point of our project was work completed by British Rail in 1969-72 which had delivered 16kW DC Thermal Arc through a $5 \mathrm{~mm}$ nozzle to improve the adhesion properties on the railhead [1]. The Imagination Factory has partnered with experts in the field to develop a mobile system using $15 \mathrm{~kW}, 2.45 \mathrm{GHz}$ microwave generated plasma to treat the railway track as to remove the 3 rd layer. The plasma can be tuned to deliver varying amounts of 'removal' by controlling its temperature \& reactivity. Currently, it is not known which plasma "recipe" is the most effective and it is possible the solution is not one size fits all due to the complex composition of the 3rd layer.

Laboratory-based tests were designed to optimise the microwave power and the performance of the plasma-based cleaning system using a phantom layer. These tests are to be benchmarked against the final tests made in the actual railway track during the Autumn season to validate the closeness of the phantom layer to the actual 3rd layer and the effectiveness of the plasma treatment. Other tests such as the hardness testing and microstructure analysis are conducted to ensure that the plasma treatment does not damage the rail track to ascertain the application of the plasma treatment in the railway industry.

\section{System Description}

The atmospheric pressure plasma was created within a dielectric tube placed in a TE01 monomode microwave cavity; the atmospheric plasma sustained in different inert gases (nitrogen, argon) as well as mixtures of inert gases with reactive molecules was jetted directly onto the railhead as to change the conditions for the wheel-rail interface - Figs. $2 \&$ 3. This technology is hoped to be a game changer in enabling predictable \& optimized braking on the railway network. Challenges encountered during the demonstration phase will be discussed.

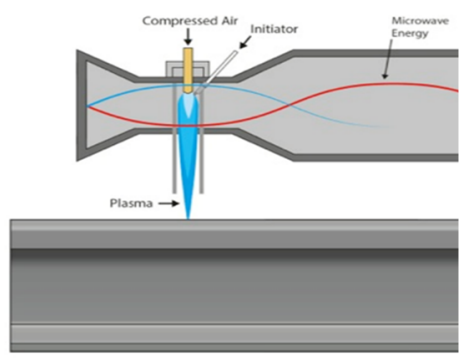

Fig. 2. Plasma Head Cross-section Schematic 


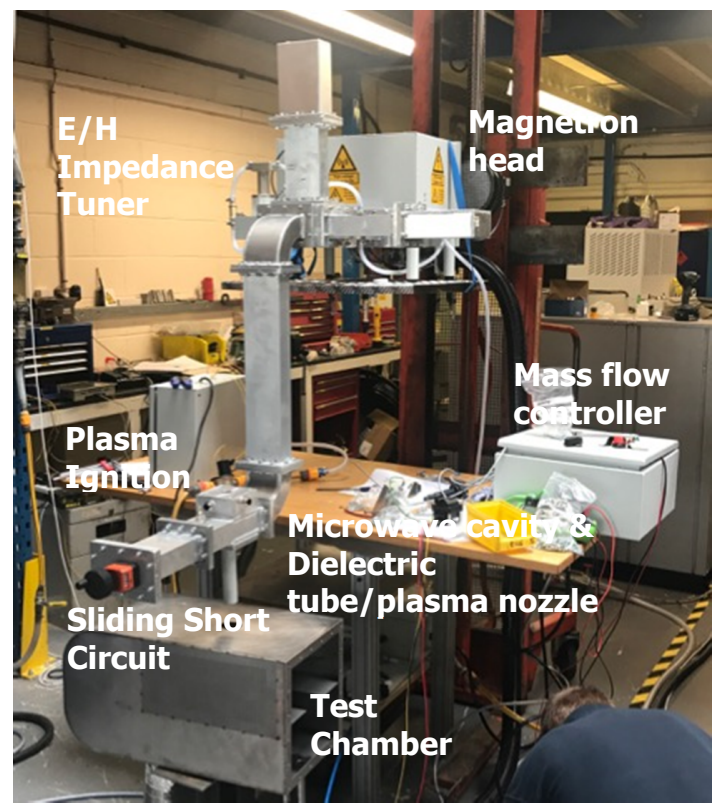

Fig. 3. Laboratory set-up

\section{Laboratory Testing}

\subsection{Development $\&$ optimization of the microwave plasma system}

The microwave system was commissioned, calibrated \& tuned at low power levels $(2 \mathrm{~kW})$. The initial plasma testing was completed with quartz glass tubes with an inside diameter ranging from 16 to $22 \mathrm{~mm}$. The outside diameter of the dielectric tube was set to fit the fixed mechanical interface in the plasma chamber. The focus of this work was to understand the relationship between gas flow rate and power input. Initially we used a measure of the rate of increase in temperature of a target block of aluminium to evaluate the relationship between flowrate, vortex entrainment \& microwave power input - Fig. 4.

The main objective of the work was to develop as high an intensity / concentration of the downstream plasma as possible. The quartz glass material had limitations with temperature and power input. It was therefore decided to embark on investigation into alternative 'microwave transparent dielectrics' which could support this goal. In addition to quartz, nonoxide ceramics as silicon carbide, aluminium nitride and boron nitride were investigated.
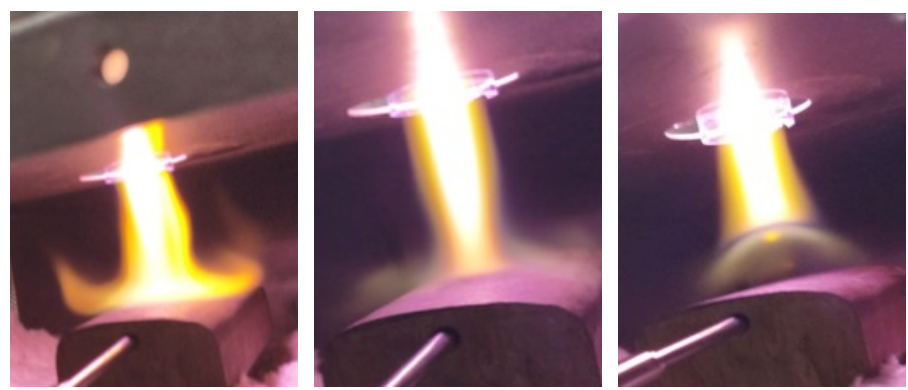

Fig. 4. Effect of gas flow on plasma afterglow delivery at $2 \mathrm{~kW}$ 
The silicon carbide ( $\mathrm{SiC}$ ) tube - SiC melting point $2830^{\circ} \mathrm{C}$ - was noted to absorb more microwave power than this setup can dissipate, which led to the melting of the tube.

Aluminium nitride (AlN), Shapal ${ }^{\mathrm{TM}}$, in addition to being a very difficult to machine ceramic had a catastrophic failure on the first plasma test - Fig. 5. This material was discounted from further development.

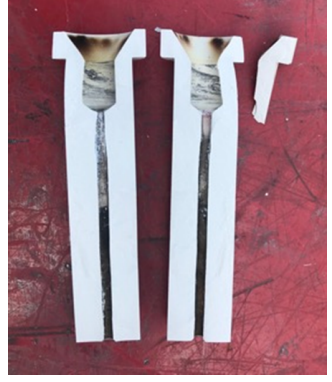

Fig. 5. Thermal shock damage of the AlN tube

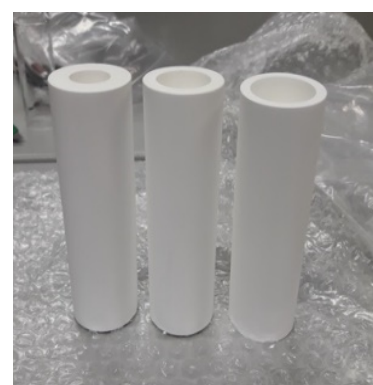

Fig. 6. BN machined tubes

The most successful material for our research - thermal shock \& plasma efficiency - was found to be BN. Various BN blends were sourced. An unbound variant AX05 proved incredible flexibility for machining \& good temperature resilience. This enabled the team to develop a range of tube ID and profiles.

\subsection{Gas flow}

The development of a vortex in the gas delivery is critical to creating a stable plasma. Computational Fluid Dynamic (CFD) analysis of the gas entrainment was completed to confirm vortex creation - Fig. 7. This included evaluation of the number of gas supply ports and pre-conditioning 'swirl' rings. These were then practically evaluated in the static testing section. A two-port solution with swirl ring was adopted for all testing.
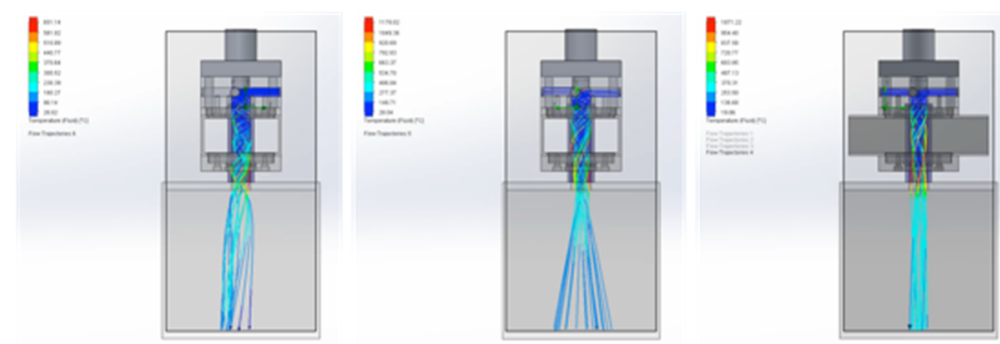

Fig. 7. CFD simulation of gas flow and gas inlet design

\subsection{Gas type investigation}

Plasma was successfully ignited and sustained in inert gases like argon and nitrogen and in mixed nitrogen \& oxygen and nitrogen $\&$ water, Figs. 8 a-c. 


\section{Neutral Plasma}

Initial tests were completed using bottled nitrogen. This was utilized mainly to reduce the likelihood of NOx creation. The performance of the nitrogen plasma is mostly related to a thermal ablation effect. Note: Purple colour indicates stable plasma, Fig. 8a.

\section{Reactive Plasma}

Two types of reactive plasmas were created by mixing nitrogen with different amounts of oxygen and water.

The addition of $\mathrm{O}_{2}$ to the $\mathrm{N}_{2}$ was done in order to promote oxidative reactions and hence, to create active species within the plasma which will oxidize the $3^{\text {rd }}$ layer - Fig. $8 \mathrm{~b}$. Note: White colour indicates stable plasma.

By bubbling $\mathrm{N}_{2}$ through a water scrubber (water at $20^{\circ} \mathrm{C}$ ), a dual reactivity (reductive \& oxidative) was created. The water molecule splits into $\mathrm{H}^{+}$fueling reduction \& $\mathrm{OH}^{-}$oxidative species in the plasma - Fig. 8c. Note: Greenish white colour indicates stable plasma

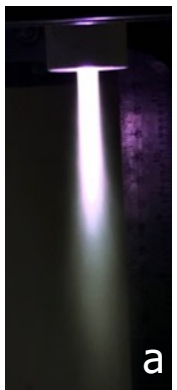

$100 \% \mathrm{~N}_{2}$

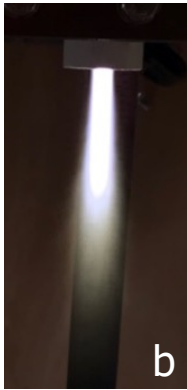

$5 \% \mathrm{O}_{2}$

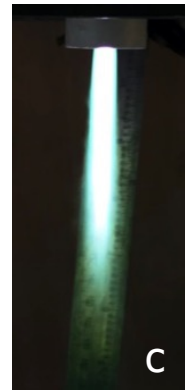

$\mathrm{H}_{2} \mathrm{O}$

Fig. 8. Plasma afterglow in different gases

\subsection{Development of infrared temperature measurement methodology}

Test methodology was developed to enable the creation of stable plasma and replicate the dynamic movement of the railhead through the established afterglow, Fig. 9.

The microwave behavior of the plasma tube materials changes with temperature, which detrimentally affects the plasma stability. It has been established that there are 3 distinct phases of plasma within the test, ignition $\rightarrow>$ stability $\rightarrow$ decay. The development of more exotic materials \& cooling designs will be continued in the next phase to reduce this effect. The evaluation of the performance of the plasma was achieved through a sample steel block being introduced through the plasma after ignition and during the stable period of testing; this was generally accepted as 2 seconds after ignition. The steel block speed can be adjusted using the pneumatic control circuit for slow, medium \& fast test speeds representative of the DMU speeds at British Rail testing facility.

The use of FLIR infrared cameras enabled the recording of the surface temperature on the block. This system enabled rapid evaluation of thermal performance for each diameter/ power/ flowrate \& gas type. The camera has limitations in frame rate $\&$ temperature range and in order to capture the full range of surface temperature multiple tests were completed with different electronic filters. An example of the measurement is shown in Fig. 10, dielectric tube internal diameter $4 \mathrm{~mm}, 15 \mathrm{~kW}$, nitrogen flow rate $35 \mathrm{~L} / \mathrm{min}$. 


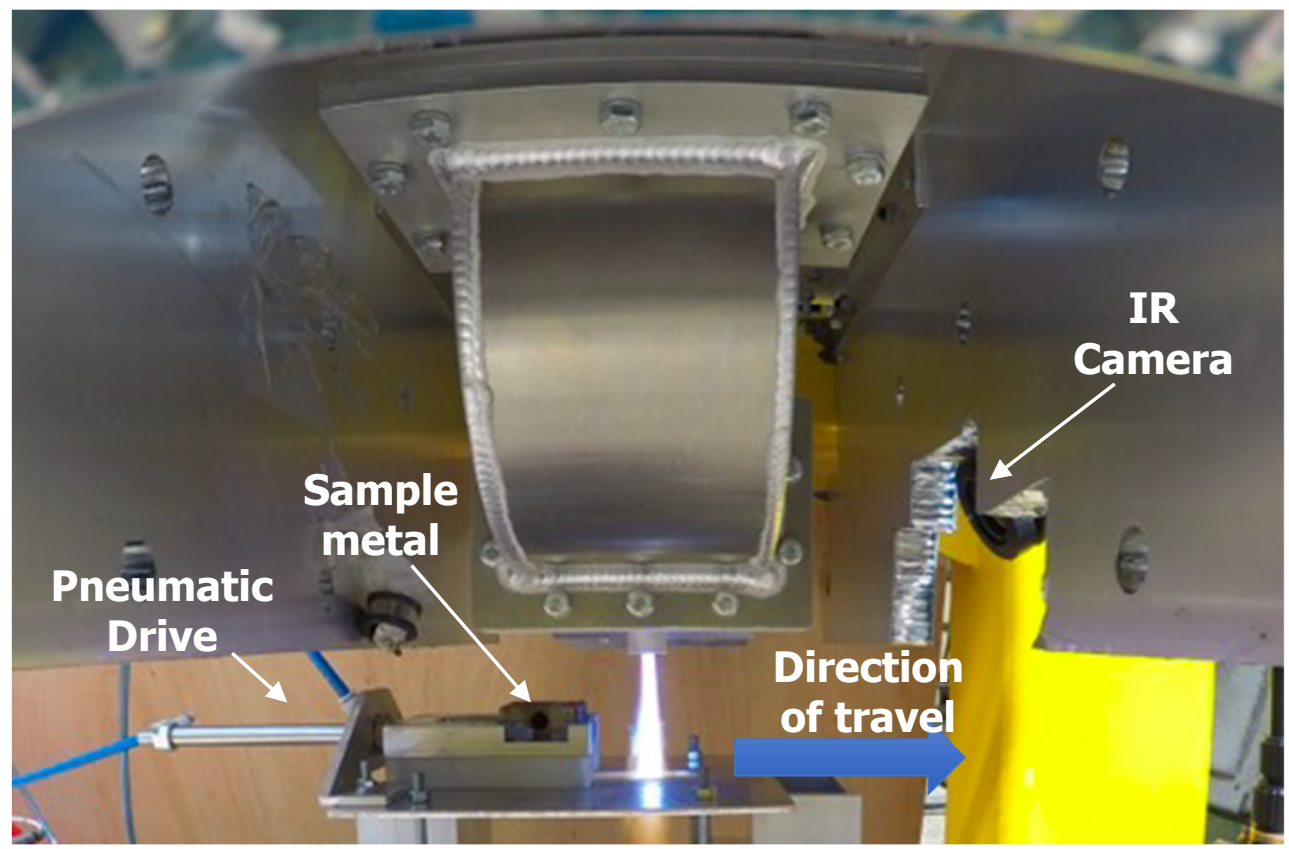

Fig. 9. Sliding shuttle \& metal block

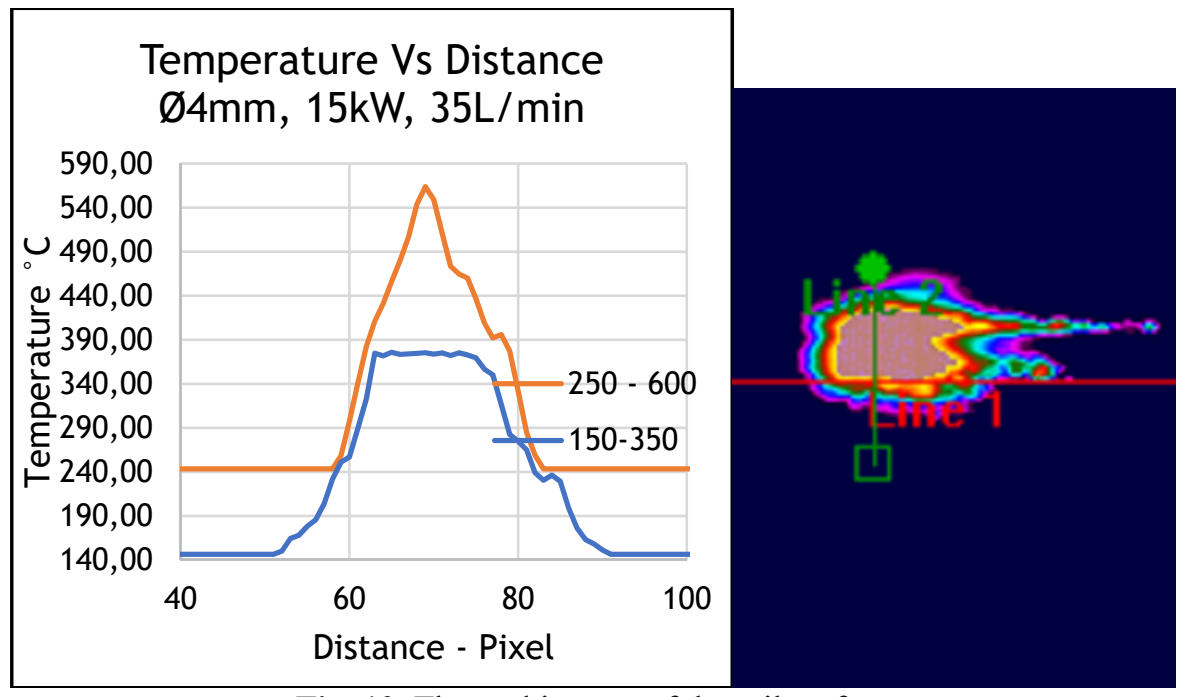

Fig. 10. Thermal images of the rail surface

\section{Mobile Testing, Results \& discussion}

The mobile test equipment, Fig. 11, was satisfactorily protected from shock damage using sprung AV mounts. Limitations of DMU speed control \& repeatability of braking cycle led to the exclusion of brake deceleration testing from the evaluation methodology for on-track testing. The track lateral movement \& relative vertical movement limited the test site to the 
straight and testing within a $20 \mathrm{~m}$ zone with vertical movement less than $8 \mathrm{~mm}$. The plasma nozzle was pre-set to a maximum height of $25 \mathrm{~mm}$ from the railhead, Fig. 12. During the testing, the track has been temporarily marked at meter increments to aid in analysis.

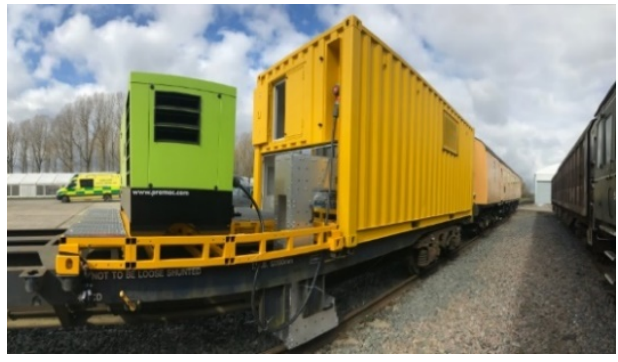

Fig. 11. Mobile test rig at British Rail testing facility

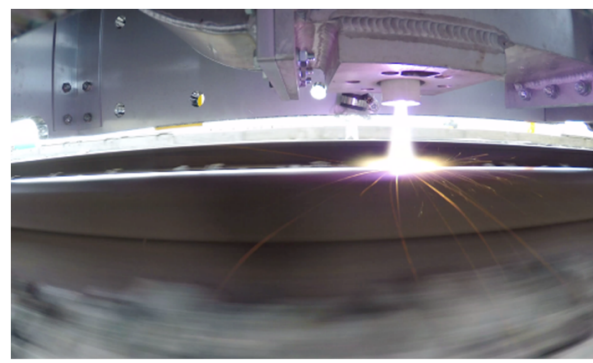

Fig. 12. Go-Pro image of plasma on the rail during mobile testing

Testing was completed for $3 \mathrm{~mm}, 5 \mathrm{~mm}, 7.5 \mathrm{~mm}$ ID plasma tubes at slow $(5 \mathrm{~km} / \mathrm{h})$, medium $(10 \mathrm{~km} / \mathrm{h}) \&$ fast $(15 \mathrm{~km} / \mathrm{h})$ speeds with power ranging from $9-15 \mathrm{~kW}$. The test area was marked with cones to aid the driver in getting to speed, maintaining speed during plasma delivery and braking to a safe stopping point.

Evidence shows a rust removal effect on the railhead with the application of plasma, Fig. 13. This is most markedly seen with the $3 \mathrm{~mm}$ ID plasma tube for all types: neutral, $\mathrm{O}_{2} \&$ $\mathrm{H}_{2} \mathrm{O}$ plasmas.
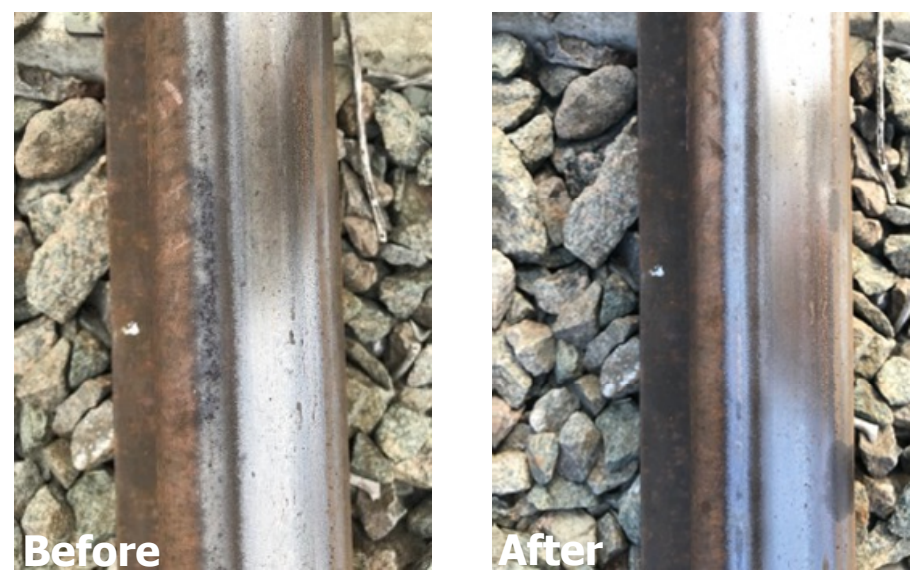

Fig. 13. Effect on railhead oxidation

As in the dynamic test protocol, FLIR infrared thermal analysis has been used to understand an indication of microwave power to speed relationship. IR \& video cameras have been side mounted on the waveguide. The video camera also enables evaluation of plasma distance to the railhead. Every test configuration has been analysed for peak temperature, width \& length of the plasma thermal area of influence for any given power input $\&$ speed - see Fig. 14. 


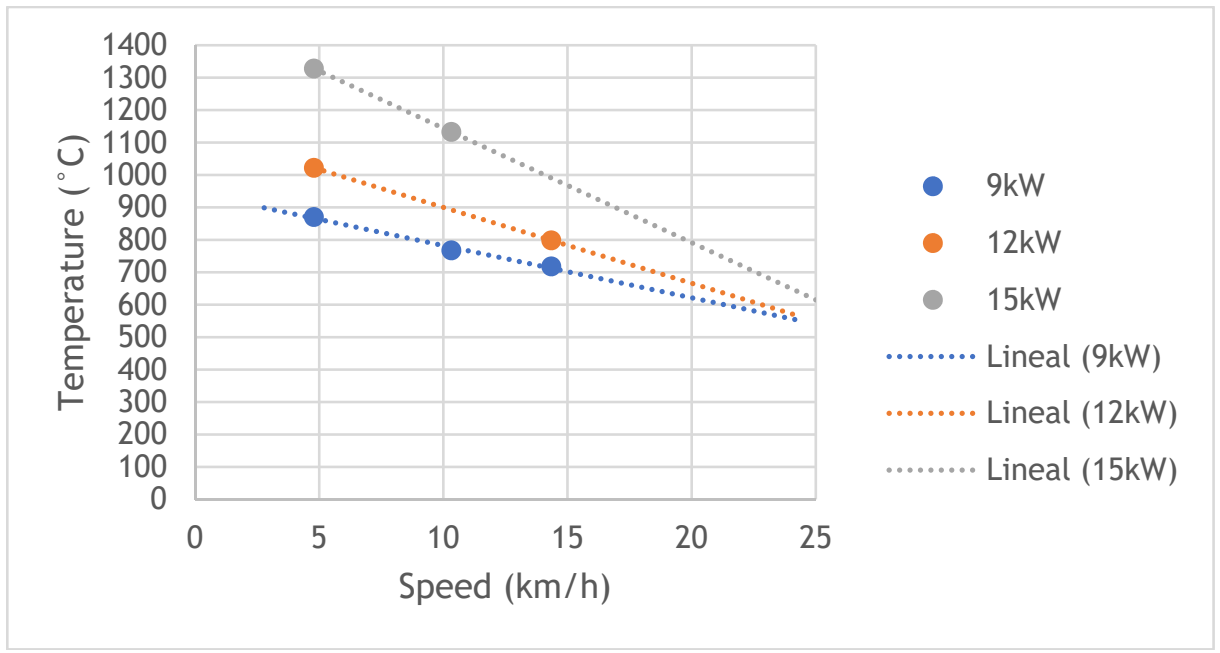

Fig. 14. Microwave power/peak temperature vs. Speed dependence, dielectric tube $3 \mathrm{~mm}$

The results clearly show a higher performance for the smaller diameter, enabling higher temperature delivery with lower power input at speed.

The peak temperature effect is believed to be most relevant for Predictable \& Optimised Braking for the on passenger mounted embodiment. This measure is indicative of energy which would be delivered onto the 3rd layer and which would create a disturbance along the running contact patch, enabling drying and removal of contaminants to give 'summer braking' conditions. The predicted energy levels are still well within the theoretical maximum proposed. This is partly due to the plasma density/energy concentration that can be achieved with the $2.45 \mathrm{GHz}$ microwave frequency.

\section{Conclusion}

An understanding \& optimization of $2.45 \mathrm{GHz}$ microwave generated atmospheric plasma in the context of track treatment has been demonstrated. The effect on 3rd layer contamination has been demonstrated in the laboratory and on the track.

Currently, $15 \mathrm{~kW}$ is the maximum power magnetron available at $2.45 \mathrm{GHz}$. In order to achieve a higher power microwave source, $896 \mathrm{MHz}$ is to be considered. When transitioning from $2.45 \mathrm{GHz}$ to $896 \mathrm{MHz}$, the plasma electron energy concentration is believed to reduce proportionally with the frequency. This, therefore, shows that there is capacity within the energy equation to accommodate available $896 \mathrm{MHz}$ magnetrons and maintain realistic electrical energy supply for onboard train systems.

\section{References}

1. Balwanz W.W., 1979, Plasma Cleaning of Surfaces. In: Mittal K.L. (eds) Surface Contamination. Springer, Boston, MA, 255-269. 


\title{
SOLID-STATE MICROWAVE PROCESSOR FOR FOOD TREATMENT
}

\author{
M.Fiore $^{1}$, N. Di Modugno ${ }^{2}$, F. Pellegrini ${ }^{3}$, M. Roselli ${ }^{4}$ \\ ${ }^{1,2}$ LEANFA Srl, Via C. A. Dalla Chiesa 6, Ruvo di Puglia, Italy \\ marco.fiore@leanfa.com,nicola.dimodugno@leanfa.com \\ ${ }^{3,4}$ Sergio Fontana Srl, Via Moscatello 2a trav., 76012 -Canosa, Italy \\ f.pellegrini@fontanasergio.it,m.roselli@fontanasergio.it
}

Keywords: microwave, solid-state, self-regulation, software, adaptive

\section{Introduction}

In a global context, the Food Processing (FP) industry is a leading manufacturing sector. Even in the periods of economic downturn, FP industry keeps being a stable, non-cyclical and robust sector with a steady growth in turnover and profitability. The popularity and sales of processed foods are still increasing, not only in the high-income Countries but even more in developing, low-medium income ones. Challenges in the market are dependent on the booming growth population, projected to hit the peak of 9Bn people by 2050: as a result, raw materials as well as natural resources will come under increased pressure to meet the growing demand for food at global level. Moreover, climate change will impact on crops, affecting food production; energy consumption will be critical for FP industries and the dramatic increase of the so-called "middle class" will generate a critical demand of higher hygiene and quality. The same will be for industry players, who will be asked to be compliant to stringent requirements in terms of food safety.

Specifically, a pressing growing need of high-quality food products is emerging, requiring food to be inherently equipped with its own bioactive elements even after the industrial processing phases for distribution. The only solution to this primary requirement is intelligent food-processing engineering minimizing the use of chemical additives while maximizing food integrity and environmental sustainability of the whole production and distribution workflow.

\section{Objective}

The present experimentation comes within a modernization trend of technologies related to processing of food and food materials, where Radiofrequency (RF) and Microwaves (MW) are often considered as alternative methods for treatment of agrofood products. In particular, the objective of the work was the definition of a modern, multi-feed MW processor based on solid-state generators.

Sergio Fontana $\mathrm{Srl}$ is a Company operating in the production of natural active principles, obtained by high-quality processing of food materials which are inherently rich in healthy bioactive compounds. Different processes are implemented, all sharing the essential need of maximally preserving the precious organoleptic properties of the incoming raw materials. Many promising applications of dielectric heating have been implemented in the FP industry; however, there are several potential problems that need to be addressed before the technology can be successfully applied to food in an industrial scale. One of the major issues of dielectric heating is the potential of incurring in hot spots within a non-homogeneous target. When this natural physical effect comes along with surface irregularities and/or inappropriate packaging, the final food quality can be severely damaged with serious economic loss. 
The microwave processor had to be designed as a flexible tool for disinfection of dry materials, possibly infested by insects or unwanted microorganisms accumulated during the previous stocking or delivery phases, and for accurate pre-heating of fresh materials to allow for optimal subsequent extraction processes.

The processor had to be conceived as a batch oven, equipped with a single front door for the load/unload operations and built to allow the presence of three parallel layers, each capable to carry two polypropylene trays containing the food materials to be processed.

The full operational flexibility of the oven, intended to work with both fresh raw materials and with dry herbs soon emphasized a clear design target: in the former case the microwave energy is dissipated into the food materials while in the latter case the dielectric loss should be ideally concentrated into the infesting organisms while safeguarding the precious bioactive elements contained in the dry products, which typically suffer from overtemperature stress.

The technical specifications of the microwave processor can be characterized by the following functions:

- availability of $3 \mathrm{~kW}$ MW power with possibility to differentiate the radiation amount among the three layers thanks to multiple feed;

- capability to extract water vapor when required;

- capability to be remotely programmed by means of standard web browsers from fixed or mobile terminals;

- capability to operate with fully customizable "recipes" allowing smart combinations of Continuous Wave $(\mathrm{CW})$ and pulsed modes, even with different radiation modes generated by each feed

- capability to measure in real-time the amount of reflected energy at every radiation port to allow self-regulating algorithms aiming at process optimization.

\section{The "kopernicook" modular architecture}

The required system has been designed by a modular architecture where the elementary module is a $250 \mathrm{~W} 2450 \mathrm{MHz}$ solid-state generator equipped with an embedded interface board allowing serial communication within a digital communication bus through RS485 standard.

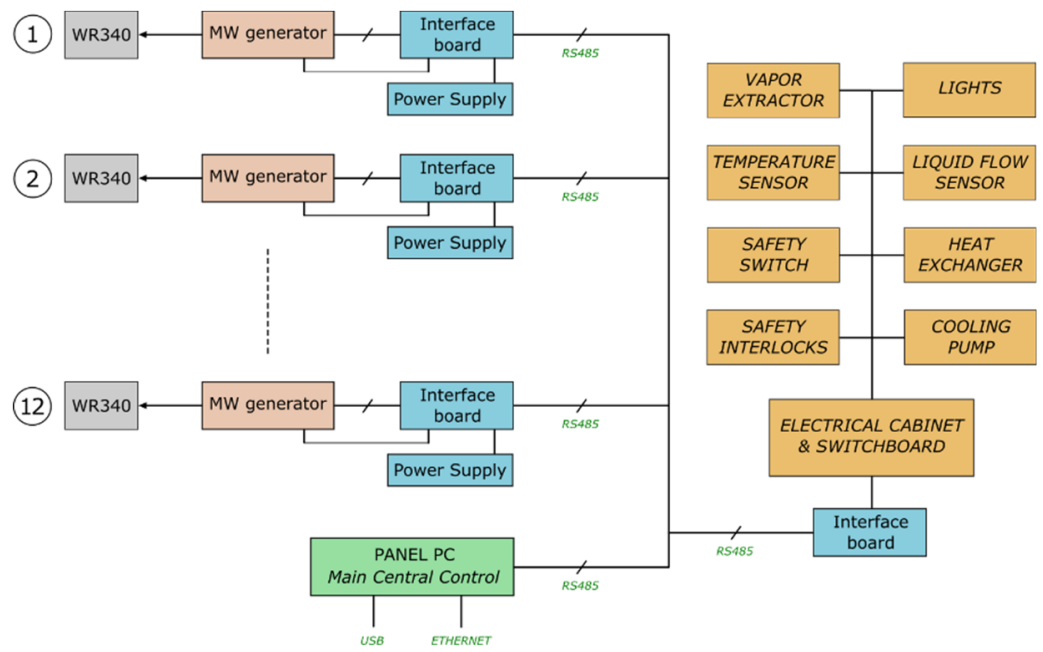

Fig. 1. Block diagram of the Kopernicook MW Processor 
The reverberating chamber, conceived to host the 6 trays distributed over the three parallel layers, has been designed with six feeds on the left wall and six on the right wall, vertically and horizontally aligned in order to have four $250 \mathrm{~W}$ feeds per each layer.

Due to the inherently multimodal behavior of the chamber, the field distribution in each layer is influenced by the contribution of each of the microwave generators and the overall field distribution also depends on the type and amount of materials contained in the trays. Moreover, due to the time-varying dielectric properties of the food materials, the field distribution also dynamically changes during the running process, determining a complex interaction with the materials that needs to be monitored and managed by the distributed control system.

Each generator is equipped by an output circulator guaranteeing safe operation even with $100 \%$ reflected power and by an autonomous microprocessor-based control system with local monitoring and management capabilities, triggering immediate protection countermeasures at the occurrence of conditions that might damage the generators hardware.
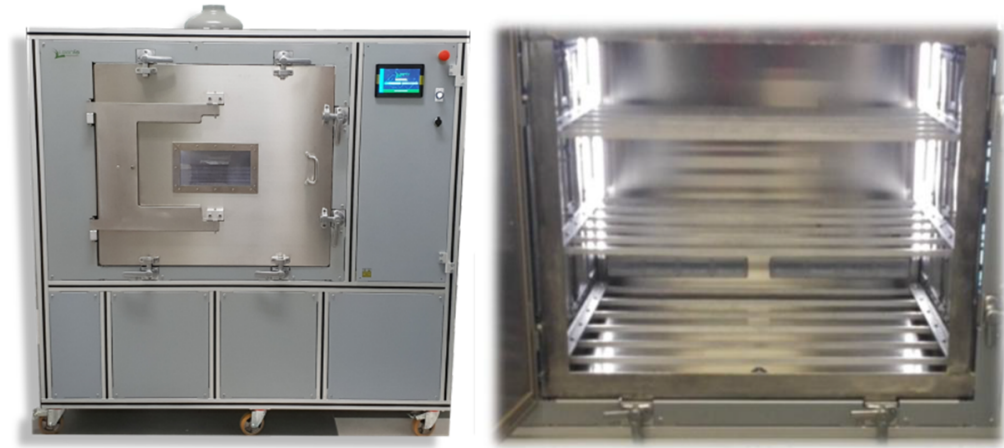

Fig. 2. Front view of the Microwave Processor and internal view of its chamber

All the operation of the microwave processor is regulated by a "Recipes Editor", a web application running on the central control unit, accessible by any web browser from remote locations. The editor allows creating "recipes" (arbitrary compositions of microwave emission phases, totally configurable by the end user) and uploading them in a dynamic database in the central control unit. The editable parameters are recipe duration, frequency, $\mathrm{CW}$ or Pulsed radiating modes, phase durations, power levels and activation/deactivation of vapor extractor, and each phase can be added in "simple" or "advanced" mode. A simple phase drives all the generators simultaneously with identical parameters, an advanced phase allows every single generator to radiate with independent parameters and both simple and advanced phases can be combined within a recipe.

The central control unit, based on an industrial-grade Panel PC, allows to select one of the uploaded recipes and to run it with the loaded materials. The software allows to monitor the operating parameters and, in particular, it shows the real-time measurement of the twelve reflected powers values.

Figure 3 shows some screenshots from the Recipes Editor application with details about parameters setting, in CW, pulsed, simple and advanced modes. 


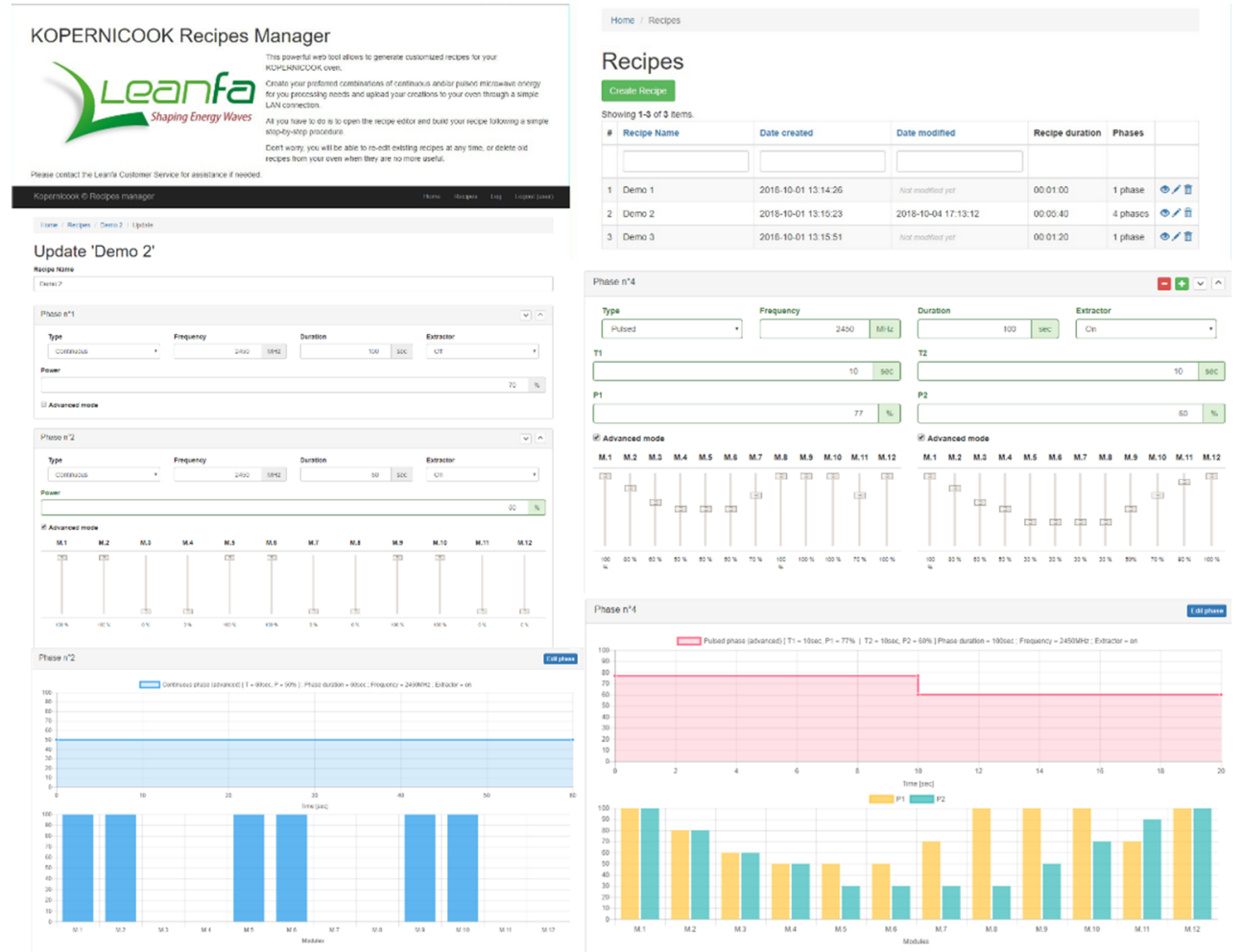

Fig. 3. Details of the Recipes Editor web application

The central control unit is not simply a collector of recipes, it is much more a high-level supervisor that, thanks to real-time monitoring of the reflected power levels at every microwave port, is capable to dynamically monitor - both in the time and space domains the capability of the materials to absorb the radiated energy and to evaluate cross-coupling contributions between the 12 ports.

This information is used to trigger a self-regulating algorithm (indicated by a brain icon on the front-panel GUI, see Fig. 4) which automatically takes control of the radiated power from each port, replacing the original recipe until certain well-tailored conditions are met.

\section{Self-regulating algorithms}

Different self-regulating algorithms have been implemented, based upon the definition, for each port, of two thresholds of reflected power level, that activate or deactivate a "foldback" power function, which is triggered by the central control system and autonomously managed by the control system of each microwave generator. Moreover, a real-time automatic selection of the optimal operating frequency (in the $2400-2500 \mathrm{MHz}$ range) has been implemented, used to achieve uniform thermal profiles with the minimum possible power consumption.

The algorithm has been designed to accomplish different goals:

- protection of the generators' hardware;

- overall minimization of the reflected energy during the process; 
- correction of deviations from the nominal expected process due to parametric shifts in the targets' physical characteristics.

The system has proven to be very robust and, thanks to the hysteresis guaranteed by userselectable reflected power thresholds, it always reaches a stable steady-state operating condition.
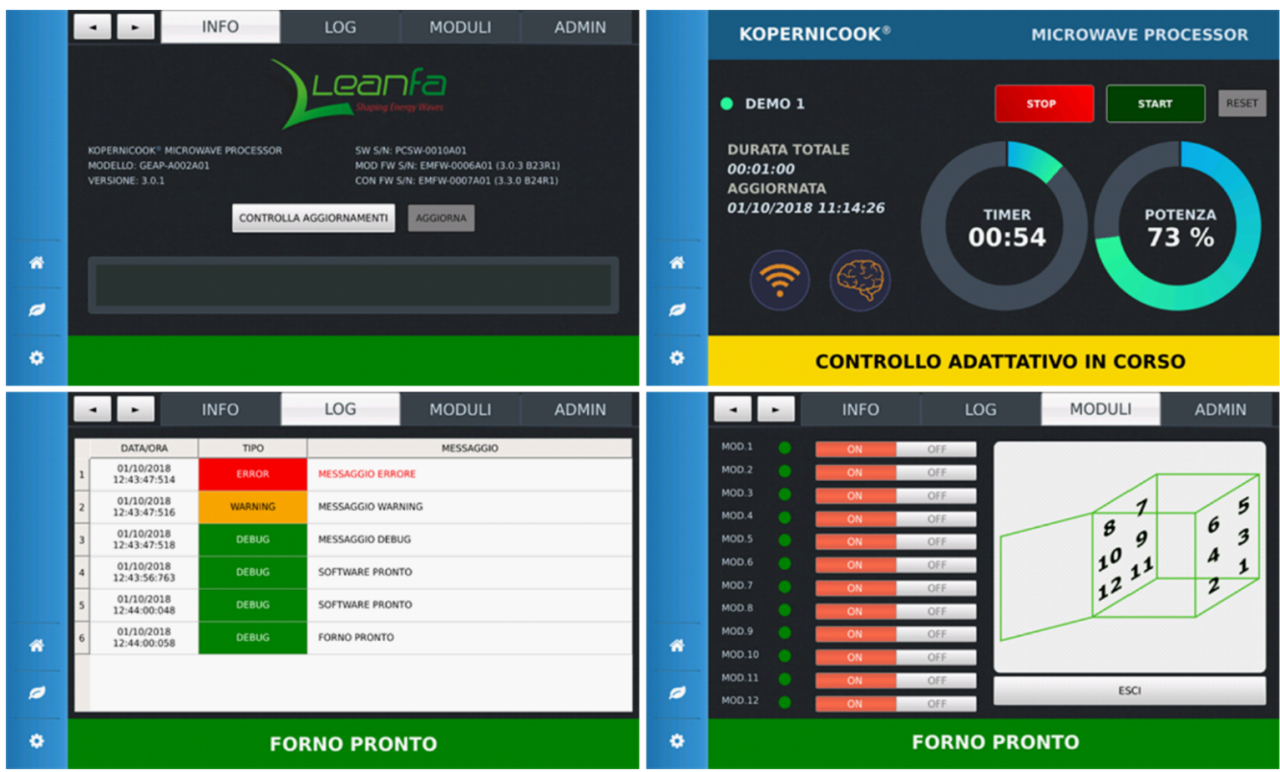

Fig. 4. Screenshots from the central control panel

The following algorithm describes one of the most effective self-regulating workflows, used with dry materials (a simplified pseudo-code is used here for schematization).

1) Central control unit:

if state $==0$

send OutPwrLev to all active gen

if (number of gen with RefPwr $\geq$ HighRefThreshold) $\leq 2$

switch off only those gen

state $=1$

else if (number of gen with RefP wr $\geq$ HighRefThreshold) $>2$

send FoldbackOn command to all active generators

state $=1$

if state $==1$

else keep state $=0$

if (number of active gen with RefPwr $>$ LowRefThreshold) $==0$

send FoldbackOff command to all running gen

state $=0$

else keep state $=1$

2) Each active generator unit in the running recipe:

if state $==0$

set $F w d P w r=O u t P w r L e v$

keep state $=0$ 


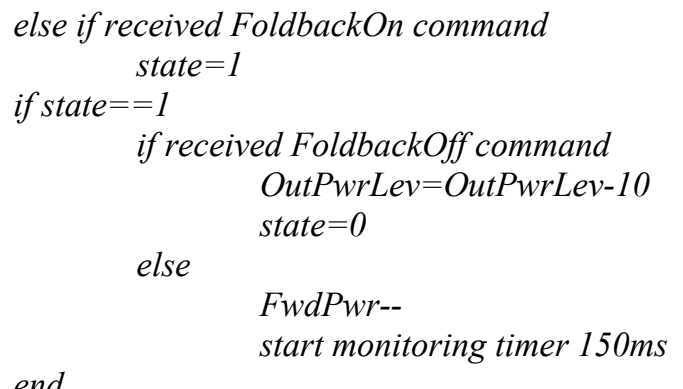

The algorithm is designed to manage the overall field distribution with a distributed intelligence approach by taking control of the process until the end of each phase in a running recipe is reached. At the beginning of the following phase in the same recipe, the control is again taken by the central control unit obeying to the nominal recipe.

\section{Results}

When enabled, the self-regulating algorithm works by automatically reducing the overall radiated power to a safe level corresponding to the user-defined threshold and later trying to recover a higher radiating level, slightly lower than the previous nominal level. The result is an optimal convergence towards minimum reflected energy, smoothly following the natural tendency of the materials to absorb less microwave power while their temperature increases and their water content decreases. After a progressive fine tuning of the recipes conceived by the engineers of Sergio Fontana Srl, the self-regulating process triggers only when the loaded materials show stronger deviations from the nominal range of physical parameters as weight, moisture and/or initial temperature.

Lower deviations (less than 10\%) have been observed on the overall radiated power with fresh materials, while higher deviations (up to $28 \%$ ) have been observed when operating with dry herbs, in accordance with the well-known sensitivity of the dielectric properties to the average moisture level.

Moreover, the distributed intelligence approach has demonstrated to allow even better regulation in case of parametric deviations limited to one or two layers in the processing chamber, allowing automatic power level differentiation according to the different capability of each layer to absorb the radiated energy.

In the following Figure 5 three different sample thermal profiles are shown, obtained with different recipes applied to fresh fruit (left and center) and to dry herbs (right).
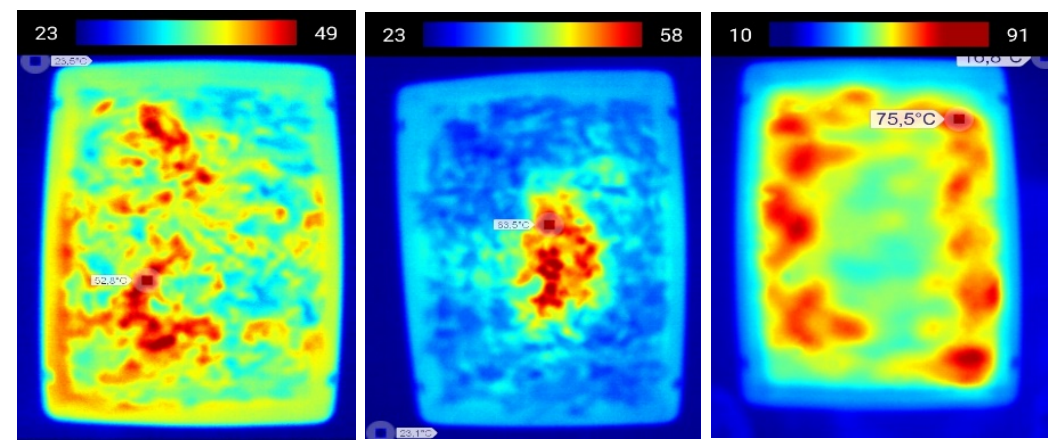

Fig. 5. Thermal profiles obtained with different fine-tuned recipes 
In particular, in-field operation has demonstrated the usefulness of pulsed radiation modes for disinfection of dry herbs, helping in concentrating the dielectric loss into the infesting organisms, while avoiding injuries on properties of the food materials generated by unwanted overtemperatures. The most homogeneous thermal results, correlated to higher disinfection rate, have been achieved thanks to the application of frequency shift using recipes with a combination of $\mathrm{CW}$ radiation modes during the first 5-7 minutes of operation and pulsed modes with decreasing duty cycles during the following 15-23 minutes, enabling water vapor extraction during the initial $30-40 \%$ of the recipe.

\section{Conclusions}

The complete absence of mechanical stirring elements in the microwave processor is compensated by the distributed architecture with 12 solid-state generators, each fully configurable in terms of radiating parameters by means of the Recipe Editor application. By using well-tailored recipes, it is possible to obtain fairly well controlled thermal profiles over the material distributed on the three layers.

Further development will be performed by progressively moving to radiation algorithms with arbitrary waveforms and by adding real-time temperature and moisture sensors to fulfil a complete monitoring network for a more accurate generation of feedback to the distributed control architecture. The technological roadmap pictured by power devices manufacturers is further encouraging new development effort into accurate hardware-software co-design aimed at facilitating the desired industrial scale-up towards efficient continuous processing systems.

\section{References}

1. OECD, "Population and growth rates, 1950-2050, actual and projected", in Local economic strategies and systems, OECD Publishing, Paris, 2014

2. Yakovlev, V. V., IEEE MTT-S International Microwave Symposium, 2015

3. Tiefeng Shi, \& Kaldi Li., IEEE/MTT-S International Microwave Symposium Digest, 2012 (generatore 157W CW 2450MHz)

4. Piyasena, P., Dussault, C. Koutchma, T., Ramaswamy, H.S., and Awuah, G.B., Critical Reviews in Food Science and Nutrition 43(6), 587-606, 2003

5. Zuber, S., Joss, M., Tresch, S., Kleingries, M., 21st International Drying Symposium, Valencia, 2018

6. Przemyslaw Korpas, Mateusz Krysicki, and Andrzej Więckowski, Multiphysics Models and Materials properties, Karlsruhe, Germany 2014

7. Awuah, G., Ramaswamy, H., \& Tang, J., Radio-Frequency Heating in Food Processing, 323-353, 2015 


\section{HYBRID MICROWAVE WITH HEAT RECOVERY FOR AN EFFICIENT DRYING PROCESS}

Willem P.R. Deleu ${ }^{1}$, Vincent Goovaerts ${ }^{1}$, Carlo Groffils ${ }^{1}$, ${ }^{1}$ MEAM test-center, Industrieweg 11193540 Herk-De-Stad, Belgium
willem.deleu@meam.be

Keywords: microwave, hybrid heating, heat recovery, drying

Microwave technology has come a long way from the sixties when the first microwave ovens were developed. At MEAM we have three distinct generation of microwave technology with increasing efficiency: The "first generation" of microwave ovens uses only microwaves to heat the substrate and with an efficiency of around 63\% in converting electrical energy to microwaves, this means $37 \%$ of the electric energy is lost as heat. ${ }^{1}$

The "second generation" is a hybrid oven, using both microwaves and hot air in the cavity. 2,3 This hot air is originating from the air cooled magnetrons (the $37 \%$ heat loss) and thus requires no extra energy input. The "third generation" is with added heat recovery either on the exhaust air and/or cooling water loop. The heat is used to preheat ingoing product and is especially useful if products needs to be defrosted before the microwave treatment. The possibility to use second and/or third generation microwave technology always depends on the industrial process so it is not always possible to use them. For this paper we look into drying processes at relative low temperatures $\left(<100^{\circ} \mathrm{C}\right)$ and the effect of the different generations of microwave technology on the efficiency of the process.

\section{Drying efficiency}

There are many possible ways to define/calculate the efficiency of a drying process. we define it here as the theoretical energy required to create $1 \mathrm{~kg}$ of steam of $100^{\circ} \mathrm{C}(2676$ $\mathrm{kJ} / \mathrm{kg})^{4}$ divided by the electric energy used to evaporate $1 \mathrm{~kg}$ of water in the microwave drying process (see Eq. 1). The primary reason for calculating this for a microwave drying process is to know the energy cost of drying, and to be able to compare it to other (microwave) drying processes. The second reason is to be able to see in a single process what the important parameters are and how they impact the energy consumption (= price).

$$
\begin{aligned}
\text { Drying efficiency } & =\frac{\text { theoretical energy required }}{\text { Electric energy used }} \\
\text { Drying efficiency }[\%] & =\frac{\mathrm{H}_{2} \mathrm{O} \text { evaporated }\left[\frac{\mathrm{kg}}{\mathrm{h}}\right] * 2676\left[\frac{\mathrm{kJ}}{\mathrm{kg}}\right]}{\text { Electric power }[\mathrm{kW}] * 3600\left[\frac{\mathrm{s}}{\mathrm{h}}\right]}
\end{aligned}
$$

Eq. 1 Drying efficiency formula

By using the fixed value of $2676 \mathrm{~kJ} / \mathrm{kg}$ steam produced, we underestimate the thermodynamic efficiency when process conditions are different: this value is lower when ingoing product temperature is higher than zero and/or outgoing air is colder than $100^{\circ} \mathrm{C}$. However, if the theoretical energy requirement was variable in function of the process conditions, we would not be able to correctly calculate energy cost gains/losses in function of changing process conditions. 
Choosing a fixed theoretical energy requirement thus allows us to compare efficiencies of different processes and/or microwave dyers with each other, even if ingoing and outgoing temperatures are different. This efficiency $[\mathrm{kg} \mathrm{H} 2 \mathrm{O}$ evaporated $* 2676 \mathrm{~kJ} . \mathrm{kg} / \mathrm{kJ}$ electric energy] is an interesting unit to work with industrially as it can be measured in real time by measuring the electric power consumption, the air flow and absolute water content of the ingoing and outgoing air.

If the ingoing and outgoing product moisture content, product flow and price per $\mathrm{kWh}$ electricity are known, it can also directly tell the energy price to dry a product to a certain level under current external and internal conditions. This allows calculating the impact of changes on the energy cost, and thus the process cost.

In every microwave dryer there is a need to remove the moisture from the cavity during the process. In the $1^{\text {st }}$ generation of microwave dryers, ambient air is sucked in and blown over the product while with $2^{\text {nd }}$ generation the hot air of the air cooled magnetrons is used instead. Using the hot air has a positive impact on the efficiency in several ways and we will illustrate it with process data obtained during production tests with two different machines.

Table 1. Values are the average of at least one hour of stable production data after startup period has ended. Process temperatures are infrared temperatures. Tests with $1^{\text {st }}$ and 3 th generation were performed respectively in May and February in Belgium, explaining the different inlet air temperature and relative humidity.

\begin{tabular}{|c|c|c|c|c|c|}
\hline & Inlet air & $\begin{array}{l}\text { Air to } \\
\text { cavity }\end{array}$ & $\begin{array}{c}\text { Air exiting } \\
\text { cavity }\end{array}$ & $\begin{array}{c}\text { Max } \\
\text { product } \\
\text { temperature }\end{array}$ & $\begin{array}{c}\text { Ingoing } \\
\text { product } \\
\text { temperature }\end{array}$ \\
\hline $1^{\text {st }}$ gen: & $16^{\circ} \mathrm{C}$ & $16^{\circ} \mathrm{C}$ & $23^{\circ} \mathrm{C}$ & & \\
\hline $\begin{array}{c}\text { MEAM } 32 \\
\text { M }\end{array}$ & $\begin{array}{l}60 \% \text { rel. } \\
\text { Humidity }\end{array}$ & $\begin{array}{l}60 \% \text { rel. } \\
\text { Humidity }\end{array}$ & $\begin{array}{l}67 \% \text { rel. } \\
\text { Humidity }\end{array}$ & $66^{\circ} \mathrm{C}$ & $16^{\circ} \mathrm{C}$ \\
\hline 3th gen: & $6{ }^{\circ} \mathrm{C}$ & $49^{\circ} \mathrm{C}$ & $45^{\circ} \mathrm{C}$ & & \\
\hline $\begin{array}{l}\text { MEAM dry } \\
\text { S } 48\end{array}$ & $\begin{array}{l}80 \% \text { rel. } \\
\text { Humidity }\end{array}$ & $\begin{array}{l}12 \% \text { rel. } \\
\text { Humidity }\end{array}$ & $\begin{array}{l}50 \% \text { rel. } \\
\text { Humidity }\end{array}$ & $51^{\circ} \mathrm{C}$ & $35^{\circ} \mathrm{C}$ \\
\hline
\end{tabular}

The function of the hot air in $2^{\text {nd }}$ generation microwave dryers is not transferring (a significant amount) of energy to the product like in conventional hot air dryers, but to improve following functions: lowering dew point of process air, insulating the product and heating up the launchers and cavity.

Hot air has a much higher capacity to transport vapor compared to cold air. In $1^{\text {st }}$ gen. a higher cold airflow over the product is required to keep the humidity below the dewpoint. The cold air will also be warmed up by the evaporated water and product $\left(16^{\circ} \mathrm{C}\right.$ ingoing air vs $23^{\circ} \mathrm{C}$ outgoing air see table 1) requiring energy that was first supplied to the product in the form of microwaves. By preheating the air we avoid this loss and also largely eliminate seasonal changes of air temperature and humidity that could have an significant impact on the process. The hot air also warms up the cavity and launchers, reducing the energy loss of the product due to irradiative losses.

Efficiency gains in going from $1^{\text {st }}$ to $2^{\text {nd }}$ generation is not due to additional (recuperated) energy but by reducing energy losses that occur in $1^{\text {st }}$ generation microwave dryers. 


\section{Evaporative cooling:}

While traditional hot air dryers use the airflow to heat the surface of the product, (hot) air can be used in microwave drying processes to decrease the surface temperature of the product by evaporative cooling. If the dielectric loss factor of the product is high enough, the limiting factor in the drying process is not be the speed of the energy transfer but rather the speed of mass transfer. As long as the absorbed microwave power is higher than the energy lost due to evaporation, the product will continue to heat up eventually reaching the maximum process temperature. When this maximum process temperature is low (e.g. sensitive food products), this put a serious limit to the drying speed.

We investigated the effect of evaporative cooling on the drying speed and the drying temperature for various products in the MEAM Explorer (figure 1). All samples were tested in three conditions, with no air current, with an cold air current $\left(12^{\circ} \mathrm{C}\right)$ and with an hot air current $\left(65^{\circ} \mathrm{C}\right)$ over the sample.

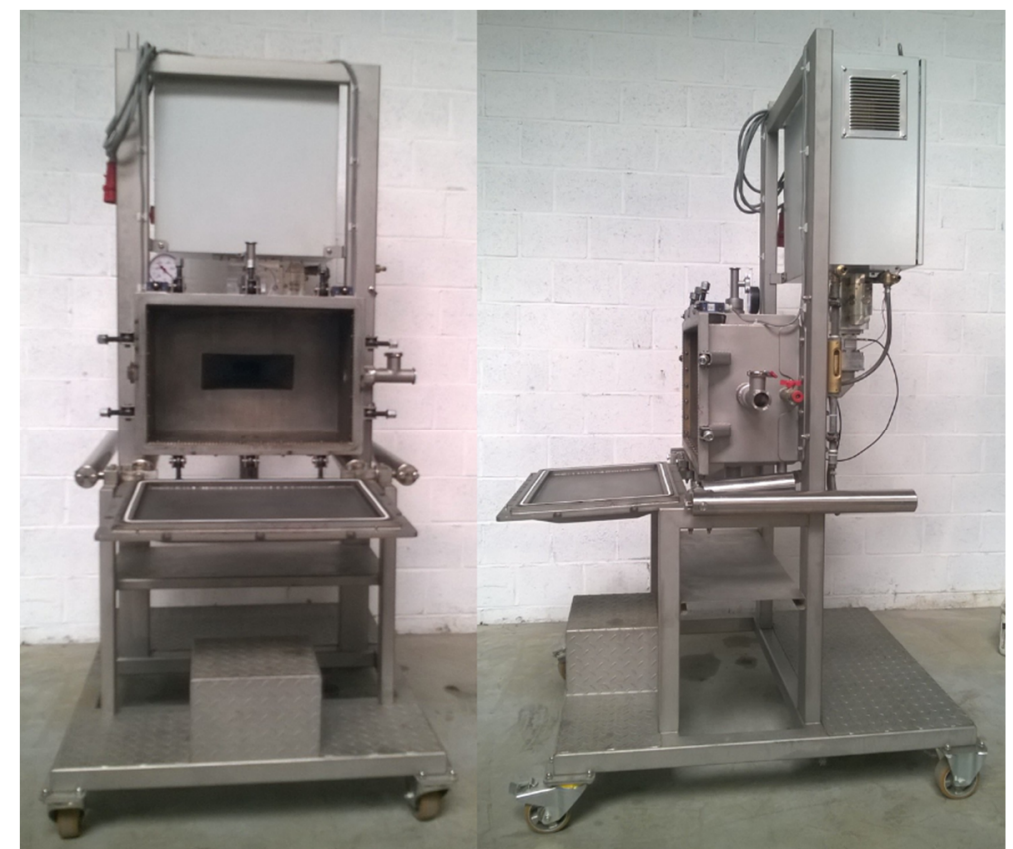

Fig. 1: MEAM Explorer: a 1.2 kW adjustable batch microwave with thermal IR camera, sample weighing and cavity openings that allow an adjustable air flow over the sample.

The first product that was dried is a granulated slag material, a by-product from metal production. This is an glassy and dense material with only water adsorbed to the surface and is thus easy to dry. Figure 1 shows three drying curves of this slag material. For this experiment, input power is regulated to keep the sample at $55^{\circ} \mathrm{C}$ while drying. Drying with a hot air current results in fast drying from $17.9 \%$ moisture to $1.69 \%$ moisture (wet weight). The input power was $1201 \mathrm{~W}$ on average. The cold air drying was slower as still $8.68 \%$ moisture (wet weight) remained after 13 min drying. Power input was $371 \mathrm{~W}$ on average. Without air current over the sample, the input power was on average $341 \mathrm{~W}$ and $12.6 \%$ moisture remained (wet weight). 


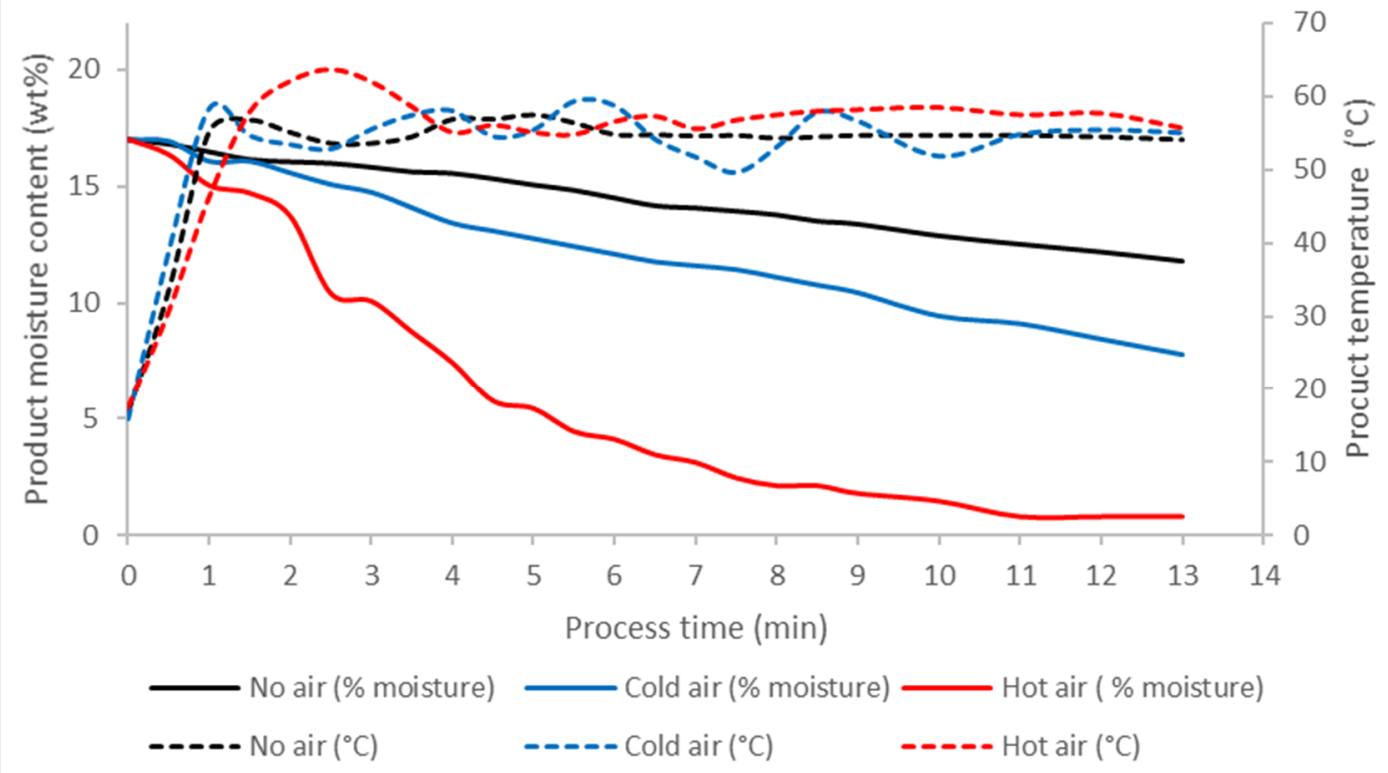

Fig. 2: Influence of air current and temperature on microwave drying curves of slag material at $55^{\circ} \mathrm{C}$.

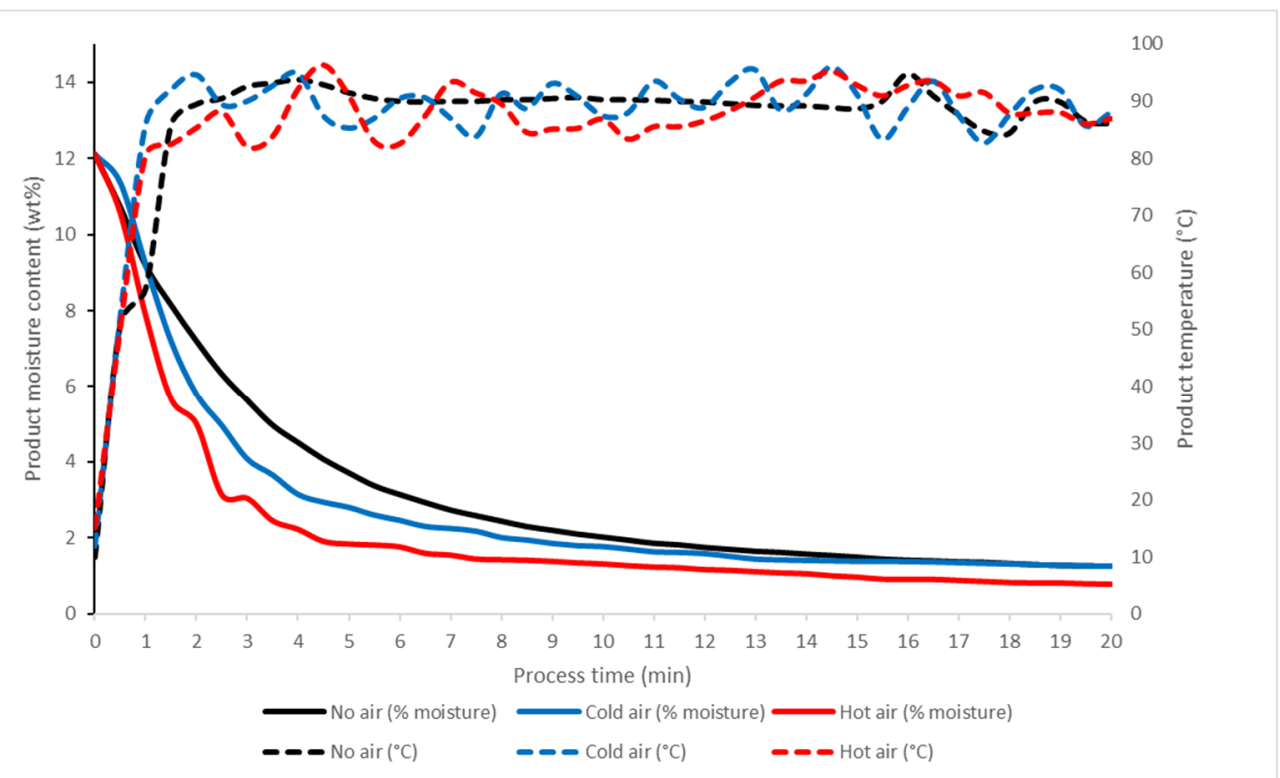

Fig. 3: Influence of air current and temperature on microwave drying curves of grass clippings at $90^{\circ} \mathrm{C}$

The second product was grass clippings that had to be dried from $12.1 \mathrm{wt} \%$ moisture to less than $1.5 \%$ moisture, the target temperature was $90{ }^{\circ} \mathrm{C}$. The test with no airflow had the slowest drying speed while the sample with an hot airflow was the fastest. Additionally, the end moisture was lower with an hot airflow. This is probably because the material is 
hygroscopic in itself, and the equilibrium between drying and moisture is shifted by using an hot airflow due to the lower partial pressure of water in hot air.

The third product was berry pomace that was dried from $40.7 \mathrm{wt} \%$ to around $10 \mathrm{wt} \%$. The drying temperature was set for $50^{\circ} \mathrm{C}$ so to prevent thermal degradation of the product.

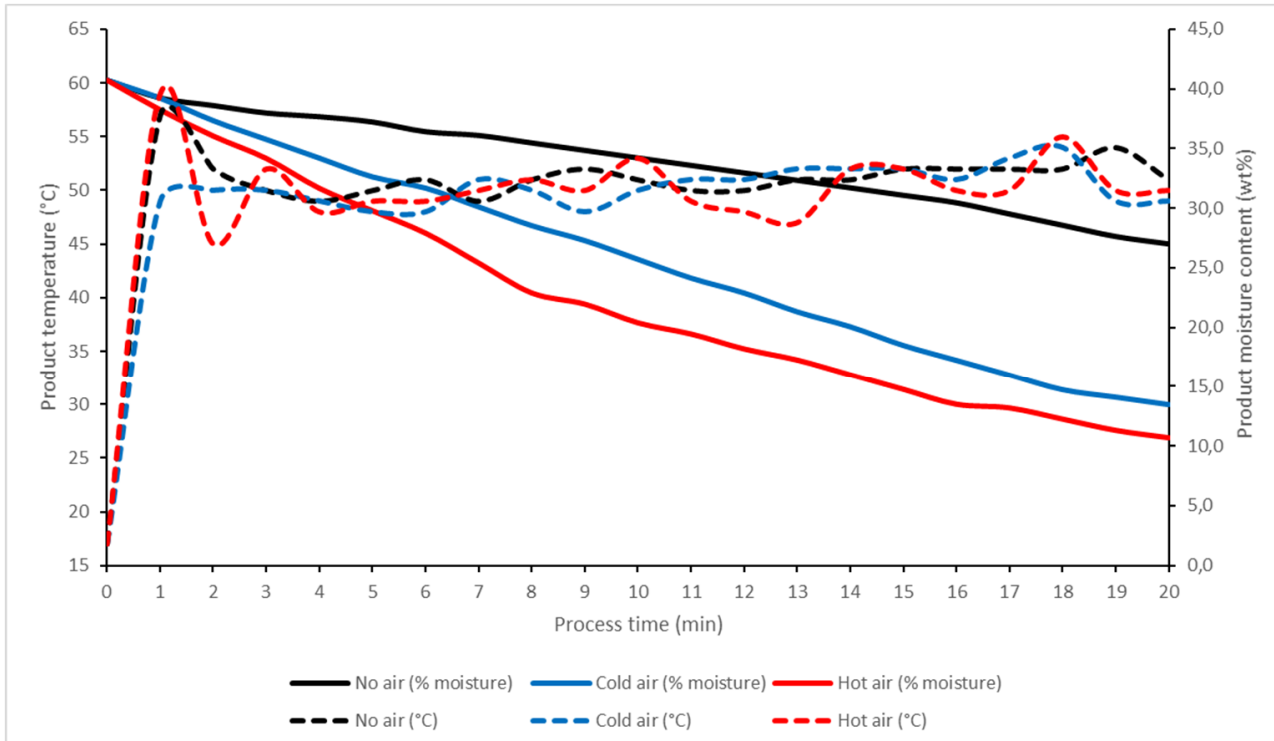

Fig. 4: Influence of air current and temperature on microwave drying curves of fruit pomace at $50{ }^{\circ} \mathrm{C}$

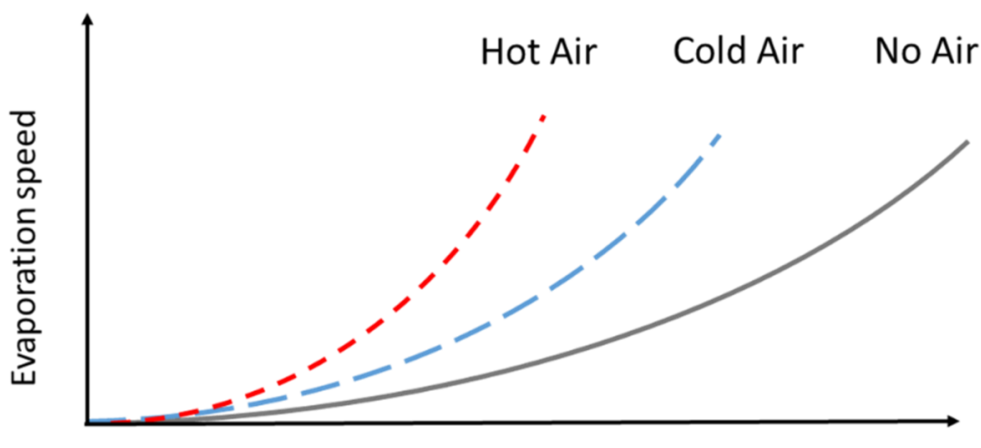

Temperature

Fig. 5: Influence of airflow on evaporation speed and temperature: At constant evaporation speed (same input MW power), the drying temperature goes down by blowing air over the

sample, with hot air being most effective. If the drying temperature is fixed, the evaporation speed can be increased by blowing air over the sample (and also increasing the input MW power). 
An air current over the sample increases evaporation rates and allows a higher input power without increasing the drying temperature or when input power is maintained, a lower drying temperature without lowering evaporation speed. The lower drying speed for the cold air samples is because hot air has a lower vapor pressure for water than cold air when both have the same absolute humidity. This will cause a faster evaporation of water in the sample with hot air and thus creates a greater evaporative cooling effect. Another way to lower vapor pressure of water is to decrease the pressure: vacuum microwave drying can operate at even lower temperatures. ${ }^{5}$

The effect of evaporative cooling is shorter process times and/or lower drying temperatures. Both are beneficial to the energy efficiency of the dryer, as shorter process times mean a smaller machine and less time to lose energy, while lower drying temperatures reduce heat loss due to a lower $\Delta \mathrm{T}$ with the environment and less energy is wasted in the form of a hot product exiting the dryer. Decreased process temperature and time have a beneficial influence on the quality of many products which is in some cases more important even than the cost of drying.

\section{Third generation: Heat recovery}

After using the waste hot air to increase evaporation rates and remove moisture from the cavity, it still contains a lot of usable energy. This energy can be used to preheat the ingoing product as long as the final temperature of the air remains above the dew point. This is why MEAM developed the "3th generation" microwave that can operate with further increased efficiency by the addition of a heat recovery unit that preheats ingoing frozen product. A stable test run of 7.30 hours was done on the MEAM dry S48 to determine the efficiency of the system. Total energy consumption of the machine at maximum capacity including electronics and fans is $68 \mathrm{~kW}$.

During the test run of $7.5 \mathrm{~h}$, on average $82 \mathrm{~kg}$ of product was dried per hour resulting in 52 $\mathrm{kg}$ of water being evaporated per hour. The drying efficiency of the machine calculated with equation 1 is $57 \%$ for the trial run. (note that this efficiency was obtained during an experimental run with an unbalanced load) The product was placed on the belt frozen at $5^{\circ} \mathrm{C}$ and after being preheating with the outgoing product air entered the cavity at $31^{\circ} \mathrm{C}$. The theoretical power needed for defrosting $\left(333 \mathrm{~kJ} / \mathrm{kg} \mathrm{H}_{2} \mathrm{O}\right)$ and heating it to $31^{\circ} \mathrm{C}(4.18$ $\mathrm{kJ} / \mathrm{kg} . \mathrm{K})$ is $8.4 \mathrm{~kW}$ with $82 \mathrm{~kg}$ ingoing product per h. This is an underestimation as the heat capacity of the dry product is not included in the calculation. When the $8.4 \mathrm{~kW}$ from heat recovery is added to the $68 \mathrm{~kW}$ total electric power of the MEAM dry S48, the total sum of ingoing power is $76.4 \mathrm{~kW}$, with heat recovery accounting for $11 \%$ of that amount.

\section{Conclusion.}

In this paper, we show three generations of microwave technology and how each successive generation has a positive influence on the efficiency of the microwave drying process. Using the hot air from air-cooled magnetrons, the $2^{\text {nd }}$ generation can cut energy losses that are inherent to the $1^{\text {st }}$ generation of microwave dryers. The hot air also increases evaporative cooling effects in the product allowing shorter process times and lower process temperatures. By using heat recovery on the wet exhaust air from the dryer, the $3^{\text {th }}$ generation microwave dryer can cut the energy cost of defrosting and preheating ingoing product accounting to more than $10 \%$ of the energy required in the process. 


\section{References}

1. Jacques Thury, Microwaves: Industrial, scientific, and medical applications, ISBN 0-89006-448-2.

2. Pieter Luypaert, (1981), Patent EP0069105 : Industrial modular microwave oven.

3. Pieter luypaert et al. (2006), Patent WO2004054324: Industrial microwave oven for the thermal treatment of products and method applied thereby in particular for killing insects in wood.

4. Ashim K. Data, Biological and bioenvironmental heat and mass transfer, ISBN 0824707753.

5. V. Goovaerts, K. Groffils, C. Groffils, $16^{\text {th }}$ International Conference on Microwave and High Frequency Heating, AMPERE 2017, Delft, The Netherlands, September 18-21, "Control of evaporation temperature in a microwave vacuum drying system”. 


\title{
HOMOGENEOUS DIELECTRIC HEATING IN LARGE MICROWAVE OVENS BY EXCITATION OF MULITIPLE EIGENMODES AT THEIR RESONANCE FREQUENCIES
}

\author{
Dominik Neumaier $^{1}$, Sabrina Sanseverino ${ }^{1}$, Guido Link ${ }^{1}$ and John Jelonnek ${ }^{1,2}$ \\ $I H M^{1}, I H E^{2}$, Karlsruhe Institute of Technology (KIT), 76131 Karlsruhe, Germany \\ dominik.neumaier@kit.edu
}

Keywords: homogeneous microwave heating, mode analysis, industrial microwave heating

\begin{abstract}
Microwave heating faces growing interest in many industries because of its large potential in increasing productivity and energy efficiency. Nevertheless, the main challenge of this heating method is to provide sufficient uniformity of the temperature distribution, in particular in industrial scale production. Several temperature maxima and minima typically occur in a sample because of the standing waves inside a cavity. An optimization-based approach is presented to increase the temperature homogeneity by exiting selected eigenmodes of the cavity. Results of a related experiment show the performance of the developed algorithm.
\end{abstract}

\section{Introduction}

Dielectric heating by microwaves offers several advantages if comparing to conventional heating. One major advantage is the volumetric heating effect. It is mostly independent from the heat conduction of the work piece. In addition, selective microwave heating is possible. It just depends on the selected loss factors of the processed materials and results in temperature profiles beneficial for specific applications. Hence, the microwave heating can significantly improve the cycle time in production and the energy efficiency in the industrial heating process. The main challenge is to achieve a sufficient temperature uniformity in the product exposed to the electromagnetic field profile in the microwave oven.

Several research activities concerning this topic were already reported. In [1] and [2] microwave applicators with magnetrons as RF (radio frequency) sources are presented. By using magnetrons, it is easily possible to change the amplitude and the pulse duration of the RF power during the heating process. On the other hand, a frequency or phase tuning of magnetrons is only limited possible if not at all impossible practically. In this frame in [1] and [2], an intelligent control algorithm is used to control the amplitudes of the different magnetrons to increase the temperature uniformity of the work piece. In contrast to the magnetron, power modules that are using solid-state amplifiers (SSA) would offer the possibility to vary accurately the amplitude, frequency and phase of the RF output power. In [3], using a simulative approach, it is shown that an intelligent change in frequency will improve the homogeneity of the temperature distribution inside a work piece. In addition, a phase shift between multiple microwave sources used can further improve the temperature homogeneity [4]. In contrast to both mentioned publications, an optimization algorithm is proposed which aims for the homogenous heating by changing both, the frequency and the amplitude of the SSA. To show this, an industrial scale cavity with a well-designed antenna is used which fulfills the following requirements: 
1. measurement the ratio of the reflected power to the transmitted power of the antenna during the process to detect the resonance frequencies,

2. amplitude (power) and frequency tuneability of the SSA,

3. direct comparison of experimental results with simulations.

\section{Experimental Setup}

Fig. 1 shows the multimode microwave oven used in the experiment. It has an industrial scale of $535 \mathrm{~mm} \times 510 \mathrm{~mm} \times 395 \mathrm{~mm}$. The corners are rounded and some deformations of the cavity exist to mount the sample holder. By using the analytical model from [5] and by assuming that the microwave oven is a perfect rectangular cavity exactly 32 eigenmodes exist in the $2.45 \mathrm{GHz}$ ISM band. For the measurement of the temperature distribution a sheet of paper $(510 \mathrm{~mm} \times 380 \mathrm{~mm})$ was used as the dielectric load. It was placed on a very low loss PTFE plate as a sample holder. The advantage of the used load is that the heating patterns can be illustrated very well, especially because the paper has a very low thermal conductivity.

In addition, for the purposed microwave cavity, an antenna system was developed based on numerical simulations with CST Microwave Studio. A loop antenna was designed (Fig. 1 (right)) and the positions of four loop antennas were optimized to excite at least $90 \%$ of those 32 eigenmodes of the unloaded cavity. In the following measurement system only the antenna, which excites most eigenmodes, was installed.

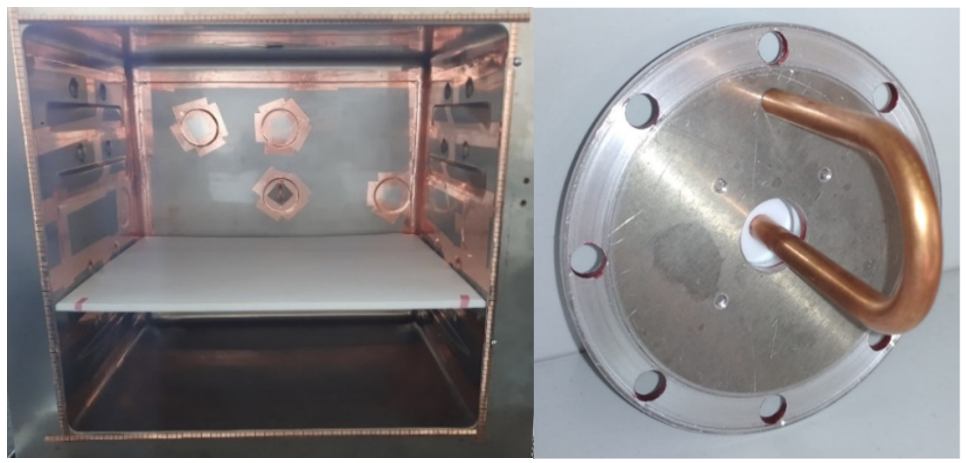

Fig. 1 Cavity geometry with a PTFE plate as a sample holder (left). Designed loop antenna (right).

Fig. 2 shows the schematic measurement setup. Firstly, the setup is capable to identify the 32 resonance frequencies of the eigenmodes that exist in the ISM Band from $2.4 \mathrm{GHz}$ to $2.5 \mathrm{GHz}$. Secondly, it allows the measurement of the power ratio of the reflected to transmitted power of the antenna while using the newly developed $300 \mathrm{~W}$ tunable RF power modules from $\mathrm{HBH}$ microwave GmbH (Germany). The RF power modules operate in the ISM frequency range from $2.4 \mathrm{GHz}$ to $2.5 \mathrm{GHz}$. The RF power modules can be used as amplifiers that are driven by a low-power signal or as free-running RF generators at a specific RF output power. It is possible to do very fast and precise frequency steps $(10 \mathrm{kHz}$ step size, $1 \mathrm{~ms}$ switching time). The RF output power is adjustable in $10 \mathrm{~W}$ steps (also PWM is supported which provide 1000 steps and 10 ns pulses). Between the antenna and the SSA a directional coupler and a circulator are located. The circulator is integrated in the main housing of the RF power module. It protects the amplifier from power reflected at the entrance of the cavity that may affect or in worst case destroy the SSA. Even more, by using the directional coupler, type Microlab CK-19N it is possible to record the reflected power. The $S_{21}$ measurement of the vector network analyzer (VNA) R\&S ZVL enables the 
measurement of the resonance frequencies of the cavity by analyzing the ratio between the reflected and transmitted power. A calibration of the directional coupler and RF power module is not necessary because only the resonance frequency is important and not the magnitude of it. At the top side of the cavity, an IR camera is installed. It allows to observe the temperature distribution of the load during the heating process. A computer is used for a fully automated system control and data recording.

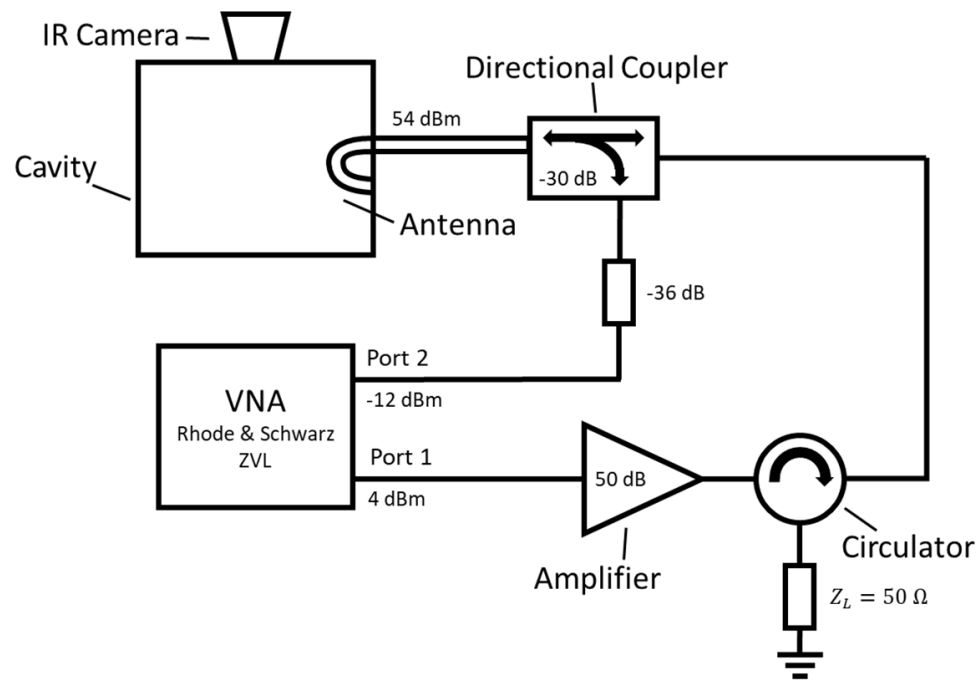

Fig. 2 Measurement setup.

The design of the loop antenna and the positioning in the cavity was done with the help of CST Microwave Studio. In this paper, only one antenna is used. The purposed system enables a later operation with up to four antennas in parallel. Fig. 3 exemplary illustrates the comparison of the simulated power distribution with the measured temperature distribution for two representative eigenmodes. It is possible to identify different high order modes of the simulation in a real measurement. The frequency shift between the measured and simulated resonance frequencies of less than $1 \%$, which appears, may be explained with geometrical inaccuracies in the simulation model. 


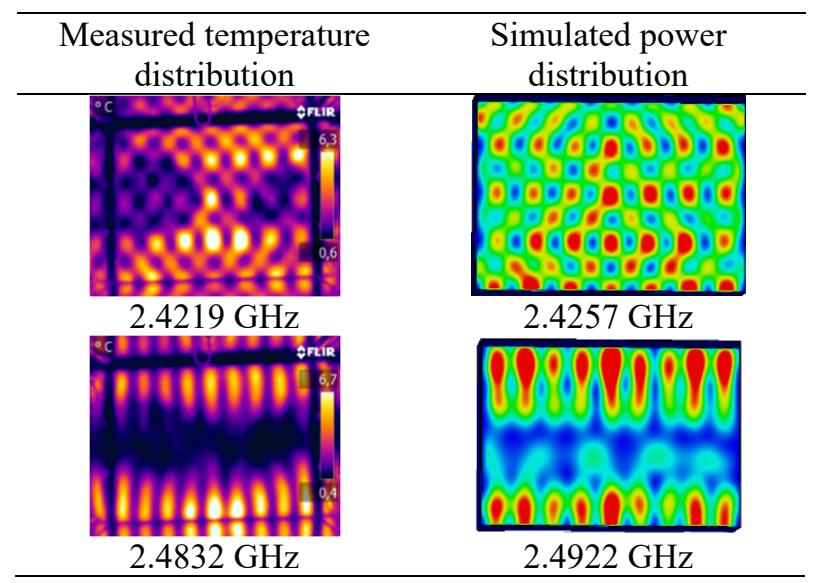

Fig. 3 Comparison of simulated with measured modes.

\section{Methods}

In following a method is described to increase the temperature homogeneity by superimposing different eigenmodes, each with its individual heating pattern. The idea is to switch very fast between different modes. Instead of changing the amplitude only the time, in which each mode is active, changes. The advantages of this is that nonlinearities of the $\mathrm{RF}$ power module do not need to be considered and it can be operated with maximum efficiency.

Fig. 4 presents the approach of the used algorithm for superimposing the eigenmodes. At first a measurement of $S_{21}$ is done in the frequency range of $2.4 \mathrm{GHz}$ to $2.5 \mathrm{GHz}$. By searching the minima of the $S_{21}$ spectrum, all resonance frequencies can be detected. The easiest way to detect how much energy the load absorbs at those eigenmodes is the measurement of the temperature increase of the load by exciting each mode the same time duration. Every resonance frequency is excited for $20 \mathrm{~s}$ and after that, the sample cooled down. The temperature difference between the start (sample is cold) and end (after $20 \mathrm{~s}$ ) temperature distribution is recorded. Every resonance frequency couples a different power in the load and therefore there is a different temperature change. To increase comparability of every eigenmode the recorded thermal images $\Delta T(x, y)$ were normalized as follows:

$$
\Delta \mathrm{T}_{\text {norm }}(\mathrm{x}, \mathrm{y})=\Delta T(x, y) / \max _{x, y}[\Delta T(x, y)],
$$

where $x$ and $y$ describe the coordinates of the picture in pixel. 


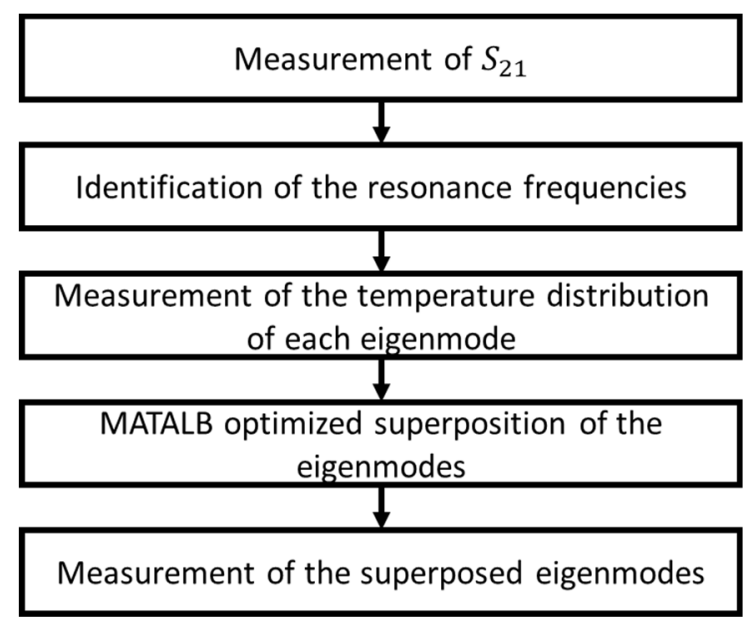

Fig. 4 Approach for superimposing the eigenmodes.

By using an optimizer, the optimal superposition of the different modes is calculated. The aim is to calculate a homogeneous superposition of the eigenmodes. Therefore the objective function

$$
J(\boldsymbol{k})=\max _{x, y}\left[\sum_{i=1}^{N}\left(k_{i} \cdot \Delta \mathrm{T}_{\mathrm{i}}(x, y)\right)\right]-\min _{x, y}\left[\sum_{i=1}^{N}\left(k_{i} \cdot \Delta \mathrm{T}_{\mathrm{i}}(x, y)\right)\right]
$$

is chosen. It describes the difference between the maximum of $\Delta T$ and the minimum of $\Delta T$ of the superimposed image. The value

$$
\boldsymbol{k}=\left[k_{1}, k_{2}, \ldots, k_{N}\right], k_{i} \in \mathbb{R}
$$

is a parameter which specifies the time duration every mode $i$ is exited. $N$ represents the number of considered eigenmodes. The optimization task

with the constraints

$$
\min _{\boldsymbol{k}}(J(\boldsymbol{k}))
$$

$$
\begin{gathered}
k_{i} \geq 0, \\
\sum_{i=1}^{N} k_{i}=1
\end{gathered}
$$

is solved in MATLAB. Only with the constraints it is guaranteed that the optimizer will not find a negative $k_{i}$, which is comparable with cooling the sample. In addition, the sum over all $k_{i}$ must be something else than zero otherwise the optimum would be zero. By choosing this value to 1 , the percentage of each used eigenmode can directly calculated with $k_{i}$.

\section{Results}

The optimization method is tested with the described measurement setup. About 30 different resonance frequencies were excited, including that modes which does not couple significant RF power into the cavity. Therefore, only eigenmodes are used for optimization, which effect at least a $3{ }^{\circ} \mathrm{C}$ temperature change after $20 \mathrm{~s}$ heating.

In Fig. 5 the normalized heating patterns of all eigenmodes are presented which are used by the optimizer and have a $k_{i} \neq 0$.

The optimizer chooses eigenmodes so that hotspots are at maximum different positions. 


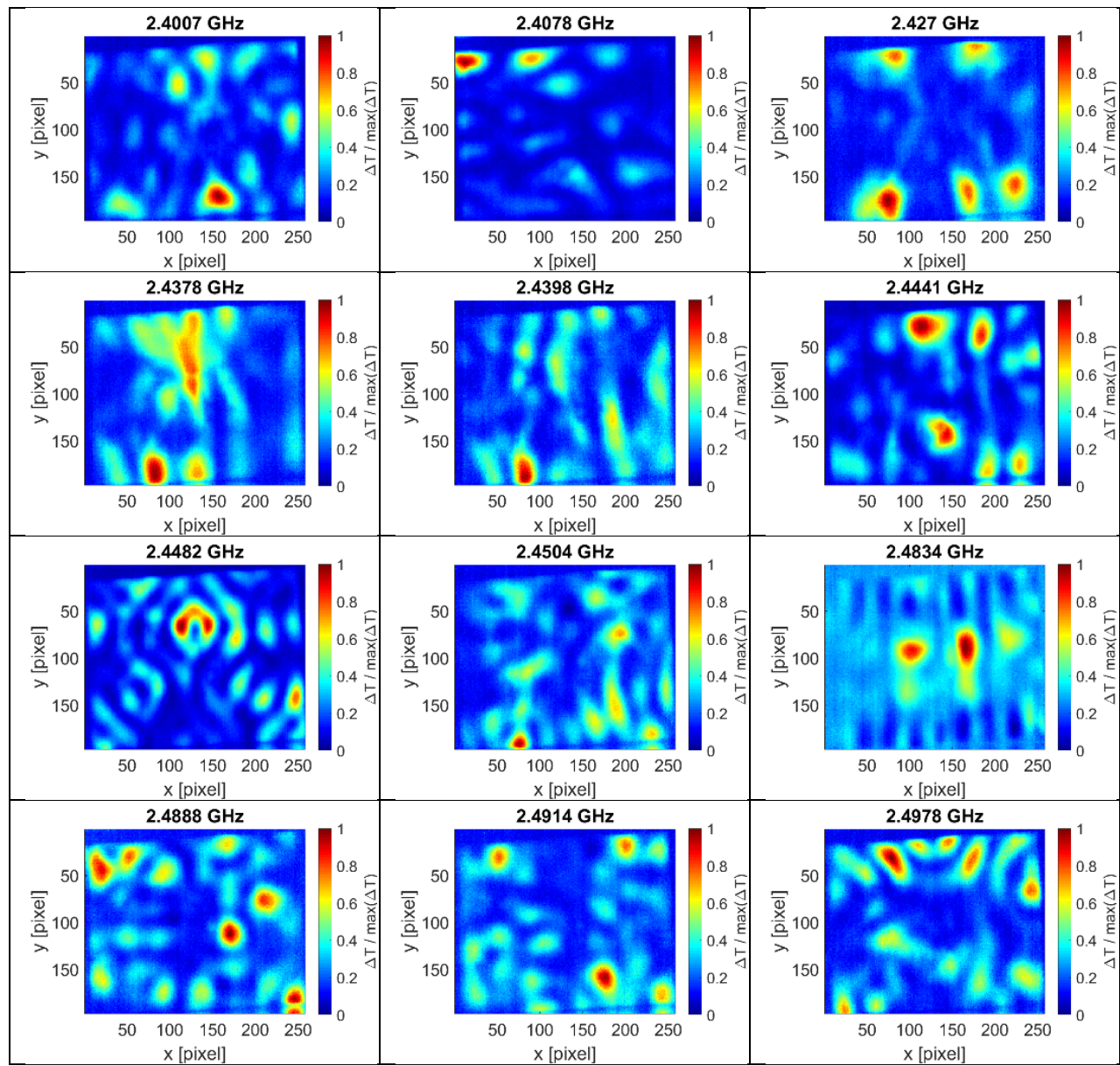

Fig. 5 Eigenmodes used by the optimizer.

The MATLAB optimized solution is presented in Fig 6 (left). The temperature uniformity with the optimized superposition is much higher than for each individual eigenmode (see Fig. 5). Thus, the optimized solution by MATLAB as described before was verified with the given setup. The period for sweeping through all modes is $0.5 \mathrm{~s}$. Every resonance frequency is excited with a time duration corresponding to its $k_{i}$. The measured results are demonstrated in Fig 6 (right). The MATLAB optimized image and the measured image are very similar to each other.
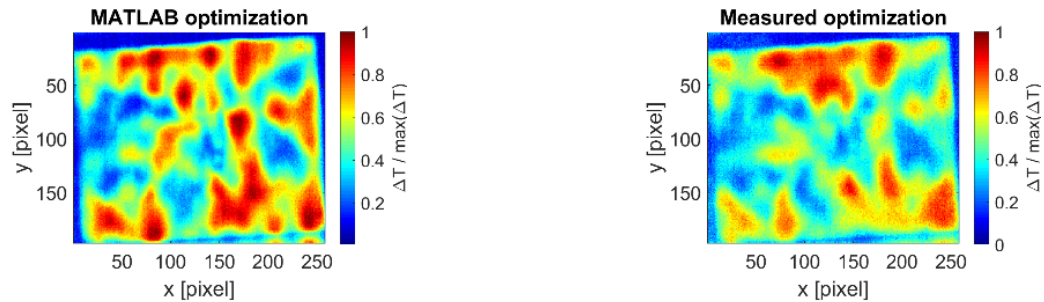

Fig 6. In MATLAB optimized superposition (left). Measured optimization (right). 
For a better comparison a criteria for homogenous heating is defined. At first, all images are normalized like in Fig. 5 and Fig 6. Then the standard deviation

$$
s=\sqrt{\frac{1}{N_{x} \cdot N_{y}-1} \sum_{x=1}^{N_{x}} \sum_{y=1}^{N_{y}}\left[\Delta \mathrm{T}_{\text {norm }}(\mathrm{x}, \mathrm{y})-1\right]^{2}}
$$

is generated for each point in the picture. $N_{x}$ and $N_{y}$ represent the number of pixels in $x$ - and $y$-direction. Instead of using the mean value as a reference, the value 1 is used. Otherwise, also a picture, which has nearly everywhere cold spots, would also create good results.

In Table 1 the results of the defined criteria are listed. All eigenmodes at their resonance frequency have a much higher standard deviation in comparison with the optimized solution. Furthermore, the results of the MATLAB optimization and in the real measurement are nearly the same.

Table 1. Criteria for homogenous heating for each eigenmode and the optimized solution.

\begin{tabular}{|c|c|c|}
\hline Frequency [GHz] & Standard deviation $\boldsymbol{s}$ & ${\text { Variance } \boldsymbol{s}^{\mathbf{2}}}^{\mathbf{G}}$ \\
\hline 2.4007 & 0.8194 & 0.6715 \\
\hline 2.4078 & 0.8716 & 0.7597 \\
\hline 2.4270 & 0.7892 & 0.6229 \\
\hline 2.4378 & 0.7520 & 0.5654 \\
\hline 2.4398 & 0.7758 & 0.6018 \\
\hline 2.4441 & 0.8081 & 0.6530 \\
\hline 2.4482 & 0.8234 & 0.6780 \\
\hline 2.4504 & 0.7682 & 0.5901 \\
\hline 2.4834 & 0.7061 & 0.4986 \\
\hline 2.4888 & 0.7541 & 0.5687 \\
\hline 2.4914 & 0.7627 & 0.5817 \\
\hline 2.4978 & 0.7647 & 0.5848 \\
\hline MATLAB optimization & 0.5352 & 0.2865 \\
\hline Measured optimization & 0.5522 & 0.3049 \\
\hline
\end{tabular}

In addition, a synthetic leather is used instead of the sheet of paper as a sample. On top of the leather, there is some glue, which has higher dielectric losses (Fig. 7 (left)). In this case, also the MATLAB optimization and the measured optimization deliver nearly the same results (Fig. 7).
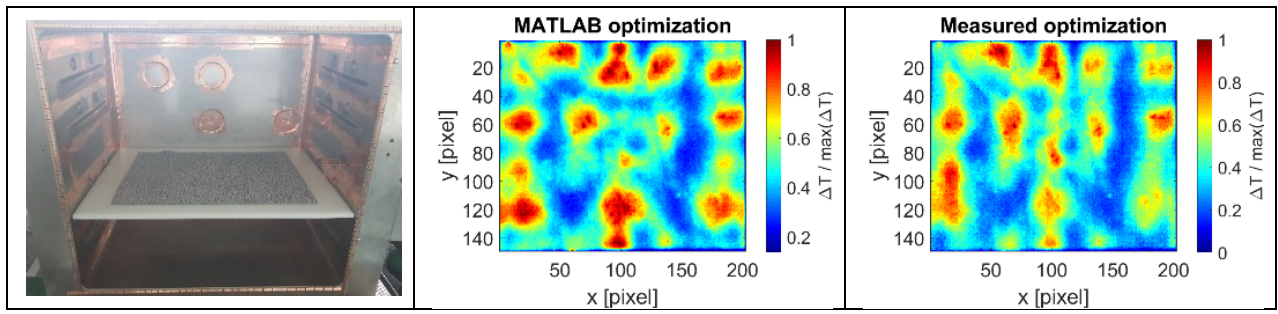

Fig. 7 Measurement of a synthetic leather with some glue on top (left). MATLAB optimization (middle). Measured optimization (right). 


\section{Conclusion and Outlook}

In this paper, an approach is demonstrated which allows to increase the homogeneity of the temperature distribution of a flat sample, that is heated by microwaves from a well controlled RF power module. At first, a setup has been designed that allows detecting and precisely characterizing available eigenmodes in the microwave applicator. Furthermore, a CST model was developed that describes the real experiment. In a first approach by use of a single antenna only, the uniformity of heating has increased. To make use of the full potential of this technique in a next step multiple antennas will be used to get access to more eigenmodes and therefore to increase the degrees of freedom. In addition, a parallel activation of different antennas is possible. The final objective is the implementation of this method into a temperature control algorithm.

\section{Acknowledgment}

The authors acknowledge the financial support by the Federal Ministry for Economic Affairs and Energy of Germany in the project ZF4204602PR6.

\section{References}

[1] Sun, Y., Adaptive and Intelligent Temperature Control of Microwave Heating Systems with Multiple Sources, PhD thesis, KIT Scientific Publishing , 2016. doi:10.5445/KSP/1000051503.

[2] Zhou, J., Li, Y., Li, N., Liu, S., Cheng, L., Sui, S., and Gao, J, A multi-pattern compensation method to ensure even temperature in composite materials during microwave curing process, Composites Part A: Applied Science and Manufacturing, vol. 107, pp. 10-20, 2018. doi: 10.1016/j.compositesa.2017.12.017

[3] Yakovlev V. V, Frequency control over the heating patterns in a solid-state dualsource microwave oven, in 2015 IEEE MTT-S International Microwave Symposium, pp. 1-4, 2015. doi: 10.1109/mwsym.2015.7166948

[4] Gambato, F, Moro, F. and Guarnieri, M., Improving microwave cooking performance by source phase shifting, in IMPI's 48th Annual Microwave Power Symposium (IMPI 48), vol. 48. Mechanicsville, VA: International Microwave Power Institute, 2014, pp. 26-29.

[5] Pozar, D. M., Microwave Engineering, 4th ed. Hoboken: Wiley, 2012 


\title{
DEVELOPING MICROWAVE CHEMISTRY UNDER PROCESS ENGINEERING PRINCIPLES
}

\author{
E.Tioni $^{1}$, J. Breysse ${ }^{1}$, P.Rousseaux ${ }^{1}$, \\ 'Processium, 30 rue Marguerite, 69100 Villeurbanne, France \\ estevan.tioni@processium.com
}

Keywords: microwave, radio-frequency heating, power applications

\section{Introduction}

It is nowadays admitted that microwaves are frequently used in organic chemistry labs [1] (even if not as much as it was predicted 20 years ago, one must say [2]). On the other side it is also certain that this technology has not yet found its place in chemical industry: application at a production scale are very scarce [3][4] and this despite the potential advantages of the technology (selective heating, high heating rate, low thermal inertia...). We believe the main reason for this is that mastering all the aspects of microwaves assisted synthesis at industrial scale demands a lot of different skills to work together: chemistry, process engineering, microwave engineering, materials science. This is so challenging that tools and methodologies for quantification of industrial microwave interest and scaling-up of lab results are missing.

The activity of Processium consists in selecting and sizing for its clients the most suitable reaction and separation techniques (and technologies) for a given process (i.e. to go from feed composition A to on-spec product composition B). It is therefore essential for this type of business to be able to analyze and compare a wide range of technologies, including innovative ones like microwave synthesis.

An overview on how microwave synthesis technology is integrated by Processium into the range of technologies available to its customers is given in this article.

\section{Each technology has its use: In which case microwaves are useful? When are they useless?}

No matter the unit operation (reaction, distillation, liquid-liquid extraction...) every technology has its own limits of application depending mainly on:

- Operating conditions

- Physico-chemical properties of the feed

- Kinetics of the reaction

- Thermodynamics of phase equilibria

Outside these limits, other technologies are more advantageous or even more easily applicable.

Rationalization of the range of applicability of each technology is key for selecting the best one. This kind of work has not been found in literature for microwave assisted organic chemistry. An example of a methodology to guide into the selection of microwave assisted synthesis is given in Fig. 1.

It should be noted that all the results presented here are based on the widely accepted assumption that microwaves simply consist of a means of heating. 


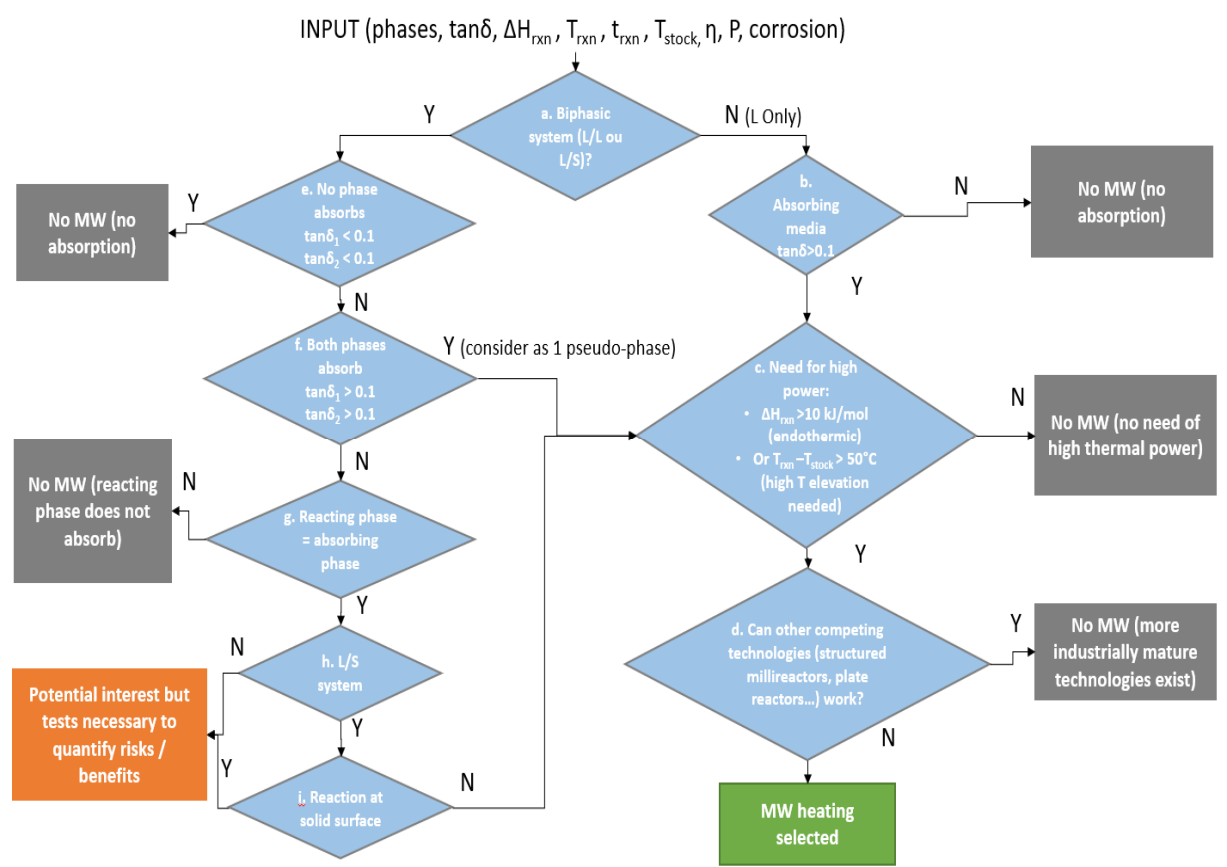

Fig. 1. Methodology for helping into the selection of microwave heating for organic reactions

The flowchart can be divided into two sections:

- $\quad$ Single-phase liquid case (right section)

- Multiphase case L/L or L/S (left section)

For the single-phase liquid case, the criteria that should be met to retain microwaves heating as an advantageous technology compared to the existing ones are:

- A need for high heating power (endothermic reaction or high temperature reaction)

- Difficult heat transfer from the walls (eg. high viscosity, thick walls due to pressure constraints, poorly conducting materials due to chemical compatibility constraints,...)

- Competing technologies not applicable (high solid content, fouling risk...)

For multiphase case the reacting phase should be the absorbing one (if both phases absorb then the media can be considered as a single pseudo-phase). This being true, two scenarios are possible:

- $\quad \mathrm{L} / \mathrm{L}$ system or L/S system with reaction at solid surface: the use of microwaves could be advantageous if, for any reason, a thermal gradient between the two phases could be maintained, and this would have a positive effect on the reaction performance (lower energy consumption, increased selectivity, accelerated

- material transfer processes, local overheating at the reaction site and consequent acceleration of the kinetics...). On the other hand, it is important to be able to control this thermal gradient and avoid local runaway. It is very difficult to predict this type of situation 'a priori': more detailed work (experimental and/or simulation) is needed to quantify benefits and risks.

- $\quad \mathrm{L} / \mathrm{S}$ system with reaction in the liquid phase: the solid is then an inert or a reactant that dissolves or a product that precipitates. The potential benefits of microwaves are related to the heating of the reaction liquid phase 


\section{Kilolab microwave reactor for data acquisition}

The main particularity of integrating microwave reaction in our panel of technologies is that, due to the lack of industrially scalable tools, Processium developed its own pilot scale equipment. This consists in a new microwave irradiated stirred tank reactor [[5]] based on the INTLI technology developed by Sairem [[6]] having the following characteristics:

- $1 \mathrm{~L}$ volume

- Equipped with double jacket, allowing direct comparison between different heating modes

- Capable of working at high $\mathrm{T}$ and $\mathrm{P}\left(250^{\circ} \mathrm{C}, 40 \mathrm{bar}\right)$

- Working in batch mode or continuous mode up to $20 \mathrm{~L} / \mathrm{h}$

- Built in non-transparent microwave materials (metals) thanks to the INTLI technology

- With close-to-ideal hydrodynamics (fig. 3)

- With perfectly characterized heat balance

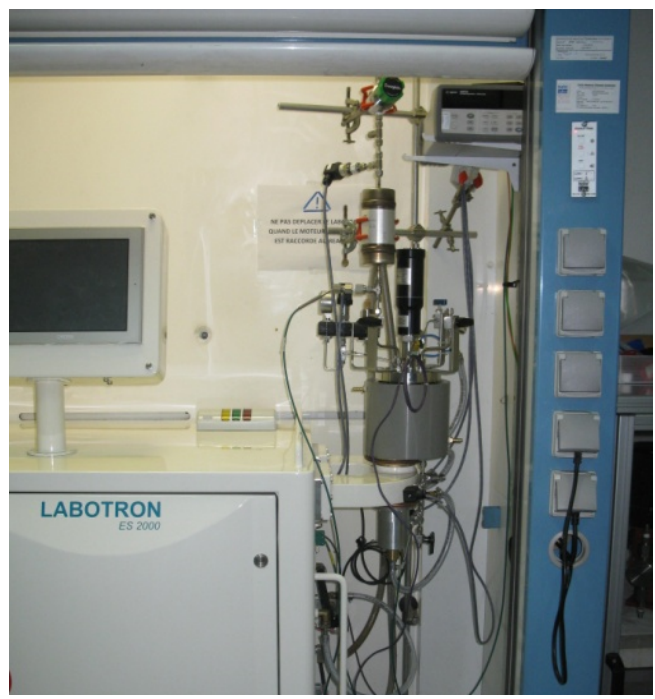

Fig. 2. Microwave reactor and generator

These characteristics have been defined in order to be able to propose an experimental tool:

- Working under a wide range of industrial conditions (high temperature and pressure)

- Allowing the scale up of the results with the classic methods of chemical reaction engineering coupled with electromagnetic simulation for industrial detailed design

- Adapted to acquisition of intrinsic chemical kinetics, without biases coming from microwave related reasons (poor temperature measurement, heterogeneous temperature distribution, value of power absorbed by the media poorly known...) 


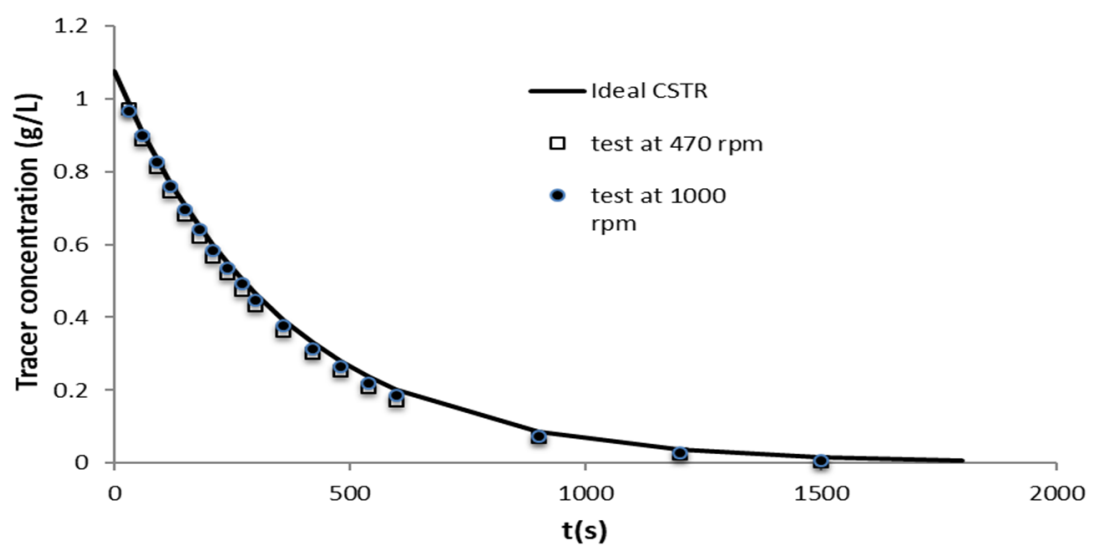

Fig. 3. RTD of the reactor at different stirrer speeds compared to an ideal CSTR

By establishing a detailed heat balance on the reactor and after few experiments having the objective to determine the model parameters (heat transfer coefficients and inert heat capacity - Table 1) it is possible, since SAIREM generator indicates continuously incident and reflected power, to know with precision at each instant the heat lost to the environment and the power used to heat up inert metal mass.

Table 1. Values of heat transfer coefficients calculated from experimental characterization

\begin{tabular}{llllll}
\hline Heated liquid & $\begin{array}{l}\text { Max MO } \\
\text { power }(\mathrm{kW})\end{array}$ & $\mathrm{T}\left({ }^{\circ} \mathrm{C}\right)$ & $\mathrm{P}($ bar $)$ & $\begin{array}{l}\mathrm{U} \\
\left(\mathrm{W} / \mathrm{m}^{2} / \mathrm{K}\right)\end{array}$ & $\begin{array}{l}\mathrm{U} \text { losses } \\
\left(\mathrm{W} / \mathrm{m}^{2} / \mathrm{K}\right)\end{array}$ \\
\hline Ethylene glycol & 2 & 180 & 10 & 908 & 70 \\
Water & 1 & 80 & 1 & 777 & 69 \\
Water & 2 & 150 & 7 & 1027 & 53 \\
Water & 2 & 80 & 1 & 1222 & 48 \\
\hline
\end{tabular}

This leads to a good knowledge of the microwave power effectively absorbed by the reaction media.

Fig. 4 shows an example of a measurement of power effectively absorbed for a liquid heated up to $150^{\circ} \mathrm{C}$. It is visible how the power effectively absorbed by the liquid media is a fraction of the microwave power produced by the generator and how it's possible to follow its evolution with time. This methodology allows then to know the real power absorbed by the media, per unit of mass, which has to be kept constant during scaleup.

Remark: noise visible in the next figure comes from noise in temperature measurement $(+/-$ $\left.1^{\circ} \mathrm{C}\right)$ and power measurement $(+/-20 \mathrm{~W})$, which are amplified by calculations. 


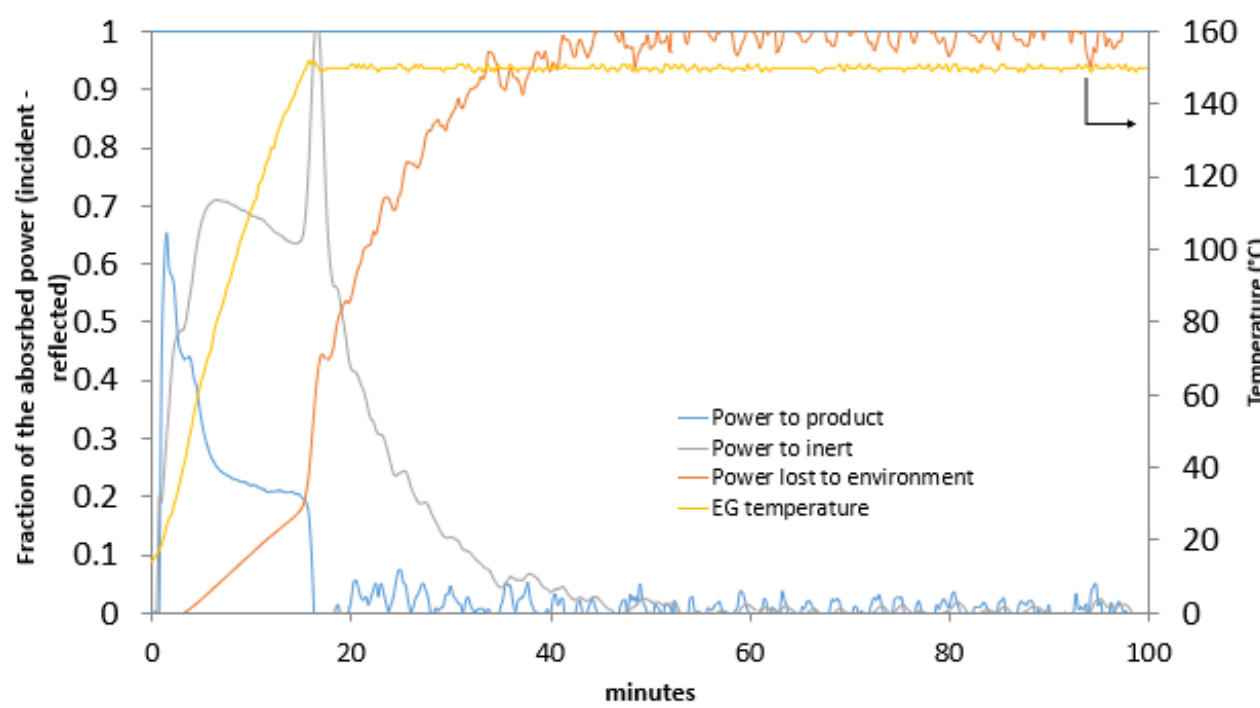

Fig. 4. Example of detailed calculation on microwaves power effectively absorbed by the heated product during a batch test

Two versions of the experimental setup are available, differing by the material of the part filling the gap between the tank and INTLI. This part should ensure sealing while being transparent to microwaves:

- Teflon version: experimentally tested with success up to $200^{\circ} \mathrm{C}$ and 20 bar, point at which Teflon starts to deform

- Ceramic version: work ongoing to identify the most suitable material, alumina and other ceramics being unsuccessfully tested (brittleness problems)

\section{Example of reaction intensification and industrial projection}

The experimental tool presented in the previous paragraphs is then suitable to be used to acquire fundamental data on intrinsic reaction kinetics under microwave irradiation. Kinetic models built on measured data are then used to size industrial reactors or to identify operating conditions optimizing the reaction performances. The following example shows how this work is conducted on a reaction of industrial interest

Production of molecule of interest via an acetylation is conducted industrially in batch mode in a few $\mathrm{m}^{3}$ reactor at $80^{\circ} \mathrm{C}$. Yield is quite good $(96 \%)$ but byproducts are highly fouling and are responsible for malfunctions in the purification section. A new synthesis path has been discovered which does not produce fouling byproducts but shows poor performances at $80^{\circ} \mathrm{C}$ (49\% yield of main product).

Microwave reactor is then used to measure kinetics and performance of the reaction at different conditions. Results are presented in Fig. 5.

It is important to note that results obtained under classic heating (by the customer at $80^{\circ} \mathrm{C}$, or during this study at $110^{\circ} \mathrm{C}$ ) are really close to the ones obtained under microware heating at the same temperature, meaning that:

- No specific microwave heating effect is measured

- The equipment can effectively be used to compare directly the performance of different heating modes 
This being said, one can notice from Fig. 5 . that yield is improved by conducting the reaction at high temperature and low residence time.

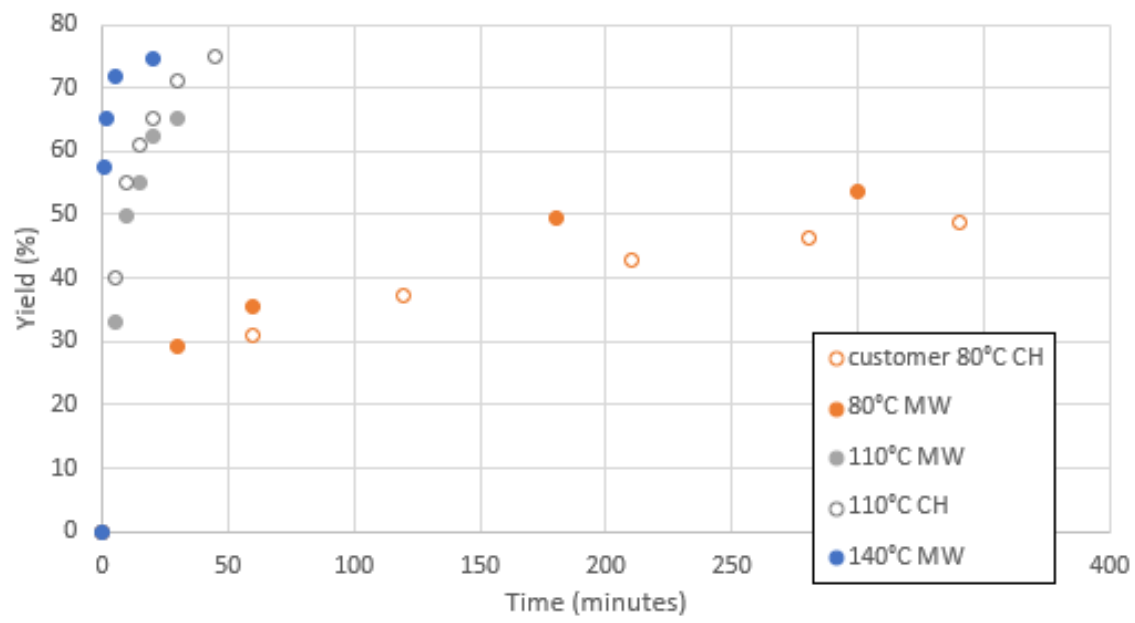

Fig. 5. Yield vs time for different batch trials; $\mathrm{MW}=$ microwave heating, $\mathrm{CH}=$ classic heating

A kinetic model has been developed based on these experimental data and used to predict optimal product yield at higher temperature. For each temperature, the volume of a theoretical continuous stirred tank industrial reactor is calculated. Effective microwaves power needed to heat the feed from room temperature to reaction temperature is also estimated. Basis for the calculations is a production of 520 tons per year of product of interest. Results are given in Table 2.

Please note that extrapolation of kinetic models outside experimental range should always be verified by experiments afterwards. In this case the work shown here is purely demonstrative.

Table 2. Theoretical reactor presizing as a function of reactor temperature

\begin{tabular}{|c|c|c|c|c|}
\hline $\mathrm{T}\left({ }^{\circ} \mathrm{C}\right)$ & $\begin{array}{l}\text { Optimal res } \\
\text { time (s) }\end{array}$ & Optimal yield (\%) & $\begin{array}{l}\text { Industrial } \\
\text { reactor volume } \\
\text { (L) }\end{array}$ & $\begin{array}{l}\text { MW power } \\
\text { absorbed by } \\
\text { product }(\mathrm{kW})\end{array}$ \\
\hline 110 & 4714 & 41.9 & 394.2 & 15.1 \\
\hline 150 & 658 & 52.4 & 43.9 & 17.4 \\
\hline 200 & 86 & 62.7 & 4.8 & 20.1 \\
\hline 250 & 17 & 70.0 & 0.9 & 23.0 \\
\hline
\end{tabular}

Working at high temperature favors the yield in product of interest. This, coupled to the relatively small size of the industrial reactor necessary to ensure the desired production, suggests that microwaves heating could be advantageous to apply industrially for this example. 


\section{Conclusions, limitations and future work}

The key to our vision is to treat microwave technology as a simple (but particular) means of heating and take the necessary precautions to be able to scale-up with conventional chemical engineering tools. Different approaches are used together to achieve this objective:

- Development of a methodology to help in microwave technology selection since the early stages

- Development of an experimental technology to acquire key data for microwave reactor sizing

- Modeling and sizing using classic tools of chemical engineering

Two main axes for future work and improvements are identified:

- Improving the reliability of the experimental tool by identifying the right ceramic material that would allow working at higher temperature and pressure

- Include electromagnetic modeling during the industrial reactor sizing step

\section{References}

[1] Diaz-Ortiz A., Prieto P., de la Hoz A., Chem. Rec., 2019, 19, 85-97

[2] Kappe C.O., Chem. Rec., 2019, 19, 15-39

[3] Aldivia, patent WO2004/066683A

[4] Tremblay JF., c\&en, 2016, 36, 24-25.

[5] Rousseaux P., Breysse J., Tioni E., patent FR3026022

[6] Sairem, patent WO2009/122101 


\title{
MICROWAVE PRETREATMENT OF LIGNOCELLULOSIC BIOMASS TO RELEASE MAXIMUM PHENOLIC ACIDS
}

\section{A. BICHOT ${ }^{1}$, M. RADOIU ${ }^{2}$, Nicolas BERNET ${ }^{1}$, Valérie MECHIN $^{3}$, J-P. DELGENÈS ${ }^{1}$, D. GARCÍA-BERNET ${ }^{1 *}$}

\author{
${ }^{1}$ LBE, Univ Montpellier, INRA, 102 Avenue des Etangs, 11100 Narbonne, France \\ ${ }^{2}$ Microwave Technologies Consulting, 153 rue des colverts, 69140 Rillieux la Pape, France \\ ${ }^{3}$ INRA Institut Jean-Pierre Bourgin, 78026 Versailles, France \\ * Corresponding author: E-mail: diana.garcia-bernet@inra.fr; tel: +33 (0)4 684664 28; \\ ORCID: 0000-0003-3163-8819
}

Keywords: pressure microwaves, biomass pretreatment, phenolic acids

\begin{abstract}
Biomass pretreatment is a critical issue for successful biorefinery deployment and thus new competitive and sustainable technologies are needed for efficient biomass bioconversion. Microwave (MW) treatment has emerged as an interesting option; nevertheless, the action mode of MW on lignocellulosic biomass is not fully understood and studies dealing with MW pretreatment do not address this important question.

The aim of this article is to provide an analysis of microwave effects on phenolic acids release from corn stalks, a biomass of industrial interest. MW treatments were performed using water as a solvent and a power density of $2.38 \mathrm{~W} / \mathrm{g}$. Incident, reflected \& absorbed power levels and reaction temperature were monitored on-line. MW treatments were compared to a conventional heating treatment, a control treatment without heating and a high $\mathrm{NaOH}$ consumption reference extraction method for phenolic acids.

Optimal MW conditions were chosen to increase phenolic acids release: pressurized test (300W and 180s) allowed the extraction of 10 times more ferulic acid than classic microwave treatment.

Finally, these different heating possibilities were compared from an economic and environmental point of view.
\end{abstract}

\section{Introduction}

The objectives fixed by world's governments concerning energy transition have aroused interest on lignocellulosic biomass utilization for bioenergy and green chemistry applications. However, due to their resistant structure, deconstructive pretreatments are necessary to render possible biological conversions of these lignocellulosic residues [1]. Microwave (MW) treatment has been reported as efficient in many biotechnology fields; biomass pretreatment for biorefinery purposes is another possible application [2]. This work presents the effects of MW pretreatment on underexploited natural agri-food biomass of economic interest: corn stalks.

Various heating types were studied including conventional heating and microwave heating at atmospheric pressure or with an increase in pressure. Effects were evaluated by a complete biomass characterization before and after treatment, with main focus on phenolic acids (PA) release and more specifically ferulic acid (FA) and para-coumaric acid ( $p \mathrm{CA})$. In the tested conditions and when compared to the high $\mathrm{NaOH}$ consumption reference extraction method for phenolic acids, the atmospheric pressure (open vessel) microwave treatment did not allow attaining high acid extraction yields. In order to increase yields, microwave treatments under pressure were carried out to reach higher temperatures while taking care as to not exceed $150^{\circ} \mathrm{C}$ - the acid denaturation temperature [3] and to avoid the formation of 
inhibitors. Energy balances were also calculated to demonstrate the advantages of MW in terms of energy consumption and processing time.

\section{Materials and methods}

\section{Raw biomass and composition analysis}

INRA IJPB (Versailles-Grignon unit, Versailles Cedex, 78026, France) supplied corn stalks (F 98902) harvested in September 2016.

Air dried samples were coarsely crushed (Viking, model GE 220, STIHL, Stuttgart, Germany) before being finely ground to $1 \mathrm{~mm}$ (using a Fritsch Pulverisette 19) and sieved to retain only particles between 200 and $1000 \mu \mathrm{m}$. Ground and sieved samples were kept in closed boxes at ambient temperature before usage. Biomass composition was determined using Van Soest protocol [4], which allows determining the amount of parietal polymers before and after treatment. Van Soest protocol is based on mass sequential partitioning of cell walls, from most extractible to less extractible, with successive extractions using different solvents (water, neutral detergent solution, acid detergent solution, and sulfuric acid $72 \%$ ). Phenolic compounds were analysed by HPLC using a HPLC-DAD Waters system: autosampler 717, multisolvent delivery system 600, Diode Array Detector 2996. All chemicals were purchased from Sigma-Aldrich Chemical. High purity water (Merck Millipore Quantum TEX) was used for all pretreatments and analysis.

\section{Biomass pretreatment}

Based on previous works [5], optimal conditions for extracting phenolic acids were determined. Alkaline water ( $\mathrm{pH}=8.5$ obtained by adding $6.7 \mathrm{mmol} \mathrm{NaOH}$ to $200 \mathrm{ml}$ water), $\mathrm{S} / \mathrm{L}$ ratio 1:21 (w:w), total volume of $200 \mathrm{ml}$, incident power of $500 \mathrm{~W}$, treatment duration of 270 s and high stirring were determined as adequate conditions to extract phenolic acids. Nevertheless, in this study water was chosen as the solvent to highlight the performance of microwave processing under mild chemical conditions. Samples underwent $1 \mathrm{~h}$ pre-soaking at ambient temperature before pretreatment.

All MW pretreatments were performed with a Minilabotron 2000 (SAIREM, FRANCE), operating at $2.45 \mathrm{GHz}$ with a maximum power of $2 \mathrm{~kW}$. The equipment was used for two different MW pretreatments: classic microwave pretreatment (CMW) and pressurized microwave pretreatment (PMW). Each pretreatment was performed in duplicate at constant incident power to ensure a power density of $2.38 \mathrm{~W} / \mathrm{g}(500 \mathrm{~W}$ and $270 \mathrm{~s}$ for CMW and $300 \mathrm{~W}$ and 180s for PMW). Temperature (excepted for PMW), incident power and reflected power were recorded during the treatment. The different elements of the devices are illustrated on Figure 1.
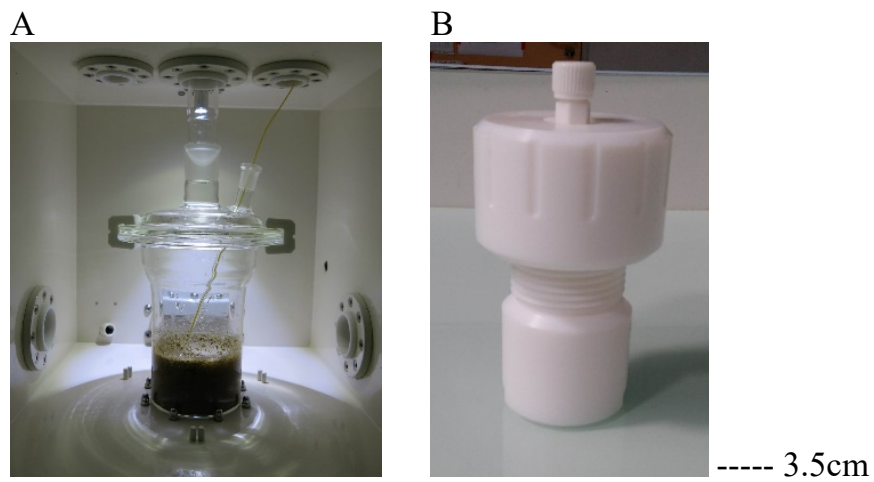

Fig. 1. Installation ready for operation: CMW (A) and PMW (B) 
A conventional heating (Conv) without using electromagnetic waves was also carried out as to compare the energy performance of the two types of heating. The reactor containing the same reaction mixture as the microwave treated sample was immersed in a heat-stabilised oil bath at $110^{\circ} \mathrm{C}$ for $360 \mathrm{~s}$. The oil bath was positioned on a laboratory heating plate.

Two control treatments (soaked biomass sample without any heating) were also carried out. The first one, using water for $1 \mathrm{~h}$ soaking, was considered as a negative control $\left(\mathrm{C}^{-}\right)$. The second one, using $\mathrm{NaOH} 10 \%(\mathrm{w} / \mathrm{w})$ for $24 \mathrm{~h}$ was considered as a positive control $\left(\mathrm{C}^{+}\right)$ allowing the extraction of a large quantity of phenolic acids [6].

After each treatment, the reactor was cooled to room temperature for $15 \mathrm{~min}$ before weighing and filtering through a $200 \mu \mathrm{m}$ sieve. Solid was washed with $300 \mathrm{~mL}$ of deionized water to remove reagents (in the case of $\mathrm{NaOH}$ treatment) and by-products inhibiting further biological reactions. Solid was placed 7 days at $40^{\circ} \mathrm{C}$ to dry. The dry matter content was measured to determine the amount of solubilized matter during processing (treatment soluble). Supernatant was filtered through cellulose filter $(2.7 \mu \mathrm{m})$ and preserved at $-20^{\circ} \mathrm{C}$ until further analysis. The overall process of a microwave test is shown in Fig. 2.
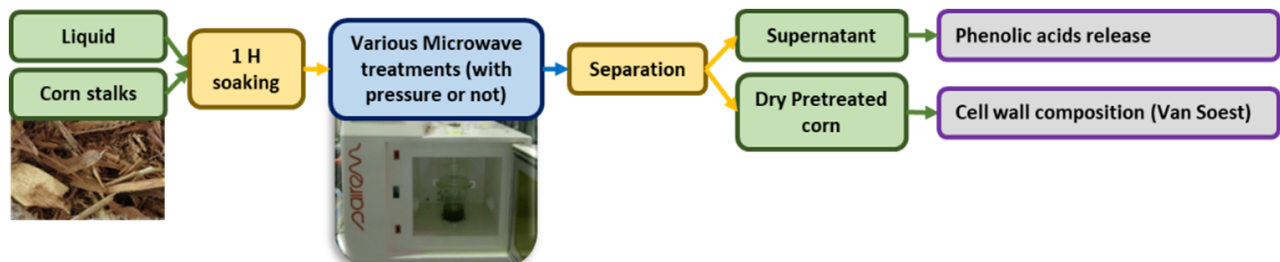

Fig. 2. Global microwave pretreatment scheme

Microwave effects on biomass composition and on phenolic acids release were analysed with ANOVA and considered significant when p-value $<0.05$ with residuals distributed according to a normal law.

\section{Energy calculations}

In order to compare Conv, CMW and PMW from an energy point of view, energy consumption for each test was measured using an amperometric clamp allowing to record intensity data. Knowing that the voltage in France is $230 \mathrm{~V}$, the power was determined by equation [1], with $\cos (\varphi)$ corresponding to the phase shift between the alternating voltage and current.

$P_{\text {real }}=$ U.I $\cos (\varphi)$

equation [1]

Considering that the microwave and the plate are purely resistive, the current and the voltage are in phase $(\varphi=0)$ and $\cos (\varphi)=1$. The power can be expressed with equation [2]:

$\mathrm{P}_{\text {real }}=\mathrm{U} . \mathrm{I}$

equation [2]

Microwave yield can then be calculated from the incident energy divided by the consumed energy following equation [3]. The yield corresponds to the mains electrical efficiency and to the magnetron efficiency. The yield was calculated for three different power levels, $1000 \mathrm{~W}, 500 \mathrm{~W}$ and $300 \mathrm{~W}$ for $800 \mathrm{~s}$, and compared to that of the pilot's manual $(65 \%)$

$\eta_{M W}=P_{i} / P_{\text {real }}$

equation [3] 
Concerning conventional heating, the maximum heating power of the plate is $1000 \mathrm{~W}$. The time required to heat the plate to $100^{\circ} \mathrm{C}$ before starting the test must be also considered equation [4]. The yield was not calculated as the plate is not optimized and a significant portion of this energy is lost due to heat losses from the oil bath.

$\mathrm{E}_{\mathrm{CONV}}=\left(\mathrm{P}_{\text {plate }} \cdot \mathrm{t}_{\text {heating }}+\mathrm{P}_{\text {plate }} \cdot \mathrm{t}_{\text {test }}\right) / \eta_{\text {plate }}$

equation [4]

\section{Results and discussion}

\section{Parietal composition}

Using Van Soest method, the parietal composition of raw biomass and each pretreated sam ple was measured, Fig. 3. The raw biomass composition corresponds to values found in lite rature [7, 8]. Fig. 3 shows that total soluble content (from treatment and from Van Soest fir st step) doesn't significantly differ according to the different treatments tested except for th e case of $\mathrm{C}+$ and PMW treatments ( $p=6.4 \mathrm{e}-09$ and $p=0.000663$ respectively). High conce ntrated $\mathrm{NaOH}$ and pressurized MW treatments have an impact on cell wall content solubili zation and more specifically on hemicellulose solubilization. Indeed, hemicellulose signific antly varies when $\mathrm{C}^{+}(p=0.00326)$ is used and is significantly different at $0.1 \%$ risk using PMW $(p=0.06359)$. Pressure and $\mathrm{NaOH}$ are well known factors affecting biomass compos ition $[9,10]$. The phenolic acids being very strongly embedded in the parietal network, if th e latter is partially destructured, then a significant release of phenolic acids can be expected

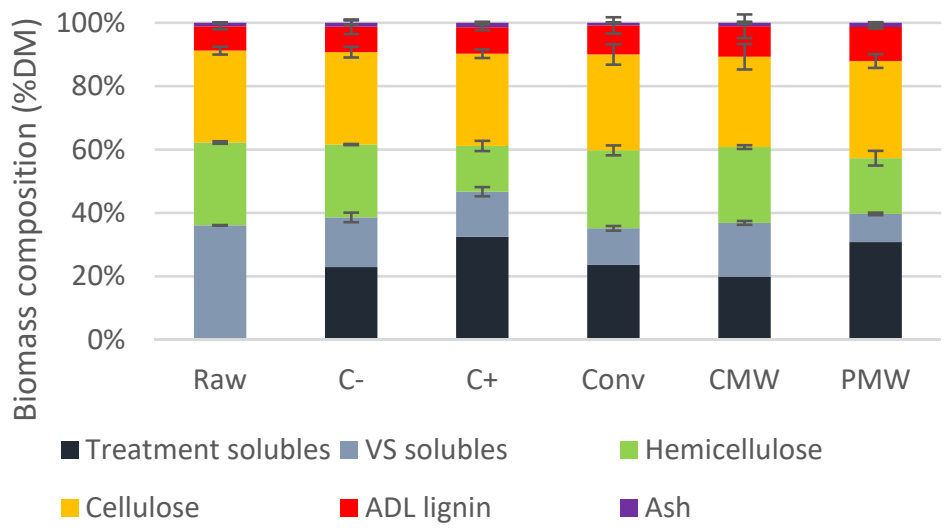

Fig. 3. Raw and pretreated biomass composition with Van Soest method

Phenolic acids were quantified in the raw biomass and the obtained values were the following: $4.2 \mathrm{mg} \mathrm{FA} / \mathrm{g} \mathrm{DM}$ and $13.1 \mathrm{mg} p \mathrm{CA} / \mathrm{gDM}$. Extraction yields in the liquid and $p$ values were calculated using equation [5] and results are summed up in Table 1.

$\mathrm{PA}_{\text {yield }}=\left(\mathrm{PA}_{\text {liberated in soluble content }} m g / g\right) /\left(\mathrm{PA}_{\text {initial }} m g / g\right)$

equation [5]

From Table 1 we can see that phenolic acids extraction significantly increased when PMW treatment was employed ( $p=0.00755)$; FA extraction yield is more than ten times higher than in $\mathrm{C}^{-}$test, which is consistent with the previous parietal composition analysis. In the case of $\mathrm{C}^{+}$treatment, FA extraction yield reached $94 \%$, which confirms the theory of almost complete extraction of FA by $\mathrm{NaOH}$. This is also visible in the case of $p \mathrm{CA}$ extraction yield: $\mathrm{C}^{+}$extraction yield reaches $45 \%$. Moreover, $p C A$ extraction yield is significantly increased 
using PMW ( $p=0.00323)$. Temperatures higher than $100^{\circ} \mathrm{C}$ are necessary to disrupt cell wall and gain access to phenolic acids [3].

Tab. 1. Phenolic acid extraction after various treatments

\begin{tabular}{l|cccc}
\hline & FA yield & FA p value & $p$ CA yield & $p$ CA $p$ value \\
\hline $\mathrm{C}^{-}$ & $0.6 \%$ & $/$ & $1.4 \%$ & $/$ \\
$\mathrm{C}^{+}$ & $94.0 \%$ & $2.45 \mathrm{e}-09$ & $45.7 \%$ & $5.47 \mathrm{e}-10$ \\
& & $* * *$ & & $* * *$ \\
Conv & $1.0 \%$ & 0.91738 & $1.2 \%$ & 0.89203 \\
CMW & $1.5 \%$ & 0.76602 & $1.1 \%$ & 0.82984 \\
PMW & $11.3 \%$ & $0.00755 * *$ & $6.2 \%$ & $0.00323 * *$ \\
\hline
\end{tabular}

\section{Energy consumption comparison}

Using equation [3], MW yield was calculated for incident power of $1000 \mathrm{~W}, 500 \mathrm{~W}$ and $300 \mathrm{~W}$ for $800 \mathrm{~s}$. No variation in intensity was detected over time and yields are represented in Fig. 4. According to Fig. 4, the MW yield follows an affine function depending on the incident MW power: the higher the incident power, the better the efficiency. The yield achieved at $1000 \mathrm{~W}$ is close to that expected for this device.

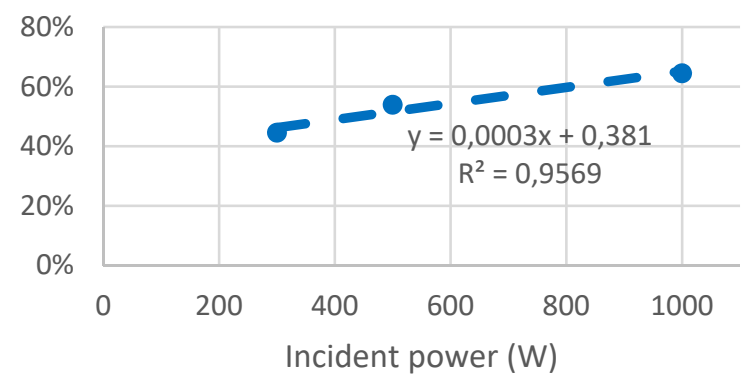

Fig. 4. MW yields vs. MW incident power

Using equations [2], [3] and [4], the total energy consumption was estimated for each treatment, Table 2 . The energy required to produce $\mathrm{NaOH}$ was not considered and as such explaining why $\mathrm{C}+$ and $\mathrm{C}$ - did not consume any energy and why were not represented.

Results listed in Table 2 show that the energy required for only one batch pretreatment is twice higher in conventional treatment $(383 \mathrm{~kJ}$ to treat $10 \mathrm{~g})$ than in pressurized treatment $(121 \mathrm{~kJ}$ to treat $2 \mathrm{~g})$. Nevertheless, it is essential to account for the quantity of treated material. In the case of PMW, the available reactor did not allow the treatment of more than $2 \mathrm{~g} / \mathrm{batch}$ while the CMW set-up allowed for $10 \mathrm{~g} / \mathrm{batch}$ treatment. Therefore, at present an extrapolation of the results at industrial scale will be not attempted. However, it is interesting to compare the two treatment methods and therefore the PMW treatment loses interest because of the high price to treat $10 \mathrm{~g}(606 / 10 \mathrm{~g})$. The design of a MW pressured reactor with a larger volume would be essential in order to scale up the process and reduce the cost. With reference to the available equipment, CMW treatment was more advantageous $(250 \mathrm{~kJ} / 10 \mathrm{~g})$ and allowed $100^{\circ} \mathrm{C}$ pretreatment temperatures to be reached quickly compared to conventional heating $(2225 \mathrm{~kJ} / 10 \mathrm{~g})$. This last treatment could be improved by installing oil bath insulation and optimized heating plate. 
Lastly, it would be interesting to know how much could be gained from the phenolic acids extracted via PMW. Due to their high added value, these molecules could compensate for the high cost of the microwave treatment process. Thus, the overall cost-effectiveness of the process would be determined.

Tab. 2. Energy consumption for each treatment: Conv = conventional heating, CMW = Classic microwave treatment at 500W, PMW = Pressurized microwave treatment at 300W

\begin{tabular}{c|ccc}
\hline & Conv & CMW $(500 W)$ & $P M W(300 W)$ \\
\hline Pretreated mass $(\mathbf{g})$ & $\mathbf{1 0}$ & $\mathbf{1 0}$ & $\mathbf{2}$ \\
$\mathrm{P}(\mathrm{W})$ & 1000 & 500 & 300 \\
$\eta$ & & $54 \%$ & $45 \%$ \\
\hline Heating duration $(\mathrm{s})$ & 1800 & & \\
Heating energy $(\mathrm{kJ})$ & 1842 & & 180 \\
\hline Batch test duration $(\mathrm{s})$ & 360 & 270 & 121 \\
Batch test energy $(\mathrm{kJ})$ & 383 & 250 & 606 \\
\hline Total energy $(\mathrm{kJ} / 10 \mathrm{~g})$ & 2225 & 250 & 0.17 \\
Total energy $(\mathrm{kWh} / 10 \mathrm{~g})$ & 0.62 & 0.07 & $\mathbf{0 . 0 2 5}$ \\
Treatment cost $(\mathbf{\epsilon} / \mathbf{1 0 g}) *$ & $\mathbf{0 . 0 9 3}$ & $\mathbf{0 . 0 1 0}$ & \\
\hline
\end{tabular}

*Considering $0.15 € / \mathrm{kWh}$ in France

\section{Conclusion}

This study demonstrates that microwaves can be used to pretreat lignocellulosic biomass. Using a PTFE reactor especially designed for this work, temperatures above $100^{\circ} \mathrm{C}$ were reached and resulted in the release of phenolic acids, molecules of high interest currently. In addition, from an economic viewpoint, the technology allows biomass to be processed quickly compared to conventional treatment. Energy consumption is significantly reduced. Nevertheless, technological advances are necessary for process scaling-up; i.e. the treatment of higher quantities of biomass in a pressurized environment and more adapted reactor. At this point, as a more in-depth economic analysis is needed, the authors would not discuss CAPEX, OPEX and ROI of such equipment.

\section{Acknowledgements}

Authors would like to thank Nicolas Holfeltz, NH Verre France for his help in designing the microwave reactor. The authors also thank Yannick Sire from INRA Pech Rouge for phenolic acids analysis.

\section{References}

1. Bichot, A., Delgenes, J.P., Mechin, V., Carrere, H., Bernet, N., Garcia-Bernet, D. 2018. Understanding biomass recalcitrance in grasses for their efficient utilization as biorefinery feedstock. Reviews in Environmental Science and Bio-Technology, 17(4), 707-748.

2. Bundhoo, Z.M.A. 2018. Microwave-assisted conversion of biomass and waste materials to biofuels. Renewable and Sustainable Energy Reviews, 82, 11491177.

3. Liazid, A., Palma, M., Brigui, J., Barroso, C.G. 2007. Investigation on phenolic compounds stability during microwave-assisted extraction. Journal of Chromatography A, 1140(1-2), 29-34.

4. Goering, H.K. and Van Soest P.J. 1970. Forage Fiber Anlysis (Apparatus, Reagents, Procedures, and some applications). USDA ARS Agricultural Handbook 379. US Government Printing Office, Washington, DC 
5. Bichot, A., Lerosty, M., Geirnaert, L., Méchin, V., Carrère, H., Bernet, N., Delgenès, J.P., García-Bernet, D. 2019. Soft microwave pretreatment to extract phydroxycinnamic acids from grass stalks. Submitted to Industrial crops and products

6. Mathew, S., Abraham, T.E. 2004. Ferulic acid: An antioxidant found naturally in plant cell walls and feruloyl esterases involved in its release and their applications. Critical Reviews in Biotechnology, 24(2-3), 59-83.

7. Provan, G.J., Scobbie, L., Chesson, A. 1994. Determination of phenolic-acids in plant-cell walls by microwave digestion. Journal of the Science of Food and Agriculture, 64(1), 63-65.

8. van der Weijde, T., Kamei, C.L.A., Torres, A.F., Vermerris, W., Dolstra, O., Visser, R.G.F., Trindade, L.M. 2013. The potential of C4 grasses for cellulosic biofuel production. Frontiers in Plant Science, 4, 18.

9. Thomas, H.L., Seira, J., Escudie, R., Carrere, H. 2018. Lime Pretreatment of Miscanthus: Impact on BMP and Batch Dry Co-Digestion with Cattle Manure. Molecules, 23(7), 13.

10. Gaspar, M., Kalman, G., Reczey, K. 2007. Corn fiber as a raw material for hemicellulose and ethanol production. Process Biochemistry, 42(7), 1135-1139. 


\title{
CONTINUOUS INDUSTRIAL-SCALE MICROWAVE-ASSISTED EXTRACTION OF HIGH-VALUE INGREDIENTS FROM NATURAL BIOMASS
}

\author{
M. Radoiu, S. Splinter, T. Popek \\ Radient Technologies Inc., 8223 Roper Road, T6E 6S4, Edmonton, Alberta, Canada \\ mradoiu@radientinc.com
}

Keywords: industrial microwaves, biomass extraction, scale-up, continuous flow processing

\begin{abstract}
An innovative technology for the continuous extraction of bioactive compounds from a wide range of biological materials has been developed, scaled up and successfully demonstrated at commercially relevant scales. The technology, known as MAP ${ }^{\mathrm{TM}}$, or Microwave-Assisted Process, robustly transfers from laboratory to continuous, industrial scale operation. In wide-ranging trials, MAP ${ }^{\mathrm{TM}}$ has comprehensively demonstrated its ability to outperform many KPIs of conventional extraction processes, while offering biomass throughput, product consistency and low operational costs not attainable by other emerging technologies.
\end{abstract}

\section{Introduction}

Plant extracts have been long used by traditional healers for providing health benefits and are nowadays suitable ingredients to produce formulated health products and nutraceuticals. Traditional (conventional) methods of extraction such as maceration, percolation, digestion, and preparation of decoctions and infusions are now been replaced by advanced extraction methods with a view of increased extraction efficiency and selectivity of bioactive compounds to meet increasing market demand. The exponentially increasing demand of herbal products and/or extracts for wider and safer applications, timely availability of highquality products with low cost of processing and higher yield are the needs of the growing herbal/nutraceutical medicine and food-based industries. To meet these challenges, there is an increased demand for alternative and more advanced extraction techniques. Advanced techniques use various means to enhance extraction, including for example microwaves, ultrasound waves, supercritical fluids, enzymes, pressurized liquids, pulsed electric fields, etc. [1]. However, to reduce the energy and material utilization inefficiencies and increase economic and environmental benefits, these advanced technologies must be more efficient than conventional extraction methods.

To enable the development of such technologies, a closer approach via process intensification and the use of microwave (MW) effects may help achieve these goals. Microwaves do not heat by the normal methods of conduction, convection and radiation from hot surfaces, but rather by direct interaction of the wave with the material being heating via dielectric loss. The resulting instant and volumetric heating can be exploited to achieve various benefits, as long as systems and equipment are designed and implemented properly. Process intensification refers to the development of novel equipment and/or methods that produce significantly higher yields or superior benefits in comparison with the existing equipment and/or methods in practice. These benefits can be realized in the form of dramatic reduction in processing times, significant improvements in product quality or quantity, decreasing the equipment size, reducing the complexity of production schemes, improving the energy efficiency, minimizing the waste production, and finally resulting in cheaper, 
safer and sustainable technologies [2]. The process intensification developments in equipment could focus on developing novel reactor design with intense mixing to promote heat- and mass-transfer while the developments in methods could focus on integrating the reaction-separation processes (minimizing process steps) with the use of alternative energy sources, including new process control techniques. For example, in the context of natural ingredients production, process intensification efforts refer to the increasing mass and heat transfer rates among the reaction products whether in extraction and/or separation and/or purification stages.

During scale-up from bench to commercial scale, a primary issue with the MW based methods is microwave penetration depth into the plant / solvent mixture, which is only a few centimetres in usual solvents. This issue can and then exclude the use of high-volume batch reactors. Because of this, the features that make the microwave-assisted process attractive (e.g. fast extraction rates, high extraction efficiency, improved energy efficiency, etc.) may not be met. Coupling microwave heating and continuous flow technology eliminates the main drawbacks of microwaves and creates a very promising way to produce high valueadded extracts since unlike the batch, the continuous flow has been demonstrated to facilitate process intensification and contribute to a safe, efficient and sustainable production. Further, if selective recovery of valuable products from the biomass can be enhanced by manipulating the unique characteristics of the microwave process intensification effects, such process can be justified from all chemical, energy and cost related aspects.

In this paper, the basis of the MAP ${ }^{\mathrm{TM}}$ technology will be described and select scale-up studies presented.

\section{Scale-up of microwave-assisted extraction: design \& validation of the MAP ${ }^{\mathrm{TM}}$ continuous-flow extractor}

For efficient continuous-flow MW extraction outcomes and scale-up, not only the MW frequency used for extraction but also the factor consideration and their linear, quadratic and interactive effects are of great value for understanding their effect on results of maximizing the compound yield while minimizing the energy loss and solvent consumption, which ultimately add to the economic value. Various factors based on the solvents, raw materials and MW parameters need to be considered for optimum extraction design - Fig. 1. With regards to MW parameters, it is important to consider that for continuous flow extraction the dielectric and thermal properties in the reaction volume are both spatially and temporally variable [3]. In addition, the variability of the properties is not the only key factor for a good coupling of the electromagnetic field with the medium. The value itself of the dielectric properties is important, since the electric field propagation and amplitude depend respectively on the real and imaginary part of the dielectric permittivity. In the special case of biomass extraction (heterogeneous reaction with solid phase) or in general when dielectric properties increase with composition or temperature, the absorption rate of microwave energy also increases, hence thermal runaway can result. Consequently, controlling heating rate and electromagnetic field homogeneity are essential for both repeatability and industrial applications.

Radient's proprietary $915 \mathrm{MHz}$ continuous-flow MAP ${ }^{\mathrm{TM}}$ extractor, Fig. 2, was designed for continuous processing of up to $200 \mathrm{~kg} / \mathrm{h}$ of biomass material. 
The reactor consists of three main components:

- The microwave generator with variable power up to $72 \mathrm{~kW}$ based on a $\mathrm{CW}$ magnetron and switch mode power supply;

- The microwave transmission line (standard WR975 waveguides) includes the isolator (circulator + water load) \& reflected power meter, automatic tuning components, and a 2-way waveguide splitter to distribute the microwave energy to the reactor (couplings);

- The microwave multimode resonant cavity, aka continuous flow reactor made of stainless steel. Within the reactor there is a mechanical agitator, designed to prevent backflow of material.

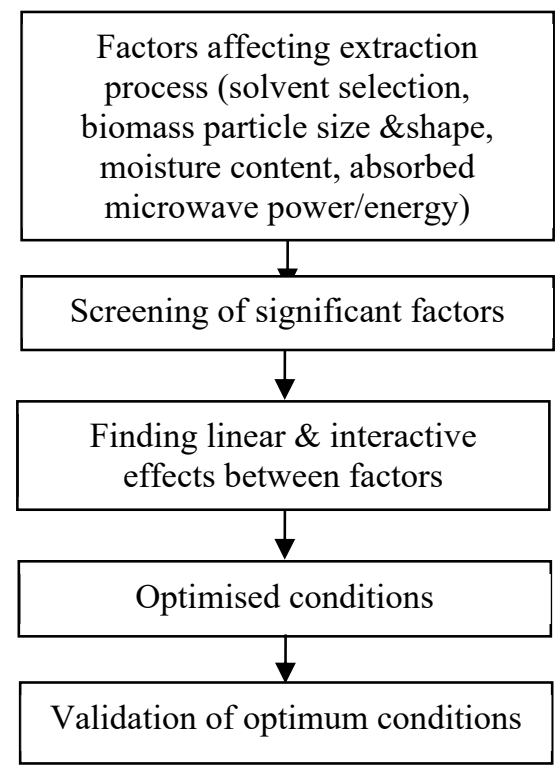

Fig. 1. Scale-up steps

Verification of the mechanical integrity of the system was confirmed by flow testing of biomass / solvent slurries. Testing and verification of the efficiency of microwave energy transfer to the extractor cavity was completed at various microwave power settings using flowing water at $870 \mathrm{~kg} / \mathrm{h}$. The microwave energy transfer to the system was verified to be $>95 \%$ in each case.

As an example of performance, continuous flow $\mathrm{MAP}^{\mathrm{TM}}$ extraction of the antioxidant SDG from flax biomass was performed using $70 \%$ ethanol / water as the solvent at two different conditions:

- $\quad 75 \mathrm{~kg} / \mathrm{h}$ flax / $5 \mathrm{~L} / \mathrm{kg}$ solvent / $15 \mathrm{~kW}$ microwave power / extractor residence time $24 \mathrm{~min}$;

- $\quad 110 \mathrm{~kg} / \mathrm{h}$ flax / $5 \mathrm{~L} / \mathrm{kg}$ solvent / $20 \mathrm{~kW}$ microwave power / extractor residence time 16 min.

The industrial-scale conditions for these runs were determined by extrapolating from optimized conditions previously obtained from batch lab-scale MAP ${ }^{\mathrm{TM}}$ experiments. The continuous flow approach eliminates the requirement for having geometric similarity 
between scales, i.e. the equipment shape and dimensions do not have to scale proportionately.

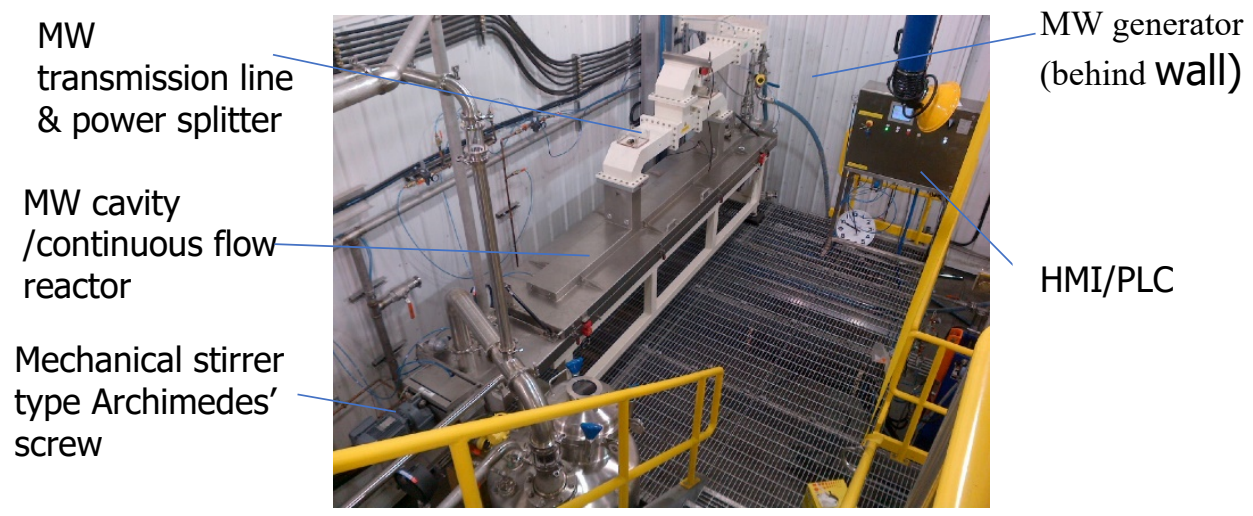

Fig. 2. Continuous-Flow Industrial-Scale MAP ${ }^{\mathrm{TM}}$ Extractor in ATEX environment

Figure 3 shows a comparison of the steady state recovery of SDG from flax obtained from lab experiments using single-stage conventional dispersed-phase mixing extraction, labscale MAP ${ }^{\mathrm{TM}}$ using the same solvent and solvent composition, and the results of the two industrial scale runs described above. From the figure, it is clear that scale-up was verified by obtaining identical results at two different flow rates and associated MAP ${ }^{\mathrm{TM}}$ energy densities. This confirmed that laboratory-scale MAP ${ }^{\mathrm{TM}}$ results can be successfully replicated at industrial scale and so verifies that the industrial-scale extractor performs as designed.

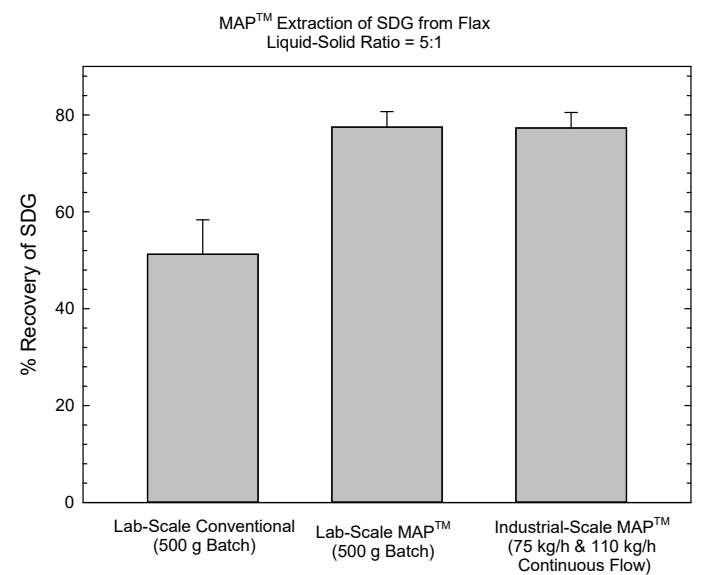

Fig. 3. Comparison of conventional extraction, lab-scale MAP ${ }^{\mathrm{TM}}$ Extraction and industrialscale MAP ${ }^{\mathrm{TM}}$ extraction of flax lignans (SDG) from flax biomass 


\section{Conclusions}

There is a necessity to explore new reactor concepts by emphasising dedicated designs that assure controllability and monitoring of the process conditions. A number of variables affect the microwave reactor output and its scale: electric field intensity, power dissipation rate and temperature of heated mixture being the most important.

It is equally important to stress that microwave technology cannot be implemented in straightforward manner to any heated industrial process. The development of microwave equipment/installations needs to be studied individually for each system in order to achieve the desired benefits. Moreover, given the interest of a chemical industry in transition from batch to continuous processing, the microwave flow reactors become increasingly interesting to the industry.

Within the ISM band (Industrial, Scientific and Medical), $915 \mathrm{MHz}$ is a frequency that presents a lot of interest for scaling up processes developed using $2.45 \mathrm{GHz}$. Industrial microwave generators operated at $915 \mathrm{MHz}$ are available up to $120 \mathrm{~kW} / \mathrm{unit}$ compared to 6 $\mathrm{kW}$ at $2.45 \mathrm{GHz}$, meaning that the quantities that can be processed are much larger using $915 \mathrm{MHz}$ equipment. In other words, a microwave process requiring for example $60 \mathrm{~kW}$, can be designed around an equipment in which the microwave power is delivered using only 1off microwave generator at $915 \mathrm{MHz}$ or 10 off generators operating at $2.45 \mathrm{GHz}$. From a viewpoint of capital investment, but also of operation and complexity of the reactor, the 915 $\mathrm{MHz}$ is by far, the more economical.

Our work was focused on investigating the scaling-up of $915 \mathrm{MHz}$ continuous flow process to industrial scales. Based on laboratory experiments and their optimisation, the continuous flow extractor for industrial biomass extraction using MAP ${ }^{\mathrm{TM}}$ was built and successfully demonstrated on various solvent - biomass matrices. The extractor comes with several benefits, including significantly increased flexibility with respect to operation:

- The contact time between the biomass and solvent before, during and after microwave treatment can be adjusted much more easily;

- It is possible to precisely control biomass residence time in the microwave zone and - if desired - separate the biomass from the solvent very quickly after treatment or continue contact for any length of time at any temperature, depending on the desired outcome.

The extractor is also easily scalable. The continuous flow approach eliminates the requirement for having geometric similarity between scales (i.e. the equipment shape and dimensions do not have to scale proportionately). Classically, even geometric similarity does not ensure thermal similarity in scaled systems - for example, heat transfer is an interface-controlled process and so the surface area relative to the volume is critical. As volumetric scale increases, the area relative to the volume decreases and the overall efficiency of heat transfer can decline considerably. There is no thermal inertia with microwaves, on the other hand. Since penetration depth is not an issue with the continuous flow design, the energy is deposited uniformly throughout the mixture resulting in rapid energy transfer and direct dielectric heating - hence the thermal inertia inherent to classical methods is not an issue.

\section{References}

1. Belwal, T., Ezzat, S.M., Rastrelli, L., Bhatt, I.D., Daglia, M., Baldi, A., Devkota, H.P., Orhan, I.E., Patra, J.K., Das, G., Anandharamakrishnan, C., Gomez-Gomez, L., Nabavi, S.F., Nabavi, S.M., Atanasov, A.G., Trends in Analytical Chemistry, 2018, 100, 82-102.

2. Stankiewicz, A.I., Moulijn, J.E., Chem. Eng. Process, 2010, 96, 22-34.

3. Estel, L., Poux, M., Benamara, N., Polaert, I., Chem. Eng. Process, 2017, 113, 5664. 


\title{
MICROWAVE PYROLYSIS OF BIOMASS IN A ROTATORY KILN REACTOR: DEEP CHARACTERIZATION AND COMPARATIVE ANALYSIS OF PYROLYTIC LIQUIDS PRODUCTS
}

\author{
Lilivet UBIERA, Isabelle POLAERT, Lokmane ABDELOUAHED, Bechara TAOUK
}

\author{
Normandie University, INSA Rouen, LSPC, EA 4704 - INSA Rouen, Avenue de l'Université \\ - Saint-Étienne-du-Rouvray cedex 76801 France \\ e-mail : isabelle.polaert@insa-rouen.fr
}

Keywords: Microwave pyrolysis, Rotatory Kiln Reactor, Flax Shives, Green fuels

\section{Introduction}

Process intensification by maximizing the production efficiency and minimizing the hazardous effects on the environment and human health is one of the main objectives nowadays, in which the biggest question is how to create a new sustainable relationship between a growing industry and our planet. In this context, both, the industry and the scientific community are increasingly concerned about the environmental deterioration and the economic risks associated with the increasing demand and dependency on fossil fuels. The pursuit of sustainable relationship between the production and consumption of energy has accelerated the research into new fuels alternatives, and mainly focused on new technologies for biomass based fuels. The potential of lignocellulosic biomass for power generation has been studied several times and it is considered as a promising renewable energy source. [8] In France, for example, around 200,000 tons of flax shives are generated annually by the flax industry, being $80 \%$ of the world's production of scutched flax fibers originated in Europe, being France, the world leader [4]. The potential of this biomass to be integrated as a power source has not been completely explored. Some of the investigations done about the exploitation of flax shives as raw material for the biofuel production by thermochemical conversion has been done in our laboratory but only for conventional heating method and to the best of our knowledge, this is the first time that the flax shives pyrolysis has been evaluated under microwave heating conditions [1][6].

Microwaves are electromagnetic radiations placed between the radio and infrared waves in the electromagnetic spectrum. The frequency of the microwaves is in a range between $300 \mathrm{MHz}$ and $300 \mathrm{GHz}$ but for industrial application the most commonly used are $2.45 \mathrm{GHz}$ and $915 \mathrm{MHz}$. The use of microwaves as a heating method for pyrolysis process has been long recognized to provide better quality bio-products in shorter reaction time due to the direct sample heating and the particular heating profile resulting from the interaction of biomass with the electric field component of an electromagnetic wave. [7][1][8]

Also, it has been reported by [2] that reactor design may have a great influence on the pyrolysis product yields. The rotatory kiln reactor provides a homogeneous heating profile inside the reactor which is desired for a fast microwave pyrolysis to avoid hot-spots. During the course of this research, flax shives were pyrolysed using a rotatory kiln reactor inside a microwave single mode cavity using a range of power between 200 and 300 watts, to reach a temperature range between $450{ }^{\circ} \mathrm{C}$ and $700^{\circ} \mathrm{C}$. The liquid bio-oil samples recovered in each case were analyzed though gas chromatography-mass spectrometry (GC-MS) and gas chromatography-flame ionization detection (GC-FID) to identify and quantify the different 
molecules produced. The aim of this work is to investigate the upgrading of flax shives via microwave pyrolysis and shed some light on its pyrolytic behaviour.

\section{Experimental section}

\section{Materials and Elemental Analysis}

The samples for this study were Flax Shives, a lignocellulosic biomass provided by "La cooperative Terre de Lin'. The samples were ground and sieved in our laboratory. The average particle size ranged between 300 and $400 \mu \mathrm{m}$.

The total $\mathrm{C}, \mathrm{H}$, and $\mathrm{N}$ were measured on sub-samples using a $\mathrm{CHN}$ elemental analyzer Flash 2000 (Thermofisher Scientific). Also a proximate analysis was done based on TGC experiments. Both results were published by part of our research group in the publication [6]

Table 1. Elemental Analysis [6]

\begin{tabular}{|c|c|c|c|c|}
\hline \multicolumn{5}{|c|}{ Elemental Analysis (\% w:w) } \\
\hline \multirow{2}{*}{ Flax Shives } & Carbon & Hydrogen & Nitrogen & Oxygen \\
\cline { 2 - 5 } & 45.70 & 5.77 & 0.41 & 48.12 \\
\hline
\end{tabular}

The analysis of the percentage of lignocellulosic biomass pseudo-components has been done based on TGA experiments.

Table 2. Flax shives composition based on TGA experiments [6]

\begin{tabular}{|c|c|c|c|c|}
\hline \multicolumn{5}{|c|}{ Proximate Analysis (\% w:w) } \\
\hline Flax Shives & Humidity & Volatile Matter & Fixed Carbon & Ash \\
\cline { 2 - 5 } & 8.28 & 69.22 & 19.97 & 2.53 \\
\hline
\end{tabular}

\section{Experimental set up}

This study used a single-mode microwave cavity. The microwaves were generated by a SAIREM magnetron at a frequency of $2.45 \mathrm{GHz}$ and a power between 0 and $2000 \mathrm{~W}$. The incident and reflected microwaves powers were recorded using two bolometers and the absorbed power was deduced from the two first measured powers. A system of three manual sliding short-circuit was used to maximize the wave-matter interaction and to maintain a range of reflective wave between 5 and $15 \%$, taking advantage of a minimum of 85 percent of the applied power.

The pyrolysis process was conducted in a rotatory kiln glass reactor, set to rotate at $65 \mathrm{rpm}$ and purged with a $600 \mathrm{~mL} / \mathrm{min}$ of nitrogen to maintain the anoxic conditions. The temperature in the reactor vessel, a glass cylinder of $17 \mathrm{~cm}$ of length and $3 \mathrm{~cm}$ of internal diameter, was measured at the end of the experiment using a thermocouple type $\mathrm{K}$ sensor. This thermocouple was introduced inside the reactor when the microwave heating system was just turned off and placed in the bottom of the glass reactor to measure the final temperature achieved and the cooling ramp. The temperature at the surface of the reactor was measured during the reaction time when the microwaves were "on" using an optical fiber to avoid interference with the electromagnetic field in the waveguide.

After purging the system, the power supply was turned on and switched to the desired microwave power level for 6 minutes. The non-condensable gases were recovered in a sampling bag to be later analysed. The cold bath and the refrigerant were both kept at a constant temperature of $-14{ }^{\circ} \mathrm{C}$. At the end of each experiment, all the reactor parts were 
weighted and the char and oil weights were experimentally deduced. The non-condensable gas weight was estimated thanks to a method using a standard gas phase GC analysis.

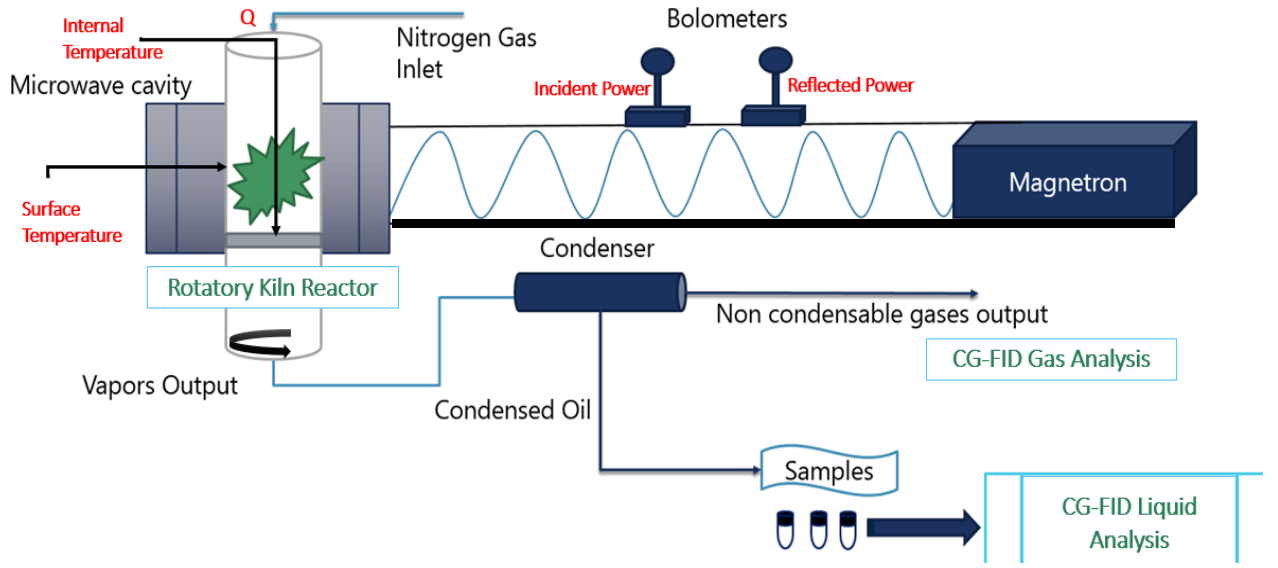

Fig. 1. Experimental Set-up

\section{Analytical procedure of the bio-oil}

The analysis of the recovered bio-oil was performed using a gas chromatograph-mass spectrometer instrument GC-MS (Varian 3900-Saturn 2100T). The column was a VF-1701 $\mathrm{ms}$ (Agilent) (60m x $0.25 \mathrm{~mm} \times 0.25 \mu \mathrm{m}$ film thickness).. The oven was held at $45{ }^{\circ} \mathrm{C}$ for 4 min then heated to $280{ }^{\circ} \mathrm{C}$ with a heating rate of $4{ }^{\circ} \mathrm{C} / \mathrm{min}$ and held for $20 \mathrm{~min}$. The carrier gas was helium with a constant flow of $1 \mathrm{ml} / \mathrm{min}$.

$1 \mu \mathrm{L}$ of the bio-oil sample diluted in pure acetone was directly injected into the heated split injector (split ratio 30:1) at $250 \mathrm{C}$. The detection was performed in a full scan mode and the components were identified with a Varian Work Station and NIST 2002 software by comparing the mass spectrum with the NIST's library.

Kovats's retention indices of the identified components were then calculated and compared to the reference value for the conformation of their identity.

After identifying the components of the bio-oil samples, a flame ionization detector (FID), GC-FID Scion 456-GC Bruker instrument was used to quantify the components. The column and temperature program used were the same as the ones used for the GC-MS analysis. The detected compounds were grouped into families of the same functional group and then one representative component in its pure form was taken to make calibration curve for the corresponding family. Five points straight line calibration curves were established for these pure representative compounds, using dodecane as internal standard.

\section{Results and discussion}

\section{Surface vs internal temperature measurement}

When using microwave processes, one of the key points is the temperature evaluation. Both surface temperature (TS) and internal temperature (Tint) were measured during experiments of biomass microwave heating. An optical fiber was used to measure the reactor surface temperature during the experiment and when the optical fiber achieved a temperature equal to $150{ }^{\circ} \mathrm{C}$, the microwave generation was turned off and then a thermocouple was inserted 
until the bottom of the reactor to measure the internal temperature. This procedure was repeated four times in order to check the stability of temperature.

The behaviour observed is presented in the figure 2. It was observed that for the same absorbed power $\left(\mathrm{P}_{\mathrm{abs}}\right)$, the temperature behaviour was similar and stable. It was also noticed that the difference of internal and surface temperature was more than the double for each measurement. When the outer surface temperature of the reactor reaches $150^{\circ} \mathrm{C}$, more than $300^{\circ} \mathrm{C}$ is reached inside the biomass and the steady state inside the reactor is reached faster than outside.

For a reactor of $3 \mathrm{~cm}$ internal diameter, several experiments were done using $4.5 \mathrm{~g}$ of biomass and several incident powers from 100 to $400 \mathrm{~W}$. For each run, the observed temperature inside the reactor, at the heart of the biomass was at least the double of the one at the outer surface of the reactor.

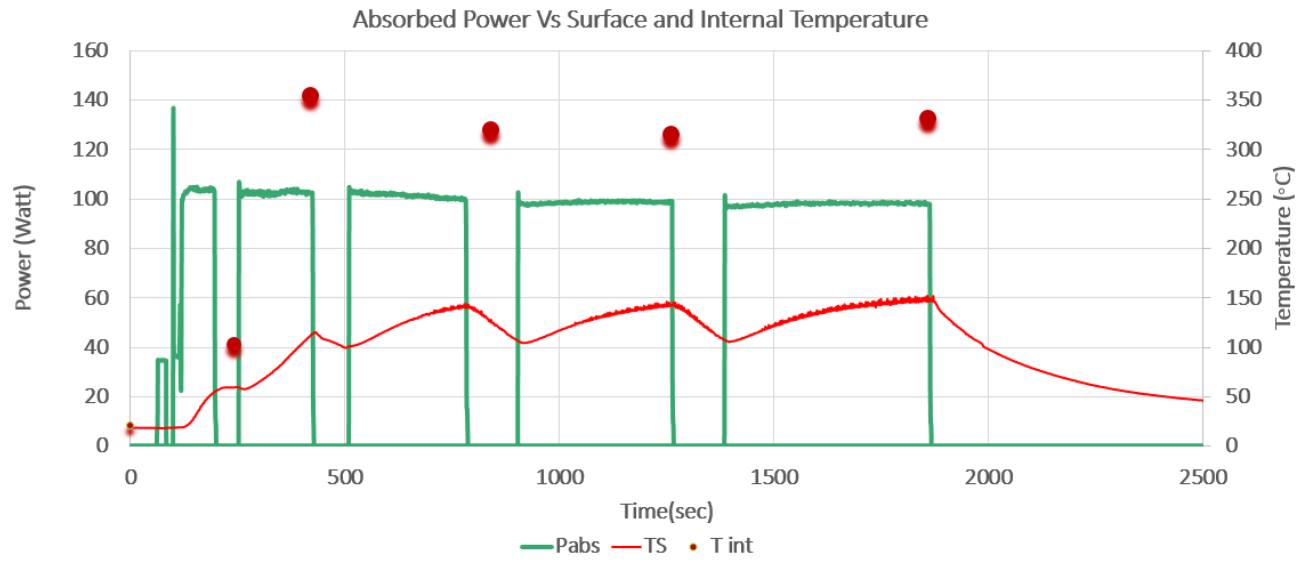

Fig. 2. Absorbed power, surface and internal temperatures during biomass microwave heating: mbiomass $_{\text {b }}=9 \mathrm{~g}$

\section{Microwave power absorption during biomass pyrolysis}

The behaviour of the absorbed, incident and reflected powers measured by bolometers was observed. The performance of the incident and reflected power mirrored the different biomass decomposition phases. For example, figure 3. shows common profiles of incident, reflected and absorbed powers during a pyrolysis experiment. The first zone inside the black square corresponds to the biomass drying period. The second zone in which both incident and reflected power are instable corresponds to the active pyrolysis zone. Inside the active pyrolysis zone, there is phenomena of devolatilization of cellulose and hemicellulose and also a significant mass loss. During this period, the sliding short circuit system is manually adjusted to maximise the absorbed power. At the end of the experiment, a new stable phase begins that can be also called the passive phase which is characterized by the slow degradation of lignin and a weak weight loss. This mechanism of three phases pyrolysis division was also observed by [1] in TGA experiments study for flax shives. 


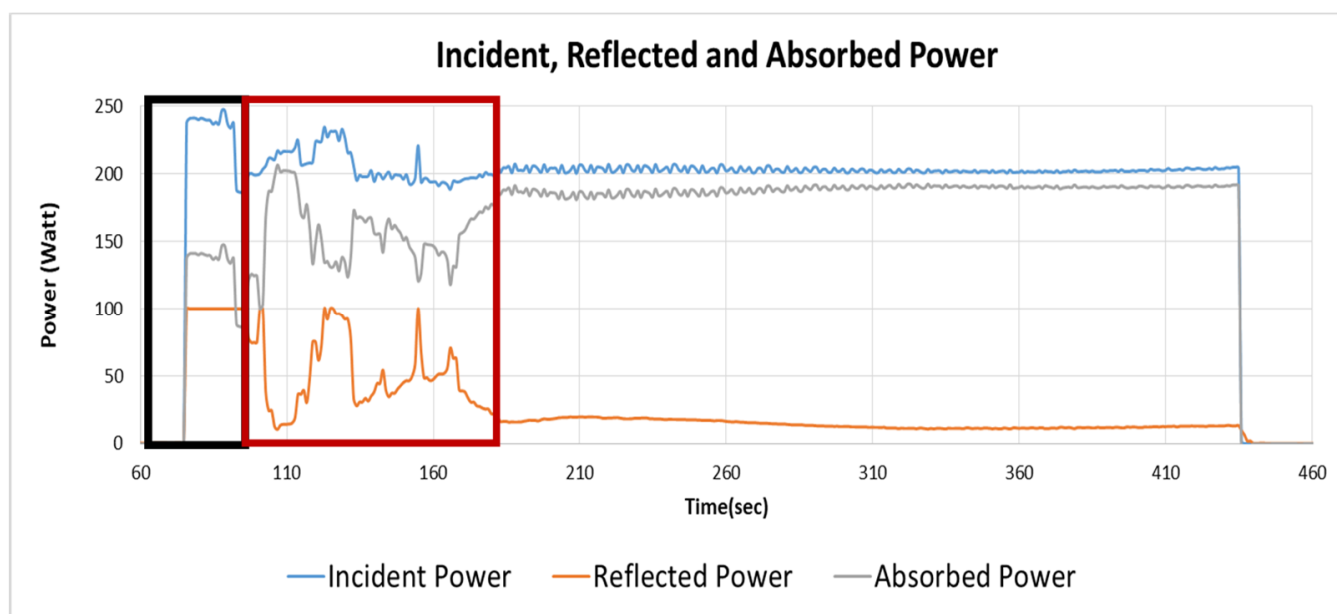

Fig. 3. Incident, reflected and absorbed power during a microwave pyrolysis process

\section{Microwave pyrolysis process results}

Several experiments were carried out with $4.5,6.75$ or $9 \mathrm{~g}$ of biomass. For each mass of biomass, the incident power was varied between 200 and $300 \mathrm{~W}$ for an average absorbed power between 160 and 220 watts respectively. The nitrogen gas flowrate was varied from 300 to $600 \mathrm{ml} / \mathrm{min}$.

Before evaluating the effect of the operational parameters, the repeatability of the microwave pyrolysis process was tested in terms of microwave absorbed power, global energy transferred and maximum temperature reached. First, 4.5 grams of biomass were treated under the same input power $(200 \mathrm{~W})$, vapors residence time and irradiation time $(7 \mathrm{~min})$. The results are presented in the table 3 .

Table 3. Repeatability of experiments

\begin{tabular}{|c|c|c|c|c|c|}
\hline Number & $\begin{array}{l}\text { Mass of } \\
\text { biomass (g) }\end{array}$ & $\begin{array}{l}\text { Average } \\
\text { absorbed } \\
\text { power }(\mathbf{W})\end{array}$ & $\begin{array}{lr}\begin{array}{l}\text { Total } \\
\text { energy }\end{array} & \text { effective } \\
\text { applied }\end{array}$ & $\begin{array}{l}\text { Maximum } \\
\text { temperature } \\
\left({ }^{\circ} \mathrm{C}\right)\end{array}$ & $\begin{array}{l}\text { Vapors } \\
\text { residence } \\
\text { time (s) }\end{array}$ \\
\hline 1 & 4.5 & 162 & 53 & 627 & 1.4 \\
\hline 2 & 4.5 & 158 & 56 & 682 & 1.4 \\
\hline 3 & 4.5 & 164 & 60 & 710 & 1.4 \\
\hline
\end{tabular}

The irradiation method was also evaluated. Different experiments were carried using continuous irradiation mode and compared with discontinuous modes. The results are presented in the table 4 . 
Table 4. Evaluation of microwave irradiation mode

\begin{tabular}{|l|l|l|l|l|l|l|l|}
\hline Number & $\begin{array}{l}\text { Mass of } \\
\text { biomass } \\
\text { (g) }\end{array}$ & $\begin{array}{l}\text { Microwave } \\
\text { irradiation } \\
\text { mode }\end{array}$ & $\begin{array}{l}\text { Irradi } \\
\text { ation } \\
\text { time } \\
\text { (minut } \\
\text { es) }\end{array}$ & $\begin{array}{l}\text { Average } \\
\text { absorbed } \\
\text { power } \\
\text { (W) }\end{array}$ & $\begin{array}{l}\text { Total } \\
\text { effective } \\
\text { energy } \\
\text { applied } \\
\text { (kJ) }\end{array}$ & $\begin{array}{l}\text { Maximum } \\
\text { tempera- } \\
\text { ture } \\
\text { ('C) }\end{array}$ & $\begin{array}{l}\text { Vapors } \\
\text { residenc } \\
\text { e } \\
\text { time } \\
\text { (s) }\end{array}$ \\
\hline 4 & 4.5 & $\begin{array}{l}\text { Continuous } \\
7 \text { min on 240W }\end{array}$ & 7 & 201 & 86 & 568 & 1.6 \\
\hline 5 & 4.5 & $\begin{array}{l}\text { Discontinuous } \\
4 \text { min on 240W } \\
1 \text { min off }\end{array}$ & 7 & 198 & 67 & 640 & 1.3 \\
\hline 3 min on 240W & & & & & & \\
\hline 6 & 4.5 & $\begin{array}{l}\text { Discontinuous } \\
4 \text { min on 200W } \\
1 \text { min off } \\
2 \text { min on 200W }\end{array}$ & 6 & 180 & 58 & 586 & 1.6 \\
\hline
\end{tabular}

In table 3, although incident power has been reproduced, the average absorbed power varies from one experiment to another. It depends on the lapse of every biomass pyrolysis phase (active or passive) during the reaction period, and the tuning effectiveness. Therefore, using the total effective energy (E) defined in equation (1) is more convenient in order to make the comparisons between experiments. $t_{0}$ is the initial irradiation time and $t_{f}$ is the time for which the microwaves are turned off.

$$
E=\int_{t 0}^{t f} P_{\text {absorbed }} d t \quad \text { Equation [1] }
$$

In table 3, this energy is correlated positively with the maximal temperature achieved by the system. In table 4, for the same average absorbed power, an important gap in the effective energy applied is noticed between a continuous and a discontinuous mode. It was shown that for the same time of irradiation, the discontinuous mode promotes a higher temperature with a lower energy, because pyrolysis takes advantage of heat accumulation in the reactor walls.

\section{Microwave pyrolysis products distribution}

For the experiments of the table 4, the products distribution is reported in table 5 and results obtained with an initial mass of 9 grams of biomass are added. The higher the applied energy is, the higher the bio-oil production is and the lowest the char production is. 
Table 5. Microwave pyrolysis products distribution using 4.5 grams or 9 grams of biomass

\begin{tabular}{|c|c|c|c|c|c|c|c|c|c|c|c|}
\hline $\begin{array}{l}\text { Mass of } \\
\text { biomass } \\
\text { (g) }\end{array}$ & $\begin{array}{c}\text { Average } \\
\text { absorbe } \\
\text { d power } \\
\text { (W) }\end{array}$ & $\begin{array}{c}\text { Energy } \\
\text { applied } \\
\text { E (kJ) }\end{array}$ & $\begin{array}{c}\text { Max } \\
\text { temperatu } \\
\text { re } \\
\left({ }^{\circ} \mathrm{C}\right)\end{array}$ & $\begin{array}{l}\text { Vapors } \\
\text { residence } \\
\text { time } \\
\text { (s) }\end{array}$ & $\begin{array}{l}\text { Oil } \\
\text { (g) }\end{array}$ & $\begin{array}{c}\text { Char } \\
\text { (g) }\end{array}$ & $\begin{array}{c}\text { Gas } \\
\text { (g) }\end{array}$ & $\begin{array}{c}\text { Oil } \\
\%\end{array}$ & $\begin{array}{l}\text { Ch } \\
\text { ar } \\
\%\end{array}$ & $\begin{array}{c}\text { Ga } \\
\text { s } \\
\%\end{array}$ & $\begin{array}{c}\text { Total } \\
\text { Product } \\
\text { recovere } \\
\text { d } \\
\%\end{array}$ \\
\hline 4,5 & 201 & 86 & 568 & 1.6 & 2.25 & 0.852 & 0.937 & 50 & 19 & 21 & 90 \\
\hline 4.5 & 198 & 67 & 640 & 1.3 & 2.14 & 0.61 & 0.771 & 48 & $14^{*}$ & 17 & 79 \\
\hline 4.5 & 180 & 58 & 586 & 1.6 & 2.017 & 1.08 & 0.727 & 46 & 24 & 17 & 87 \\
\hline 9 & 222 & 94 & 678 & 6 & 4.32 & 1.89 & - & 48 & 21 & -** & - \\
\hline 9 & 186 & 70 & 600 & 6 & 3.7 & 2.7 & - & 41 & 23 & $-* *$ & - \\
\hline 9 & 156 & 57 & 457 & 6 & 2.7 & 3.06 & - & 30 & 34 & $-* *$ & - \\
\hline
\end{tabular}

*Mass of char was partially recovered. **Mass of gas not available.

The effect of the initial mass of biomass put inside the reactor was evaluated. 9, 6.75 and 4.5 grams were treated during $6 \mathrm{~min}, 200 \mathrm{~W}$ of incident power and nitrogen flowrate of 600 $\mathrm{ml} / \mathrm{min}$. For a similar range of energy absorbed, the initial mass has a strong influence on the maximum temperature achieved and products distribution (see table 6). High loads lead to higher temperature because thermal losses are less and it has a negative influence on the bio-oil production. Gasification is clearly favoured at high temperature at the expense of oil production.

Table 6. Microwave pyrolysis products distribution: effect of the biomass loading

\begin{tabular}{|c|c|c|c|c|c|c|c|c|c|c|c|}
\hline $\begin{array}{c}\text { Mass of } \\
\text { biomass } \\
(\mathbf{g})\end{array}$ & $\begin{array}{c}\text { Average } \\
\text { absorbed } \\
\text { power } \\
(\mathbf{W})\end{array}$ & $\begin{array}{c}\text { Energy } \\
\text { applied } \\
\mathbf{E}(\mathbf{k J})\end{array}$ & $\begin{array}{c}\text { Maximum } \\
\text { temperature } \\
\left({ }^{\circ} \mathbf{C}\right)\end{array}$ & $\begin{array}{c}\text { Vapors } \\
\text { residence } \\
\text { Time } \\
(\mathbf{s})\end{array}$ & $\begin{array}{c}\text { Oil } \\
\mathbf{( g )}\end{array}$ & $\begin{array}{c}\text { Char } \\
(\mathbf{g})\end{array}$ & $\begin{array}{c}\text { Gas } \\
\mathbf{( g )}\end{array}$ & $\begin{array}{c}\text { Oil } \\
\mathbf{\%}\end{array}$ & $\begin{array}{c}\text { Char } \\
\mathbf{\%}\end{array}$ & $\begin{array}{c}\text { Gas } \\
\mathbf{\%}\end{array}$ & $\begin{array}{c}\text { Total } \\
\text { product } \\
\text { recovered } \\
\mathbf{\%}\end{array}$ \\
\hline $\mathbf{4 . 5}$ & 159 & 60 & 710 & 1.3 & 2.4 & 0.85 & 0.91 & 52 & 19 & 20 & 91 \\
\hline $\mathbf{6 . 7 5}$ & 164 & 63 & 794 & 2.1 & 2.9 & 1.4 & 1.1 & 43 & 21 & 17 & 81 \\
\hline $\mathbf{9}$ & 177 & 64 & 805 & 2.6 & 2.8 & 1.8 & 2.1 & 31 & 20 & 24 & 75 \\
\hline
\end{tabular}

\section{Microwave pyrolysis bio-oil characterization}

The liquid bio-oil samples recovered in each case were analyzed to identify and quantify the different molecules produced. As an example, results are reported in table 7 for the three experiments of table 4. Molecules were grouped into 11 families, as shown in figure 4. Figure A and B correspond respectively to the results obtained by the GC-FID analysis for the first and the second line of table 7. Results for the third line are similar to the last ones. In general, the bio-oil samples are composed of about 40-50 wt. \% of carboxylic acids, 15 20 wt. $\%$ of phenols, $12-14$ wt. \% of ketones and 8-10 wt. \% of esters majorly. Carboxylic acids are the dominating chemical group present, followed by phenols. 


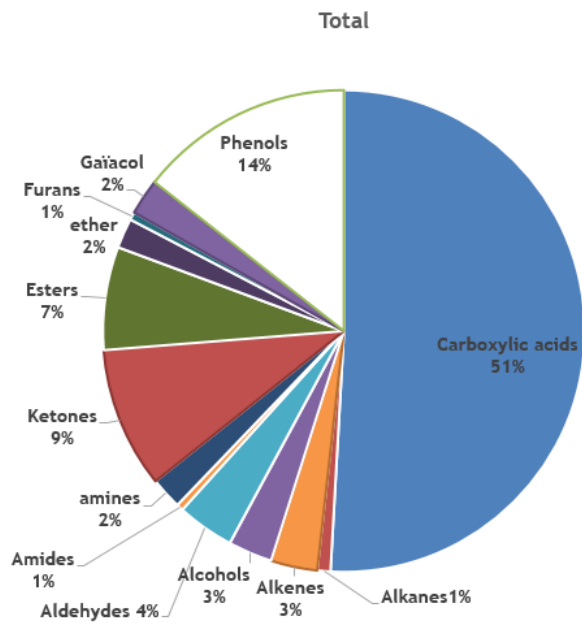

Figure A

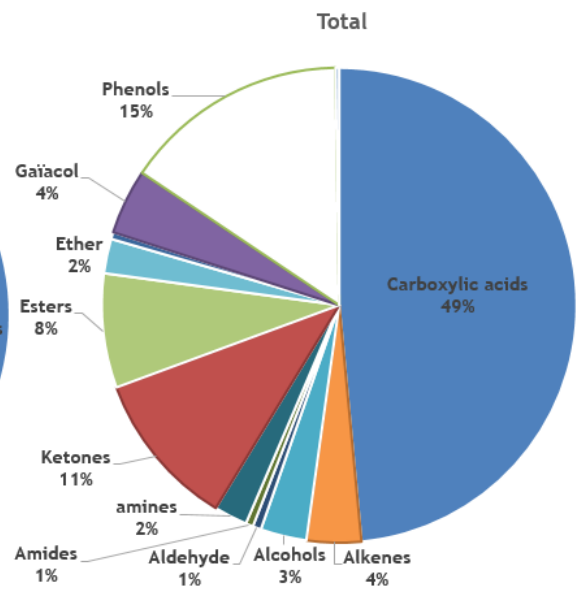

Figure B

Fig. 4. Chemical families in flax shives pyrolytic bio-oil

Both experiments have a similar average of absorbed power but different effective energies applied. from the percentages in table 7, it is clear that the distribution of chemical families is a little bit affected by the absorbed energy and temperature reached. The carboxylic acid production and therefore the oxygen rate are favored by an increasing absorbed energy where ketones formation is decreased. The percentage of phenols are stable in each case but the higher percentage of alkenes is found when lower energy is applied.

Table 7. Microwave pyrolytic bio-oil: effect of the effective applied energy

\begin{tabular}{|c|c|c|c|c|c|c|c|c|c|c|}
\hline $\begin{array}{c}\text { Mass of } \\
\text { biomass } \\
\text { (g) }\end{array}$ & $\begin{array}{c}\text { Average } \\
\text { absorbed } \\
\text { power } \\
\text { (W) }\end{array}$ & $\begin{array}{l}\text { Energy } \\
\text { applied } \\
\text { E (kJ) }\end{array}$ & $\begin{array}{c}\text { Maximum } \\
\text { temperature } \\
\left({ }^{\circ} \mathrm{C}\right)\end{array}$ & $\begin{array}{c}\text { Quantity of } \\
\text { compounds } \\
\text { detected by } \\
\text { GC-DID }\end{array}$ & $\begin{array}{c}\text { Oxygen } \\
\text { rate } \\
\%\end{array}$ & $\begin{array}{c}\text { Carboxylic } \\
\text { acids } \\
\%\end{array}$ & $\begin{array}{c}\text { Phenols } \\
\%\end{array}$ & $\begin{array}{c}\text { Ketones } \\
\%\end{array}$ & $\begin{array}{c}\text { Esters } \\
\%\end{array}$ & $\begin{array}{c}\text { Alkenes } \\
\%\end{array}$ \\
\hline 4.5 & 201 & 86 & 568 & 270 & 39.2 & 51 & 14 & 9 & 7 & 2 \\
\hline 4.5 & 198 & 67 & 640 & 272 & 37.8 & 49 & 15 & 11 & 7 & 3 \\
\hline 4.5 & 180 & 58 & 586 & 237 & 32.8 & 47 & 15 & 12 & 6 & 4 \\
\hline
\end{tabular}

\section{Conclusions}

The effect of the microwave heating using a rotatory kiln glass reactor for flax shives was investigated during the course of this study. The different steps of the pyrolysis reaction interfere in the stability of the microwave absorbed power and a precise tuning is required during the course of the pyrolysis. Microwave heating leads to high temperatures and heating rates and the rotary kiln reactor configuration favours homogeneity of the process.

When applying different incident powers resulting into different global applied energies, moderate differences on the products distribution were found. But, the higher the absorbed 
energy is, the higher bio-oil production is. In conclusion, the developed microwave pyrolysis process gives a clear way to produce an exploitable bio-oil from flax shives.

\section{References}

1. Abdelouahed, L., Leveneur, S., Vernieres-Hassimi, L., Balland, L., \& Taouk, B. Journal of Thermal Analysis and Calorimetry, 2017, Vol. 129, pp. 1201-12013

2. Beneroso, D., Monti, T., Kostas, E., \& Robinson, J2017, Chemical Engineering Journal, pp. 481- 498.

3. Bridgwater, A. Biomass Bioenergy, 2012, Vol. 38, pp. 68-94.

4. EIA (Energy Information Administration), Annual Energy Outlook 2010. 2010

5. https://www.europeanflax.com/. (2019, Juin 15) . Retrieved from Europuean Flax and Hemp.

6. Mohabeer, C., Abdelouahed, L., Marcotte, S., \& Taouk, B. J. Anal. Appl. Pyrolysis, 2017, Vol. 127, pp. 269-277.

7. Robinson, J., Dodds, C., Stavrinides, A., S. Kingman, Katrib, j., Wu, Z., Overend, R. Energy Fuels, 2015, Vol. 29, pp. 1701-1709.

8. Shiung Lam, S., \& Chase, H. A. Energies, 2012, Vol. 5, pp. 4209-4232 


\title{
GROWTH OF NANNOCHLORIS ALGAE IN THE PRESENCE OF MICROWAVES (CONTINUOUS REACTOR)
}

\author{
I. Calinescu ${ }^{1}$, A. Vintila ${ }^{1,2}$, A. Diacon ${ }^{1}$, M. Vinatoru ${ }^{1}$, P. Chipurici ${ }^{1}$, Ana-Maria Galan ${ }^{2}$, \\ Sanda Velea ${ }^{2}$
}

1 Faculty of Applied Chemistry and Material Science, University "Politehnica » of Bucharest, 1-7 Gh. Polizu, Bucharest, 011061, Romania

${ }^{2}$ National Institute for Research \& Development in Chemistry and Petrochemistry ICECHIM, 202 Spl. Independentei, 060021, Bucharest, Romania

Ioan.calinescu@upb.ro

Keywords: microwave, bioeffect, nannochloris, PUFA, carotenoids

\begin{abstract}
Algae are very effective in capturing the sun's energy, carbon dioxide from the atmosphere, and nutrients to turn them into useful substances. Certain algae contain significant amounts of unsaturated oils but also carotenoids. The commercial value of these compounds can be greatly increased by increasing the degree of unsaturation of oils and the carotenoid concentration. One of the possibilities to alter the growth rate of algae and metabolite composition is microwave irradiation. By microwave-controlled irradiation of the nutrient and algae flux, which is recirculated through the photobioreactor and through a glass reactor located in a TE-type monomod cavity, the algal oil content increased and the change in oil fraction composition was important. Significantly increased the concentration of polyunsaturated acids with 16 and 18 carbon atoms. As far as carotenoids are concerned, the algae nannochloris has a higher carotenoid content over many known vegetables holding carotene or lycopene (carrots or tomatoes). Besides oil increasing microwave treatment produced a significant increase in carotenoid content of algae. They can be extracted together with omega-3-rich algal oil and are the basis of very valuable dietary supplements.
\end{abstract}

\section{Introduction}

Algae are very effective in capturing the sun's energy, carbon dioxide from the atmosphere, and nutrients to turn them into useful substances (carbohydrates, oils, proteins, etc.). Besides the main metabolites, there are also secondary metabolites, such as carotenoids (astaxanthin, $\beta$-carotene, lutein, lycopene, and canthaxanthin [1].

Both major and compounds existing in small amounts in algae are useful. Oils and carbohydrates could provide biofuels, proteins can get products with nutritional value and from carotenoids can be prepared food supplements. Obtaining biofuel from algae has not yet proved to be economically viable $[2,3]$. A much higher interest might be getting food supplements from algae. To increase their value as ingredients for food supplements, algal oils should have a higher degree of unsaturation (rich in omega 3) and an increased carotenoid content to be an important additional benefit in over all processing of algae.

There are studies that refer to the influence of environmental factors on algae composition [2], but the microwave influence on algae growth, especially algal metabolites composition change is very poor studied.

One of the first descriptions of microwave effect on algae is that of Hamid and Badour in 1973[4]. The authors study the effect of microwaves on algae at high temperatures (50 to $100{ }^{\circ} \mathrm{C}$ ) resulting in proteolysis and complete failure of the alga to photosynthesize and 
grow, and at $35-40^{\circ} \mathrm{C}$, exerted a stimulating effect on photosynthesis and subsequent algal growth as compared to conventional heating within the same temperature range.

Another important factor in stimulating algal growth is the wavelength of electromagnetic radiation, thus $7.1 \mathrm{~mm}$ wavelength (exposure for $30 \mathrm{~min}$ at $2.2 \mathrm{~mW} / \mathrm{cm}^{2}$ ) enhanced the growth of a blue-green algae Spirulina platensis by $50 \%$, whereas $8.34 \mathrm{~mm}$ wavelength produced no changes compared with sham control [Tambiev et al., 1989 in [5]].

In this paper, besides the experiments for the activation of algal growth in discontinuous reactors [6] additional work was conducted in a continuous photobioreactor. The goal was checking not only the growth of microalgae but also their content in polyunsaturated oil and in carotenoids.

\section{Experimental section}

\section{Microalgae Data}

Nannochloris sp. 424-1 is a strain deposited under the number CCAP 251/10 in the Culture Collection of Algae and Protozoa (CCAP) at the Scottish Marine Institute, Aryll, UK. The strain was obtained from a wild strain by mutation-selection, after cultivation on media with metronidazole [7].

\section{Culture Medium}

The culture medium for Nannochloris $s p$. was Zarrouk medium, with the following composition $(\mathrm{g} / \mathrm{L}) 16.8 \mathrm{NaHCO}_{3}, 0.5 \mathrm{~K}_{2} \mathrm{HPO}_{4}, 2.5 \mathrm{NaNO}_{3}, 1.0 \quad \mathrm{~K}_{2} \mathrm{SO}_{4}, 1.0 \mathrm{NaCl}, 0.2$ $\mathrm{MgSO}_{4} .7 \mathrm{H}_{2} \mathrm{O}, 0.04 \mathrm{CaCl}_{2} .2 \mathrm{H}_{2} \mathrm{O}, 1 \mathrm{~mL} / \mathrm{L}$ of trace metal solution, and $5 \mathrm{~mL} / \mathrm{L}$ of chelated iron.

\section{Cultivation Conditions}

Microalgae were cultivated into a Biostat PBR 2S photobioreactor (a 3L volumetric type with a photosynthesis module), the photobioreactor being connected in series with the Miniflow 200SS microwave system. Microwave treatment was performed in a $25 \mathrm{~mL}$ volumetric glass coil mounted in the TE monomod cavity.

To perform each experiment, 0.3 L inoculum Nannochloris sp and 2.7 L Zarrouk growth medium were used respectively. Cultivation was carried out under photoautotrophic conditions: cultivation temperature $28^{\circ} \mathrm{C}$, continuous illumination $85 \mu \mathrm{mol} /\left(\mathrm{m}^{2} * \mathrm{~s}\right)$, continuous aeration with synthetic air mixture with $7 \% \mathrm{CO} 2(\mathrm{v} / \mathrm{v})$. All cultures were treated with microwave the day after inoculation. The culture was recirculated in the photoreactor using a pump flow rate of $0.0217 \mathrm{~L} / \mathrm{s}$.

\subsection{Cell Counting}

The algal cultures cell density was determined by direct cell counting daily by using a Thoma Hemocytometer [8]. The data obtained was reported as cell per mL.

\subsection{Biomass assay}

The quantity of the algal biomass obtained per $\mathrm{mL}$ of sample was determined by measuring the dry weight. The algal suspension was centrifuged at $8000 \mathrm{rpm}$ for 10 minutes using a Rotina 380R centrifuge from Hettich Zentrifugen. The biomass obtained was washed two times with distilled water and after that the biomass obtained was dried in the oven at $105^{\circ} \mathrm{C}$ for $1 \mathrm{~h}$ and then placed in vacuum desiccant for 30 minutes. The sample was weighed and then introduced in the oven for 30 minutes. The procedure was repeated until the weight of the sample was constant. For the determination and characterization of the oil content of the lipid fraction of the microalgae, an own method, as described in [9] was used. 


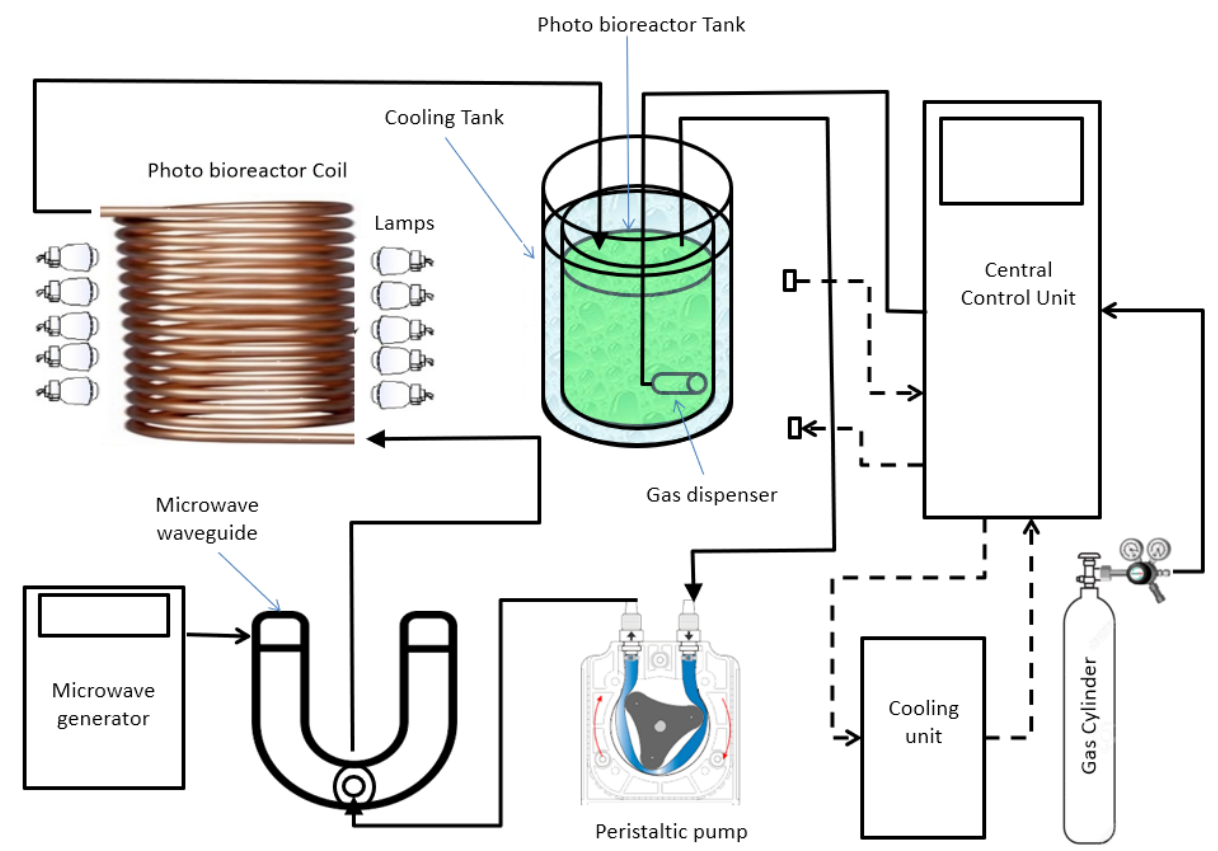

Fig. 1. Setup used for ultrasound assisted growing of algae

To determine the percentage of oil (transesterifiable lipids - free fatty acids and triglycerides) available in dry biomass. Equation 2 was used:

$\%$ oil in dry biomass $=\frac{C_{\text {esters }} \cdot \text { Vol }_{\text {esters }}}{\text { Weight }_{\text {dry biomass }}}$ where:

$\mathrm{C}_{\text {esters }}=$ the concentration of methyl esters of fatty acids obtained by GC-MS analysis $[\mathrm{g} / \mathrm{mL}]$;

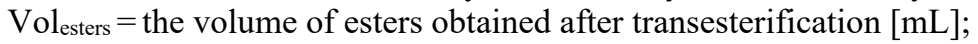

Weight $_{\text {dry biomass }}=$ the weight of dry biomass $[\mathrm{g}]$.

\subsection{Algal pigments determination}

The chlorophyll and carotenoids concentration was determined by UV-Vis spectroscopy in accordance with standard protocols [10]. The determination involved the extraction of 0.25 $\mathrm{g}$ of dried algal biomass with $10 \mathrm{~mL}$ of ethanol (95\%) (1:40 weight: volume ratio) at $55^{\circ} \mathrm{C}$ using an ultrasonic probe Hielscher UP200St (equipped with a titanium probe of $13 \mathrm{~mm}$ using $60 \%$ amplitude and $5 \mathrm{~s}$ on, $5 \mathrm{~s}$ off operating conditions) and a magnetic stirrer set for $800 \mathrm{rpm}$. The extract was centrifuged after $1 \mathrm{~h}$ of extraction and diluted with ethanol. The UV-Vis absorption spectra were registered using an Able Jasco V-550 spectrophotometer. The equations used to quantify the chlorophyll $\mathrm{a}$, chlorophyll $\mathrm{b}$ and beta-carotene content were:

$[\text { Chl a }]_{\frac{m g}{L}}=13,36 A_{664,1}-5,19 A_{648,6}$

$[\text { Chl b }]_{\frac{m g}{L}}=27,43 A_{648,6}-8,12 A_{664,1}$ 
$[\text { beta }- \text { carotene }]_{\frac{m g}{L}}=\frac{1000 A_{470}-2,13[\text { Chl a }]-97,64[\text { Chl b }]}{209}$

For a clearer presentation of the results, the final pigment concentration was calculated against dried algal biomass and expressed as $\mathrm{mg}$ pigment/g dried algae.

\section{Result and discussion}

In the first stage, experiments were carried out with the change of the microwave power. Microwave treatment was performed for 4 hours / day on days 2, 3, 4 and 5 with 5, 10 and $20 \mathrm{~W}$ microwave power (for the $25 \mathrm{~mL}$ serpentine, the SAR values are : 200, 400 and 800 $\mathrm{W} / \mathrm{kg}$ respectively). Cultures were monitored at all times, and the cell density was read daily. The obtained values (cell number and total dry algae) are shown in Table 1.

From the analysis of the data presented in Table 1 it can be seen that the small microwave powers ( 5 and $10 \mathrm{~W}$ ) have a favorable influence on the number of cells and the total quantity of dried dry algae, while at the power of $20 \mathrm{~W}$ both the number of cells and the algae mass obtained is lower than the control sample.

Considering that the power of $10 \mathrm{~W}$ seems to be the best choice, it has been decided to continue experimenting with the use of microwave treatment at a power of $10 \mathrm{~W}$ for just 2 or 3 days (also with a 4-hour treatment duration /day) with stopping algae growth after these days. The results on cell number evolution are shown in Table 1.

Table 1 Experimental data on microwave irradiated microalgae cultures.

\begin{tabular}{|c|c|c|r|r|r|r|}
\hline & \multicolumn{7}{|c|}{ MW power, W / days of irradiation } \\
\cline { 2 - 7 } & Control & $5 / 4$ & $10 / 4$ & $20 / 4$ & $10 / 2$ & $10 / 3$ \\
\hline SAR (W/kg) & 0 & 200 & 400 & 800 & 400 & 400 \\
\hline Time (days) & \multicolumn{7}{|c|}{ Cell number / $10^{7}$} \\
\hline 0 & 0.14 & 0.10 & 0.10 & 0.10 & 0.01 & 0.01 \\
\hline 1 & 0.70 & 0.62 & 0.56 & 0.62 & 0.54 & 0.50 \\
\hline 2 & 1.31 & 1.09 & 1.09 & 1.09 & 0.98 & 1.05 \\
\hline 3 & 3.97 & 2.51 & 3.11 & 3.11 & 2.32 & 1.95 \\
\hline 4 & 7.25 & 5.33 & 7.81 & 5.33 & & 6.56 \\
\hline 5 & 10.64 & 11.65 & 11.14 & 8.00 & & \\
\hline 6 & 14.19 & 14.81 & 15.77 & 11.14 & & \\
\hline 7 & 16.77 & 17.82 & 19.30 & 12.73 & & \\
\hline 8 & 17.82 & 18.92 & 20.46 & 13.02 & & \\
\hline \multicolumn{7}{|c|}{ The final amount of dry algae (g/L) } \\
\hline
\end{tabular}

It was further determined the amount of lipid fraction and the percentage and composition of oil obtained from the microalgal biomass samples. The results obtained for the case of microwaves irradiated at 5, 10 and 20 watts for 4 days are presented in Table 3. 


\section{High Frequency Heating}

Valencia, Spain, September 9-12, 2019

Table 3 Oil and lipid content for microwave irradiated samples at 5, 10 and 20 watts

\begin{tabular}{|l|c|c|c|c|}
\multicolumn{2}{c}{ for 4 days } \\
\hline & \multicolumn{4}{c|}{ MW power, W / days of irradiation } \\
\hline Lipids content $(\% \mathrm{w} / \mathrm{w})$ & Control & $5 / 4$ & $10 / 4$ & $20 / 4$ \\
\hline Oil content $(\% \mathrm{w} / \mathrm{w})$ & 25.03 & 29.12 & 29.42 & 30.44 \\
\hline Carotenoid content $(\mathrm{mg} / \mathrm{g})$ & 10.15 & 11.40 & 13.80 & 12.35 \\
\hline
\end{tabular}

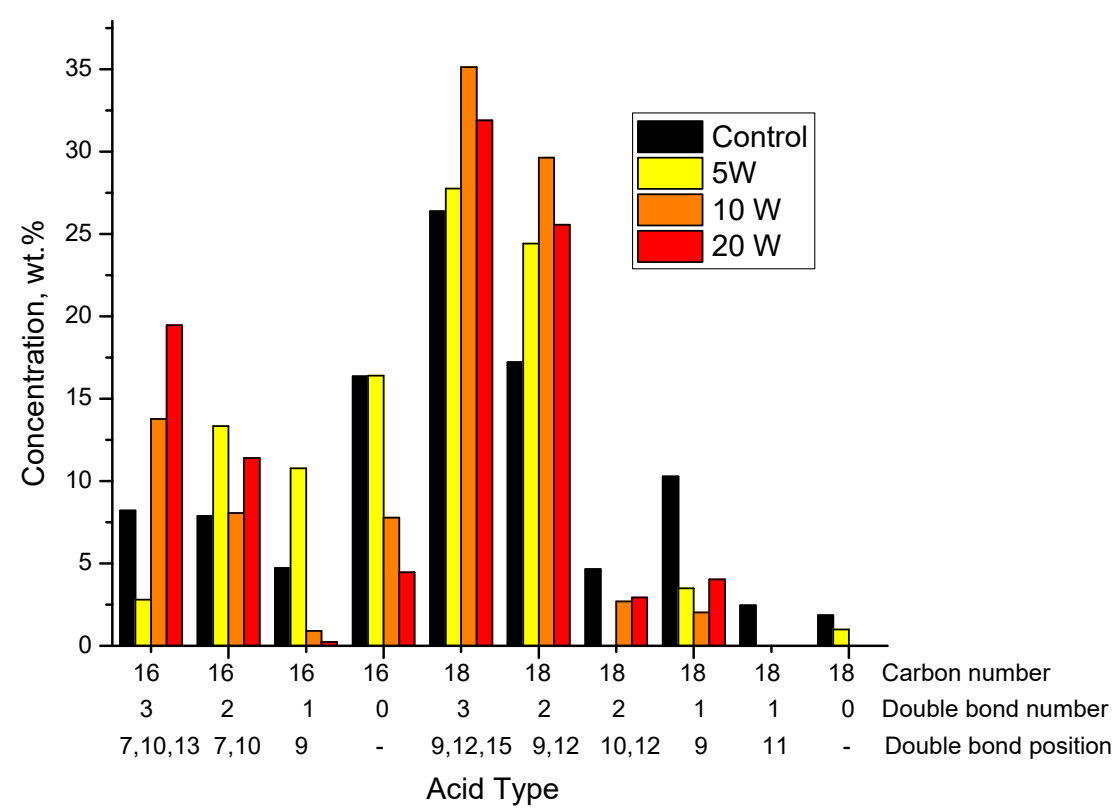

Fig.2. Fatty acid distribution of oil extracted from algae grown for 8 days microwave irradiated at 5, 10 and 20 watts for 4 days.

From the analysis of the data presented in Table 3 and figure 2, it can be noticed an increase in the microalgal biomass oil concentration in all microwave-treated samples. A significant increase is observed in the sample treated with a power of $10 \mathrm{~W}$. As for the distribution of fatty acids the most significant increases are seen in polyunsaturated acids: 16: $3,18: 3$ and 18:2. These increases are particularly noticeable for the $10 \mathrm{~W}$ power. Acids whose concentrations decreases are those with low unsaturation: 16: 0, 18: 1 and 18: 0 .

The carotenoid concentration has also increased with microwave treatment. The best value is also obtained at a $10 \mathrm{~W}$ irradiation.

In order to determine the cell growth period in which the modification of the fatty acid structure occurs, we compared the analysis of treated samples with a microwave power of $10 \mathrm{~W}$ (4 hours / day) for 2 days, 3 days and 4 days respectively for the control sample. The results are shown in table 4 and figure 3. 
Table 4 Oil and lipid content for microwave irradiated samples at 10 watts for 2,3 and 4 days

\begin{tabular}{|l|c|c|c|c|}
\hline & \multicolumn{5}{|c|}{ MW power, W / irradiation days / } \\
& \multicolumn{4}{|c|}{ growing days } \\
\hline & Control & $10 / 4 / 8$ & $10 / 3 / 4$ & $10 / 2 / 3$ \\
\hline Lipids content $(\% \mathrm{w} / \mathrm{w})$ & 25.03 & 29.42 & 21.42 & 19.42 \\
\hline Oil content $(\% \mathrm{w} / \mathrm{w})$ & 10.15 & 13.80 & 7.32 & 5.52 \\
\hline
\end{tabular}

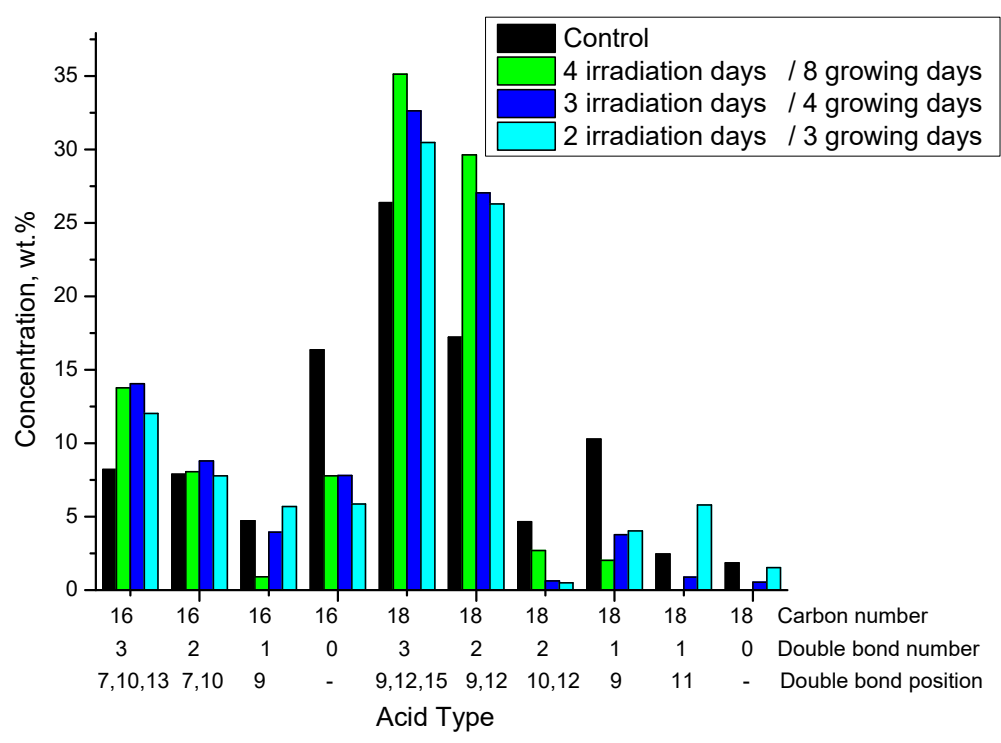

Fig.3. Fatty acid distribution of oil extracted from algae grown under a microwave irradiation of $10 \mathrm{~W}$ for 2,3 or 4 days.

By analyzing the data obtained, a progressive increase in polyunsaturated fatty acid content (16:3, 18:3 and 18:2) can be observed. Growth is very strong after the second day of microwave irradiation and continues on day three and fourth, but with a slower growth rate. Similarly, decreasing the concentration of saturated acids (16:0 and 18:0) or monounsaturated (18:1) is very important after 2 days of microwave irradiation and then the decrease is much lower.

\section{Conclusion}

$\checkmark \quad$ The growth of microalgae of the Nannochloris sp type is influenced by the applied microwave treatment;

$\checkmark$ Growth of the algae was carried out in a Zarrouk growth medium containing the algae through a microwave heated serpentine and through the light irradiated photobiorector;

$\checkmark$ Microwave treatment was performed starting with the next day of growth, for 4 hours / day, with different powers; 
$\checkmark$ Thus, the best microwave treatment condition was obtained which was adequate at a power of $10 \mathrm{~W}$ (SAR $400 \mathrm{~W} / \mathrm{kg})$;

$\checkmark \quad$ Under these conditions an increase in algal mass from 3.03 to $3.35 \mathrm{~g} / \mathrm{L}$, an increase in oil content from 10.15 to $13.8 \%$, but especially a significant increase in the content of polyunsaturated fatty acids (18:3,18:2 and 16:3) and in the concentration of carotenoids from 3.03 to $4.12 \mathrm{mg} / \mathrm{g}$;

$\checkmark$ The use of microwave treatment for just 2 or 3 days showed that the change in the composition of the algal oil begins even after the first day of treatment and continues at a lower rate even after days 2,3 or 4 of treatment.

\section{Acknowledgement}

The authors acknowledge the financial support received from the Competitiveness Operational Programme 2014-2020, Action 1.1.4: Attracting high-level personnel from abroad in order to enhance the RD capacity, project: P_37_471, „Ultrasonic/Microwave Nonconventional Techniques as new tools for nonchemical and chemical processes", financed by contract: 47/05.09.2016

\section{References}

1. Gong, M. and A. Bassi, Carotenoids from microalgae: A review of recent developments. Biotechnology Advances, 2016. 34(8): p. 1396-1412.

2. Juneja, A., R. Ceballos, and G. Murthy, Effects of Environmental Factors and Nutrient Availability on the Biochemical Composition of Algae for Biofuels Production: A Review. Energies, 2013. 6(9): p. 4607-4638.

3. Adeniyi, O.M., U. Azimov, and A. Burluka, Algae biofuel: Current status and future applications. Renewable and Sustainable Energy Reviews, 2018. 90: p. 316-335.

4. Hamid, M.A.K. and S.S. Badour, The Effects of Microwaves on Green Algae. Journal of Microwave Power, 1973. 8(3): p. 268-273.

5. Pakhomov, A.G., et al., Current state and implications of research on biological effects of millimeter waves: A review of the literature. Bioelectromagnetics, 1998. 19(7): p. 393-413.

6. Popescu, A., et al., Microwaves bioeffects on microalgae growth, in 15th International Conference on Microwave and High Frequency Heating AMPERE 2015. 2015: Krakow, Poland.

7. Oancea, F., et al., Nannochloris sp. stain for obtainment of bio-based aviation fuel. 2015.

8. Thoma cell counting chamber. 12.06.2019]; Available from: http://insilico.ehu.es/counting_chamber/thoma.php.

9. Galan, A.M., et al., Development of a new method for determination of the oil content from microalgae lipid fraction. Revista de Chimie, 2017. 68(4): p. 671674.

10. Lichtenthaler, H.K. and C. Buschmann, Chlorophylls and Carotenoids: Measurement and Characterization by UV-VIS Spectroscopy. Current Protocols in Food Analytical Chemistry, 2001. 1(1): p. F4.3.1-F4.3.8. 


\title{
SCALABLE MICROWAVE WASTE-TO-FUEL CONVERSION
}

\author{
M. Robinson ${ }^{1}$, Z. Popovic ${ }^{1}$ \\ ${ }^{1}$ Electrical, Computer and Energy Engineering, University of Colorado, Boulder, U.S.A. \\ Megan.Robinson@,colorado.edu,Zoya.Popovic@,colorado.edu
}

Keywords: microwave, pyrolysis, waste management, high-efficiency SSPAs

\section{Introduction}

This paper presents an efficiency study for scalable microwave waste management. When waste with carbon content is subjected to volume power densities on the order of $0.25 \mathrm{~W} / \mathrm{cm}^{3}$ at $\mathrm{GHz}$ frequencies, it converts to solid coke fuel with oil and gas bi-products that can further be for fuel, leaving no trace. For an efficient process, a well-controlled uniform RF field should be maintained in a non-uniform and time-variable material. We are developing a 2.45-GHz active microwave cavity with solid-state $(\mathrm{GaN})$ spatially power combined sources for lower volumes, Fig.1. In the energy balance calculations, the input energy into the system consists of the waste chemical energy and the DC electrical energy used to obtain the RF power with an efficiency that can reach $70 \%$ for $\mathrm{kW}$ power levels. The efficiency of RF power conversion to heating rate in the waste mass is calculated from full-wave simulations for four waste mixtures, input power, and operating temperature. The output energy estimates are collected from various pyrolysis process descriptions, e.g. [1], with the total energy being that of the solid fuel $(35 \mathrm{MJ} / \mathrm{kg})$ and oil caloric values, e.g. $40 \mathrm{MJ} / \mathrm{kg}$ for plastics and about $10-15 \mathrm{MJ} / \mathrm{kg}$ for non-plastics [2]. A byproduct is flue gas, which can be converted to synthetic gas fuel (Syngas) as described in, e.g. [3].

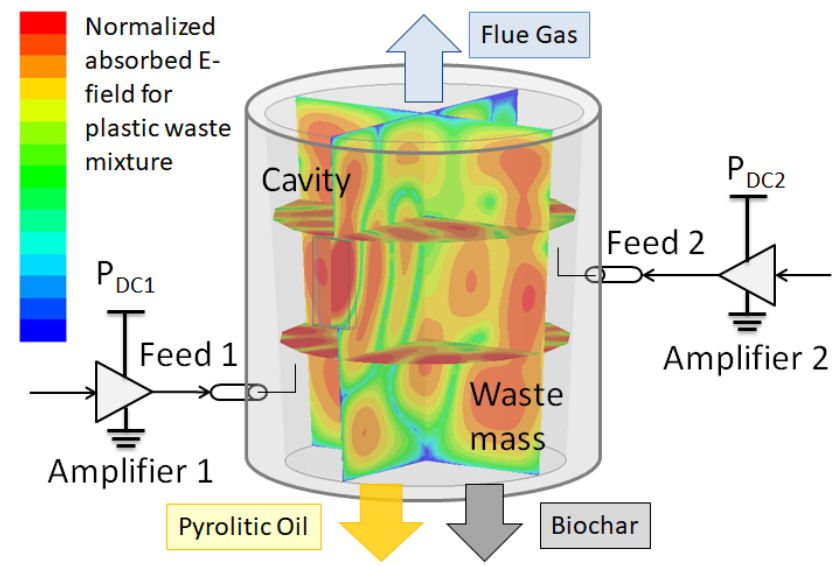

Fig. 1. Block diagram of an active microwave cavity for waste to fuel conversion. The cavity is fed by several power amplifiers with probe outputs. Absorption of microwave energy results in waste conversion into solid fuel (biochar), oil which can be converted to liquid fuel, and flue gas which can be converted to synthetic gas fuel. The relative phase between the feeds, mass mixing, frequency modulation and mode mixing can be used to ensure high uniformity of the electric field within the waste mass. 


\section{DC to RF Conversion}

The two methods to obtain $\mathrm{kW}$-level power at $\mathrm{GHz}$ frequencies are solid-state power amplifiers and tubes. In the $2 \mathrm{GHz}$ range, $\mathrm{GaN}$ transistors have demonstrated high power levels with high efficiency, e.g. [4],[5] and can be further power combined. Magnetrons have also been power combined with power combining efficiencies above $90 \%$ to achieve power levels around $30 \mathrm{~kW}[6]$. The analysis in this paper is not specific to a particular type of RF power generation, and is described by a conversion efficiency which is assumed to be $70 \%$.

\section{Electric Field Simulations}

The fields inside the waste volume produce heat through Joule losses. The thermal power produced in the cavity depends on the filling material properties, relative permittivity $\left(\varepsilon_{r}\right)$, conductivity $(\sigma)$, geometry of cavity and type of excitation, and is calculated from:

$$
P_{T h}=\iiint|E(x, y, z)|^{2} \sigma(x, y, z) d V
$$

It is desirable to keep the power density of Joule losses uniform through the heating process, because that usually results in the best time-average efficiency. This analysis was done using a single waveguide port excitation. There is a tradeoff between large volumes which can have a high level of field uniformity and small volumes which are effectively heated with a single feed. Several rectangular and cylindrical cavities, with volumes ranging from $0.0008 \mathrm{~m}^{3}$ to $0.15 \mathrm{~m}^{3}$, were compared using a full-wave eigen-mode solver (Ansys HFSS) to determine the modal content around the operating frequency of $2.45 \mathrm{GHz}$. The chosen cavity has the shape of an actual trash-can, cylindrical in shape and with one base larger, Fig.2. For the simulations, the radii of the bases are chosen to be $R_{l}=12.7 \mathrm{~cm}$ and $R_{2}=11.2 \mathrm{~cm}$, with a height of $H=28.2 \mathrm{~cm}$. The feed is an S-band (WR340) waveguide port with a lower edge $13 \mathrm{~cm}$ above the bottom of the cavity. A larger version of this system will include multiple waveguide ports that are strategically placed to uniformly excite the waste volume under varying permittivity and loss conditions.

Equation (1) can be simplified if the waste volume is assumed to consist of sub-volumes that have uniform conductivities, where the integral becomes a sum:

$$
P_{T h}=\sum_{k=0}^{n} \sigma|E(x, y, z)|^{2} \Delta V
$$

The fields can be found using a full-wave EM solver, given a specific geometry, electrical properties, and excitation. Plots of the magnitude of the electric field throughout the cavity are shown for two extremes of the four cases in Fig. 2. The breakdown of the food mixture was not detailed in reference [8] and for this analysis a mixture of $45 \%$ meats and starches, $35 \%$ fruits and vegetables, and $20 \%$ fats was assumed. This food mixture, high permittivity and loss, shows absorption near the feed and would improve with multiple excitations. Whereas the low-density polyethylene, LDPE, and polystyrene mixture, low permittivity and loss, has high electric field magnitude throughout the cavity. Despite the high field magnitude the low loss of the material limits the power that can be absorbed and reduces the heating efficiency. 


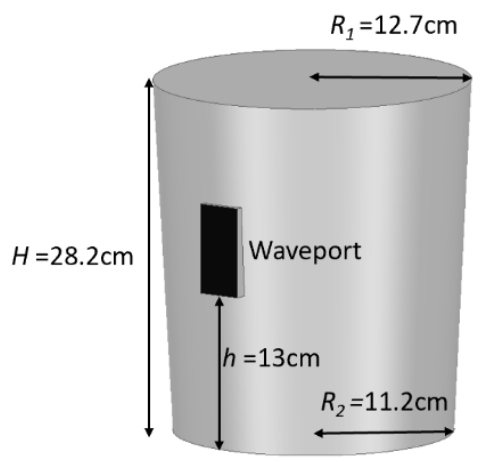

(a)
Magnitude of Electric Field

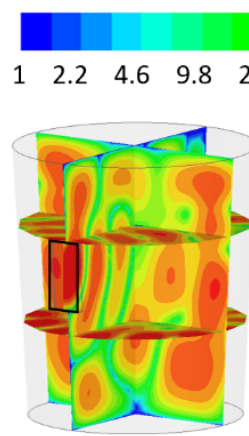

(b)

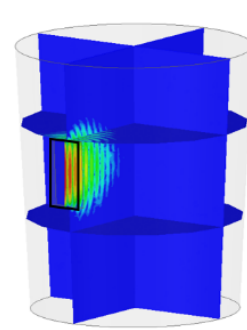

(c)

Fig 2. Simulated magnitude of the electric field for a single waveguide port excitation of a cavity completely filled with various materials. (a) Simulated cavity with dimensions labeled. (b) Cavity filed with food mixture $\varepsilon_{\mathrm{r}}=42$ and $\tan \delta=.3$, and (c) Cavity filled with LDPE and polystyrene $\varepsilon_{r}=2.25$ and $\tan \delta=.0003$. The field value is normalized to a $1 \mathrm{~W}$ incident power at $2.45 \mathrm{GHz}$ on waveguide port.

\section{Thermal Heating Rate Simulations}

The electrical and chemical processes that occur when there is an electromagnetic field inside a material are linked through the heating rate caused by Joule losses as:

$$
\sigma|E(x, y, z)|^{2}=\rho c \frac{d T(x, y, z)}{d t}-\nabla \cdot(\kappa \nabla T(x, y, z))
$$

where $\rho$ is the mass density of the filling material, $c$ is the specific heat of the material, $T$ is the temperature at a specific point within the material, and $\kappa$ is the thermal conductivity. An analytical solution for the heating rate is complicated since the temperature and electric field are functions of position. Therefore, multi-physics simulations were used to determine the temperature increase due to Joule losses and account for the anticipated energy leakage through the cavity walls with thermal insulation as shown in Fig. 3.

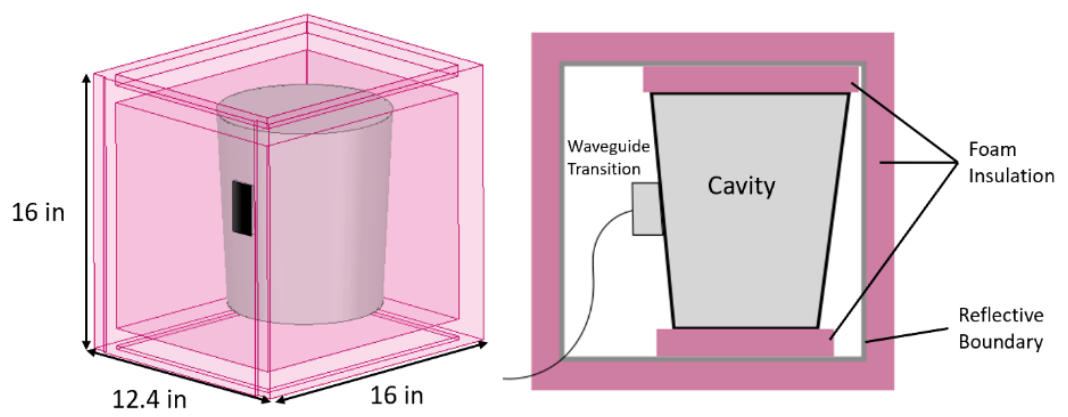

Fig. 3. Image of thermal simulation set up and a side cut of the insulation used in the simulation. The cavity filling is coupled to the field simulation as a thermal source. The cavity walls are $1 \mathrm{~mm}$ thick tin-plated steel, the reflective surround has a reflectivity of $94 \%$ and the foam insulation is 1 in thick XPS rigid polystyrene. 
Using Ansys Workbench to connect the electromagnetic field data from Ansys HFSS and thermal excitation in Ansys Mechanical, heating rates are determined for several input power levels and two internal cavity operating temperatures, the results are shown in Table 1 . These will be used to make an upper estimate of the RF power needed to obtain the specified heating rate required for pyrolysis processes.

Table 1. Simulated heating rates for given material input power and temperature

\begin{tabular}{cccc}
\hline Material & $\begin{array}{c}\text { Input RF } \\
\text { Power }(\mathrm{W})\end{array}$ & $\begin{array}{c}\text { Heating Rate at } 25^{\circ} \mathrm{C} \\
\left({ }^{\circ} \mathrm{C} / \mathrm{min}\right)\end{array}$ & $\begin{array}{c}\text { Heating Rate at } 400^{\circ} \mathrm{C} \\
\left({ }^{\circ} \mathrm{C} / \mathrm{min}\right)\end{array}$ \\
\hline $\begin{array}{c}\text { LDPE and } \\
\text { Polystyrene }\end{array}$ & 500 & .8 & .01 \\
\hline Textiles & 3000 & 5 & 1 \\
\cline { 2 - 4 } & 500 & 16 & 14 \\
\hline Food & 3000 & 75 & 65 \\
\cline { 2 - 4 } & 500 & 100 & 600 \\
\hline Paper & 3000 & 650 & 40 \\
& 500 & 44 & 310 \\
\hline
\end{tabular}

\section{Pyrolysis Process}

The approximation for input materials, output materials, and process requirements for the system are obtained from four reference papers [7-10] which describe pyrolysis for various materials. The cited experiments, with input parameters detailed in Table 2, deal with slow pyrolysis systems that are sealed and operated without catalysts over one hour or longer time periods.

Table 2. Input parameters for several pyrolysis examples with single materials

\begin{tabular}{cccccc}
\hline Material & $\begin{array}{c}\text { Input Caloric } \\
\text { Value } \\
(\mathrm{MJ} / \mathrm{kg})\end{array}$ & $\begin{array}{c}\text { Heating } \\
\text { Rate } \\
\left({ }^{\circ} \mathrm{C} / \mathrm{min}\right)\end{array}$ & $\begin{array}{c}\text { Duration } \\
\text { of Heating } \\
(\mathrm{min})\end{array}$ & $\begin{array}{c}\text { Max } \\
\text { Temperature } \\
\left({ }^{\circ} \mathrm{C}\right)\end{array}$ & $\begin{array}{c}\text { Time at } \\
\text { Max Temp. } \\
(\mathrm{min})\end{array}$ \\
\hline $\begin{array}{c}\text { LDPE and } \\
\text { Polystyrene [7] }\end{array}$ & 44 & 10 & 40 & 425 & 60 \\
Textiles [8] & 28 & 5 & 105 & 550 & 60 \\
Food [9] & 15.7 & 10 & 52 & 500 & 240 \\
Paper [10] & 13.6 & 10 & 85 & 873 & 60 \\
\hline
\end{tabular}

The compounds, composition, and corresponding caloric values of the chemical outputs for the various pyrolysis processes were determined in the references using spectroscopic methods. A summary of the chemical outputs for each process are summarized in Table 3. 
Table 3. Output Parameters for Pyrolysis

\begin{tabular}{ccccccc}
\hline Material & $\begin{array}{c}\text { Gas } \\
\text { Caloric } \\
\text { Value } \\
(\mathrm{MJ} / \mathrm{kg})\end{array}$ & $\begin{array}{c}\text { Percent } \\
\text { weight } \\
\text { Gas } \\
(\%)\end{array}$ & $\begin{array}{c}\text { Oil } \\
\text { Caloric } \\
\text { Value } \\
(\mathrm{MJ} / \mathrm{kg})\end{array}$ & $\begin{array}{c}\text { Percent } \\
\text { weight } \\
\text { Oil } \\
(\%)\end{array}$ & $\begin{array}{c}\text { Biochar } \\
\text { Caloric } \\
\text { Value } \\
(\mathrm{MJ} / \mathrm{kg})\end{array}$ & $\begin{array}{c}\text { Percent } \\
\text { weight } \\
\text { Biochar } \\
(\%)\end{array}$ \\
\hline $\begin{array}{c}\text { LDPE and } \\
\text { Polystyrene }\end{array}$ & 50.8 & 10 & 40.4 & 89.5 & $\mathrm{~N} / \mathrm{A}$ & 0.05 \\
$\begin{array}{c}\text { [7] } \\
\text { Textiles [8] }\end{array}$ & N/A & 45.44 & 11.49 & 24.7 & 31.73 & 13.28 \\
Food [9] & 0.22 & 7.41 & 8.01 & 32.29 & 7.55 & 60.3 \\
Paper [10] & N/A & 15.0 & 21.8 & 19.0 & 11.7 & 43.0 \\
\hline
\end{tabular}

\section{Total Efficiency}

Four different waste materials and mixtures are analyzed and the conversion efficiencies are calculated for filled cavities with dimensions as in Fig.2. The input and output parameters from Tables 2 and 3 are used for the material inside the cavity. The CW RF power required for each waste material to attain the heating rates from Table 2 are estimated by extrapolating the applied power and heating rate simulations from Table 1. Assuming a $70 \% \mathrm{DC}$ to RF efficiency the DC power is calculated for each material. The DC energy is computed by multiplying the DC power by the operating times in Table 2 . The conversion efficiency is defined as

$$
\eta=\frac{W_{C h, O U T}}{W_{C h, I N}+W_{D C}}
$$

where $W_{C h, O U T}$ and $W_{C h, I N}$ are the output and input chemical energies, and $W_{D C}$ is the input dc electrical energy. Fig. 4 shows the simulated electric field magnitude for the 4 cases at $2.45 \mathrm{GHz}$, and Table 4 summarizes the calculated efficiency using equations (2), (3) and (4).

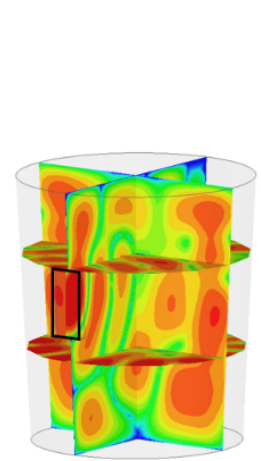

(a)

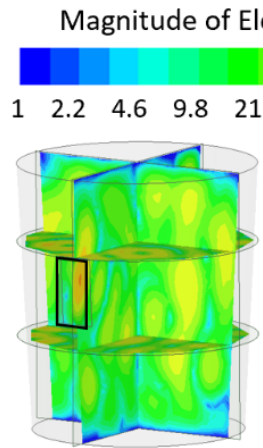

(b)

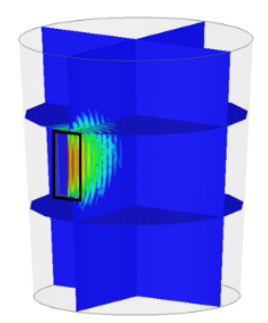

(c)

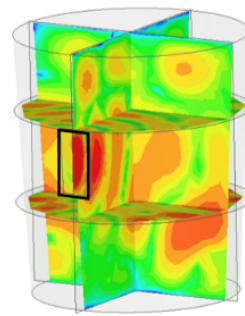

(d)

Fig.1. Simulated magnitude of the electric field, with $1 \mathrm{~W}$ incident power at $2.45 \mathrm{GHz}$, inside cavities completely filled with (a) LDPE and Polystyrene, $\varepsilon_{\mathrm{r}}$ of 2.25, $\tan \delta$ of .0003, thermal conductivity of $.28 \mathrm{~W} / \mathrm{m}^{\circ} \mathrm{C}$, density of $950 \mathrm{~kg} / \mathrm{m}^{3}$ and specific heat of 2300 $\mathrm{J} / \mathrm{kg}^{\circ} \mathrm{C}$, (b) 
Textiles, $\varepsilon_{\mathrm{r}}$ of 3 , tan $\delta$ of .0003 , thermal conductivity of $.05 \mathrm{~W} / \mathrm{m}^{\circ} \mathrm{C}$, density of $1100 \mathrm{~kg} / \mathrm{m}^{3}$ and specific heat of $.33 \mathrm{~J} / \mathrm{kg}^{\circ} \mathrm{C}$, (c) Food mixture, $\varepsilon_{\mathrm{r}}$ of 42 , $\tan \delta$ of .3, thermal conductivity of $.85 \mathrm{~W} / \mathrm{m}^{\circ} \mathrm{C}$, density of $950 \mathrm{~kg} / \mathrm{m}^{3}$ and specific heat of $1.7 \mathrm{~J} / \mathrm{kg}^{\circ} \mathrm{C}$, and (d) Paper, Er of 2.2, $\tan \delta$ of .03 , thermal conductivity of $.05 \mathrm{~W} / \mathrm{m}^{\circ} \mathrm{C}$, density of $1150 \mathrm{~kg} / \mathrm{m}^{3}$ and specific heat of $.33 \mathrm{~J} / \mathrm{kg}^{\circ} \mathrm{C}$.

Table 4. Summary of Full System Efficiencies

\begin{tabular}{cccccc}
\hline Material & $\begin{array}{c}\text { Input } \\
\text { Caloric } \\
\text { Value } \\
(\mathrm{MJ})\end{array}$ & $\begin{array}{c}\text { Output } \\
\text { Caloric } \\
\text { Value } \\
(\mathrm{MJ})\end{array}$ & $\begin{array}{c}\text { DC } \\
\text { Energy } \\
(\mathrm{MJ})\end{array}$ & $\begin{array}{c}\text { Conversion } \\
\text { Efficiency (\%) }\end{array}$ & $\begin{array}{c}\text { Output Energy } \\
\text { compared to } \\
\text { Input DC } \\
\text { Energy (\%) }\end{array}$ \\
\hline $\begin{array}{c}\text { LDPE and } \\
\text { Polystyrene }\end{array}$ & 545 & 548 & 96.8 & 85.4 & 566 \\
Textiles & 480 & 121 & 26.1 & 23.9 & 464 \\
Food & 202 & 92 & 12.2 & 43.0 & 754 \\
Paper & 136 & 92 & 16.0 & 60.5 & 575 \\
\hline
\end{tabular}

\section{Conclusion}

In summary, simulations of microwave heating at $2.45 \mathrm{GHz}$ indicate that it can be effectively used for pyrolysis of various types of waste material. The simulations are performed with a cylindrical thermally-insulated over-moded metal cavity with a single waveguide feed. Future work includes adding more feeds and an investigation power and volume scaling using solid-state sources for smaller volumes and magnetrons for larger volumes where higher power is required.

\section{Acknowledgement}

This work was funded by DARPA under contract W911NF-18-1-0073.

\section{References}

1. D. Czajczyńska, "Potential of pyrolysis processes in the waste management sector," Thermal Science and Engineering Progress, vol. 3, p. 171. Sept., 2017.

2. J.A. Onwudili, "Composition of products from the pyrolysis of polyethylene and polystyrene in a closed batch reactor: effects of temperature and residence time," Journal of Analytical and Applied Pyrolysis, vol. 86 p. 293-303. Nov., 2009.

3. S. Chunshan, "Tri-reforming of methane: a novel concept for synthesis of industrially useful synthesis gas with desired $\mathrm{H} 2 / \mathrm{CO}$ ratios using $\mathrm{CO} 2$ in flue gas of power plants without CO2 separation," Prepr. Pap.-Am. Chem. Soc., Div. Fuel Chem 49, no. 1 (2004): 128.

4. https://www.qorvo.com/products/p/QPD1025

5. H. Jeong, T. Yoon, H. Yoo, H. Jung, S. Cho, "A Highly Efficient and Compact 6kW GaN Solid-State Microwave Generator for CW 2.45GHz Applications," 2019 IEEE International Microwave Symp. (IMS), pp.1-4, June 2019, Boston, U.S.A.

6. C. Liu, H. Huang, Z. Liu, F. Huo and K. Huang, "Experimental Study on Microwave Power Combining Based on Injection-Locked 15-kW S-Band Continuous-Wave Magnetrons," IEEE Transactions on Plasma Science, vol. 44, no. 8, pp. 1291-1297, Aug. 2016. 
7. J.A. Onwudili, "Composition of products from the pyrolysis of polyethylene and polystyrene in a closed batch reactor: effects of temperature and residence time," Journal of Analytical and Applied Pyrolysis, vol. 86 p. 293-303. November, 2009.

8. S. Barış̧1, "The disposal of combed cotton wastes by pyrolysis," International Journal of Green Energy, vol. 11 p. 255-266. June, 2013.

9. S. Opatokun, "Product based evaluation of pyrolysis of food waste and its digestate," Energy, vol. 92 p. 349-354. December, 2015.

10. A. Sarkar, R. Chowdhury, "Co-pyrolysis of paper waste and mustard press cake in a semi-batch pyrolyser-optimization and bio-oil characterization," International Journal of Green Energy, Vol. 13 p. 373-382, October 


\title{
OPTIMIZATION OF MICROWAVE ASSISTED DELIGNIFICATION OF WOOD RESIDUES BY SURFACE RESPONSE METHODOLOGY
}

\author{
A. Trifan ${ }^{1}$, I. Calinescu ${ }^{1}$, M. Vinatoru ${ }^{1}$, A. I. Gavrila ${ }^{1}$, \\ ${ }^{1}$ University Politehnica of Bucharest, Faculty of Applied Chemistry and Material Sciences, \\ Bioresources Departament, 1-7 Polizu Gh. Street, Bucharest, Romania \\ adrian.trifan@upb.ro
}

Keywords: microwave, lignin, wood residue

\section{Introduction}

Due to effects such as global warming, environmental pollution and health hazards directly linked with the intensive exploitation of fossil fuels, there is an urgent need to find alternative energy resources. Vegetal biomass is a renewable resource with high availability worldwide. Current research is focused on developing efficient processes to transform this resource into bio-fuels and value-added fine chemicals [1, 2]. Vegetal biomass contains cellulose, hemicellulose and lignin in variable amounts. Pre-treatment plays a major role in breaking down the lignin barrier for the recovery of cellulose from lignocellulosic biomass. The effectiveness of a pre-treatment should be assessed following some criteria: minimizing energy input and the formation of inhibitors; maximizing the yield of fermentable sugars and preservation of cellulosic and hemicellulosic fractions [3]. There are several options of pre-treatment for lignocellulosic biomass and among them the most promising are: mineral acids, alkali, organosolv, wet oxidation, $\mathrm{CO}_{2}$ and steam explosion. A considerable amount of lignocellulosic biomass is generated as waste byproduct through agricultural practices and processing. Thus, the objective of this research is to improve the yield of enzymatic hydrolysis of wood residues by removal of lignin using alkaline wash assisted by microwave heating.

\section{Experimental}

Wood chips residue from a furniture factory (in Bucharest) were used for the experiments. The wood residue was milled and sieved. The $0.315-0.5 \mathrm{~mm}$ fraction was retained for the experiments. The wood residues were subjected to successive extractions with toluene:ethanol 1:2 solvent and then with water, for 6 hours, in a Soxhlet extractor, in order to remove wax and other extractives which can act as inhibitors for delignification and enzymatic hydrolysis. The wood residue was then dried for $48 \mathrm{~h}$ at $40^{\circ} \mathrm{C}$.

\subsection{Determination of the composition of wood residue}

The lignin and structural carbohydrates content of the wood residue (extracted) was determined according to NREL 42618 standard [4] by acid hydrolysis in two steps. In the first step, the wood residue is put into contact for 1 hour with $72 \%$ sulfuric acid. For the second step the solution is diluted to $4 \%$ sulfuric acid and autoclaved for 1 hour at $121^{\circ} \mathrm{C}$. Acid insoluble lignin is filtered, dried and weighted. The solution is neutralized and passed through $0,45 \mathrm{~mm}$ nylon filter before HPLC analysis for the determination of monosaccharides. The HPLC analysis was performed with a Jasco 2000+ System equipped with an RI detector. The monosaccharides were separated in isocratic conditions with 0,5 $\mathrm{ml} / \mathrm{min}$ ultrapure water as eluent, at $80^{\circ} \mathrm{C}$, on a Carbosep Corgel $87 \mathrm{P}(7.8 \times 300 \mathrm{~mm})$ 
Column. The results obtained for the characterization of the wood residue are presented in table 1 .

Table 1. Composition of wood residue from furniture manufacturing

\begin{tabular}{|l|l|c|}
\hline Chemical composition & Determination method & $\%$ \\
\hline Cellulose & Glucose - HPLC & 51.3 \\
\hline Hemicellulose & Xylose - HPLC & 19.6 \\
\hline Lignin & Klason lignin - gravimetry after acid hydrolysis & 25.5 \\
\hline \multirow{2}{*}{ Extractives } & Toluen+Ethanol extractives - gravimetry & 2.8 \\
\cline { 2 - 3 } & Water extractives - gravimetry & 0.8 \\
\hline
\end{tabular}

\subsection{Microwave alkaline treatment}

The treatments of the extracted wood residue were carried out in alkaline conditions, for 30 minutes, in a pressurized microwave reactor (Synthwave-Milestone). This $800 \mathrm{~mL}$ reactor is able to provide severe conditions: high temperatures (up to $300^{\circ} \mathrm{C}$ ) and pressures (up to 200 bar). The treatments were performed with a volume of $250 \mathrm{~mL}$ solution of $0.4 \mathrm{M} \mathrm{NaOH}$, $1 \mathrm{~mL}$ of Fatty acid methyl esthers (FAME), variable amount of extracted wood residue, different temperatures and 7 bar Argon pressure.

Figure 1 presents a one of the diagrams in which the experimental conditions (temperature, pressure and microwave power) are monitored and registered during alkaline treatment in the Synthwave microwave reactor. In order to maintain a constant temperature of $60^{\circ} \mathrm{C}$ inside the microwave reactor, the microwave power delivered inside the reactor was kept at 500$800 \mathrm{~W}$ in the heating region of the profile and at $150-200 \mathrm{~W}$ in the constant region of the temperature profile.

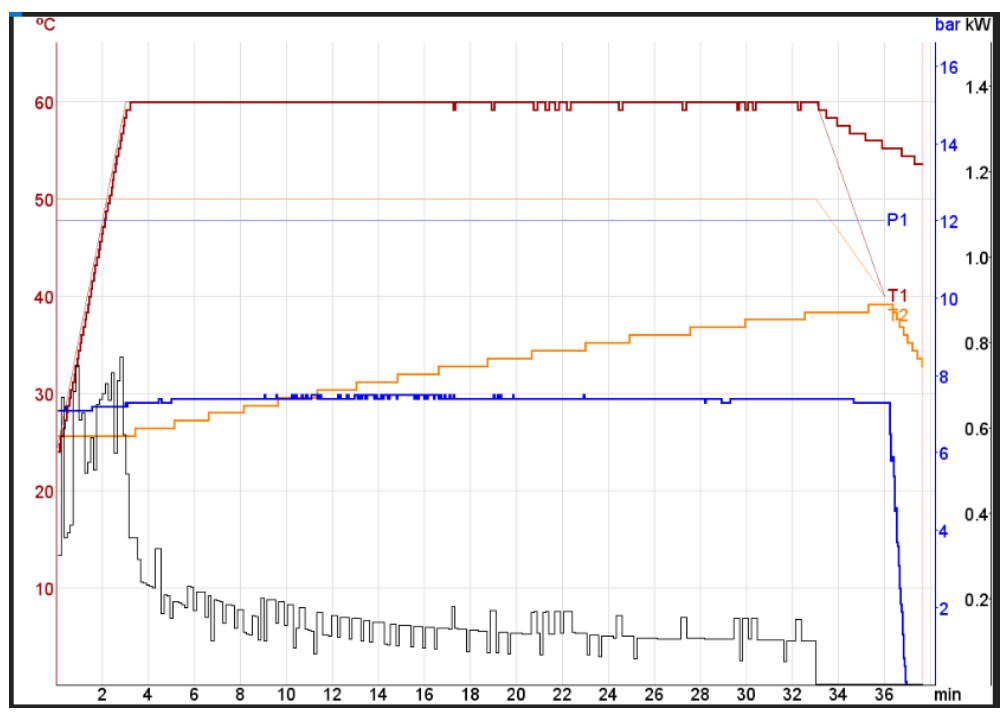

Fig. 1. Monitoring of alkaline treatment of wood residues in Synthwave microwave reactor: (Graph colour: temperature in red; pressure in blue; microwave power in black) 


\subsection{Lignin content}

The efficiency of the alkaline wash was assessed by monitoring the concentration of lignin (determined by UV absorbance at $320 \mathrm{~nm}$ against a calibration curve) from the alkaline washing solution.

\subsection{Determination of sugars from enzymatic hydrolysis}

The solid biomass obtained after the alkaline treatment was washed with distilled water until neutral $\mathrm{pH}$ and subjected to enzymatic hydrolysis. Enzymatic hydrolysis was performed at $50^{\circ} \mathrm{C}$, in a shaking water bath, with $1 \mathrm{~g}$ of treated wood residue, $25 \mathrm{~mL}$ of buffer solution $\mathrm{pH}=5$ (citric acid/ $\mathrm{Na}_{2} \mathrm{HPO}_{4}$ ) and $0.6 \mathrm{~mL}$ Celluclast $1.5 \mathrm{~L}$ enzyme. Sugar concentrations were determined every $24 \mathrm{~h}$ for three days to monitor the yield of enzymatic hydrolysis. Sugar concentration (as glucose equivalents obtained per one gram of dry mass) was determined following a modified dinitrosalicylic acid assay [5].

\subsection{Central composite design - lignin and glucose equivalents by enzymatic hydrolysis}

The treatment conditions were established according to the results obtained from a screening and characterization study carried out before constructing the experimental matrix for optimization (in Design Expert 11).

Table 2. The experimental matrix and the values determined for lignin and glucose equivalents from enzymatic hydrolysis of wood residue

\begin{tabular}{|c|c|c|c|c|c|c|c|}
\hline & & Factor 1 & Factor 2 & $\begin{array}{c}\text { Response } \\
1\end{array}$ & Response 2 & Response 3 & Response 4 \\
\hline \multirow[t]{2}{*}{ Std } & Run & A:temperature & $\| \begin{array}{c}\text { B: wood } \\
\text { residue }\end{array}$ & Lignin & $\begin{array}{c}\text { Glucose } \\
\text { Equivalents- } \\
\text { day } 1(\mathrm{EG} 1)\end{array}$ & $\begin{array}{c}\text { Glucose } \\
\text { Equivalents- } \\
\text { day } 2 \text { (EG2) }\end{array}$ & $\begin{array}{c}\text { Glucose } \\
\text { Equivalents- } \\
\text { day } 3(\mathrm{EG} 3)\end{array}$ \\
\hline & & ${ }^{0} \mathrm{C}$ & $\mathrm{g}$ & $\begin{array}{c}\mathrm{mg} / \mathrm{g} \\
\text { wood } \\
\text { residue }\end{array}$ & $\begin{array}{l}\mathrm{mg} / \mathrm{g} \text { wood } \\
\text { residue }\end{array}$ & $\begin{array}{l}\mathrm{mg} / \mathrm{g} \text { wood } \\
\text { residue }\end{array}$ & $\begin{array}{l}\mathrm{mg} / \mathrm{g} \text { wood } \\
\text { residue }\end{array}$ \\
\hline 10 & 1 & 60 & 3.75 & 19.3595 & 78.27 & 95.69 & 99.38 \\
\hline 12 & 2 & 60 & 3.75 & 19.8549 & 78.11 & 97.84 & 102.25 \\
\hline 8 & 3 & 60 & 5.52 & 15.5074 & 83.2 & 105.42 & 106.1 \\
\hline 2 & 4 & 80 & 2.5 & 26.6245 & 82.74 & 99.31 & 104.48 \\
\hline 11 & 5 & 60 & 3.75 & 18 & 80.78 & 94.25 & 100.38 \\
\hline 5 & 6 & 32 & 3.75 & 9.9068 & 77.24 & 87.7 & 92.98 \\
\hline 3 & 7 & 40 & 5 & 11.5167 & 73.04 & 87.79 & 93.45 \\
\hline 6 & 8 & 88 & 3.75 & 31.9907 & 98.61 & 109.98 & 114.77 \\
\hline 1 & 9 & 40 & 2.5 & 12.1358 & 86.04 & 101.2 & 105.05 \\
\hline 7 & 10 & 60 & 1.98 & 22.3591 & 88.75 & 92.27 & 100.28 \\
\hline 4 & 11 & 80 & 5 & 24.6122 & 93.63 & 104.39 & 115.51 \\
\hline 9 & 12 & 60 & 3.75 & 17.9974 & 85.91 & 96.46 & 104.87 \\
\hline
\end{tabular}


The screening allowed the careful selection of the most important factors that affect the lignin removal from wood residue: liquid to solid ratio (quantity of wood residue) and temperature. All experiments were carried out in duplicate. A Central Composite Design (CCD) model type was selected to be fitted with the experimental data for optimization. All experiments were carried out in duplicate. Table 2 contains the CCD experimental matrix with 12 experiments carried out with different settings of the independent factors (wood residue quantity and temperature) and the values determined experimentally for the two responses (lignin concentration and glucose equivalents).

\section{Results and discussion}

Fitting analysis of different model types to experimental values of lignin concentration indicate that the best model is linear (table 3$)$. The correlation coefficients $\left(\mathrm{R}^{2}\right.$ adjusted $=0.95$ and $\mathrm{R}^{2}$ predicted $\left.=0.91\right)$ are very close to 1 and there is a very small difference between their values.

Table 3. Fit summary for the concentration of lignin

\begin{tabular}{||r|r|r||r|r|r||}
\hline Source & Sequential p-value & Lack of Fit p-value & Adjusted R & Predicted R & \\
\hline \hline Linear & $<0.0001$ & 0.1993 & 0.9510 & 0.9170 & Suggested \\
\hline \hline $2 \mathrm{FI}$ & 0.6581 & 0.1674 & 0.9463 & 0.8842 & \\
\hline \hline Quadratic & 0.4026 & 0.1421 & 0.9471 & 0.8255 & \\
\hline \hline Cubic & 0.1752 & 0.1674 & 0.9668 & 0.5855 & Aliased \\
\hline
\end{tabular}

ANOVA of the linear model fitted for the concentration of lignin (table 4) confirm that the selected model is significant and the model is fitted. The factors that have significant effect on the concentration of lignin are the two independent factors considered: temperature and wood residue quantity.

Table 4. ANOVA of the linear model fitted for the concentration of lignin

\begin{tabular}{|c|c|c|c|c|c|c|}
\hline Source & $\begin{array}{l}\text { Sum of } \\
\text { Squares }\end{array}$ & $\mathrm{df}$ & $\begin{array}{c}\text { Mean } \\
\text { Square }\end{array}$ & F-value & $\mathrm{p}$-value & \\
\hline Model & 451.39 & 2 & 225.69 & 107.68 & $<0.0001$ & significant \\
\hline A-temperature & 432.41 & 1 & 432.41 & 206.31 & $<0.0001$ & \\
\hline B-wood residue & 18.98 & 1 & 18.98 & 9.05 & 0.0147 & \\
\hline Residual & 18.86 & 9 & 2.10 & & & \\
\hline Lack of Fit & 16.15 & 6 & 2.69 & 2.98 & 0.1993 & not significant \\
\hline Pure Error & 2.71 & 3 & 0.9034 & & & \\
\hline Cor Total & 470.25 & 11 & & & & \\
\hline
\end{tabular}

The second response - glucose equivalents from enzymatic hydrolysis of microwave alkaline treated wood residue - was monitored for 3 consecutive days (EG1, EG2 and EG3 
in table 2). The data were fitted for each individual response. In table 5 is presented the fitting for the data collected in the $3^{\text {rd }}$ day of enzymatic hydrolysis. It can be observed that a two factor interaction model is suggested.

Table 5. Fit summary for the glucose equivalents determined after 3 days of enzymatic hydrolysis of microwave alkaline treated wood residue

\begin{tabular}{|r|r|r|r|r|r||}
\hline Source & Sequential p-value & Lack of Fit p-value & Adjusted R & Predicted R & \\
\hline \hline Linear & 0.0083 & 0.1142 & 0.5787 & 0.2789 & \\
\hline \hline $2 \mathrm{FI}$ & 0.0027 & 0.4381 & 0.8563 & 0.7050 & Suggested \\
\hline Quadratic & 0.3818 & 0.4143 & 0.8610 & 0.6358 & \\
\hline \hline Cubic & 0.2414 & 0.5684 & 0.8976 & 0.6564 & Aliased \\
\hline
\end{tabular}

ANOVA of the model fitted for the glucose equivalents (table 6) confirm that the selected model is significant and the model is fitted. The factors that have significant effect on the concentration of lignin are the temperature and the interaction of temperature with wood residue quantity. The quantity of wood residue as single term does not have an significant effect on response as shown by its p-value which is well above significance level of 0.05 .

Table 6. ANOVA of the model fitted for the glucose equivalents from enzymatic hydrolysis of microwave alkaline treated wood residue (3 days)

\begin{tabular}{|l||r|r|r|r|r|l||}
\hline \multicolumn{1}{|c|}{ Source } & Sum of Squares & df & Mean Square & F-value & p-value & \\
\hline \hline Model & 477.35 & 3 & 159.12 & 22.86 & 0.0003 & significant \\
\hline \hline A-temperature & 341.99 & 1 & 341.99 & 49.12 & 0.0001 & \\
\hline B-wood residue & 7.34 & 1 & 7.34 & 1.05 & 0.3347 & \\
\hline AB & 128.03 & 1 & 128.03 & 18.39 & 0.0027 & \\
\hline \hline Residual & 55.69 & 8 & 6.96 & & \\
\hline Lack of Fit & 38.22 & 5 & 7.64 & 1.31 & 0.4381 & not significant \\
\hline \hline Pure Error & 17.47 & 3 & 5.82 & & & \\
\hline \hline Cor Total & 533.04 & 11 & & & & \\
\hline
\end{tabular}

The exploration of the experimental surfaces (figure 2) of the fitted models indicate the dominant effect of temperature as independent factor. Although this suggests that an increase of temperature would provide higher values of lignin concentration removed from the wood residue, previous experiments that were carried out showed a negative effect of very high temperatures on the performance of enzymatic hydrolysis. This can be explained by the fact that with the increase of temperature, degradation of lignin and structural carbohydrates is enhanced generating compound with inhibitory effect to the enzymes from hydrolysis [6]. 


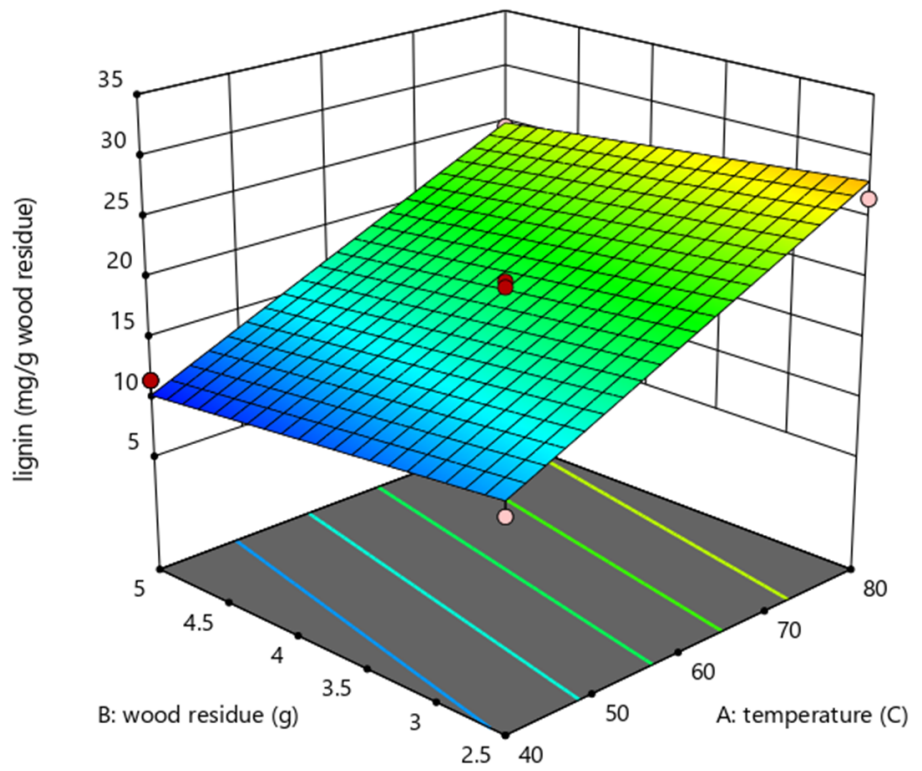

(a)

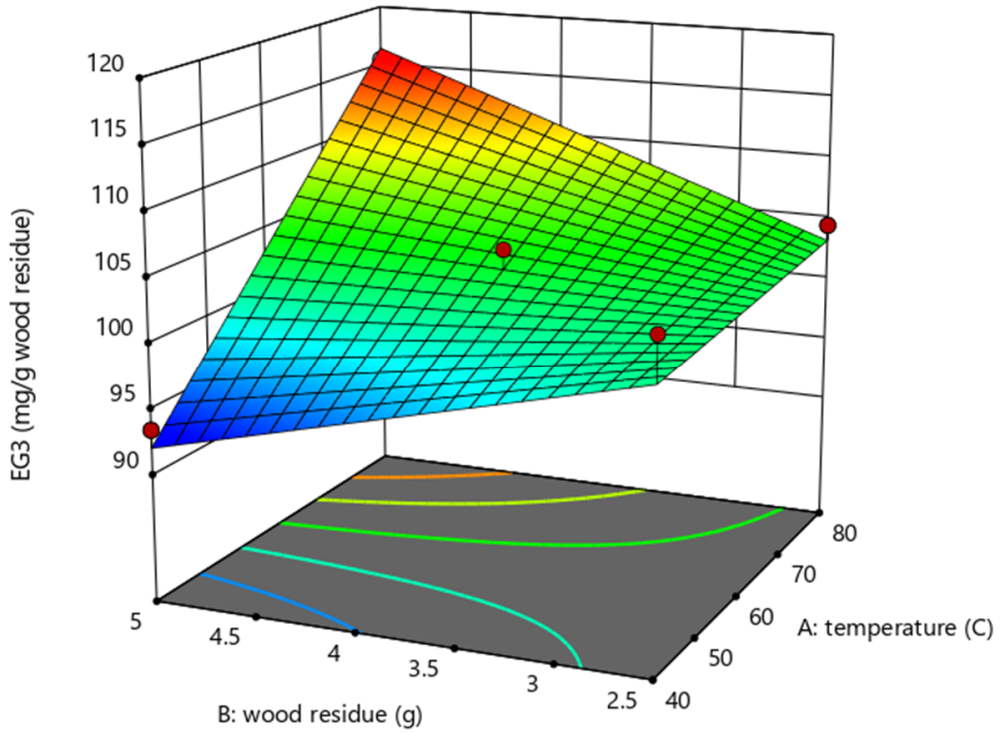

(b)

Fig. 2. 3D plot of response surfaces function of the independent factors with significant effect for: a) lignin concentration; b) glucose equivalents from enzymatic hydrolysis of microwave alkaline treated wood residue (3 days)

Optimization of experimental conditions within the experimental space was carried according to the following criteria: minimization of temperature and maximization of the solid residue quantity, lignin concentration and glucose equivalents. There are 8 solutions that satisfy the conditions imposed for the desirability function built for optimization and all 
of them are found in the same region of the response surface. One of the optimal solution (22.09 mg lignin / g wood residue and 111.67 glucose equivalents / g wood residue) indicated by the model for these optimization criteria is obtained for the following coordinates: $72^{\circ} \mathrm{C}$ and $5 \mathrm{~g}$ of wood residue (figure 3 ).
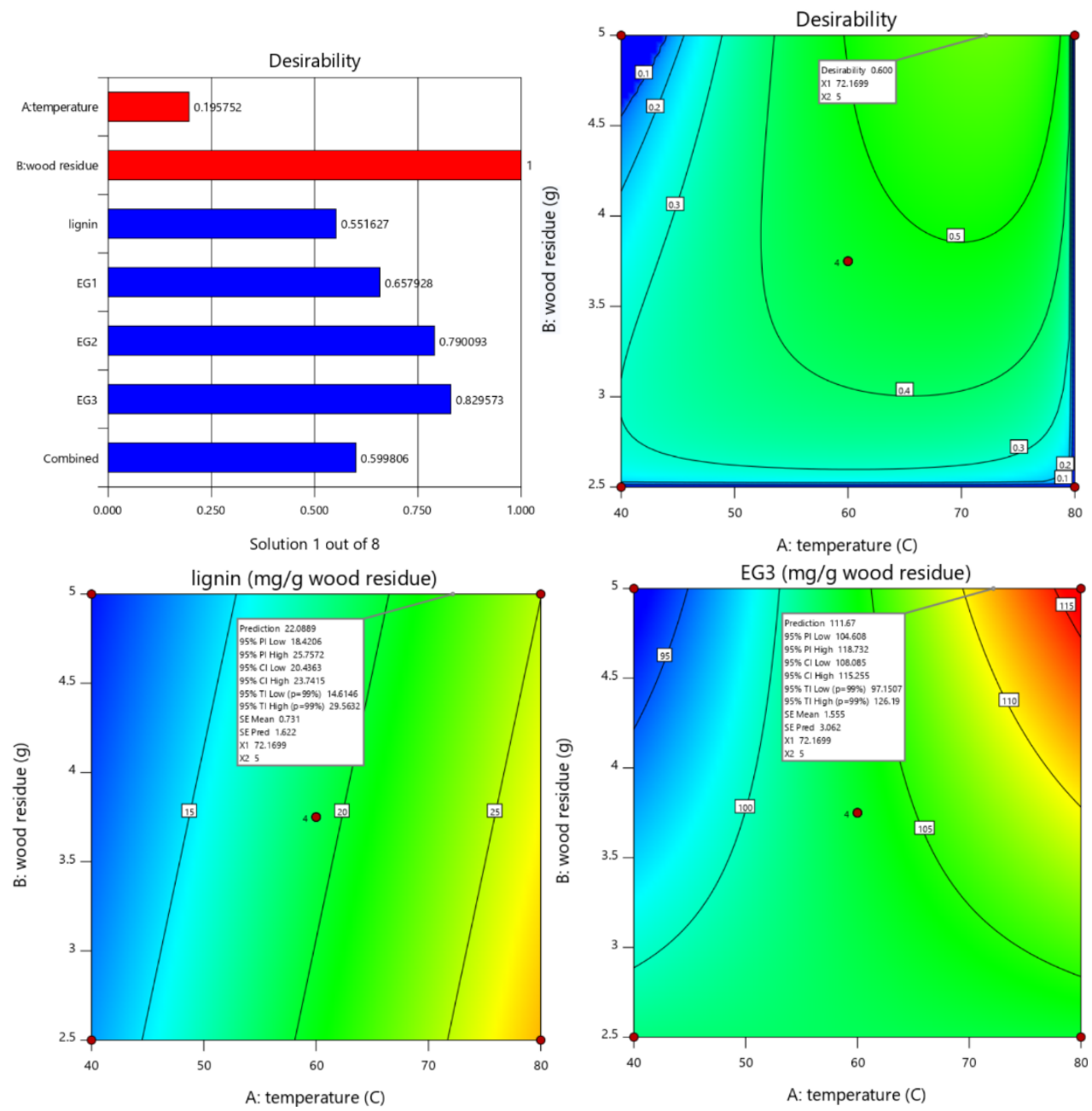

Fig. 3. Optimization (maximization) of the responses (lignin and glucose equivalents) function of the independent variables (temperature and quantity of wood residue)

\section{Conclusions}

This study is focused on improving the enzymatic hydrolysis of wood residue from furniture manufacturing by microwave alkaline treatment. Response surface methodology was used in order to find the best settings of the independent parameters (temperature and wood residue quantity) considered for the alkaline treatment. The optimization function (desirability) indicated a narrow range of settings for the temperature and the quantity of wood residue at which optimal performance is achieved: $72^{\circ} \mathrm{C}$ and $5 \mathrm{~g}$ of wood residue. 


\section{Acknowledgment}

The authors acknowledge the financial support received from the Competitiveness Operational Programme 2014 - 2020, Action 1.1.4: Attracting high-level personnel from abroad in order to enhance the RD capacity, ID project: P_37_471, MY SMIS 105145, Ultrasonic/Microwave nonconventional techniques as new tools for nonchemical and chemical processes, financed by contract: 47/05.09.2016.

\section{References}

1. Fan, J., Santomauro, F., Budarin, V. L., Whiffin, F., Abeln, F., Chantasuban, T., Gore-Lloyd, D., Henk, D., Scott, R. J., Clark, J., Chuck, C. J., J. Clean. Prod., 2018, 198, 776-784.

2. Baksi, S., Ball, A. K., Sarkar U., Banerjee, D., Wentze, A., Heinz, A., Preisig, H. A., Kuniyal, J. C., Birgen, C., Saha, S., Wittgens, B., Markussen, S., Int. J. Biol. Macromolec., 2019, 129, 634-644.

3. Pinkert, A., Goeke, D. F., Marsh, K N., Pang, S., Green Chem., 2011, 13, 31243136.

4. Sluiter, A., Determination of structural carbohydrates and lignin in biomass, NREL, 2015, 42618: 16.

5. Wood, I. P., Elston, A., Ryden, P., Bancroft, I., Roberts, I. N., Waldron, K. W., Biomass Bioenergy, 2012, 44, 117-121.

6. Jonsson, L. J., Matin, C., Biores. Technol., 2016, 199, 103-112. 


\title{
EFFECTIVENESS OF A MICROWAVE FLUIDISED BED DRYER IN ERADICATION OF SEED-BORNE BOTRYTIS GREY MOLD OF LENTIL
}

\author{
S. Taheri ${ }^{1}$, G. Brodie ${ }^{2}$, D. Gupta ${ }^{3}$, \\ 1,2,3 School of Food and Agriculture, Faculty of Veterinary and Agricultural Science, \\ Melbourne, Victoria, Australia \\ Isaeideh.taheri@gmail.com; ${ }^{2}$ grahamb@unimelb.edu.au
}

Keywords: Microwave Fluidized Bed, lentil, Botrytis Grey Mold

\begin{abstract}
A single mode microwave cavity, with a $2.45 \mathrm{GHz}$ microwave source, was modified to have a microwave fluidized bed and its potential to eliminate Botrytis grey mold (BGM) pathogen of lentil seeds was evaluated. Air speed was maintained at a constant value and was just enough to fluidize $100 \mathrm{~g}$ of red lentil seeds in the sample holder. Two wet-based (w.b.) seed moisture contents (MC) of $10.5 \%$ and $18.5 \%$ were prepared and the process parameters were selected as air temperature at 50 and $60^{\circ} \mathrm{C}$; microwave power at $0,300,400 \mathrm{~W}$ for $18.5 \%$ $\mathrm{MC}$ and $0,400,500 \mathrm{~W}$ for $10.5 \% \mathrm{MC}$; and exposure times of 5 and $10 \mathrm{~min}$. The effect of the process parameters on seed moisture loss, seed germination, the electrical conductivity of seed soaking water and the percentage of infected seeds (IS\%) were analyzed. The most effective factors on moisture loss, after seed moisture content, was exposure time, followed by microwave power and air temperature. While the final bed temperature was mostly affected by air temperature, and then by microwave power. Furthermore, based on general full factorial regression and Pareto chart of standardized effects, moisture content had a major influence on the reduction of IS\%. Seed pathogen inoculum reduction, without significant seed viability loss, was obtained by applying microwave power of $300 \mathrm{~W}$ and set air temperature of $60^{\circ} \mathrm{C}$ (actual inlet air temperature of $57 \pm 1^{\circ} \mathrm{C}$ ) on seeds with $\mathrm{MC}$ of $18.5 \%$ for $10 \mathrm{~min}$. This gave a $27 \%$ reduction in IS\%, from $82 \%$ to $55 \%$. Thus, these results showed that applying microwave fluidized bed dryer was able to reduce BGM pathogen in lentil seeds and it could be considered as part of integrated disease management.
\end{abstract}

\section{Introduction}

Botrytis cinerea $(B C)$ is one of the important seed-borne pathogens causing botrytis grey mold (BGM) disease of chickpea and lentil crops, one of the economically important seedborne diseases in Australia. It can survive in the form of micro and macroconidia, mycelium, sclerotia and chlamydospores (Yigal Elad 2007). Lindbeck, Bretag et al. (2009) showed that $\mathrm{BC}$ is mostly located externally on lentil and chickpea seeds. They mentioned that seeds, which are infected during early stages of growth, may have an internal infection and will be discoloured and shrivelled, so they could be recognized by their appearance. However, those infected later, close to the harvest time, do not have any symptoms and might be used for sowing in the next season. The later seeds are mostly externally infected.

BGM control in lentil crop consists of integrated management including the application of resistant cultivars, healthy seeds, biological agents, seed treatment, foliar treatment with fungicides and cultural practices (Beniwal and Trapero-Casas 1994, Yigal Elad 2007). Lentil varieties grown in Australia do not have complete resistance against BGM and need fungicide application to control the disease (Lindbeck, Bretag et al. 2008). Therefore, using chemicals for seed and foliar treatments has remained the easiest and the most popular way of BGM control in this crop. However, there are still concerns about BGM pathogen's ability to develop resistance as well as environmental and health impacts due to over usage of 
fungicides (Fillinger and Elad 2016). There have been some attempts to find a physical treatment for control or eradication of BGM pathogen. Burgess (1997) showed that BC is mostly present externally in chickpea seeds and can be controlled by moist heat at $45^{\circ} \mathrm{C}$ for $10 \mathrm{~min}$ or $50^{\circ} \mathrm{C}$ for $5 \mathrm{~min}$. These treatments decreased seed infection to 2.5 and $0 \%$ respectively with no adverse effect on the seed germination and seedling emergence. However, the same treatment on the freshly harvested seeds reduced the infection to $18 \%$, which could be the result of initial inoculum or changes in heat tolerance of the pathogen during treatment time. They also indicated that infected seeds did not necessarily have any visual symptoms to distinguish them from healthy seeds. This highlighted the need for seed testing. Marquenie, Lammertyn et al. (2002) applied UV-C, thermal treatment and their combination to inactivate spores of $\mathrm{BC}$. They used first-order kinetic models to describe the inactivation of the spore, with temperature and UV dose as the independent factors for thermal and UV-C treatment respectively. They observed that there was no survival of conidia at $45^{\circ} \mathrm{C}$ after $15 \mathrm{~min}$ and at $48^{\circ} \mathrm{C}$ after $5 \mathrm{~min}$. It was concluded that the combination of UV and thermal treatment allowed inactivation at lower temperature and lower UV doses. Nevertheless, there is still a lack of information on a proper physical method for elimination of BC from important legume crop seeds, such as lentil.

The objective of this experiment was to investigate the possibility of control or eradication of seed-borne $B C$ from lentil seeds in a microwave fluidized-bed drier. For this purpose, the effect of different process parameters on moisture loss, temperature, seed viability and infected seed percentage (IS\%) were investigated.

\section{Materials and Methods}

\section{Lentil Seeds preparation}

Red lentil seeds (Type Bolt) were purchased from PBseeds Co, Horsham, Victoria, Australia. The initial moisture content was measured by drying $10 \mathrm{~g}$ of unground seeds at $130^{\circ} \mathrm{C}$ for $20 \mathrm{~h}$ (Tang and Sokhansanj 1991). The 100 -seeds weight was $4.27 \pm 0.09 \mathrm{~g}$ and their average diameter and thickness, which were measured using a digital calliper, were $5 \pm$ 0.19 and $2.4 \pm 0.14 \mathrm{~mm}$ (average of twenty seeds). To reach the desired moisture content, a calculated volume of sterilised distilled water was added to a known amount of dried seeds in a resealable polyethylene bag and after thorough shaking, they were kept in a refrigerator for at least one week and used in the experiment within one month, to make sure the seed viability did not change in storage, especially for moisture contents of above $17 \%$. During the first week of storage, before the start of the experiment, seeds were mixed two times per day to make sure moisture was uniformly distributed among the seeds.

\section{Inoculation of lentil seeds}

Botrytis cinerea (174/02) spores, isolated from lentil seeds in Australia in 2002, were provided by Dr. Jenny Davidson, from the South Australian Research and Development Institute (SARDI). The isolate was subcultured on potato dextrose agar (PDA) and incubated at $22^{\circ} \mathrm{C}, 12 \mathrm{~h} / 12 \mathrm{~h}$ dark/light cycle under fluorescent (OSRAM TLD/18W) and near ultraviolet (UV) lights (PHILIPS BLB/18W). After two weeks, when the cultures were full of conidia, the spore solution was prepared by flooding with sterilized distilled water (SDW) (with $0.01 \%$ tween 20 ) on plates of BC on PDA and harvesting the spores by gently rubbing the surface of the culture using a glass rod. About $100 \mathrm{ml}$ of spore solution, with $10^{5}-10^{6}$ spore $/ \mathrm{ml}$, was obtained from two plates.

Inoculation of the lentil seeds was done according to the method described by Burgess, Bretag et al. (1997). Briefly, lentil seeds were surface sterilized with Sodium Hypochlorite $(1 \%)$ for one minute, followed by rinsing three times with sterile distilled water. Sterilized 
seeds were placed on a sterilized cheesecloth in a safety biological cabinet to let the excess water dry before putting them inside an autoclaved Scott bottle. The spore solution was diluted with sterilized distilled water to reach a concentration of $10^{4}-10^{5} \mathrm{spore} / \mathrm{ml}$. This solution was poured onto the lentil seeds in the Scott bottle to reach $10^{3}$ spores $/ \mathrm{seed}$. The spore solution's volume was enough to wet all the seeds (for red lentil $25 \mathrm{ml} / 100 \mathrm{~g}$ ). After pouring the spore solution, the bottle was tightly closed and mixed thoroughly and left overnight in the dark to make sure the spores are attached to the seeds. The bottle was shaken two times to make sure the spores are attached to all the seeds. On the next day, the inoculated seeds were spread on sterilized aluminum foil in a biological safety cabinet and weighed regularly to reach the required moisture content of 10.5 and $18.5 \%(\mathrm{wb})$. To reach the initial moisture content, they were left for three nights. After reaching the desired moisture contents, seeds were transferred in the Scott bottles, kept at $5^{\circ} \mathrm{C}$ and used in the experiment within one month. Before each treatment, seeds were taken out of the refrigerator and left overnight in the room to reach room temperature.

\section{Microwave fluidized bed system and treatment of the lentil seeds}

Schematic of the system is illustrated in Figure 1.

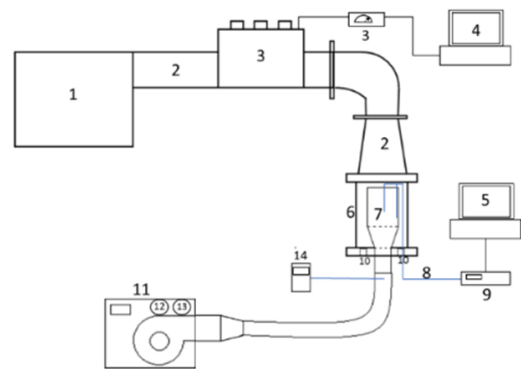

Figure 1 Microwave fluidized-bed system for drying and disinfection of particles, 1magnetron, 2- waveguide, 3- three-stub tuner, 4- absorbed power monitoring, 5- data acquisition system and microwave power control, 6- microwave cavity, 7- sample holder (fluidized bed), 8- fibre optic probes, 9- temperature monitor, 10- vents, 11- air blower and heater, 12- air speed potentiometer, 13- air temperature potentiometer, 14- inlet air temperature monitor

For each treatment, $90 \mathrm{~g}$ of healthy seeds were mixed with $10 \mathrm{~g}$ of inoculated seeds, which were marked with a red marker. The total of $100 \mathrm{~g}$ of seeds was transferred into the sample holder with a perforated bottom. Three fiber optic sensors were placed in the hot spot (top center of the bed), the cold spot (corner of the cylindrical sample holder) and at the bottom of the sample holder, where the air enters. This was considered as inlet air temperature. Hot and cold spots were obtained from the results of simulation of microwave power distribution in $\mathrm{XFdtd}^{\circledR}$ 7.5.0 (Remcom, Inc., State College, PA) using dielectric properties (Taheri, Brodie et al. 2018) and physical properties (Tang, Sokhansanj et al. 1991, Gharibzahedi, Ghahderijani et al. 2014) of red lentil seeds. Autodesk Inventor Professional 2017 (Autodesk Inc., USA) was used to design the 3-D geometry of lentil seeds with dimensions of 6.8 and $2.4 \mathrm{~mm}$ and $36 \mathrm{~mm}$ bed thickness (15 layers of lentil seeds), which was then placed in the sample holder inside the microwave cavity to perform the simulation.

Here, the real dimensions of the lentil seeds were not considered, because the real dimensions were too small, and the computer's space was not enough to solve the finite- 
difference-time-domain equations. However, the thickness of $36 \mathrm{~mm}$ was the same as $100 \mathrm{~g}$ of red lentils at $18.5 \% \mathrm{MC}$, which was used in the experiment. The amount of sample was selected, firstly, for having a proper power density, which did not increase the hot spot temperature quickly and harm the seeds and secondly, for having a thickness less than the penetration depth of the microwave at $2450 \mathrm{MHz}$ in lentil seeds (Taheri, Brodie et al. 2018). As the seeds are fluidized by hot air, fiber optic probs did not show the real seed surface temperatures and it is assumed that the measured temperatures are an average of air and seeds surface temperature, which is henceforth called bed temperature. Air speed was adjusted to fluidize the bed. Turning the potentiometer, a fracture below $560 \mathrm{l} / \mathrm{min}$ turned the fluid bed into a static bed.

\section{Evaluation of lentil seed viability and vigour after treatments}

Seed viability was examined by the standard germination test. Twenty seeds per replicate and a total of 60 seeds per treatments were placed on Whatman filter paper no.1 in 9-cm Petri dishes. Each plate contained 10 seeds which were wetted by $4 \mathrm{ml}$ of sterilized distilled water. Germination of the seeds was examined after two and five days. Vigour of the seeds was evaluated by measuring electrical conductivity (EC) of the seed soaking water according to ISTA ( 2016). Following each treatment, 50 lentil seeds were counted and, accurately weighed and placed in $250-\mathrm{ml}$ Erlenmeyer flasks. One hundred $\mathrm{ml}$ distilled water was poured in each container to cover the seeds and provide enough depth to soak the seeds. The containers' heads were covered with aluminium foil and after $24 \mathrm{~h} \pm, 15$ minutes electrical conductivity of the soaking water was measured using an EC probe (model HI98304, HANNA Instruments Inc.; Melbourne, Australia) with a resolution of $0.01 \mathrm{mS} / \mathrm{cm}$ and accuracy of $\pm 2 \%$ F.S. at $25^{\circ} \mathrm{C}$. The result was calculated according to equation (1). Distilled water was used as a blank for background reading of conductivity and the test was carried out in triplicate.

$$
\begin{aligned}
\text { Conductivity }\left(\mu S \mathrm{~cm}^{-1} \mathrm{~g}^{-1}\right) \\
\text { conductivity reading }\left(\frac{\mu S}{\mathrm{~cm}}\right)-\text { background reading }
\end{aligned}
$$

\section{Evaluation of BGM pathogen viability after treatments}

Botrytis selective media (BSM) was prepared according to Burgess (1997) and Edwards and Seddon (2001). The media contained (g/l): glucose 2; PDA 5; Agar 10; tannic acid 5; Ridomil 0.02; Pentachloronitrobenzene (PCNB) solution 0.5\% in ethanol 4; Zineb 0.00091; and streptomycin sulphate 0.1. a mixture of glucose. The PDA and Agar were autoclaved before adding to the rest of the ingredients.

After each treatment, the contaminated seeds, which had been marked, were separated and 30 seeds per replicate with a total of 90 seeds per treatment were subcultured on BSM. The plates were incubated at $23^{\circ} \mathrm{C}$ and after 4 and 10 days were examined to see the pathogen's growth. BC was recognized, based on the appearance of the mycelium and their conidia under microscope (after 2 weeks) as well as the colour change of the selective media to brown, which was the result of tannic acid oxidation.

\section{Evaluation of thermal resistance of the BMC pathogen in a water bath}

To see the susceptibility of the pathogen to thermal treatment at different seed moisture contents, five grams of inoculated lentil seeds at moisture contents of $10.5 \%$ (initial), $12.5 \%$, $14.5 \%, 16.5 \%$ and $18.5 \%(\mathrm{wb})$ were placed inside a resealable polyethylene bag and immersed in a water bath at temperatures $60,65,67,70,75^{\circ} \mathrm{C}$ for $10 \mathrm{~min}$. The seeds were 
sub-cultured on BSM and examined for the percentage of infected seeds after 4 and 10 days. Five-Minute exposure time was also examined for those temperatures, which were effective in $100 \%$ elimination of the pathogens. The results of these treatments helped the selection of the microwave power to treat the seeds with selected moisture content. The powers were selected to raise the seed surface temperatures to those which were lethal to the pathogen so that the bed temperature was at least $5^{\circ} \mathrm{C}$ above these temperatures.

\section{Experimental design and statistical analysis}

To investigate the effect of different process parameters on moisture loss, final temperature, germination, and frequency of pathogen isolation from the seeds or percentage of infected seeds (IS\%), an experiment was designed with four factors, each with 2 or 3 levels and with 3 replications. Factors and levels were selected as follow: moisture content (MC) at 10\% (dry form) and $18 \%$ wet base (wb); air temperature set at 50 and $60{ }^{\circ} \mathrm{C}$; microwave output power at $0,200,300$ for $18 \% \mathrm{MC}$ and $0,400,500$ for $10 \% \mathrm{MC}$; and exposure times of 5 and $10 \mathrm{~min}$. The set air temperature of 50 and $60^{\circ} \mathrm{C}$ was measured as $50 \pm 1$ and $57 \pm 1^{\circ} \mathrm{C}$ at the inlet of the sample holder.

This experiment was set out as a randomized full factorial design in Minitab version 18.1 (Minitab Inc., Pennsylvania, USA). The effect of microwave power, air temperature, exposure time and initial moisture content on final sample temperature, moisture loss, seed viability and percentage of infected seeds were investigated using a general factorial regression and analysis of variance. The most effective factors were found from a Pareto chart of the standardized effects with $\alpha=0.05$. Significant differences from the control were analyzed by one-way analysis of variance. All the statistical analysis was performed in MINITAB Statistical Software version 18.1 (Minitab, Inc., Pennsylvania, USA) and presented data are mean of three replicates.

\section{Results and discussion}

\section{Simulation of power density inside the single mode cavity}

Results of microwave power dissipation inside $100 \mathrm{~g}$ of lentil seeds are shown in Figure 2. As can be seen in the power distribution in the bottom layer of the lentil seeds (Figure 2 (a)), the hot spot of the microwave radiation is predicted to be in the centre of the sample and the cold spot is along the circumference of the cylindrical sample holder. The same distribution was obtained for the top layer of the samples (data not shown). In Figure 2 (b), the maximum and average predicted microwave power density are represented. Here, the maximum microwave power is predicted to be on the top layer of lentil samples and the minimum power is absorbed in the middle layers, $20-25 \mathrm{~mm}$ distance from the top. Therefore, the hot spot was in the centre of the top layer and the cold spot in the corner of the middle layer. Fibre optic probes were placed in these two locations to obtain hot and cold spot temperatures during the treatments. Additionally, hot air was assumed to be evenly distributed in the bed after entering through the perforated plate. 
a

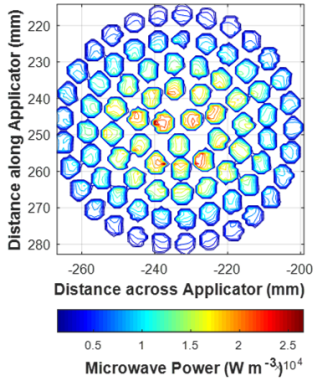

b

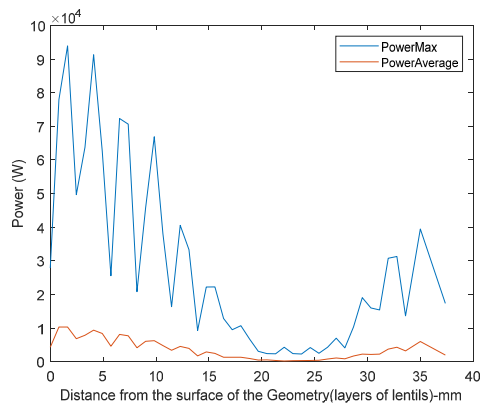

Figure 2 Power dissipation in lentil seeds, the result of simulation; (a) absorbed power distribution in a cross section of the bottom layer of lentil seeds; (b) vertical maximum and average power density in $100 \mathrm{~g}$ of lentil seeds in a cylindrical sample holder $\left(\mathrm{W} / \mathrm{m}^{3}\right)$

\section{Thermal sensitivity of the BGM pathogen in a water bath}

Results of thermal sensitivity of the BGM pathogen on lentil seeds, with different seed MCs, are shown in Figure 3. A 10 min exposure time led to complete eradication of the pathogens from the lentil seeds at $65^{\circ} \mathrm{C}$ for seed $\mathrm{MC}$ of $18.5 \%, 16.5 \%$, and $14.5 \%$, at $70^{\circ} \mathrm{C}$ for $12.5 \%$ seed $\mathrm{MC}$ and at $75^{\circ} \mathrm{C}$ for $10.5 \% \mathrm{MC}$ (Figure $3(\mathrm{a})$ ). A temperature of $65^{\circ} \mathrm{C}$ was also lethal to seed pathogen after 5 min exposure of the contaminated seeds at $18.5 \%$ and $16.5 \% \mathrm{MC}$. However, seeds with MC of $14.5 \%, 12.5 \%$, and $10.5 \%$ required temperatures of $70^{\circ} \mathrm{C}, 75^{\circ} \mathrm{C}$ and $80^{\circ} \mathrm{C}$, respectively to remove the pathogen completely, after $5 \mathrm{~min}$. Taheri, Brodie et al. (2019) also found that Ascochyta blight pathogen of lentil seeds could be reduced more at higher seed moisture content and the same final temperature, by applying microwave treatment.

It must be noted that, here, the seeds were not directly exposed to water, which creates $100 \%$ water activity and lowers the lethal temperature to its minimum. The seeds were sealed in the resealable polyethylene bag and the moisture was not allowed to scape, and water activities could be considered as the seeds water activity at the corresponding moisture content at each temperature. If the exposure time is enough to reach the equilibrium in the closed bags, relative humidity $(\mathrm{RH})$ might be considered close to the water activity.
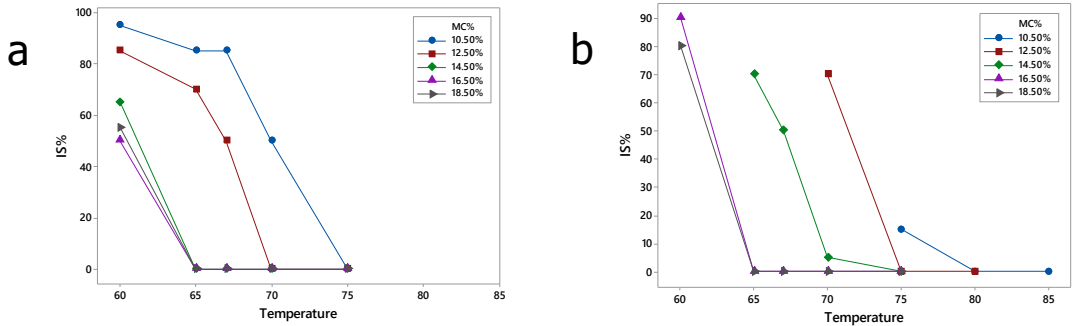

Figure 3 Percentage of botrytis grey mold infected lentil seeds after treatments in a water bath at different temperatures $\left({ }^{\circ} \mathrm{C}\right.$ ) after (a) 10 min and (b) 5 min. IS\%- infected seeds percentage; $\mathrm{MC} \%$-seed moisture content(w.b.) 


\section{Effect of microwave power, air temperature and exposure time on moisture loss and final temperatures of lentil seeds}

Results of the Pareto chart of the standardized effects from general factorial regressions ( $\alpha=$ 0.05) for moisture loss and bed temperature are represented in Figure 4, (a) and (b) respectively. The $\mathrm{x}$-axis of these charts shows the coefficient of each factor in the regression model divided by its standard error. The factors, with the standardized effect of more than 2.04 (red vertical line), have significant effects on the response and the more the standardized effect is, the more effective the factor will be. Pareto chart of moisture loss (Figure 4(a)) suggested that all the factors including seed moisture content, microwave power, air temperature and exposure time significantly affected the seed moisture loss, whereas, seed moisture content being most effective factor followed by exposure time. Additionally, microwave power had more contribution to moisture removal than the air temperature. According to Figure 4, (b) lentil seeds bed temperature was mostly affected by air temperature followed by microwave power, while other factors did not have any significant effect. Final lentil bed temperature is shown in Figure 5. The lentil seeds' bed temperature corresponded with increasing air temperature and microwave power. Its maximum value was $70^{\circ} \mathrm{C}$ at the power of $400 \mathrm{~W}$ for $18.5 \% \mathrm{MC}$ and $500 \mathrm{~W}$ for $10.5 \% \mathrm{MC}$ and air temperature of $60^{\circ} \mathrm{C}$. Lentil seeds' surface temperatures were different from the bed temperature as the bed temperature was an average of seed surface and air temperature.

a

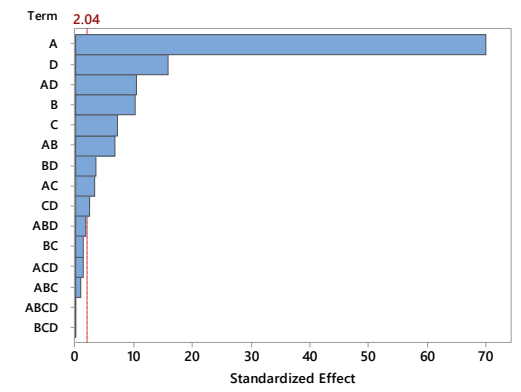

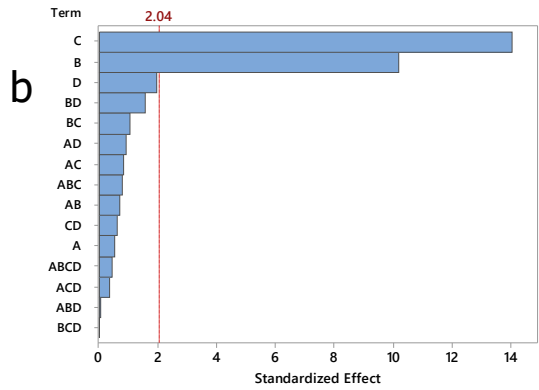

Figure 4 Pareto Chart of the standardized effects for (a) moisture loss and (b) bed temperature with $\mathrm{a}=0.05 ; \mathrm{A}=$ moisture content $(10.5 \%, 18.5 \%), \mathrm{B}=$ microwave power $(0,300,400 \mathrm{~W}$ for $18.5 \% \mathrm{MC}$ and $0,400,500 \mathrm{~W}$ for $10.5 \% \mathrm{MC}), \mathrm{C}=$ air temperature $(50$, $\left.60^{\circ} \mathrm{C}\right), \mathrm{D}=$ exposure time $(5,10 \mathrm{~min})$ 


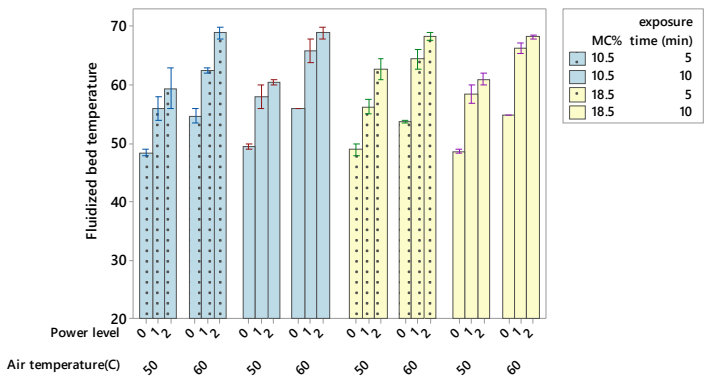

Figure 5 Final bed temperature at two set air temperatures of 50 and $60^{\circ} \mathrm{C}$, two exposure times of 5 and $10 \mathrm{~min}$, microwave powers of $0,300,400 \mathrm{~W}$ for $18.5 \% \mathrm{MC}$ (power level of $0,1,2$ respectively) and $0,400,500 \mathrm{~W}$ for $10.5 \% \mathrm{MC}$ (power level of $0,1,2$ respectively); bars are SE from the mean; MC-moisture content

Effect of microwave power, air temperature and exposure time on seed viability and percentage of infected lentil seeds at each seed moisture content as a categorical factor Generally, initial seed moisture content had by far the most influence on IS\% using general full factorial regression with $\mathrm{R}^{2}=79 \%, \mathrm{~F}-$ Value $=42.62$ and P-Value $=0.000$. Therefore, statistical analysis for seed viability and IS\% were carried out separately for each of moisture contents of $10.5 \%$ and $18.5 \%$. The Pareto chart of standardized effects for germination percentage and infected seeds percentage counted after 4 days (IS\%-4d) for treated lentil seeds at $18.5 \% \mathrm{MC}$ is shown in Figure 6, (a) and (b) respectively. Both microwave power and air temperature had a significant effect on seed germination and IS\%-4d. However, the effect of microwave power on IS\%-4d was more than the effect of air temperature, while they affect seed germination almost equally. According to the results of main effect plots for the seeds at $18.5 \% \mathrm{MC}$, IS\% decreased with increasing microwave power, air temperature and exposure time. Similarly, seed germination was negatively affected by increasing air temperature and microwave power from 300 to $400 \mathrm{~W}$, while increasing microwave power from 0 to 300 had a positive effect on seed germination. For $10.5 \% \mathrm{MC}$, the general factorial regression did not provide a good fit and hence, no conclusion was derived about the effect of process parameters on IS\% and seed viability. 

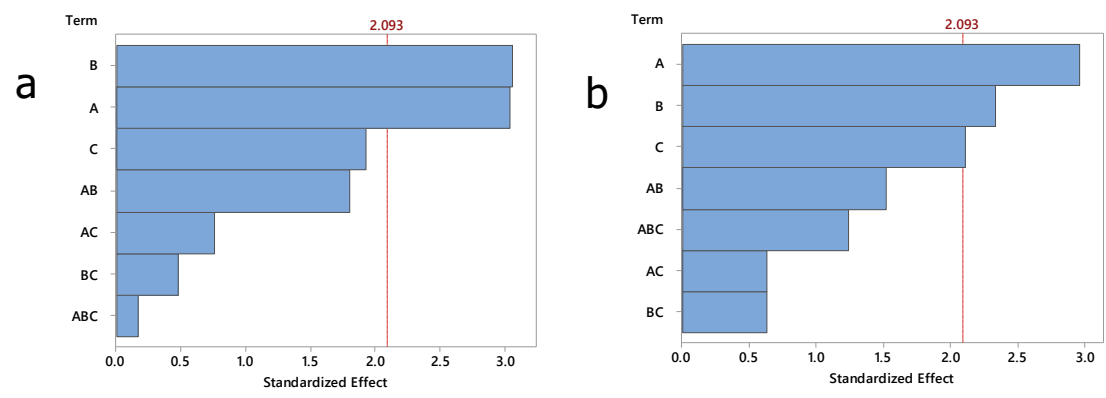

Figure 6 Pareto Chart of the standardized effects for (a) germination percentage and (b) infected seeds percentage counted after $4 \mathrm{~d}$ of lentil seeds at $18.5 \% \mathrm{MC} ; \mathrm{A}=$ microwave power $(0,300,400 \mathrm{~W}), \mathrm{B}=$ air set temperature $\left(50,60^{\circ} \mathrm{C}\right), \mathrm{C}=$ exposure time $(5,10 \mathrm{~min})$;

$$
\alpha=0.05
$$

Table 1 represents the results of germination percentage ( $\mathrm{G} \%)$, infected seeds percentage counted after 4 days (IS\% 4d) and 10 days (IS\% 10d), and electrical conductivity of soaking water (EC) after microwave fluidized bed treatments of lentil seeds at two moisture contents of $10.5 \%$ and $18.5 \%$ with exposure times of 5 and $10 \mathrm{~min}$. For seeds at $10.5 \% \mathrm{MC}$, none of the treatments affected germination, electrical conductivity or IS\%, even at bed temperature of close to $70^{\circ} \mathrm{C}$ (seed surface temperature of $75-80^{\circ} \mathrm{C}$ ), which was obtained by microwave power of $500 \mathrm{~W}$ and air temperature of $60^{\circ} \mathrm{C}$ and exposure time of $10 \mathrm{~min}$, while in the experiment with the water bath, the pathogen was eliminated at $75^{\circ} \mathrm{C}$. The difference between these results could stem from the difference between relative humidity $(\mathrm{RH})$ around the seeds at the time of treatments. Equilibrium RH around the seeds (water activity of the seeds) at $10.5 \% \mathrm{MC}$ at $60^{\circ} \mathrm{C}$ is between 60 to $75 \%$ (Menkov 2000) and as the water activity (and corresponding equilibrium $\mathrm{RH}$ ) of the carbohydrate/protein rich foods increases by increasing temperature (Syamaladevi, Tang et al. 2016), RH of the seeds sealed in the bag in the water bath at $10.5 \% \mathrm{MC}$ and temperature of $75^{\circ} \mathrm{C}$ will be more than $60 \%$. However, in microwave fluidized bed treatments, RH of air at the mentioned speed and temperature of $60{ }^{\circ} \mathrm{C}$ was measured as $11 \%$, when ambient $\mathrm{RH}$ was about $50 \%$, and this amount can be even less when the microwave is also used in combination with hot air. Therefore, the very low $\mathrm{RH}$ in the microwave fluidized bed could be the reason for increasing the lethal temperature of the seed pathogen. The other reason, which was mentioned by Syamaladevi, Tang et al. (2016), might be very fast desiccation of the pathogen in the microwave fluidized bed which could increase the pathogen's resistance to irreversible damage due to high temperatures.

Results for seeds with $18.5 \% \mathrm{MC}$ showed that by applying microwave power of $300 \mathrm{~W}$ and air temperature of $60^{\circ} \mathrm{C}$ for $10 \mathrm{~min}$, IS\% counted after $10 \mathrm{~d}(\mathrm{IS} \%-10 \mathrm{~d})$ reduced from $82 \%$ to $55 \%$ without any significant loss of viability. IS $\%$ - $10 \mathrm{~d}$ was reduced from $82 \%$ to $51.6 \%$ by applying microwave power of $400 \mathrm{~W}$ and air temperature of $60^{\circ} \mathrm{C}$ for $10 \mathrm{~min}$; however, germination was also decreased from $95 \%$ to $60 \%$. No significant change in electrical conductivity was observed as a result of these treatments. Here again, the difficulty of eliminating the seed pathogen could be related to the lower RH around the seeds. Nevertheless, it was more feasible to reduce the pathogen's load at higher MC of $18.5 \%$ than the dry seeds.

BGM pathogen growth on the seeds in BSM was evaluated on day 4 and 10, as after 4 days the pathogen mycelium, as well as the separated brown areas on BSM (tannin oxidation) were clearly discriminated and were comparable with the non-treated seeds. After 10 days, the mycelial growth was more and IS\% in almost all the plates, including the control. The 
difference between the treated and control samples was less than that on day 4 . After 2 weeks of incubation, conidia were observed on the mycelium, however, the mycelium grew beyond the initially contaminated seeds and also infected healthy lentil seeds. Conclusively, reduction of IS\% was considered after 4 (start of the growth) and 10 days (when healthy seeds could still be separated).

\section{Conclusion}

Microwave fluidized bed processing method was developed in this study to evaluate its potential for eradication of BGM pathogen from lentil seeds. The effect of process parameters on moisture loss, bed temperature, seed germination and infected seed percentage (IS\%) were investigated. Most effective factors on moisture loss and bed temperature were seed moisture content and air temperature, respectively and microwave power was the most effective factor in IS\% reduction. Air temperature and microwave power equally affected seed germination. Additionally, it was observed that eradication of BGM pathogen from lentil seeds in a microwave fluidized bed was more difficult than doing this in a sealed polyethylene bag in a water bath at the same temperature, which was concluded to be due to the difference in RH of the air around the seeds at the time of treatment. It is therefore recommended to investigate the effect of increasing RH of inlet air on seed pathogen reduction in future studies. BGM pathogen reduction, without significant seed viability loss in a microwave fluidized bed, was obtained by applying microwave power of $300 \mathrm{~W}$ and set air temperature of $60^{\circ} \mathrm{C}$ on seeds with a moisture content of $18.5 \%$ for $10 \mathrm{~min}$. This gave a $27 \%$ reduction in IS\% from $82 \%$ to $55 \%$. Therefore, microwave fluidized bed dryer can be considered as part of integrated disease management of BGM of lentil if the seed moisture content and process parameters are standardized for effective irradiation of the pathogen inoculum from the lentil seeds. 


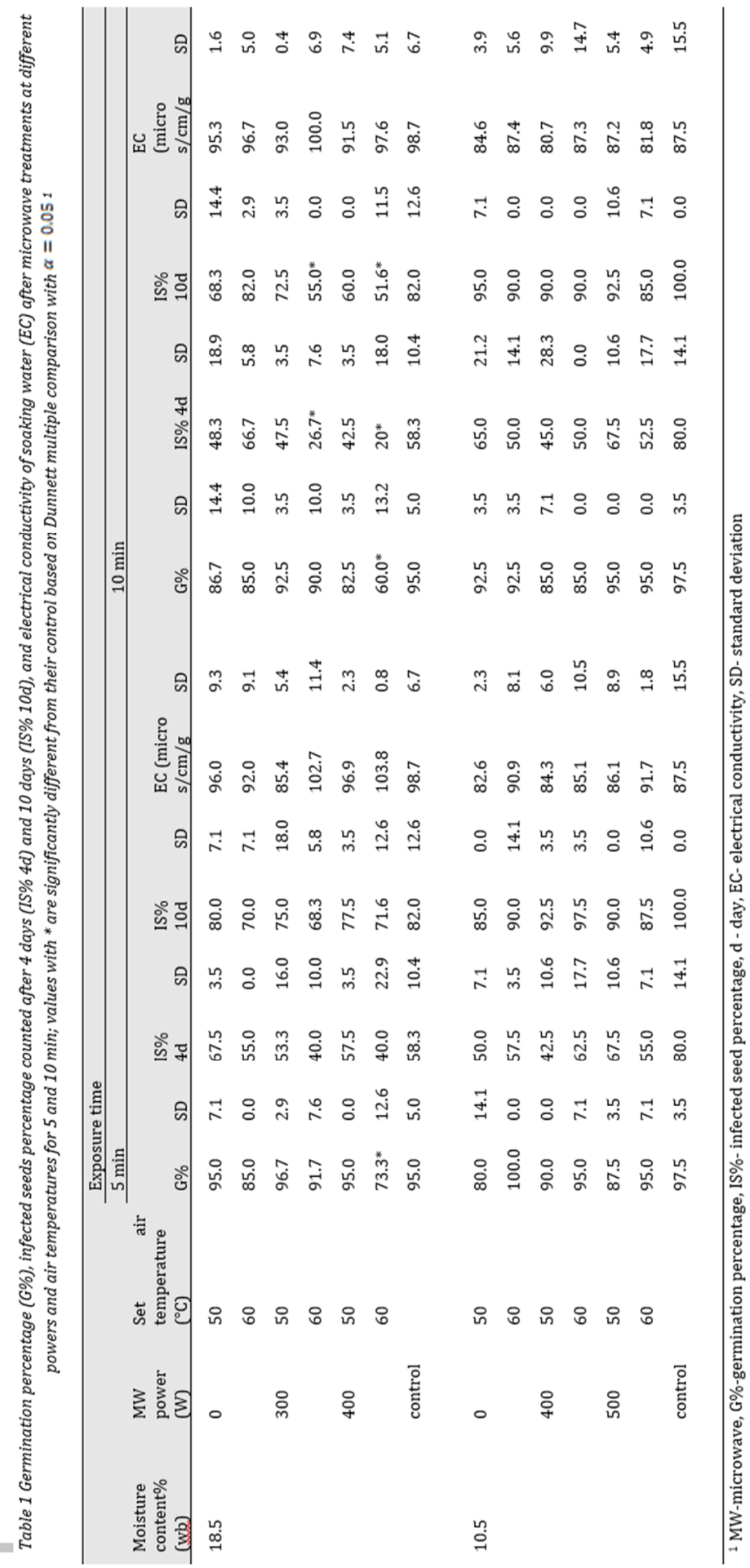




\section{References}

Beniwal, S. and A. Trapero-Casas (1994). Integrated control of diseases of cool season food legumes. Expanding the Production and Use of Cool Season Food Legumes, Springer: 642665 .

Burgess, D., T. Bretag and P. Keane (1997). "Biocontrol of seedborne Botrytis cinerea in chickpea with Gliocladium roseum." Plant Pathology 46(3): 298-305.

Burgess, D. R. (1997). "Seed-to-seedling transmission of Botrytis cinerea in chickpea and disinfestation of seed with moist heat." Australian journal of experimental agriculture 37(2). Edwards, S. and B. Seddon (2001). "Selective media for the specific isolation and enumeration of Botrytis cinerea conidia." Letters in applied microbiology 32(2): 63-66.

Fillinger, S. and Y. Elad (2016). Botrytis: The Fungus, the Pathogen and Its Management in Agricultural Systems, Springer.

Gharibzahedi, S. M. T., M. Ghahderijani and Z. S. Lajevardi (2014). "Specific heat, thermal conductivity and thermal diffusivity of red lentil seed as a function of moisture content." Journal of Food Processing and Preservation 38(4): 1807-1811.

ISTA ( 2016). International Rules for Seed Testing Zürichstr. 50, CH-8303 Bassersdorf, Switzerland, International Seed Testing Association.

Lindbeck, K., T. Bretag and R. Ford (2009). "Survival of Botrytis spp. on infected lentil and chickpea trash in Australia." Australasian Plant Pathology 38(4): 399-407.

Lindbeck, K. D., T. W. Bretag and M. A. Materne (2008). "Field screening in Australia of lentil germplasm for resistance to botrytis grey mould." Australasian Plant Pathology 37(4): 373-378.

Marquenie, D., J. Lammertyn, A. H. Geeraerd, C. Soontjens, J. F. Van Impe, B. M. Nicolaï and C. W. Michiels (2002). "Inactivation of conidia of Botrytis cinerea and Monilinia fructigena using UV-C and heat treatment." International Journal of Food Microbiology 74(1): 27-35.

Menkov, N. D. (2000). "Moisture sorption isotherms of lentil seeds at several temperatures." Journal of Food Engineering 44(4): 205-211.

Syamaladevi, R. M., J. Tang, R. Villa-Rojas, S. Sablani, B. Carter and G. Campbell (2016). "Influence of water activity on thermal resistance of microorganisms in low-moisture foods: a review." Comprehensive Reviews in Food Science and Food Safety 15(2): 353-370.

Taheri, S., G. Brodie, M. V. Jacob and E. Antunes (2018). "Dielectric properties of chickpea, red and green lentil in the microwave frequency range as a function of temperature and moisture content." Journal of Microwave Power and Electromagnetic Energy: 1-17.

Taheri, S., G. I. Brodie, D. Gupta and R. H. R. Dadu (2019). "Effect of Microwave Radiation on Internal Inoculum of Ascochyta Blight in Lentil Seeds at Different Seed Moisture Contents." Transactions of the ASAE 62(1): 33-43.

Tang, J. and S. Sokhansanj (1991). "Determination of moisture content of whole kernel lentil by oven method." Transactions of the ASAE 34(1): 255-0256.

Tang, J., S. Sokhansanj, S. Yannacopoulos and S. Kasap (1991). "Specific heat capacity of lentil seeds by differential scanning calorimetry." Transactions of the ASAE 34(2): 5170522.

Yigal Elad, B. W., Paul Tudzynski, Nafiz Delen (2007). "Botrytis: Biology, Pathology and Control." 


\title{
MICROWAVE DRYING OF CORN SEEDS: EFFECT OF TEMPERATURE ON DRYING TIME, ENERGY CONSUMPTION AND GERMINATION RATE
}

\author{
Á. H. Moreno ${ }^{1 *}$, R. Hernández-Maqueda ${ }^{2}$, I. Ballesteros², C. Torres-Miñoº \\ ${ }^{1}$ Facultad de Ciencias de la Ingeniería y Aplicadas. Universidad Técnica de Cotopaxi, Av. \\ Simón Rodríguez s/n. Barrio San Felipe, Latacunga, Ecuador \\ ${ }^{2}$ Facultad de Ciencias Agropecuarias y Recursos Naturales. Universidad Técnica de \\ Cotopaxi, Av. Simón Rodríguez s/n. Barrio San Felipe, Latacunga, Ecuador \\ angel.hernandez@utc.edu.ec \\ rafael.hernandez@utc.edu.ec \\ maria.ballesteros@utc.edu.ec \\ carlos.torres@utc.edu.ec
}

Keywords: microwave drying, corn seeds, drying time, energy consumption, germination rate

\begin{abstract}
In this work, the microwave drying of corn seeds at different temperatures has been studied. The main objective of this study is to evaluate the effect of the temperature on drying time, energy consumption and the germination rate of corn seeds after drying with microwave energy. The tests were performed in a domestic microwave oven at three drying temperatures $\left(35,55\right.$ and $\left.75^{\circ} \mathrm{C}\right)$. It was observed that an increase in the drying temperature causes a simultaneous decrease in drying time $(\sim 78 \%)$ and in the consumption of energy $(\sim 79 \%)$ required to dry the seeds. However, there is also an unacceptable decrease $(\sim 87 \%)$ in the germination rate of the seeds with the drying temperature. In order to increase the germination rates of corn seeds, after the drying process, it is necessary to employ drying temperatures between 35 and $50^{\circ} \mathrm{C}$.
\end{abstract}

\section{Introduction}

Corn or Maize (Zea mays L.), belongs to the grass family Poaceae (Gramineae), is the most extensively cultivated, produced and consumed cereal in the world [1]. Corn is considered as a staple food in many parts of the world and is also an important source of phytochemical compounds, such as carotenoids, phenolic compounds which could reduce the risk of several diseases such as atherosclerosis or cancer. Due to the presence of nutrients and phytochemicals it is considered as a healthy food [2]. Moreover, corn has a great economic importance worldwide not only as human food, but also as animal feed and as feedstock for a large quantity of industrial products and biofuels [1].

In Ecuador, the cultivation of corn crops has increased in the recent years. According to statistical information provided by the agricultural sector ESPAC 2018, Ecuador has increased its maize production levels considerably, even though, it is still deficient and needs to import large quantities of this cereal. In 2018, the area cultivated to dry hard corn nationwide was 383.399 hectares and this produced, approximately, 1.3 million tons of corn [3], a significant proportion of which was acquired by the national industry for the production of protein food. Moreover, based on reports of the Ecuadorian Central Bank [4], $55 \%$ of the credits granted to householders were destined to the production of several corn 
varieties, reflecting the importance of this crop in the country. For these reasons it is important to improve agricultural techniques in order to ensure an increase in the yield of corn crops.

Corn ears are usually harvested at a moisture content ranging from $22 \%$ to $40 \%$ (wb) in order to reduce the risk of frost, insect, disease damage and to maintain seed quality [5], [6], [7]. Corn harvested at these high moisture contents requires rapid drying for safe storage, to prevent respiration, germination, mold damage and insect infestation. Moreover, fungal spoilage and mycotoxin contamination are of major concern during corn storage [8]. To reduce or prevent the production of mycotoxins, drying is recommended immediately after harvesting [8]. It is very important to control the stage of drying and conservation of the seeds since poor management at this stage would result in substantial financial loss for the farmers.

The drying of corn, as other grains and seeds, can be performed naturally or artificially. Natural drying is the main method used by farmers in Cotopaxi as well as in other parts of Ecuador to dry the seeds, this consists in exposing them to the air, thereby taking advantage of solar energy. However, this method of drying has several disadvantages, such as we have pointed out in previous works [9], [10], [11], [12].

Several types of dryers have been developed for the drying of grains and cereals [13], [14], [15]. None of them, however, have proved suitable to satisfy all the needs and drying requirements of the different seeds. The grain drying system may be classified as Solar drying, Batch drying and Continuous-flow drying systems [14]. The dryers most commonly used to dry seeds are fixed bed dryers (batch) and sliding bed dryers, in their various configurations (concurrent, counter-current, and cross flows) [13], [14]. One of the first types of dryers used for drying corn seeds was the forced air dryer and many different innovative designs for forced-air corn drying have evolved in response to the needs of corn producers and processors [15]. However, this type of dryer has the disadvantage of requiring large amounts of thermal energy to vaporize the high moisture content of corn seeds. Therefore, the cost of producing this energy is the main disadvantage of this method.

In the drying process it is very important to control the temperature and humidity, since both factors are key for the growth viability of the seed. Most of authors consider that temperatures higher than $50^{\circ} \mathrm{C}$ are lethal for the viability of a seed, depending on the variety studied [6]. Moreover, the drying temperature has a significant effect on mechanical properties [16] and can cause mechanical damage to the seeds, in the form of stress cracking or kernel breakage [17]. Therefore, the control of temperature must be taken into account when designing an alternative drying method to sun drying. Microwave drying has great potential in this respect because this technique allows a good control of the process temperature, an increase in energy efficiency and a reduction of its carbon footprint [11], [18], [19].

Previous studies on the microwave drying of corn seeds have shown its effectiveness as a drying method [20], [21], although it should also be noted that the process parameters, in particular the temperature, play a very important role in determining the properties and quality of this grain [20]. Accordingly, the main objective of the present work is to evaluate the effect of the temperature on drying time, energy consumption and the germination rate of corn seeds after drying with microwave energy. 


\section{Materials and Methods}

\section{Materials}

The maize seeds used in this work were obtained by shelling $40 \mathrm{~kg}$ of ears that were purchased from farmers in Latacunga, Ecuador.

\section{Drying equipment and drying procedure}

The drying process of the corn seeds was carried out in a rotating turntable domestic multimode microwave oven (LACOR Model 69330) with a $900 \mathrm{~W}$ magnetron, using a frequency of $2.45 \mathrm{GHz}$ and a voltage of $220 \mathrm{~V}$. The microwave device is also equipped with a type $\mathrm{K}$ thermocouple connected to a PID controller Eurotherm $3216 \mathrm{~L}$ which controls the amount of power delivered and allows the temperature to be monitored and controlled.

In this oven, $100 \mathrm{~g}$ of corn seeds, with an initial humidity of between 16 and $20 \%(\mathrm{wb})$, was placed in a microwave plastic container (105 mm long, $105 \mathrm{~mm}$ wide and $45 \mathrm{~mm}$ high). The container, uncovered, was placed on the microwave oven dish (315 $\mathrm{mm}$ in diameter), and the thermocouple was immersed in the bed of seeds. The corn seeds were heated up to 3 drying temperatures $\left(35,55\right.$ and $\left.75{ }^{\circ} \mathrm{C}\right)$. All the experiments were carried out with the turntable in stationary mode to avoid fluctuations in the measurements of seed temperature. The seeds were weighed every 30 minutes, using a GASON balance (model Z1s, 300g x $0.01 \mathrm{~g}$ ), and the drying process was considered completed when a humidity of $12 \%$ was obtained. For each drying temperature studied, the experiments were carried out in duplicate.

\section{Energy consumption}

In each experiment, the electrical energy consumption was measured using a FLUKE 1735 energy analyzer. This analyzer allows the measurement of different electrical parameters, including current, voltage, power (active, reactive and apparent), frequency and energy consumption at specific time intervals. In this work the measurements of these parameters were recorded every 30 seconds.

\section{Germination tests}

The viability of the corn seeds dried in the microwave oven up to $12 \%$ humidity (wb), at the three temperatures under study, was evaluated by means of a Petri dish germination test. To perform this test, a sample of the dried seeds (20 seeds) was placed on a filter paper and a $20 \mathrm{~mL}$ volume of distillate water of was added to achieve sufficient humidity for them to sprout. The seeds were incubated at a temperature of $25^{\circ} \mathrm{C}$. For each temperature studied, a total of 5 replicates of the test were made. To evaluate the results of the germination test, the emergence of the radicle was taken into account and the time and percentage of germination of each of the 5 trials was determined. The average germination percentage for each drying temperature was then calculated. 


\section{Drying rate}

The drying rate (DR) of the seeds is the loss of mass (moisture) during the drying process per unit of time and per unit of dry mass $\left(\mathrm{g} \mathrm{H}_{2} \mathrm{O} /(\mathrm{g}\right.$ dry mass $\left.* \mathrm{~s})\right)$. The DR was determined according to the calculation procedure described in a previous work [12].

\section{Results and Discussion}

The results obtained from the drying of corn seeds in a multimode microwave oven at three different temperatures, as well as their effect on the drying time, energy consumption and viability of the seeds are as follows. The effect of the drying temperature on the three variables evaluated is shown in Fig. 1.

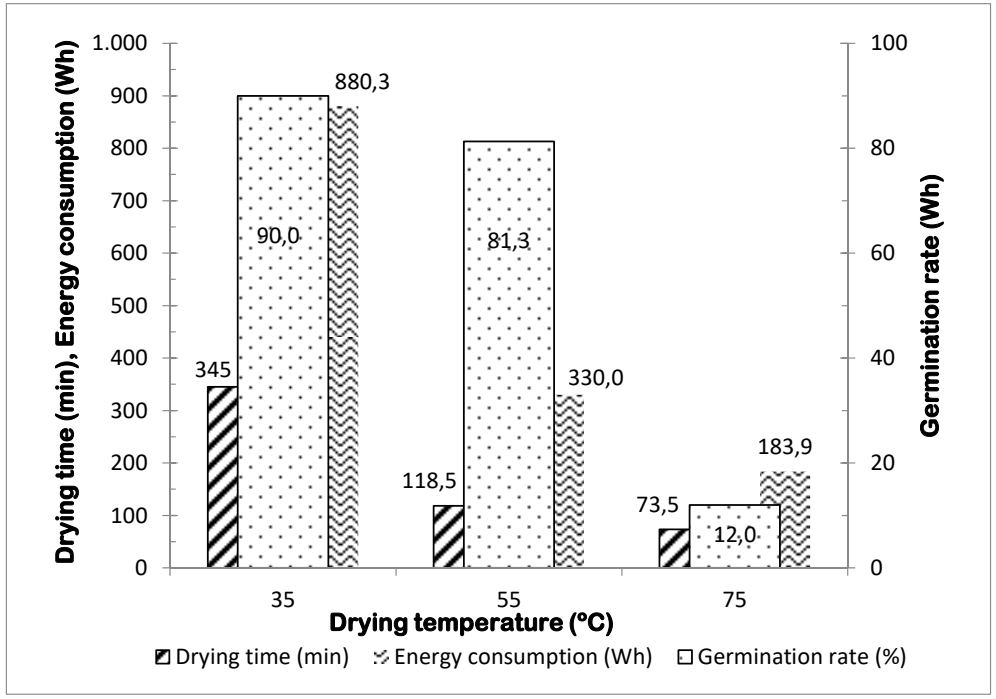

Fig. 1. Results of the microwave drying experiments of the corn seeds at different temperatures and their germination tests.

As can be seen in Fig1, as the drying temperature increases (from 35 to $75^{\circ} \mathrm{C}$ ) the drying times needed to reach a humidity of $12 \%$ decrease (from 345 to $74 \mathrm{~min}$ ), as a consequence of the increase in the drying rate of the seeds. This result agrees with the findings of other authors on microwave drying of corn seeds [20] and apples [22]. As the drying temperature increases, the maximum values of mass loss (maximum drying rate) increase, reaching magnitudes of $0.045,0.154$ and $0.565 \mathrm{~g}$ water/g dry mass $/ \mathrm{s} * 10^{-2}$ for drying temperatures of 35,55 and $75^{\circ} \mathrm{C}$, respectively. The high percentages of reduction of the drying time for the seeds with temperature, i.e. between 65 and $79 \%$ (See Table 1), are positive developments for reducing operating costs of the drying process.

Fig. 1 also illustrates the effect of the drying temperature on the energy consumption of microwave drying of corn seeds. It can be seen that as the drying temperature increases, the energy consumption required for the drying process of the seeds decreases significantly (between 62.5 and 79.1\%), especially in the case of the highest drying temperature (Table 1). This reduction in energy consumption with temperature is due to the much shorter drying times required to dry the seeds. The significant reduction of energy consumption in the 
drying process of the seeds is another very positive step towards the reduction of the operating costs of the drying process.

Table 1. Results of the microwave drying experiments of the corn seeds at different temperatures and their germination tests.

\begin{tabular}{cccc}
\hline $\begin{array}{c}\text { Drying temperature } \\
\left({ }^{\circ} \mathrm{C}\right)\end{array}$ & $\begin{array}{c}\text { Reduction in } \\
\text { drying time (\%) }\end{array}$ & $\begin{array}{c}\text { Reduction in energy } \\
\text { consumption (\%) }\end{array}$ & $\begin{array}{c}\text { Reduction in } \\
\text { germination rate (\%) }\end{array}$ \\
\hline 35 & - & - & - \\
55 & 65.7 & 62.5 & 9.7 \\
75 & 78.7 & 79.1 & 86.7 \\
\hline
\end{tabular}

Finally, it can be observed in Fig. 1 that at a drying temperature of $35^{\circ} \mathrm{C}$ the percentage of germination of the seed is good $(90 \%)$. However, when the drying temperatures increase, from 35 to $75^{\circ} \mathrm{C}$, there is significant reduction in the germination rate of the corn seeds (from $90 \%$ to $12 \%$ ). This behavior is due to the capacity of the seeds to germinate decreases because there is a reduction in the synthesis of proteins responsible for embryo respiration [23]. Moreover, at higher temperatures the seeds undergo mechanichal damage, such as internal tensions, cracks, breakages, and fractures [16], which also affects their ability to germinate. In fact, the reduction in germination rate $(86.7 \%)$ that occurs at $75^{\circ} \mathrm{C}$ is unacceptably high. In order to avoid affecting the germination capacity of the seeds and, consequently, obtain high germination rates in the microwave drying process of corn seeds, the drying temperatures must be maintained between 35 and $50^{\circ} \mathrm{C}$. This drying temperature interval has also been confirmed as optimal by other researchers [20].

\section{Conclusions}

From an analysis of the results obtained in this work the following conclusions can be drawn. The drying temperature has a significant effect on the microwave drying process and the germination rate of corn seeds. An increase in the drying temperature causes a simultaneous decrease in drying time $(\sim 78 \%)$ and in the consumption of energy $(\sim 79 \%)$ required to dry the seeds. This saving of time and energy makes the drying process of corn seeds much more competitive due to the decrease in operating costs. However, there is also an unacceptable decrease $(\sim 87 \%)$ in the germination rate of the corn seeds with the drying temperature. Therefore, for the implementation of microwave drying of corn seeds on a commercial scale the most suitable temperature to obtain high germination rates, is in the $35-50^{\circ} \mathrm{C}$ range. These seeds should not be dried at temperatures higher than $55^{\circ} \mathrm{C}$, due to the significant decrease in their germination rate.

\section{Acknowledgements}

The financial support received from Universidad Técnica de Cotopaxi (Ref. PI.EPSHMS.CIYA.2017) is greatly acknowledged. The authors also thank Cristian Hinojosa, Lili Tigasi and Daysi Pila for their contribution to the experimental work. 


\section{References}

[1] S. García-Lara and S. O. Serna-Saldivar, "Corn History and Culture", in Corn. Chemistry and Technology, 3rd ed., S. O. Serna-Saldivar, Ed. Oxford, UK: AACC International Press, 2019, ch. 1, pp. 1-18.

[2] T. R. Shah, K. Prasad, P. Kumar, "Maize - A potential source of human nutrition and health: A review", Cogent Food Agric., vol. 2, no. 2, pp. 1-9, 2016.

[3] INEC, "Encuesta de Superficie y Producción Agropecuaria Continua -ESPAC 2018", Instituto Nacional de Estadística y Censos (INEC), Quito, Ecuador, Abril, 2019.

[4] BCE, "Reporte de coyuntura del sector agropecuario". Publicaciones Técnicas Banco Central Ecuador (BCE), no. 90, III, pp. 1-38, Enero, 2018.

[5] J. S.Wall, C. James, and G. L. Donaldson, "Corn proteins: chemical and physical changes during drying of grain”, Cereal Chem., vol. 52, no. 6, pp. 779-790, 1975.

[6] R. J. Navratil, J. S. Burris, "The effect of drying temperature on corn seed quality", Can. J. Plant Sci., vol. 64, no. 3, pp. 487 - 496, July, 1984.

[7] U. Herter, J. S. Burris, "Preconditioning reduces the susceptibility to drying injury in corn seed", Can. J. Plant Sci., vol. 69, no, 3, pp. 775 - 789, July, 1989.

[8] S. N. Chulze, "Strategies to reduce mycotoxin levels in maize during storage: a review Part A Chemistry, analysis, control, exposure \& risk assessment”, Food Addit. Contam., vol. 27, no. 5, pp. 651-657, 2010.

[9] Á. H. Moreno, R. Hernández Maqueda, I. Ballesteros, "Microwave drying of seeds and vegetable products: a viable option for Ecuador", in Proc. of the 3rd Global Congress on Microwave Energy Applications (3GCMEA), J. Fayos-Fernández and J. MonzóCabrera, Eds., Cartagena, Spain, 2016, pp. 103-108.

[10] Á. H. Moreno, R. Hernández Maqueda, I. Ballesteros, "Microwave Drying of Seeds of Agricultural Interest for Ecuador", AMPERE Newsl., no. 92, pp. 28-32, April, 2017.

[11] Á. H. Moreno, R. Hernández Maqueda, I. Ballesteros, "Secado industrial con energía microondas", in Aplicaciones industriales del calentamiento con energía microondas, J. A. Menéndez, Á. H. Moreno, Eds., Latacunga, Ecuador: Univ. Téc. de Cotopaxi, 2017, Ch. 5, pp. 85-118.

[12] R. Hernández Maqueda, I. Ballesteros, S. Jiménez Jácome, Á. H Moreno, “Microwave drying of amaranth and quinoa seeds: effects of the power density on the drying time, germination rate and seedling vigour", J. Microw. Power Electromagn. Energy, vol. 52, no. 4, pp. 299-311, 2018.

[13] M. A. S. Barrozo, A. Mujumdar, J. T. Freire, “Air-Drying of Seeds: A Review”, Drying Technol., vol. 32, no. 10, pp. 1127-1141, 2014.

[14] B. K Bala, Drying and Storage of Cereal Grains. Second Edition. Wiley-Blackwell, 2016.

[15] C. J. Bern, G. Quick, F. L. Herum, "Harvesting and Postharvest Management", in Corn. Chemistry and Technology, 3rd ed., S. O. Serna-Saldivar, Ed. Oxford, UK: AACC International Press, 2019, ch. 5, pp. 109-145.

[16] S. Abasi, S. Minaei, "Effect of Drying Temperature on Mechanical Properties of Dried Corn", Drying Technol., vol. 32, no. 7, pp. 774-780, 2014.

[17] S. Mason, N. D'croz-Mason, “Agronomic Practices Influence Maize Grain Quality”, $J$. Crop Prod., vol. 5, no. 1-2, pp. 75-91, 2002.

[18] R. F. Schiffmann, "Microwave and Dielectric Drying", in Handbook of the Industrial Drying $4^{\text {th }}$ Ed., A. S. Mujumdar, Ed., Boca Raton, FL., US, 2015, Ch. 13, pp. 283-302.

[19] P. Rattanadecho, N. Makul, "Microwave-Assisted Drying: A Review of the State ofthe-Art”, Drying Technol., vol. 34, no. 1, pp. 1-38, 2016. 
[20] G. R. Nair, Z. Li, Y. Gariepy, V. Raghavan, "Microwave Drying of Corn (Zea mays L. ssp.) for the Seed Industry", Drying Technol., vol. 29, no. 11, pp. 1291-1296, 2011.

[21] S. Gürsoy, R. Choudhary, D. G Watson, "Microwave drying kinetics and quality characteristics of corn”, Int. J Agric. \& Biol. Eng., vol. 6, no. 1, pp. 90-99, March, 2013.

[22] Z. Li, G. S. V. Raghavan, V. Orsat, "Temperature and power control in microwave drying", J. Food Eng., vol. 97, no. 4, pp. $478-483,2010$.

[23] G. Riley, "Effects of high temperature on the germination of maize (Zea mays L.)", Planta, vol. 151, no. 1, pp. 68-74, 1981. 


\title{
GENERALISATION AND EVALUATION OF MACROSCOPIC MODELS FOR MICROWAVE SUSCEPTORS IN CONTACT WITH HEATED FOODS
}

\author{
M. Celuch ${ }^{1}$, K. Wilczynski², M. Olszewska-Placha ${ }^{1}$, \\ ${ }^{1}$ QWED Sp. z o.o., Krzywickiego 12/1, 02-078 Warsaw, Poland \\ ${ }^{2}$ Faculty of Physics, Warsaw University of Technology, Koszykowa 75, 00-662 Warsaw, \\ Poland \\ mceluch@qwed.eu
}

Keywords: microwave susceptors, microwave heating, electromagnetic modelling, conformal FDTD method, active packaging

\begin{abstract}
The aim of this paper is to evaluate the accuracy of macroscopic models of thin conductive layers used for electromagnetic modelling of microwave susceptors. The equivalent model assumes using a surrogate layer made of dielectric of higher thickness and maintained value of surface resistance. Such an approach allows decreasing computer effort required for electromagnetic analysis with $3 \mathrm{D}$ discrete methods. The present work extends to real-life scenarios, wherein a thin microwave susceptor is placed below food. Evaluation of the macroscopic model accuracy and limits of application is conducted with the aid of analytical calculations and conformal finite difference time domain (FDTD) simulations. The results of the analysis are generalised to give practical guidelines for microwave oven and food packaging designers and manufacturers.
\end{abstract}

\section{Motivation and background}

Thin conductive layers are widely encountered in microwave technology. An example of a daily life application are susceptors in microwaveable food packages [1], [2], [3], which are used in order to increase power dissipation and heating efficiency in food. Moreover, conductive layers (e.g. based on silver nano-particles) are present as the coating of Carbon Fiber Reinforced Polymers (CFRPs) [4]. Those kinds of coatings do not increase the weight of vehicles, therefore they are very useful for aircraft industry and enable to reduce fuel consumption, as well as increase lightning damage protection.

Electromagnetic modelling has been recently recognised by microwave oven and food packaging designers and manufacturers as an important stage of the design process. The most convenient and popular methods for virtual design are finite difference time domain (FDTD) [5] and finite element (FEM) [6] methods. However, introducing a thin conductive layer such as a microwave susceptor, with thickness (below 1 um [7], [8]) by orders of magnitude less than the operating frequency of a microwave oven, into a finite-mesh (as inherent in e.g. FDTD and FEM) typically requires a dedicated equivalent macroscopic model allowing for computationally effective and accurate electromagnetic (EM) and thermal simulations.

Thin conductive layers, characterised by their surface resistance $\left(R_{s}\right)$, are adequately represented with a dielectric surrogate layer of higher thickness and proportionally scaled conductivity, maintaining the value of $R_{s}$ :

$$
R_{s}=\left(d_{0} \sigma_{0}\right)^{-1}=(d \sigma)^{-1},
$$


where $\sigma 0, d_{0}$ stand for values of the original metal and $\sigma, d$ are the scaled parameters of the dielectric surrogate. Systematic evaluation of macroscopic models of microwave susceptors used for enhancing the heating efficiency of microwavable food packages has been reported in [9]. Our studies therein have focused on validity, accuracy, and practical application limits of the proposed macroscopic models of thin metallic layers, in terms of power dissipated in susceptor placed in free space and irradiated by EM wave, at all angles of incidence. In this work we extend our studies to real-life simulation scenarios, in which a microwave susceptor is in contact with food. The discussion is supported with both analytical and numerical conformal FDTD calculations. The accuracy and application limit of the macroscopic model are investigated for all incidence angles and both, TE and TM polarisations of the impinging EM wave, for real foods.

\section{Power dissipation in a surrogate without food}

In Fig. 1 we provide 2D maps of relative power dissipated in a surrogate layer for exemplary values of sheet resistance. The values have been chosen to cover a wide spectrum of applications of thin conductive layers: $1 \Omega$ - absorbing panels, $35 \& 105 \Omega$ - popcorn and pizza susceptors at room temperature, $400 \Omega$ - food susceptors breaking at above $240 \mathrm{deg}$, $20000 \Omega$ - susceptors after use. The results of this study are obtained analytically with the use of equations, which are discussed in detail in Section III.

Fig. 2 shows vertical cuts through the maps of Fig. 1, i.e., the dependence of relative power dissipated in a surrogate on the surrogate's thickness. Figs. 1 and 2 indicate that the values of $\mathrm{Rs}=30 \div 400 \Omega$ enable the best absorption for most field patterns (provided that the susceptor is in free space). This is further confirmed by the continuous plots of relative power dissipated in an ultra-thin susceptor, as a function of the susceptor's sheet resistance, presented in Fig. 3.

Note that analytical calculations indicating $\mathrm{Rs}=30 \div 400 \Omega$ as the optimum range for microwave heating have been reported earlier [1], however, those calculations were limited to normal incidence (TEM waves). Our study extends the proof to oblique incidence. It also shows that for higher incidence angles, TM waves heat more efficiently lower-resistance susceptors, while TE waves heat more efficiently higher-resistance susceptors. These observations are complementary to the investigation of equivalent angles of incidence in multi-modal domestic ovens, and thereby can help design of microwaveable food packages.

From the viewpoint of macroscopic surrogates, the results provided in Figs. 1 and 2 clearly show the range of admissible scaling of the surrogate thickness. The scaling remains neutral to power dissipation, provided that the scaled surrogate thickness does not exceed $1 \mathrm{~mm}$, which at $2.45 \mathrm{GHz}$ corresponds to ca. 0.05 wavelength. In [9], the analytical results were further validated with conformal FDTD simulations using QuickWave 3D software [11]. 


$$
1-\left|\mathrm{S}_{11}\right|^{2}-\left|\mathrm{S}_{21}\right|^{2} @ \mathrm{f}=2.45 \mathrm{GHz} \quad \epsilon_{\text {food }}=1, \sigma_{\text {food }}=0
$$
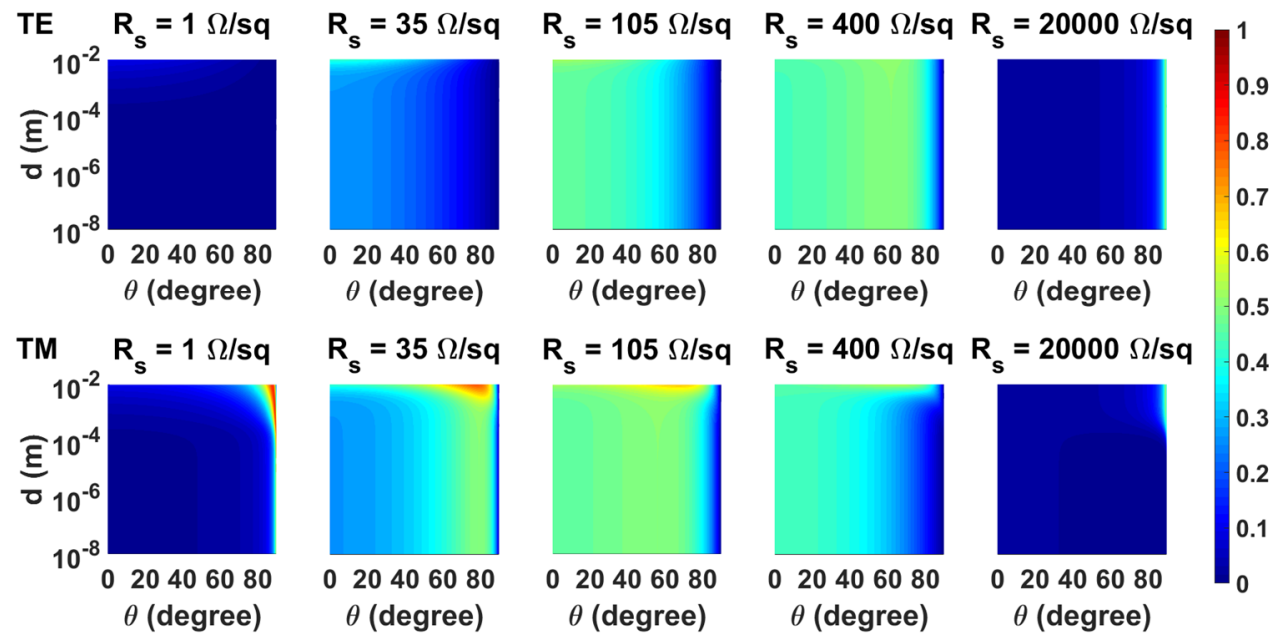

Fig. 1. Maps of relative dissipated power $\left(1-\left|S_{11}\right|^{2}-\left|S_{21}\right|^{2}\right)$ in a susceptor in free space (without food) for varying angles of incidence $\theta$ and thickness $d$ of the surrogate.
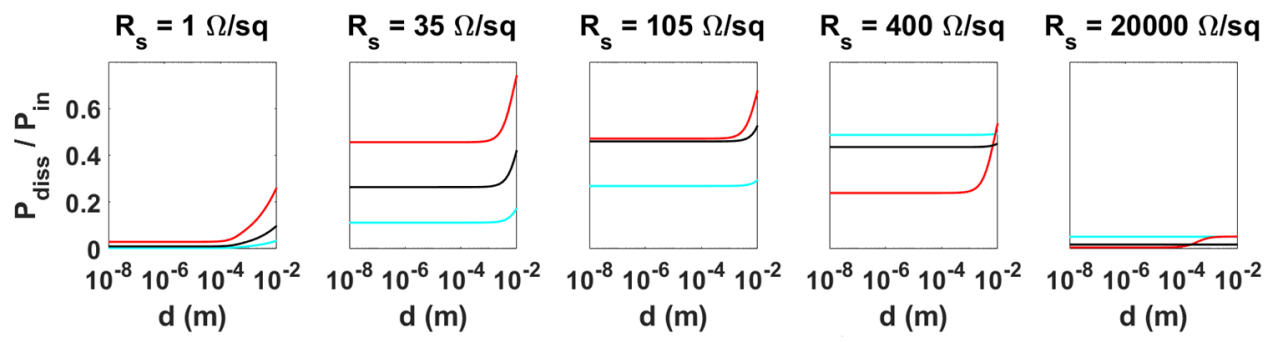

$$
\text { - TE }\left(70^{\circ}\right) \text { - TM }\left(70^{\circ}\right) \text { - TEM }
$$

Fig. 2. Vertical cuts through the maps of Fig. 1, for normal incidence (TEM), transverse electric (TE) and transverse magnetic (TM) polarisation at $70 \mathrm{deg}$.
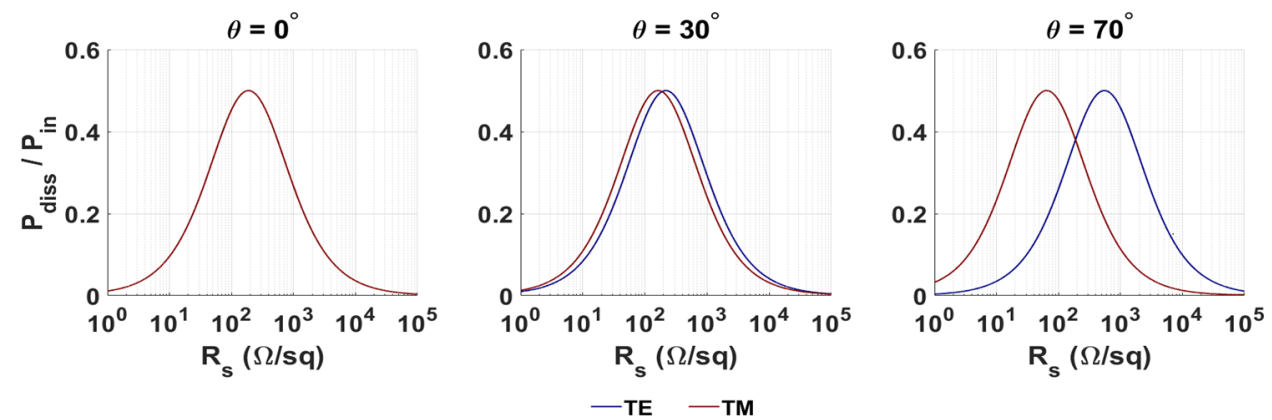

Fig. 3. Relative dissipated power in a susceptor placed in free space and irradiated at different angles, by waves of different polarisations. 


\section{Power dissipation jointly in the surrogate and food}

We now extend our study to the case of a susceptor being in contact with a slice of food, which is a typical situation in microwaveable food packages. The stacked structure as in Fig. 4, illuminated with EM wave at varying angle of incidence $\theta$ is considered. In the model, susceptor and food are assumed to have constant and isotropic values of permittivity and conductivity and the food is made of a non-magnetic material.

The electromagnetic multi-layered scenario shown on the left of Fig. 4 can be conveniently analysed with the transmission line theory, where it reduces to the flow graph shown on the right of Fig. 4. The graph accounts for three impedance steps at media boundaries, which the EM wave faces when propagating through the structure.

The system of Fig. 5 can be reduced to a two-port network, described with the S-matrix:

$$
\begin{aligned}
S_{11}= & \Gamma_{A B}+T_{A B} T_{B A} \cdot \tau_{B}^{2} \cdot\left(\Gamma_{B C}+\Gamma_{C D} \cdot \tau_{C}^{2}\right) \cdot \Delta, \\
S_{22}= & \Gamma_{D C}+T_{C D} T_{D C} \cdot \tau_{C}^{2} \cdot\left(\Gamma_{C B}+\Gamma_{B A} \cdot \tau_{B}^{2}\right) \cdot \Delta, \\
& S_{21}=T_{A B} T_{B C} T_{C D} \cdot \tau_{B} \tau_{C} \cdot \Delta, \\
& S_{12}=T_{D C} T_{C B} T_{B A} \cdot \tau_{B} \tau_{C} \cdot \Delta,
\end{aligned}
$$

where:

$$
\Delta=\left(1-\Gamma_{B A} \Gamma_{B C} \cdot \tau_{B}^{2}-\Gamma_{C B} \Gamma_{C D} \cdot \tau_{C}^{2}-\Gamma_{B A} \Gamma_{C D} \cdot \tau_{B}^{2} \tau_{C}^{2}\right)^{-1},
$$

$\Gamma_{i j}$ and $T_{i j}$ parameters stand for reflection and transmission coefficients (for wave amplitudes) respectively from $i$-th to $j$-th layer (where $i, j$ stand for $A-D$ ):

$$
\Gamma_{i j}=\left\{\begin{array}{l}
\frac{z_{j}-Z_{i}}{Z_{i}+Z_{j}}, \text { for TEM } \\
\frac{z_{j} \cos \theta_{i}-Z_{i} \cos \theta_{j}}{Z_{j} \cos \theta_{i}+Z_{i} \cos \theta_{j}}, \text { for TE } \\
\frac{Z_{j} \cos \theta_{j}-Z_{i} \cos \theta_{i}}{Z_{i} \cos \theta_{i}+Z_{j} \cos \theta_{j}}, \text { for TM }
\end{array} \quad T_{i j}=\left\{\begin{array}{l}
\frac{2 Z_{j}}{z_{i}+Z_{j}}, \text { for TEM } \\
\frac{2 Z_{j} \cos \theta_{i}}{Z_{j} \cos \theta_{i}+Z_{i} \cos \theta_{j}}, \text { for TE } \\
\frac{2 z_{j} \cos \theta_{i}}{z_{i} \cos \theta_{i}+Z_{j} \cos \theta_{j}}, \text { for TM }
\end{array}\right.\right.
$$

and $\tau_{i}$ stands for a wave transmission coefficient through $i$-th layer:

$$
\tau_{i}=e^{-\gamma_{i} \cdot d_{i} \cdot \cos \theta_{i}}
$$

In above equations $Z_{i}=\sqrt{\frac{\mu_{i}}{\varepsilon_{i}}}$ is a wave impedance of the material in $i$-th layer, $\gamma_{i}=$ $2 \pi j f \sqrt{\mu_{i} \varepsilon_{i}}$ is a propagation constant at the frequency of $f, d_{i}$ stands for the thickness of the layer, and $\cos \theta_{i}$ is defined as follows:

$$
\cos \theta_{i}=\sqrt{1-\left(\mu_{i} \varepsilon_{i} c^{2}\right)^{-1} \sin ^{2} \theta_{0}},
$$

where $\theta_{0}$ stands for an incidence angle in air and $c$ for speed of light. In the above equations $\mu_{i}$ and $\varepsilon_{i}$ stand for complex permeability and permittivity of material in $i$-th layer, which for the purpose of this paper are defined as $\mu_{i}=\mu_{0}$ and $\varepsilon_{i}=\varepsilon_{r i} \varepsilon_{0}-\frac{j \sigma_{i}}{2 \pi f}$. Index $i \equiv 0$ denotes vacuum parameters. 


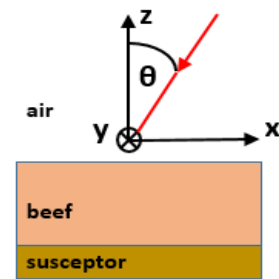

air

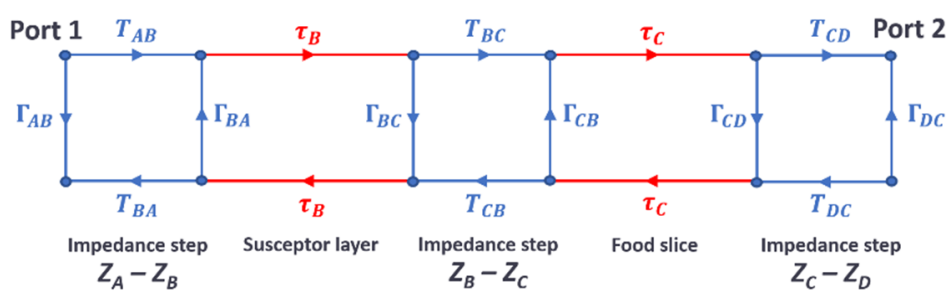

Fig. 4. Four-layer EM model (left) and (right) its microwave flow graph: A and D layers stand for air, B for a susceptor, and C for a food slice.

Based on the above analysis and equations, total relative power dissipated, jointly in a susceptor and $1 \mathrm{~cm}$-thick slice of beef $\left(\varepsilon_{r}=48.2, \sigma=2.194 \mathrm{~S} / \mathrm{m}\right.$ at room temperature), defined as $\left(1-\left|S_{12}\right|^{2}-\left|S_{22}\right|^{2}\right)$, is calculated for varying thicknesses of the dielectric surrogate and angles of incidence. The results are presented in Fig. 5 in a form of 2D maps. It is clearly seen that using a surrogate layer of thickness up to $1 \mathrm{~mm}$ does not affect the dissipated power, compared to the original ultra-thin susceptor. Thereby, the practical limit of application of the macroscopic model of thin conductive sheets derived in [9] for susceptors in free space remains valid for real-life scenarios, in which susceptor is in contact with food. The results further prove that the heating will be more efficient for TM than for TE-waves, and the highest absorption is obtained for high angles of TM wave incidence.

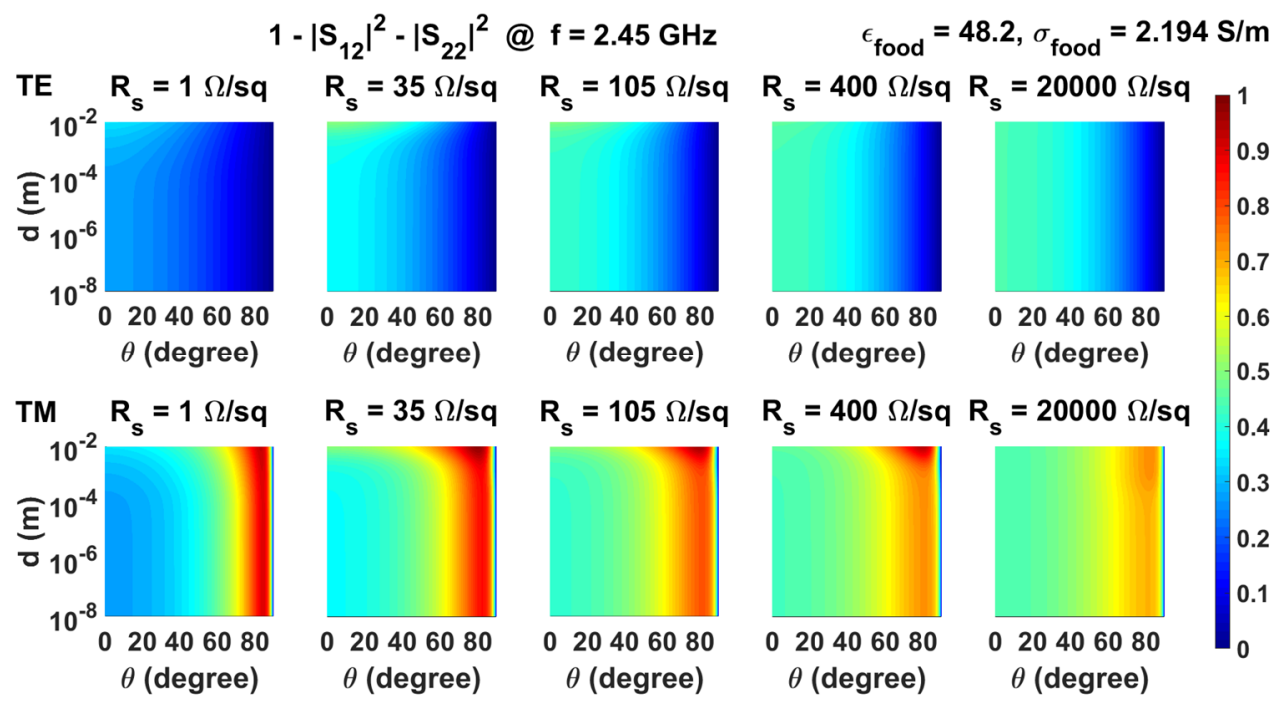

Fig. 5. Maps of total relative power dissipated jointly in a susceptor and $1 \mathrm{~cm}$-thick slice of beef $(\varepsilon=48.2 \sigma=2.194 \mathrm{~S} / \mathrm{m})$, for EM waves incident at varying angle $\theta$ from air onto beef, and varying thickness $d$ of the surrogate. 


\section{Power dissipated separately in the surrogate and the food}

In this section we extend the analysis of the scenario of Fig. 4, by considering power dissipated in each layer of the model. We consider power transmitted through an arbitrary plane parallel to the layers:

$$
P=\operatorname{Re}\left(\boldsymbol{E} \times \boldsymbol{H}^{*}\right) \cdot \hat{\mathbf{z}}=\left(\frac{\cos \theta}{Z}\right)^{*} \cdot\left(E^{+}+E^{-}\right) \cdot\left(E^{+}-E^{-}\right)^{*},
$$

where $\boldsymbol{E}, \boldsymbol{H}$ stand for electric and magnetic fields in the plane, $\hat{\boldsymbol{z}}$ is a unit vector normal to the layers, $\cos \theta$ was defined with equation (8), $Z$ is impedance, $E^{+}$and $E^{-}$stand for complex amplitudes of electric field of the incident and reflected waves at the considered plane. Power dissipated in $i$-th layer can be calculated as a difference of power transmitted through the opposite planes of the layer:

$$
P_{i}=P_{i}^{\text {in }}-P_{i}^{\text {out }}
$$

For EM wave incident at port 1, electric field amplitudes in layer B (see Fig. 5) can be calculated as follows:

$$
\begin{array}{ll}
E_{\text {in }}^{+}=\frac{1}{T_{B A}}\left(1+\Gamma_{B A} S_{11}\right) \cdot E_{0}^{+}, & E_{\text {out }}^{+}=E_{\text {in }}^{+} \cdot \tau_{B}, \\
E_{\text {in }}^{-}=\frac{1}{T_{B A}}\left(-\Gamma_{A B}+S_{11}\right) \cdot E_{0}^{+}, & E_{\text {out }}^{-}=E_{\text {in }}^{-} \cdot \tau_{B}^{-1},
\end{array}
$$

and in layer $\mathrm{C}$ (further from the incident port):

$$
\begin{gathered}
E_{\text {out }}^{+}=\frac{1}{T_{C D}} S_{21} \cdot E_{0}^{+}, \quad E_{\text {in }}^{+}=E_{\text {out }}^{+} \cdot \tau_{C}^{-1}, \\
E_{\text {out }}^{-}=\Gamma_{C D} \cdot E_{\text {out }}^{+}, \quad E_{\text {in }}^{-}=E_{\text {out }}^{-} \cdot \tau_{C},
\end{gathered}
$$

where $E_{0}^{+}$stands for an amplitude of an incident wave at port 1. Analogical equations can be derived in the case of a wave incident at port 2 , if one reverts indices of layers and ports.

The above conducted analysis and formulated equations are confirmed with the use of conformal FDTD modelling performed in QuickWave 3D software [11]. In order to simulate normal incidence (TEM) of EM wave on the considered multi-layer stacked structure (Fig. 4), a parallel-plate line model is used. For the purpose of modelling plane wave with an arbitrary incidence angle, we use a rectangular waveguide with a square cross section $a \times a$ supporting TE11 and TM11 modes. In order to obtain an arbitrary angle $\theta$ of EM wave incidence in air, the waveguide dimension $a$ needs to be adjusted following the formula:

$$
a=\frac{c}{f \cdot \sin \theta \cdot \sqrt{2}},
$$

where $c$ stands for the speed of light in vacuum.

Standard stair-case formulation of the FDTD method requires that the surrogate thickness occupies at least one full FDTD cell, which for thin layers significantly increases simulation time as the FDTD time step decreases. QuickWave 3D software implements conformal FDTD, which allows for adopting thin layers filling only a part of the FDTD cell, thereby alleviating the stair-case limitation and achieving the geometric flexibility classically attributed to FEM formulations. In the conducted EM analysis, FDTD cell size in the direction of wave propagation is set to $1 \mathrm{~mm}$ and $10 \mu \mathrm{m}$-thick surrogate is considered. 
Fig. 6 presents comparison of analytical and conformal FDTD results for dissipated power, calculated separately for the susceptor and raw beef, as a function of surface resistance of the susceptor. Analytical data are fully consistent with the electromagnetic simulation results. It is seen that the absorption in susceptor remains maximal for its sheet resistance in the range of $\mathrm{Rs}=30 \div 400 \Omega$, similarly as for the susceptor in free space. However, total value of dissipated power tends to increase monotonously with the increasing sheet resistance of the susceptor (except for very high incidence angle and TM polarisation, where a subtle optimum occurs around the optimum of the susceptor heating). Two physical effects are therefore observed, when the surface resistance of the susceptor increases: first, the susceptor heats more and then, the susceptor stops heating but its backing of the food causes more efficient volumetric heating of the food itself.

Note that in real-life microwaveable food packages, the susceptors are manufactured so that they break or otherwise increase their surface resistance during the heating [1], [10]. Our graphs in Fig. 6 help therefore explain how the initial surface heating by the susceptor is followed by the volumetric heating of the actual load; in typical microwaveable foods the volumetric heating phase is further enforced by the increase of food losses with temperature, in typical foods [10].

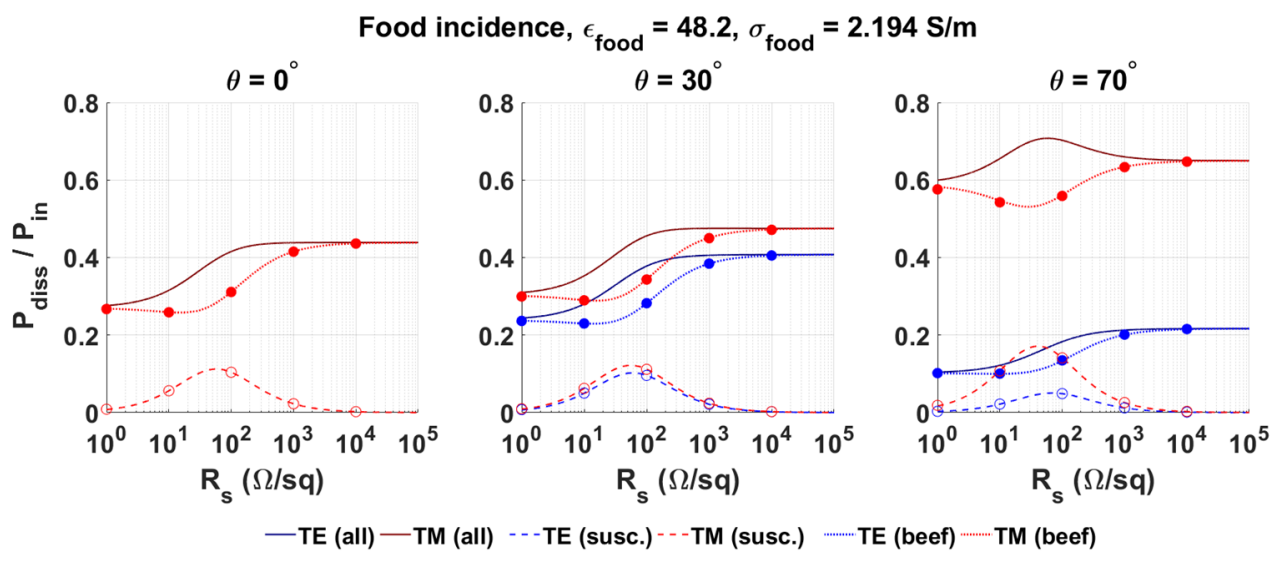

Fig. 6. Relative power dissipated separately in $10 \mu \mathrm{m}$ susceptor (susc) and $1 \mathrm{~cm}$ raw beef (beef) layers; and jointly (all) in both layers, for EM waves incident from air onto food backed by the susceptor. Analytical results are plotted by lines; dots and circles indicate conformal FDTD results for food and susceptor, respectively.

\section{Conclusions}

In the present work an application of macroscopic models in a form of dielectric surrogate layers of significantly higher thickness for microwave susceptors analysis is considered. In extension to earlier studies, the discussion addresses real-life scenarios, where a microwave susceptor is in contact with food, as it typical microwaveable food packages. The evaluation of the macroscopic model is conducted with the aid of analytical calculations and conformal FDTD simulations, accounting for various angles of EM wave incidence and wave polarisation, surface resistance of microwave susceptor, and surrogate thickness. The systematic evaluation of the equivalent macroscopic model in terms of power dissipated in the structure allows to derive a practical limit of $1 \mathrm{~mm}$ surrogate thickness (at $2.45 \mathrm{GHz}$ ), for application of the surrogate model in the modelling scenarios where the susceptor 
remains in contact with food. Moreover, the studies aiming at extracting power dissipated separately in food and susceptor resulted in formulating a range of susceptors' surface resistance assuring the highest power absorption in susceptor, and the highest absorption in the overall package, which constitutes practical guidelines for food packaging designers and manufacturers.

\section{References}

1. M. R. Perry and R. R. Lentz, "Susceptors in microwave packaging," in Development of packaging and products for use in microwave ovens, $1^{\text {st }}$ ed., W. Lorence and P. S. Pesheck, London, United Kingdom: Woodhead Publishing Limited and CRC Press, 2009, 207-236.

2. T. H. Bohrer, "Shielding and field modification - thick metal films," in Development of packaging and products for use in microwave ovens, $1^{\text {st }}$ ed., W. Lorence and P. S. Pesheck, London, United Kingdom: Woodhead Publishing Limited and CRC Press, 2009, 237-266.

3. P. Risman, "Modelling the effects of active packaging of microwave foods," in Development of packaging and products for use in microwave ovens, $1^{\text {st }}$ ed., W. Lorence and P. S. Pesheck, London, United Kingdom: Woodhead Publishing Limited and CRC Press, 2009, 349-371.

4. Ha, M. S., Kwon, O. Y., Choi, H. S., Composites Research, 2010, 23, 31-36.

5. A. Taflove, M. Celuch-Marcysiak, and S. Hagness, "Local Subcell Models of Fine Geometrical Features," in Computational Electrodynamics - The Finite-Difference Time-Domain Method, $3^{\text {rd }}$ Edition, A. Taflove and S. Hagness, Boston-London: Artech House, 2005, pp. 407-462.

6. P. P. Silvester and R. L. Ferrari, Finite Elements for Electrical Engineers, $3^{\text {rd }}$ Ed., Cambridge University Press, 1996.

7. W. Gwarek, "Modeling and measurements of susceptors for microwave heating applications", at: IEEE IMS Workshop "Recent Advances in Microwave Power Applications and Techniques RAMPAnT”, Boston, June 2009.

8. Krupka, J., Gwarek, W., "Measurements and modeling of planar metal film patterns deposited on dielectric substrates",IEEE Microwave and Wireless Components Letters, 2009, 19, 134-136.

9. M. Celuch, K. Wilczynski, M. Olszewska-Placha, "Macroscopic Models of Thin Conductive Layers: Systematic Evaluation for Microwave Heating and Shielding Applications", IEEE International Microwave Symposium, Boston, May 2019, 4750 .

10. P. O. Risman, "Microwave oven and food compatibility: historical background, the present situation and future possibilities" private communication; also presented at: 42 $2^{\text {nd }}$ Annual Microwave Power Symp. (IMPI), New Orleans, LA, June 2008.

11. The QuickWave EM Software (1997-2019) website. [Online] Available: http://www.qwed.eu 


\title{
ENHANCED MICROWAVE ASSISTED PROCESSING OF 2D CYLINDRICAL POROUS FOOD DIELECTRIC
}

\author{
S. Kumari ${ }^{1}$, S. K. Samanta ${ }^{1}$, K. Patel ${ }^{1}$ \\ ${ }^{1}$ Indian Institute of Technology Patna, Bihta, Patna-801103, India \\ sushma.pcb16@iitp.ac.in; sksamanta@iitp.ac.in; kushpatel1407@gmail.com
}

\begin{abstract}
:
The importance of microwave assisted thermal processing for the food industry relies on the fact of the procedure being economical and swift along with ensuring high quality product. The sample has been assumed as a porous solid food with meat being the continuous phase and oil or water being the dispersed phase. The resultant matrix of meat-oil or meat-water is considered to be a 2D cylinder being subjected to microwave irradiation in two different interaction schemes i.e. lateral and radial. The thermal effect of the interaction is calculated by applying Galerkin finite element analysis to simultaneously solve the Maxwell's equation and heat transfer equation using pertinent boundary conditions. The preliminary solution gives the value of average power absorption at different sample sizes which exhibit few peak points depicting enhanced heating and power absorption. Further study consists of analyzing the spatial distribution of power and temperature along with the transient temperature difference and mean temperature evolution within the sample corresponding to designated observation points. The heating strategy exhibiting high heating rate and low thermal nonuniformity is recommended as the preferred optimum condition. For both meat-oil and meatwater porous solids, the radial irradiation is recommended as the optimal heating strategy corresponding to smaller sample diameter (OP: 1) whereas lateral irradiation is recommended for larger sample diameter (OP: 2). Overall the present work serves as a guide for enhanced microwave assisted heating of porous food samples in the shape of $2 \mathrm{D}$ cylinders.
\end{abstract}

Keywords: Microwave, Porous food, Optimum heating, Average power absorption

\section{Introduction:}

The electromagnetic waves with the wavelengths ranging from $1 \mathrm{~m}$ to $1 \mathrm{~mm}$ are known as microwaves and the typical frequency range is $300 \mathrm{MHz}$ to $300 \mathrm{GHz}$. The value of dielectric loss of a material is the determining factor in conversion of electrical energy into heat energy ${ }^{1-3}$. The volumetric heat generation effect of microwave has gained attention in different processing industries such as food, pharmaceutical, ceramics, polymer and many more $^{3-5}$. The exigencies of a swift, qualitative and economical procedure for the food industry was addressed by the microwave assisted processing such as heating, drying, thawing, sterilization, tempering, etc ${ }^{4,6,7}$.

The importance of proper investigation on processing of food prior to its industrial scaling is discussed by Karim and Hawlader (2005) where an analysis shows a loss of 30-40 \% of global food production in developing nations as a result of processing faults ${ }^{8}$. So, researchers are striving to come up with novel physics-based models to best imitate food samples. Ayappa et al. (1992) was the first to report a combined analysis of microwave and thermal transport for $1 \mathrm{D}$ and 2D solid samples ${ }^{9}$. The analysis and formulation for the occurrence of resonance at a specific sample size and dimension was formed by Ayappa et al. (1991) ${ }^{1}$. Basak and Ayappa (1997) performed thawing of solid samples observing higher melting rate during resonances at some specific thicknesses ${ }^{10}$. 
The mathematical imitation of a food is slightly difficult as food is amorphous and hygroscopic in nature and structurally non-homogenous ${ }^{11}$. A food material in itself is a complex sample with multiple components and varying dielectric properties which makes its mathematical modeling arduous. Many authors have come up with models but they tend to take assumptions to reduce mathematical complications. The most realistic characterization of food can be as a porous material with the dielectric response calculated as the operative dielectric properties of solid frame and the fluid present in the voids. Only a few studies have been reported on the heating effect of typical 1D porous-food dielectric with oil and air as dispersed medium using various metallic, ceramic or composite supports $^{7,12}$. A few 2D analyses of microwave heating of food samples studied till date have not considered the effect of porosity of the material ${ }^{4,13}$. Basak (2007) has carried out a detailed analysis to study the effect of lateral and radial irradiations on microwave heating of 2D solid meat cylinders and oil samples ${ }^{4}$. The effect of shape on the microwave heating dynamics of food materials was analyzed for different shapes like circular and square by Bhattacharya and Basak (2017) $)^{13}$.

Here we have carried out a detailed theoretical analysis on enhanced microwave heating of typical food samples (porous meat) in the form of 2D cylinders in the presence of lateral and radial irradiations. The food sample has been assumed to be a highly lossy porous dielectric with the meat as the continuous phase and oil or water as the dispersed phase of the porous solid structure. The resulting porous materials such as meat-oil and meat-water, where oil and water act as the fluid medium of the porous body, have been analyzed with operative dielectric properties for various porosity values $(\phi=0.1,0.25$ and 0.4$)$.

\section{Theoretical modeling and solution strategy:}

\section{Microwave propagation: Power and temperature formulations}

The extent of the absorption of microwave irradiation by the porous food dielectric has been analyzed through the electromagnetic field equation which assists the study of temperature distribution owing to different irradiation schemes i.e. lateral and radial. The scheme of lateral mode of irradiation assumes the sample to have unidirectional interaction (left in this study) due to being stationary in a uniform plane wave, whereas the radial irradiation scheme implies the sample to have a multidirectional interaction due to being rotated.

The electromagnetic field consists of mutually perpendicular electric and magnetic field with magnitude varying in the $\mathrm{z}$-direction i.e. the direction of wave propagation considered in this study (see Fig. 1). The propagation of microwave due to uniform electric field, $E_{x}$ varying only in the $y-z$ plane in a $2 \mathrm{D}$ porous solid can be given by Maxwell's equation as shown below ${ }^{1}$.

$$
\nabla^{2} E_{x}+\kappa^{2} E_{x}=0
$$

The propagation constant, $\kappa$, depends on dielectric constant $\kappa^{\prime}$, dielectric loss $\kappa^{\prime \prime}$ and frequency of electromagnetic wave, $f$, which can be given as:

$$
\kappa=2 \pi f\left(\sqrt{\kappa^{\prime}+i \kappa^{\prime \prime}}\right) / c
$$

The $c$ in the above equation is the speed of light.

The sample having a porous structure has been assumed to contain a dispersed phase and a continuous phase with both having different dielectric and thermal properties. The operative dielectric property of the sample can be evaluated by the Fricke's conductivity model as given below:

$\kappa_{o p}^{*}=\frac{\kappa_{c}^{*}\left[\kappa_{d}^{*}(1+a \phi)+a \kappa_{c}^{*}(1-\phi)\right]}{\kappa_{d}^{*}(1-\phi)+\kappa_{c}^{*}(a+\phi)}$ 
where, $\kappa_{c}^{*}$ and $\kappa_{d}^{*}$ are relative complex dielectric properties of the continuous and dispersed phases, respectively and are assumed as temperature independent. The value of ' $a$ ' is assumed to be 2 for the present case study which is due to the assumption of spherical dispersion.

Energy balance equation within the porous food sample due to microwave assisted heat source can be expressed as

$\rho C_{p} \frac{\partial T}{\partial t}=k \nabla^{2} T+P(z)$

where $\rho$, the operative density, $C_{p}$, the operative specific heat and $k$, the operative thermal conductivity can be expressed as operative properties such as:

$\rho=(1-\phi) \rho_{c}+\phi \rho_{d}$

$C_{p}=(1-\phi) C_{p c}+\phi C_{p d}$

and

$k=(1-\phi) k_{c}+\phi k_{d}$

The power per unit volume can be obtained by:

$$
P=\left(2 \pi f \varepsilon_{0} \kappa_{o p}^{\prime \prime}(\phi) E_{x} E_{x}^{*}\right) / 2
$$

where, $\varepsilon_{0}$ is permittivity of free space, $\phi$ is the porosity, $\kappa_{o p}^{\prime \prime}$ is the operative dielectric loss of the porous food and $E_{x}^{*}$ is the complex conjugate of the electric field. The above equation on being integrated over the entire domain considering $\mathrm{S}$ as the cross-sectional area and $\Gamma$ as the outer surface of the porous solid can be given as:

$P_{a v}=\frac{1}{S} \iint_{\Gamma} P(\Omega) d \Omega$

The insulation condition (no flux condition) has been used for outer surface as the boundary condition.
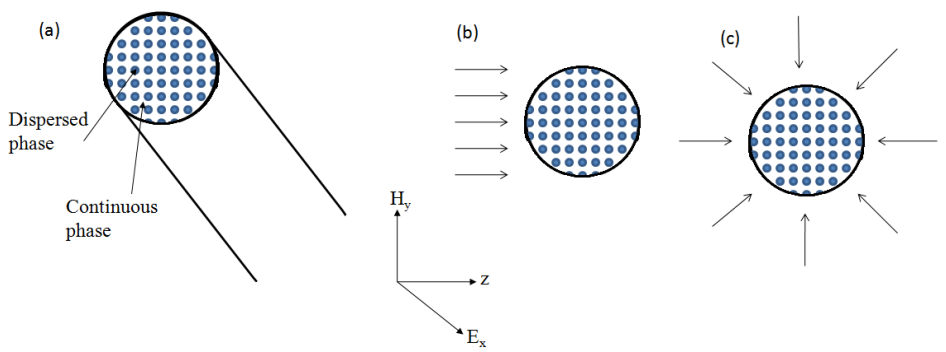

Fig. 1. Schematic illustrations of 2D porous food cylinder incident with (a) uniform plane waves, (b) lateral irradiation and (c) radial irradiation.

\section{Solution strategies}

The field equation given by Maxwell's and heat balance equation are solved simultaneously using Galerkin finite element method assuming appropriate boundary conditions $[1,7]$. The common element at the interface of the two phases satisfies the continuity condition of field variables and fluxes. To discretize the time domain, Crank-Nicholson method is used, and the nonlinear residual equations are solved using Newton Raphson method. The simulations are carried out for meat as the continuous phase and oil or water as dispersed phase of the porous cylinder considering the initial temperature as 
$275 \mathrm{~K}$, ambient temperature as $300 \mathrm{~K}$ and the microwave irradiation of $1,1.5$ and $2 \mathrm{~W} . \mathrm{cm}^{-2}$ intensities and $2450 \mathrm{MHz}$ frequency. The effective intensity of incident radiation from the source has been assumed to be identical for both the lateral and radial irradiation schemes.

\section{Results and discussion:}

A theoretical analysis has been performed to study the thermal effects on the 2D cylindrical porous food dielectrics subjected to microwave irradiation of $2450 \mathrm{MHz}$ frequency. The analysis comprises of studying the effect of different porosity conditions $(\phi=0.1,0.25$ and 0.4 ), different intensities $\left(I_{0}=1,1.5\right.$ and $\left.2 \mathrm{~W} . \mathrm{cm}^{-2}\right)$ and different dispersed phases (oil, water) on the heating strategy. The study of average power absorption within a porous cylindrical sample with respect to diameter of the sample gives a preliminary understanding of enhanced heating and power absorption within the sample. Owing to the main objective of comparing the heating strategy of both lateral and radial irradiation mode, the peaks obtained for either of the mode leads to selection of the sample size for both modes and being labeled as observation points (OP). The information on the average power absorption needs further exploration through the spatial distribution of power and temperature to understand the occurrence of resonances due to the constructive inferences of the transmitted and reflected waves. The exploration of distribution of heat inside the sample leads the way to the selection of the optimum heating strategy based on factor of high thermal uniformity and heating rate. The plots of average temperature and temperature difference with respect to time give the heating rate of the procedure and the extent of thermal non-uniformity or the thermal runaway, respectively. The heating strategy favoring low thermal non-uniformity and high heating rate is optimum. However, the decision is always inclined towards uniform distribution of temperature.

\section{Meat-oil porous dielectric at $\mathrm{I}_{0}=1 \mathrm{Wcm}^{-2}$ and $\phi=0.4$}

The microwave warming of porous meat-oil dielectric at frequency $f=2450 \mathrm{MHz}$, intensity $I_{0}=1 \mathrm{Wcm}^{-2}$ and porosity $\phi=0.4$ has been analysed with the aim of determining the best suited heating strategy. The plot of average power absorption with respect to the size of the sample exhibits the smaller samples to absorb more power corresponding to lateral irradiation mode and larger samples to absorb more power during radial irradiation mode. The peaks obtained due to constructive interferences of the waves are chosen as observation point (OP) for detailed analysis. The OP:1 and OP:2 are selected corresponding to sample diameter, $d_{c}=1.78$ and $3.00 \mathrm{~cm}$, respectively as shown in Fig. 2 . The OP: 1 corresponds to average power, $\mathrm{P}_{\mathrm{av}}=1.330$ and $0.940 \mathrm{~W} . \mathrm{cm}^{-3}$ for lateral and radial modes of irradiation, respectively. In addition, the average power absorption for OP: 2 exhibits the value of $\mathrm{P}_{\mathrm{av}}=$ 0.461 and $0.852 \mathrm{~W} . \mathrm{cm}^{-3}$ for lateral and radial schemes, respectively. It may also be noted that the effect of resonance dies down significantly for $d_{c} \geq 3 \mathrm{~cm}$ and the average power is found to decrease almost asymptotically for both lateral and radial irradiation cases. The inset of the plot includes the spatial distribution of power inside the sample. The sample at OP: 1 subjected to lateral irradiation displays two maxima in spatial power, one being 2.5 $\mathrm{W} . \mathrm{cm}^{-3}$ at the left side and the other being $3 \mathrm{~W} . \mathrm{cm}^{-3}$ at the right side whereas the radial irradiation displays a single central maxima of $2.5 \mathrm{~W} . \mathrm{cm}^{-3}$. OP: 2 displays single central maxima for both lateral and radial irradiation modes of 1 and $4 \mathrm{~W} . \mathrm{cm}^{-3}$, respectively. The thermal spatial distribution analysis at $\mathrm{t}=120$ seconds for meat-oil porous cylinders corresponding to lateral and radial irradiations at OP: 1 and OP: 2 have been displayed in Fig. 3(a). The maximum temperature for OP: 1 is observed at both the left and right faces and is around 350 and $390 \mathrm{~K}$, respectively for lateral irradiation. However, at the same OP, 
a central maxima of around $350 \mathrm{~K}$ for radial irradiation is observed. Moreover, for the OP: 2 , both the lateral and radial irradiations display a central maxima of 310 and $380 \mathrm{~K}$, respectively. This highlights the unpredictability of hotspot formation in the sample with different schemes and parameters. To further decide the optimum strategy, a study on the transient temperature difference and mean temperature evolution is performed.

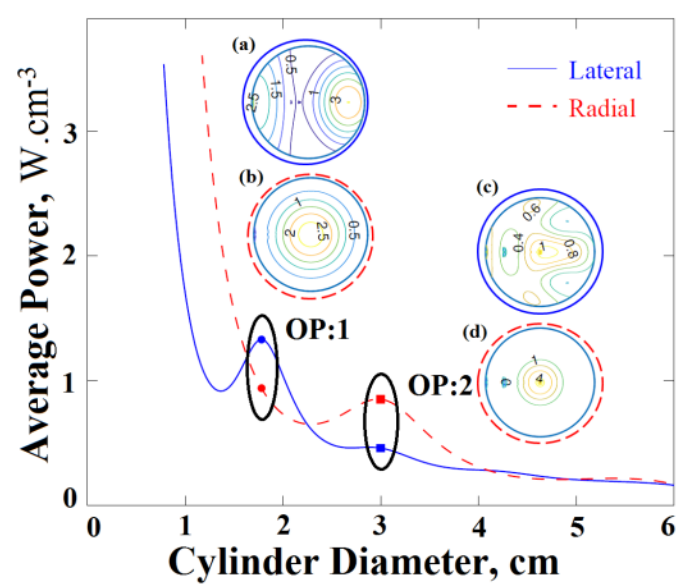

Fig. 2. Average power $\left(\mathrm{W} . \mathrm{cm}^{-3}\right)$ vs. cylinder diameter for various meat-oil samples due to lateral and radial irradiations corresponding to $\phi=0.4 ; f=2450 \mathrm{MHz} ; I_{0}=1 \mathrm{~W} . \mathrm{cm}^{-2}$. The inset is the representation of the power distribution inside the cylindrical sample (a) for lateral heating at $d_{c}=1.78 \mathrm{~cm}$, (b) for radial heating at $d_{c}=1.78 \mathrm{~cm},(\mathbf{c})$ for lateral heating at $d_{c}=$ $3.00 \mathrm{~cm}$ and (d) for radial heating at $\mathrm{d}_{\mathrm{c}}=3.00 \mathrm{~cm}$.
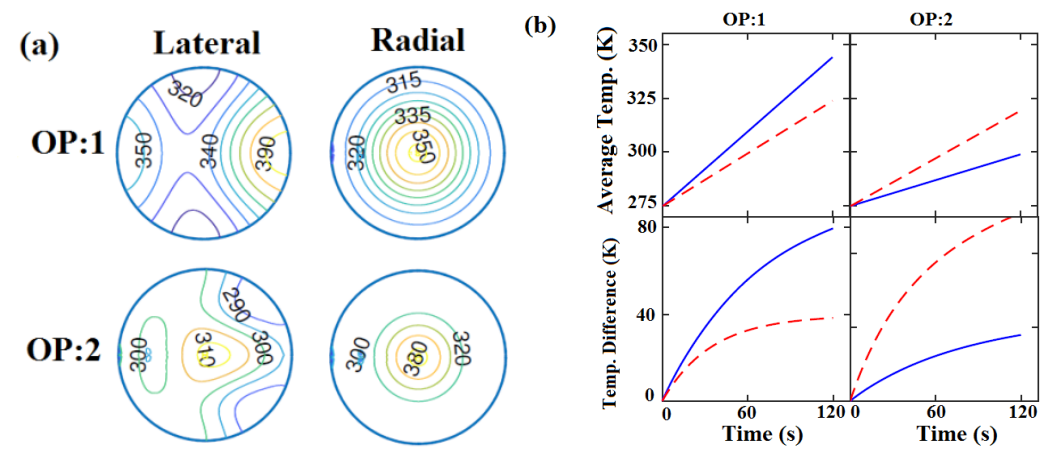

Fig. 3. (a) Spatial representation of thermal mapping inside the meat-oil food cylinders during microwave assisted warming at $\phi=0.4 ; f=2450 \mathrm{MHz} ; I_{0}=1 \mathrm{~W} . \mathrm{cm}^{-2}$ for 120 seconds.

(b) Average temperature $(\mathrm{K})$ in the meat-oil food cylinders vs. time (s) [upper panel];

Temperature difference $(\mathrm{K})$ in the meat-oil food cylinders vs. time (s) [lower panel].

The temperature difference vs. time which is a measure of the degree of non-uniformity of temperature distribution within the sample and average temperature vs. time that denotes the heating rate have been presented in the lower and upper panels of Fig. 3(b), respectively. An optimal heating strategy can be characterized as one with higher heating rate and smaller thermal runaway. At OP: 1, the heating rate is slightly higher for lateral irradiation however, 
the thermal non-uniformity is very large making the radial irradiation as the desired strategy. Although, at OP: 2 the lateral irradiation exhibits lower heating but lower thermal nonuniformity makes it the preferred heating strategy.

\section{Meat-water porous dielectric at $I_{0}=1.5 \mathrm{Wcm}^{-2}$ and $\phi=0.1$}

The analysis of microwave warming of porous meat-water food dielectric at a frequency of $2450 \mathrm{MHz}$, intensity $I_{0}=1.5 \mathrm{Wcm}^{-2}$ and porosity $\phi=0.1$ has been performed with the objective to determine the best heating strategy. Fig. 4 exhibits the average power absorption with respect to the sample diameter along with the spatial distribution of power at the chosen peaks labeled as OPs. The OP: 1 has been marked at sample diameter $d_{c}=1.30 \mathrm{~cm}$ with average power $\mathrm{P}_{\mathrm{av}}=2.736$ and $2.060 \mathrm{~W} \cdot \mathrm{cm}^{-3}$ for lateral and radial irradiation modes, respectively. The next OP i.e. OP: 2 for lateral and radial heating schemes is noted at $d_{c}=$ $2.12 \mathrm{~cm}$ with average power $\mathrm{P}_{\mathrm{av}}=0.920$ and $2.196 \mathrm{~W} . \mathrm{cm}^{-3}$, respectively.

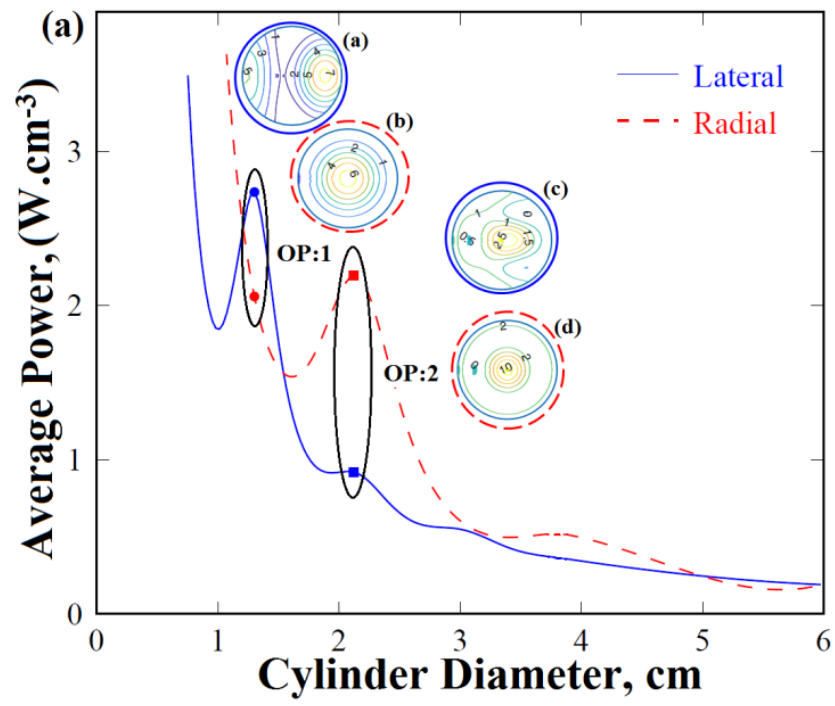

Fig. 4. Average power (W. $\left.\mathrm{cm}^{-3}\right)$ vs. cylinder diameter for various meat-water samples due to lateral and radial irradiations corresponding to $\phi=0.1 ; f=2450 \mathrm{MHz} ; I_{0}=1.5 \mathrm{~W} . \mathrm{cm}^{-2}$. The inset is the representation of the power distribution inside the cylindrical sample (a) for lateral heating at $\mathrm{d}_{\mathrm{c}}=1.30 \mathrm{~cm}$, (b) for radial heating at $\mathrm{d}_{\mathrm{c}}=1.30 \mathrm{~cm}$, (c) for lateral heating at $\mathrm{d}_{\mathrm{c}}=2.12 \mathrm{~cm}$ and (d) for radial heating at $\mathrm{d}_{\mathrm{c}}=2.12 \mathrm{~cm}$.

A detailed spatial distribution of temperature within the sample and study of the transient temperature difference and mean temperature evolution and distribution within the sample have also been performed for different OPs selected (see Fig. 5). The temperature contour in Fig. 5 (a) in the case of OP: 1 present a right sided maxima of $410 \mathrm{~K}$ for lateral irradiation and a central maxima of $370 \mathrm{~K}$ for radial irradiation. The OP: 2 displays a central maxima for both the irradiation modes with $320 \mathrm{~K}$ and $420 \mathrm{~K}$ for lateral and radial irradiations, respectively.

The investigation at OP: 1 for meat-water sample recommended the radial irradiation as the preferred heating strategy due to higher thermal uniformity even with slightly lower heating rate (see Fig.5 (b)). In addition, since OP: 2 exhibits lower heating rate but higher thermal 
uniformity for the lateral irradiation hence it is recommended as the preferred heating strategy.
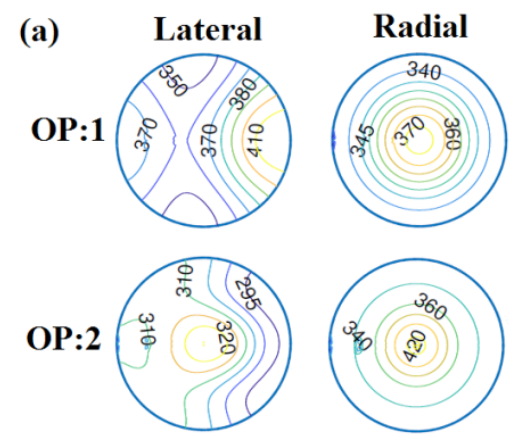

(b)

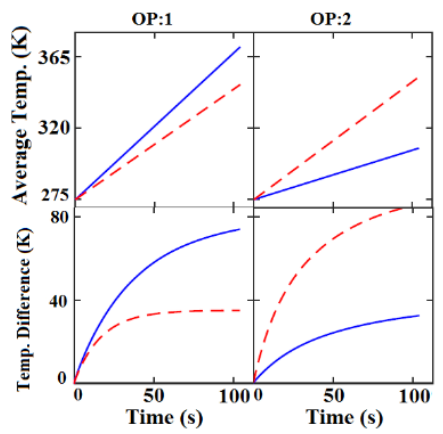

Fig 5. (a) Spatial representation of thermal mapping inside the meat-water food cylinders during microwave assisted warming at $\phi=0.1 ; f=2450 \mathrm{MHz} ; I_{0}=1.5 \mathrm{~W} . \mathrm{cm}^{-2}$ for 100 seconds. (b) Average temperature (K) in the meat-water food cylinders vs. time (s) [upper panel]; Temperature difference $(\mathrm{K})$ in the meat-water food cylinders vs. time (s) [lower panel].

Overall, the dielectric properties of the porous food samples and its dimension $\left(d_{c}\right)$ can be considered as two of the major parameters that are creating the diverse scenario of heating rate and thermal runaway within the samples. In addition, the final choice of lateral/radial irradiation is a trade-off between two parameters namely higher heating rate and minimal thermal runaway where minimal thermal runaway is generally given more emphasis owing to the importance of preservation of the quality of the sample. Similar work related to other food systems, porosity conditions and intensity of the microwave irradiation has also been carried out, however, results are not reported here for the brevity of the manuscript. The present analysis works as a guide for enhanced microwave assisted processing of 2D porous food dielectrics.

\section{Conclusions:}

An extensive analysis for investigating the thermal effect of microwave irradiation on 2D porous food cylinders with meat as continous phase and oil or water as dispersed phase has been perfomed. The analysis entails the importance of the role of lateral and radial irradiation schemes for obtaining an optimum heating strategy. Preliminarily, the extent of absorption of average power with respect to the sample size (cylinder diameter) has been studied for obtaining the power absorption and distribution within meat samples. Further, a detailed mathematical analysis on spatial temperature distributions and the transient temperature difference and mean temperature evolution within the sample for both the lateral and radial irradiation schemes has been carried out to decide an optimal heating strategy for any specific OP chosen from the precedent analysis. The plot of average temperature and temperature difference against time present us the heating rate and thermal non-uniformity, respectively. The recommended optimal heating strategies decide the procedure to have high heating rate with minimal thermal runaway (thermal non-uniformity).

Based on the recommendation for the optimal heating strategy for both meat-oil and meatwater porous solids, radial irradiation is recommended as the optimal heating strategy corresponding to smaller sample diameter (OP: 1) whereas lateral irradiation is recommended for larger sample diameter (OP: 2). Observing the overall scenarios, the choice of lateral/radial irradiations depends primarily on the sample dielectric properties and 
sample dimension $\left(d_{c}\right)$ and the suitability of any specific irradiation as an optimal heating strategy is measured based on the heating rate and thermal runaway for each case. Hence, the sample dielectric properties, sample dimension, non-uniformity in temperature distribution and heating rate come out to be the determining factors for efficient heating process for porous food dielectrics. Finally, the present study can be extended for other porous solids with different compositions of continuous and dispersed phases.

\section{References}

[1] Ayappa KG, Davis HT, Crapiste G, Davis EA, Gordon J. Chem Eng Sci. 1991;46:1005-16.

[2] Samanta SK, Basak T. Food Res Int. 2010;43:148-66.

[3] Samanta SK, Basak T. Food Res Int. 2009;42:1337-50.

[4] Basak T. Chem Eng Sci. 2007;62:3185-96.

[5] Verma P, Samanta SK. Environ Chem Lett. 2018;16:969-1007.

[6] Chandrasekaran S, Ramanathan S, Basak T. Food Res Int. 2013;52:243-61.

[7] Aparna K, Basak T, Balakrishnan AR. Int J Heat Mass Transf. 2007;50:3072-89.

[8] Karim MA, Hawlader MNA. Int J Heat Mass Transf. 2005;48:4914-25.

[9] Ayappa KG, Davis HT, Davis EA, Gordon J. Aiche J. 1992;38:1577-92.

[10] Basak T, Ayappa KG. Aiche J. 1997;43:1662-74.

[11] Khan MIH, Joardder MUH, Kumar C, Karim MA. Crit Rev Food Sci Nutr. 2016;57:1-19.

[12] Basak T, Aparna K, Meenakshi A, Balakrishnan AR. Int J Heat Mass Transf. 2006;49:4325-39.

[13] Bhattacharya M, Basak T. Innov Food Sci Emerg Technol. 2017;39:247-66. 


\title{
MICROWAVE PROCESSING OF FOOD SAMPLES: INFLUENCE OF CAVITY DESIGN AND DIELECTRIC PROPERTIES
}

\author{
C. D. Albuquerque, S. Curet, L. Boillereaux \\ UMR GEPEA CNRS 6144 - ONIRIS, CS 82225, 44322 Nantes-France \\ sebastien.curet@oniris-nantes.fr
}

Keywords: microwave heating, modelling, impedance matching, temperature distribution.

\begin{abstract}
Microwave-material interactions and electromagnetic propagation phenomena are important to optimize the microwave heating process of food samples. In this study, a 3D numerical modelling of a TE10 rectangular waveguide including microwave antenna and impedance matching elements is proposed. The microwave applicator is aimed to process both solid and liquid food samples. The model illustrates the standing wave patterns and microwave absorbed power within the cavity by taking into account the influence of the screw tuner, quartz windows, shorting plunger, and additional dielectric support plates.

The results reveal the importance to consider the real cavity design and the precise dielectric characterization to predict accurate temperature profiles within the food product during the microwave heating. Such a model can be now be used to optimize the food sample geometry to achieve minimum reflected power and better heating uniformity.
\end{abstract}

\section{Introduction}

Microwave heating processes for food products are used industrially for various unit operations such as tempering, drying, pasteurization ${ }^{1}$. During a microwave heating process, the energy contained into an electromagnetic wave is converted into heat within dielectric materials. This selective volumetric heating mechanism allows a considerable reduction in processing time and increases the energy efficiency of the process ${ }^{2}$. However, one of the main challenges is the heterogeneous electric field distribution within the food matrix which could result in hot and cold spots ${ }^{3}$. Since electromagnetic field measurements within a microwave cavity are particularly difficult to perform, the numerical modeling and simulation appear as a promising tool to understand, predict and control the process, as well as, to optimize the design of equipment and packaging ${ }^{4}$. As many factors influence the degree of interaction between the microwaves and the material, the model needs to take into account both geometrical details of the microwave cavity and the physical properties of the materials under consideration. These include dielectric, thermal and physical properties of the product and the dielectric materials (volume, geometry and location) and the design of the microwave cavity ${ }^{5}$. The purpose of this study is to investigate the influence of the cavity design and dielectric properties during the microwave processing of solid and liquid food products.

\section{Design of the microwave cavity}

A solid-state microwave generator (SG 524, model AG340M3, Alter, Reggio Emilia, Italy) enables to supply a monochromatic wave transmitted along the $z$-direction of a TE10 rectangular waveguide (cross-section $86 \mathrm{~mm} \times 43 \mathrm{~mm}$ ) at a frequency of $2.45 \mathrm{GHz}$ with adjustable output power. The device includes an antenna placed at the waveguide transition, a 3-stub tuner with adjustable screws, two quartz windows to protect the upper and lower sides of the microwave applicator, a liquid food product flowing continuously within a 
quartz tube and a shorting plunger. Impedance matching is important to insure maximum transfer of microwave power from the microwave generator to the dielectric

loads. In this work, both the screw tuners and the waveguide shorting plunger are used to perform impedance matching between the waveguide and the dielectric samples by reducing the microwave reflected power. The experimental device is detailed in Fig. 1.
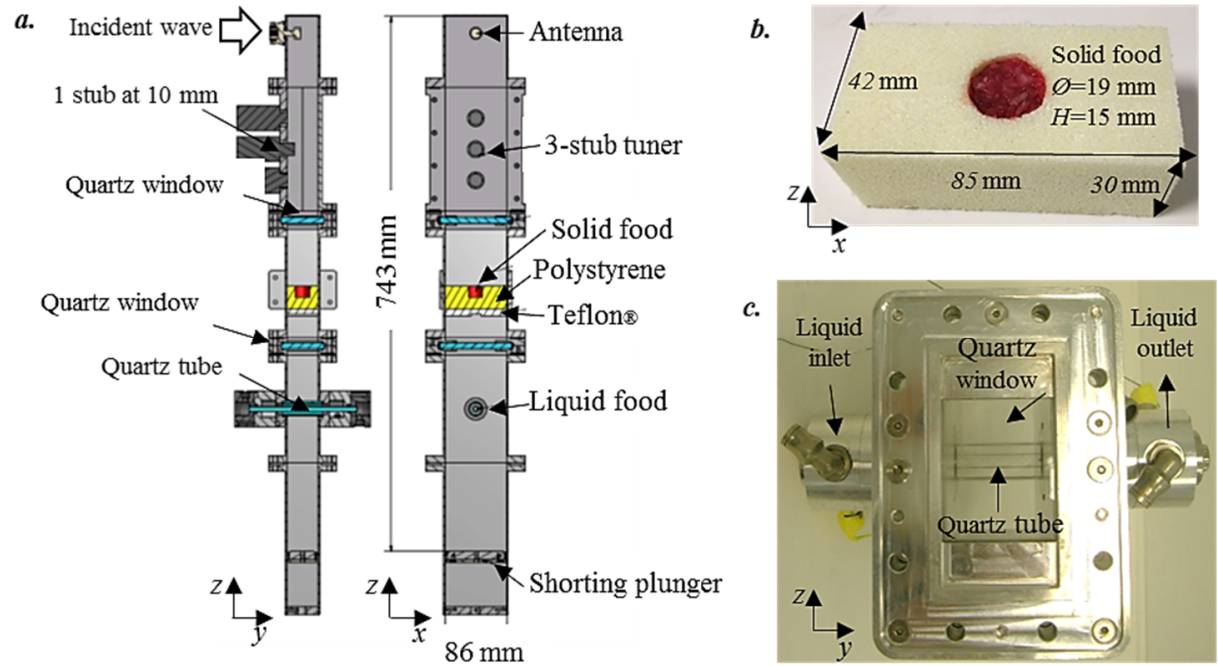

Fig. 1 (a) Schema of the microwave experimental apparatus (b) solid food product within a polystyrene block (c) quartz window and liquid food within a quartz tube.

The microwave applicator consists in $5 \mathrm{~g}$ of solid food sample and a continuous flow of liquid food within a quartz tube $(6 \mathrm{~mm}$ inlet diameter, $2 \mathrm{~mm}$ thick, liquid flowrate $300 \mathrm{~L} / \mathrm{h})$. The quartz tube crosses the small side of the rectangular waveguide (Fig 1c).

To improve thermal insulation of the solid food sample, the product was inserted on a cylindrical orifice (19 $\mathrm{mm}$ diameter, $15 \mathrm{~mm}$ length) in the centre of the polystyrene block (85 mm x $42 \mathrm{~mm} \times 30 \mathrm{~mm}$ ) filling the cross-section of the waveguide (Fig 1b). In addition, a Teflon ${ }^{\circledR}$ support plate is inserted at the bottom of the polystyrene block. The temperature is measured at two locations within the sample with sheathed type-K thermocouples. Temperatures are recorded by a data logger (OM-CP QuadTemp 2000, OMEGA, France). Impedance matching conditions are reached by setting one screw displacement at $10 \mathrm{~mm}$ for a full cavity length of $743 \mathrm{~mm}$ (Fig 1a).

\section{Model design}

The real geometry of the experimental device is considered for the $3 \mathrm{D}$ numerical modelling. The model is solved using the $\mathrm{COMSOL}^{\circledR}$ Multiphysics 5.3a software which uses finite element method for discretization of equations. Mesh refinements is performed at narrow regions of the geometry to insure a sufficient numerical precision. The selected mesh size consisted in a total of 1.000 .760 tetrahedral elements to obtain the mesh independence of numerical results (Fig. 2). 


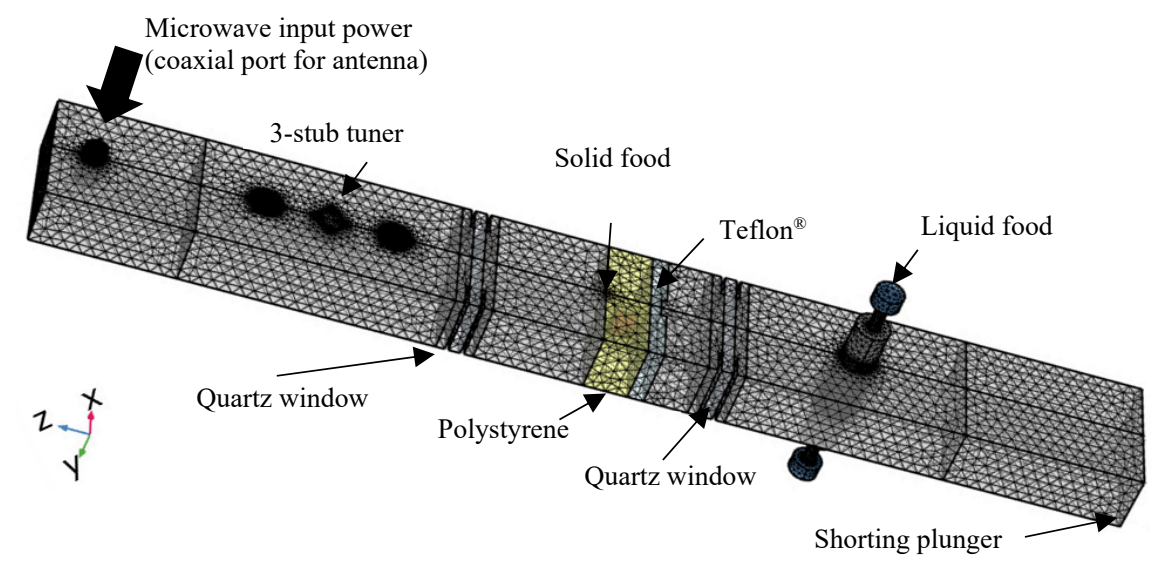

Fig. 2 3D numerical modelling of the full microwave rectangular waveguide

The model is aimed at predicting both electric field distributions in the TE10 mode rectangular waveguide and temperature profiles in a solid food product. To achieve this objective, the following assumptions are introduced:

(i) The initial temperature of the food sample is uniform;

(ii) The processed solid food sample is considered as a homogeneous isotropic cylinder with constant thermophysical properties;

(iii) The dielectric properties of the materials are constant;

(iv) The shrinkage and mass transfer of the solid food sample are negligible;

(v) The upper surface of the solid sample is surrounded by air (zero dielectric losses).

The Maxwell's equations are solved numerically to predict the electromagnetic field distribution into the rectangular waveguide. Input microwave power is supplied at the antenna via a coaxial port $(20 \mathrm{~W})$. External waveguide walls are considered as perfect electric conductors to propagate a standing wave within the waveguide.

The general equation for electric field propagation within a material is defined as follows ${ }^{6}$ : $\nabla^{2} E+\omega^{2} \mu \varepsilon\left(1-j \frac{\sigma}{\omega \varepsilon}\right) E=0 \quad$ with $\varepsilon=\varepsilon_{0} \varepsilon_{r}^{\prime}-j \varepsilon_{0} \varepsilon^{\prime \prime}{ }_{r}$ and $\mu=\mu_{0} \mu_{r}$

Where $\square{ }^{\prime} r$ is the relative dielectric constant of the material, $\square{ }^{\prime}{ }_{r}$ the loss factor, $\square 0$ the permittivity of a vacuum, $\mu_{r}$ the relative permeability and $\mu_{0}$ the permeability of a vacuum. The dielectric properties of the various materials are depicted in Table 1.

The internal heat generation term $\left(Q_{a b s}\right)$ quantifies the amount of power dissipated into the material by dielectric losses. The heat generation term is computed from the local electric field strength at any location of the sample domain from the resolution of the Maxwell's equations.

$$
Q_{a b s}=\frac{1}{2} \omega \varepsilon_{0} \varepsilon_{r}^{\prime \prime}\left|E_{\text {local }}\right|^{2}
$$

For a solid food product and a heat generation due to microwaves, the classical heat transfer equation without any convection term is reduced to:

$$
\rho C_{p} \frac{\partial T}{\partial t}=\operatorname{div}(k \nabla T)+Q_{a b s}
$$


This equation depends on the thermophysical properties of the food sample. The specific heat $\left(3.69 \mathrm{~kJ} \mathrm{~kg}^{-1} \mathrm{~K}^{-1}\right)$ and density $\left(1130 \mathrm{~kg} / \mathrm{m}^{3}\right)$ were determined experimentally and thermal conductivity $\left(0.41 \mathrm{~W} \mathrm{~m}^{-10} \mathrm{C}^{-1}\right)$ was taken from literature for meat sample ${ }^{7}$. The upper surface of the food product is submitted to an external convective heat flux expressed as a boundary condition:

$$
k . \nabla T=h_{\text {air }} \cdot\left(T-T_{\infty}\right), \quad \forall(x, y, z), \forall t>0, \quad T_{\infty}=20^{\circ} \mathrm{C}
$$

The convective heat transfer coefficient was estimated experimentally $\left(6.5 \mathrm{~W} / \mathrm{m}^{2} / \mathrm{K}\right)$ by the lumped capacitance method.

Table 1. Relative dielectric constant $\left(\square r^{\prime}\right)$ and dielectric loss factor ( $\square r$ '”) obtained experimentally by resonant cavity technique (polystyrene, Teflon ${ }^{\circledR}$ ) and open-ended coaxial probe for solid and liquid food samples), for each material at $20^{\circ} \mathrm{C}$. Dielectric properties for quartz were taken from literature ${ }^{8}$.

\begin{tabular}{lcccccc}
\hline $\begin{array}{l}\text { Dielectric } \\
\text { properties }\end{array}$ & Air & Solid food & Polystyrene & Quartz & Teflon ${ }^{\circledR}$ & Liquid food \\
\hline$\square{ }^{\prime}{ }_{r}$ & 1 & 40.3 & 1 & 4.2 & 1.7 & 77.6 \\
$\square{ }^{r}$ & 0 & 15.1 & $2.510^{-5}$ & 0 & $3.410^{-4}$ & 12.3 \\
\hline
\end{tabular}

The model is solved numerically as a two-way coupling: the electromagnetic field distribution is firstly computed within the whole geometry following a harmonic propagation at $2.45 \mathrm{GHz}$. Then, the transient heat equation is considered to compute the temperature distribution within the solid food sample by considering the microwave absorbed power.

The model is solved with a Dell ${ }^{\circledR}$ Precision ${ }^{\mathrm{TM}}$ Workstation computer, equipped with $2 \times$ Intel $^{\circledR}$ Xeon processors ( 8 cores), at $2.5 \mathrm{GHz}$, with $256 \mathrm{~GB}$ of RAM, running on Windows ${ }^{\circledR} 8$ Professional, 64 bits.

Four scenarios are considered to investigate both the dielectric properties influence of materials and the cavity design on the electric field distribution.

Scenario 1: Waveguide transition with an antenna (coaxial port), solid and liquid food, and shorting plunger. Dielectric properties of other materials are assumed to be close to air; Scenario 2: Scenario 1 with the addition of one screw tuner with $10 \mathrm{~mm}$ displacement; Scenario 3: Scenario 2 by considering the dielectric properties of the quartz material; Scenario 4: Scenario 3 by considering dielectric properties of polystyrene and Teflon ${ }^{\circledR}$.

Following the four previous scenarios, both electric field distribution and temperature map within the solid food sample are investigated. To take into account the uncertainty of the temperature probe locations in the $(\mathrm{x}, \mathrm{z})$ plane, an experimental position error of $\pm 1 \mathrm{~mm}$ is considered at the two probe locations.

\section{Results and discussion}

For each considered scenario, a power balance is performed to compute the microwave forward, reflected and absorbed power within the dielectric samples.

For the first scenario without the screw tuner modelling, the cavity is not adapted $(64 \%$ of the forward power is reflected). By taking into account the real design of the cavity 
(scenario 4), the reflection coefficient is reduced to a minimum value of $9 \%$ (ratio of reflected power versus microwave input power). This value is found in agreement with experimental data.

For each scenario, a power balance is realized and the values are presented in Table 2.

Table 2. Power balance within the system for a microwave input power of $20 \mathrm{~W}$.

\begin{tabular}{cccc}
\hline Scenario & $\begin{array}{c}\text { Reflected } \\
\text { power }\end{array}$ & $\begin{array}{c}\text { Absorbed power within } \\
\text { the solid food sample }\end{array}$ & $\begin{array}{c}\text { Absorbed power within } \\
\text { the liquid food sample }\end{array}$ \\
\hline 1 & 13 & 3.6 & 3.4 \\
2 & 10 & 5 & 5 \\
3 & 6 & 2.4 & 11.5 \\
4 & 1.8 & 4 & 14.1 \\
\hline
\end{tabular}

Results indicates that impedance matching increases the energy efficiency of the system by reducing the reflected power $(13 \mathrm{~W}$ vs. $1.8 \mathrm{~W})$ and by increasing the absorbed power within the liquid sample $(3.4 \mathrm{~W}$ vs. $14.1 \mathrm{~W})$. However, the solid food sample absorbs different microwave power depending on the design of the equipment. The modelling of the screw tuner clearly influences the predicted electric field distribution. The quartz, polystyrene and Teflon $^{\circledR}$ materials are known as microwave-transparent materials due to their very low dielectric loss factor. Nevertheless, these materials have a non-negligible dielectric constant value (Table 2). Thus, they are able to polarize the electric field resulting in modification of the electric field distribution within the waveguide, as illustrated in Fig. 3.

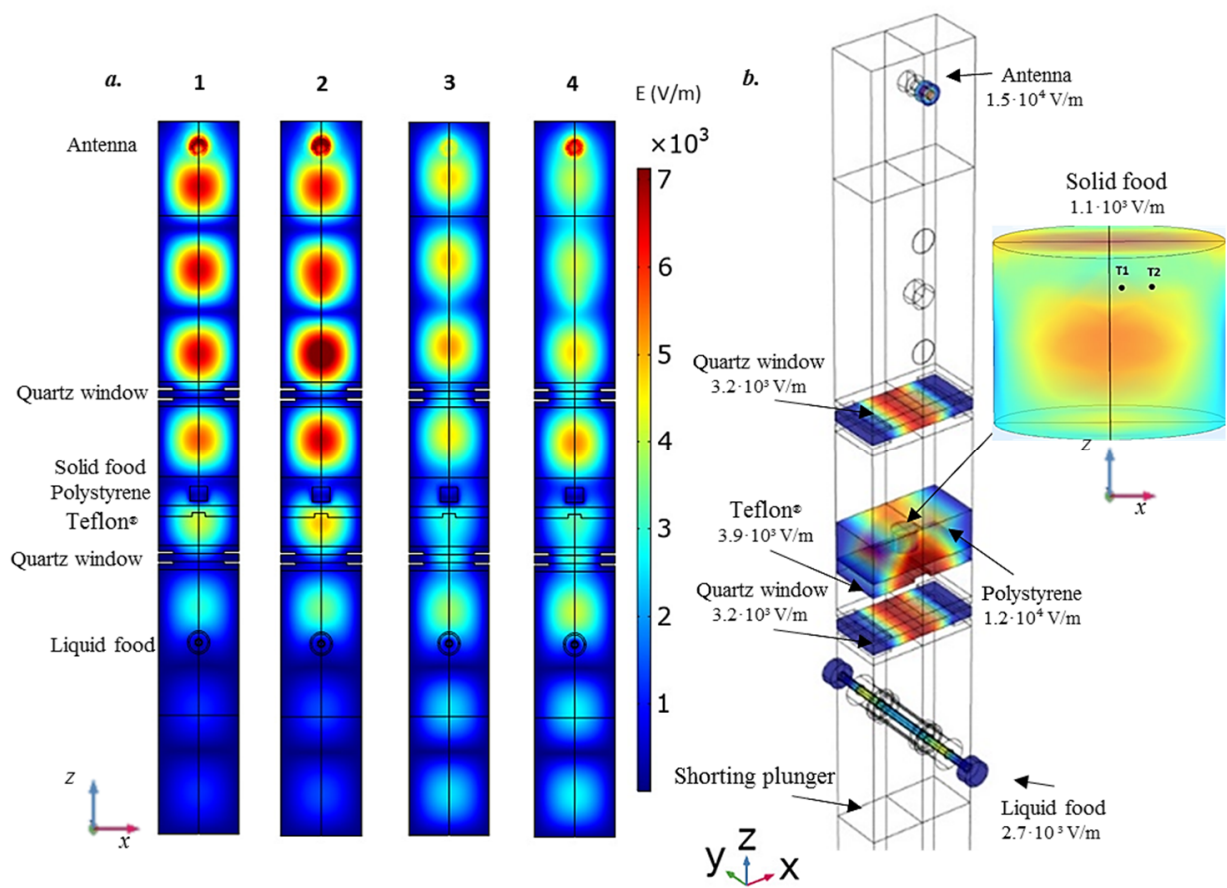

Fig. 3 (a) Electric field distribution within the rectangular waveguide (b) Electric field patterns within dielectric materials with maximum electric field strength values. 
Under our operating conditions, the incident wave is superimposed with the reflected wave which leads to a standing wave pattern. For each scenario, the electric field distribution is displayed on a line located at the center of the rectangular waveguide and along the microwave propagation direction (from the antenna to the short plunger location).
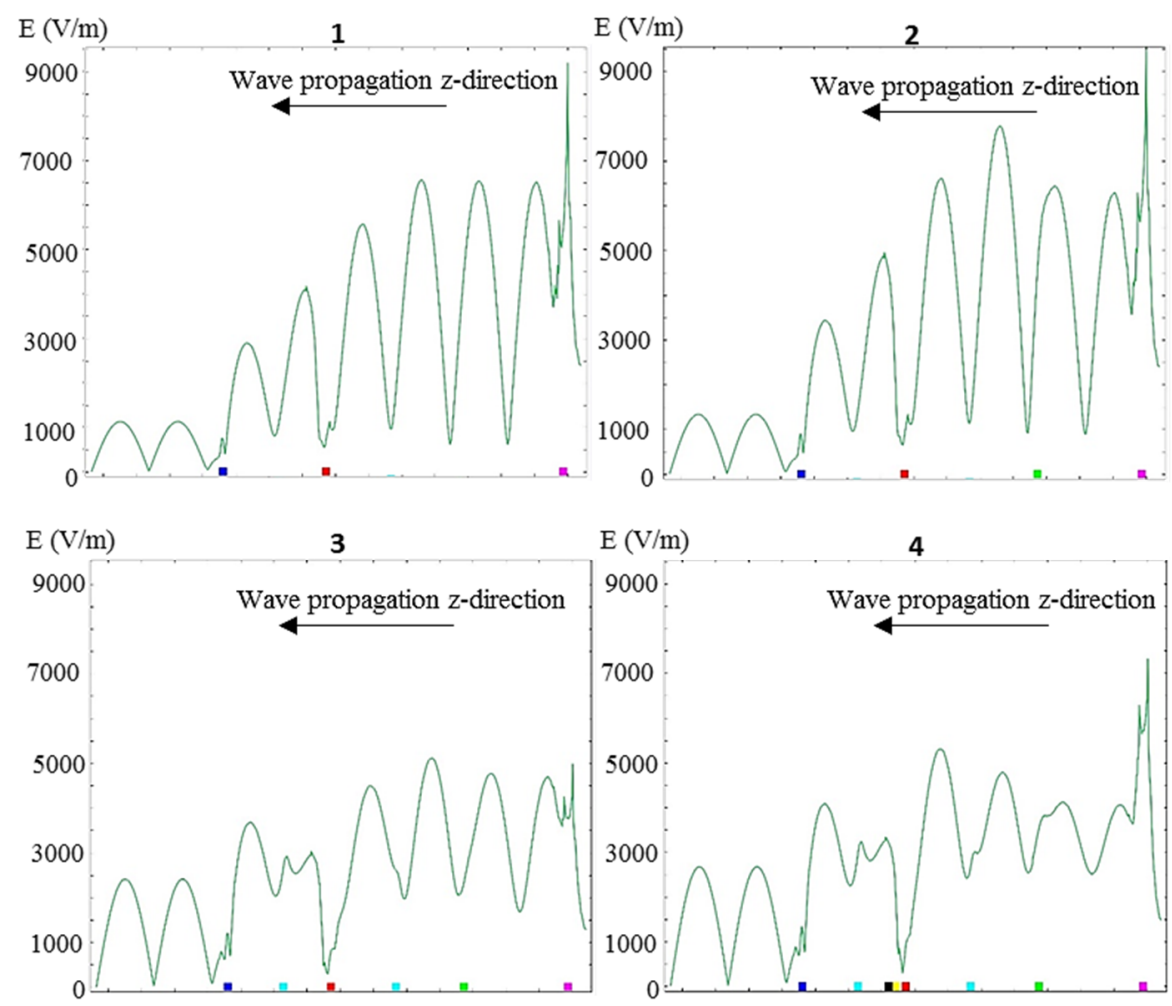

Subtitle

$\square$ Liquid food $\square$ Teflon॰ Polystyrene $\square$ Solid food $\square$ Quartz window $\square$ Stub $\square$ Antenna

Fig. 4 Standing wave patterns for each scenario

Fig. 4 clearly illustrates the influence of the antenna, screw tuner, quartz, polystyrene and Teflon ${ }^{\circledR}$ materials on the electric field distribution. Due to its small size, the solid food sample is always located at a region where the electric field strength is minimal.

Temperature distribution with the solid food matrix is then evaluated by considering a microwave heating process at $20 \mathrm{~W}$ during 131 seconds. Experimental temperature measurements at two locations are compared with simulated temperature according to the four previously detailed scenarios (Fig. 5). The numerical simulation clearly indicates a heterogeneous temperature distribution within the solid food sample, with the presence of hot and cold spots for all four scenarios. For each case, a hot spot is found in the upper center and a cold spot in the lower side of the sample, as shown in the Fig. 5. In the second scenario (Fig. 5-2), the solid food sample absorbs more microwave power (5W, Table 2), resulting in a large temperature gradient within the sample volume. The temperature in the hot spot reaches $82^{\circ} \mathrm{C}$ and in the cold spot only $29^{\circ} \mathrm{C}$. 

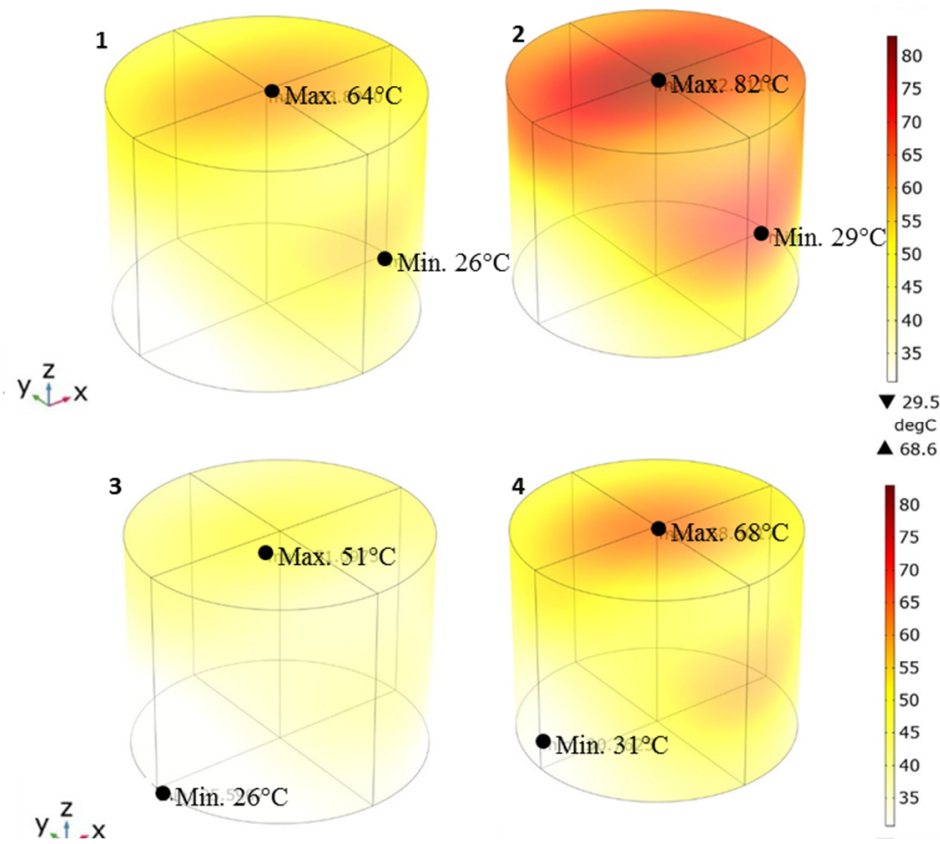

Fig. 5 3D temperature distribution in the solid food sample for each scenario.
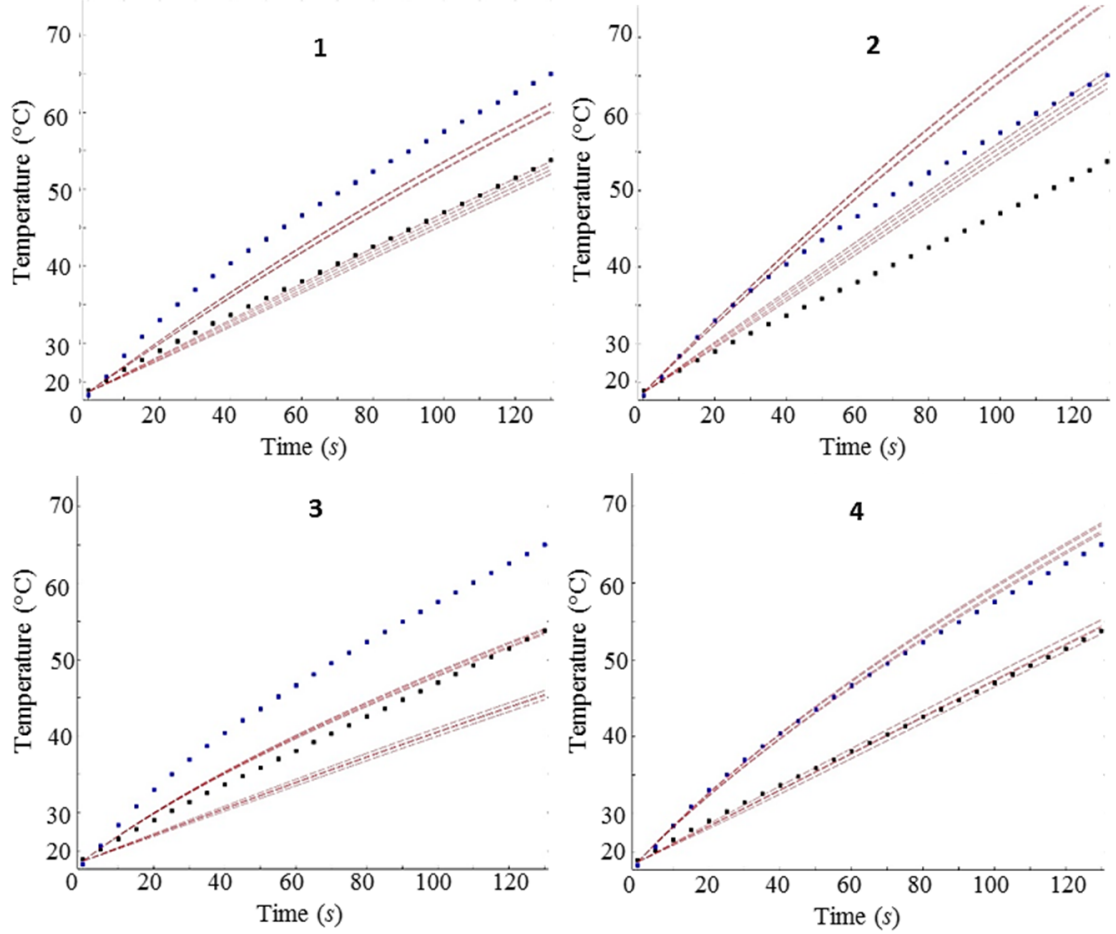

Fig. 6 Experimental vs. predicted temperatures. Red broken lines represent the simulated temperatures at two locations with probe position uncertainties and blue (T1) and black (T2) diamonds represent the experimental data. 
For scenario 1 and 3, the temperature measured experimentally is always higher than the simulated ones. For scenario 4, the simulation results show a good correlation with the experimental data (Fig. 6-4) when all the geometrical details of the rectangular waveguide are taken into account for the 3D numerical modelling.

\section{Conclusion}

In this study, a 3D numerical modelling of a microwave heating process in a single-mode rectangular waveguide is performed. The results are analyzed following different scenarios to illustrate the influence of the cavity design and dielectric properties of tested materials on both the absorbed power and temperature distribution within food samples. In the case of a standing wave pattern inside a microwave cavity, this study demonstrates the importance to consider all the geometrical details of the cavity and the accurate dielectric properties characterization of the materials to predict the electric field distribution. The proposed methodology could now be extended to design a single mode microwave applicator with optimized geometry to reduce temperature gradients inside the food matrix using external heat sources, such as infrared or hot air.

\section{Acknowledgements}

The authors are grateful to the National Council for Scientific and Technological Development (CNPq, Brazil) for Clarissa Detomi Albuquerque's PhD scholarship and financial support (process number 232767/2014-9). The authors would also like to thank O. Tantot (XLIM, UMR CNRS 7252) for the dielectric characterization of materials.

\section{References}

[1] Ahmed, J. \& Ramaswamy, H. Microwave Pasteurization and Sterilization of Foods. in Handbook of Food Preservation (ed. Taylor \& Francis Group, L.) 691711 (2007). doi:10.1201/9781420017373.ch28

[2] Kostas, E. T., Beneroso, D. \& Robinson, J. P. The application of microwave heating in bioenergy: A review on the microwave pre-treatment and upgrading technologies for biomass. Renew. Sustain. Energy Rev. 77, 12-27 (2017).

[3] Motasemi, F. \& Afzal, M. T. A review on the microwave-assisted pyrolysis technique. Renew. Sustain. Energy Rev. 28, 317-330 (2013).

[4] Pitchai, K., Birla, S. L., Subbiah, J., Jones, D. \& Thippareddi, H. Coupled electromagnetic and heat transfer model for microwave heating in domestic ovens. J. Food Eng. 112, 100-111 (2012).

[5] Zhang, H. \& Datta, A. K. Coupled electromagnetic and thermal modeling of microwave oven heating of foods. J. Microw. Power Electromagn. Energy 35., $71-85$ (2000).

[6] Curet, S., Rouaud, O. \& Boillereaux, L. Effect of Sample Size on Microwave Power Absorption Within Dielectric Materials: 2D Numerical Results vs. ClosedForm Expressions. AIChE J. 55, 1569-1583 (2009).

[7] Pan, Z. \& Paul Singh, R. Physical and thermal properties of ground beef during cooking. LWT - Food Sci. Technol. 34, 437-444 (2001).

[8] Salema, A. A. \& Afzal, M. T. Numerical simulation of heating behaviour in biomass bed and pellets under multimode microwave system. Int. J. Therm. Sci. 91, 12-24 (2015). 
17th International Conference on Microwave and High Frequency Heating

\section{EM modelling and numerical techniques}
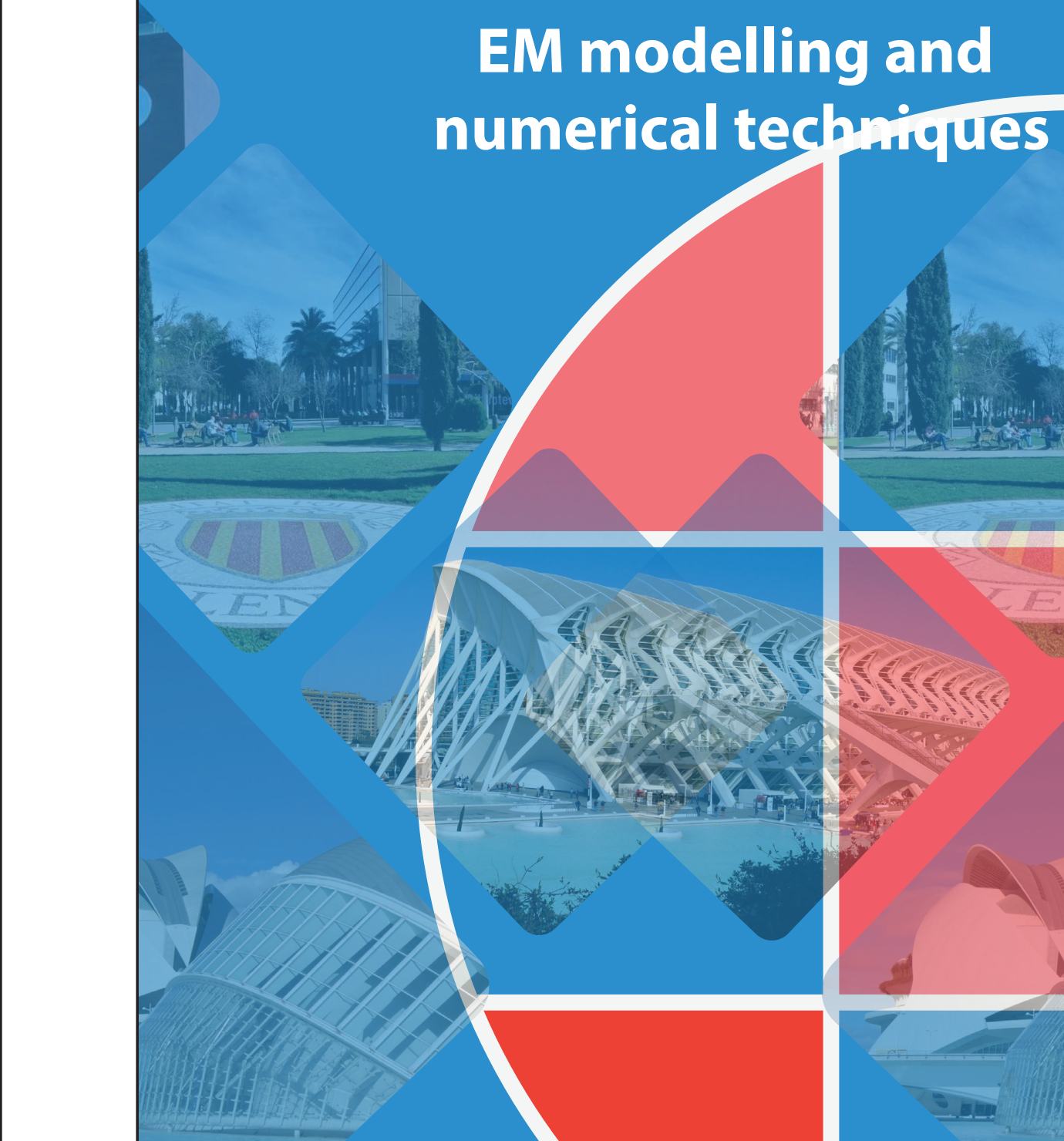


\title{
NUMERICAL INVESTIGATION ON THE EFFECTS OF SINGLE-MODE MICROWAVE TREATMENT ON ROCK BREAKAGE SYSTEM
}

\author{
K. Teimoori ${ }^{1}$, F. Hassani ${ }^{1}$, A.P. Sasmito ${ }^{1}$, and S.A. Ghoreishi-Madiseh ${ }^{2}$ \\ ${ }^{1}$ Department of Mining and Materials Engineering, McGill University, 3450 University, \\ Frank Dawson Adams Bldg., Montreal, QC H3A OE8, Canada \\ ${ }^{2}$ Norman B Keevil Institute of Mining Engineering, University of British Colombia, \\ Vancouver, BC, V6T 1Z4, Canada \\ khashayar.teimoori@mail.mcgill.ca \\ ferri.hassani@mcgill.ca \\ agus.sasmito@mcgill.ca \\ ali.madiseh@ubc.ca
}

Keywords: microwave heating, rock preconditioning, single-mode microwave treatment, Maxwell's theory

\begin{abstract}
In this study, a rock model which consists of a conceptual block (host rock and ore sample) is numerically modeled by using the finite element method. The rock model is subjected to several single-mode microwave treatments with different power levels, distances from the antenna, and exposure times in order to calculate and compare the corresponding effects including temperature distribution and mechanical stress/damage profiles. The main objective of the present study is to analyze the distribution of temperature and mechanical stress at the boundary of two different attached rocks when exposed to microwaves. This enables comparing the intensity of the distribution with respect to the applied microwave input operating parameters and, consequently, understanding rock preconditioning. The results of the present study verify that an increase in temperature by microwave treatment facilitates the rock weakening process. Also, a more efficient selection of the distance from the antenna and the power level can maximize the overall impact of the microwave treatment on rock preconditioning which ultimately helps with the rock breakage mechanism.
\end{abstract}

\section{Introduction}

The application of microwave heating has been investigated to precondition rocks prior to breakage by mechanical means such as a tunneling machine. The concept is that the microwave irradiation will heat the rock. The rock consists of different minerals with different thermal expansions at different rates, causing microcracks in the boundary of the minerals upon heating. Such microcracks consequently reduce the rock strength. This reduction will contribute to a higher penetration rate of the cutting machine and a reduction of wear on the cutting discs.

The technology of microwave-induced rock breakage has been considered a potential method for rock fracturing. The idea of applying microwaves to rocks began with a patented study conducted by Lindroth et al. in 1991. This process is referred to as "rock preconditioning" [1-3]. Fig. 1 illustrates the idea of microwave-assisted rock breakage as a potential alternative to conventional methods.

Understanding the complex process of rock breakage under microwave treatment requires a knowledge of the fundamental principles of electromagnetic waves, heat transfer theory, thermo-mechanical stress analysis, rock mechanics and fracture mechanics. Coupling these 
physical phenomena enables the study of all factors influencing the microwave-assisted rock breakage operation. Each of these factors may substantially change the process and its corresponding results.

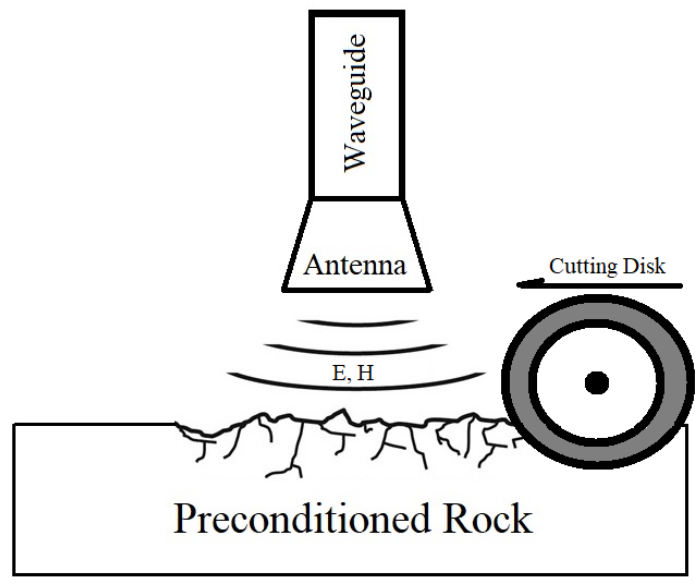

Fig. 1. The concept of microwave-assisted rock breakage

Over the past twenty years, several studies have highlighted the effects of microwave treatment on different rocks and minerals by performing both experimental tests and numerical simulations. Although several numerical studies have been done in recent years to investigate the effects of applied microwave irradiation on different rocks and minerals, most of the experiments were performed only to identify the potentiality and importance of microwave energy as an application in the rock breakage system. Also, none of the numerical simulations have focused on the effects of applying different operating parameters of microwave energy on thermal and mechanical responses of a rock model made up of differently attached rock samples under microwave exposure. The nature of the grain boundaries between the rock samples is not well understood, but it has been suggested that it is an area of disorder between two ordered species which can be considered as a potential area of weakness [4].

Since the rock breakage process by microwave energy depends mainly on different microwave operating parameters, a numerical modeling approach can easily evaluate the corresponding effects of the applied microwave, i.e. temperature gradients and initiation of mechanical stresses. Evaluation of these effects enables understanding of (1) how microwave energy affects minerals, and (2) what the effects of different microwave operating parameters on rock preconditioning are. Therefore, following the recent development of a fully coupled FE modeling approach by K. Teimoori et. al, [5], the current study uses the validated FE modeling approach to simulate a rock model consisting of a conceptual block of R1 host rock and R2 ore sample under microwave treatments with different operating parameters. The numerical models of the present study investigate microwave heating, thermal conduction, and thermally induced mechanical stresses on the boundaries of the two attached conceptual rock samples. Finally, the results of temperature and mechanical stress distribution are analyzed and compared with respect to different microwave input operating parameters.

The current study is part of an ongoing project that began in 2005 in the Geomechanics Laboratory at McGill University. The project's goal was to evaluate the influence of microwave irradiation with different operating parameters on different hard rocks. Ultimately, the objective of the project is to develop a new rock fracturing technique referred 
to as "non-explosive continuous rock breakage operation", where microwave energy is radiated from a pyramidal open-ended horn antenna onto the rock surface.

\section{Methodology}

Single-mode microwave cavities consist of a metallic enclosure and a waveguide horn. These metallic parts were designed so that the electromagnetic field polarization from a microwave signal will undergo several reflections. The superposition of the propagated waves in different directions gives rise to a standing wave pattern [6]. This pattern is very well defined inside the cavity's space. Consequently, the precise knowledge of different configurations of the electromagnetic waves enables the dielectric material (rock model) to be placed in the position at which maximum heating rate and subsequent maximum mechanical stress can be achieved. With that information in mind, a single-mode microwave cavity was chosen for numerical simulations in the present study. Therefore, the rock models of this study were exposed to microwaves with a frequency of $2.45 \mathrm{GHz}$ at $3 \mathrm{KW}$ and $9 \mathrm{KW}$ power levels, and at 30 seconds and 60 seconds exposure times when the distance from the antenna changes from $5 \mathrm{~cm}$ to $10 \mathrm{~cm}$. After simulations, the results of temperature and mechanical stress distribution were obtained in the R1 and R2 samples.

\subsection{Model development}

Fig. 2 presents the modeled single-mode microwave cavity and the rock model. The R1 rock host is rectangular with side-length of $40 \times 40 \mathrm{~cm}$, and the R2 ore sample has side-length of $2 \times 2 \mathrm{~cm}$ and height of $37 \mathrm{~cm}$. The rock models are placed on samples of glass insulator with side-lengths of $30 \times 30 \mathrm{~cm}$ and different heights (depending upon distances from the antenna). The rock models were used to conduct surface treatments in a single-mode cavity and with unidirectional loading conditions.

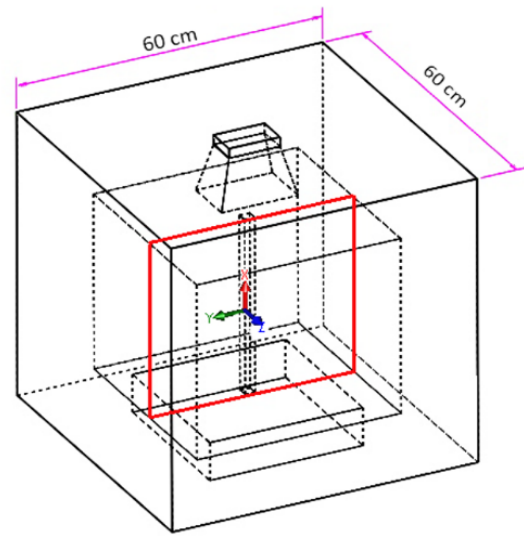

(a)

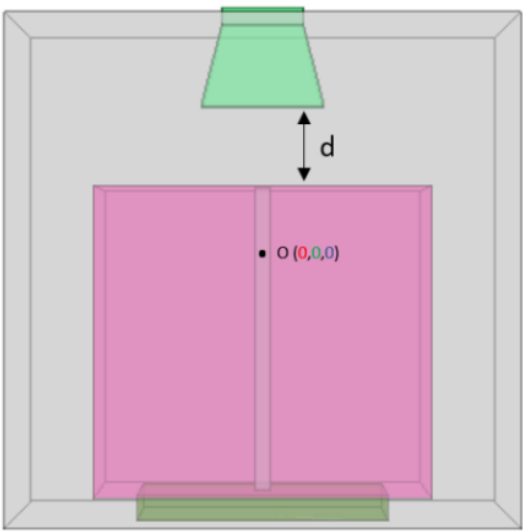

(b)

Fig. 2. The modelled single-mode microwave cavity including the R1 host rock and R2 ore sample, which is referred to as "rock model". (a) The 2D cut plane (shown by red) at the intersection of R1 and R2, and (b) the microwave equipment set-up with the rock model placed inside the microwave cavity and below the horn; the distance from the waveguide horn (antenna), $\mathrm{d}(\mathrm{cm})$, is shown by a black arrow. Here, the host rock is shown in pink, the ore sample in yellow, the glass insulator - beneath the rock model - in dark green, the waveguide horn in cyan, and the cavity walls in gray. 
As shown in Fig. 2, two different conceptual rock samples, R1 and R2, were assigned as the rock model inside the single-mode microwave cavity. The inclusion of these two different materials in the rock model was to investigate the effects of microwave treatments with different operating parameters on the boundaries between an ore sample and its surrounding rock host. To demonstrate the results of the simulations and to compare the obtained data with respect to different power levels, exposure times and distances from the antenna, a $2 \mathrm{D}$ cut plane at the intersection/boundary between the R1 and R2 samples was created for each simulation. This 2D cut plane is shown in Fig. 2a by red. By plotting the gradients of temperature and mechanical stress distribution on the 2D cut planes within the rock models, it will be possible to analyze and compare data with respect to the input microwave powers, distances from the antenna, and exposure times. In fact, the numerical models of two different materials presented in this paper can be considered an initial step to understand the mechanisms involved in such a complex system of rock-ore composition with three multiphysics interactions, and with respect to different microwave parameters.

\subsubsection{Governing equations}

Rocks are non-conductive materials usually made up of bonds of charged particles. The charged particles can absorb electricity when subjected to externally applied electromagnetic waves. In this case, fundamental electromagnetic theories apply and the solution to Maxwell's equations is required for this case study. Therefore, the problem of electromagnetic theory on a macroscopic level is that of solving Maxwell's equations subject to certain boundary conditions.

The amount of heat and mechanical stress produced in a rock sample when subjected to microwave treatment depends on the following parameters: power level, exposure time, and distance from the antenna (for single-mode microwave cavity type). The development of a numerical model to describe the electromagnetic-thermal-mechanical (ETM) multiphysics interaction in rocks requires careful consideration of the heating mechanism when microwave irradiation is applied. Therefore, it is important to express all three multiphysics mathematically.

Equations 1 to 3 were solved for electromagnetic, thermal and mechanical multiphysics to arrive at the final distribution of electric and magnetic fields, temperature, and maximum principal stress in the rock model as follows:

Electromagnetic waves $(\mathbf{E})$ :

$\nabla \times \mu_{r}^{-1}(\nabla \times \mathbf{E})-k_{0}^{2}\left(\epsilon_{r}-\frac{j \sigma}{\omega \epsilon_{0}}\right) \mathbf{E}=0, \quad k_{0}=\omega \sqrt{\varepsilon_{0} \mu_{0}}$

where,

E: is the electric field $(\mathrm{V} / \mathrm{m})$

$k_{0}$ : is the wave number $(\mathrm{rad} / \mathrm{m})$

$\mu_{r}:$ is the relative permeability

$\epsilon_{r}:$ is the relative permittivity

$\sigma:$ is the electrical conductivity

$\omega:$ is the angular frequency

$\varepsilon_{0} \& \mu_{0}$ : are the electric permittivity and magnetic permeability of free vacuum 
Heat transfer $(\mathbf{T})$ :

$\rho C_{P} \frac{\partial T}{\partial t}-\nabla \cdot(k \nabla \mathbf{T})=Q$

where,

$\rho:$ is the rock density $\left(\mathrm{kg} / \mathrm{m}^{3}\right)$

$C_{p}$ : is the heat capacity at constant pressure $\left(\mathrm{J} / \mathrm{kg}^{\circ} \mathrm{C}\right)$

$k$ : is the thermal conductivity $\left(\mathrm{W} / \mathrm{m}^{\circ} \mathrm{C}\right)$

$Q:$ is the heat source $\left(\mathrm{W} / \mathrm{m}^{3}\right)$

Solid mechanics (u):

$\rho \frac{\partial^{2} \mathbf{u}}{\partial t^{2}}=\mathbf{f}_{\mathrm{V}}-\nabla \cdot s$

where,

$\mathbf{f}_{\mathrm{V}}$ : is the deformation force $(N)$

$s:$ is the Cauchy stress $\left(N / m^{2}\right)$

\subsection{Numerics}

The finite element (FE) method is used to calculate the transient temperature, electromagnetic fields, and the mechanical stress/damage profiles in the rock models. The final mode, given by equations (1) to (3), together with appropriate boundary conditions, constitutive relations, and input material properties of the rock samples given in Table 1 constitute a highly coupled nonlinear differential algebraic system for the dependent variables $\mathrm{E}, \mathrm{T}$, and $\mathrm{u}$.

\begin{tabular}{ccc}
\hline Property name & $\mathrm{R} 2$ & $\mathrm{R} 1$ \\
\hline Thermal conductivity $(\mathrm{W} / \mathrm{m} \cdot \mathrm{K})$ & $37.9[4]$ & $1.55[5]$ \\
Heat capacity at constant pressure $(\mathrm{J} /(\mathrm{kg} \cdot \mathrm{K}))$ & $517[4]$ & $900[5]$ \\
Density $\left(\mathrm{kg} / \mathrm{m}^{3}\right)$ & $5018[4]$ & $2870[5]$ \\
Coefficient of thermal expansion $(1 / \mathrm{K})$ & $2.95 \mathrm{e}-6[7]$ & $5.4 \mathrm{e}-6[8]$ \\
Poisson's ratio $(1)$ & $0.16[4]$ & $0.28[5]$ \\
Young's modulus $(\mathrm{GPa})$ & $147[9]$ & $73[5]$ \\
Electrical conductivity $(\mathrm{S} / \mathrm{m})$ & $0.01[10]$ & $0.00005[11]$ \\
Relative permeability $(1)$ & $0.9-0.4 \mathrm{j}[12]$ & $1[5]$ \\
Relative permittivity $(1)$ & $7.6-1.3528 \mathrm{j}[12]$ & $7.77-0.85 \mathrm{j}[5]$ \\
\hline
\end{tabular}

Table 1. Input data of electrical, thermal, and mechanical properties of R1 and R2 samples for numerical models

The commercial FE solver, COMSOL multiphysics, was chosen to implement the derived models because of its versatility in handling general coupled nonlinear partial differential equations for fully coupled ETM multiphysics interaction. For time discretization, the backward differentiation formula (BDF) solver was employed as an implicit solver that uses backward differentiation formulas with second order of accuracy. The MUltifrontal Massively Parallel Sparse (MUMPS) direct solver was also employed as a sparse solver that is optimized for solving the system of equations in parallel, as this solver offers additional options for customization of parallel computing. A mesh-independent solution was ensured by comparing with results obtained using a coarse mesh consisting of 7,278 boundary elements, followed by several mesh adaptations until the difference in computed solid material's temperature was below $2 \%$, with a final mesh size of 7,492 . The geometries for all numerical models were resolved for 1,438,592 number of degrees of freedom (DOFs), plus 12,158 internal DOFs, to ensure mesh independent solutions. The computations carried out on a $3.5 \mathrm{GHz}$ PC with $128 \mathrm{~GB}$ RAM, required around $\sim 6047 \mathrm{~s}$ (1 hour, 40min, $47 \mathrm{~s}$ ) for the simulations. 


\section{Results and Discussion}

For better visual understanding of the numerical models' results, a 3D distribution of the electric field, temperature, and maximum principal stress is plotted when microwave irradiation of $3 \mathrm{KW}$ power, 30 seconds exposure, and $5 \mathrm{~cm}$ distance from the antenna is applied to a rock model (see Fig. 3).

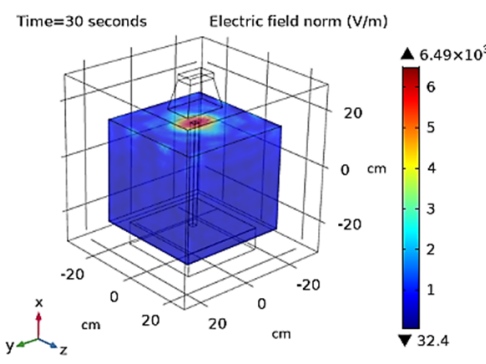

(a)

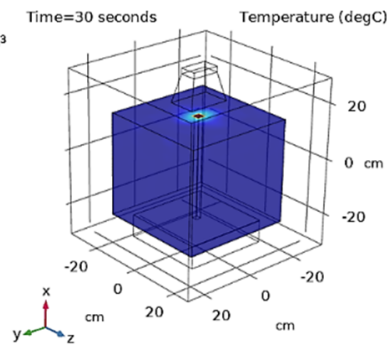

(b)

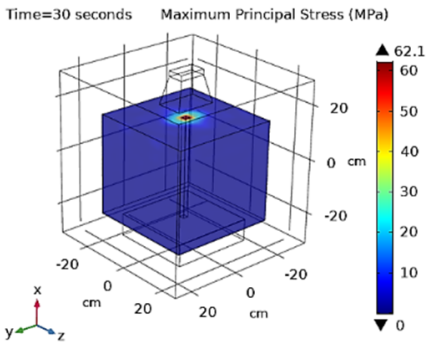

(c)

Fig. 3. Three-dimensional distribution of (a) electric field $(\mathrm{V} / \mathrm{m}),(\mathrm{b})$ temperature $\left({ }^{\circ} \mathrm{C}\right)$, and (c) maximum principal stress $(\mathrm{MPa})$ in a rock model when microwave irradiation of $3 \mathrm{KW}$ is applied at $5 \mathrm{~cm}$ distance from the antenna for 30 seconds exposure

As can be seen in Fig. 3, the distribution of temperature and maximum principal stress gradients differs between the R1 and R2 samples. To illustrate this phenomenon, a 2D cut plane was made at the coordinate of $Z=1 \mathrm{~cm}$, where the distributions occur on the boundaries between the two rock samples. It should be noted that rocks are usually anisotropic and consist of different minerals; therefore, the distribution of temperature and mechanical stresses on the boundaries of the two different rock samples varies depending upon their material properties and the amount of applied microwave irradiation. These results are shown in Figs. 4 and 5.

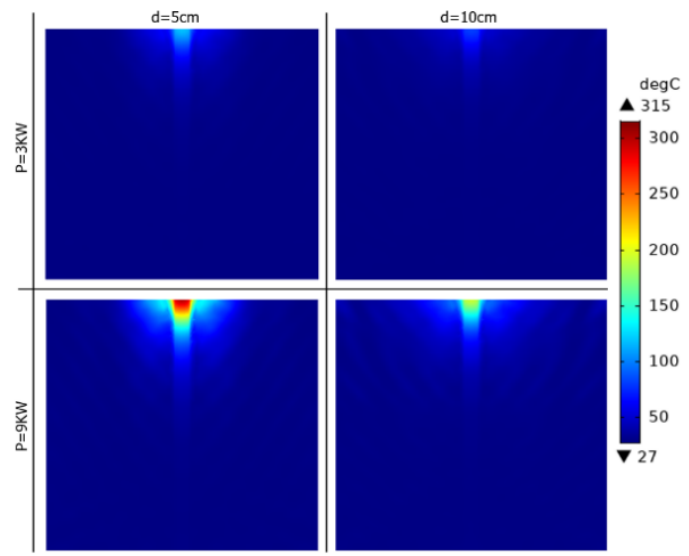

(a) $\mathrm{t}=30$ seconds

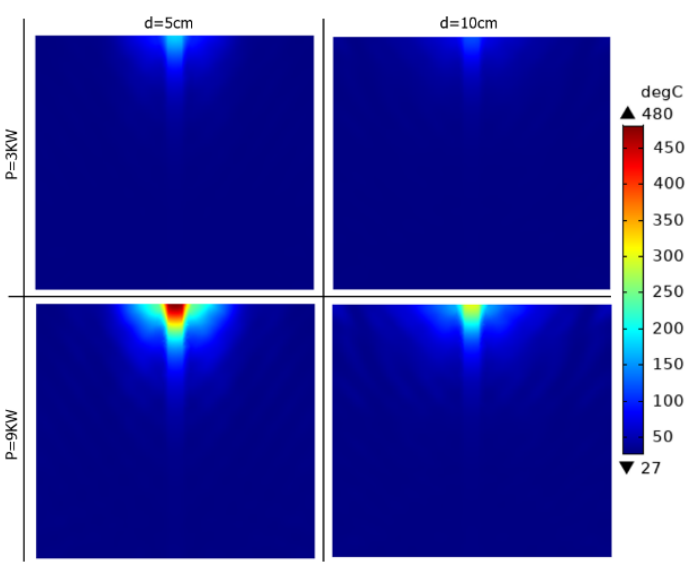

(b) $\mathrm{t}=60$ seconds

Fig. 4. Two-dimensional temperature distribution for power levels of $3 \mathrm{KW}$ (top) and $9 \mathrm{KW}$ (bottom), and distances of $5 \mathrm{~cm}$ (left) and $10 \mathrm{~cm}$ (right) at (a) $\mathrm{t}=30$ seconds and (b) $\mathrm{t}=60$ seconds exposure 
The temperature distribution in Fig. $4 \mathrm{a}$ and $4 \mathrm{~b}$ has a direct relationship to the microwave power. This means that by increasing power, a rise in temperature is observed; however, the distance from the antenna decreases the temperature. As the distance from the antenna increases from $5 \mathrm{~cm}$ to $10 \mathrm{~cm}$, the temperature distribution becomes lower. A higher distance from the antenna distributes the patterns of temperature more evenly. The exposure time is also considered to play a substantial role in the heating process; as expected, an increase in exposure time can result in higher temperature and mechanical stress values. This can be verified by comparing Fig. 4a with Fig. 4b, and Fig. 5a with Fig. 5b, when exposure time increases from 30 seconds to 60 seconds.

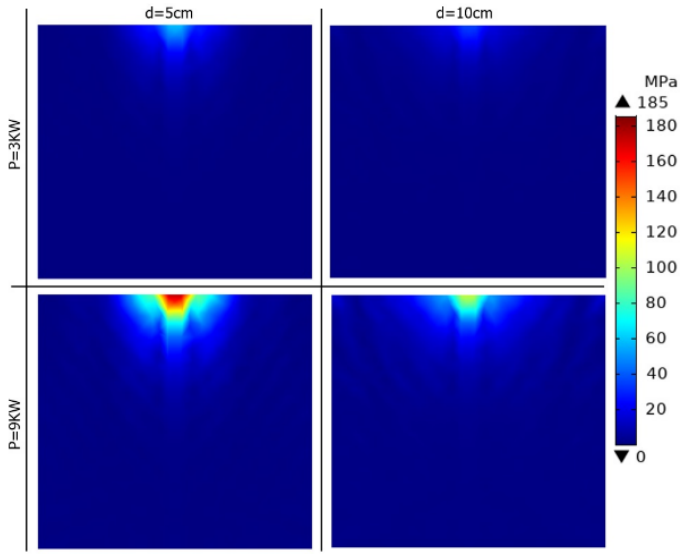

(a) $\mathrm{t}=30$ seconds

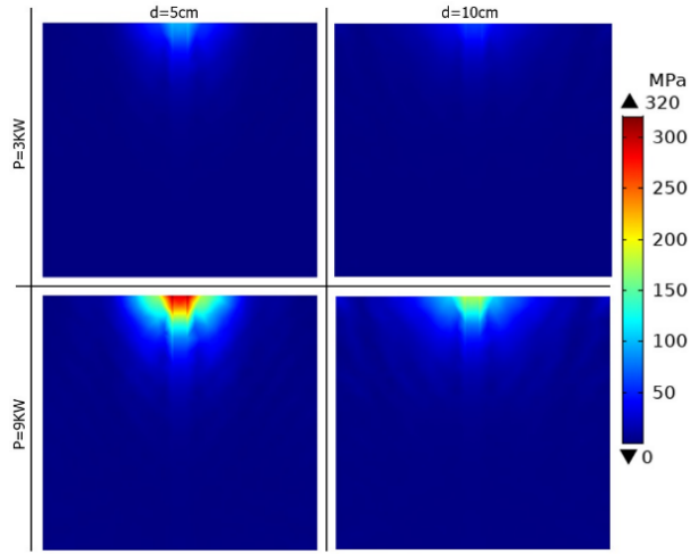

(b) $\mathrm{t}=60$ seconds

Fig. 5. Two-dimensional maximum principal stress distribution for power levels of $3 \mathrm{KW}$ (top) and 9KW (bottom), and distances of $5 \mathrm{~cm}$ (left) and $10 \mathrm{~cm}$ (right) at (a) $\mathrm{t}=30$ seconds and (b) $\mathrm{t}=60$ seconds exposure

A pattern of simulated maximum principal stresses developed within the modelled R2 ore sample inside the R1 host rock after simulations is shown in Fig. 5. The results show that the maximum principal stress occurred very close to the grain boundary between R1 and R2 samples. Also, as the distance from the antenna increases, the intensity of the maximum principal stress decreases. Consequently, there is an inverse relationship between the power level and the distance from the antenna. Furthermore, as the power level increases, the intensity of maximum principal stress increases. Increasing the power level from $3 \mathrm{KW}$ to $9 \mathrm{KW}$ provided a sharper and more distributed mechanical stress gradients. It should be noted that when the mechanical stress exceeds the strength of the material, then plastic deformation and crack propagation begins. In the current study, the highest value of maximum principal stress achieved $(315 \mathrm{MPa})$ was when $9 \mathrm{KW}$ power was applied for 60 seconds exposure. This value should be considered a failure given that rocks generally fail at approximately $240 \mathrm{MPa}$ (see ref [5]). By comparing Fig. 5a with Fig. 5b for the same $(\mathrm{P}=9 \mathrm{KW}$ and $\mathrm{d}=5 \mathrm{~cm})$ configuration, the highest value of maximum principal stress achieved was $174 \mathrm{MPa}$ and $315 \mathrm{MPa}$. This means that for a high power level, the exposure time needed to cause thermally induced microcracking, and subsequent breakage, is shorter and the energy is very efficiently used to produce breakage. This finding is very consistent with the results from [5]. A higher input power level of microwaves contributes to more complex physical and mechanical reactions, which include the elevation of internal pressure and decomposition of particles along the boundary between the R1 host and the R2 ore sample. This can be thought of as the fundamental mechanism of cracking in rocks composed of different materials; as 
thermal stresses increase, unstable growth of cracks along the boundaries of different minerals will be observed.

\section{Conclusion}

In this paper, the thermally induced mechanical stresses on the boundaries of two conceptual attached rock samples are numerically simulated by the finite element method, and the dependence of the failure mechanism on microwave input operating parameters is studied. The present study shows that differential volumetric expansion of a rock model, which includes a host rock and an ore sample, during microwave heating increases mechanical stresses along grain boundaries. Also, exposure time is a parameter that can influence this phenomenon; more exposure time will result in more mechanical stresses and subsequent cracking/spallation.

The results from the current study can be thought of as groundwork for the future of microwave energy application in civil and mining engineering related to rock breakage. The numerical results suggest that microwave energy has a significant application for rock breakage systems and, therefore, for continuous excavation machinery. In terms of modelling and simulation, this work provides some tools and insights into the design and performance of microwave-assisted heating of a rock model that is made up of two attached types of rocks.

\section{Acknowledgements}

Financial support for this work from the Natural Sciences and Engineering Research Council (NSERC) of Canada, the McGill Engineering Doctoral Award (MEDA) Award is gratefully acknowledged.

\section{References}

[1] Lindroth, D. P., Morrell, R. J., and Blair, J. R., "Microwave Assisted Hard Rock Cutting”, U.S. Patent, No. 5,003,144. 26 Mar. 1991.

[2] Hassani, F., Nekoovaght, P. M., and Gharib, N., "The Influence of Microwave Irradiation on Rocks for Microwave-Assisted Underground Excavation”, J. Rock Mech. Geotech. Eng., 2016, 8(1), pp. 1-15.

[3] Lu, G. ming, Li, Y. hui, Hassani, F., and Zhang, X., "The Influence of Microwave Irradiation on Thermal Properties of Main Rock-Forming Minerals”, Appl.

Therm. Eng., 2017, 112, pp. 1523-1532.

[4] Wang, Y., and Djordjevic, N., "Thermal Stress FEM Analysis of Rock with Microwave Energy”, Int. J. Miner. Process., 2014, 130, pp. 74-81.

[5] Teimoori, K., Hassani, F., and Sasmito, A. P., "Multiphysics Study of Microwave Irradiation Effects on Rock Breakage System”, IJRMMS, 2019, 1(514), pp. 1-32.

[6] Whittles, D. ., Kingman, S. ., and Reddish, D., "Application of Numerical Modelling for Prediction of the Influence of Power Density on Microwave-Assisted Breakage”, Int. J. Miner. Process., 2003, 68(1-4), pp. 71-91.

[7] Press, D. C., "Thermal Expansion of Fluorspar and Iron Pyrite”, Proc. Indian Acad. Sci. - Sect. A, 1949, 30(6), pp. 284-294.

[8] Hartlieb, P., Toifl, M., Kuchar, F., Meisels, R., and Antretter, T., "ThermoPhysical Properties of Selected Hard Rocks and Their Relation to MicrowaveAssisted Comminution”, Miner. Eng., 2016, 91, pp. 34-41.

[9] Whitaker, M. L., Liu, W., Wang, L., and Li, B., "Acoustic Velocities and Elastic Properties of Pyrite (FeS 2) to 9.6 GPa”, J. Earth Sci., 2010, 21(5), pp. 792-800.

[10] Pearce, C. I., "Electrical and Magnetic Properties of Sulfides", Rev. Mineral. Geochemistry., 2006, 61(1), pp. 127-180. 
[11] Carmichael, R. S., "Practical Handbook of Physical Properties of Rocks and Minerals", CRC press, 2018.

[12] Peng, Z., Hwang, J.-Y., Kim, B.-G., Kim, J.-Y., and Wang, X., "Microwave Permittivity, Permeability, and Penetration Depth of Pyrite”, Characterization of Minerals, Metals, and Materials., 2014, pp. 371-378. 


\title{
THEORETICAL ANALYSIS ON EFFICIENT MICROWAVE WARMING OF HUMAN BLOOD
}

\author{
S. Kumari ${ }^{1}$, S. K. Samanta ${ }^{1}$, B. Saxena ${ }^{1}$ \\ ${ }^{1}$ Indian Institute of Technology Patna, Bihta, Patna-801103, India \\ sushma.pcb16@iitp.ac.in; sksamanta@iitp.ac.in; brijsaxena1994@gmail.com
}

\begin{abstract}
:
To study the thermal impact of microwave irradiation on the human blood, a meticulous analysis has been performed with the aim of rapid generation of uniformly warmed blood assumed as a 2D cylinder. The analysis preliminarily involves the comparison of average microwave power absorption by the sample for various sample sizes on being subjected to two different irradiation schemes i.e. lateral and radial. The Galerkin finite element analysis has been applied to simultaneously solve the Maxwell's equation and heat transfer equation using pertinent boundary conditions to study the thermal effect of the microwave interaction with blood sample. Prelusive analysis of average power absorption and the subsequent detailed analysis of the heating rate and thermal non-uniformity through the plots of average temperature and temperature difference against time, respectively, have led to selection of optimum heating strategies for various blood samples corresponding to suitably selected sizes. Considering the frequency of $2450 \mathrm{MHz}$ and intensity of $1 \mathrm{~W} . \mathrm{cm}^{-2}$, the lateral irradiation is found to be more favorable for samples having moderate or large diameter, whereas the radial irradiation is found to be more suitable for small samples. Considering all the aspects, the present work recommends an efficient way for enhanced microwave assisted heating of human blood samples (2D cylindrical geometry) with known or measurable dielectric properties.
\end{abstract}

Keywords: Microwave, Human blood, Optimum heating, Thermal runaway, 2D cylinders

\section{Introduction:}

The microwave is a non-ionizing electromagnetic irradiation with the feature of volumetric processing $^{1-3}$. The frequency range of a microwave irradiation lies between $300 \mathrm{MHz}$ to 300 $\mathrm{GHz}$ in the electromagnetic spectrum. The interaction of microwave irradiation with a dielectric substance depends upon its factor of dielectric constant and loss values ${ }^{2}$. The value of dielectric loss determines the quantity of conversion of the electromagnetic energy to heat energy on interaction. The thermal effect of microwave irradiation has gained attention in different processing industries such as food, pharmaceutical, ceramics, polymer, medical sciences and many more ${ }^{2,4-6}$. The diverse applications of microwave in the field of medical science have always been the topic of extensive investigation from decades and have both therapeutic and diagnostic utility ${ }^{4-6}$.

The importance of warming of pre-transfusion blood has been a topic of great investigation ${ }^{7,8}$. The prominence of warming was accepted after several investigations demonstrated the complications of cold blood transfusion such as hemorrhagic shocks, ventricular fibrillation, etc $^{7-9}$. These studies highly favored the need to research and investigate all the aspects of warming of pre-transfusion blood. The human blood needs to be warmed rapidly taking in cogitation the preservation of its sterility and quality by employing microwave assisted warming technique ${ }^{10}$. The microwave assisted warming of human blood has been a suggested alternative in the category of dry technique of blood warming 8,11 . 
Although, the microwave warming of human blood started decades back, however the procedure was not widely accepted owing to the reports of causalities due to the transfusion of microwave warmed denatured blood ${ }^{12-14}$. So, the aim of this study is to perform a mathematical analysis using the information available in present literature about the dielectric properties of blood and analyze precisely the exact heating time and strategy for known sample size and ambience of the system ${ }^{15-17}$.

Microwave blood warmers drew two major stipulations for the acceptance of microwave assisted blood warming technique: The decimation of the occurrence of over-heating and the elimination of any other attributes of microwave which would damage the cell functionality ${ }^{18,19}$. So, they suggested circumvention of overheating to avoid any damage by microwave warming and the need of further detailed investigation to make it a clinically useful tool ${ }^{18,19}$.

In this work, a thermal analysis has been carried out which involves the comparison of heating effects of microwave irradiation with frequency $(f)=2450 \mathrm{MHz}$ and intensities $\left(I_{0}\right)$ $=1,1.5$ and $2 \mathrm{~W} \cdot \mathrm{cm}^{-2}$, on human blood samples of various sizes and on being subjected to two different irradiation schemes i.e. lateral and radial. The exploration of heating rate and thermal non-uniformity through the plots of average temperature and temperature difference against time, respectively, will lead to the selection of the optimum heating strategies for various blood samples corresponding to suitably selected sizes.

\section{Theoretical formulations:}

\section{Power and temperature calculations:}

The evaluation of the extent of absorption of microwave irradiation by the blood sample has been performed through the electromagnetic field equation. The evaluation aids the calculation of temperature distribution inside the sample when subjected to different modes of irradiation i.e. lateral and radial. The lateral irradiation mode is the situation designed with an assumption of a stationary sample in a uniform plane wave having interaction from one direction (left in this study), whereas radial irradiation mode assumes the sample to rotate with the surety of uniform interaction from all the directions.

The electromagnetic field representing microwave irradiation consists of electric and magnetic fields which are perpendicular to each other and have the variation of the magnitude in the direction of propagation i.e. $\mathrm{z}$ direction (see Fig. 1). The electric field propagation equation is represented by the Maxwell's equation ${ }^{1}$ i.e.

$\nabla^{2} E_{x}+\kappa^{2} E_{x}=0$

where, $E_{x}$ is the electric field which varies in $\mathrm{y}-\mathrm{z}$ plane and $\kappa$, the propagation constant, depends on dielectric constant $\kappa^{\prime}$, dielectric loss $\kappa^{\prime \prime}$ of the sample, frequency $f$ of the irradiation and speed of light $c$. The relation is given as:

$\kappa=\frac{2 \pi f}{c} \sqrt{\kappa^{\prime}+i \kappa^{\prime \prime}}$

Further, the calculation of the change in the phase of the wave and the field intensity decay rate is carried out using the relation between wavelength $\left(\lambda_{s}\right)$ and penetration depth $\left(\delta_{p}\right)$ with the dielectric properties of the sample, respectively as ${ }^{1}$ : 


$$
\begin{aligned}
& \lambda_{s}=\frac{c}{\sqrt{\frac{\kappa^{\prime} f^{2}\left(\left(1+\tan ^{2} \delta\right)^{1 / 2}+1\right)}{2}}} \\
& \delta_{p}=\frac{c / \pi}{\sqrt{\frac{\kappa^{\prime} f^{2}\left(\left(1+\tan ^{2} \delta\right)^{1 / 2}-1\right)}{2}}}
\end{aligned}
$$

where, $\tan \delta=\frac{\kappa^{\prime \prime}}{\kappa^{\prime}}$ represents the loss tangent.

The dissipation of power in the sample is evaluated using the Poynting theorem and can be given as:

$\nabla \cdot S=-\frac{1}{2} \omega \varepsilon_{0} \kappa^{\prime \prime} E_{x} \cdot E_{x}^{*}+i \omega\left(\frac{\mu_{0}}{2} H_{y} \cdot H_{y}^{*}+\frac{\varepsilon_{0} \kappa^{\prime}}{2} E_{x} \cdot E_{x}^{*}\right)$

The real part of the equation gives the power dissipation and the imaginary part gives the stored electric and magnetic power. So, the local power dissipation is ${ }^{1}$ :

$P=-\operatorname{Re}(\nabla \cdot S)=\frac{1}{2} \omega \varepsilon_{0} \kappa^{\prime \prime} E_{x} \cdot E_{x}^{*}$

The time dependent heat equation for the system involves a heat conduction equation with heat source term due to microwave power $(P)$ can be given as: $\rho C_{p} \frac{\partial T}{\partial t}=\nabla \cdot(k \nabla T)+P$

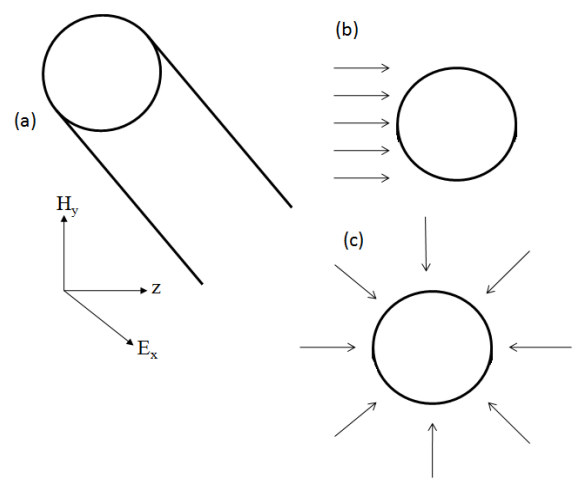

Fig. 1. Schematic illustrations of 2D human blood cylinders incident with (a) uniform plane waves, (b) lateral irradiation and (c) radial irradiation.

The insulation condition (no flux condition) has been used for outer surface as the boundary condition.

\section{Calculation strategies:}

The simultaneous evaluation of the heat equation and electric field equation with pertinent boundary conditions is performed through Galerkin finite element method. The time domain is discretized using Crank-Nicholson method, and Newton Raphson method is used to solve the non-linear residual equations with the first time step of $1 * 10^{-4}$ seconds and subsequently 0.1 seconds. The initial temperature of the blood sample and the ambient temperature for the analysis are considered to be $273 \mathrm{~K}$ and $300 \mathrm{~K}$, respectively. 


\section{Results and discussion:}

The thermal effect of microwave irradiation on human blood samples without any additives has been investigated. The samples have been assumed to be cylindrical in shape and have been subjected to microwave irradiation by lateral and radial mode of incidences of the irradiation. A prolegomenon investigation on the average microwave power the human blood absorbed for a given sample size is performed. The plot obtained revealed several peak points which are as a result of constructive interferences between transmitted and reflected waves of the microwave irradiation (see Fig. 2). The peak points of average power absorption at the emphasized conditions of lateral and radial irradiations have been further analyzed to study the spatial distribution of power and temperature.

\section{Microwave warming of human blood with intensity, $I_{0}=1 \mathrm{~W} \cdot \mathrm{cm}^{-2}$}

The peaks observed in the plot are caused due to constructive interference of the propagating microwave and show the maximum values of the average power absorbed by the human blood. The observation points are selected on the basis of either of the irradiation techniques achieving a maxima in the average power absorption observed. The $1^{\text {st }}$ observation point (OP) considering the initial sample size, $d_{c}>1 \mathrm{~cm}$ is observed at $d_{c}=1.154 \mathrm{~cm}(O P: 1)$ for lateral and radial irradiation with average power of $\mathrm{P}_{\mathrm{av}}=2.004$ and $1.612 \mathrm{~W} . \mathrm{cm}^{-3}$, respectively. The next OP occurs at $\mathrm{d}_{\mathrm{c}}=1.894 \mathrm{~cm}(\mathrm{OP}: 2)$ with average power of $\mathrm{P}_{\mathrm{av}}=0.687$ and $1.765 \mathrm{~W} . \mathrm{cm}^{-3}$ for lateral and radial irradiations, respectively. The third observation point for lateral and radial irradiation is obtained at $\mathrm{d}_{\mathrm{c}}=3.378 \mathrm{~cm}(\mathrm{OP}: 3)$ with corresponding average power absorption $\mathrm{P}_{\mathrm{av}}=0.264$ and $0.408 \mathrm{~W} . \mathrm{cm}^{-3}$, respectively. The OP: 1 exhibited a higher value of average power absorption for lateral heating scheme whereas the OP: 2 and OP: 3 depict the radial heating scheme to exhibit higher value of average power absorptions.

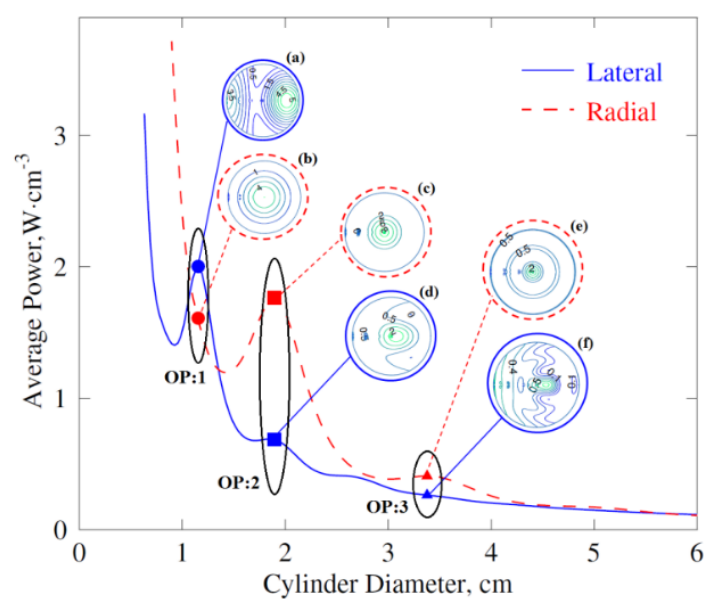

Fig. 2. The representation of average power absorption $\left(\mathrm{W} . \mathrm{cm}^{-3}\right)$ in the human blood sample with respect to sample size $(\mathrm{cm})$ during microwave assisted warming at frequency of $2450 \mathrm{MHz}$ and intensity of $1 \mathrm{~W} . \mathrm{cm}^{-2}$. The inset is the representation of the power distribution inside the cylindrical sample (a) for lateral heating at $\mathrm{d}_{\mathrm{c}}=1.154 \mathrm{~cm}$, (b) for radial heating at $d_{c}=1.154 \mathrm{~cm},(\mathbf{c})$ for radial heating at $\mathrm{d}_{\mathrm{c}}=1.894 \mathrm{~cm},(\mathbf{d})$ for lateral heating at $d_{c}=1.894 \mathrm{~cm}$, (e) for radial heating at $d_{c}=3.378 \mathrm{~cm}$ and (f) for lateral heating at

$$
\mathrm{d}_{\mathrm{c}}=3.378 \mathrm{~cm} \text {. }
$$


The details of distribution of power absorption need to be further analyzed extensively due to the sample being prone to denaturation on exposure to high power and non-uniform heating. The OPs are further analyzed in detail regarding the uniformity of power and temperature dissemination along with rate of heating. The insets in the Fig. 2 show the spatial distribution of absorbed power by the human blood with respect to the size of the sample subjected to microwave irradiation in both lateral and radial modes. The power distribution contour for lateral irradiation at OP: 1 shows two cruxes of high power absorption on either sides of the sample with left at $3.5 \mathrm{~W} . \mathrm{cm}^{-3}$ and right side at $5 \mathrm{~W} \cdot \mathrm{cm}^{-3}$ (see Fig. 2(a)). These are the possible causes of constructive interferences of the waves inside the sample being subjected to the microwave irradiation. The power at the centre of the sample ranges from 1.5 to $0.5 \mathrm{~W} . \mathrm{cm}^{-3}$ depicting low power absorption. Moving on to OP: 2 and being consistent with lateral mode of irradiation, the power absorbed by the sample is observed to shift towards the centre and reduce to $2 \mathrm{~W} \cdot \mathrm{cm}^{-3}$ (see Fig. 2 (d)). The OP: 3 for the lateral irradiation exhibits thoroughly concentrated average power absorption at the centre with higher values of $0.5 \mathrm{~W} . \mathrm{cm}^{-3}$ (see Fig. 2 (f)). On studying the radial mode of irradiation at the 3 OPs, a single high power absorption crux residing at the centre of the sample and reduce when traversing towards the edge. The value of power varies from $4 \mathrm{~W} . \mathrm{cm}^{-3}, 8 \mathrm{~W} . \mathrm{cm}^{-3}$ and 2 W.cm ${ }^{-3}$ for OP: 1, 2 and 3, respectively (see Fig. 2 (b), (c) and (e)).

The Fig. 3(a) is the surface contour exhibiting the distribution of temperature on being exposed to the microwave irradiation for 80 seconds with $I_{0}=1 \mathrm{~W} \cdot \mathrm{cm}^{-2}$. The lateral mode of irradiation at OP: 1 displays a maximum temperature of $335 \mathrm{~K}$ on the right side of the sample and minimum temperature of $305-310 \mathrm{~K}$ and radial mode a central maxima of about $318 \mathrm{~K}$. The OP: 2 manifests a single central maxima of $296 \mathrm{~K}$ and minima of $282 \mathrm{~K}$ towards the right sided outer edge on lateral and a central maxima of $340 \mathrm{~K}$ on radial microwave exposure. The lateral irradiation mode at OP: 3 displays a left inclined maxima of around $283 \mathrm{~K}$ whereas the radial mode presents a central maxima of $290 \mathrm{~K}$.

(a)
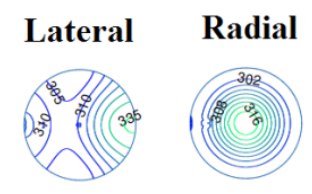

OP:2
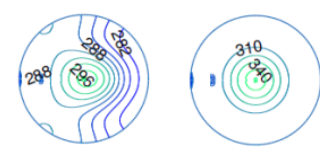

OP:3
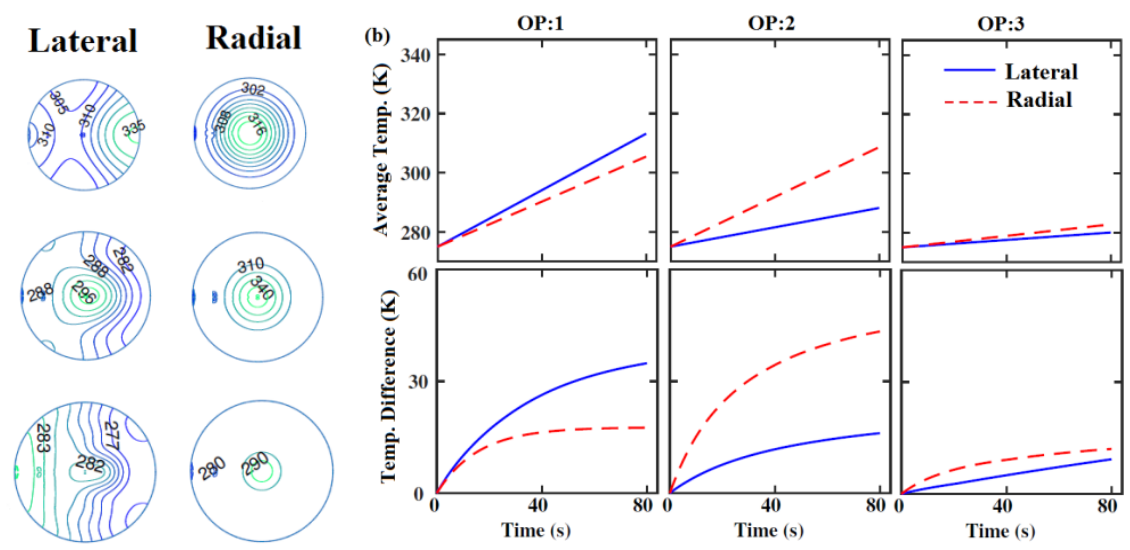

Fig. 3. (a) Spatial representation of thermal mapping inside the sample during microwave assisted warming at frequency of $2450 \mathrm{MHz}$ and intensity of $1 \mathrm{~W} \cdot \mathrm{cm}^{-2}$ for 80 seconds. (b)

Average temperature (K) in the human blood vs. time (s) [upper panel]; Temperature difference $(\mathrm{K})$ in the human blood vs. time (s) [lower panel].

The Fig. 3 (b) gives the plot of average temperature (K) in the blood sample with respect to time (s) measuring the heating rate and the temperature difference $(\mathrm{K})$ in blood sample with respect to time (s) representing the non-uniformity of the temperature distribution or the thermal runaway occurring in the sample. The optimum heating strategy out of either lateral 
or radial irradiation mode for a chosen set of sample size, intensity and exposure duration is set on the criteria of high heating rate and low thermal non-uniformity. The OP: 1 put forth the lateral irradiation mode having a slightly higher heating rate with a very high thermal non-uniformity making it the undesired heating strategy. However, lateral irradiation is preferred for OP: 2 due to very high thermal runaway even with higher heating rate owing to the delicate nature of blood samples and have identical situation for OP: 3. A similar analysis is performed to the samples at different time steps with different input intensities for understanding the pattern for microwave warming for different sizes of human blood sample.

\section{Conclusions:}

The mathematical analysis was performed with the objective to understand the thermal effects of microwave irradiation on very delicate human blood samples assumed as a $2 \mathrm{D}$ cylindrical geometry. The study is performed by solving the equation of electromagnetic wave propagation along with the equation of energy balance and pertinent boundary conditions. The sample was subjected to two different modes of interaction of the microwave with the sample i.e. lateral and radial. The initial analysis put forward the change in nature of average power absorption occurring at different sample sizes due to the resonance effects of the electromagnetic wave interaction. The peaks observed are chosen as observation points for detailed analysis about the spatial distribution of power and temperature inside the sample. The microwave warming of human blood at $2450 \mathrm{MHz}$ and $1 \mathrm{~W} . \mathrm{cm}^{-2}$ gives the plot of average power with respect to the sample size exhibiting the lateral mode to with higher average power absorption for small sizes samples. However, the average power is more absorbed through the radial irradiation mode for medium to large sized samples. Further spatial analysis of the temperature and power is performed displaying the number of maxima obtained along with its position. The plot of average temperature and temperature difference with respect to time leads to the selection of the condition of high heating rate and low thermal non-uniformity as the parameter to decide the optimum heating strategy for either lateral or radial irradiation mode. The OP: 1 indicates the radial mode and the OP: 2 and 3 indicate the lateral mode of irradiation as the optimum strategy for the microwave blood warming. In conclusion we can say that the efficient warming technique is dependent on the dielectric property of the sample, exposure duration, the property of the wave and many more. The analysis acts as a guideline for development of a safe and rapid warming technique for human blood or other delicate crystalloid fluids using microwave irradiation with consideration to all the strategies and sample properties.

\section{References}

1. Ayappa KG, Davis HT, Davis EA, Gordon J. Aiche J. 1992;38:1577-92.

2. Samanta SK, Basak T. Food Res Int. 2010;43:148-66.

3. Samanta SK, Basak T. Food Res Int. 2009;42:1337-50.

4. Vrba J. Electromagn Biol Med. 2005;24:441-8.

5. Rosen A, Stuchly MA, Vorst A Vander. IEEE Trans Microw Theory Tech. 2002;50:963-74.

6. Yang WJ, Mochizuki S, Yang PPT. J Mech Med Biol. 2002;2:53-65.

7. Boyan CP. Ann Surg. 1964;160:282-6.

8. Iserson K V, Huestis DW. Transfusion. 1991;31:558-71.

9. Restall CJ, Leonard PF, Taswell HF, Holaday RE. Anesth Analg. 1967;46:625-8.

10. Smith CE, Wagner K. Int TraumaCare. 2008;18:71-9.

11. Campbell NL, Drewe J. IEEE MTT-S Int. 1981;479-81.

12. Arens JF, Leonard GL. JAMA. 1971;218:1045-6. 
13. Hirsch J, Menzebach A, Welters ID, Dietrich GV, Katz N, Hempelmann G. Clin Chem. 2003;49:792-9.

14. Linko K, Hynynen K. Acta Anaesthesiol Scand. 1979;23:320-8.

15. Takeda A, Takata K, Wang J, Fujiwara O. Meas 2011, Proceedings 8th Int Conf Smolenice, Slovakia. 2011;387-90.

16. Salahuddin S, O'Halloran M, Porter E, Farrugia L, Bonello J, Sammut C V, et al. IEEE Trans Dielectr Electr Insul. 2017;24:3283-9.

17. Rauf A. Int J Sci Environ Technol. 2013;2:1396-400.

18. Schwaitzberg SD, Allen MJ, Connolly RJ, Grabowy RS, Carr KL, Cleveland RJ. J Investig Surg. 1991;4:505-10.

19. Herron DM, Grabowy R, Connolly R, Schwaitzberg SD. J Trauma Acute Care Surg. 1997;43:9291364. 


\title{
MATHEMATICAL MODELING OF MICROWAVE HEATING OF PRODUCTS WITH CENTRAL SYMMETRY
}

\author{
V.A. Karelin ${ }^{1,2}$, V1.V. Salomatov ${ }^{1,2}$, \\ ${ }^{1}$ Novosibirsk State University, Pirogova 2, Novosibirsk 630090, Russia \\ ${ }^{2}$ Kutateladze Institute of Thermophysics SB RAS, Kutateladze 2, Novosibirsk 630090, Russia \\ vad2hen@mail.ru
}

Keywords: microwave, radio-frequency heating, power applications, modeling, central symmetry

Microwave heating has found widespread use in the energy, construction, forestry, chemical and food industries, etc. There are a number of publications that discuss the main mechanisms that occur during microwave heating and microwave drying [1,2]. For a better understanding of these processes and the development of highly efficient microwave installations, mathematical modeling is needed. As a rule, nonlinear models that most adequately describe these phenomena use a numerical algorithm for calculations. The authors of this work are engaged in approximate analytical approaches for microwave heating and microwave drying of bodies, which, with controlled error, allow displaying the main processes and estimating such heating and drying parameters as: temperature and moisture distribution, heating time, drying rate, reaching maximum values and etc.

In this paper, a model of microwave heating of a body in the form of a ball with uniform irradiation with microwave energy in the conditions of radiation-convective interaction of the product with the environment is considered. The absorption of the microwave inside the material is given by the law of the Bouguer. In this case, a number of simplifications were made: the electrophysical and thermophysical properties of the material are constant, the material is homogeneous in composition and properties. The equations defining this problem are as follows:

$$
\begin{aligned}
& \frac{\partial T(r, t)}{\partial t}=a\left[\frac{\partial^{2} T(r, t)}{\partial r^{2}}+\frac{2}{r} \frac{\partial T(r, t)}{\partial r}\right]+\frac{q_{0}}{c \rho} e^{-k\left(r_{0}-r\right)} \\
& 0<t<t_{d} ; 0<r<r_{0} ; T_{0}<T<T_{d} \\
& \lambda \frac{\partial T\left(r_{0}, t\right)}{\partial r}=\varepsilon \sigma_{0}\left[T^{4}\left(r_{0}, t\right)-T_{c}^{4}\right]+\alpha\left[T\left(r_{0}, t\right)-T_{c}\right] \\
& \lambda \frac{\partial T(0, t)}{\partial r}=0 \\
& T(r, 0)=T_{0}
\end{aligned}
$$

Using the conditions for the uniqueness of the problem, we represent this system in the following dimensionless variables.

$\xi=\frac{r}{r_{0}} ; F o=\frac{a t}{r_{0}^{2}} ; \theta(\xi, F o)=\frac{T(r, t)}{T_{c}} ; P o=\frac{q_{0} r_{0}^{2}}{\lambda T_{c}} ; B i=\frac{\alpha r_{0}}{\lambda} ; S k=\frac{\varepsilon \sigma_{0} T_{c}^{3} r_{0}}{\lambda} ; B u=k r_{0}$

As a result, the system (1-5) will go to the following form:

$$
\begin{aligned}
& \frac{\partial \theta}{\partial F o}=\frac{\partial^{2} \theta}{\partial \xi^{2}}+\frac{2}{\xi} \frac{\partial \theta}{\partial \xi}+P o e^{-B u(1-\xi)} \\
& -\frac{\partial \theta(1, F o)}{\partial \xi}=S k\left[\theta(1, F o)^{4}-1\right]+B i[\theta(1, F o)-1]=K i(F o) \\
& \frac{\partial \theta(0, F o)}{\partial \xi}=0 \\
& \theta(\xi, 0)=\theta_{0}
\end{aligned}
$$


Apply the Laplace transform to this system:

$$
\begin{aligned}
& s \theta_{L}(\xi, s)-\theta_{0}=\theta_{L}^{\prime \prime}(\xi, s)+\frac{2 \theta_{L}^{\prime}(\xi, s)}{\xi}+\frac{P o}{s} e^{-B u(1-\xi)} \\
& -\theta_{L}^{\prime}(1, s)=K i_{L}(s) \\
& \theta_{L}^{\prime}(0, s)=0
\end{aligned}
$$

We will find the solution of this system as the sum of the solution of a homogeneous problem and the solution with the inhomogeneous term taken into account:

$$
\theta_{L}(\xi, s)=Y_{1}(\xi, s)+Y_{2}(\xi, s)
$$

The solution of a homogeneous problem in general: $Y_{1}(\xi, s)=\frac{A e^{\sqrt{s} \xi}+B e^{-\sqrt{s} \xi}}{\xi}$

The non-uniform part should be: $Y_{2}(\xi, s)=C(\xi) e^{-B u(1-\xi)}+\frac{\theta_{0}}{s}$

Next, we substitute a general solution $\theta_{L}(\xi, s)=\frac{A e^{\sqrt{s} \xi}+B e^{-\sqrt{s} \xi}}{\xi}+C(\xi) e^{-B u(1-\xi)}+\frac{\theta_{0}}{s}$ of inhomogeneous problem in the system (10-12), we find the constants:

$$
C=\frac{P o\left(2 B u-B u^{2} \xi+s \xi\right)}{\left(B u^{2}-s\right)^{2} s \xi}+\frac{e^{-(B u+\sqrt{s}) \xi} C_{1}}{\xi}+\frac{e^{-(B u-\sqrt{s}) \xi} C_{2}}{2 \sqrt{s} \xi}
$$

As a result, after substituting the constant $\mathrm{C}$ :

$$
\theta_{L}(\xi, s)=\frac{A e^{\sqrt{s} \xi}+B e^{-\sqrt{s} \xi}+\frac{2 P o B u e^{-B u}}{\left(B u^{2}-s\right)^{2} s} e^{B u \xi}+C_{1} e^{-B u} e^{-\sqrt{s} \xi}+C_{2} \frac{e^{-B u}}{2 \sqrt{s}} e^{\sqrt{s} \xi}}{-\frac{P o}{\left(B u^{2}-s\right) s} e^{-B u(1-\xi)}+\frac{\theta_{0}}{s}}
$$

Constants with corresponding exponent degrees can be combined:

$$
\theta_{L}(\xi, s)=\frac{A^{\prime} e^{\sqrt{s} \xi}+B^{\prime} e^{-\sqrt{s} \xi}+\frac{2 P o B u e^{-B u}}{\left(B u^{2}-s\right)^{2} s} e^{B u \xi}}{\xi}-\frac{P o}{\left(B u^{2}-s\right) s} e^{-B u(1-\xi)}+\frac{\theta_{0}}{s}
$$

From conditions $(11,12)$ we get:

$$
\begin{gathered}
A^{\prime}=M(P o, B u, s)-\frac{2 P o B u e^{-B u}}{\left(B u^{2}-s\right)^{2} s} \\
B^{\prime}=-M(P o, B u, s)
\end{gathered}
$$

Where $M(P o, B u, s)=\frac{K i_{L}+\frac{2 P o B u e^{-B u} e^{\sqrt{s}}(1-\sqrt{s})}{\left(B u^{2}-s\right)^{2} s}+\frac{2 P o B u^{2}-2 P o B u}{\left(B u^{2}-s\right)^{2} s}-\frac{P o B u}{\left(B u^{2}-s\right) s}}{-\sqrt{s}\left(e^{\sqrt{s}}+e^{-\sqrt{s}}\right)+e^{\sqrt{s}}-e^{-\sqrt{s}}}$

As a result:

$$
\theta_{L}(\xi, s)=\frac{\left(M(P o, B u, s)-\frac{2 P o B u e^{-B u}}{\left(B u^{2}-s\right)^{2} s}\right) e^{\sqrt{s} \xi}-M(P o, B u, s) e^{-\sqrt{s} \xi}+\frac{2 P o B u e^{-B u}}{\left(B u^{2}-s\right)^{2} s} e^{B u \xi}}{\xi}
$$

This solution has a rather complicated dependence on the parameter $\mathrm{s}$ and it is not possible to take the inverse Laplace transform. However, from the terms not containing $e^{\sqrt{s} \xi}, e^{-\sqrt{s} \xi}$ the inverse transformation is possible to obtain: 


$$
\theta_{L}(\xi, F o)=\frac{2 P o e^{-B u+B u \xi}\left(1-e^{B u^{2} F o}+B u^{2} e^{B u^{2} F o} F o\right)}{B u^{3} \xi}+\frac{P o\left(e^{B u^{2} F o}-1\right)}{B u^{2}} e^{-B u(1-\xi)}
$$

In order to obtain the inverse Laplace transform from the rest of the solution, we find the asymptotic expansions for large and small values of the parameter s. This will make it possible to find approximate solutions for large and small values of the time parameter, which allows determining the temperature by layer with high accuracy and depending on time.

\section{Large values of s (small Fo)}

For large values of s:

$M(P o, B u, s)=\frac{K i_{L}+\frac{2 P o B u e^{-B u_{e} \sqrt{s}}(1-\sqrt{s})}{\left(B u^{2}-s\right)^{2} s}}{e^{\sqrt{s}}(1-\sqrt{s})}=\frac{K i_{L}}{e^{\sqrt{s}}(1-\sqrt{s})}+\frac{2 P o B u e^{-B u}}{\left(B u^{2}-s\right)^{2} s}$

Consider the terms containing $e^{\sqrt{s} \xi}, e^{-\sqrt{s} \xi}$ :

$$
\begin{aligned}
& \frac{\left(M(P o, B u, s)-\frac{2 P o B u e^{-B u}}{\left(B u^{2}-s\right)^{2} s}\right) e^{\sqrt{s} \xi}-M(P o, B u, s) e^{-\sqrt{s} \xi}}{\xi} \frac{K i_{L}}{e^{\sqrt{s}}(1-\sqrt{s})} e^{\sqrt{s} \xi}-\left(\frac{K i_{L}}{e^{\sqrt{s}}(1-\sqrt{s})}+\frac{2 P o B u e^{-B u}}{\left(B u^{2}-s\right)^{2} s}\right) e^{-\sqrt{s} \xi} \\
& \xi
\end{aligned}
$$

For large values of the s parameter:

$$
\frac{K i_{L}}{e^{\sqrt{s}}(1-\sqrt{s}) \xi} e^{\sqrt{s} \xi}
$$

Inverse transform through convolution from a given addend:

$\theta(\xi, F o)=-\frac{1}{\xi} \int \frac{K i(y)}{\sqrt{\pi(F o-y)}} e^{\frac{-(\xi-1)^{2}}{4(F o-y)}}$

To obtain an explicit calculated expression, decompose Ki(y) near y = Fo into a Taylor series:

$$
K i(y)=K i(F o)+(y-F o) K i^{\prime}(F o)+\ldots
$$

Restricting ourselves to the first member of the expansion, we have:

$\theta(\xi, F o)=-K i(F o) \frac{1}{\xi} \int \frac{e^{\frac{-(\xi-1)^{2}}{4(F o-y)}}}{\sqrt{\pi(F o-y)}} d y$

After taking the integral, we finally get:

$$
\begin{gathered}
\theta_{L}(\xi, F o)=K i(F o) D(\xi, F o)+\frac{2 P o e^{-B u+B u \xi}\left(1-e^{B u^{2} F o}+B u^{2} e^{B u^{2} F o} F o\right)}{B u^{3} \xi} \\
+\frac{P o\left(e^{B u^{2} F o}-1\right)}{B u^{2}} e^{-B u(1-\xi)}+\theta_{0}
\end{gathered}
$$


Where $\mathrm{D}$ is the function obtained after integration.

Next, we find the boundary temperature $\theta_{L}(1, F o)$ from the previous equation.

A typical view of the dependence of temperature on the depth of the layer, as well as the temperature at the boundary, depending on the time is given below:

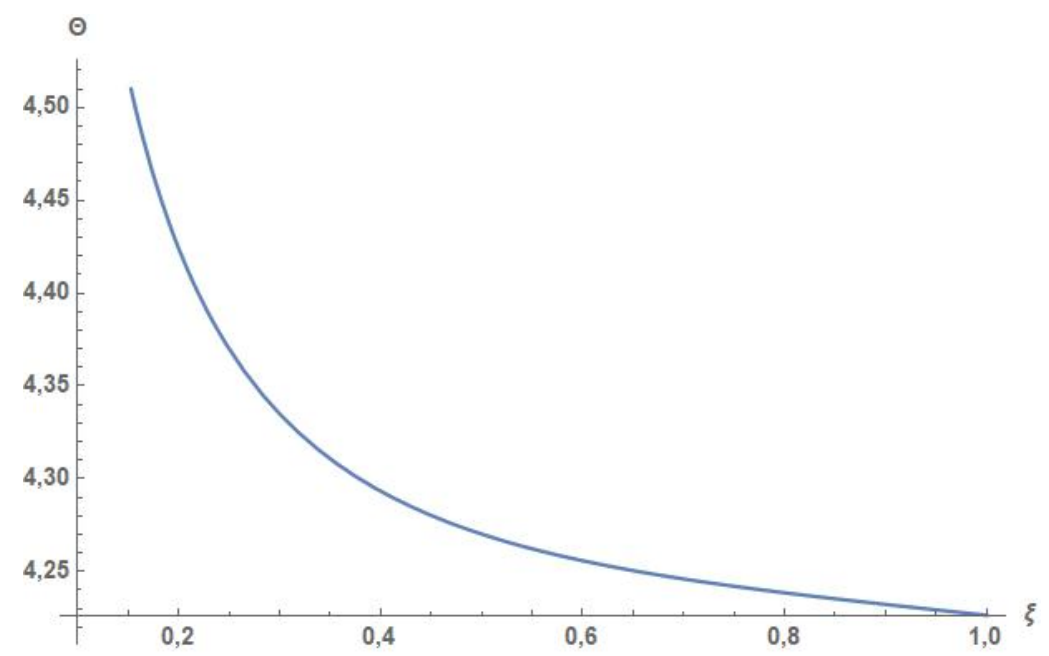

Fig. 1. The dependence of the dimensionless temperature on the surface coordinates $(\xi=1$ corresponds to the edge of the ball)

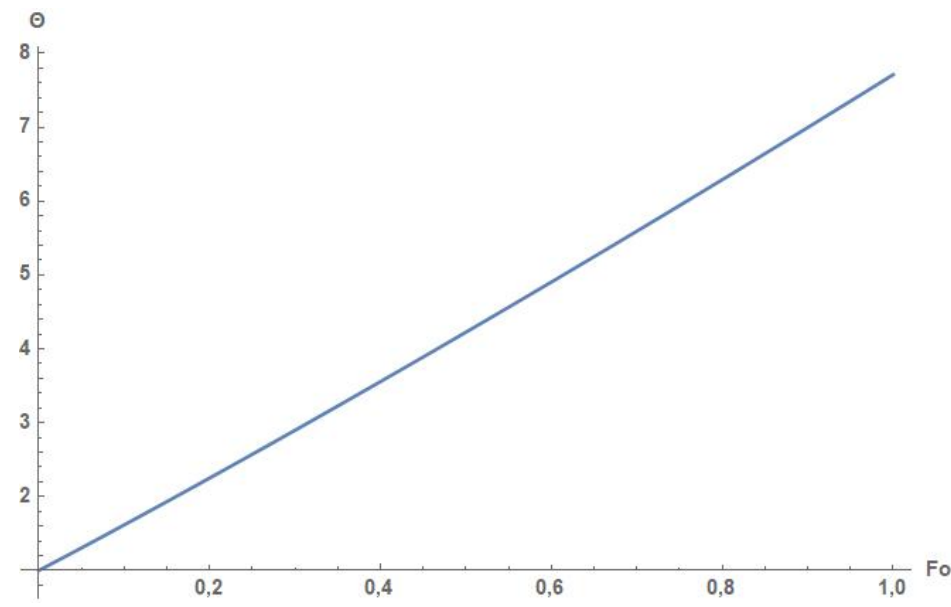

Fig. 2. The dependence of the dimensionless temperature on time at the boundary of the ball. 


\section{Small values of s (large Fo)}

For small values of s:

$M(P o, B u, s)=\frac{K i_{L}+\frac{2 P o\left(e^{-B u}+B u-1-\frac{B u^{2}}{2}\right)}{B u^{3} s}}{-\sqrt{s} s}$

Consider the terms containing $e^{\sqrt{s} \xi}, e^{-\sqrt{s} \xi}$ for small values of the parameter s:

$2 M \sqrt{s}-\frac{2 P o e^{-B u}}{B u^{3} s \xi}=2 \frac{K i_{L}+\frac{2 P o\left(e^{-B u}+B u-1-\frac{B u^{2}}{2}\right)}{B u^{3} s}}{-s}-\frac{2 P o e^{-B u}}{B u^{3} s \xi}$

Find the inverse Laplace transform from this part:

$-2 K i-\frac{2 P o F o\left(e^{-B u}+B u-1-\frac{B u^{2}}{2}\right)}{B u^{3}}-\frac{2 P o e^{-B u}}{B u^{3} \xi}$

As a result, for final solution:

$$
\begin{aligned}
\theta(\xi, F o)=-2 K i & -\frac{2 P o F o\left(e^{-B u}+B u-1-\frac{B u^{2}}{2}\right)}{B u^{3}} \\
& -\frac{2 P o e^{-B u}-2 P o e^{-B u+B u \xi}\left(1-B u^{2} F o+B u^{2} e^{B u^{2} F o} F o\right)}{B u^{3} \xi} \\
& +\frac{P o\left(e^{B u^{2} F o}-1\right)}{B u^{2}} e^{-B u(1-\xi)}+\theta_{0}
\end{aligned}
$$

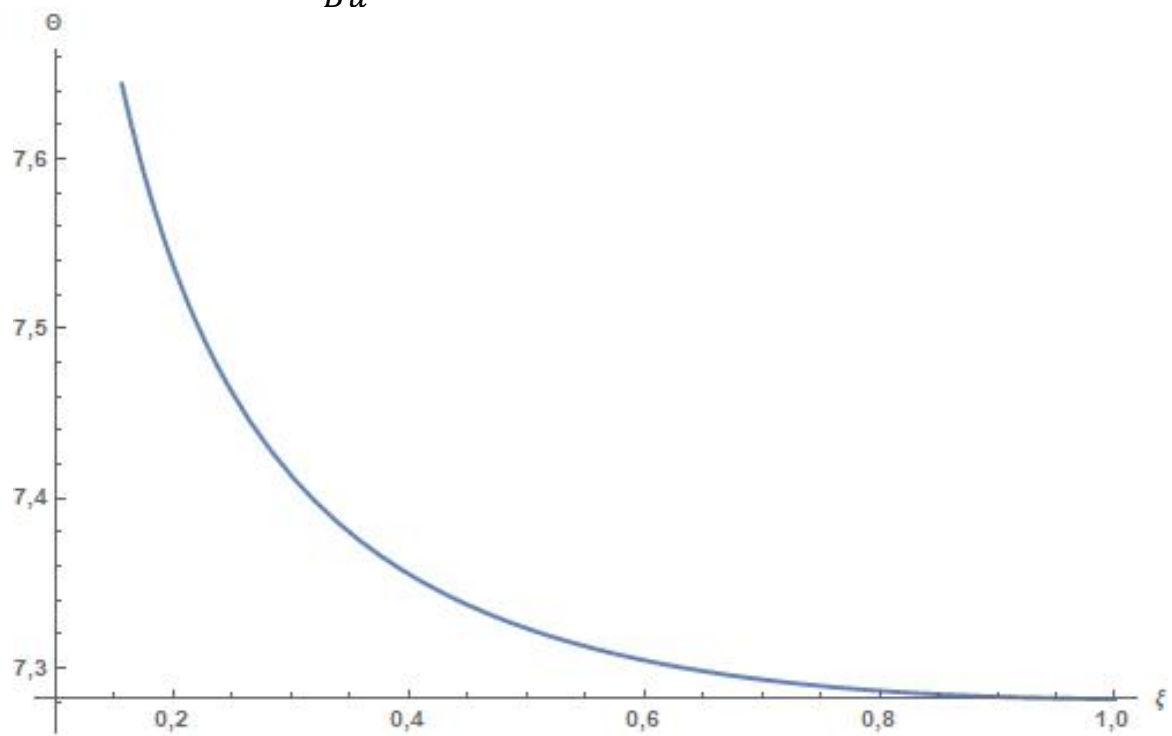

Fig. 3. The dependence of the dimensionless temperature on the surface coordinates $(\xi=1$ corresponds to the edge of the ball) 
Comparing with the numerical solution of a similar problem, it was found that for the corresponding solutions for large and small Fo, they give an error of no more than $7 \%$ with Fo $<0.1$ and Fo $>0.3$, respectively. These solutions allow you to qualitatively take into account the main processes occurring during the heating of a spherically symmetric particle.

As a result, using asymptotic procedures, approximate solutions were obtained for the temperature field depending on the time and intensity of processing, the electrical and thermal properties of the material, which allow to find the warm-up time to a certain temperature, the start time of drying, the maximum temperature coordinate, etc.

\section{Acknowledgement}

The statement of the problem and the solution for the stage of sphere heating were performed within the framework of the state task IT SB RAS, the search for approximate analytical solutions was done with the support of the Russian Foundation for Basic Research, grant No. 17-08-00752

\section{References}

1. Feng Gao, Comparison of microwave drying and conventional drying of coal. A thesis submitted to the Department of Mining Engineering in conformity with the requirements for the degree of Master of Applied Science Queen's University Kingston, Ontario, Canada, 2010, 167.

2. Khaidurova A.A., Fedchishin V.V., Konovalov N.P. Mikrovolnovaya sushka buryh uglej i povyshenie ih tekhnologicheskih harakteristik [Microwave drying of brown coals and increasing their technological characteristics]. Izv. Universities. Problems of energy. - Kazan: Publishing house KGEU. - 2010. - P. 31-35. In Rus. 


\title{
PLANE WAVE IRRADIATION OF A LAYERED SYSTEM: RESONANCE-BASED CONTROL OVER THERMAL RUNAWAY
}

\author{
A.A. Mohekar ${ }^{1}$, B.S. Tilley ${ }^{1,2}$, V.V. Yakovlev ${ }^{2}$ \\ ${ }^{1}$ Department of Mechanical Engineering, Worcester Polytechnic Institute, Worcester, \\ MA, USA \\ ${ }^{2}$ Center for Industrial Mathematics and Statistics, Department of Mathematical Sciences, \\ Worcester Polytechnic Institute, Worcester, MA, USA \\ aamohekar@wpi.edu,tilley@wpi.edu,vadim@wpi.edu
}

Keywords: computational modeling, EM heating, thermal runaway

\section{Introduction}

Traditional electromagnetic (EM) heating applications include thermal processing of food products, microwave assisted chemistry, and high temperature treatment of materials [1-3]. Currently, under development is a new technology of high-power EM heat exchangers (HX) intended for power beaming applications in wireless energy transfer [4, 5]. The key function of EM HX is to efficiently convert EM energy into heat (and, subsequently, into useful mechanical work). One of the challenges in front of the designers of such high-power devices is the nonlinear phenomenon of thermal runway.

The loss factor $\epsilon^{\prime \prime}$ is a key mechanism behind heat generation during EM heating. For typical ceramics, $\epsilon^{\prime \prime}$ increases exponentially with temperature, and that can potentially initiate thermal runaway putting the heating process out of control and damaging the material. Equilibrium EM heating can be represented by a parametric plot of the average steady-state temperature as function of the applied power that is known as a power response curve. In a layered structure, for the wavelengths of the incident wave that are much larger than the layer's thickness, the power response curve is an $S$-shaped bifurcation diagram $[6,7]$ (or $S$ curve) with two stable branches. Typically, temperatures are low at the lower branch of the $S$-curve and may be very high (up to $1,500-2,000 \mathrm{~K}$ ) at the upper branch [8].

The recent analytical $[8,9]$ and numerical [10] models show that, for a triple (lossless-lossylossless) layered system, when thickness of the structure is comparable with the wavelength, an electric field resonance can be achieved in the lossy layer. This resonance causes the $S$ curve to acquire another (middle) stable branch and become a double $S$-curve. In this case, thermal runway triggered at lower power can stabilize at the middle branch (500-1000 K). That suggests that during thermal runaway temperature may be controlled by the electric field resonance and be limited to some reasonable (not damaging) values. Our goal is to determine if thermal runaway in an EM HX can be controlled with this mechanism in a layered layout $[4,5]$.

The models in [8-10] considered a triple layered geometry where symmetric irradiation from both sides of the layers was applied. In this paper, we analyze a more practical case, where the plane wave is applied to a grounded two-layer (lossless-lossy) structure. The grounded end of the lossy layer may cause total reflection of the EM waves. We show that the resonance producing a double $S$-curve can be achieved in the lossy layer by choosing its thickness such that a constructive interference between the incident and reflected waves is achieved. First, we analytically solve the EM-thermal coupled problem with a thin-domain approximation $[8,9]$ to get a power response curve. To validate the results, we simulate a numerical model in COMSOL Multiphysics and compare the power response curves produced by both approaches. Finally, a parametric study is carried out to identify factors affecting the shape of the double $S$-curve. 


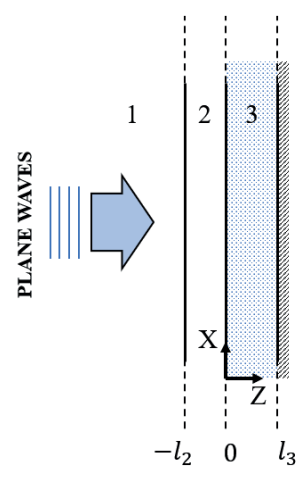

Fig. 1. Layout of the layered structure: region 1 is free space, region 2 is a lossless dielectric material, and region 3 is a lossy ceramic.

\section{Formulation of the Problem}

We consider a layered structure shown in Fig. 1. An incident plane wave travels along the positive Z-direction, is polarized along the Y-direction, and is uniform in the X-direction. Maxwell's equations for the considered problem are reduced to the one-dimensional Helmholtz equation

$$
\frac{\partial^{2} E_{j}}{\partial z^{2}}+k_{0}^{2} \mu_{r_{j}}\left[\epsilon_{r_{j}}^{\prime}-i \frac{\sigma_{e_{j}}\left(T_{j}\right)}{\omega \epsilon_{0}}\right] E_{j}=0, \quad j=1,2,3
$$

where subscript $j$ represents the region of the solution, $E=\bar{E} / E_{0}, \bar{E}$ is the dimensional form of the electric field, $E_{0}$ is the strength of the plane wave, $z=\bar{Z} / L_{z}, \bar{z}$ is the dimensional length along $Z, L_{z}$ is the length scale in $Z, k_{0}=2 \pi L_{z} / \lambda_{0}$ is the non-dimensional wave number, $\mu_{r}$ is the relative permeability, $\epsilon_{r}^{\prime}$ is the relative permittivity of the medium, $\sigma_{e}$ is the effective electrical conductivity, $T$ is temperature, $\omega$ is the angular frequency, and $\epsilon_{0}$ is permittivity of free space. Since region 2 is a lossless dielectric, $\sigma_{e_{2}}=0$.

Since $\sigma_{e}$ is temperature-dependent, we solve the thermal energy equations to evaluate $T$. We introduce non-dimensional form $T=\left(\bar{T}-T_{A}\right) / T_{A}$, where $\bar{T}$ is the dimensional temperature and $T_{A}$ is the ambient temperature, choose the conduction time scale as $L_{x}^{2} / \alpha_{3}$, where $\alpha_{3}$ is the thermal diffusivity of region 3 and $L_{x}$ is the length scale along X-direction, and assume that the electric field is uniform in the X-direction, which means that the heat flux along $\mathrm{X}$ is zero $\left(\frac{\partial T}{\partial x}=0\right)$ and $T$ is only function of $t$ and $z$. With these assumptions, the 1D timedependent non-dimensional energy equations become

$$
\begin{gathered}
\eta^{2} \frac{\partial T_{2}}{\partial t}=\alpha \frac{\partial^{2} T_{2}}{\partial z^{2}} \\
\eta^{2} \frac{\partial T_{3}}{\partial t}=\frac{\partial^{2} T_{3}}{\partial z^{2}}+\eta^{2} P \sigma_{3}\left(T_{3}\right)\left|E_{3}\left(T_{3}\right)\right|^{2},
\end{gathered}
$$

where $\eta=L_{z} / L_{x}$ is the aspect ratio of the geometry, $T_{2}$ and $T_{3}$ are non-dimensional temperatures in regions 2 and 3, respectively, $\alpha=\alpha_{2} / \alpha_{3}$ is the ratio of thermal diffusivities of regions 2 and 3 , respectively, $P=\frac{L_{x}^{2} \sigma_{0} E_{0}^{2}}{2 k_{3} T_{A}}$ is non-dimensional power, $k_{3}$ is thermal conductivity of region $3, \sigma_{3}=\sigma_{e_{3}}\left(T_{3}\right) / \sigma_{0}$ is non-dimensional electrical conductivity, and $\sigma_{0}=$ $\omega \epsilon_{0}$. The length scale in the Z-direction, i.e., thickness of the lossy layer, is chosen to be $l_{3}$. We solve the coupled system (1)-(3) with the EM boundary conditions 


$$
\begin{gathered}
\frac{\partial E_{2}}{\partial z}+i k_{0} E_{2}=2 i k_{0}, \quad \text { at } z=-\frac{l_{2}}{l_{3}}=-l, \\
E_{2}=E_{3}, \frac{\partial E_{2}}{\partial z}=\frac{\partial E_{3}}{\partial z}, \quad \text { at } z=0, \\
E_{3}=0, \quad \text { at } z=1,
\end{gathered}
$$

and the thermal boundary conditions

$$
\begin{gathered}
k \frac{\partial T_{2}}{\partial z}=\eta^{2} B i T_{2}, \quad \text { at } z=-l \\
k \frac{\partial T_{2}}{\partial z}=\frac{\partial T_{3}}{\partial z}, T_{2}=T_{3}, \quad \text { at } z=0, \\
\frac{\partial T_{3}}{\partial z}=0, \quad \text { at } z=1,
\end{gathered}
$$

where $\eta^{2} B i=h l_{3} / k_{3}$ is the scaled Bio number, $h$ is the heat transfer coefficient at $z=-l$, and $k=k_{2} / k_{3}$ is the ratio of thermal conductivities of regions 2 and 3 , respectively.

Boundary conditions (5) describe continuity of the electric field and its derivative at the interfaces between the regions, (6) suggests that the layered structure is attached to the ground/metal plate at $z=1$. We assume that thermal lossless to the surrounding are scaled with $\eta^{2}$; since $\eta$ is assumed to be small, thermal losses are also small. Eq. (7) describes the thermal losses to the surrounding by Newton's law of cooling, (8) states that the interfaces between the regions are in perfect thermal contact, i.e., heat flux and temperature are continuous, and (9) is adiabatic boundary condition at the metal plate.

We first solve the coupled system (1)-(3) analytically using a thin domain approximation $(\eta \ll 1)$ and compute the power response curve. Then we solve the system in COMSOL Multiphysics and compare the power response curves produced by both the models.

\section{Assumptions}

Since the main objective of this paper is demonstration of a principle possibility of the control over thermal runaway in the structure in Fig. 1, we do not consider here practical materials leaving that for future consideration. Thermal properties as well as $\epsilon_{r}$ are assumed to be temperature independent, and all the materials are non-magnetic $\left(\mu_{r}=1\right)$. Since another objective of this study is to find desirable permittivity of region 2 , we consider a wide range of values for $\varepsilon_{r}$. As it is shown below, the power response curves are independent of thermal parameters of regions 2 and 3 , so we choose specific heat $C_{p}$, density $\rho$, and thermal conductivity $k$ of region 2 to be the same as those of zirconia. The material properties used in computations are listed in Table 1. For computation of power response curves, we take $\eta$ $=0.1$. The relation between $\sigma_{3}$ and $T$ is determined by curve fitting using the least squares.

\section{Thin Domain Approximation}

The 1D Helmholtz equation (1) can be solved exactly in terms of $T$ and the general solution has the form

$$
E_{j}(z)=a_{j} e^{i \xi_{j} z}+b_{j} e^{-i \xi_{j} z}
$$

where $\xi_{j}=\sqrt{k_{0}^{2}\left[\epsilon_{r_{j}}^{\prime}-i \frac{\sigma_{e_{j}}\left(T_{j}\right)}{\omega \epsilon_{0}}\right]}$, and the coefficients $a_{j}$ and $b_{j}$ depend on the boundary conditions (4)-(6). Under the thin-domain approximation $\eta \ll 1$, we can expand temperatures in the form of asymptotic series

$$
T_{j}=T_{j}^{(0)}+\eta T_{j}^{(1)}+\eta^{2} T_{j}^{(2)}+o\left(\eta^{3}\right) .
$$


Table 1. Properties of the Materials in Region 3 (Zirconia, [11]) and Region 2.

\begin{tabular}{cccccc}
\hline Region & $\epsilon_{r}{ }^{\prime}$ & $\sigma_{e}(\bar{T})[\mathrm{S} / \mathrm{m}]$ & $\rho\left[\mathrm{Kg} / \mathrm{m}^{3}\right]$ & $C_{p}[\mathrm{~J} / \mathrm{kgK}]$ & $k[\mathrm{~W} / \mathrm{mK}]$ \\
\hline 2 & $6.69-133.8$ & 0 & 2848 & 217 & 0.2 \\
3 & 6.69 & $0.00046 e^{2.285\left[\frac{\left(T-T_{A}\right)}{T_{A}}\right]}$ & 2848 & 217 & 0.2 \\
\hline
\end{tabular}

Substituting $T_{j}$ in (4)-(5), we get zero order terms and first order terms as

$$
\frac{\partial^{2} T_{j}^{(0)}}{\partial z^{2}}=0, \frac{\partial^{2} T_{j}^{(1)}}{\partial z^{2}}=0 .
$$

Applying boundary conditions (7)-(9), we see that both zero and first order solutions are independent on $z$, and the leading order solution can be written as

$$
T_{j}^{(0)}=T^{(0)}(t) \text {. }
$$

Second order correction term results in the system of equations

$$
\begin{gathered}
\alpha \frac{\partial^{2} T^{(2)}}{\partial z^{2}}=\frac{\partial T^{(0)}}{\partial t} \\
\frac{\partial^{2} T^{(3)}}{\partial z^{2}}=\frac{\partial T^{(0)}}{\partial t}-P \sigma_{3}\left(T^{(0)}\right)\left|E_{3}\left(T^{(0)}\right)\right|^{2} .
\end{gathered}
$$

Integrating $(10)$ over $(-l, 0)$ and $(11)$ over $(0,1)$, and applying boundary conditions (7)-(9), we get an ordinary differential equation governing average temperature in the form

$$
\left(\frac{k l}{\alpha}+1\right) \frac{\partial T}{\partial t}=P \sigma_{3}(T)|| E_{3} \|_{2}^{2}-B i T,
$$

where ||$E_{3} \|_{2}^{2}=\int_{0}^{1}\left|E_{3}(z)\right|^{2} d z,\left|E_{3}(z)\right|^{2}=E_{3}(z) E_{3}^{*}(z)$, and $E_{3}^{*}$ is the complex conjugate of $E_{3}$. At steady state, solution of (12) can be written down as

$$
P=\frac{B i T}{\sigma_{3}(T)|| E_{3} \|_{2}^{2}}, \quad \text { or } P_{\text {in }}=\frac{h\left(\bar{T}-T_{A}\right)}{\gamma l_{3} \sigma_{e_{3}}(\bar{T})|| E_{3} \|_{2}^{2}},
$$

where $T$ and $\bar{T}$ are now average non-dimensional and dimensional steady-state temperatures, respectively, $\sigma_{e_{3}}$ is the dimensional effective loss factor of region 3 , and $E_{3}$ is the nondimensional electric field in region 3. The second formula in (13) for the dimensional power density of the incident wave $P_{i n}=E_{0}^{2} / 2 \gamma$ (here $\gamma$ is the characteristic impedance of free space) is obtained by substitution of the dimensional quantities in the first formula. So (13) defines the power response curve as a parametric plot of $P$ (or $P_{i n}$ ) and $T$ (or $\bar{T}$ ). We see that the power responses are indeed independent of thermal properties of regions 2 and 3.

\section{COMSOL Multiphysics Model}

We now solve the coupled system (1)-(3) along with the boundary conditions (4)-(9) in COMSOL Multiphysics. We use quadratic Lagrange elements to spatially discretize the geometry and determine maximum size of the element according to the meshing criteria discussed in [10]. Also, due to a high degree of nonlinearity, we use COMSOL's adaptive time-dependent solver and steady-state is assumed to be reached when an absolute difference between average temperatures at previous and current time step falls below $10^{-4}$. This solver discretizes temporal gradients using second order backward difference method. To get a 


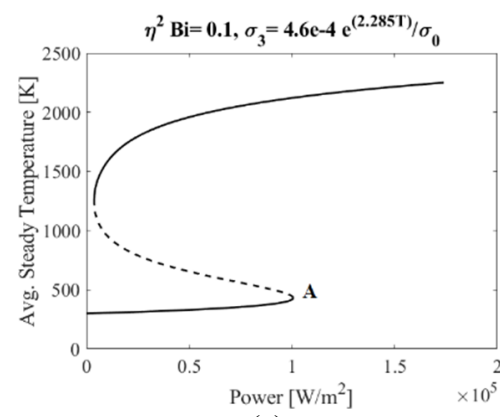

(a)

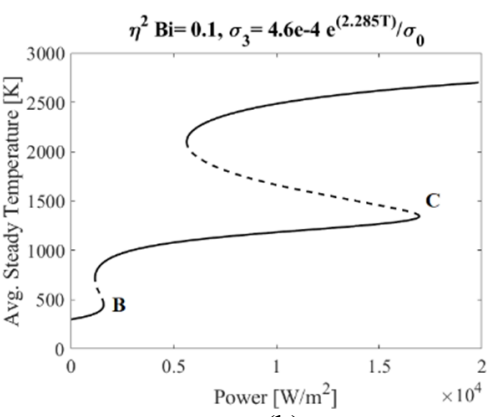

(b)

Fig. 2. Power response curves without (a) and with (b) an electric field resonance in region 3 for $\varepsilon_{2}=71$; stable (solid curves) and unstable solutions (dashed curves).

power response curve in the COMSOL model, simulation is performed for different values of $P_{i n}$, and the average steady-state temperature is plotted as a function of $P_{i n}$.

\section{Computational Results}

\section{A. Effect of Electric Field Resonance}

Gaone et al in $[8,9]$ thoroughly studied the effect of the electric field resonance on the power response curve of a layered structure. For a triple layer (lossless-lossy-lossless), a power response curve becomes a double $S$-curve if electric field resonance is achieved in the lossy layer. The resonance criteria considered in [8-10] are given for any odd multiple $n_{1}, n_{2}$ as

$$
l_{2}=\frac{n_{1} \lambda_{2}}{4} \text { and } l_{3}=\frac{n_{2} \lambda_{3}}{2}
$$

where $\lambda_{2}$ and $\lambda_{3}$ are the wavelengths of EM wave in region 2 and 3, respectively.

For the geometry considered in this paper, we follow the criteria (14) and choose $n_{1}=1$ and $n_{2}=3$. The ground/metal plate boundary acts as a perfect reflector of the EM waves in region 3 . The total reflection causes constructive interference between incident and reflected waves, and resonance builds up in the lossy layer. From Fig. 2, we see that as we achieve electric field resonance in the lossy layer, $S$-curve transitions into a double $S$-curve.

A branch of a power response curve is stable (or unstable) if it has a positive (or negative) slope [7]. It can be seen from Fig. 2(a) that, when the incident power of the EM wave is higher than critical power at point $\mathrm{A}$, thermal runaway is triggered there, but stabilized at the upper stable branch ( $2000 \mathrm{~K})$. However, from Fig. 2(b), we see that the double $S$-curve has a new stable (middle) branch between lower and upper branch. When incident power is above critical power at $\mathrm{B}$ but less than power at $\mathrm{C}$, thermal runway may stabilize at the middle branch $(\sim 1000 \mathrm{~K})$. We also notice that because of the electric field resonance, more EM energy is trapped inside the lossy layer as compared to a non-resonant case. Therefore, critical power levels (powers corresponding to points B and C) are significantly lowered.

\section{B. Comparison with COMSOL Model}

We now compare the power response curves generated by the analytical and COMSOL models. From Fig. 3, we see that, for $h=2.54 \mathrm{~W} /\left(\mathrm{m}^{2} \mathrm{~K}\right)$, the curves do not match well with each other. The disagreement is due to the differences in corresponding assumptions. The analytical model approximates the solution for a very thin and long channel assuming uniform temperatures in the Z-direction. On the other hand, COMSOL model considers spatial variation of temperature in $Z$. The effect of spatially varying temperature on the power response curve was discussed in [10]. We now maintain $h=0.64 \mathrm{~W} /\left(\mathrm{m}^{2} \mathrm{~K}\right)$, and 


\section{High Frequency Heating}

Valencia, Spain, September 9-12, 2019

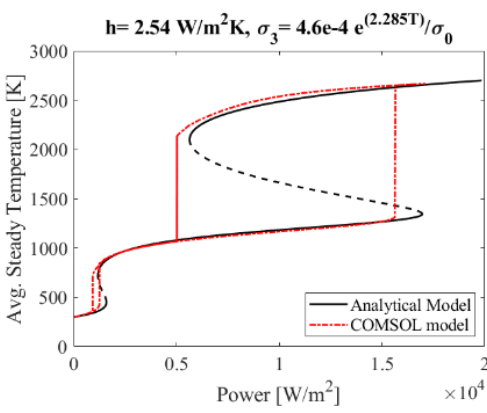

(a)

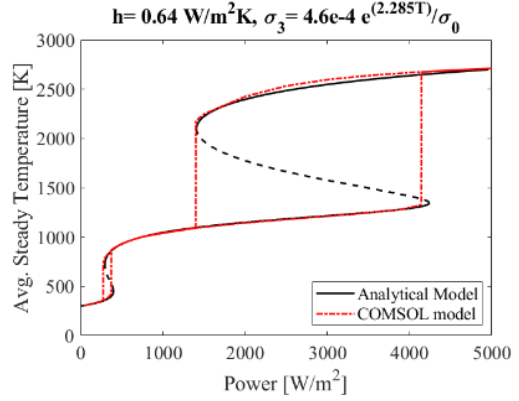

(b)

Fig. 3. Power response curves obtained by the analytical and COMSOL models for $\varepsilon_{r 2}=71, \eta^{2} B i=0.1(\mathrm{a})$, and $\eta^{2} B i=0.025$ (b).

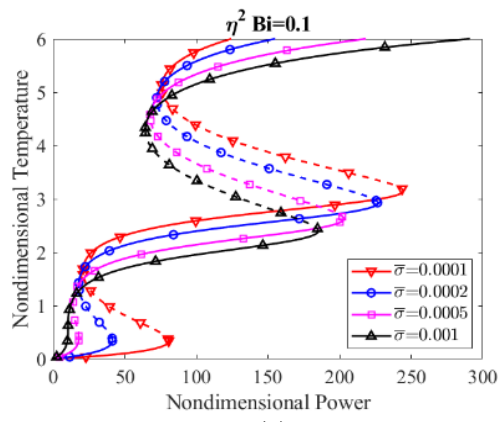

(a)

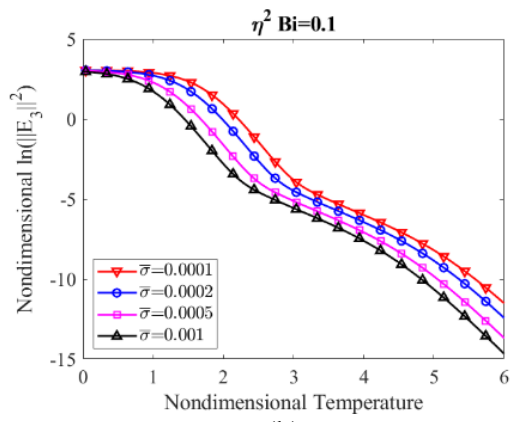

(b)

Fig. 4. Power response curves (a), and $\ln || E_{3} \|^{2}$ (b) as functions of temperature for $\beta=3, \varepsilon_{r 2}=71$ and varying factor $\bar{\sigma}$.

further limit thermal lossless to the surrounding making spatial variation of temperatures more uniform. We expect that as $h \rightarrow 0$, the response curves given by both models get closer. From Fig. 3(b), we observe that the power response curves are in satisfactory agreement.

\section{Parametric Study}

In this section we discuss how $\sigma_{3}, \eta^{2} B i$, and $\epsilon_{r_{2}}$ affect the shape of the power response curve. Throughout this parametric study, thermal properties are the same as in Table 1. For many typical ceramics, the loss factor increases exponentially with temperature, therefore, we assumed $\sigma_{3}$ to have a form of $\bar{\sigma} e^{\beta T}[8-10]$.

First, we hold $\beta$ constant and vary $\bar{\sigma}$. From Fig. 4(a) it is seen that when $\bar{\sigma}$ is increased, the region of unstable solution diminishes, and for $\bar{\sigma}=0.001$ the only stable solution is found in the low power region. To understand why an unstable solution disappears in low power region with increasing $\bar{\sigma}$, we take natural logarithm of both sides of (13) and take its derivative with respect to temperature

$$
\frac{d}{d T}[\ln (P)]=\frac{1}{T}-\beta-\frac{d}{d T}\left[\ln || E_{3}||^{2}\right] .
$$

A branch is stable (or unstable) if 


$$
\frac{1}{T}>(\text { or }<) \beta+\frac{d}{d T}\left[\ln || E_{3}||^{2}\right]
$$

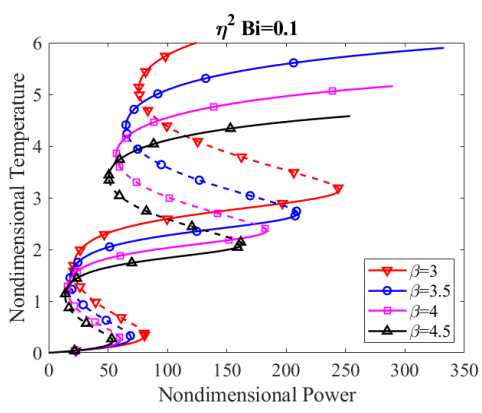

(a)

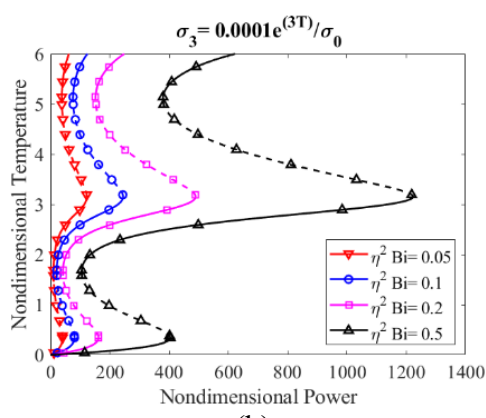

(b)

Fig. 5 Power response curves for $\epsilon_{r_{2}}=71$ and varying $\beta$ (a) and $\eta^{2} B i$ (b).

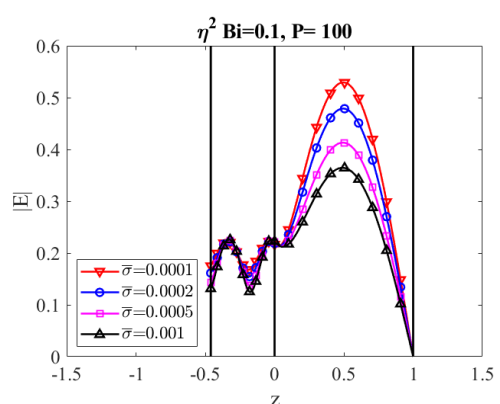

(a)

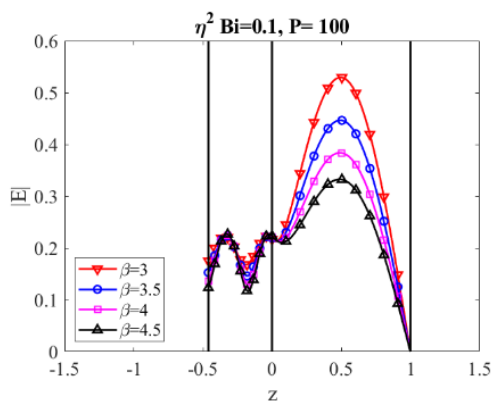

(b)

Fig. 6. Magnitude of the non-dimensional electric field as a function of $z$ for $\beta=3$ and varying $\bar{\sigma}$ (a) and for $\epsilon_{r_{2}}=71$ and varying $\beta$ (b).

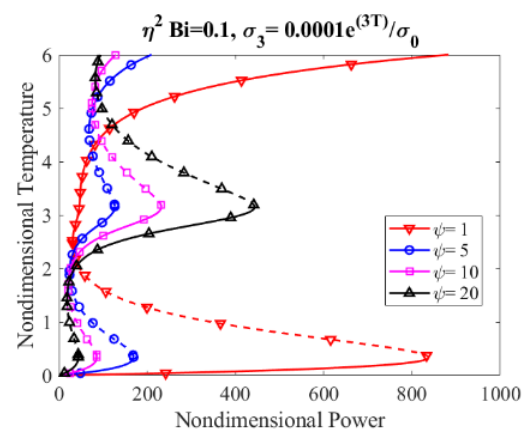

Fig. 7. Power response curves for varying $\psi=\epsilon_{r_{2}} / \epsilon_{r_{3}}$.

From Fig. 4(b), we notice that in the lower unstable temperature range, $-\frac{d}{d T}\left[\ln || E_{3} \|^{2}\right]$ is larger when $\bar{\sigma}=0.001$ compared to other cases. This makes combined contribution of $\beta$ 
and $\frac{d}{d T}\left[\ln || E_{3} \|^{2}\right]$ in (15) smaller than $1 / T$; as a result, only a stable solution exits in the lower temperature region. From (15), we observe that the range of stable temperatures may increase if $\beta$ is increased. We confirm this in Fig. 5(a), where the length of the stable branches increases with increasing $\beta$. It can be also seen that with increasing $\bar{\sigma}$ or $\beta$, the EM losses also increase, which causes $\left|E_{3}\right|$ to decrease, as seen in Fig. 6. Since less EM energy is available for the source of thermal energy, temperatures of the middle and top branches keep dropping down, as seen in Fig. 4(a) and Fig. 5(a).

Describing the effects of thermal losses to the surrounding, we see from Fig. 5(b) that as $\eta^{2} B i$ goes up, the length of the middle branch also increases. This behavior may be expected since we see from (13) that $P$ is proportional to $B i$. We define the ratio of permittivities of regions 2 and 3 as $\psi=\varepsilon_{r 2} / \varepsilon_{r}$. Keeping $\varepsilon_{r 3}$ constant, we change $\varepsilon_{r 2}$ by varying $\psi$. When $\varepsilon_{r 2}$ changes, the thickness of region 2 also changes by (14). From Fig. 7, we see that when $\psi=$ 1 , the electric field resonance breaks, and an $S$-curve emerges. We also observe that the length of the middle branch increases with $\psi$. This is expected because the strength of the resonance in region 3 is greatest when $\varepsilon_{2}$ is large and $\varepsilon_{r 3}$ is small $[8,9]$. Thus for a double $S$-curve to have a shape such that thermal runaway could be stabilized at the middle branch, having higher permittivity of the outer layer is preferable.

\section{Conclusions}

We have described two (1D analytical and numerical) models of a grounded two-layer system undergoing EM heating by plane waves. It has been demonstrated that the resonance producing a double $S$-curve can be achieved with a one-sided irradiation. Because of the electric field resonance, thermal runaway starts at lower power levels, but may stabilize at the middle branch of the double $S$-curve. With the thin-domain asymptotic approximation, the system of equations is reduced to a time-dependent ordinary equation. Its steady-state solution gives the power response curve. A numerical model has been built in COMSOL Multiphysics. Double $S$-curves generated by both the models are found to be in satisfactory agreement. We have shown that temperatures of the middle and upper branches may be decreased by increasing the electrical conductivity of the lossy layer as less EM energy is available for heat generation. It has been demonstrated that the length of the middle branch increases with thermal losses to the surrounding. Thus the resonance-based control is, in principle, possible for the considered structure (with the ground/metal surface on the back of region 3), but it depends on the relation between dielectric constants of regions 2 and 3 .

\section{Acknowledgement}

The authors are grateful for the support from the US Air Force Office of Scientific Research, Award FA9550-18-1-0528.

\section{References}

[1] Willert-Porada, M., Ed., Advanced in Microwave and Radio Frequency Processing, Springer, 2006.

[2] Tao, J., Ed., Microwave and RF Power Applications, Cépaduès Éditions, 2011.

[3] Leadbeater, N.E., Ed., Microwave Heating as a Tool for Sustainable Chemistry, CRC Press, 2010.

[4] Jawdat, B., et al, Proc. 2017 IEEE Wireless Power Transfer Conf., 978-1-50904595-3/17.

[5] Kumi, P., et al, In: Scientific Computing in Electrical Engineering, G. Nicosia, V. Romano, Eds, Springer, 2019 (in press).

[6] Kriegsmann, G.A., J. of Applied Physics, 1992, 71, 1960-1966. 
[7] Pelesko, J.A., G.A. Kriegsmann, J. of Eng. Math., 1997, 32, 1-18.

[8] Gaone, J.M., et al, J. of Eng. Math., 2019, 114, 65-86.

[9] Gaone, J.M., et al, IEEE MTT-S Intern Microwave Symp. Dig. (Honolulu, HI, June 2017), 978-1-5090-6360-4.

[10] Mohekar, A.A., et al, IEEE MTT-S Intern. Micro. Symp. Dig. (Philadelphia, PA, June 2018), pp. 1389-1392.

[11] V.V. Yakovlev, et al, In: Microwave and RF Power Applications, J. Tao, Ed., Cépaduès Éditions, 2011, pp. 303-306. 


\title{
A NEW CONCEPT TO IMPROVE MICROWAVE HEATING UNIFORMITY THROUGH DATA-DRIVEN PROCESS MODELLING
}

Jing Zhou, Yingguang Li*, Di Li

National Key Laboratory of Science and Technology on Helicopter Transmission, Nanjing University of Aeronautics and Astronautics, No.29, Yudao Street, Nanjing, China liyingguang@nuaa.edu.cn

Keywords: microwave processing, temperature distribution, heating pattern, neural network

\begin{abstract}
For a long time, the heating pattern of the workpiece within a multimode microwave oven was considered to be highly sophisticated. As a consequence, the uneven microwave heating problem can only be partly alleviated by a random movement between the electromagnetic field and the workpiece, like the rotating turntable or mode stirrers. In this paper, we reported that the heating behavior has a specific correspondence with the power ratio of multiple microwave sources under certain conditions. The influence factors of this relationship and the corresponding mechanisms were systematically studied by both theoretical analysis and experimental investigations. On this basis, a data-driven process model was introduced to learn the material's dynamic temperature behaviors during microwave heating process, and a new concept to improve the microwave heating uniformity by temperature monitoring and active compensation was presented.
\end{abstract}

\section{Introduction}

Microwave energy with the ability to rapidly heat dielectric media has been widely used in domestic and industrial fields [1]. However, the distribution of the electric field in a multimode applicator is usually not uniform, since it is a random combination of all the modes excited in a given frequency range [2]. It is just like the sun shine through the dense woods forming mottled reflections on the ground, without any regularity. In combination with a linear or nonlinear thermal response of dielectric absorption this may result in local thermal runway or cold spots in processed materials [3]. Major problems, such as poor end quality, microbial safety concerns, and overheating, are related to the non-uniform heating process [4].

Up to now, the relationship between temperature behaviors and microwave system settings has been rarely researched in the multimode applicator. Here, microwave system settings are parameters regarding power level, frequency and phase etc., of microwave sources. Traditional model-based method inevitably with a series of approximations and omissions for efficient computation, on the one hand, cannot accurately predict the temperature behaviors under a given boundary condition, especially in the case of heating anisotropic media and multi-scale problems included. On the other hand, the microwave system settings cannot be computed as well after the desired temperature behavior is given. As a result, existing methods attempt to solve the uneven heating problem mainly focused on the relative movement between the electric field and the processed materials. For example, mode stirs and variable-frequency microwave sources [5-6] are designed to change the static electric fields into dynamic ones [7-8], and the turntable is to move the processed object through the areas of high and low power fields alternately [9-10]. 
The above method can, to some extent, improve the temperature uniformity on the surface of the composite parts. However, with a random compensation effect, these methods cannot solve the problem in principle. In this paper, we validated that under certain conditions there is a fixed relationship between the heating behavior and the power ratio of multiple microwave sources from both theoretical analysis and experiment results. The influence factors of this relationship and the corresponding mechanisms were systematically studied as well. On this basis, a data-driven process model was introduced to learn the material's dynamic temperature behaviors during microwave heating process, and a new concept to improve the microwave heating uniformity by temperature monitoring and active compensation was presented.

\section{Experiment}

The microwave oven used in this experiment has an octagonal applicator with two microwave sources on each side, as shown in Fig.1 [11]. For each microwave source, the frequency is stabilized at $2.45 \mathrm{GHz}$ and the power range is $0 \sim 800 \mathrm{~W}$. A fiber optical fluorescence measurement system (Beijing DongFang RuiZe Technology Co., Ltd.) and an infrared thermal imager (FLIR A300) were integrated into the equipment for temperature measurement.

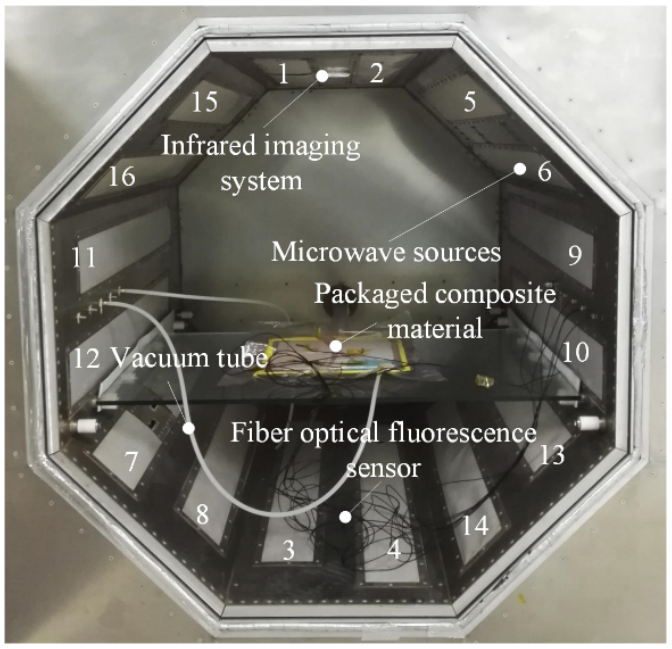

Fig.1 Microwave heating system used in this work.

A $250 \times 250 \times 2.5 \mathrm{~mm}^{3}\left([0]_{25}\right)$ carbon fiber composite laminate (nonmagnetic) was prepared and used as the workpiece in this work. Aluminum foils were stuck on the edge of the laminate to prevent arcing. The carbon fiber reinforced epoxy prepreg (UIN10000), with the thickness of $0.1 \mathrm{~mm}$, was purchased from Weihai Guangwei Composites Co., Ltd. The density and fiber volume fraction of this material were $1560 \mathrm{~kg} / \mathrm{m}^{3}$ and $60 \%$, respectively. The recommended curing temperature was $120^{\circ} \mathrm{C}$.

\section{Results and discussion}

At each time $k, k \geq 0$, any heating process can be described as:

$\mathbf{T}(k+1)=\mathbf{T}(k)+\mathbf{H R}(k) \cdot t_{k}$ 
where $\mathbf{T}(k+1)$ and $\mathbf{T}(k)$ are the temperature distribution at time $k+1$ and $k, \mathbf{H R}(k)$ and $t_{k}$ are the heating rate distribution adopted at time $k$ and its fuction time. Therefore, if $\operatorname{HR}(k)$ can be controlled to compensate the unven $\mathbf{T}(k)$, a uniform microwave heating process can be guaranteed. For convinience, the distribution of the heating rate (or heating rate ratio) on the surface of the workpiece was defined as heating pattern (HP). In order to realize an effective temperature control process, the influence factor of the HP and its influencing mechanism should be studied.

\subsection{Constant HP-MCS relationship in non-transforming materials}

For non-transforming materials (nonmagnetic), the heat transfer model during the microwave heating process can be considered as:

$\rho c_{p} \frac{d T}{d t}=2 \pi f \varepsilon^{\prime \prime} E^{2}+Q_{\mathrm{cd}}+Q_{\mathrm{cv}}+Q_{\mathrm{rd}}$

where $\rho, c_{p}$ and $\varepsilon^{\prime \prime}$ are the density, specific heat capasity and dielectric loss factor of the material; $d T / d t$ is the heating rate; $f$ is the micorwave frequency; $E$ is the electric field strength. $Q_{\mathrm{cd}}, Q_{\mathrm{cv}}$ and $Q_{\mathrm{rd}}$ are the heat flux due to conduction, convection and radiation which usually can be considered as constants or even ignored compared with the absorbed microwave power, when the material properties remain constant. Thus, the HP is directly proportional to the distribution of the absorbed microvave power [11].

To investigate the influence factor of the HP, a large number of heating experiments were carried out under different conditions. Firstly, single microwave source (No.1 to No.16) was used to heat the composite laminate. For each feeding source, the heating experiment was repeated for three times. For each heating process, the maximum temperature was limited below $55^{\circ} \mathrm{C}$ to ensure that the polymerizing reaction of the composite was not triggered and the material properties can be approximated as constants (i.e., non-transforming materials). When the temperature was dropped to room temperature, another heating process was started. Fig. 2 shows the representative HP under the single microwave source. It can be seen that the temperature distribution is extremely uneven where many hot spots and cold spots exist without any regularity. However, the temperature distribution or HP is almost unchanged when the microwave source is confirmed. The above phenomenon can be explained by the following equation which considers the case of a rectangular cavity.

$f_{m n l}=\frac{1}{2 \sqrt{\mu \varepsilon}} \sqrt{\left(\frac{m}{\mathrm{a}}\right)^{2}+\left(\frac{n}{b}\right)^{2}+\left(\frac{l}{d}\right)^{2}}$

where $f_{m n l}$ is the resonant frequency of the electromagnetic modes that can be excited in the microwave cavity; $\mu$ and $\varepsilon$ denote the distribution of the permeability and permittivity for the medium inside the cavity; a,$b$ and $d$ represent the dimension of the cavity in the $x, y$ and $z$ direction; $m, n$ and $l$ are integers which determine the field type of electromagnetic modes. Once the microwave cavity and the object being heated are chosen, the dimension of the cavity (,$b$ and $d$ ), the distribution of the permeability $\mu$ and permittivity $\varepsilon$ are constants, and the field type of electromagnetic modes ( $m, n$ and $l$ ) only depends on the working state of each microwave source including power level, frequency and phase etc. Among them, the control of microwave frequency or phase is very expensive by using traveling-wave tubes and solid-state amplifiers. As an alternative, the power control of microwave sources is a much more economic and feasible way, since most 
industrial microwave heating devices are equipped with multiple magnetrons and it is precisely these equipment that need high heating uniformity. For convenience, the power (or power ratio) of multiple microwave sources are defined as microwave control strategy (MCS). For single microwave source, the electric field directly depends on its location on the resonant cavity. This is the reason why the HP remains unchanged for the same feeding source but was different between different feeding sources (see Fig.2). Additionally, a linear increase in temperature can be observed during each heating process, except for some influence caused by heat dissipation at relatively high temperatures. That is, the heating rate remains constant when the power of the feeding source remains constant, which demonstrates that the effect of the heat conduction, convection and radiation can really be considered as constants or ignored. Considering the space limitation, only the results of No.5 and No.15 microwave source are given in this paper. Similar results were also reported by Sun who used the silica gel membrane (non-transforming) as the material [12].
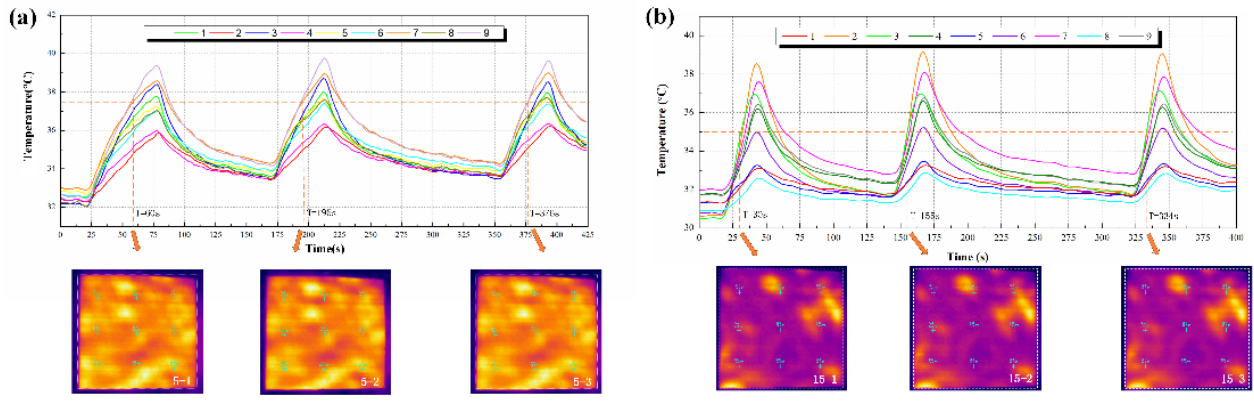

Fig.2 Heating pattern under source No.5 (a) and No.15 (b).

To investigate the situation where multiple sources are included, similar heating experiments with multiple sources were carried out. Representative HP can be found in Fig.3. It can be seen that the result was similar to that of the single source heating experiment. As mentioned above, if the properties of the workpiece remain constant, a fixed HP can be excited by a specific MCS, i.e., $\mathrm{F}(\mathbf{M C S})=\mathbf{H P}$.

In order to further understand whether different feeding sources will interfere with each other, the HP generated by multiple sources was compared with the algebraic sum of the HP under the involved individual feeding source. As shown in Fig.4, various degrees of difference between these two cases can be clearly observed. If only a few feeding sources, for example two or three, work together, the difference between the two curves is small. However, as the number of the feeding source increases, an obvious interference appears between different sources. For instance, a big difference exists in Fig.4 (d) on both the value and the trend between the two curves. This phenomenon can be explained by the power superposition principles. 


\section{High Frequency Heating}

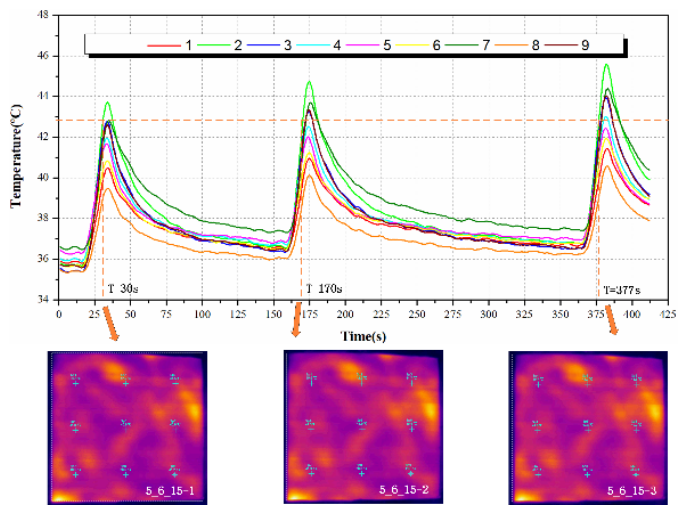

Fig.3 Heating pattern under the combination of source No.5, No.6 and No.15.
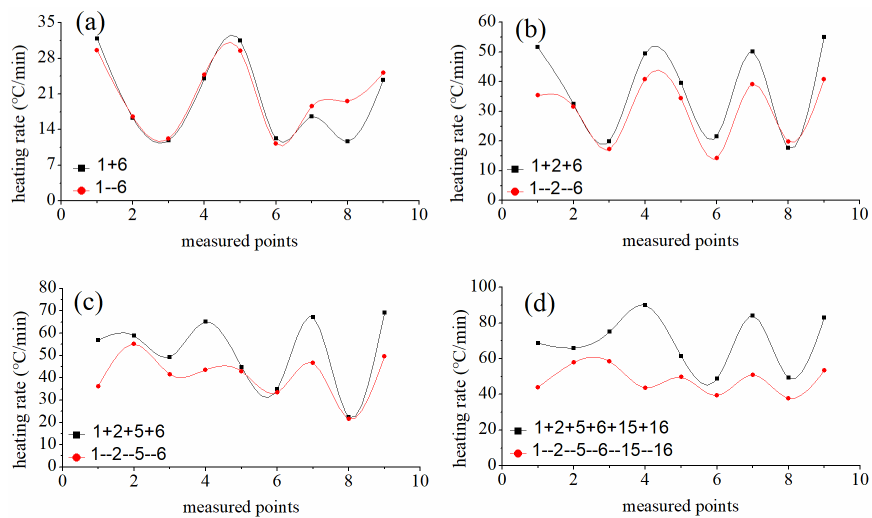

Fig.4 Results of the power superposition experiment. $M--N$ means the HP was generated by source $M$ and source $N$ working together. $M+N$ means the algebraic sum of the HP under source $M$ and source $N$ individually.

Generally speaking, the power superposition principles can be classified into two types, i.e., scalar superposition principle and vector superposition principle. In the first situation, the microwave from different sources is incoherent. Consider the microwave heating system used in this experiment with 16 sources, the total power $P_{i}$ at the point $i$ can be expressed as:

$P_{i} \propto\left|E_{i}^{1}\right|^{2}+\left|E_{i}^{2}\right|^{2}+\mathrm{L}+\left|E_{i}^{16}\right|^{2}$

where $E_{i}^{j}$ is the electrical field provided by source $j$ at the location $i$. In this case, the total power or heating rate at each point on the composite surface is linear with the microwave power provided by each feeding source. This is related to the situation where only a few microwave sources are involved and little probability of interference between microwave sources can be resulted. In the second situation where the microwave from different sources will couple with each other, the total power $P_{i}$ at the point $i$ can be expressed as:

$P_{i} \propto\left|\mathbf{E}_{i}^{1}+\mathbf{E}_{i}^{2}+\mathrm{L}+\mathbf{E}_{i}^{16}\right|^{2}$ 
This clearly indicates that the power superposition is no longer linear, and is more appropriate for the situation where multiple microwave sources are included. As mentioned above, we believe that both the scalar superposition principle and vector superposition principle exist during the microwave heating process. As the number of the operating source increases, the vector superposition contributes more than the scalar one.

It should be noted that in above experiments all microwave sources were worked at the same power level. In the situation where multiple feeding sources work with different power ratios, it can be easily deduced that the HP will vary a lot as well. This is because the magnitude of the electromagnetic mode contributed by each feeding source is inconsistent, although the type and the number of the electromagnetic mode are confirmed once the combination of operating sources is selected.

\subsection{Dynamic HP-MCS relationship in phase change materials}

For phase change materials (nonmagnetic) like polymer composites, the HP will be further influenced by the exothermic curing reaction of the resin matrix.

$\rho c_{p} \frac{d T}{d t}=2 \pi f \varepsilon^{\prime \prime} E^{2}+Q_{\mathrm{cd}}+Q_{\mathrm{cv}}+Q_{\mathrm{rd}}+\rho H_{u} \frac{d \alpha}{d t}$

where $H_{u}$ is the ultimate enthalpy of cure of the composite; $\alpha$ is the degree of cure. In order to investigate how much influence can be resulted during the phase change materials' heating process, a complete microwave curing process was carried out under a fixed MCS. As shown in Fig.5, unlike the heating process within the confined temperature range, an obvious variation in the distribution of the hot spots and cold spots can be observed during the curing process, especially when the temperature of the composite approaches to $120^{\circ} \mathrm{C}$. This is becaused when the curing temperature is reached, the exothermic polymerizing reaction will be triggered for the resin matrix. On the one hand, the released heat will disturb the oringnal HP. On the other hand, basic parameters such as the specific heat capacity, thermal conductivity, permitivitty etc., will change rapidly with the degree of cure in this process.

For the purpose of intuitively reflecting the dynamics during the composite microwave curing process, the curing behaviour of the used composite material was characterized by the differential scanning calorimeter (DSC, Netzsch 204F1), while the specific heat capacity and the thermal conductivity were characterized by TA Q2000 and Netzsch LFA 457. Corresponding results are shown in Fig. 6 which clearly demonstrate that an amount of heat will be released during the composite curing process, accompanying with the variation of some basic properties. What's worse, the change of the material properties will influence the electric field in return according to Eq. 2 and Eq.6. It is these problems that make it almost impossible to accurately predict the HP under a given MCS using the traditional modelbased methods, and vice versa. As mentioned above, the dynamic HP-MCS relationship in phase change materials should be modified to $\mathrm{F}_{\alpha}(\mathbf{M C S})=\mathbf{H P}$. That is, the function $\mathrm{F}_{\alpha}$ holds for a specific value of degree of cure $\alpha$ in a small time interval. 


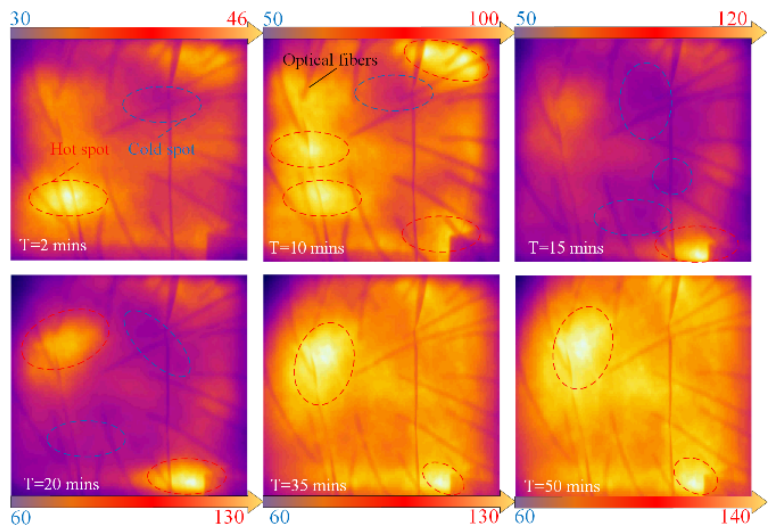

Fig.5 Temperature distribution during the composite microwave curing process under a fixed MCS.
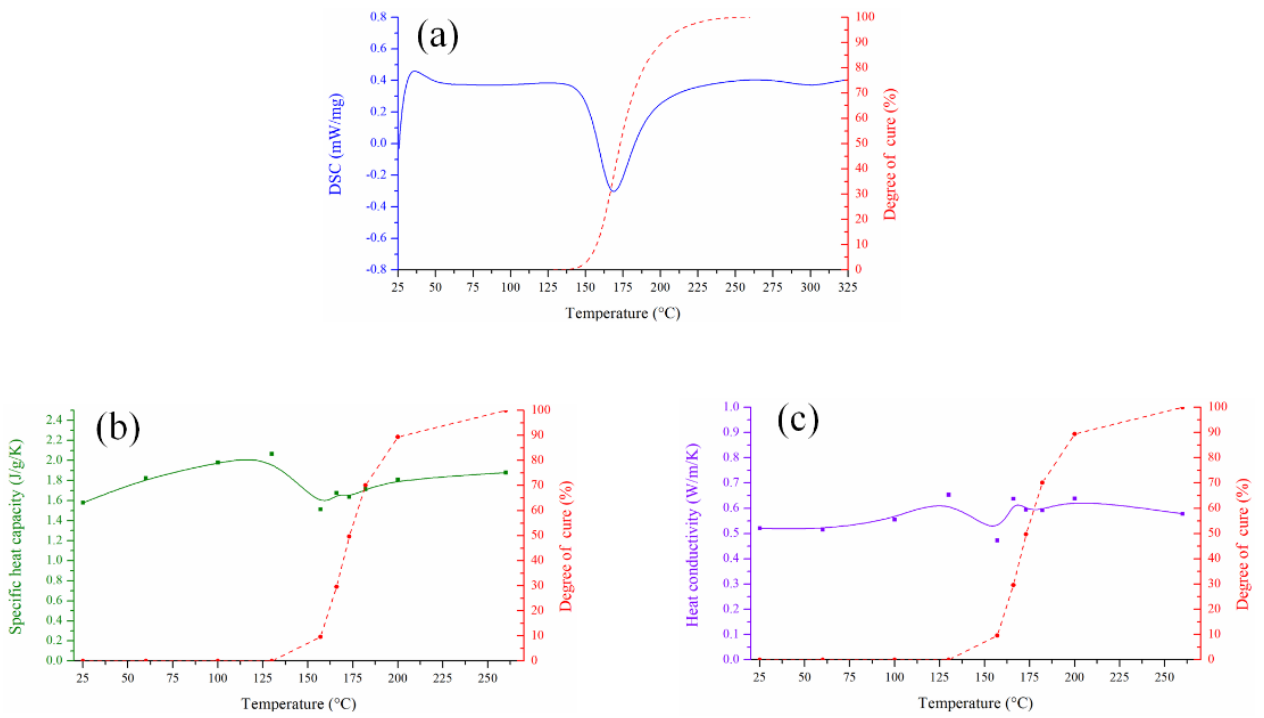

Fig.6 Dynamic properties during the composite curing process: (a) heat generation and variation of degree of cure; (b) variation of the specific heat capacity; (c) variation of the thermal conductivity (perpendicular to fiber direction).

\section{Our solutions for active heating uniformity control}

Based on the above findings, we proposed an online learning based intelligent temperature control method to realize a homogeneous microwave curing process [13]. In this method, a trainable neuron network kept learning the dynamic HP-MCS relationship through analyzing a series of (HP,MCS) datapairs collected online, which continuously provided accurate solutions to the uneven temperature distribution monitored in real time by active compensation. Experimental results indicated that the average temperature difference of a carbon fiber/bismaleimide composite plate $\left(500 \times 400 \times 2 \mathrm{~mm}^{3}\right)$ can be reduced by $\sim 42.3 \%$ compared with existing representative methods. 


\section{Conclusion}

The relationship between HP and MCS was constructed. The influence factors of this relationship and the corresponding mechanisms were systematically studied by both theoretical analysis and experimental investigation. On this basis, a new concept to improve microwave heating uniformity through data-driven process modelling was presented. Our method may pave the way for realizing a homogeneous microwave heating process.

\section{References}

1. Zhong J., Liang S., Yuan Y., et al. IEEE Transactions on Microwave Theory and Techniques, 2016, 64(8), 2467-2477.

2. Domínguez-Tortajada E., Monzó-Cabrera J., Díaz-Morcillo A. IEEE transactions on microwave theory and techniques, 2007, 55(1), 85-91.

3. Link G., Ramopoulos V. Chemical Engineering and Processing-Process Intensification, 2018, 125, 334-342.

4. Vadivambal R., Jayas D S. Food and bioprocess technology, 2010, 3(2), 161-171.

5. H.S.L., Ku T., Yusaf. Progress in Electromagnetics Research B, 2008, 5, 185-205.

6. C. Davis, R. Tanikella, T. Sung, et al. Electronic Components and Technology Conference, 2003 Proceedings, 53 ${ }^{\text {rd }}$, IEEE, 2003, 1718-1723.

7. Plaza-González P., Monzó-Cabrera J., Catalá-Civera J M., et al. IEEE Transactions on Microwave Theory and Techniques, 2005, 53(5), 1699-1706.

8. Sebera V., Nasswettrová A., Nikl K. Drying Technology, 2012, 30(13), 1388-1396.

9. Geedipalli S S R., Rakesh V., Datta A K. Journal of Food Engineering, 2007, 82(3), 359-368.

10. Chen J., Pitchai K., Birla S., et al. Food and bioproducts processing, 2016, 99, 116 127.

11. Zhou J., Li Y., Li N., et al. Composites Part A: Applied Science and Manufacturing, 2018,107,10-20.

12. Sun Y., KIT Scientific Publishing, 2016, 10.5445/KSP/1000051503.

13. Zhou J., Li Y., Li D., Chemical Engineering Journal, 2019, 370, 455-465.

\section{Acknowledgements}

This project was supported by National Science and Technology Major Project of China (Grant no. 2017ZX04002001). 


\title{
MILLIMETER WAVE ABSORPTION IN HYDROXYAPATITE AND 3YSZ CERAMICS IN WIDE TEMPERATURE RANGE
}

\author{
A. Eremeev, S. Egorov, V. Kholoptsev \\ Institute of Applied Physics, Russian Academy of Sciences, 46 Ulyanov str., Nizhny \\ Novgorod, 603950, Russia \\ aeremeev@appl.sci-nnov.ru
}

Keywords: millimeter wave, absorption, ceramics

\section{Introduction}

In the field of ceramic-based materials processing the last three decades has been marked by significant academic and industry interest in Additive Manufacturing (AM) technology due to its capability to produce ceramic parts with complex geometry and customizable materials properties. Conceptually, AM technology is a layer-by-layer fabrication of three dimensional physical parts directly from computer-aided design [1]. Solidification of the parts prepared from substances containing ceramic powder may be performed either by conventional heat treatment of a part as whole or by directed energy deposition. Both these strategies can be implemented using gyrotron-based millimeter-wave facilities allowing alternatively both the uniform heating of large-size parts in multi-mode cavities and local heating by focused wave-beams [2].

AM technology proves a growing potential in biomedicine. Hydroxyapatite and yttriastabilized zirconia-based ceramics are widely used in biomedical applications due to their high biocompatibility. The knowledge of their microwave (MW) absorption variation with temperature and porosity as the materials are densified, is necessary to optimize the scheme of microwave heating.

Results of microwave absortption measurements in powder compacts of hydroxyapatite and yttria-stabilized zirconia are presented in this paper.

\section{Experimental procedure}

The samples in the shape of disks $8 \mathrm{~mm}$ in diameter and about $2.5 \mathrm{~mm}$ in thickness were compacted by uniaxial pressing under a pressure of $200 \mathrm{MPa}$ from commercially available hydroxyapatite (HA) GAP-85d (Polistom, Russia), and yttria-stabilized zirconia (3YSZ) powder (Tosoh corp., Japan). Phase composition of 3 YSZ was $12.5 \%$ of monoclinic and $87.5 \%$ of tetragonal phase. Green densities of the samples were $1.56 \mathrm{~g} / \mathrm{cm}^{3}(49.3 \%$ of theoretical value) for HA and $2.86 \mathrm{~g} / \mathrm{cm}^{3}$ (47.3\% of theoretical value) for $3 \mathrm{YSZ}$.

The measurements were performed at $24 \mathrm{GHz} 3 \mathrm{~kW}$ gyrotron system [2, 3]. For comparison the measurements of complex dielectric permittivity of hydroxyapatite at frequency of 180 $\mathrm{GHz}$ were made by spectrometer based on a high-Q $\left(\mathrm{Q}_{0} \sim 10^{6}\right)$ open Fabry-Perot resonator [4]. Phase composition of the samples was analysed with X-ray difractometer Rigaku Ultima IV.

Samples for measurements were placed into the gyrotron system applicator and surrounded with low loss porous alumina based thermal insulation (AL-30, ZIRCAR Ceramics, USA). Some experiments with preliminary sintered samples were performed without thermal insulation.

Schematic of the applicator and the sample position in it are shown in Fig.1. Sample temperature was measured with B-type thermocouple. Applicator door was equipped with a water cooled optical window made as a double quartz glass with flowing water inside. 

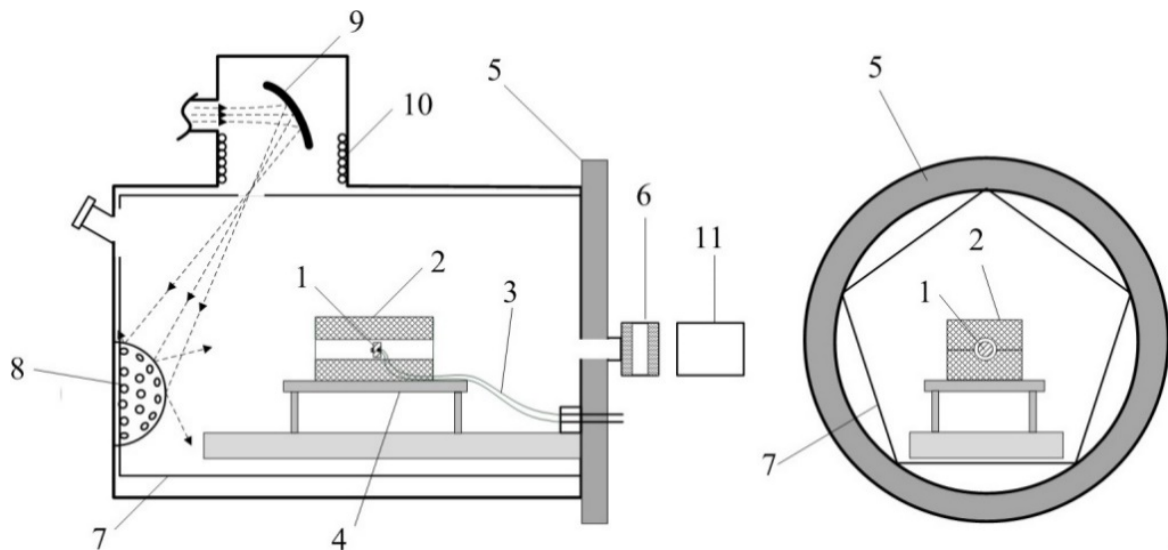

Fig. 1. Schematic of Gyrotron System Applicator: specimen (1), thermal insulation (2), thermocouple (3), support table (4), applicator door (5), optical window (6), pentaprism

(7), mode stirrer (8), focusing mirror (9), MW absorber (10), infrared camera (11).

The thermal insulation had a through hole of $10 \mathrm{~mm}$ in diameter allowing observation of the sample with infrared CMOS camera 3iCube IC4133IR (New Electronic Technology, Germany). Transfer and recording to a computer, and the display on the monitor were carried out using the software Stream Pix 7 (NORPIX, Canada). Thus, the design of the applicator and insulation allowed performing optical measurements of both the sample size and temperature distribution over the sample surface.

Upper part of the applicator contains a metal mirror directing the microwave radiation into a main chamber of the applicator through a coupling hole of $50 \mathrm{~mm}$ in diameter, and a microwave absorber based on a set of Teflon tubes with flowing water which absorbs the scattered radiation. The power of the radiation absorbed in the upper chamber of the applicator is measured by using a system consisting of water temperature meters at the input and output of the absorber, as well as a calibration heater with known power.

The applicator is equipped with a mode stirrer, representing an excentrically rotating hemisphere with hemispherical hollows drilled in it. Pentaprism shape of the applicator main chamber inner surface along with rotation of the mode stirrer provides uniform microwave field distribution over the volume of the applicator.

Measurements were made by calorimetric method, when microwave power absorbed in the sample is determined basing on the differences of the heating/cooling rates and MW power densities at the moments of intentional abrupt change of the microwave power at different sample temperatures. The method allows to measure absorption coefficients in real time during the sintering process. An example of the hydroxyapatite sample heating is presented in Fig. 2. Required temperature-time dependence was loaded to the gyrotron system control computer before start of the heating process. This dependence was automatically maintained by the gyrotron system control unit but from time to time an operator manually abruptly reduced the value of high voltage applied to the gyrotron in order to lower MW power fed the applicator. After some time (about $20 \mathrm{~s}$ ) the operator returned the control unit to the automatic control regime, and the sample temperature is automatically increased up to prescribed value. Then the procedure was repeated at the next value of the sample temperature. 


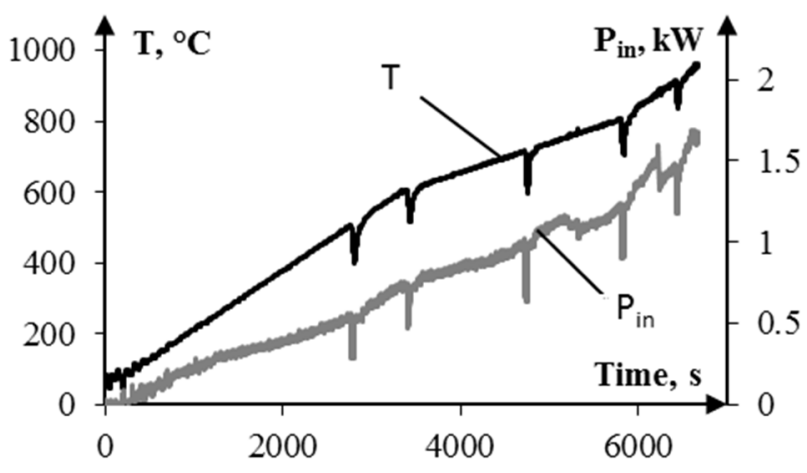

Fig. 2. Heating of hydroxyapatite sample: sample temperature (T), MW power fed the applicator $\left(\mathrm{P}_{\text {in }}\right)$.

\section{Results and discussion}

Energy balance equation is:

$C_{s} m\left[\frac{d T}{d t}\right]_{b(a)}=\alpha \Pi_{b(a)} S_{\text {sample }}-P_{\text {loss }}$,

where $C_{s}$ is specific heat capacity of the sample material, $\mathrm{m}$ is a mass of the sample, $\left[\frac{d T}{d t}\right]_{b(a)}$ is a heating/cooling rate, $\alpha$ is an absorption coefficient, $\Pi_{b(a)}$ is a MW power density incident to the sample, $\mathrm{S}_{\text {sample }}$ is a surface area of the sample, $\mathrm{P}_{\text {loss }}$ is a power in heat losses, subscripts $b$ and $a$ denote moments of time just before and just after the intentional change of MW power correspondingly.

Heat capacities $C_{s}$ as a functions of temperature were taken from [5] for HA, and from [6, 7] for 3 YSZ.

From (1) the absorption coefficient can be expressed as:

$\alpha=C_{s} m \frac{\left[\frac{d T}{d t}\right]_{b}-\left[\frac{d T}{d t}\right]_{a}}{\left(\Pi_{b}^{T E}-\Pi_{a}^{T E}\right) S_{\text {sample }}}=C_{s} m \frac{\left[\frac{d T}{d t}\right]_{b}-\left[\frac{d T}{d t}\right]_{a}}{\psi\left(\Pi_{b}^{a p p}-\Pi_{a}^{a p p}\right) S_{\text {sample }}}$

Here $\Pi_{b(a)}^{T E}$ are MW power densities inside the thermal insulation, $\Pi_{b(a)}^{a p p}$ are the power densities outside the thermal insulation, in the volume of the applicator, and $\psi$ is a ratio of MW power inside the thermal insulation to the power outside the thermal insulation:

$\psi=\frac{\Pi_{b(a)}^{T E}}{\Pi_{b(a)}^{a p p}}$

Power density in the volume of the applicator can be evaluated basing on its Q-factor:

$\Pi^{a p p}=\frac{c P_{\text {in }}}{\omega V_{\text {app }}} Q$

where $\mathrm{c}$ is the speed of light in free space, $\mathrm{P}_{\text {in }}$ is MW power fed the applicator, $\omega$ is the angular microwave frequency, $\mathrm{V}_{\text {app }}$ is the volume of the applicator, $\mathrm{Q}$ is the $\mathrm{Q}$-factor.

Q-factor for empty applicator can be calculated theoretically:

$\frac{1}{Q_{\text {empty }}}=\frac{1}{Q_{\text {ohm }}}+\frac{1}{Q_{c}}+\frac{1}{Q_{w}}$,

where $Q_{\text {ohm }}$ is ohmic Q-factor related to the absorption of microwave radiation in the applicator walls, $\mathrm{Q}_{\mathrm{c}}$ and $\mathrm{Q}_{\mathrm{w}}$ are coupling $\mathrm{Q}$-factors determining by the loss of MW radiation in the coupling hole and optical window correspondingly.

The Q-factors can be calculated by formulas [8]:

$Q_{c}=\frac{8 \pi V_{a p p}}{\lambda S_{c}}, \quad Q_{w}=\frac{8 \pi V_{a p p}}{\lambda S_{w}}, \quad Q_{o h m}=\frac{V_{a p p}}{s_{a p p} \delta_{s}}$, 
where $\lambda$ is a wavelength of MW radiation, $S_{c}$ and $S_{w}$ are the surface areas of the coupling hole and optical window correspondingly, $S_{\text {app }}$ is a surface area of the applicator, $\delta_{s}=c / \sqrt{2 \pi \omega \sigma}$ is a skin depth, $\sigma=1.49 \cdot 10^{7}(\mathrm{Ohm} \cdot \mathrm{m})^{-1}$ is the specific conductivity of the chamber wall material (aluminium alloy). Substituting the applicator parameters $\left(\mathrm{V}_{\text {app }}=5.7 \cdot 10^{4} \mathrm{~cm}^{3}, \mathrm{~S}_{\text {app }}=9 \cdot 10^{3} \mathrm{~cm}^{2}\right)$ one can obtain:

$Q_{\text {empty }}=2.5 \cdot 10^{4}$

Q-factor of the applicator can be estimated in real time during the heating process using the approach described in [3]. According to the definition of the Q-factor:

$Q_{i}=\frac{W \omega}{P_{i}}$,

where $\mathrm{Q}_{\mathrm{i}}$ is the $\mathrm{Q}$-factor of the $i$-th loss channel, $\mathrm{W}$ is the electromagnetic energy stored in the applicator volume, and $\mathrm{P}_{\mathrm{i}}$ is the power going to the $i$-th loss channel, one can conclude: $Q=Q_{c} \frac{P_{c}}{P_{\text {in }}}$,

where $\mathrm{P}_{\mathrm{c}}$ is $\mathrm{MW}$ power lost in the coupling hole, $\mathrm{P}_{\mathrm{in}}$ is a microwave power injected to the applicator.

As the upper part of the applicator is equipped with effective MW power absorber along with the system for measuring the absorbed power, the value of $\mathrm{P}_{c}$ was measured in real time during each experiment. The value of the power injected to the applicator $\mathrm{P}_{\text {in }}$ was determined from the gyrotron operating parameters basing on preliminary calibration experiments. Obtained by this approach Q-factor for empty furnace was $\mathrm{Q}_{\text {empty }}=(2.2 \pm 0.1) \cdot 10^{4}$, which is in very good agreement with the theoretical value (7). Typical dependence of applicator Qfactors on the sample temperature, when the applicator is loaded with both the sample and the thermal insulation is shown in Fig. 3 (a). It is seen that the values of Q-factor of the loaded applicator are close to the Q-factor of empty applicator.

After the Q-factor of the applicator is known, MW power density in the volume of the applicator can be found according to (4), but as it was mentioned above, MW power density inside the thermal insulation could differ from the power density outside the thermal insulation.

The following procedure was used to evaluate MW power density inside the thermal insulation. At the first stage, the samples of both composites were sintered in gyrotron system using described above thermal insulation. At the second stage the sintered samples were placed in the thermal insulation and heated in the regimes similar to shown in Fig. 2. At the last, third stage, the same samples were heated without thermal insulation, when the sample was fixed in open space with a purposely bent thermocouple wires. To reach high temperatures the last heating was made in vacuum, with a residual pressure of $15 \mathrm{~Pa}$. When heating without thermal insulation the values of absorptivity of the sintered samples $\alpha_{s}(T)$ can be defined from formula (2) with $\psi=1$.

Knowing this absoprtivity allows to evaluate the value of $\psi$ from (2), when heating the same sintered sample in the thermal insulation:

$\psi=C_{s} m \frac{\left[\frac{d T}{d t}\right]_{b}-\left[\frac{d T}{d t}\right]_{a}}{\alpha_{s}(T) \cdot\left(\Pi_{b}^{a p p}-\Pi_{a}^{a p p}\right) S_{\text {sample }}}$,

where $\alpha_{s}(T)$ is a value of absorption coefficient of the sintered sample, which was defined from experiments without thermal insulation.

The dependence of $\psi$ on the sample temperature is presented in Fig. 3 (b) for both compositions: hydroxyapatite (HA) and yttria-stabilized zirconia (3YSZ).

Absorptivity of initially raw samples were evaluated basing on (2) with ratio $\psi$ defined above. During heating the samples shrunk because of sintering process and the value of the sample surface area $\mathrm{S}_{\text {sample in }}(2)$ decreased. 


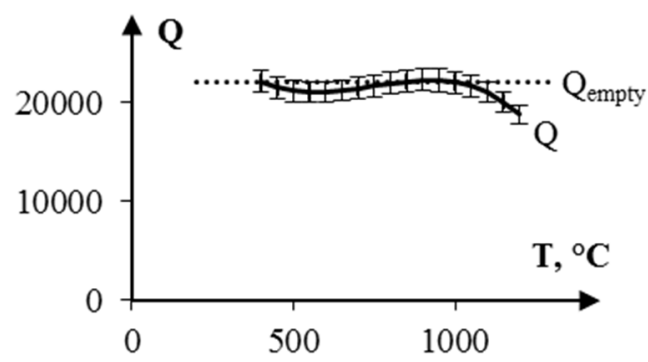

(a)

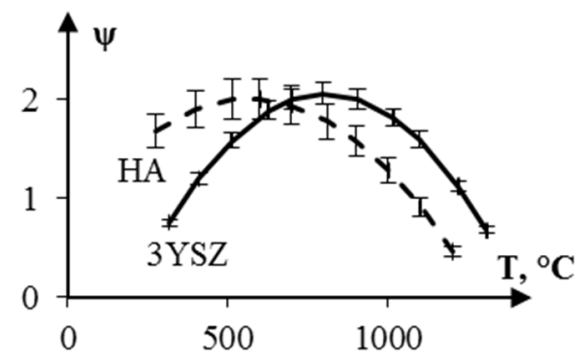

(b)

Fig. 3. Q-factor of the applicator (a) and ratio $\psi\left(\right.$ b) versus sample temperature, $Q_{\text {empty }}$ is Qfactor of empty applicator.

Basing on optical images from CMOS camera the sample diameter was measured in real time, allowing substitution of the real time values of $S_{\text {sample }}$ to (2).

Finally, as all parameters in (2) have been defined, the absorptivities of raw samples of the both compositions were obtained. The results along with the absorptivities of sintered samples are presented in Fig. 4.

Calculated above absorptivities depend not only on the materials properties, but also on the shape and dimensions of the samples. To reveal the intrinsic properties of the materials one should know the electric field inside the sample. For the samples with dimensions on the order of the wavelength and at isotropic irradiation as a rough estimation the electric field in the volume of the sample can be considered approximately equal to the value of electric field outside the sample. In this case:

$\alpha \Pi S_{\text {sample }}=\frac{\omega}{8 \pi} \varepsilon^{\prime \prime}|E|^{2} V_{\text {sample }}$,

where $\Pi=\frac{c}{8 \pi}|E|^{2}$ is MW power density, $\varepsilon^{\prime \prime}$ is an imaginary part of the complex permittivity, $\mathrm{E}$ is an electric field of the electromagnetic wave.

From (11) it is easy to get:

$\varepsilon^{\prime \prime}=\frac{c}{\omega h_{\text {sample }}} \alpha$,

where $h_{\text {sample }}$ is a thickness of the sample. Note that $h_{\text {sample }}$ decreases during the sintering process of raw samples.

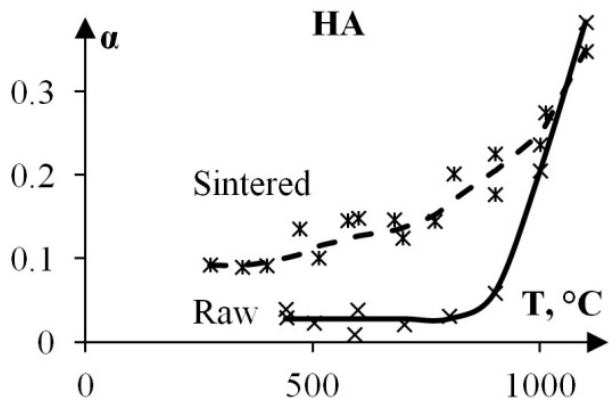

(a)

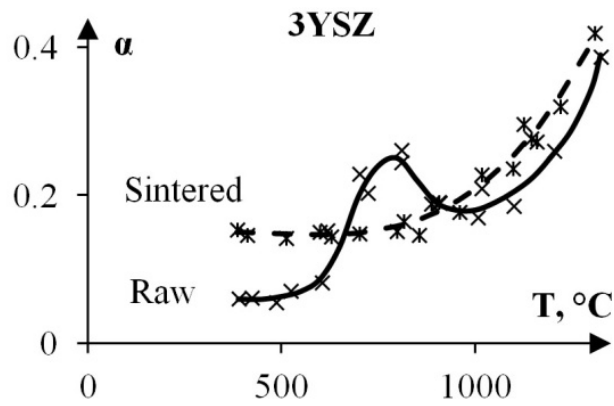

(b)

Fig. 4. Samples absorptivity: HA (a), 3YSZ (b), raw samples (solid lines), sintered samples (dashed lines). 


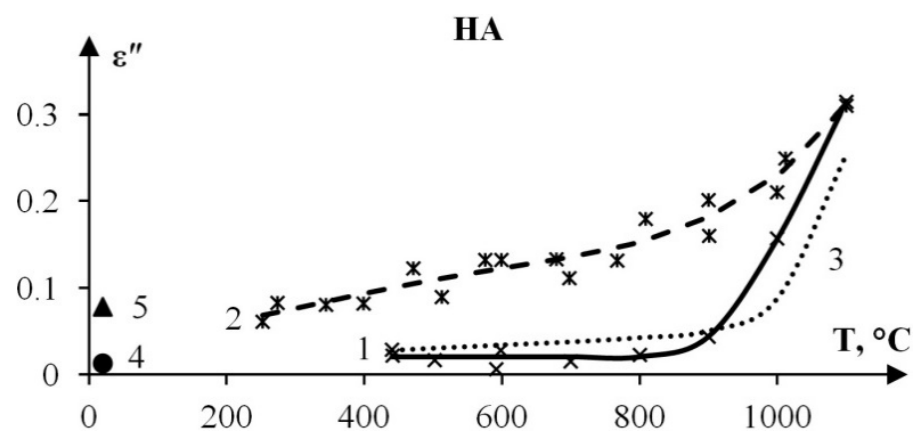

Fig. 5. Imaginary part of hydroxyapatite permittivity: raw samples (1), sintered samples (2), Effective Medium Approximation (3), raw sample at $180 \mathrm{GHz}$ (4), $180 \mathrm{GHz}$ sintered sample at $180 \mathrm{GHz}(5)$.

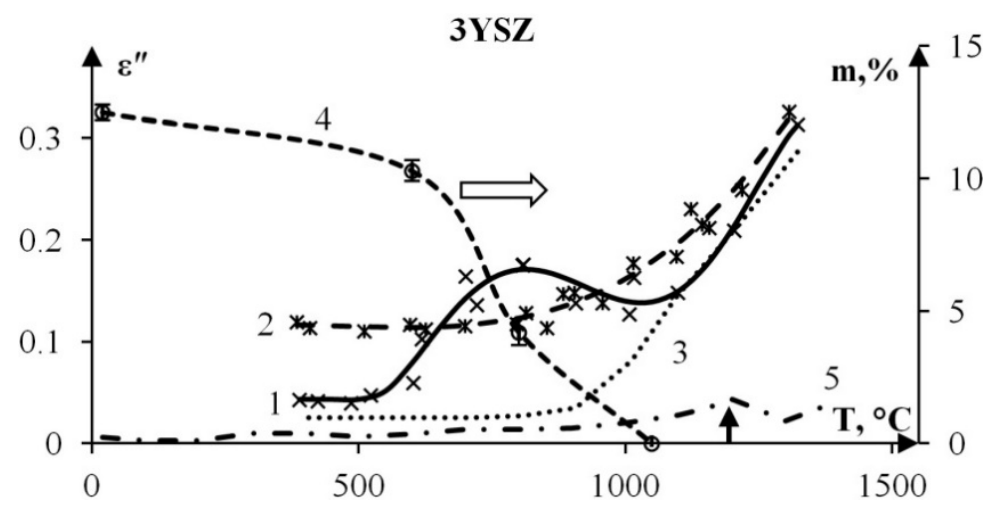

Fig. 6. Imaginary part of 3 YSZ permittivity: raw samples (1), sintered samples (2),

Effective Medium Approximation (3), content of monoclinic phase (4), pure zirconia powder at $3.8 \mathrm{GHz}$ [11] (5), solid arrow shows m-t transformation area for pure zirconia.

Figs. 5, 6 show the results of calculations of $\varepsilon^{\prime \prime}$ using formula (12) and taking into account shrinkage of the samples.

Measurements of the complex permittivity of HA sintered in conventional furnace by FabryPerot technique [4] at room temperature gave the following results:

$\varepsilon^{\prime}=2.82, \varepsilon^{\prime \prime}=0.013 \pm 0.001$ (raw sample);

$\varepsilon^{\prime}=9.42, \varepsilon^{\prime \prime}=0.078 \pm 0.008$ (sintered sample).

These values are included to Fig. 5. It is seen that the values for $180 \mathrm{GHz}$ and $24 \mathrm{GHz}$ are in good agreement with each other.

In [9] the results of DC conductivity measurements of zirconia - yttria sintered compositions heating by MW radiation at frequency of $24 \mathrm{GHz}$ are presented. It was found that DC conductivity depends strongly on the thermal insulation material in which the sample was placed. At temperature $\mathrm{T}=500{ }^{\circ} \mathrm{C}$ for composition 3YSZ DC conductivity of the sample placed into 2YSZ container was $0.093(\mathrm{Ohm} \cdot \mathrm{m})^{-1}$, and in the case when the sample was placed into alumina container the conductivity was $0.22(\mathrm{Ohm} \cdot \mathrm{m})^{-1}$. Calculation of high frequency conductivity from our data $\left(\sigma=\frac{\omega}{4 \pi} \varepsilon^{\prime \prime}\right)$ gives the value of $0.15(\mathrm{Ohm} \cdot \mathrm{m})^{-1}$, which is just in between the values of DC conductivity mentioned above. It means that the mechanisms of both DC and high frequency conductivities are similar. 
Imaginary part of the permittivity for HA and 3YSZ raw samples show significantly different behaviour with temperature: while for HA $\varepsilon^{\prime \prime}$ smoothly increases with temperature, $\varepsilon^{\prime \prime}$ of 3 YSZ has a peak with its maximum at $800^{\circ} \mathrm{C}$.

According to X-ray analysis observed peak in $\varepsilon^{\prime \prime}$ corresponds to transition from monoclinic (m) to tetragonal $(\mathrm{t})$ phase, which started at $600{ }^{\circ} \mathrm{C}$ and finished at less than $1000{ }^{\circ} \mathrm{C}$ (see Fig. 8). After $1000{ }^{\circ} \mathrm{C}$ only tetragonal phase was found. These data are in accordance with [10], where the detailed phase diagram of zirconia - yttria system was presented. For the composition of zrconia with $3 \mathrm{~mol} \%$ yttria $\mathrm{m}$ - $\mathrm{t}$ transition occurred in the temperature interval from $560{ }^{\circ} \mathrm{C}$ to $800{ }^{\circ} \mathrm{C}$ [10]. Note that for pure zirconia this transition takes place at temperature of $1200{ }^{\circ} \mathrm{C}$.

It is known that $\mathrm{m}-\mathrm{t}$ transition in pure zirconia causes nonmonotonic temperature dependence of the $\varepsilon^{\prime \prime}$ at high frequencies [11]. Fig. 6 presents data from [11], illustrating peak in $\varepsilon^{\prime \prime}$ during monoclinic - tetragonal transition at frequency of $3.8 \mathrm{GHz}$ for loose zirconia powder (density $22 \%$ of theoretical value). Taking into account this behaviour and shift of $\mathrm{m}-\mathrm{t}$ transition to lower temperatures for zirconia - yttria composition it is reasonable to suppose that observed in our experiments peak in $\varepsilon^{\prime \prime}$ is a result of $\mathrm{m}-\mathrm{t}$ transformation.

Difference between imaginary part of the permittivity of raw and sintered samples for both compositions clearly seen in Figs. 5, 6 can be explained by their different porosity. To prove it a series of calculations of the materials permittivities based on Effective Medium Approximation (EMA) [12] were performed. According to EMA approach effective dielectric permittivity $\varepsilon_{\mathrm{e}}=\varepsilon_{\mathrm{e}}{ }^{\prime}+\mathrm{i} \varepsilon_{\mathrm{e}}{ }^{\prime \prime}$ of the porous media satisfies the equation:

$\sum_{i=1}^{n} p_{i} \frac{\varepsilon_{i}-\varepsilon_{e}}{\varepsilon_{i}-2 \varepsilon_{e}}=0$,

where $\mathrm{p}_{\mathrm{i}}$ and $\varepsilon_{\mathrm{i}}=\varepsilon_{\mathrm{i}}{ }^{\prime}+\mathrm{i} \varepsilon_{\mathrm{i}}{ }^{\prime \prime}$ are the volume ratio and complex dielectric permittivity of the $\mathrm{i}$-th component of the medium (including pores), correspondingly, $\mathrm{n}$ is a number of components. In the calculations obtained above values of imaginary parts $\varepsilon_{i}^{\prime \prime}$ of the both sintered materials were used. Real parts of the permittivity $\varepsilon_{\mathrm{i}}{ }^{\prime}$ were assumed independent on the temperature.

For HA a value $\varepsilon^{\prime}=9.42$ measured at frequency of $180 \mathrm{GHz}$ was used. As for 3 YSZ literature date for $\varepsilon^{\prime}$ were used. For monoclinic zirconia a big scatter of data in the range from 12 to 22 is presented in literature, for tetragonal sirconia the data are near $40[13,14]$. In our calculation the $\varepsilon^{\prime}=17.9$ for monoclinic phase and $\varepsilon^{\prime}=34.5$ for tetragonal phase [14] were used.

The results of calculations are presented in Figs. 5, 6. Calculated dependency of the imaginary part of the permittivity $\varepsilon^{\prime \prime}$ for hydroxyapatite is in good accordance with the experimental curve (see Fig. 5). As for 3 YSZ calculated value of $\varepsilon^{\prime \prime}$ smoothly increases with temperature, which doesn't correspond to the experimental data. Taking into account that in formula (13) $\varepsilon_{\mathrm{i}}^{\prime \prime}$ for the sintered material, which is completely in tetragonal phase, was substituted, the discrepancy between measured and calculated behaviour of $\varepsilon^{\prime \prime}$ can be explained by the nonmonotonic nature of zirconia permittivity imaginary part at $\mathrm{m}-\mathrm{t}$ transformation described above.

\section{Conclusion}

Absorptivities of both raw and sintered samples of hydroxyapatite and 3YSZ compositions at frequency of $24 \mathrm{GHz}$ in wide range of temperatures have been measured.

Imaginary parts of permittivities as functions of temperatures for both compositions were evaluated.

Nonmonotonic dependence of $3 \mathrm{YSZ} \varepsilon^{\prime \prime}$ on temperature caused possibly by monoclinic tetragonal phase transition in zirconia was found. 
Calculations of $\varepsilon^{\prime \prime}$ based on Effective Medium Approximation are in good agreement with experimental values for hydroxyapatite, but don't describe the peak in $\varepsilon^{\prime \prime}$ for 3 YSZ.

\section{Acknowledgement}

This research was supported by Russian Basic Research Foundation, grant \# 18-29-11045.

\section{References}

1. Vaezi, M., et al., Int. J. Adv. Manuf. Technol., 2013, 67, 1721-1759.

2. Bykov, Yu., et al., Radiophysics and Quantum Electronics, 2019, 61 (10) 752762.

3. Volkovskaya, I.I., et al., Radiophysics and Quantum Electronics, 2018, 61 (4), $286-295$.

4. V. V. Parshin, V.V., et al, Radiophysics and Quantum Electronics, 2009, 52 (8), 525-535

5. Egan, E.P., et al.., Journal of the American Chemical Society, 1950, 72 (6), 24182421.

6. Samsonov, G.V., Physical and Chemical Properties of Oxides: Handbook (in Russian), 1978, 110.

7. Grigoriev, I.S., Physical quantities: Handbook (in Russian), 1991, 212

8. Kimrey, H.D., Janney, M.A., Mater. Res. Soc. Symp. Proc.,1988, 124, 367-372.

9. Kishimoto, A., et al, Scripta Materialia, 2011, 64, 860-863

10. Scott, H.G., J. of Materials Science, 1975, 10, 1527-1535.

11. Baeraky, T.A., Egypt. J. Sol., 2004, 27 (1), 25 - 34.

12. D. J. Bergman, D.J., Stroud, D, Solid State Phys., 1992, 46, 147 - 269.

13. Molla, J., Preprint CEIMAT-736, 1994.

14. Yongduk, O., et al, Additive Manufacturing, 2019, 27, 586 - 594. 


\section{MICROWAVE RAPID HEATING SYSTEM USING CARBON HEATING TUBE}

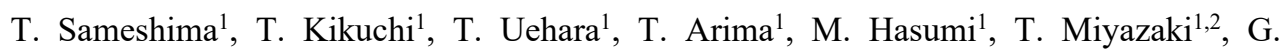
Kobayashi $^{2}$, and I. Serizawa ${ }^{3}$

${ }^{1}$ Tokyo University of Agriculture and Technology, Graduate School of Engineering, 2-2416, Naka-cho, Koganei, Tokyo, Japan

${ }^{2}$ Techno Research., Ltd., Koganei, Tokyo, Japan

${ }^{3}$ ORC MANUFACTUREING CO., LTD., Chino, Nagano, Japan

Email: tsamesim@cc.tuat.ac.jp

Keywords: Electromagnetic interference, Highly efficient heat treatment, PID temperature control, Silicon solar cell

A microwave heating system with a carbon heating tube (CHT) is reported. 2.45-GHz microwave at $200 \mathrm{~W}$ was introduced to a 300-dimameter metal cavity, in which the CHT made by $60-\mathrm{mm}$-long and $4-\mathrm{mm}$ diameter quartz tube filled with $2-\mu \mathrm{m}$ diameter carbon particles and $\mathrm{Ar}$ gas at $1400 \mathrm{~Pa}$ was set at the central position. The numerical simulation with a finite element moment method demonstrated the standing wave of the electric field caused by three-dimensional Fresnel interference effect. The lowest average electric field intensity of $5 \mathrm{kV} / \mathrm{m}$ in the cavity space was achieved in the case of the electrical conductivity of carbon ranging from 10 to $55 \mathrm{~S} / \mathrm{m}$. Heating a CHT with $55 \mathrm{~S} / \mathrm{m}$ was experimentally realized to $1200^{\circ} \mathrm{C}$ by microwave irradiation at $200 \mathrm{~W}$. This heating method was applied to activate $1.0 \times 10^{15}-\mathrm{cm}^{-2}$ boron and phosphorus implanted regions in n-type crystalline silicon substrate to fabricate pn junction and solar cells. The $\mathrm{CHT}$ heating at $1200^{\circ} \mathrm{C}$ realized a solar cell characteristic with a conversion efficiency of $14.8 \%$ under illumination of air mass 1.5 at $0.1 \mathrm{~W} / \mathrm{cm}^{2}$.

\section{Introduction}

Heat treatment has been widely applied for semiconductor device fabrication processing such as activation of dopant atoms to form the pn junction and crystallization to realize high quality crystalline state [1-6]. Many heating technologies have been developed such as laser annealing, plasma jet annealing, and rapid thermal annealing [7-10]. We have recently developed a lamp of carbon heating tube (CHT), in which 2- $\mu$ m-diameter-carbon powders were filled with inert gas in a quartz tube whose edges were then closed by thermal welding [11]. Conductive carbon is effectively heated via free carrier absorption of the electromagnetic energy with excellent thermal properties of low specific heat and high heat proof [12-14]. We have found that the low packing density of carbon powders about 0.09 allows effective absorption of microwave [12].

In this paper, we report characterization of the electric field distribution in the cavity in the steady state during microwave irradiation in the CHT heating system analyzed by a numerical simulator constructed with the three-dimensional finite element moment method to determine the effective condition of microwave absorption by the CHT. Then we demonstrate CHT heating with different temperatures controlled by a home-madeproportional-integral-differential feedback (PID) circuit which regulated the magnetron power source with a signal of the thermometer. We demonstrate application of the present heating system to activation of silicon surface region implanted with boron and phosphorus atoms in n-type silicon substrates. Decrease in the sheet resistivity and increase in the effective minority carrier lifetime $(\tau$ eff), and solar cell characteristics are reported. 


\section{Experimental}

\section{2-1. CHT heating system}

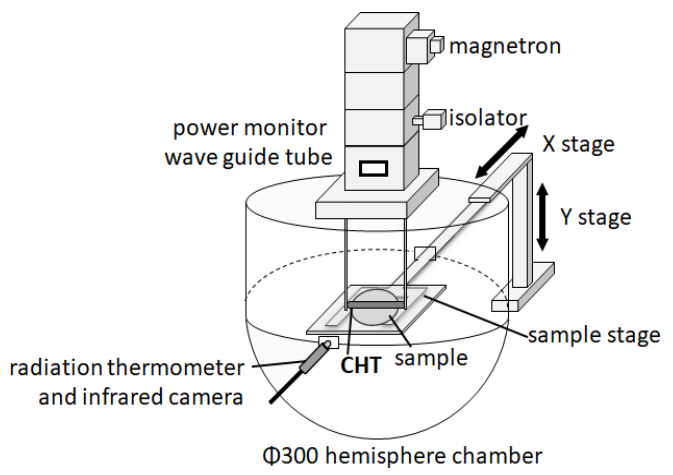

(a)

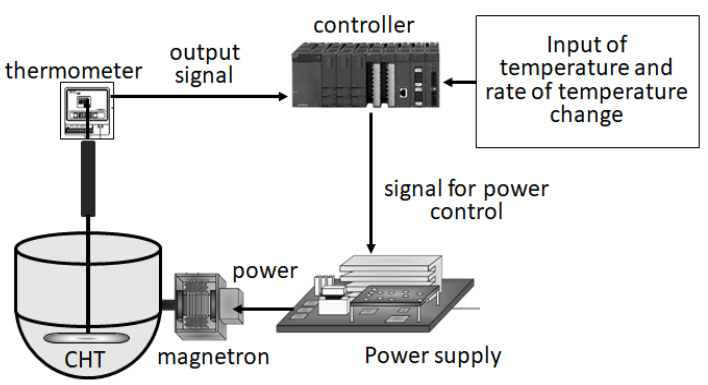

(b)

Fig. 1. Schematic images of (a) CHT heating system and (b) PID temperature control system.

Figure 1(a) shows a schematic image of the CHT heating system. For fabricating CHTs, quartz tubes with 60-mm long and 4-mm inter diameter and 6-mm outer diameter were prepared. $2-\mu \mathrm{m}$-diameter-carbon powders with a 0.1 -g weight were put in the quartz tubes, in which the packing density was controlled as 0.09 with an electrical conductivity of 55 $\mathrm{S} / \mathrm{m}$, which was determined by the previous work as the best value for heating [12]. Ar inert gas was filled in the quartz tube at $1400 \mathrm{~Pa}$. The edges of the quartz tubes were closed by thermal welding. Two quartz rods were jointed at the edges of the CHT by thermal welding to hold the CHT. A CHT was placed in the heating system which consists of a magnetron for generating 2.45-GHz microwave with an electrical power source, an isolator for blocking the reflectance of microwave backward the magnetron, a power monitor, an impedance matching box for reducing microwave reflectance, a wave-guide tube, and a microwave cavity made by Al metals. The cavity had a structure with a cylindrically upper part with a diameter of $300 \mathrm{~mm}$ and a spherically lower part to promote interferingly multiple reflection of the microwave in the cavity and to have the CHT effectively absorb the microwave. Those works resulted in a reflected power less than $4 \mathrm{~W}$ in the case of an input power of $500 \mathrm{~W}$. The CHT effectively absorbed the microwave power. In contrast, the power monitor gave the same value of the input and reflected power when the cavity had no CHT. The cavity completely reflected the microwave power. The cavity also had a window port for observing 
light emission from CHT heated by microwave. A thermometer detecting 900-nm wavelength radiation light CHINO IR-FAS was used to monitor the temperature of the CHT in real time. An infrared digital camera was also used to observe CHT heating behavior. A mechanically moving stage was also installed in the cavity, as shown in Fig. 1(a) to move a sample just below the CHT for demonstration of heating samples by the CHT. An electrical circuit was developed to control the CHT temperature, as shown by a schematic image in Fig. 1(b). The analog voltage signal of the thermometer is monitored in real time by a PID circuit controller [15] which was installed with a plan of temperature increasing and decreasing ratios, a target temperature and its duration in its memory in advance. The controller sent a signal to the power source of the magnetron to increase or decrease the microwave power for coinciding the $\mathrm{CHT}$ temperature with the initial temperature plan.

\section{2-2. Numerical calculation}

The electric field distribution in the cavity in the steady state during microwave irradiation was numerically analyzed by a simulator constructed with the three-dimensional finite element moment method combined with the Cholesky decomposition [16]. The grid system of the cavity and waveguide was formed with the equilateral-triangle elements with the sides ranging from 1 to $4 \mathrm{~mm}$ for the numerical calculation. They were assumed as perfect electric conductor which reflects completely the microwave. The CHT grid was formed with 1-mmside-equilateral-triangle elements. The electrical conductivity of carbon was widely changed from 0.1 to $10000 \mathrm{~S} / \mathrm{m}$ to find an effective condition of microwave absorption by the CHT. The calculation of electric field distribution in the cavity was also conducted in the case of no CHT.

\section{2-3. Solar cell fabrication}

4-inch-diameter 500 - $\mu$ m-thick $17-\Omega \mathrm{cm}$ n-type silicon substrates coated with 100 -nmthick thermally grown $\mathrm{SiO}_{2}$ layers were prepared. The ion implantation of boron atoms was conducted with a dose of $2.0 \times 10^{15} \mathrm{~cm}^{-2}$ at $25 \mathrm{keV}$ to the top silicon surface of the substrates. Phosphorus atoms with $2.0 \times 10^{15} \mathrm{~cm}^{-2}$ at $75 \mathrm{keV}$ was also implanted to the rear surface. The boron and phosphorus atoms with a dose of $1.0 \times 10^{15} \mathrm{~cm}^{-2}$ were effectively incorporated in the top and rear silicon surface regions. The samples were cut to 4 sectored pieces. They were placed in the heating system just below the CHT and moved at $0.12 \mathrm{~mm} / \mathrm{s}$. The CHT temperature was controlled by PID feedback method at 1000,1100 , and $1200^{\circ} \mathrm{C}$. After heating, the thermally grown $\mathrm{SiO}_{2}$ layer was removed using hydrofluoric acid. To estimate the sheet resistivity and effective minority carrier lifetime $\tau_{\text {eff, we measured the }} 9.35 \mathrm{GHz}$ microwave transmittance of the samples in dark field and 635-nm-light illumination [17]. Comb-type Al electrodes were formed on the top surface and the rear surface was entirely coated with $\mathrm{Al}$ electrodes by vacuum evaporation. 100-nm-thick $\mathrm{AlO}_{\mathrm{x}}$ layers was subsequently deposited on the top surfaces as anti-reflection layer. The samples were heated in $\mathrm{H}_{2} \mathrm{O}$ vaper at $0.8 \mathrm{MPa}$ at $230^{\circ} \mathrm{C}$ for $3 \mathrm{~h}$ to decrease defect states in the doped regions [18]. The electrical current density as a function of applied voltage $(\mathrm{J}-\mathrm{V})$ was measured under illumination of air mass (AM) 1.5 light at $0.1 \mathrm{~W} / \mathrm{cm}^{2}$.

\section{Results and discussion}

Figure 2 shows calculated cross-sectional electric field distributions in the cavity with different effective electrical conductivities of CHT of (a) 0 (no CHT), (b) 0.1, (c) 10, (d) 55, (e) 100 , and (f) $1000 \mathrm{~S} / \mathrm{cm}$ when the $200 \mathrm{~W}$ microwave was continuously introduced in the cavity. The calculation resulted in the standing wave of the electric field in every case because of the three-dimensional Fresnel interference effect in the cavity. The numerical 


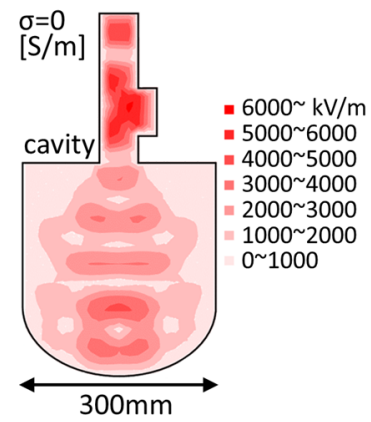

(a)

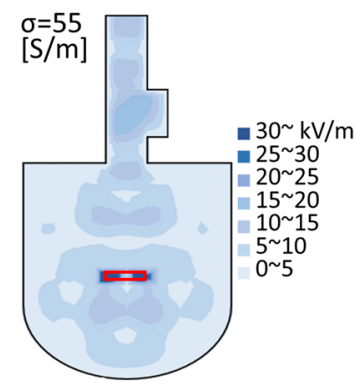

(d)

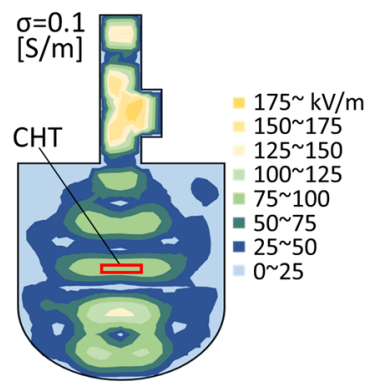

(b)

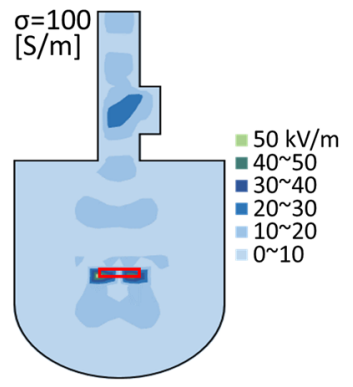

(e)

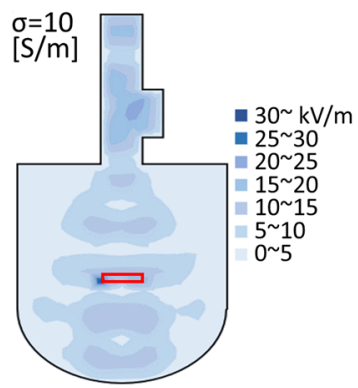

(c)

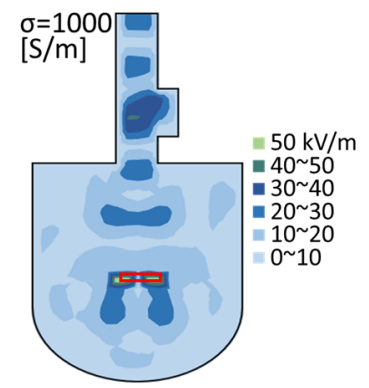

(f)

Fig. 2. Calculated cross-sectional electric field distributions with different effective electrical conductivities of CHT of (a) 0 (no CHT), (b) with 0.1 , (c) 10, (d) 55, (e) 100, and (f) $1000 \mathrm{~S} / \mathrm{cm}$ when $200 \mathrm{~W}$ microwave was continuously introduced.

calculation resulted in existence of a high electric field intensity ranging from 1000 to 6000 $\mathrm{kV} / \mathrm{m}$ in the no CHT case, as shown in Fig. 2(a). In contrast, the electric field intensity decreased to very low values in the case of the cavity with CHTs. In the most of region of the cavity, the electric field ranged from 25 to $125 \mathrm{kV} / \mathrm{m}$ for an electrical conductivity of 0.1 $\mathrm{S} / \mathrm{m}$, as shown in Fig. 2(b). This means that the microwave power was effectively absorbed by the CHT. The pattern of distribution of the electric field was similar to that of the no CHT case. Microwave was absorbed during three-dimensionally interfering in the cavity. The electric field intensity was very low when the electrical conductivity ranged from 10 to 100 $\mathrm{S} / \mathrm{m}$, as shown in Figs. 2(c) to (f). Especially the electric field intensity distributed from 5 to $25 \mathrm{kV} / \mathrm{m}$ in the case of 10 and 55-S/m-CHT, as shown in Figs. 2 (c) and (d). It was reduced to at least $0.1 \%$ of the initial intensity of the cavity space without CHT. This indicates that the microwave power in the cavity was almost completely absorbed by the CHT. Absorption of microwave power depends on the electrical conductivity. On the other hand, the electric field intensity increased when the electrical conductivity increased to $1000 \mathrm{~S} / \mathrm{m}$, as shown in Fig. 2(f). A CHT with a very high electrical conductivity (high packing density of carbon particles) is not suitable probably because of high reflectivity caused by a high extinction coefficient. 


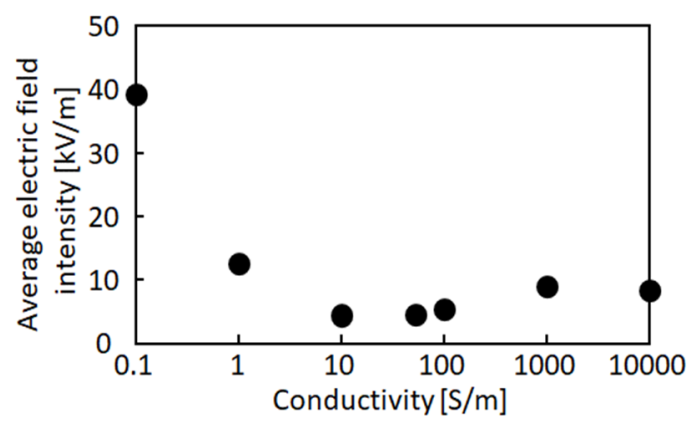

Fig. 3. Average electric field intensity in the cavity space as a function of the electrical conductivity of the CHT.

Figure 3 shows the average electric field intensity in the cavity space as a function of the electrical conductivity of the CHT. Although the average electric field intensity was very high of $1173 \mathrm{KV} / \mathrm{m}$ in the case of no CHT, it decreased to $40 \mathrm{kV} / \mathrm{m}$ by installing $0.1 \mathrm{~S} / \mathrm{m}$ $\mathrm{CHT}$, as shown in Fig. 3. The minimum average electric field intensity was $5 \mathrm{kV} / \mathrm{m}$ when the electrical conductivity ranged between 10 and $55 \mathrm{~S} / \mathrm{m}$. This means that the effective absorption of the microwave power by CHT reduced the electric field intensity in the cavity. The average electric field intensity gradually increased as the electrical conductivity further increased. The calculation supports experimental results of low microwave refection and effective heating of the CHT [11,12].

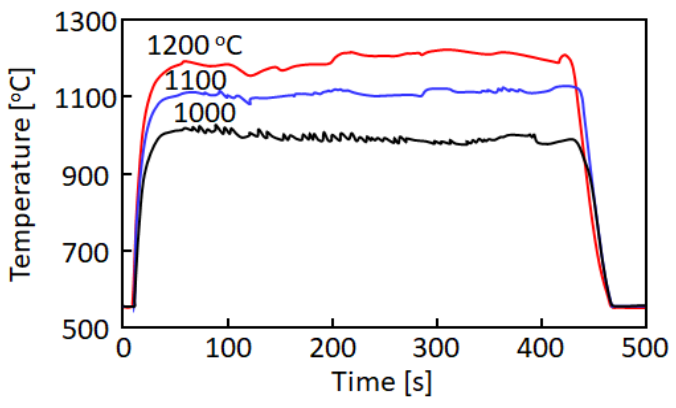

Fig. 4. Temperature changes with time of the CHT for three setting temperatures of 1000 , 1100 , and $1200^{\circ} \mathrm{C}$ during moving samples just below the $\mathrm{CHT}$ at $0.12 \mathrm{~mm} / \mathrm{s}$.

Figure 4 shows temperature changes with time of the $\mathrm{CHT}$ for three setting temperatures of 1000,1100 , and $1200^{\circ} \mathrm{C}$ under moving the silicon samples at $0.12 \mathrm{~mm} / \mathrm{s}$ just below the CHT. The PID control system well kept the setting temperatures by real-time changing the microwave power. Temperature fluctuation about $50 \mathrm{~K}$ was observed. This is probably because the condition of PID control with best time constant has not established yet. Further development of PID system is planned to precisely design temperature sequence for the CHT heating system. 


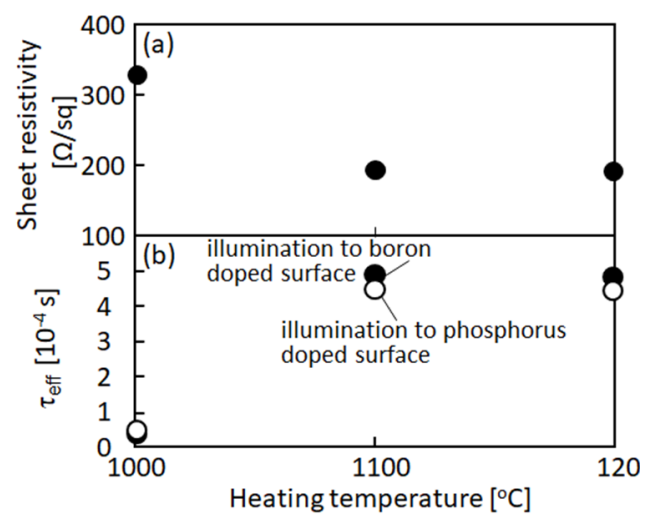

Fig. 5. (a) Sheet resistivity of the ionimplanted regions and (b) $\tau_{\text {eff }}$ in the case of 635-nm light illumination to the boron (solid circles) and phosphorus (open circles) doped surfaces.

Figure 5 shows (a) the sheet resistivity of the ion-implanted regions and (b) $\tau_{\text {eff }}$ in the case of 635-nm light illumination to the boron (solid circles) and phosphorus (open circles) implanted surfaces as functions of CHT heating temperature. The sheet resistivity of asimplanted sample was high of $626 \Omega$ /sq because of the low electron carrier density in the silicon bulk, it decreased to 215,148 , and $146 \Omega / \mathrm{sq}$, as the CHT heating temperature increased to 1000,1100 , and $1200^{\circ} \mathrm{C}$. Those experimental data resulted in that the implanted regions were activated with sheet resistivity of 327,194 , and $190 \Omega /$ sq, as shown in Fig. 5(a). The carrier generation were successfully achieved by CHT heating. $\tau_{\text {eff }}$ was also increased by CHT heating. Although they were low of $6.8 \times 10^{-6}$ and $9.1 \times 10^{-6} \mathrm{~s}$ in the cases of light illumination to the as-boron and as-phosphorus implanted surfaces, $\tau_{\text {eff }}$ increased to $3.3 \times 10^{-5}, 4.9 \times 10^{-4}$, and $4.8 \times 10^{-4} \mathrm{~s}$ in the cases of light illumination to the boron implanted surfaces as the CHT heating temperature increased to 1000,1100 , and $1200^{\circ} \mathrm{C}$. It also increased to $5.5 \times 10^{-5}, 4.5 \times 10^{-4}$, and $4.4 \times 10^{-4} \mathrm{~s}$ in the cases of light illumination to the phosphorus implanted surfaces as the CHT heating temperature increased to 1000,1100, and $1200^{\circ} \mathrm{C}$. These experimental lifetime results indicate that defect states caused by ion implantation were effectively reduced. Our numerical analysis resulted in that the recombination defect densities were $3.2 \times 10^{10}$ and $3.5 \times 10^{10} \mathrm{~cm}^{-2}$ for boron $\left(\mathrm{p}^{+}\right)$and phosphorus $\left(\mathrm{n}^{+}\right)$implanted surfaces in the case of $1200^{\circ} \mathrm{C} \mathrm{CHT}$ heating [17].

Figure 6 shows linear plotted J-V characteristics of solar cells fabricated with CHT heating at temperatures of 1000,1100 , and $1200^{\circ} \mathrm{C}$. Typical diode rectified characteristics were observed in dark field measurement. The AM 1.5 light illumination realized photovoltaic characteristics. The short circuit current density and open circuit voltage increased as the CHT heating temperature increased, as shown in Fig. 6. The conversion efficiency increased to $10.1,13.9$, and $14.8 \%$, as the CHT heating temperature increased. The $1200^{\circ} \mathrm{C}$ heating realized the best performance with the short circuit current density, open circuit voltage, fill factor, and conversion efficiency of $39 \mathrm{~mA} / \mathrm{cm}^{2}, 0.55 \mathrm{~V}, 0.69$, and $14.8 \%$, respectively. These results indicate that the temperature-controlled $\mathrm{CHT}$ heating has a capability of activating dopant-implanted regions, reducing the defect states, realizing high performance silicon solar cells. 


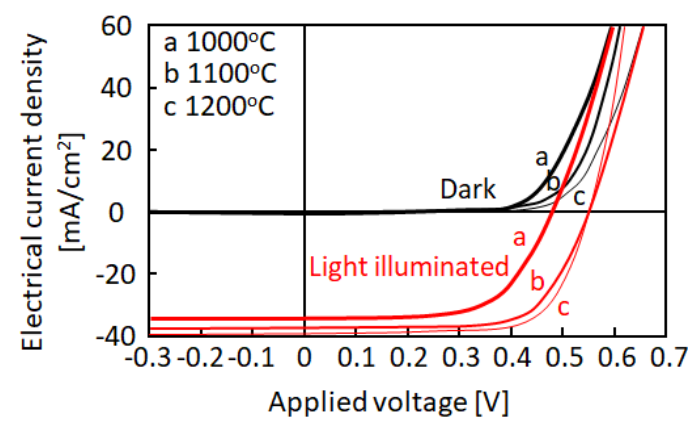

Fig. 6. Linear plotted J-V characteristics of solar cells.

\section{Conclusions}

We demonstrated CHT heating equipment which consisted of 2.45-GHz microwave irradiation wave guide tube into $300-\mathrm{mm}$-diametered semispherical metal cavity to accumulate the microwave, which was effectively absorbed by CHT made by a 4-mmdiameter and 60-mm-long quartz tube filled with conductive carbon particles at an average electrical conductivity of $55 \mathrm{~S} / \mathrm{m}$ in Ar gas at $1400 \mathrm{~Pa}$. The numerical simulation with a finite element moment method demonstrated the standing wave of the electric field caused by three-dimensional Fresnel interference effect. The lowest average electrical field intensity 5 $\mathrm{kV} / \mathrm{m}$ in the cavity space was achieved in the case of the electrical conductivity of carbon ranging from 10 to $55 \mathrm{~S} / \mathrm{m}$ when the $200 \mathrm{~W}$ microwave was continuously introduced. The PID feedback circuit regulated the microwave power by the signal of thermometer detecting the intensity of blackbody irradiation emitted from CHT heated by microwave to control the CHT heating temperature. The n-type silicon surface regions with $1.0 \times 10^{15} \mathrm{~cm}^{-2}$ boron and phosphorus ion implanted were heated at 1000,1100 , and $1200^{\circ} \mathrm{C}$ by $\mathrm{CHT}$ heating. The sheet resistivity of implanted regions decreased to $190 \Omega /$ sq by $1200^{\circ} \mathrm{C}$ heating and the density of defect states also decreased to $3.2 \times 10^{10}$ and $3.5 \times 10^{10} \mathrm{~cm}^{-2}$ for boron $\left(\mathrm{p}^{+}\right)$and phosphorus $\left(\mathrm{n}^{+}\right)$implanted surfaces. Via formation of $\mathrm{Al}$ metal electrodes and $\mathrm{AlO}_{\mathrm{x}^{-}}$ antireflection layer resulted in solar cell characteristics with the short circuit current density, open circuit voltage, fill factor, and conversion efficiency of $39 \mathrm{~mA} / \mathrm{cm}^{2}, 0.55 \mathrm{~V}, 0.69$, and $14.8 \%$, respectively, under illumination of air mass 1.5 at $0.1 \mathrm{~W} / \mathrm{cm}^{2}$.

\section{Acknowledgments}

This work was partially supported by Japan Science and Technology Agency ASTEP (No. AS3015022S).

\section{References}

1. S. M. Sze, Physics of Semiconductor Devices, 3rd ed., New York, WileyInterscience, 1985, 510-547.

2. Y. Taur, T, Ning, Fundamental of Modern VLSI Physics, 2nd ed., Cambridge, Cambridge University Press, 1998, 9-105.

3. S. Inoue, K. Sadao, T. Ozawa, Y. Kobashi, H. Kwai, T. Kitagawa, T. Shimoda, Tech. Dig. IEDM, 2000, 197.

4. K.Shibata, H. Takahashi, Workshop on Active Matrix Liquid Crystal Displays'01, 2001, 219-222. 
5. M. A. Green, Nat. Energy 1, 2016, Article number 15015.

6. M. Dahlinger, B. Bazer-Bachi, T. C. Röder, J. R. Köhler, R. Zapf-Gottwick, J. H. Werner, Energy Procedia, 2013, 38, 250-253.

7. T. Sameshima, S. Usui, M.Sekiya, IEEE Electron Device Lett., 1986, 7, 276-278.

8. T. Serikawa, S. Shirai, A. Okamoto, S. Suyama, Jpn. J. Appl. Phys., 1989, 28, L1871-L1873.

9. S. Higashi, H. Kaku H. Murakami, S. Miyazaki, H. Watakabe, N. Aando, T. Sameshima, 1st Thin Film Materials \& Devices Meeting, 2004, 25-28.

10. G. Mannino, Appl. Phys. Lett., 2001, 78, 889.

11. T. Sameshima, T. Miyazaki, G. Kobayashi, T. Arima, T. Kikuchi, T. Uehara, T. Sugawara, M. Hasumi, I. Serizawa, IEEE Access, 2019, 7, 23798-23805.

12. S. Kimura, K. Ota, M. Hasumi, A. Suzuki, M. Ushijima, T. Sameshima, Appl. Phys. $A, 2016, \mathbf{1 2 2}, 695$.

13. M. Yoshikawa, N. Nagai, M. Matsuki, H. Fukada, G. Katagiri, H. Ishida, A. Ishitani, I. Nagai, Phys. Rev. B, 1992, 46, 7169-7174.

14. Sameshima, N. Andoh, Jpn. J.Appl. Phys., 2005, 44, 7305-7308.

15. Aidan O'Dwyer, Handbook of PI and PID Controller Tuning Rules, 3rd ed., London, Imperial College Press, 2009, 4-17.

16. J. H. Richmond, IEEE Trans. Antennas \& Propagation, 1966, 14, 782-786.

17. T. Sameshima, H. Hayasaka, T. Haba, Jpn. J.Appl. Phys., 2009, 48, 021204-1-6.

18. T. Sameshima, M. Satoh, Jpn. J. Appl. Phys., 1997, 36, L687-L689. 


\title{
LOW-COST MEASUREMENT SYSTEM AND FILTER FOR REDUCTION OF EMC INTERFERENCES IN RADIO-FREQUENCY APPLICATIONS
}

\author{
R. Sallier ${ }^{1,2}$, L. Nietner ${ }^{1}$, U. Roland ${ }^{1,2,4}$, U. Trommler ${ }^{1,2}$, M. Kraus², F. Holzer ${ }^{2}$, C. Hoyer ${ }^{2,3}$, \\ and D. Schlayer ${ }^{4}$ \\ ${ }^{1}$ Leipzig University of Applied Sciences, Karl-Liebknecht-Str. 132, 04277 Leipzig, \\ Germany \\ ${ }^{2}$ Helmholtz Centre for Environmental Research - UFZ, Department of Environmental \\ Engineering, Permoserstr. 15, 04318 Leipzig, Germany \\ ${ }^{3}$ Otto Richter GmbH, Seelenbinderstr. 80, 12555 Berlin, Germany \\ ${ }^{4}$ Advisory Board RWTec Innovation Network, https://www.ufz.de/rwtec \\ rene.sallier@htwk-leipzig.de,lutz.nietner@htwk-leipzig.de,ulf.roland@ufz.de
}

Keywords: filter, balun, interference reduction, radio-frequency (RF) heating, prototype, electromagnetic compatibility (EMC)

\begin{abstract}
For drying building structures, dielectric heating using RF energy (13.56 MHz) is an efficient and fast alternative to conventional heating methods relying solely on heat transfer from the surface to the interior of the masonry. Another innovative application of volumetric RF heating is chemical-free pest control in wood-based materials. For such applications where the RF systems often cannot be completely shielded, the propagation or coupling of the electromagnetic waves into wires that are placed inside the field cannot be neglected which represents a certain risk. Depending on the different electromagnetic coupling mechanisms, several interference signals can be generated. In order to protect electrical equipment in the surrounding, these interferences have to be significantly reduced by electrical filters. For this purpose, a cost-effective and robust measuring concept was developed. With the help of this system, the common mode $\left(i_{\mathrm{CM}}\right)$ interference could be identified as the dominant part and the differential mode $\left(i_{\mathrm{DM}}\right)$ interference as a minor disturbance. Based on the experimental evaluation, a cost-effective filter for the respective disturbance was constructed and successfully tested.
\end{abstract}

\section{Introduction}

An innovative approach of applying radio waves (RF) for dielectric heating and drying building structures was presented in [1]. This application provides an efficient and fast alternative to conventional heating methods, which depend to a large extend on heat transfer from the heated surface into the inner volume of the material. Another innovative approach of dielectric heating via RF is the chemical-free pest control in wood-based materials [2]. Fig. 1 illustrates these two applications for the use of RF for dielectric heating. 


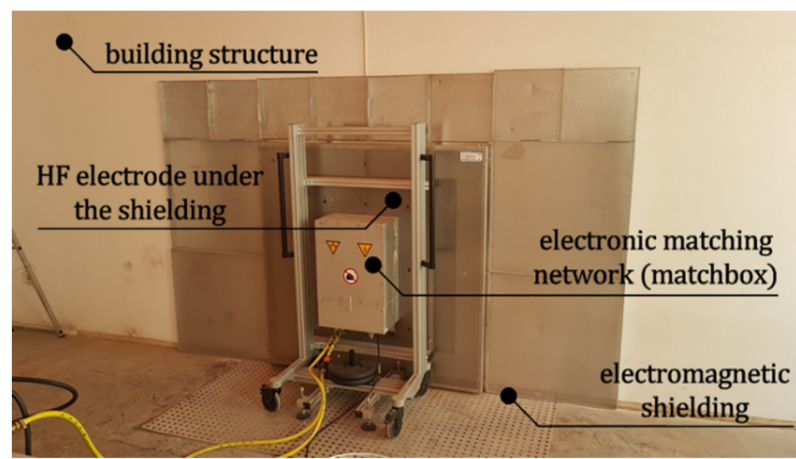

a)

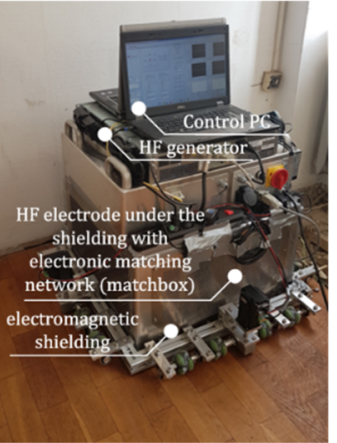

b)

Fig. 1 Electrode and matchbox systems for dielectric heating a) of building structures and

b) for chemical-free pest control in wood-based materials

In all applications of high-frequency (HF) electromagnetic waves, e.g. RF, the electromagnetic compatibility (EMC) has to be carefully considered. The undesired generation, propagation and reception of electromagnetic energy has to be prevented or suppressed as much as necessary, since detrimental effects such as electromagnetic interference (EMI) or even physical damage of equipment could occur [3]. Therefore, in Germany installations emitting RF disturbance have to be evaluated with regard to EMC for conducted interference voltages in compliance with the generic standard DIN 61000-6-3: 2011 (emission for residential, commercial and commercial areas). Since a radio-frequency of $13.56 \mathrm{MHz}$ is used in the presented applications without completely shielding, the propagation or coupling of the RF wave in existing wires in the electromagnetic field has to be examined. Depending on the different electromagnetic coupling mechanisms, different interference signals can be generated on this wire [3]. One established way in order to protect connected electrical equipment is to reduce these disturbances by means of so-called EMC filters (which are electrical filters). In order to be able to implement and use an effective electrical filter for the various types of disturbances, it is essential to identify and understand the types of coupling mechanisms and the associated disturbances. For this purpose, a simple measurement concept was developed, which works with cost-effective measuring devices in comparison to the standard expensive devices used in the HF measurement technology. On the basis of system analysis and its validation by applying a new measurement concept, cost effective filters were developed for the respective disturbance. Furthermore, the used measuring instruments are more robust compared to the conventional measuring instruments, so that they can be used in situ under field conditions.

\section{Fundamentals}

As described in Chapter I, there are different electromagnetic coupling mechanisms, which generate different disturbances in wires located in or in close vicinity of electromagnetic fields. Through a first principal functional analysis with regard to the structure of the used system, a first prediction can be made about the types of disturbance to be expected analyzing the electromagnetic coupling mechanisms. The basic structure of an RF system utilized, e.g., for drying building structures as shown in Fig. 1, is presented in Fig. 2. 


\section{High Frequency Heating}

Valencia, Spain, September 9-12, 2019

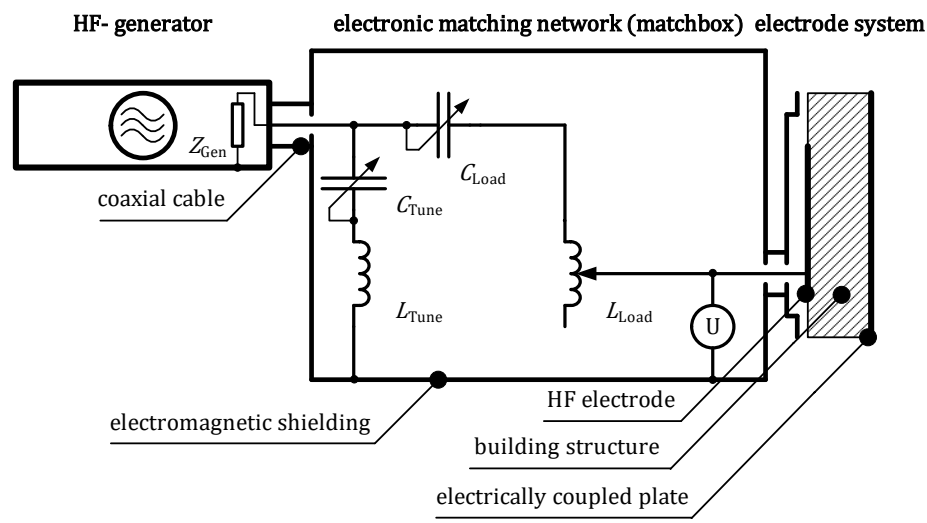

Fig. 2 Schematic representation of an RF system used for dielectric heating of building structures or chemical-free pest control

From Fig. 2, the network characteristics of the electrode system with the treated building structure acting as dielectric can be demonstrated. In more detail, the electrode system can be represented by a series connection of two capacitors (Fig. 3 a)) and, thus, it is possible to describe the total capacitance by using a single capacitor (Fig. 5 a)). When a wire (two- or three-wire leads) is located between the electrodes or in the vicinity of the electrode system, disturbances are coupled into this wire in accordance with the mentioned coupling mechanisms. The occurring coupling mechanisms are the capacitive - by E field (Fig. $3 \mathrm{~b}$ ) and Fig. 4 b)) and the inductive coupling $-\mathrm{H}$ field (Fig. 3.b)) correlated with the arrangement of the electrodes systems vs. wire leads.

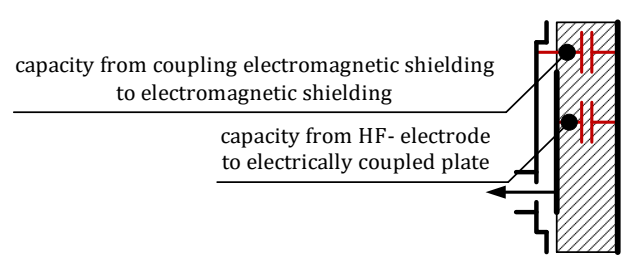

a)

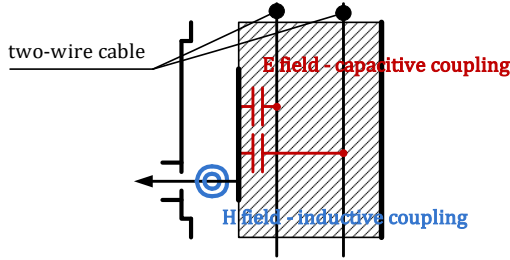

b)

Fig. 3 a) RF Electrode system with building structure as dielectric and resulting capacities, b) acting coupling mechanisms 


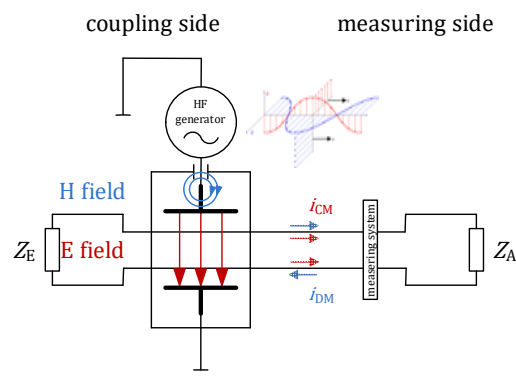

a)

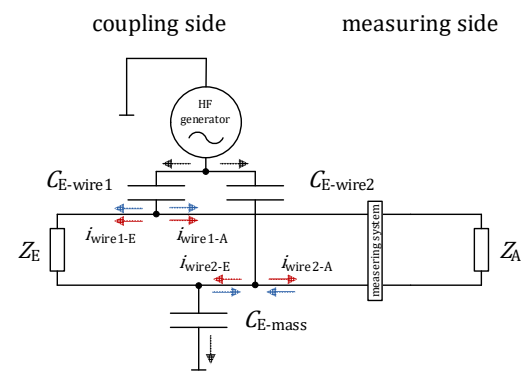

b)

Fig. 4 a) Simplified schematic representation of the coupling mechanisms in a two- or three-wire lead system, b) simplified scheme of the E field coupling paths

As already described, the whole system of the series connection of two capacitors can be transferred into a system consisting of only one capacitor which is installed between the RF voltage side and the ground (see Fig. 4 a)). $Z_{\mathrm{E}}$ and $Z_{\mathrm{A}}$ are the input and output impedances, respectively, of the lead wires in the electromagnetic field. The two dominant coupling mechanisms (via E field and $\mathrm{H}$ field) result primarily from the arrangement of the electrodes relative to the wires. In order to identify the contributions of the two disturbances due to the different coupling mechanisms, it is crucial to detect the disturbances also separately employing an appropriate measurement (see Fig. 5 a)). The coupling mechanisms can be fundamentally described using Maxwell's equations [4, 5].

\section{Measurement system}

To determine the respective disturbance currents for common mode $\left(i_{\mathrm{CM}}\right)$ and differential mode $\left(i_{\mathrm{DM}}\right)$, a measuring system consisting of three current clamps was constructed with the respective consideration of the current directions of the clamps (Fig. 5). The measurement of these disturbance currents with current clamps is possible without significant influences on the system to be measured since the measurement principle is based on inductive coupling. The system which was used for RF heating is a $5 \mathrm{~kW}$ system from Trumpf Hüttinger (Freiburg/Brsg., Germany). The measurements were carried out for a power range between 0 and $500 \mathrm{~W}$.

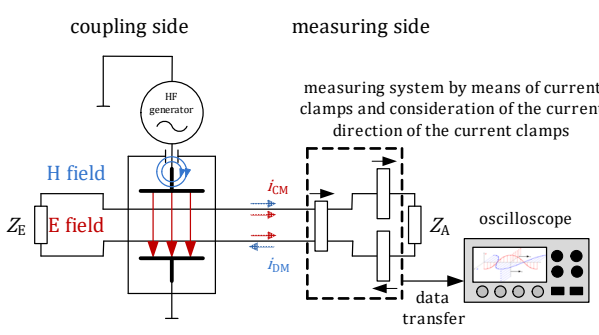

a)

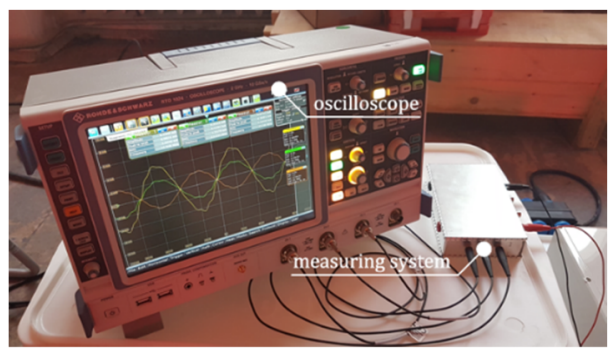

b)

Fig. 5 a) Arrangement for measuring the common mode and differential mode currents in a two- or three-wire lead system exposed to electromagnetic fields, b) measuring system 
The integral disturbance currents were measured with three Tektronix current measuring probes (CT1) in combination with a Rohde \& Schwarz oscilloscope (RTO 1024, probe input $>10 \mathrm{M} \Omega$ ). The disturbance currents $i_{\mathrm{CM}}$ and $i_{\mathrm{DM}}$ were determined from the measured values applying the following equations:

and

$$
i_{\text {wirel }}=i_{\mathrm{CM}}+i_{\mathrm{DM}}
$$

and

$$
i_{\mathrm{wire} 2}=i_{\mathrm{CM}}-i_{\mathrm{DM}}
$$

$$
i_{\text {wire } 12}=2 i_{\mathrm{CM}} \text {. }
$$

The disturbance currents $i_{\mathrm{CM}}$ and $i_{\mathrm{DM}}$ can already be determined from the first two equations. The third equation is used to validate the $i_{\mathrm{CM}}$ result derived from the first two equations. For this purpose, the forward wire (wire1) and return wire (wire2) of a two-wire lead were routed through a common clamp meter (green graph in Fig. 6). Thus, the components of $i_{\mathrm{CM}}$ of the lead in the current clamp add up and the components of $i_{\mathrm{DM}}$ were compensated due to opposite signs. With the help of the other two current clamps, the currents in the forward $\left(i_{\text {wire } 1}\right)$ and return $\left(i_{\text {wire2 }}\right)$ wires were determined. The disturbance currents calculated from the equations are represented by the red and blue curves in Fig. 6. Both measurement options of $i_{\mathrm{CM}}$ show, within the measurement accuracy, a very good agreement as also shown in Fig. 6 by the green and the red curves.

This diagram exemplarily shows the resulting disturbance currents for the application illustrated by Fig. 1 a. Analyzing these measurements, it can be clearly seen that the dominant mode of disturbance in this case is common mode (CM). Thus, the measurement of disturbance currents supports the results of the system analysis given in Chapter II. From Fig. 6, it has to be also concluded that the disturbance currents in the wires due to an exposure to an electromagnetic field can not be ignored. Of course, the position of the wire with respect to the electrode system plays a significant role for the amplitude of the disturbance currents as well as for the correlation with the generator output voltage. At a certain level of disturbance currents, the communication within the electrical equipment may be disturbed or even electrical devices could be affected. Thus, there is a need to analyze, control and, if necessary, to reduce these disturbance currents.

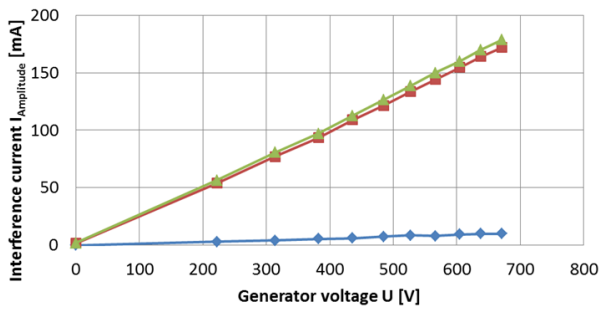

$\rightarrow$ I_DM $\rightarrow$ - I_CM - I_CM from common mode measurement

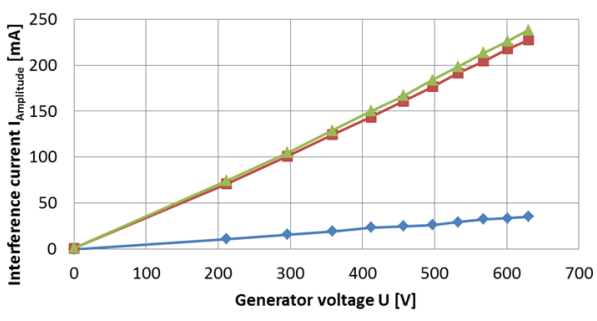

$\rightarrow 1 \mathrm{DM} \rightarrow-\mathrm{C} C \mathrm{CM} \rightarrow \mathrm{I}$ CM from common mode measurement

Fig. 6 Identification of common (CM) and differential mode (DM) interferences without filter and two different measuring modes used for common interference: wire directly under the electrode (left) and wire beside the electrode (distance about $1.5 \mathrm{~m}$ from the RF electrode, right) 


\section{Filter}

On the basis of the disturbance current analysis in Chapter II and the results obtained for an application described in Chapter III showing a dominance of CM over DM conventional filter concepts such as, e.g., suction circuits are less suitable or even not effective at all [6]. This is due to the lack of potential difference between both wires. Thus, a new filter concept must be employed for this type of disturbance current. A well-known approach in the world of radio amateurs is the so-called balun (balanced-unbalanced, Fig. 7 and [7]). Such baluns are used in high-frequency technology mainly as balancing unit. Baluns consist of electrically poor or non-conductive ferrimagnetic materials as core material, e.g. 4W620. For each type of disturbance (CM oder DM), a filter effect can be realized with a corresponding coil arrangement. In Fig. 7, the winding directions are shown for a CM and DM lock, respectively.

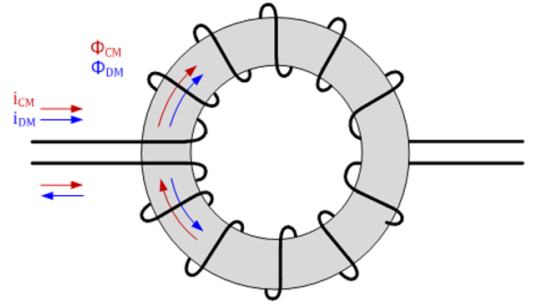

a)

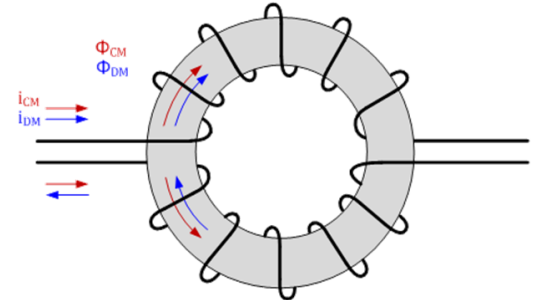

b)

Fig. 7 a) CM lock and b) DM lock

The filter effect of a balun [7,8] is based on the well-known principle of induction. When a periodic current flows through a wire, a periodic magnetic field is formed around this wire. According to Ampère's law, the current $I$ is calculated as a ring integral over the magnetic field strength $H$ with the path $s$ leading to the induction voltage $\Theta$. By winding the wire into a coil, $\Theta$ can be multiplied. The voltage $\Theta$ generates a magnetic flux $\Phi$ that is also dependent on the magnetic resistances in the considered circle. The current direction of the coil determines the direction of $\Phi$. Thus, a periodic magnetic flux is generated in the ferrite material depending on the winding direction and the direction of the periodic disturbance current. For coils with ring cores and without air gap, the inductance $L$ can be determined, according to [9], by the following equation:

$$
L=\frac{1}{2 \pi} \cdot \mu_{0} \cdot \mu_{i} \cdot N^{2} \cdot h \cdot \ln \left(\frac{d_{a}}{d_{i}}\right)
$$

It can be simplified by introducting the geometry parameter $A_{\mathrm{L}}$ leading to

$$
L=A_{L} \cdot N^{2} \text {. }
$$

Here, $N$ is the number of coil turns, $h$ the length of the core, $d_{\mathrm{a}}$ and $d_{\mathrm{i}}$ are the outer and inner diameters, respectively, $\mu_{0}$ is the magnetic field constant $\left(4 \pi 10^{-7} \mathrm{Vs} \mathrm{A}^{-1} \mathrm{~m}^{-1}\right)$ and $\mu_{\mathrm{i}}$ as relative permeability of the material. Based on these theoretical principles and applying the 4W620 ferrite material after several tests with the impedance value from the datasheet of the provider $\left(\mu_{\mathrm{i}}=620\right)$, two baluns were realized. The baluns, one for reducing the $\mathrm{CM}$ 
disturbance (CM lock) and one for reducing the DM disturbance (DM lock), were installed in one chassis (see Fig. 9 a)).

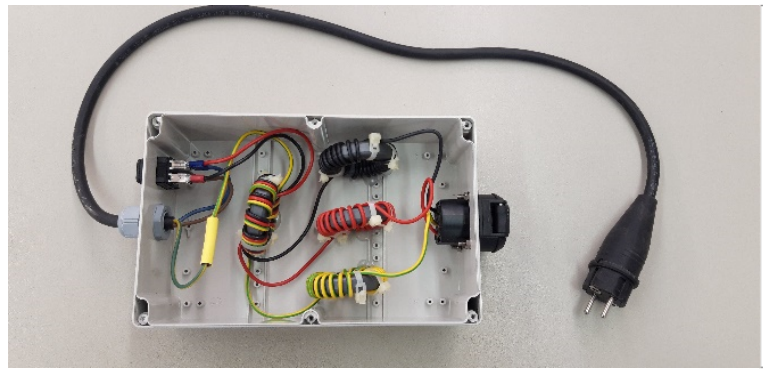

a)

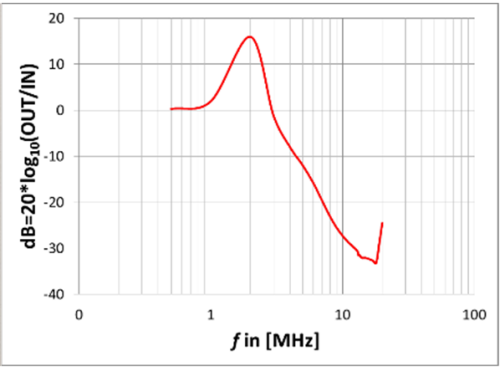

b)

Fig. 9 a) Realized filter consisting of a CM lock and a DM lock, b) transfer function of the total balun, measured between phase and ground wires

Measurements of $i_{\mathrm{CM}}$ and $i_{\mathrm{DM}}$ using the realized filter (Fig. 9 a)) showed a good matching result within the measurement accuracy as can be seen from Fig. 10 (green and red graphs for $i_{\mathrm{CM}}$ and blue graph for $i_{\mathrm{DM}}$ ). But, more important, the graphs in Fig. 10 show the resulting markedly lower disturbance currents after using the new filter exemplarily for the application of drying brickwork by RF heating [1] presented in Fig. 1 a).
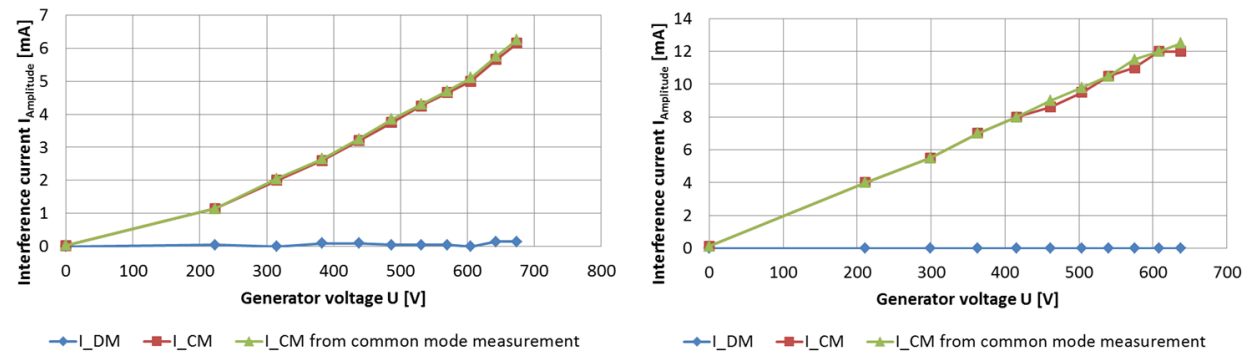

Fig. 10 Identification of CM and DM interferences with the new filter determined by applying two different measuring modes for common interference: wire directly under the electrode (left) and b) wire beside the electrode (right)

From a comparison of the disturbance current amplitudes presented in Fig. 6 and Fig. 10, it can be concluded that the realized integral filter has a marked filter effect with an average

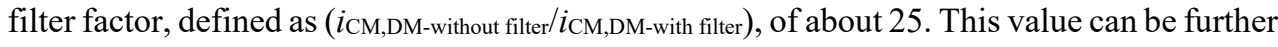
enhanced using the developed principle thus providing an important tool for RF applications in the future.

\section{CONCLUSION}

The reduction of electromagnetic noise is an important aspect for ensuring electromagnetic compatibility (EMC) for RF methods in many application fields. It is especially crucial for situations where complete surrounding of the electrodes and the treated dielectric by an electromagnetic shielding (Faraday cage) is not possible. Often, this corresponds to 
processes with large application potential for RF heating such as drying of brickwork and building structures or chemical-free pest control, e.g. of parquet floor.

In order to develop appropriate solutions for EMC, typical RF heating systems were carefully analyzed with respect to the emission of electromagnetic waves. The main coupling mechanisms were identified which are most relevant for situations where electrical wires cross the treatment or are installed in the vicinity of the electrode system.

Based on detailed investigation of common and differential mode disturbances, the common mode was identified as the more critical influence. For doing so, appropriate measuring regimes were developed and applied, exemplarily for RF drying applications. Thus, a robust and cost-effective measuring system based on current clamps was developed and successfully tested for the in situ detection of RF interferences and identification of coupling mechanisms.

An important conclusion from the measurements is the necessity of developing suitable filter systems for the RF applications. Therefore, based on the concept of baluns, a filter was developed which reduced the coupling disturbance current by a factor of approximately 25 . Further developments can enhance this parameter. In practise, this filter reduced coupling disturbance to an extent that downstream devices (e.g. computers and temperature monitors) were protected against possible disturbance and the communication between the devices at the site was not endangered.

\section{References}

1. Roland, U., F. Holzer et al., COMPEL, 2018, 37, 1933-1942

2. Hoyer, C., C. Pfütze et al., Chem. Eng. Technol., 2018, 41, 108-115

3. Nedtwig, J., M. Lutz, „WEKA Praxishandbuch Elektromagnetische Verträglichkeit“", Berlin, 1999, ISBN 3-8111-7890-3

4. Schwab, A.J., „Elektromagnetische Verträglichkeit“, Springer Verlag, Heidelberg, 1996

5. Morgan, D., „A Handbook for EMC Testing and Measurement“, IEE Electrical Measurement Series 8, 1996

6. Chen, W.-K., „The Circuits and Filters Handbook“, CRC Press, IEEE Pres., 1995, ISBN 0.8493-8341-2

7. Ellis, G., „Electronic Filter Analysis and Synthesis” Artech House, 1994, ISBN 089006-616-7

8. Tietze, U., C. Schenk, E. Gramm, „Halbleiter Schaltungstechnik“, Springer Verlag, Heidelberg, 2016, ISBN 978-3-662-48354-1

9. Schindler, G., Presentation „Magnetische Bauteile und Baugruppen“, attempo, Rottenburg, Version 1.1, 25.06.2010 


\section{DO A SCIENCE EXPERIMENT FOR FUTURE SCIENTISTS}

\section{Y. Kanematsu ${ }^{1}$, T. Matsumura ${ }^{2}$}

${ }^{1}$ Minerva Light Laboratory, 1-7 Hikaridai Seika, Soraku, Kyoto 619-0237, Japan mll.minerva@gmail.com

Keywords: microwave, chemical experiments, educational and voluntary activities.

It has been realized that various chemical reactions are accelerated under irradiation of MW. Such Microwave chemistry is known as timesaving, clear and eco-friendly. MW ovens are world-wide domestic tools for cooking which can serve meals quickly. Regardless of its convenience, few understand the essential mechanism of MW ovens. For better understanding of MW chemistry, authors think it is necessary for to introduce elementary knowledge by holding a 1-day program of experiments by using microwave (MW) ovens. "Science with microwave oven", 1-day program which we developed and named "Hirameki Tokimeki Science" was supported by Japan Society for the promotion of Science, has been performed over four years.More than 100 students of elementary and junior-high school have joined the program. Here we report the program, response from students.Program of experiments: "1: Dyeing handkerchief with onion peer $(* 1), 2$ : Cooking of pizza quickly yeast-leavened, 3: Preparation of shining slime with fluorescein dye synthesized in nonsolvent reaction. 4. Plasma in MW oven (*2), etc."Students realized how MW accelerated chemical reactions and that dyeing under MW was faster and more fixed compared with the conventional methods. Besides, they could enjoy lunch with pizza-and dealing with the slime, both they made. They had a good time with a bit of scientific knowledge.
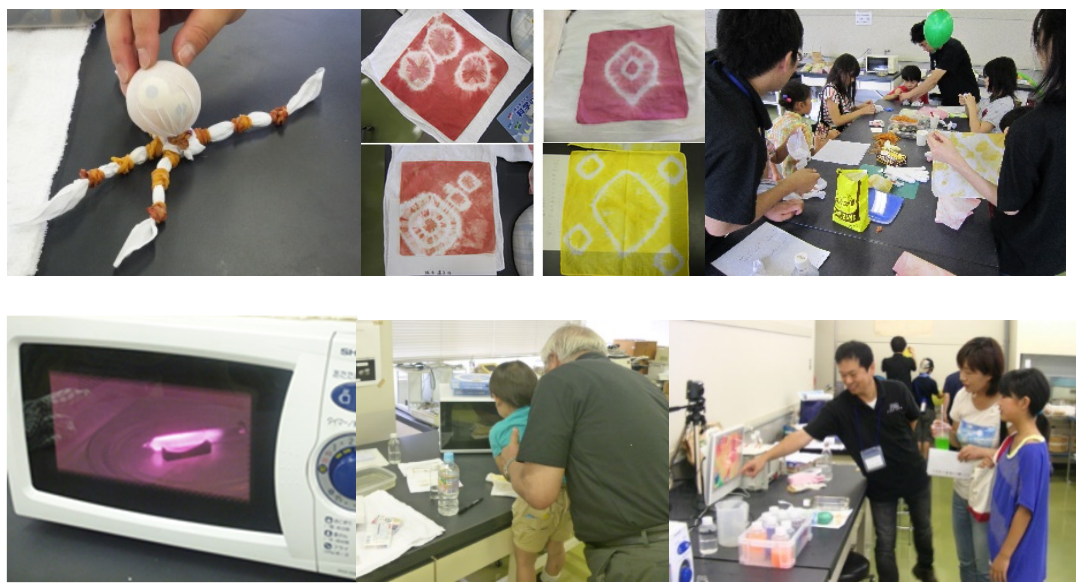

"Science with microwave oven" program surely gives students mysterious interest and dream for Science. That is "Inspire and Spark!" 
Through 1-day program, we can make science more familiar with students, and it will cause young students to become more interested in science, lead them to future research workers. In addition to the "Hirameki Tokimeki (Inspiration and Spark) Program, we have done Volunteer activities at Ishinomaki, one of the most damaged cities at the Higashi Nihon Big Earthquake, in 2011.

\section{Contents of Program}

\section{Introduction: What is microwave?}

2. Microwave plasma in microwave oven.

3. Speedy dying with plants.

4. Rapid cooking Pizza

5. Let's make shining slime!

6. Resume

\section{Introduction: What is microwave?}

Microwaves are a form of electromagnetic radiation with wavelengths ranging from about one meter to one millimeter; with frequencies between $300 \mathrm{MHz}(1 \mathrm{~m})$ and $300 \mathrm{GHz}(1 \mathrm{~mm})$.

One of the most familiar use of microwave is a mobile phone and a microwave oven. MW oven, discovered by American engineer, Percy Spencer of Raytheon Co. in 1946 has been spread over the world and used as kitchen appliance after Sharp Co. developed low-cost microwave oven with turn table in 1966. In this project, we use microwave oven to do microwave experiments in care with safety guide.

30 students, who joined the experiment, are divided into six groups, with one teaching assistant for each group.

\section{Microwave plasma in microwave oven}

The electricity of microwave oven. This experiment is aimed to realize the existence of electricity in a microwave oven. A core of sharp pencil, carbon fiber, sealed test tubes, are used.
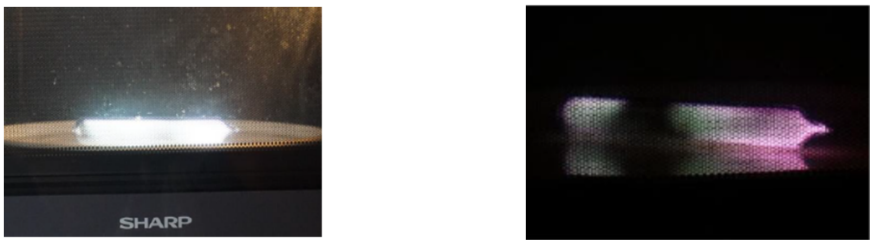

Brilliant 1 color of plasma in test tubes. 


\section{Procedure of dyeing with Plants}

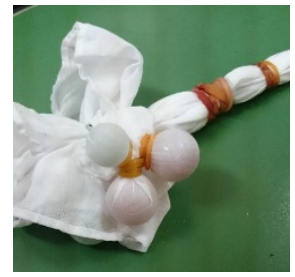

1. Design with chopstick marbles rubber bands

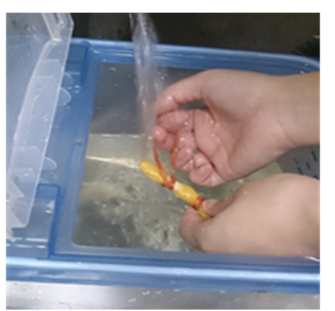

4.washing

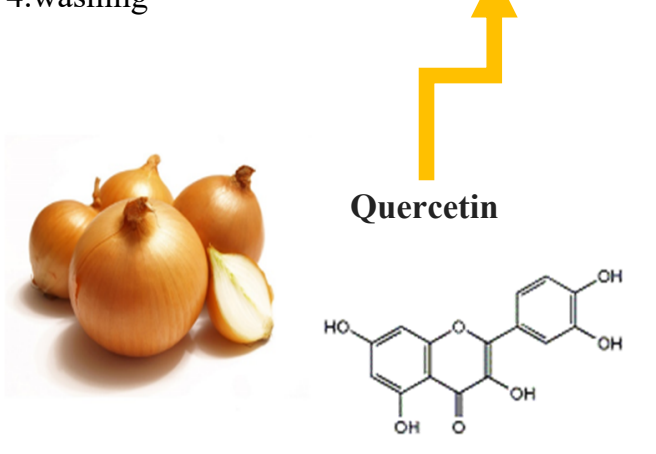

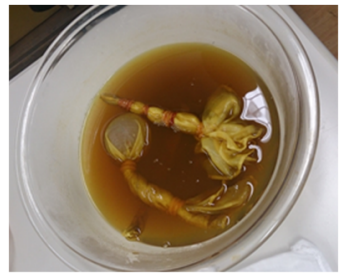

2. Immerse in the dyeing solution.

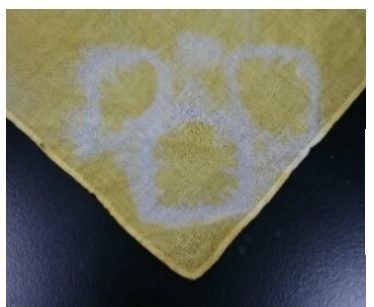

5. Dyed

Handkerchief

\section{Quercetin}

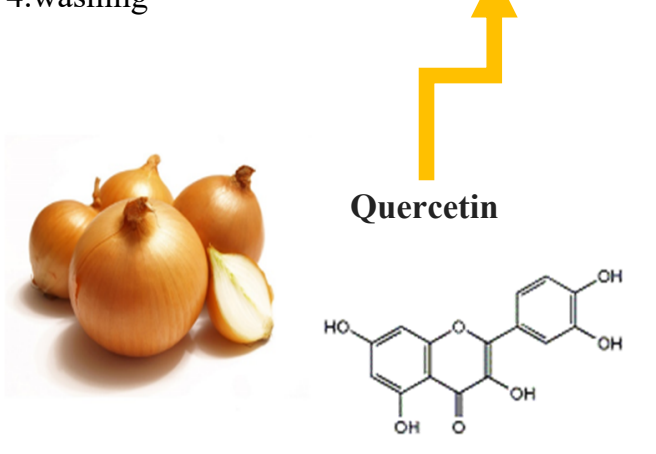

3. 5 minutes in an microwave oven 


\section{4 : Rapid cooking of Pizza}

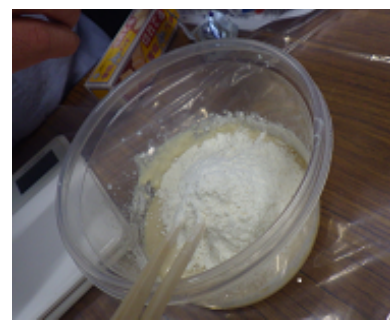

Mixing powder, milk, and butter

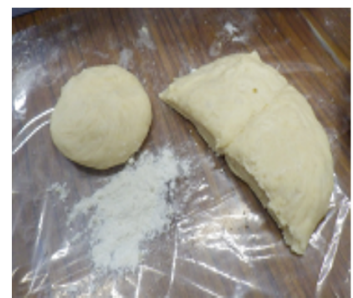

Punch the dough down

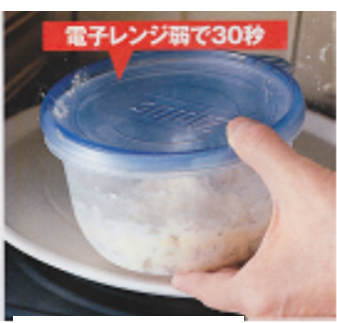

$30 \mathrm{sec}$.

in Oven

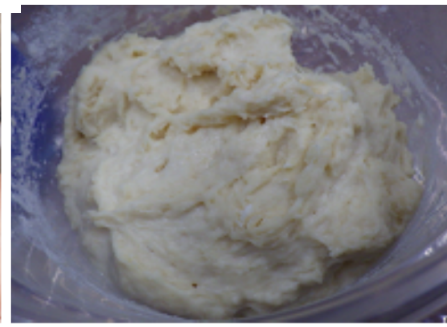

After 10min. the end of the first fermentation

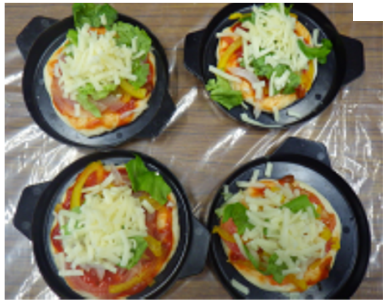

2 min. oven

Voila!

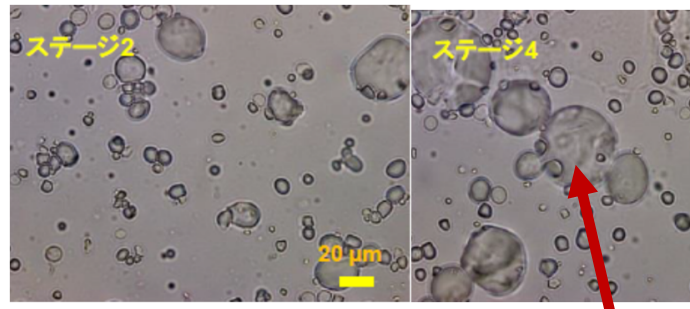

As fermentation proceeds, the number and

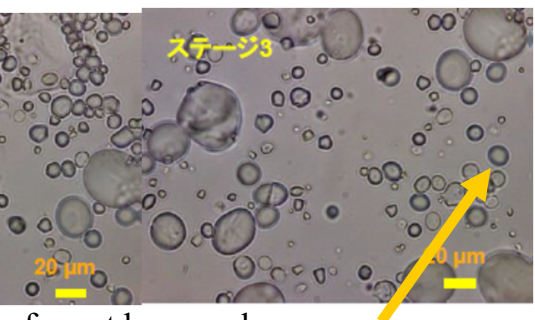

Large, awkward shapes are flour 


\section{5 : Let's make shining slime!}

Powdered phthalic anhydride and resorcinol were placed in a beaker and irradiated with $\mathrm{MW}(500 \mathrm{~W})$ without acid $\left(\mathrm{H}_{2} \mathrm{SO}_{4}\right)$. The powdered mixture started to melt in 2 minutes. The molten substance, became orange color in 3 minutes and red-orange in 5 minutes. Adding $\mathrm{Na}_{2} \mathrm{~B}_{4} \mathrm{O}_{7} \cdot 10 \mathrm{H}_{2} \mathrm{O}$, the color changes to brilliant green luminescent.<smiles>O=C1OC(=O)c2ccccc21</smiles>

Prehalic anhydride<smiles>Oc1ccccc1C=Cc1ccccc1O</smiles>

Hydroęcen bonded resorcinol dimer

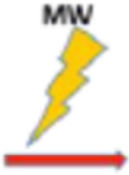

H
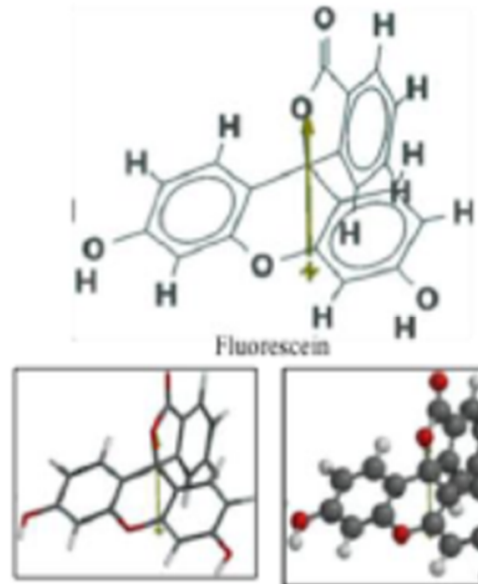

Tube model

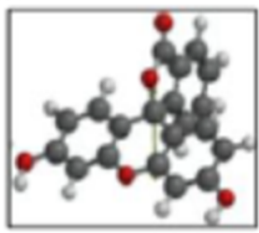

Ball and stick model

Mechanism of MW synthesis of fluorescein without catalyst.

\section{Let's make shining slime !}

Phtalic anhydride(A) and resorcinol(B) are microwaved to react chemically to $200^{\circ} \mathrm{C}$ and gives a sticky brown solution.

Mixture of $A$ and $B$ in a

bottle
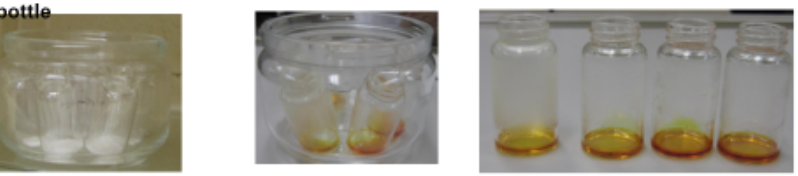

Before heating

Heating 3 minutes

Heating 6 minutes

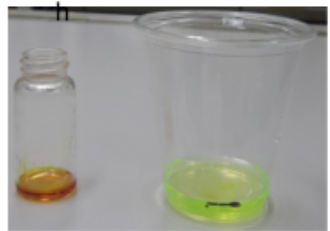

Add a bit of the solution into borax solution to emit $\Leftrightarrow$ Fluorescence Study conditions for emission
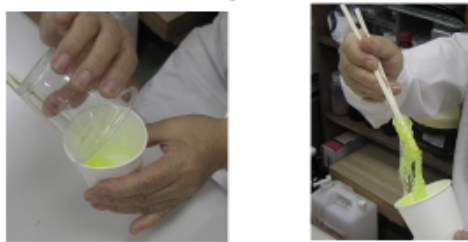

Add a portion of borax solution into laundry starch (polyvinyl alcohol) ) under stirring, the mixture gets hard. Add a bit of fluorescence produces shining slime 


\section{Resume for the study "DO A SCIENCE EXPERIMENT FOR FUTURE SCIENTISTS"}

After the experiment, Students accept the future doctoral degree from organizer.

Students are delight with new experience with microwave oven and surprised with the miracle activity of microwave science.

Thus, "Science with microwave oven" program surely gave students mysterious interest and dream for Science. That is "Inspire and Spark!"

\section{Acknowlegment}

We express our thanks to Teaching members of "Hirameki Tokimeki Science" and Volunteer members ISHINOMKI for their collaboration. 


\title{
MICROWAVE ANNEALING OF POWDER METALS WITHOUT SINTERING
}

\author{
R. Bures ${ }^{1}$, M. Faberova ${ }^{1}$, M. Dilyova ${ }^{2}$ \\ ${ }^{1}$ Institute of Materials Research of Slovak Academy of Sciences, Watsonova 47, 04001 \\ Kosice, Slovakia \\ ${ }^{2}$ Faculty of Manufacturing Technologies of the Technical University of Košice, Bayerova 1, \\ 08001 Presov, Slovakia \\ rbures@saske.sk
}

Keywords: microwave heating, annealing, powder metallurgy, metallic powder compressibility

\section{Introduction}

Powder metallurgy (PM) is a technology for producing solid bodies made of powder materials, for which compressibility is an essential parameter. The compressibility of powder materials is determined by the chemical and phase composition and the particle size and shape. The process for producing powder particles has a significant impact on compressibility since it affects the level of residual mechanical stresses, strain hardening, oxygen and carbon content of the produced powders. Powder annealing is a common technology applied for improving compressibility of produced metallic powders. The goal of annealing is to relax the mechanical stresses generated by the rapid cooling as well as to remove the carbon and oxides formed in the atomisation process on the surface of the metal powder particles. Annealing temperature of iron based powders ranges from 700 to $1200{ }^{\circ} \mathrm{C}$. The annealing to improve compressibility is carried out in an inert gas reducing atmosphere, usually argon containing from 5 to $25 \%$ of hydrogen. If carbon removing is required, then annealing runs under pure hydrogen. The holding time at the annealing temperature is usually in the range of 1 to 2 hours. Water atomized powder is heat treated in a continuous furnace as a "cake" on a belt. A typical set of annealing parameters is as follows: temperature $=1060^{\circ} \mathrm{C}$, time $=1 \mathrm{~h}$, throughput $=2 \mathrm{t} / \mathrm{h}, \mathrm{H}_{2}$-consumption $=160 \mathrm{~m}^{3} / \mathrm{h}\left(80 \mathrm{~m}^{3} / \mathrm{t}\right)$. The annealing of the atomized powder is the most energy-consuming step in the PM production chain [1]. The energy efficiency of the powder annealing is $14 \%$ compared to the $59 \%$ induction melting efficiency or $28 \%$ for the water atomisation process [2]. The annealing yields a powder in the form of a sinter-cake which is necessary to be transformed into a powder again. Sinter-cake is broken e.g. by mechanical milling in disc mill or attritor [3]. Milling process introduces mechanical stresses to the powder.

There is technology to prevent sinter-cake creating and eliminate the need for mechanical milling after annealing. Patent US3668024 [4] describe the way of controlling the furnace atmosphere that prevents welding of the particles. So that the sinter-cake can be readily broken up after annealing. The powder is continuously passed through an annealing furnace and heated to temperature of 780 to $1150{ }^{\circ} \mathrm{C}$, while exposed to reducing gas. The dew point of the furnace atmosphere is maintained at a value slightly below equilibrium value throughout the length of the heating zone by adjustment of the rate of flow of the gas to the furnace. The dew point of the supplied and exhausted reducing gas is monitored and controlled throughout the annealing process. The disadvantage of this method is the necessity of precise monitoring and control of the dew point of the process gases, as well as higher consumption of process gases compared to the countercurrent gas flow in the furnace. Another method of preventing caking [5] is to form a mixture of annealed powder with an easily removable inert powder material e.g. sodium chloride. The inert powder separates the metal powder particles during annealing. After annealing, the inert material is removed by 
washing the powder mixture with a solvent. A disadvantage of such a process is the wet removal of the inert component.

The most efficient, economical and standard method used in the practice is the annealing followed by fine mechanical disintegration of the sinter-cake [3,6-8]. This process is effective to improve compressibility by removing hard oxidic compounds. When using powders in applications requiring magnetically soft materials, the disadvantage of this process is the grinding of the sinter-cake, which causes a re-increase in coercivity due to the insertion of lattice defects and mechanical stresses.

Microwave (MW) heating of materials is used for the synthesis, sintering, curing and heat treatment of ceramic, metal, polymer and composite materials. The main advantages of microwave processing are rapid heating, selectivity of heating and shortening of process times $[9,10]$. Susceptor assisted microwave annealing was proposed for fabrication of silicon based semiconductors in US20120196453A1 [11]. Acceleration of the heat treatment process has been proposed for some electrochemical devices e.g. Li-ion batteries in US20130266741A1 [12]. Microwave annealing has been used in a method for defect recovery of semiconductors for integrated circuits applications US20150294881A1 [13]. Microwave annealing technology of two different materials, resulting in an interface with the desired properties, was designed to produce CMOS semiconductors US006051283A [14].

Concern about global warming motivates the analysis of whole powder metallurgy production chain from the viewpoint of environmental impact. The state of the art show that there is significant opportunity for reducing energy using higher energy efficient technology in PM production. The intensification and shortening of process time are the main features of microwave processing that have been successfully applied in several areas of material engineering. The aim of this work was to investigate the possibility of using microwave heating to improve the compressibility of ferromagnetic metal powder systems while reducing the coercivity.

\section{Experimental materials and methods}

Ferromagnetic powder alloys were investigated to evaluate the influence of microwave annealing on coercive force and compressibility. Investigated metallic powders were subjected to mechanical treatment by milling or die pressing. Microwave annealing (MWA) was provided in single mode microwave cavity at frequency of $2.45 \mathrm{GHz}$. Cylindrical microwave cavity with dimensions in diameter of $28 \mathrm{~mm}$ and height of $80 \mathrm{~mm}$ was equipped with Impedance analyser HiPom and IR pyrometer Optris CT (supplied by UPV-ITACA). Annealed powder was placed in high purity alumina crucible with diameter size of $10 \mathrm{~mm}$ and height of $20 \mathrm{~mm}$. Hydraulic press LabTest 5.600 Zl was used to prepare green compact samples, as well as for the compressibility measurements. High energy vibrating ball milling was provided by Lab Wizz 320 equipment. Coercive force $\left(\mathrm{H}_{\mathrm{c}}\right)$ was measured by coercive force meter ATS-320.

\section{Results and discussion}

Iron powder ASC100.29 (supplied by Hogänäs) was uniaxially pressed in closed die at pressure of $800 \mathrm{MPa}$. Obtained green compact were crushed to powder in a mortar and than MW annealed. MWA process $\log$ in Fig. 1 shows smooth regulation regardless of temperature and process atmosphere. Coercive force was measured step by step after each process. Results of coercive force measurement are presented in Tab. 1. It is well known that ASC100.29 is the powder characterized by very good compressibility. Coercive force increases from $210 \mathrm{~A} / \mathrm{m}$ (as received ASC.29 powder) to value above $320 \mathrm{~A} / \mathrm{m}$ in green compact. Coercive force slightly decreases after green compact crushing due to partial stress 
relieving in compressed powder particles. MWA at $500^{\circ} \mathrm{C}$ for 15 minutes under argon resulted in the highest efficiency (46\%) of the MWA process in terms of reduction of $\mathrm{H}_{\mathrm{c}}$. Shorter time (10 min.) slightly reduces the efficiency (34\%), while lowering the temperature $\left(400^{\circ} \mathrm{C}\right)$ has a more pronounced effect $(8 \%)$. In case of technically pure iron powder as ASC100.29, ambient air atmosphere is not suitable for MW annealing.

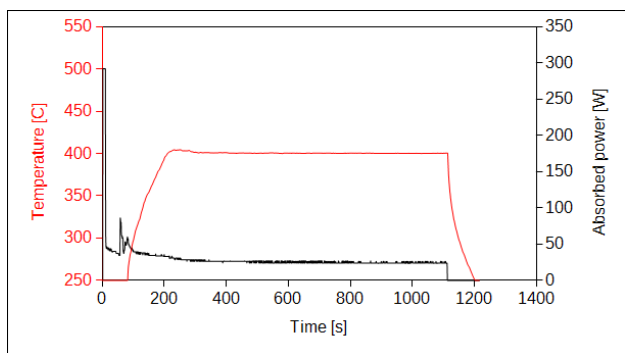

MWA $400^{\circ} \mathrm{C}$, argon

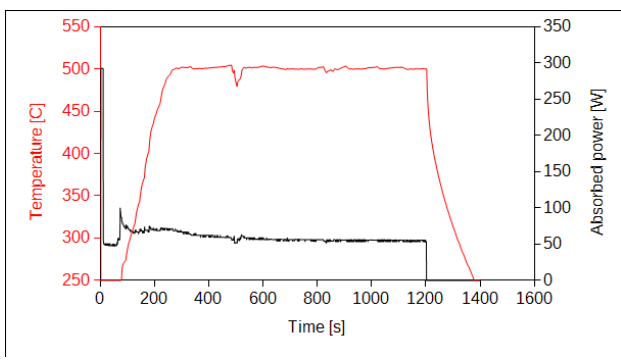

MWA $500^{\circ} \mathrm{C}$, air

Fig. 1. Iron powder ASC100.29 - microwave annealing process log.

Table 1. Coercive force $\mathrm{H}_{\mathrm{c}}$ of iron powder ASC100.29.

\begin{tabular}{ccccccc}
\hline $\begin{array}{c}\mathrm{H}_{\mathrm{c}-\text { green }} \\
{[\mathrm{A} / \mathrm{m}]}\end{array}$ & $\begin{array}{c}\mathrm{H}_{\mathrm{c}-\text { pow }} \\
{[\mathrm{A} / \mathrm{m}]}\end{array}$ & $\begin{array}{c}\mathrm{H}_{\mathrm{c}-\mathrm{MWA}} \\
{[\mathrm{A} / \mathrm{m}]}\end{array}$ & $\begin{array}{r}\eta-\mathrm{H}_{\mathrm{c}} \\
{[\%]}\end{array}$ & $\begin{array}{r}\text { TMwA } \\
{\left[{ }^{\circ} \mathrm{C}\right]}\end{array}$ & $\begin{array}{r}\text { tMwA } \\
{[\mathrm{min}]}\end{array}$ & $\begin{array}{c}\text { Process } \\
\text { gas }\end{array}$ \\
\hline 362 & 340 & 185 & 46 & 500 & 15 & argon \\
340 & 290 & 267 & 8 & 400 & 15 & argon \\
333 & 349 & 230 & 34 & 500 & 10 & argon \\
324 & 307 & 600 & -95 & 500 & 15 & air \\
\hline
\end{tabular}

$\mathrm{H}_{\mathrm{c}}$ - Coercive force at $30 \mathrm{kA} / \mathrm{m}$, green - Green compact, pow - Powder obtained by green compact crushing, $\eta-\mathrm{H}_{\mathrm{c}^{-}}$Efficiency of MWA related to coercive force

Iron-silicon alloy is a metallic material with limited plastic deformation ability. Higher silicon content leads to lower deformation ability of FeSi alloy, while the coercivity decreases. Atomised Fe6.8Si powder alloy with median particle size of $355 \mu \mathrm{m}$ and $180 \mu \mathrm{m}$ was used in this experiment. Fe6.8Si powder with larger particle size distribution was milled for $2 \mathrm{~min}$ or $15 \mathrm{~min}$ respectively. Coercive force and compressibility of all Fe6.8 powders (as received and milled) were measured and than MWA were applied. In all cases, MW annealing was carried out at $500{ }^{\circ} \mathrm{C}$ for 20 min under argon. Time-temperature-absorbtion diagrams in Fig. 2 show smooth regulation of the temperature. Absorbed power curve is slightly different for as received powder and milled powder. The results of compressibility and $\mathrm{H}_{\mathrm{c}}$ analysis are summarised in Table 2. The decrease in $\mathrm{H}_{\mathrm{c}}$ can be attributed to stress relieving and structure recovering MWA, since the residual oxides could not be reduced in an inert atmosphere of Ar. Coercive force of the rapid solidified atomised Fe6.8Si powder decreased from 68 to $41 \mathrm{~A} / \mathrm{m}$ after MWA. Increasing the milling process time results in an increase in $\mathrm{H}_{\mathrm{c}}$ and a reduction in the particle size of the ground powder. The coercive force of the milled powder increases due to the accumulation of structural defects in the powder particles. The highest efficiency of MWA was achieved in case of milled powder due to positive effect of MWA on recovery processes in this powder alloy. Efficiency of MWA process is higher in powder systems consisting of larger particles. The highest efficiency of MWA related to $\mathrm{H}_{\mathrm{c}}(64 \%)$ was achieved for short time milled Fe6.8Si powder. In this case, 
short milling time causes deformation of spherical particles, while particle size reduction was minimal.

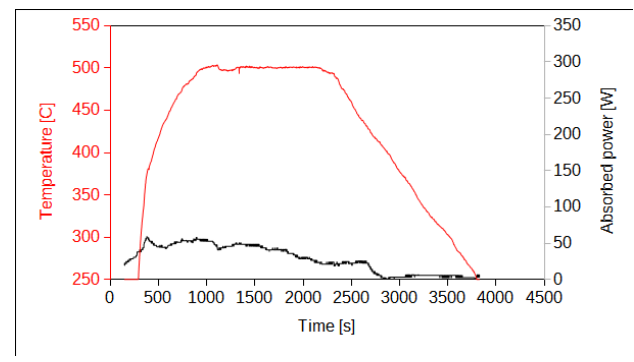

Fe6.8Si $355 \mu \mathrm{m}$ atomised

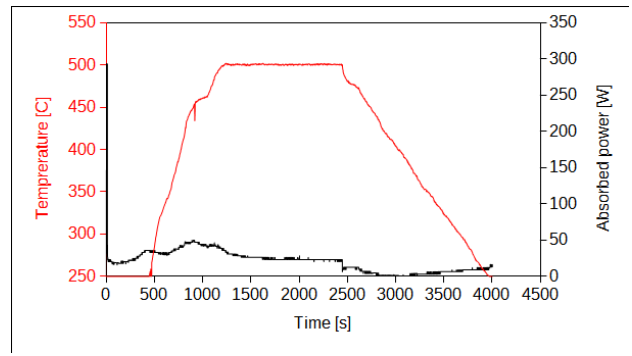

Fe6.8Si $355 \mu \mathrm{m}$ milled for $15 \mathrm{~min}$.

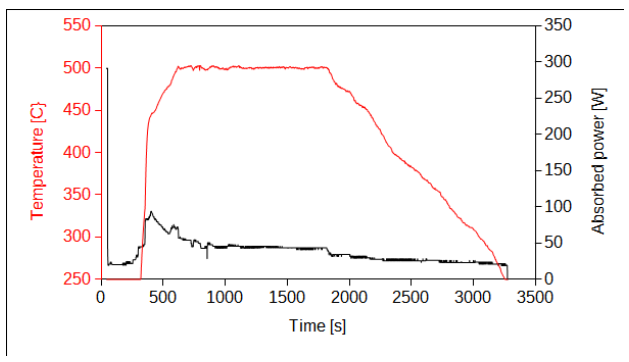

Fe6.8Si $180 \mu \mathrm{m}$ atomised

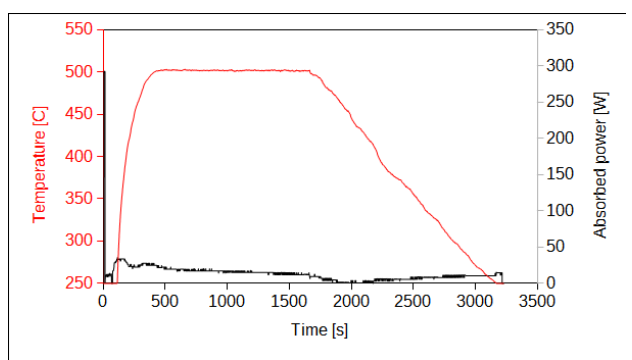

Fe6.8Si $355 \mu \mathrm{m}$ milled for $2 \mathrm{~min}$.

Fig. 2. Fe6.8Si microwave annealing process log.

Table 2. Coercive force $\mathrm{H}_{\mathrm{c}}$ of pressed and MW annealed Fe6.8Si powder material.

\begin{tabular}{|c|c|c|c|c|c|c|}
\hline Powder material & $\begin{array}{c}\mathrm{H}_{\mathrm{c}} \\
\text { source } \\
{[\mathrm{A} / \mathrm{m}]}\end{array}$ & $\begin{array}{c}\mathrm{H}_{\mathrm{c}} \\
\mathrm{MWA}^{*} \\
{[\mathrm{~A} / \mathrm{m}]}\end{array}$ & $\begin{array}{r}\eta-H_{c} \\
{[\%]}\end{array}$ & $\begin{array}{c}\beta \\
\text { source } \\
{[\%]}\end{array}$ & $\begin{array}{c}\beta \\
\mathrm{MWA}^{*} \\
{[\%]}\end{array}$ & $\begin{array}{c}\eta-\beta \\
{[\%]}\end{array}$ \\
\hline \multirow{2}{*}{$\begin{array}{l}\text { Fe6.8Si } 355 \mu \mathrm{m} \\
\text { as received }\end{array}$} & 68 & 41 & \multirow[t]{2}{*}{40} & \multirow[t]{2}{*}{22.85} & \multirow[t]{2}{*}{24.9} & \multirow[t]{2}{*}{9} \\
\hline & \pm 3 & \pm 2 & & & & \\
\hline \multirow{2}{*}{$\begin{array}{l}\text { Fe6.8Si } 355 \mu \mathrm{m} \\
\text { milled } 15 \mathrm{~min}\end{array}$} & 565 & 210 & \multirow[t]{2}{*}{63} & \multirow[t]{2}{*}{43.35} & \multirow[t]{2}{*}{40.6} & \multirow[t]{2}{*}{-6} \\
\hline & \pm 4 & \pm 2 & & & & \\
\hline \multirow{2}{*}{$\begin{array}{l}\text { Fe6.8Si } 355 \mu \mathrm{m} \\
\text { milled } 2 \mathrm{~min}\end{array}$} & 366 & 132 & \multirow[t]{2}{*}{64} & \multirow[t]{2}{*}{21.73} & \multirow[t]{2}{*}{24.1} & \multirow[t]{2}{*}{10} \\
\hline & \pm 2 & \pm 2 & & & & \\
\hline Fe6.8Si 180 & 76 & 68 & 11 & 25.37 & 24.9 & 2 \\
\hline as received & \pm 3 & \pm 2 & & & & \\
\hline
\end{tabular}

$\mathrm{H}_{\mathrm{c}}$ - Coercive force at $30 \mathrm{kA} / \mathrm{m}, \beta$ - Relative compressibility, $\eta$ - Efficiency in relation to $\mathrm{H}_{\mathrm{c}}$ and $\beta,{ }^{*} \mathrm{MWA}-500^{\circ} \mathrm{C}, 20 \mathrm{~min}$, argon atmosphere 
Compressibility of Fe6.8Si powder is influenced by particle size and shape as well as mechanical properties of the powder particles. Fig. 3 shows relative compressibility of investigated Fe6.8Si powders. Relative compressibility was measured as volume reduction in relation to pressing pressure during continual compression test. Falling curve represents volume reduction in dependence on applied pressing pressure from 0 to $1 \mathrm{GPa}$. Raising part of the pressing curve reflects relaxation of the green compact in the closed die in dependence on decreased pressing pressure. Compressibility analysis shows increase in compressibility of the Fe6.8Si powder with large particles (as received or milled for 2 minutes). The compressibility of the 15 min powder was increased due to the changed shape and especially the particle size, rather than because of MWA. The highest efficiency of MWA related to compressibility $(10 \%)$ was achieved for short time milled Fe6.8Si powder.
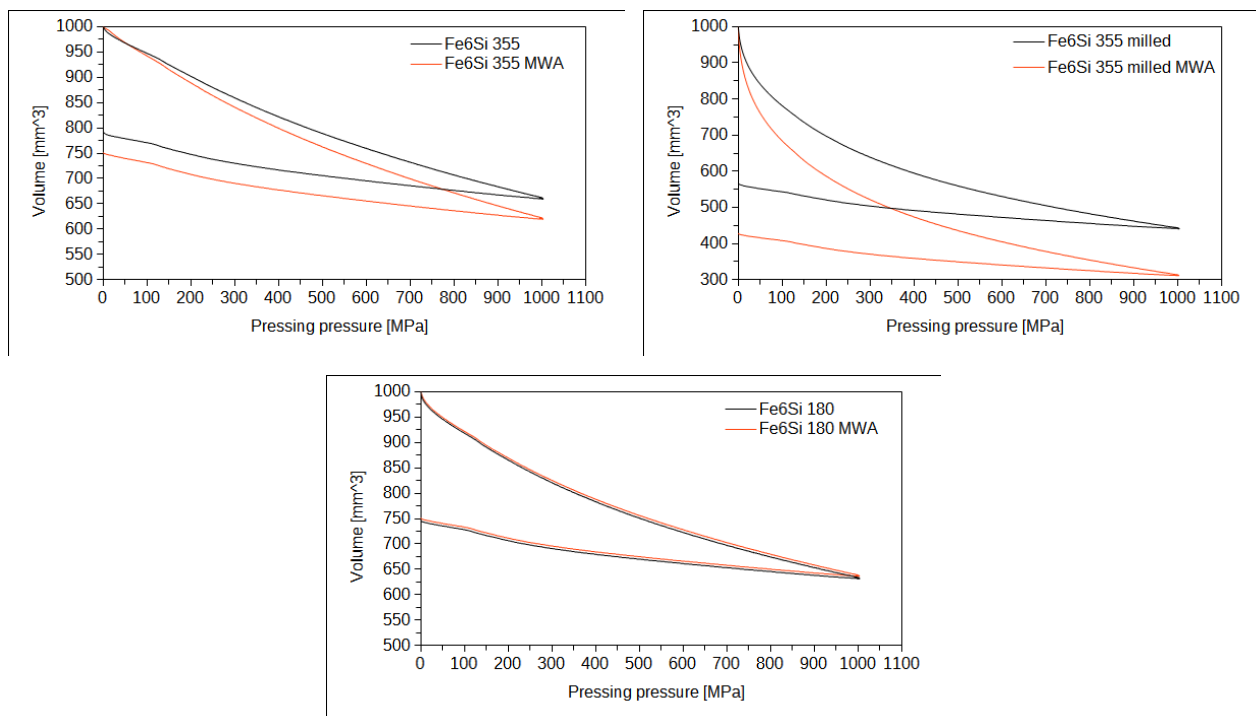

Fig. 3. Compressibility of Fe6.8Si powders.

Amorphous soft magnetic powder alloy $\mathrm{Fe}_{73.5} \mathrm{Cu}_{1} \mathrm{Nb}_{3} \mathrm{Si}_{15.5} \mathrm{~B}_{7}$ (Vitroperm ${ }^{\circledR} 800$, Vacuumschmelze) was used in another experiment. VITROPERM is an amorphous material in the beginning stages of the manufacturing process. In order to produce the nanocrystalline two-phase structure, the amorphous circular tape cores are tempered at about $550{ }^{\circ} \mathrm{C}$ [15]. Vitroperm as received powder was cold die pressed at pressing pressure from $400 \mathrm{MPa}$ to $1000 \mathrm{MPa}$. It was impossible to obtain consolidated bulk sample. Vitroperm powder pressed at pressure of $800 \mathrm{MPa}$ was $\mathrm{MW}$ annealed at temperature of $440^{\circ} \mathrm{C}$ for $20 \mathrm{~min}$ in air. Coercive force of Vitroperm powder increases from $8 \pm 0.5 \mathrm{~A} / \mathrm{m}$ (as received) to $61 \pm 1 \mathrm{~A} / \mathrm{m}$ (pressed powder). MWA leads to the decrease in $\mathrm{H}_{\mathrm{c}}$ to $46 \pm 1 \mathrm{~A} / \mathrm{m}$. The same powder was MW annealed once more at temperature of $500^{\circ} \mathrm{C}$ for $10 \mathrm{~min}$ in air to investigate a trend of $\mathrm{H}_{\mathrm{c}}$ value. Coercive force after second MWA $(58 \pm 2 \mathrm{~A} / \mathrm{m})$ increases in comparison to first MWA, but the value is still lower in comparison with pressed powder $(61 \pm 1 \mathrm{~A} / \mathrm{m})$. New batch of Vitroperm powder pressed at pressure of $800 \mathrm{MPa}$ with $\mathrm{H}_{\mathrm{c}}$ value of $63 \pm 1 \mathrm{~A} / \mathrm{m}$ was MWA at temperature $400{ }^{\circ} \mathrm{C}$ for $20 \mathrm{~min}$ under argon. Coercive force of $\mathrm{MW}$ annealed Vitroperm achieve $43 \pm 1 \mathrm{~A} / \mathrm{m}$. MW annealed Vitroperm powders were successfully pressed to the cylinder shape with diameter of $10 \mathrm{~mm}$ at pressure of $800 \mathrm{MPa}$. Relative density of 
two step in air MW annealed powder achieved $71.5 \%$ compared to the value of $69.3 \%$ in case of MWA at temperature of $400{ }^{\circ} \mathrm{C}$ under argon. Initial state of crystallization begins at temperature of $480^{\circ} \mathrm{C}$ [16]. Results indicates that ability to be compressed was obtained by partial devitrification of initially amorphous powder sample. The most important is the effect of $\mathrm{Cu}$ clustering, which starts prior to crystallization, it is in detail described in [17].

Permalloy, having the composition: $79 \% \mathrm{Ni}, 16 \% \mathrm{Fe}, 5 \% \mathrm{Mo}$ and $0.5 \% \mathrm{Si}$ (also known as Supermalloy), is well known for its high performance as a soft magnetic material, characterized by high permeability and low coercivity. Atomised Permalloy 80 powder (ESPI Metals) was investigated together with laboratory prepared Supermalloy powder. Supermalloy (the same alloy composition as Permalloy 80) was prepared by mechanical alloing (MA) using long term dry ball milling. MA and atomised powder were consolidated at pressing pressure $800 \mathrm{MPa}$ in closed die. Coercive force of the prism shape green compacts with size of $4 \times 5 \times 20 \mathrm{~mm}$ was measured. Green compacts were crushed in mortar to obtain the recycled powder. These powders were MW annealed. MWA process of the atomised Permalloy was smooth regulated in comparison with unstable MWA of MA Supermalloy powder, as it is shown in Fig. 4.

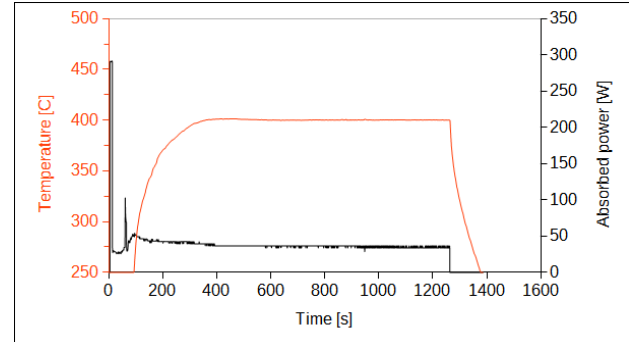

MWA - Permalloy 80

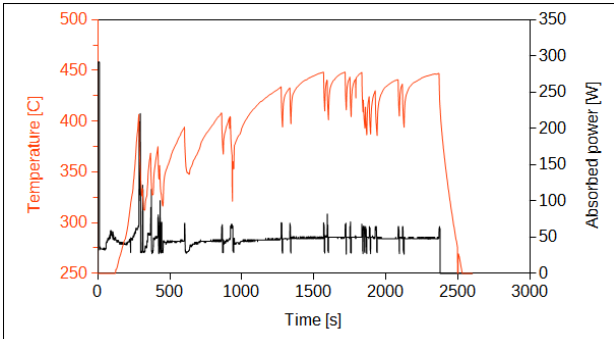

MWA - MA Supermalloy

Fig. 4. NiFeMo powder alloys - microwave annealing process log.

Results of coercive force step by step measurements of initial powders, consolidated green sample, crushed green sample and MW annealed powder are presented in Tab. 3. Permalloy powder system can be MW annealed with high efficiency (about $70 \%$ ) regardless of powder initial state particle size and shape. Irregular MA Supermalloy powder with median particle size of $70 \mu \mathrm{m}$ achieves lower coercive force (99) compared to spherical atomised Permalloy powder with median particle size of $45 \mu \mathrm{m}$.

Table 3. Coercive force $\mathrm{H}_{\mathrm{c}}[\mathrm{A} / \mathrm{m}]$ of MW annealed Ni79Fe16Mo5Si0.5 powder alloys.

\begin{tabular}{c|c|c|c|c|c}
\hline Material & powder1 & Green & powder2 & MWA & $\begin{array}{c}\eta-\mathrm{H}_{\mathrm{c}} \\
{[\%]}\end{array}$ \\
\hline Permalloy atomised & $134 \pm 2$ & $666 \pm 2$ & $456 \pm 2$ & $138 \pm 2.5$ & 70 \\
Supermalloy milled & $347 \pm 0.2$ & $436 \pm 0.2$ & $340 \pm 4.5$ & $99 \pm 1.5$ & 71 \\
\hline
\end{tabular}

Powder 1 - initial state, Powder2 - crashed green compact, $\eta-\mathrm{H}_{\mathrm{c}}$ - MWA efficiency

High entropy alloy based on equiatomic composition FeSiBAlNiMo (HEA) was prepared by mechanical alloying. The as milled powder alloy was amorphous, as it was presented elsewhere [18]. Coercive force of MA HEA powder was $915 \mathrm{~A} / \mathrm{m}$, while MWA at $580^{\circ} \mathrm{C}$ 
for 30 min under argon reduce $H_{c}$ value to $300 \mathrm{~A} / \mathrm{m}$. Efficiency of $\mathrm{H}_{\mathrm{c}}$ reduction by MWA process is similar to conventional soft magnetic powder alloys as FeSi and Permalloy.

Oxide dispersion strengthened steel (ODS) was another investigated non-conventional powder alloy. ODS was prepared by mechanical milling as ferritic powder steel with Y2O3 strengthening phase. ODS powder systems are characterized by very limited cold compressibility. Investigated MA ODS was impossible to consolidate using uniaxial cold die pressing in the range of pressing pressure from 250 to $1200 \mathrm{MPa}$. MWA enabled to achieve a compressibility to relative density of $67.1 \%$ at pressing pressure $500 \mathrm{MPa}$ without spring back and sufficient manipulation strength of the green compact.

In most of the studied powder systems, the regulation of the temperature-time mode of microwave heating was easy to control. The results of experimental microwave annealing of $\mathrm{Fe}, \mathrm{FeSi}, \mathrm{NiFeMo}$ and some amorphous and nanocrystalline powder alloys showed the possibility to achieve improved compressibility and reduced coercive force without the formation of sinter-cake. The effectiveness of reducing coercive force was more significant compared to the efficiency of increasing compressibility. Microwave annealing in inert or air atmospheres may be particularly beneficial in the production of powders for magnetically soft applications.

\section{Conclusions}

In this work, the application of microwave heating to metal powder annealing with the focus on ferromagnetic metals and soft magnetic alloys was investigated. Results of all investigated MWA powder systems documents high potential of MW processing for PM technology. Extra benefit can be expected in the field of powder soft magnetic alloys and composites, where low coercivity of powders is required. Microwave annealing can be applied in powder production, where sinter-cake milling is not applicable. Short process time is the known advantage of MW processes. Additional benefit of MW annealing of powders without sintering can be the possibility to use low cost protective furnace atmosphere, even the air in some cases. In 2019 patent No. 288686 [19] has been granted to the method of microwave annealing of soft magnetic powders.

\section{Acknowledgement}

This work was supported by projects: APVV 0115-15 and VEGA 2/0108/18.

\section{References}

1. Arnhold, V., Kruzhanov, V., 2016, In European Congress and Exhibition on Powder Metallurgy. European PM Conference Proceedings; Shrewsbury, EPMA 2016, 1-10.

2. Azevedo, J.M.C., Serrenho, A.C., Allwood, J.M., Powder Technology, 2018, 328, 329336.

3. Chao, H.-Ch., et al., Process for the production of high apparent density water atomized steel powders: US 3,900,309. United States Patents, 1975

4. Waukesha, M.J., et al., Method of annealing metal powder: US 3,668,024. United States Patents Office, 1972

5. Von Batchelder, F.W., Stauss, H.E., Rev. Sci. Instrum., 2004, 22, 396-397

6. Johnen, D., et al., Method and arrangement for reduction annealing of iron powder: US 5,395,463. United States Patent, 1995

7. Jang, P., Korean J. Met. Mater., 2018, 56, 680-685

8. Lindskog, P.F., et al., Method of manufacturing low-alloy steel powder having a low content of oxidic constituents: US 3,764,295. United States Patents Office, 1973

9. Loharkar, P.K., Ingle, A., Jhavar, S., J. Mater. Res. Technol., 2019, 8, 3306-3326 
10. Borrell, A., Salvador, M.D.,2018, Sintering Technology-Method and Application, Chapter 1, Intech Open, http://dx.doi.org/10.5772/intechopen.78831

11. Terry, L.A., Systems and method s for susceptor assisted microwave annealing: US 2012/0196453 A1. United States - Patent Application Publication, 2012

12. Song, D., et al., Microwave rapid thermal processing of electrochemical devices: US 2013/0266741 A1. United States - Patent Application Publication, 2013

13. Tsai, Ch.H., Wann, C.H., Microwave anneal (MWA) for defect recovery: US 2015/0294881 A1. United States - Patent Application Publication, 2015

14. Lee, K.L., et al., Microwave annealing: US 6,051,283. United States Patent, 2000

15. www.vacuumschmelze.com/index.php?id $=139$

16. Günes, T., J. Non-Cryst. Solids., 2019, 513, 97-104

17. Pradeep, K.G., Herzer, G., Choi, P., Raabe, D., Acta Materialia, 2014, 68, 295-309

18. Bureš, R., et al., Acta Phys. Pol. A, 2017, 131, 771-773

19. Bureš, R., Fáberová, M.. The method of microwave annealing of soft magnetic powders: Patent N. 288686. Banská Bystrica : Industrial Property Office of the SR, 2019 


\title{
OPTIMIZING ASPHALT MIXTURES TO BE HEATED BY MICROWAVES
}

\author{
J. Gallego ${ }^{1}$, F. Gulisano ${ }^{1}$, L. Picado ${ }^{2}$, J. Crucho ${ }^{2}$, \\ ${ }^{1}$ Technical University of Madrid,C/ Profesor Aranguren 3, Madrid, Spain \\ ${ }^{2}$ CERIS, Instituto Superior Tecnico, U. de Lisboa,. Avda. Rovisco Pais 1, Lisbon, Portugal \\ juan.gallego@upm.es
}

Keywords: microwave, heating, asphalt mixtures, self-healing

\begin{abstract}
Over the last ten years the concept of self-healing in asphalt mixtures for road pavements has been reaching great importance due to the fact that this capability allows a material to recuperate original properties after failure. This new generation of asphalt paving materials would allow road agencies to save no renewable resources as aggregates and bitumen, making the road investments more efficient.

Probably the most promising method for the in-situ heating of the pavements is the radiation by microwaves. By heating the pavement, the asphalt binder gets melt and refills the cracks in the material, recuperating a part of its initial structural parameters. Unfortunately, asphalt mixture components (aggregates and binder) are low sensitive to microwave energy. This is why it may be advantageous to incorporate in the mixture additives to improve the sensibility to the radiation with microwaves.

In this investigation two additives were studied: graphene and electric arc furnace (EAF) slag coming from the steel industry. These additives were incorporated at several contents and the efficiency of the heating process was evaluated by the ratio ${ }^{\circ} \mathrm{C} / \mathrm{kwh} / \mathrm{kg}$ in order to optimize the content of the additive in the asphalt mixture.

Additionally, an evaluation of the electrical conductivity of the asphalt mixture at different contents of additives were carried out to study the possible relation between the conductivity of the mixture and its sensibility to microwave radiation.

As a result of the investigation, it seems that microwave radiation can be a real option to promote in situ self-healing of asphalt pavements.
\end{abstract}

\section{Introduction}

Asphalt mixture is the most common material used for road pavement construction in the world and is composed of aggregates and bitumen. However, traffic loads combined with environmental conditions deteriorate asphalt pavement. Cracking is one of the most common sign of asphalt mixture distress, and is caused by cycling loading (fatigue), bitumen aging (oxidation) and temperature variation [1]. Such a damage reduces the mechanical strength and the durability over time [2], and affects the safety of driving [3]. As a result, the pavement should be regularly maintained to restore its original properties. Fortunately, asphalt mixture is a self-healing material, and its healing properties are directly linked to temperature and to the rest periods [4]. When the asphalt pavement is exposed to a temperature above a certain threshold (between $50{ }^{\circ} \mathrm{C}$ and $70{ }^{\circ} \mathrm{C}$ ), bitumen starts behaving as a Newtonian fluid and can flow through the cracks, filling them. This phenomenon can be explained by the diffusion of the molecules between the two sides of the crack, which would create connection points that partially restore the continuity of the material [5]. Such a technology permits increasing the lifetime of the pavement and will also lead savings of 
$\mathrm{CO}_{2}$ emission and less energy consumption for production and maintenance pavements [6]. However, natural self-healing process may require several days to restore the original properties of the pavement, which in practice is impossible due to traffic flow and weather conditions. For this reason, in the last years several researchers proposed different technology with the aim of accelerating the self-healing process. One of the most promising technologies is the microwave heating. Microwave radiation has found different applications in pavement engineering, such as recycling of asphalt pavement [7] and deicing application [8] [9], although most of the researchers used microwave heating to promote self-healing process [10][11][12][13][14][1]. When asphalt mixture is exposed to microwave radiation, heat is generated through conversion of the energy of the electromagnetic field [15]. Under the influence of an alternative electromagnetic field, the polar molecules of asphalt mixture attempt to line up (polarize) with the field. The motion of these molecules is limited by inertia and resisting forces which restrict the motion of molecules and generate random motion and inter-molecular friction that produces heat [7]. Unfortunately, the microwave heating efficiency of ordinary asphalt mixture is very low, due to the low microwave absorbing properties, and for this reason some researchers utilized different additives to improve microwave absorbing properties of asphalt mixture [16], such as steel wool [11] [12], steel slag [17] [13], ferrite [14] and carbon nanotubes [18]. The efficiency of a material in absorbing microwave energy can be described by its dielectric properties [19], usually expressed by the complex permittivity:

$$
\varepsilon^{*}=\varepsilon^{\prime}-i \varepsilon^{\prime \prime}
$$

where $\varepsilon^{\prime}$ is the dielectric constant, representing the amount of energy that can be stored in the material in the form of electric field, $\varepsilon$ " indicates how much of that energy a material can dissipate in the form of heat. Another parameter used for determining the microwave absorbing efficiency is the loss tangent:

$$
\tan \delta=\sigma_{s} /\left(\omega \varepsilon^{\prime}\right)+\varepsilon^{\prime \prime} / \varepsilon^{\prime}
$$

It can be expressed as a sum of two terms [20]: The first term, $\sigma_{s} /\left(\omega \varepsilon^{\prime}\right)$, depends on the conductivity of the material $\left(\sigma_{s}\right)$, and describes loss due to collisions of electrons with other electrons, while the second term, $\varepsilon$ "' $\varepsilon$ ' describes how much energy supplied by an external electric field is dissipated as motion and heat. In conductors, the first term dominates the second, while in dielectrics the second term dominates the first. Semiconductors maintain a relative balance between the two terms. In the case of asphalt mixture dielectric properties are affected not only by frequency and temperature, but also by other properties of the mixture, such as density, moisture and type of aggregates [21]. Several researchers have simulated the electro-magneto-thermal phenomenon of microwave heating in asphalt mixture by using different simulation models, and different parameters have been considered, such as complex permittivity, complex permeability and some thermal properties [15] [8][17][7].

In this research, two kind of additives were studied with the aim of optimizing the microwave heating efficiency: Graphene and Electric Arc Furnace (EAF) Slag. The efficiency of the microwave heating process has been evaluated in terms of the ratio ${ }^{\circ} \mathrm{C} / \mathrm{kWh} / \mathrm{kg}$. This is, the increase of temperature $\left({ }^{\circ} \mathrm{C}\right)$ achieved by the radiation of $1 \mathrm{~kg}$ of asphalt mixture with $1 \mathrm{kWh}$ of microwave energy in fixed conditions. Asphalt mixtures were prepared with different contents of additive, in order to find the optimum percentage in terms of energy efficiency. Additionally, an evaluation of the electrical resistivity of the asphalt mixture at different contents of additives were carried out to study the possible relation between the conductivity of the mixture and its sensibility to microwave radiation. This technology is a promising 
field in the sector of microwave heating applications, although it has not been deeply explored.

\section{Materials}

In total, eight different asphalt mixtures were manufactured in the study: one reference mixture without additives; three asphalt mixture with EAF slag in different contents by total volume of aggregates $(3 \%, 6 \%$ and $9 \%)$; four asphalt mixture with graphene in different contents by total mass of bitumen $(0.5 \%, 1 \%, 1.5 \%$ and $2 \%)$. A conventional asphalt mixture AC20 35/50 was chosen as reference. It was manufactured with limestone aggregates, and its particle size composition is presented in Table 1 . The bitumen content of all the mixtures was $4.6 \%$ by weight of aggregate. Cylindrical specimens (approximately $100 \mathrm{~mm}$ in diameter and $60 \mathrm{~mm}$ in height) were prepared at $165^{\circ} \mathrm{C}$ and then compacted with a Marshall hammer (EN 12697-30: Specimen preparation by Impact compactor) applying 75 bowls on each side at $155^{\circ} \mathrm{C}$. Two Slag fractions were used to replace limestone aggregates: 2/0.5 $\mathrm{mm}$ and $0.5 / 0.063 \mathrm{~mm}$. The chemical composition of EAF slag is reported in Table 2 . Graphene Nanoplatelets (GRAPHENIT-XL) with a bulk density of $0.04 \mathrm{~g} / \mathrm{ml}$ was used, and the elemental analysis is reported in Table 3.

Table 1. Grading curve of the asphalt mixtures

\begin{tabular}{cccccccc}
\hline Sieve $(\mathrm{mm})$ & 22 & 16 & 8 & 4 & 2 & 0.5 & 0.063 \\
$\%$ Passing & 100 & 83 & 56 & 42 & 34 & 19 & 5 \\
\hline
\end{tabular}

Table 2. Chemical composition of EAF slag

\begin{tabular}{cc}
\hline Chemical composition & $\%$ \\
\hline $\mathrm{Al}_{2} \mathrm{O}_{3}$ & 8.81 \\
$\mathrm{CaO}$ & 24.28 \\
$\mathrm{Fe}_{2} \mathrm{O}_{3}$ & 40.49 \\
$\mathrm{MgO}$ & 3.02 \\
$\mathrm{MnO}$ & 4.72 \\
$\mathrm{SiO}_{2}$ & 12.60 \\
$\mathrm{P}_{2} \mathrm{O}_{5}$ & 0.36 \\
Other substances & 5.72 \\
\hline
\end{tabular}

Table 3. Elemental Analysis of Graphene

\begin{tabular}{cc}
\hline Chemical composition & $\%$ \\
\hline $\mathrm{C}$ & 96.41 \\
$\mathrm{H}$ & 0.07 \\
$\mathrm{~N}$ & 0.48 \\
$\mathrm{~S}$ & 0.48 \\
$\mathrm{O}$ & 1.05 \\
\hline
\end{tabular}




\section{Methods}

\subsection{Microwave heating}

To heat the asphalt mixture specimens, a microwave oven was used with an output of $1200 \mathrm{~W}$ and a $230 \mathrm{~V}, 50 \mathrm{~Hz}$ power supply. The oven can produce microwaves of up to $700 \mathrm{~W}$ with a frequency of $2.45 \mathrm{GHz}$. However, in this research a medium power of $350 \mathrm{~W}$ was used. The asphalt mixture specimens were cut into two semi-cylindrical halves; then, both pieces were placed together in the oven and heated for five heating times: $30 \mathrm{~s}, 60 \mathrm{~s}, 90 \mathrm{~s}, 120 \mathrm{~s}$ and 150 s. After each heating time the internal temperatures were measured with an infrared gun. Eight temperatures were taken, randomly chosen in the test sample, and the average was calculated. The initial air temperature was $25^{\circ} \mathrm{C}$. Additionally, an electricity meter was used to measure the energy consumption of the oven during the heating of the specimens. The microwave heating process is shown in Fig. 1.

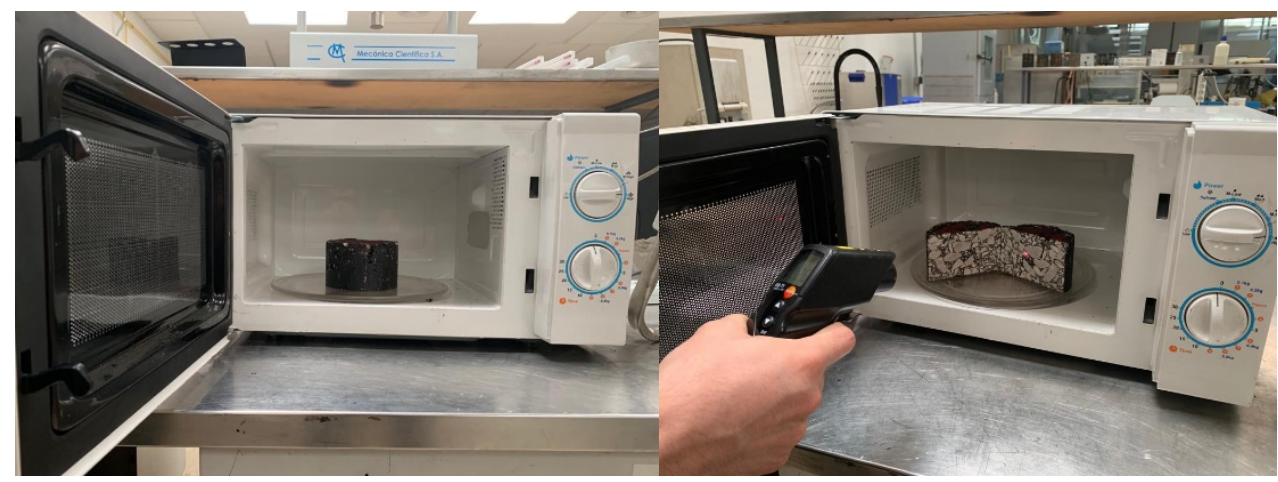

Fig. 1. Asphalt mixtures heating.

\subsection{Electrical resistivity}

The electrical resistance of the asphalt mixtures was measured with the two-probe method by using a megohmmeter with 5 ranges $(50 \mathrm{~V}-1000 \mathrm{~V})$. The asphalt specimen, with a thickness of $\sim 4 \mathrm{~cm}$ was placed between two copper plate electrodes with dimensions $15 \mathrm{x}$ $15 \mathrm{~cm}$ connected with the megohmmeter. Graphene powder was used to fill the gaps between the plate electrodes and the specimens and to ensure a perfect contact between them. The selected voltage was applied to each asphalt specimen for 5 minutes, and then the measurement was taken, as shown in Fig. 2. The electrical resistivity was calculated applying the second Ohm's law:

$$
\rho=(R \cdot S) / l(3)
$$

Where $R$ is the electrical resistance of each specimen in $\Omega ; S$ is the electrode-specimen contact area measured in $\mathrm{m}^{2}$ and $l$ is the thickness of the asphalt sample in $\mathrm{m}$. 

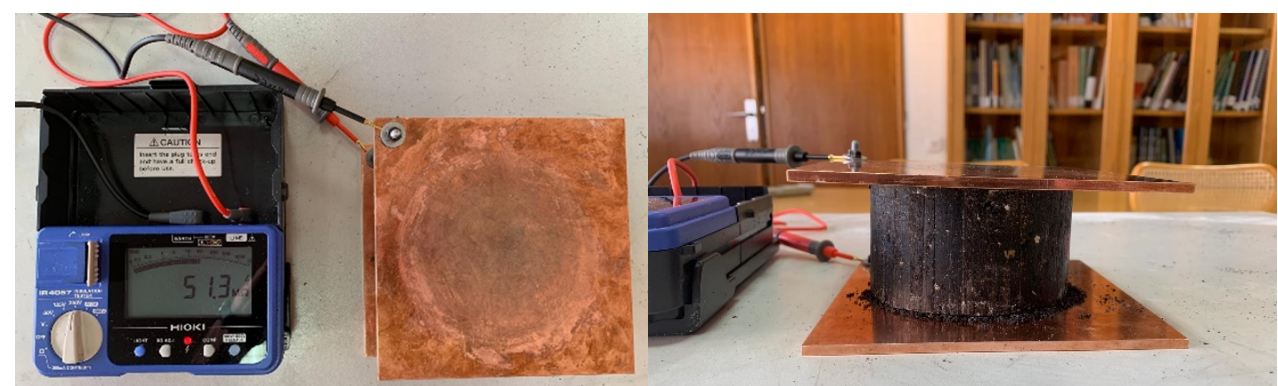

Fig. 2. Measurement of the electrical resistivity of asphalt specimens

\section{Results}

\subsection{Heating efficiency}

Fig. 3 shows the relationship between the internal temperature of the asphalts mixtures and the total energy consumed during the heating. As can be seen, adding EAF slag or graphene to the asphalt mixture produce an increase in the heating efficiency, in terms of the ratio ${ }^{\circ} \mathrm{C} / \mathrm{kWh} / \mathrm{kg}$. The higher the amount of additive, the faster the temperature increase with energy. For a total energy of $0.03 \mathrm{kWh} / \mathrm{Kg}$, corresponding to a heating time of $150 \mathrm{~s}$, the asphalt mixture with $9 \%$ of slag reaches $88{ }^{\circ} \mathrm{C}$, while the mixture with $2 \%$ of graphene reaches $95{ }^{\circ} \mathrm{C}$. On the contrary, the reference mixture (with no additives) reaches $60{ }^{\circ} \mathrm{C}$
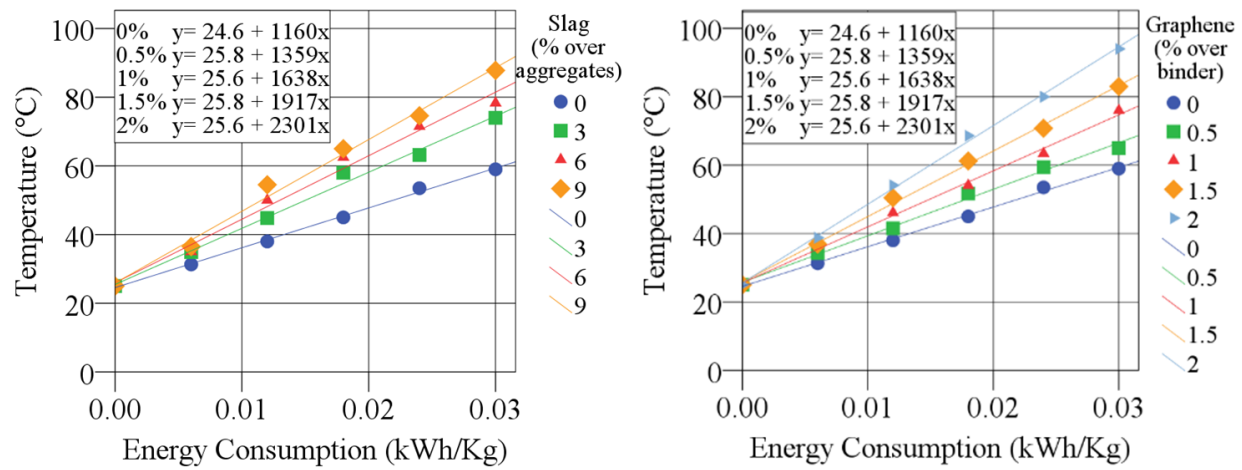

Fig. 3. Temperature analysis of asphalt mixtures with (a) EAF slag and (b) with graphene

However, two aspects must be considered when adding slag or graphene in the asphalt mixtures: the cost and the mechanical performance of the mixtures. The inclusion of EAF slag doesn't imply additional cost, as the cost is similar to the prices of natural aggregates [16], and the mechanical behavior of mixtures with steel slag aggregate is generally better than that of the samples with natural aggregate [22]. In contrast, the addition of graphene is very expensive, and there are currently very few studied about the mechanical performance of these kind of mixtures.

\subsection{Electrical resistivity}

Fig 4 shows the electrical resistivity of the asphalt mixture specimens with different contents of EAF slag and graphene. The inclusion of additives produces a decrease in the electrical 
resistivity of the mixture, and therefore an increase in the electrical conductivity. As for the slag, by adding a total amount of $6 \%$ to the mixture, the benefit in terms of electrical resistivity reduction is not very high, while a substitution of $9 \%$ allows to obtain a value of $1.7 \times 10^{6} \Omega \cdot \mathrm{m}$, corresponding to a total reduction of $99 \%$ respect to the reference mixture $\left(2.3 \times 10^{8} \Omega \cdot \mathrm{m}\right)$. The resistivity of the mixtures containing graphene slightly decrease with the increase of additive content, until reaching a value of $1.3 \times 10^{8} \Omega \cdot \mathrm{m}$ by adding $2 \%$ of graphene. Comparing these results with the ones obtained with the heating curves, it seems that the electrical conductivity of the mixture has not a direct relationship with the heating efficiency. Further research must be conducted to analyze the influence of other parameters, such as the complex permittivity, complex permeability and thermal properties of the asphalt mixture.
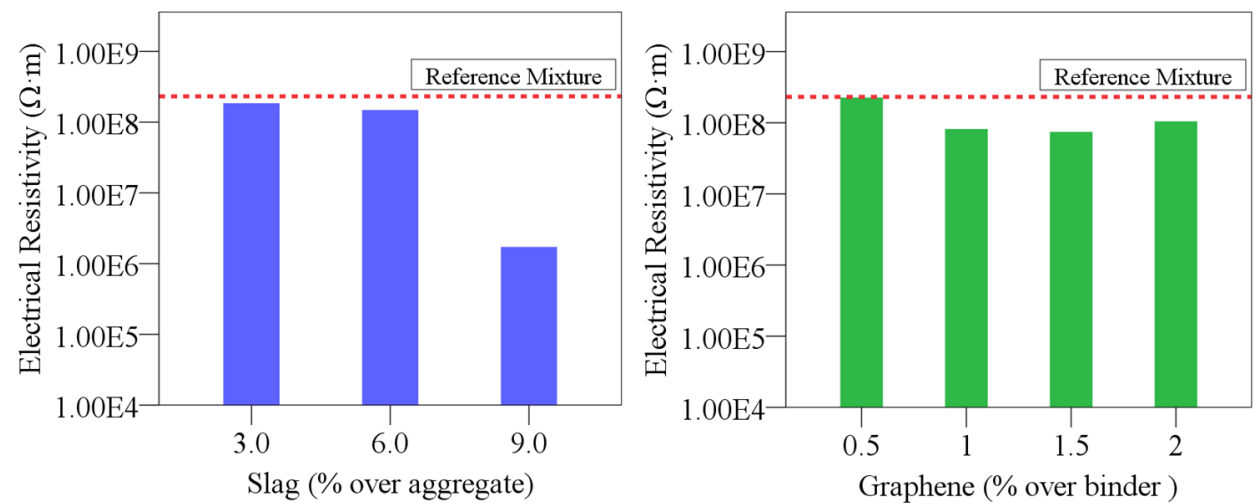

Fig. 4. Electrical resistivity of asphalt mixtures with (a) EAF slag and (b) with graphene

\section{Conclusion}

In this paper, EAF slag and graphene were incorporated in the asphalt mixture, in order to increase the microwave heating efficiency. From the results obtained, several conclusions can be drawn. Both additives produce an increase in the microwave heating efficiency, in terms of the ratio ${ }^{\circ} \mathrm{C} / \mathrm{kWh} / \mathrm{kg}$. However, the lower price of EAF slag compared with graphene makes preferable the use of slag for these applications from an economic point of view. In future research, a comparison between the mechanical performance and the selfhealing properties of the two additives should be conducted beyond the heating properties showed in this investigation.

Additionally, an evaluation of the electrical resistivity of the asphalt mixture at different contents of additives were carried out to study the possible relation between the conductivity of the mixture and its sensibility to microwave radiation. The inclusion of additives produces a decrease in the electrical resistivity of the mixture, and the addition of $9 \%$ of EAF slag provide the best benefits in terms of resistivity reduction. However, the results show that this parameter is not directly related to the microwave heating efficiency. In future research, dielectric and thermal properties of the mixture should be analyzed, in order to study the microwave absorption properties of asphalt mixtures. In conclusion, the use of microwave energy for heating purpose is a very promising technology for asphalt pavement engineering. 


\section{Acknowledgments}

The collaboration for this investigation between the University of Lisbon and the Technical University of Madrid was possible thanks to Grant n. PDI-18-0HXIUI-55-QZMWQL for Investigation Stays of Senior Researchers in Foreign Universities and an additional Grant for PhD students by the Agustín of Betancourt Foundation.

\section{References}

[1] A. Gonzalez, J. Norambuena-Contreras, L. Storey, and E. Schlangen, "Effect of RAP and fibers addition on asphalt mixtures with self-healing properties gained by microwave radiation heating," Constr. Build. Mater., vol. 159, pp. 164-174, 2018.

[2] J. Norambuena-Contreras, R. Serpell, G. V. Vidal, A. Gonzalez, and E. Schlangen, "Effect of fibres addition on the physical and mechanical properties of asphalt mixtures with crack-healing purposes by microwave radiation," Constr. Build. Mater., vol. 127, pp. 369-382, 2016.

[3] C. Li, S. Wu, Z. Chen, G. Tao, and Y. Xiao, "Enhanced heat release and self-healing properties of steel slag filler based asphalt materials under microwave irradiation," Constr. Build. Mater., vol. 193, pp. 32-41, Dec. 2018.

[4] Á. García, "Self-healing of open cracks in asphalt mastic," Fuel, vol. 93, pp. 264-272, Mar. 2012.

[5] D. N. Little and A. Bhasin, "Exploring Mechanism of Healing in Asphalt Mixtures and Quantifying its Impact," Springer, Dordrecht, 2007, pp. 205-218.

[6] Q. Liu, Á. García, E. Schlangen, and M. van de Ven, "Induction healing of asphalt mastic and porous asphalt concrete," Constr. Build. Mater., vol. 25, no. 9, pp. 3746-3752, Sep. 2011.

[7] A. Benedetto and A. Calvi, "A pilot study on microwave heating for production and recycling of road pavement materials," Constr. Build. Mater., vol. 44, pp. 351-359, Jul. 2013.

[8] L. Ding, X. Wang, W. Zhang, S. Wang, J. Zhao, and Y. Li, "Microwave Deicing Efficiency: Study on the Difference between Microwave Frequencies and Road Structure Materials," Appl. Sci., vol. 8, no. 12, p. 2360, Nov. 2018.

[9] Y. Sun, S. Wu, Q. Liu, J. Hu, Y. Yuan, and Q. Ye, "Snow and ice melting properties of self-healing asphalt mixtures with induction heating and microwave heating," Appl. Therm. Eng., vol. 129, pp. 871-883, Jan. 2018.

[10] J. Norambuena-Contreras, A. Gonzalez, J. L. Concha, I. Gonzalez-Torre, and E. Schlangen, "Effect of metallic waste addition on the electrical, thermophysical and microwave crack-healing properties of asphalt mixtures," Constr. Build. Mater., vol. 187, pp. 1039-1050, 2018.

[11] J. Gallego, M. A. del Val, V. Contreras, and A. Paez, "Heating asphalt mixtures with microwaves to promote self-healing," Constr. Build. Mater., vol. 42, pp. 1-4, 2013.

[12] J. Norambuena-Contreras and A. Garcia, "Self-healing of asphalt mixture by microwave and induction heating," Mater. Des., vol. 106, pp. 404-414, 2016.

[13] T. M. Phan, D.-W. Park, and T. H. M. Le, "Crack healing performance of hot mix asphalt containing steel slag by microwaves heating," Constr. Build. Mater., vol. 180, pp. 503-511, Aug. 2018.

[14] X. Zhu, Y. Cai, S. Zhong, J. Zhu, and H. Zhao, "Self-healing efficiency of ferrite-filled asphalt mixture after microwave irradiation," Constr. Build. Mater., vol. 141, pp. 12-22, Jun. 2017.

[15] H. Wang, Y. Zhang, Y. Zhang, S. Feng, G. Lu, and L. Cao, "Laboratory and Numerical 
Investigation of Microwave Heating Properties of Asphalt Mixture," Materials (Basel)., vol. 12, no. 1, p. 146, Jan. 2019.

[16] J. Gallego, M. A. Del Val, V. Contreras, A. Páez, and A. Páez, "Use of additives to improve the capacity of bituminous mixtures to be heated by means of microwaves," Mater. Construcción, vol. 67, no. 325, p. 110, Feb. 2017.

[17] W. Liu, P. Miao, and S.-Y. Wang, "Increasing Microwave Heating Efficiency of Asphalt-Coated Aggregates Mixed with Modified Steel Slag Particles," J. Mater. Civ. Eng., vol. 29, no. 10, p. 04017171, Oct. 2017.

[18]I. Pérez et al., "Self-healing of asphalt mixes, containing conductive modified bitumen,using microwave heating," in 6th Eurasphalt \& Eurobitume Congress, 2016.

[19] A. A. Al-Ohaly and R. L. Terrel, Effect of microwave heating on adhesion and moisture damage of asphalt mixtures. 1988.

[20] C. Bishop, "The Relationship Between Loss, Conductivity, and Dielectric Constant." 2001.

[21] E. J. Jaselskis, J. Grigas, and A. Brilingas, "Dielectric Properties of Asphalt Pavement," J. Mater. Civ. Eng., vol. 15, no. 5, pp. 427-434, Oct. 2003.

[22] M. Skaf, J. M. Manso, Á. Aragón, J. A. Fuente-Alonso, and V. Ortega-López, "EAF slag in asphalt mixes: A brief review of its possible re-use," Resour. Conserv. Recycl., vol. 120, pp. 176-185, May 2017. 
17th International Conference on Microwave and High Frequency Heating

\section{Techniques for monitoring microwave processes}




\title{
H2-REDUCTION BEHAVIOR OF FeS-CaO MIXTURE DURING MICROWAVE HEATING
}

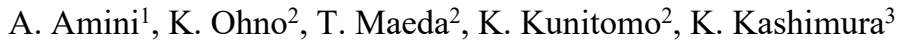 \\ ${ }^{1}$ Department of Materials Process Engineering, Graduate School of Engineering, Kyushu \\ University, 744 Motooka, Nishi-Ku, Fukuoka, Japan \\ ${ }^{2}$ Department of Materials Science and Engineering, Faculty of Engineering, Kyushu \\ University, 744 Motooka, Nishi-Ku, Fukuoka, Japan \\ ${ }^{3}$ Faculty of Engineering, Chubu University, 1200 Matsumoto-cho, Kasugai, Aichi 487- \\ 8501, Japan \\ ahmadreza.amini.ir@gmail.com
}

Keywords: microwave heating, $\mathrm{H}_{2}$-reduction, kinetic study

\begin{abstract}
Kinetics of iron production via hydrogen-reduction of $\mathrm{FeS}-\mathrm{CaO}$ mixture during microwave heating was investigated at 750,570 , and $460{ }^{\circ} \mathrm{C}$ to clarify the dominant rate-controlling mechanism. Results revealed that the interfacial chemical reaction is rapidly progressed during microwave irradiation and gas diffusion is the dominant rate-controlling mechanism with an activation energy of $22.3 \mathrm{~kJ} \mathrm{~mol}^{-1}$. This study demonstrated that the microwave irradiation enhances the rate of chemical reaction during iron production from FeS-CaO mixture.
\end{abstract}

\section{Introduction}

Reduction of sulfide minerals in the presence of lime $(\mathrm{CaO})$ has been studied in previous literature ${ }^{[1,2]}$. Hara et al. ${ }^{[1]}$ produced metallic $\mathrm{Cu}, \mathrm{Co}$ and $\mathrm{Fe}$ from a sulfide concentrate via a carbothermic reduction in the presence of $\mathrm{CaO}$ at $1000^{\circ} \mathrm{C}$. Jha et al. ${ }^{[3]}$ showed that the reduction of $\mathrm{FeS}: \mathrm{CaO}: \mathrm{C}=1: 1: 2$ mixture initiates after a heat treatment at $850{ }^{\circ} \mathrm{C}$ for $>90$ min. On the other hand, reduction rate of iron oxide in $\mathrm{H}_{2}$ is much higher than that in $\mathrm{CO}$ ${ }^{[4,5]}$. Accordingly, hydrogen-reduction of $\mathrm{FeS}$ in the presence of lime is likely an alternative method for iron production from iron sulfide minerals that can be considered as a twostage reaction ${ }^{[6]}$; ion exchange reaction, Eq. (1), and reduction reaction, Eq. (2) ${ }^{[7]}$. The total reaction is represented by Eq. (3).

$$
\begin{array}{lll}
\mathrm{FeS}_{(\mathrm{s})}+\mathrm{CaO}_{(\mathrm{s})}=\mathrm{FeO}_{(\mathrm{s})}+\mathrm{CaS}_{(\mathrm{s})} & \triangle \mathrm{G}_{1}{ }^{\circ}=-4615-0.205 \mathrm{~T} & \mathrm{~J}^{\circ} \mathrm{mol}^{-1} \\
\mathrm{FeO}_{(\mathrm{s})}+\mathrm{H}_{2(\mathrm{~g})}=\mathrm{Fe}_{(\mathrm{s})}+\mathrm{H}_{2} \mathrm{O}_{(\mathrm{g})} & \triangle \mathrm{G}_{2}{ }^{\circ}=16240-8.45 \mathrm{~T} & \mathrm{~J} . \mathrm{mol}^{-1} \\
\mathrm{FeS}_{(\mathrm{s})}+\mathrm{CaO}_{(\mathrm{s})}+\mathrm{H}_{2(\mathrm{~g})}=\mathrm{Fe}_{(\mathrm{s})}+\mathrm{CaS}_{(\mathrm{s})}+\mathrm{H}_{2} \mathrm{O}_{(\mathrm{g})} & \triangle \mathrm{G}_{3}{ }^{\circ}=11625-8.655 \mathrm{~T} & \mathrm{~J} . \mathrm{mol}^{-1}
\end{array}
$$

In addition, microwave irradiation is an energy-efficient and a rapid-heating method to decrease activation energy of chemical reactions owing to both thermal and non-thermal effects of microwave photons. The non-thermal effect of microwave irradiation on the rate of chemical reactions, has attracted the attention of researchers on microwave irradiation energy in terms of speeding up chemical reactions ${ }^{[8-10]}$. The thermal effect of microwave irradiation has the potential to mitigate $\mathrm{CO}_{2}$ emission and decrease the amount of carbonaceous materials required for carbothermic reduction of iron resources due to its specific characteristics, such as rapid and selective heating, volumetric heating, and highefficiency heating.

As a novel idea for a further mitigation of $\mathrm{CO}_{2}$ emission, hydrogen-reduction of $\mathrm{FeS}-\mathrm{CaO}$ mixture during microwave irradiation is investigated in the present study to combine the advantages of microwave irradiation and using $\mathrm{H}_{2}$ as reducing agent. 


\section{Materials and Methods}

Reagent $\mathrm{CaCO}_{3}$ powder (purity, $99.5 \%$, calcined at $1300{ }^{\circ} \mathrm{C}$, crushed and ground to obtain a $\mathrm{CaO}$ with grain size of less than $45 \mu \mathrm{m}$ ) and $\mathrm{FeS}$ powder (purity, 99\%, crushed and ground to the grain size of less than $45 \mu \mathrm{m}$ ) were mixed well by a stainless steel spoon to prepare a powder mixture of $\mathrm{FeS}$ : $\mathrm{CaO}$ with stoichiometric mole ratio of $1: 1$. The mixture $(2 \mathrm{~g})$ was pressed to a cylindrical shape briquette sample $(10 \mathrm{~mm}$ diameter, $10 \mathrm{~mm}$ height, $2.54 \mathrm{~g}^{\mathrm{c}} \mathrm{cm}^{-3}$ apparent density) using a cold hydraulic press. The reaction progress was estimated based on the weight change of samples during microwave treatment in $\mathrm{H}_{2}$ (1 NL. min $^{-1}$ ) using a traditional multi-mode microwave generator with a maximum output power of $1.5 \mathrm{~kW}$ at $2.45 \mathrm{GHz}$. In the present study, the microwave heating system was equipped with thermobalance to prepare a unique apparatus for a novel continuous study of the hydrogen-reduction during microwave irradiation. The sample was placed in a silica basket (silica is a transparent material during microwave irradiation), and the basket was suspended in a silica tube (inner diameter, $35 \mathrm{~mm}$ ) by silica chains connected to the thermobalance which installed on top of the microwave apparatus. Temperature of the sample was measured using an infrared radiation thermometer with the measurement range of $330-1500{ }^{\circ} \mathrm{C}$. The experiments were conducted at various temperatures by applying the microwave out-put powers of 1275,1125 , and $975 \mathrm{~W}$. After the certain durations, the microwave heating system was turned off and the atmosphere was changed to $\mathrm{N}_{2}$ (1 NL. min $^{-1}$ ) to cool down the sample. In the present study, the microwave treatment was conducted at least twice at each output power and the average temperature was considered as the temperature corresponding to the applied power. The average temperature of 750 , 570 , and $460{ }^{\circ} \mathrm{C}$ were attained after a 1200 s-treatment during microwave irradiation at 1275,1125 , and $975 \mathrm{~W}$, respectively.

\section{Reduction degree}

Reduction degree (X, dimensionless) was calculated according to Eq. 4:

Reduction degree $(X)-\frac{W_{t}-W_{t}-W_{h t}}{W_{i} \times W_{O}}$

where $\mathrm{W}_{\mathrm{i}}(\mathrm{g}), \mathrm{W}_{\mathrm{t}}(\mathrm{g})$, and $\mathrm{W}_{\mathrm{ht}}(\mathrm{g})$ are the initial weight of the sample, the weight of the sample after treatment for $t$ seconds, and the weight change of the sample after treatment for $t$ seconds owing to the dehydration reaction. $W_{O}$ (dimensionless) is the stoichiometric weight ratio of oxygen in the sample, which is 0.111 . Fig. 1 shows the reduction degree of samples during microwave irradiation in $\mathrm{H}_{2}$ at $1275 \mathrm{~W}\left(750^{\circ} \mathrm{C}\right), 1125 \mathrm{~W}\left(570{ }^{\circ} \mathrm{C}\right)$, and $975 \mathrm{~W}\left(460^{\circ} \mathrm{C}\right)$ for $1200 \mathrm{~s}$. 


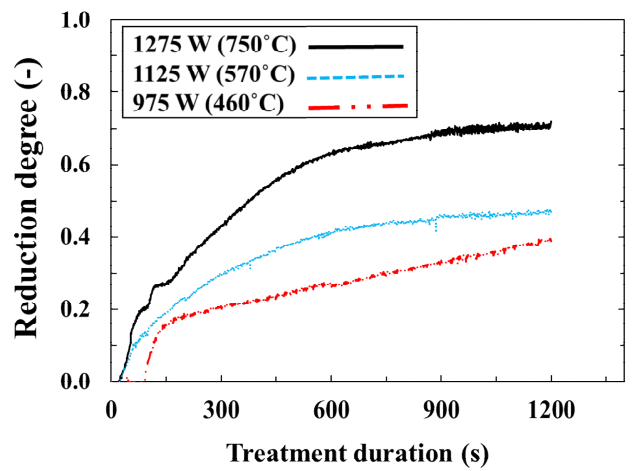

Fig. 1. Reduction degree of samples during microwave irradiation at 750,570 , and $460{ }^{\circ} \mathrm{C}$ for $1200 \mathrm{~s}$.

A noticeable increase in the reduction degree observed in the first $600 \mathrm{~s}$ of treatment in $\mathrm{H}_{2}$ whereas no significant change occurred during a longer processing time. Therefore, in the present kinetic study, the initial $600 \mathrm{~s}$ of the treatment was investigated owing to the insignificant change in reduction degree after $600 \mathrm{~s}$. Reduction reaction during treatment of the sample in $\mathrm{H}_{2}$ is attributed to the oxygen removal as $\mathrm{H}_{2} \mathrm{O}$ according to Eq. 2 that subsequently results in phase transformations in the sample. X-ray diffraction pattern of un-treated sample and that of microwave treated sample at $750{ }^{\circ} \mathrm{C}$ for certain durations are shown in Fig. 2.

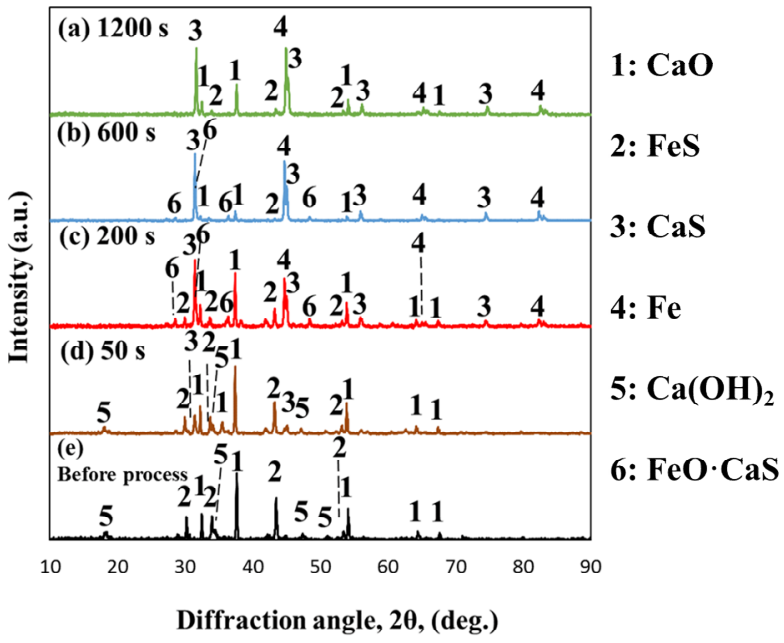

Fig. 2. XRD patterns of (a-d) microwave treated sample in $\mathrm{H}_{2}$ at $1275 \mathrm{~W}\left(750{ }^{\circ} \mathrm{C}\right)$ for certain durations and (e) that of un-treated sample.

$\mathrm{Ca}(\mathrm{OH})_{2}$ was detected in the pattern of un-treated sample (Fig. 2(e)), although the crushed $\mathrm{CaO}$ powder was kept in a vacuum desiccator chamber to protect it from hydration. This is attributed to the hydration of a portion of $\mathrm{CaO}$ during sample preparation steps such as cold-pressing of the powder mixture. Treatment of sample in the inert gas $\left(\mathrm{N}_{2}, 1 \mathrm{NL} \cdot \mathrm{min}^{-1}\right)$ confirmed a decrease in weight of the sample in the first $80 \mathrm{~s}$ of microwave treatment at 750 and $570{ }^{\circ} \mathrm{C}$, and in the first $100 \mathrm{~s}$ of microwave processing at $460{ }^{\circ} \mathrm{C}$. Therefore, weight change in these times is likely attributed to either reduction reaction or dehydration of $\mathrm{Ca}(\mathrm{OH})_{2}$ according to Eq. $5^{[11]}$. 


$$
\mathrm{Ca}(\mathrm{OH})_{2(\mathrm{~s})}=\mathrm{CaO}_{(\mathrm{s})}+\mathrm{H}_{2} \mathrm{O}_{(\mathrm{g})}
$$

$\mathrm{CaS}$ and $\mathrm{Fe}$ were detected in pattern of samples treated for $>200 \mathrm{~s}$ indicating a great progress in both ion exchange reaction (Eq. 1) and reduction reaction (Eq. 2). Furthermore, an intermediate oxysulfide phase ( $\mathrm{FeO} \cdot \mathrm{CaS})$ was detected in the XRD pattern of sample treated for 200 and $600 \mathrm{~s} \mathrm{(Fig.} \mathrm{2(b)} \mathrm{and} \mathrm{(c)).} \mathrm{This} \mathrm{phase} \mathrm{is} \mathrm{an} \mathrm{intermediate} \mathrm{phase} \mathrm{during}$ ion exchange reaction and confirms a great progress in this reaction at $750{ }^{\circ} \mathrm{C}$. Formation of oxysulfide phases is also reported during carbothermic reduction of sulfide minerals in the presence of lime in previous studies ${ }^{[3,6,12]}$.

Fig. 3 shows the SEM-EDX images of large FeS particles from reacted part of a sample microwave treated at $750{ }^{\circ} \mathrm{C}$ for $600 \mathrm{~s}$. It seems the ion exchange reaction (Eq. 1) is initiated on the surface of FeS particles and forms iron oxide. Then, the iron oxide (product of the ion exchange reaction) is reduced by $\mathrm{H}_{2}$ to form a layer of metallic iron which surrounds the un-reacted part of FeS particles.

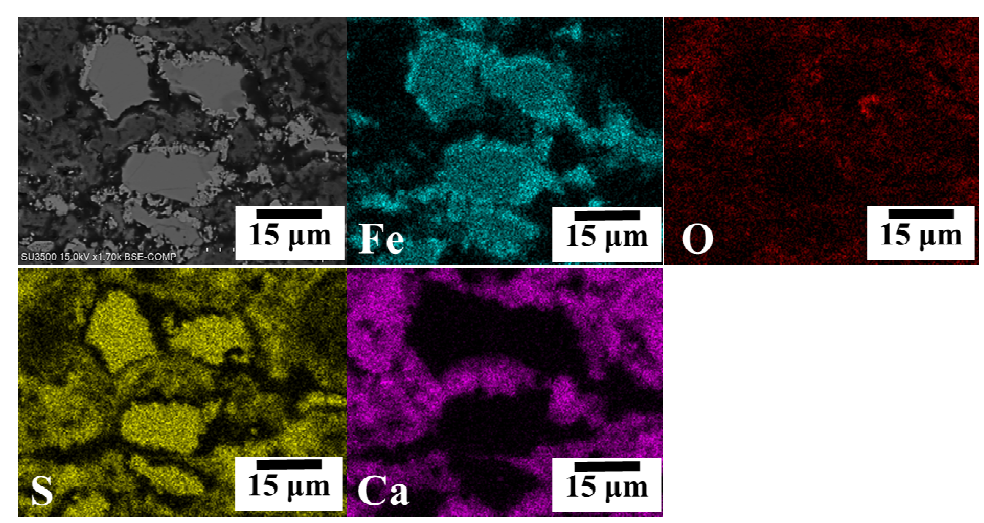

Fig. 3. SEM-EDX images of large FeS particles in samples microwave treated at $750{ }^{\circ} \mathrm{C}$ for $600 \mathrm{~s}$.

\section{Morphology observations}

Macroscale observation of samples microwave treated for $50 \mathrm{~s}$ at $1275 \mathrm{~W}\left(750{ }^{\circ} \mathrm{C}\right)$ is illustrated in Fig. 4. A boundary was recognized between the reacted part (bright gray, outer part) and un-reacted core (dark gray). Further observations showed that the boundary is moved toward the center of the sample with an increase in processing time that resulted in the shrinking of the un-reacted core. Accordingly, the total reaction is progressed topochemically and the un-reacted core model can be employed for kinetic study of iron production during microwave treatment of $\mathrm{FeS}-\mathrm{CaO}$ mixture in $\mathrm{H}_{2}$. 


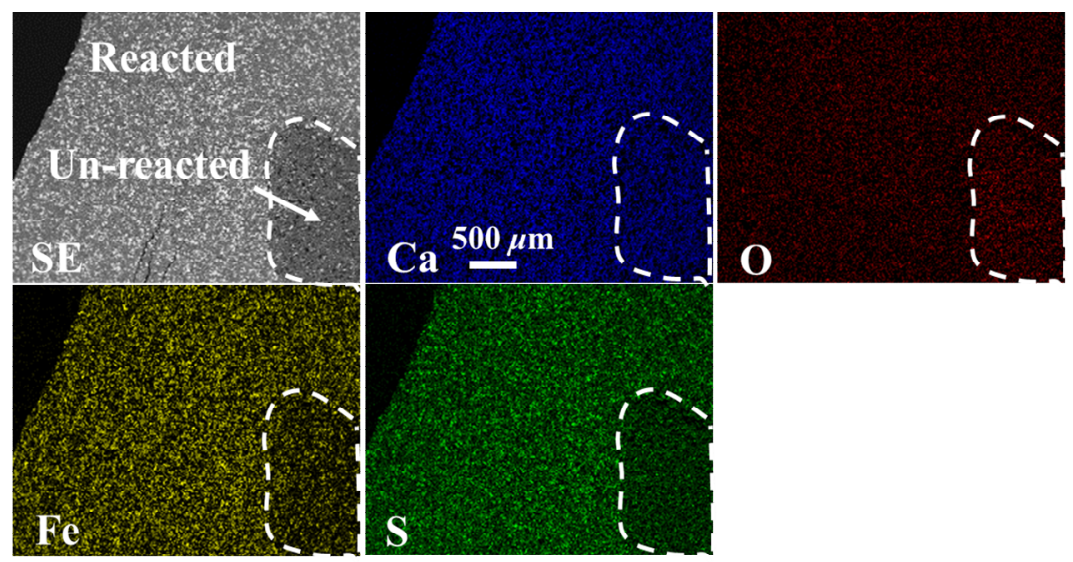

Fig. 4. SEM-EDX macrographs of microwave treated samples at $750{ }^{\circ} \mathrm{C}$ for $50 \mathrm{~s}$.

\section{Kinetic model}

Un-reacted core model has been largely applied in previous investigations ${ }^{[13-16]}$ to study the kinetics of chemical reactions which progress topochemically. In this model, four mechanisms can contribute to reaction progress: gas diffusion in micro pores of solid product, gas-solid interfacial chemical reaction, mass transfer, and a mix of these mechanisms. To evaluate kinetic parameters in dominant rate-controlling mechanism, mathematical kinetic equations have been derived [17] based on the characteristics of reactants and products in gas-solid reactions. The derived kinetic equations corresponding to the reaction rate-controlling mechanisms for spherical shape particles are summarized as follows:

Gas diffusion in micro pores (GD):

$1-3(1-X)^{2 / 3}+2(1-X)=\frac{6 D_{a}}{C_{R} r_{i}^{2}}\left(\frac{K}{1+K}\right)\left(C_{A b}-C_{A e}\right) t$

Interfacial chemical reaction (ICR):

$1-(1-X)^{1 / s}=\frac{k\left(C_{A b}-C_{A B}\right)}{C_{R} r_{!}} t$

Mass transfer (MT):

$X=\frac{3 k_{f}}{C_{R} r_{i}}\left(\frac{K}{1+K}\right)\left(C_{A b}-C_{A Q}\right) t$

Mixed GD, ICR, and MT:

$\frac{1}{C_{R} r_{i}}\left(\frac{K}{1+K}\right)\left(C_{A b}-C_{A e}\right) \frac{t}{f}-\frac{3-3 f+f^{2}}{3 k_{f}}=\frac{r_{i}}{6 D_{\tilde{z}}}\left(3 f-2 f^{2}\right)+\frac{K}{k(1+K)}$

Where X (dimensionless), $\mathrm{K}$ (dimensionless), $\mathrm{D}_{\mathrm{e}}\left(\mathrm{cm}^{2} \cdot \mathrm{s}^{-1}\right)$, and $\mathrm{k}_{\mathrm{f}}\left(\mathrm{cm} \cdot \mathrm{s}^{-1}\right)$ are the reduction degree, the equilibrium constant for reduction reaction, the effective diffusion coefficient of gas in micro pores, and the mass transfer coefficient in the gas film, respectively. $\mathrm{C}_{\mathrm{Ab}}$ and $\mathrm{C}_{\mathrm{Ae}}\left(\mathrm{mol} . \mathrm{cm}^{-3}\right)$ are the concentration of reducing gas in bulk and at the equilibrium state, respectively. $\mathrm{k}\left(\mathrm{cm} . \mathrm{s}^{-1}\right)$ is the rate constant of chemical reaction, $\mathrm{t}(\mathrm{s})$ is the treatment duration, $r_{i}(\mathrm{~cm})$ is the radius of sample, and $f$ is equal to $\left(1-(1-X)^{1 / 3}\right) . C_{R}\left(m^{-1} \mathrm{~cm}^{-3}\right)$ is the concentration of reducible oxygen in the sample, that is $0.0177 \mathrm{~mol} . \mathrm{cm}^{-3}$. 


\section{Kinetic analysis}

With regards to the microscopic observations that confirm the topochemical progress of reaction in the present study, un-reacted core model was employed to clarify the dominant rate-controlling mechanism in reduction reaction (Eq. 2) during treatment under $\mathrm{H}_{2}$ atmosphere. To simplify calculations, the radius of the assumed spherical sample $\left(\mathrm{r}_{\mathrm{i}}, \mathrm{cm}\right)$ was calculated based on the radius $(\mathrm{r}, \mathrm{cm})$ and height $\left(\mathrm{h}_{\mathrm{b}}, \mathrm{cm}\right)$ of the cylindrical shape briquette sample according to Eq. $10{ }^{[18]}$. The equilibrium constant $(\mathrm{K})$ of $0.409,0.272$, and 0.192 were attained using Eq. 11 at 750,570 , and $460{ }^{\circ} \mathrm{C}$, respectively. The mass transfer coefficient $\left(\mathrm{k}_{\mathrm{f}}\right)$ of 14.9, 10.9, and 8.69 were attained using the Sherwood (Sh), Reynolds $(\mathrm{Re})$, and Schmidt (Sc) dimensionless numbers at 750,570 , and $460^{\circ} \mathrm{C}$, respectively.

$r_{l}=\left(\frac{3}{4} r_{b}^{2} h_{b}\right)^{1 / 3}$

$\ln K=1.016-1953.33 T^{-1}$

The kinetic study was conducted up to $600 \mathrm{~s}$ treatment duration owing to no significant weight change after treatment for duration $>600 \mathrm{~s}$.

Considering the kinetic equations (6-9), Chemical reaction, mass transfer, gas diffusion, and mixed rate-controlling mechanisms at 750,570 , and $460{ }^{\circ} \mathrm{C}$ were plotted to attain the linearity $\left(\mathrm{R}^{2}\right)$ of each plot, as presented in Table $\mathbf{1 .}$

Table 1: Coefficient determination $\left(\mathrm{R}^{2}\right)$ of kinetic mechanisms

\begin{tabular}{lcccc}
\hline & Gas diffusion & Interfacial chemical reaction & Mass transfer & Mixed \\
\hline $\mathrm{R}^{2}$ at $750{ }^{\circ} \mathrm{C}$ & 0.9732 & 0.8968 & 0.699 & 0.9654 \\
$\mathrm{R}^{2}$ at $570{ }^{\circ} \mathrm{C}$ & 0.9859 & 0.8295 & 0.7116 & 0.9835 \\
$\mathrm{R}^{2}$ at $460^{\circ} \mathrm{C}$ & 0.9524 & 0.7545 & 0.7021 & 0.068 \\
\hline
\end{tabular}

The coefficient determination (linearity) of gas diffusion in micro pores mechanism is larger than that of other mechanisms at all temperatures. This indicates that the dominant mechanism for controlling of the reduction rate during microwave irradiation in $\mathrm{H}_{2}$ is gas diffusion in micro pores.

Microwave irradiation enhances the rate of interfacial chemical reaction where the gas diffusion acts as dominant resistance for the reduction reaction from the onset of microwave irradiation. This is likely attributed to the non-thermal (catalytic) effect of the microwaves on speeding up the chemical reactions, as reported previously ${ }^{[8-10,19]}$. On the other hand, such behavior is likely related to the different interaction between particles and microwaves. In the microwave irradiation, heat can be generated from within the particle (volumetric heating) owing to the penetrative power of microwaves ${ }^{[20]}$ resulting in larger progress of the chemical reaction during microwave heating owing to the volumetric heating characteristic of microwaves which improves the interfacial chemical reaction through the sample.

\section{Activation energy}

The effective diffusion coefficients $\left(\mathrm{D}_{\mathrm{e}}\right)$ were attained at 750,570 , and $460{ }^{\circ} \mathrm{C}$ by calculating the slope of linear trend lines in the gas diffusion plot, as illustrated in Fig. 6. 


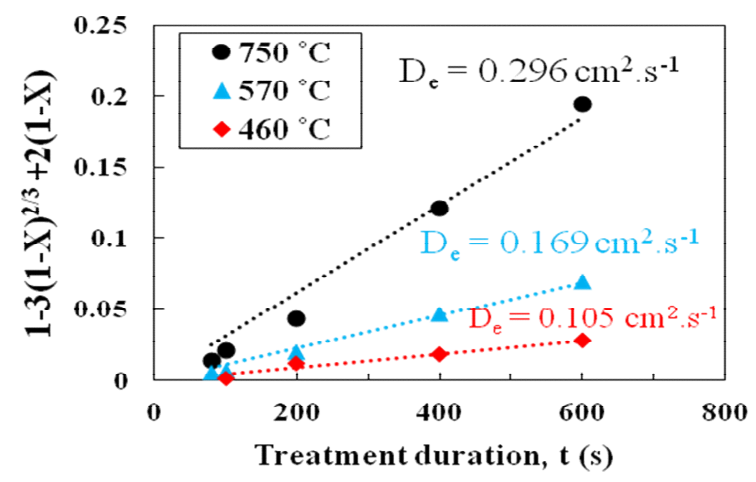

Fig. 6. Diffusion coefficient at 750,570 , and $460{ }^{\circ} \mathrm{C}$.

To calculate activation energy for gas diffusion $\left(\mathrm{E}_{\mathrm{D}}, \mathrm{kJ}\right)$ during microwave processing, the Arrhenius equation was applied wherein the $\ln \mathrm{D}_{\mathrm{e}}$ (Arrhenius equation) was plotted versus $10^{4} / \mathrm{T}$. The slope of lines gives the activation energy of $22.3 \mathrm{~kJ} \cdot \mathrm{mol}^{-1}$.

In the present study, the chemical reaction is likely progressed rapidly during microwave irradiation owing to the extraordinary effects of microwave irradiation (thermal and nonthermal) on speeding up the chemical reactions ${ }^{[8-10,19]}$ and the overall reaction is controlled by gas diffusion after the onset of microwave irradiation.

\section{Conclusions}

Kinetics of iron production during hydrogen reduction of $\mathrm{FeS}-\mathrm{CaO}$ mixture was investigated under microwave irradiation to clarify the dominant rate-controlling mechanisms. The results are summarized as follows:

1. Reduction of FeS-CaO mixture in $\mathrm{H}_{2}$ can be considered as a two-step reaction: An ion exchange reaction between $\mathrm{FeS}$ and $\mathrm{CaO}$ that forms iron oxide on the surface of FeS particles. Then, $\mathrm{H}_{2}$ reduces the iron oxide (product of the ion exchange reaction) to form a layer of metallic iron which surrounds the un-reacted part of FeS particles.

2. Hydrogen-reduction of FeS-CaO mixture during microwave irradiation is a topochemical reaction and un-reacted core model can be applied for kinetic analysis.

3. After the onset of microwave irradiation, the dominant rate-controlling mechanism is gas diffusion in micro pores with activation energy of $22.3 \mathrm{~kJ} \cdot \mathrm{mol}^{-1}$. This is likely attributed to the volumetric heating characteristic of microwaves and the extraordinary effect of microwave irradiation on speeding up the chemical reactions, particularly at lower temperatures.

\section{References}

1. Hara, Y.S.R., A. Jha, Miner. Process. Extr. Metall., 2013, 122, 146-56.

2. Hara, Y.R.S., A. Jha, Energy Technology 2015, Springer International Publishing, Cham, 2015, 83-91.

3. Jha, A., P. Grieveson, Scand. J. Metall., 1992, 21, 50-62.

4. Liu, W., J.Y. Lim, M.A. Saucedo, A.N. Hayhurst, S.A. Scott, J.S. Dennis, Chem. Eng. Sci., 2014, 120, 149-66.

5. Kim, K.Y., E.J. Jang, D.Y. Kim, S.M. Jung, Ironmak. Steelmak., 2017, 44, 6-16.

6. Hara, Y.S.R., Int. J. Miner. Metall. Mater., 2014, 21, 1-11.

7. Kuila, S.K., R. Chatterjee, D. Ghosh, Int. J. Hydrogen Energy, 2016, 41, 9256-9266.

8. Ferrari, A., J. Hunt, A. Lita, B. Ashley, A.E. Stiegman, J. Phys. Chem. C, 2014, 118, 9346-9356.

9. Zhou, J., W. Xu, Z. You, Z. Wang, Y. Luo, L. Gao, C. Yin, R. Peng, L. Lan, Sci. Rep., $2016,6,25149$. 
10. Kashimura, K., M. Sato, M. Hotta, D. Kumar Agrawal, K. Nagata, M. Hayashi, T. Mitani, N. Shinohara: Mater. Sci. Eng. A, 2012, 556, 977-979.

11. Schaube, F., L. Koch, A. Wörner, H. Müller-Steinhagen, Thermochim. Acta, 2012, 538, 9-20.

12. Jha, A., S. Tang, A. Chrysanthou, Metall. Mater. Trans. B Process Metall. Mater. Process. Sci., 1996, 27, 829-840.

13. Noguchi, D., K. Ohno, T. Maeda, K. Nishioka, M. Shimizu, ISIJ Int., 2013, 53, 13501357.

14. Bai, M.-H., H. Long, S.-B. Ren, D. Liu, C.-F. Zhao, ISIJ Int., 2018, 58, 1034-1041.

15. Bai, M., H. Long, L. Li, D. Liu, S.-B. Ren, C.-F. Zhao, J. Cheng, Int. J. Hydrogen Energy, 2018, 43, 15586-15592.

16. Fu, D., G. Tang, Y. Zhao, J. D’Alessio, C.Q. Zhou, Int. J. Heat Mass Transf., 2016, 103, 77-86.

17. Valipour, M.S., Sci. Iran., 2009, 16, 108-124.

18. Usui, T., M. Ohmi, S. Hirashima, N. Kitagawa, Tetsu-to-Haganè, 1987, 73, 19561963.

19. Hunt, J., A. Ferrari, A. Lita, M. Crosswhite, B. Ashley, A.E. Stiegman, J. Phys. Chem. C, 2013, 117, 26871-26880.

20. Gupta, M., E. Wong Wai Leong, Microwaves and Metals, John Wiley \& Sons, 2007. 


\title{
DIELECTRIC MONITORING OF THE PAN FIBER STABILIZATION PROCESS
}

\author{
J. Hofele ${ }^{1}$, M. Jung ${ }^{2}$, G. Link ${ }^{1}$ and J. Jelonnek ${ }^{1,3}$
}

\author{
$I H M^{1}, I H E^{3}$, Karlsruhe Institute of Technology (KIT), 76131 Karlsruhe, Germany \\ Korea Institute of Carbon Convergence Technology ${ }^{2}$ (KCTECH), Jeonju-si, 54853 Korea \\ julia.hofele@kit.edu
}

Keywords: dielectric measurements, microwave heating, in-situ monitoring, PAN fiber, stabilization, carbon fiber production

\begin{abstract}
The production of carbon fibers based on Polyacrylonitrile (PAN) precursor fibers is a very energy intensive process. In order to increase the energy efficiency, microwave plasma processes and, alternatively, dielectric heating with microwaves are in the focus. In this latter case, the knowledge of the dielectric properties over temperature is the key in the design of an appropriate microwave heating system. A measurement system was developed that is capable to monitor in-situ the change of the dielectric properties during the stabilization process of the PAN fiber. First results show that the loss tangent of the PAN fiber is strongly varying with temperature. Additionally, the dielectric properties are changing during the stabilization process due to the chemical transformation.
\end{abstract}

\section{Introduction}

Carbon fiber composites are the key components of future lightweight applications. But, due to the energy intensive production of carbon fibers, the final material costs are not competitive if compared to steel or aluminum even though the mechanical properties are superior [1]. Sunter et al. specify the practical minimum energy needed for the carbon fiber production to be $330 \mathrm{MJ} / \mathrm{kg}$ [2] whereas Fruehan et al. state $10 \mathrm{~kJ} / \mathrm{kg}$ for steel [3], respectively. The production process of carbon fibers starts from spinning the Polyacrylonitrile (PAN) fiber, which is then passing two major stages: the initial stabilization and the final carbonization stage. During the stabilization stage, different chemical reactions take place such as oxidation, cyclization and dehydration. Depending on the chemical composition, the reactions take place at temperatures between $180^{\circ} \mathrm{C}$ and $280^{\circ} \mathrm{C}$. The chemical transformation is an exothermic chemical reaction that has to be considered in the control of the heating rates and holding times. The most significant energy saving is expected at the stabilization stage [4]. Hence, new energy efficient production processes for the stabilization stage are necessary. Microwave heating might be one of the solutions. In [5] Zhang et al. compare microwave and conventional heating of PAN fibers. They state that microwave heating has a shortening influence on the reaction time and improves the fiber surface. In [5] the fibers are attached to a ceramic rod but no dielectric properties are provided neither for the ceramic rod nor for the pan fibers. It can be assumed that the microwave was predominantly heating the ceramic rod, which heated the fiber via thermal conduction. In [6] dielectric properties of PAN fibers are presented, but only to a limited range in temperature and also without information about the final stabilization degree, as the cooling down was not recorded.

For the successful design of an appropriate direct heating system, the knowledge of the temperature-dependent dielectric properties of the raw material together with the chemical process during the production is mandatory. In the following, a measurement system to 
measure the temperature dependent dielectric properties during the complete stabilization stage and its results are described.

\section{Experimental Setup}

The complete measurement system is shown in Fig. 1. The measurement is performed in a cylindrical TM010-mode cavity using the perturbation method at $2.45 \mathrm{GHz}$ with a quality factor of the empty cavity larger than 15000 . A $12 \mathrm{k}$ PAN fiber bundle is located in a quartz tube that is placed along the maximum electric field of the TM010-mode. A HP 8720D Network Analyzer measures the resonance frequency and quality factor of the system.

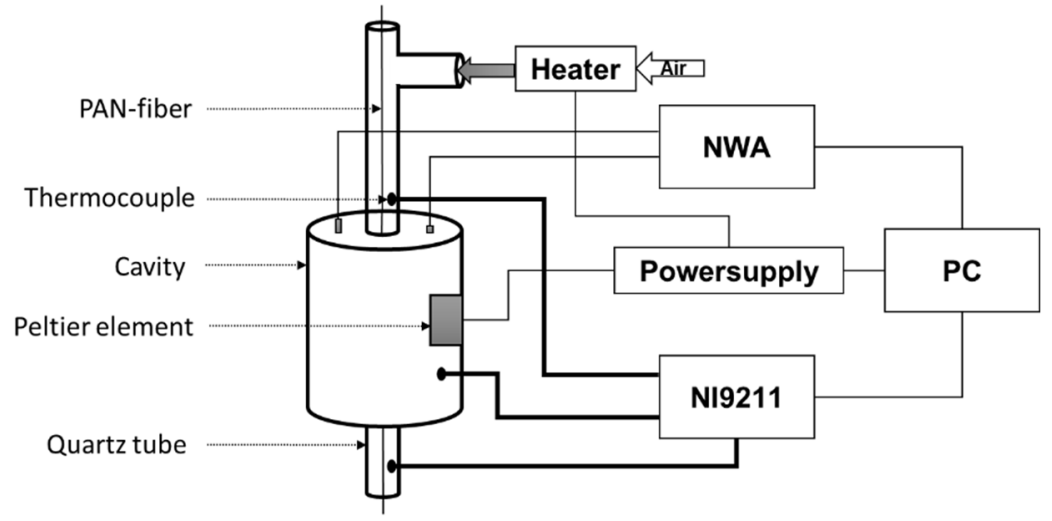

Figure 1. Measurement setup

The dielectric properties are calculated from the following equations [7]:

$$
\begin{aligned}
& \varepsilon^{\prime}=\frac{1}{A} \frac{f_{\text {ref }}-f_{s}}{f_{\text {ref }}} \frac{V_{c}}{V_{s}}+1 \\
& \tan \delta=\frac{1}{B} \frac{Q_{\text {ref }}-Q_{s}}{Q_{\text {ref }} Q_{s}} \frac{V_{c}}{V_{s}}
\end{aligned}
$$

where $\varepsilon^{\prime}$ is the relative dielectric constant, $\tan \delta$ is the relative dielectric loss tangent, $f_{\text {ref }}$ and $\mathrm{f}_{\mathrm{s}}$ are the unperturbed and perturbed resonance frequencies, respectively. $\mathrm{Q}_{\mathrm{ref}}$ and $\mathrm{Q}_{\mathrm{s}}$ are the unperturbed and perturbed quality factors. $V_{c}$ and $V_{s}$ are the volumes of the cavity and the sample. The calibration factors A and B depend on the sample and cavity geometries as well as on the final cavity wave pattern. The calibration of A and B is done with the help of CST Microwave Studio within the range of the expected dielectric properties.

For the temperature dependent measurements a heater, type MK-45R from Zinser GmbH, Germany is used to heat the air and was modified in order to use an analog control for the heater power. Pressurized air of one bar is used as input air flow. The air is heated up as it passes the heating cartridge and is channeled into the quartz tube where it heats the PAN fiber. A NI-9211 thermocouple input module is used to measure the temperature by use of type $\mathrm{K}$ thermocouples with an accuracy of $+/-2.2{ }^{\circ} \mathrm{C}$ and a measurement sensitivity of $0.07{ }^{\circ} \mathrm{C}$. Two thermocouples are placed in the quartz tube at the entry and the exit point of the cavity to measure the air flow temperature. A third one is used to measure the cavity 
temperature. Using a $180 \mathrm{~W}$ Peltier element the cavity temperature can be stabilized to a preset value of typically $20^{\circ} \mathrm{C}$ with an accuracy of $+/-0.3{ }^{\circ} \mathrm{C}$.

The accuracy of the measured dielectric properties is influenced by the following main parameters: temperature of the cavity and quartz tube, positioning of the quartz tube, simulation accuracy, NWA measurement, estimation of the sample volume.

Since the cavity perturbation with the PAN fiber is rather small, the precise measurement of the dielectric constant depends strongly on the accuracy of the measured resonance frequency of the empty cavity. This can be significantly improved by stabilizing the cavity temperature to $20^{\circ} \mathrm{C}$. A change in the quartz temperature leads to a frequency shift. Thus to distinguish the effect of the PAN fibers from the effect of the quartz tube, the temperature dependent frequency shift of an empty quartz tube is measured separately for each set of process parameters and used as reference. The impact of the quartz tube position is minimized by using the junction of the quartz tube as a stopping mechanism when inserted into the cavity. The simulation error can be neglected compared to the error from the sample volume and the NWA measurement.

Over all known errors the permittivity $\varepsilon$ is measured with an accuracy of $+/-0.65$ and the loss tangent $\tan \delta$ with $+/-0.001$ for the virgin PAN fiber at room temperature. During the stabilization process, the accuracy drops with increasing temperature. The worst case for the accuracy of the permittivity is $+/-2.1$ and $+/-0.06$ for the loss tangent. The stabilized fiber is measured at room temperature with an accuracy of $+/-1.3$ and $+/-0.02$, respectively. During the heating routine, the loss tangent increases which leads to the decline of the accuracy. This is due partly to the current measurement speed. The accuracy for the whole process improves when a fitting for the measurement curve is used, but this will extend the measurement time. It is preferred to measure faster in order to get a more precise temperature control. In a next step, it would be possible to switch to a more accurate analysis.

As a first test, the temperature is measured along the length of the cavity. In Fig. 2, the results of three consecutive runs show a temperature drop along the length due to heat losses to the Teflon insulation ring, the cavity wall, the quartz tube and the surroundings. The temperature drop inside the cavity is in the range of $10^{\circ} \mathrm{C}$. During the dielectric measurement, the thermocouples can not be placed too close to the inside of the cavity, as the metal tip is perturbing the electric field. In order to be sure to get the final temperature inside the cavity, a $10^{\circ} \mathrm{C}$ higher final temperature is selected. 


\section{High Frequency Heating}

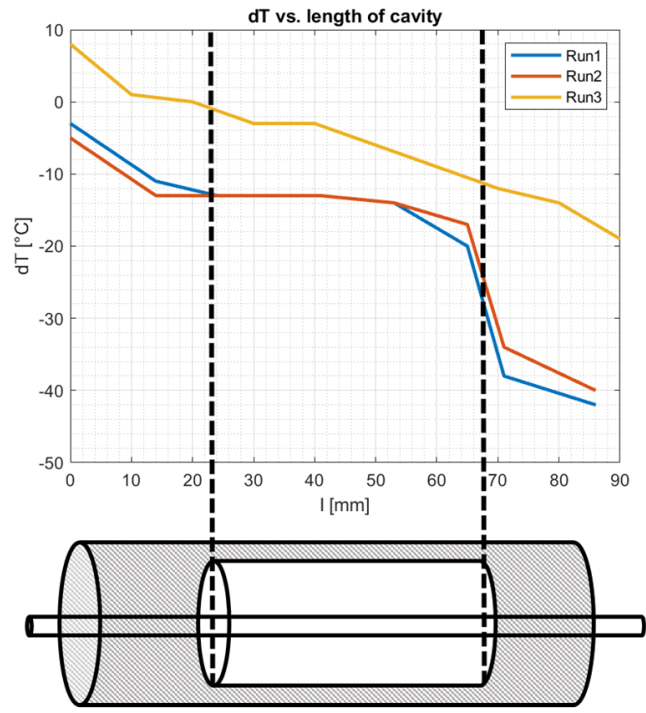

Figure 2. Temperature along the length of the cavity

\section{Experimental Results}

According to the DSC measurements, to be seen in Fig. 3, the stabilization process of the PAN fibers starts between $220-240{ }^{\circ} \mathrm{C}$. Two local maxima can be distinguished. As reported by Heine [8], the first peak can be assigned to the cyclisation, whereas the second peak is a combination of dehydration and oxidation. Thus the different reactions of which the stabilization process consists, start at different temperatures. The DSC measurements also show, that the heating rate as an influence on the heat flow produced by the exothermic reaction.

A process parameter set for the dielectric properties analysis consists of a heating rate, final temperature and holding time. The temperature measured by the thermocouple at the entry point of the cavity is defined as final temperature, as the heater control is using it as input. The sets, which are chosen based on the DSC measurements, are noted in Table 1. The temperature, resonance frequency and quality factor are measured every two seconds.

Table 1. Process parameter sets used for dielectric measurements

\begin{tabular}{cccccccc}
\hline $\begin{array}{c}\text { Process } \\
\text { Parameter Set }\end{array}$ & $\mathbf{1}$ & $\mathbf{2}$ & $\mathbf{3}$ & $\mathbf{4}$ & $\mathbf{5}$ & $\mathbf{6}$ & $\mathbf{7}$ \\
\hline $\begin{array}{c}\text { Heating Rate } \\
{\left[{ }^{\circ} \mathrm{C} / \mathrm{min}\right]}\end{array}$ & 10 & 5 & 2 & 10 & 10 & 10 & 10 \\
\hline $\begin{array}{c}\text { Final Temperature } \\
{\left[{ }^{\circ} \mathrm{C}\right]}\end{array}$ & 260 & 260 & 260 & 280 & 300 & 260 & 260 \\
\hline $\begin{array}{c}\text { Holding Time } \\
{[\mathrm{min}]}\end{array}$ & 50 & 50 & 50 & 50 & 50 & 75 & 170 \\
\hline
\end{tabular}




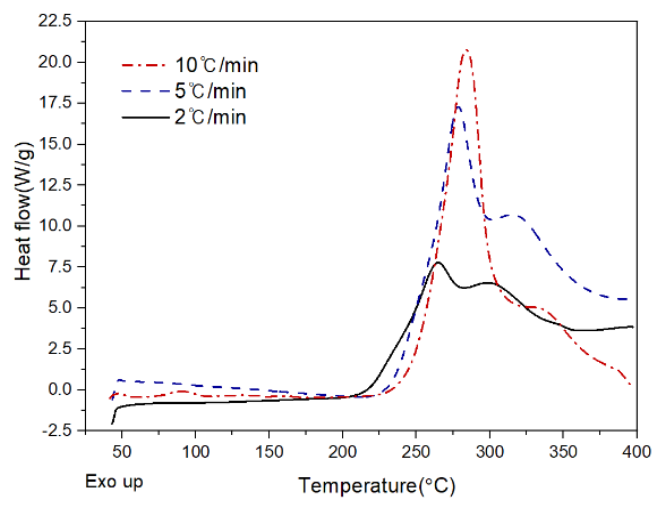

Figure 3. DSC measurement results of PAN fiber for different heating rates $\mathrm{CKCTECH}$

a) Varying holding time, Time dependent
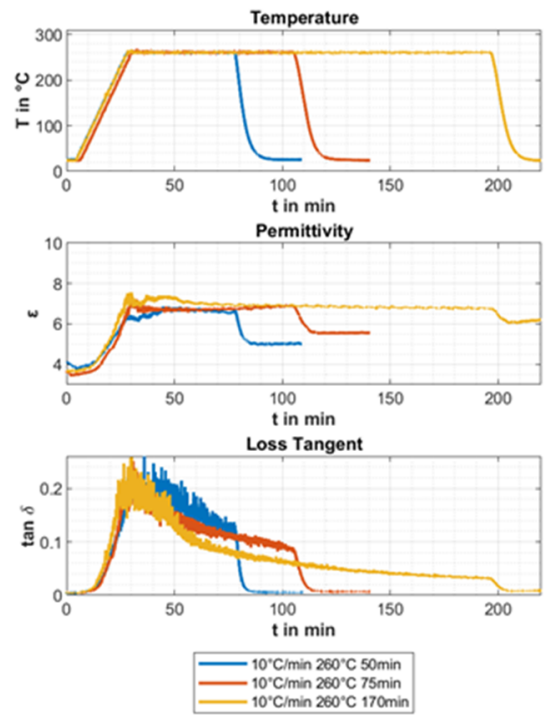

b) Varying holding time, Temperature dependent
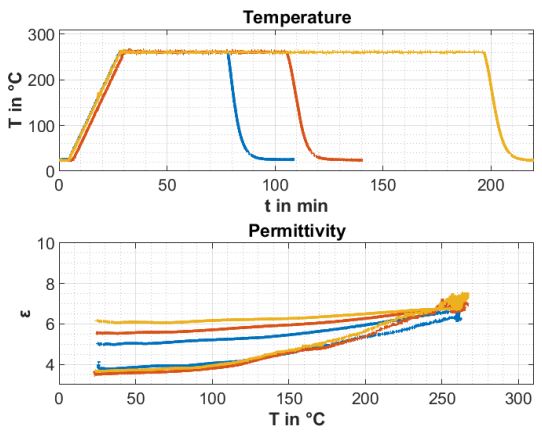

Loss Tangent

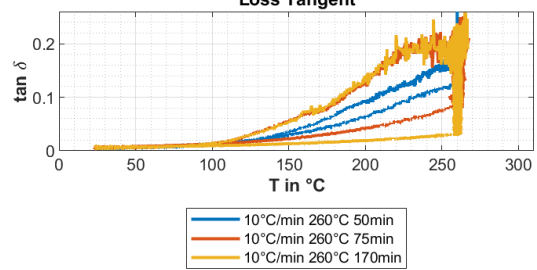

Figure 4. Dielectric properties for different measurement sets versus time and temperature 
c) Varying heat rate, Temperature dependent

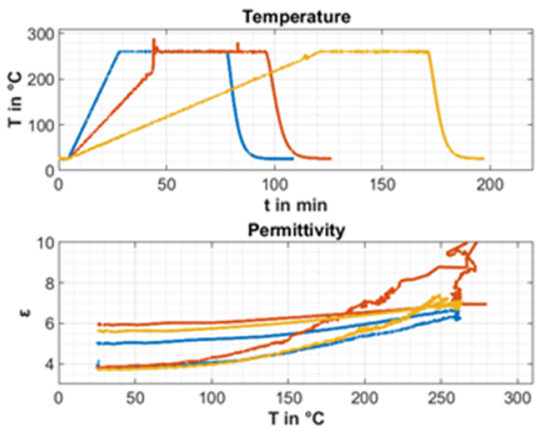

Loss Tangent

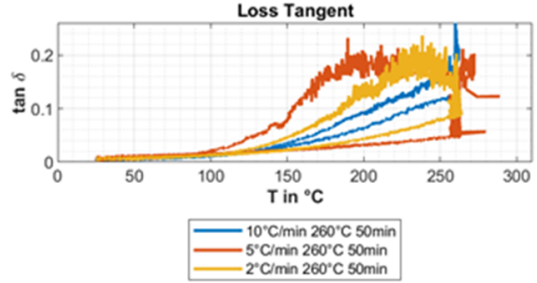

d) Varying final temperature, Temperature dependent
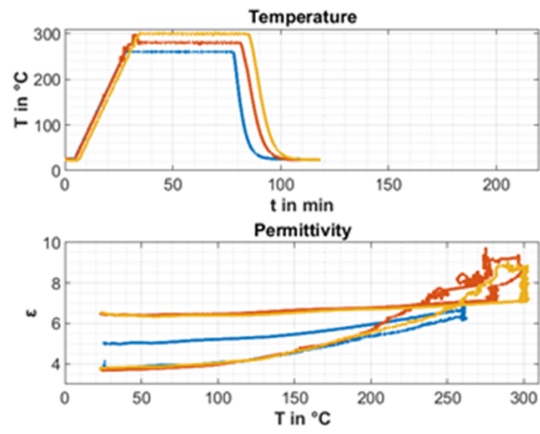

Loss Tangent

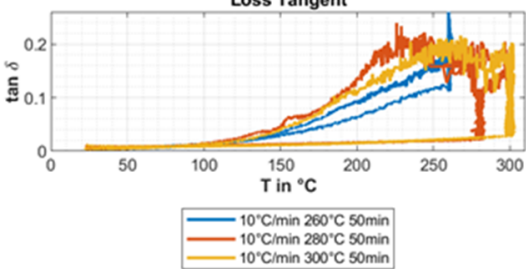

Figure 4. Dielectric properties for different measurement sets versus time and temperature

A strong temperature dependence can be seen for both the loss tangent and the permittivity in Fig. 4 respectively. As heating rate, final temperature and holding time have a great influence on the chemical reaction, they also affect the dielectric properties. Considering all process parameter sets, it can be said that the permittivity and loss tangent increase with an increasing temperature. The permittivity rises only about to the double value, whereas the loss tangent rises to about the twentyfold value. Both decrease again with the ongoing chemical reaction that unfolds during the holding time. The dielectric properties after the stabilization vary for different heating rates, final temperatures and holding times due to different stabilization degrees.

A heating rate of about $10^{\circ} \mathrm{C} / \mathrm{min}$ leads to a smaller change in loss tangent and permittivity compared to slower heating rates. This is may be due to the shorter overall process time. For increasing heating rates, the holding time has to be longer or the final temperature higher in order to achieve the same result. The correspondent values can be found in Table 2 . The process parameter set " 0 " contains the information of the untreated virgin PAN fiber. The varying stabilization degrees are also evident from the densities. It is shown in Table 2. Literature values for stabilized PAN fibers of different chemical compositions are in the range from $1.34 \mathrm{~g} / \mathrm{cm}^{3}$ to $1.39 \mathrm{~g} / \mathrm{cm}^{3}$ [9] (SGL Carbon). Similar densities result for the process parameter sets " 3 " and " 6 ". For the dielectric properties the only publication were found in [6], however the chemical composition is unknown, hence, a direct comparison is not possible.

A final temperature higher than $260^{\circ} \mathrm{C}$ leads to a higher dielectric permittivity, but no changes in the permittivity can be found when increased more, whereas the loss tangent is highest for the highest end temperature. 
Table 2. Comparison of the properties of virgin PAN and stabilized fibers

\begin{tabular}{ccccccccc}
\hline $\begin{array}{c}\text { Process Parameter } \\
\text { Set }\end{array}$ & $\mathbf{0}$ & $\mathbf{1}$ & $\mathbf{2}$ & $\mathbf{3}$ & $\mathbf{4}$ & $\mathbf{5}$ & $\mathbf{6}$ & $\mathbf{7}$ \\
\hline Heating Rate $\left[{ }^{\circ} \mathrm{C} / \mathrm{min}\right]$ & - & 10 & 5 & 2 & 10 & 10 & 10 & 10 \\
\hline Final Temperature $\left[{ }^{\circ} \mathrm{C}\right]$ & - & 260 & 260 & 260 & 280 & 300 & 260 & 260 \\
\hline Holding Time $[\mathrm{min}]$ & - & 50 & 50 & 50 & 50 & 50 & 75 & 170 \\
\hline Density $\left[\mathrm{g} / \mathrm{cm}^{3}\right]$ & 1,17 & 1,27 & 1,41 & 1,36 & 1,42 & 1,46 & 1,37 & 1,45 \\
\hline Permittivity & 3,8 & 5,0 & 6,2 & 5,6 & 6,5 & 6,5 & 5,6 & 6,1 \\
\hline Loss tangent $\mathrm{x} 10^{-3}$ & 4 & 6 & 10 & 8 & 9 & 11 & 6,5 & 8,5 \\
\hline
\end{tabular}

\section{Conclusion}

A measurement system is presented, that allows to track the change of the dielectric properties of PAN fiber during the stabilization stage. The measurements during that process show a significant temperature dependency of the dielectric properties. More importantly, the measurement system allows to follow the chemical process during the stabilization stage of the PAN fiber. That gets visible from the decrease of the loss tangent and the permittivity during the process even though the final temperature is reached and kept constant. That follow-up in the change of the loss tangent will allow to recognize e.g. hot spots in the process and to determine the end of the stabilization stage. As different process parameter sets lead to varying dielectric properties, the next step will be to determine the degree of the stabilization precisely with the help of other measurement techniques such as FTIR. In addition, it is planned to compare the dielectric properties of industrial stabilized fibers to the current results. Clearly, the knowledge of the temperature dependent dielectric properties provides a much better view on to the transients in the chemical process during microwave heating of PAN fiber during the stabilization phase.

\section{Acknowledgment}

The authors acknowledge the financial support by the Federal Ministry for Economic Affairs and Energy of Germany in the project REINFORCE (project number ZF4204603SY7) and the DSC measurements done by the project partner $\mathrm{KCTECH}$.

\section{References}

1. Frank, E. et al., Angewandte Chemie International Edition, Wiley Online Library, 2014, vol. 53, 5262-5298.

2. Sunter, D. et al., The manufacturing energy intensity of carbon fiber reinforced polymer composites and its effect on life cycle energy use for vehicle door lightweighting, 20th International Conference on Composite Materials, 2015.

3. Fruehan R.J. et al., Theoretical Minimum Energies To Produce Steel for Selected Conditions, U.S. Department of Energy, 2000.

4. Liddell, H. et al., Bandwidth Study on Energy Use and Potential Energy Saving Opportunities in U.S. Carbon Fiber Reinforced Polymer Manufacturing. U.S. Department of Energy, 2017.

5. Zhang, C. et al., Comparison of microwave and conventional heating methods for oxidative stabilization of polyacrylonitrile fibers at different holding time and heating rate, Ceramics International, 2018, Vol. 44, Issue 12, 14377-14385. 
6. Paulauskas, F. L. et al., Temperature-Dependent Dielectric Measurements of Polyacrylonitrile Fibers During Air, SAMPE 2004 Materials and Processing Technology.

7. Chen, L. F., Microwave Electronics: Measurements and Material Characterization, 2004, J. Wiley \& Sons

8. Heine, M., Optimierung der Reaktionsbedingungen von thermoplastischen Polymerfasern zur Kohlenstoffaser-Herstellung am Beispiel von Polyacrylnitril, Dissertaion, 1989, Falk-Verlag Ötigheim

9. Takaku, A. et al., Tensile properties of carbon fibers from acrylic fibers stabilized under isothermal conditions. J. Appl. Polym. Sci.,1985, 30: 1565-1571. 


\title{
IN-SITU SPECTROSCOPY AND TWO-COLOR THERMOGRAPHY DURING MICROWAVE IRRADIATION IN MATERIALS PROCESSING
}

\author{
J. Fukushima ${ }^{1}$ and H. Takizawa ${ }^{1}$,
}

${ }^{1}$ Graduated school of Engineering, Dept. of Appl. Chem., Tohoku University, 6-6-07 Aoba Aramaki, Sendai, Miyagi 980-8579, Japan

fukushima@aim.che.tohoku.ac.jp

Keywords: microwave selective heating, plasma exciting, in-situ measurement, high spatial resolution

\begin{abstract}
In $\gamma-\mathrm{Fe}_{2} \mathrm{O}_{3}+\mathrm{C}$ system, a plasma discharge (such as $\mathrm{CN}$ and $\mathrm{N}_{2}$ ) during microwave E-field irradiation and local temperature distribution is are investigated by using in situ emission spectroscopy device and high-resolution two-color thermography. The time development of excited plasma in E-field irradiation demonstrate that excited $\mathrm{N}_{2}, \mathrm{C}$ and $\mathrm{CN}$ were observed at first but $\mathrm{CN}$ excitation was sustained at least 3 seconds. In microwave $\mathrm{H}$-field irradiation, $\gamma-\mathrm{Fe}_{2} \mathrm{O}_{3}$ powders were selectively heated compared to carbon. Excited $\mathrm{CN}$ results in enhancement of the reduction reaction in addition to thermal reduction in E-field irradiation, and selective heating of $\gamma-\mathrm{Fe}_{2} \mathrm{O}_{3}$ suggests a reduction reaction proceeds in thermally nonequilibrium condition in $\mathrm{H}$-field irradiation.
\end{abstract}

\section{Introduction}

Microwave processing has many advantageous such as rapid heating and selective heating, resulting it is expected to apply for high-temperature processing including material synthesis $^{1-3}$ and sintering ${ }^{4,5}$. Concentration of microwave E-field between material particles is considered to cause the enhancement of sintering ${ }^{6}$ and chemical reaction under microwave irradiation. For example, it is usually required $1700{ }^{\circ} \mathrm{C}$ to synthesize AIN by carbothermal reduction method using $\mathrm{Al}_{2} \mathrm{O}_{3}$ as a starting material, but microwave processing can proceed this process at $1200{ }^{\circ} \mathrm{C}^{7}$. In particular, microwave smelting has an attracted attention because of the great merit of microwave processing. For example, microwave process can produce a pig iron about $1350{ }^{\circ} \mathrm{C}^{8}$. The process temperature is very low to smelt the phosphorus oxides: the produced slag was decreased in microwave process. Moreover, the reduction of copper oxide $(\mathrm{CuO})$ occurred during microwave irradiation, and the reduction behavior was different between irradiation by the microwave electric field and the magnetic field ${ }^{9}$. In addition, our previous work investigated the effect of microwave E- and H-field on the reduction reaction by in situ visible image and emission spectra during microwave irradiation ${ }^{10}$. These studies suggest that the effects of plasma and microwave electric and magnetic fields itself can contribute to ironmaking reactions, and these effects result in lowtemperature reactions and a shortening of the period of the carbothermal reduction reaction. To understand the mechanism of microwave smelting process, it is necessary to understand an occurrence behavior of plasma, which leads to a chemical reaction related to radical species generated by a local E-field concentration. In addition, in carbothermal reduction reaction, which is typical smelting method, a raw material was composed of several $\mathrm{mm}$ powders. It is suggested that a selective heating in the powder scale occurs. However, to discuss this selective heating behavior on this scale, it is necessary to realize a quantitative temperature measurement system with independent of the emissivity of the material and several mm spatial resolution. 
In this study, we conducted an in-situ spectroscopy and two-color thermography to verify these non-equilibrium effects during microwave irradiation.

\section{Experimental procedure}

In-situ spectroscopic system was explained elsewhere ${ }^{10}$. The authors was used $\gamma-\mathrm{Fe}_{2} \mathrm{O}_{3}$ (99\% purity, 20-40 nm, Kojundo Chemical Laboratory Co., Ltd., Saitama, Japan) and pure carbon (99.9\%, $50 \mu \mathrm{m}$, Kojundo Chemical Laboratory Co., Ltd., Saitama, Japan) as starting powders. The powders were mixed together $\left(\gamma-\mathrm{Fe}_{2} \mathrm{O}_{3}: \mathrm{C}=2: 3\right.$ mol.\%) with alumina mortar and pestle with small amount of ethanol. The mixture powders $(0.15 \mathrm{~g})$ were pressed into a pellet $(\varnothing 6 \mathrm{~mm})$ at a pressure of $4 \mathrm{MPa}$. Microwave irradiation system was composed of magnetron (IMH-20A259, IDX Co., Ltd., Tochigi, Japan, $2455 \pm 15 \mathrm{MHz}$ ), isolator, three stub tuner, TE102 single-mode cavity and plunger. Sample pellet was set in the quartz test tube. During microwave irradiation, pure nitrogen gas was flowed at $0.1 \mathrm{~L} / \mathrm{min}$. Microwave was irradiated with the sample, which is placed at the maximum point of E-filed and H-field intensity in the cavity. The sample temperature of the bottom of the pellet was measured by infrared (IR) thermometer (FTK9-P300A-30L21, Japansensor, Co., Tokyo, Japan).

Visible images and emission spectra were obtained by using an integrated microscopic imaging spectrometer (IMIS, Bunkoukeiki Co., Ltd., Tokyo, Japan) during microwave process ${ }^{11}$. This system can measure the images and spectra on the upper part of the pellet. The spectrometric wavelength ranged from $308 \mathrm{~nm}$ to $492 \mathrm{~nm}$.

In addition, temperature distribution of the sample at upper part of the pellet was obtained by two-color thermography system (Thermera-NIR2, Mitsui photonics Ltd., Tokyo, Japan) during microwave $\mathrm{H}$-field irradiation in five minutes.

Obtained sample was analyzed by X-ray diffraction (XRD) and Field-type emission scanning electron microscopy (FE-SEM) and energy dispersion spectroscopy (EDS). In both analysis, upper surface of the pellet was analyzed; irradiated sample was not pulverized before sample analysis.

\section{Results and discussion}

During E-field irradiation, peripheral edges of the pellet was only heated to a high temperature, and the maximum temperature of the center of the pellet was up to $800^{\circ} \mathrm{C}$, which value was indicated by IR measurement. Microwave power at holding temperature at $800{ }^{\circ} \mathrm{C}$ was about $200 \mathrm{~W}$.

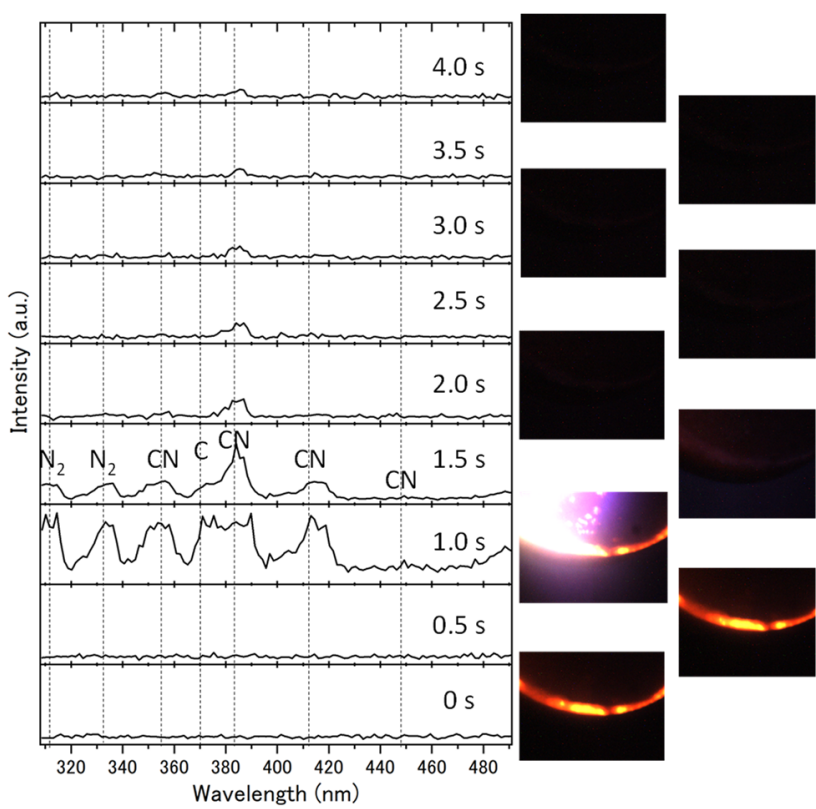

Fig. 1. Visible image of the sample pellet and emission spectra within 4 seconds during microwave E-field processing. 
First of all, we investigated that the time development of plasma when $\gamma-\mathrm{Fe}_{2} \mathrm{O}_{3}+\mathrm{C}$ pellet was irradiated with microwave E-field. Figure 1 shows the visible image of the sample pellet and plasma spectra within 4 seconds. At $0.0 \mathrm{~s}$, peripheral edge was heated up, but no peaks was observed in a spectral pattern: the spectroscopic spectrum at $0.0 \mathrm{~s}$ was almost flat between $308-492 \mathrm{~nm}$. At $1.0 \mathrm{~s}$, white $\sim$ purple plasma was generated as shown in visible image. From the spectra, these peaks were derived from $\mathrm{N}_{2}, \mathrm{CN}$ and $\mathrm{C}$. Then, this plasma seemed to be disappeared from the visible image, but $\mathrm{N}_{2}, \mathrm{CN}$, and $\mathrm{C}$ spectra were remained. On the other hand, hot region on the peripheral edge were almost disappeared, indicating that the temperature at these area decreased. At $2.0 \mathrm{~s}, \mathrm{~N}_{2}$ and $\mathrm{C}$ spectra were almost disappeared, but $\mathrm{CN}$ peaks was remained for 2 minutes. This excited $\mathrm{CN}$ enable to enhance the reduction reaction in addition to thermal reduction.

Figure 2 shows the visible image of the sample pellet and plasma spectra within 4 seconds in microwave $\mathrm{H}$-field irradiation. Unlike E-field process, the temperature of the pellet was increased up to $1200{ }^{\circ} \mathrm{C}$ in $\mathrm{H}$-field irradiation. Moreover, almost all the visible area of the pellet was heated up uniformly, indicating that uniform

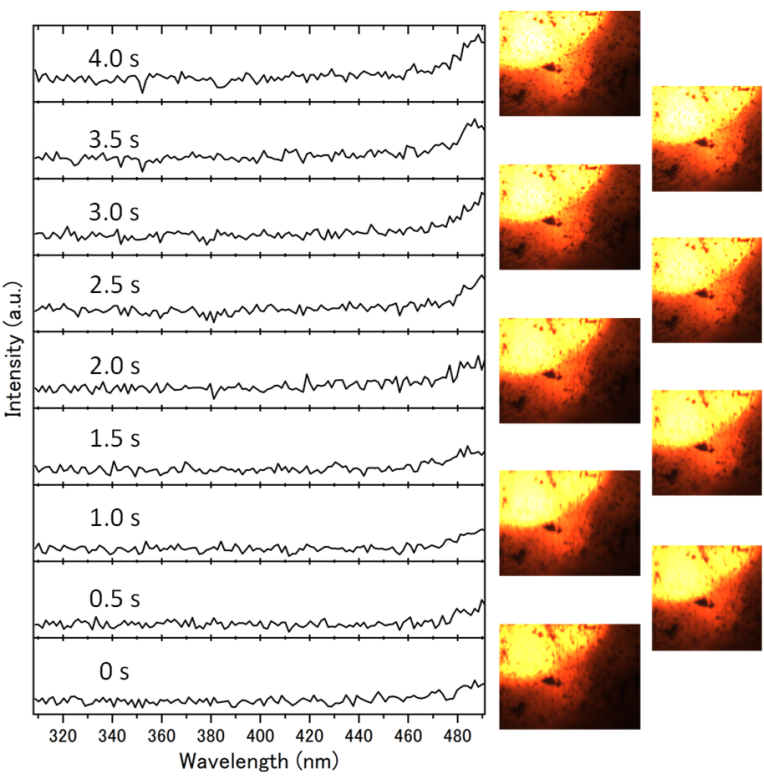

Fig. 2. Visible image of the sample pellet and emission spectra within 4 seconds during microwave $\mathrm{H}$-field processing.

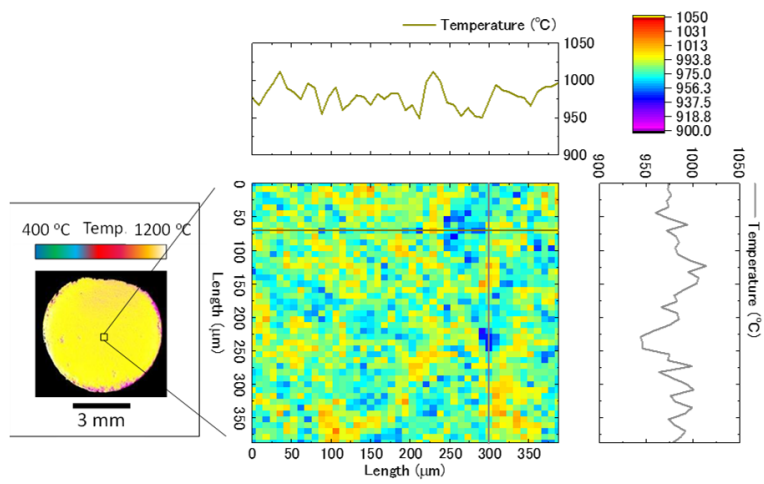

Fig. 3. Two-color thermography at $1000^{\circ} \mathrm{C}$ (measured by IR thermometer) and the local temperature distribution image 
heating was achieved as compared to E-field heating process. The images and spectra was measured at about $1100{ }^{\circ} \mathrm{C}$, and the microwave power at that time was about $400 \mathrm{~W}$. As shown in Fig. 2, continuous Planck radiation was only observed in the high-wavelength range. Furthermore, spectra intensity of these continuous Planck radiation was increased with the IR temperature increasing. During H-field experiment, no peaks was observed; thus, in H-field irradiation, plasma did not occur.

In-situ spectroscopy during microwave process suggest that plasma or excited radicals will affect the reduction reaction when the sample was irradiated with E-field. On the other hand, in H-field, the reduction reaction seems to proceed only thermal reaction.

Then, in order to evaluate the selective heating effect on ironmaking process, the authors focused on in situ measurement of the reduction reaction in $\mathrm{Fe}-\mathrm{O}-\mathrm{C}$ system during microwave irradiation by using two-dimensional two-color thermography. Figure 3 shows that the two-color thermography at $1000{ }^{\circ} \mathrm{C}$ (measured by IR thermometer) and the local temperature distribution image. The two-color thermography image demonstrated that the pellet temperature was almost uniform, but the temperature at peripheral edge of the pellet was little lower than center part of the pellet. The temperature distribution image at about $400 \mu \mathrm{m}$ square part in the pellet was shown in the center of Fig. 3. From the figure, temperature gradient was observed in such a local scale. The temperature profile of the horizontal line indicated in the temperature distribution image was shown at the top of Fig. 3 . The profile suggested that the sample temperature dropped at around $x=275 \mu \mathrm{m}$. The width of this area was corresponds to the size of the pristine carbon powders (about $50 \mu \mathrm{m}$ ). The temperature profile at vertical line was also shown in Fig. 3 right. The temperature was reached a low at around $y=250 \mu \mathrm{m}$. The width of this localized area was also about $50 \mu \mathrm{m}$. These profiles suggested that the carbon powders were existed. In this system, single-phase $\gamma-\mathrm{Fe}_{2} \mathrm{O}_{3}$ and carbon absorbs microwaves strongly. From the above results, in mixture case, $\gamma$ $\mathrm{Fe}_{2} \mathrm{O}_{3}$ is selectively heated in $\mathrm{H}$ field irradiation. Although carbon also absorbs microwaves, a selective heating was occurred in the scale of $\mu \mathrm{m}$ powder.

XRD results of the pristine powders and the sample pellet after microwave irradiation were shown in Fig. 4. The pristine powders certainly consisted of $\gamma-\mathrm{Fe}_{2} \mathrm{O}_{3}$ and carbon, and after microwave process, carbon peaks remained but $\gamma-\mathrm{Fe}_{2} \mathrm{O}_{3}$ became iron and wustite. Therefore, the reduction reaction was not complete in five minutes.

Figure 5 shows FE-SEM image and element mapping by EDS. The sample had many macro pores. Element mapping exhibit that carbon remained; the results is

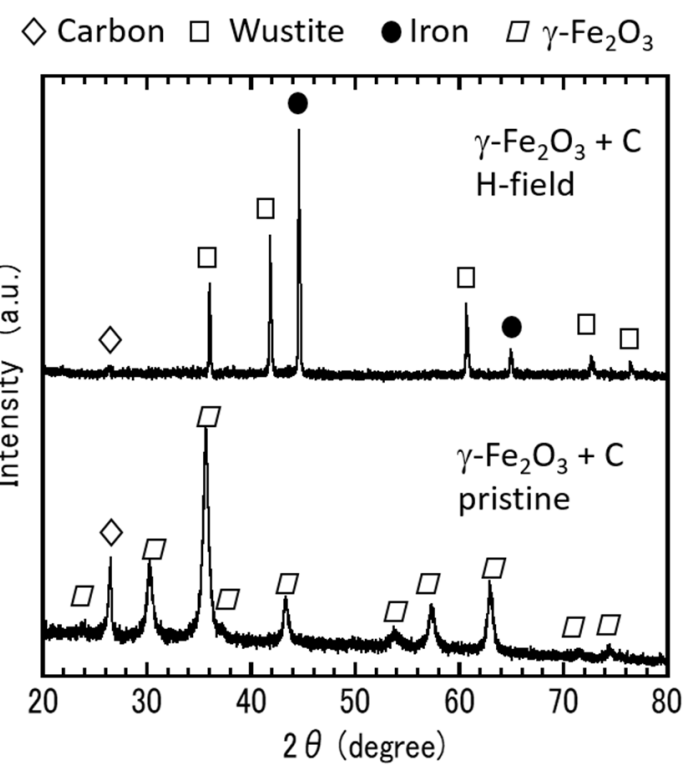

Fig. 4. XRD results of the pristine powders and the sample pellet after microwave irradiation consistent with the XRD results. 
Oxygen mapping demonstrate that the amount of oxygen was low at around remained carbon. This results indicate that the reduction reaction proceeded at the contact point
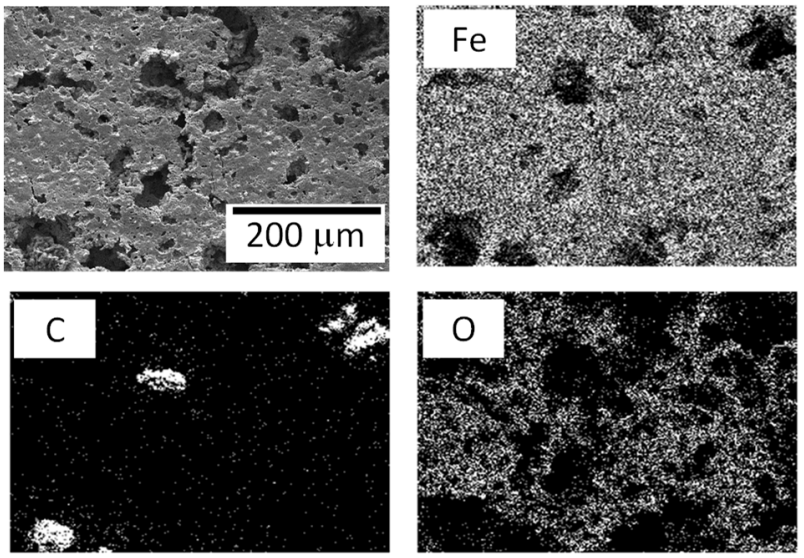

Fig. 5. FE-SEM image and element mapping by EDS between carbon and $\gamma$ $\mathrm{Fe}_{2} \mathrm{O}_{3}$.

Here we discuss the selective heating effect in this system. In $\mathrm{H}$-field heating, $\quad \gamma-\mathrm{Fe}_{2} \mathrm{O}_{3} \quad$ was selectively heated and carbon was heated by thermal conduction from heated $\gamma-\mathrm{Fe}_{2} \mathrm{O}_{3}$. On the other hand, temperature distribution was sustained during microwave $\mathrm{H}$-field irradiation, and the reduction reaction of iron oxides proceeded under thermally non-equilibrium condition.

\section{Conclusion}

The study investigated that the time development of excited plasma in E-field irradiation and selective heating of $\gamma-\mathrm{Fe}_{2} \mathrm{O}_{3}$ in $\mathrm{H}$-field irradiation by using in-situ measurement system which enable to obtain emission spectra and high-resolution two-color thermographic image. In microwave E-field irradiation, excited $\mathrm{N}_{2}, \mathrm{C}$ and $\mathrm{CN}$ were observed, and $\mathrm{CN}$ excitation was sustained at least 3 seconds. This excited $\mathrm{CN}$ causes enhancement of the reduction reaction in addition to thermal reduction. In microwave $\mathrm{H}$-field irradiation, $\gamma-\mathrm{Fe}_{2} \mathrm{O}_{3}$ powders were selectively heated compared to carbon.

\section{Acknowledgement}

This work was supported by a JSPS Grant-in-Aid for Scientific Research (S) No. JP17H06156.

\section{References}

[1] Takeuchi, T. et al., Catalysts, 2017, 7, 65

[2] Kashimura, K. et al., Journal of Alloys and Compounds, 2013, 550 239-244.

[3] Komarneni, S. et al., Mater. Res. Bull., 1992, 27 1393-1405

[4] Sutton, W., Am. Ceram. Soc. Bull., 1989, 68, 376-386.

[5] Roy, R. et al., Nature, 1999, 399, 668

[6] Rybakov, K.I. et al, Abbr. J. Am Ceram. Soc., 2013, 94, 1003-1020.

[7] Chikami, H., Fukushima, J. et al., J. Am Ceram. Soc., 2018, 101, 4905-4910.

[8] Ishizaki, K. et al., ISIJ International, 2006, 46, 1403-1409.

[9] Fukushima J. et al., Materials Chemistry and Physics, 2013, 172, 47-53

[10] Fukushima, J. et al., Metals, 2018, 8, 49-58.

[11] Matsubara, A. et al., Plasma Fusion Res., 2010, 5, 041. 


\title{
FASTER PLASTICIZERS PRODUCTION BY MICROWAVE IRRADIATION
}

\section{A. Jermolovicius ${ }^{1}$, E. V. S. Pouzada ${ }^{1}$, E. R. de Castro ${ }^{1}$, R. B. do Nascimento ${ }^{1}$, J. T. Senise ${ }^{1}$ \\ ${ }^{1}$ Instituto Mauá de Tecnologia, Praça Mauá, 1, São Caetano do Sul, SP 09580-900, Brazil. jermolovicius@maua.br}

Keywords: microwave assisted chemical process, plasticizers, effect of microwaves on chemical kinetics.

\begin{abstract}
In this work the microwave effect of enhancing chemical reactions was applied to maleic anhydride esterification with 2-ethylhexanol-1 catalyzed by p-toluene sulfonic acid. The analysis of this reaction was simplified considering the industrial practice of withdrawn the reaction water, which makes the whole reaction act as a pseudo irreversible one. A statistical design of experiments was applied to determine an empirical chemical kinetics equation with three levels of catalyst concentration and two levels related to the kind of heating (microwave and conventional); six chemical kinetics equations (three for microwave heating and three for conventional heating) were determined. A batch/plug flow simulation was done and a spreadsheet developed for the reactor's behavior. The most interesting result was the confirmation of a synergic effect between microwaves and acid catalysis that accelerated this esterification reaction, transforming it in a fast reaction.
\end{abstract}

\section{Introduction}

Chemical industry may be conceived as a reactor surrounded by various process equipment as pumps, evaporators, dryers, distillers, crystallizers etc. All of them are necessary to transform raw materials into products. The reactor is the equipment where raw materials molecular structures are changed through a chemical reaction and the remainders act to purify the product produced inside the reactor.

Chemical kinetics data are fundamental for a good reactor project [1] because they provide the basic information to design a suitable reactor volume that fits the chemical reaction necessity of processing time, temperature, products distribution etc. Unfortunately, this kind of data are rare [2] and even rarer are data for chemical kinetics enhanced by microwaves.

Nowadays, it is well known the microwave effect that accelerates chemical reactions [3-5], but data for the complete chemical equation (equation with reaction order for all reactants, activation energy, pre exponential Arrhenius factor) are still very rare [2].

This paper was written with the aim to disclose how chemical kinetics equation may be determined and applied to the project of microwave irradiated reactors and/or conventional reactor.

\section{Plasticizers}

Plasticizers are esters of organic acids or acid anhydrides, which are used as additives in the plastics, concrete and ceramics industries to increase plasticity and or decrease viscosity of a material. Several products may act as a plasticizer, but only approximately fifty are commercial products and most of them are for PVC applications [6].

Di-2-ethylhexil maleate (DEM) was selected as an example for this study. It is produced by reaction of maleic anhydride (MA) with 2-ethylhexanol-1 (EH), usually catalyzed by acids, as p-toluene sulfonic acid (PTSA) [7]. There is also a second two step esterification reaction: firstly, MA reacts with EH producing mono-2-ethylhexyl maleate (MEM) and then MEM reacts with more EH to produce DEM. These equations are shown in (1) and (2), respectively. In industry is usual to work with a global reaction, that is the sum of these two partial reactions, as shown in (3). 
Reaction 1 is fast and irreversible, because it deals with anhydride and alcohol, producing only one product, the MEM. The second reaction is reversible because it is a reaction between a carboxylic acid and an alcohol, producing an ester (the DEM) and water, which may establish a dynamic equilibrium with their raw materials.

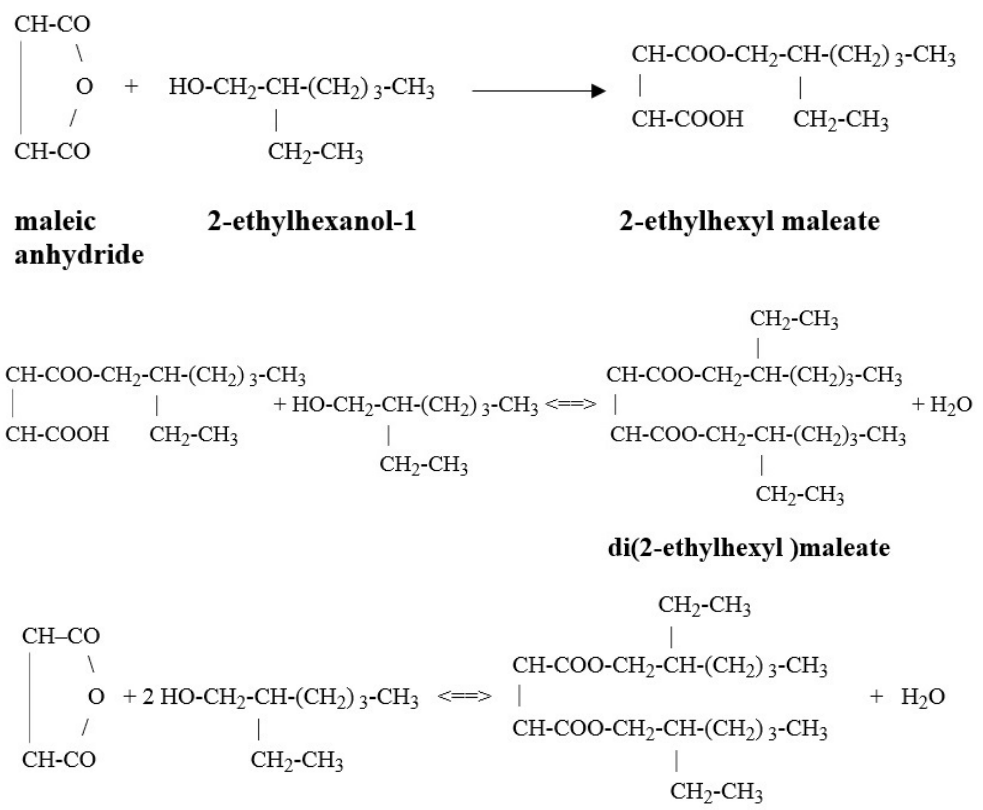

The reversibility of the second reaction is a technical problem in industry, because it reduces the total conversion of reactants. Increasing conversion of MA is done by adding xylene to the load, as a solvent, and distilling an azeotropic mixture of xylene and reaction water [7]. Elimination of this reaction water, shift the reaction equilibrium to products formation, becoming a pseudo-irreversible reaction, as shown in (4), and increasing yield in DEM.

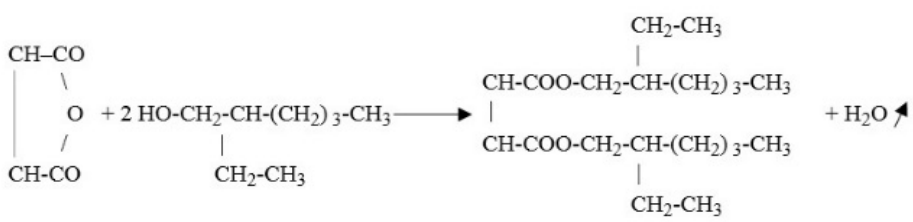

Equation (4) is the representation used in industry for controlling production of DEM and it was adopted for this study.

\section{Reaction velocity}

Chemical reaction engineering deals with reaction velocities to select and design chemical reactors for a desired capacity of a product production [1]. All project strategies are based on the chemical reaction behavior, which is well expressed by chemical kinetics equations. Supposing a non-elementary reaction (5),

$$
\mathrm{A}+\mathrm{B} \rightarrow \mathrm{R}
$$

then its reaction velocity may be expressed by (6) [1].

$$
-r_{A}=k \cdot C_{A}^{n a} \cdot C_{B}^{n b}
$$


where, $-r_{A}$ is the reaction velocity, $k$ is the velocity constant, $C_{A}$ and $C_{B}$ are the concentrations of the reactants $\mathrm{A}$ and $\mathrm{B}$, and $\mathrm{n}_{\mathrm{a}}$ and $\mathrm{n}_{\mathrm{b}}$ are the individual reaction order, related to $\mathrm{A}$ and $\mathrm{B}$. The $k$ factor is a pseudo constant, because it depends on the reaction temperature according to Arrhenius law [8]:

$$
k=k_{0} \cdot \exp (-E / R T)
$$

where, $k_{0}$ is the Arrhenius pre-exponential factor, $E$ is the activation energy, $R$ is the universal gas constant and $T$ is the temperature. Other factors can also influence this value, for example: use of catalyst and its concentration, reaction mechanism, mixing, irradiation [10]. Usually, kinetics equation is determined for specific conditions of temperature, catalysis and reactor operation. This restriction is to simplify the experimental determination of reaction velocities.

A simulation of reaction 5, shown in Figures 1-3, exemplify how kinetics parameters influence the reaction rate. Fig. 1 shows that, for a specific value of velocity constant, reactions with lower orders are faster. Fig. 2 shows that, for a specific reaction order, concentrated reactants produce faster reaction. Fig. 3 shows that higher values of velocity constant produce faster reactions.

It is possible to observe (Fig. 2) that, sometimes, high level of reactants conversion may be reached in a few minutes and also that total conversion may happen in a long reaction time. In industrial practice, the usual reactant concentration is about 10-20 M and the economic conversion is about $92 \%$ which may be reached after several hours. For this reason, in this case, the reaction is classified, in industry, as a slow reaction although its high conversions during the initial operation time, even if catalyzed.

High reactant concentrations may reduce the reaction time to reach the $92 \%$ conversion level, but it is not economical for industries. Then the option is to change the reaction conditions, as temperature, mixing, contact time. Obviously, the best procedure is to enhance the reaction rate by changing the reaction mechanism, say, changing the values of activation energy, reaction order of reactants, pre-exponential Arrhenius constant. This option means that alterations in the reaction mechanism and catalysis are the first options.

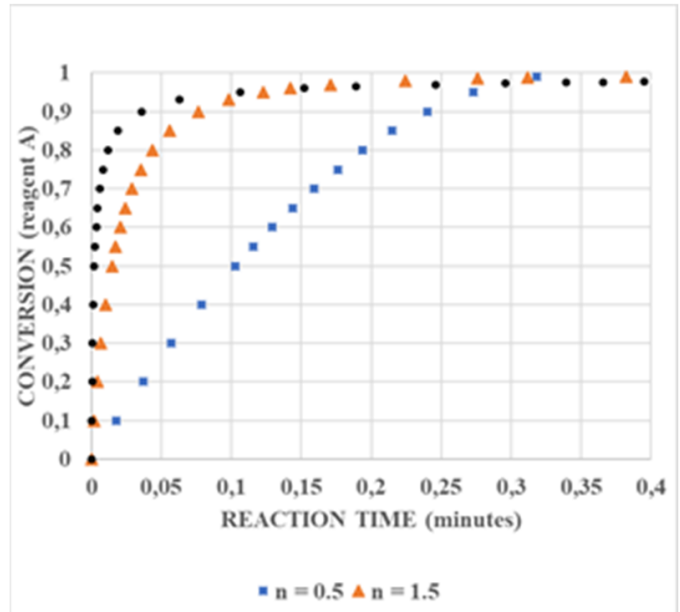

Fig.1. Reaction order $(0.5,1.5$ and 2.5$)$ effect on conversion $\left(k=17.9 ; \mathrm{C}_{\mathrm{A}}=\mathrm{C}_{\mathrm{B}}=10 \mathrm{M}\right)$. 
Nowadays, there is a new option: the microwave enhanced chemistry. There are several reports and review papers on reaction acceleration by microwaves [3-5]. Most of the reported experiments were done in small scale; unfortunately, this kind of data are not suitable for industry reaction conditions (especially in petrochemical industry). The knowledge of chemical kinetics equation of (conventional or microwave enhanced) chemical reactions can help the scale up process and the design of microwave irradiated chemical reactors.

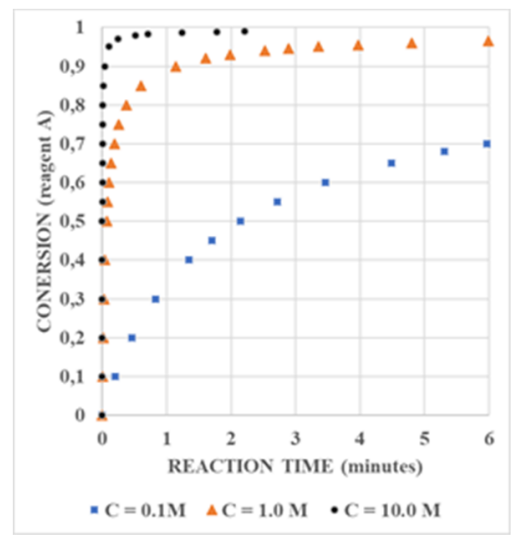

Fig.2. Reactants concentration $(0.1,1.0$ and $10.0 \mathrm{M})$ effect on conversion (reaction order $2.5, k=17.9$ ).

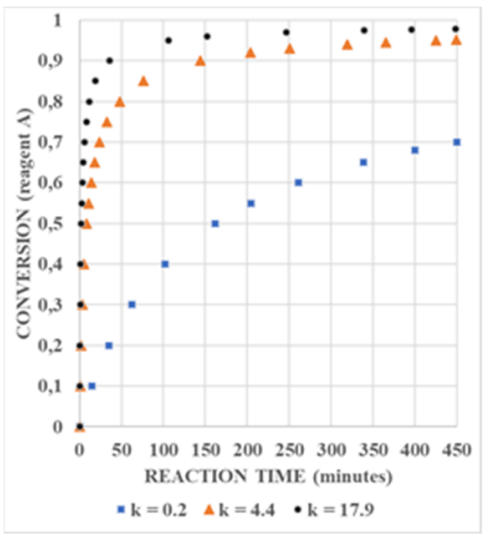

Fig.3. Velocity constant $(0.2,4.4$ and 17.9$)$ effect on conversion (reaction order $2.5, \mathrm{C}_{\mathrm{A}}=\mathrm{C}_{\mathrm{B}}=0.1 \mathrm{M}$ ).

\section{Experimental}

\section{Reactor and operational procedure in chemical kinetics experiments}

A procedure for the determination of chemical kinetics was developed in an early work [10] and is described in this paper. The reactions were performed in a well-stirred batch reactor of $500 \mathrm{~mL}$ boron silicate glass kettle vessel with internal baffles used to prevent whirlpool formation, with a top cover with four holes 24/40. A shaft of boron silicate glass rod and a 
paddle of Teflon were used. The stirrer's explosion proof electrical motor was assembled over the top of the reactor. A total reflux Allinh condenser (boron silicate glass, $60 \mathrm{~cm}$ ) with a Dean Stark flask $(25 \mathrm{~mL})$ was assembled at the vessel top. The condenser was cooled with water at room temperature. The sample collector was a $2 \mathrm{~mm}$ inner diameter boron silicate glass tube immersed into the reagents and with the other end side connected to a vacuum vessel. It was fitted at the same hole used to feed the reagents. A digital fiber optic thermometer sensor, protected by a thermometric well of boron silicate glass, was used to monitor the temperature of reactor load. The whole assembly was enclosed in an aluminum wall multimode cavity with a mode stirrer, connected to a microwave variable-power generator of $2.45 \mathrm{GHz}$. A directional coupler and a power meter measured the microwave transmitted and reflected power. During the experiments with conventional electric heating, the reactor was wrapped with an electric tape with temperature control.

The experiments were performed with loads of $300 \mathrm{~mL}$ of solution of MA and PTSA in xylene, which were heated up to the reaction temperature. Simultaneously the right quantity of EH was heated to 139 or $143{ }^{\circ} \mathrm{C}$ (according to the test planned, as shown in Table 1) in an electrical heated separatory funnel. When the temperature in the reactor and in the separatory funnel reached 139 or $143{ }^{\circ} \mathrm{C}$ the $\mathrm{EH}$ was introduced into the reactor over the MA solution and the time count started. This pre-heating of raw materials was done because it was observed in a preliminary experiment that 12 minutes were necessary to heat the load from room temperature up to $143^{\circ} \mathrm{C}$.

The azeotrope vapor of xylene and water was condensed in the Allinh condenser and the condensed azeotrope broken in two phases: an upper one with xylene and a lower with water. These phases were decanted in the Dean Stark flask and the condensed xylene was returned to the reactor and the water drawn and discarded. At the beginning of each esterification, the Dean Stark was filled up with dry xylene. During the esterification, the Dean Stark flask water level was maintained at a maximum level of $2 \mathrm{~mL}$ by intermittently drawing off the water.

The samples were taken in a 1 minute time step, during the total processing time of 10 minutes; they were analyzed with a near infrared (NIR) spectrometer calibrated for maleic anhydride.

The concentration of reactants solutions and the reaction temperature of each test are described in the next section. The solvent used was dry xylol. The experiments with conventional electric heating and with microwave irradiation were done at the same temperatures. The effective microwave power was $1.33 \mathrm{~W} / \mathrm{g}$ for $139{ }^{\circ} \mathrm{C}$ and $1.69 \mathrm{~W} / \mathrm{g}$ for $143^{\circ} \mathrm{C}$.

\section{Experimental determination of complete chemical kinetics equation}

Simplifying the kinetics analysis, the esterification of MA by EH, catalysed by PTSA, with withdrawn of reaction water, was assumed as a pseudo irreversible reaction. This simplification is expressed in (4).

Assuming that (a) the concentration of PTSA is constant, (b) the reaction is non-elementary and (3) that it is processed isothermally, then it was possible to write a chemical kinetics equation with three variables (1/T, CMA an CEH) as in (8),

$$
-r_{M A}=k_{0} \cdot \exp (-E / R T) \cdot C_{M A}^{n a} \cdot C_{E H}^{n b}
$$

where $\mathrm{r}_{\mathrm{MA}}$ is the rate of consumption of MA, $\mathrm{k}_{\mathrm{o}}$ is the Arrhenius pre exponential factor, $\mathrm{E}$ is the activation energy $(\mathrm{kcal} / \mathrm{mol}), \mathrm{T}$ is the reaction temperature $(\mathrm{K}), \mathrm{C}_{\mathrm{MA}}$ and $\mathrm{C}_{\mathrm{EH}}$ are molar concentrations of MA and HE respectively, $n_{a}$ and $n_{b}$ are the reaction orders for MA and HE respectively. Then, equation 8 was linearized by logarithm application and resulted in equation 9, which is a multilinear equation and may be obtained by regression analysis. 


$$
\log \left(-r_{M A}\right)=\log \left(k_{0}\right)-(E / R T) \cdot \log (e)+n_{a} \log \left(C_{M A}\right)+n_{b} \log \left(C_{E H}\right)
$$

Initial velocity method [1] and multilinear regression based on $2^{3}$ complete factorial design [11] were applied together to establish an experimental chemical kinetic equation; care was take to use logarithms as regression parameters of the initial reaction rate, concentration variables and the inverse of temperature in Kelvin. Table 1 shows the experimental design for determination of one chemical kinetics equation. The laboratory procedure for this experiment was described above.

Table 1. Experimental conditions for the factorial design.

\begin{tabular}{ccccccc}
\hline $\mathrm{a}$ & $\mathrm{b}$ & $\mathrm{c}$ & $\mathbf{C}_{\mathbf{M A}}(\mathbf{M})$ & $\mathbf{C}_{\mathbf{E H}}(\mathbf{M})$ & $\mathbf{T}\left({ }^{\circ} \mathbf{C}\right)$ & $\mathbf{1} / \mathbf{T}(\mathbf{1} / \mathbf{K})$ \\
\hline+ & - & - & 2 & 0.5 & 139.2 & 0.002426 \\
- & - & - & 1 & 0.5 & 139.2 & 0.002426 \\
+ & - & + & 2 & 0.5 & 142.8 & 0.002404 \\
- & - & + & 1 & 0.5 & 142.8 & 0.002404 \\
+ & + & - & 2 & 1 & 139.2 & 0.002426 \\
- & + & - & 1 & 1 & 139.2 & 0.002426 \\
+ & + & + & 2 & 1 & 142.8 & 0.002404 \\
- & + & + & 1 & 1 & 142.8 & 0.002404 \\
\hline
\end{tabular}

The above described experimental procedure allows us to draw the curve of MA concentration decay, during the time of test. Each curve has ten results of MA concentration determined every minute, for ten minutes. This curve was numerically derived to obtain instantaneous values of the reaction speed. From these data, the initial velocity (at zero time) is used to process the regression analysis.

This experimental design was repeated six times in two sets of experiments: one with conventional electric heating and another with microwave heating. Each set had three levels for PTSA concentration: $0.012 \mathrm{M}, 0.006 \mathrm{M}$ and without PTSA. Each repetition was done in duplicate. The order of execution within a repetition was random with no restrictions.

\section{Reactor simulation for DEM production}

It is difficult to visualize the global effect of chemical kinetics on a reactor design by analyzing only their kinetic parameters. The effect of these parameters on the reactor's physical dimensions is very abstract to conceive. This situation consumed long time to be solved. The solution found was to analyze the reactor behavior instead of its chemical kinetic equation parameters. Therefore, an Excel spreadsheet was developed to allow the simulation of the reactant conversion for different chemical kinetic equations and different operation conditions.

For the simulation of the present case of DEM production it was assumed that the reactor is ideal, operating in isothermal condition and with negligible change of the volume of the reactants [1]. This spreadsheet basically resolves the equations for batch or plug flow reactor. Considering all the above restrictions, then batch and plug flow reactors have the same project equations [1].

The chemical equation is introduced in the spreadsheet and the variables as reactants concentration, temperature and molar relation between reactants can be changed. As a result, it shows the reaction time necessary to obtain a specified conversion of the limiting reagent in a batch or plug flow reactor. 


\section{Results and discussion}

The initials results were the consumption curves for MA. There were six pairs of duplicates, one pair for each combination of two kinds of heating and three PTSA concentrations. These data were numerically derived to produce the MA consumption rate curves which led to the initial velocity figure, and, finally, the empirical chemical kinetics for MA esterification by EH with PTSA catalyzing and microwave/conventional electric heating, as stated by (8). Table 2 shows these data.

In the case of conventional electric heating the activation energy decreases to approximately $90 \%$ of its initial value (without PTSA) as the PTSA concentration increases. This is the expected effect; it seems that this decrease follows a parabolic curve. An unexpected result occurred when microwave heating was applied. The activation energy decreased only $25 \%$ of its initial value and has a maximum value in the middle of PTSA concentration range, when it increased approximately $59 \%$ of its initial value. This fact requires a more detailed future study.

Table 2. Chemical kinetics parameters for MA esterification with EH in (8).

\begin{tabular}{cccccc}
\hline PTSA (M) & Heating & $\mathbf{k}_{\mathbf{0}}$ & $\mathbf{E}$ (kcal/mol) & $\mathbf{n}_{\mathbf{M A}}$ & $\mathbf{n}_{\mathbf{E H}}$ \\
\hline 0 & Electrical & $2.29 \mathrm{E}+78$ & 149.31 & 0.89 & 2.47 \\
0 & MW & $6.66 \mathrm{E}+14$ & 25.65 & 1.59 & 2.24 \\
0.006 & Electrical & $1.39 \mathrm{E}+25$ & 46.30 & 0.56 & 2.18 \\
0.006 & MW & $6.90 \mathrm{E}+21$ & 40.73 & 1.04 & 1.98 \\
0.012 & Electrical & $2.96 \mathrm{E}+08$ & 15.87 & 0.25 & 0.65 \\
0.012 & MW & $2.36 \mathrm{E}+52$ & 19.31 & 0.25 & 0.05 \\
\hline
\end{tabular}

The Arrhenius' pre-exponential factor had different responses to the PTSA concentration increase. For the conventional electric heating case, this value showed a strong decrease while for microwave heating it increased and, at 0.012 M PTSA concentration, its value was near to the conventional electric heating value without PTSA.

The reaction orders decreased with the PTSA concentration increase for both kind of heating. The order with respect to MA decreases, apparently, as a linear law. The order related to EH decreased, apparently, as a second order curve. Using microwave heating, the order related to MA is higher than that of the conventional heating; the order related to $\mathrm{EH}$ is lower than that of the conventional heating. The global order, up to 0.006 M PTSA concentration, is higher than the value of the conventional electric heating, but, beyond this point, an inversion occurred and the global order using microwaves decreased approximately $67 \%$ of the conventional heating value order. In practical terms all those observations did not show clearly this effect; putting differently: the best operation condition for DEM production was not determined.

The conversion of MA was simulated for all six options (three PTSA concentrations and two kinds of heating) using the developed spreadsheet. It was assumed an isothermal operation at $140{ }^{\circ} \mathrm{C}$ with a load of MA $10 \mathrm{M}$, EH $20 \mathrm{M}$, PTSA 0.006 (or $0.012 \mathrm{M}$ ) and dry xylene as solvent. The results are shown in Fig. 4, including a window with time scale magnified to show the first minute of the reaction. 


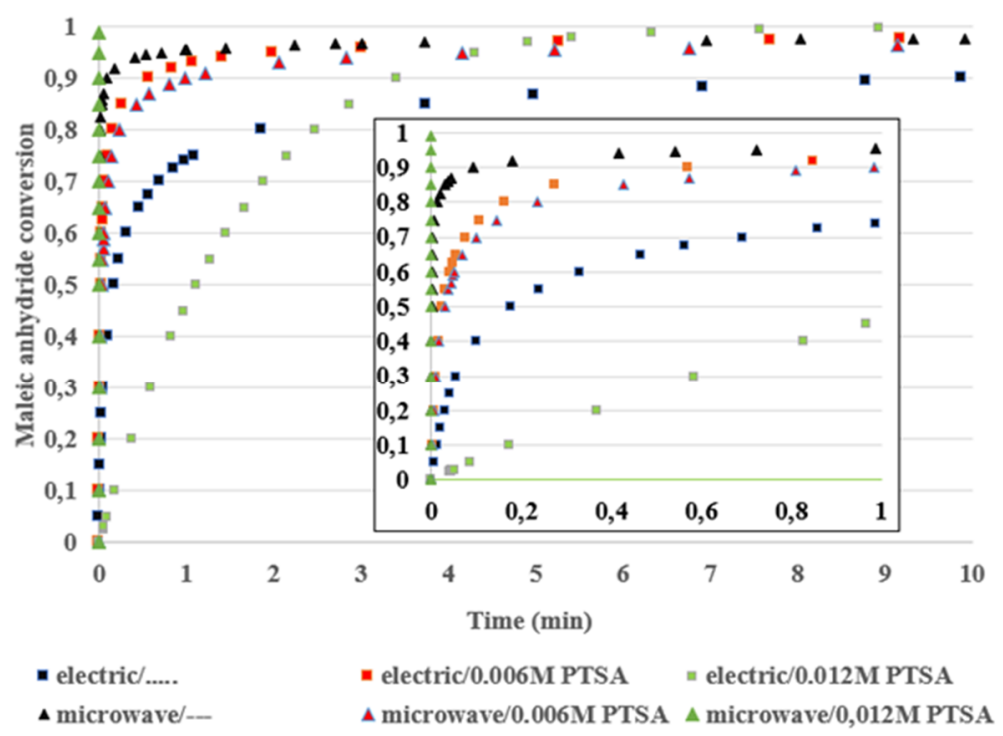

Fig. 4. Simulation of MA conversion I esterification with EH, catalyzed by PTSA and at $140{ }^{\circ} \mathrm{C}$, based on empirical chemical kinetic equations.

Curves of the electric heating show that, without PTSA, the reaction is a slow one for the industry point of view, because of the $80 \%$ plateau. Introducing PTSA at $0.006 \mathrm{M}$, it becomes a little bit faster but not enough to change the slow attribute. With PTSA at $0.012 \mathrm{M}$, it shows an initial 3-min time interval with slower figures compared to the uncatalyzed reaction, and afterwards it went to a complete conversion in nine minutes.

Introducing the microwave heating, without PTSA, the reaction becomes faster than the conventional heated reaction without PTSA. This shows a catalytic effect of microwaves alone on this esterification, being stronger than the effect of PTSA at $0.006 \mathrm{M}$. After three minutes, the microwave enhanced reaction and the conventional heated reaction, both catalyzed with PTSA $0.006 \mathrm{M}$, show the same reaction speed and both may be considered slow reactions.

No enhancement was observed with PTSA at $0.006 \mathrm{M}$ when microwave was applied as shown by the overlapping of the three curves: electric and microwave heating with PTSA at $0.006 \mathrm{M}$ and microwaves heating without PTSA. In fact, the addition of PTSA at $0.006 \mathrm{M}$ under microwave radiation had a bad result compared to the pure microwave application without catalyst. This phenomenon may be related to the unusual behavior of the activation energy observed in the reaction with microwaves and PTSA at $0.006 \mathrm{M}$ in Table 2 and analyzed above. Consequently, a plain microwave application is better than adding catalyst PTSA.

A complete conversion was reached at $5 \times 10^{-42}$ minutes by applying microwaves with PTSA at $0.0012 \mathrm{M}$. This curve is overlapping the vertical axis in Fig. 4. This is a real fast reaction and may be understood as a flash reaction. This phenomenon may be related to a synergic effect between microwaves and PTSA that reduces global reaction order and increase Arrhenius' pre exponential factor, as observed in Table 2.

\section{Conclusion}

Microwaves enhanced the esterification of MA with EH, either with or without catalysis of PTSA, as shown in Fig. 4. An important result is that a probable synergic effect between microwaves and PTSA can transform this reaction into a fast one, also shown in Fig. 4. 
Another interesting phenomenon was observed: the reaction under microwave irradiation and PTSA at $0.006 \mathrm{M}$ had the same velocity as the uncatalyzed enhanced esterification and microwave irradiated with PTSA at $0.006 \mathrm{M}$. So, it is better to apply plain radiation than use PTSA at $0.006 \mathrm{M}$. This fact may be explained by the odd behavior of the activation energy in the interaction of microwaves and PTSA at $0.006 \mathrm{M}$, as shown in Table 2. Careful observation of Table 2 show that microwaves can change the mechanism of this esterification because all parameters vary their values at different exposition to microwave energy.

\section{Acknowledgements}

The authors would like to thank C. Tognela. Support from Instituto Mauá de Tecnologia - IMT - and from Fundação de Amparo à Pesquisa do Estado de São Paulo FAPESP - (Grant 2017/24238-7) are gratefully acknowledged.

\section{References}

1. Levenspiel, O. Chemical Engineering Reaction. 3ed, USA, John Wiley, 1999.

2. Elsevier, Science Direct. www.sciencedirect.com, (accessed in 7 April, 2019).

3. Senise, J.T., Jermolovicius, L. A. J. Microw. Optoelectron. 2004, 3, 97-112.

4. Stefanidis, G. D., Muñoz, A. N., Sturm, G. S. J., Stankiewicz, A. Rev. Chem. Eng. 2014, 30, 233-259.

5. Kappe.O. Chem. Rec., 2018, 18, 1-26.

6. Ash, M., Ash, I. Plasticizers, Stabilizers and Thickeners. USA, Ed. Arnold, 1989.

7. Groggins, P. H. Unit Processes in Organic Synthesis. 5ed, Tokyo, McGraw-Hill, Kogakusha, 1958.

8. Castellan, G. W. Physical Chemistry. USA, Addison-Wesley, 1970.

9. Glasstone, S. Tratado de Quimica Fisica. 6 ed, España, Aguilar, 1966.

10. Jermolovicius, L. A., Senise, J. T., Castro, E. R. Proc. AMPERE 2005.

11. Montgomery, D. C. Design and Analysis of Experiments. 6 ed, USA, John Wiley, 2005. 


\title{
RAPID SYNTHESIS OF HIGHLY LUMINESCENT CARBON QUANTUM DOTS USING LOW-PRESSURIZED MICROWAVE SOLVOTHERMAL HEATING
}

\author{
K. Hagiwara and S. Horikoshi ${ }^{1}$
}

Department of Materials and Life Sciences, Faculty of Science and Technology, Sophia University, 7-1 Kioi-cho, Chiyoda-ku, Tokyo, Japan

k-hagiwara-7ge@eagle.sophia.ac.jp, 'horikosi@sophia.ac.jp

Keywords: Microwave Solvothermal Heating; Carbon quantum dots, Low-Pressurized synthesis

\section{Abstract}

In this research, we ameliorated the problems of carbon quantum dots synthesis and luminescence (quantum yields) by the novel synthesis protocol using microwave chemistry. Specifically, we synthesized high quantum yields carbon quantum dots $(61 \%)$ by utilizing a microwave chemical synthesis, synthesizing at low pressure condition (lower than 5 atom) and short reaction time $(3 \mathrm{hrs})$. The achievement of this high quantum yields made it clear that the contribution of polyethylene glycol (PEG) shell to carbon quantum dots is large. It was confirmed from the DLS and TEM image that the particle size of the synthesized particles was 8 to $13 \mathrm{~nm}$. On the other hand, the relationship between the polymerization degree of added PEG and the quantum yields to the addition amount is summarized. The quantum yields of carbon quantum dots without addition of PEG was $16.7 \%$, while it was improved at $61.1 \%$ when $0.6 \mathrm{~g}$ of PEG 6000 (Molecular weight: 6000) was added. Furthermore, we compared microwave with conventional method, and it was revealed that higher luminescent and narrower sized carbon quantum dots were synthesized in microwave heating than conventional method. This result presumably comes from the nature of microwave selective heating of carbon precursor.

\section{Introduction}

Since the first serendipity of carbon quantum dots (also called as carbogenic dots, carbon dots and carbon nanodots) ${ }^{1}$, it has been expected to be used for imaging materials for reusable living bodies ${ }^{2,3}$ (e.g. Hela cells) and detector for heavy metal ions ${ }^{4,5}$ (e.g. $\mathrm{Cu}^{2+}$, $\mathrm{Hg}^{2+}$ ), and many experimental protocols to synthesize carbon quantum dots have been reported, such as laser ablation ${ }^{6-8}$, electrochemical oxidation ${ }^{9-12}$, ultrasonic synthesis $^{13-16}$, and microwave heating ${ }^{17-20}$. However, in most of the reported methods, high temperature and pressure conditions and/or formation of solid-rich intermediates during the reaction are required to synthesize carbon quantum dots, which hinder the widespread of practical use of this material. Particularly, an acid-base reaction to synthesize carbon quantum dots using microwave heating leads the formation of solid-rich intermediates. For instance, succinic acid and tris (2-aminoethyl) amine are polymerized by microwave in the step of hydronation, resulting in the pyrolysis of black-colored polymer, followed by the purification using freeze-drying for 3 days ${ }^{20}$. This acid-base reaction also induces the long purification time and lowly luminescent products. To ameliorate these tasks, modified methodologies, such as solvothermal method using aromatic compounds ${ }^{21,22}$ and surface passivation using polymers ${ }^{5,6,23}$, have been reported recently.

Specifically, solvothermal method makes it possible to lower the production of toxic gas in the reaction system. For example, in 2018, L. Zhu and co-authors achieved in solvothermal-synthesizing carbon quantum dots with HATU/ other carbon sources and many kinds of low boiling point solvents, and in purifying the product using centrifugation ${ }^{21}$. 
This solvothermal method suppressed the production of solid-rich intermediates during the reaction and reduced purification time of the product. Also, surface passivation is known to bring higher quantum yields carbon quantum dots solution. For instance, Y. P. Sun and coworkers succeeded in functionalizing the surface of synthesized carbon quantum dots with PEG, resulting in the increase of quantum yields of carbon quantum dots ${ }^{6}$. Nonetheless, the existing carbon quantum dots synthetic methods have yet to be at the practical levels; the quantum yields are still lower than metal/semiconductor quantum dots, and synthetic condition is over $5 \mathrm{hrs}$ under more than $30 \mathrm{atms}^{24}$. Furthermore, carbon quantum dots synthesis using microwave solvothermal method, comparing to conventional solvothermal method, has not been reported yet, hence effects of microwave heating on the synthesis of carbon quantum dots have yet to be unveiled.

We successfully synthesized highly yellow luminescent carbon quantum dots (QYs: $61 \%$ at $580 \mathrm{~nm}$ ) by microwave solvothermal heating under much lower pressure condition (approximately 5 atm), using high boiling point solvent, ethylene glycol (b.p. $\left.197.6^{\circ} \mathrm{C}\right)^{25}$. This novel methodology also revealed the surface passivation of carbon quantum dots is highly dependent on the polymerization degree/amount of PEG. Furthermore, carbon precursor/ intermediate of carbon quantum dots highlighted a high absorbability of microwave, contributing to microwave superiority of carbon quantum dots synthesis over conventional solvothermal heating.

\section{Methodology}

In this research, highly luminescent carbon quantum dots were synthesized with 2step experimental strategies.

Synthesis of carbon precursor: White-colored solid anthracene $(0.143 \mathrm{~g}, 0.8 \mathrm{mmol})$ was added into $\mathrm{HNO}_{3}$ aq. $(20 \mathrm{~mL})$ in a three-necked round bottom flask $(50 \mathrm{~mL})$. The mixture was refluxed in $80{ }^{\circ} \mathrm{C}$ using microwave multimode chemical synthesis system (Wavemajic, EYELA; Frequency: $2.45 \mathrm{GHz}$ ). For comparison of microwave heating with conventional heating, oil bath heating was also conducted under the same conditions. In both microwave heating and conventional heating, samples were synthesized every hour for 12 hrs, and their changes over time were compared. Note that all the experiments were conducted in duplicates in the drying hood. After cooled to r.t., the mixture was centrifugated at $4000 \mathrm{rpm}$ for $5 \mathrm{~min}$. The resultant yellow 9.10-Dinitroanthracene was obtained by drying the precipitate at $60{ }^{\circ} \mathrm{C}$ with an electric furnace, followed by filtration with excess pure water, and Identification of the compound was performed by ${ }^{1} \mathrm{H}-\mathrm{NMR}$.

Synthesis of highly luminescent carbon quantum dots: 9.10-Dinitroanthracene $(0.107 \mathrm{~g}, 0.4 \mathrm{mmol})$ was added to $20 \mathrm{~mL}$ of ethylene glycol in a vial container. The mixture was turned into yellow solution after ultrasonicated $(20 \mathrm{kHz})$ for $5-10 \mathrm{~min}$ at room temperature. The yellow solution was removed and put into the reaction tube $(25 \mathrm{~mL}$ glass tube), then polyethlene glycol (PEG) and $\mathrm{NaOH}(0.24 \mathrm{~g})$ were added into the yellow solution. The polymerization degree of PEG used in this experiment was 600, 1500 and 6000 , respectively. The solution was rapidly heated to $210{ }^{\circ} \mathrm{C}$ using microwavesolvothermal method and kept at $210{ }^{\circ} \mathrm{C}$ for $3 \mathrm{hrs}$. In microwave solvothermal heating, single-mode applicator (Monowave 300, Anton Paar Ltd.; Frequency: $2.45 \mathrm{GHz}$ ) was utilized. Conventional solvothermal method was also carried out to for comparison with microwave solvothermal method. Impure carbon quantum dots solution (color: dark-blown) was obtained after rapidly cooling at r.t.. To remove impurities, the carbon quantum dots solution was centrifugated at $4000 \mathrm{rpm}$ for $5 \mathrm{~min}$ and dispersed in ethylene glycol. In order to investigate the solubility of the synthesized carbon quantum dots, carbon quantum dots synthesized without PEG were dissolved in four kinds of solvents; water, methanol, acetone and hexane. The ratio between carbon quantum dots solution and each solvent was fixed as 
1: 1 , and the surface status of carbon quantum dots was determined by FT-IR (FT/IR-4600, JASCO). The photoluminescence was evaluated by performing UV-Vis spectrometric measurement (V-7300, JASCO), 3D spectrum analysis (FP-8600, JASCO), and PL measurement (FP-8600, JASCO), and particle size distribution was characterized using TEM observation (H-7650, Hitachi High-Technologies; Accelerating voltage: $120 \mathrm{kV}$ )) and DLS analysis (Zetasizer nano ZS, Malvern). To determine microwave enhancement of carbon quantum dots $(0.60 \mathrm{~g}$ of PEG added $)$, temperature-dependent relative dielectric losses were measured with network analyzer (apparatus: E5071C, probe: 85070-20037, Keysight technology).

\section{Results and discussion}

Highly luminescent carbon quantum dots were synthesized by microwave solvothermal heating. In the determination of solubility of carbon quantum dots in four kinds of solvents (water, methanol, acetone and hexane), three ranges of emission colors (purple, blue and yellow) under UV irradiation were observed (Figure 1 (a)). On the ground that aromatic compounds emit from 370-440 nm (especially, anthracene strongly emits from $400-415 \mathrm{~nm})^{26}$, purple light was determined as the unreacted carbon precursor, 9.10Dinitroanthracene, or intermediates of carbon quantum dots. The other emission sources, on the other hand, were highly dissolved in water and water-soluble organic solvents (ethanol and acetone) but not in hexane, and were finally determined as carbon quantum dots. This observation implies that the surface of synthesized carbon quantum dots is polar and hydrophilic. It was also found that synthesized carbon quantum dots were completely dispersed into water, while, in the other water-soluble organic solvents, they were divided into two types of carbon quantum dots; emitting in the blue region and yellow emission region. For the further determination of the surface structure of synthesized carbon quantum dots, FT-IR measurement was utilized. According to Figure 1 (b), $-\mathrm{OH}$ and $-\mathrm{NO}_{2}$ moieties were observed at 2500-3600, 1520-1680, 1290-1360 $\mathrm{cm}^{-1}$ respectively, indicating that waterdispersibility of the synthesized carbon quantum dots derives from the surface structure of $\mathrm{OH}$ (hydroxy base), which is polar and hydrophilic. From these results, it was suggested that two types (blue and yellow emissions) of carbon quantum dots were both synthesized and characterized as water-dispersible due to the surface substituents of $-\mathrm{OH}$ moiety.
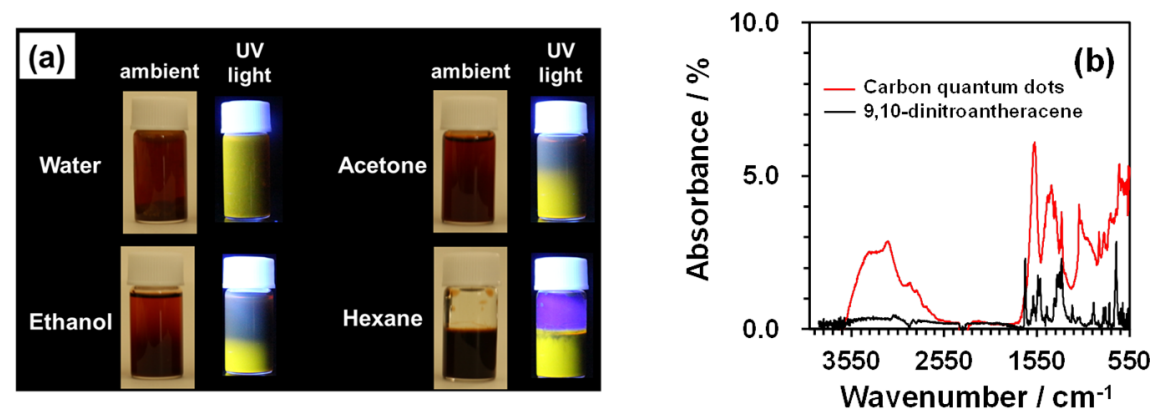

Figure 1. (a) solubility of carbon quantum dots to several kinds of solution: water, ethanol, acetone and hexane were each added to impure carbon quantum dots solution. The ratio between solution and a solvent was fixed as 1:1. (b) FT-IR spectra of non-PEG added carbon quantum dots solution. Each peak was assigned both automatically.

From the viewpoint of highly polarized surface of carbon quantum dots, purified carbon quantum dots were dispersed in water or ethylene glycol after centrifugation and used to measure the further analyses. From 3D-reverse, PL and UV-Vis spectroscopies, the 
optimum excitation wavelength for synthesized carbon quantum dots was determined as 275 $\mathrm{nm}$ by 3D-reverse analysis and PL spectra (Figure 2 (a)). Thus, the excitation wavelength of carbon quantum dots solution synthesized with various polymerization degree/ amount of PEG addition were also adjusted to $275 \mathrm{~nm}$ when PL spectra, full width at half maximum and quantum yields were measured. PL spectra was also utilized for the determination of the nature of carbon quantum dots. According to Table 1, a tendency of broader full width at half maximum was seen in all the experimental conditions, whistle quantum yields of each condition was of great difference. Quantum yields were improved dramatically as the added polymerization degree/ amount of PEG was changed, and we assume that this tendency derives from either the nature of microwave heating or high temperature condition. Moreover, it was observed that TEM image and DLS measurement show that the size distribution of synthesized carbon quantum dots is quite narrow (around 8-13 nm), indicating that a broad peak at approximately $580 \mathrm{~nm}$ depends on the nature of carbon source (e.g. surface emission), not on the widely distributed size of carbon quantum dots (Figure 2 (b)). This wide full width at half maximum can result from either large number of absorbance peaks or broadly distributed sizes of carbon quantum dots.
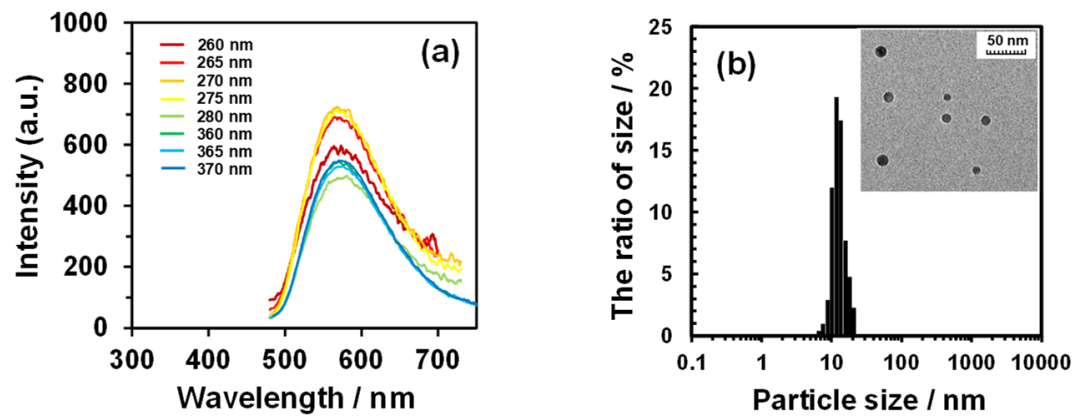

Figure 2. (a) PL spectrum and (b) TEM image of carbon quantum dots synthesized without the addition of PEG.

Table 1. Comparison between the amounts and kinds of polyethylene glycol (PEG) and the quantum yields (QYs) of synthesized carbon quantum dots solutions.

\begin{tabular}{cccc}
\hline Molecular weight of PEG & Weight amount /g & $\begin{array}{c}\text { FWHM / } \\
\mathrm{nm}\end{array}$ & QYs / \% at 275 nm \\
\hline$/ /$ & 0 & 108.8 & 16.7 \\
\hline 600 & 0.06 & 116.3 & 32.5 \\
\hline 600 & 0.15 & 115.5 & 42.9 \\
\hline 1500 & 0.15 & 114.4 & 39.9 \\
\hline 6000 & 0.15 & 114.8 & 43.1 \\
\hline 6000 & $\mathbf{0 . 6 0}$ & 112.2 & $\mathbf{6 1 . 1}$ \\
\hline
\end{tabular}

As aforementioned, high boiling point solvent, ethylene glycol, was utilized in this research. Note that this solvent plays an important role in lowering the inner pressure of reaction vessel, succeeding in the synthesis of highly luminescent carbon quantum dots and causing microwave-enhanced rapid heating of the solution. Particularly, ethylene glycol is well known as highly microwave absorbing solvent $\left(\varepsilon^{\prime \prime}=16.46\right.$ at $\left.25.0^{\circ} \mathrm{C}\right)$ and promoted the rapid heating of microwave: the heating rate of the solution was optimized as approximately 
$120{ }^{\circ} \mathrm{C} / \mathrm{min}$ in microwave heating and $1{ }^{\circ} \mathrm{C} / \mathrm{min}$ in conventional heating, resulting in the achievement of the 120 -fold faster heating rate. Thus, optimized total reaction time was set as $3 \mathrm{hrs}$ in microwave and $5 \mathrm{hrs}$ in autoclave, achieving 0.6-fold shorter total reaction time of carbon quantum dots in microwave solvothermal method. Moreover, it was confirmed that carbon quantum dots synthesized by two types of heating media, microwave and conventional, have apparent difference in qualities. For instance, we also synthesized carbon quantum dots with $0.60 \mathrm{~g}$ of PEG 6000 added in autoclave (conventional heating), and lower luminescence (QYs: $42.3 \%$ ) and broader size-distribution (diameter: $10 \mathrm{~nm}$ and 300-1000 $\mathrm{nm})$ were apparently observed in the conventional synthesis of carbon quantum dots in comparison with microwave-synthesized carbon quantum dots (QYs: 61.1 \%, diameter: 2-9 $\mathrm{nm}$ ) (Figure 3 (a)). We assume that this obvious difference was caused by microwave superiority, which is either rapid heating of microwave or specific microwave absorption of the other sources. By intentionally changing the heating rate in microwave method to 1 ${ }^{\circ} \mathrm{C} / \mathrm{min}$ (total reaction time: $5 \mathrm{hrs}$ ) as conventional method, it was clarified that slowly microwave-heated carbon quantum dots also attained approximately $61 \%$ of the quantum yields. This result highlights that the difference between microwave and conventional heating is related with specific microwave absorption of the other sources. Hence, in order to reveal the accuracy of hypothesized specific microwave superiority, the dependence of relative dielectric losses of ethylene glycol and the carbon precursor mixed solution on temperature was measured by network analyzer (Figure 3 (b)). It was revealed that the microwave absorbability of ethylene decreases as temperature increases, whistle that of the carbon precursor mixed solution stagnates even in the increase of temperature. This indicates that the carbon precursor and nuclei of carbon quantum dots should be the key factor to obtain highly luminescent carbon quantum dots, and microwave selective heating in the reaction temperature $\left(210^{\circ} \mathrm{C}\right)$ made a contribution to the highly heated carbon precursor/ nuclei of carbon quantum dots.
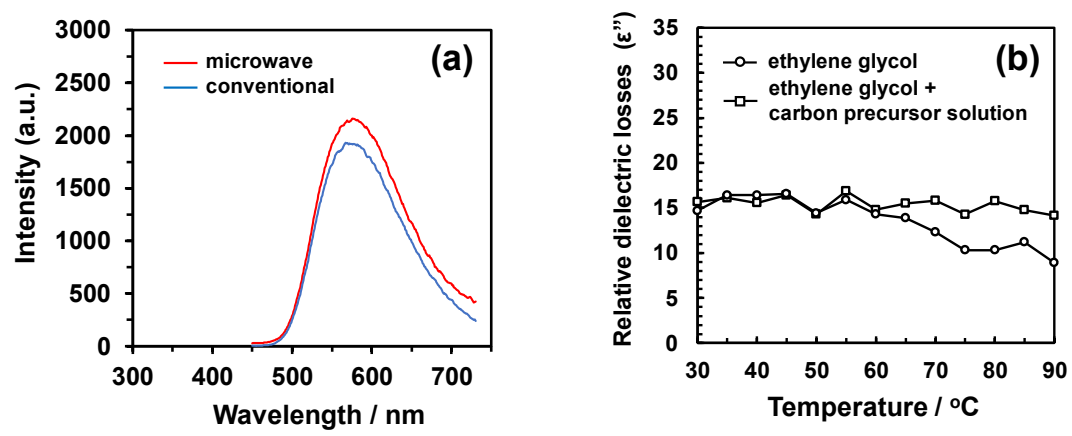

Figure 3. (a) Comparison of PL spectrum between microwave and conventional heating, and (b) comparison of relative dielectric losses between ethylene glycol and carbon quantum dots precursor solution.

\section{Concluding remarks}

We succeeded in achieving the uniform particle size distribution most required for nanomaterials, because the microwave method can rapidly heat the sample solution. This success made it clear that a rapid heating could result in high quantum yields. Particularly, the achievement of highly luminescent carbon quantum dots even by intentionally modified microwave slow heating clarified that relatively high microwave absorbability of carbon source in the reaction temperature is of great significance for microwave selective heating. 
Moreover, it was shown in this research that the mechanism of improvement of quantum yields differentiated from the polymerization degree/ amount of PEG.

\section{Acknowledgements}

We fully thank Prof. Kida of Kumamoto University for technical advice of this research. We also thank Prof. Uchida and Ass. Prof. Suzuki of Sophia University for the continuous support of analytical guidance. We also appreciate Prof. Torigoe of Tokyo University of Science for the TEM analysis.

\section{References}

1. X. Xu et al., J. Am. Chem. Soc., 2004, 126, 12736-12737.

2. H.M. Xiong et al., ACS Nano., 2016, 10, 484-491.

3. M. Zou et al., Mater. Lett., 201266 222-224.

4. B. Yang et al., Angew. Chemie - Int. Ed., 2013, 52, 3953-3957.

5. Chi Y et al., Anal. Chem., 2012, 84, 6220-6224.

6. Y. P. Sun et al., J. Am. Chem. Soc., 2006, 128, 7756-7757.

7. X. Wang et al., J. Phys. Chem. C., 2008, 112, 18295-18298.

8. N. Koshizaki et al., Chem. Commun., 2011, 47, 932-934.

9. K.P. Loh et al., ACS Nano., 2009, 3, 2367-2375.

10. Z. Ding et al., J. Am. Chem. Soc., 2007, 129, 744-745.

11. Z. Kang, Y. Liu, S.T. Lee et al., Angew. Chemie - Int. Ed., 2010, 49, 4430-4434.

12. Y. Liu, Z. Kang, S.T. Lee et al., ACS Appl. Mater. Interfaces, 2013, 5, 5080-5084.

13. Z. Kang et al, J. Mater. Chem., 2012, 22, 17470-17475.

14. J. Lee et al., ACS Appl. Mater. Interfaces, 2014, 6, 3365-3370.

15. Z. Kang et al, J. Chem., 36 (2012) 861-864.

16. S. Zhuo, M. Shao and S. T. Lee, ACS Nano, 2012, 6, 1059-1064.

17. W. Liu et al., J. Mater. Chem. B, 2014, 2, 46-48.

18. W. Guan, W. Gu et al., Int. J. Nanomedicine, 2014, 9, 5071-5078.

19. X. Sun, J. Mater. Chem., 2011, 21, 11726-11729.

20. I. In et al., RSC Adv., 2017, 7, 12663-12669.

21. L. Zhu et al, Chem. Sci., 2018, 9, 1323-1329.

22. X. G. Liu et al., Appl. Phys. Lett., 2015, 107, 213102-1-213102-5.

23. Y. Chen, Y. Yao et al., ACS Macro Lett., 2013, 2, 1033-1037.

24. X.B. Yin et al., Angew. Chemie., 2013, 19, 2276-2283.

25. K. Hagiwara, S. Horikoshi, 2019, will be published.

26. D. Jacewicz et al., Anal Lett., 2012, 46, 349-355. 


\title{
MICROWAVE-ASSISTED SYNTHESIS AND SPECTROSCOPIC PROPERTIES OF NOVEL PYRIDINE-BASED FLUORESCENT MOLECULAR PROBES
}

\author{
P. Fiedor ${ }^{1}$, J. Ortyl ${ }^{1,2}$, M. Galek ${ }^{2}$ \\ ${ }^{1}$ University of Technology in Cracow, Warszawska 24, 31-155 Cracow, Poland \\ ${ }^{2}$ Photo HiTech, Bobrzyńskiego 14, 30-348 Cracow, Poland \\ pfiedor@chemia.pk.edu.pl
}

Keywords: microwave chemistry, synthesis, spectroscopy, fluorophores, molecular probes

\section{Introduction:}

Fluorescent molecular probes become interesting analytical tools in determination and labeling of chemical compounds, and physical properties such as viscosity and polarity. Currently known fluorescent molecular probes can selectively and regardless of the environment detect only few molecules. Their applicability for determination of microviscosity and micro-polarity is limited to narrow range and specific condition. Therefore design and synthesis of novel molecular probes with extended range of operation are highly needed [1].

Derivatives of 2-amino-4,6-diphenyl-pyridine-3-carbonitrile can find application in different fields of science. Depending on the structure of this fluorophore, those compounds exhibit high sensitivity to changes in polarity and viscosity of environment, also concentration of specific cations, and $\mathrm{pH}$ can be determined by measuring of fluorescence spectrum of 2-amino-4,6-diphenyl-pyridine-3-carbonitriles [2]. Knowing that spectroscopic parameters strongly depends on substituents present at phenyl rings of those compounds it is important to examine this influence by synthesis and direct research on synthesized compounds [2]. To enable such studies, cheap, quick and efficient method of synthesis of 2amino-4,6-diphenyl-pyridine-3-carbonitrile derivatives must be used. Variety of synthesis paths for this group of compounds has been proposed at literature, most of them base on two steps reaction $[3,4,5]$. At first step simple Knovenagel condensation of benzaldehyde with malonodinitrile was conducted at methanol solution with catalytic addition of sodium hydroxide. Second step is conducted at high temperature in toluene for $4 \mathrm{~h}$. This traditional way takes long time and consume unnecessary amounts of solvents and substrates [5]. Some solutions of this problems was reported according to literature. Different heterogenic catalytic systems were proposed to solve problem of wasting solvents and materials. Main problem of this approach is that, proposed catalysts are expensive and are hard to reach or obtain [6].

In case employing microwave heating, single step reaction can be performed without or with trace amount of solvent. This approach simplify the procedure of preparation of many derivatives of 2-amino-3-cyanopyridines. Such synthesis strategy was reported, but only very little of derivatives were proven to form at such conditions. The advantage of this method is that, crystalline product was formed after reaction, and after recrystallization from ethanol or 2-propanol pure compounds were obtained $[1,7]$. 


\section{Results and Discussion:}

Traditionally synthesis of 2-amino-4,6-diphenyl-pyridine-3-carbonitriles was divided into two steps. First stage was Knoevenagel condensation between malonodinitrile and proper derivative of benzaldehyde at methanol solution in presence of catalytic amount of sodium hydroxide [5]. Condensation was conducted for $30 \mathrm{~min}$ at room temperature. After that time product was filtrated and washed with cold methanol. Yield of this stage for all investigated structures were higher than $90 \%$.

Second step was a reaction between obtained alikilidene malonodinitirile (1 eq) with proper derivative of acetophenone (1 eq) in presence of ammonium acetate (1.1 eq). Reaction was conducted in toluene (10 eq) at $100^{\circ} \mathrm{C}$ for $4 \mathrm{~h}$ [5]. Reaction path and conditions are presented on scheme 1. After second step of the reaction some impurities were present on TLC, due to that condition, purification on column chromatography was necessary.<smiles>[R]c1ccc(C=O)cc1</smiles>

1<smiles>[R]c1ccc(C=C(C#N)C#N)cc1</smiles>

3<smiles>N#CCC#N</smiles>

2

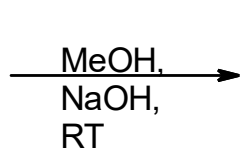
RT

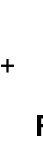<smiles>[R2]c1ccc(C(C)=O)cc1</smiles>

4<smiles>[R]c1ccc(C=C(C#N)C#N)cc1</smiles>

3<smiles>[R]c1ccc(-c2cc(-c3ccc([R])cc3)c(C#N)c(N)n2)cc1</smiles>

\section{$\mathbf{R}_{1}, \mathbf{R}_{2}=\mathrm{CN}, \mathrm{OCH}_{3}, \mathrm{SCH}_{3}$}

PhMe, $\mathrm{NH}_{4} \mathrm{OAc}$, $100^{\circ} \mathrm{C}, 4 \mathrm{~h}$

Scheme 1. Reacon route for traditional synthesis of 2-amino-4,6-diphenyl-pyridine-3carbonitrile derivatives.

Yield of synthesis for derivatives of 2-amino-4,6-diphenyl-pyridine-3-carbonitriles obtained on traditional path are presented in the table 1.

Table 1. Yield of synthesis of 2-amino-4,6-diphenyl-pyridine3-carbonitrile conducted at traditional path.

\begin{tabular}{cccc}
\hline Product & $\mathbf{R}_{\mathbf{1}}$ & $\mathbf{R}_{\mathbf{2}}$ & Yield in traditional synthesis \\
\hline $\mathbf{P 0}$ & $\mathrm{H}$ & $\mathrm{H}$ & $30 \%$ \\
\hline $\mathbf{P 1}$ & $\mathrm{CN}$ & $\mathrm{H}$ & $14 \%$ \\
\hline $\mathbf{P 2}$ & $\mathrm{CN}$ & $\mathrm{OCH}_{3}$ & $30 \%$ \\
\hline $\mathbf{P 3}$ & $\mathrm{CN}$ & $\mathrm{SCH}_{3}$ & $33 \%$ \\
\hline $\mathbf{R} 1$ & $\mathrm{H}$ & $\mathrm{CN}$ & $24 \%$ \\
\hline $\mathbf{R 2}$ & $\mathrm{OCH}_{3}$ & $\mathrm{CN}$ & $15 \%$ \\
\hline $\mathbf{R 3}$ & $\mathrm{SCH}_{3}$ & $\mathrm{CN}$ & $23 \%$ \\
\hline $\mathbf{S 1}$ & $\mathrm{OCH}_{3}$ & $\mathrm{SCH}_{3}$ & $55 \%$ \\
\hline $\mathbf{S 2}$ & $\mathrm{SCH}_{3}$ & $\mathrm{OCH}_{3}$ & $80 \%$ \\
\hline
\end{tabular}


General procedure for microwave synthesis was single step reaction between malonodinitrile (1 eq), and proper derivatives of acetophenone (1 eq), benzaldehyde ( 1 eq) with addition of ammonium acetate (1.1 eq). Reactions were conducted into small amount of toluene (3 eq). To investigate influence of phase transfer catalyst presence in reaction mixture TBAB was added in quantity of 0.04 eq. Reactions were carried out in a Alasca MWD2925GC domestic microwave oven operating at $2450 \mathrm{MHz}$ at a maximum power of $800 \mathrm{~W}$. Time of reaction was selected by studies of reaction progress via TLC to be $210 \mathrm{~s}$. Microwave synthesis path and conditions are presented on scheme 2. After reaction, when resulting mixture was cooled down, product crystalized directly from reaction mixture, in most cases there were no additional impurities visible on TLC. After filtration, washing with cold toluene and recrystallization from methanol products were pure for further investigation.<smiles>[R]c1ccc(C=[OH+])cc1</smiles><smiles>[R]c1ccc(C(C)=O)cc1</smiles>
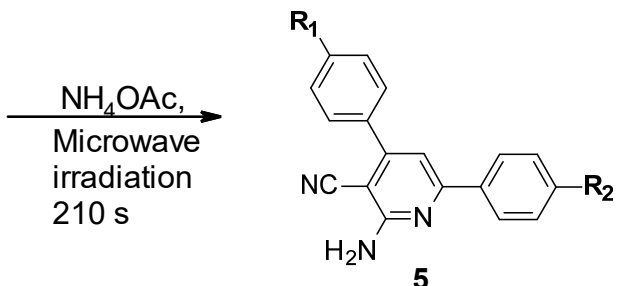

$\mathrm{R}_{1}, \mathrm{R}_{2}=\mathrm{CN}, \mathrm{OCH}_{3}, \mathrm{SCH}_{3}$

Scheme 2. Reaction route for microwave synthesis of 2-amino-4,6-diphenyl-pyridine-3carbonitrile derivatives.

Yield of microwave assisted synthesis of derivatives of 2-amino-4,6-diphenyl-pyridine-3carbonitriles obtained by path presented on scheme 2 are presented in the table 2 . As can be observed one pot synthesis assisted by microwave irradiation gives the same, or higher yields in apolar solvent, and even better results were observed in the presence TBAB.

Table 2. Yield of microwave assisted synthesis of 2-amino-4,6-diphenyl-pyridine-3carbonitrile conducted at traditional path.

\begin{tabular}{ccccc}
\hline Product & $\mathbf{R}_{1}$ & $\mathbf{R}_{2}$ & $\begin{array}{c}\text { Yield with } \\
\text { toluene }\end{array}$ & $\begin{array}{c}\text { Yield with toluene } \\
\text { and TBAB }\end{array}$ \\
\hline P0 & $\mathrm{H}$ & $\mathrm{H}$ & $35 \%$ & $33 \%$ \\
\hline $\mathbf{P 1}$ & $\mathrm{CN}$ & $\mathrm{H}$ & $30 \%$ & $32 \%$ \\
\hline $\mathbf{P 2}$ & $\mathrm{CN}$ & $\mathrm{OCH}_{3}$ & $42 \%$ & $50 \%$ \\
\hline P3 & $\mathrm{CN}$ & $\mathrm{SCH}_{3}$ & $57 \%$ & $58 \%$ \\
\hline $\mathbf{R} 1$ & $\mathrm{H}$ & $\mathrm{CN}$ & $32 \%$ & $30 \%$ \\
\hline $\mathbf{R 2}$ & $\mathrm{OCH}_{3}$ & $\mathrm{CN}$ & $36 \%$ & $43 \%$ \\
\hline $\mathbf{R 3}$ & $\mathrm{SCH}_{3}$ & $\mathrm{CN}$ & $27 \%$ & $30 \%$ \\
\hline $\mathbf{S 1}$ & $\mathrm{OCH}_{3}$ & $\mathrm{SCH}_{3}$ & $43 \%$ & $72 \%$ \\
\hline S2 & $\mathrm{SCH}_{3}$ & $\mathrm{OCH}_{3}$ & $45 \%$ & $86 \%$ \\
\hline
\end{tabular}


As can be observed at table 2, microwave synthesis shorten reaction time and enhance yields for almost all investigated compounds. At this conditions it was possible to synthesize sufficient amount of compounds in short time, without wasting materials and solvents.

To confirm structure of obtained derivatives ${ }^{1} \mathrm{H}$ NMR spectra were recorded at DMSO-D 6 solutions on Avance III HD $400 \mathrm{MHz}$ (Bruker) spectrometer. Collected data are presented in table 3 .

Table 3. Characterization of 2-amino-4,6-diphenyl-pyridine-3-carbonitriles derivatives by ${ }^{1} \mathrm{H}$ NMR spectra.

\begin{tabular}{|c|c|}
\hline Product & ${ }^{1}$ H NMR (400 MHz, DMSO-D $)$ \\
\hline P0 & $\begin{array}{l}\delta 8.17-8.11(\mathrm{~m}, 2 \mathrm{H}), 7.72-7.66(\mathrm{~m}, 2 \mathrm{H}), 7.60-7.54(\mathrm{~m}, 3 \mathrm{H}), \\
7.52-7.47(\mathrm{~m}, 3 \mathrm{H}), 7.29(\mathrm{~s}, 1 \mathrm{H}), 7.03(\mathrm{~s}, 2 \mathrm{H}) .\end{array}$ \\
\hline P1 & $\begin{array}{l}\delta 8.17-8.11(\mathrm{~m}, 2 \mathrm{H}), 8.07-8.02(\mathrm{~m}, 2 \mathrm{H}), 7.91-7.86(\mathrm{~m}, 2 \mathrm{H}), \\
7.53-7.47(\mathrm{~m}, 3 \mathrm{H}), 7.33(\mathrm{~s}, 1 \mathrm{H}), 7.13(\mathrm{~s}, 2 \mathrm{H}) .\end{array}$ \\
\hline $\mathbf{P 2}$ & $\begin{array}{l}\delta 8.15-8.09(\mathrm{~m}, 2 \mathrm{H}), 8.07-8.01(\mathrm{~m}, 2 \mathrm{H}), 7.90-7.84(\mathrm{~m}, 2 \mathrm{H}), \\
7.27(\mathrm{~s}, 1 \mathrm{H}), 7.09-7.01(\mathrm{~m}, 4 \mathrm{H}), 3.82(\mathrm{~s}, 3 \mathrm{H}) .\end{array}$ \\
\hline $\mathbf{P 3}$ & $\begin{array}{l}\delta 8.13-8.07(\mathrm{~m}, 2 \mathrm{H}), 8.06-8.02(\mathrm{~m}, 2 \mathrm{H}), 7.90-7.85(\mathrm{~m}, 2 \mathrm{H}), \\
7.38-7.33(\mathrm{~m}, 2 \mathrm{H}), 7.31(\mathrm{~s}, 1 \mathrm{H}), 7.11(\mathrm{~s}, 2 \mathrm{H}), 2.53(\mathrm{~s}, 3 \mathrm{H}) .\end{array}$ \\
\hline $\mathbf{R} 1$ & $\begin{array}{l}\delta 8.36-8.28(\mathrm{~m}, 2 \mathrm{H}), 8.01-7.93(\mathrm{~m}, 2 \mathrm{H}), 7.74-7.65(\mathrm{~m}, 2 \mathrm{H}), \\
7.62-7.52(\mathrm{~m}, 3 \mathrm{H}), 7.41(\mathrm{~s}, 1 \mathrm{H}), 7.14(\mathrm{~s}, 2 \mathrm{H}) .\end{array}$ \\
\hline $\mathbf{R 2}$ & $\begin{array}{l}\delta 8.34-8.28(\mathrm{~m}, 2 \mathrm{H}), 8.00-7.93(\mathrm{~m}, 2 \mathrm{H}), 7.71-7.65(\mathrm{~m}, 2 \mathrm{H}), \\
7.38(\mathrm{~s}, 1 \mathrm{H}), 7.14-7.10(\mathrm{~m}, 2 \mathrm{H}), 7.08(\mathrm{~s}, 2 \mathrm{H}), 3.84(\mathrm{~s}, 3 \mathrm{H}) .\end{array}$ \\
\hline $\mathbf{R 3}$ & $\begin{array}{l}\delta 8.34-8.29(\mathrm{~m}, 2 \mathrm{H}), 8.00-7.94(\mathrm{~m}, 2 \mathrm{H}), 7.68-7.62(\mathrm{~m}, 2 \mathrm{H}), \\
7.46-7.40(\mathrm{~m}, 2 \mathrm{H}), 7.39(\mathrm{~s}, 1 \mathrm{H}), 7.13(\mathrm{~s}, 2 \mathrm{H}), 2.55(\mathrm{~s}, 3 \mathrm{H}) .\end{array}$ \\
\hline S1 & $\begin{array}{l}\delta 8.13-8.06(\mathrm{~m}, 2 \mathrm{H}), 7.69-7.62(\mathrm{~m}, 2 \mathrm{H}), 7.39-7.33(\mathrm{~m}, 2 \mathrm{H}), \\
7.24(\mathrm{~s}, 1 \mathrm{H}), 7.15-7.08(\mathrm{~m}, 2 \mathrm{H}), 6.93(\mathrm{~s}, 2 \mathrm{H}), 3.85(\mathrm{~s}, 3 \mathrm{H}) 2.54 \\
(\mathrm{~s}, 3 \mathrm{H}) .\end{array}$ \\
\hline $\mathbf{S 2}$ & $\begin{array}{l}\delta 8.14-8.09(\mathrm{~m}, 2 \mathrm{H}), 7.61-7.49(\mathrm{~m}, 2 \mathrm{H}), 7.09(\mathrm{~s}, 1 \mathrm{H}), 6.86- \\
6.80(\mathrm{~m}, 2 \mathrm{H}), 6.79-6.71(\mathrm{~m}, 2 \mathrm{H}), 6.66(\mathrm{~s}, 2 \mathrm{H}), 3.83(\mathrm{~s}, 3 \mathrm{H}) 2.56 \\
(\mathrm{~s}, 3 \mathrm{H}) .\end{array}$ \\
\hline
\end{tabular}

\section{Absorbtion and fluorescence characteristics}

Molecular absorption spectra of obtained fluorophores were recorded in acetonitrile, using the SilverNova spectrometer (StellarNet, Inc., USA) in combination with a broadband tungsten-deuterium UV-Vis light source (from StellarNet, Inc., USA), and a quartz cuvette with $1.0 \mathrm{~cm}$ optical path. Next, the absorbance data were converted into extinction coefficients, expressed in classical units $\left[\mathrm{dm}^{3} \cdot \mathrm{mol}^{-1} \cdot \mathrm{cm}^{-1}\right]$. 


\section{High Frequency Heating}

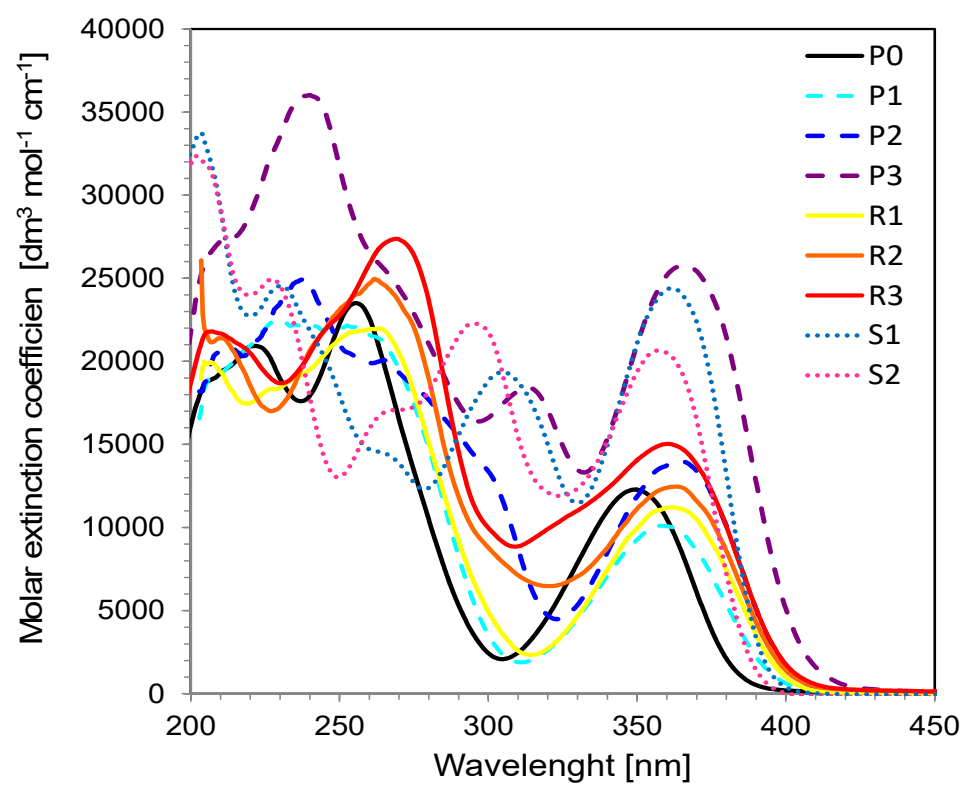

Figure 1. Molar extinction coefficient of investigated compounds investigated at acetonitrile solution.

The effect of substituents on absorption of the 2-amino-4,6-diphenyl-pyridine-3carbonitrile derivatives are clearly visible, significant differences in absorption and fluorescent spectra were observed especially between basis compound P0 and compound with best absorption characteristics $\mathrm{P} 3$ where batochromic shift of $20 \mathrm{~nm}$ is observed. Molar extinction coefficient of investigated compounds also distinguish obtained compounds depending on the structure. Best values are also assigned to compound P3, but also S1 and S2 reach very high value in comparison to others 2-amino-4,6-diphenyl-pyridine-3carbonitrile derivatives.

Fluorescence measurements were carried out using the same miniature spectrometer. The spectral characteristics of the sensors were measured in acetonitrile at room temperature $\left(22^{\circ} \mathrm{C}\right)$ using $1.0 \mathrm{~cm}$ thick quartz cells. As a source of excitation, the UV-LED $320 \mathrm{~nm}$ (UVTOP315-BL-TO39, Roithner Laser Technik GmbH, Austria) light was used. 


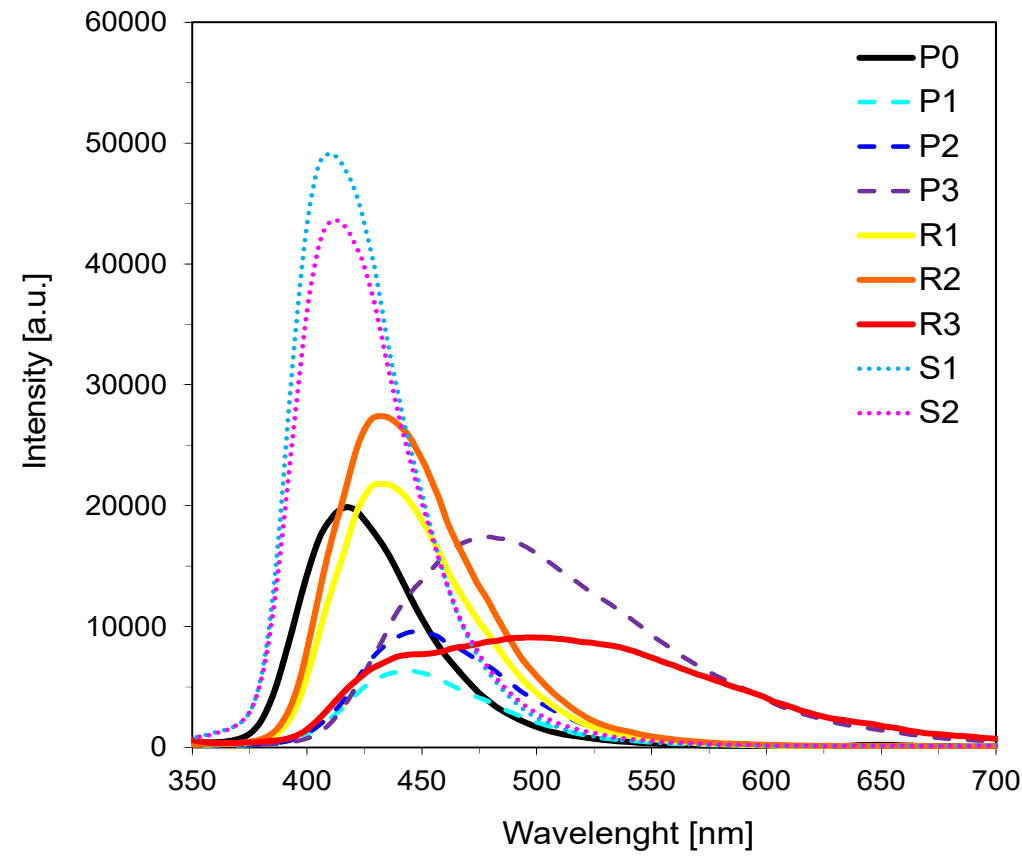

Figure 2. Fluorescence spectra of obtained compounds recorded at acetonitrile solution with excitation at 320nm with UV LED.

The introduction of two electron donor groups (methylthio and methoxy group) to the compound S1 and S2 resulting in significant increment of fluorescence intensity. Structure, where molecule contain both electron donor and electron acceptor moiety exhibit fluorescent spectra shifted to longer wavelength what can be useful in case of application as fluorescent molecular probes, where optical window must be found for every single application.

\section{Conclusion}

Traditional long time synthesis of 2-amino-4,6-diphenyl-pyridine-3-carbonitrile require higher volume of solvents used directly in synthesis, and also more complicated solvent-wasting purification.

Microwave synthesis is great convenience for synthesis of different derivatives as a base for screening tests. Thanks to extremely fast reactions with satisfying yields, and fact that reactions can be conducted with minimal necessary amounts of solvents and substratum a significant quantity of different compounds are prepared and analyzed in short period of time. Screening test of this group of fluorophores shown, that if fluorescence intensity is considered as most important factor for application of fluorescent probe, both substituents placed at external aromatic ring of obtained derivative should have electron donor characteristics. On the other hand, when longer wavelength of fluorescence is necessary both electron donor, and electron acceptor moieties should be simultaneously placed into fluorophore structure. 
Obtained compounds shows great spectral properties for application as fluorescent molecular probes into different fields of science, but their structure can be still refined by investigating other moieties and substituent placed at different places of fluorophore.

\section{References}

1. P. Gameiro, B. Castro, M. Rangel, A. M. G. Silva Eur. J. Org. Chem., 2012, 29, 5810-5817.

2. J. Ortyl, P. Fiedor, A. Chachaj-Brekiesz, M. Pilch, E. Hola, M. Galek, Sensors, 2019, 19.

3. F. Belhadja, Z. Kiboua, N. Cheikha,d, N. Choukchou-Brahama, D. Villeminb, Tetrahedron Letters, 2015, 56, 5999-6002.

4. R. Gupta, A. Jain, M. Jain, R. Joshi, Bull. Korean Chem. Soc, 2010, 3, 3180-3182.

5. S. Kambe, K. Saito, A. Sakurai, H. Midorikawa, Synthesis, 1980, 5, 366-368.

6. D. Khalili, Tetrahedron Letters, 2016, 57, 1721-1723.

7. S. Paul, R. Gupta, A. Loupyb, J. Chem. Research, 1998, 330-331.

8. J. Ortyl, K. Sawicz, R. Popielarz, J. Polym. Sci. A, 2010, 48, 4522-4528.

\section{Acknowledgement}

The authors are grateful to the Foundation for Polish Science (Warsaw, Poland) - Project REINTEGRATION (Contract No. REINTEGRATION/2016-1/4 - "Synthesis and photochemistry/photophysics studies of the intelligent luminescent molecular sensors for selective detection in biochemistry and chemistry") for financial support of the FPT research.

The authors also acknowledge the support of the PROM programme no. PPI/PRO/2018/1/00013/U/001 which is co-financed by the European Social Fund under the Knowledge Education Development Operational Programme. 


\title{
TUNGSTEN CARBIDE NANOPOWDER SYNTHESIS UNDER THE EXPOSURE OF 24 GHZ GYROTRON RADIATION ON THE NANOCOMPOSITE OF THE W-C SYSTEM OBTAINED IN A THERMAL PLASMA
}

\author{
A. Vodopyanov ${ }^{1,2}$, A. Samokhin ${ }^{2}$, N. Alekseev ${ }^{2}$, M. Sinayskiy ${ }^{2}$, A. Sorokin ${ }^{1}$, S. Sintsov $^{1}$ \\ ${ }^{1}$ Institute of Applied Physics RAS, 46 Ul'yanov st., Nizhny Novgorod, Russia \\ ${ }^{2}$ Baikov Institute of Metallurgy and Material Science RAS, 49 Leninskiy av., Moscow, Russia \\ avod@yandex.ru
}

Keywords: microwave, gyrotron, tungsten carbide, nanoparticles, synthesis microwave

\section{Introduction}

Nanoscale tungsten carbide WC powders are of practical interest for creating nanostructured hard alloys with enhanced physical and mechanical characteristics, wearresistant nanostructured coatings, electro-catalysts in fuel cells, metal melt modifiers [1-8]. An efficient method for producing tungsten carbide nanopowders is a plasma-chemical synthesis of a multicomponent $\mathrm{W}-\mathrm{C}$ powder nanocomposite with its subsequent heat treatment. It results in the formation of tungsten carbide WC [2, 9-11]. Experimental studies have shown the possibility of producing tungsten carbide WC nanopowder using this method. Heat treatment in the electric furnace at a temperature of $1100-1200^{\circ} \mathrm{C}$ for a few hours is necessary for the complete conversion of the nanocomposite into the target product. The heating is accompanied by an increase of nanoparticles in size.

Currently, much attention is paid to the heating of reagents by microwaves to conduct solid-phase chemical reactions [12-19]. Microwave heating provides energy deposition in the volume of the processed material, this significantly increases the heating rate compared to traditional heating in furnaces, where energy is transferred from the surface to the volume of material. Numerous studies have shown that the use of microwave heating for chemical reactions in solids makes it possible to increase the rate by order of magnitude, to increase the yield of target products and to reduce the impurities. The use of microwave heating with a radiation frequency of $2.45 \mathrm{GHz}$ for the synthesis of tungsten carbide WC from powder mixtures of tungsten or tungsten trioxide with carbon leads to a reduction in the reaction time [20-27]. It also provides the possibility to obtain the target product in the form of a nano-sized powder using tungsten precursors having a particle size in the nanometer range.

In the most cases, studies of chemical syntheses using microwave heating were carried out in an electromagnetic field with a frequency of $2.45 \mathrm{GHz}$, but it is noted that an increase in the frequency of the waves may result in additional effects of material synthesis $[13,19$, 28].

The purpose of the research was an experimental study of the possibility of obtaining tungsten carbide WC from a powder multicomponent nanocomposite system W-C, obtained by plasma-chemical synthesis when processed in the microwave field with two different frequencies: $2.45 \mathrm{GHz}$ and $24 \mathrm{GHz}$.

\section{Experimental Technique}

The W-C nanopowder processing experiments were conducted on two available microwave sources: the gyrotron complex with a nominal output power of $7 \mathrm{~kW}$ in $\mathrm{CW}$ mode with radiation frequency of $24 \mathrm{GHz}$ and a magnetron with a radiation frequency of $2.45 \mathrm{GHz}$ and an average power of $0.8 \mathrm{~kW}$. The use of two sources of microwave radiation made it possible to investigate the dependence of the properties of the treated nanopowder $\mathrm{W}-\mathrm{C}$ on the wavelength of the heating field while other conditions being equal. 
The gyrotron complex can be structurally divided into a treatment chamber with a microwave radiation focusing system and a microwave system. Fig. 1a shows the experimental setup. The gyrotron radiation with a frequency of $24 \mathrm{GHz}$ in is introduced by a circular waveguide with a diameter of $32.6 \mathrm{~mm}$ through a sealed window into the processing chamber. The microwave radiation converted from the TE11 mode of a circular waveguide into a Gaussian beam. Then it was focused by a parabolic 90 -degree mirror ( 1 in Fig. 1A). The Gaussian beam was focused on the surface of the processed nanopowder placed in a quartz crucible ( 2 in Fig. 1A). The amount of nanopowder loaded in a quartz crucible and processed in one experiment was about $10 \mathrm{~g}$. There is a region of the focal waist at a distance of $32 \mathrm{~cm}$ from the center of the parabolic mirror. The cross-sectional area of the beam was $3 \mathrm{~cm}^{2}$. The power of the gyrotron radiation varied from 100 to 400 watts in the experiments described. The sample surface temperature was 1050-1200 C. The processing chamber was sealed; all the flanges entrances on its body are standard CF vacuum fittings. The flange entrance ( 3 in Fig. 1A) was made according to the CF250 standard and has a vacuum quick access door with a viewing port covered with a metal grid. Visual control of the sample heating processes and the measurement of the surface temperature were performed using a RAYTEK Marathon MR1S two-color pyrometer through the viewing port. Also, this door used to load and unload the crucible with nanopowder. Samples of nanosized powder $\mathrm{W}-\mathrm{C}$ were processed in a stream of nitrogen at atmospheric pressure.

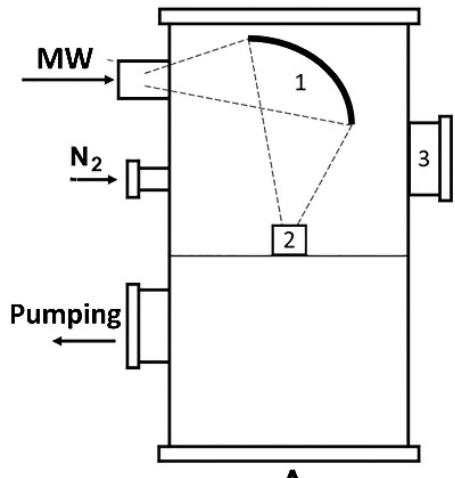

A

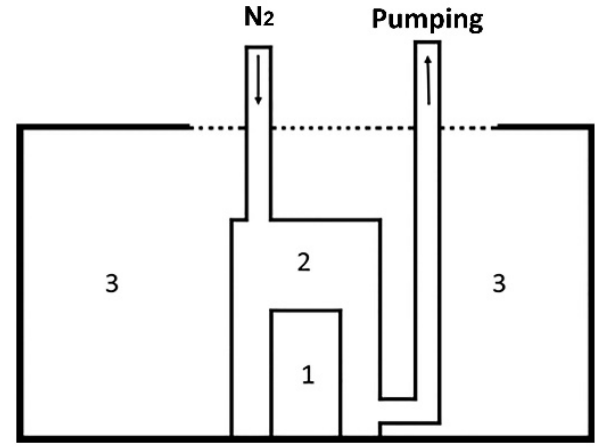

B

Fig. 1. Schemes of experimental installations.

Fig. 1B shows the setup for heating a W-C nanopowder with microwave radiation with a frequency of $2.45 \mathrm{GHz}$. The processing chamber housing is a commercial microwave oven (3 in Fig. 1B). There was a quartz reactor (2 in Fig. 1B) inside the microwave oven which isolates the ceramic crucible (1 in Fig. 1B) with the nanopowder being treated from the external air atmosphere.

The quartz reactor has 2 entrances for nitrogen purge and pumping out. The pressure inside the reactor was maintained slightly excessive relative to the atmosphere to ensure continuous nitrogen purging.

The powders obtained in the experiments were analyzed using following techniques: phase composition using an X-ray diffractometer Rigaku Ultima IV; total carbon content using a LECO CS-600 analyzer; the specific surface of the powders using a Micromeritics TriStar 3000 specific surface analyzer; morphology and particle size of powders using a Scios FEI electron microscope. 


\section{The starting materials}

A nanopowder of the W-C system was used for processing in the microwave field. This nanopowder was obtained by the interaction of tungsten oxide $\mathrm{WO}_{3}$ powder with methane in a stream of hydrogen-nitrogen plasma generated in an electric arc plasma torch [9]. The initial nanopowder consists of the carbide phases $\mathrm{WC}_{1-\mathrm{x}}$ and $\mathrm{W}_{2} \mathrm{C}$ mainly with the presence of metallic W and free carbon. The total carbon content in the nanopowder was $6.3 \mathrm{wt} . \%$. The specific surface area was $22 \mathrm{~m}^{2} / \mathrm{g}$. The nanopowder consists of highly aggregated particles with sizes less than $100 \mathrm{~nm}$ (see Fig. 2). The nanopowder contains particles of micron size range as impurities, consisting of tungsten oxide and metal tungsten, the mass content of such particles was about $10 \%$. Processing of nanopowders was carried out in a nitrogen environment. The experiments high purity $(99,9999 \%)$ compressed nitrogen and evaporated liquid nitrogen was used.

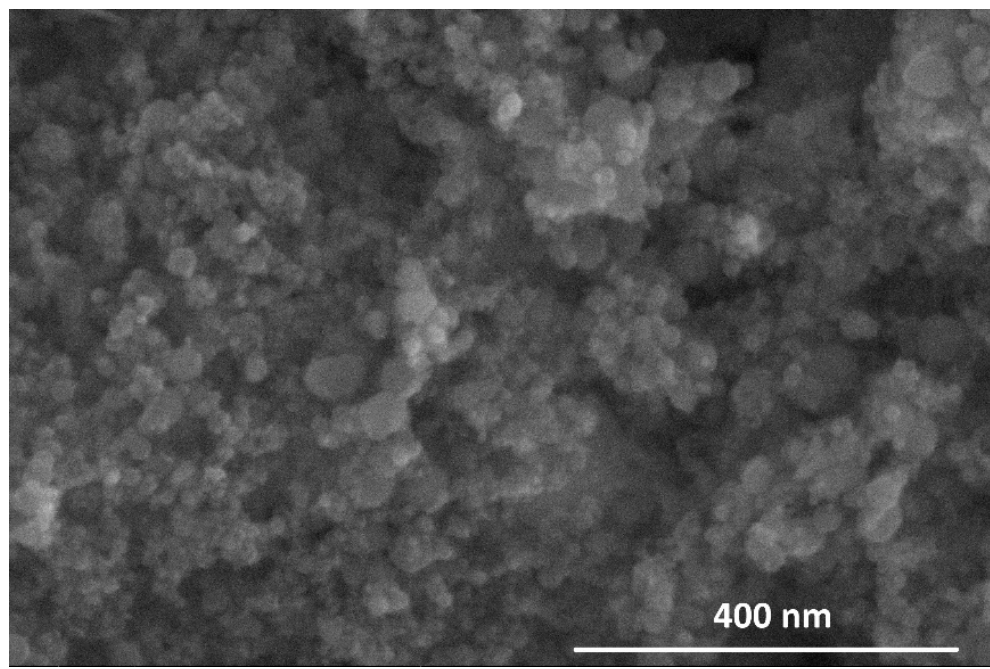

Fig. 2. Microphoto of initial nanopowder.

\section{Results and Discussion}

One of the main factors determining the course of solid-phase chemical reactions during direct heating of reagents in a microwave field is the absorption efficiency of reagents and reaction products, which determine its temperature. Experiments have shown that the original nanopowder absorbs well the used microwave radiation with both frequencies of $2.45 \mathrm{GHz}$ and $24 \mathrm{GHz}$. A sample of the original nanopowder with a mass of 10-12 $\mathrm{g}$ was heated to a temperature of $1100^{\circ} \mathrm{C}$ for exposure time on the order of several seconds. The temperature variation during the process was $50-70^{\circ} \mathrm{C}$ with a significant change in the phase composition of the nanopowder as a result of the transformation of $\mathrm{WC}_{1-\mathrm{x}}->\mathrm{WC}$. This fact indirectly indicates the proximity of the absorption properties of these carbides at the frequencies of microwave radiation used in the experiments.

Results of experiments performed at a constant microwave power of $260 \mathrm{~W}$ with a frequency of $24 \mathrm{GHz}$ shows that chemical transformation of the original nanopowder occur from the first minutes of the microwave processing and is characterized by a decrease of the tungsten carbide $\mathrm{WC}_{1-\mathrm{x}}$ content and an increase of the $\mathrm{WC}$ carbide (see Fig. 3). The highest transformation rate was observed in the first minutes. During the subsequent time (up to 12.5 minutes) the residual content of carbide $\mathrm{WC}_{1-\mathrm{x}}$ practically does not change with a certain 
increase in the content of carbide WC. The maximum processing time was $12.5 \mathrm{~min}$, however, during this time, the complete conversion of the components of the original nanopowder to tungsten carbide WC was not achieved, which may be due to a decrease in the total carbon content in the nanopowder during microwave processing (see Fig. 4). Tungsten carbide WC is a stoichiometric compound and has no homogeneity region; therefore, lower carbides are formed with a lower carbon content with a decrease in the total carbon content in the reaction system. The decrease in total carbon content could be due to its chemical ablation as a result of the interaction of free carbon and tungsten carbides with oxygen impurities present in the nitrogen used to purge the powder treatment chamber. The experiment showed the effect of the nitrogen type used for purging the composition of the nanopowder treated. The parameters were the following: the frequency of microwaves 24 $\mathrm{GHz}$, heating power was $260 \mathrm{~W}$, treating time $5 \mathrm{~min}$. The sample treated in a stream of evaporated liquid nitrogen characterized by a higher conversion of $\mathrm{WC}_{1-\mathrm{x}}$ to $\mathrm{WC}$. The presence of oxygen provides the possibility to transfer carbon required for the formation of $\mathrm{WC}$ from $\mathrm{WC}_{1-\mathrm{x}}$ through the gas phase through the reactions $\mathrm{O}_{2}+\mathrm{Cs}_{\mathrm{S}}-\mathrm{CO}, \mathrm{CO}->\mathrm{Cs}_{\mathrm{S}}+$ $\mathrm{CO}_{2}$.

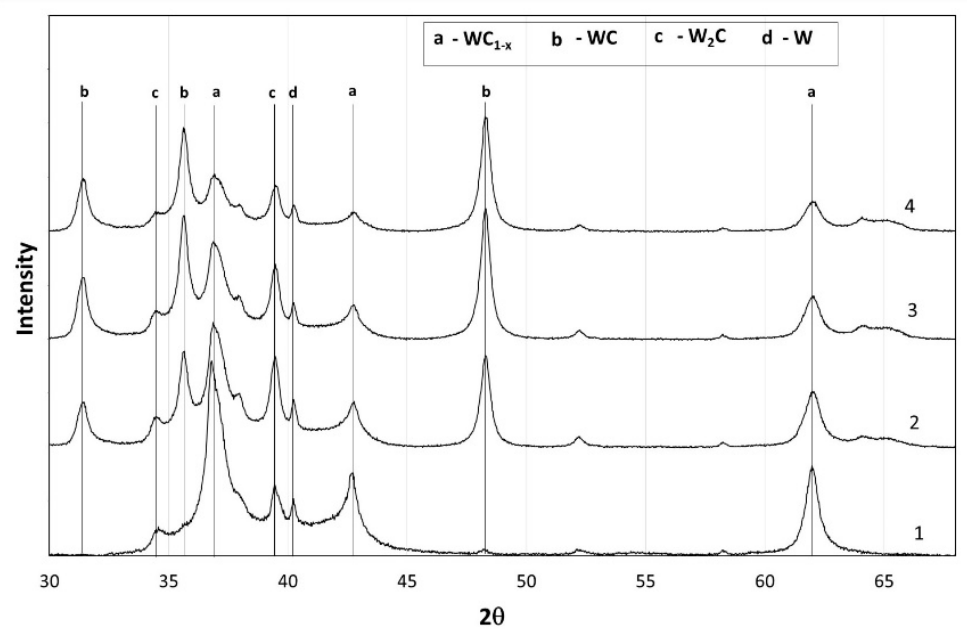

Fig. 3. X-ray diffraction pattern of nanopowders after the microwave treatment $(24 \mathrm{GHz}$, $260 \mathrm{~W}$ ) during the various time. 1 - initial, 2 - 2 min., 3 - 5 min., $4-12.5 \mathrm{~min}$. 


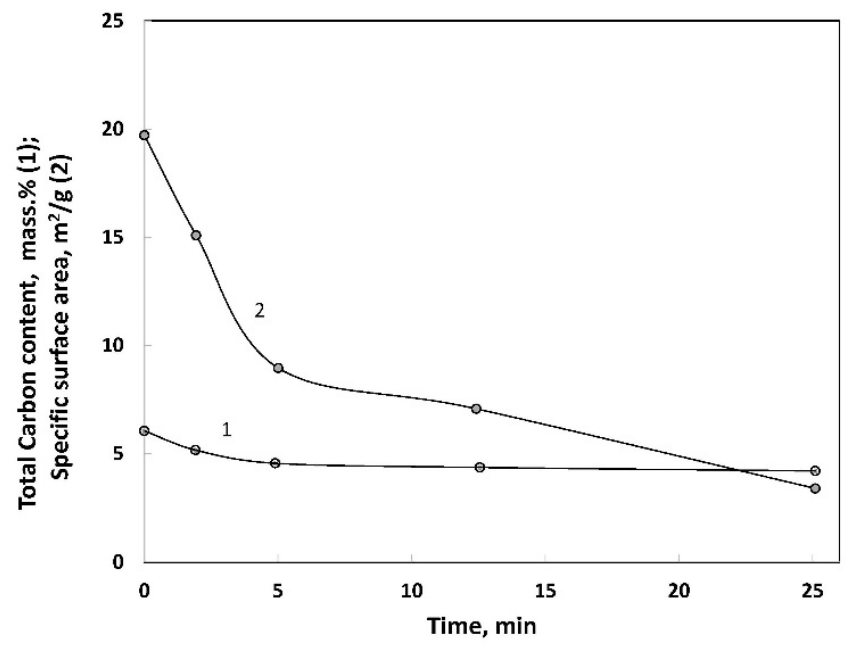

Fig. 4. Change in time for total carbon content and for the specific surface in nanopowders at the microwave treatment $(24 \mathrm{GHz}, 260 \mathrm{~W})$.

The specific surface of the powder being processed decreases when tungsten carbide $\mathrm{WC}$ is formed under microwave heating with a frequency of $24 \mathrm{GHz}$ (see Fig. 4) and the specific surface area decreases from $22 \mathrm{~m}^{2} / \mathrm{g}$ to $12 \mathrm{~m}^{2} / \mathrm{g}$ in $25 \mathrm{~min}$ of processing, while the particle size remains in the corresponding nanoscale range (see Fig. 5A).

An increase in the power of microwaves with a frequency of $24 \mathrm{GHz}$ with a constant processing time of 5 min leads to an increase in the output of tungsten carbide WC (see Fig. $6)$.

The phase composition and the morphology of the obtained products are mostly the same when processing samples in the microwaves with a frequency of $2.45 \mathrm{GHz}$ with a power of $800 \mathrm{~W}$ and when processing samples in the microwaves with a frequency of 24 GHz (see Fig. 5b and Fig. 7). A significant change in the phase composition (transformation of $\mathrm{WC}_{1-\mathrm{x}}->\mathrm{WC}$ ) also occurs during the first minutes, and from 3 to 8 minutes the WC content increases much slowly (see Fig. 7). There is a decrease in the total carbon content and a decrease in the specific surface area of the powder during processing when used microwaves with a frequency of $2.45 \mathrm{GHz}$ likely when used microwaves with a frequency of $24 \mathrm{GHz}$.
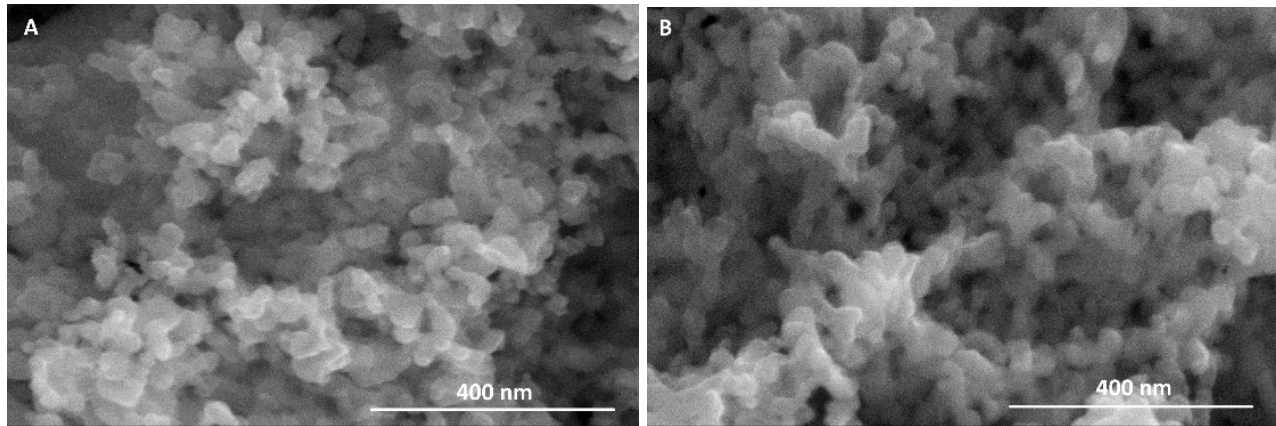

Fig. 5. Microphoto of nanopowders after the microwave treatment. A - $24 \mathrm{GHz}, 370 \mathrm{~W}, 5$ min. $\mathrm{B}-2.45 \mathrm{GHz}, 800 \mathrm{~W}, 5 \mathrm{~min}$. 


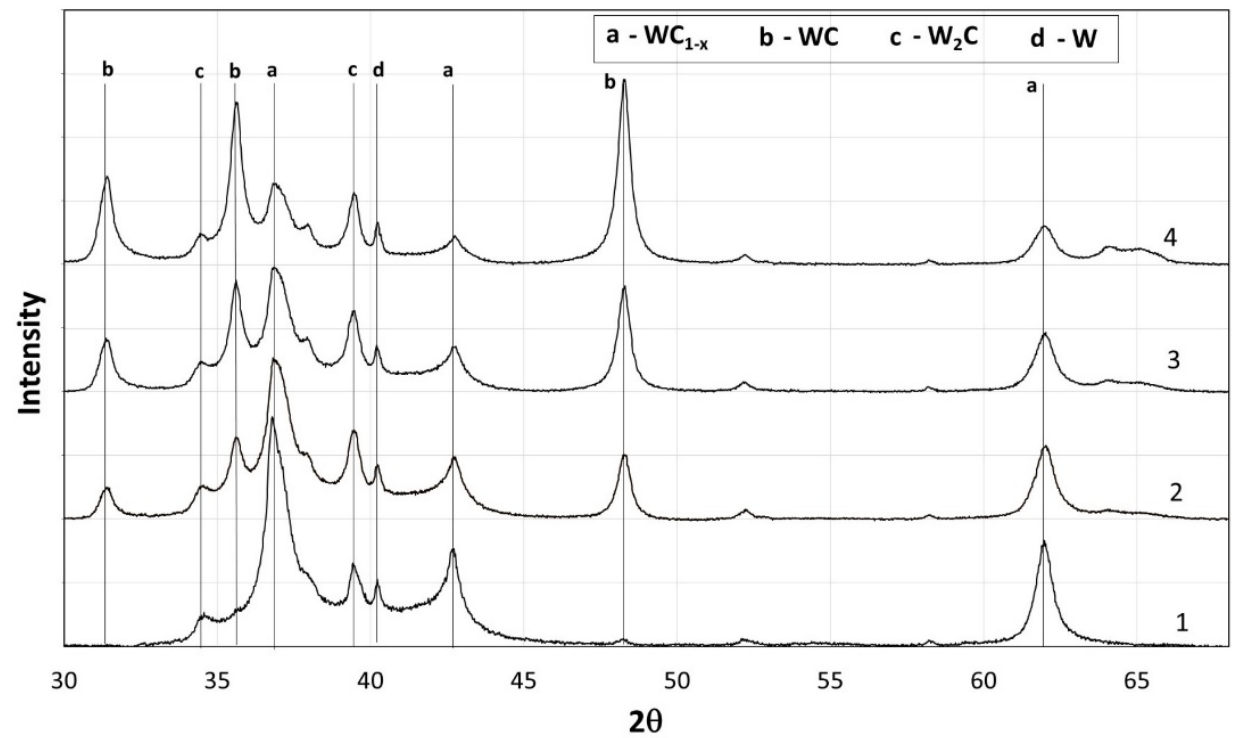

Fig. 6. X-ray diffraction pattern of nanopowders after the microwave treatment $(24 \mathrm{GHz}, 5$ min.) at various power. 1 - initial, $2-260 \mathrm{~W}, 3-310 \mathrm{~W}, 4-370 \mathrm{~W}$

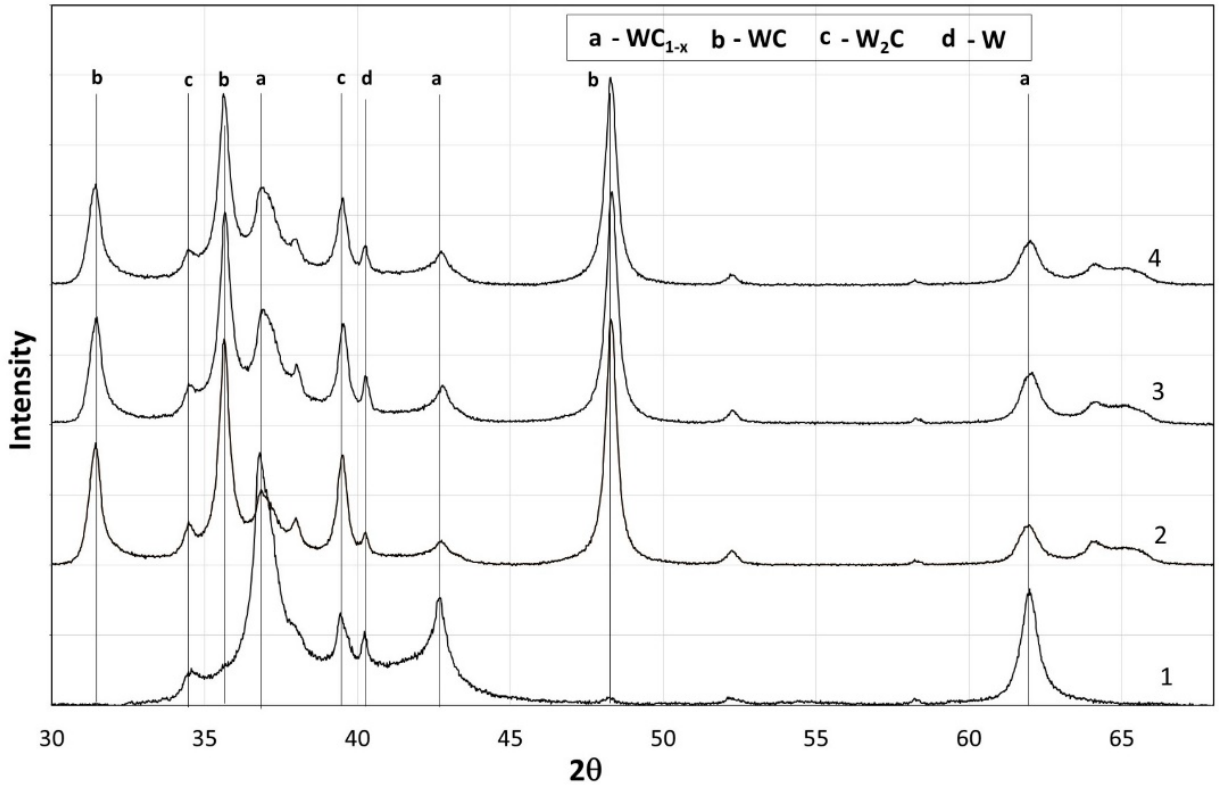

Fig. 7. X-ray diffraction pattern of nanopowders after the microwave treatment $(2.45 \mathrm{GHz}$, $800 \mathrm{~W}$ ) during the various time. 1 - initial, $2-3 \mathrm{~min} ., 3-5 \mathrm{~min} ., 4-8 \mathrm{~min}$.

\section{Conclusion}

Experimental studies performed have demonstrated the possibility of direct heat treatment of multicomponent nanopowders of the W-C system obtained by plasma-chemical synthesis, when exposed to a microwave field with frequencies of 2.45 and $24 \mathrm{GHz}$. The formation of nanoscale particles of tungsten carbide WC occurs when microwaves used for 
processing while there is a decrease in the value of the specific surface area of the nanopowder. The process of chemical and phase transformation is affected by the composition of the gas medium, which components can provide gas-phase carbon transfer.

Subsequent studies will determine the possibility to achieve $100 \%$ yield of WC while maintaining the particle size of the powder in the nanometer range.

The work was carried out within the framework of the Program \#14 "Physical chemistry of adsorption phenomena and actinide nanoparticles" of the Presidium of the Russian Academy of Sciences.

\section{References}

1. Panov V.S. Metal Ceramics, 2015, 53(11-12), 643-654.

2. Z. Zak Fang, Xu Wang, Taegong Ryu, et al.. Int. J. of Refr. Met. and Hard Mater., 2009, 27(2), 288-299.

3. Kurlov A.S., Gusev A.I. Tungsten Carbides. Struct., Prop., and Appl. in Hardmetals, Springer 2013, 242 p.

4. Kalita, V.I., Komlev, D.I., Yashin, V.B., et al., Fiz. Khim. Obrab. Mater. (in rus), 2011, 5, 32-43.

5. Sokolov G.N., Zorin I.V., Artemev A.A., et al.. Inorg. Mater.: Appl. Research, 2015, 6(3), 240-248.

6. Linnik A.A., Pankratov A.S., Kobernik N.V. Izv. Vissh. Ucheb. Zaved. Mashinostroenie (in rus), 2013, 6, 66-71.

7. Nikiforov A., Petrushina I., Christensen E., Alexeev N.V., Samokhin A.V., Bjerrum N.. Int. J. of Hydrogen Energy, 2012, 37 (24), 18591-18597.

8. S. Emina, C. Altinkayab, A. Semercib, H. Okuyucub, A. Yildizb, P. Stefanovic.. Appl. Catalysis B: Environmental, 2018, 236, 147-153.

9. Samokhin A. V., Alekseev N. V., Kornev S. A. Plasma Chem. and Plasma Proc., 2013, 33(3), 605-616.

10. Taegong Ryu, H.Y. Sohn, Kyu Sup Hwang, Zhigang Z. Fang. J. Mater. Sci., 2008, 43, 5185-5192.

11. T. Ryu, H. Y. Sohn, K. S. Hwang, Zh. Z. Fang. J. Am. Ceram. Soc., 2009, 92(3), 655-660.

12. Kitchen H.J., Vallance S.R., Kennedy J.L., et al. Chem. Rev. 2014, 114, 1170-1206.

13. Y. Makino. ISIJ Int., Vol. 47 (2007), No. 4, pp. 539-544.

14. K. J. Rao, B. Vaidhyanathan, M. Ganguli, P. A. Ramakrishnan. Chem. of Mater., 1999, 11 (4), 882-895.

15. Kappe C.O. Am Lab. 2001, 33, 13-19.

16. Dąbrowska S. Chudoba T., Wojnarowicz J .Łojkowski W. A Review. Crystals 2018, 8, 379-405.

17. Leonelli C., Lojkowski W. Chem. Today, 2007, 25 (3), 34-38.

18. Leonelli C., Komarneni S. Inorganics, 2015, 3, 388-391.

19. Imam M. A., Lewis D., Bruce R. W., Fliflet A. W., Kurihara L.K. Mater. Sc. Forum, 2003, 426-432, 4111-4116.

20. K. Essaki, E. J. Rees, G.T. Burstein. J. Am. Ceramic Soc.2009, 93 (3), $692-695$.

21. A. Karimzadeh, B. Arman, H. Masoud, S.Email, M. Sh. Bafghi, K. Yanagisawa. Int. J. of Miner. Metall. and Mater., 2017, 24 (2), 202-207.

22. Nikolaenko I., Kedin N., Shveikin G., Polyakov E. Int. J. of Mater. Res. 2014, 105 (3), 314-317.

23. J. L. Lu, Z. H. Li, S. P. Jiang, et al. J.of Power Sources, 2012, 202, 56- 62.

24. Vallance S.R., Kitchen H.J., Ritter C., et al. Green Chem., 2012, 14, 2184-2192. 
25. Behnami A.K., Hoseinpur A., Sakaki M., et al. Int. J. of Miner. Metall., and Mater., 2017, 24 (2), 202-207.

26. Wu A.-H., Tang J.-C., Ye N., et al. Mater. Sc. and Enginer. Powd. Metall. 2014, 19(6), 862-866.

27. Vallance S.R., Kingman S., and Gregory D.H. Adv. Mater. 2007, 19, 138-142.

28. Bykov Yu.V., Rybakov K.I., Semenov V.E.. J. Phys. D: Appl. Phys., 2001, 34, 5575. 


\section{HIGH EFFICIENCY MICROWAVE FLOW CHEMISTRY TOWARD SYNTHESIS OF FUNCTIONAL MATERIALS AND PHARMACEUTICAL CORES}

Joshua P. Barham $^{1 *}$, Emiko Koyama ${ }^{1}$, Jun-ichi Sugiyama ${ }^{1}$, Yasuo Norikane ${ }^{1}$, Hiromichi Egami $^{2}$, Yoshitaka Hamashima ${ }^{2}$

${ }^{1}$ National Institute of Advanced Industrial Science and Technology, Tsukuba Central 5, 11-1 Higashi, Tsukuba, Ibaraki, 305-8565, Japan

${ }^{2}$ School of Pharmaceutical Sciences, University of Shizuoka, 52-1, Yada, Suruga-ku, Shizuoka, Shizuoka, 422-8526, Japan

*E-mail: j.barham@aist.go.jp; j.barham@saidagroup.jp

Keywords: microwave, flow, single-mode, radio-frequency heating, solid state microwave

Microwave (MW) heating benefits organic synthesis by affording higher product yields in shorter time periods than conventional heating $(\mathrm{CH})$, yet it suffers from poor scalability and is limited to polar solvents in typical batch mode reactors. ${ }^{1,2}$ An auto-frequency tunable microwave (AFT MW) continuous flow (CF) reactor has been developed and commercialized by SAIDA FDS Inc. and Pacific Microwave Technologies (PMT). ${ }^{3}$ ATF MW compensates for changes in the microwave absorption properties (permittivity, $\square$ ) with increasing temperature, ${ }^{1,2,4}$ affording excellent temperature and process control. Moreover, it is applicable to heating of non-polar solvents. Organic synthesis using an ATF MW CF reactor is a rapidly developing research field in Japan and beyond. ${ }^{5,6,7}$ Herein, we report recent applications of a microwave flow reactor using a solid-state semiconductor $\mathrm{MW}$ generator toward high efficiency synthesis of functional materials and pharmaceutical cores. Attention is drawn to examples where AFT MW heating has been compared to $\mathrm{CH}$ in flow, and where its application has expediated reaction optimization.

\section{CF synthesis comparing AFT MW heating with CH}

\subsection{Heterogeneous reactions}

Sajiki and co-workers at Gifu Pharmaceutical University disclosed a dehydrogenative aromatization under heterogeneous Pt catalysis using the AFT MW CF reactor (Fig. 1). ${ }^{8}$ When methylcyclohexane (MCH, $220 \mathrm{~mL})$ was passed through a straight-tube reactor filled with Pt catalyst mounted on carbon beads and heated by AFT MW (10 W applied power) at $0.5 \mathrm{~mL} / \mathrm{min}$ for $12 \mathrm{~h}$ of recirculation, $34 \mathrm{~L}$ of pure $\mathrm{H}_{2}$ gas was collected. This represented an energy-efficient method for $\mathrm{H}_{2}$ production, because the reflected MW power was only $1 \mathrm{~W}$ (90\% heating efficiency) with a catalyst TON of ca. 68,000 . The space-time yield of this system is ca. $1000 \mathrm{~mol} \mathrm{~L}_{\mathrm{cat}}^{-1} \mathrm{~h}^{-1}$, far superior to the rivalling organic chemical hydride (K$\mathrm{Pt} / \mathrm{Al}_{2} \mathrm{O}_{3}$-catalyzed) technology at $46 \mathrm{~mol} \mathrm{~L}_{\text {cat }}{ }^{-1} \mathrm{~h}^{-1} .{ }^{8}$ This process has great promises for $\mathrm{H}_{2}$ storage and transfer hydrogenation applications, because hydrogenation of aromatic rings (the reverse reaction) can be readily achieved under Pd catalysis. Therefore, it is an example of a functional material synthesis. The results of AFT MW heating (catalyst $\mathrm{T}=248^{\circ} \mathrm{C}$ ) vs. $\mathrm{CH}$ (oil bath $\mathrm{T}=248{ }^{\circ} \mathrm{C}$ ) were compared after $30 \mathrm{~min}$ of $\mathrm{CF}$. The former conditions gave ca. $2.0 \mathrm{~L}$ of $\mathrm{H}_{2}$ (ca. $25 \%$ yield) while the latter conditions gave ca. $0.2 \mathrm{~L}$ of $\mathrm{H}_{2}$ (ca. $2 \%$ yield). Thermography revealed that this is due to selective heating of the Carbon Bead support of the Pt catalyst by AFT MW, compared to unselective heating of the whole reactor by $\mathrm{CH}$. 

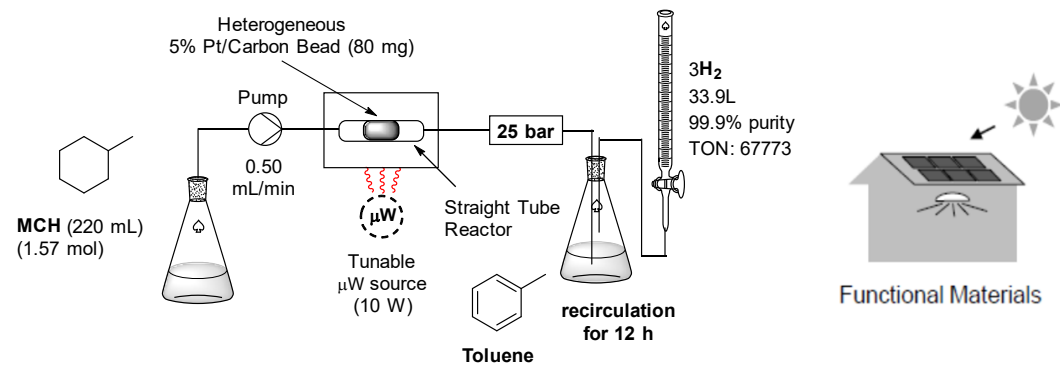

Fig. 1. Dehydrogenative aromatization of methylcyclohexane catalyzed by heterogeneous Pt/Carbon Bead disclosed by Sajiki and co-workers. ${ }^{8}$
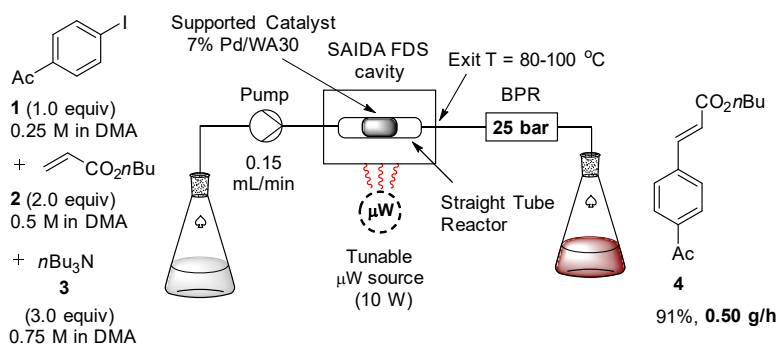

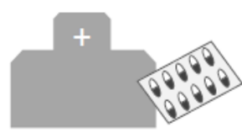

Pharmaceuticals

Fig. 2. MW CF Heck reaction under heterogeneous Pd-supported catalysis.

Sajiki and co-workers reported a heterogeneous Mizuroki-Heck reaction using supported Pd catalysis in a straight tube within the AFT MW CF reactor. ${ }^{9}$ In one example, product 4 was afforded in up to $91 \%$ yield, $0.5 \mathrm{~g} / \mathrm{h}$. When the aryl iodide substrate 1 was changed, different exit temperatures were observed under the same applied MW power. This observation implicated the selective MW heating of reagents in the presence of solvent. Accordingly, a slight improvement in yield was observed when the reaction was performed using AFT MW heating $(92 \% 4,4 \% 1)$ vs. $\mathrm{CH}(87 \% 4,8 \% 1)$ under the same $\mathrm{CF}$ conditions. ${ }^{9}$ The MizorokiHeck reaction furnishes multi-substituted alkenes as ubiquitous intermediates in organic chemistry and in the pharmaceutical industry. ${ }^{10}$

\subsection{Homogeneous reactions}

At AIST, we applied AFT MW heating to the CF synthesis of $\mathrm{IC}_{60} \mathrm{MA}$ and $\mathrm{IC}_{60} \mathrm{BA}$ via a mismatched (electron-rich + electron-rich) Diels-Alder-type conjugation reaction between $\mathrm{C}_{60}$ and indene. ${ }^{5}$ The synergy of AFT MW heating and $\mathrm{CF}$ was realized in the ability to synthesize $\mathrm{IC}_{60} \mathrm{MA}$ and $\mathrm{IC}_{60} \mathrm{BA}$ in productivities as high as $0.7 \mathrm{~g} / \mathrm{h}(47 \%$ yield $)$ and $0.5 \mathrm{~g} / \mathrm{h}$ ( $32 \%$ yield), respectively. This is important because $\mathrm{IC}_{60} \mathrm{MA}$ and $\mathrm{IC}_{60} \mathrm{BA}$ have been demonstrated as superior electron acceptors with enhanced solubility vs. phenyl-C61-butyric acid methyl ester (PCBM) as the benchmark electron acceptor in polymer solar cells. ${ }^{11}$ Moreover, non-polar solvent $o$-xylene could be heated to $270^{\circ} \mathrm{C}$ which is next to impossible in other (non-AFT) MW reactors. The use of $o$-xylene in place of high-boiling polychlorinated aromatic solvents improves the cost-efficiency and green chemistry of these reactions. ${ }^{5}$ At room temperature, the MW absorption property, $\tan \square$, of the reaction mixture (o-Xylene + indene $+\mathrm{C}_{60}$ ) was very similar to that of the solvent only (o-Xylene) (Table 1$)$, indicating there should be no selective reagent heating. Indeed, under the same conditions of $\mathrm{R}_{\mathrm{T}}$ and exit temperature, the CF reaction gave very similar results for AFT MW heating 
vs. $\mathrm{CH}$ (Table 2). Here, the $\mathrm{CH}$ reactor was a ceramic furnace fitted with thermocouples (Fig. 4, left). External thermocouples 1-4 are fitted to the reactor tube surface and external thermocouple 5 is fitted to the inner furnace wall. 'Saida' is the internal thermocouple measuring the exit temperature of the flowing solution inside; its readings corresponded well with thermocouple 1 (Fig. 4, right). External thermocouple 6 (not shown) is fitted to the end of the cooling loop prior to the BPR.

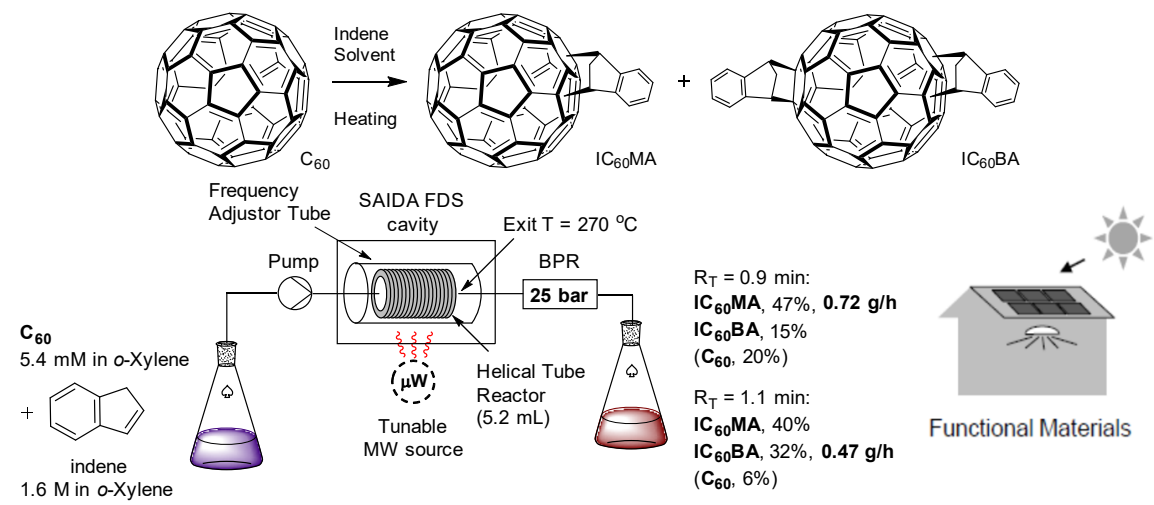

Fig. 3. Top: $\mathrm{C}_{60} /$ indene conjugation reaction scheme with structures of $\mathrm{IC}_{60} \mathrm{MA}$ and $\mathrm{IC}_{60} \mathrm{BA}$. Bottom: MW $\mathrm{CF} \mathrm{C}_{60}$ /indene conjugation reaction.

Table 1. Permittivity Data for $\mathrm{C}_{60} /$ indene conjugation reaction components

\begin{tabular}{cccc}
\hline Entry & Sample & $\begin{array}{c}\square{ }^{\prime}-\mathrm{j} \square{ }_{\mathrm{r}}{ }^{\prime \prime}(2.45 \\
\mathrm{GHz})\end{array}$ & $\tan \square$ \\
\hline 1 & $o$-Xylene & $2.70-\mathrm{j} 0.08$ & 0.030 \\
2 & $o$-Xylene $+\mathrm{C}_{60}$ & $2.72-\mathrm{j} 0.09$ & 0.033 \\
3 & $o-X y l e n e+$ indene & $2.84-\mathrm{j} 0.11$ & 0.038 \\
4 & $o$-Xylene $+\mathrm{C}_{60}+$ indene & $2.84-\mathrm{j} 0.11$ & 0.039 \\
\hline
\end{tabular}

All samples were recorded at $\mathrm{rt}$ at $2.45 \mathrm{GHz} . \mathrm{H}_{2} \mathrm{O}$ was employed as a standard.

Table 2. Effect of AFT MW heating vs. $\mathrm{CH}$ on yields of $\mathrm{IC}_{60} \mathrm{MA}$ and $\mathrm{IC}_{60} \mathrm{BA}$ from the $\mathrm{CF}$ reaction of $\mathrm{C}_{60}$ and indene.

\begin{tabular}{cccccc}
\hline Entry & $\mathrm{T}^{\mathrm{a}}\left({ }^{\circ} \mathrm{C}\right)$ & Heating Type & \multicolumn{3}{c}{ Yields $^{\mathrm{b}}$} \\
\cline { 4 - 6 } & & & $\mathrm{C}_{60}$ & $\mathrm{IC}_{60} \mathrm{MA}$ & $\mathrm{IC}_{60} \mathrm{BA}$ \\
\hline 1 & 260 & $220 \mathrm{~W} \mathrm{MW}$ & 26 & 46 & 11 \\
2 & 260 & N/A $(\mathrm{CH})$ & 25 & $41[42]$ & 9 \\
3 & 270 & $220 \mathrm{~W} \mathrm{MW}$ & 20 & 47 & 15 \\
4 & 270 & N/A $(\mathrm{CH})$ & 21 & $46[46]$ & 12 \\
\hline
\end{tabular}

$\mathrm{T}=$ temperature. $\mathrm{R}_{\mathrm{T}}=$ residence time. All reactions used a $0.90 \min \mathrm{R}_{\mathrm{T}}, 5.4 \mathrm{mM} \mathrm{C} 60,1.6 \mathrm{M}$

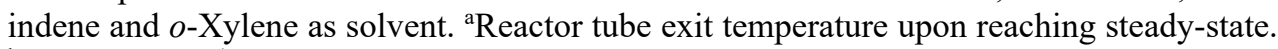
${ }^{\mathrm{b}} \mathrm{HPLC}$ yields. ${ }^{1} \mathrm{H}$ NMR yields in parenthesis. 
Amides are one of the most important moeities in organic chemistry and in pharmaceuticals, but amide bond formations typically require inefficient or hazardous coupling reagents. ${ }^{12}$ Novel methods for construction of functionalization of amides are desirable to researchers and industry. At the University of Shizuoka, we applied AFT MW CF reactor to a basecatalyzed C-alkylation reaction of NMP with styrene (Fig. 5). ${ }^{13}$ AFT MW heating in CF allowed $180^{\circ} \mathrm{C}$ to be achieved at $\mathrm{R}_{\mathrm{T}}=0.4 \mathrm{~min}$ for a given reaction mixture $(0.44 \mathrm{M}$ styrene, $0.26 \mathrm{M} \mathrm{NaO} t \mathrm{Bu}$ and $0.26 \mathrm{M} 18$-crown-6 in NMP), resulting in a productivity of $65 \mathrm{~g} / \mathrm{h}$ as an upper limit. At lower $\mathrm{R}_{\mathrm{T}}$, the maximum applied MW power $(200 \mathrm{~W})$ is insufficient to reach $180{ }^{\circ} \mathrm{C}$. The MW absorption property $\tan \square$ of the reaction mixture (NMP $+\mathrm{NaO} t \mathrm{Bu}$ +18 -crown-6 + styrene) vs. solvent only (NMP) were very

similar at room temperature (Table 3 ), indicating there should be no selective reagent heating. In this case, however, under the same conditions of $\mathrm{R}_{\mathrm{T}}$ and exit temperature, the reaction gave superior results for the AFT MW heating vs. $\mathrm{CH}$ in $\mathrm{CF}$ (Table 4).
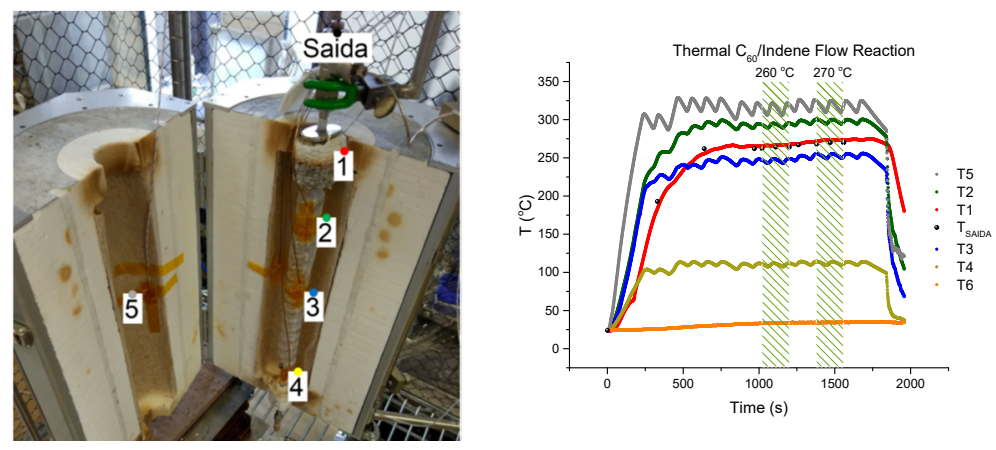

Fig. 4. Left: Ceramic furnace reactor fitted with external thermocouples 1-5 and internal thermocouple 'Saida'. Right: Temperature data for $\mathrm{CH}$ experiments in Table 2.

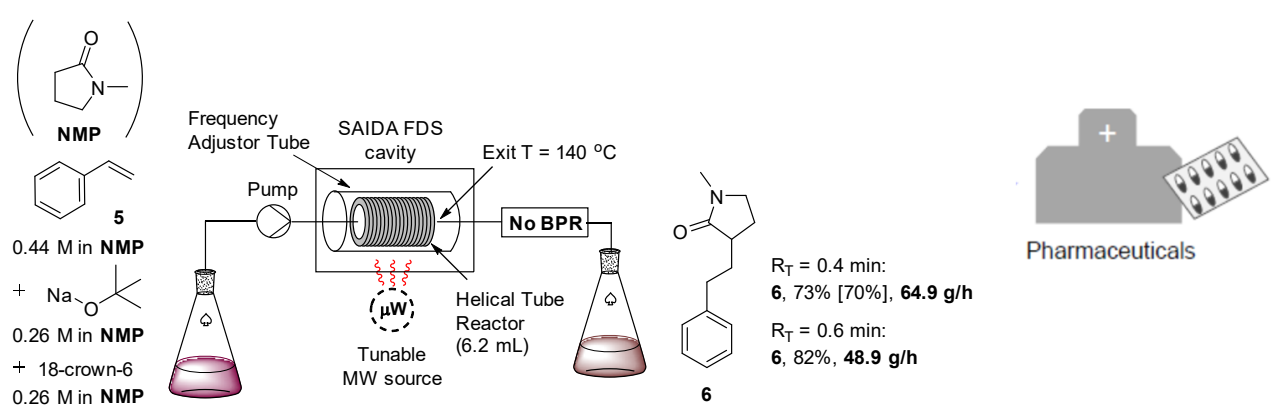

Fig. 5. Top: $\mathrm{C}_{60} /$ indene conjugation reaction scheme with structures of $\mathrm{IC}_{60} \mathrm{MA}$ and IC 60 BA. Bottom: MW CF $\mathrm{C}_{60} /$ indene conjugation reaction. 
Table 3. MW CF base-catalyzed C-alkylation reaction of NMP with styrene.

\begin{tabular}{cccc}
\hline Entry & Sample & $\begin{array}{c}\square{ }^{\prime}-\mathrm{j} \square \mathrm{r} \text { ' }(2.45 \\
\mathrm{GHz})\end{array}$ & $\tan \square$ \\
\hline 1 & $\mathrm{NMP}$ & $14.15-\mathrm{j} 12.56$ & 0.888 \\
2 & $\mathrm{NMP}+\mathrm{NaO} t \mathrm{Bu}$ & $13.56-\mathrm{j} 11.42$ & 0.842 \\
3 & $\mathrm{NMP}+\mathrm{NaO} t \mathrm{Bu}+18$-crown-6 & $11.60-\mathrm{j} 9.72$ & 0.838 \\
4 & $\mathrm{NMP}+\mathrm{NaO} t \mathrm{Bu}+18$-crown-6 + styrene & $12.08-\mathrm{j} 9.92$ & 0.821 \\
5 & $\mathrm{NMP}+18$-crown-6 & $12.89-\mathrm{j} 11.29$ & 0.876 \\
6 & $\mathrm{NMP}+$ styrene & $13.49-\mathrm{j} 11.37$ & 0.843 \\
7 & $\mathrm{NMP}+\mathrm{NaO} t$ Bu + styrene & $12.36-\mathrm{j} 10.48$ & 0.848 \\
8 & $\mathrm{NMP}+18$-crown-6 + styrene & $13.15-\mathrm{j} 10.79$ & 0.821 \\
\hline
\end{tabular}

All samples were recorded at $\mathrm{rt}$ at $2.45 \mathrm{GHz} . \mathrm{H}_{2} \mathrm{O}$ was employed as a standard.

In the case of MW, thermography was used to measure the reactor surface temperature gradient from bottom to top of the reactor tube (locations 1-16, Fig. 5, left, center) with calibration as previously described. ${ }^{6}$ The surface temperature increased in a non-linear fashion and the surface temperature of locations near the reactor exit were in agreement with the internal exit temperature (Fig. 5, right). Together with the dielectric constant of the Duran pipe $\left(\square \mathrm{r}^{\prime}=4.6,1 \mathrm{MHz}, 25^{\circ} \mathrm{C}\right)^{6}$ being much lower than $\mathbf{N M P}\left(\square{ }_{\mathrm{r}}{ }^{\prime}=14.2,2.45 \mathrm{GHz}, 25^{\circ} \mathrm{C}\right)$, this indicates the surface temperature is similar to the internal temperature and confirms the direct nature of radiative heating of the reaction mixture by $\mathrm{MW}$. $\mathrm{CH}$ requires much higher surface temperatures (locations 5,15, Fig. 5, right) to deliver the same internal exit temperature. The high concentration and high-polarity solvent (NMP) yields more effective heat transfer by MW to the fast-flowing reaction mixture over the same given residence time than that of $\mathrm{CH}$. This 'thermal' $\mathrm{MW}$ effect is less pronounced for the $\mathrm{C}_{60}$ /indene conjugation reaction, where heating of the Duran reactor tube and $o$-Xylene occurs in competition (such an effect has been previously reported for Duran and CPME). ${ }^{6}$ Dielectric properties may change at elevated temperatures, so a 'non-thermal' microwave effect cannot be exclusively ruled out.

Table 4. Effect of AFT MW heating vs. thermal heating on yields of monoadduct 6 from the $\mathrm{CF}$ base-catalyzed $\mathrm{C}$-alkylation reaction of NMP with styrene.

\begin{tabular}{cccc}
\hline Entry & $\mathrm{T}^{\mathrm{a}}\left({ }^{\circ} \mathrm{C}\right)$ & MW power $(\mathrm{W})$ & ${\text { Yield } \mathbf{6}^{\mathrm{b}}}^{\mathrm{b}}$ \\
& & & \\
\hline 1 & 140 & $0-200^{\mathrm{c}}$ & $30^{\mathrm{d}}$ \\
2 & 140 & N/A (thermal) & $14^{\mathrm{e}}$ \\
\hline
\end{tabular}

$\mathrm{T}=$ temperature. $\mathrm{R}_{\mathrm{T}}=$ residence time. All reactions used a $0.55 \mathrm{~min} \mathrm{R}_{\mathrm{T}}, 0.44 \mathrm{mM}$ styrene,

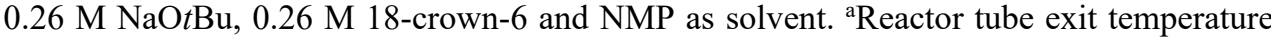
upon reaching steady-state. ${ }^{\mathrm{b}} \mathrm{NMR}$ yields. ${ }^{\mathrm{c}} \mathrm{A}$ temperature control algorithm was employed which varied the applied MW power until desired steady-state temperature was reached. ${ }^{\mathrm{d}}$ Average of 2 replicates. ${ }^{\mathrm{e}}$ Average of 3 replicates. 

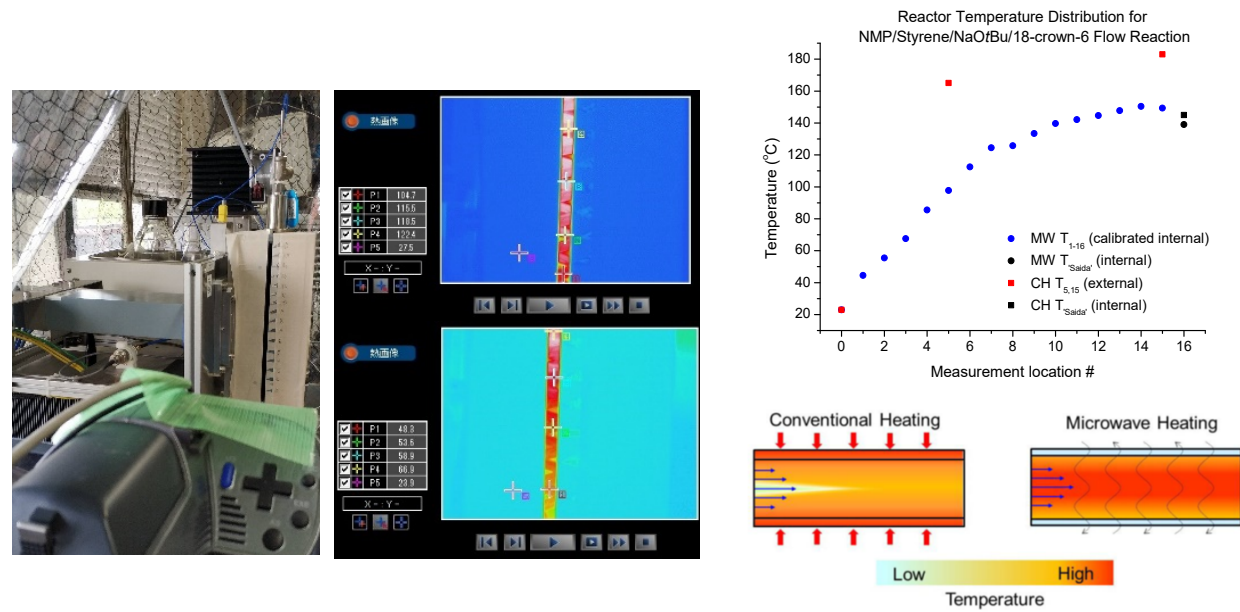

Fig. 5. Left: Thermography apparatus showing measurement locations $1-16$. Location 0 at the assumed to be $\mathrm{rt}\left(23^{\circ} \mathrm{C}\right)$. Center: Thermography picture and data (uncalibrated, locations 1,3,5,7 and 8,10,12,14 are shown from bottom to top). Right: Reactor temperature distribution for $\mathrm{NMP} / \mathrm{Styrene} / \mathrm{NaO} t \mathrm{Bu} / 18$-crown- $6 \mathrm{CF}$ reaction and illustration of inverted thermal gradient for MW $\mathrm{CF}$ vs. $\mathrm{CH} \mathrm{CF}{ }^{7}$

\section{Applications of AFT MW heating toward rapid optimization studies}

Mase and co-workers at Shizuoka University reported a kilo-scale alcohol acetylation using the AFT MW CF reactor (up to ca. $9.0 \mathrm{~kg}$ /day of ester 9, Fig. 6) ${ }^{14}$ In order to achieve the optimal conditions, they fitted the MW CF reactor with a flowIR cell, monitored the yield in-line and fed the data to a 9+4+1 optimization method (Fig. 7). They identified the upper and lower limits of MW applied power and flow rate as variables and yield as the response. They divided the range of each variables by 3 to arrive at 9 experimental runs (black spots). The point which gave the best yield was divided into four and four further experiments performed (grey spots). A final experiment was performed according to the expected optimum condition (white spot). In DoE analysis, factorial designs are generally more efficient and reliable compared to OFAT methods or the $9+4+1$ method. ${ }^{15}$ At AIST, we utilized a two-factorial face-centred central composite design (CCD) for the optimization of the $\mathrm{C}_{60}$ /indene conjugation (Fig. 8). ${ }^{5}$ The 8 experiments for the cube design combined with the 6 experiments for the star design and several $(5 \mathrm{x})$ central points were used to build the $\mathrm{CCD}$, which imparted a deep understanding of the relative importance of temperature, [indene] and $\mathrm{R}_{\mathrm{T}}$ on the $\mathrm{C}_{60}$ conversion ( $\mathrm{R}_{\mathrm{T}}$ was most important).

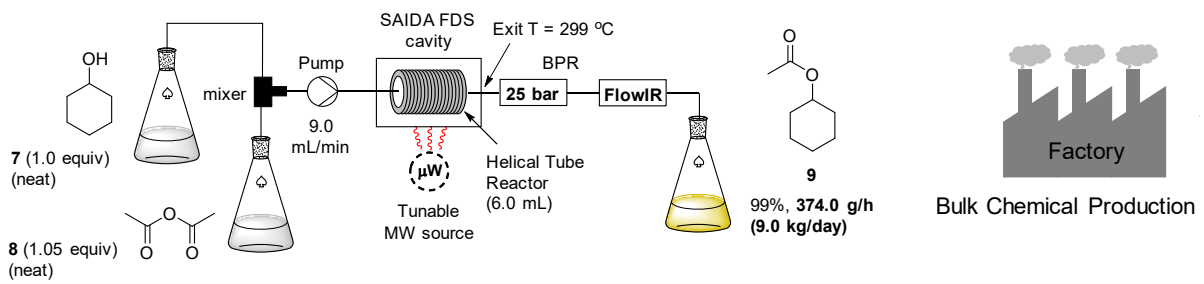

Fig. 6. MW CF acetylation of cyclohexanol with acetic anhydride. 


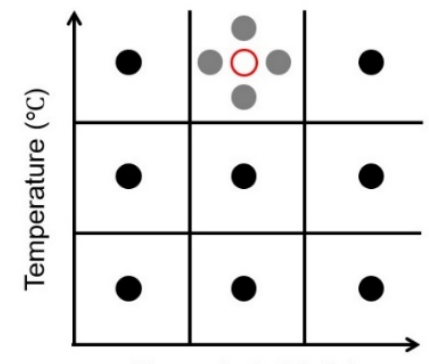

Flow rate $(\mathrm{mL} / \mathrm{min})$

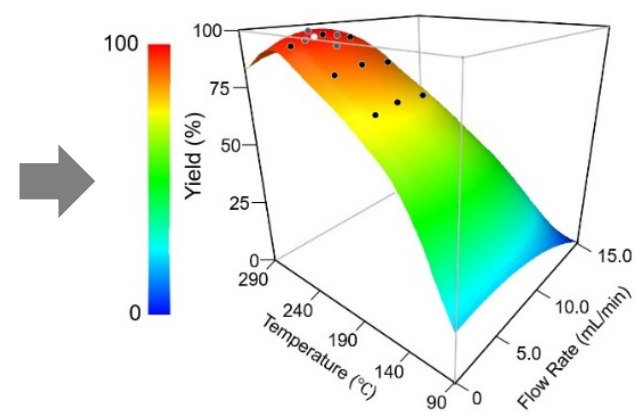

Fig. 7. 9+4+1 OFAT method construction and application to an alcohol acetylation reaction. Note: data differs from the reported study and is shown for illustration.
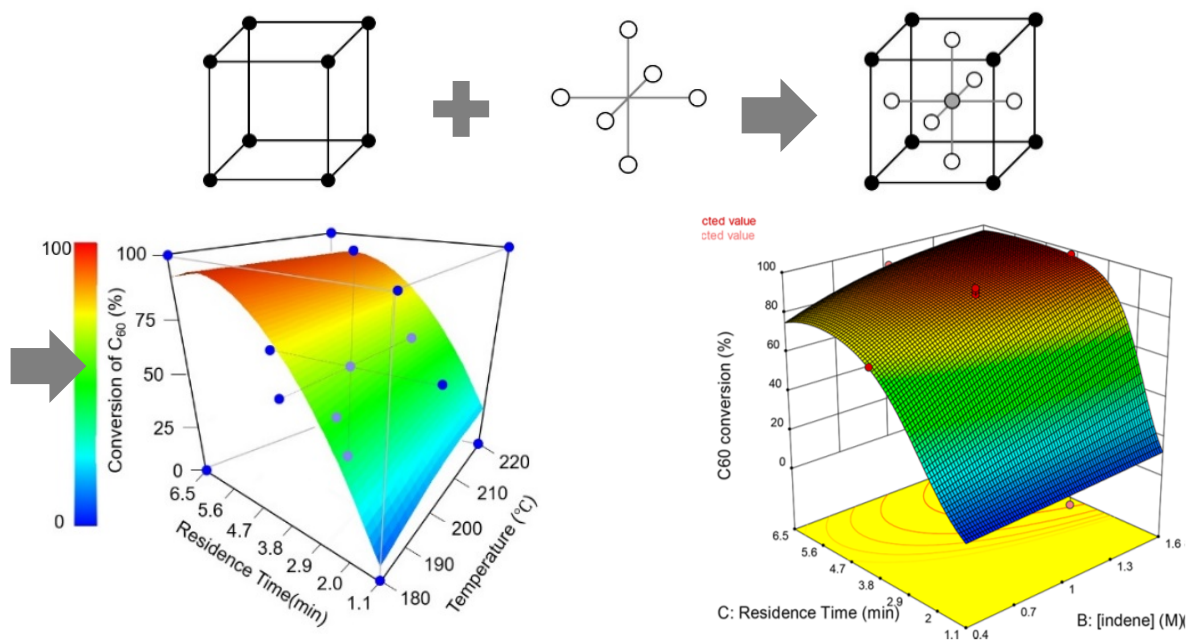

Fig. 8. Top: Construction of a two-factorial face-centred central composite design (CCD) from a two-level full factorial cubic design and a face-centred star design. Bottom (left): Illustrative application. Bottom (right): Application to $\mathrm{C}_{60} /$ indene conjugation reaction.

The solid state oscillator's ability to continuously detect and match the temperaturedependent microwave absorption resonant frequency allowed rapid and controlled changes in $\mathrm{T}$ which culminated in rapid data acquisition (on average, $<5 \mathrm{~min}$ intervals between runs of different temperatures). In contrast to OFAT methods, the factorial nature of the CCD allows it to reveal multifactor interactions, although such interactions were not significant in the $\mathrm{C}_{60}$ /indene conjugation reaction. The successful construction of the $\mathrm{CCD}$ requires the results of reactions under 'extreme' conditions at the edges of the design space and is in itself a testament to the robustness of AFT MW CF technology. Elsewhere, AFT-MW CF technology has been leveraged for the OFAT-type optimization of a Johnson-Claisen rearrangement ${ }^{16}$ and the synergy of AFT MW CF with software programs that automatically vary the flow rate and MW power has been employed to rapidly optimize an ortho-Claisen rearrangement reaction of allyl 1-napthyl ether. ${ }^{6}$ 


\section{Summary}

AFT MW applicators allow better temperature control and energy efficiency to CF reactions than other (non-AFT) MW applicators and they can successfully heat non-polar solvents such as $o$-Xylene and CPME. The reaction temperature can be rapidly changed which expediates reaction optimization and allows conservation of reaction mixture. The combination of AFT MW heating and CF has enabled high efficiency synthesis of functional materials and pharmaceutical cores.

\section{Acknowledgements}

The authors gratefully acknowledge the technical support from the National Institute of Advanced Industrial Science and Technology and the University of Shizuoka, as well as financial support from NEDO, Shizuoka Prefectural Government. Joshua P. Barham is grateful for financial support from JSPS (KAKENHI Grant Number JP18F19030). The authors are grateful for Shizuoka University (Prof. Noboyuki Mase's group) and Gifu Pharmaceutical University (Prof. Hironao Sajiki's group) for their research in this area.

\section{References}

1. Stuerga, D. in Microwaves in Organic Synthesis, $2^{\text {nd }}$ Ed., Wiley-VCH, 2006, pp 929.

2. Grant, E., Halstead, B. J. Chem. Soc. Rev. 1998, 27, 213-224.

3. a) Saida, H., Odajima, H., Ohneda, S., Yokozawa, S. (SAIDA FDS Inc.) 2012, WO/2012/043753; b) saidagroup.jp/fds; c) pacificmicrowave.com

4. Chandrasekaran, S., Ramanathan, S., Basak, T. AIChE J. 2012, 58, 330-363.

5. Barham, J. P., Tanaka, S., Koyama, E., Ohneda, N., Okamoto, T., Odajima, H., Sugiyama, J., Norikane, Y. J. Org. Chem., 2018, 83, 4348-4354.

6. Koyama, E., Ito, N., Sugiyama, J., Barham, J. P., Norikane, Y., Azumi, R., Ohneda, N., Ohno, Y., Yoshimura, T., Odajima, H., Okamoto, T. J. Flow Chem., 2018, 8, 147-156.

7. Barham, J. P., Koyama, E., Norikane, Y., Ohneda, N., Yoshimura, T. Chem. Rec., 2019, 19, 188-203.

8. Ichikawa, T., Matsuo, T., Tachikawa, T., Yamada, T., Yoshimura, T., Yoshimura, M., Takagi, Y., Sawama, Y., Sugiyama, J., Monguchi, Y., Sajiki, H. ACS Sustainable Chem. Eng. 2019, 7, 3052-3061.

9. Ichikawa, T., Mizuno, M., Ueda, S., Ohneda, N., Odajima, H., Sawama, Y., Monguchi, Y., Sajiki, H. Tetrahedron Lett. 2018, 74, 1801-1816.

10. Budarin, V. L., Shuttleworth, P. S., Clark, J. H., Luque, R. Curr. Org. Synth. 2010 , 7, 614-627.

11. He, Y., Chen, H.-Y., Hou, J., Li, Y., J. Am. Chem. Soc. 2010, 132, 1377-1382.

12. Sabatini, M. T., Boulton, L. T., Sheppard, T. D. Sci. Adv. 2017, 3, e1701028.

13. Barham, J. P., Tamaoki, S., Egami, H., Ohneda, N., Okamoto, T., Odajima, H., Hamashima, Y. Org. Biomol. Chem., 2018, 16, 7568-7573.

14. Vámosi, P., Matsuo, K., Masuda, T., Sato, K., Narumi, T., Takeda, K., Mase, N. Chem. Rec., 2019, 19, 77-84.

15. Weissman, S. A., Anderson, N. G. Org. Process Res. Dev. 2015, 19, 1605-1633.

16. Egami, H., Tamaoki, S., Abe, M., Ohneda, N., Yoshimura, T., Okamoto, T., Odajima, H., Mase, N., Takeda, K., Hamashima, Y. Org. Process Res. Dev. 2018, 22, 1605-1633. 


\section{About the authors}
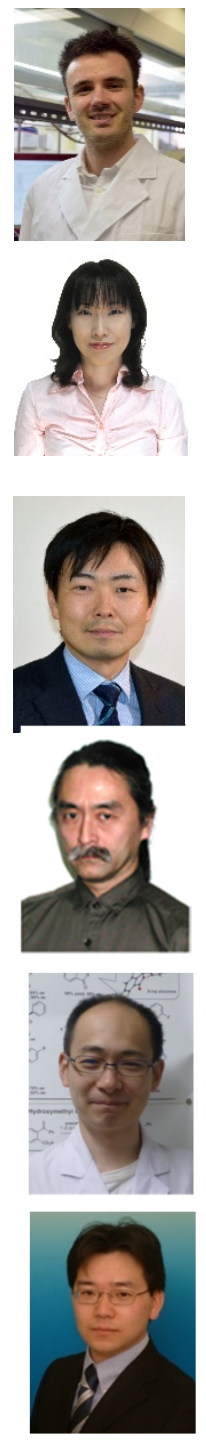

Joshua P. Barham, JSPS Research Fellow at AIST: Expert in photochemical and microwave flow chemistry in organic synthesis. Ph.D in Chemistry from the University of Strathclyde/GlaxoSmithKline Collaborative Ph.D Programme. Previously with SAIDA FDS Inc./University of Shizuoka as a NEDO Japan Trust Research Fellow.

Emiko Koyama, Researcher at AIST: Expert in microwave flow chemistry in organic synthesis. Ph.D in Engineering from Tokyo Institute of Technology. Previously with University of Tsukuba as an Assistant Professor.

Yasuo Norikane, Group Leader at AIST and Associate Professor at the University of Tsukuba: Expert in photochemistry in organic synthesis. Ph.D in Science from the University of Tsukuba. Previously with Scripps as a Postdoctoral Research Fellow.

Jun-ichi Sugiyama, Researcher at AIST: Expert in microwave heating theory and chemistry. Ph.D in Engineering from Tokyo Institute of Technology. Previously with Yamagata University as an Assistant Professor.

Hiromichi Egami, Associate Professor at University of Shizuoka: Expert in microwave flow chemistry in organic synthesis. Ph.D in Science from Kyushu University. Previously with the ERATO Sodeoka Live Cell Chemistry Project as a Postdoctoral Research Fellow and Group Leader.

Yoshitaka Hamashima, Full Professor at University of Shizuoka: Expert in microwave flow chemistry in organic synthesis. Ph.D in Pharmacy from the University of Tokyo. Previously with Tohoku University as an Assistant Professor and RIKEN as a Senior Researcher. 


\title{
MICROWAVE-ASSISTED PREPARATION OF 1D NCs FOR PHOTODEGRADATION PROCESS OF ORGANIC DYES
}

\author{
A. Żaba ${ }^{1}$, K. Matras-Postolek ${ }^{1 *}$, S. Sovinska ${ }^{1}$, D. Bogdal ${ }^{1}$ \\ ${ }^{1}$ Cracow University of Technology, Faculty of Chemical Engineering and Technology, \\ Warszawska 24, 31-155 Krakow, Poland, \\ matras@chemia.pk.edu.pl
}

Keywords: microwave, $\mathrm{ZnS}$, Mn doping, photoactivity

\begin{abstract}
One-dimensional (1D) zinc sulphide ( $\mathrm{ZnS})$ manganese-doped and un-doped nanocrystals (NCs) were successfully fabricated in only 15-20 minutes by solvothermal reactions under microwave irradiation using ethylenediamine and hydrazine, respectively, as a stabilizer. The obtained NCs were systematically characterized in terms of structural and optical properties using X-ray diffraction (XRD), transmission electron microscopy (TEM), diffuse reflectance UV-Vis spectroscopy (DR UV-Vis), Fourier-transform infrared spectroscopy (FT-IR) analysis. The photocatalytic activity of the $\mathrm{ZnS}$ and $\mathrm{ZnS}: \mathrm{Mn}$ NCs was evaluated by the degradation of Rhodamine 6G (R6G). The results show that the Mn-doped NCs samples had higher coefficient of degradation of organic dyes under ultraviolet irradiation (UV).
\end{abstract}

\section{Introduction}

Since 1972, when Fujishima and Honda reported [1] the photocatalytic water splitting by titanium dioxide $\left(\mathrm{TiO}_{2}\right)$ electrode, inorganic semiconductors have been considered a promising material for photocatalytic degradation of pollutants due to photocatalytic reactions [2], [3]. Due to their good properties, the materials like $\mathrm{TiO}_{2}, \mathrm{ZnO}$, ternary oxides such a $\mathrm{CuWO}_{4}, \mathrm{ZnFe}_{2} \mathrm{O}_{4}, \mathrm{CaFe}_{2} \mathrm{O}_{4}, \mathrm{CuBi}_{2} \mathrm{O}_{4}$ and $\mathrm{CuNb}_{3} \mathrm{O}_{8}$ have been investigated as the best materials for photocatalytic application [2]-[6]. In the last decade, researchers have focused on the photocatalytic materials based on the II-VI group nanocrystals (NCs), for example sulphides of the metals from those groups [2], [3], [5], [7], [8]. One of the most investigated sulphides for photocatalytic application is cadmium sulphide (CdS). Due to its band gap, which is about $2.4 \mathrm{eV}, \mathrm{CdS}$ is considered a promising material for optoelectronic applications and also for the previously mentioned photocatalytic application, therefore many forms of CdS were synthesized and investigated [8]-[11]. Unfortunately, due to rapid recombination of electrons and holes, bare CdS semiconductors have a low efficiency of hydrogen production. Additionally, such materials, due to their high activity to light irradiation, lead to the corrosion of the semiconductors [12]. To avoid these problems, the $\mathrm{CdS}$ semiconductors are combined with other semiconductors. In such a case, the photogenerated electrons from the CdS semiconductors are transferred to electron levels of noble metals or are delocalized and transferred between the conduction bands of the semiconductors [2], [8], [9], [13], [14].

The second most popular metal sulphides used for photocatalytic application is zinc sulphide $(\mathrm{ZnS})[2]$. It is a non-toxic semiconductor from the II-VI group with the band gap of about $3.7 \mathrm{eV}$. Because of these characteristics, $\mathrm{ZnS}$ nanoparticles are considered promising materials for optoelectronic, electronic, photocatalytic and biomedical applications. There is a lot of examples of the synthesis of different forms with different dimensions of the $\mathrm{ZnS}$ NCs [15]. 
One example is a $\mathrm{ZnS}$ nanoribbon film obtained by Wang et al. [16]. They used their $\mathrm{ZnS}$ NCs for degradation of X-3B dye. After $100 \mathrm{~min}$, even about $89 \%$ of dye was degraded with one sample of the $\mathrm{ZnS}$ nanoribbons. The most popular shape of the $\mathrm{ZnS}$ NCs used in the photodegradation are quantum dots and nanospheres [17]-[20][21]-[29]. For example, Sharma et al. [18] synthesized ZnS nanoparticles capped and uncapped with thioglycerol by chemical precipitation method and degraded bromophenol blue, crystal violet and reactive red with the obtained materials. To improve the photocatalytic properties, the $\mathrm{ZnS}$ NCs are dotted with some metals, for example iron [30] and more popular copper [31][34], nickel [34], [35] and manganese [34]. The addition of such compounds influences the applicable surface of the photocatalyst and also accelerates the migration rate of electron/hole to the surface of the nanoparticles. These two phenomena increase the photocatalytic properties of the ZnS NCs.

In this work, we used microwave irradiation to obtain one dimensional $\mathrm{ZnS}$ NCs and $\mathrm{ZnS}$ NC synthesis with ethylenediamine and hydrazine, respectively, as a stabilizer. Both materials were obtained with and without Mn-doping. Due to the use of the microwave irradiation, the reaction time was only 15-20 minutes. The obtained materials were used to degrade organic dye - Rhodamine B under UV light.

\section{Materials}

All chemicals were used without further purification. The zinc nitrate hexahydrate $\left(\mathrm{Zn}\left(\mathrm{NO}_{3}\right)_{2} \cdot{ }_{6} \mathrm{H}_{2} \mathrm{O}\right)(>99 \%)$, thiourea $\left(\mathrm{CH}_{4} \mathrm{~N}_{2} \mathrm{~S}\right)(99 \%)$, manganese acetate tetrahydrate $\left(\mathrm{Mn}(\mathrm{CH} 3 \mathrm{COO})_{2} \cdot{ }_{4} \mathrm{H}_{2} \mathrm{O}\right) \quad(>99 \%)$, ethylenediamine $(\mathrm{C} 2 \mathrm{H} 8 \mathrm{~N} 2) \quad(>99 \%)$, hydrazine monohydrate (N2H4) ( 98\%) and Rhodaimne B (RhB) (99\%) were purchased from SigmaAldrich.

\section{Synthesis of the $\mathrm{ZnS}$ and $\mathrm{ZnS}$ :Mn NCs with hydrazine}

The synthesis of the ZnS:Mn NCs was carried out in a essel in a microwave reactor Magnum II (ERTEC, Poland) with a maximum applied power of $600 \mathrm{~W}$ and frequency of $2.45 \mathrm{GHz}$. In a typical procedure, zinc nitrate hexahydrate $(1.25 \mathrm{mmol})$, thiourea $(2.5$ $\mathrm{mmol})$ and manganese (II) acetate tetrahydrate $(0.125 \mathrm{mmol})$ were dissolved in a solution of hydrazine monohydrate $(20 \mathrm{ml})$ and distilled water $(10 \mathrm{ml})$ in the PTFE vessel. The mixture was stirred at room temperature for $10 \mathrm{~min}$. After that, the PTFE vessel was placed into the reactor and heated in various temperatures at 6-8 bar for $20 \mathrm{~min}$. Next, the reaction mixture was cooled to room temperature. After cooling, the obtained materials were centrifuged and washed with methanol three times. Then, the nanoparticles were dried at $40^{\circ} \mathrm{C}$ in an oven for $24 \mathrm{~h}$. In a typical reaction procedure, about $0.12 \mathrm{~g}$ of dry sample was obtained. The ZnS NCs were obtained as above without manganese acetate.

\section{Synthesis of the $\mathrm{ZnS}$ and $\mathrm{ZnS}$ :Mn NCs with ethylenediamine}

The synthesis of the ZnS:Mn NCs was carried out in a PTFE vessel in a microwave reactor Magnum II (ERTEC, Poland) with a maximum applied power of $600 \mathrm{~W}$ and frequency of $2.45 \mathrm{GHz}$. In a typical procedure, zinc nitrate hexahydrate $(4 \mathrm{mmol})$, thiourea $(8 \mathrm{mmol})$ and manganese (II) acetate tetrahydrate $(0.04 \mathrm{mmol})$ were dissolved in a solution of ethylenediamine $(20 \mathrm{ml})$ and distilled water $(10 \mathrm{ml})$ in a PTFE vessel. The mixture was stirred at room temperature for $10 \mathrm{~min}$. After that, the PTFE vessel was placed into the reactor and heated in various temperatures for $30 \mathrm{~min}$. Next, the reaction mixture was cooled to room temperature. After cooling, the obtained materials were centrifuged and washed with methanol (with or without ultrasonic bath) three times. Then, the nanoparticles were dried at $40^{\circ} \mathrm{C}$ in the oven for $24 \mathrm{~h}$. The $\mathrm{ZnS}$ NCs were obtained as above without manganese acetate. 


\section{Characterization and measurements of the nanomaterials.}

All materials were characterized by XRD, FT-IR, S/TEM, UV-Vis analyses. The surface of the NCs was characterized with FT-IR. The morphology of the nanostructures was examined with transmission electron microscopy (TEM). The measurements were performed on a FEI Tecnai Osiris S/TEM. The crystallographic structure of the samples was investigated with an X-ray diffraction (XRD) method using Bruker D8 Phaser Diffractometer with $\mathrm{CuK} \alpha$ radiation $(\lambda=1.5406 \AA)$. The surface of the NCs was confirmed with a Nicolet 6700 FT-IR spectrometer. The absorbance measurements of the powders were recorded using a Shimadzu UV-2700 spectrophotometer with ISR-2600 Plus Integrating Sphere Attachment. The emission and excitation of $\mathrm{ZnS}$ and $\mathrm{ZnS}: \mathrm{Mn}$ NCs were characterized with Spectrofluorometer Fluorolog-3 from Horiba company.

\section{Photocatalytic investigation of the nanomaterials}

The photocatalytic activities of the $\mathrm{ZnS}$ and $\mathrm{ZnS}: \mathrm{Mn}$ NCs were tested by the decomposition of $\mathrm{RhB}$ in water solution under ultraviolet $(254 \mathrm{~nm})$ (UV) irradiation. The appropriate amount of $\mathrm{ZnS}$ ( $\mathrm{ZnS}: \mathrm{Mn}$ ) was dispersed in $60 \mathrm{ml}$ of a Rhodamine B solution (concentration $5 \mathrm{mg} / \mathrm{L}$ ). The solutions of dye with nanoparticles were placed under UV illumination for four hours. About $2 \mathrm{ml}$ of the solution was taken every 20 minutes and characterized with UVViSNIR (200-1100 nm) spectrometer from StellarNet Incorporation. The concentration of RhB in solutions was determined by monitoring the changes in the maximal absorbance at approximately $550 \mathrm{~nm}$. The photocatalytic activities were implemented with a $6 \mathrm{~W}$ UV Spectroline lamp (Model ENF-260C/FE, $50 \mathrm{~Hz}, 0.17$ AMPS, $254 \mathrm{~nm}$ ). The blank control test without the $\mathrm{ZnS}$ or $\mathrm{ZnS}: \mathrm{Mn} \mathrm{NCs}$ was also made.

\section{Results and discussion}

The $\mathrm{ZnS}$ and $\mathrm{ZnSMn}$ NCs were synthesized in a microwave assisted synthesis with ethylenediamine or hydrazine as a synthesis stabilizer. The detailed information about the synthesis and the calculated bandgap of the samples are presented in Table 1.

The obtained $\mathrm{ZnS}$ and $\mathrm{ZnS}: \mathrm{Mn}$ NCs were characterized and analyzed with XRD, TEM, ATR-IR, DR UV-vis measurements. The crystal structure of $\mathrm{ZnS}$ and $\mathrm{ZnS}: \mathrm{Mn}$ was characterized with an X-Ray Difractions method. The results are presented on Figure 1. All samples have a wurtzite structure. Figure 1 a shows that the materials synthesized with a microwave assisted heating method have all characteristic peaks for the $\mathrm{ZnS}$ wurtzite structure and crystal planes of (100) (002) (101) (102) (103) and (103) from the ZnS wurtzite JCPDS 96-110-0045 are present. The addition of manganese, different temperature and type of heating have no influence on crystal structure of the obtained $\mathrm{ZnS}$ NCs. 
Table 1. Detailed experimental parameters for the synthesis of $\mathrm{ZnS}$ and $\mathrm{ZnS}: \mathrm{Mn}$ and their band gap energy.

\begin{tabular}{|c|c|c|c|c|c|}
\hline Sample & $\begin{array}{c}\mathbf{Z n}^{2+}: \mathbf{S}^{2-}: \mathbf{M n}^{2+} \\
\text { molar ratio }\end{array}$ & $\begin{array}{c}\text { Temper } \\
\text { ature } \\
{\left[{ }^{\circ}\right]}\end{array}$ & $\begin{array}{c}\text { Pressure } \\
{[\mathbf{b a r}]}\end{array}$ & Stabilizer & $\begin{array}{c}\text { Bandgap } \\
{[\mathbf{e V}]}\end{array}$ \\
\hline H170Mn & $1: 1: 0.01$ & 170 & $6-8$ & hydrazine & 3.80 \\
\hline H170 & $1: 1: 0$ & 170 & $6-8$ & hydrazine & 3.78 \\
\hline H160Mn & $1: 1: 0.01$ & 160 & $6-8$ & hydrazine & 3.64 \\
\hline H160 & $1: 1: 0$ & 160 & $6-8$ & hydrazine & 3.64 \\
\hline H17011:2 & $1: 2: 0.01$ & 170 & $6-8$ & hydrazine & 3.56 \\
\hline EN170 & $1: 2: 0$ & 170 & $6-8$ & ethylenediamine & 3.41 \\
\hline EN170Mn0.01 & $1: 2: 0.01$ & 170 & $6-8$ & ethylenediamine & 2.75 \\
\hline EN180 & $1: 2: 0$ & 180 & $7-9$ & ethylenediamine & 3.3 \\
\hline EN180Mn0.01 & $1: 2: 0.01$ & 180 & $7-9$ & ethylenediamine & 2.97 \\
\hline EN160 & $1: 2: 0$ & 160 & $6-8$ & ethylenediamine & 3.3 \\
\hline EN160Mn0.01 & $1: 2: 0.01$ & 160 & $6-8$ & ethylenediamine & 2.44 \\
\hline EN170Mn0.001 & $1: 2: 0.001$ & 170 & $6-8$ & ethylenediamine & 2.93 \\
\hline EN170Mn0.005 & $1: 2: 0.005$ & 170 & $6-8$ & ethylenediamine & 2.86 \\
\hline EN170Mn0.02 & $1: 2: 0.02$ & 170 & $6-8$ & ethylenediamine & 2.44 \\
\hline EN170Mn0.1 & $1: 2: 0.1$ & 170 & $6-8$ & ethylenediamine & 3.33 \\
\hline
\end{tabular}


a

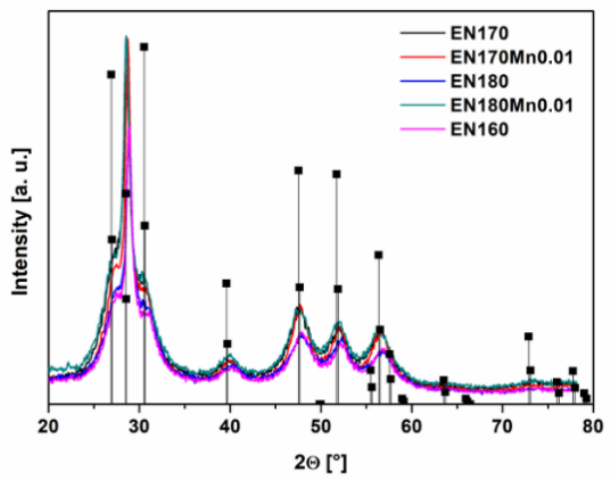

b

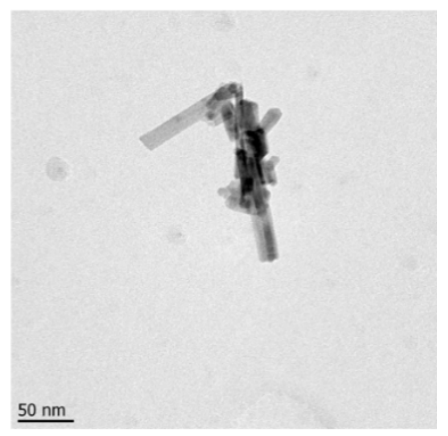

Fig. 1. XRD diffraction patterns of $\mathrm{ZnS}$ and $\mathrm{ZnS}: \mathrm{Mn}$ of the samples obtained with ethylenediamine (a) compared to the ZnS wurtzite JCPDS 96-110-0045 and (B) TEM images.

TEM images of the ZnS and ZnS:Mn NCs were also taken. As we can see on Figure 1b, the $\mathrm{ZnS}: \mathrm{Mn}$ NCs obtained with ethylenediamine in the microwaved assisted reaction at $170^{\circ} \mathrm{C}$ have one dimensional structures. As synthesized, the nanorods had a diameter of about $10 \mathrm{~nm}$ and the length of about $200 \mathrm{~nm}$. Similarly, the $\mathrm{ZnS}$ and ZnS:Mn NCs synthesized with hydrazine have a one dimensional morphology and a wurtzite structure.

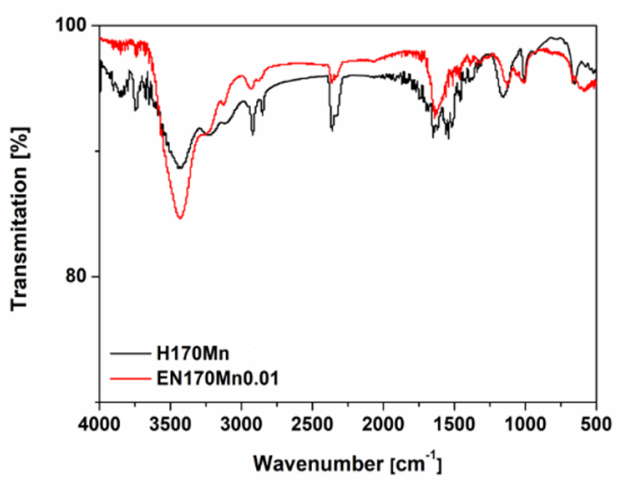

Fig. 2. FT-IR patterns of H170Mn0.01 and EN170Mn0.01

To investigate the surface of the $\mathrm{ZnS}$ and $\mathrm{ZnS}: \mathrm{Mn}$ NCs, the FT-IR measurements were performed. Figure 2 shows that the FT-IR patterns of the ZnS:Mn NCs synthesized with ethylenediamine have a very similar FT-IR pattern to the ZnS:Mn NCs from the synthesis with hydrazine, indicating the presence of the stabilizer agent on the surface of the NCs. For $v=2841 \mathrm{~cm}-1$ and $2924 \mathrm{~cm}^{-1}$, the peaks are visible which correspond to the asymmetrical stretching vibrations of the methylene group. The peaks for scissoring and stretching vibrations of the amine group are observed at 1638 and $3443 \mathrm{~cm}-1$ respectively. Peak at $v=$ $1142 \mathrm{~cm}^{-1}$ correspond to stretching of the $\mathrm{C}-\mathrm{N}$ bond and the peak visible at $670 \mathrm{~cm}-1$ is from the $\mathrm{C}-\mathrm{H}$ bond. 
The photocatalytic activities of the $\mathrm{ZnS}$ and $\mathrm{ZnS}: \mathrm{Mn}$ NCs synthesized with different capping agents were determined in the degradation of Rhodamine B under UV light. Figure 3 a and $\mathrm{b}$ demonstrates the changes of the concentration of Rhodamine $\mathrm{B}$ with the presence of the ZnS:Mn NCs stabilized with ethylenediamine (a) and hydrazine (b).
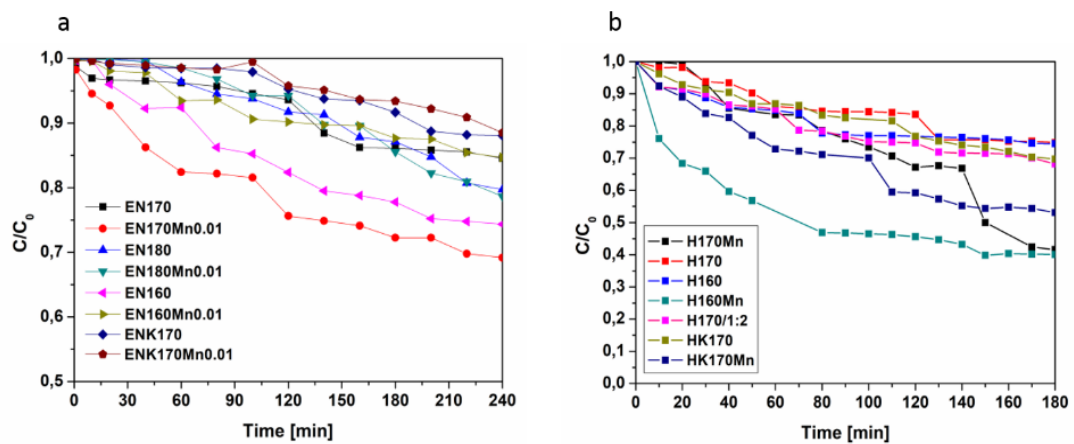

Fig. 3. Catalytic degradation of Rhodamine B with the $\mathrm{ZnS}$ and $\mathrm{ZnS}: \mathrm{Mn}$ NCs from the synthesis with ethylenediamine (a) and hydrazine (b).

The curves present the dependence of the dye's normalized UV absorption at the maximum of absorbance versus illumination time. It can be seen that the degradation ratios for the microwave-assisted synthesis stabilized with hydrazine are bigger than the ZnS:Mn NCs stabilized with ethylenediamine. About $55 \%$ of the dye was degraded after $3 \mathrm{~h}$, when the nanoparticles from the microwave-assisted synthesis with hydrazine were used. Also the addition of manganese improves the photocatalytic properties in both cases.

\section{Acknowledgement}

This work was financially supported by the National Centre for Research and Development within the $\mathrm{V}$ edition of the LIDER program (project number LIDER/009/185/L5/13/NCBR/2014).

\section{References}

1. A. Fujishima, K. Honda, Nature, 1972, 238, 37-38.

2. T. Jafari, E. Moharreri, A. S. Amin, R. Miao, W. Song, S. L. Suib, Molecules, 2016, 21, 900-928.

3. M. B. Wilker, K. J. Schnitzenbaumer, G. Dukovic, Isr. J. Chem., 2012, 52, 1002-1015

4. S. Rehman, R. Ullah, A. M. Butt, N. D. Gohar, J. Hazard. Mater., 2009, 170, 560-569.

5. K. Sivula, R. van de Krol Nat. Rev. Mater., 2016, 1.

6. H. Tong, S. Ouyang, Y. Bi, N. Umezawa, M. Oshikiri, Adv. Mater., 2012, 24, 229 251.

7. Y. Shiga, N. Umezawa, N. Srinivasan, S. Koyasu, E. Sakai, M. Miyauchi, Chem. Commun., 2016, 52, 7470-7473.

8. K. Zhang, Liejin Guo, Catal. Sci. Technol., 2013, 3, 1672-1690.

9. Q. Fan, Y. Huang, C. Zhang, J. Liu, L. Piao, Y. Yu, S. Zuo, B. Li, Catal. Today, 2016, 264, 250-256.

10. Q. Wang, J. Lian, J. Li, R. Wang, H. Huang, B. Su, Z. Lei, Sci. Rep., 2014, 5, 1-9.

11. K. Giribabu, R. Suresh, R. Manigandan, Bull. Korean Chem. Soc., 2012, 33, 2910 2916. 
12. I. Majeed, M. A. Nadeem, M. Al-Oufi, M. A. Nadeem, G. I. N. Waterhouse, A. Badshah, J. B. Metson, H. Idriss,, Appl. Catal. B Environ,. 2016, 182, 266-276.

13. Y. Zhu, Y. Wang, Z. Chen, L. Qin, L. Yang, L. Zhu, P. Tang, T. Gao, Y. Huang, Z. Sha, G. Tang, Appl. Catal. A Gen., 2015, 498, 159-166.

14. Z. S. Liu, B. T. Wu, Y. B. Zhu, F. Wang, L. G. Wang, J. Colloid Interface Sci., 2013, 392, 337-342.

15. X. Fang, T. Zhai, U. K. Gautam, L. Li, L. Wu, Y. Bando, D. Golberg, Prog. Mater. Sci., 2011, 56, 175-287.

16. C. Wang, Y. Ao, P. Wang, S. Zhang, J. Qian, J. Hou, Appl. Surf. Sci., 2010, 256, 4125-4128.

17. Z. Fang, S. Weng, X. Ye, W. Feng, Z. Zheng, M. Lu, S. Lin, X. Fu, P. Liu, ACS Appl. Mater. Interfaces, 2015, 7, 13915-13924.

18. M. Sharma, T. Jain, S. Singh, O. P. Pandey, Sol. Energy, 2012, 86, 626-633.

19. X. Hao, Y. Wang, J. Zhou, Z. Cui, Y. Wang, Z. Zou, Appl. Catal. B Environ., 2018, 221, 302-311.

20. X. Li, C. Hu, H. Liu, J. Xu, B. Wan, X. Wang, Phys. E Low-Dimensional Syst. Nanostructures, 2011, 43, 1071-1075.

21. D. Samanta, T. I. Chanu, S. Chatterjee, Mater. Res. Bull., 2017, 88, 85-90.

22. M. Muruganandham, R. Amutha, E. Repo, M. Sillanpää, Y. Kusumoto, M. AbdullaAl-Mamun, J. Photochem. Photobiol. A Chem., 2010, 216, 133-141.

23. L. Yin, D. Zhang, J. Ma, X. Kong, J. Huang, H. Zhang, C. Liu, Powder Technol., 2016, 301, 1085-1091.

24. F. Chen, Y. Cao, D. Jia, Ceram. Int., 2015, 41, 6645-6652.

25. X. Wu, K. Li, and H. Wang, J. Alloys Compd., 2009, 487, 537-544.

26. L. Yin, D. Zhang, D. Wang, X. Kong, J. Huang, F. Wang, Y. Wu, Mater. Sci. Eng. B Solid-State Mater. Adv. Technol., 2016, 208, 15-21.

27. F. Dong, Y. Guo, J. Zhang, Y. Li, L. Yang, Q. Fang, H. Fang, K. Jiang, Mater. Lett., 2013, 97, 59-63.

28. M. Kaur, C. M. Nagaraja, Mater. Lett., 2015, 154, 90-93.

29. Q. Ma, Y. Wang, J. Kong, H. Jia, Ceram. Int., 2016, 42, 2854-2860.

30. R. Chauhan, A. Kumar, R. Pal Chaudhary, Spectrochim. Acta Part A Mol. Biomol. Spectrosc., 2013, 113, 250-256.

31. H. Labiadh, T. Ben Chaabane, L. Balan, N. Becheik, S. Corbel, G. Medjahdi, R. Schneider, Appl. Catal. B Environ., 2014, 144, 29-35.

32. J. Kaur, M. Sharma, O. P. Pandey, Superlattices Microstruct., 2015, 77, 35-53.

33. R. Chauhan, A. Kumar, R. Pal Chaudhary, J. Lumin., 2014, 145, 6-12.

34. H. R. Pouretedal, A. Norozi, M. H. Keshavarz, A. Semnani, J. Hazard. Mater., 2009, 162, 674-681.

35. M. Jothibas, C. Manoharan, S. Johnson Jeyakumar, P. Praveen, I. Kartharinal Punithavathy, J. Prince Richard, Sol. Energy, 2018, 159, 434-443. 


\title{
LOW TEMPERATURE DEGRADATION BEHAVIOUR OF 10Ce-TZP/Al $\mathrm{O}_{3}$ BIOCERAMICS OBTAINED BY MICROWAVE SINTERING TECHNOLOGY
}

\author{
L. Gil ${ }^{1}$, M. D. Salvador ${ }^{1}$, F. L. Penaranda-Foix ${ }^{2}$, R. Rosa ${ }^{3}$, P. Veronesi ${ }^{3}$, C. Leonelli ${ }^{3}$, A. \\ Borrell $^{1}$
}

\begin{abstract}
${ }^{1}$ Instituto de Tecnología de Materiales, Universitat Politècnica de València, Camino de Vera $s / n, 46022$, Valencia, Spain

${ }^{2}$ Instituto de Aplicaciones de las Tecnologías de la Información y de las Comunicaciones Avanzadas (ITACA), Universitat Politècnica de València, Camino de Vera s/n, 46022, Valencia, Spain

${ }^{3} M A G \_$Microwave Application Group, Dipartimento di Ingegneria "Enzo Ferrari", Università degli Studi di Modena e Reggio Emilia, Via P. Vivarelli 10, 41125 Modena, Italy logiflo@upv.es
\end{abstract}

Keywords: microwave sintering, biomaterials, mechanical properties, ageing process

\section{Introduction}

Zirconia is one of the most widely used ceramic materials as a biomaterial due to its outstanding mechanical properties and biocompatibility. This ceramic has three crystalline phases: monoclinic, tetragonal and cubic; but there is a spontaneous phase transformation during cooling - from the tetragonal to the monoclinic phase - which is accompanied by a volume expansion. This transformation produces significant residual stresses that induce micro-cracking and make the material useless for structural applications ${ }^{1}$. For this reason, the addition of a dopant is required to stabilize the tetragonal phase in zirconia at room temperature. $\mathrm{Y}_{2} \mathrm{O}_{3}$ is the most commonly used stabilizer, although in this study zirconia is doped with $\mathrm{CeO}_{2}$, as it manages to improve some properties, such as fracture toughness and degradation ${ }^{2}$.

Despite stabilizing zirconia with the addition of an oxide, phase transformation can occur spontaneously under humid conditions at temperatures of $20^{\circ} \mathrm{C}-300{ }^{\circ} \mathrm{C}$, leading to a drastic drop in its long-term mechanical properties. This phenomenon is generally known as low temperature degradation (LTD), hydrothermal degradation or aging ${ }^{3}$.

Different factors can affect the aging behavior in zirconia materials, such as grain size and shape, porosity or stabilizer content, among others ${ }^{4}$. Some of these factors depend on the sintering process and its mechanisms. Commonly, ceramics are consolidated thanks to a heat treatment, where mass transfer mechanisms are activated. The most crucial parameters are temperature and dwell time, which set mechanical properties like hardness and its microstructure as grain size, for instance. The conventional sintering method is carried out in an industrial furnace, where the heat transfer mechanisms are conduction, convection and radiation. In this type of sintering, the heat flows from the surface to the bulk of the material. Conventional sintering requires high temperatures and long processing times and hence high energy consumption. Therefore, non-conventional sintering methods are currently being developed, such as microwave heating technology. Microwave sintering is based on the absorption of electromagnetic radiation, which causes the increase of the material temperature. The mechanism of this method differs from conventional sintering, as the 
temperature gradient is on the contrary - from inside to outside. It is known as volumetric heating ${ }^{5,6}$.

Most of the research carried out to date uses $2.45 \mathrm{GHz}$ microwave applicators, however another frequency has been evaluated in this work, in particular $5.8 \mathrm{GHz}$, in order to evaluate the possible differences.

This research is focused on the study of the low temperature degradation of ceria doped zirconia/alumina composites and the effect of microwave sintering and the used frequency. In order to assess the phase change, hardness, surface roughness and monoclinic-phase transformation progression are evaluated as a function of degradation time.

\section{Materials and methods}

\section{A. Sample preparation}

Ceria doped zirconia-alumina composites were employed in this work. The starting powders were $10 \mathrm{Ce}-\mathrm{TZP}\left(\mathrm{ZrO}_{2}\right.$ with $\left.10 \mathrm{~mol} \% \mathrm{CeO}_{2}\right)$ provided by Daiichi Kigenso Kagaku Kogyo and $\mathrm{Al}_{2} \mathrm{O}_{3}$ (SPA 0.5) from Sasol. The composition $10 \mathrm{Ce}-\mathrm{TZP} / \mathrm{Al}_{2} \mathrm{O}_{3}$ was 65 vol. $\%$ of $10 \mathrm{Ce}-$ TZP and 35 vol. $\%$ of $\mathrm{Al}_{2} \mathrm{O}_{3}$, and it was selected on the basis of a previous study ${ }^{7}$.

The powders were pressed uniaxially at $200 \mathrm{MPa}$ and sintered by microwave technology (MW) at two different frequencies $(2.45 \mathrm{GHz}$ and $5.8 \mathrm{GHz})$. The MW sintering conditions were $1300{ }^{\circ} \mathrm{C}$ during $10 \mathrm{~min}$ of holding time. The specimens were also densified by conventional sintering (CS) in an electrical furnace at $1500{ }^{\circ} \mathrm{C}$ and $120 \mathrm{~min}$ of dwell time.

\section{B. Microwave cavities}

Two microwaves equipments used are shown in Fig. 1. The first heating test was carried out in a cylindrical cavity operating in single-mode ( $\mathrm{TE}_{111}$ ) at $2.45 \mathrm{GHz}$ frequency (Fig. 1a). In order to monitor the temperature, an optical pyrometer was used, which was previously calibrated in this temperature range. This cavity has been designed by ITACA ${ }^{8}$.

The $5.8 \mathrm{GHz}$ single-mode applicator (Fig. 1b) used for this work had a cavity with rectangular geometry (WR159). In this case, the temperature was measured simultaneously using a sapphire fiber (MIKRON M680 Infraducer, Mikron Infrared Inc., Santa Clara, CA, USA), that directly touched the free upper surface of the sample and was connected to a signal conditioner. These tests were performed in the Microwave Application Group at the University of Modena and Reggio Emilia in Italy ${ }^{9}$.

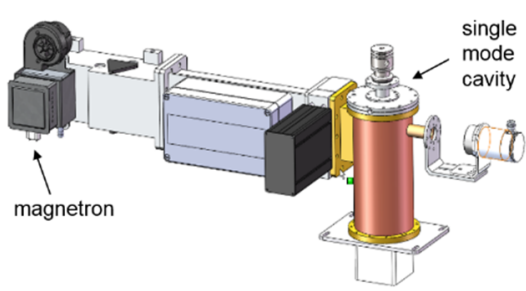

(a)

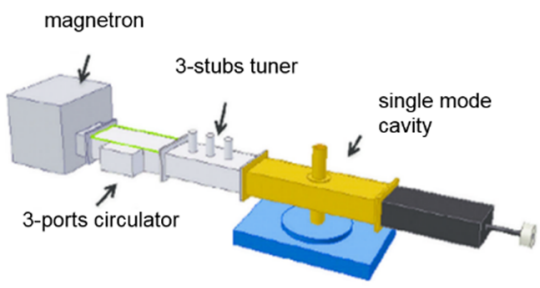

(b)

Fig. 1. Microwave systems operating at (a) $2.45 \mathrm{GHz}$ and (b) $5.8 \mathrm{GHz}$.

\section{Degradation assessment}

Characterization of aged samples was assessed with a phase content analysis, roughness and assessment of mechanical properties. LTD test of $10 \mathrm{Ce}-\mathrm{TZP} / \mathrm{Al}_{2} \mathrm{O}_{3}$ was performed under conditions which promotes the hydrothermal degradation. Samples were aged in a steam autoclave at $120^{\circ} \mathrm{C}$ and 1.2 bar. These conditions are based on several studies which claim 
that $1 \mathrm{~h}$ corresponds to approximately 3 years in contact with human fluids. Degradation assessment was carried out as a function of time exposed in the autoclave.

A Raman spectrometer Horiba-MTB Xplora was employed in order to analyze the phase content. Measurement was made on the polished exposed surface for a Raman range 120 $700 \mathrm{~cm}^{-1}$.

Monoclinic phase in the degraded samples was estimated with the following equation, which was proposed by $\operatorname{Lim}^{10,11}$ :

$$
V_{m}=\frac{I_{m}^{181}+I_{m}^{190}}{0.33 \cdot\left(I_{t}^{147}+I_{t}^{265}\right)+I_{m}^{181}+I_{m}^{190}}
$$

Where; $V_{m}$ is the m-phase volume fraction, $I_{m}^{181}, I_{m}^{190}$ represent the integrated area of the monoclinic peaks at 181 and $190 \mathrm{~cm}^{-1}$ and $I_{t}^{147}, I_{t}^{265}$ are the integrated area of the tetragonal peaks at 147 and $265 \mathrm{~cm}^{-1}$.

Surface topography and roughness were analyzed by atomic force microscopy (AFM) in tapping mode (Multimode, Veeco, Plainview, NY). Regarding mechanical properties, the hardness of the material has been measured by a nanoindenter (G-200; Agilent Technologies, Barcelona, Spain) with a Berkovich tip.

\section{Results and discussion}

Raman spectra for $10 \mathrm{Ce}-\mathrm{TZP} / \mathrm{Al}_{2} \mathrm{O}_{3}$ sintered by $\mathrm{MW}-2.45 \mathrm{GHz}, \mathrm{MW}-5.8 \mathrm{GHz}$ and $\mathrm{CS}$ are shown in the Fig. 2. As can be seen, no peak of the monoclinic phase is observed after 80 hours of exposure to LTD conditions. 

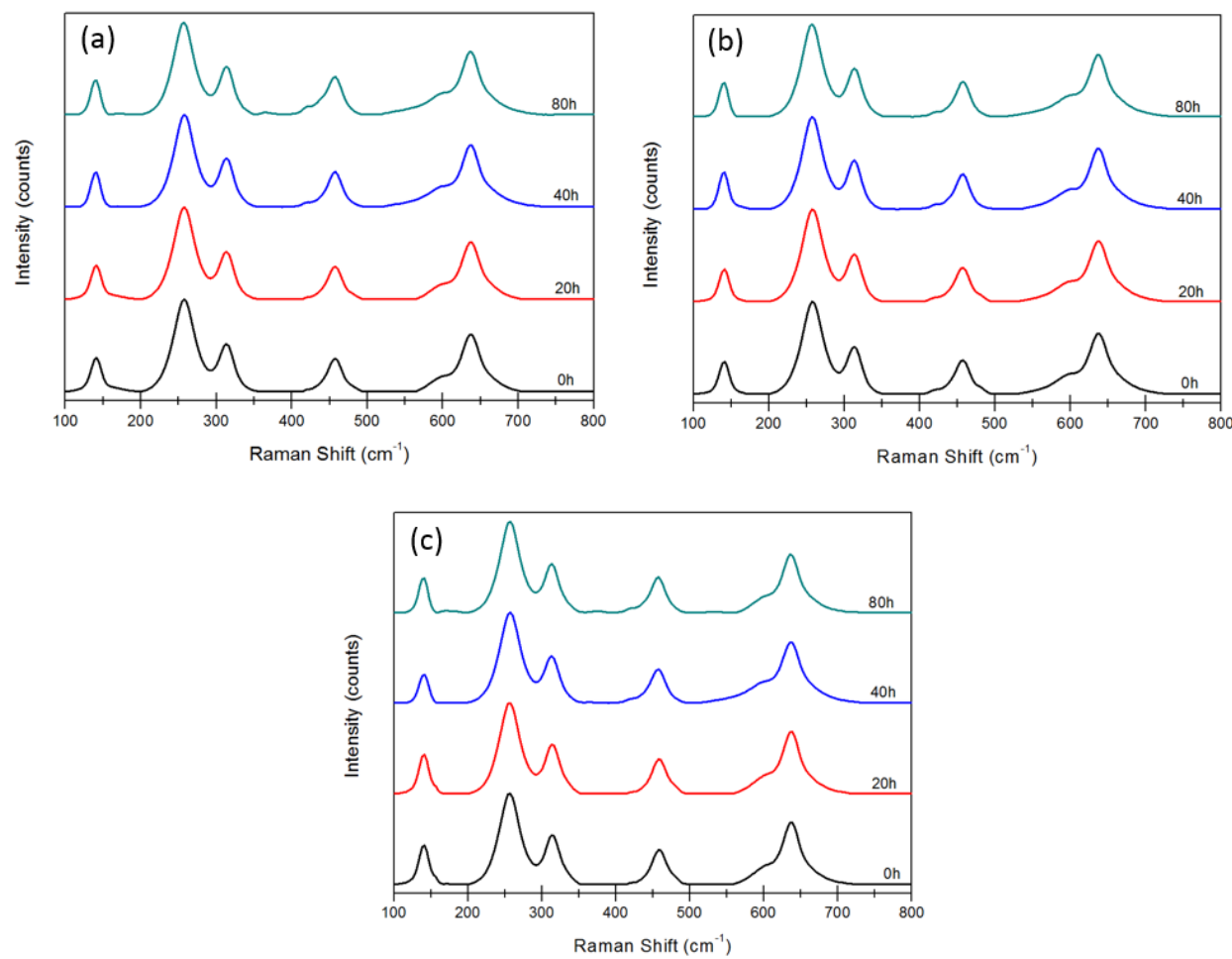

Fig. 2. Raman spectra obtained for $10 \mathrm{Ce}-\mathrm{TZP} / \mathrm{Al}_{2} \mathrm{O}_{3}$ sintered at different degradation times: (a) MW-2.45GHz, (b) MW-5.8GHz and (c) CS.

As there are no monoclinic peaks in the Raman spectra, it is not possible to quantify the percentage of monoclinic phase. Due to the measurement error in the Raman spectra, the percentage of monoclinic does not exceed 5\%. In addition, the sintering method has not influenced on the degradation of $10 \mathrm{Ce}-\mathrm{TZP} / \mathrm{Al}_{2} \mathrm{O}_{3}$, as since in none of them appears monoclinic phase.

Fig. 3 shows the topographic AFM images for the conventional sintered sample. It can be verified that the roughness of the sample increases as the hours of LTD exposure rises. However, this increase is not significant, as the percentage of monoclinic is less than $5 \%$. 

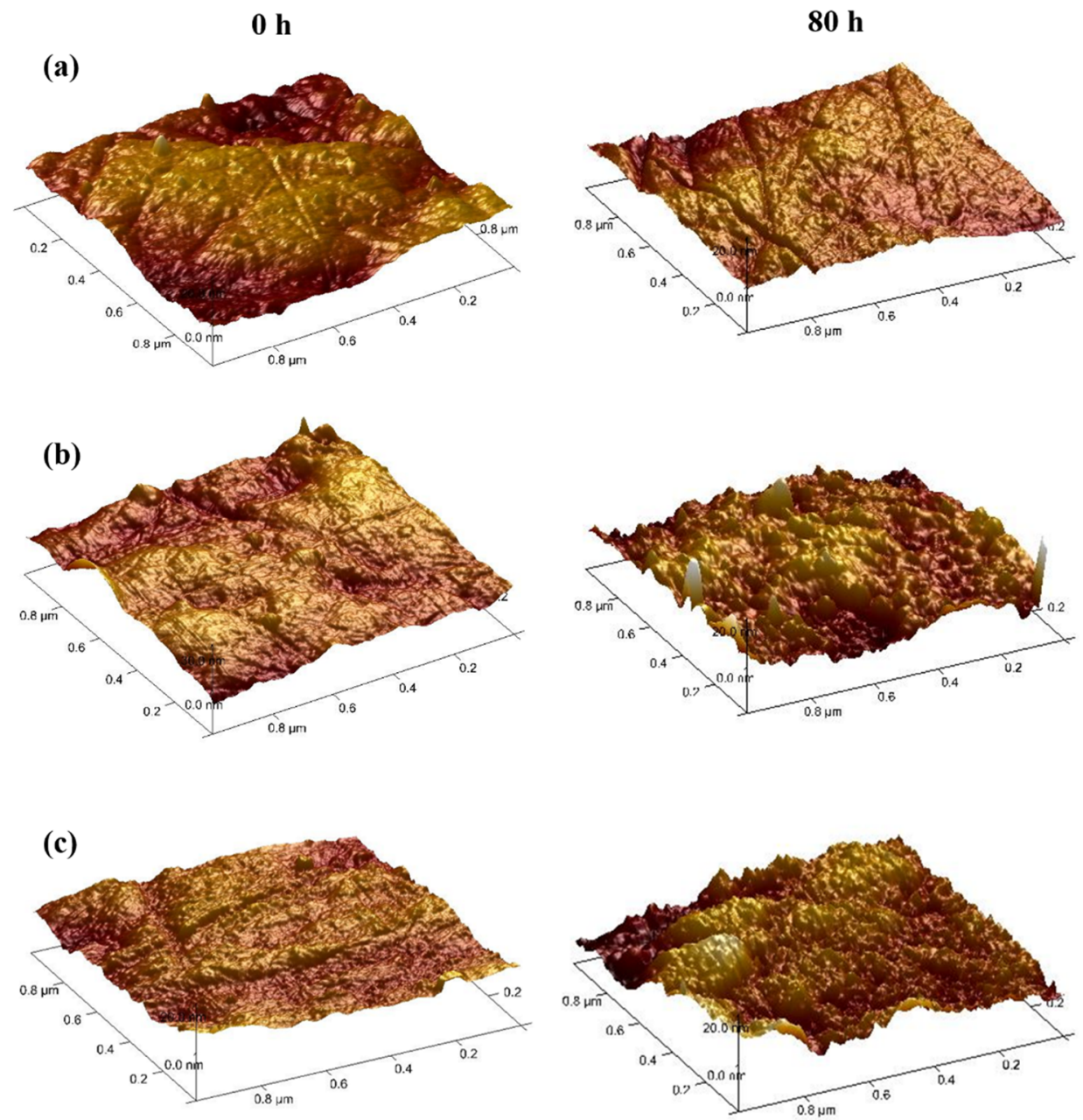

Fig. 3. Topographical AFM images at $0 \mathrm{~h}$ (left) and $80 \mathrm{~h}$ (right) of LTD exposure of 10Ce$\mathrm{TZP} / \mathrm{Al}_{2} \mathrm{O}_{3}$ sintered (a) $\mathrm{MW}-2.45 \mathrm{GHz}$ (b) $\mathrm{MW}-5.8 \mathrm{GHz}$ and (c) $\mathrm{CS}$.

Regarding hardness values (see Fig. 4), there are not high differences between samples at 0 $\mathrm{h}$ and $80 \mathrm{~h}$ of LTD exposure (not more of $1 \mathrm{GPa}$ ). It proves that there is practically no aging in $10 \mathrm{Ce}-\mathrm{TZP} / \mathrm{Al}_{2} \mathrm{O}_{3}$ composites. 


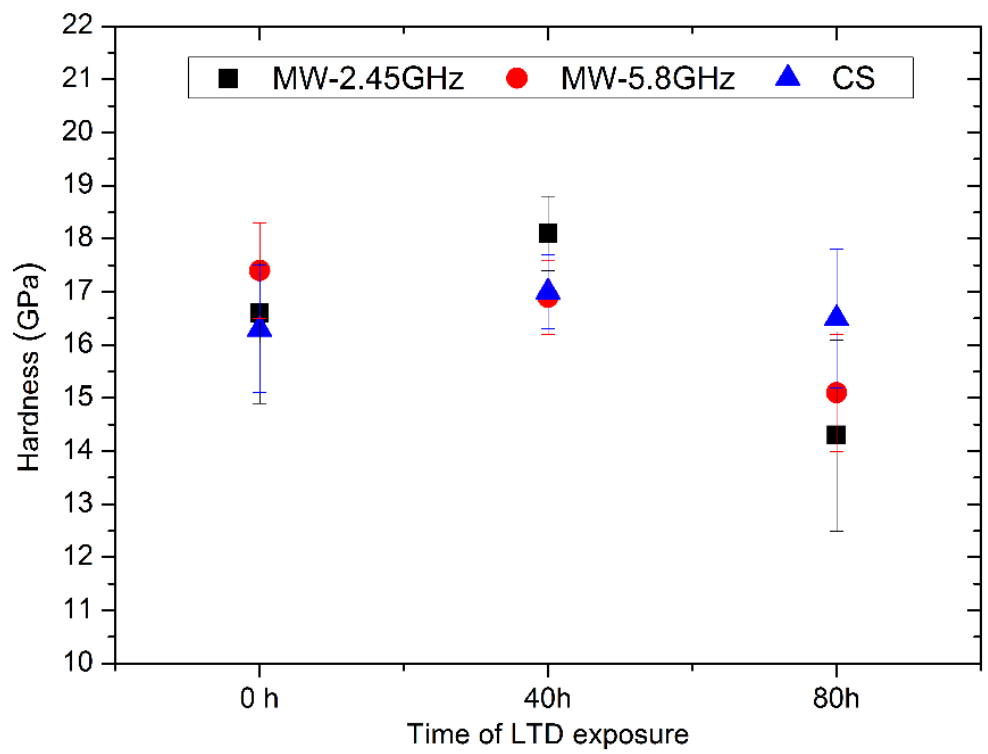

Fig. 4. Hardness values for $10 \mathrm{Ce}-\mathrm{TZP} / \mathrm{Al}_{2} \mathrm{O}_{3}$ sintered by $\mathrm{MW}$ and $\mathrm{CS}$ as a function of LTD exposure time

In the literature, a composite with similar proportions of zirconia and alumina but using $\mathrm{Y}_{2} \mathrm{O}_{3}$ as dopant, is already degraded after $80 \mathrm{~h}$ exposure ${ }^{12}$. Therefore, the use of the $\mathrm{CeO}_{2}$ stabilizer has been shown to prevent low hydrothermal degradation. Therefore, 10Ce$\mathrm{TZP} / \mathrm{Al}_{2} \mathrm{O}_{3}$ can be used for applications where resistance to aging is required.

Moreover, the effect of the sintering method on the aging could not be verified in this work, because until $80 \mathrm{~h}$ exposed to LTD does not appear monoclinic. However, it is possible that at higher times, differences between CS and MW can be observed ${ }^{3}$.

Lastly, $80 \mathrm{~h}$ of exposure to LTD is over enough, since it corresponds to more than 200 years in the human body, thus achieving the purpose of the prosthesis.

\section{Conclusion}

Low temperature degradation of $10 \mathrm{Ce}-\mathrm{TZP} / \mathrm{Al}_{2} \mathrm{O}_{3}$ has been investigated in the present study, as well as, the effect of two different sintering method (MW and CS) and frequency (2.45 $\mathrm{GHz}$ and $5.8 \mathrm{GHz}$ ) on the aging of this material. After the analysis of the Raman spectra, the following can be claimed:

- The use of $\mathrm{CeO}_{2}$ as a dopant prevents the ageing of $10 \mathrm{Ce}-\mathrm{TZP} / \mathrm{Al}_{2} \mathrm{O}_{3}$.

- No differences in LTD between MW and CS nor between the two frequencies used for MW (2.45 and $5.8 \mathrm{GHz})$ up to $80 \mathrm{~h}$ of exposure have been evidenced.

To sum up, microwave sintering technology allows to obtain highly densified 10Ce$\mathrm{TZP} / \mathrm{Al}_{2} \mathrm{O}_{3}$ composites with a resistance to degradation similar to those obtained by conventional. Therefore, microwave technique is an exceptional alternative for sintering this material, as it requires lower sintering temperatures $\left(1300{ }^{\circ} \mathrm{C}\right)$ and dwelling time $(10 \mathrm{~min})$ than conventional sintering $\left(1500^{\circ} \mathrm{C}\right.$ and $120 \mathrm{~min}$, respectively), leading to a reduction in energy costs and processing times and, consequently, the microwave technique has a lower environmental impact. 


\section{Acknowledgement}

The authors thank the Generalitat Valenciana for the financial support provided to the PROMETEU/2016/040 project. A. Borrell is grateful to the Spanish Ministry of Economy and Competitiveness for her RyC contract (RYC-2016-20915).

\section{References}

1. Kelly, J. R., Denry, I. 2008, 24, 289-298.

2. Kohorst, P., Borchers, L., Strempel, J., Stiesch, M., Hassel, T., Bach, F. W., Hübsch, C. Acta Biomater., 2012, 8, 1213-1220.

3. Presenda, Á., Salvador, M. D., Moreno, R., Borrell, A. J. Am. Ceram. Soc., 2015, 98, 3680-3689.

4. Lawson, S. J. Eur. Ceram. Soc., 1995, 15, 485-502.

5. Rybakov, K. I., Olevsky, E. A., Krikun, E. V. J. Am. Ceram. Soc., 2013, 96, 10031020.

6. Upadhyaya, D. D., Ghosh, A., Gurumurthy, K. R., Prasad, R. R. Ceram. Int., 2001, 27, 415-418.

7. $\quad$ Lopez-Píriz, R., Fernández, A., Goyos-Ball, L., Rivera, S., Díaz, L. A., FernándezDomínguez, M., Prado, C., Moya, J. S., Torrecillas, R. Materials (Basel)., 2017, 10, 614 .

8. Gil-Flores, L., Salvador, M. D., Penaranda-Foix, F. L., Fernández, A., Suarez, M., Rosa, R., Veronesi, P., Leonelli, C., Borrell, A. Ceram. Int., 2019.

9. Veronesi, P., Colombini, E., Rosa, R., Leonelli, C., Garuti, M. Chem. Eng. Process. Process Intensif., 2017, 122, 397-403.

10. Lim, C. S., Finlayson, T. R., Ninio, F., Griffiths, J. R. J. Am. Ceram. Soc., 1992, 75, 1316-1712.

11. Muñoz Tabares, J. A., Anglada, M. J. J. Am. Ceram. Soc., 2010, 93, 1790-1795.

12. Gremillard, L., Chevalier, J., Martin, L., Douillard, T., Begand, S., Hans, K., Oberbach, T. Acta Biomater., 2018, 68, 286-295. 


\title{
COMPARISON IN MECHANICAL PROPERTIES OF ZIRCONIUM TITANATE (ZrTIO4) SYNTHETIZED BY ALTERNATIVE ROUTES AND SINTERED BY MICROWAVE (MW)
}

\author{
R. Guillén ${ }^{1}$, A. Borrell ${ }^{1}$, M. Salvador ${ }^{1}$, F. L. Penaranda-Foix ${ }^{2}$, R. Moreno ${ }^{3}$ \\ ${ }^{1}$ Instituto de Tecnología de Materiales (ITM), Universitat Politècnica de València, Camino \\ de Vera s/n 46022, Valencia, Spain \\ ${ }^{2}$ Instituto de Aplicaciones de las Tecnologías de la Información y de las Comunicaciones \\ Avanzadas (ITACA), Universitat Politècnica de València, Camino de Vera s/n, 46022, \\ Valencia, Spain \\ ${ }^{3}$ Instituto de Cerámica y Vidrio, CSIC, Kelsen 528049 Madrid, Spain \\ reguipi@upv.es
}

Keywords: Microwave sintering, Synthesis methods, Mechanical properties

\begin{abstract}
This paper shows the mechanical properties of zirconium titanate $\left(\mathrm{ZrTiO}_{4}\right)$ synthetized by SOL-GEL and Lyophilization methods. In bout cases, the materials were fabricated to different sintering method: Microwave and Conventional. The sinterization parameter for conventional were $1500^{\circ} \mathrm{C} / 2 \mathrm{~h}$ and for microwave $1200^{\circ} \mathrm{C} / 10 \mathrm{~min}$. The material obtained was characterized by micro-indentation technique and FESEM analysis of microstructure. It was observed that the microwave sintering process produce comparable density and uniform microstructure with conventional, using less time and energy.
\end{abstract}

\section{Introduction}

At present, $\mathrm{ZrTiO}_{4}$ nanopowders are used as a dielectric material in the electroceramic field, applications of catalysis, microwave telecommunications devices, pigments, composites, etc. One of the most interesting applications is the potential as structural material and similar applications that require a high thermal resistance. However, all the properties of zirconium titanate are still a subject of interest for the industrial field.[1][2]

There are several routes of synthesis of $\mathrm{ZrTiO}_{4}$; among them is the sol-gel method and lyophilization. These methods have been used to make powders or small pieces of zirconium titanate. However, structural applications require materials in large quantities, so it is necessary to identify the differences between the methods of synthesizing and allowing the preparation of powders suitable for the generation of green materials for subsequent sintering process.[3]

To develop a new generation of nanomaterials with microstructural differences it is necessary to innovate in the sintering process. Years ago, the use of conventional oven for sintering material was the usual procedure. Nowadays, non-conventional methods as Microwave sintering (MW) are a bright way to produce high dense materials, using heating rates in reduce dwell times and lower consumption using 70\%-80\% less energy[4]. 
This reactive sintering technique achieves excellent mechanical properties, homogeneous microstructure employing lower sintering temperatures. All these energy and economic advantages generate a new vision for the future on ceramic materials and their industrial production.

The main objective of this study is investigated and compacted of the mechanical properties of the materials synthesized by sol-gel method and lyophilization and sintered by microwave and conventional processes.

\section{Materials and experimental procedure}

\subsection{Material}

Commercial undoped m-ZrO 2 (TZ-0, Tosoh Tokyo, Japan) and anatase- $\mathrm{TiO}_{2}$ (Aeroxide $\mathrm{P} 25$, Degussa, Germany) were used as starting powders to obtain $\mathrm{ZrTiO}_{4}$ materials. Starting zirconia and anatase powders have average particle diameters of $300 \mathrm{~nm}$ and $40 \mathrm{~nm}$, and specific surface areas of $14 \mathrm{~m}^{2} \mathrm{~g}^{-1}$ and $52 \mathrm{~m}^{2} \mathrm{~g}^{-1}$, respectively. The approach used involves the preparation of a total solids loading suspension of 30 vol. $\%$, with 15 vol. $\%$ of $\mathrm{m}-\mathrm{ZrO}_{2}$ and 15 vol.\% of a- $\mathrm{TiO}_{2}$, using a poly(acrylic acid) based polyelectrolyte (Duramax D3005 Rohm and Haas, USA) as a dispersant to a total concentration of $5.2 \mathrm{wt} . \%$ on a dry solids basis. This high content of deflocculant is due to the nanometric size of titania that can be effectively dispersed with a concentration of $4 \mathrm{wt} . \%$ as demonstrated in previous work [5].

\subsection{Powder mixture preparation}

For the preparation of the materials, $\mathrm{m}-\mathrm{ZrO}_{2}$ powders were firstly added to the proper amount of distilled water containing the dispersant with the help of a high shear mixer (L2R, Silverson, Chesham, UK). Afterwards a- $\mathrm{TiO}_{2}$ nanopowders were dispersed with the help of the high shear mixer and an ultrasonic probe (UP 400 S, Hielscher, Stuttgart, Germany). Sonication was performed using several cycles of $1 \mathrm{~min}$, being the maximum sonication time of $8 \mathrm{~min}$ and a frequency of $24 \mathrm{kHz}$, since further sonication cycles led to reagglomeration. The final suspension was frozen in a liquid nitrogen bath and then dried in a freeze drier (Cryodos-50, Telstar, Spain) for $24 \mathrm{~h}$. Powders obtained were sieved using a $37 \mu \mathrm{m}$ mesh nylon sieve before sintering.

\section{3 $\mathrm{ZrTiO}_{4}$ powders sintering}

The material was sintered by conventional (CS) in an electrical furnace at $1500{ }^{\circ} \mathrm{C}$ with a heating rate of $10{ }^{\circ} \mathrm{C} / \mathrm{min}, 2$ and 6 hours of dwelling time at the maximum temperature. Microwave (MW) sintering at $1200{ }^{\circ} \mathrm{C}$ with a heating rate of $100{ }^{\circ} \mathrm{C} / \mathrm{min}$ and $15 \mathrm{~min}$ dwelling time at the maximum temperature. These selected parameters are based on previous studies in our research group, where sintering conditions were optimized for Y-TZP materials [6].

\subsection{Material Characterization}

Sintered density and open porosity were determined by the Archimedes' method, introducing the samples into water according to the ASTM C373-88. At the same time, Mechanical properties were evaluated on surfaces polished down to one $\mu \mathrm{m}$ using diamond paste. Hardness $(\mathrm{H})$ was evaluated via the micro indentation technique.

Specimen microstructures were characterised by means of field emission-scanning electron microscopy (FE-SEM, Gemini Ultra 55 model, Zeiss). A 30 minutes thermal etching was performed at $100{ }^{\circ} \mathrm{C}$ below the maximum temperature to reveal grain boundaries. The linear 
intercept method was used to measure the average grain sizes of material [7]. Approximately 100 grains were considered for each phase.

\section{Results and discussion}

The density values for all samples sintered via microwave (MW) and conventional sintering (CS) are shown in the Table 1. In a general view, the results show that the sintering processes by MW and CS at different temperatures and dwell times have values of densification around 90-100\%.

\section{Table 1}

Sintering conditions, density and grain size values of $\mathrm{ZrTiO}_{4}$ sintered under different microwave and conventional conditions.

\begin{tabular}{|c|c|c|c|c|c|}
\hline Material & $\begin{array}{c}\text { Sintering } \\
\text { method }\end{array}$ & $\begin{array}{c}\text { Temperature } \\
\left({ }^{\circ} \mathrm{C}\right)\end{array}$ & $\begin{array}{c}\text { Dwell } \\
\text { time } \\
\text { (min) }\end{array}$ & $\begin{array}{c}\text { Density } \\
\text { (\%) }\end{array}$ & $\begin{array}{c}\text { Grain } \\
\text { size } \\
(\mu \mathrm{m})\end{array}$ \\
\hline \multirow{3}{*}{$\begin{array}{c}\mathrm{ZrTiO}_{4} \\
\text { (Sol Gel) }\end{array}$} & \multirow{2}{*}{$\mathrm{CS}$} & 1500 & 120 & 98.0 & 1.95 \\
\hline & & 1500 & 360 & 98.9 & 2.26 \\
\hline & MW & 1200 & 15 & 99.7 & 1.00 \\
\hline \multirow{3}{*}{$\begin{array}{c}\mathrm{ZrTiO}_{4} \\
\text { (Lyophilized) }\end{array}$} & \multirow[t]{2}{*}{$\mathrm{CS}$} & 1500 & 120 & 85.5 & 1.64 \\
\hline & & 1500 & 360 & 93.3 & 1.33 \\
\hline & MW & 1200 & 15 & 95.1 & 1.00 \\
\hline
\end{tabular}

The density of the material $\mathrm{ZrTiO} 4$ synthetized by Sol-Gel method represent the higher values in compare with Lyophilized. In the same way, the result of hardness indicates that Sol-gel material is superior. Even though, dwell time influence in their performance. For example, the sol-gel materials sintered by CS displays that with more exposure of dwell time the hardness value decrease (Figure 1). Conversely, the Lyophilized material rise the hardness value with more exposure of dwell time. 


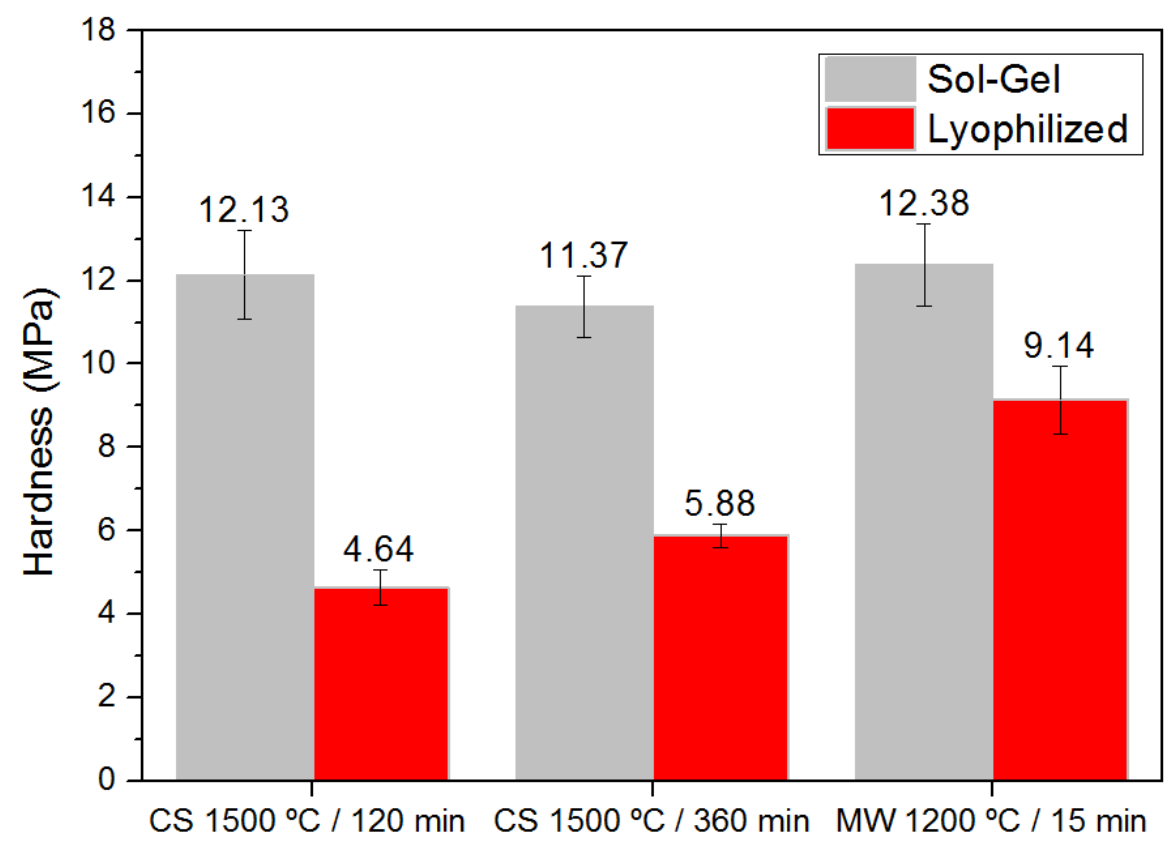

Figure 1. Hardness evaluation of $\mathrm{ZrTiO} 4$ materials.

Subsequently, is important to emphasize the behavior of each material base on the sintering method. Both materials display an improvement in the physical properties. Density and harness grown significantly by microwave sintering method.

Figure 2 shows the behavior of the microstructure of the materials. The material sintered by MW at $1200{ }^{\circ} \mathrm{C}$ at dwell time of 15 min shown a bulk material. The grain size is smaller in compare with CS. Sol- Gel material by CS indicates that with long dwell times the grain size grows.

With a temperature of $1500^{\circ} \mathrm{C}$ and 2 hours of dwell time, the grain pass from $1.95 \mu \mathrm{m}$ to $2.26 \mu \mathrm{m}$ with 6 hours dwell time. On the other hand, Lyophilized material indicates that with long dwell times the grain size decreases. At the same dwell times, the reduction is from 1.64 to $1.33 \mu \mathrm{m}$. 

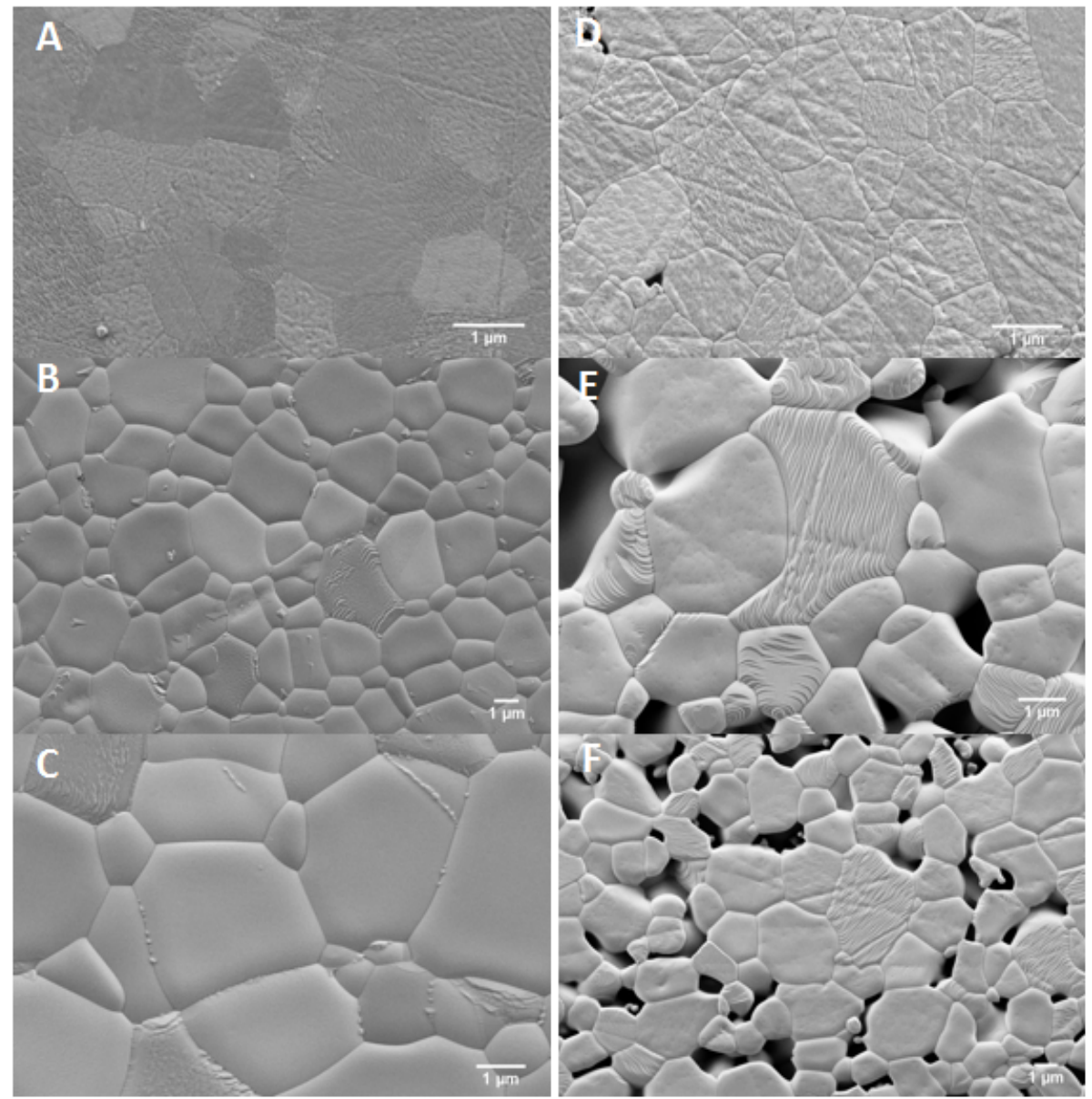

Figure 2. FESEM images of $\mathrm{ZrTiO} 4$ microstructure sintered by $\mathrm{MW}$ and $\mathrm{CS}$. A, B and C corresponds to Sol-Gel material. D, E and F corresponds to Lyophilized material. A and D sintered by MW at $1200{ }^{\circ} \mathrm{C} / 15 \mathrm{~min}$. B and E sintered by CS at $1500{ }^{\circ} \mathrm{C} / 120 \mathrm{~min}$. $\mathrm{C}$ and $\mathrm{F}$ sintered by $\mathrm{CS}$ at $1500{ }^{\circ} \mathrm{C} / 360 \mathrm{~min}$.

\section{Conclusions}

In conclusion, zirconium titanate bulk ceramic material was successfully obtained from SOL-GEL powders and consolidated by MW at temperature of $1200{ }^{\circ} \mathrm{C}$ with a dwell time of 15-min. The density achieved in $\mathrm{ZrTiO}_{4}$ synthetized by sol-gel and sintered by microwave is slightly high in comparison to conventional sintering process (99,7\% versus $98,0 \%)$. The grain size obtained by MW has a measure of $1 \mu \mathrm{m}$. However, the value of grain size treated at different temperatures in CS are double with respect to MW (1,95-2,26 $\mu \mathrm{m})$. Improvement in the hardness value of the material by sintering MW.

The Lyophilized material showed better properties when is exposed at long dwell time. The lyophilized material showed better properties when exposed in a prolonged residence time. In a different way, Sol-gel with more time of permanence its properties decrease. 


\section{Acknowledgements}

The authors would like to thank to the Generalitat Valenciana for financial support received for Santiago Grisolía program scholarship (GRISOLIAP/2018/168). A. Borrell acknowledges the Spanish Ministry of Economy and Competitiveness for her RyC contract (RYC-2016-20915).

\section{References}

[1] Y. Park and Y. Kim, "Influence of cooling rate on the physical properties of tin modified zirconium titanate," J. Mater. Sci. Lett., vol. 15, no. 10, pp. 853-855, 1996.

[2] A. Borrell et al., "ZrTiO4 materials obtained by spark plasma reaction-sintering," Compos. Part B Eng., vol. 56, pp. 330-335, 2013.

[3] A. Borrell and M. D. Salvador, "Advanced ceramic materials: processed and applications," in Advanced ceramic materials: processed and applications, UPV, 2018, pp. 6-9.

[4] A. Borrell, F. L. Penaranda-Foix, M. D. Salvador, J. M. Catala-Civera, and M. Miranda, "Microwave Technique: A Powerful Tool for Sintering Ceramic Materials," Curr. Nanosci., vol. 10, no. 1, pp. 32-35, 2014.

[5] M. Vicent Cabedo, E. Sanchez, I. Santacruz, and R. Moreno, "Dispersion of TiO 2 nanopowders to obtain homogeneous nanostructured granules by spray-drying," $J$. Eur. Ceram. Soc. - J EUR CERAM SOC, vol. 31, pp. 1413-1419, 2011.

[6] A. Borrell, M. D. Salvador, F. L. Peñaranda-Foix, and J. M. Cátala-Civera, "Microwave sintering of Zirconia materials: Mechanical and microstructural properties," Int. J. Appl. Ceram. Technol., vol. 10, no. 2, pp. 313-320, 2013.

[7] ASTM, "ASTM E112-13: Standard test methods for determining average grain size," ASTM Int., pp. 1-28, 2013. 


\title{
FEATURES OF MICROWAVE FIRED UTILITARIAN STONEWARE
}

\author{
T. Santos ${ }^{1,2}$, N. F. Santos ${ }^{2}$, C. S. F. Gomes ${ }^{3}$, L. Hennetier ${ }^{4}$, V. A. F. Costa ${ }^{1}$, L. C. Costa ${ }^{2}$ \\ 1 Centre for Mechanical Technology and Automation, Department of Mechanical \\ Engineering, University of Aveiro, 3810-193 Aveiro, Portugal \\ ${ }^{2}$ I3N and Department of Physics, University of Aveiro, 3810-193 Aveiro, Portugal \\ ${ }^{3}$ GeoBioTec, Research Unit of FCT, University of Aveiro, 3810-193 Aveiro, Portugal \\ ${ }^{4}$ Technological Centre for Ceramic and Glass Industries, 3025-307 Coimbra, Portugal \\ tiago.santos@ua.pt
}

Keywords: microwave heating, stoneware, microstructure, SEM, XRD, water absorption.

\begin{abstract}
Both high temperature phases and microstructure transformations of stoneware products microwave and conventionally (gas and electric) fired are investigated aiming at a better understanding of factors affecting their mechanical and aesthetic properties. The microstructure of microwave fired stoneware has similar characteristics to those of conventionally fired samples, microwave firing requiring lower temperatures to achieve a similar structure. X-Ray diffraction and scanning electron micrographs show that the most relevant transformations are complete for lower temperatures when using microwave heating. Microwave radiation is presented as an alternative heating technology for stoneware firing, leading to similar, or even better, mechanical properties and similar microstructural characteristics, requiring lower firing temperatures and shorter processing times.
\end{abstract}

\section{Introduction}

Stoneware, such as porcelain and other clay-based materials, is a composite material consisting of a glass matrix that embraces two main crystalline phases, quartz and mullite. It is a ceramic material, usually produced through a single firing stage at temperatures close to $1200{ }^{\circ} \mathrm{C}[1,2]$. Like porcelain, it can be double fired, through a first firing stage at 1000 ${ }^{\circ} \mathrm{C}$ followed by the firing of the glazed body at $1325^{\circ} \mathrm{C}[3]$. The firing temperatures depend on the constituent raw materials, which can have significant variations: $30-40 \%$ of clay, 5 $-20 \%$ of quartz and $45-55 \%$ of feldspars. Secondary minerals such as flint, talc and zircon may be added to lower the melting temperature and to increase the whiteness of the finished product $[1,2,4]$. It is a product normally colored, presenting some impurities like hematite, goethite, maghemite, lepidocrocite, the purity of the raw materials being not so crucial as for porcelain $[4,5]$.

The stoneware industry is highly hydrocarbons dependent, natural gas being the most used primary energy source for products drying and firing. Searching for a more decarbonized industry, microwave radiation is presented as an alternative heating technology, which shows to be a more environmentally friendly firing method for a wide variety of ceramic materials $[6,7]$. 


\section{Experimental methodology- Firing}

Samples were fired in both microwave and electric furnaces at temperatures of 500, 800, $1000,10501100,1140,1180,1225$ and $1300{ }^{\circ} \mathrm{C}$. The heating cycles, from room to maximum temperature, were processed from 40 up to 75 minutes. The cooling phase depends on the thermal inertia of each furnace. Results were compared with a reference, corresponding to the average of a set of 10 mugs fired in the tunnel kiln of Grestel Company at $\sim 1200{ }^{\circ} \mathrm{C}$ in 200 minutes. One test was carried out in the microwave furnace using a firing cycle of 115 minutes.

Microwave furnace: Samples were fired in a 6 magnetrons multimode furnace, each one operating with a nominal power of $900 \mathrm{~W}$ at a frequency of $2.45 \mathrm{GHz}$. A pyrometer and a thermocouple were installed in the microwave furnace for temperature measurement, monitoring and control. Calibration of the pyrometer was made in an electric furnace for more accurate temperature measurements. The thermocouple used in the microwave furnace was temporarily installed in the electric furnace, presenting a temperature difference of the order of 2 to $5{ }^{\circ} \mathrm{C}$ when comparing with that indicated by the electric furnace thermocouple. The microwave firing process was automated through the development of a Labview program searching for homogeneous heating, by creating a sequence of combined permutations of the magnetrons operating at a given moment of the heating process. The system operates in a Microwave Hybrid Heating ( $\mathrm{MHH}$ ) mode [8], as it uses a SiC plate acting as the base for the stoneware mugs and as a microwave susceptor. For lower temperatures, the material is heated indirectly, due to the heat released from the susceptor. After reaching its critical temperature, the stoneware absorbs the microwave radiation efficiently and is directly heated by the absorption of the microwave radiation [9].

The interior of the microwave furnace has a sandwich insulating wall, with $75 \mathrm{~mm}$ thickness. The layout displaying the distribution of the mugs over the $\mathrm{SiC}$ plate, inside the microwave furnace, is shown in Fig. 1.

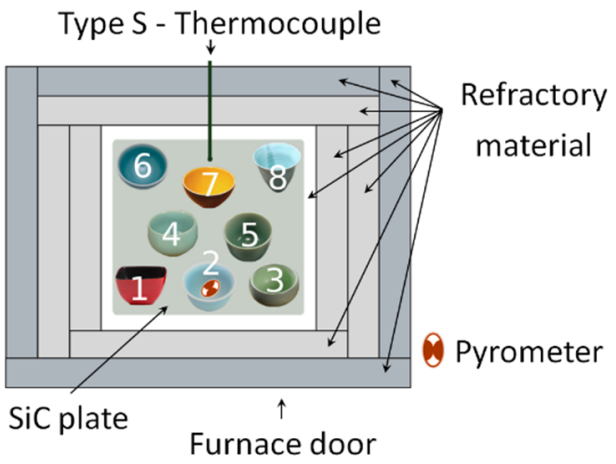

Fig. 1. Mugs distribution inside the microwave furnace. 
Electric furnace: The electric furnace is a standard furnace with a nominal power of 4.5 $\mathrm{kW}$. Due to the reduced availability of the samples' material and the reduced size of the electric furnace, only an aliquot taken from stoneware mugs was electrically fired. The temperature during the firing process was measured by the electric furnace thermocouple.

\section{Employed techniques and analysed features}

Rupture energy analysis: Stoneware mugs were tested by impact at the rim, according to ASTM 368/ISO 12980. Samples were repeatedly impacted increasing hammer's energy until fracture occurrence [10]. Results are presented in Fig. 2.

Water absorption analysis: Water absorption was determined based on the mass of aliquots samples impregnated with boiling water, following the porcelain tableware standard (BS5416) [11]. Aliquots were drawn from two different parts (the base and the rim) of the mugs. Results are presented in Fig. 2.

Simultaneous Thermo Analysis (STA): Differential Scanning Calorimetry (DSC) and ThermoGravimetry Analyses (TGA) were performed on stoneware powder from room temperature up to $1250{ }^{\circ} \mathrm{C}$. Some of the transformations occurring in the stoneware during firing were studied and results compared with those found in the literature. Results are presented in Fig. 3.

Optical microscopy analysis: Olympus $\mathrm{BH} 2$ optical microscope was used to perform microstructure (closed porosity) analysis. Prior to optical analysis, stoneware aliquots were grinded with sandpaper and water, and subjected to a final polishing with a $1 \mu \mathrm{m}$ ultra pure alumina suspension. Results are presented in Fig. 4.

Scanning Electron Microscopy (SEM - EDS) analysis: After optical analysis the aliquots were etched with a $10 \% \mathrm{HF}$ solution during $60 \mathrm{~s}$, then carbon coated and analyzed using the TESCAN-Vega 3 SBM SEM instrument. Results are presented in Fig. 5.

X-Ray diffraction (XRD) analysis: For detection of the phases present during firing, the powder aliquots of the samples fired at different temperatures, including green and gas fired references, were analyzed by powder X-Ray Diffraction (XRD). Data were collected using Empyrean Panalytical Diffractometer ( $\mathrm{CuK} \alpha$ radiation) with a Ni filter. Intensity data of diffraction peaks were provided by the continuous counting method in the range of $5^{\circ}$ to $80^{\circ}$ of $2 \theta$ angles. Results are presented in Fig. 6.

\section{Results}

The rupture energy and the water absorption determined on aliquots drawn from mugs rim and from mugs base are presented in Fig. 1. The rupture energy of the reference is over the curve of the microwave fired samples. Aliquots taken from the base of microwave fired mugs show lower water absorption than the reference. Aliquots taken from the rim of microwave fired mugs provided results contradicting the results corresponding to the basal ones. Comparing results with those of the electrically fired samples, it is clear that microwave fast fired samples present characteristics closer to that of the reference than the 
electrically fired ones, Table 1 . At $1180{ }^{\circ} \mathrm{C}$ the electrically fired samples provide water absorptions values of $7.5 \%$, much higher than the water absorption obtained for microwave fired and reference samples. The firing test with a 115 minutes cycle (marked in Fig. 1 in green) did not present significant differences when compared to the samples fired in 70 minutes cycles, the rupture energy being lower for this case.

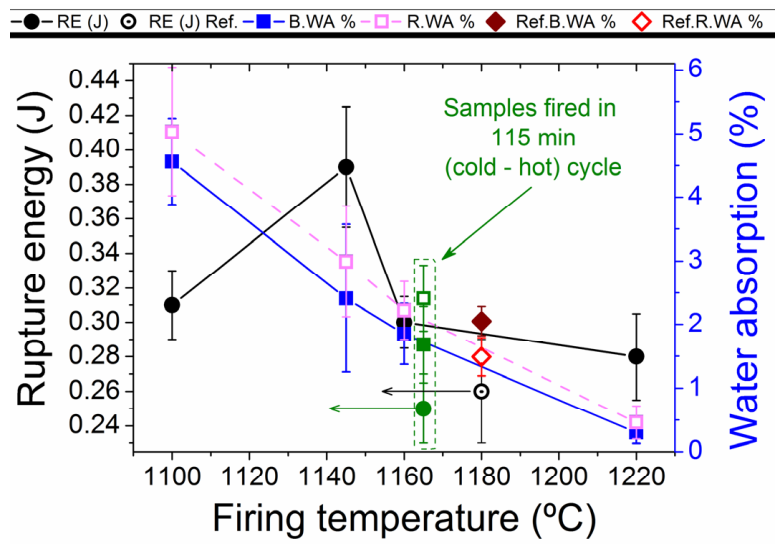

Fig. 2. Rupture energy (RE (J)) and water absorption (WA \%) as a function of the firing temperature (measured by the pyrometer). Ref. refers to the reference samples. B.WA\% refers to the water absorption of aliquots from the mugs base. R.WA\% refers to the water absorption of aliquots from the mugs rim.

Table 1. Water absorption of aliquots fired in the electric furnace.

\begin{tabular}{cc}
\hline Temperature $\left({ }^{\circ} \mathrm{C}\right)$ & Water Absorption $(\%)$ \\
\hline 1100 & 18.6 \\
1140 & 10.3 \\
1180 & 7.5 \\
1225 & 2.9 \\
1300 & 0.2 \\
\hline
\end{tabular}

Differential Scanning Calorimetry (DSC) and ThermoGravimetry (TG) analyses presented in Fig. 3, show that the loss of free and bound water represent about $1.8 \%$ of the total weight loss. Below $450{ }^{\circ} \mathrm{C}$ and under oxidizing conditions [12], burning of organic matter may occur, mainly in less pure clays. This could explain the continuous TG decrease, associated also to the continuous release of water from the stoneware raw materials. It is followed by the release of structural water from kaolinite leading to the formation of metakaolinite, endothermic reaction expressed by a peak at $510{ }^{\circ} \mathrm{C}$. The onset of the kaolinite structure collapse is observable from $\sim 450^{\circ} \mathrm{C}$ upwards, which according to [13] might extend up to temperatures close to $1000{ }^{\circ} \mathrm{C}$. This transformation comprises a weight loss of $5.6 \%$. The $\alpha \rightarrow \beta$ transformation of quartz occurs at $573{ }^{\circ} \mathrm{C}$ [4]. The pre-mullite and liquid phase formations occur at approximately $990^{\circ} \mathrm{C}$ and $1100^{\circ} \mathrm{C}$, respectively $[2,4]$. Formation of the liquid phase lowers to the eutectic temperature of $985^{\circ} \mathrm{C}$ [14], or of $990 \pm 20^{\circ} \mathrm{C}$ according to [15], for the $\mathrm{K}_{2} \mathrm{O}-\mathrm{Al}_{2} \mathrm{O}_{3}-\mathrm{SiO}_{2}$ system. 
The residual quartz, which is part of the initial composition, only reacts with the liquid phase at higher temperatures, beginning to be dissolved for temperatures above $1200{ }^{\circ} \mathrm{C}[4,14]$.

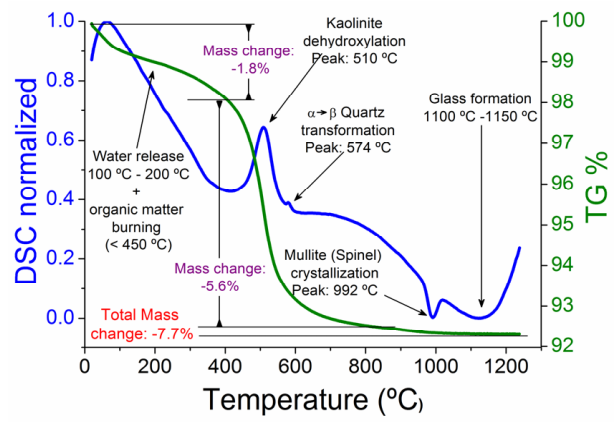

Fig. 3. Differential Scanning Calorimetry (DSC) and ThermoGravimetry (TG) curves of dry stoneware raw materials composition.

At temperatures close to $990{ }^{\circ} \mathrm{C}$ mullite is formed due to a solid state reaction consisting of a rearrangement of the constituents, silica and alumina, released from metakaolinite. Above $1200{ }^{\circ} \mathrm{C}$ recrystallization occurs, with the consequent development of mullite crystals exhibiting needles' shape. With the dissolution of quartz the liquid phase content increases and its viscosity decreases, having an enhanced effect on the growth of mullite crystals [4,16]. In Fig. 4 the set of images show the microstructures of aliquots of several samples fired between $1000{ }^{\circ} \mathrm{C}$ and $1300{ }^{\circ} \mathrm{C}$ in both the microwave (MW) and electric (E) furnaces. The gas fired reference is also shown for comparison.

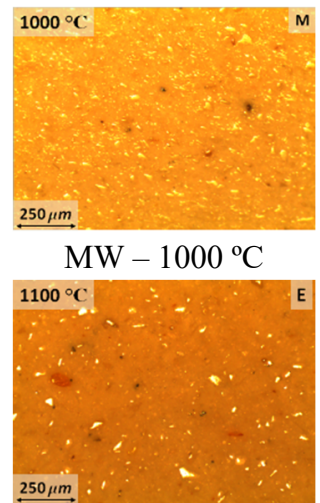

$\mathrm{E}-1100^{\circ} \mathrm{C}$

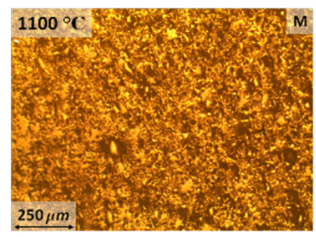

$\mathrm{MW}-1100^{\circ} \mathrm{C}$



$\mathrm{E}-1225^{\circ} \mathrm{C}$

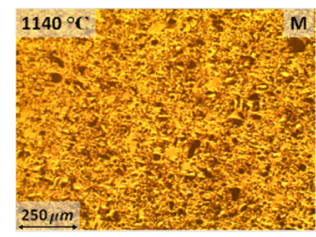

$\mathrm{MW}-1140{ }^{\circ} \mathrm{C}$

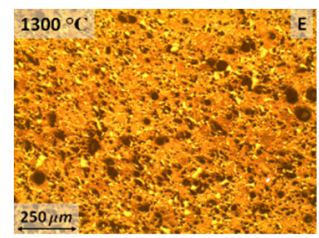

$\mathrm{E}-1300^{\circ} \mathrm{C}$

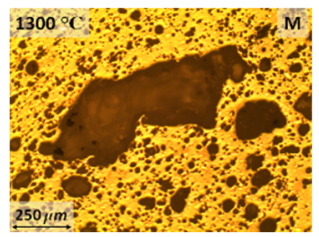

$\mathrm{MW}-1300^{\circ} \mathrm{C}$

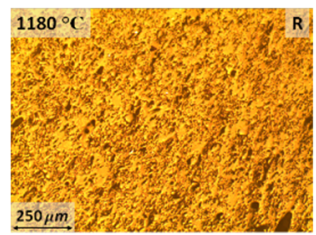

Gas Ref. $-1180^{\circ} \mathrm{C}$

Fig. 4. Optical microscope images of samples microwave fired (M), electrically fired (E) and gas fired (Gas Ref.) at several temperatures.

From Fig. 4 the microwave fired samples, in fast cycles of $70 \mathrm{~min}$ or less, have a more developed microstructure that those electrically fired, microwave fired samples at $1140{ }^{\circ} \mathrm{C}$ already presenting features very similar to those of the reference. The electrically fired samples at this same temperature have an underdeveloped microstructure, the sample fired at $1225^{\circ} \mathrm{C}$ being closer to the reference. For a better and deeper understanding of the main 
reasons for the observations made so far, SEM observations were carried out (Fig. 5). At $1050^{\circ} \mathrm{C}$ an advanced state of melting and dissolution could be observed in feldspar grains from samples fired in the microwave furnace. This melting and dissolution is just incipient in the electrically fired samples, which still present structures/agglomerates of platy particle relics derived from kaolinite structural collapse, and that are just starting transformation to mullite. At $1140{ }^{\circ} \mathrm{C}$ the mullite is more developed in the microwave fired samples than in the electrically fired ones, already presenting mullite type-II, yet not so developed as in the reference sample.
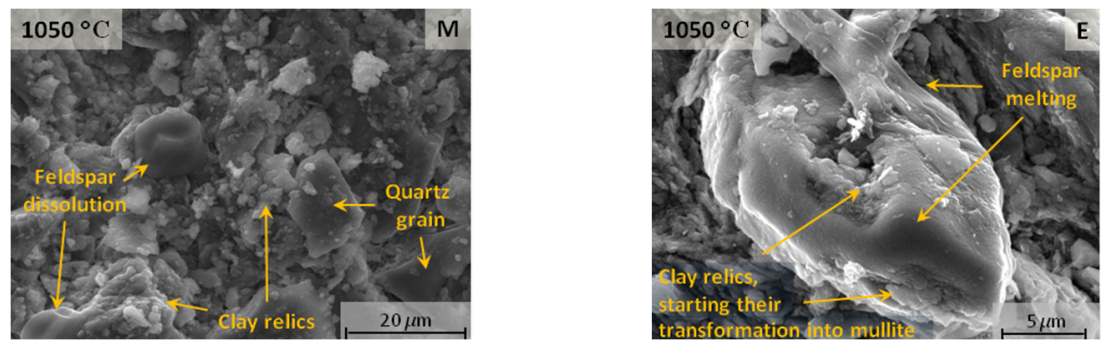

Microwave (left) and electrically (right) fired samples at $1050{ }^{\circ} \mathrm{C}$
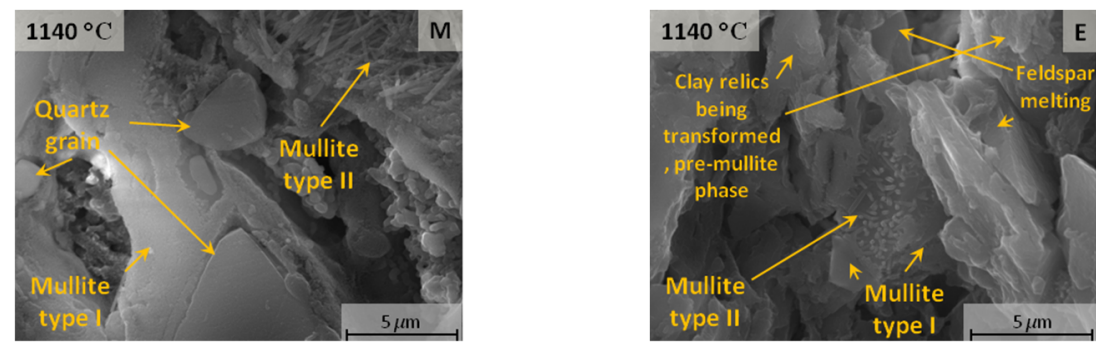

Microwave (left) and electrically (right) fired samples at $1140^{\circ} \mathrm{C}$

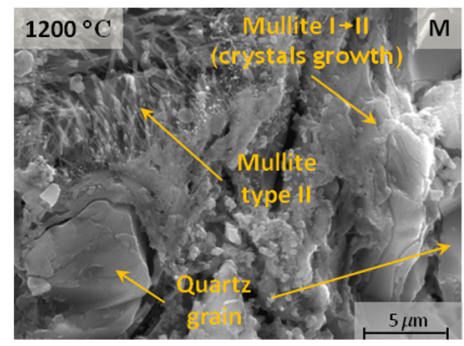

$\mathrm{MW}-1200{ }^{\circ} \mathrm{C}$

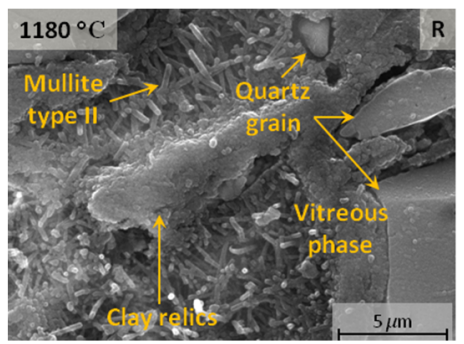

Gas Ref. $-1180^{\circ} \mathrm{C}$

Fig. 5. SEM images of microwave fired, electrically fired and gas fired reference samples, fired at $1050{ }^{\circ} \mathrm{C}, 1140^{\circ} \mathrm{C}, 1180{ }^{\circ} \mathrm{C}$ and $1200^{\circ} \mathrm{C} .(\mathrm{M})=$ Microwave; $(\mathrm{E})=$ Electrically; $(\mathrm{R})=$ Gas reference.

The results of the XRD analysis presented in Fig. 6 show that the electrically fired samples exhibit less intense mullite peaks than those microwave fired at the same temperature, the diffractograms of microwave fired samples at $1140{ }^{\circ} \mathrm{C}$ being very similar to the one of the reference sample. XRD results also show that some transformations occur earlier, i.e., for lower temperatures, in the case of microwave fired samples. Kaolinite dehydroxylation is a 
paradigm, the collapse of kaolinite structure being completely accomplished at $500{ }^{\circ} \mathrm{C}$. Electrically fired samples still show residual kaolinite at $800^{\circ} \mathrm{C}$, although not visible in Fig. 6. The same is observed for talc and muscovite, which structural changes comparatively occur at lower temperatures for microwave firing.
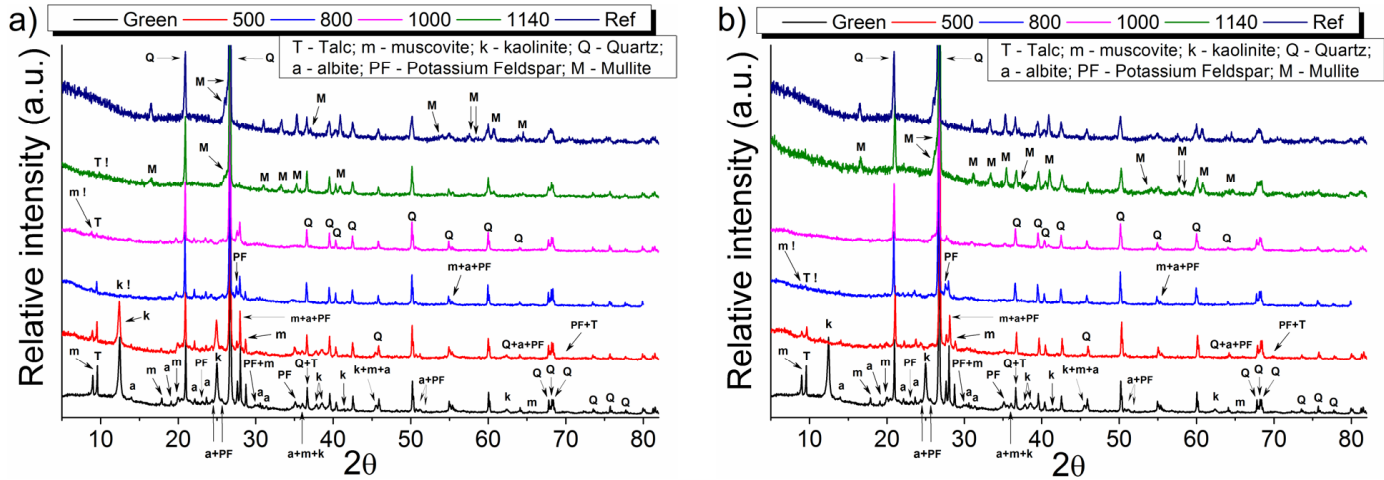

Fig. 6. X-ray diffractograms of aliquots fired at $500{ }^{\circ} \mathrm{C}, 800{ }^{\circ} \mathrm{C}, 1000^{\circ} \mathrm{C}$ and $1140{ }^{\circ} \mathrm{C}$ : a) in electric furnace, and b) in microwave furnace. Dried ("Green") stoneware reference and the gas fired reference ("Ref”) are also presented.

\section{Discussion}

The water absorption analysis shows that aliquots taken from the basal surface of microwave fired mugs provide values below those of the reference; the opposite is observed for the aliquots taken from the mugs rim. This might be related to the temperature differences between the mugs' base and rim, which depends on the heat technology, allowing the higher temperature in the mug's base than in the mug's rim. Since $\mathrm{SiC}$ is a very good microwave absorber, the mug's base contacting the $\mathrm{SiC}$ plate can be at a higher temperature than the mug's rim, and being denser at the base they are less water permeable there.

Microstructural analysis shows that the microstructures of the microwave processed samples are similar to those of the gas reference, for a firing cycle of only $35 \%$ of that required by the reference and for a temperature $\sim 50{ }^{\circ} \mathrm{C}$ lower (Fig. 4). For the same temperatures, the electrically fired aliquots will require more time to reach the same features or characteristics as the reference. Microwave fired samples at $1300^{\circ} \mathrm{C}$ present greater closed porosity, already presenting coalescence and the lost of pores rounded shape. Electrically fired samples at $1300{ }^{\circ} \mathrm{C}$ only show the beginning of the closed porosity growth, still very close to the microstructure of the reference sample.

SEM analysis show that at $1050{ }^{\circ} \mathrm{C}$ there is some feldspar grains dissolution in microwave fired samples, $60{ }^{\circ} \mathrm{C}$ above the formation of the liquid phase at $990 \pm 20^{\circ} \mathrm{C}$ according to [15]. Although it has not been clearly detected through SEM analysis, from XRD analysis it is shown the existence of mullite type- $\mathrm{I}$ in samples fired at $1050{ }^{\circ} \mathrm{C}$, as some peaks characteristic of the presence of mullite in the microwave fired samples are already visible. From XRD analysis, the electrically fired samples at $1140{ }^{\circ} \mathrm{C}$ present mullite peaks less intense than those microwave fired, the last ones having very similar diffractogram to the one of the reference sample. From SEM analysis, at $1140^{\circ} \mathrm{C}$, the mullite is more developed in the microwave fired samples than in the electrically fired ones, already presenting mullite type-II, yet not so developed as in the reference sample. XRD analysis shows also that for temperatures lower than the beginning of the vitrification temperature, some transformations 
occur considerably earlier in the microwave fired samples. Kaolinite dehydroxylation is a case study, already fully accomplished at $500^{\circ} \mathrm{C}$. Electrically fired samples still show residual kaolinite at $800{ }^{\circ} \mathrm{C}$, not observable from the figures. It could be also observed that talc and muscovite structures full transformation and collapse occur for lower temperatures in microwave fired samples than in electrically fired samples.

\section{Conclusions}

Microwave stoneware firing provides similar, or even better, macroscopic features comparatively to those obtained by electrical and gas firing technologies. Microstructure analysis, optical and SEM, provide a better understanding of what was observed on the macroscopic features, microwave fired samples showing more advanced stages of development comparatively to the electrically fired ones, for the same temperatures and/or firing times. DRX analysis shows that some transformations are completed at lower temperatures when microwave firing is used, kaolinite, talc and muscovite transformations being the most notorious cases. The potassium and sodium feldspars seem to completely melt earlier in the microwave fired samples than in the electrically fired ones, presenting less intense peaks. At $1140{ }^{\circ} \mathrm{C}$ the mullite crystals become more developed in the microwave fired samples than in the electrically fired ones, already presenting mullite type-II, with a diffractogram similar to that of the reference sample. The full transformations occur at temperatures closer to those referred to in the literature when microwave firing is used when compared with electric firing.

\section{Acknowledgments}

This work was funded by FEDER funds through the COMPETE 2020 Programme and National Funds through FCT - Portuguese Foundation for Science and Technology under the project UID/CTM/50025/2019. The authors express their sincere thanks to Grestel, Produtos Cerâmicos S.A. (Portugal) and to its staff member Eng. Carlos Pinto for his help and for providing the samples required for this study. The authors also express their sincere thanks to Doctor Manuel Graça from Department of Physics and I3N of University of Aveiro for its help in SEM measurements, to Doctor Maria Rosário from Central Laboratory of Analysis/CICECO of University of Aveiro for its help in the XRD analysis. Author V. A. F. Costa acknowledges the Portuguese Foundation for Science and Technology for the financial support provided through project UID/EMS/00481/2013-FCT, and CENTRO-010145-FEDER-022083.

\section{References}

[1] P. Rado, An introduction to the technology of pottery, Pergamon, 1969.

[2] C. Zanelli, M. Raimondo, et. al, J. Non. Cryst. Solids., 2011, 357, 3251-3260.

[3] Royal Copenhagen, General information about porcelain - Report, n.d.

[4] W.M. Carty, U. Senapati, J. Am. Ceram. Soc., 1998, 81, 3-20.

[5] M.R. Hosseini, A. Ahmadi, Appl. Clay Sci., 2015, 107, 238-245.

[6] R. Wroe, in: IEE Semin. New Dev. Ceram. Manuf., 1998, 1-9.

[7] D.E. Clark, W.H. Sutton, Annu. Rev. Mater. Sci., 1996, 26, 299-331.

[8] D.E. Clark, D.C. Folz, et. al, MRS Bull, 1993, 18, 41-46.

[9] M. Bhattacharya, T. Basak, Energy, 2016, 97, 306-338. 
[10] ASTM C368 e 88, American Society for Testing and Materials, 2011.

[11]BS 5416 - British Standard Specification for China Tableware, 1990.

[12] J.S. Johnson, J. Clark, et. al, J. Archaeol. Sci., 1988, 15, 403-414.

[13] G.W. Brindley, M. Nakahira, Clay Miner. Bull., 1957, 3, 117-119.

[14] Schüller K. H., Porcelain, Ceram. Monogr. - A Handb. Ceram. ,1979, 1-6.

[15] C.R.R. and H.F.M. Ernest M. Levin, Phase diagrams for ceramists, 3rd ed., American Chemical Society, 1964.

[16] H.-Y. Lu, W.-L. Wang, et. al, J. Am. Ceram. Soc., 2005, 87, 1843-1847. 


\title{
Fe/MgO POWDER COMPOSITE SINTERED BY MICROWAVE HEATING
}

\author{
R. Bureš ${ }^{1}$, M. Fáberová1, Z. Birčáková1, V. Koval'1, P. Kollár ${ }^{2}, J^{1}$. Füzer², M. Jakubčin², \\ P.Slovenský2
}
${ }^{1}$ Institute of Materials Research of Slovak Academy of Sciences, Watsonova 47, 04001 Košice, Slovak Republic
${ }^{2}$ Institute of Physics, Faculty of Science of P.J. Šafárik University, Park Angelinum 9, 04154
Košice, Slovak Republic
mfaberova@saske.sk

Keywords: microwave heating, powder sintering, metal-ceramics composite

\section{Introduction}

Composite materials based on ferromagnetic powder particles surrounded by electric insulation phase are known as soft magnetic composites (SMC). It is a group of 3D isotropic materials characterized by low coercivity, low energy losses at medium to higher frequencies and high resistivity [1]. SMC materials can contain organic, inorganic or hybrid electric insulation constituents [2]. Inorganic oxide ceramic compounds are suitable for SMC fabrication, because of their high temperature stability and high resistivity. Application of ceramic oxides is inseparable from limited ability to achieve high density of SMC's. Powder metallurgy (PM) technology is common way of SMC fabrication. The most simple and economic method is press and sintering technology. Residual porosity of PM materials depend on effectivity of densification process during pressing and sintering. In case of metallic powder materials it is possible to achieve high density already in the stage of cold pressing of powders. Short sintering is required to create tight bond among close packed powder particles. Hard and brittle ceramics is incompressible. Powder ceramic is consolidated using low pressure followed by long term sintering to achieve neck creation between ceramic powder particles. Metallic/ceramics coated powders are characterized by limited ability to plastic deformation in dependence on ceramic content and their volume distribution. Soft magnetic applications require to use thin and compact ceramic coating layer on the ferromagnetic particle [3]. This spatial distribution of the ceramic secondary phase have to be preserved during pressing and sintering technology steps to maximize magnetic properties [4]. Ideal SMC microstructure consists from the metallic primary phase and the regular ceramic network. Limited plastic deformation and requirement of well bonded ceramic network is the motivation to search new methods of SMC fabrication. One of perspective consolidation methods is the microwave sintering.

Study of the interaction mechanism of metal-ceramic composites during microwave sintering led to identification of so called micro-focusing effect. Densification of metalceramic during the microwave sintering is selective process induced by the mixed interaction mechanisms on the particle-free surface and interface as it was shown in Al-SiC powder composite [5]. Local focus effect of electromagnetic fields and its influence on microwave sintering of metal-ceramic composite was investigated in $\mathrm{Sn}-\mathrm{SiO}_{2}$ composite. This focus effect depends on the geometric configurations of particles, and may be an important factor for the microstructure evolution during microwave sintering. [6].

The SMC materials are hierarchic microstructurally inhomogeneous composites ordered to 3D continuous secondary-dielectric phase with isolated primary-ferromagnetic phase [7]. This ordering correspond to microstructurally inhomogeneous, but macroscopically 
homogeneous microstructures. Subject of the paper is an investigation of microwave sinterering behavior of the $\mathrm{Fe} / \mathrm{MgO}$ powder material as well as an influence of the microwave heating on soft magnetic and mechanical properties of the sintered composites.

\section{Material and methods}

Iron powder ASC100.29 (Höganäs, AB) with particle size distribution $\mathrm{d}_{0.1}=45 \mu \mathrm{m}, \mathrm{d}_{0.5}=100$ $\mu \mathrm{m}$ and $\mathrm{d}_{0.9}=195 \mu \mathrm{m}$ was used as ferromagnetic constituent. Electric insulation constituent was provided by $\mathrm{MgO}$ (US Research Nanomaterials, Inc.) with narrow particle size distribution $1 \mu \mathrm{m}$ of mean size. Powder mixtures were prepared using resonant acoustic mixing method in Resodyn ${ }^{\mathrm{TM}}$ LabRAM mixer. Hydraulic press machine LabTest $5.600 \mathrm{Zl}$ was used for cold pressing to prepare green body. Green compacts were sintered by conventional heating (CS) in chamber muffle furnace. Microwave (MW) sinterability analysis was realised in single mode microwave cavity at frequency of $2.45 \mathrm{GHz}$. Cylindrical cavity with dimensions in diameter of $28 \mathrm{~mm}$ and height of $80 \mathrm{~mm}$ was equipped with Impedance analyser HiPom and IR pyrometer Optris CT (supplied by UPV-Institute for Information and Communication Technologies). Microwave sintering was provided by multimode microwave laboratory system Hamilab V3000 (Synotherm) equipped with Raytek IR pyrometer. Density was measured by Archimedes method. Hardness measurement was provided by Vickers hardness tester Leco LM700AT. Elastic properties were measured by impulse excitation technique using Buzz-o-Sonic system. Resistivity was measured by four point probe method using Mitsubishi Loresta AX equipment. Complex permitivity and permeability were measured by impedance spectroscopy method using HP 4294A impedance analyser. Coercive force meter DX-320 was used for coercive force measurement. Transverse rupture strength was measured by three point bending test method using universal testing machine LabTest $5.600 \mathrm{Zl}$.

\section{Results and discussion}

Iron and magnesia oxide powder were dry mixed for 1 minute in LabRAM mixer to prepare $\mathrm{Fe}+2$ wt. $\% \mathrm{MgO}$ and $\mathrm{Fe}+5$ wt. $\% \mathrm{MgO}$ mixtures. Intensive acoustic mixing packed $\mathrm{Fe}$ and $\mathrm{MgO}$ in a micro-agglomerates as it is shown in Fig. 1. The mixtures were uniaxially cold pressed in closed die at pressing pressure $600 \mathrm{MPa}$ to the shape of cylinder size of $\phi 10 \times 3 \mathrm{~mm}$, prism bar size of $4 \times 5 \times 20 \mathrm{~mm}$ and ring shape size of $\phi_{\text {in }} 28 \times \phi_{\text {out }} 25 \times 3 \mathrm{~mm}$. Cylinder shape samples were sintered in the single mode MW cavity to investigate sinterability of the prepared mixtures. 


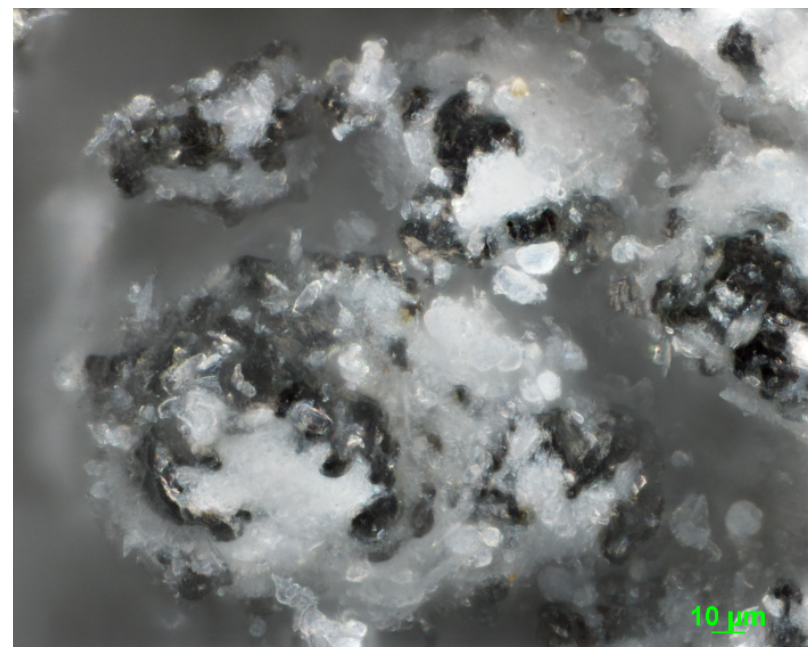

Fig. 1. $\mathrm{Fe}+5 \mathrm{wt} . \% \mathrm{MgO}$ dry mixed to micro-agglomerates.

In Figures 2-4 it is shown the time-temperature and MW energy absorption diagram of the single mode $\mathrm{MW}$ sintering process in air atmosphere at $600^{\circ} \mathrm{C}$. Pure iron green compact can be treated using MWS, whereas MW heating is more stable at lower relative density, Fig. 2. High density achieved at high pressing pressure $(900 \mathrm{MPa})$ leads to instability, plasma formation and thermal run away due to low resistivity at low porosity of the iron green compact. Pressing pressure has exactly the reverse effect on $\mathrm{MgO}$ heating as it is shown in Fig. 3. It was achieved limited temperature in MWS of low density $\mathrm{MgO}$ pressed at pressure of 100 and $300 \mathrm{MPa}$. $\mathrm{MgO}$ green compact pressed at $600 \mathrm{MPa}$ was possible to heat in $\mathrm{MW}$ to $600^{\circ} \mathrm{C}$. It can be expected that higher pressing pressure can improve stability of MW heating, but it is impossible to apply higher pressure due to spring back effect in pure $\mathrm{MgO}$ ceramic powder system. $\mathrm{Fe} / \mathrm{MgO}$ green compacts were pressed at $600 \mathrm{MPa}$ of pressing pressure. Results of SM MWS in Fig. 4 shows very good stable MW heating in case of $\mathrm{Fe} / 2 \mathrm{MgO}$ material. $\mathrm{Fe} / 5 \mathrm{MgO}$ can be controlled heated in $\mathrm{MW}$ with a little higher temperature deviation in comparison with $\mathrm{Fe} / 2 \mathrm{MgO}$. The results of complex dielectric permittivity analysis are presented in Table 1. Imaginary part of complex permittivity is increasing with electric conductivity of the interphase regions in powder system based on conductive metallic particles with insulation layer. Depending on the value of the $\varepsilon " 16400$ or 7800 for $\mathrm{Fe} / 2 \mathrm{MgO}$ and $\mathrm{Fe} / 5 \mathrm{MgO}$ respectively, the power absorbed in the interphases can be comparable to the power absorbed in the metal particles. The boundary losses act to smoothen the resonant absorption peaks, making it possible to implement stable microwave heating processes as it was demonstrated by simulation within semi-analytic model [8]. 


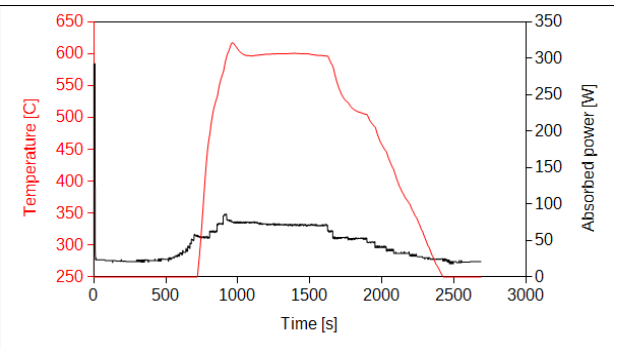

Fe green - $300 \mathrm{MPa}$

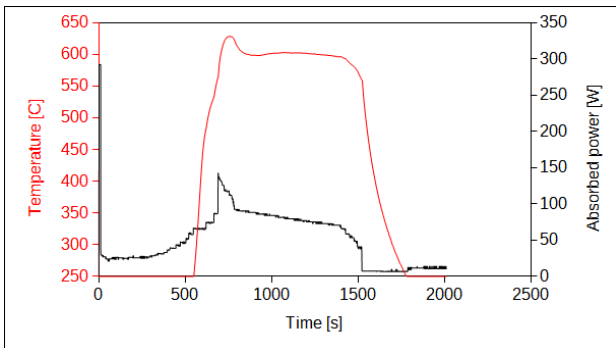

Fe green - $600 \mathrm{MPa}$

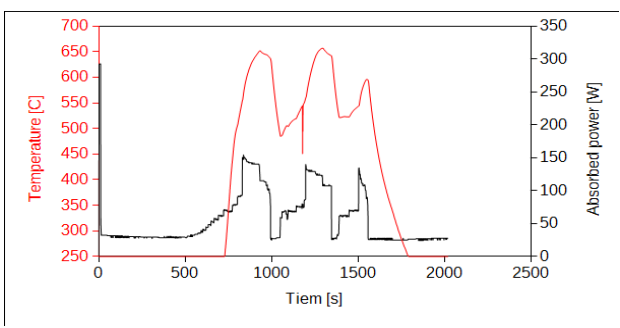

Fe green - $900 \mathrm{MPa}$

Fig. 2. Single mode microwave sintering analysis - Fe green compacts.

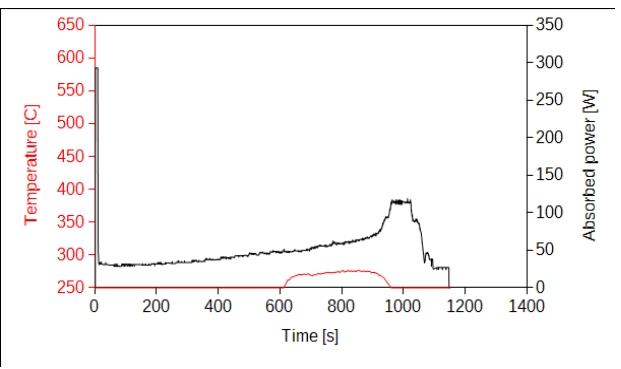

$\mathrm{MgO}$ green - $100 \mathrm{MPa}$

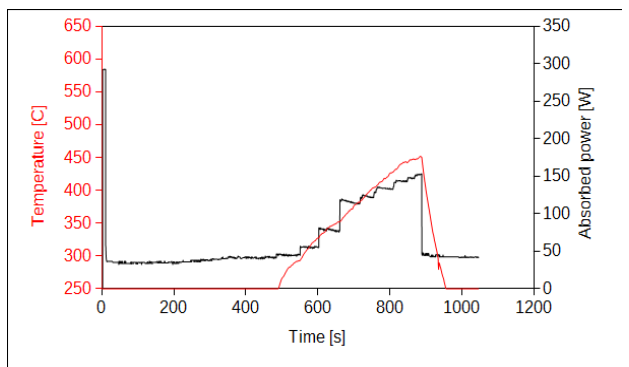

$\mathrm{MgO}$ green - $300 \mathrm{MPa}$

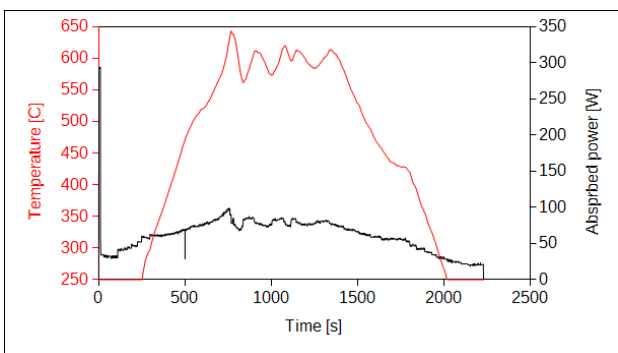

$$
\mathrm{MgO} \text { green }-600 \mathrm{MPa}
$$

Fig. 3. Single mode microwave sintering analysis $-\mathrm{MgO}$ green compacts. 


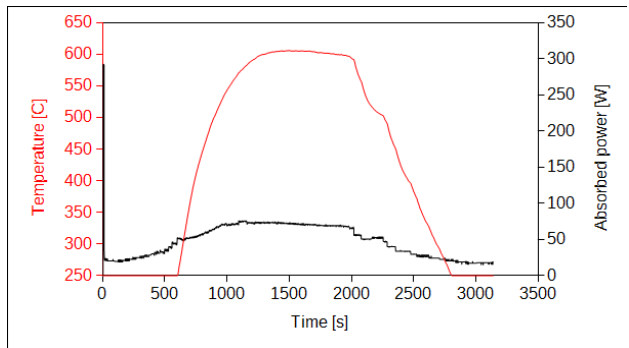

$\mathrm{Fe} / 2 \mathrm{MgO}$ green

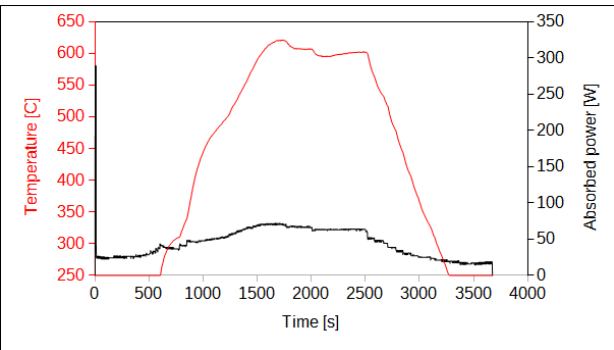

$\mathrm{Fe} / 5 \mathrm{MgO}$ green

Fig. 4. Single mode microwave sintering analysis $-\mathrm{Fe} / \mathrm{xMgO}$ green compacts.

Table 1. Dielectric properties of $\mathrm{Fe} / \mathrm{xMgO}$ green compacts.

\begin{tabular}{ccccc}
\hline sample & $\varepsilon^{\prime} @ 1 \mathrm{MHz}$ & $\varepsilon^{\prime \prime} @ 1 \mathrm{MHz}$ & $\varepsilon^{\prime} @ 10 \mathrm{MHz}$ & $\varepsilon^{\prime \prime} @ 10 \mathrm{MHz}$ \\
& {$[-]$} & {$[-]$} & {$[-]$} & {$[-]$} \\
\hline Fe-green & 23 & 5244 & 33 & 561 \\
MgO-green & 1.53 & 0.43 & 1 & 0.3 \\
Fe2MgO-green & 16 & 16400 & 6 & 1500 \\
Fe5MgO-green & 10 & 7800 & 4.8 & 816 \\
\hline
\end{tabular}

$\varepsilon^{\prime}$-Real part of complex permitivity, $\varepsilon^{\prime \prime}-$ Imaginary part of complex permitivity at specific frequency

On the basis of the MW sinterability evaluation the multimode MW sintering process was set. One sintering batch consisted from 3 cylinders, 3 prism bars and 3 rings of $\mathrm{Fe} / \mathrm{MgO}$ green samples. Experimental batch of samples was placed into the dense alumina crucible, then into the second low density alumina chamber and finally into the third alumina fiber chamber. Chambers and crucible were covered by top with the slot for pyrometric temperature measurement. This gradient density alumina assembly was closed in the double wall stainless steel water cooled multimode cylindrical microwave process chamber, in Fig. 5 , powered by $3 \times 1 \mathrm{~kW}$ magnetrons. MW output power was automatically driven based on temperature measurement feedback to the required temperature. Smooth process control was achieved by programmed corrections in the temperature regions where a change of MW energy absorption was predicted by the single mode MW analysis. Two composites $\mathrm{Fe} / 2 \mathrm{MgO}(\mathrm{M} 2)$ and $\mathrm{Fe} / 5 \mathrm{MgO}(\mathrm{M} 5)$ prepared using multimode $\mathrm{MWS}$ at $600^{\circ} \mathrm{C}$, for $15 \mathrm{~min}$., with $10^{\circ} \mathrm{C} / \mathrm{min}$ of heating and cooling rate, in dry air atmosphere. Analogous conventional sintered samples $\mathrm{C} 2$ and $\mathrm{C} 5$ were prepared using muffle furnace at $600^{\circ} \mathrm{C}$, for $60 \mathrm{~min}$., with $10^{\circ} \mathrm{C} / \mathrm{min}$ of heating and cooling rate, in air. 


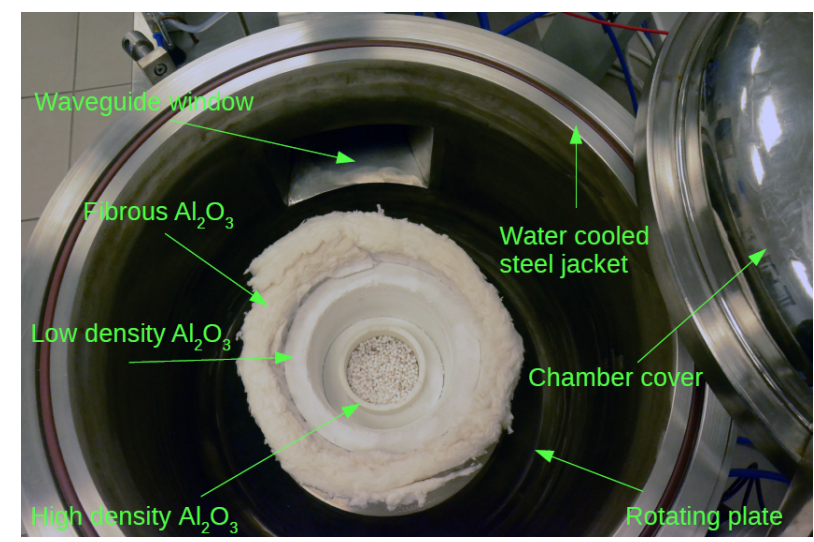

Fig. 5. Multimode microwave process chamber assembling.

Microwave sintering led to higher relative sintered density in comparison with conventional sintering as it is shown in Table 2. Elastic properties represented by Young modulus values are in good agreement with relative density. Flexural strength of $\mathrm{Fe} / 2 \mathrm{MgO}$ composite shows increasing value after MWS as well as statistical scatter is also higher in comparison with $\mathrm{C} 2$ sample. In case of MWS $\mathrm{Fe} / 5 \mathrm{MgO}$ composite, TRS value is lower with high value of statistical scatter than C5 sample. Results of the microhardness measurement indicates a reason of mechanical strength behavior. Two families of the microhardness values were found in the the case of $\mathrm{MW}$ sintered $\mathrm{Fe} / 5 \mathrm{MgO}$ composite. The value of $4.1 \mathrm{GPa}$ fit the tendency to decreasing microhardness value after MWS. Another significantly different value of $0.9 \mathrm{GPa}$ of MWS $\mathrm{Fe} / 5 \mathrm{MgO}$ composite indicate new phase creation in the microwave sintered composite at higher content of $\mathrm{MgO}$.

The results of electric and magnetic properties in dependence on sintering process technology are presented in Table 3 . The value of DC resistivity of the $\mathrm{Fe} / 2 \mathrm{MgO}$ composite is almost not influenced with sintering technology. It is not a case of $\mathrm{Fe} / 5 \mathrm{MgO}$ composite, which resistivity is decreasing by microwave sintering. Real part of the complex permeability at higher frequency $(1 \mathrm{MHz})$ has the same tendency as TRS value. MWS increases the real part of permeability value in comparison with CS with the exception of $\mathrm{Fe} / 5 \mathrm{MgO}$ composite. Real part of the complex permeability at lower frequency $(1 \mathrm{kHz})$ is increased by MWS. MWS causes increasing of coercive force values in both M2 and M5 composites. 
Table 2. Mechanical properties of the sintered composites.

\begin{tabular}{ccccc}
\hline sample & $\begin{array}{c}\mathrm{D}_{\mathrm{r}} \\
{[\%]}\end{array}$ & $\begin{array}{c}\mathrm{HV} 10 \\
{[\mathrm{GPa}]}\end{array}$ & $\begin{array}{c}\mathrm{E} \\
{[\mathrm{GPa}]}\end{array}$ & $\begin{array}{c}\text { TRS } \\
{[\mathrm{MPa}]}\end{array}$ \\
\hline $\mathrm{C} 2$ & 82.92 & 4.94 & 122.4 & 103.9 \\
& \pm 0.23 & & \pm 0.8 & \pm 2.2 \\
$\mathrm{C} 5$ & 80.82 & 5.71 & 92.0 & 77.9 \\
& \pm 0.71 & & \pm 2.8 & \pm 5.0 \\
\hline M2 & 84.88 & 3.64 & 133.2 & 109.4 \\
& \pm 0.66 & & \pm 4.7 & \pm 17.0 \\
M5 & 82.27 & $4.1 ; 0.9^{*}$ & 93.8 & 71.7 \\
& \pm 0.80 & & \pm 12.8 & \pm 21.0 \\
\hline
\end{tabular}

$\mathrm{D}_{\mathrm{r}}-$ Relative density, HV-Vickers hardness, E-Young modulus, TRS-Transverse Rupture Strength, "two groups of the hardness values were found

Table 3. Electric and magnetic properties of the sintered composites.

\begin{tabular}{|c|c|c|c|c|}
\hline sample & $\begin{array}{c}\rho \\
{[\Omega . \mathrm{cm}]}\end{array}$ & $\begin{array}{c}\mathrm{H}_{\mathrm{c}} \\
{[\mathrm{A} / \mathrm{m}]}\end{array}$ & $\begin{array}{c}\mu^{\prime} @ 1 \mathrm{kHz} \\
{[-]}\end{array}$ & $\begin{array}{c}\mu^{\prime} @ 1 \mathrm{MHz} \\
{[-]}\end{array}$ \\
\hline $\mathrm{C} 2$ & $\begin{array}{c}4.56 \mathrm{e}-4 \\
\pm 3.53 \mathrm{e}-5\end{array}$ & $\begin{array}{c}304.7 \\
\pm 7.7\end{array}$ & 55 & 10 \\
\hline $\mathrm{C} 5$ & $\begin{array}{c}5.24 \mathrm{e}-2 \\
\pm 6.47 \mathrm{e}-3\end{array}$ & $\begin{array}{c}321.5 \\
\pm 8.7\end{array}$ & 27 & 24 \\
\hline M2 & $\begin{array}{c}4.71 \mathrm{e}-4 \\
\pm 2.53 \mathrm{e}-5\end{array}$ & $\begin{array}{c}426.5 \\
\pm 7.0\end{array}$ & 71 & 12 \\
\hline M5 & $\begin{array}{c}6.39 \mathrm{e}-3 \\
\pm 5.23 \mathrm{e}-4\end{array}$ & $\begin{array}{l}575.2 \\
\pm 9.2\end{array}$ & 40 & 21 \\
\hline
\end{tabular}

$\rho$-Resistivity, $\mathrm{H}_{\mathrm{c}}-$ Coercive force at $30 \mathrm{kA} / \mathrm{m}, \mu^{\prime}-$ Real part of complex permeability at specific frequency

Results of the complex permittivity measurements in Table 4 show a tendency in dielectric properties in dependence on heating technology. Real part of complex permittivity is increased by MWS as well as imaginary part of complex permittivity is decreased at the same time in case of $\mathrm{Fe} / 2 \mathrm{MgO}$ composite. Different behavior were found in case of $\mathrm{Fe} / 5 \mathrm{MgO}$ composite. Real part of complex permittivity of the M5 is significantly lower in comparison with CS composites, while the imaginary part of complex permittivity is very little influenced by a heating technology.

Table 4. Dielectric properties of Fe/xMgO sintered composites.

\begin{tabular}{ccccc}
\hline sample & $\varepsilon^{\prime} @ 1 \mathrm{MHz}$ & $\varepsilon^{\prime \prime} @ 1 \mathrm{MHz}$ & $\varepsilon^{\prime} @ 10 \mathrm{MHz}$ & $\varepsilon^{\prime \prime} @ 10 \mathrm{MHz}$ \\
& {$[-]$} & {$[-]$} & {$[-]$} & {$[-]$} \\
\hline C2 & 1.6 & 4800 & 5 & 710 \\
C5 & 14 & 1050 & 14 & 109.2 \\
\hline M2 & 2 & 2400 & 6.4 & 480 \\
M5 & 4 & 1040 & 4.8 & 100.8 \\
\hline
\end{tabular}

$\varepsilon^{\prime}-$ Real part of complex permitivity, $\varepsilon^{\prime \prime}$-Imaginary part of complex permitivity at specific frequency 
Light optical microscopy observation of the sintered composites is presented in Fig. 6 . Lower content of the $\mathrm{MgO}$ in $\mathrm{Fe} 2 \mathrm{MgO}$ powder material leads to relatively regular spatial distribution of the $\mathrm{MgO}$ in sintered composite. Increasing $\mathrm{MgO}$ content causes higher tendency to agglomeration of the secondary phase. MWS process helped to preserve distribution of $\mathrm{MgO}$ and suppress formation of iron-iron connections in $\mathrm{Fe} 2 \mathrm{MgO}$ composite. In case of $\mathrm{Fe} 5 \mathrm{MgO}$ composite, strong agglomeration process were supported by MWS. Microstructure observations confirm partial formation of a new phase in the interphase structure region between $\mathrm{Fe}$ and $\mathrm{MgO}$ in $\mathrm{MWS} \mathrm{Fe} 5 \mathrm{MgO}$ composites. Non-stoichiometric $\mathrm{MgFe}_{2} \mathrm{O}_{4}$ formation can be expected in connection of $\mathrm{MgO}$ with Fe particles [9], because iron particles contained residual oxides as well as sintering process was done in air atmosphere.

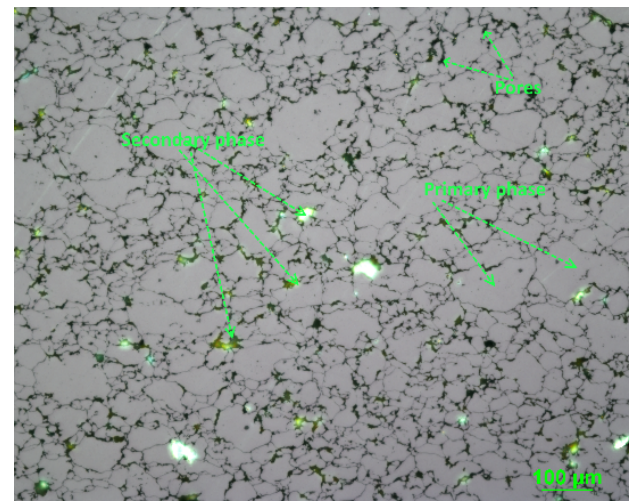

$\mathrm{Fe} / 2 \mathrm{MgO}-\mathrm{CS}$

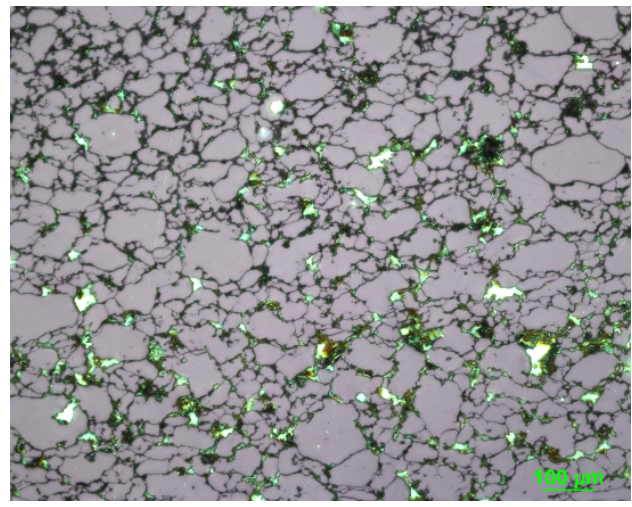

$\mathrm{Fe} / 5 \mathrm{MgO}-\mathrm{CS}$

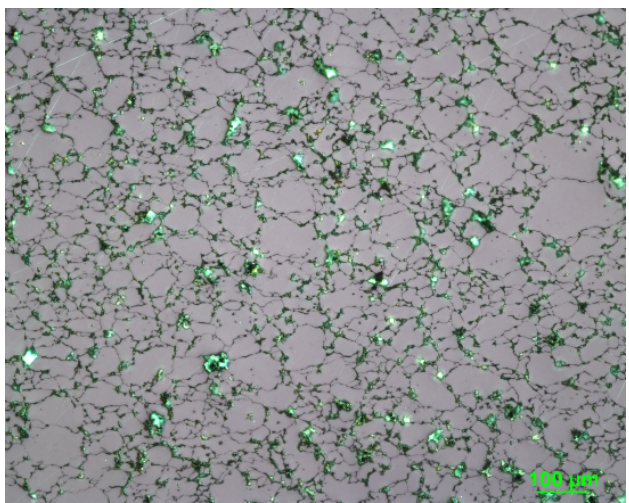

$\mathrm{Fe} / 2 \mathrm{MgO}-\mathrm{MW}$

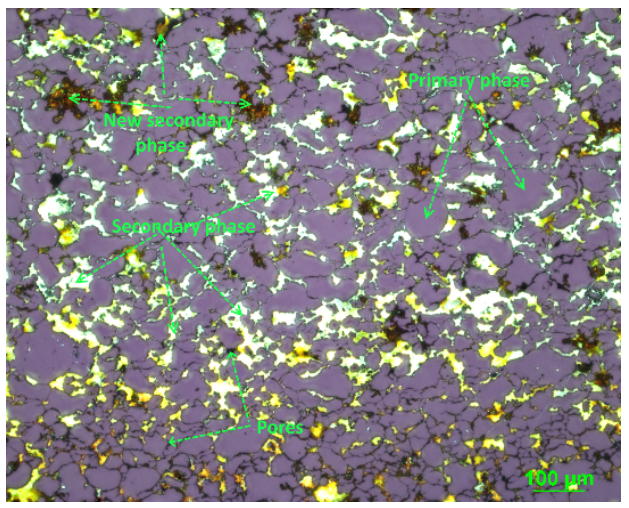

$\mathrm{Fe} / 5 \mathrm{MgO}-\mathrm{MW}$

Fig. 6. Microstructure of microwave and conventionally sintered composites observed in polarised light by light optical microscopy.

There is a focus effect of microwave E-fields between the metallic-ceramic particles. For example, the peak microwave E-field within the neck region between the ceramic and metallic particles is about 24.8 times of the applied field as it was shown Y.C. Li [5,6]. This effect will accelerate the mass diffusion and promote the sintering process, and may be an important mechanism for new phase creation or it can create specific distribution of 
secondary phase in the composite matrix. This behaviour can be supported or suppressed by small changes in content ratio of the dielectric constituent in the composite. Yoshikawa present recent studies and introduced some topics on negative permittivity of metallic materials and some specific phenomena related with the so-called "microwave non-thermal effect" [10]. This can be a way to clarify different properties of the FeMgO composites achieved in microwave sintering and conventional sintering process.

\section{Conclusions}

Powder soft magnetic composites $\mathrm{Fe} / 2 \mathrm{MgO}$ and $\mathrm{Fe} / 5 \mathrm{MgO}$ were prepared using press and sintering method. Sinterability of Fe-MgO powder system was investigated using single mode microwave sintering and complex permittivity measurement. Mechanical, elastic, electric and magnetic properties of microwave sintered composites were investigated. FeMgO SMC material can be effectively produced using microwave sintering process. Sintered composites differ in physical and mechanical properties in dependence on applied sintering technology as well as in dependence on $\mathrm{MgO}$ content ratio. Microwave sintering technology led to different microstructure formation in $\mathrm{Fe} / 5 \mathrm{MgO}$ composite.

\section{Acknowledgement}

This work was supported by projects: APVV 0115-15 and VEGA 2/0108/18.

\section{References}

[1] Périgo, E.A., Weidenfeller, B., Kollár, P., Füzer, J., Appl. Phys. Rev., 2018, 5, 031301-1-37

[2] Bures, R., Strečková, M., Fáberová, M., Kollár, P., Füzer, J., Arch. Metall. Mater., 2017, 62 (2B), 1149-1154

[3] Bures, R., Fáberová, M., Kurek, P., PMP, 2018, 18, 103-110

[4] Pittini-Yamada, Y., Périgo, E.A., de Hazan, Y., Nakahara, S., Acta Mater., 2011, 59, 4291-4302

[5] Li, Y.C., Xu, F., Hu, X.F., Kang, D., Xiao, T.Q., Wu, X.P., Acta Mater., 2014, 66, 293-301

[6] Li, Y., Xu, F., Luan, Y., Yan, X., Dong, B., Shen, Y., Hu, X., J. Alloys Compd., 2016, 687, 943-953

[7] Huang, L.J., Geng, L., Peng, H-X., Prog. Mater. Sci., 2015, 71, 93-168

[8] Rybakov, K.I., Volkovskaya, I.I., Ceram. Int., 2019, 45, 9567-9572

[9] Deraz, N.M., Abd-Elkader, O.H., Int. J. Electrochem. Sci., 2013, 8, 8632-8644

[10] Yoshikawa, N., IOP Conf. Ser.: Mater. Sci. Eng., 2018, 424, 012041-4 


\title{
SINTERING OF MLCC'S BARIUM TITANATE WITH MICROWAVES
}

Juan A. Aguilar-Garib, Osvaldo Tijerina-García, Javier Garza-Guajardo

Universidad Autónoma de Nuevo León, FIME, Pedro de Alba s/n, Cd. Universitaria, San Nicolás de los Garza, NL, México

Juan.aguilargb@uanl.edu.mx

Keywords: microwave sintering, barium titanate, MLCC

\begin{abstract}
A comparison of microwave and conventional, in an electric resistance furnace, sintered layers of dielectric base barium titanate $\left(\mathrm{BaTiO}_{3}\right)$ of the kind employed for multilayer ceramic capacitors (MLCC) was performed. Two kinds of samples were used for each processing method; the layers alone without electrodes, and the green MLCC with the layers and electrodes interdigitated. Samples were exposed to microwaves for 20 minutes and heated up to $1050^{\circ} \mathrm{C}$ and $1150^{\circ} \mathrm{C}$ for sintering in a crucible with graphite that acted as reduction agent and microwave susceptor. Conventional sintering was performed in the same arrangement but lasted 120 minutes since it was found that 20 minutes was not enough time to achieve sintering. Heating rate in both cases was $10^{\circ} \mathrm{C} / \mathrm{min}$. It was observed that the layers without the electrodes achieve about the same densification for both processes, while in the case of the green MLCC's the results were variable, ranging from sample that became dust, to cracked samples and some well sintered ones. At least in the microwave case, it is possible that the variability of the results is due to the importance of the location of the sample in the cavity that in turn affects the electric field pattern, especially because the presence of the electrodes that can cause overheating around them.
\end{abstract}

\section{Introduction}

Multilayer ceramic capacitors (MLCC) are one of the most used passive components in consumer electronics. They are fabricated with a base metal electrode technology, where successive layers of dielectric and electrodes. Barium titanate $\left(\mathrm{BaTiO}_{3}\right)$ based ceramic is among the most common dielectric materials employed as dielectric, which is formed in thin layers by type casting of a barbotine. Then the electrodes are printed over the layer, and in turn they are stacked to obtain an interdigitated electrode structure (Figure 1).
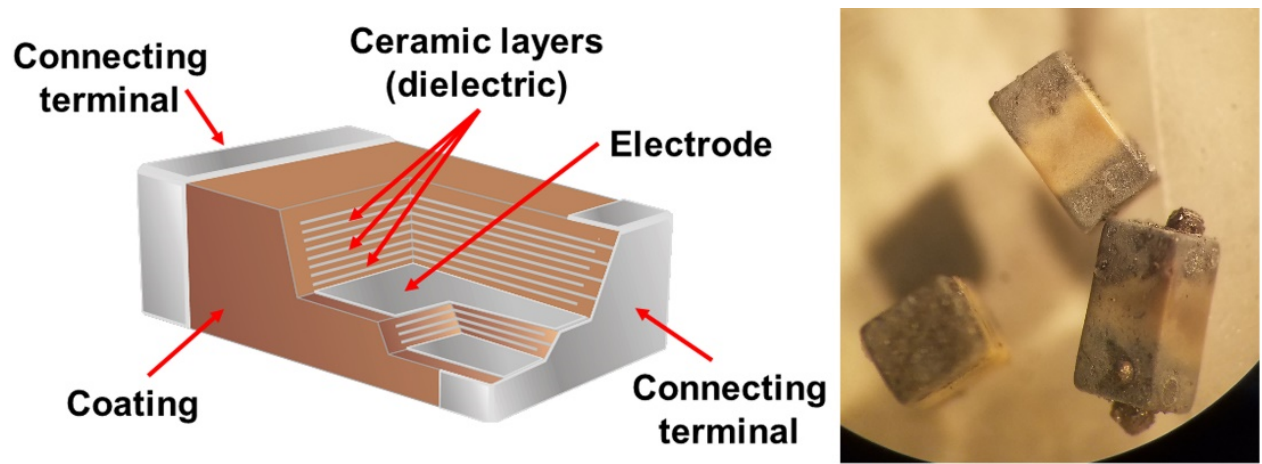

Fig. 1. Scheme of the electrode structure of a MLCC (left). Actual MLCC's, the size is approximately $1.5 \mathrm{~mm}$ X $0.8 \mathrm{~mm}$ X $0.8 \mathrm{~mm}$ (right). 
This structure is then sintered under an atmosphere with the dual purpose of heating and provide reductive conditions to prevent electrode oxidation and the conditions for achieving the final properties of the MLCC.

There are several reports about microwave processing of ceramics [1], some under claims of uniform heat transfer and higher efficiency [2], and reaction rates compared to conventional heating, as well as non-thermal effects of microwaves in the processing [3]. The purpose of this research is to sinter layers of $\mathrm{BaTiO}_{3}$, and the green MLCC's with microwaves and compare them with those sintered conventionally.

One important issue when comparing both processes is that in the conventional case, the maximum temperature and heating rate is governed by conduction of the sample with the boundary conditions dictated by the furnace. The only way for increasing the heating rate is increasing the furnace temperature, but there is the risk to get inappropriate sintering conditions, such as melting of the surface. Heating below this point in conventional heating is relatively easy using the control of the furnace. However, in the case of microwaves, the heating rate is driven by the sample absorption, or the susceptor, hence, often the temperature excesses the set point, and the results obtained from each processing method are different because the thermal conditions where different and it is difficult to raise a conclusion from this kind of tests. Temperature control was considered in the experiments.

Another aspect to consider is that sintering the layers of $\mathrm{BaTiO}_{3}$ with microwaves could resemble the conventional heating, while sintering the MLCC's certainly would produce a different field pattern in the layer due to the metallic electrode, so that the dielectric could exhibit high thermal gradients.

\section{Experimental}

The microwave cavity was feed with a $2.45 \mathrm{GHz}$ magnetron, $1100 \mathrm{~W}$ nominal power, coupled to a WR284 waveguide system with a circulator, dummy load, and a directional coupler, so that forward and reflected powers were registered (Figure 2). The magnetron was taken out from a kitchen oven. These devices use to adjust power output by averaging the on cycling time, keeping the power on for a fraction of time according to the desired power. $50 \%$ nominal power means a $50 \%$ of the time on. In this case the electronics was modified so that that it was possible to adjust the power output all the time that the on/off control dictated according to the heating slope. It is quite common that the actual power of these magnetrons is below the nominal one, and the maximum value is rather variable.

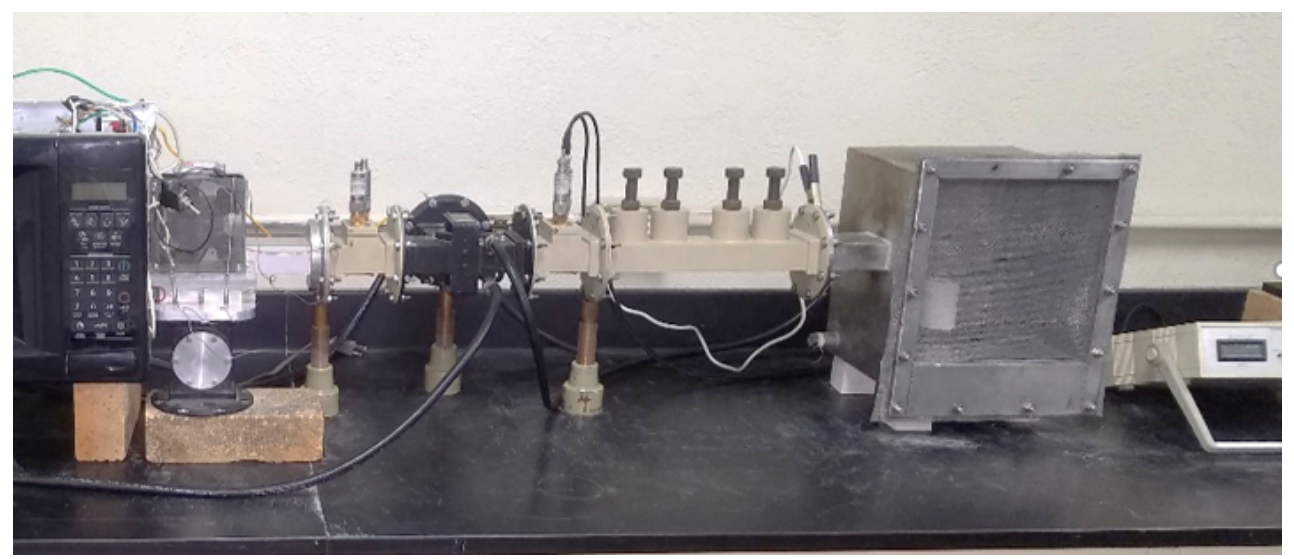

Fig. 2. Experimental arrangement showing the microwave cavity (on the right). 
The temperature was measured with a shield thermocouple and controlled with an on/off device as in reference [1]. It was confirmed that the thermocouple was in such position that did not exhibit self-heating. Optical pyrometry was also employed for having this confirmation. The $\mathrm{BaTiO}_{3}$ was prepared in layers by tape casting and stacked in the same form than the MLCC's, but without the electrodes. The samples of $\mathrm{BaTiO}_{3}$ without electrodes were placed in a crucible with $5 \mathrm{~g}$ of graphite as thermal susceptor and reducing agent, and then set in the microwave cavity for being sintered at $1050^{\circ} \mathrm{C}$ and $1150^{\circ} \mathrm{C}$. These temperatures are often reported in literature [4-6]. The samples were exposed to microwaves for 20 minutes, at a heating rate $10^{\circ} \mathrm{C} / \mathrm{min}$. For conventional heating, the samples were placed in the same crucible with graphite as well in an electric resistance furnace, but in this case, it is only for providing the reduction conditions. It was found that the 20 minutes exposition was not enough for conventional sintering, hence, these tests lasted 120 minutes each with the same heating rate. The green capacitors were treated in the same arrangement and conditions

\section{Results and discussion}

The temperature control was an important issue because the sample was getting hot too quickly so that the proposed heating rate was exceeded. Therefore, it was necessary to adjust the delivered power and then increase it as needed. The appearance of the system heated to $1150^{\circ} \mathrm{C}$ can be seen in figure 3 .

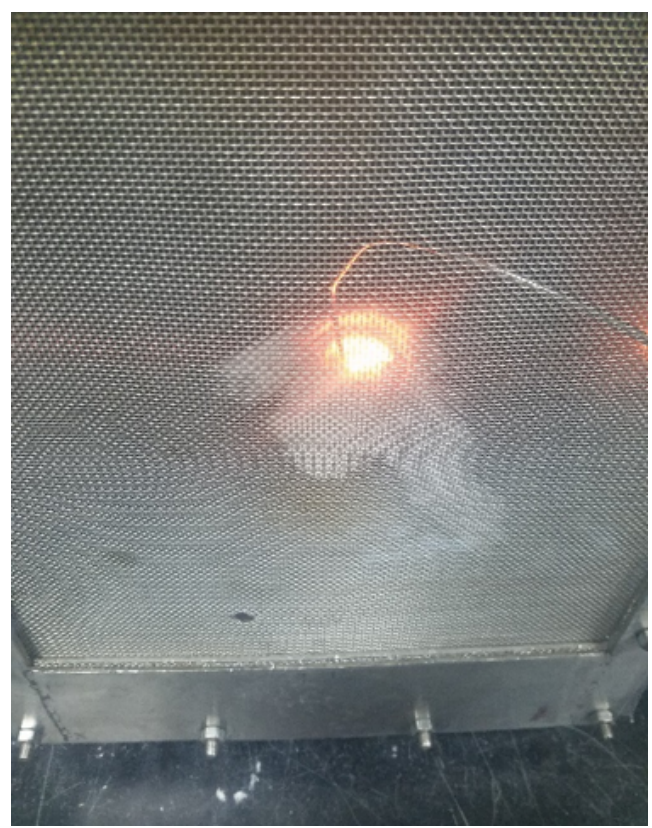

Fig. 3. Appearance of the sample heated at $1150^{\circ} \mathrm{C}$

Figure 4 shows a plot of the earlier stage of heating showing the forward and reflected power against time (sampling time, 3 seconds), while figure 5 shows the whole test but with a sampling time of 1 minute. The cooling time is also here, that is why there is no power at the end, the goal temperature was kept by 20 minutes. 
Notice how forward power is low at the beginning and higher as temperature increased, this adjusted was performed manually. Once that it was possible to control the temperature, the different tests were conducted.

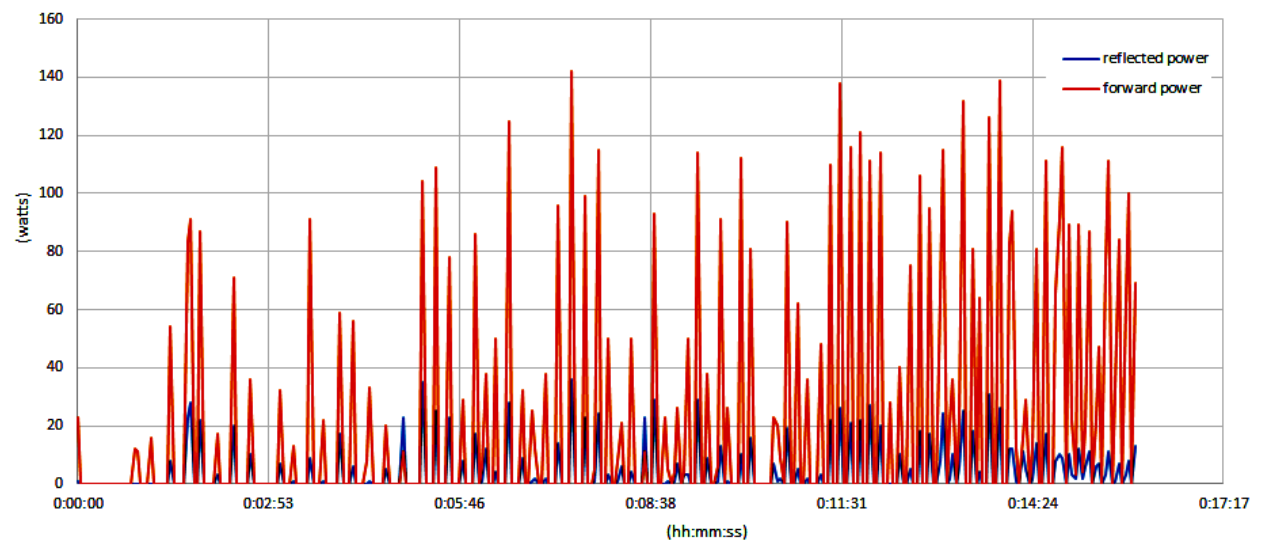

Fig. 4. Example of the measured forward and reflected power during one of the experiments ( 3 seconds of sampling time).

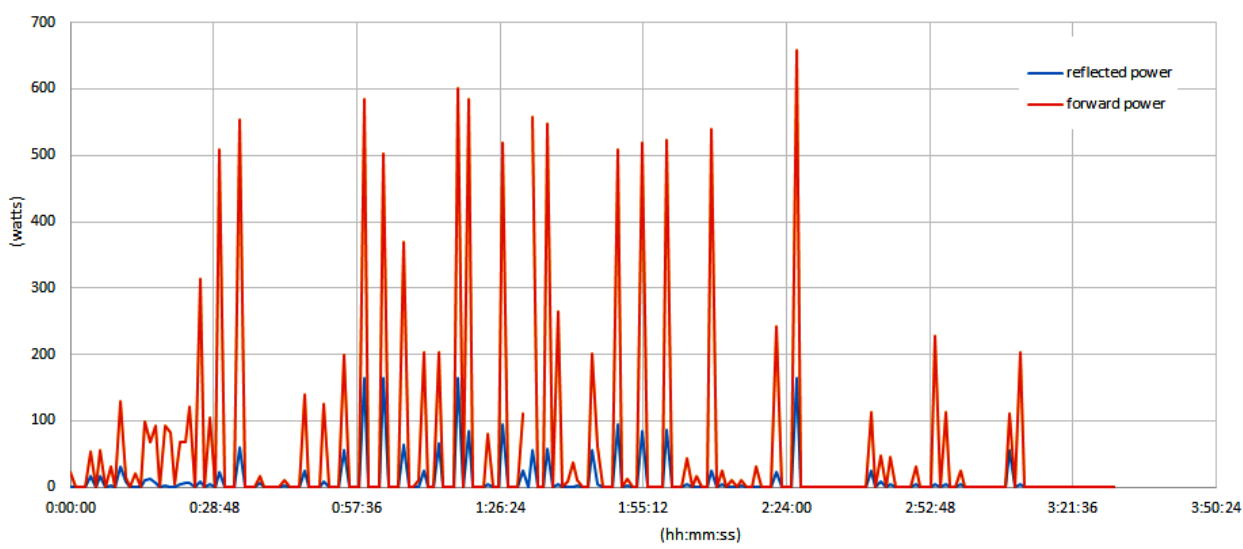

Fig. 5. Example of the measured forward and reflected power during one of the experiments ( 1 minute of sampling time).

The sintering degree can be determined by the rate of achieved density against theoretical density, which in this case is $6.02 \mathrm{~g} / \mathrm{cm}^{3}$. Density was determined by Archimedes method, and the results are shown in table 1.

Table 1. Relative density of the sintered layers.

\begin{tabular}{ccc}
\hline Temperature & Conventional & Microwave \\
\hline $1050^{\circ} \mathrm{C}$ & 0.92 & 0.91 \\
$1150^{\circ} \mathrm{C}$ & 0.88 & 0.92 \\
\hline
\end{tabular}

Sintering the MLCC's produce different results than layers alone, since some of the samples became dust and others developed cracks, just few of them were sintered consistently. Figure 6 shows the aspect of the microwave sample that is sintered with cracks, meaning that this MLCC would not work properly. In this case sintering was compared qualitatively through 
hardness measurements, but they are not conclusive. The same figure shows a MLCC heated in the electric resistance furnace, that also looks sintered, but with a different color. It was expected that only the samples processed in with microwaves would develop cracks, bit as it can be seen in figure 7, the samples processed conventionally also developed them. Since in the microwave case the samples were cooled down at a controlled rate, they were still exposed to microwaves in cycles (figure 5) during cooling. In the conventional case, the samples were cooled down in the furnace. Therefore, it is expected that at least in this late case there are not thermal stresses.
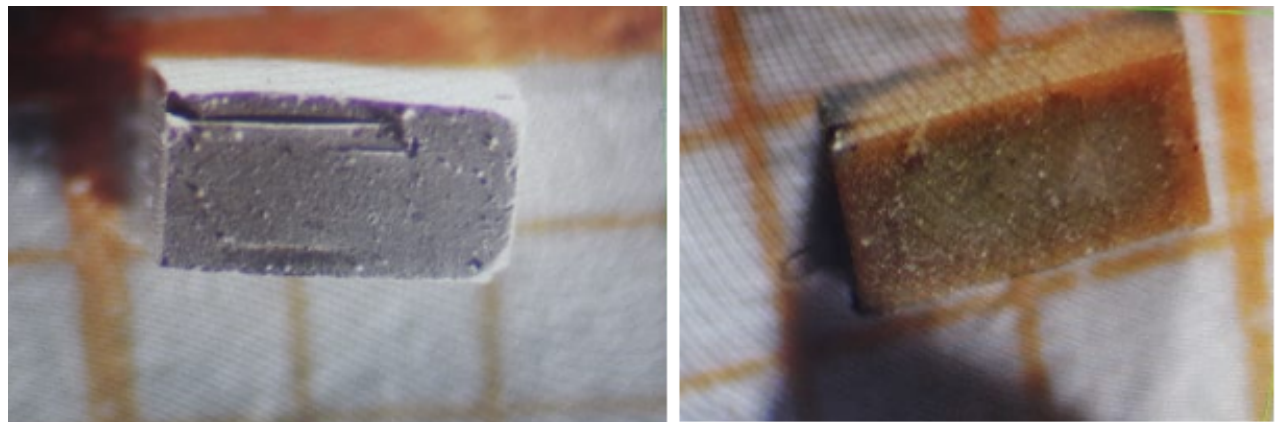

Fig. 6. MLCC sintered with microwaves (left) and conventionally (right). Grid is $1 \mathrm{~mm}$.

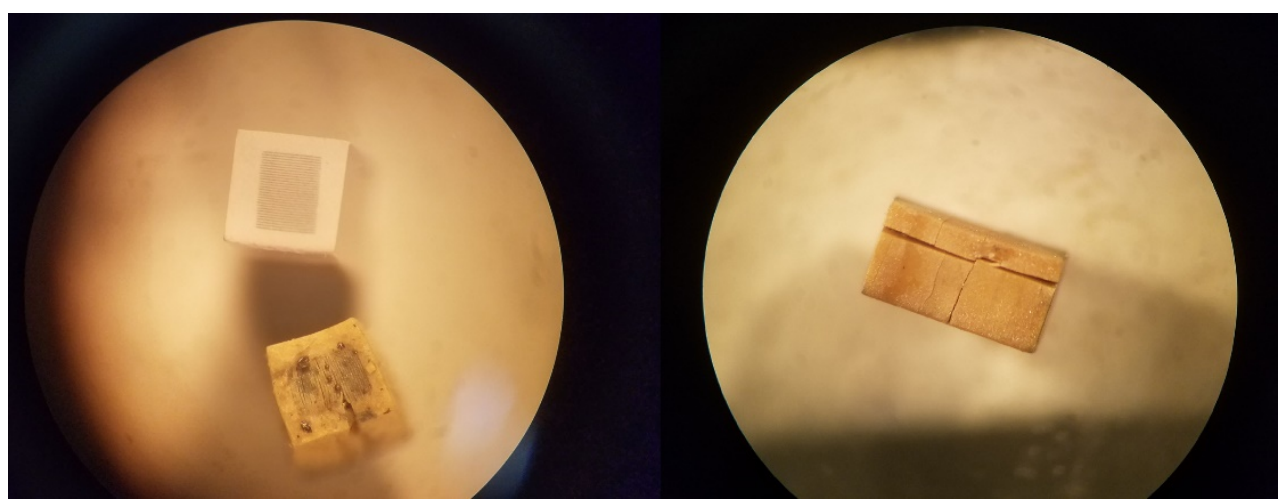

Fig. 7. MLCC sintered with microwaves (left) and conventionally (right).

There are reports of accelerated shrinkage on sintering with microwaves [5]. Despite the combination of having small samples compared to the penetration depth of the microwaves, there is evidence of great thermal gradients in the MLCC's. The temperature that is controlled always is taken in a single location or averaged, then inside the samples the temperatures can be higher. That would explain that sintering takes less time with microwaves than conventionally.

\section{Final remarks}

Although it was confirmed that sintering of ceramics is possible with microwaves, in the case of MLCC's there are several issues that challenges thermal uniformity and the possibility to produce them with microwaves. The only item that was addressed was the achieved density of the ceramic, but still the dielectric properties must be considered for building a capacitor. 


\section{References}

1. J. Aguilar-Garib, Advances in Induction and Microwave Heating of Mineral and Organic Materials, IntechOpen, 2011, 243-268.

2. K. Bai, H-G Kim, Journal of Materials Science Letters, 1994, 13, 806-809.

3. J. Binner, B. Vaidhyanathan, J. Wang, D. Price, M. Reading, Journal of Microwave Power and Electromagnetic Energy, 2008, 42, 47-63.

4. R.K. Patel, P. Kumar, C. Prakash, D.K. Agrawal, Ceramics International, 2012, 38, 1585-1589.

5. S. Derling, H.-P. Abicht, Journal of Microwave Power and Electromagnetic Energy, 1996, 31, 221-227.

6. A. Domer-Reisel, S. Schöps, A. Lenk, G. Schmutzler, Advanced Engineering Materials, 2007, 9, 400-405. 


\title{
SELECTIVE FORMATION OF LINEAR ALPHA-OLEFINS VIA MICROWAVE CATALYTIC CRACKING OF STRAIGHT-CHAIN ALKANES
}

\author{
V.A. Bolotov ${ }^{1}$, S.F. Tikhov ${ }^{1}$, K.R. Valeev ${ }^{1}$, V.T. Shamirzaev ${ }^{1}$, V.N. Parmon ${ }^{1,2}$ \\ ${ }^{1}$ Boreskov Institute of Catalysis, Siberian Branch, Russian Academy of Sciences, \\ pr. Lavrentieva 5, 630090, Novosibirsk, Russia \\ ${ }^{2}$ Novosibirsk National Research State University, Novosibirsk-90, 2 Pirogova Str., 630090, \\ Novosibirsk, Russia
}

bolotov@catalysis.ru

Keywords: microwave heating, catalyst, linear alpha-olefins

\section{Introduction}

Linear even-carbon-number alpha-olefins (LAO) with four or more carbon atoms are important compounds of high demand in chemical industry as precursors of a wide range of value-added chemicals [1]. The linear alpha-olefins are used as co-monomers for polyethylene production, for the production of alcohols (mainly in detergents and plasticizers) and for synthesis of polyalphaolefins (used in synthetic lubricants). Butene-1, hexene-1, octene-1 and decene-1 are mainly used to produce high-density polyethylene (HDPE), linear low-density polyethylene (LLDPE) and poly(vinyl chloride) (PVC) plasticizers to impart the stress-crack resistance. $\mathrm{C}_{10}-\mathrm{C}_{14}$ alpha olefins can be used to synthesize linear alkylbenzene (LAB) sulfonates (detergents). Synthetic detergents produced from LAO have enhanced biodegradation characteristics. A conventional route to produce alpha olefins is oligomerization of ethylene, when thermal cracking of naphtha mainly to low-molecular weight paraffin gases is followed by their dehydrogenation to produce low-molecular olefins, the latter being converted to larger alpha olefin molecules through oligomerization. The process provides production of high quality alpha-olefins but is very costly. If not oligomerization, linear alpha-olefins can be produced by thermal cracking of waxy paraffins but the product is not pure and contains numerous internal olefins, dienes and paraffin impurities. The process is conducted in the vapor phase at relatively low cracking temperatures and needs rapid quenching to prevent side reactions such as isomerization or cyclization.

In our previous work [2], we showed that the selectivity to alpha-olefins one can increase considerably via catalytic cracking of n-alkanes under selective MW heating of catalysts. The observed phenomena can be accounted for by an efficient "quenching" of target cracking products via inhibiting undesirable side reactions in the "cold" liquid phase.

In the present work, some general regularities (effect of the temperature, particle size and the morphology of catalyst) of the MW cracking of liquid n-alkanes are presented. Porous composite ceramic matrix $\mathrm{Al}_{2} \mathrm{O}_{3} / \mathrm{Al}$ (ceramometals) and various carbon materials $(\mathrm{CM})$ were studied as supports and precursors of the catalysts. The MW cracking was conducted with n-hexadecane $\mathrm{C}_{16} \mathrm{H}_{34}$ and n-octacosane $\mathrm{C}_{28} \mathrm{H}_{58}$. The influence of the morphology of ceramometals and carbon materials on the structural and group composition of the products was studied.

\section{Experimental}

The MW cracking of high-molecular hydrocarbons was achieved using model straight-chain systems: $99 \%$ n-hexadecane $\mathrm{C}_{16} \mathrm{H}_{34}$ (Acros) (density $0.773 \mathrm{~g} / \mathrm{cm}^{3}$, b.p. $286.5^{\circ} \mathrm{C}$ ) and 99.5 $\%$ n-octacosane $\mathrm{C}_{28} \mathrm{H}_{58}$ (Acros) (density $0.8067 \mathrm{~g} / \mathrm{cm}^{3}$, b.p. $432{ }^{\circ} \mathrm{C}$ ). The $\mathrm{MW}$ cracking was 
carried out using a lab scale set up (operating frequency $2470 \pm 10 \mathrm{MHz}$, power up to $1 \mathrm{~kW}$ ) based on a rectangular single-mode cavity with high Q-factor $(\sim 6000)$, which is excited by oscillation mode $\mathrm{TE}_{102}$, described in [3]. The MW cracking was studied using a non-flow quartz reactor.

The effective heating was provided by introducing to reaction volume various carbon materials $(\mathrm{CM})$ and porous composite ceramic matrix $\mathrm{Al}_{2} \mathrm{O}_{3} / \mathrm{Al}$ (ceramometals) having high dielectric losses. Mesoporous carbon material Sibunit (produced at the Institute for Hydrocarbon Processing, Russia) and carbon nanotubes (CNTs) were used. CNTs were obtained on an $\mathrm{Fe}-\mathrm{Mo}-\mathrm{Co} / \mathrm{Al}_{2} \mathrm{O}_{3}$ catalyst from a propane - butane mixture at $700^{\circ} \mathrm{C}$. The CNT length reached $10^{4} \mathrm{~nm}$, and the outer diameter of CNTs was within $7-26 \mathrm{~nm}$, the dimension of internal channels was within $4-12 \mathrm{~nm}$. Ceramometals were synthesized through the hydrothermal oxidation (HTO) of aluminum powder according to a published procedure [4]. The samples were mixed with paraffin's at the weight ratio paraffin: Sibunit(ceramometals) $=6: 1$; paraffin $:$ CNTs $=15: 1$. The morphology was varied by crushing the initial granules followed by fractioning; in some cases the CM surface was modified by depositing a thin layer of amorphous silica by the method described elsewhere [5]. An industrial catalyst for hydrocarbon cracking (from GASPROMNEFT - Omsk Refinery) was used as the reference sample. The samples under study were heated selectively during MW cracking; MW power was varied to maintain the hydrocarbon temperature close to the boiling point, i.e. the process occurred in the liquid phase.

Experimental efficiency of the conversion of the MW energy to reagent heating was determined by the method suggested elsewhere [6]. The parameters were measured directly in the MW reactor at room temperature and MW power of $1 \mathrm{~mW}$.

\section{Results and discussion}

Table 1 shows morphological properties of samples and maximal efficiencies $\eta_{\max }$ of MW heating obtained at the used MW set up, as well as operational efficiencies $\eta$ of $0.5 \mathrm{~g}$ (in case CNTs $-0.2 \mathrm{~g}$ ) samples observed during MW cracking.

Table 1. Properties of samples under study

\begin{tabular}{|c|c|c|c|c|c|c|c|}
\hline No. & Composition & $\begin{array}{l}\text { Fractional } \\
\text { composition }\end{array}$ & $\begin{array}{l}\mathrm{S}_{\mathrm{BET}}, \\
\mathrm{m}^{2} / \mathrm{g}\end{array}$ & $\begin{array}{c}\mathrm{D}_{\text {por }} \\
\AA\end{array}$ & $\begin{array}{c}\mathrm{V}_{\text {пор }} * 10^{3} \\
, \mathrm{cc} / \mathrm{g}\end{array}$ & $\begin{array}{l}\eta \\
\%\end{array}$ & $\begin{array}{l}\eta_{\max } \\
, \%\end{array}$ \\
\hline 1 & Sibunit & $1.0-1.6 \mathrm{~mm}$ & 288 & 47.9 & 14.7 & 76.6 & 81.4 \\
\hline 2 & $12.6 \% \mathrm{SiO}_{2} /$ Sibunit & $1.0-1.6 \mathrm{~mm}$ & 216 & 45 & 26.2 & 69.2 & 76.2 \\
\hline 3 & Sibunit & $0.2-0.4 \mathrm{~mm}$ & 324 & 52.2 & 5.5 & 75.1 & 80.6 \\
\hline 4 & CNT & ---- & 250 & ---- & ---- & 86.4 & 89.6 \\
\hline 5 & $4 \% \mathrm{Co} /$ Sibunit & $1.0-1.6 \mathrm{~mm}$ & 264 & 46 & 13.4 & 72.1 & 78.9 \\
\hline 6 & Industrial catalyst & $0.2-0.4 \mathrm{~mm}$ & --- & ---- & ---- & 23.8 & 29.2 \\
\hline 7 & $\begin{array}{c}\text { ASD-1 } \\
\left(\mathrm{Al}_{2} \mathrm{O}_{3} / \mathrm{Al}, \text { pressed }\right)\end{array}$ & $0.1-0.25 \mathrm{~mm}$ & 45.9 & 54.7 & 69.64 & 74.3 & 90.6 \\
\hline 8 & $\begin{array}{c}\text { ASD-1 } \\
\left(\mathrm{Al}_{2} \mathrm{O}_{3} / \mathrm{Al}, \mathrm{HTO} 1 \mathrm{~h}\right)\end{array}$ & $0.5-1 \mathrm{~mm}$ & 41.3 & 37.1 & 49 & 70.2 & 87.1 \\
\hline & $\begin{array}{c}\text { n-hexadecane } \mathrm{C}_{16} \mathrm{H}_{34} \\
\text { n-octacosane } \mathrm{C}_{28} \mathrm{H}_{58}\end{array}$ & & & & & $\begin{array}{l}<2 \\
<1\end{array}$ & $\begin{array}{l}-- \\
--\end{array}$ \\
\hline
\end{tabular}

All samples (except for cracking catalyst) are highly efficient ( $>50 \%$ ) to the MW energyto-heat conversion, while the silica modification of the surface makes a small decrease in the heating efficiency. Hence, it is possible to feed energy to the catalytic system and to 
create thermodynamically preformed conditions owing to the selective MW heating of the catalysts.

GC-MS techniques were used for qualitative and quantitative analysis of the cracking products. It was established that alpha-olefins $\left(\mathrm{C}_{2}-\mathrm{C}_{23}\right)$ and n-alkanes $\left(\mathrm{C}_{2}-\mathrm{C}_{26}\right)$ were the main cracking products under selective MW heating of the used supports and catalysts (Figure 1).

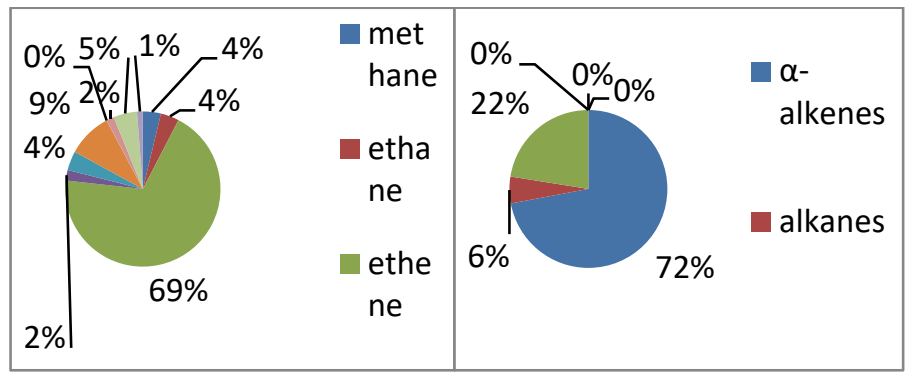

Fig. 1. Composition of products after microwave cracking of n-hexadecane with Sibunit as the MW absorbing catalyst. Left: gaseous products. Right: liquid products

The main gaseous products of the MW cracking over the cracking catalyst were ethylene, propylene and iso-butane, and the main condensed products were iso-alkanes (Figure 2).

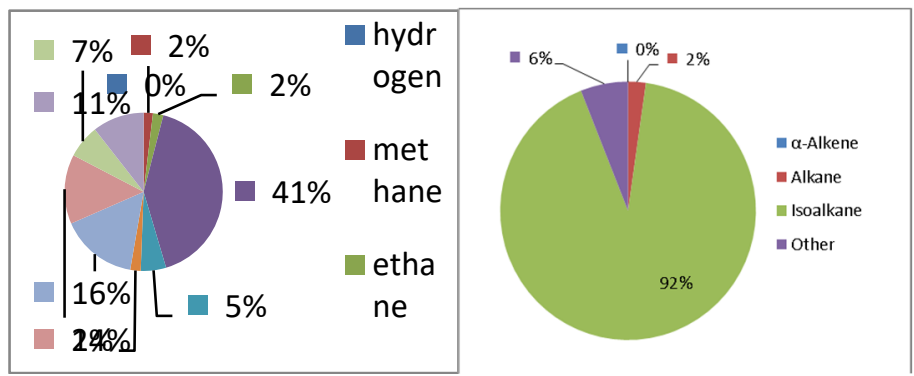

Fig. 2. Composition of products after microwave cracking of n-hexadecane with cracking catalyst as the MW absorbing catalyst. Left: gaseous products. Right: liquid products

Supporting of iron group metals on CM resulted in changes in the structural and group composition of the cracking products and in an increase in the contents of olefin isomers and aromatic hydrocarbons (Figure 3). 


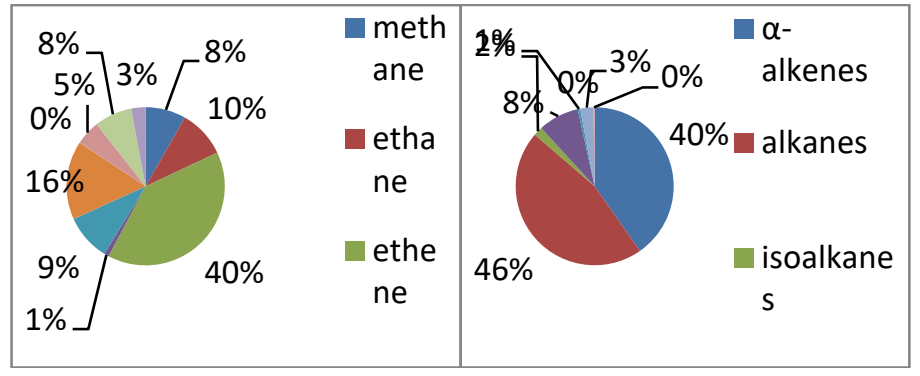

Fig. 3. Composition of products after microwave cracking of n-octacosane with $4 \% \mathrm{Co} /$ Sibunite as the MW absorbing catalyst. Left: gaseous products. Right: liquid products

The quantitative analysis of the products demonstrated that the liquid-phase process is more selective to alpha-olefins at the MW catalytic cracking than at the thermal cracking at the hydrocarbon temperature close to the boiling point at the same reaction time (Table 2).

Table 2. Observed conversion and selectivity to alpha-olefins of high-molecular hydrocarbons over different catalysts

\begin{tabular}{cllllll}
\hline & \multicolumn{3}{c}{ Microwave cracking } & \multicolumn{3}{c}{ Thermal cracking } \\
\cline { 2 - 7 } No. & \multicolumn{1}{c}{$\mathrm{n}$-Hexadecane $\left(\mathrm{C}_{16} \mathrm{H}_{34}\right)$} & \multicolumn{5}{c}{ n-Octacosane $\left(\mathrm{C}_{28} \mathrm{H}_{58}\right)$} \\
\cline { 2 - 7 } & $\begin{array}{c}\text { Conversio } \\
\mathrm{n} \%\end{array}$ & $\begin{array}{c}\text { Selectivity } \\
\%\end{array}$ & $\begin{array}{c}\text { Conversio } \\
\mathrm{n} \%\end{array}$ & $\begin{array}{c}\text { Selectivity } \\
\%\end{array}$ & $\begin{array}{c}\text { Conversio } \\
\mathrm{n} \%\end{array}$ & $\begin{array}{c}\text { Selectivity } \\
\%\end{array}$ \\
\hline 1 & 18.1 & 46.7 & - & - & - & - \\
2 & 15.8 & 63.0 & - & - & - & - \\
3 & 15.9 & 43.0 & 44.1 & 47.7 & 32.5 & 4.61 \\
4 & 9.3 & 45.0 & - & - & & \\
5 & - & - & 30.2 & 40.3 & - & - \\
6 & 16.4 & 16.5 & 63.6 & - & 90.4 & 23.1 \\
7 & 1.5 & 48.4 & 18.7 & 32.7 & - & - \\
8 & 9.4 & 46.9 & - & - & - & - \\
\hline
\end{tabular}

The qualitative product composition was similar to the composition of products of the convectional thermal cracking that indicates the radical-chain mechanism of the MW cracking when the homolytic cleavage of $\mathrm{C}$ bond on the catalyst surface produces two alkyl radicals. An analysis of the results showed that the key factor affecting the conversion in the case of the radical-chain mechanism of the MW cracking is the surface area but not only the chemical composition of the catalyst. Notice that silica modification of the CM surface was shown to suppress spark discharge (usually observed at the MW heating of $\mathrm{CM}$ ); hence, the thermal cleavage of $\mathrm{C}-\mathrm{C}$ bonds on the $\mathrm{CM}$ surface rather than in the plasma discharge contributes the most to the formation of radicals.

\section{Conclusions}

Studies of the MW cracking revealed that the conversion n-hexadecane and n-octacosane could reach 18 and $44 \%$, respectively, over Sibunit and increased up to $63 \%$ in the presence of the cracking catalyst. It was shown that the selectivity to liquid alpha-olefin could be more than $48 \%$ under MW heating of ceramometals. The results obtained demonstrated that the 
selective MW heating during liquid-phase cracking resulted in an increase in the selectivity to alpha-olefins against that of thermal cracking.

\section{Acknowledgement}

The research was supported by RSF (project No. 17-73-30032).

\section{References}

1. George R. Lappin and Joe D. Sauer, Alpha olefins applications handbook, Marcel Dekker, New York, 1989, 454

2. V.A. Bolotov, et al., Journal of Microwave Power and Electromagnetic Energy, 2012, 1, 39-46.

3. Yu. D. Chernousov, et al., Instruments and Experimental Techniques, 2019, 2, 289-294

4. S.F. Tikhov, et al.,, Metal, ceramic and polymeric composites for various uses, INTECH, Croatia, 2011, 195-210

5. V.V. Chesnokov, et al., Composite Structures., 2012, 1, 23-32

6. V.A. Bolotov, et al., 15th International Conference on Microwave and High Frequency Heating (AMPERE2015), 2015, 67 


\section{A COMPARATIVE STUDY OF MICROWAVE AND BARRIER DISCHARGE PLASMA FOR THE REGENERATION OF SPENT ZEOLITE CATALYSTS}

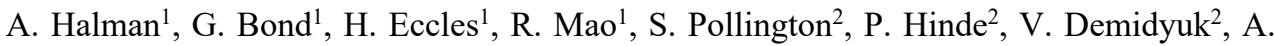 Gkelios $^{2}$
${ }^{1}$ Centre for Materials Science, University of Central Lancashire, Preston, PR1 2H,.UK.
${ }^{2} J o h n s o n$ Matthey Technology Centre, PO Box1, Belasis Avenue, Billingham, Cleveland, $T S 231 L B, U K$.
gbond@uclan.ac.uk

Keywords: microwave, barrier discharge, plasma, catalyst, toluene disproportionation.

\section{Introduction}

Due to their acid characteristics and pore structure, which can induce high product selectivity; zeolite catalysts are used extensively in industry to catalyse reactions involving hydrocarbons. However, these catalysts can suffer from deactivation due to cracking reactions that result in the deposition of carbon leading to poisoning of the acid sites and blocking of the pores [1]. Depending upon the reaction and the particular catalyst involved, this deactivation may take place over several months or even years but in some cases occurs in minutes. Therefore, zeolite catalysts are frequently reactivated / regenerated. This generally comprises of a thermal treatment involving air which results in oxidation of the carbon [2]. However, the oxidation of carbon is highly exothermic, and if not carefully controlled, results in the generation of exceedingly high localized temperatures which can destroy the zeolite structure and lead to subsequent loss of catalyst activity. More conservative thermal treatments can result in incomplete regeneration and again a catalyst displaying inferior activity.

This paper explores the use of non-thermal plasma which had been generated using either microwaves or via a barrier discharge to regenerate spent zeolite catalysts. The catalyst, Hmordenite, was tested for the disproportionation of toluene (Figure 1) using conventional heating. The spent catalyst was then regenerated using a plasma or conventional thermal treatment before having its activity re-evaluated for the toluene disproportionation reaction as previous.
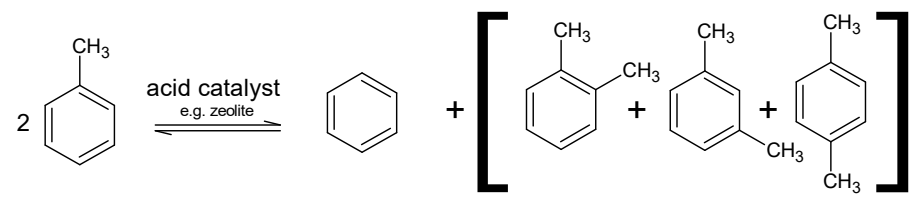

Fig. 1. Reaction Scheme for Toluene Disproportionation.

\section{Experimental}

\section{Catalyst Testing}

Virgin mordenite ( 700 mg) was packed into a fixed-bed borosilicate microreactor containing a thermocouple well and plugged with glass wool (total reactor bed volume: 1.79 $\mathrm{cm}^{3}$ ). The microreactor was enclosed within a temperature programmed furnace and connected to a nitrogen supply $\left(20 \mathrm{ml} \mathrm{min}^{-1}\right)$ regulated by a mass flow controller. The 
catalyst was calcined for 1 hour at $300^{\circ} \mathrm{C}$ after which the temperature was increased to $500^{\circ} \mathrm{C}$ prior to switching the toluene reactant feed on. The toluene was supplied by a gas saturator fitted with a sinter which was attached to the gas line before the microreactor. The saturator was submerged in an ice bath to regulate the temperature of the toluene and provide a constant partial pressure of toluene in the reactor feed gas. The toluene disproportionation reaction was studied for a period of 600 minutes. The output gas line was heated using heating tape (to $150^{\circ} \mathrm{C} \pm 3^{\circ} \mathrm{C}$ ) which was connected to a controller.

The exit gas was periodically sampled using air actuated sampling valves and the reactant / products analysed using online gas chromatography / mass spectrometry (GC/MS). The GC was fitted with a Supelco SupelcowaxTM 10 fused silica capillary column $(30 \mathrm{~m})$. This provided base line resolution of all products including the individual xylene isomers.

\section{Catalyst Regeneration}

Three regeneration methods were employed, all or which involved treating the spent catalyst in an oxidising environment in order to remove the deposited carbon from the surface of the catalyst. Two plasma techniques were employed: one using a plasma generated under low pressure utilising microwaves $(2.45 \mathrm{GHz})$ in a TE010 single mode cavity [3], the other utilising high voltages in a barrier discharge system to generate plasma at atmospheric pressure.

Microwave plasma experiments were performed in a 30\% oxygen in argon atmosphere (30 $\left.\mathrm{ml} \mathrm{min}^{-1}\right)$. Experiments were performed to optimise the regeneration conditions by independently varying the microwave power and consequently the wall temperature of the reactor which was monitored with an infrared pyrometer $\left(150-350^{\circ} \mathrm{C}\right)$. In separate experiments the pressure was also altered by varying the pumping conditions in order to achieve pressures in the range 5-50 mbar. During which the reactor wall temperature was maintained constant at $250^{\circ} \mathrm{C}$. Regeneration was carried out for a period of 7 hours.

The dielectric barrier discharge experiments were performed in $20 \%$ oxygen in argon at atmospheric pressure $\left(100 \mathrm{ml} \mathrm{min}^{-1}\right)$ in a reactor configuration similar to that used by Di et. al. [4]. The peak to peak voltage used was $20 \mathrm{kV}$ at a frequency of $10 \mathrm{~Hz}$, the power consumption was $17 \mathrm{~W}$. The discharge gap between the electrodes was $5 \mathrm{~mm}$. Regeneration of the spent catalyst was carried out for a period of 8 hours.

A thermal regeneration was also carried out using a tube furnace set to $500^{\circ} \mathrm{C}$. The sample was regenerated in air $\left(20 \mathrm{ml} \mathrm{min}^{-1}\right)$ for a period of 24 hours. Previous studies had shown that the reduced regeneration times utilising conventional heating did not result in complete removal of the deposited carbon.

Regenerated catalysts were then re-tested for their activity for the toluene disproportionation reaction. Post-mortem analysis was performed using X-ray diffraction to determine if the regeneration technique had resulted in degradation to the zeolite structure. The efficacy of the regeneration techniques was checked using thermogravimetric analysis (TGA) to determine the extent of carbon removal from the catalyst.

\section{Thermogravimetric analysis (TGA)}

Carbon content of deactivated and regenerated catalysts was determined using TGA (Mettler Toledo TGA1 STARe System), with the mass loss at $500^{\circ} \mathrm{C}$ attributed to loss of coke. Samples were analysed using a complex thermal programme that involved heating the sample in air to a temperature of $800^{\circ} \mathrm{C}$; which had previously been validated for this type of analysis [5]. 


\section{Powder X-ray diffraction (XRD)}

$\mathrm{XRD}$ analysis was carried out on catalyst samples to determine any crystalline changes that regeneration may have had on the sample. Samples were ground using a mortar and pestle prior to analysis using a Bruker D2 Phaser diffractometer and nickel filtered copper $\mathrm{K} \alpha$ radiation $(0.154 \mathrm{~nm})$.

\section{Results and Discussion}

\section{Thermal Regeneration of Spent H-mordenite}

Toluene conversion over a new (virgin) sample of H-mordenite is shown in Figure 2. It can be seen that the activity decreased from an initial conversion of approximately $61 \%$ to $8 \%$ over a period of 600 minutes. The decrease in catalytic activity can be attributed to competing cracking reactions that result in the deposition of carbon on the surface of the catalyst. The catalyst on discharge from the reactor had visibly changed from its original white colour to black. TGA analysis of the spent sample showed that $4.55 \mathrm{wt} \%$ carbon had been deposited during the course of the reaction. The TGA analysis also indicated that a regeneration temperature of $500^{\circ} \mathrm{C}$ was necessary to remove in excess of $95 \%$ of the carbon. Studies to determine the time necessary to achieve this level of regeneration indicated that a reaction time in excess of 16 hours was required and this was standardised to 24 hours for all conventionally heated regenerations. The thermally regenerated catalyst was re-tested to determine its activity for toluene disproportionation as a function of time (Figure 2). The results showed that the regenerated catalysts displayed a slightly lower initial activity of approximately $58 \%$ and then displayed a very similar activity time profile to the virgin catalyst. The slightly reduced initial activity may be the result of a small amount of carbon still remaining in a highly graphitic form after regeneration, TGA studies of the regenerated catalyst did show that $0.36 \mathrm{wt} \%$ carbon remained in the structure. The XRD pattern of the thermally regenerated catalyst (Figure 5) indicates that the structure of the zeolite was not affected by the regeneration process. However, the initial benzene to xylene ratio is noticeably less than the in the virgin catalyst (Figure 6). For pure toluene disproportionation the benzene to xylene ratio should be approximately 1.0 [6]. However, it has been shown to be highly dependent upon operating conditions [7]. Under the operating conditions that have been chosen for this study where the aim was to achieve high rates of carbon deposition a benzene to xylene ratio less than 1 was observed. However, comparing the benzene to xylene ratio of the virgin and thermally regenerated catalyst it can be seen that the thermally regenerated catalyst exhibits a lower benzene to xylene ratio which is indicative that the catalyst has fewer strong acid sites which are known to catalyse the cracking reaction [8]. 


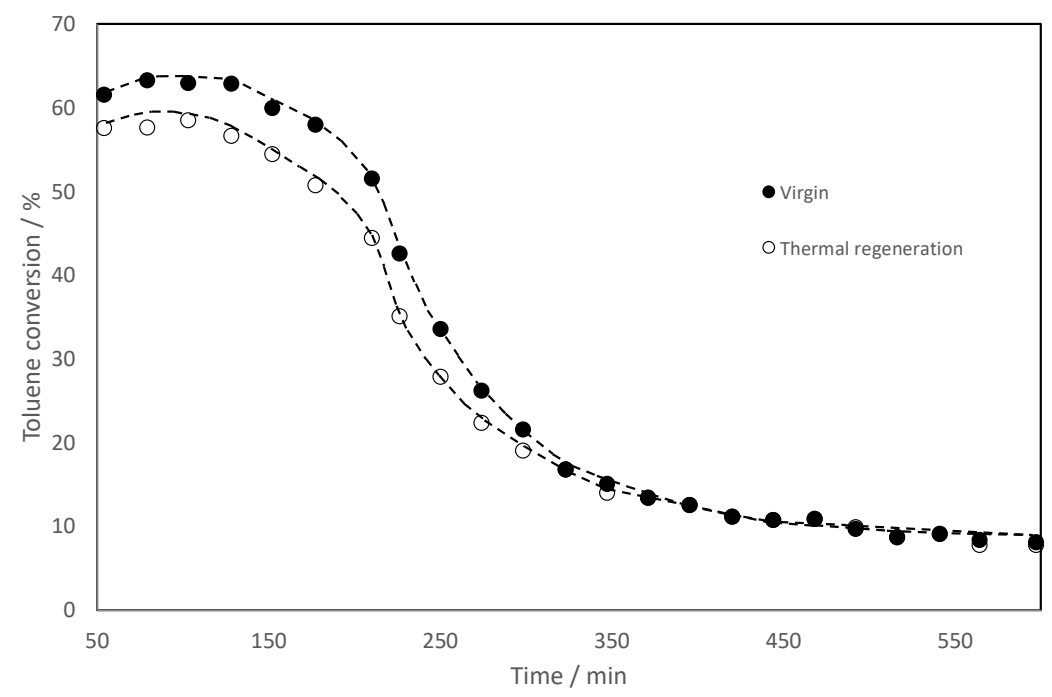

Fig.2. Plot of toluene conversation over a virgin and thermally regenerated H-mordenite catalyst.

Microwave Plasma Regeneration of Spent H-mordenite

The microwave powers required to attain wall temperatures in the range $150-350{ }^{\circ} \mathrm{C}$ are contained in Table 1. Figure 3 contains the activity time profiles for the re-testing of the regenerated catalysts. The figure clearly shows that the microwave power / temperature has a dramatic effect on the resulting catalyst activity. The catalyst regenerated at $150^{\circ} \mathrm{C}$ shows a modest initial activity ( $55 \%$ ), however, the catalyst rapidly deactivates with the activity falling to less than $10 \%$ over a period of 200 minutes. While from its external appearance the catalyst extrudate appeared to have undergone regeneration due to their white colour; when the catalysts were sectioned they clearly contained un-oxidised carbon in their core (Figure 3A). Therefore, the regenerated catalyst will have a reduced number of active (acid) sites which accounts for the rapid deactivation. The initial benzene to xylene ratio is indicative that some strong acid sites have been lost.

The catalyst regenerated at $250^{\circ} \mathrm{C}$ is interesting. The TGA data confirmed that almost all the carbon had been removed during the regeneration. The initial benzene to xylene ratio (Figure 6) is significantly lower than the virgin, thermal and microwave plasma $150^{\circ} \mathrm{C}$ regenerated catalysts. This provides evidence that the catalyst has fewer strong acid sites. Therefore, the catalyst is less likely to catalyse the cracking reaction. The consequence of which is that there is less carbon deposition and therefore the catalyst deactivates more slowly. The XRD pattern (Figure 5) confirms that the structural integrity of the catalyst is maintained during the regeneration. 


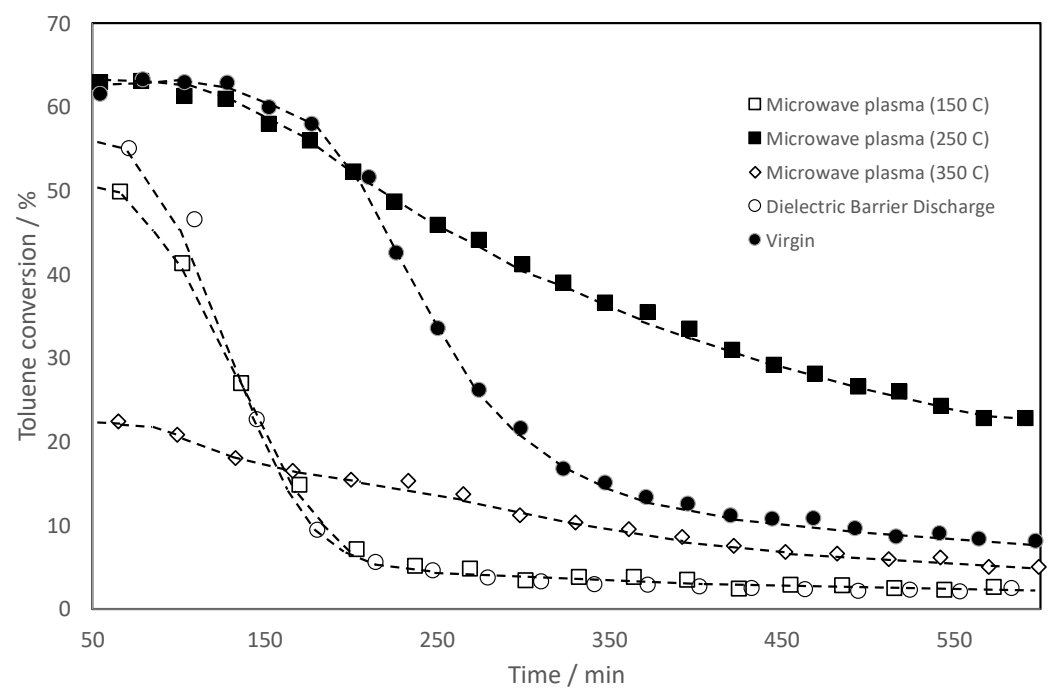

Fig.3. Toluene conversion over H-mordenite catalysts that have been regenerated using microwave plasma at varying temperatures / powers.

The catalyst regenerated at $350^{\circ} \mathrm{C}$ does not retain structural integrity as can be seen from the loss of intensity of the H-Mordenite peaks in the diffractogram (Figure 5). The initial benzene to xylene ratio is exceptionally low but so is the activity. This is indicative that a significant portion of the acid sites have been lost during the regeneration process.

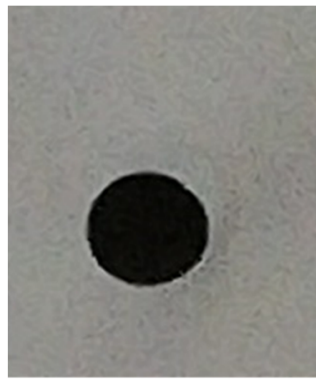

A

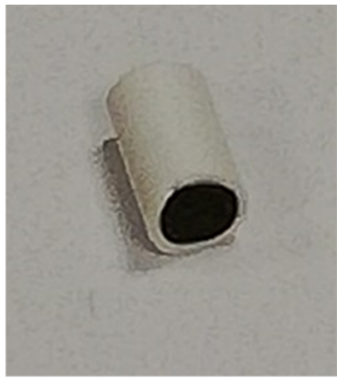

B

Fig.4. A Photograph of sectioned H-mordenite extrudate after microwave plasma regeneration at $150^{\circ} \mathrm{C}$, B Photograph of sectioned $\mathrm{H}$-mordenite extrudateafter dielectric barrier discharge plasma regeneration.

Table 1. Microwave power required to produce pre-set wall temperatures

\begin{tabular}{c|c}
\hline Wall temperature $/{ }^{\circ} \mathrm{C}$ & Microwave power $/ \mathrm{W}$ \\
\hline 150 & 125 \\
250 & 195 \\
350 & 400 \\
\hline
\end{tabular}




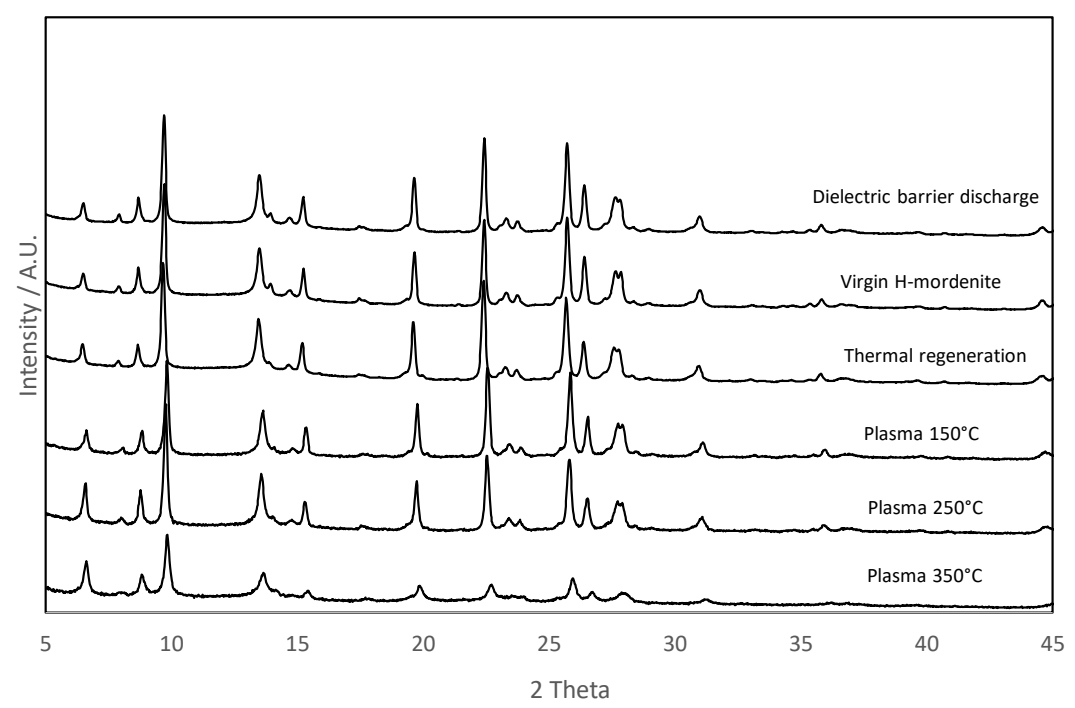

Fig.5. X-ray Diffractograms of virgin, thermal, microwave plasma and dielectric barrier discharge plasma regenerated catalysts.

The results from re-testing spent H-mordenite catalysts that had undergone microwave plasma regeneration in which the pressure of the microwave plasma had been varied between 5 and 50 mbar whilst keeping the reactor wall temperature constant at $250^{\circ} \mathrm{C}$ are shown in Figure 7. The microwave power used at both 5 mbar and 50 mbar was approximately 200 $\mathrm{W}$. The results confirm that microwave plasma offers a means of producing catalysts with resistance to deactivation. However, the pressure does appear to play a role. The catalyst regenerated at $5 \mathrm{mbar}$ has a greater resistance to deactivation than the catalyst regenerated at the higher pressure. The catalyst regenerated at $50 \mathrm{mbar}$ has a similar deactivation profile to the virgin catalyst, this is likely a result of the regeneration process not resulting in a significant reduction of acid sites as can be seen from the relatively high initial benzene to xylene ratio and consequently, there is significant cracking resulting in carbon deposition and deactivation of the catalyst. 


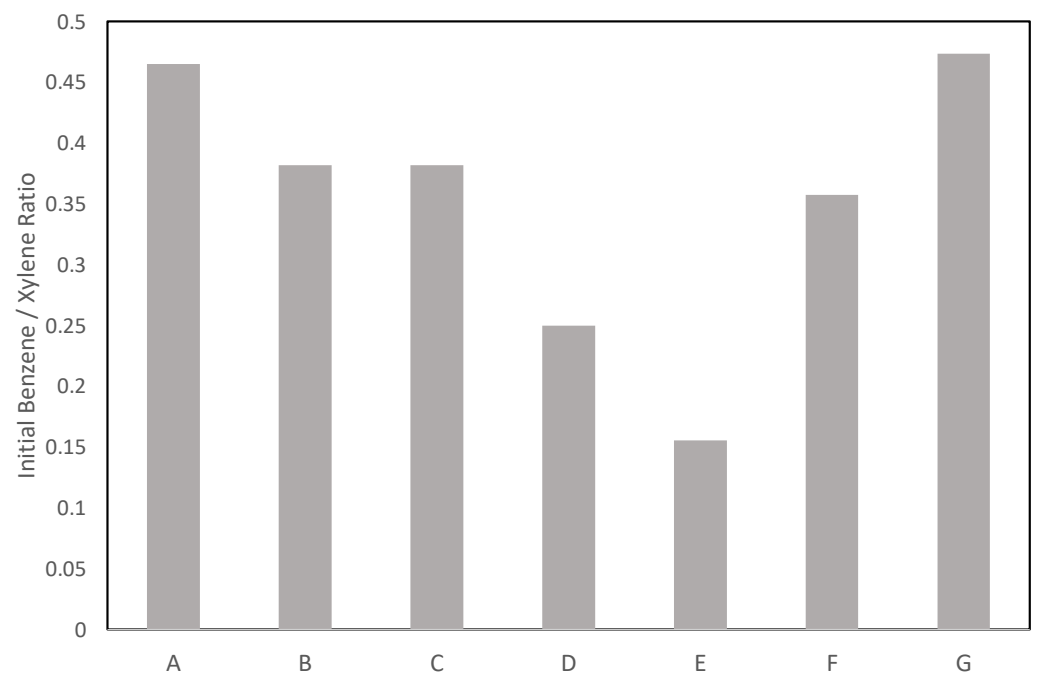

$\mathrm{A}=$ Virgin, $\mathrm{B}=$ Thermal regeneration, $\mathrm{C}=$ Microwave plasma $150^{\circ} \mathrm{C}, \mathrm{D}=$ Microwave plasma $250^{\circ} \mathrm{C}, \mathrm{E}=$ Microwave plasma $350^{\circ} \mathrm{C}, \mathrm{F}=$ Dielectric barrier discharge, $\mathrm{G}=$ Microwave plasma $250^{\circ} \mathrm{C}$ and $50 \mathrm{mbar}$.

Fig.6. Initial benzene to xylene ratios over H-mordenite catalysts that had been regenerated under different conditions.

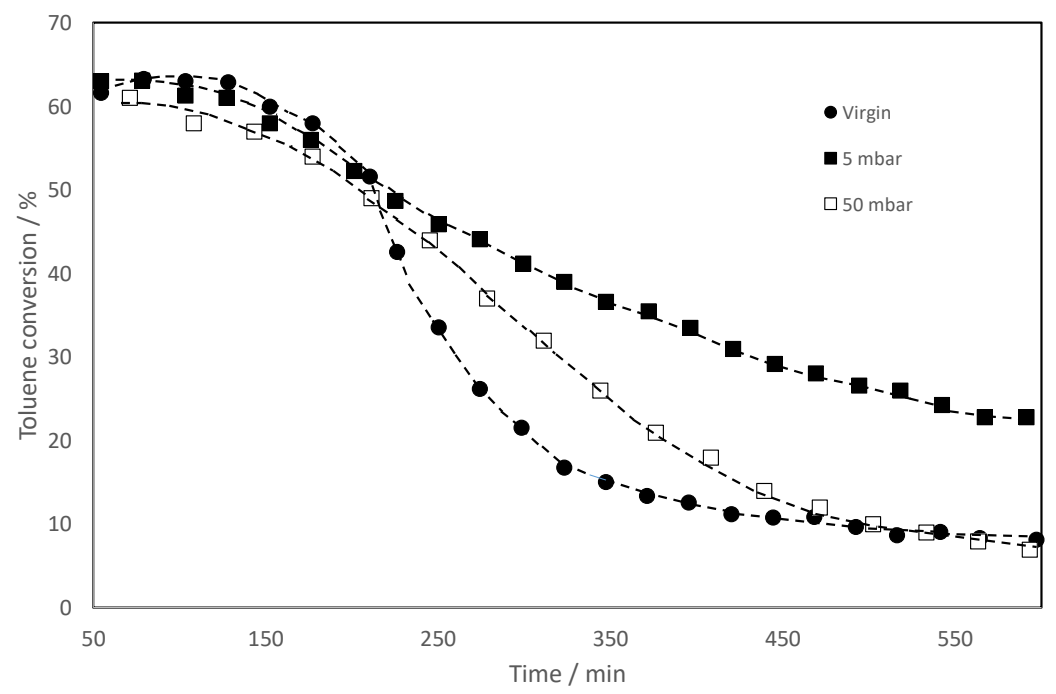

Fig.7. Toluene conversion over H-mordenite catalysts that have been regenerated using microwave plasma at varying pressures.

Dielectric barrier discharge plasma regeneration of spent H-mordenite

The sample of spent $\mathrm{H}$-mordenite which was regenerated using plasma generated using a dielectric barrier discharge method, displays an activity profile very similar to the sample regenerated using microwave plasma at $150^{\circ} \mathrm{C}$ (Figure 3). Considering the images shown in Figure 4 this is hardly surprising, as both catalysts have clearly not undergone full 
regeneration. The initial benzene to xylene ratio is also not dissimilar and consequently the number of strong acid sites would be expected to be similar.

\section{Conclusion}

The results from this study show that plasma regeneration of spent zeolite catalysts can be highly effective, not just through reducing the time required for regeneration but also through modifying the acid sites. The modification of strong acid sites, most probably occurs through dehydration of Brønsted acid sites. The modification to these strong acid sites reduces cracking reactions and consequently the amount of carbon that is deposited on the catalyst surface. This reduced carbon deposition results in extended catalyst lifetime. The study shows the microwave power / temperature used is critical as a power which is too low results in incomplete regeneration and a power which is too high can lead to loss of the structural integrity of the zeolite.

The study using plasma generated using dielectric discharge is at this stage inconclusive. If a more intense / energetic plasma was used, then it may be possible to achieve full regeneration. This fully regenerated catalyst may then display similar characteristics to the microwave plasma catalyst regenerated at $250^{\circ} \mathrm{C}$.

\section{References}

[1] Wu J., Leu L., Appl. Catal., 1983, 7, 283-294.

[2] M. Guisnet and P. Magnoux, Deactivation of Zeolites by Coking. Prevention of Deactivation and Regeneration. In: Zeolite Microporous Solids: Synthesis, Structure, and Reactivity. E.G. Derouane, F Lemos, C. Naccache, F. Ramôa Ribeiro, Eds. Pages 437-456. Springer 1992.

[3] Parkes, G.M.B., Bond, G., Barnes, P.A. and Charsley, E.L., Rev. Sci Instr., 2000, $71,168$.

[4] Di, L. Xu, Z., Wang, K. and Zhang, X. Catal. Today, 2013, 211, 109-113.

[5] Zhang, H., Shao, S., Xiao, R., Shen, K. and Zeng, J., Energy and Fuels., 2013, 28, 52-57.

[6] Kareem, A., Chand, S., and Mishra, I.M., J. Sci. Ind. Res., 2001, 60, 319-327.

[7] Tsai, T.C., Liu, S.B. and Wang, I., Appl. Catal. A. Gen., 1999, 181, 355-398.

[8] Gates, B. C., J. R. Katzer, and G. C. A. Schuit. Cracking. In: Chemistry of Catalytic Processes. B. C. Gates, J.R. Katzer and G.C.A. Schuit, Eds. Pages 1111. New York: McGraw-Hill, 1979. 
17th International Conference on Microwave and High Frequency Heating

\section{Design of microwave applicators and circuits}




\section{AUTOMATIC MEASUREMENT OF MAGNETRON RIEKE DIAGRAMS}

\section{Bilik ${ }^{1}$}

${ }^{1}$ Faculty of Electrical Engineering and Information Technology, Slovak University of Technology, Ilkovicova 3, 81219 Bratislava, Slovak Republic vladimir.bilik@s-team.sk

Keywords: Rieke diagram, magnetron, high-power vector reflectometer

\section{Introduction}

One of the most important characteristics of a magnetron is its Rieke diagram [1], [2]. The diagram visualizes the dependence of the generated frequency $f_{g}$ and the net power $P_{L}$ delivered to load on a specifically defined magnetron load reflection coefficient $\Gamma_{R}$. The diagram is a (rotated) polar chart in the complex plane of $\Gamma_{R}$, plotted as a family of isolines of constant $f_{g}$ and of constant $P_{L}$ (Fig. 1). Rieke diagrams are essential in the design of magnetrons and in magnetron applications without isolators, such as domestic or professional microwave ovens.

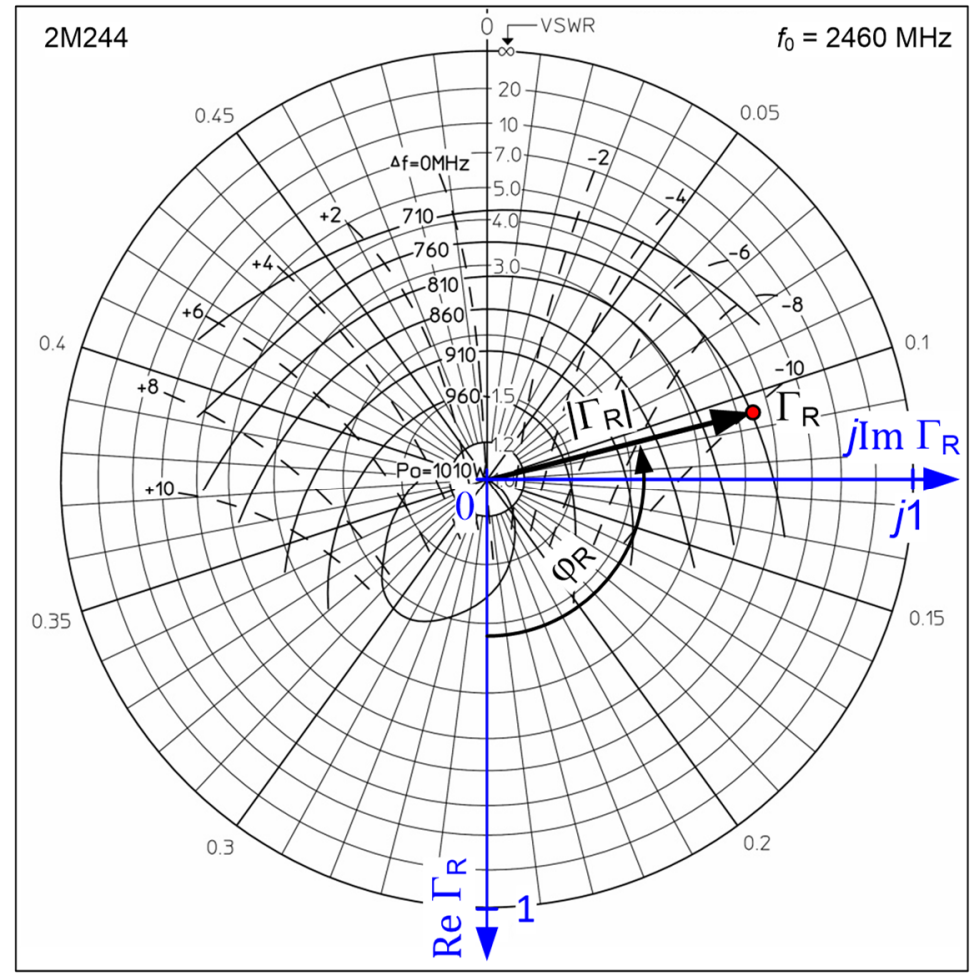

Fig. 1. Example Rieke diagram.

Traditionally, the construction of Rieke diagrams (see e.g. [1], [3], [4]) has been a tedious and time-consuming task that requires highly specialized equipment. This has prevented 
systematic studies of the dependence of Rieke diagrams on operating conditions, such as, for example, anode current and its ripple, filament current, and mounting repeatability.

We have devised a procedure centered around a standard, commercially available, highpower automatic impedance matching device (autotuner). This procedure enables fully automatic measurement and plotting of the isolines of choice. The measurement process consists of stepping through a grid of $n$ suitably predetermined reflection coefficients $\Gamma_{R}$, covering a desired area of the polar chart. Each $\Gamma_{R}$ is measured accurately by the autotuner, along with the corresponding $f_{g}$ and $P_{L}$. A dedicated MATLAB routine then reads this raw data, approximates them by 2-dimensional splines, and uses the splines to plot smoothed isocontours for chosen constant values of $f_{g}$ and $P_{L}$, thus completing the Rieke diagram construction. In what follows, we present the details of this procedure as well as a real-life example.

\section{Reference Launcher}

A magnetron's Rieke diagram-related load reflection coefficient, conveniently named the Rieke reflection coefficient [3]

$$
\Gamma_{R}=x_{R}+j y_{R}=\left|\Gamma_{R}\right| \exp \left(j \varphi_{R}\right)
$$

is defined as the reflection coefficient observed looking toward the load in an agreed magnetron-to-rectangular-waveguide coupling structure called the reference launcher (Fig. 2). The launcher is characterized by three main parameters: the waveguide inner dimensions $a_{r}$ (not shown in Fig. 2) and $b_{r}$, and the distance $d_{s}$ of the magnetron antenna axis from the short-circuited waveguide end. The distance $d_{R}$ between the antenna axis and the waveguide output is not critical; it should only be long enough to sufficiently attenuate higher-order modes potentially excited by the antenna. Conceptually, the reference launcher can serve to directly measure $\Gamma_{R}$, as shown in Fig. $2 \mathrm{~b}$.

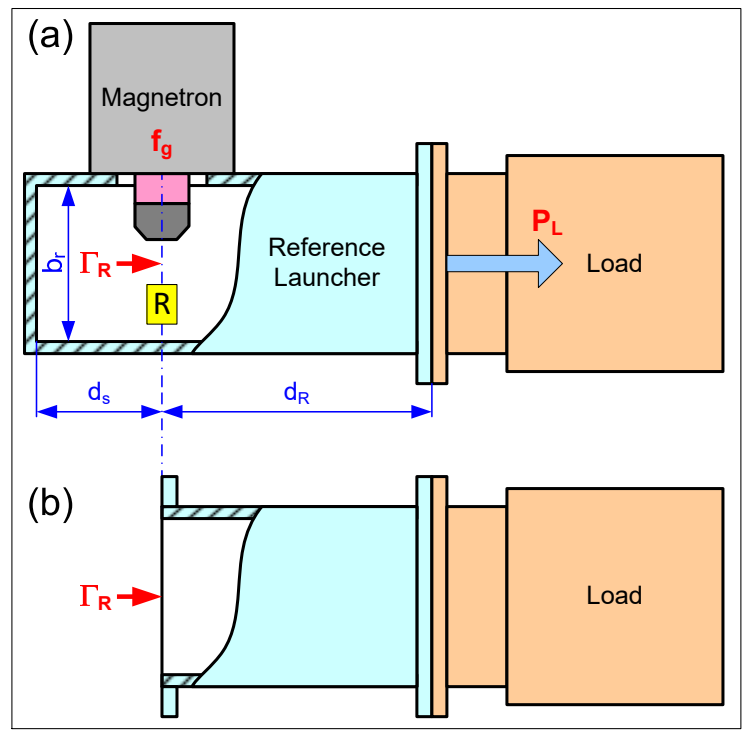

Fig. 2. a) Magnetron in reference launcher. b) Definition of Rieke reflection coefficient $\Gamma_{R}$. 
Basic reference launcher dimensions for $2.45-\mathrm{GHz}$ magnetrons with powers up to about 2 $\mathrm{kW}$ have been standardized by the Electronic Industries Association of Japan (EIAJ). The launcher uses a waveguide with the inner dimensions $a_{r}=95.3 \mathrm{~mm}$ and $b_{r}=54.6 \mathrm{~mm}$. We will refer to it as the launcher waveguide (LWG). The antenna-short distance is $d_{s}=18.6$ $\mathrm{mm}$. The antenna-flange distance $d_{R} \geq 150 \mathrm{~mm}$ ensures that the attenuation of the lowest higher-order mode (TE01) exceeds $33 \mathrm{~dB}$.

\section{Experimental Setup}

A block diagram of the experimental setup is shown in Fig. 3a. The magnetron under test is coupled to a reference launcher and fed from a high-voltage DC power supply. A lowreflection transition (taper) connects the launcher with the standard WR340 waveguide autotuner. The autotuner is terminated in a high-power matched load, such as a waterload. In this configuration, the autotuner can accomplish a task inverse to impedance matching: realizing any desired Rieke reflection coefficient $\Gamma_{R}$. During the measurement process, the autotuner accurately measures each $\Gamma_{R}$ it produces, along with the corresponding generated frequency $f_{g}$ and net delivered power $P_{L}$. These are the three data a collection of which is necessary for creating a Rieke diagram. The whole process is controlled from an external computer (PC).

The setup is fairly simple: the only specialized components required are the reference launcher and the tapered LWG-to-WR340 transition" ${ }^{1}$.

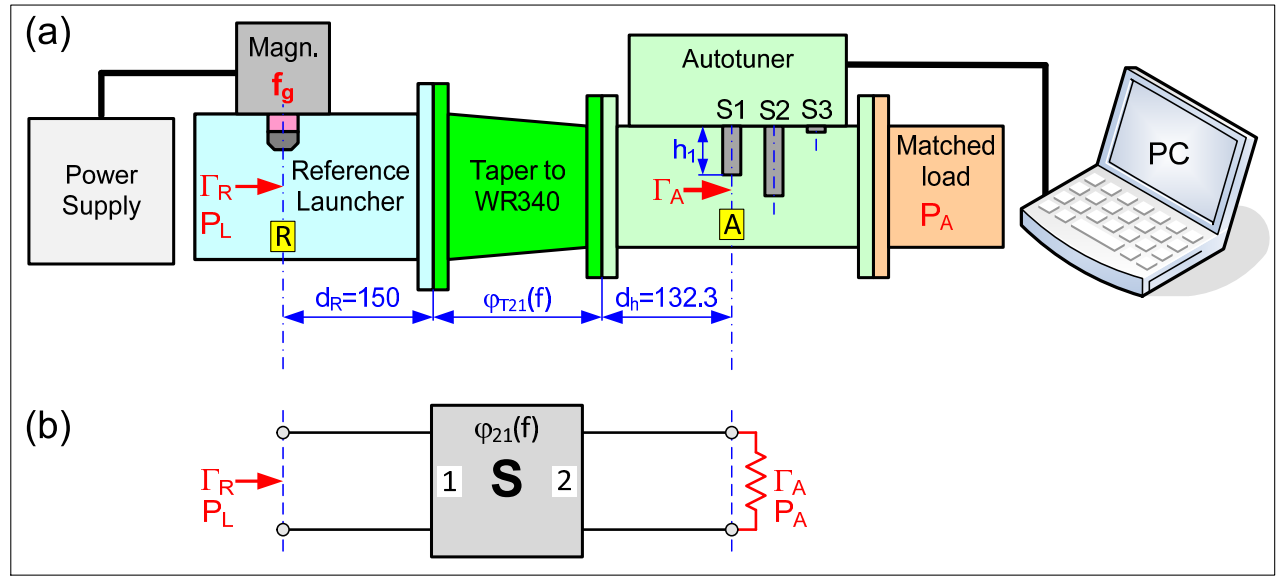

Fig. 3. Rieke diagram measurement setup.

\section{Reflection Coefficient and Delivered Power Transformation}

The high-power vector reflectometer incorporated in the autotuner inherently measures the reflection coefficient $\Gamma_{A}$ at a reference plane A as well as the net power $P_{A}$ absorbed in the load. In order to compute $\Gamma_{R}$ and $P_{L}$, we need to know the scattering matrix $\boldsymbol{S}$ of the circuit between planes R and A (Fig. 3b). The transform is accomplished by the formulas [7]

\footnotetext{
${ }^{1}$ For measurements with magnetron antenna probes, an LWG-based calibration kit is also required [5], [6].
} 


$$
\begin{aligned}
& \Gamma_{R}=S_{11}+\frac{S_{12} S_{21} \Gamma_{A}}{1-S_{22} \Gamma_{A}}=\frac{S_{11}-D \Gamma_{A}}{1-S_{22} \Gamma_{A}} \\
& P_{L}=P_{A} \frac{\left|1-S_{22} \Gamma_{A}\right|^{2}-\left|S_{11}-D \Gamma_{A}\right|^{2}}{\left|S_{21}\right|^{2}\left(1-\left|\Gamma_{A}\right|^{2}\right)}
\end{aligned}
$$

where $D=S_{11} S_{22}-S_{12} S_{21}$ is the determinant of the $\boldsymbol{S}$-matrix. The inverse transform to (1) is

$$
\Gamma_{A}=\frac{\Gamma_{R}-S_{11}}{S_{22} \Gamma_{R}-D}
$$

In our case (a typical situation), the circuit between planes $\mathrm{R}$ and $\mathrm{A}$ is a cascade of a launcher waveguide section with length $d_{R}$, a well-matched LWG-to-WR340 transition, and a section of WR340 waveguide with length $d_{h}$. Neglecting the circuit losses and mismatches (justified for a properly designed transition) the S-parameters are

$$
S_{11}=0 ; \quad S_{22}=0 ; \quad S_{12}=S_{21}=\exp \left(j \varphi_{21}\right)
$$

which results in

$$
\Gamma_{R}=\Gamma_{A} \exp \left(j 2 \varphi_{21}\right) ; \quad P_{L}=P_{A}
$$

reducing the transform to merely a phase-shifting (rotation) of $\Gamma_{A}$. The $S_{21}$ phase angle is

$$
\varphi_{21}(f)=-\frac{2 \pi d_{R}}{\lambda_{g r}}+\varphi_{T 21}-\frac{2 \pi d_{h}}{\lambda_{g}}
$$

where $\lambda_{g r}$ is the launcher guide wavelength, $\lambda_{g}$ is the WR340 guide wavelength, and $\varphi_{T 21}$ is the taper transmission coefficient phase. All three quantities are frequency-dependent.

The usefulness of expressing the conversion in terms of $S$-parameters lies in the fact that the PC program controlling the measurement process is capable of loading a text file with tabulated frequency dependence of $S_{i j}$, performing the mappings (1) and (2) internally, and then immediately outputting the Rieke reflection coefficient $\Gamma_{R}$ and the delivered power $P_{L}$.

\section{Realizing Reflection Coefficients}

The procedure of obtaining a Rieke diagram calls for realizing a sufficiently dense set of predetermined Rieke reflection coefficients $\Gamma_{R}$, and consequently, autotuner input reflection coefficients $\Gamma_{A}$. Any $\Gamma_{A}$ within the tuner's capability can be realized by proper tuning stub insertions $h_{1}, h_{2}, h_{3}$ of a match-terminated autotuner. The method of determining the insertions is intimately linked with the autotuning algorithm. An equivalent circuit for an impedance matching arrangement with a matched generator $\left(\Gamma_{G}=0\right)$ is shown in Fig. 4 . The tuner is a loss-free adjustable circuit characterized by its $\mathrm{S}$-matrix $\boldsymbol{S}_{\boldsymbol{A}}$ defined for reference planes 1 and 2 . A load with reflection coefficient $\Gamma_{3}$ is connected at plane 3 , that is usually separated from plane 2 by a transmission line with electric length $\theta$. To achieve a matching of load $\Gamma_{3}$, the tuner must be adjusted so that its input reflection coefficient is $\Gamma_{1}=0$. The theory [8] proves that in this case, the condition $\Gamma_{2}=\left(\Gamma_{x}\right)^{*}$ is satisfied at plane 2 , where $\Gamma_{2}$ is seen looking into port 2 of the tuner and $\Gamma_{x}=\Gamma_{3} \exp (-j 2 \theta)$ is seen looking toward the load. The asterisk indicates the complex conjugation. In fact, $\Gamma_{2}$ is the reflection coefficient "realized" by the reversed tuner. The matched termination at the other port (port 1) is 
provided by the generator. Based on these facts, the following algorithm can be formulated for realizing any input reflection coefficient $\Gamma_{A}$ :

- Choose a desired input reflection coefficient $\Gamma_{A}$ and frequency $f$.

- Run the autotuning algorithm, using as input values the conjugated and rotated reflection coefficient

$$
\Gamma_{3}=\Gamma_{A}^{*} \exp (j 2 \theta)
$$

and frequency $f$. The algorithm will output the tuning stub insertions $h_{1 m}, h_{2 m}, h_{3 m}$.

- $\quad$ "Reverse" the tuner, i.e. set the tuning stubs to insertions $h_{1}=h_{3 m}, h_{2}=h_{2 m}, h_{3}=h_{1 m}$. Then the tuner input reflection coefficient at frequency $f$ will be $\Gamma_{1}=\Gamma_{A}$.

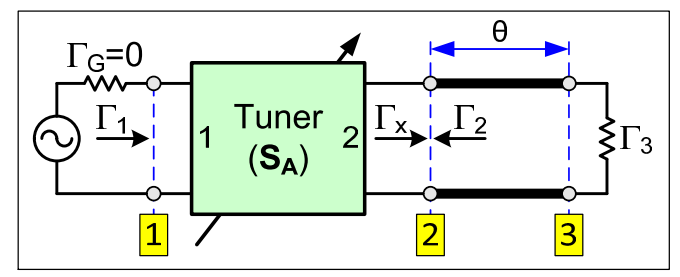

Fig. 4. Equivalent circuit for an impedance matching arrangement.

\section{Rieke Diagram Measurement Procedure}

The procedure for obtaining a Rieke diagram consists of the following basic steps:

1. Define a grid of Rieke reflection coefficients $\Gamma_{R i}, i=1 \ldots n$, that cover with a sufficient density the desired area of the polar chart. The grid need not be regular; it can be, for instance, finer wherever we need a greater detail.

2. Using (3), create from $\Gamma_{R i}$ a set of corresponding tuner input reflection coefficients $\Gamma_{A i}$, $i=1 \ldots n$, to be realized by the autotuner.

3. Create the stubs motion scenario: a sequence $\{\boldsymbol{h}\}_{i} \equiv\left\{h_{1}, h_{2}, h_{3}\right\}_{i}, i=1 \ldots n$, of autotuner stub insertions realizing the set $\Gamma_{A i}$.

4. Run the scenario, i.e. step through the sequence $\{\boldsymbol{h}\}_{i}, i=1 \ldots n$, and, for each setting:

a) Move the tuning stubs to the corresponding insertions.

b) Use the autotuner to measure the reflection coefficient $\Gamma_{A}$, the generated frequency $f_{g}$, and the power $P_{A}$ absorbed in the load (note that the measured $\Gamma_{A}$ will generally differ from the scenario value).

c) Transform $\Gamma_{A}$ to the Rieke reflection coefficient $\Gamma_{R}$, and transform $P_{A}$ to the net power $P_{L}$ delivered by the magnetron. For all practical purposes, the simplified equations (4) - (6) are appropriate for this purpose.

In this way, raw data for constructing a Rieke diagram are obtained. The data is a collection of $n$ triplets $\left\{\Gamma_{R}, f_{g}, P_{L}\right\}_{i}, i=1 \ldots n$, with $\Gamma_{R}=x_{R}+j y_{R}$ being, in general, scattered irregularly and arbitrarily in the complex plane.

5. Choose a range of constant values of $f_{g}$ and of $P_{L}$ for which the Rieke diagram isolines should be drawn.

6. Apply a mathematical routine that

a) uses the acquired data to create tabulated functions $f_{g}=f\left(x_{R}, y_{R}\right), P_{L}=f\left(x_{R}, y_{R}\right)$; and 
b) approximates the two obtained functions by a 2D smoothing spline surface each.

7. Using the approximations, make plots of smoothed isocontours for the chosen values of $f_{g}$ and $P_{L}$, thus completing the desired Rieke diagram construction.

We have developed a dedicated MATLAB routine that implements steps 6 and 7.

\section{Experiments}

To illustrate the developed procedure, we performed experiments using a Panasonic 2M244 magnetron (nominal frequency $f_{0}=2460 \mathrm{MHz}$, nominal power into a match $P_{0}=1010 \mathrm{~W}$ ). The datasheet Rieke diagram (Fig. 1) was reportedly obtained using a single-phase full-wave rectifier without filter, with peak anode voltage $4.35 \mathrm{kV}$. However, in our experiments, we used an available single-phase half-wave rectifier that was only able to reach $900 \mathrm{~W}$ rather than the rated magnetron power $P_{0}$. The time dependence $P_{L}(t)$ of power delivered to a matched load is shown in Fig. 5.

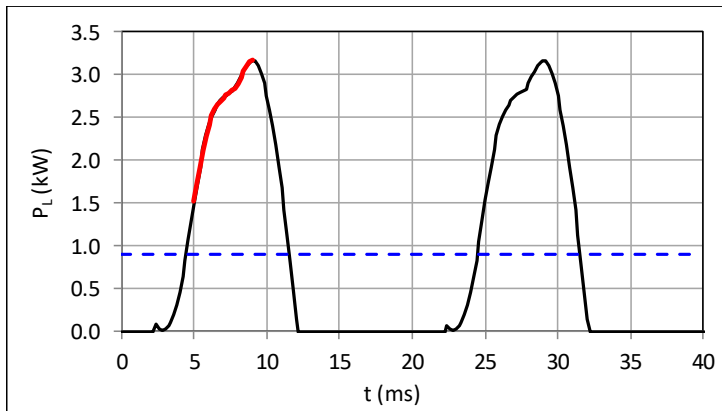

Fig. 5. Time dependence of power $P_{L}$ delivered to a matched load. Dashed line - mean power $(900 \mathrm{~W})$. Red portion - frequency counting interval.

A photograph of the experimental setup is shown in Fig. 6; it corresponds to the block diagram of Fig. 3. The setup employs an S-TEAM WR340 autotuner STHT 1.6. Results of each individual measurement were obtained by processing 201 samples taken over $40 \mathrm{~ms}$, i.e. two periods of the power waveform shown in Fig. 5. Frequency was averaged over the red-highlighted 4-ms interval. To improve the terminating match, we preceded the waterload by an auxiliary tuner with fixed stub insertions.

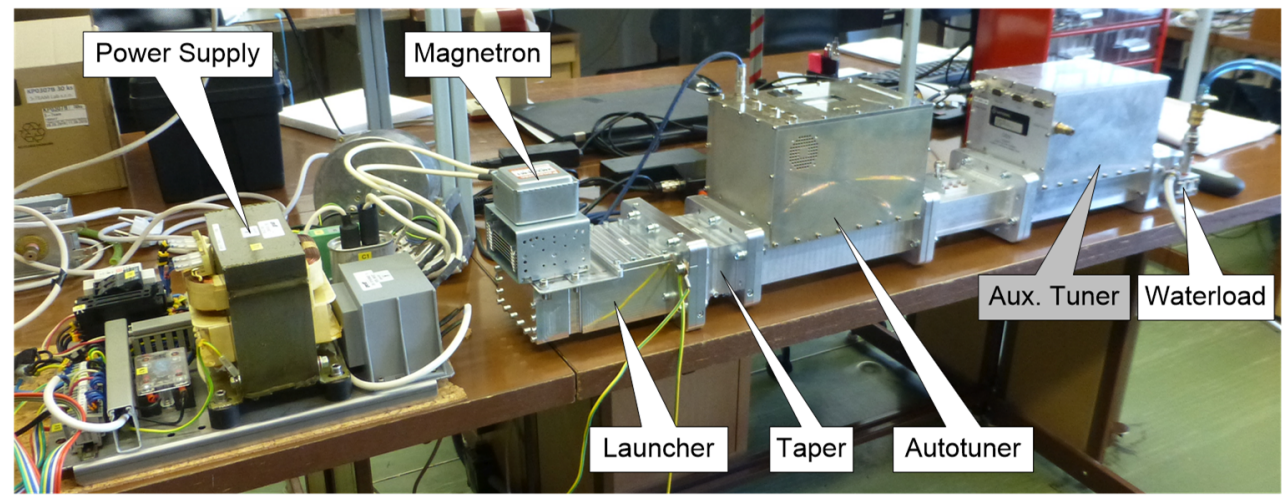

Fig. 6. Experimental setup. 
We used a linear taper with optimized length $82.5 \mathrm{~mm}$. We determined its scattering parameters $S_{T i j}$ in the $2400-2500 \mathrm{MHz}$ range by both electromagnetic simulation and measurement. The difference of the transmission phase $\varphi_{T 21}$ was below $1.4^{\circ}$. The measured magnitude $\left|S_{T 21}\right|$ deviates from theoretical unity by less than 0.005 ; the input mismatches $\left|S_{T 11}\right|$ and $\left|S_{T 22}\right|$ are below 0.01 . Therefore, the assumptions $\left|S_{T 21}\right|=1$ and $\left|S_{T 11}\right|=\left|S_{T 22}\right|=0$ were justified.

We implemented the transformation matrix $\boldsymbol{S}$ (Fig. 3) by applying (4) and (6) for frequencies in the range $2400-2500 \mathrm{MHz}$ in $1-\mathrm{MHz}$ increments, and storing the results in a text file that was then read in by the PC controller SW.

To ready the system for repeated Rieke diagram measurements, we prepared a rectangular grid of tuner input reflection coefficients $\Gamma_{A}$ with maximal magnitude 0.7 and step 0.05 in both dimensions (Fig. 7a). We used the resulting $n=614$ values in the control SW to generate the corresponding stubs motion scenario for an assumed frequency of $2460 \mathrm{MHz}$.

During the measurement process, the control SW steps through the scenario. For each step, the stubs are moved to the prescribed insertions, and then a wait period (in our case $300 \mathrm{~ms}$ ) is introduced to allow the magnetron to settle. Then, a "burst" of measurements (in our case 10 of them) is made and recorded. This cycle is repeated for the whole scenario. For our settings, the complete process took about 24 minutes. All individual results are stored in a text file (in our case $10 \times 614$ data lines) for detailed process analysis. In addition, a separate ".rkd" text file was created with only $n=614$ data lines, each containing the burst averages and standard deviations for the quantities of interest $\left(\Gamma_{R}, f_{g}\right.$, and $\left.P_{L}\right)$. These averages are the actual input data to be used for creating the Rieke diagram; the deviations are useful for detecting possible magnetron instabilities (e.g. jumps in frequency and power).

An example of a measured collection of the Rieke reflection coefficients, as stored in an rkd file, is shown in Fig. 7b. Evidently, the data tend to be "expelled" from the magnetron sink area, where the generated frequency is very sensitive to load variations.
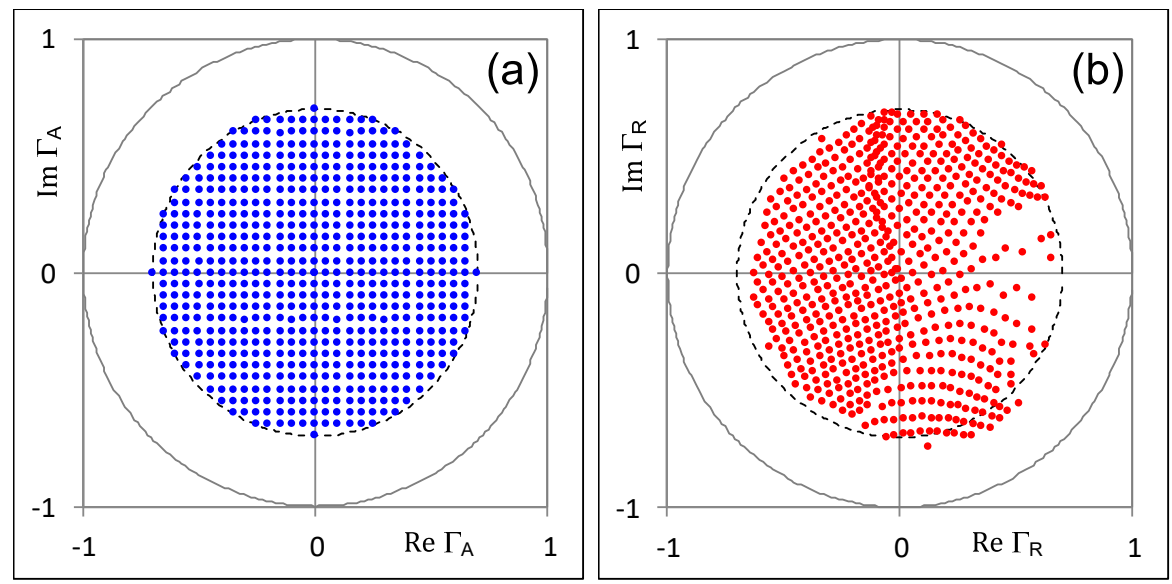

Fig. 7. a) - A grid of desired tuner input reflection coefficients $\Gamma_{A}$; b) - A corresponding set of measured Rieke reflection coefficients $\Gamma_{R}$.

We used the generated rkd file as an input to a MATLAB program implementing steps 6 and 7 of the procedure outlined above. Fig. 8 shows examples of the input data and their approximation by $2 \mathrm{D}$ smoothing spline surfaces, as obtained by the MATLAB function 
tpaps. Using the approximations, smoothed isocontours (horizontal cuts of the surfaces in Fig. 8) can be plotted (e.g. via the MATLAB function contour) for any desired constant value of $P_{L}$ and $\Gamma_{R}$ (solid curves in Fig. 9).

In a similar way, we processed a digitized datasheet Rieke diagram (dashed lines in Fig. 9). The measured and datasheet patterns manifest general similarity but also significant discrepancies. The most likely reason may be the insufficient DC power supply that we used. For explanation of the differences, a systematic study is required but is beyond the scope of this paper. Nevertheless, we have provided an efficient tool that enables, among others, studies of this kind.

\section{Conclusions}

We have developed an experimental setup, a measurement procedure, and a MATLAB data processing program that enable fully automatic measurement and plotting of magnetron Rieke diagrams. The fundamental component of the workplace is a standard, commercially available, high-power autotuner. After a one-time processing for preparation of the necessary text files (transformation $\boldsymbol{S}$-matrix and stubs motion scenario), the measurement is a matter of practically a single button click. Depending on the length of the scenario, the data acquisition process takes typically several tens of minutes, with no involvement required from the operator. Given the data, smoothed Rieke diagram contours can be generated practically immediately.

The proposed system and methodology have the potential to enable systematic statistical studies of the dependence of Rieke diagrams on magnetron operating conditions, for which mass data collection and rapid evaluation are essential.
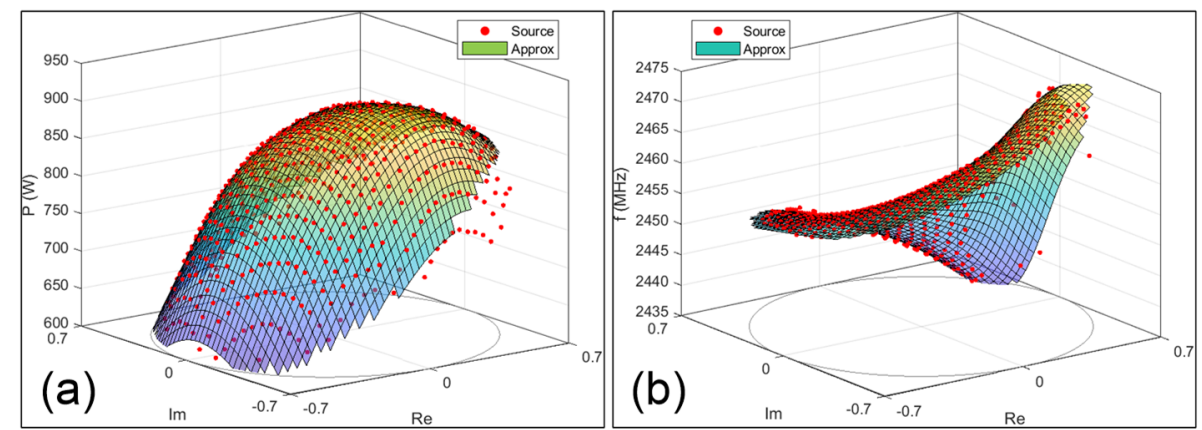

Fig. 8. Input data (red dots) and their spline approximation for (a) delivered power $P_{L}$ and (b) generated frequency $f_{g}$. The bottom surface is $\Gamma_{R}$-plane. 

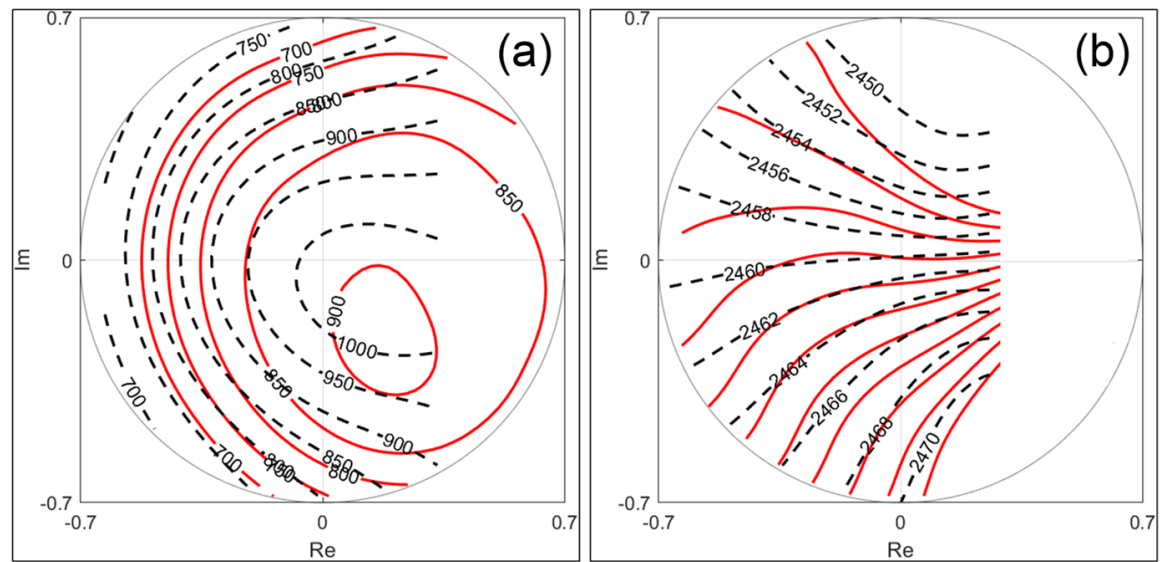

Fig. 9. Rieke diagram contours for (a) delivered power $P_{L}$ and (b) generated frequency $f_{g}$. The dashed lines correspond to the 2M244 magnetron datasheet.

\section{References}

1. Smith, G., Microwave Magnetrons, MIT RadLab Series, Vol. 6, New York: McGraw-Hill, 1948, Sec. 7-5, 18-9.

2. Meredith, R. J., Engineers' Handbook of Industrial Microwave Heating, London: The IEE, 1998, Sec. 9.3.

3. Takahashi, H., I. Namba, K. Akiyama, J. Microwave Power, 1979, 14, 261-267.

4. Yixue, W., Z. Zhaotang, Proc. ICMMT'98, 1998, 795-798.

5. Bilik, V, Proc. $3^{\text {rd }}$ Global Congress on Microwave Energy Applications $3 G C M E A, 2016$.

6. Bilik, V, Proc. $16^{\text {th }}$ Int. Conf. on Microwave and High Frequency Heating AMPERE, 2017.

7. Engen, G., Microwave Circuit Theory and Foundations of Microwave Metrology, London: P. Peregrinus, 1992, 15-39.

8. Bilik, V, Proc. 48 ${ }^{\text {th }}$ Annual Microwave Power Symposium IMPI 48, 2014, 6-9. 


\title{
DEVELOPMENT OF COAXIAL TYPE FLOW MICROWAVE REACTOR
}

T. Matsumura ${ }^{1}$, M. Kishihara ${ }^{2}$, H.Urushihara ${ }^{2}$,

\author{
${ }^{1}$ Minerva Light Laboratory, 1-7Hikaridai, Seika, Soraku, Kyoto Japan \\ ${ }^{2}$ Okayama Prefectual University, 111 Kuboki, Soja, Okayama, 719-1197 Japan \\ mll.minerva@gmail.com
}

Keywords: microwave, solid state generator, coaxial reactor

We have developed various type microwave chemical reactors with solid state microwave generators $^{1), 2)}$. In this report, flow microwave chemical reactors with coaxial cavities with $2.45 \mathrm{GHz}$ and $5.8 \mathrm{GHz}$ microwave is described.

1. Design of Coaxial Cavity Chamber for $2.45 \mathrm{GHz}$ microwave

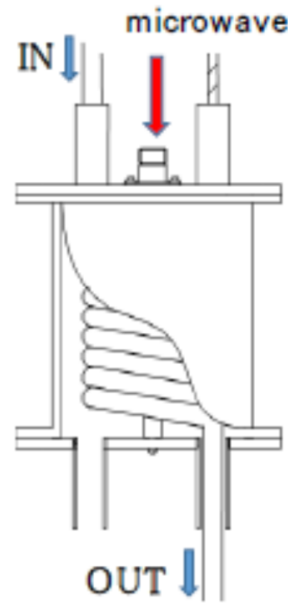

Fig. 1. Structure of coaxial cavity chamber for microwave chemical reaction system.

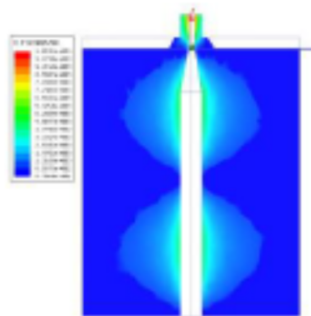

(a)

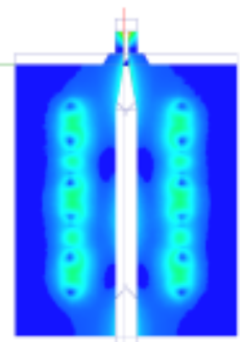

(b)

Fig. 2. Field distributions in the coaxial cavity chamber at $2.45 \mathrm{GHz}$. (a) Electrical field distribution (without glass tube). (b) Magnetic field distribution (with ethylene glycol flowing glass tube).

Figure 1 shows a structure of the coaxial cavity chamber for the $2.45 \mathrm{GHz}$ microwave chemical system. It consists of a cylindrical cavity of $100 \mathrm{~mm}$ inside diameter with a metal rod at the center, containing a spiral glass tube to flow solvents. When the input port of the microwave is placed at the end of the metal rod, TEM mode is excited in the cylindrical chamber due to the presence of the metal rod.

Figure 2 (a) displays an electrical field distribution in the coaxial cavity chamber simulated at $2.45 \mathrm{GHz}$. The two maximum positions of the electric field are found. In this work, the height of the cavity is selected such that the standing wave has two maximum positions in the electric field. In this case, $120 \mathrm{~mm}$ is used. In this case N-type connector is used.

To achieve matched state and avoid excess heating of the input connector, a conical aperture is introduced between the $\mathrm{N}$-type connector and the coaxial cavity. In addition, the end of the center rod is tapered. In order to obtain a matched state, some external matching networks should be connected. In this work, a slug tuner is used. 
Figure 2 (b) shows a magnetic field distribution in the coaxial cavity chamber with the glass tube simulated at $2.45 \mathrm{GHz}$. As a solvent in the glass tube, ethylene glycol is assumed. It is found that the magnetic field is uniform in the glass tube. So, the equal microwave heating is expected in the coaxial cavity chamber. This configuration was confirmed suitable for rapid and continuous microwave syntheses of various functional metal complexes. Experimental results are presented for synthesizing $\operatorname{Ir}(\mathrm{III})$ complexes for OLED dopants and $\mathrm{Ru}(\mathrm{II})$ complexes for various sensors.

2. Microwave syntheses of $\operatorname{Ir}(\mathrm{III})$ complex and $\operatorname{Ru}(\mathrm{II})$ complex with a flow microwave reactor with a coaxial cavity.

$\mathrm{Ir}(\mathrm{III})$ and $\mathrm{Ru}(\mathrm{II})$ complexes are important functional materials as phosphor illuminants or phosphor sensors. As for syntheses of these metal complexes, it is a hard process to dissociate $\mathrm{M}-\mathrm{Cl}$ bond of $\mathrm{MCl}_{3}$ to create new bonds with organic ligands; accordingly effective new synthetic methods of these complexes are strongly required. Conventional synsesis of $\operatorname{Ir}(\mathrm{III})$ and $\mathrm{Ru}(\mathrm{II})$ complexes are tedious, time-consuming, andlow yeild of products.

Using microwave reaction, these difficulties are resolved; rapid and high yield synthetic methods have been reported . 3).4)

Microwave syntheses of these metal complexes are performed with the newly developed coaxial flow microwave reactor as shown in Fg.3.

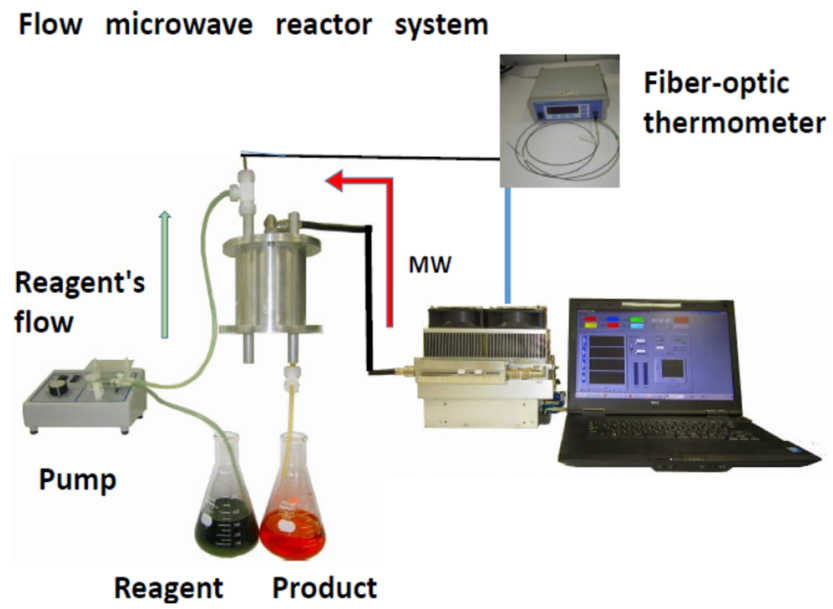

Fig.3 A coaxial flow microwave reactior system

Firpic, [2-(4,6-difluorophenyl)pyridinato- $\left.\mathrm{C}^{2}, N\right]$ picolinato)iridium(III) which is one of blue OLED dopants, was synthsized with the coaxial flow microwave reactor system. The reaction porcess is compared with the conventional synthesis in the table. 
Table. Comparison of classical and flow microwave reactor syntheses.

\begin{tabular}{|c|c|c|c|c|}
\hline $\begin{array}{l}\text { Synthetic } \\
\text { procedure }\end{array}$ & Reaction time & $\begin{array}{l}\text { Power } \\
\text { consumption }\end{array}$ & $\begin{array}{l}\text { Purification } \\
\text { procedure }\end{array}$ & Yield \\
\hline $\begin{array}{l}\text { Classical } \\
\text { synthesis }\end{array}$ & 18.5 hours & $2800 \mathrm{WH}$ & $\begin{array}{l}\text { Column Separation } \\
\text { Long time, Much Solvents }\end{array}$ & $53 \%$ \\
\hline $\begin{array}{l}\text { Flow } \\
\text { microwav } \\
\text { e } \\
\text { method }\end{array}$ & 0.75 hours & $75 \mathrm{WH}$ & $\begin{array}{l}\text { Recrystallization } \\
\text { Short time, } \\
\text { Small amount Solvents }\end{array}$ & $>75 \%$ \\
\hline
\end{tabular}

\section{Property of $5.8 \mathrm{GHz}$ flow microwave reactor}

In the similar manner as the coaxial reaction chamber of $2.45 \mathrm{GHz}$, a coaxial reaction chamber for $5.8 \mathrm{GHz}$ IMS band is designed with $51 \mathrm{~mm}$ diameter and $50 \mathrm{~mm}$ height. The electric field distributions in the chamber and the temperature profiles of solvent are simulated using the commercial simulator (COMSOL Multiphysics) for $5.8 \mathrm{GHz}, 5 \mathrm{~W}$ microwave input.

The structure of the coaxial line type reaction chamber is shown in Fig.3. Metallic rods of diameter $c_{d}$ and height $m_{h}$ are installed as a center conductor in the cylindrical metal cavity (diameter $m_{d}$, height $m_{h}$ ). The tip of the center conductor $c_{l}$ has the form of a truncated cone of diameters $u_{d 1}$ (upper base) and $c_{d}$ (lower base). A lid of thickness $l_{h}$ is attached to the top of the cylindrical cavity. A truncated conical hole with diameters $u_{d 2}$ (upper base) and diameter $b_{d 2}$ (lower base) is used for the coaxial input port of microwave. Selecting the size of the coaxial cavity appropriately, the places where the incident and reflected microwaves are added are generated. By arranging a flow channel (PTFE) which has the outer diameter $o_{d}$ (and the inner diameter $i_{d}$ ) in a spiral shape with the interval $p_{i}$

at the distance $r$ from the center conductor, it is possible to raise the temperature of the solvent in the channel in the chamber.
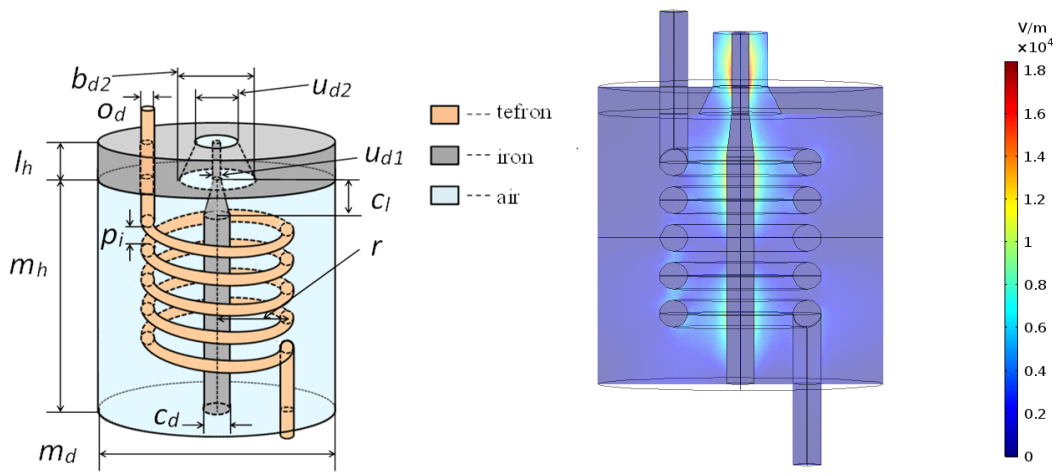

Fig.3 Structure of reaction chamber. Fig .4 Simulated electric field distribution.

For the input port of the microwave, an $\mathrm{N}$ type coaxial connector is assumed, and the upper base $u_{d 2}=10 \mathrm{~mm}$ of the truncated cone shape of the lid and the diameter $u_{d 1}=3.1 \mathrm{~mm}$ of the upper base of the truncated cone part are determined. The dimensions are adjusted so as to realize the low microwave reflection characteristics. As a result, the lower base $b_{d 2}=16$ $\mathrm{mm}$ of the truncated cone shape of the lid, the thickness $l_{h}=5 \mathrm{~mm}$, the diameter of the central 
axis $c_{d}=5 \mathrm{~mm}$, the length from the tip $c_{l}=8 \mathrm{~mm}$ are determined. The electric field distribution obtained by the simulation is shown in Fig.4.

4. Numerical Simulation of Temperature Profiles

The temperature of water for $5.8 \mathrm{GHz}, 5 \mathrm{~W}$ microwave input is examined using the COMSOL. The flow channel installed inside the cavity is assumed to be made of PTFE. However, in this simulation, the thickness PTFE is ignored, that is, the water is arranged in the spiral form at the distance $r=12 \mathrm{~mm}$ from the center conductor and the interval $p_{i}=2$ $\mathrm{mm}$, with the outer diameter $o_{d}=5 \mathrm{~mm}$. The temperature distribution after 300 seconds is shown in Fig.3. The temperature-time profiles at each point P1, P2, P3, P4, and P5 in Fig.3 is shown in Fig.4. In the simulation, the complex relative permittivity of water, $72-\mathrm{j} 20$, thermal conductivity, $0.615 \mathrm{~W} /(\mathrm{mK})$, specific heat capacity $4.2 \mathrm{~kJ} /(\mathrm{kg} \mathrm{K})$, initial temperature $20^{\circ} \mathrm{C}$ is assumed. From Figs 5 and 6 , it is found that the temperature of water rises over 90 ${ }^{\circ} \mathrm{C}$ at $\mathrm{P} 3$ point.

The temperature-time profiles of water were simulated. From the results, it was confirmed that the temperature rises to $95^{\circ} \mathrm{C}$ in 300 seconds, when the microwave power of $5 \mathrm{~W}$ is input. This structure is smaller than that of $2.45 \mathrm{GHz}$ and can be used in less space.

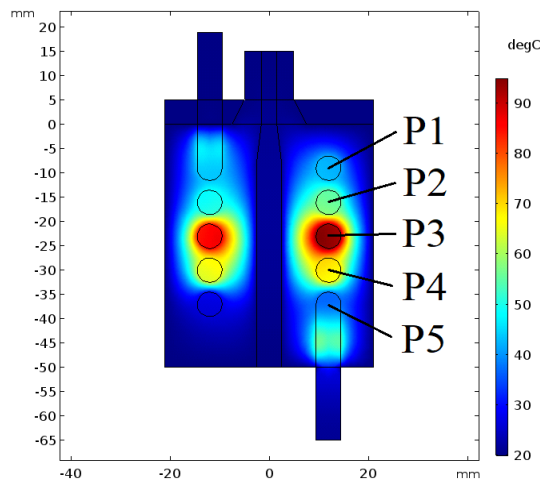

Fig. 5 Simulated temperature distribution.

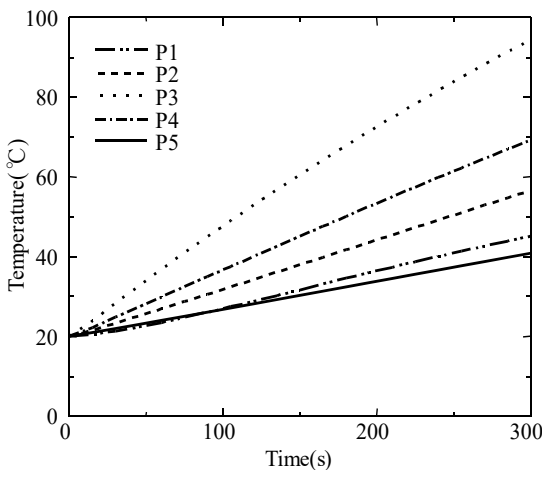

Fig.6 Temperature - time profiles of water under microwave irradiation $(5.8 \mathrm{GHz}, 5 \mathrm{~W})$.

Temperature profiles for experiments are shown in Figure 7 using ethyleneglycol and ethyl alcohol. Thus, the effect of microwave at $5.8 \mathrm{GHz}$, with the higher frequency, the thermal effect became larger with hiher effciency. 


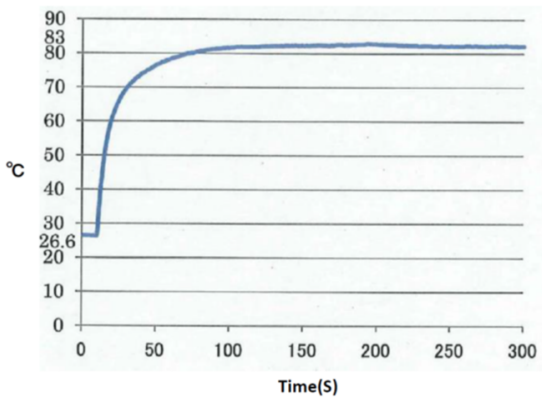

(a) Ethyleneglycol

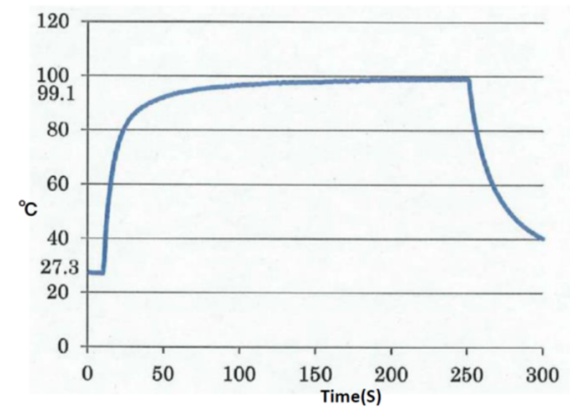

(b) Ethyl alcohol

Fig.7. Temperature Profile for (a)Ethyleneglycol and (b)Ethyl alcohol

\section{References}

[1] T. Matsumura-Inoue et. al, Chemical Engineering 51(8), 590-596, 2006-08

[2] Y. Utsumi, A. Yamaguchi, T. Matsumura-Inoue, and M. Kishihara, Sensors and

[3] Actuators B: Chemical, vol.242, pp.384-388, April 2017

[4] T. Matsumura-Inoue, M. Tanabe, T. Minami, T. Ohashi, Chem. Lett., 1994,2443-2446

[5] T.Matsumura-Inoue, Y.Yamamoto, N.Yoshikawa, M.Terashima, Y.Yoshida, A.Fujii, K.Yoshino, Optical Materials,27,183-191(2004) 


\title{
ON THE POSSIBILITIES OF PERMITTIVITY CALCULATION IN A CERTAIN BANDWIDTH FROM SINGLE FREQUENCY RESULTS
}

\author{
Antonio José Lozano Guerrero ${ }^{1}$, Juan Monzó-Cabrera ${ }^{1}$, Alejandro Díaz-Morcillo ${ }^{1}$ \\ ${ }^{1}$ Departamento de Tecnologías de la Información y las Comunicaciones, Universidad \\ Politécnica de Cartagena, Cartagena (Murcia) Spain 30202. \\ antonio.lozano@upct.es
}

Keywords: inverse technique, microwave, permittivity

\section{Introduction}

The permittivity of a material can be obtained from resonant measurements in an accurate way [1] at a single frequency (where the resonance occurs). In this work we propose and explore the possibilities of obtaining the permittivity of materials from resonant measurements in a certain frequency bandwidth around the resonance frequency. With this purpose a Debye model useful for polar liquids [1] jointly with a certain conductivity, are studied to evaluate this possibility jointly with inverse techniques.

The dielectric permittivity is a complex number

$$
\varepsilon^{*}=\varepsilon^{\prime}-j \varepsilon^{\prime \prime}
$$

and its dependence with the frequency can be obtained from [2] for polar liquids

$$
\varepsilon^{*}(\omega)=\varepsilon_{\infty}+\frac{\varepsilon_{S}-\varepsilon_{\infty}}{1+j \omega \tau}
$$

where: $\varepsilon^{\prime}$ is the dielectric constant, $\varepsilon^{\prime \prime}$ is the loss factor, $f$ is the frequency $(\mathrm{Hz}), \varepsilon_{S}$ and $\varepsilon_{\infty}$ are the dielectric constants at DC (low frequency) and at very high frequency, respectively. $\omega=2 \pi f(\mathrm{rad} / \mathrm{s})$ is the angular frequency. $\mathrm{T}(\mathrm{s})$ is the relaxation time of the system. It is defined as the time it takes the polarization of the material falling to 36.79 $\%$ of its value when the electric field is no longer applied.

To include the conductivity $\sigma(\mathrm{S} / \mathrm{m})$ in the Debye model [2] we have added to the permittivity model the term [3]

$$
\frac{\sigma}{\mathrm{j} \omega \varepsilon_{0}}
$$

where $\varepsilon_{0}=8.8542 \times 10^{-12}(\mathrm{~F} / \mathrm{m})$ is the free space permittivity and $j$ is the imaginary constant.

To include the temperature in the fitting models for expressions (1-2) we have used from [1] the following linear expressions for $\varepsilon_{s}$ and $\varepsilon_{\infty}$, and the exponential one for relaxation time T:

$$
\begin{aligned}
& \varepsilon_{\mathrm{s}}=\mathrm{a} \cdot \mathrm{T}+\mathrm{b} \\
& \varepsilon_{\infty}=c \cdot T+d
\end{aligned}
$$




$$
\tau=\tau_{0} \mathrm{e}^{\mathrm{E} / \mathrm{T}}
$$

where $T$ is the temperature and $E$ the activation energy. Coefficients $\left(a, b, c, d, \tau_{0}, E\right)$ were obtained by minimizing the fitness function (7).

$$
\text { feval }=\sqrt{\frac{\sum_{\mathrm{i}=1}^{\mathrm{N}}\left|\varepsilon_{\text {measured }}^{*}(\mathrm{i})-\varepsilon_{\text {model l ed }}^{*}(\mathrm{i})\right|^{2}}{\mathrm{~N}}}
$$

$\varepsilon_{\text {measured }}^{*}(i)$ is each frequency point for which the permittivity has been measured and $\varepsilon_{\text {model l ed }}^{*}(i)$ is each frequency point for which the permittivity has been modelled. $N$ is the number of non-equally temperature spaced samples which in our case is 7 .

\section{Method}

Samples were heated in a commercial microwave oven to near its boiling temperature. Its dielectric properties were measured at a frequency near $2 \mathrm{GHz}$ with a dielectrometer, which uses vials, ITACA (ITACA Dielkitv, Dielectrometer for vials, DIMAS, Spain.). Obtained data were processed by using Matlab (Mathworks, Natick, USA). As can be seen in Figure 1 an optical fiber from (Neoptix, Nomad Touch, Canada) was used to obtain the temperature. Measurements were acquired for temperature, frequency and permittivity while the temperature decreased. Various measurement rounds were carried out in order to test the repeatability of the procedure. Since permittivity changed with temperature resonant frequency also did as expected.

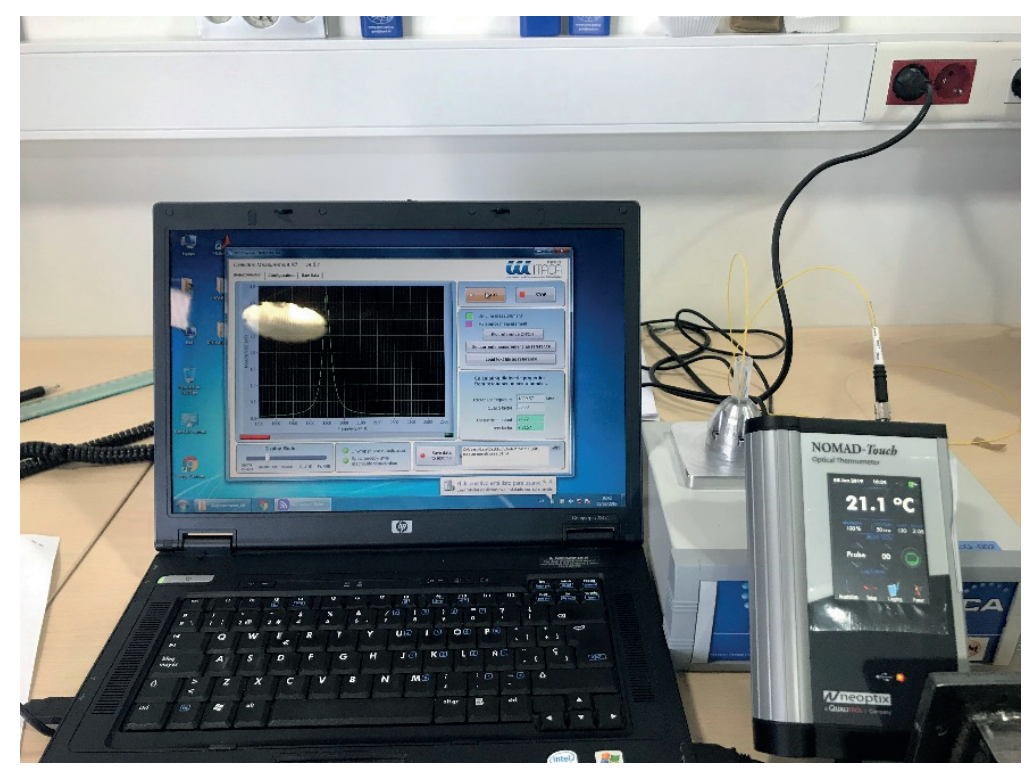

Fig. 1. Set up for the dielectrrometer with a fiber optic temperature probe. 
Concerning the optimization technique genetic algorithms are a powerful and wellestablished tool that has been used for the past 20 years successfully when a global optimum needs to be found. Extracting inversely the permittivity of materials at microwave frequencies has been studied in [4]. The parameters to be optimized depend on the unknowns for the Debye model including the term for the conductivity at the different temperatures. Upper and lower bound limits were established around the distilled water values except for the conductivity. To obtain the fitted models around 100 generations and a population of about 5000 were used in the studies. Genetic Algorithms jointly with gradient descend method have been used in Matlab (Mathworks, Natick, USA). Since most of the materials provide limited $\varepsilon_{\infty}$, this parameter can be fixed to a finite value. For instance: 1 , to reduce uncertainty and an excessive degree of freedom which may lead to wrong solutions.

\section{Results}

Optimized parameters are shown in Table I for distilled water provided in [1] and the ones obtained in this study. Jointly, the conductivity at each of the temperatures $(296.5,303$, $313.3,322.3,333.5,343.4$ and $357.3 \mathrm{~K}$ ) was also optimized. As we can see they do not differ much from results in [1] and they have been adjusted to our own measurements using only results from the available measurements.

Table 1. Parameter values for the Debye model for distilled water.

\begin{tabular}{ccccccc}
\hline Parameters & $\boldsymbol{a}$ & $\boldsymbol{b}$ & $\boldsymbol{c}$ & $\boldsymbol{d}$ & $\tau_{0}(\mathrm{~s})$ & $\boldsymbol{E}$ \\
\hline From [1] & -0.35 & 183.68 & -0.03 & 14.40 & $1.61 \mathrm{e}-15$ & 2470 \\
This work & -0.27 & 155.15 & 0 & 1 & $3.46 \mathrm{e}-15$ & 2278 \\
\hline
\end{tabular}

In Figure 2 and Figure 3, the dielectric constant and the loss factor of distilled water are shown respectively at different temperatures $(296.5,303,313.3,322.3,333.5,343.4$ and $357.3 \mathrm{~K})$. It can be observed how the dielectric constant and the loss factor generally decrease with temperature at the measurement frequencies. To fit the results a Debye model (2) including the ionic conductivity from (3) has been used. Expressions from (4-6) have been included and coefficients for $\left(a, b, c, d, \tau_{0}, E\right)$ were the parameters to be optimized jointly with the conductivity at each of the temperatures enforcing $\varepsilon_{\infty}=1$.

The fitting model results are represented with the discontinuous line in Figures 4 and 5 from $1.5 \mathrm{GHz}$ to $100 \mathrm{GHz}$ and reproduce the permittivity values better at frequencies close to the measurement ones. For these frequencies several values for $\varepsilon_{\mathrm{s}}, \varepsilon_{\infty}$ and $\mathrm{T}$ may provide reasonable optimums for (7) provided that the measured frequency range is limited to $1905 \mathrm{MHz}$ to $1950 \mathrm{MHz}$ and taking into account that our measurements show a noisy behavior. For this reason, upper and lower bounds for the relaxation time parameters have been adjusted around the theoretical ones to provide reasonable results. For the optimized conductivity $(296.5,303,313.3,322.3,333.5,343.4$ and $357.3 \mathrm{~K})$ results show a good repeatability and can be observed in Figure 6 . Values near the expected $0 \mathrm{~S} / \mathrm{m}$ for the distilled water are obtained. 


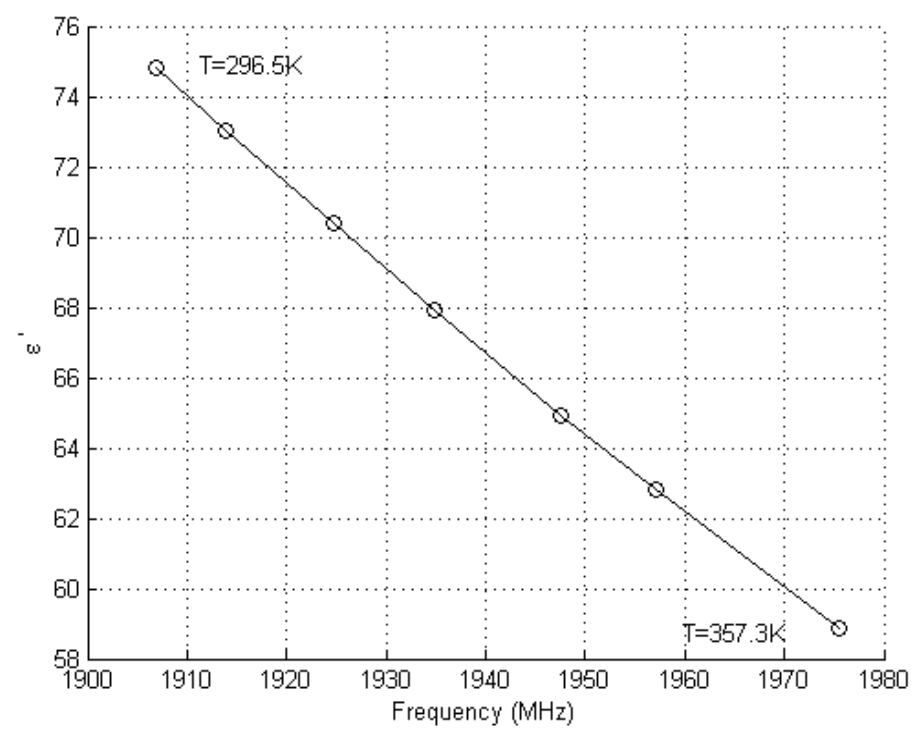

Fig. 2. Measured dielectric constant of distilled water at different temperatures.

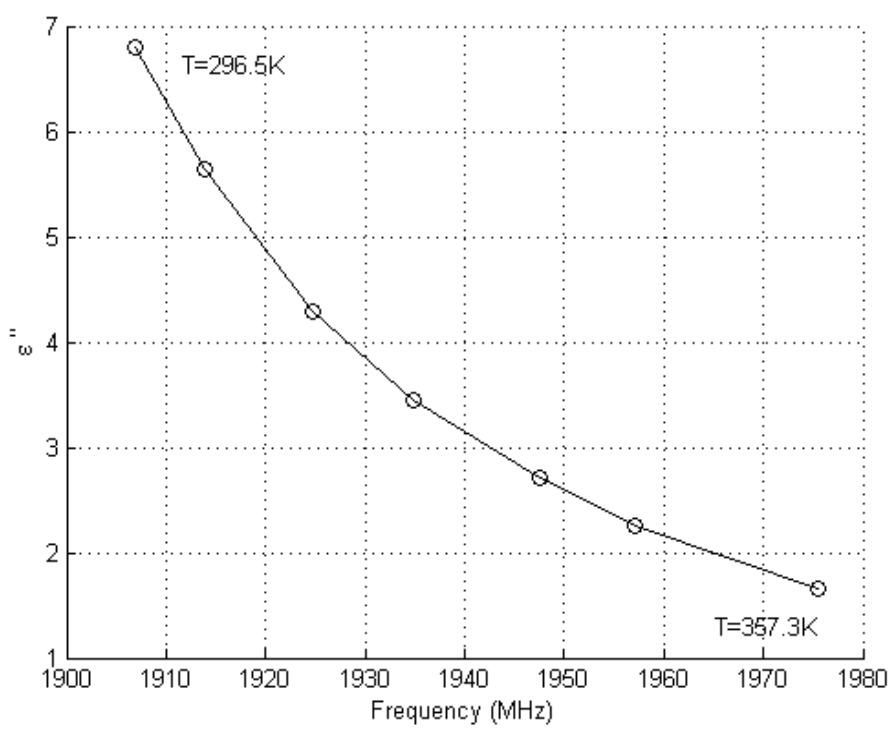

Fig. 3. Measured loss factor of distilled water at different temperatures. 


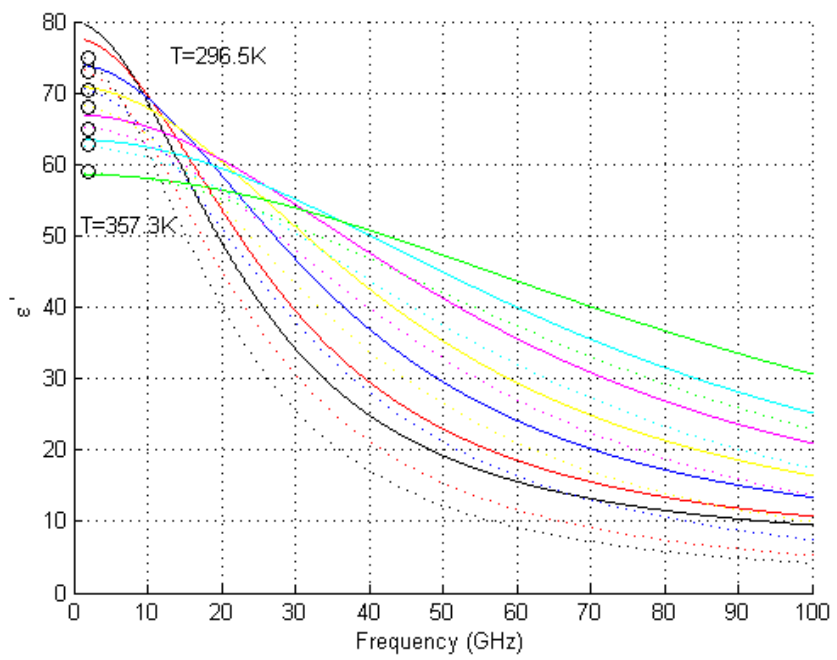

Fig. 4. Results for the dielectric constant of distilled water at different temperatures (Continuos line: model in [1], discontinuous line: proposed method, o: measurements).

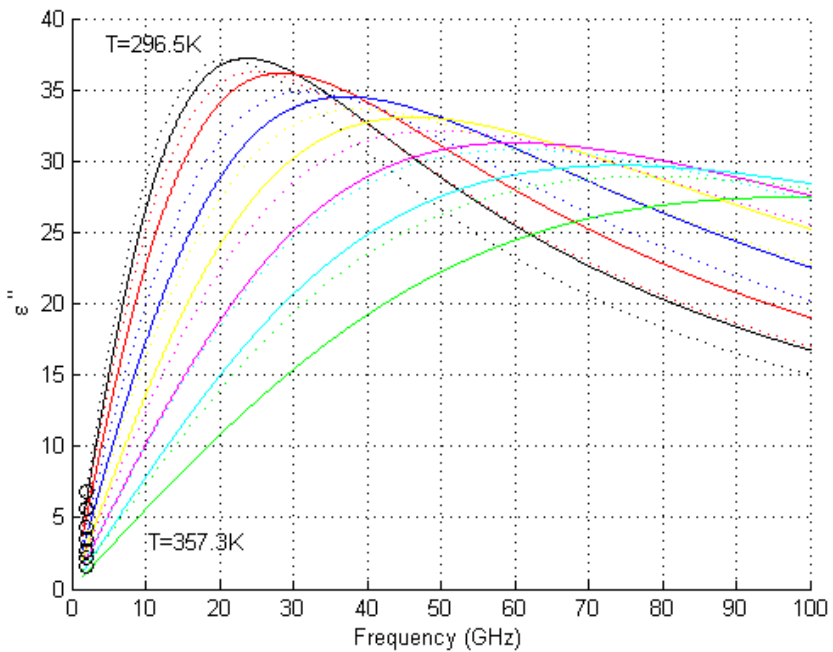

Fig. 5. Results for the loss factor of distilled water at different temperatures. (Continuos line: model in [1], discontinuous line: proposed method, o: measurements). 


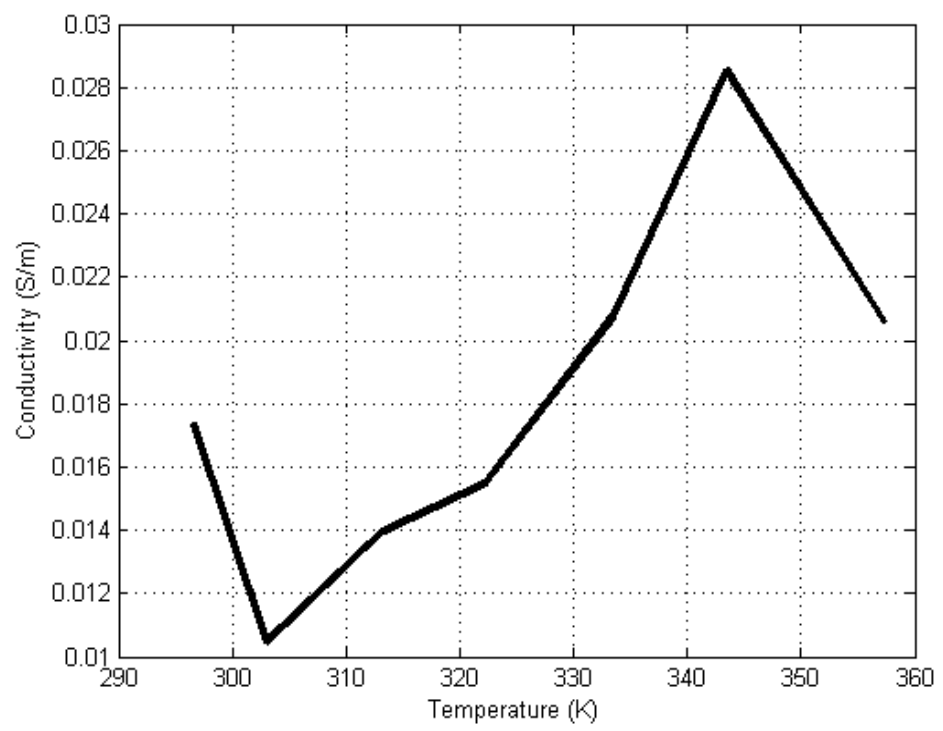

Fig. 6. Conductivity for the distilled water at different temperatures.

However uncertainty for the parameters related with the relaxation time is high, as it could be expected, since the measurement frequencies are far from the frequency value related with the relaxation time using the relationship

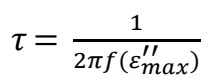

This time value (inverse of the angular frequency at which the loss factor is maximum) leads to a frequency around $20 \mathrm{GHz}$ at room temperature for the distilled water, far from measured values around $1.9 \mathrm{GHz}$.

\section{Conclusions}

In this work we have studied the idea of obtaining the permittivity of a material at different temperatures from measurements using a resonant cavity in a certain wider bandwidth. Since known models such as the Debye are available it is possible to provide an estimation of the permittivity in a broader bandwidth using the cited measurements, the permittivity model and an optimization technique. Genetic algorithms have been used successfully for this purpose. However, care must be taken since selecting wider lower and upper optimization limits may lead to unexpected solutions. As it could have been expected results for the provided solution are worse for farer frequencies from the measurement one. The main uncertainty becomes for the relaxation time since, at the measured frequencies, different combinations for this parameter may lead to reasonable values for the fitness function and more work is envisaged in this direction. 


\section{References}

1. Álvarez, A., Fayos-Fernández, J., Monzó-Cabrera, J., Cocero, M.J. and Mato R.B. (2017), Journal of Food Engeeneering, 197, 98-106.

2. Debye, P. (1929) Chemical Catalogue Company, New York.

3. Tofighi, M. R. (2009), IEEE Transactions on Microwave Theory and Techniques, 17, 2588-2596.

4. Lozano-Guerrero, A.J., Monzó-Cabrera, J., Pedreño-Molina, J.L., ArtésHernández, F., Díaz-Morcillo, A. (2016), Global Congress on Microwave Energy Applications, Cartagena, (SPAIN). 


\title{
APPLICATION OF DIELECTRIC RESONATORS TO SURFACE IMPEDANCE MEASUREMENTS OF MICROWAVE SUSCEPTORS
}

\author{
M. Celuch ${ }^{1}$, J. Rudnicki ${ }^{1}$, J. Krupka ${ }^{2}$, W. Gwarek ${ }^{1}$ \\ ${ }^{1}$ QWED Sp. z o.o., Krzywickiego 12/1, 02-078 Warsaw, Poland \\ ${ }^{2}$ Faculty of Electronics and Information Technology, Warsaw University \\ of Technology, Nowowiejska 15/19, 00-665 Warsaw, Poland \\ mceluch@gwed.eu
}

Keywords: microwave susceptors, active packaging, material measurements, microwave dielectric resonators, microwave heating

\begin{abstract}
This paper describes the application of dielectric resonators (DR) to the measurements of surface impedance of microwave susceptors. We demonstrate that the single-post (SiPDR) configuration is applicable to plain susceptors before use, while the split-post (SPDR) configuration - to crazed susceptors after use. Attention is given to the full characterisation of active packaging, that is, the influence of paper support on the overall electric losses is also investigated. The measurements can be preformed with various form-factor VNAs, including benchtop VNAs and hand-held FieldFox, though the most economical setup is constructed with a dedicated computer-controlled microwave signal oscillator system available under the name of Q-Meter. Finally, an extension of dielectric resonator measurements to surface imaging is presented, achieved by incorporating the resonator in a $2 \mathrm{D}$ automatic scanner.
\end{abstract}

\section{Susceptors and their microwave characterisation}

Microwave susceptors are thin metallic layers incorporated into microwaveable food packages for the enhancement and control of the heating process [1], [2], [3]. Their most typical function is to increase the microwave power dissipation at the specific stages of the heating, though in some cases they are used to shield selected parts of the food. A daily-life example is a microwave popcorn bag: at the room temperature, the seeds of corn and the solidified grease are both lossless, and it is only thanks to the susceptor that the microwave heating initiates. The grease (and then the corn) warm up by the classical heat diffusion, their electric losses increase, and volumetric heating begins. The susceptor, on the other hands, breaks due to thermal expansion of its supporting material, no longer conducts the surface currents, and hence no longer heats [4].

The parameter which fully characterises a microwave susceptor, from the viewpoint of its microwave behaviour, is surface resistance - also called sheet resistance - denoted by symbol $R_{s}$ and expressed in Ohm per square. For a plain uniform susceptor of thickness $d_{0}$ and conductivity $\sigma_{0}$ we have:

$$
R_{s}=\left(d_{0} \sigma_{0}\right)^{-1}
$$

In a separate study to be presented at the conference, and based on [5], we mathematically prove that the actual values of conductivity and thickness are indeed irrelevant, and the effective value of $R_{s}$ unambiguously determines the power dissipated in the susceptor as well as its complex reflection and transmission coefficients, as long thickness $d$ of the surrogate 
layer modelling the original susceptor does not exceed ca. 0.02 wavelength $(1 \mathrm{~mm}$ at the ISM frequency of $2.45 \mathrm{GHz}$ ) and the following relation is conserved:

$$
R_{s}=\left(d_{0} \sigma_{0}\right)^{-1}=(d \sigma)^{-1},
$$

where $\sigma$ is the scaled conductivity of the surrogate.

Microwave measurements of surface resistance of susceptors are of practical importance for the understanding and design of microwaveable food packages. They have been subject of different approaches summarised, for example, in [1]. Traditionally those measurements are performed by transmission line methods: the susceptor is mounted between the flanges of two waveguide to coax adapters and its surface impedance is deembedded from Sparameters measured with a vector network analyser (VNA). Such setups require professional microwave laboratory equipment, which in turn needs to be operated by a trained microwave engineer. These deficiencies have stimulated our search for simpler and cheaper methods of susceptor measurements.

In this work, we discuss an approach based on dielectric resonator (DR) methods, originally developed for microwave measurements of low-loss dielectric materials. In particular, the split-post dielectric resonator (SPDR; Fig. 1) method after [6] has become a formal IEC standard [7] for low-loss laminar dielectrics. In [8], the SPDR method has been extended to high-resistivity semiconductor wafers and an alternative single-post dielectric resonator (SiPDR; Fig. 2) has been developed for semiconductors having resistivity less than $100 \Omega \mathrm{cm}$. While DR - just like waveguide - test fixtures can be operated with a VNA, they do not require all VNA capabilities. Hence to further decrease the cost and increase the easeof-use, we propose the DR setups operating with dedicated Microwave Frequency Q-Meters, controlled by a laptop [9].

The possibility to apply DRs to the characterisation of microwave susceptors has been noted in a workshop presentation [10] but remains unpublished and further unexplored. We build upon the concepts of [10] and discuss the application of SiPDR to plain susceptors before use, and of SPDR to crazed susceptors after use. We also present a new 2D scanner, which allows DR imagining of large inhomogeneous surfaces.

\section{Principles of SPDR and SiPDR application to susceptors}

The principle behind dielectric resonator characterisation of materials resides in making two measurements of resonant frequencies and Q-factors:

- one of the empty resonator, producing $f_{0}$ and $Q_{0}$,

- and one of the resonator loaded with the sample under test (SUT), producing $f_{m}$ and $Q_{m}$.

Dielectric resonators considered in this work are designed so that the electric field distribution inside the empty resonator denoted by $E_{0}$ remains approximately unchanged, after SUT is inserted, and constant along the SUT's height $h$. The following relations then apply, which allow calculating the complex permittivity $\varepsilon_{m}=\varepsilon_{m}{ }^{\prime}-\mathrm{j} \varepsilon_{m}$ " of the SUT material:

$$
\begin{aligned}
& \frac{f_{0}-f_{m}}{f_{0}} \approx \frac{h}{2 C} \iiint_{S}\left[\varepsilon_{m}^{\prime}(x, y)-1\right]\left|E_{0}(x, y)\right|^{2} d S \\
& \frac{1}{Q_{m}}-\frac{1}{Q_{0}} \approx \frac{h}{C} \iint_{S} \varepsilon_{m}^{\prime \prime}(x, y)\left|E_{0}(x, y)\right|^{2} d S
\end{aligned}
$$




$$
C=\iiint_{V}\left|E_{0}(x, y)\right|^{2} d V
$$

where $V$ is DR's volume and $S$ is SUT's lateral surface. The above equations can be manipulated to provide the value of surface resistance of thin material sheets deposited on semi-insulating substrates, in which case the resonator loaded with the semi-insulating substrate is considered as the "empty" one. While the nonlinear manipulations exceed beyond the scope of this paper, their graphical plots can be found in e.g. [8].

In the split-post dielectric resonator configuration after [6], the electric field demonstrates maximum strength in the area between the two dielectric posts. Hence, there is a strong interaction between the resonator and the SUT. The SPDR method has therefore been recognised as a standard for the characterisation of low-loss dielectric materials [norm], on average providing the accuracy of $0.3 \%$ for real permittivity and $3 \%$ for the loss tangent (see [7][11] for more detailed error estimates). For higher-loss materials, the resonance is damped and thereby the highest measurable conductivity is of the order of $1 \mathrm{~S} / \mathrm{m}$, corresponding to the lowest sheet resistance of $5 \mathrm{k} \Omega /$ sq. Hence SPDRs can be used for typical susceptors after use or during use. For plain susceptors before use, whose sheet resistance is expected to be significantly below $5 \mathrm{k} \Omega / \mathrm{sq}$, an alternative single-post configuration (SiPDR) previously developed for semiconductor wafers [8] becomes applicable. In SiPDR the electric field decays rapidly in the direction from the dielectric post towards the metal plate, where it sets to zero, see Fig. 2. The sample weakly interacts with the resonant fields allowing one to measure sheet resistances as low as $0.2 \mathrm{~m} / \mathrm{sq}$.
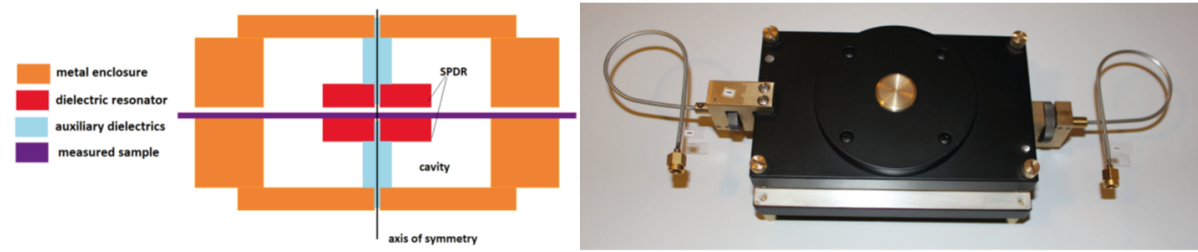

Fig. 1. Split-Post Dielectric Resonator: schematic (left) and photo (right) of an example implementation [11].
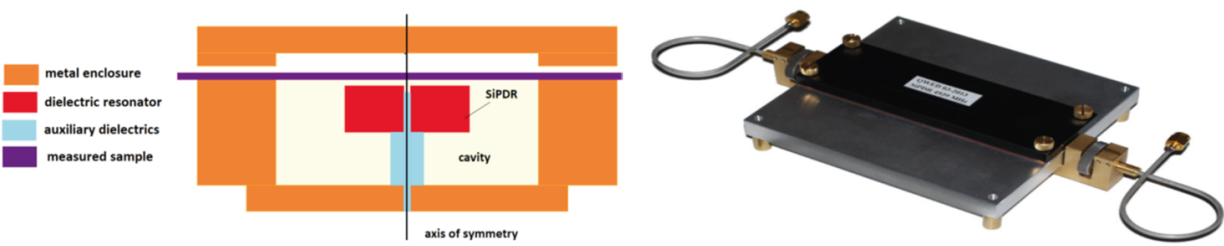

Fig. 2. Single-Post Dielectric Resonator: schematic (left) and photo (right) of an example implementation [11].

\section{Example measurements of microwave popcorn packages}

To validate our existing SPDR and SiPDR in application to microwave susceptors, we bought a few tens of ready-to-use packages of popcorn. We divided them into several batches of four bags each. For each batch, the smallest and biggest values of the measured surface resistance are recorded in Table 1. 
From the packages of the first batch, we cut out the part of the paper bag containing the susceptor and measured it in SiPDR (measurement in SPDR was impossible, due to too high losses of the sample). The following three batches were heated for, respectively, $60 \mathrm{sec}$, $120 \mathrm{sec}$, and $240 \mathrm{sec}$; then the part of the bag containing the susceptor was cut out and measured in SPDR. (Here, some of the samples could be measured in SiPDR, but at the limit of SiPDR application, where high sensitivity to measurement conditions led to big deviations between the results.)

All the above four batches of the measurements characterised "susceptor on paper" and are recorded in the second column of Table 1. The values for the original unheated bags are in agreement with our expectations based on [1][10]. However, during the heating the surface resistance increases slower than expected in [10]. This could be explained by the contributions of losses from the paper, which is in contact with grease and becomes wet and sticky after the heating. To verify this hypothesis, we treated further batches analogously as above, but after the heating, we decomposed each package and measured the actual PET susceptor and the paper substrate separately. The results are recorded in the fourth and third columns of Table 1, respectively. They confirm that after $240 \mathrm{sec}$ of the heating, the greased paper and the crazed susceptor equally contribute to the overall electric losses of the package, which (based on the equations from [5]) cause by over an order in magnitude less of dissipated power than the original unheated susceptor.
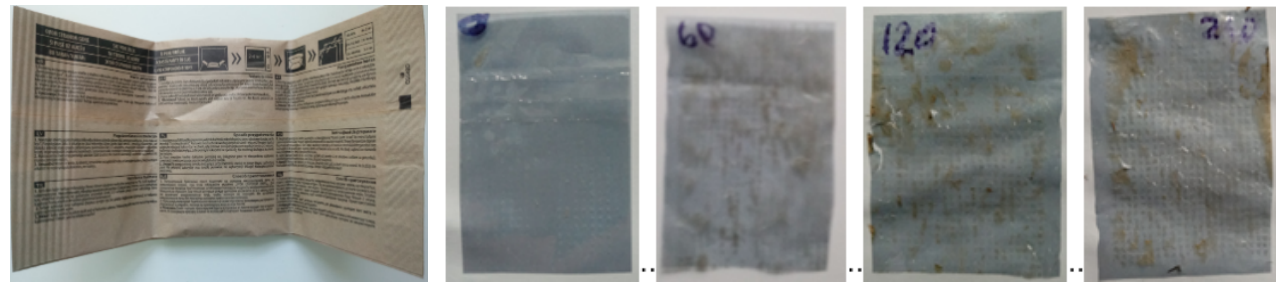

Fig. 3. From left to right, examples of: popcorn bag, original unheated susceptor and susceptors heated for $60 \mathrm{sec}, 102 \mathrm{sec}, 240 \mathrm{sec}$.

Table 1. Measurements (blue - SPDR, red - SiPDR)) of surface resistivity [ $\Omega / \mathrm{sq}]$ of the extracted elements of popcorn packages: susceptor on support paper; only paper, and only susceptor. Results in blue are from SPDR, red from SiPDR. From each batch of the measurements, minimum and maximum values are recorded.

\begin{tabular}{cccc}
\hline $\begin{array}{c}\text { Heating time } \\
{[\mathrm{sec}]}\end{array}$ & Susceptor on paper & Paper & Susceptor \\
\hline 0 & 141.10 & 86432 & 194.13 \\
0 & 258.75 & 87197 & 254.97 \\
60 & 3398.0 & 90233 & 2245.0 \\
60 & 4682.9 & 92795 & 3054.9 \\
120 & 7231.2 & 92265 & 4721.3 \\
120 & 8085.2 & 92993 & 5510.0 \\
240 & 6691.4 & 92559 & 9868.4 \\
240 & 6875.3 & 94605 & 11893.0 \\
\hline
\end{tabular}




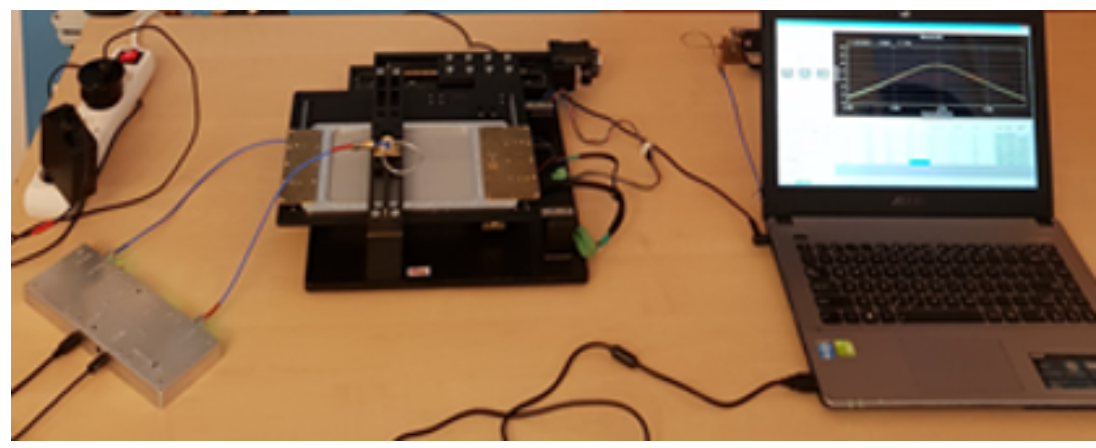

Fig. 4. A complete 2D surface imaging setup comprising Q-Meter, scanner, and laptop.

\section{IV.Conclusions and future work}

In the present work our existing split-post and single-post dielectric resonators, originally developed for low-loss laminar dielectrics and for resistivity measurements of semiconductor wafers at a nominal frequency of $5 \mathrm{GHz}$, have been applied to the measurements of surface resistance of microwave susceptors. It has been shown that the susceptors found in ready-to-use microwaveable popcorn packages are within the measurability range of SiPDR. Their surface resistance obtained with SiPDR and a dedicated Q-Meter is of the order of $100 \div 200 \Omega / \mathrm{sq}$, which is consistent with the more classical transmission line measurements requiring a professional VNA [1]. The susceptors crazed after use have been measured in SPDR, with surface resistances as high as $10 \mathrm{k} \Omega / \mathrm{sq}$ - which demonstrates a broader range of measurable values, when compared to the reported transmission line measurements performed up to $400 \Omega / \mathrm{sq}[1]$.

Our measurements taken with the Q-Meter have been confirmed in a laboratory setup consisting of the same resonators and a fully-fledged benchtop VNA. Both measurement setups produce the results in excellent agreement. Therefore, a suite compring one Q-Meter and two replaceable resonators (SPDR and SiPDR) is recommended for fast and easy characterisation of microwave susceptors, also in a manufacturing or environment food research laboratories where VNAs are unavailable. The proposed suite is low-cost (especially when compared to a VNA purchase), portable, and controlled from a standard laptop.

Finally, in Table 1 we note ca. 20\% deviations between the measurements of different susceptors, heated for the same time. Since each susceptor was measured only once, we cannot say whether those were the differences between the different susceptors, or any individual susceptor may exhibit such variations over its surface. Therefore, further work is planned on investigating surface non-uniformities of susceptors. To this end, surface imaging of susceptors will be performed with a newly constructed 2D scanner, which incorporates a $10 \mathrm{GHz}$ SPDR, as shown in Fig. 4. Microwave measurements can be taken with a Q-Meter or a new hand-held FieldFox VNA from Keysight; in both cases, the setup remains portable and controlled from a laptop. Extended results of the work will be presented at the Conference. 


\section{Acknowledgement}

This project has received funding from the European Union's Horizon 2020 research and innovation programme (H2020-NMBP-07-2017) under grant agreement MMAMA No. 761036.

\section{References}

1. M. R. Perry and R. R. Lentz, "Susceptors in microwave packaging," in Development of packaging and products for use in microwave ovens, $1^{\text {st }}$ ed., W. Lorence and P. S. Pesheck, London, United Kingdom: Woodhead Publishing Limited and CRC Press, 2009, pp. 207-236.

2. T. H. Bohrer, "Shielding and field modification - thick metal films," in Development of packaging and products for use in microwave ovens, $1^{\text {st }}$ ed., W. Lorence and P. S. Pesheck, London, United Kingdom: Woodhead Publishing Limited and CRC Press, 2009, pp. 237-266.

3. P. Risman, "Modelling the effects of active packaging of microwave foods," in Development of packaging and products for use in microwave ovens, $1^{\text {st }}$ ed., W. Lorence and P. S. Pesheck, London, United Kingdom: Woodhead Publishing Limited and CRC Press, 2009, pp. 349-371.

4. P. Risman, "Microwave popcorn popping - a multi-step process", private communication, 2019.

5. M. Celuch, K. Wilczynski, and M. Olszewska-Placha, "Macroscopic models of thin conductive layers: systematic evaluation for microwave heating and shielding applications", Proc. IEEE Intl. Microwave Symp. IMS 2019, Boston, MA, June 2019, pp. 47-50.

6. J. Krupka, A. P. Gregory, O. C. Rochard, R. N. Clarke, B. Riddle, and J. Baker-Jarvis, "Uncertainty of complex permittivity measurements by split-post dielectric resonator technique", J. Eur. Ceramic Soc., vol. 21, 2001, pp. 2673-2676.

7. IEC61189-2-721: 2015 standard.

8. J. Krupka and J. Mazierska, "Contactless measurements of resistivity of semiconductor wafers employing single-post and split-post dielectric-resonator techniques," IEEE Trans. Instr. Meas., vol. 56, no. 5, Oct. 2007, pp. 1839-1844.

9. P. Korpas, L. Usydus, and J. Krupka J (2012), "Automatic split post dielectric setup for measurements of substrates and thin conducting and ferroelectric films", Ferroelectrics, 434:1, pp. 113-120, 201. DOI: 10.1080/00150193.2012.732779

10. W. Gwarek, "Modeling and measurements of susceptors for microwave heating applications", at: IEEE IMS Workshop "Recent Advances in Microwave Power Applications and Techniques RAMPAnT', Boston, MA, June 2009.

11. http://www.qwed.eu/resonators.html\#ResonatorsFamily, accessed 10 June 2019. 


\section{MEASUREMENT OF THE DIELECTRIC PROPERTIES OF LIQUID CRYSTAL MATERIAL FOR MICROWAVE APPLICATIONS}

Juan R. Sánchez ${ }^{1}$, Vicente Nova ${ }^{2}$, Carmen Bachiller ${ }^{2}$, Belén Villacampa ${ }^{3}$, Alberto de la $\mathrm{Rua}^{4}$, Rainer Kronberger ${ }^{4}$, Felipe Peñaranda ${ }^{1}$, and Vicente E. Boria ${ }^{2}$

${ }^{1}$ ITACA, Universitat Politècnica de València, Camino de Vera s/n, Valencia, Spain.

${ }^{2}$ ITEAM, Universitat Politècnica de València, Camino de Vera $s / n$, Valencia, Spain.

${ }^{3}$ Dto de Fisica de la Materia Condensada. Universidad de Zaragoza. Zaragoza, Spain.

${ }^{4}$ High Frequency Laboratory. TH Köln,. Betzdorfer Str. 2, Koeln, Germany.

Email:jrsanchez@itaca.upv.es

Keywords: dielectric characterization, liquid crystal, microwave, split-cylinder resonator.

\section{Introduction}

The use of Liquid Crystal (LC) in telecommunications for developing reconfigurable microwave devices is spreading [1-4]. LCs anisotropic materials with properties between crystalline solids and liquids. Their rod-like molecules present nematic phase, having orientational order but no positional one. The long axis of the molecules has a preferred position, which can be changed by applying an external electric or magnetic field.

In the macroscopic scale, the direction of the molecules is represented by a vector called director $\vec{n}$, which is parallel to the longitudinal axis of the LC molecules. The dielectric permittivity values of the nematic LCs are defined by the angle formed between the director vector and an external RF electric field $\vec{E}$. The values represented by $\varepsilon_{r \perp}$ and $\varepsilon_{r \|}$ are, respectively, the permittivity values when $\vec{n}$ is perpendicular and parallel to $\vec{E}$ [5]. Moreover, loss tangents $\tan \delta_{\perp}$ and $\tan \delta_{||}$are also defined corresponding to those directions.

Different methods can be used for this characterization. On the one hand, transmission line methods developed at millimiter-wave and microwave frequencies [6-8]. On the other hand, resonant methods, which can obtain dielectric properties at single frequency points have been also developed [9-12]. However, all of them need to apply an electric or magnetic field for measuring both polarization of the LC molecules. In this paper, it is proposed an alternative for measuring parallel and perpendicular dielectric properties without any external electromagnetic field. It is based on the split-cylinder resonator method.

\section{Method description}

The split-cylinder resonator consists of two cylindrical halves, where the sample is placed between them. The empty resonator has a specific resonance frequency and quality factor (Q-factor), and when the sample is placed in the middle, these parameters change. The relative change between the resonator without and with sample allows to calculate the dielectric permittivity and the loss tangent of the material. In the case of a LC material, the samples are confined in a cell formed by two glasses and the LC is sited between them as shown in Fig.1. To form the cell, firstly an alignment surface must be created in the inner side of the each glass. This surface consists of microgrooves created by depositing a polyimide layer and afterwards rubbing with a natural velvet. This will provoke the LC molecules to be aligned in the microgrooves direction. Next, the glasses are adhered by using four separators in the corners and the LC is placed in the gap between them filling the cell by capillarity. 
This process allows to obtain a cell formed by three layers (two glasses and the LC sample material). For this reason, classical methods for characterization [13-14] have to be modified for extracting the dielectric properties of the LC material sample placed between the two glasses. The glasses are characterized following the classical methods, therefore for each cell, the properties of the glasses are known. Thus, the modal analysis method described [1517] is used, where the structure is divided in canonical circuital elements and the only unknown is the dielectric properties of the LC material.

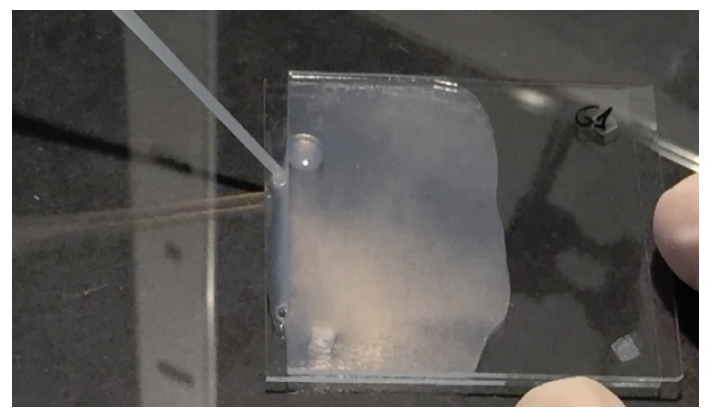

Fig. 1. Filling of a Liquid Crystal cell by capillarity.

The chosen working mode of the resonator is based on the field distribution. Mode $\mathrm{TE}_{111}$ has a quasi-straight field lines distribution, allowing to measure the two polarizations of the molecules of LC just rotating $90^{\circ}$ the cell inside the cavity without applying any external field, as shown in Fig. 2.

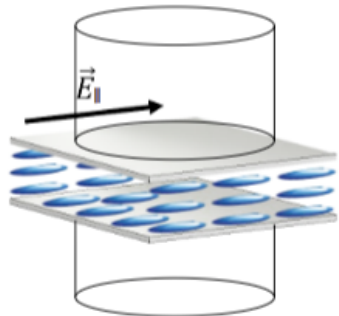

(a)



(c)

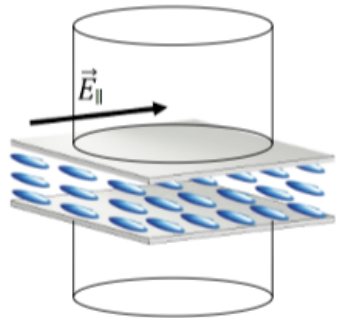

(b)

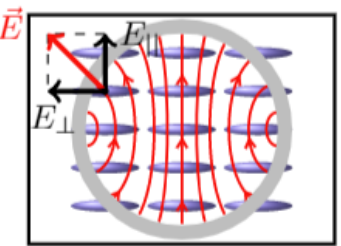

(d)

Fig. 2. (a) Placement of LC cell is Position 1. (b) Placement of LC cell is Position 2.

(c) E-field and LC molecules in Position 1. (d) E-field and LC molecules in Position 2. 
However, as shown in Fig. 1, the non-ideal straight field distribution, position 1 and 2 of the LC cell do not correspond to the parallel and perpendicular permittivity directly. The values can be extracted from the following equations:

$$
\begin{aligned}
& \varepsilon_{\text {rpos } 1} \iint|\vec{E}| d S=\varepsilon_{r \perp} \iint E_{\perp} d S+\varepsilon_{r||} \iint E_{||} d S \\
& \varepsilon_{\text {rpos } 2} \iint|\vec{E}| d S=\varepsilon_{r \|} \iint E_{\perp} d S+\varepsilon_{r \perp \mid} \iint E_{||} d S
\end{aligned}
$$

where:

- $\quad \varepsilon_{\text {rpos } 1}$ and $\varepsilon_{\text {rpos } 2}$ are the extracted complex permittivities with the cells in position 1 and 2.

- $\quad|\vec{E}|$ is the module of the electric field calculated in each point of the cell.

- $\quad E_{\|}$is the component of the electric field in parallel direction.

- $\quad E_{\perp}$ is the component of the electric field in the perpendicular direction.

\section{Results}

For performing the measurement a set-up composed of a VNA and a split-cylinder resonator is used, as shown in Figure 9. Four different nematic LCs have been prepared and measured by using different cells: QYPD036, QYPD142, and QYPD193 from Qingdao QY Liquid Crystal Co and GT3-23002 from Merck. The measurements have been carried out at two different frequencies $(5 \mathrm{GHz}$ and $11 \mathrm{GHz})$ at room temperature $\left(25^{\circ} \mathrm{C}\right)$.

The resonance frequency and the Q-factor for positions 1 and 2 have been measured for each LC cell. These measurements have been performed several times in order to extract an error value. With these values and following the method explained above, the complex dielectric permittivity values are obtained. Then, the transformation explained in (1) is applied to obtain the parallel and perpendicular dielectric permittivity and the loss tangent.

There are some error sources that affect the extraction of the complex permittivity: the features of the cylinder (radius, height, conductivity, and feeding loops), the measured EM response (resonance frequency and quality factor), the thickness of the glasses and the thickness of liquid crystal. The impact of the cylinder dimensions is calibrated and compensated with a first measurement of the empty cylinder. However, the tolerance in the measurement of the other parameters is translated into a set of uncertainties $\left(\sigma_{i}\right)$ and their error contribution to the complex permittivity results is quantified. Then, if the error contribution of these parameters is independent and known, the total uncertainty of the complex dielectric permittivity can be expressed as the quadrature sum [18] described in equation (2). Finally, the complex permittivity error can be easily propagated to obtain the loss tangent uncertainty.

$$
\sigma_{i}=\left(\sum\left|\frac{\delta \varepsilon}{\delta \text { error }}\right| \sigma_{\text {error }}^{2}\right)^{1 / 2}
$$

The results corresponding to $5 \mathrm{GHz}$ and $11 \mathrm{GHz}$ are presented in Tables 1 and 2, respectively. The highest value of anisotropy for the Qingdao LCs family, is 0.81 at $5 \mathrm{GHz}$ (QYPD193) and 0.98 at $11 \mathrm{GHz}$ (QYPD036). The highest values of the loss tangents are 
also recorded for these two materials. Therefore, choosing an LC for designing a reconfigurable microwave device must be a trade-off between the dielectric anisotropy and the related loss, depending on the required specifications.

Table 1. Dielectric properties of LCs at $5 \mathrm{GHz}$.

\begin{tabular}{ccccc}
\hline LC & $\varepsilon_{r \|}$ & $\tan _{\|} \mathrm{x} 1000$ & $\varepsilon_{r \perp}$ & $\tan _{\perp} \mathrm{x} 1000$ \\
\hline QYPD036 & $3.08 \pm 0.19$ & $17.06 \pm 2.08$ & $2.28 \pm 0.15$ & $75.45 \pm 7.89$ \\
QYPD142 & $2.72 \pm 0.17$ & $7.95 \pm 1.09$ & $2.27 \pm 0.16$ & $58.51 \pm 7.20$ \\
QYPD193 & $3.03 \pm 0.19$ & $7.15 \pm 0.97$ & $2.22 \pm 0.15$ & $91.90 \pm 7.55$ \\
GT3-23002 & $2.59 \pm 0.15$ & $5.13 \pm 1.79$ & $1.94 \pm 0.14$ & $39.33 \pm 10.90$ \\
\hline
\end{tabular}

Table 2. Dielectric properties of LCs at $11 \mathrm{GHz}$.

\begin{tabular}{ccccc}
\hline LC & $\varepsilon_{r \|}$ & $\tan _{\|} \times 1000$ & $\varepsilon_{r \perp}$ & $\tan \delta_{\perp} \times 1000$ \\
\hline QYPD036 & $3.22 \pm 0.19$ & $3.95 \pm 0.67$ & $2.24 \pm 0.15$ & $57.51 \pm 6.80$ \\
QYPD142 & $3.05 \pm 0.18$ & $5.80 \pm 1.06$ & $2.36 \pm 0.16$ & $40.88 \pm 4.92$ \\
QYPD193 & $3.26 \pm 0.20$ & $1.97 \pm 0.11$ & $2.40 \pm 0.17$ & $63.31 \pm 4.40$ \\
GT3-23002 & $3.25 \pm 0.21$ & $2.50 \pm 0.83$ & $2.35 \pm 0.17$ & $22.05 \pm 3.81$ \\
\hline
\end{tabular}

\section{Conclusions}

In this work, the split-cylinder resonator method has been used for the characterization of LCs at microwave frequencies. The method is based on the measurement of the resonance frequency and quality factor of the two states of the LC molecules. To achieve these two states, no electric or magnetic fields are needed, the cell must be turned $90^{\circ}$ inside the cavity. The dielectric properties (permittivity and loss tangent) of four different LC samples, GT323002 from Merck and QYPD193, QYPD142, and QYPD036 from Qingdao QY Liquid Crystal Co, have been obtained. The highest values of the dielectric anisotropy are presented for the samples QYPD036 and QYPD193, together with the highest values of the corresponding loss tangent parameters. Furthermore, it is observed for all the LCs that the loss tangent decreases and the dielectric anisotropy increases at higher frequencies. This must be taken into account in the development of reconfigurable microwave devices.

\section{References}

[1] M. Yazdanpanahi and D. Mirshekar-Syahkal, "Millimeter-wave liquid-crystal-based tunable bandpass filter," in 2012 IEEE Radio and Wireless Symp., Jan 2012, pp. 139142 .

[2] M. Jost, C. Weickhmann, T. Franke, A. E. Prasetiadi, W. Hu, M. Nickel,O. H. Karabey, and R. Jakoby, "Tunable hollow waveguide devices for space applications based on liquid crystal," in 2015 SBMO/IEEE MTT-S Int. Microw. and Optoelectronics Conf. (IMOC), Nov 2015, pp. 1-5.

[3] S. Strunck, O. H. Karabey, C. Weickhmann, A. Gaebler, and R. Jakoby, “Continuously tunable phase shifters for phased arrays based on liquid crystal technology," in 2013 IEEE Int. Symp. on Phased Array Systems and Technol., Oct 2013, pp. 82-88.

[4] K. Wang and $\mathrm{K}$. Wu, "Liquid crystal enabled substrate integrated waveguide variable phase shifter for millimeter-wave application at $60 \mathrm{GHz}$ and beyond," in2015 IEEE MTT-S Int. Microw. Symp., May 2015,pp. 1-4. 
[5] O. H. Karabey, Electronic Beam Steering and Polarization Agile Planar Antennas in Liquid Crystal Technology (Springer Theses).Springer, 2013.

[6] S. Mueller, A. Penirschke, C. Damm, P. Scheele, M. Wittek, C. Weil, and R. Jakoby, "Broad-band microwave characterization of liquid crystals using a temperaturecontrolled coaxial transmission line,'IEEE Trans.Microw. Theory Techn., vol. 53, no. 6, pp. 1937-1945, June 2005.

[7] S. Mueller, M. Koeberle, F. Goelden, A. Penirschke, A. Gaebler, A. La-panik, W. Haase, and R. Jakoby, "W-band characterization of anisotropic liquid crystals at room temperature," in2008 38th Eur. Microw. Conf., Oct 2008, pp. 119-122.

[8] S. Bulja, D. Mirshekar-Syahkal, R. James, S. E. Day, and F. A.Fernandez, "Measurement of dielectric properties of nematic liquid crystals at millimeter wavelength, "IEEE Trans. Microw. Theory and Techn., vol. 58, no. 12, pp. 3493-3501, Dec 2010.

[9] C. V. L.F. Chen, C.K. Ong and V. Varadan, "Chapter 5 - Resonator Methods," in Microwave Electronics: Measurement and Materials Characterization. Hoboken, UK: John Wiley \& Sons, Incorporated, 2004.

[10] A. Penirschke, S. Muller, P. Scheele, C. Weil, M. Wittek, C. Hock, and R. Jakoby, "Cavity perturbation method for characterization of liquid crystals up to $35 \mathrm{GHz}$," in34th Eur. Microw. Conf., 2004, vol. 2, Oct2004, pp. 545-548.

[11] R. Kowerdziej, J. Krupka, E. Nowinowski-Kruszelnicki, M. Olifierczuk, and J. Parka, "Microwave complex permittivity of voltage-tunable nematic liquid crystals measured in high resistivity silicon transducers," Applied Physics Letters, vol. 102, no. 10, p. 102904, 2013. [Online].Available: https://doi.org/10.1063/1.4795534

[12] R. Kowerdziej, J. Parka, J. Krupka, M. Olifierczuk, E. Nowinowski-Kruszelnicki, L. Jaroszewicz, and O. Chojnowska, "Dielectric properties of highly anisotropic nematic liquid crystals for tunable microwave components," Applied Physics Letters, vol. 103, no. 17, p. 172902, 2013. [Online]. Available: https://doi.org/10.1063/1.4826504

[13] G. Kent, "Nondestructive permittivity measurement of substrates," IEEE Trans. Instrum. Meas., vol. 45, no. 1, pp. 102-106, Feb 1996.

[14] M. D. Janezic and J. Baker-Jarvis, "Full-wave analysis of a split-cylinder resonator for nondestructive permittivity measurements,'IEEE Trans.Microw. Theory Techn., vol. 47, no. 10, pp. 2014-2020, Oct 1999.

[15] F. L. Penaranda-Foix and J. M. Catala-Civera, "Circuital analysis of cylindrical structures applied to the electromagnetic resolution of resonant cavities," in Passive Microwave Components and Antennas,V. Zhurbenko, Ed.Rijeka: IntechOpen, 2010, ch. 7. [Online].Available: https://doi.org/10.5772/9400

[16] F. L. Penaranda-Foix, M. D. Janezic, J. M. Catala-Civera, and A. J. Canos, "Fullwave analysis of dielectric-loaded cylindrical waveguides and cavities using a new four-port ring network, 'IEEE Trans. Microw. Theory Techn., vol. 60, no. 9, pp. 2730 2740, Sept 2012.

[17] D. Marques-Villarroya, F. L. Penaranda-Foix, B. Garcia-Banos, J. M.Catala-Civera, and J. D. Gutierrez-Cano, "Enhanced full-wave circuit analysis for modeling of a split cylinder resonator," IEEE Trans. Microw. Theory Techn., vol. 65, no. 4, pp. 11911202, April 2017.

[18] J. Taylor, An introduction to Error Analysis. Sausalto, CA, USA: University Science Books, 1997. 